\title{
REVIEW OF PARTICLE PROPERTIES
}

\section{Particle Data Group}

M. ROOS

Department of High Energy Physics, University of Helsinki, SF.001 70 Helsinki-17, Finland

F.C. PORTER

Department of Physics, California Institute of Technology, Pasadena, CA 91125, USA

M. AGUILAR-BENITEZ, L. MONTANET, Ch. WALCK

CERN, European Organization for Nuclear Research, CH-I2I1 Geneva 23, Switzerland

R.L. CRAWFORD

Department of Natural Philosophy, University of Glasgow, G12 $8 Q Q$ Glasgow, Scotland, UK

R.L. KELLY, A. RITTENBERG, T.G. TRIPPE, C.G. WOHL, G.P. YOST

Lawrence Berkeley Laboratory' ${ }^{1}$, University of California, Berkeley, CA 94720, USA

\section{T. SHIMADA}

Meiji Universiry, Kanda-Surugadai 1-1, Chiyoda-ku, Tokyo 101, Japan

\section{M.J. LOSTY}

Division of Physics, National Research Council, Ottawa, Ontario KIA OR6, Canada

\section{G.P. GOPAL}

Rutherford and Appleton Laboratories, Chilton, Didcot, Oxon $O X 110 Q X$, England

\section{R.E. HENDRICK}

Department of Pliysics. St. Bonaventure University, St. Bonaventure, NY 14778, USA

\section{R.E. SHROCK}

Department of Physics, State University of New York at Stony Brook, Stony Brook. Long Island, NY II794, USA

\section{R. FROSCH}

Swiss Institute for Nuclear Research, SIN, CH-5234 Villigen, Switzerland

\section{L.D. ROPER}

Department of Physics, Vinginia Polytechnic Institute and State University, Blacksburg, VA 2406I, USA

and

\section{B. ARMSTRONG}

(Technical Associate)

Lawrence Berkeley Laboratory 1 , University of Califormia, Berkeley, CA 94720, USA

1 The Berkeley Particle Data Group is supported by the Director, Office of Energy Research, Office of High Energy and Nuclear Physics, Division of High Energy Physics of the US Department of Energy under Contract No. DE-AC03-76SF00098 and by the US National Sciince Foundation. 
This review of the properties of leptons, mesons, and baryons is an updating of Review of Particle Properties, Particle Data Group [Rev. Mod. Phys. 52 (1980) No. 2, Part II]. Data are evaluated, listed, averaged, and summarized in tables. Numerous tables, figures, and formulae of interest to particle physicists are also included. A data booklet is available.

Table of Contents

I. Introduction, credits, consultants

1I. Selection of data

III. Nomenclature
A. Quantum numbers
B. Particle names

IV. Conventions and parameters for atrong interactions
A. Partial-wave amplitudes and resonance parameters

B. Sign conventions for resonance couplings

C. Types of partial-wave analyses

D. Production of resonances

V. Criteria for resonances

VI. Conventions and parametcrs for weak and electromagnetic decays
A. Muon-decay parameters
B. K-decay parameters
C. $n$-decay parameters
D. Baryon decay parameters

VII. Statistical Procedures

A. Unconstrained averaging

B. Constrained fits

Acknowledgments

References (for above sections)

Tables of particle properries

stable particles

Aöendum

Mesons

Baryons

Miscellaneous tables, figures and formulae

Physical and numerical constants (rev.)

Clebsch-Gordan coefficients, spherical harmonics and $d$ runctions

SU(3) isoscalar factors, $\mathrm{SU}(n)$ multiplicities, and properties of quarks (rev.)
Weak interactions of quarks and leptons (rev.) 26

Relativistic kinematics

Lorentz invariant phase space formulae 30

C.M. energy and momentum versus beam momentum 31

Probability and statistics $\quad 32$

Particle detectors, absorbers and ranges (rev.) 34

Electromagnetic relations $\quad 43$

Radioactivity and radiation protection $\quad 43$

Perjodic table of the elements (rev.) 44

Plots of cross sections and related quantities (rev.) 45

Dato cord listings

Illustrative key

Stable particles

Leptons

Mesons

Baryons

$x v$

$x v i i$

$x$ vii

$\mathrm{xix}$

$x x$

$\mathrm{xx}$

Mesons, $S=0$

$S= \pm 1$

charmed mesons

bottom (beauty) meson

51

54

54

61

90

102

122

166

175

176

Baryons, $S=0$

$S=+1$

177

232

235

$S=-2 \quad 275$

$S=-3 \quad 281$

bottom (beauty) baryon $\quad 282$

dibaryons

Appendix I. Test of $\Delta J=1 / 2$ sule for byperon decays Appendix II. SU(3) classitication of resonances

\section{Introduction, credits, consultants}

This review is an updating through December 1981 of our previous review of particle properties [Particle Data Group (1980)]. As in previous editions we have

attempted to make the text as complete and seif-contained as possible.

As usual, the results of our compilation are presented in two sections, the Tables of Particle Properties and the Data Card Listings. The Tables summarize 
the properties of only those particles whose existence is in our judgment experimentally well founded and which have a high probability of standing the test of time. This is a conservative judgment, and surely some genuine resonances are onitted, awaiting confirmation (see section $\mathrm{V}$ below).

The Data Card Listings give up-to-date information, with references, on all reported particles. whether considered weil established or not. The Listings also contain mini-reviews on questions of interest.

A history of the Particle Data Group, with a discussion of procedures and problems, has been given by Rosenfeld (1975), and a short survey of the history of some of the constants we compile may be found in Appendix III.

As in previous editions, we include a section of miscellaneous tables, figures, and formulae. These are aimed at the practicing high energy physics experimentalist. We welcome all suggestions and comments regarding topics for deletion or inclusion. etc. This year we have revised many of these items, but no new ones have been added.

A pockei-sized Particle Properties Data Jooklet, containing the Tables and a reprint of the figures and formulae from the first part of the review, is available on request. For North and South America. Australia, and the Far East , write to Technical Information Department, Lawrence Berkeley Laboratory, Berkeley, CA 94720, USA. For all other areas, write to CERN Scientific Information Service. CH-1211 Geneva 23. Switzerland.

As usual, we wish to emphasize that we compile the experimental results of others. It is inappropriate to give us the credit for their countless hours of effort. We urge that references be given directly to the original data, and we provide complete references in the Data Card Listings for that purpose.

The responsibilities for the various sections can be broken down as follows:

(1) Stable particles: R. Frosch, T. Shimada, R.E. Shrock. T.G. Trippe, C.G. Wohl, and G.P. Yost.

(2) Meson resonances: M. Aguilar-Benitez, M.J. Losty, L. Montanet, F.C. Porter, M. Roos, and Ch. Walck.

(3) Baryon resonances: R.L. Crawford, G.P. Gopal. R.E. Hendrick, R.L. Kelly, M.J. Losty, L.D. Roper, and C.G. Wohl.

(4) General, including sext: All authors.
Consultants: To overcome gaps in our coverage, both intellectual and geographical, we have solicited the help of consultants:

R.A. Arndt (Virginia Polytechnic Institute and State University).

S. Aronson (BNL),

W.B. Atwood (SLAC),

C. Baltay (Columbia University),

A. Barbaro-Gaitieri (LBL),

B. Barish (California Institute of Technology),

A.V. Barnes (LBL),

D. Besset (Stanford University),

C. Bricman (CERN),

R. Cahn (LBL),

M.S. Chanowitz (LBL),

J.M. Dorfan (SLAC).

J. Engler (DESY).

G. Felỏman (SLAC),

V. Flaminio (University of Pisa),

F. Foster (University of Lancaster).

M.K. Gaillard (LBL),

G. Goldhaber (LBL),

M. Goldhaber (BNL),

Y. Goldschmidt-Clermont (CERN),

R. Hagstrom (Argonne National Laboratory),

K. Hashimoto (Virginia Polytechnic Institute and State University),

J.H. Hubbell (US National Bureau of Standards),

D.A. Jensen (University of Massachusetts at Amherst),

J. Learned (University of Hawaii),

G.M. Lewis (University of Glasgow),

W.G. Moorhead (CERN),

D.R.O. Morrison (CERN),

P. Némethy (LBL),

P. Oddone (LBL).

O.E. Overseth (University of Michigan),

S.I. Parker (University of Hawaii),

M. Perl (SLAC),

D.N. Schramm (University of Chicago),

M. Shaevitz (Nevis Laboratory).

R.I. Steinberg (University of Pennsylvania),

B.N. Taylor (US National Bureau of Standards),

J.A. Thompson (University of Pittsburgh).

N.A. Törnqvist (University of Helsinki),

G.H. Trilling (LBL).

R.D. Tripp (LBL).

W.P. Trower (Virginia Polytechnic Institute and State University). 
The usefulness of this compilation depends in large part on the interaction between the users and the authors and consultants. We appreciate comments, criticisins, and suggestions for improvements of all stages of data retrieval, processing, evaluation, and presentation.

\section{Il. Selection of data}

All particles are considered to fall into one of the three groups:

(1) Stable particles, defined to be those imınune to decay via the strong interaction, including the photon, the leptons, the $\eta$, the $D$ and $F$ charmed mesons, the $\Lambda_{c}$ charmed baryon, etc.

(2) Meson resonances, including the $\psi$. the $\chi$, and the $\Upsilon$ particles.

(3) Baryon resonances.

These groups are maintained within the two main parts of the compilation:

(1) Tables of Particle Properties.

(2) Data Card Listings.

The Data Card Listings contain the original information (data, references, etc.), weighted averages. comments, and "mini-reviews". Immediately preceding the Data Card Listings is an illustrative key thereto. We attempt to give complete Data Card Listings up to our closing date (January 1, 1982) for all journals listed in the Illustrative Key. As a general rule, we do not include results from preprints or unpublished conference reports. Exceptions to this rule are made on a case-by-case basis.

Many of our encoded results, those set off in parentheses, are not used for averaging. The reasoning is then often given in a footnote below the data. If the reason is not gjven, it is one of the following:

The result was presented with no error stated.

The result comes from a preprint or conference seport. It is our experience that such results (and particularly the errors) often change before final publication. Accordingly we keep these new results in parentheses until they are published (or explicitly verified to us by the authors).

It involves some assumptions that we do not wish to : zcorporate.

It is of poor quality, e.g. bad signal-to-noise ratio.

It is inconsistent with other results, e.g. because of different methods employed, rendering averaging meaningless.

It is not independent of other results, e.g. it is a result from one of several partial-wave analyses all using the same data, again rendering averaging meaningless.

Upper limits are not averaged (except in rare cases which are re-expressible as numbers with gaussian errors).

When the data for a particle have received special treatment or present special problems, this is noted in a mini-review in the Data Card Listings.

As time goes by, some early results lose all their weight in the averages. We may ther: remove them from the Listings without further comment. We usually do not remove the corresponding reference cards, however, so that our reference sections preserve the historical record. In this edition the meson section has undergone an extensive "house-cleaning". As a result it appears more readable (or so we hope). The earlier data may be found in the 1980 edition).

The Tables of Particie Properties contain "best" values obtained from the data in the Data Card Listings by various methods. The statistical procedures of section VIl are used to combine independent data which have gaussian errors. Upper linnits in the Tables usually represent the strongest limit available from a single experiment. The extent to which these methods are tempered by critical judgruent is explained in the footnotes to the Tables. In general, however, the footnotes are less complete than is the collection of notes and minireviews in the Data Card Listings. The reader is thus encouraged to become familiar with the Data Card Listings and, ultimately, with the original references.

\section{Nomenclature}

\section{A. Quantum numbers}

The symbois $I^{G}\left(J^{P}\right) C_{\mathrm{n}}$ represent:

$I=$ isospin,

$G=G$ parity,

$J=$ spin (also s),

$P$ = space parity,

$C_{\mathrm{n}}=$ charge-conjugation parity for the neutral member of the isospin multiplet.

We also use:

$B$ = baryon number, 
$S=$ strangeness,

$C=$ charm,

$l=$ orbital angular momentum.

1. Mesons. The charge-conjugation operator $\mathrm{C}$ turns particle into antiparticle and has eigenvalues \pm 1 only for neutral states; so it is useful to define an operator G which has eigenvalues for charged states too. This is usually ${ }^{\ddagger 1}$ defined by

$\mathbf{G}=\mathbf{C} \exp \left(\mathrm{i} \pi I_{y}\right)$.

A neutral nonstrange, noncharmed state is an eigenstate of exp(i $\left.\pi I_{y}\right)$ with eigenvalue $(-1)^{I}$. Then we can write the eigenvalue equation for the whole multiplet as

$G=C_{\mathrm{n}}(-1)^{I}$.

where $C_{n}$ ( $n$ for neutral) is the eigenvalue $C$ would have if applied to the neutral member of the multiplet. Thus, for a $\pi^{0}, \mathrm{C}$ has the eigenvalue +1 , and since $I$ $=1, G=-1$. For a charged $\left.p^{i}\right\urcorner$, there are no eigenvalues corresponding to $\mathrm{C}$ and to the isospin rotation, but eqs. (1) and (2) still give $G=-1$.

Consider a meson as a bound state of fermionantifermion, e.g. quark-antiquark $\bar{q} q$, with orbital angular momentum $l$, and with the two fermion spins coupling to give a spin $s$. Then one can show that the charge-conjugation eigenvalue [defined as in eq. (2)] is

$C_{\mathrm{n}}=(-1)^{l+s}$.

Eqs. (2) and (3) combine to give

$G=(-1)^{l+s+I}$.

The parity is

$P=-(-1)^{l}$.

Eqs. (3) and (5) combine to give

$C_{\mathrm{n}} P=-(-1)^{S}$,

so all singlets $\left({ }^{1} \mathrm{~S}_{0},{ }^{1} \mathrm{P}_{1}, \ldots\right)$ have $C_{\mathrm{n}} P=-1$. and al] triplets $\left({ }^{3} \mathrm{~S}_{1}, \ldots\right)$ have $C_{\mathrm{n}} P=+1$. For proofs of the above, see our 1969 text [Particle Data Group (1969)]

11 Most texts define it as in eq. (1); see e.g. Gasiorowicz (1966); however, sometines the rotation is taken about $I_{x}$. The difference between the two conventions is mentioned in a footnote in Källen (1964). and Appendix by C. Zemach.

If. instead of $\bar{q} q$. we consider the meson as a state of boson-antiboson (e.g. $\mathrm{A}_{2} \rightarrow \overline{\mathrm{K} K}$ ), it turns out that some signs cancel, and eqs. (3) and (4) [not eq. (5)!] apply unchanged. Of course, the mesons are often spinless, so $s$ is zero, but the equations are more general. Eqs. (3) and (4) can be considered as selection rules forbidding many decays.

We now use eqs. (3) and (4) to introduce the concept of "Abnormal- $C_{n}$ " mesons, i.e. mesons that cannot be composed of qq. For this, it is sufficient to consider the SU(3) subgroup of the full unitary group of flavors, containing the $u, d$, and s quarks in a $\{3\}$ representation.

This triplet of quarks is of course defined to have isospin and hypercharge properties such that $\bar{q} q \mathrm{can}$ combine (according to the SU(3) relations $\{3\} \otimes\{3\}$ $=\{8\} \oplus\{1\})$ so as to form only octets and singlets. The non-observation of "exotic" mesons (i.e., mesons in larger $S U(3)$ representations, or mesons requining at least a q $\bar{q} q \bar{q}$ structurc) is of course a direct consequence of the naive quark model. It is less obvious that even some octets are forbidden by the model. namely those with $\left(J^{P}\right) C_{n}=\left(0^{*}\right)-.\left(1^{-}\right)+,\left(2^{+}\right) \cdots, \ldots$ Such states are not observed. and this is an additional success of the naive quark model classification scheme.

When the naive quark model is extended to QCD, one expects gg gluonium mesons also. Since the gluon $\mathrm{g}$ is a flavor singlet. all gluonium states nust be flavor singlets which can be expected to mix with nearby $\bar{q} q$ singlets. Nu gluonium states have been definitely established yet.

In what follows. do not confuse "Abnormal $\cdot C_{\mathrm{n}}$ " with "Normal" or "Abnormal" $J^{P}$, both of which are allowed by the quark model. The series $J^{P}=0^{+} .1^{-}$, $2^{+}, \ldots$ is called Normal because $P=(-1)^{J}$ as for normal spherical harmonics, and $J^{P}=0^{-}, 1^{+}, \ldots$ is called Abnormal.

The top part of table 1 shows all the low angular momentum states that can be formed from qq. Note that half of the $J^{P}$ states can be formed by both a triplet and a singlet $\bar{q} q$ state. e.g. ${ }^{3} \mathrm{P}_{1},{ }^{1} \mathrm{P}_{1}$, or ${ }^{3} \mathrm{D}_{2}$, ${ }^{1} \mathrm{D}_{2}$, in spectroscopic notation $\left[{ }^{(2 s+1)} l_{f}\right]$. Fq. (3) shows that ${ }^{3} \mathrm{P}_{1}$ and ${ }^{1} \mathrm{P}_{1}$ have opposite $C_{\mathrm{n}}$, so the $\overline{\mathrm{q}} \mathrm{q}$ model allows both. But the states ${ }^{3} \mathrm{P}_{0}$ and ${ }^{3} \mathrm{P}_{2}$ have no $' P$ counterparts. According to eq. (6) they have $C_{\mathrm{n}} P=+1$, and with the $\overline{\mathrm{q} q}$ model there is no way to form a state with a $J^{P}$ of ${ }^{3} \mathrm{P}_{0.2}$ (i.e. $J^{P}=$ Normal) and 
Tabie I

Orbital excitations of the $\bar{q} q$ system, and corresponding mesons. For the distinction between $A$ brormal $J^{P}$ and Abnormal $C_{n}$, see text following eq. (6) in section III. Strange and charmed mesons share the same values of $J^{P}$ as the $J=0$ and 1 states shown, but are not eigenstates of $\mathrm{G}$. The second column, which gathers tcaether $\left(J^{P}\right) \mathrm{N}$ or $\mathrm{A} C_{\mathrm{n}} P$, is a redundant intermediate step intended to make the table easier to rcad. The table repeats itself for each radial excitation.

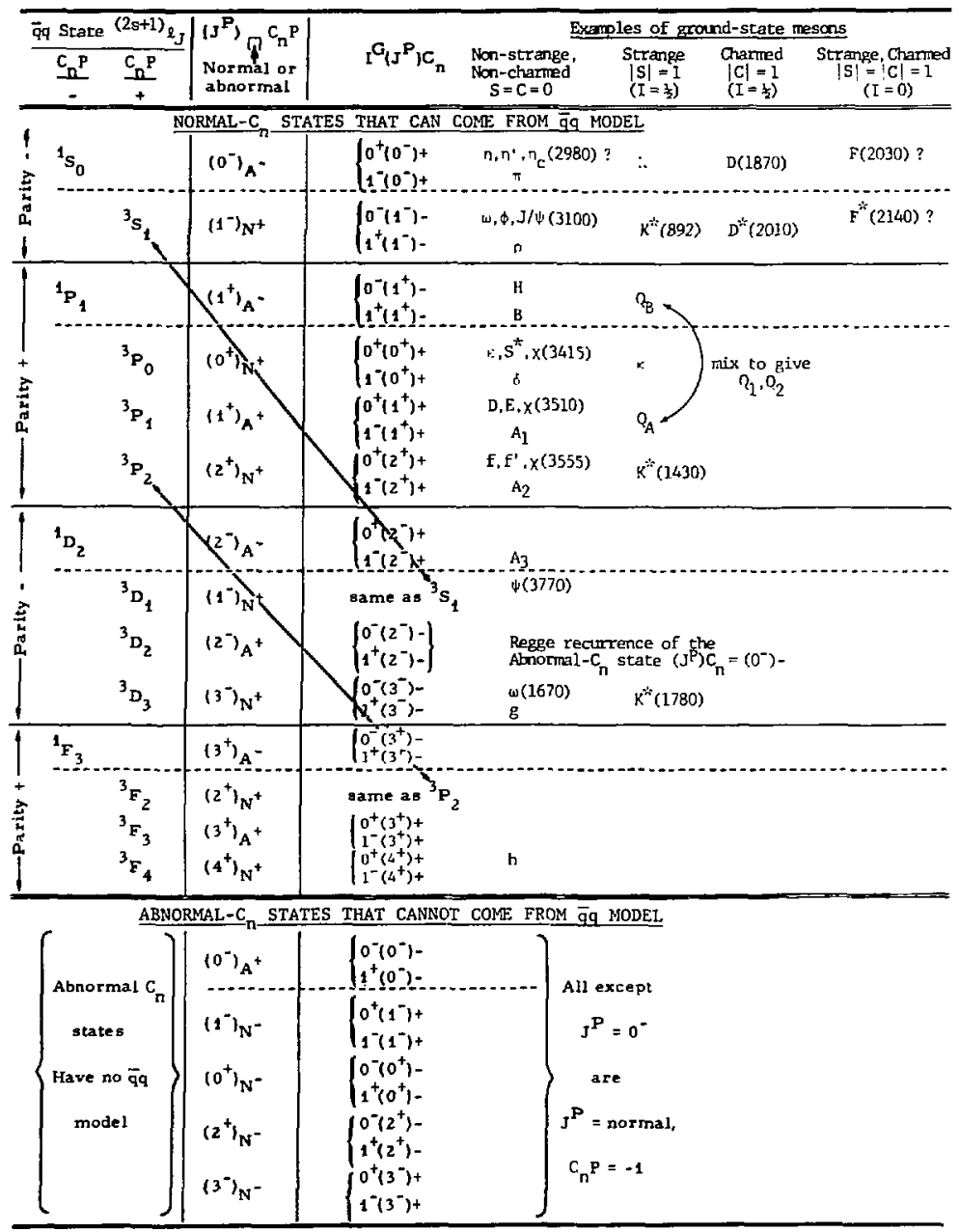


with $C_{\mathrm{n}} P=-1$. As mentioned, such octets have not been found. With the help of table 1 one can also see that the special state ${ }^{1} \mathrm{~S}_{0}, C_{\mathrm{n}} P=+1$, cannot be formed, so has Abnormal $C_{\mathrm{n}}$.

When, in addition to the $l$-excitation, there are radial excitations of the $\vec{q} q$ system, table 1 repeats itself, and we need a radial quantum number $n$ for each repetition ( $n=1$ for the ground state). Examples of first radial excitations, $n=2$, are $\rho^{\prime}(1600), \psi(3685)$, and $\Upsilon(10020)$. Examples of further possible radial excitations can be found in the $\psi$ and $\Upsilon$ families.

2. General remarks. Well-established quantum numbers are underlined in the Tables of Particle Properties (except for stable particles, where most of the quantum numbers are established). We have used what evidence is available (sometimes flimsy) to guess many of the remaining ones, and we have indicated with "?" ones (in the Baryon Table) for which there is almost no evidence.

As is customary, we define antiparticles as the result of operating with CPT on particles, so both share the same spins, masses, and mean lives. Whenever there is a particularly interesting test of $C P T$ invariance we include it in the Stable Particles Table.

\section{B. Particle names}

If a meson has a well-accepted colloquial name, we use it. If not, we name it by a single symbol which specifies its $J^{P}$."Normality", its isospin $I$, its strangeness $S$ and charm $C$, and, for a non-st range, noncharmed meson, its $G$ parity.

The name conventions for mesons are given in the first part of table 2.

For some pairs of mesons with supposedly identical quantum numbers, we also use primes; e.g. $\eta, \eta^{\prime} ; f, f^{\prime}$; $\rho, \rho^{\prime}$. Note that primes and subscripts do not carry any further specific meaning.

For baryons no attempt has been made to attach a subscript about $J$ and $P$. The name conventions are given in the second part of table 2 . For stable bary. ons of each $I$ and $S$ we use the symbol standing alone; for resonances, the mass is in parentheses [i.e. $\mathrm{N}(1680)$, $\Lambda(1405), \Sigma(1775)$, etc.]. The $J^{P}$ assignments are reported in the Baryon Table as $\frac{1}{2}+, \frac{3}{2}-, \frac{5}{2}+$, etc., and also by the symbols $P_{11}, D_{13}, F_{15}$, which refer to the $\pi \mathrm{p}$ or $\mathrm{Kp}$ partial-wave amplitude in which the resonant state occurs (the first subscript refers to the isospin
Table 2

Particle name conventions.

\begin{tabular}{|c|c|c|c|c|}
\hline Name & $I$ & $\boldsymbol{s}$ & $c$ & $G$ \\
\hline \multicolumn{5}{|l|}{$\begin{array}{l}\text { Mesons, } \\
J^{P} \text { "Normal" }\end{array}$} \\
\hline$\omega, \phi, \psi, \Upsilon a)$ & 0 & 0 & 0 & - \\
\hline $\mathbf{E}$ & 0 & 0 & 0 & + \\
\hline$\delta$ & 1 & 0 & 0 & - \\
\hline$p$ & 1 & 0 & 0 & + \\
\hline $\mathrm{K}^{*}, \kappa$ & $1 / 2$ & \pm 1 & 0 & \\
\hline$D^{*}$ & $1 / 2$ & 0 & \pm 1 & \\
\hline $\mathrm{F}^{*}$ & 0 & \pm 1 & \pm 1 & \\
\hline \multicolumn{5}{|c|}{$\begin{array}{l}\text { Mesons, } \\
J^{P} \text { "Abnormal" }\end{array}$} \\
\hline$n$ & $\mathbf{0}$ & 0 & 0 & + \\
\hline$\pi$ & 1 & 0 & 0 & - \\
\hline $\mathbf{K}, \mathbf{Q}$ & $1 / 2$ & \pm 1 & 0 & \\
\hline D & $1 / 2$ & 0 & \pm 1 & \\
\hline $\mathbf{F}$ & 0 & \pm 1 & \pm 1 & \\
\hline \multicolumn{5}{|l|}{ Baryons } \\
\hline $\mathrm{N}$ & $1 / 2$ & 0 & 0 & \\
\hline$\Delta$ & $3 / 2$ & 0 & 0 & \\
\hline $\mathrm{Z}_{\mathrm{I}}$ & 0,1 & +1 & 0 & \\
\hline$\Lambda$ & 0 & -1 & 0 & \\
\hline$\Sigma$ & 1 & -1 & 0 & \\
\hline$\Xi$ & $1 / 2$ & -2 & 0 & \\
\hline$\Omega$ & 0 & -3 & 0 & \\
\hline$\Lambda_{\mathfrak{c}}$ & 0 & 0 & 1 & \\
\hline$\Sigma_{\mathrm{c}}$ & 1 & 0 & 1 & \\
\hline
\end{tabular}

a) We use the symbol $\omega$ for those $I^{G}=0^{-}$mesons which are mainiy $u \bar{u}$ and d d quark states; $\phi$ for those which are mainly $\bar{s}$ quark states, $\psi$ for mainly ce states, and $\gamma$ for mainly b̄ states.

state: $2 \times I$ for $\mathrm{N}, \Delta$, and $\Xi$, and just $I$ for $Z, \Lambda$, and $\Sigma)$. When two or more baryons have identical quantum numbers we warn the reader by adding primes to the spectroscopic symbol as explained in footnote (a) of the Baryon Table.

\section{Conventions and parameters for strong interactions}

\section{A. Partial-wave amplitudes and resonance parameters}

The vast majority of information concerning baryon resonances comes in the form of partial-wave analyses. In addition data concerning meson resonances ( $\pi \pi$, $\mathrm{K} \pi, \pi \pi \pi$ ) are, with increasing frequency, being subjected to partial-wave analyses. We thus find it natural to introduce the resonance parameters which we com- 
pile in terms of a Brejt-Wigner approximation for the partial-wave amplitude.

In general the elastic amplitude for a given angular momentum $l$ may be written as

$T_{11}=[\eta \exp (2 \mathrm{i} \delta)-1] / 2 \mathrm{j}$,

where $\eta$ is the absorption parameter $(0 \leqslant \eta \leqslant 1)$ and $\delta$ is the phase shift. The subscripts 11 on $T$ denote scattering from channel 1 to channel 1 (e.g. $\pi \pi \rightarrow \pi \pi$ or $\overline{\mathrm{K}} \mathrm{K} \rightarrow \overline{\mathrm{K}} \mathrm{K}$ ).

In fig. 1 we show an Argand plot of the elastic partial wave amplitude $T_{11}$. It illustrates geometrically how the real parameters $\eta$ and $\delta$ are related ts the real and imaginary parts of $T_{11}$. Many examples of such Argand plots may be found in the Baryon Data Card Listings.

Consider the so-called non-relativistic Breit-Wigner approximation for $T_{11}$ :

$T_{11}=\frac{1}{2} \Gamma_{1} /\left(M-E-\frac{1}{2} \mathrm{i} \Gamma\right)$,

where $E$ is the c.m. energy or invariant mass, $\Gamma_{1}$ and $\Gamma$ are the elastic and total widths, and $M$ is the resomance mass. Eq. (2) is, of course, not the only possible description of a resonant amplitude; but it suffices to illustrate the properties of partial-wave amplitudes which we associate with resonance behavior in the absence of any background in the same partial wave (see, e.g., the $\pi \mathrm{N} D_{15}$ and $F_{15}$ waves in the Baryon Data Card Listings). Usually the widths contain barrier-penetration factors which can vary rapidly with energy. Near threshold, $\Gamma_{1}(E)$ should start up as $q^{2 l+1}$

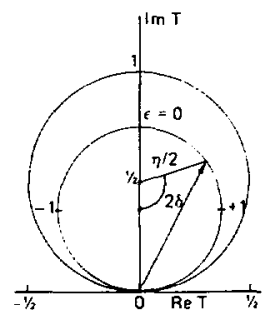

(a)

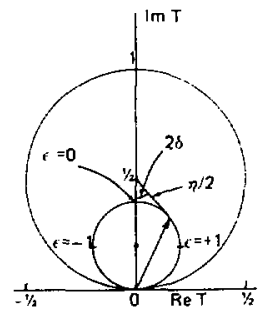

(b)
Fig. 1. Argand plots for the elastic pastial wave amplitude $T_{11}$. The outer eircles are the unitarity bound $(\eta=1)$. The inner circles correspond to the Brejt-Wigner approximation of eq. (2) for (a) $x_{1}=\Gamma_{1} / \Gamma=0.75$ and (b) $x_{1}=0.4$. Note: $\epsilon=2(M-E) / \Gamma$. (also true for the inelastic width $\Gamma_{\beta}$ ). Various $E$ dependences are then used for $\Gamma_{1}$, mostly of the form

$\Gamma_{1}(E) \propto(q R)^{2 l+1} /\left[\right.$ const $\left.+\ldots+(q R)^{2 l}\right]$;

see Jackson (1964), Pisút and Roos (1968), and

Barbaro-Galtieri (1968).

The BW approximation to the amplitude for an in. elastic process leading from channel 1 to channel $\beta$ $(\pi \pi \rightarrow \overline{\mathrm{K} K}$ or $\overline{\mathrm{K}} N \rightarrow \Sigma \pi$, for example) is

$$
\begin{aligned}
T_{\mathrm{I} \beta} & =\frac{1}{2}\left(\Gamma_{1} \Gamma_{\beta}\right)^{1 / 2} /\left(M-E-\frac{1}{2} \mathrm{i} \Gamma\right) \\
& =\left(x_{1} x_{\beta}\right)^{1 / 2}\left[\frac{1}{2} \Gamma /\left(M-E-\frac{1}{2} \mathrm{i} \Gamma\right)\right],
\end{aligned}
$$

where

$\Gamma=\sum_{1}^{N} \Gamma_{\beta}, \quad x_{\beta}=\Gamma_{\beta} / \Gamma$

and $x_{1}$ (called the elasticity) is often written $x_{\mathrm{L}}$. (Note that in the Data Card Listings we use the symbol $P_{\beta}$ to denote $x_{\beta}$.) The channel cross section $\sigma_{1 \beta}$ for the reaction $1 \rightarrow \beta$, for spin 0 -spin $1 / 2$ scattering, is

$\sigma_{1 \beta}=4 \pi \lambda^{2}\left(J+\frac{l}{2}\right)\left|T_{1 \beta}\right|^{2}$,

where $J=l \pm \frac{1}{2}$.

The inportant features of eq. (4) which characterize resonant behavior in the Argand diagram $\left(\operatorname{Im} T_{1 \beta}\right.$ versus $\operatorname{Re} T_{1 \beta}$ ) are:

energy variation given by circles with diameter $\left(x_{1} x_{\beta}\right)^{1 / 2}$ and maximum amplitude at $E=M$ of

$T_{1 \beta}^{\operatorname{mix}}=\mathrm{i}\left(x_{1} x_{\beta}\right)^{1 / 2}$

a maximuin in the speed near resonance. given approximately by

"Speed" $($ res $)=\left|\mathrm{d} T_{1 \beta} / \mathrm{d} E\right|_{E=M}=2\left(x_{1} x_{\beta}\right)^{1 / 2} / \Gamma(E)$,

for slowly varying $\Gamma(E)$. These features may be related to the $\eta, \delta$ representation of $T_{11}$. Thus when $E=M, \delta$ is either $90^{\circ}\left(x_{1}>\frac{1}{2}\right)$ or $0^{\circ}\left(x_{1}<\frac{1}{2}\right)$ and $\eta$ dips to its ninimun value.

These simple properties can be used to judge the presence or absence of resonance behavior in an Argand plot, but do not necessarily constitute the criteria we use (see section V). It must also be kept in mind that eqs. (2) and (4) are only approximations to the "true" amplitude. The simple picture given above can be distorted by yarious effects: 
the presence of "background" in the same partial wave as the resonance,

two resonances in the same partial wave overlapping in energy,

the resonant energy $M$ being close to an inelastic channel threshold, in which case a $K$-matrix-like parametrization is more appropriate,

the speed of the resonance being very slow so that the resonance is very broad, and the Breit-Wigner formula a bad approximation.

\section{B. Sign conventions for resonance couplings}

Consider the partial width $\Gamma_{\beta}$ of a resonance decaying into the channel $\beta$. We can always define a coupling constant such that

$\Gamma_{\beta} \propto G_{\beta}^{2}$.

In this case the inelastic amplitude in the BreitWigner approximation, eq. (4), will go as

$T_{1 \beta} \propto G_{1} G_{\beta} /\left(M-E-\frac{1}{2} \mathrm{i} \Gamma\right)$,

where $G_{1}$ is the coupling constant for the elastic channel. In the context of exact SU(3) symmetry the relative signs of the product $G_{1} G_{\beta}$ for different resonances are of ten useful as a consistency check on SU(3) assignment of baryon resonances. See Appendix I for further details.

In the Data Card Listings for baryon resonances, we tabulate measuted values for $\left(x_{1} x_{\beta}\right)^{1 / 2} \propto G_{1} G_{\beta}$. When the sign of the amplitude is determined, it is given; $a b$ sence of an explicit sign indicates that it is undetermined (not that it is positive). For $\Lambda$ and $\Sigma$ resonances, the signs are chosen according to the convention advocated by Levi-Setti (1969) and used in the table of SU(3) Isoscalar Factors presented in this review. Thus the signs multiplying the Breit-Wigner amplitudes for $\overline{\mathrm{K}} \mathrm{N} \rightarrow \Sigma(1385) \rightarrow \Sigma \pi, \Lambda \pi$ and $\overline{\mathrm{K} N} \rightarrow \Lambda(1405) \rightarrow \Sigma \pi$ are simply the product of the phases of the appiopriate isoscalar factors. This convention is shown in fig. 2 , adapted from Levi-Setti (1969).

\section{Types of partial-wave analyses}

Partial-wave analyses (PWA) are classified into three categories in the Data Card Listings: energy-independent partial-wave analyses (IPWA). energy-dependent partial-wave analyses (DPWA), and model-dependent partial-wave analyses (MPWA), in increasing order of the number of explicit supplementary hypotheses that are used to extract the amplitudes from experimental data.

\section{SU(3) RELATIVE SIGN OF RESONANT AMPLITUDES}

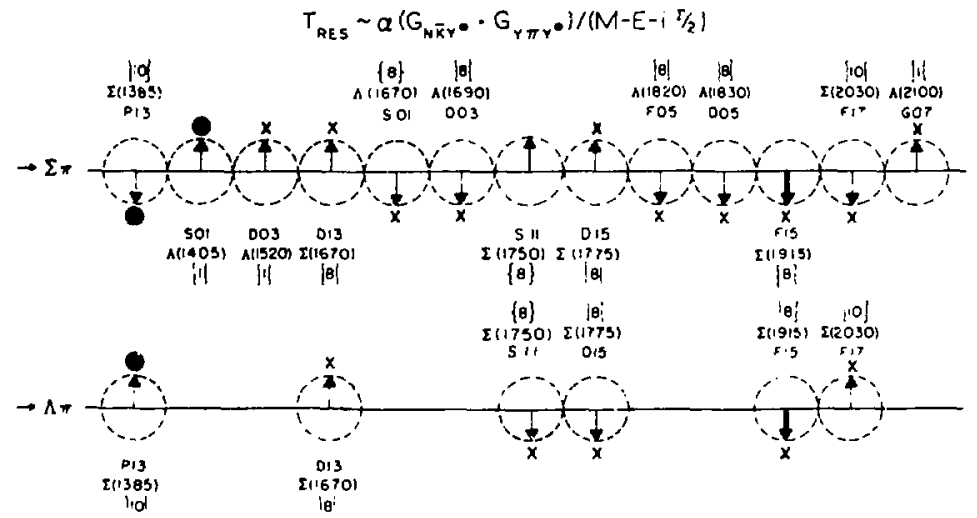

Fig. 2. P!ot adapted from Levi-Setti (1969) showing the sign convention adopted here for the $\Sigma \pi$ and $\Lambda \pi$ amplitudes. Once the signs of one $I=0$ and one $f=1$ amplitude are fixed, the others can be measured relative to these two. Arrows here indicate signs predicted by SU(3); $x$ marks indicatc the observed phases; $\bullet$ indicates phase chosen according to sign convention described in text. The $\Sigma(1915)$ predictions have been changed from Levi-Setti's original figure. 
In an IPWA, data at different energies are analyzed separately. Usually each partial wave included in the fit is allowed to vary freely (subject to unitarity constraints) over some large region, and waves whose angular momenta are above some cutoff value are assumed to be negligible. The sharp cutoff in angular momentum resolves continuum ambiguities in the solution (such as the overall phase ambiguity), but there remains a finite number of indistinguishable "best" solutions (i.e., solutions corresponding to identical physical observables) which have been codified by Barrelet (1972). In addition, there are generally some nearby solutions (and their associated Barrelet ambiguities) which have chi-squared values close to the minimum one.

At the end of the analysis a choice is made among these many solutions, usually on the basis of energy continuity. A popular criterion for making this choice is the shortest path $t$, inique in which the total "length" of the preferred solution is chosen to be a minimum. The definition of "length" used here is not universal but is usually closely related to the total geometrical length of the lines representing the various partial-wave amplitudes in Argand plots (see the baryon section of the Data Card Listings for examples of Argand plots). Various other criteria which are also used in some analyses are, e.g., matching with known solutions at low energies, the presence of known reso. nances in the final results, and limited inelasticity in high partial waves.

In a DPWA, data at different energies are fit simultaneously by using an energy-dependent parametrization of the partisl-wave amplitudes. The parametrization is usually chosen to include both resonances and nonresonant background of some sort and an attempt is made to keep it as "model independent" as possible. Often the data are grouped into several energy bins which are fit separately rather than trying to fit the whole energy range under consideration simultaneously. One of the main advantages of DPWA over IPWA is that sparse data spread over many different energies can be analyzid, e.g.. nearly all $S=-1$ analyses are DPWA. In addition, the built-in energy continuity helps to resolve the ambiguities that plague IPWA and eases the problems associated with resonance parameter extraction. The price one pays for these advantages lies in the danger of systematic error in the am. plitudes and poor fits to the data if the parametrization is poorly chosen or insufficiently flexible.

An MPWA also uses an energy-dependent parametrization, but one based on explicit mode-dependent theoretical assumptions such as Regge exchanges. This technique is usually applied to reactions where the data are incomplete. There is, of course, no sharp distinction between DPWA and MPWA, and a well chosen MPWA parametrization may actually be less biased than a model-independent but poorly chosen DPWA parametrization.

\section{Production of resonances}

Hereby, we mean the cbservation of statistically significant peaks in invariant mass plots or, loosely, in integrated cross sections. Many meson resonances are of this type. We expect most of these peaks to be associated with Breit-Wigner behavior in appropriate Argand plots; thus the $\rho$ meson peak in $\pi \pi$ mass plots is firmly related to the $l=1, l=1 \pi \pi$ phase shift passing through $90^{\circ}$. The shape of the $\rho$ meson is reasonably well described by the relativistic Breit-Wigner formula with the three purameters $M, \Gamma(M)$. and $R$ of eq. (3).

From mass plots we can determine $M . \Gamma$. and the approximate branching ratios

$x_{\alpha} / x_{\beta}=\Gamma_{\alpha} / \Gamma_{\beta}$.

In the case of total cross sections, the peak above background gives us, using the optical :heorem. the product $\left(J+\frac{1}{2}\right) x_{\mathrm{c}}$ :

$\sigma^{101}(E=M)=4 \pi \lambda^{2}\left(J+\frac{1}{2}\right) x_{\mathrm{c}}$.

\section{Criteria for resonances}

An experimentalist who sees indications of a resanance in some energy (or mass) region will of course want to know what has been seen in that region in the past; hence, we strive to have the Data Card Listings serve as an archive for all substantial claims for resonances. (However, we do not intend to preserve a perennial record of claims proved to be wrong. Some such claims have been removed from the Meson Lis. tungs in 198... We refer the interested reader to our 1980 edition for our complete archives.)

For the Tables of Particle Properties. on the other hand, we wish to be more conservative than for the 
Listings and to include only those peaks or resonances which we feel have a large chance of survival. An arrow $(\rightarrow)$ at the left of the Tables of Particle Properties indicates that a questionable candidate has been omitted from the Table, but that it can be found in the corresponding part of the Data Card Listings. One's betting odds for survival are of course subjective; therefore no precise criteria can be defined. Very slow speeds ( $\epsilon$ and $\kappa$ ) make it quite difficult to decide what is a resonance and what is not. For more detailed discussions. see the mini-reviews in the Listings. In what follows we shall at tempt to specify some guidelines.

(a) When energy-independent partial-wave analyses are available (mostly for $\mathrm{N}^{*}$ 's), approximate BreitWigner behavior of the amplitude appears to us to be the most satisfactory test for a resonance. We can check that the Argand plot follows roughly a lefthand circle, and that the "speed" of the amplitude also shows a maximum near the resonance energy: further, there should be data well above the resonance. showing that the speed again decreases. Indeed proper behavior of the partial-wave amplitude could accredit a resonance even if its elasticity is too small to make a noticeable peak in the cross section.

Of course even if Argand plots are available. it may still be a matter of opinion as to what behavior constitutes a resonance. Such an example is the $Z_{0}(1780)$ state seen in $\mathrm{KN}$ total cross-section experiments and in partial-wave analysis. The partial-wave analyses of Giacomelli (1974) and Martin (1975) find preferred solutions which exhibit a resonance-like loop in the $P_{01}$ wave near $1740 \mathrm{MeV}$. H: wever. Giacomelli et al. and Martin point out that, despite the resonantlike appearance of the loop, the evidence for resonant energy dependence is inconclusive. Thus we omit the $\mathrm{Z}_{0}(1780)$ from the Baryon Table. A similar quandary has existed for some time concerning the $Z_{1}(1900)$. and it too has been omitted from the Tables.

(b) When there are insufficient data to perform energy-independent analyses, one often resorts to energy-dependent partial-wave analyses (mostly for $Y^{*}$ 's). In this case Breit-Wigner behavior is an input. We therefore require that resonance solutions be found by several different analyses, preferably in different channels ( $\overline{\mathrm{K}} \mathrm{N} \rightarrow \overline{\mathrm{K}} \mathrm{N}, \pi \Sigma$, etc.). before putting the claim in the tible.

(c) Partial-wave analyses of three-body final states $(\pi N \rightarrow \pi \pi N)$ are now available. While these analyses are based on the isobar model ( $\pi \mathrm{N} \rightarrow \rho \mathrm{N}, \pi \Delta$, etc.) and are subject to theoretical objections of varying importance. they provide increasingly reliable information on inelastic decay modes of otherwise established resonances.

(d) Most mesons. $\bar{\Xi}^{*}$ peaks, and high-mass $\mathrm{N}^{*}$ and $\mathrm{Y}^{*}$ peaks fall into a category for which no partial-wave analyses exist. In general we accept such peaks if they are experimentally reliable. of high statistical significance or observed in several different production processes.

Thus, we enter into the Tables of Particle Properties only states for which there is experimentally convincing evidence, and we expect that most of these will be confirmed as resonances.

\section{Conventions and parameters for weak and electromagnetic decays}

\section{A. Munn-decay parameters}

The $\mu$-decay parameters describe the momentum spectrum ( $\rho$ and $\eta$ ). the asymmetry ( $\xi$ and $\delta$ ). and the helicity $(h)$ of the electron in the process $\mu^{-} \rightarrow \mathrm{e}^{-}$ $+\nu_{\mu}+\bar{\nu}_{\mathrm{e}}$. Assuming a locil and lepton-conserving interaction. the matrix element in the charge-retention form may be written as

$$
\sum_{i}\left\langle\overline{\mathrm{e}}\left|\Gamma_{i}\right| \mu\right\rangle\left\langle\bar{\nu}_{\mu}\left|\Gamma_{i}\left(C_{i}+C_{i}^{\prime} \gamma_{5}\right)\right| \nu_{\mathrm{e}}\right\rangle .
$$

where the summation is taken over $i=\mathrm{S}, \mathrm{V}, \mathrm{T}, \mathrm{A}, \mathrm{P}$. Using the definitions and sign conventions of Sachs and Sirlin (1975) and Scheck (1978) for the Lorentzcovariant operators, we have for the momentum parameters:

$$
\begin{aligned}
& \rho=\left(3 g_{\mathrm{A}}^{2}+3 g_{\mathrm{V}}^{2}+6 g_{\mathrm{T}}^{2}\right)^{\prime} D . \\
& \eta=\left(g_{\mathrm{S}}^{2}-g_{\mathrm{P}}^{2}+2 g_{\mathrm{A}}^{2}-2 g_{\mathrm{V}}^{2}\right) / D:
\end{aligned}
$$

for the asymmetry parimeters:

$$
\begin{gathered}
\xi=-6 g_{\mathrm{S}} g_{\mathrm{P}} \cos \phi_{\mathrm{SP}}-8 g_{\mathrm{A}} g_{\mathrm{V}} \cos \phi_{\mathrm{AV}}+14 g_{\mathrm{T}}^{2} \cos \phi_{\mathrm{TT}} \\
D \\
\delta=\left(-6 g_{\mathrm{A}} g_{\mathrm{V}} \cos \phi_{\mathrm{AV}}+6 g_{\mathrm{T}}^{2} \cos \phi_{\mathrm{TT}}\right) / D \xi:
\end{gathered}
$$

and for the parameter describisig the helicity of the electron: 
$h=-\frac{2 g_{\mathrm{S}} g_{\mathrm{P}} \cos \phi_{\mathrm{SP}}+8 g_{\mathrm{A}} g_{\mathrm{V}} \cos \phi_{\mathrm{AV}}+6 g_{\mathrm{T}}^{2} \cos \phi_{\mathrm{TT}}}{D}$

Here

$D=g_{\mathrm{S}}^{2}+g_{\mathrm{P}}^{2}+4 g_{\mathrm{V}}^{2}+6 g_{\mathrm{T}}^{2}+4 g_{\mathrm{A}}^{2}$.

$g_{i}^{2}=\left|C_{i}\right|^{2}+\left|C_{i}^{\prime}\right|^{2}$,

and

$\cos \phi_{i j}=\operatorname{Re}\left(C_{j}^{*} C_{j}^{\prime}+C_{i}^{\prime} C_{j}^{*}\right) / g_{i} g_{j}$.

The quantities $g_{i}$ are defined to be real non-negative numbers, and the $\phi_{i j}$ are phase angles between the $i$ type and $j$-type interactions. Linder the assumption of two-component neutrinos $C_{\mathrm{V}}^{\prime}=-C_{\mathrm{V}}$ and $C_{\mathrm{A}}^{\prime}=-C_{\mathrm{A}}$, the $S, P$, and $T$ terms vanish, and $\phi_{A V}$ is the phase angle between $C_{\mathrm{A}}$ and $C_{\mathrm{V}}$ in the complex plane.

By using the above equations and the experimental determinations of $\rho, \eta, \xi, \delta$, and $h$, limits can be placed on $g_{\mathrm{S}} / g_{\mathrm{V}}, g_{\mathrm{A}} / g_{\mathrm{V}}, g_{\mathrm{T}} / g_{\mathrm{V}}, g_{\mathrm{P}} / g_{\mathrm{V}}$, and $\phi_{\mathrm{AV}}$ The results, given in the Data Card Listings, assume neither two-component neutrinos nor time-reversal invariance. If, however, two-component neutrinos are assumed, then sin $\phi_{\mathrm{AV}}$ is the amplitude of time-reversal violation. Note that most experiments study only the upper end of the spectrum where $\rho$ and $\eta$ are highly correlated, so they can oniy report $\rho$ for $\eta \equiv 0$ and $\eta$ for $\rho=\frac{3}{4}$. The values for $\rho$ and $\eta$ we use here were obtained by combining measurements of both upper and lower ends of the spectrum and turn out to be nearly uncorrelated.

Note also that the radiative corrections are unam. biguous only when $g_{\mathrm{S}}=g_{\mathrm{T}}=g_{\mathrm{P}}=0$. The same limits on $g_{\mathrm{A}} / g_{\mathrm{V}}$ and $\phi_{\mathrm{AV}}$ are obtained, however, as when $\xi_{\mathrm{S}}, g_{\mathrm{T}}$, and $g_{\mathrm{P}}$ are left free.

Current values for the asymmetry parameters as well as $\left|g_{\mathrm{A}} / g_{\mathrm{V}}\right|$ and $\phi_{\mathrm{AV}}$ are given in the Addendum to the Stable Particle Table. In addition, upper limits on $\left|g_{S} / g_{V}\right|,\left|g_{T} / g_{\mathrm{V}}\right|$ and $\left|g_{\mathrm{P}} / g_{\mathrm{V}}\right|$ are given in the $\mu$ section of the Stable Particle Data Card Listings.

\section{B. $K$-decay parameters}

1. Dalitz plot for $K \rightarrow 3 \pi$ decays. The Dalitz plot distribution for the $\tau$ mode $\left(\mathrm{K}^{ \pm} \rightarrow \pi^{ \pm} \pi^{ \pm} \pi^{\mp}\right)$, the $\tau^{\prime}$ mode $\left(K^{ \pm} \rightarrow \pi^{0} \pi^{0} \pi^{ \pm}\right)$, and the $\tau^{0}$ mode $\left(K_{L}^{0} \rightarrow \pi^{+} \pi^{-} \pi^{0}\right)$ of $K$ decay can be parametrized by iseries expansion such as that introduced by Weinberg (1960). We use the form

$$
\begin{gathered}
|M|^{2} \propto 1+g \frac{s_{3}-s_{0}}{m_{\pi^{+}}^{2}}+h\left(\begin{array}{c}
s_{3}-s_{0} \\
m_{\pi^{+}}^{2}
\end{array}\right)^{2} \\
+i \frac{s_{2}-s_{1}}{m_{\pi^{+}}^{2}}+k\left(\frac{s_{2}-s_{1}}{m_{\pi^{+}}^{2}}\right)^{2}+\ldots,
\end{gathered}
$$

where $m n_{\pi^{+}}^{2}$ has been introduced so as to make the coefficients $g, h, j$, and $k$ dimensionless, and

$s_{i}=\left(P_{\mathrm{K}}-P_{i}\right)^{2}=\left(m_{\mathrm{K}}-m_{i}\right)^{2}-2 m_{\mathrm{K}} T_{i}, \quad i=1,2,3$,

$s_{0}=\frac{1}{3} \sum_{i} s_{i}=\frac{1}{3}\left(m_{K}^{2}+m_{1}^{2}+m_{2}^{2}+m_{3}^{2}\right)$.

Here the $P_{i}$ are four-vectors, $m_{i}$ and $T_{i}$ are the mass and kinetic energy of the $i$ th pion, and the index 3 is used for the odd pion.

The coefficient $g$ is a measure of the slope in the variable $s_{3}$ (or $T_{3}$ ) of the Dalitz plot, while $h$ and $k$ measure the quadratic dependence on $s_{3}$ and $\left(s_{2}-s_{1}\right)$, respectively. The coefficient $j$ is related to the asym: metry of the plot and must be zero if $C P$ invariance holds ( $C$ stands for charge conjugation throughout the discussion in this section). Note also that if $C P$ is good, $g$ must be the same for $\tau^{+}$and $\tau^{--}$, and similarly for $h$ and $k$.

Since different experiments use different forms for $|M|^{2}$, in order to compare the experiments we have converted to $g, h, j$, and $k$ whatever coefficients have been measured. See the mini-review in the $\mathrm{K}^{ \pm}$section of the Stable Particle Data Card Listings for details on this point. The results are given in the Addendurn to the Stable Particle Table and in the $K^{ \pm}$and $K_{L}^{0}$ sections of the Stable Particle Data Card Listings.

Relations among $\tau^{ \pm}, \tau^{\prime \pm}$, and $\tau^{0}$ are predicted by the $\Delta I=\frac{1}{2}$ rule.

2. Form factors in $K_{\mathrm{Q} 3}$ leptonic decays. Assuming that only the vector current contributes to these decays, we write the matrix element as

$$
\begin{aligned}
& M \propto f_{+}(t)\left[\left(P_{\mathrm{K}}+P_{\pi}\right)_{\mu} \overline{\mathrm{u}}_{\mathrm{Q}} \gamma_{\mu}\left(1+\gamma_{5}\right) \mathrm{u}_{\nu}\right] \\
& \quad+f_{-}(t)\left[m_{\mathrm{Q}} \overline{\mathrm{u}}_{\mathrm{Q}}\left(1+\gamma_{5}\right) \mathrm{u}_{\nu}\right],
\end{aligned}
$$

where $P_{K}$ and $P_{\pi}$ are the four-momenta of $K$ and $\pi$ mesons; $m_{Q}$ is the lepton mass; $f_{+}$and $f_{-}$are dimensionless form factors which can depend only on $t$ $=\left(P_{\mathrm{K}}-P_{\pi}\right)^{2}$, the square of the four-momentum 
transfer to the leptons, $f_{+}$and $f_{-}$are relatively real if time-reversal invariance holds for these decays. $K_{\mu 3}$ experiments measure $f_{+}$and $f_{-}$, while $\mathrm{K}_{\mathrm{e} 3}$ experiments are sensitive only to $f_{+}$because the presence of the lepton mass makes the $f$ term negligible.

(a) $K_{\mu 3}$ experiments. Analyses of $\mathrm{K}_{1,3}$ uata frequently assume a linear dependence of $f_{+}$and $f_{-}$on $t$, i.e.

$f_{ \pm}(t)=f_{ \pm}(0)\left[1+\lambda_{ \pm}\left(t / m_{\pi}^{2}\right)\right]$.

Most $\mathbf{K}_{\mu 3}$ data are adequately described by eq. (3) for $f_{+}$and a constant $f_{-}$(i.e. $\lambda_{-}=0$ ). There are two equivalent parametrizations commonly used in these analyses:

(1) $\lambda_{+}, \xi(0)$ parametrizaticn. Analyses of $\mathrm{K}_{\mu 3}$ data often introduce the ratio of the two form factors

$\xi(t)=f_{-}(t) / f_{+}(t)$.

The $K_{\mu 3}$ decay distribution is then described by the two paiameters $\lambda_{+}$and $\xi(0)$ (assuming time reversal invariance and $\lambda_{-}=0$ ). These parameters can be determined by three different methods:

Method $A$. By studying the Dalitz plot or the pion spectrum of $\mathbf{K}_{\mu 3}$ decay. The Dalitz plot density is [see. e.g. Chounet et al. (1972)]:

$\rho\left[E_{\pi}, E_{\mu}\right) \sim f_{+}^{2}(t)\left[A+B \xi(t)+C \xi(t)^{2}\right]$,

where

$A=m_{\mathrm{K}}\left(2 E_{\mu} E_{\nu}-m_{\mathrm{K}} E_{\pi}^{\prime \prime}\right)+m_{\mu}^{2}\left(\frac{1}{4} E_{\pi}^{\prime}-E_{\nu}\right)$.

$B=m_{\mu}^{2}\left(E_{\nu}-\frac{1}{2} E_{\pi}^{\prime}\right)$.

$C=\frac{1}{4} m_{\mu}^{2} E_{\pi}^{\prime}$.

$E_{\pi}^{\prime}=E_{\pi}^{\mathrm{max}}-E_{\pi}=\left(m_{\mathrm{K}}^{2}+m_{\pi}^{2}-m_{\mu}^{2}\right) / 2 m_{\mathrm{K}}-E_{\pi}$.

Here $E_{\pi} . E_{\mu}$, and $E_{\nu}$ are. respectively the pion, muon, and neutrino energies in the kaon center of mass. The density $\rho$ is fit to the data to determine the values of $\lambda_{+}, \xi(0)$, and their correlation.

Method $B$. By measuring the $\mathrm{K}_{\mu 3} / \mathrm{K}_{\mathrm{c} 3}$ branching ratio and comparing it with the theoretical ratio [see. e.g.. Fearing et al. (1970)] as given in terms of $\lambda_{+}$and $\xi(0)$, assuming $\mu-\mathrm{e}$ universality:

$$
\begin{aligned}
& \Gamma\left(\mathrm{K}_{\mu 3}^{ \pm}\right) / \Gamma\left(\mathrm{K}_{\mathrm{c} 3}^{\ddagger}\right)=0.6457+1.4115 \lambda_{+}+0.1264 \xi(0) \\
& \quad+0.0192 \xi(0)^{2}+0.0080 \lambda_{+} \xi(0), \\
& \Gamma\left(K_{\mu 3}^{0}\right) / \Gamma\left(K_{\mathfrak{c} 3}^{0}\right)=0.6452+1.3162 \lambda_{+}+0.1245 \xi(0) \\
& \quad+0.0186 \xi(0)^{2}+0.0064 \lambda_{+} \xi(0) .
\end{aligned}
$$

This cannot determine $\lambda_{+}$and $\xi(0)$ simultaneously but simply fixes a relationship between them.

Method $C$. By measuring the muon polarization in $\mathrm{K}_{\mu 3}$ decay. In the rest frame of the $\mathrm{K}$, the $\mu$ is expected to be polarized in the direction $A$ with $P=A$ / $|A|$, where $A$ is given [Cabibbo and Maksymowicz (1964)] by

$$
\begin{aligned}
\boldsymbol{A} & =a_{1}(\xi) \boldsymbol{p}_{\mu} \\
& -a_{2}(\xi)\left[\frac{\boldsymbol{p}_{\mu}}{m_{\mu}}\left(m_{\mathrm{K}}-E_{\pi}+\frac{\boldsymbol{p}_{\pi} \cdot \boldsymbol{p}_{\mu}}{\left|\boldsymbol{p}_{\mu}\right|^{2}}\left(E_{\mu}-m_{\mu}\right)\right)+\boldsymbol{p}_{\pi}\right] \\
& +m_{\mathrm{K}} \operatorname{Im} \xi(t)\left(\boldsymbol{p}_{\pi} \times \boldsymbol{p}_{\mu}\right) .
\end{aligned}
$$

If time-reversal invariance holds, $\xi$ is real, and thus there is no polarization perpendicular to the $\mathrm{K}$-decay plane. Polarization experiments measure the weighted average of $\xi(t)$ over the $t$ range of the experiment. where the weighting accounts for the variation with $t$ of the sensitivity to $\xi(t)$.

(2) $\lambda_{+}, \lambda_{1)}$ parametrization. Some of the more recent $K_{\mu 3}$ analyses have parametrized $i$ : termis of the form factors $f_{\dot{r}}$ and $f_{0}$ which are associated with vector and scalar exchange. respectively, to the lepton pair. $f_{0}$ is related to $f_{+}$and $f_{--}$by

$$
f_{0}(t)=f_{+}(t)+\left[t /\left(m_{\mathrm{K}}^{2}-m_{\pi}^{2}\right)\right] f_{-}(t) \text {. }
$$

Here $f_{0}(0)$ must equal $f_{+}(0)$ unless $f_{-}(t)$ diverges at $t=0$. The earlier assumption that $f_{+} \overrightarrow{\text { is linear in } t \text { and }}$ $j_{-}$is constant leads to $f_{0}$ linear in $t$ :

$f_{0}(t)=f_{0}(0)\left[\mathrm{I}+\lambda_{0}\left(t / m_{\pi}^{2}\right)\right]$.

With the assumption that $f_{0}(0)=f_{+}(0)$. the two parametrizations. $\left(\lambda_{+}, \xi(0)\right)$ and $\left(\lambda_{+}, \lambda_{0}\right)$ are equivalent as long as correlation information is retained. $\left(\lambda_{+}, \lambda_{0}\right)$ correlations tend to be less strong than $\left(\lambda_{+}, \xi(0)\right)$ correlations.

The experimental results for $\xi(0)$ and its correlation with $\lambda_{+}$are listed in the $K^{ \pm}$and $K_{L}^{0}$ sections of the Stable Particle Data Card Listings in section XIA, $\mathrm{XIB}$, or XIC depending on whether method $\mathrm{A} . \mathrm{B}$, or $\mathrm{C}$ discussed above was used. The corresponding values of $\lambda_{+}$are listed in subsection $L+M$.

Because current experiments tend to use the $\left(\lambda_{+}\right.$, $\lambda_{0}$ ) parametrization, we have added a subsection LO for $\lambda_{0}$ results. Wherever possible we have converted $\xi(0)$ results into $\lambda_{0}$ results and vice versa.

(b) $K_{e 3}$ experiments. Analysis of $\mathrm{K}_{\mathrm{e} 3}$ data is simpler than that of $K_{\mu 3}$ because the second term of the 
matrix element assuming a pure vector current [eq. (2) above] can be neglected. Here $f_{+}$is usually assumed to be linear in $t$, and the linear cuefficient $\lambda_{t}$ of eq. (3) is determined.

If we remove the assumption of a pure vector cur. rent, then the matrix element for the decay, in addition to the terms in eq. (2), would contain

$$
\begin{aligned}
& +2 m_{\mathrm{K}} f_{\mathrm{S}} \overline{\mathrm{u}}_{\mathrm{k}}\left(1+\gamma_{5}\right) \mathrm{u}_{\nu} \\
& +\left(2 f_{T} / m_{\mathrm{K}}\right)\left(P_{\mathrm{K}}\right)_{\lambda}\left(P_{\pi}\right)_{\mu} \overline{\mathrm{u}}_{\mathrm{v}, \sigma_{\lambda \mu}}\left(1+\gamma_{5}\right) \mathrm{u}_{\nu},
\end{aligned}
$$

where $f_{\mathrm{S}}$ is the scalar form factor, and $f_{\mathrm{T}}$ is the tensor form factor. In the case of the $\mathrm{K}_{\mathrm{e}} 3$ decays where the

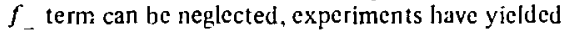
limits on $\left|f_{\mathrm{S}} / f_{+}\right|$and $\left|f_{\mathrm{T}} / f_{+}\right|$.

The $\mathrm{K}_{\mathrm{L}, 3}$ results for $\lambda_{+} \cdot\left|f_{\mathrm{S}} / f_{+}\right|$, and $\mid f_{T} / f_{2} ;$ ire listed in the subsections $\mathrm{L}+\mathrm{M}$. FS, and FT. respectively, of the $K^{ \pm}$and $K_{L}^{0}$ sections of the Stable Particle Data Card Listings.

See also the Note on $K_{3}^{+}$and $K_{13}^{0}$ Form Factors in the $\mathrm{K}^{\ddagger}$ section of the Stahle Particle Data Card Listings for additional discussion of the $K_{\mu 3}^{0}$ parameters. correlations. and conversion between parametrizalion and also for a comparison of the experimental results.

\section{CP violation in $K^{0}$ decays. We list parameters} for four different reactions in which $C P$ can be tested [for details, see Okun and Rubbia (1967). Steinberger (1969), and Wolfenstein (1969)].

(a) $K_{S} \rightarrow \pi^{+} \pi^{-} \pi^{0}$. The quantity measured here is the ratio of amplitudes

$$
A_{\mathrm{S}}\left(\mathrm{K}_{\mathrm{S}} \rightarrow \pi^{+} \pi^{--} \pi^{0}\right) / A_{\mathrm{L}}\left(\mathrm{K}_{\mathrm{L}} \rightarrow \pi^{+} \pi^{-} \pi^{0}\right) \equiv{ }_{x}+i \mathrm{y} .
$$

If $C P T$ invariance holds and there is no $I=3$ state present, then $x$ can be neglected and $C P$ violation would be observed as a nonzero $y$. We give the result for eq. (4) in the $K_{\mathrm{L}}^{0}$ section of the Stable Particle Table.and under Branching Ratio $R_{4}$ in the $K_{S}^{0}$ section of the Stable Particle Data Card Listings. Our procedure is to assume that $x=0$, and to list $\left(A_{\mathrm{S}} / A_{\mathrm{L}}\right)^{2}$ in the form of a branching ratio.

(b) Charge asymunetry in $K_{l} \rightarrow 3 \pi$ decays. As mentioned above, the presence of a $1 \mathrm{erm}$ in $\left(s_{2}-s_{1}\right)$ in expression (1) describing the Dalitz plot distribution for $\tau^{ \pm}, \tau^{0}$ decays of $\mathrm{K}$ mesons would be an indication of $C P$ violation. Experimenters have used several forms for this $C P$-violation term. As described in the mini. review in the $K^{ \pm}$section of the Stable Particle Data
Card Listings, we have converted all results to coefficient $j$ in eq. (1) above. The latter is listed among the $C P$-violating parameters at the back of the $K_{\mathrm{L}}^{0}$ section of the Stabie Particle Data Card Listings. Note that only upper linits have been reported for this quantity.

(c) Asymetry in the $K_{l} \rightarrow \pi^{*} \ell^{ \pm} v$ decays. The quatitity measured and compiled here is

$\delta=\begin{aligned} & \Gamma\left(\mathrm{K}_{\mathrm{L}} \rightarrow \pi \ell^{+} \nu\right) \quad \mathrm{I}\left(\mathrm{K}_{\mathrm{L} .} \rightarrow \pi^{+} \ell \nu\right) \\ & \mathrm{I}\left(\mathrm{K}_{\mathrm{L}} \rightarrow \pi^{-} \ell^{+} \nu\right)+\Gamma\left(\mathrm{K}_{\mathrm{I} .} \rightarrow \pi^{+} \ell^{-} \nu\right)\end{aligned}$

This asymmetry violates $C P$ invariance. If $C P T$ is good. for a pare $\mathrm{K}_{1}^{0}$. heam. $\delta$ can he written as

$\delta=2\left[\left(1-|x|^{2}\right) /\left(|1-x|^{2}\right)\right] \operatorname{Re} E$.

where $x$ is the $\Delta S=\Delta Q$-violating paraneter cefined in section $B 4$, and $E$ is the paraneter of the expansion

$$
\begin{aligned}
& \left|K_{\mathrm{I}}\right\rangle=\left\{(1+\epsilon)|K\rangle-(1-\epsilon)|\bar{K}\rangle \mid /\left[2\left(1+|\epsilon|^{2}\right)\right\}^{1 / 2} \cdot(5 a)\right. \\
& \left|K_{S}\right\rangle=[(1+\epsilon)|K\rangle+(1-\epsilon)|\bar{K}\rangle] /\left[2\left(1+|\epsilon|^{2}\right)\right\}^{1 / 2} \cdot(5 b)
\end{aligned}
$$

We give $\delta$ in the Addendum to the Stable Particle Table. In addition. in the $\mathrm{K}_{1}^{0} C P$-violation section of the Stable Particle Data Card Listings. we lisi $\delta$ sepiraltely for $\mathrm{K}_{\mathrm{L}}^{()} \rightarrow \pi \mu \nu$ and $\mathrm{K}_{\mathrm{L}}^{0} \rightarrow \pi \mathrm{e} \nu$.

(d) $K_{l} \rightarrow 2 \pi$ decay. The relevant paraneters are

$$
\begin{aligned}
\eta_{+} & =A\left(\mathrm{~K}_{\mathrm{L}} \rightarrow \pi^{+} \pi^{\cdots}\right) / A\left(\mathrm{~K}_{\mathrm{S}} \rightarrow \pi^{+} \pi \mid\right. \\
& =\left|\eta_{+}\right| \exp \left(\mathrm{i} \phi_{+} .\right) . \\
\eta_{00} & =A\left(\mathrm{~K}_{\mathrm{l}_{-}} \rightarrow \pi^{0} \pi^{0}\right) / A\left(\mathrm{~K}_{\mathrm{S}} \rightarrow \pi^{0} \pi^{0}\right) \\
& =\left|\eta_{\left(\hat{H}_{0}\right.}\right| \exp \left(\mathrm{i} \phi_{000}\right) .
\end{aligned}
$$

e. defined in eqs. (5) above. and

$\epsilon^{\prime}=\frac{1}{2} i \sqrt{2} \exp \left[i\left(\delta_{2} \quad \delta_{0}\right)\right] \operatorname{In}\left(A_{2} / A_{0}\right)$.

Herc $A_{i}$ and $\delta_{i}$ are the amplitude and phase of $\pi \pi$ scattering at the $\mathrm{K}$ mass, defined by

$\left\langle(I=0|T| \mathrm{K}\rangle=\exp / i \delta_{0}\right) A_{0}$.

$\langle l=2|T| \mathrm{K}\rangle=\exp \left(\mathrm{i}_{2}\right) A_{2}$.

Wu and Yang (1964) have derived the relationships

$\eta_{+-}=\epsilon+\epsilon^{\prime}, \quad \eta_{00}=\epsilon-2 \epsilon^{\prime}$.

We give $\eta_{+} \ldots \eta_{00}, \phi_{+}$. and $\phi_{00}$ in the Addendum to the Stable Particle Table. The phases are measured directly, whereas the magnitudes $\eta_{+} \ldots$ and $\eta_{00}$ are derived parameters. We use, as far as we can, the 
directly measured quantities as input and calculate $\eta_{+-}$and $\eta_{00}$ from the values given by our constrained fits. Therefore, if one looks at the Data Card Listings, most of the $|\eta|$ measurements appear in the form of branching ratios, with appropriate comments. We ther: give the values of $\eta_{+-}$and $\left|\eta_{00}\right|^{2}$ in a separate list at the end of the $\boldsymbol{C}$-viclating parameters section of the $\mathbf{K}_{\mathbf{L}}^{0}$ section of the Stable Particle Data Card Listings.

4. $\Delta S=\Delta Q$ rule in $K^{0}$ decays. The relative amount of $\Delta S \neq \Delta Q$ component present is measured by the parameter $x$, defined as

$x=A\left(\overline{\mathrm{K}}^{0} \rightarrow \pi^{-} \ell^{+} \nu\right) / A\left(\mathrm{~K}^{0} \rightarrow \pi^{-} \ell^{+} \nu\right)$.

We list $\operatorname{Rc}\{x\}$ and $\operatorname{lm}\{x\}$ for both $K_{\mathrm{c} 3}$ and $K_{\mu 3}$ at the end of the Stable Particle Data Card Listings and give values in the Addendum to the Siable Particle Table.

\section{T-decay parameters}

1. C-yiolation in ndecays. As a test of possible $C$ violation in electromagnetic interactions, a number of experiments have looked for possible charge asymmetries in the decays $\eta \rightarrow \pi^{+} \pi^{-} \pi^{0}$ and $\eta \rightarrow \pi^{+} \pi^{-} \gamma$. We list the following parameters:

(a) The left-right asymmetry

$A=\left(N^{+}-N^{-}\right) /\left(N^{+}+N^{-}\right)$,

where $N^{( \pm)}$means the number of events with the $\pi^{(\cdot)}$ erergy greater than the $\pi^{(\mp)}$ energy in the $\eta$ rest frame.

(b) The sextant asymmetry

$A_{\mathrm{s}}=\frac{N_{1}+N_{3}+N_{5}-N_{2}-N_{4}-N_{6}}{N_{1}+N_{2}+N_{3}+N_{4}+\bar{N}_{5}+N_{6}}$

for the decay $\eta \rightarrow \pi^{+} \pi^{-} \pi^{0}$. The numbers refer to the sextants of the Dalitz plot [see, for example, Layter (1972)]. $A_{\mathrm{s}}$ is sensitive to an $I=0 C$-violating asymmetry.

(c) The quadrant asymmetry $A_{\mathrm{q}}$, defined in a similar way as $A_{5}$, but with each sector of the Dalitz plot now containing $\pi / 2$ rather than $\pi / 3$ radians. $A_{\mathrm{q}}$ is sensitive to an $I=2 C$-violating final state.

(d) The d-wave contribution to the $C$-violating $: n$ plitude in the decay $\eta \rightarrow \pi^{+} \pi^{-} \gamma$. The upper limit ${ }^{{ }^{-}} r$ this contribution is measured by the parameter $\beta$, de. fined by

$\mathrm{d} N / \mathrm{d}|\cos \theta| \propto \sin ^{2} \theta\left(1+\beta \cos ^{2} \theta\right)$, where $\theta$ is the angle between the $\pi^{+}$and the $\gamma$ in the di-pion center of mass. A term proportional to $\cos ^{2} \theta$ could also be due to $\mathrm{p}$ - and $\mathrm{f}$-wave interference.

We list $A$ for the decay modes $\eta \rightarrow \pi^{+} \pi^{-} \pi^{0}$ and $\eta$ $\rightarrow \pi^{+} \pi^{-} \gamma, A_{\mathrm{s}}$ and $A_{\mathrm{q}}$ for the decay $\eta \rightarrow \pi^{+} \pi^{-} \pi^{0}$, and $\beta$ for the decay $\eta \rightarrow \pi^{+} \pi^{-} \gamma$ in the $\eta$ section of the Stable Particle Data Card Listings.

2. Dalitz plot for $\eta \rightarrow \pi^{+} \pi^{-} \pi^{0}$. The Dalitz plot for the decay $\eta \rightarrow \pi^{+} \pi^{-} \pi^{0}$ may be fit by the distribution $|M(x, y)|^{2} \propto 1+a y+b y^{2}+c x+d x^{2}+e x y$.

Here,

$x=\sqrt{3}\left(T_{+}-T_{-}\right) / Q, \quad y=\left(3 T_{0} / Q\right)-1$,

$T_{+}, T_{-}, T_{0}$ are the kinetic energies of the $\pi^{+}, \pi^{-}$, and $\pi^{0}$ in the $\eta$ rest system, and $Q=m_{\eta}-m_{\pi^{+}}-m_{\pi^{-}}-m_{\pi^{0}}$. The coefficient of the term linear in $x$ is sensitive to $C$-violation due to an $I=0$ or $I=2$ final state. We list papers presenting determinations of the parameters $a$, $b, c$, and $d$ in the $\eta$ section of the Stable Particle Data Card Listings. However, we do not tabulate values of these parameters because the assumptions made by different authors are not compatible and do not allow comparison of the numerical values.

3. Dalitz plot for $\eta \rightarrow \pi^{+} \pi^{-} \gamma$. The Dalitz plot for the decay $\eta \rightarrow \pi^{+} \pi^{-} \gamma$ rnay be fit to the expression $|M|^{2} \propto 1+2 \alpha 2$,

where

$z=\frac{2}{3} \sum_{i=1}^{3}\left[3\left(m_{\eta}-3 m_{n}\right)^{-1}\left(E_{i}-\frac{1}{3} m_{\eta}\right)\right]^{2}=\rho^{2} / \rho_{\max }^{2}$.

Here $E_{i}$ is the energy of the $i$ th pion in the $\eta$ rest frame, and $\rho$ is the distance to the center of the Dalitz plot. We list the parameter $\alpha$ in the $\eta$ section of the Stable Particle Data Card Listings.

\section{Baryon-decay parameters}

I. A/V ratio for baryon leptonic decays. Consider the decay

$\mathrm{B}_{\mathrm{i}} \rightarrow \mathrm{B}_{\mathrm{f}}+\ell+\nu$.

Assuming V, A theory, neglecting "induced" scalar, "induced" pseudoscalar, and axial weak-magnetism terms, and neglecting the $q^{2}$ dependence of the form factors, the baryon part of the matrix element for 
these decays may be written [Goldberger and Treiman (1958)] as

$\left\langle B_{\mathrm{f}} \mid \gamma_{\lambda}\left(g_{\mathrm{V}}-g_{\mathrm{A}} \gamma_{5}\right)+\left(g_{\mathrm{W}} / m_{\mathrm{B}_{\mathrm{i}}}\right) \sigma^{\lambda \nu} q_{\nu} / \mathrm{B}_{\mathrm{i}}\right\rangle$,

where $B_{i}$ and $B_{f}$ represent initial and final baryons, $g_{\mathrm{A}}$ and $g_{\mathrm{V}}$ the axial and vector coupling constants, $g_{\mathrm{W}}$ the weak magnetism coupling constant, and $q_{\nu}$ the sum of the lepton momenta. Here the Pauli representation is used for the $\gamma$ matrices. The ratio $g_{\mathrm{A}} / g_{\mathrm{V}}$ may be wriiten as

$g_{\mathrm{A}} / g_{\mathrm{V}}=\left|g_{\mathrm{A}} / g_{\mathrm{V}}\right| \exp (\mathrm{i} \phi)$,

where $\phi$ is $0+n \pi$ if time reversal holds [see Jackson et al.(1957)].

Experiments on the leptonic decays of baryons other than the neutron havc generally assumed $\phi$ to be either 0 or $\pi$, and have thus measured the magnitude and sign of $g_{\mathrm{A}} / g_{\mathrm{V}}$. In studying neutron beta decay, however, experiments have been sensitive enough to measure $\phi$ more precisely, and we include the phase angle in our Listings for this case. It is consistent with time-reversal invariance, and by using the above definition of the matrix element with the Pauli representations, the value of $g_{A} / g_{V}$ in neutron beta decay is negative.

Due to statistical limitations the weak magnetism form factor $g_{W}$ is usually assumed from CVC and SU(3), so that usually only $g_{A}$ and $g_{V}$ are determined experimentally. This determination is accomplished in a variety of ways.

(a) The lepton-neutrino angular correlation provides a measure of the absolute value of $g_{A} / g_{V}$ [for relevant formulas, see, e.g., Albright (1959)].

(b) The up-down asymmetry of the lepton from polarized baryon decays provides a measure of $g_{\mathrm{A}} / g_{\mathrm{V}}$ with its sign [for relevant formulas, see, e.g., Aibrighi (1959)].

(c) The lepton spectrum, given enough statistics, provides a measure of $g_{\mathrm{A}} / g_{\mathrm{V}}$ with its sign [for relevant formulas, see, e.g., Bender (1968)]. The lepton spectrum also provides a measure of $g_{W} / g_{A}$ if the CVC$\mathrm{SU}(3)$ assumption is relaxed.

(d) The polarization of the decay baryon, from polarized or unpolarized initial baryon, also provides $g_{\mathrm{A}} / g_{\mathrm{V}}$ with its sign [for formulas, see, e.g., Willis and Thompson (1968)].

(e) The presence of a term proportional to

$\sigma_{B_{\mathrm{i}}} \cdot\left(p_{\mathrm{c}} \times p_{p}\right)$,

xvi where the initial baryon is polarized or

$\sigma_{\mathrm{B}_{\mathrm{f}}} \cdot\left(\boldsymbol{p}_{\mathrm{c}} \times \boldsymbol{p}_{\mathrm{v}}\right)$,

where the polarization of the decay baryon 15 observed provides a measure of the deviation of $\phi$ from $c$ or $\pi$, and is thus a test of time-reversal invariance [see, e.E., Willis and Thompson (1968)].

We compile the ratio $g_{A} / g_{V}$ with its sign, for those decays for which it has been measured.

All the coupling constants and decay rates for baryon leptonic decays are related by Cabibbo's theory [Cabibbo (1963)], extended to six quarks (and three mixing angles) by Kobayashi and Maskawa (1973). A recent fit to this theory has been done by Shrock and Wang (1978).

\section{Asymmetry parameters in nondeptonic hyporon} decays. The transition matrix for the hyperon decay may be written as

$M=s+p(\sigma \cdot q)$,

where $s$ and $p$ are the parity-changing and the parityconserving amplitudes, respectively; $\sigma$ is the Pauli spin operator, and $q$ is a unit vector along the direction of the decay baryon in the hyperon rest frame.

The asymmetry parameters are defined by the relations

$\alpha=? \operatorname{Re}\left(s^{*} p\right) /\left(|s|^{2}+|p|^{2}\right)$,

$\beta=2 \operatorname{Im}\left(s^{*} p\right) /\left(|s|^{2}+|p|^{2}\right)$,

$\gamma=\left(|s|^{2}-|p|^{2}\right) /\left(|s|^{2}+|p|^{2}\right)$.

With the transition matrix (6), the angular distribution of the decay batyon, in the hyperon rest system, is of the form

$I=1+\alpha P_{Y} \cdot q$,

where $P_{Y}=\langle Y|\sigma| Y\rangle$ is the hyperon polarization.

In the notation of Lee and Yang (1957) the polarjzation $\boldsymbol{P}_{\mathrm{B}}$ of the decay baryons is $\$ 2$

$P_{\mathrm{B}}=\frac{\left(\alpha+P_{\mathrm{Y}} \cdot q\right) q+\beta\left(P_{\mathrm{Y}} \times q\right)+\gamma q \times\left(P_{Y} \times q\right)}{1+\alpha P_{Y} \cdot q}$,

\footnotetext{
+2 Note that Lee and Yang (1957) contains a misprint. The minus sign in the definition of $\beta$ should be replaced by a 2 . In addition, our unit vector $q$ is the direction of the baryon. whereas their unit vector $p$ is the direction of the pion.
} 
where $\boldsymbol{P}_{\mathrm{B}}$ is defined in that rest system of the baryon obtained by a Lorentz transformation along $q$ from the hyperon rest system in which $\boldsymbol{q}$ and $\boldsymbol{P}_{Y}$ are defined. Note that $\alpha$ is the helicity of the decay baryon for unpolarized hyperons.

The three parameters $\alpha, \beta$, and $\gamma$ satisfy the relation $\alpha^{2}+\beta^{2}+\gamma^{2}=1$.

It is then convenient to describe hyperon nonleptonic decays in terms of the two independent parameters $\alpha$ and the angle $\phi$ defined by

$\beta=\left(1-\alpha^{2}\right)^{1 / 2} \sin \phi$.

$\gamma=\left(1-\alpha^{2}\right)^{1 / 2} \cos \phi$,

which has a more nearly gaussian distribution than $\beta$ or $\gamma$. Evidently

$-\frac{1}{2} \pi \leqslant \phi \leqslant \frac{1}{2} \pi$ for $\gamma>0$,

$+\frac{1}{2} \pi \leqslant \phi \leqslant \frac{3}{2} \pi$ for $\gamma<0$.

In discussing time-reversal invariance, the quantity of interest is $\Delta$, defined by

$\alpha=2|s||p| \cos \Delta /\left(|s|^{2}+|p|^{2}\right)$,

$\beta=-2|s||p| \sin \Delta /\left(|s|^{2}+|p|^{2}\right)$;

that is, $\Delta$ is the phase angle of $s$ relative to $p$. Evidently

$-\frac{1}{2} \pi \leqslant \Delta \leqslant \frac{1}{2} \pi$ for $\alpha>0$,

$+\frac{1}{2} \pi \leqslant \Delta \leqslant \frac{3}{2} \pi \quad$ for $\alpha<0$.

Under the assumption of time-reversal invariance, the angle $\Delta$ must satisfy the relation

$\Delta=\delta_{s}-\delta_{p}$,

modulo $\pi$, where $\delta_{s}$ and $\delta_{p}$ are the pion-baryon scattering phase shifts at the appropriate energy and for the appropriate isospin state. For $\Lambda$ decay, assuming the validity of the $\{\Delta I\}=\frac{1}{2}$ rule,

$\Delta=\delta_{s}-\delta_{p}=(7.0 \pm 1.0) \mathrm{deg}^{13}$.

In the Stable Particle Data Card Listings we give $\alpha$ and

43 This value for $\delta_{s}-\delta_{p}$ is derived from the phase-shift analyses by $A y e d(1976)$. The error is our estimation of the uncertainty allowing for possible correlations. $\phi$ for each decay since they are the most closely related to the experiments and are essentially uncorrelated. Whenever necessary we have changed the signs of the reported values, so as to agree with our conventions. In the Stable Particle Table we give $\alpha, \phi$, and $\Delta$ with errors; and for convenience we also give the central value of $\gamma$, without an error.

\section{Statistical procedures}

We divide this discussion on obtaining averages and errors into two sections: and

A. The unconstrained case, or "simple averaging",

B. The constrained case.

In what follows, the term "error" means one standard deviation ( $1 \sigma)$; that is, for cent ral value $\bar{x}$ and error $\varepsilon \bar{x}$, the range $\bar{x} \pm \delta \bar{x}$ constitutes a $68.3 \%$ confidence interval.

\section{A. Unconstrained averaging}

We are returning this year to the use of a stanciard gaussian procedure (with "scale factor") as our only method of averaging the data. The Student's distribution procedure, introduced in 1976 as a second meth od of averaging, has been discontinued. This results primarily from our observation that, although the data are better represented by a Student's distribution, the standard deviation ( $=$ the $68.3 \%$ confidence limit) of this Student's distribution turns out to be equal to the gaussian standard deviation. If one would chouse to quote, e.g., $90 \%$ confidence limits, however, the gaussian piocedure would give too small errors.

We begin by assuming that measurements of a given quantity abe; a gaussian distribution, and thus we calculate a weighted average and error

$$
\bar{x} \pm \delta \bar{x}=\left(\sum_{i} w_{i} x_{i} / \sum_{i} w_{i}\right) \pm\left(\sum_{i} w_{i}\right)^{-1 / 2},
$$

$w_{i}=\left[1 /\left(\delta x_{i}\right)^{2}\right]$,

where $x_{i}$ and $\delta x_{i}$ are the value and error, respectively, reported by the ith experiment, and the sums run over $N$ experiments. We also calculate $\chi^{2}$ and compare it with its expectation value of $N-1$.

If $\chi^{2} /(N-1)$ is less than or equal to 1 , and there are no known problems with the data, we accept the above results. 
If $\chi^{2} /(N-1)$ is very large, or if there is prior knowledge of extremely large inconsistencies between experiments, we may choose not to average the data at all. Alternatively, we may quote the calculated average, but then give an educated guess as to the error; such a guess is generally a quite conservative estimate designed to take into account known problems with the data.

Finally, if $\chi^{2} /(N-1)$ is greater than 1, but not to such a large extent, we still average the data, but then try to make up for this fact in two ways:

(i) We plot an ideogram to guide the reader in deciding which data might be rejected before selected averages are made. An example of such an ideogram is given in fig. 3 below. Each experiment appearing in the plot is represented by a gaussian with central value $x_{i}$, error $\delta x_{i}$, and area proportional to $1 / \delta x_{i}$. The chojce of area is a sonewhat arbitrary one: it is based on the assumption that an experimenter will work to reduce his (or her) systematic errors until they are slightly smaller (but seldom much smaller) than the statistical errors. Thus, as a bubble chamber physicist gets more events, he (or she) will use them both to reduce the statistical errors and to study the biases. Our

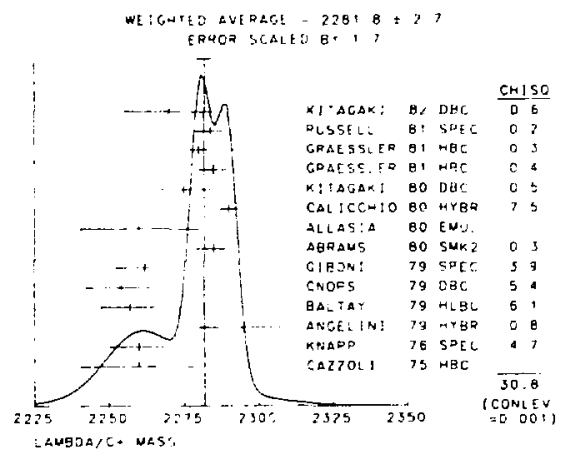

Fig. 3. Ideogram of measurements of the $\lambda_{\mathrm{C}}^{+}$mass. The vertical line indicates the position of the weighted average. while the horizontal bar atop the line gives the error in the average after scaling by the SCALE factor. Only those experiments indicated by + error flags were precise enough to be alcepled in the calculation of the SCALE factor: the column on the far right gives the $x^{2}$ contribution of each of these experiments. The less precise experiments were included in the calculation of the weighted arerage, but not SCALE; they have $\perp$ crror Mags. confidence that a significant systematic error has not been made in a given experiment, as :nmnared with other contradictory experiments, then tends to go up as $1 / \delta x_{i}$.

But why not assign a weight $1 / \delta_{i}^{2}$, as is done when computing a weighted average? We feel that this is equivalent to assuming that large systematic errors are as infrequent as large statistical fluctuations, and that this is unrealistic.

We emphasize the difference between least-squares averaging (where the weighting factor is the inverse square of the error) and the ideograms prepared for visual display. The former arithmetic is of course best if one has statistically distributed input, and yiclds a narrow gaussian distribution centered at the weighted mean. The idcogram (often multipeaked and certainly not gaussion) is based on the opposite hypothesis that some of the input is systematically in error. The idea behind least-squares averaging is that experiments 1,2 . 3, etc., are all valid (so we should multiply their probabilities). Our ideograms are based on the assumption that 1 or 2 or 3 , etc., is valid, "hedged" with $1 / \delta x_{i}$ betting odds; we then add their probabilities. Both approaches cannot simultaneously be right; we leave it to the reader to choose. A glance at the ideogram will show, however, that the discrepancy is often not severe for reasonably distributed input.

(ii) The second way in which we try to take account of $\chi^{2} /(N-1)$ being greater than 1 is to scale up our quoted error $\delta \bar{x}$ in eq. (1) by a factor

$\operatorname{SCALE}=\left[x^{2} /(N-1)\right]^{1 / 2}$.

Our reasoning is as follows. Since we do not know which onc or more of the experiments are wrong, we assume that all experimentalists underestimated their errors by the same scale factor (2). If we scale up all input errors by this factor, $\chi^{2}$ returns to $N-1$, and of course the output error scales up by the same factor.

If all the experiments have errors of about the same size, the above (straightforward) procedure for calculating SCALE is carricd out. If, however, we are to combine experiments with widely varying errors, we must modify the procedure slightly. This is because it is the more precise experiments that most influence not only the average value $\bar{x}$, but also the error $\delta \bar{x}$. Now, on the average, the low-precision experiments each contribute about unity to both the numerator and the denominator of SCALE. hence the $\chi^{2}$ contri- 
bution of the sensitive experiments is diluted, i.e., reduced. Therefore, we evaluate SCALE by using only experiments for which the errors are not much greater than those of the more precise experiments. Explicitly, to calculate SCALE we use only the most sensitive experiments, i.e., those with errors less than $\delta_{0}$, where the ceiling $\delta_{0}$ is (arbitrarily) chosen to be

$\delta_{0}=3 N^{1 / 2} \delta \bar{x}$.

Here $\delta \bar{x}$ is the unscaled error of the mean of all the experiments. Note that if each experiment had the same error $\delta x_{i}$, then $\delta \bar{x}$ would be $\delta x_{i} / N^{1 / 2}$, so each individual experiment would be well under the ceiling on SCALE.

This scaling approach has the property that if there are two values with comparable errors separated by much more than their stated errors (with or without a number of other experiments of lower accuracy), the error on the mean value $\delta \tilde{x}$ is increased so that it is approximately half the interval between the two discrepant values.

We wish to emphasize the fact that our scaling procedures for errors in no way affect central values. In addition. if one wishes to recover the unscaled error $\delta \bar{x}$, one need only divide the given error by the SCALE factor for that error.

\section{B. Constrained fits}

Except for trivial cases, all branching ratios and rate measurements are analyzed by the computer program AHR. This program makes a simultaneous least-squares fit to ail the data, and outputs the partialdecay fractions $\vec{P}_{i}$. width $\Gamma$. partial widths $\Gamma_{i}$, and their error matrix.

The original version of AHR was written by J. Peter Berge. It is documented separately, and we wish here only to give the simplest nontrivial example that permits us to comment on the error matrix and the scale factor.

Assume that a state has only three partial-decay fracticis. $P_{1}, P_{2}$, and $P_{3}\left(\Sigma P_{i}=1\right)$, which have been measured in four different ratios. $R_{1}, \ldots, R_{4}$, where, e.g. $R_{1}=P_{1} / P_{2}, R_{2}=P_{1} / P_{3}$, etc. ${ }^{\$ 4}$ Further assume that eacl ratio has been measured by $N$ experiments

\footnotetext{
:4 We ean handle any $R$ of the form $R=\Sigma \alpha_{i} P_{i} / \Sigma \beta_{i} P_{i}$. where $\alpha_{i}$ and $\beta_{i}$ are constants, usually 1 or 0 . The forms $R=P_{i} \cdot P_{j}$ and $R=\left(\mu_{i} \cdot P_{j}\right)^{1 / 2}$ are also allowed.
}

(we designate cach experiment with a subscript $x$, e.g., $R_{1 x}$ ). Then AHR finds the best values of $P_{1}, P_{2}$, and $P_{3}$ by minimizing $\chi^{2}$, namely

$\chi^{2}=\sum_{r=1}^{4}\left[\sum_{x=1}^{N}\left(\begin{array}{c}R_{r x}-R_{1}\left(P_{1}, P_{2}, P_{3}\right) \\ \delta R_{r x}\end{array}\right)^{2}\right]$.

In addition to the fitted values $\bar{P}_{i}$, the program calculates an error matrix $\left\langle\delta \bar{P}_{i} \delta \bar{P}_{j}\right\rangle$. We tabulate the diagonal elements $\delta \bar{P}_{i}=\left\langle\delta \bar{P}_{i} \delta \bar{P}_{i}\right\rangle^{1 / 2}$ [except that some errors are scaled according to eq. (2) as discussed below]. In the listings we give the complete error matrix: we also calculate the fitted value of each ratio, for comparison with the input data, and list it below the relevant input, along with a simple unconstrained average of the same input.

Two furlher comments on the example above.

(1) There was no connection between measurements of the width and the branching ratios. But often we also have information on partial widths $\Gamma_{i}$ as well as total width $\Gamma$. In this case AHR must introduce $\Gamma$ as a parameter into the fit, along with the relations $\Gamma_{i}=\Gamma P_{i} . \mathbf{\Sigma} \Gamma_{i}=\Gamma$. When appropriate, we tabulate the $\Gamma_{i}$ along with the $P_{i}$ and give error matrices. in the listings.

(2) Note that we do not allow for correlations between input data. We do try to pick those ratios and widths which are as independent and as close to the original data as possible.

In asymmetric errors, we use a continuous function of $\delta(P)^{+}$and $\delta(P)^{-}$in the fitting. When no errors are reported. we merely list the dita for inspection.

Hyperon-decay parameters. The program AHR handles any type of input, $\alpha, \phi, \lambda, \beta$, or $\gamma$, according to the definitions of section VI. If for a particular hyperon decay there are data for more than two of the decay parameters, they ale analyzed by usir $\leftleftarrows$. te constraint

$\alpha^{2}+\beta^{2}+\gamma^{2}=1$.

Inconsistent constrained data. According to our simple example, which led to eq. (3), the double sum for $\lambda^{2}$ is summed over experiments $x^{*}=1$ to $\lambda$. leaving a single sum over ratios

$x^{2}=\sum_{r} x_{r}^{2}$ 
Even before fitting, some of the $\chi_{r}^{2}$ may be too large. But if we scaled them hefore fitting, then the scaling would move the central value, contrary to our policy. So we do not scale until after the first fit; then, knowing the fitted $\chi_{r}^{2}$ and its expectation value $\left\langle\chi_{r}^{2}\right\rangle$ we form SCALE factors (just as before), i.e.,

$$
(\text { SCALE) })_{r}^{2}=\chi_{r}^{2} /\left\langle\chi_{r}^{2}\right\rangle \text {, }
$$

and if any (SCALE), is greater than 1 , all $N$ of the measurements of that particular ratio are equally penalized by having their errors increased by (SCALE), Program AHR then recycles on all the data, those with errors unchanged as well as those with errors increased. We then get new values, $\delta \bar{P}_{i}^{\prime}$ for the errors in the partial-decay modes.

Because of the constraint $\left(\Sigma P_{i}=1\right)$ some SCALE factors may still be greater than I even after this second pass. If this is so, the whole procedure (i.e., increasing errors by the new SCALE factors and recy. cling through AHR) is repeated until AHR has converged.

At the end of AHR's final pass we have two measures of the errors for the $\bar{P}_{i}$. One is, of course, the $\delta \bar{P}_{i}^{\prime}$, i.e., the errors in the final fitted values $\bar{P}_{i}^{\prime}$ which include the effects of scaling the input errors. The other measure of the errors is $\left(\bar{P}_{i}-\bar{P}_{i}^{\prime}\right)$, i.e., the shift in the central values of the $i$ th mode between the first (unscaled) fit and the final (scaled) fit. In practice we find that on the average these two measures of the uncertainty are about equal. Rather than selecting just one or the other, our tabulated errors are given by the combination

$\left(\delta \bar{P}_{i}\right)_{\mathrm{tab}}=\left[\delta \bar{P}_{i}^{\prime 2}+\left(\bar{P}_{i}-\bar{P}_{i}^{\prime}\right)^{2}\right]^{1 / 2}$,

where $\bar{P}_{i}$ is the fitted value of the $i$ th partial-decay mode before scaling, $\bar{P}_{i}^{\prime}$ is its value after scaling, and $\delta \bar{P}_{i}^{\prime}$ is the error in $\bar{P}_{i}^{\prime}$. The SCALE factors we finally list in such cases are defined by

$$
(\mathrm{SCALE})_{i}=\left(\delta \bar{P}_{i}\right)_{\mathrm{tab}} / \delta \bar{P}_{i} \text {. }
$$

However, in line with uur policy of not letting SCALE affect the central values, we give the values of $\bar{P}_{i}$ obtained from the original (unscaled) fits. [The differences between the $\bar{P}_{i}$ calculated with either the scaled or the unscaled errors are, of course, aiways within the tabulated errors, $\left(\delta \bar{P}_{i}\right)_{\text {tab }}$.]

\section{Acknowledgments}

The Particle Data Group wishes to acknowledge with appreciation the contributions made by Naomi Barash-Schmidt throughout most of the years of the Review of Particle Properties.

We thank all those who have assisted in the many phases of preparing this Review. In particular, we acknowledge the usefulness of feedback from the physics community, especially those who have made suggestions or pointed out errors. The European members of the Particle Data Group wish to acknowledge the generous support of CERN, in particular Division $E P$ and $L_{1} \quad A$. Günther and his services.

\section{References}

C.H. Albright, Phys. Rev. 115 (1959) 750.

R. Ayed, CEA-N-192, Sacliay thesis (1976).

A. Barbaro-Galtieri, Baryon resonances, in: Advances in particle physics, eds. R.L. Cool and R.E. Marshak (Wiley. New York, 1968) Vol. 2, sec specifically, table IV and figs. 10 and 12.

E. Barrelet, Nuovo Cimento 8A (1972) 33 I.

I. Bender, V. Linke and H.J. Rothe, Z. Phys. $212(1968) 190$.

N. Cabibbo. Phys. Rev. Lett. 10 (1963) 531.

N. Cabibbo and A. Maksy mowicz. Phy's. Lett. 9 (1964) 352.

L.M. Chounct, J.M. Gaillard and M.K. Gaillard. Phys. Rep. 4C (1972) 199.

H.W. Fcaring. E. Fischbach and J. Smilh, Phys. Rev. D2 (1970) 542 .

S. Gasiorowicz, Elementary particic physics (Wiley, New York, 1966).

G. Giacomelli et al. Nucl. Phys. B71 (1974) 138.

M.L. Goldberger and S.B. Treiman, Phys. Rev. $11(1958) 354$.

J.D. Jackson, S.D. Treiman and H.W. Wyld Jr.. Phys. Rev. $106(1957) 517$.

J.D. Jackson, Nuovo Cimento 34 (1964) 1644.

G. Källèn, Elementary particle pliysies (Addison-Wesley, Reading, MA, 1964).

T. Kinoshita and A. Surlin, Plyys, Rev, 108 (1957) 844.

M. Kobayashi and T. Maskawa, Prog. Theor. Phys. 49 (1973) 652.

J.G. Laytcr, J.A. Appel. A. Kotlewski, W. Lec, S. Stcin and J.J. Thaler, Phys. Rev. Lett. 29 (1972) 316.

T.D. Lee and C.N. Yang. Phys. Rev. 10s (1957) 1615.

R. Levi-Setti, Rapporteur talk Lund Intern. Conf. on Particle physies (Lund, June 1969).

B.R. Martin, Nuel. Phys. B94 (1975) 413.

L.B. Okun and C. Rubbja, Proc. Heidelberg Conf. on Élementary particles (1967) p. 301. 
Particle Data Group: N. Barash-Schmidt, A. Barbaro-Galtieri, L.R. Price, A.H. Rosenfeld, P. Söding, C.G. Wohl, M. Roos and G. Conforto, Rev. Mod. Phys. 41 (1969) 109.

Particle Data Group: R.L. Kelly, C.P. Horne, M.J. Losty, A. Rittenberg, T. Shimada, T.G. Trippe, C.G. Wohl, G.P. Yost, N. Barash-Schmidt, C. Bricman, C. Dionisi, M. Mazzucato, L. Montanet, R.L. Crawford, M. Roos and B. Armstrong, Rev. Mod. Phys. 52 (1980) S1.

J. Pisút and M. Roos, Nucl. Phys. B6 (1968) 325.

L.D. Roper, R.M. Wright and B.T. Feld, Phys. Rey, 138 (1965) B190.

A.H. Rosenfeld, Ann. Rev. Nucl. Sci. 25 (1975) 555.

A.M. Sachs and A. Sirlin, in: Muon physics II, eds. C.S. Wu and V. Hughes (Academic Press, New York, 1975) p. 49.
F. Scheck, Phys. Rep. 44 (1978) 187.

R.E. Shrock and Ling-Lie Wang, Phys. Rev. Lett. 41 (1978) 692.

J. Steinberger, CERN Topical Conf, on Weak interactions, CERN 69-7 (1969) p. 291.

S. Weinberg, Phys. Rev. Lett. 4 (1960) 87

$W$. Willis and $J$. Thompson, Leptonic decays of elementary particles, in: Advances in particle physics, eds. R.L. Cool and R.E. Marshak (Wiley, New York, 1968) Vol. 1, p. 295.

L. Wolfenstein, in: Theory and phenomenology in particle physics, ed. A. Zichichi (Academic Press, New York, 1969) F. 218.

T.T. Wu and C.N. Yang, Phys. Rev. Lett. 12 (1964) 380. 


\title{
TABLES OF PARTICLE PROPERTIES
}

\author{
April 1982
}

M. Aguilar-Benitez, R.L. Crawford, R. Frosch, G.P. Gopal, R.E. Hendrick, R.L. Kelly, M.J. Losty. L. Montanet.

F.C. Porter, A. Rittenberg, M. Roos, LD. Roper, T. Shimada, R.E. Shrock, T.G. Trippe, Ch, Walck, C.G. Woht, G.P. Yost

(Closing date for data; Jan. 1, 1982)

\section{Stable Particle Table}

\section{For additional paraneters, see Addendum to this table.}

Quantities in ilalics have changed by more than one (old) standard deviation since Api '?1980.

\begin{tabular}{|c|c|c|c|c|c|c|c|}
\hline \multirow[t]{2}{*}{ Particle } & \multirow{2}{*}{$\left.\mathbf{I}_{(\mathbf{J}} \mathbf{G}^{\mathbf{P}}\right) \mathbf{C}_{\mathbf{n}}{ }^{a}$} & \multirow{2}{*}{$\begin{array}{l}\text { Mess }^{\circ} \\
\text { (MeV) }^{2} \\
\text { Mass } \\
\left(\mathrm{GeV}^{2} \text { ) }\right.\end{array}$} & \multirow{2}{*}{$\begin{array}{c}\text { Mean life }{ }^{b} \\
\text { (sec) } \\
\text { ct } \\
\text { (cm) }\end{array}$} & \multicolumn{4}{|c|}{ Partial decay mode } \\
\hline & & & & Mode & Fraction $^{b}$ & & $\begin{array}{c}\mathbf{P}^{\text {or }} \\
\mathrm{P}_{\max } \\
(\mathrm{M} \mathrm{V} / \mathrm{C})\end{array}$ \\
\hline \multicolumn{8}{|c|}{ PHOTON } \\
\hline $\boldsymbol{\gamma}$ & $0.1\left(1^{-}\right)-$ & $\left(<6 \times 10^{-22}\right)$ & -...-. & stable & & & \\
\hline \multicolumn{8}{|c|}{ LEPTONS } \\
\hline$y_{e}$ & $J=\frac{1}{2}$ & $(<0.000046)^{d}$ & $\begin{array}{l}\text { stable } \\
\left(>3 \times 10^{8} \mathrm{~m}_{\nu_{\mathrm{e}}}(\mathrm{MeV})\right)\end{array}$ & stable & & & \\
\hline $\mathbf{e}$ & $J=\frac{1}{2}$ & $\begin{array}{r}0.5110034 \\
\pm 0.0000014 \\
\end{array}$ & $\begin{array}{l}\text { stable } \\
\left(>? \times 10^{22} y\right)\end{array}$ & stable & & & \\
\hline$\nu_{\mu}$ & $J=\frac{1}{2}$ & $0(<0.52)$ & $\begin{array}{l}\text { stable } \\
\left(>1.1 \times 10^{5} \mathrm{~m}_{\nu_{\mu}}(\mathrm{MeV})\right.\end{array}$ & stable & & & \\
\hline$\mu$ & $J=\frac{1}{2}$ & $\begin{array}{c}105.65943 \\
\pm 0.00018 \\
\mathrm{~m}^{2}=0.01116392\end{array}$ & $\begin{array}{l}2.19714 \times 10^{-6} \\
\pm 0.00007 \\
c \tau=6.5868 \times 10^{4}\end{array}$ & $\begin{array}{l}\quad\left(\text { or } \mu^{+} \rightarrow \mathrm{CC}\right) \\
\mathrm{e}^{-\bar{\nu} \nu} \\
\mathrm{e}^{-\bar{\nu} \nu \gamma} \\
{\left[\mathrm{e}^{-} \nu_{\mathrm{e}} \bar{\nu}_{\mu}\right.} \\
\mathrm{e}^{-\gamma} \\
\mathrm{e}^{-} \mathrm{e}^{+} \mathrm{e}^{-} \\
\mathrm{e}^{-} \gamma \gamma\end{array}$ & 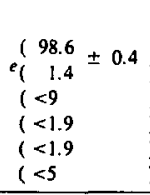 & $\begin{array}{l}j \% \\
; \\
; \% \\
3 \times 10^{-10} \\
3 \times 10^{-9} \\
j \times 10^{-8}\end{array}$ & $\begin{array}{l}53 \\
53 \\
53 \\
53 \\
53 \\
53 \\
\end{array}$ \\
\hline$y_{T}$ & $J=\frac{1}{2}$ & $<250$ & & & & & \\
\hline $\boldsymbol{\tau}$ & $J=\frac{1}{2}$ & $\begin{array}{c}1784.2 \\
\pm 3.2 \\
\mathrm{~m}^{2}=3.18\end{array}$ & $\begin{array}{l}(4.6 \pm 1.9) \times 10^{-13} \\
c \tau=0.014\end{array}$ & 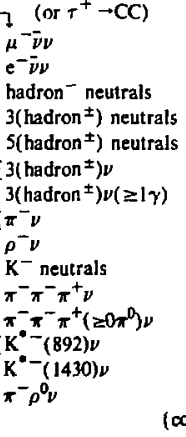 & 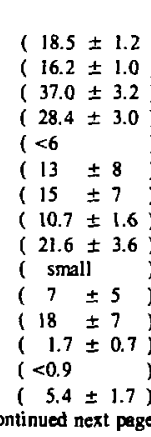 & 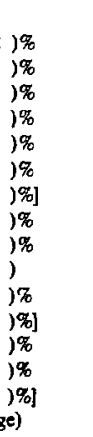 & $\begin{array}{l}887 \\
726 \\
864 \\
864 \\
669 \\
316 \\
718\end{array}$ \\
\hline
\end{tabular}


Stable Particle Table (cont'd)

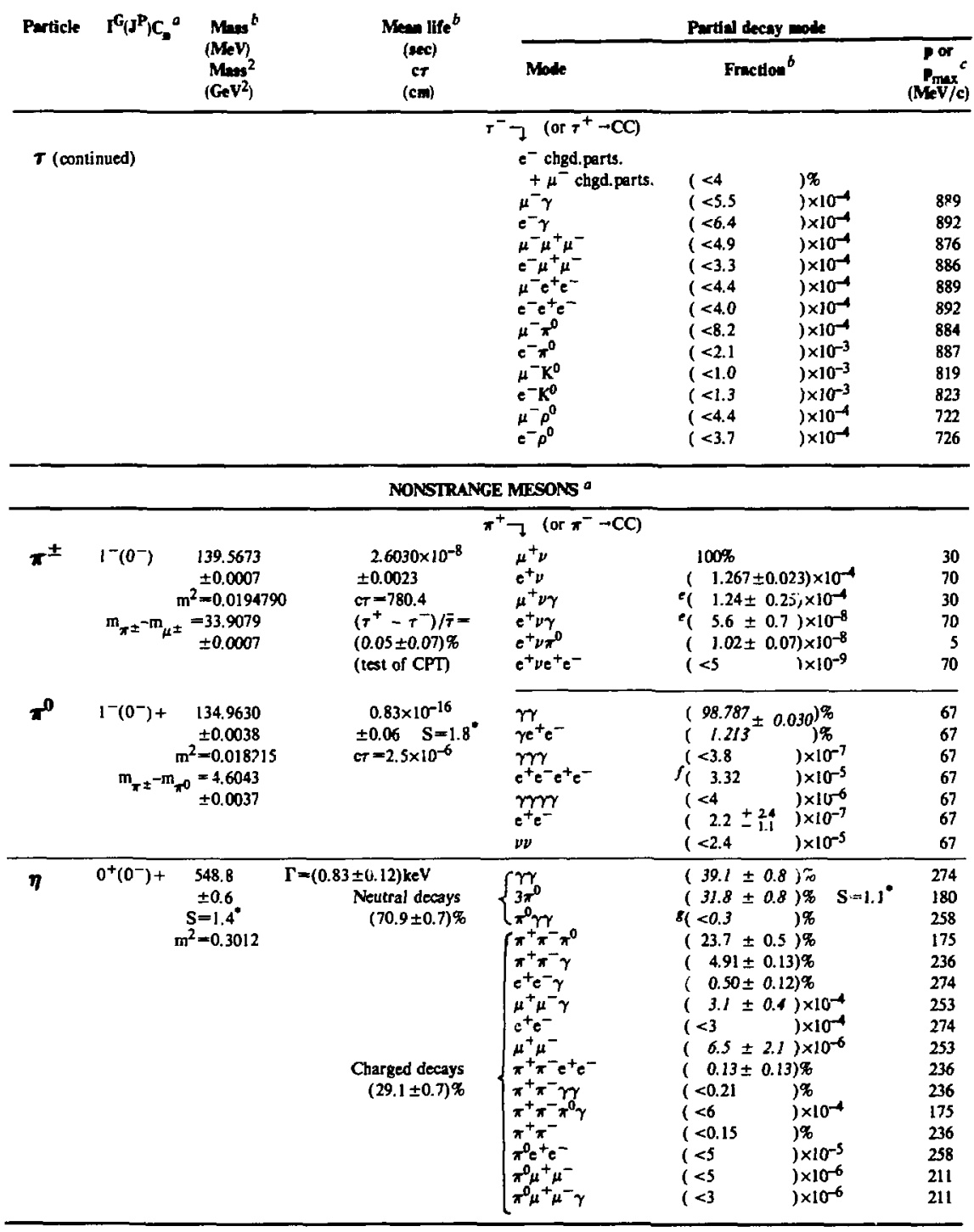


Stable Particle Table (cont'd)

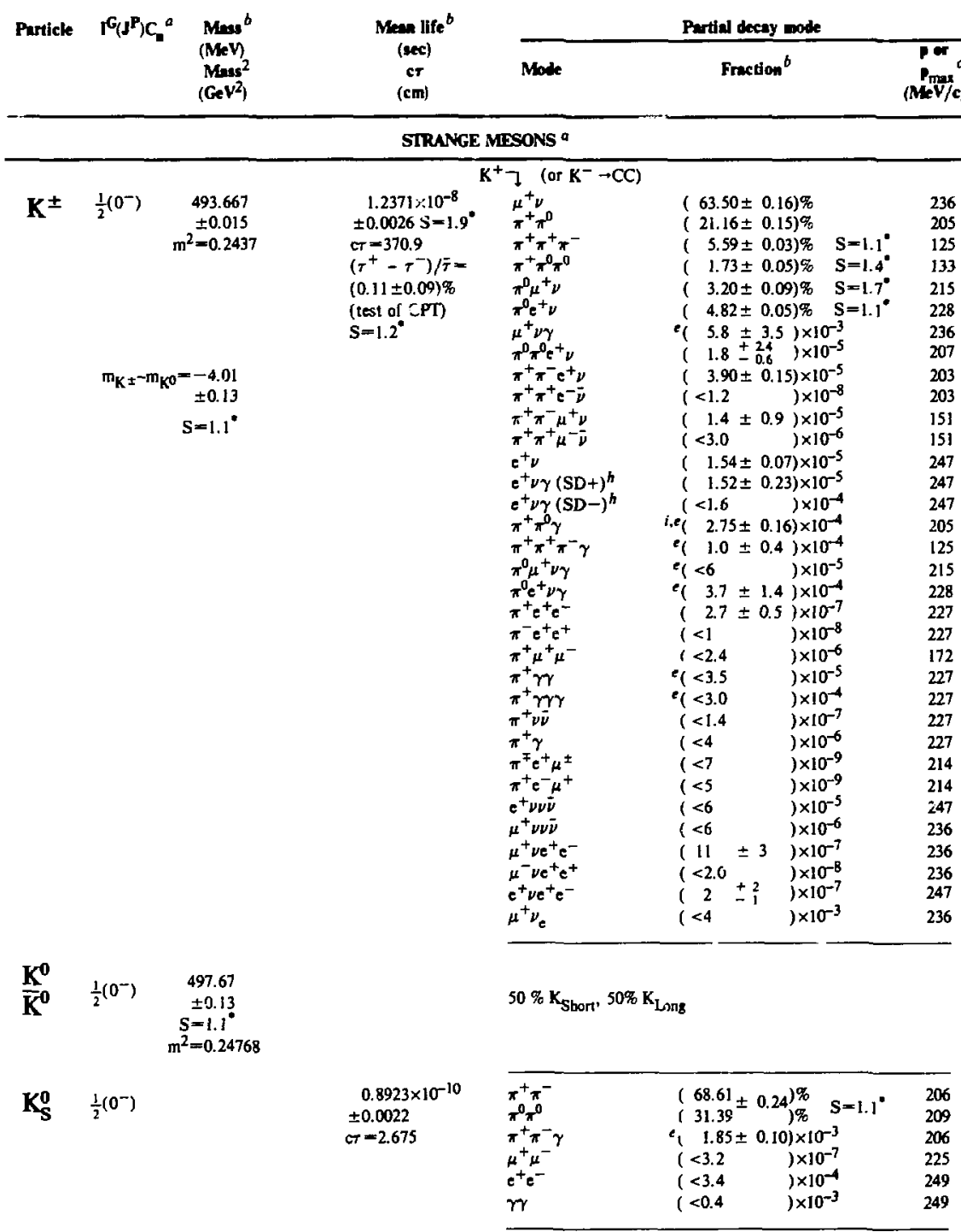


Stable Particle Table (cont'd)

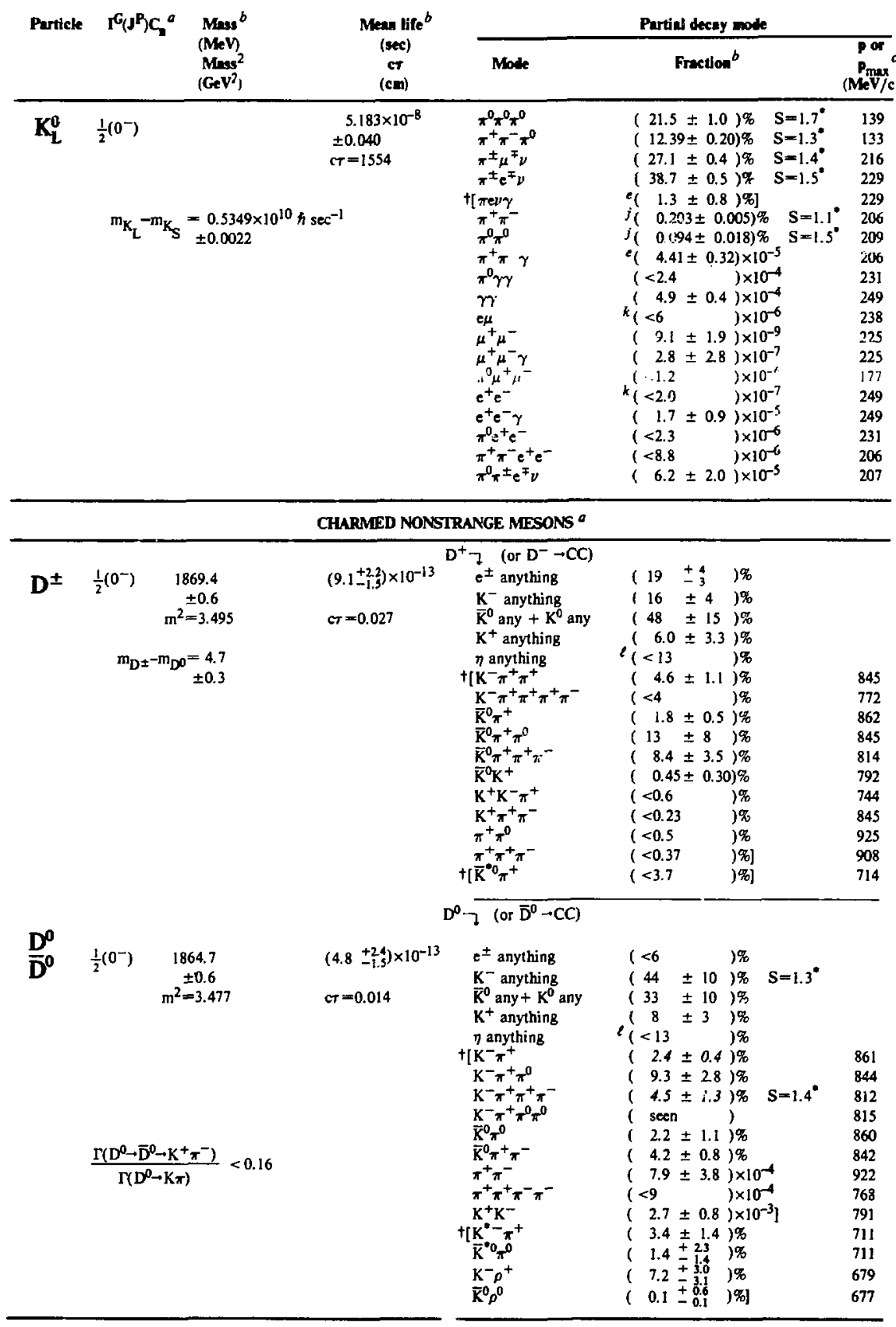


Stable Particle Table (cont'd)

\begin{tabular}{|c|c|c|c|c|c|c|c|}
\hline \multirow[t]{2}{*}{ Particle } & \multirow{2}{*}{$\mathbf{I}_{(\mathbf{J}} \mathbf{P}_{)} \mathbf{C}_{\mathbf{n}}{ }^{a}$} & \multirow{2}{*}{$\begin{array}{c}\text { Mess }^{b} \\
\text { (MeV) } \\
\text { Masss }^{2} \\
\left(\mathrm{GeV}^{2} \text { ) }\right.\end{array}$} & \multirow{2}{*}{$\begin{array}{c}\text { Meas life } \\
\text { (sec) } \\
\text { ct } \\
(\mathrm{cm})\end{array}$} & \multicolumn{4}{|c|}{ Partial decay mode } \\
\hline & & & & Mode & Fra & & $\begin{array}{l}P_{\text {or }} \\
P_{\max } \\
(\mathrm{MeV} / \mathrm{c}\end{array}$ \\
\hline \multicolumn{8}{|c|}{ CHARMED STRANGE MESON ${ }^{a}$} \\
\hline $\mathbf{F}^{ \pm}$ & $0\left(0^{\circ}\right)^{m}$ & $\begin{array}{r}2021 \\
\pm 15\end{array}$ & $\left(2.2_{-1.1}^{+28}\right) \times 10^{-13}$ & $\begin{array}{l}7\left(\text { or } \mathbf{F}^{-} \rightarrow C C\right) \\
\eta \pi^{+} \\
\eta \pi^{+} \pi^{+} \pi^{-} \\
\eta^{\prime} \pi^{+} \pi^{+} \pi^{-} \\
\rho^{+} \phi\end{array}$ & 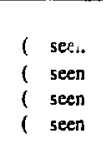 & $\begin{array}{l}\text { ) } \\
\text { ) }\end{array}$ & $\begin{array}{l}930 \\
885 \\
713 \\
467\end{array}$ \\
\hline \multicolumn{8}{|l|}{$\rightarrow \mathrm{B}$} \\
\hline \multicolumn{8}{|c|}{ NONSTRANGE BARYONS ${ }^{\circ}$} \\
\hline p & $\frac{1}{2}\left(\frac{1}{2}^{+}\right)$ & $\begin{aligned} 938.2796 \\
\pm 0.0027 \\
m^{2}=0.880369\end{aligned}$ & stable $\left(\geq 8 \times 10^{30} y\right)$ & $\begin{array}{l}\text { stable } \\
\left|q_{p}\right|-\left|q_{e}\right|<10^{-2} \mid\end{array}$ & $\left|q_{e}\right|^{n}$ & & \\
\hline $\mathbf{n}$ & $\begin{array}{l}\frac{1}{2}\left(\frac{1}{2}^{+}\right) \\
m_{p}-m_{n}=\end{array}$ & $\begin{aligned} & 939.5731 \\
& \pm 0.0027 \\
\mathrm{n}^{2} & =0.882798 \\
= & -1.29343 \\
& \pm 0.00004\end{aligned}$ & $\begin{array}{l}925 \pm 11 \\
c \tau=2.77 \times 10^{13}\end{array}$ & $\begin{array}{l}\mathrm{pe}^{-\bar{\nu}} \\
\mathrm{p} \nu \bar{\nu} \text { (chg.noncons.) } \\
\left|\mathrm{q}_{n}\right|<10^{-21}\left|\mathrm{q}_{e}\right|^{n}\end{array}$ & $\begin{array}{l}100 \% \\
1<9\end{array}$ & )$\times 10^{-24}$ & 1 \\
\hline \multicolumn{8}{|c|}{ STRANGENESS -1 BARYONS ${ }^{\circ}$} \\
\hline $\boldsymbol{\Lambda}$ & $\begin{array}{l}o\left(\frac{1}{2}^{+}\right) \\
m_{\Lambda^{-}}-m_{2}= \\
\end{array}$ & $\begin{array}{c}1115.60 \\
\pm 0.05 \\
S=1.2^{*} \\
\mathrm{n}^{2}=1.2446 \\
=-76.86 \\
\pm 0.08\end{array}$ & $\begin{array}{l}2.632 \times 10^{-10} \\
\pm 0.020 \mathrm{~S}=1.6^{\circ} \\
\mathrm{GT}=7.89\end{array}$ & $\begin{array}{l}\mathrm{p} \pi^{-} \\
\mathrm{n} \pi^{0} \\
\mathrm{pe} e^{-\nu} \\
\mathrm{p} \mu^{-\nu} \\
\mathrm{p} \pi^{-} \gamma\end{array}$ & $\begin{array}{l}64.2 \\
(35.8 \pm \\
(8.35 \pm \\
\text { ( } 1.57 \pm \\
8.5 \pm\end{array}$ & $\begin{array}{l}0.5 \% \\
\% \% \\
0.15) \times 10^{-4} \\
0.35) \times 10^{-4} \\
1.4) \times 10^{-4}\end{array}$ & $\begin{array}{l}100 \\
104 \\
163 \\
131 \\
100\end{array}$ \\
\hline$\Sigma^{+}$ & $\begin{array}{l}1\left(\frac{1}{2}^{+}\right) \\
m_{\Sigma^{+}}-m_{z^{-}}=\end{array}$ & $\begin{array}{c}1189.36 \\
\pm 0.06 \\
S=1.8^{\circ} \\
n^{2}=1.4146 \\
=-7.97 \\
\pm 0.07 \\
S=1.3^{\circ}\end{array}$ & $\begin{array}{l}0.800 \times 10^{-10} \\
\pm 0.004 \\
c \tau=2.40\end{array}$ & $\begin{array}{l}\mathrm{p}^{0} \\
\mathrm{n} \pi^{+} \\
\mathrm{p \gamma} \gamma \\
n \pi^{+} \gamma \\
\Lambda \mathrm{e}^{+} \nu \\
\mathrm{n} \mu^{+} \nu \\
\mathrm{ne}{ }^{+} \nu \\
\mathrm{pe}^{+} \mathrm{e}^{-}\end{array}$ & 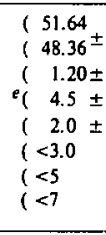 & $\begin{array}{l}0.30 \% \\
0.13) \times 10^{-3} \\
0.5) \times 10^{-4} \\
0.5) \times 10^{-5} \\
) \times 10^{-5} \\
) \times 10^{-6} \\
) \times 10^{-6}\end{array}$ & $\mathrm{~S}=1.4^{\circ} \begin{array}{r}189 \\
185 \\
225 \\
185 \\
71 \\
202 \\
224 \\
225\end{array}$ \\
\hline$\Sigma^{\mathbf{v}}$ & 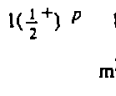 & $\begin{array}{c}1192.46 \\
\pm 0.08 \\
\mathrm{a}^{2}=1.4220\end{array}$ & $\begin{array}{l}5.8 \times 10^{-20} \\
\pm 1.3 \\
c \tau=1.7 \times 10^{-9}\end{array}$ & $\begin{array}{l}\Delta \gamma \\
\Lambda \mathrm{e}^{+} \mathrm{e}^{-} \\
\Lambda \gamma \gamma\end{array}$ & $\begin{array}{l}100 \% \\
\varepsilon_{1} \quad 5.45 \\
i<3\end{array}$ & $\begin{array}{l}3 \times 10^{-3} \\
3 \%\end{array}$ & $\begin{array}{l}74 \\
74 \\
74\end{array}$ \\
\hline$\Sigma^{-}$ & $\begin{array}{l}\mathrm{I}\left(\frac{1}{2}^{+}\right) \\
\mathrm{m}_{\Sigma^{0-}} \mathrm{m}_{\Sigma^{-}}=\end{array}$ & $\begin{array}{l}1197.34 \\
\pm 0.05 \\
2^{2}=1.4336 \\
=-4.88 \\
\pm 0.06\end{array}$ & $\begin{array}{l}1.482 \times 10^{-10} \\
\pm 0.011 \mathrm{~S}=1.3^{0} \\
\mathrm{cT}=4.44\end{array}$ & $\begin{array}{l}\mathrm{n} \pi^{-} \\
\mathrm{ne} \mathrm{e}^{-\nu} \\
\mathrm{n} \mu^{-\nu} \\
\Lambda \mathrm{e}^{-} \nu \\
\mathrm{n} \pi^{-} \gamma\end{array}$ & 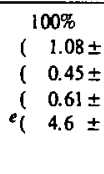 & $\begin{array}{l}0.04) \times 10^{-3} \\
0.04) \times 10^{-3} \\
0.05) \times 10^{-4} \\
0.6) \times 10^{-4}\end{array}$ & $\begin{array}{r}193 \\
230 \\
210 \\
79 \\
193\end{array}$ \\
\hline
\end{tabular}




\section{Stable Particle Table (cont'd)}

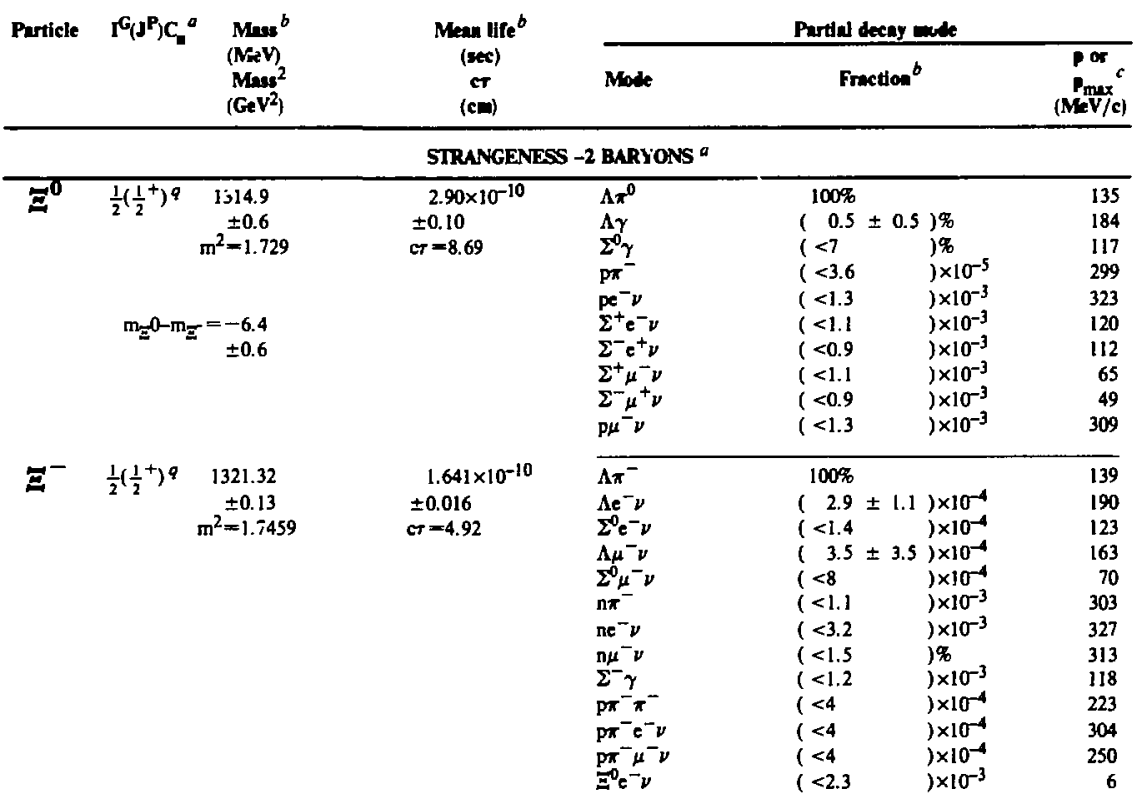

STRANGENESS -3 BARYON ${ }^{\circ}$

\begin{tabular}{|c|c|c|c|c|c|c|c|}
\hline $\mathbf{\Omega}^{-}$ & $\alpha\left(\frac{3}{2}^{+}\right)^{q}$ & $\begin{array}{c}1672.45 \\
\pm 0.32 \\
\mathrm{~m}^{2}=2.7971\end{array}$ & $\begin{array}{l}0.819 \times 10^{-10} \\
\pm 0.027 \\
c r=2.46\end{array}$ & $\begin{array}{l}\Delta K^{-} \\
\Xi^{0} \pi^{-} \\
\Xi^{-} \pi^{0} \\
\Xi^{0} e^{-} \nu \\
\Xi^{0}(1530) \pi^{-} \\
\Lambda \pi^{-} \\
\Xi^{-} \gamma\end{array}$ & $\begin{array}{l}(68.6 \pm 1.3 \\
(23.4 \pm 1.3 \\
(8.0 \pm 0.8 \\
(-1 \\
(-2 \\
(<1.3 \\
(<3 .)\end{array}$ & $\begin{array}{l}j \% \\
j \% \\
j \% \\
\% \times 10^{-3} \\
3 \times 10^{-3} \\
1 \times 10^{-3}\end{array}$ & $\begin{array}{l}211 \\
294 \\
290 \\
319\end{array}$ \\
\hline
\end{tabular}

NONSTRANGE CHARMED BARYON ${ }^{\circ}$

\begin{tabular}{|c|c|c|c|c|c|c|}
\hline$\Delta_{c}^{+}$ & $O\left(\frac{1}{2}^{+}\right)^{r}$ & $\begin{array}{r}2282.2 \\
\pm 3.1 \\
5=1.8 \\
\mathrm{~m}^{2}=5.21\end{array}$ & $\begin{array}{l}\left(1.1_{-0.4}^{+0.9}\right) \times 10^{-13} \\
c \tau=0.003\end{array}$ & $\begin{array}{l}\mathrm{pK}^{-} \pi^{+} \\
\mathrm{pK}^{0} \\
\mathrm{p}^{0} \pi^{+} \pi^{-} \\
\Lambda \pi^{+} \\
\Lambda \pi^{+} \pi^{+} \pi^{-} \\
\mathbf{2}^{0} \pi^{+} \\
+\mid \mathrm{pK}^{+0} \\
\Delta^{++} \mathbf{K}^{-} \\
\mathrm{pK}^{+-} \pi^{+} \\
\mathrm{e}^{+} \text {anything } \\
+\left[\mathrm{pe}^{+} \text {anything }\right. \\
\Lambda \mathrm{e}^{+} \text {anything }\end{array}$ & 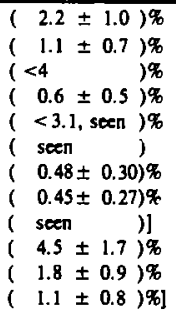 & $\begin{array}{l}820 \\
870 \\
751 \\
861 \\
804 \\
822 \\
682 \\
707 \\
576\end{array}$ \\
\hline
\end{tabular}

\footnotetext{
$\rightarrow \Delta^{0}$

$\rightarrow$ seirches for massive neutrinos and lepton mixing

$\rightarrow \nu$ bounds from astrophysics and cosmology

$\rightarrow$ heavy lepton searches

$\rightarrow$ weak gauge boson searches

$\rightarrow$ free quark searches

$\rightarrow$ megnetic monopole searches

$\rightarrow$ charm searches and evidence

$\rightarrow$ bottom hadron searches

$\rightarrow$ top hadron searches

$\rightarrow$ other stubie particle searches
} 
ADDENDUM TO

Stable Particle Table

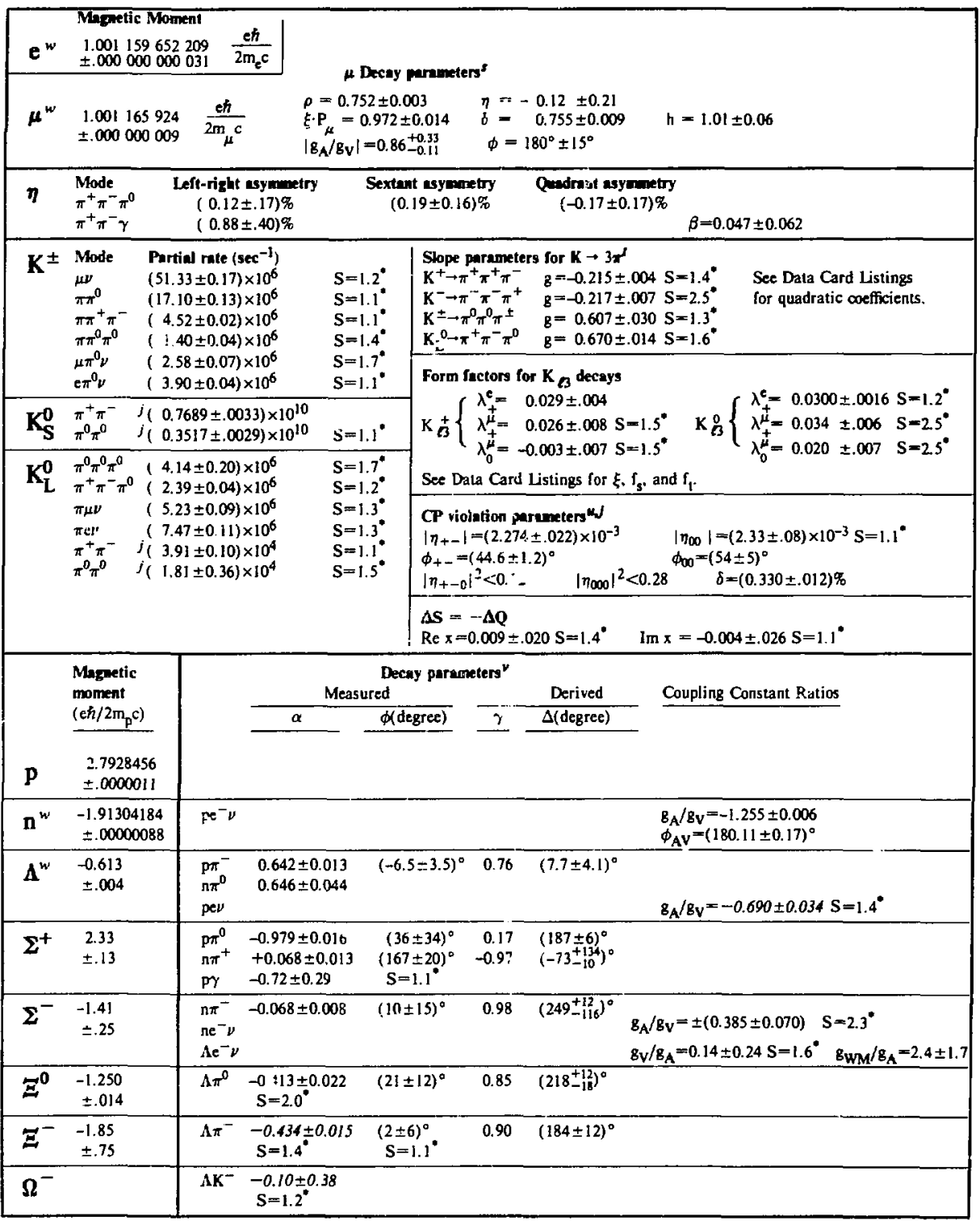




\section{Stable Particle Table (cont'd)}

$\rightarrow$ Indicates an entry in the Stabte Particle Data Card Listings not entered in the Stable Particle Table.

- $S=$ Scale factor $=\sqrt{\chi^{2} /(\mathrm{N}-1)}$, where $\mathrm{N} \approx$ number of experiments. S should be $\approx 1$. If $\mathrm{S}>1$, we have enlarged the error of the mean, $\delta \overline{\bar{x}}$; i.e., $\delta \overline{\mathrm{x}}-S \delta \overline{\mathrm{x}}$. This convention is still inadequate, since if $S \gg 1$ the experiments are probably inconsistent, and therefore the real uncertainty is probably even greater than Sא. $\mathbf{x}$. See text, and ideograms in Stable Particle Data Card Listings.

+ Square brackets indicate subreactions of some previous unbracketed decay mode(s). Reactions in one set of brackets may overlap with reactions in another set of brackets.

a. The baryon itumber $B$, strangeness $S$, and charm $C$ of the hadrons which appear in the tables are as follows:

\begin{tabular}{|c|c|c|c|c|c|}
\hline Mesons $(B=0)$ & $\mathbf{S}$ & C & Baryons $(B=1)$ & s & $\mathrm{C}$ \\
\hline$\pi, \eta$ & 0 & 0 & $p . n$ & 0 & 0 \\
\hline $\mathrm{K}^{+}, \mathrm{K}^{0}$ & +1 & 0 & $\Lambda, \Sigma$ & -1 & 0 \\
\hline $\mathrm{K}^{-} \cdot \overline{\mathrm{K}}^{0}$ & -1 & 0 & $\Xi$ & -2 & 0 \\
\hline$D^{+}, D^{0}$ & 0 & $+I$ & $\Omega^{-}$ & -3 & 0 \\
\hline$D^{-}, \bar{D}^{0}$ & 0 & -1 & $\mathrm{~A}_{\mathrm{c}}^{+}$ & 0 & +1 \\
\hline $\mathbf{F}^{+}$ & +1 & +1 & & & \\
\hline $\mathrm{F}^{-}$ & -1 & -1 & & & \\
\hline
\end{tabular}

b. Quoted upper limits correspond to a $90 \%$ confidence level.

c. In decays with more than two bodies, $\mathrm{P}_{\max }$ is the maximum nomentum that any particle can have.

d. $99 \%$ confidence level. Lower limit from same experiment. $>14 \mathrm{eV}$, not yet confirmed. See Stable Particle Data Card Listings.

e. See Stable Particle Data Card Listings for energy limits us - 1 in this measurement

f. Theoretical value; see also Stable Particle Data Card Lisings.

g. See note in Stable Particle Data Card Listings.

h. Structure-dependent part with positive (SD+) and nrgative (SD-) photon helicity.

$i$. The direct emission branching fraction is $(1.56 \pm .34) \times 10^{-5}$.

j. The $\mathrm{K}_{\mathrm{S}}{ }^{0} \rightarrow \pi \pi$ and $\mathrm{K}_{\mathrm{L}}{ }^{0} \rightarrow \pi \pi$ rates (and branching fractions) are from our branching fraction and rate fits and do not include results of $K_{-}{ }^{0}-K_{S}{ }^{0}$ interference experiments. The $\left|\eta_{+-}\right|$and $\left|\eta_{\infty 0}\right|$ values given in the addendum are these rates combined with the $\left|\eta_{+-}\right|$and $\left|\eta_{00}\right|$ results from interference experiments.

k. The stronger limit $<2 \times 10^{-9}$ of Clark et al., Phys. Rev. Lett. 26, 1667 (1971) is not listed because of possible (but unknown) systematic errors. See Stable Particle Data Card Listings.

ใ. This is a weighted average of $D^{ \pm}(44 \%)$ and $D^{0}(56 \%)$ branching fractions.

m. Quantum numbers shown are favored but not yet established. See Stable Particle Data Card Listings.

n. Limit from neutrality-of-matter experiments. Assumes $\left|q_{n}\right|=\left|q_{p}\right|-\left|q_{e}\right|$.

p. $J^{P}$ not measured for $\Sigma^{0}$. Assumed same as $\Sigma^{ \pm}$to allow isotriplet association.

q. $P$ for $\Xi$ and $J^{P}$ for $\Omega^{-}$not yet measured. Values shown are $S U(3)$ predictions.

r. $\mathrm{J}^{\mathrm{P}}$ for $\boldsymbol{\Lambda}_{c}^{+}$not yet measured. Values shown are SU(4) predictions.

s. $\left|g_{A} / g_{V}\right|$ defined by $g_{A}^{2}=\left|C_{A}\right|^{2}+\left|C_{A}^{\prime}\right|^{2}, g_{V}^{2}=\left|C_{V}\right|^{2}+\left|C_{V}^{\prime}\right|^{2}$, and $\Sigma<\bar{e}\left|\Gamma_{i}\right| \mu><\bar{\nu}\left|\Gamma_{i}\left(C_{i}+C_{i}^{\prime} \gamma_{5}\right)\right| \nu>; \phi$ defined by $\cos \phi=-\operatorname{Re}\left(C_{A}{ }^{*} C^{\prime}{ }_{V}+C^{\prime}{ }_{A} C_{V}^{*}\right) / 8_{A} B_{V}, P_{\mu}$ is muon longitudinal polarization [for more details, see text Section VI $A$ ].

1. The definition of the slope parameter of the Dalitz piot is as follows [see also text Section VI B.1]: $|M|^{2}=1+g\left(\frac{s_{3}-s_{0}}{m_{\pi^{+}}^{2}}\right)$

u. The definition for the CP violation parameters is as follows [see also text Section VI B.3]:

$$
\begin{aligned}
& \eta_{+-}=\left|\eta_{+-}\right| e^{\mathrm{i} \phi_{+-}}=\frac{\mathrm{A}\left(\mathrm{K}_{\mathrm{L}}^{0} \rightarrow \pi^{+} \pi^{-}\right)}{\mathrm{A}\left(\mathrm{K}_{\mathrm{S}}^{0} \rightarrow \pi^{+} \pi^{-}\right)} \quad \eta_{00}=\left|\eta_{00}\right| e^{\mathrm{i} \phi_{00}}=\frac{\mathrm{A}\left(\mathrm{K}_{\mathrm{L}}^{0} \rightarrow \pi^{0} \pi^{0}\right)}{\mathrm{A}\left(\mathrm{K}_{\mathrm{S}}{ }^{0} \rightarrow \pi^{0} \pi^{0}\right)} \\
& \delta=\frac{\Gamma\left(K_{L}^{0} \rightarrow \ell^{+}\right)-\Gamma\left(K_{L}^{0} \rightarrow \ell^{-}\right)}{\Gamma\left(K_{L}^{0} \rightarrow \ell^{+}\right)+\Gamma\left(K_{-}^{0} \rightarrow \ell^{-}\right)} . \quad\left|\eta_{+-0}\right|^{2}=\frac{\Gamma\left(K_{S}^{0} \rightarrow \pi^{+} \pi^{-} \pi^{0}\right) C P \text { viol. }}{\Gamma\left(K_{L}^{0} \rightarrow \pi^{+} \pi^{-} \pi^{0}\right)}, \quad\left|\eta_{000}\right|^{2}=\frac{\Gamma\left(K_{S}^{0} \rightarrow \pi^{0} \pi^{0} \pi^{0}\right) C P \text { viol. }}{\Gamma\left(K_{L}^{0} \rightarrow \pi^{0} \pi^{0} \pi^{0}\right)} .
\end{aligned}
$$

v. The definition of these quantities is as follows [for more details on sign convention, see text Section VI B]:

$$
\begin{aligned}
& \alpha=\frac{2|\mathrm{~s}||\mathrm{p}| \cos \Delta}{|\mathrm{s}|^{2}+|\mathrm{p}|^{2}} \mid \beta=\sqrt{1-\alpha^{2} \sin \phi} \quad \mathrm{B}_{\mathrm{A}}, \mathrm{g}_{\mathrm{V}}, \mathrm{B}_{\mathrm{WM}} \text { defined by }\left\langle\mathrm{B}_{\mathrm{f}}\left|\gamma_{\lambda}\left(\mathrm{g}_{V}-\mathrm{B}_{\mathrm{A}} \gamma_{5}\right)+\left(\mathrm{g}_{\mathrm{WM}} / \mathrm{m}_{\mathrm{B}_{\mathrm{i}}}\right) \sigma^{2 y} \mathrm{q}_{\mathrm{y}}\right| \mathrm{B}_{\mathrm{i}}\right\rangle \\
& \beta=\frac{-2|s||p| \sin \Delta}{|s|^{2}+|p|^{2}} \mid \gamma=\sqrt{1-\alpha^{2}} \cos \phi \quad \phi_{A V} \text { defined by } g_{A} / g_{V}=\left|g_{A} / B_{V}\right| e^{i \phi_{A V}}
\end{aligned}
$$

$w$. For limits on electric dipole moment, see Data Card Listings. Forbidden by $P$ and $T$ invariance. 


\section{Meson Table}

April 1982

In addition to the entries in the Meson Table, the Meson Data Card Listings contain all substantial claims for meson resonances. See contents of Meson Data Card Listings below.

Quantities in italics are new or have changed by more than one (old) standard deviation since April 1980.
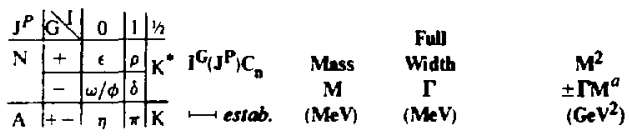

\begin{tabular}{|c|c|c|}
\hline \multicolumn{3}{|c|}{ Partial decay ande } \\
\hline Mode & $\begin{array}{c}\text { Fraction(क) } \\
{[\text { Uper linits }(\%) \text { are } 90 \% \mathrm{CL}]}\end{array}$ & $\begin{array}{c}\text { Por } b \\
\text { Pmas/c) }\end{array}$ \\
\hline
\end{tabular}

NONSTRANGE MESONS

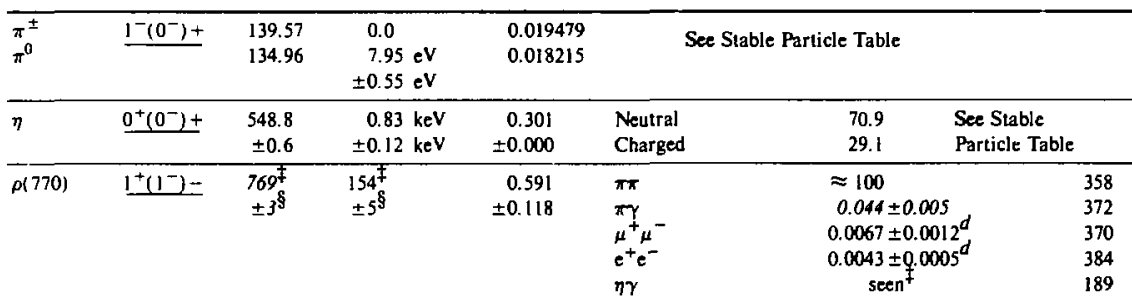

$M$ and $\Gamma$ from neutral mode.

For upper limits, see footnote $e$

\begin{tabular}{|c|c|c|c|c|c|c|c|}
\hline$\omega(783)$ & $0^{-}\left(1^{-}\right)-$ & $\begin{array}{c}782.6 \\
\pm 0.2 \\
S=1.1^{*}\end{array}$ & $\begin{array}{r}9.9 \\
\pm 0.3\end{array}$ & $\begin{array}{r}0.612 \\
\pm 0.008\end{array}$ & $\begin{array}{l}\pi^{+} \pi^{-} \pi^{0} \\
\pi^{0} \gamma \\
\pi^{+} \pi^{-} \\
\pi_{\mu}^{+} \mu^{-} \\
\mathrm{e}^{+} \mathrm{e}^{-} \\
\eta \gamma \\
\text { For upper }\end{array}$ & $\begin{aligned} & 89.9 \pm 0.5 \\
& 8.7 \pm 0.5 \\
& 1.4 \pm 0.2 \\
& 0.010 \pm 0.002 \\
& 0.0072 \pm 0.0007 \mathrm{~S}=1.3^{*} \\
& \text { seen }^{+} \\
& \text {otnote } f\end{aligned}$ & $\begin{array}{l}327 \\
380 \\
366 \\
349 \\
391 \\
199\end{array}$ \\
\hline$\eta^{\prime}(958)$ & $0^{+}\left(0^{-}\right)+{ }^{\ddagger}$ & $\begin{array}{r}957.57 \\
\pm 0.25\end{array}$ & $\begin{array}{r}0.28 \\
\pm 0.10\end{array}$ & $\begin{array}{c}0.917 \\
\pm 0.0003\end{array}$ & $\begin{array}{l}\eta \pi \pi \\
\rho^{0} \gamma \\
\omega \gamma \\
\gamma \gamma \\
\mu^{+} \mu^{-} \gamma \\
\text { For upper }\end{array}$ & $\begin{array}{c}65.3 \pm 1.6 \\
30.0 \pm 1.6 \\
2.8 \pm 0.5 \\
1.9 \pm 0.2 \\
0.009 \pm 0.002 \\
\text { otnote } g\end{array}$ & $\begin{array}{l}23 ! \\
170 \\
159 \\
479 \\
467\end{array}$ \\
\hline$S^{*}(975)$ & $0^{+}\left(0^{+}\right)+$ & $\begin{array}{c}975^{\circ} \\
\pm 4 \\
S=1.4^{\circ}\end{array}$ & $\begin{array}{l}33^{c} \\
\pm 6\end{array}$ & $\begin{array}{r}0.951 \\
\pm 0.032\end{array}$ & $\begin{array}{l}\pi \pi \\
K \bar{K}\end{array}$ & $\begin{array}{l}78 \pm 3 \\
22 \pm 3\end{array}$ & 467 \\
\hline
\end{tabular}

See note on $\pi \pi$ and $K \bar{K} S$ wave.

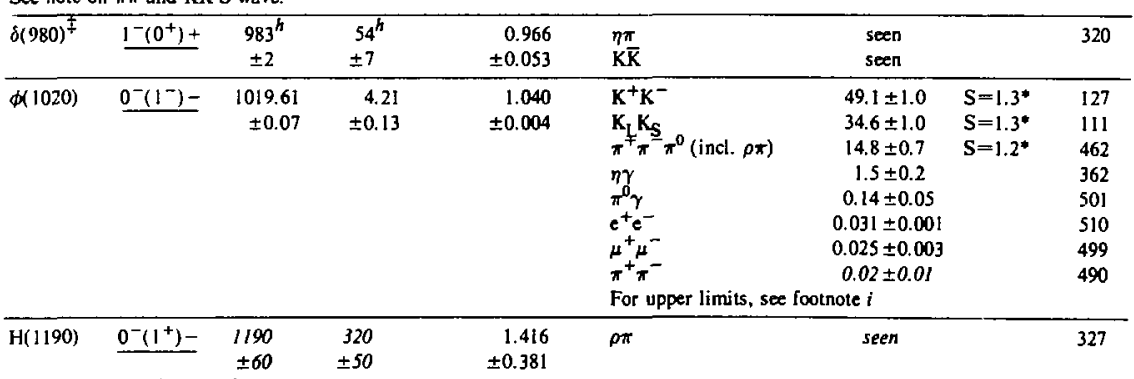

Seen in one experiment only.

\begin{tabular}{|c|c|c|c|c|c|c|}
\hline $\mathrm{B}(1235)$ & $1^{+}\left(1^{+}\right)-$ & $\begin{array}{l}1233 \\
\pm 10^{8}\end{array}$ & $\begin{array}{l}137 \\
\pm 10^{\S}\end{array}$ & $\begin{array}{r}1.52 \\
\pm 0.17\end{array}$ & $\begin{array}{l}\omega \pi \\
{[D / S \text { amplitude ratio }=0.29 \pm 0.05]} \\
\text { For upper limits, see footnote } j\end{array}$ & 349 \\
\hline
\end{tabular}




\section{Meson Table (cont'd)}

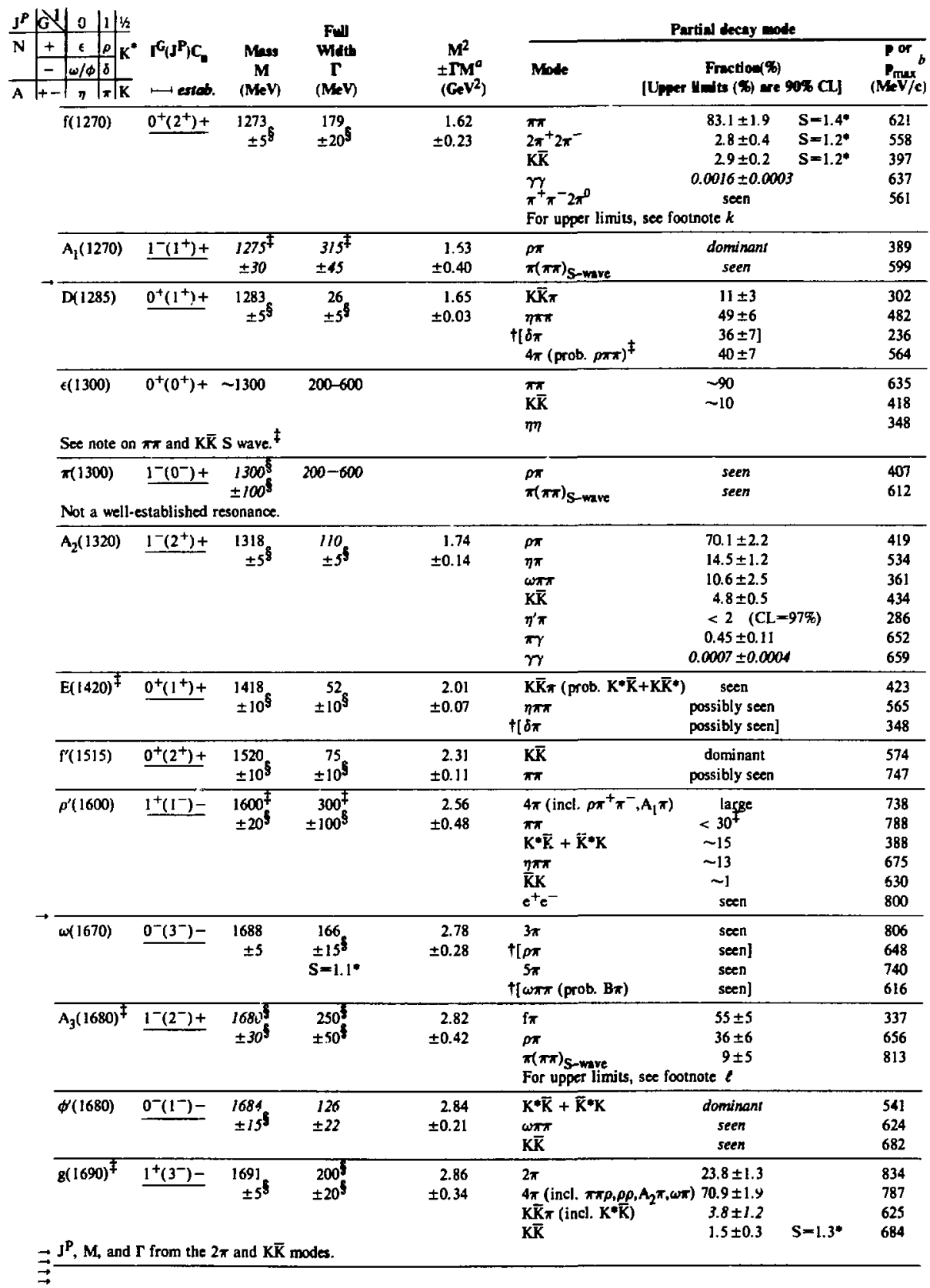


11

\section{Meson Table (cont'd)}

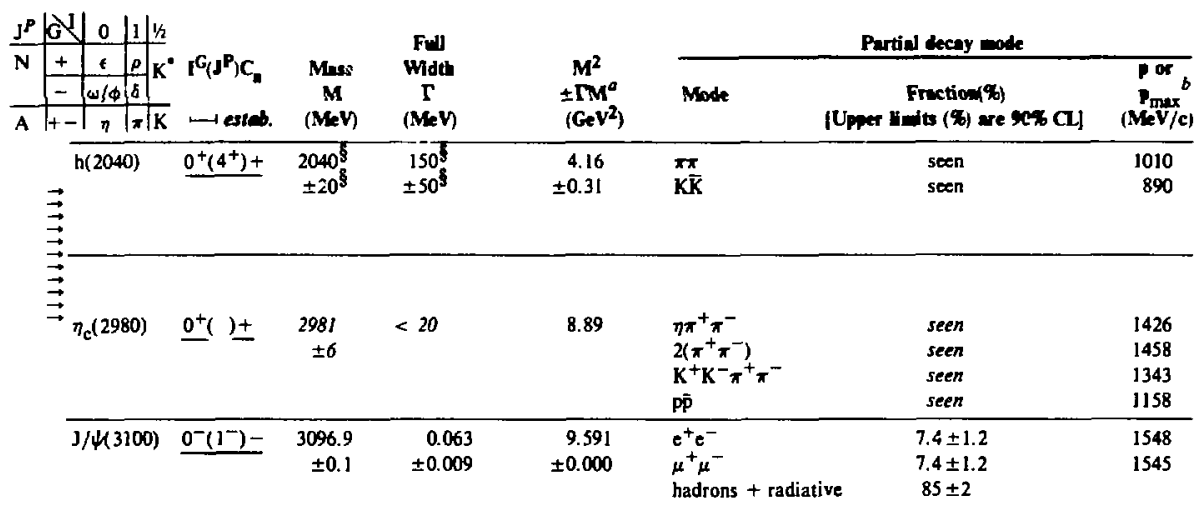

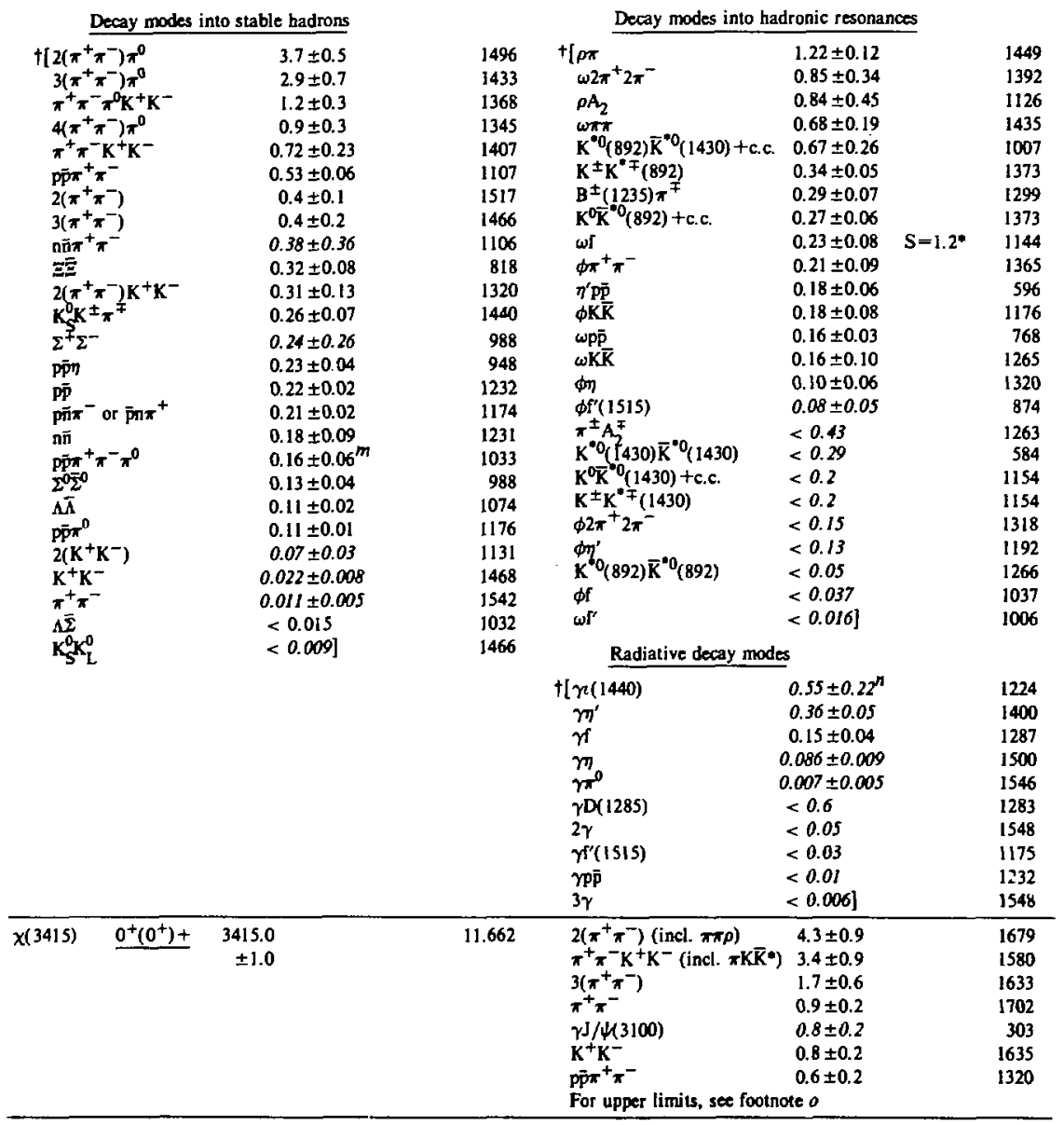


Meson Table (cont'd)

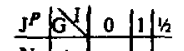

\begin{tabular}{|c|c|c|c|c|}
\hline $\mathbf{Y}$ & \begin{tabular}{|c|c}
+ & $t$ \\
- & $\omega / \phi$ \\
\end{tabular} & $\frac{p}{6} \mathrm{~K}$ & 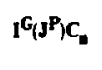 & Men \\
\hline & $+-1 \pi$ & $\prod_{\pi / K}$ & • estab. & (MeV) \\
\hline & $\begin{array}{l}p_{c} \text { or } \\
\chi(35\end{array}$ & is & $\underline{0}^{+}\left(1^{+}\right)+$ & $\begin{array}{r}3510.0 \\
\pm 0.6\end{array}$ \\
\hline
\end{tabular}

Partid decay mode

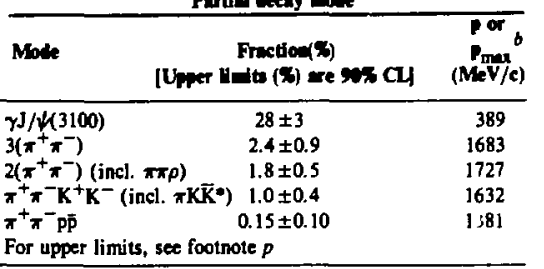

\begin{tabular}{|c|c|c|c|c|c|c|c|}
\hline$x(3555)$ & $0^{+}\left(2^{+}\right)+$ & $\begin{array}{r}3555.8 \\
\pm 0.6\end{array}$ & & 12.644 & $\begin{array}{l}\gamma \mathrm{J} / K^{3}(3100) \\
\left.2\left(\pi^{+} \pi^{-}\right) \text {(incl. } \pi \pi \rho\right) \\
\pi^{+} \pi^{-} \mathrm{K}^{+} \mathrm{K}^{-}\left(\text {incl } \pi \mathrm{KK}^{\infty}\right) \\
3\left(\pi^{+} \pi^{-}\right) \\
\pi^{+} \pi^{-} \mathrm{p} \overline{\mathrm{p}} \\
\pi^{+} \pi^{-} \\
\mathrm{K}^{+} \mathrm{K}^{-}\end{array}$ & $\begin{aligned} 15.7 \pm 1.7 \\
2.3 \pm 0.5 \\
20 \pm 0.5 \\
1.2 \pm 0.8 \\
0.35 \pm 0.14 \\
0.20 \pm 0.11 \\
0.16 \pm 0.12\end{aligned}$ & $\begin{array}{r}429 \\
1750 \\
1656 \\
1706 \\
1410 \\
1772 \\
1708\end{array}$ \\
\hline & & & & & For upper limits, see footno & ote $q$ & \\
\hline$\psi(3685)$ & $0^{-}\left(1^{-}\right)$ & $\begin{array}{r}3686.0 \\
\pm 0.1\end{array}$ & $\begin{array}{r}0.215 \\
\pm 0.040\end{array}$ & $\begin{array}{r}13.587 \\
\pm 0.001\end{array}$ & $\begin{array}{l}\mathrm{e}^{+} \mathrm{e}^{-} \\
\mu^{+} \mu^{-} \\
\text {hadrons + radiative }\end{array}$ & $\begin{array}{r}0.9 \pm 0.1 \\
0.8 \pm 0.2 \\
98.1 \pm 0.3\end{array}$ & $\begin{array}{l}1843 \\
1840\end{array}$ \\
\hline
\end{tabular}

$m_{k(3685)}-m_{k 3100)}=589.06 \pm 0.13$
Radiative decay modes

$\begin{array}{ll}+[\gamma \chi(3415) & 8.2 \pm 1.4 \\ \gamma \chi(3510) & 8.0 \pm 1.3 \\ \gamma \chi(3555) & 7.4 \pm 1.3 \\ \gamma \pi_{c}(2980) & 0.43 \pm 0.26 \\ \gamma \eta^{\prime}(3590) & 0.2 \text { to } 1.3 \\ \gamma \pi^{\prime} & <0.5(\mathrm{CL}=95 \%) \\ \gamma \pi & <0.02 \\ \gamma \eta^{\prime} & <0.02 \\ \gamma(1440) & \left.<0.018^{n}\right]\end{array}$

Decay modes into hadrons

261
172
128
638
91
1841
1802
1719
1570

$4+\pi$

$3 / 4 \pi 0 \pi$

$\mathrm{J} / 4 \pi$

$2\left(\pi^{+} \pi^{-}\right) \pi^{0}$

$\pi^{+} \pi^{-} \mathrm{K}^{+} \mathrm{K}^{-}$

$\mathrm{J} / 4 \pi^{\mathrm{D}}$

$\mathrm{p} \overline{\mathrm{p}} \pi^{+} \pi^{-}$

$K^{* 0}(892) K^{ \pm} \pi^{\mp}$

$2\left(\pi^{+} \pi^{-}\right)$
$M^{2}$ $\pm \operatorname{Im}_{(\mathbf{M}}$ 12.320

$12.320 \quad \gamma \mathrm{J} / \mathrm{k}(3100)$

$2(\pi+\pi)$

$\gamma \mathrm{J} / \mathrm{K}(3100)$

$\pi^{+} \pi^{-} \mathbf{K}^{+} \mathbf{K}^{-}$(incl $\pi \mathrm{K}^{*}{ }^{*}$

$\mathrm{K}^{+} \mathrm{K}$

$0^{0} x^{+} \pi^{-}$

$\hat{\mathbf{p}}$

$3\left(\pi^{+} \pi^{-}\right)$

$\mathrm{K}^{+} \mathrm{K}^{-}$

$\pi^{+} \pi^{-}$

$\rho \pi$

$\bar{A}$

14.213

$\pm 0.094$

$e^{+} e^{-}$
$\mathbf{D}^{-} \overline{\mathbf{D}}$

$m_{\psi(3770)}-m_{\psi(3685)}=83.9 \pm 2.4$

$S=1.8 *$

\begin{tabular}{|c|c|c|c|c|c|c|c|}
\hline$\nvdash(4030)$ & $(1-)$ & $\begin{array}{r}4030^{8} \\
\pm 5^{8}\end{array}$ & $\begin{array}{r}52 \\
\pm 10 \\
\end{array}$ & $\begin{array}{r}16.241 \\
\pm 0.210\end{array}$ & $\begin{array}{l}\mathrm{e}^{+} \mathrm{e}^{-} \\
\text {hadrons }\end{array}$ & $\begin{array}{l}0.0014 \pm 0.0004 \\
\text { dominant }\end{array}$ & 2015 \\
\hline K(4160) & $\left(1^{-}\right)-$ & $\begin{array}{r}4159 \\
\pm 20\end{array}$ & $\begin{array}{r}78 \\
\pm 20\end{array}$ & $\begin{array}{r}17.297 \\
\pm 0.324\end{array}$ & $\begin{array}{l}\mathrm{e}^{+} \mathrm{e}^{-} \\
\text {hadrons }\end{array}$ & $\begin{array}{l}0.0010 \pm 0.0004 \\
\text { dominant }\end{array}$ & 2079 \\
\hline$\overline{\psi(4415)}$ & $\left(1^{-}\right)-$ & $\begin{array}{r}4415 \\
\pm 6\end{array}$ & $\begin{array}{r}43 \\
\pm 20^{5} \\
\end{array}$ & $\begin{array}{r}19.492 \\
\pm 0.190 \\
\end{array}$ & $\begin{array}{l}\mathrm{e}^{+} \mathrm{e}^{-} \\
\text {hadrons }\end{array}$ & $\begin{array}{l}0.0010 \pm 0.0003 S=1.4^{*} \\
\text { dominant }\end{array}$ & 2208 \\
\hline$\Upsilon(9460)$ & $\left(1^{-}\right)-$ & $\begin{array}{r}9456 \\
\pm 10\end{array}$ & $\begin{array}{r}0.042 \\
\pm 0.015 \\
\end{array}$ & $\begin{array}{r}89.416 \\
\pm 0.0004\end{array}$ & $\begin{array}{l}\mu^{+} \mu^{-} \\
e^{+} e^{-}\end{array}$ & $\begin{array}{l}3.2 \pm 0.7 \\
2.8 \pm 1.1\end{array}$ & $\begin{array}{l}4727 \\
4728\end{array}$ \\
\hline$\tau(10020)$ & $\begin{array}{l}\left(1^{-}\right)- \\
m_{\mathrm{r}(10020}\end{array}$ & $\begin{array}{r}10016 \\
\pm 10 \\
-m_{\mathrm{T}(9460)}\end{array}$ & $\begin{array}{r}0.030 \\
\pm 0.010 \\
-559 \pm 3 \\
\end{array}$ & $\begin{array}{r}100.320 \\
\pm 0.0003\end{array}$ & $\begin{array}{l}\mu^{+} \mu^{-} \\
e^{+} e^{-} \\
T(9460) \pi \pi\end{array}$ & $\begin{array}{l}\text { seen } \\
1.7 \pm 0.6 \\
30 \pm 6\end{array}$ & $\begin{array}{r}5007 \\
5008 \\
472\end{array}$ \\
\hline$\Upsilon(10350)$ & $\left(1^{-}\right)-$ & $\begin{array}{r}10347 \\
\pm 10\end{array}$ & & 107.060 & $e^{+} e^{-}$ & seen & 5174 \\
\hline
\end{tabular}

$m_{\Upsilon(10350)}-m_{\Upsilon(9460)}=891 \pm 1$ 
Meson Table (cont'd)

\begin{tabular}{|c|c|c|c|c|c|c|c|c|}
\hline & 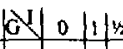 & & & & & \multicolumn{3}{|c|}{ Partial decay mode } \\
\hline $\mathbf{N}$ & \begin{tabular}{|c|c|c|c}
+ & $\epsilon$ & $\rho$ & $K$ \\
- & $\omega / \phi$ & $\delta$ & \\
+- & $\eta$ & $\pi$ & $K$
\end{tabular} & 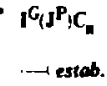 & $\begin{array}{c}\text { Mess } \\
\mathbf{M} \\
\text { (MeV) }\end{array}$ & $\begin{array}{c}\text { width } \\
\Gamma \\
\text { (MeV) }\end{array}$ & $\begin{array}{c}M^{2} \\
\pm \mathbf{M M}^{a} \\
\left(\mathrm{GeV}^{2}\right)\end{array}$ & Mode & $\begin{array}{c}\text { Fractiou(\%) } \\
\text { [Uper binits (\%) are } 90 \% \text { C.] }\end{array}$ & $\begin{array}{c}P_{\text {or }} b \\
(\mathrm{MeV} / \mathrm{c})\end{array}$ \\
\hline & $\mathrm{T}(10570)$ & (1-)- & $\begin{array}{r}10569 \\
\pm 10\end{array}$ & $\begin{array}{r}14 \\
\pm 5\end{array}$ & $\begin{array}{r}111.704 \\
\pm 0.15\end{array}$ & $e^{+} e^{-}$ & $0.0019 \pm 0.0008$ & 5285 \\
\hline & & $\mathrm{m}_{\mathrm{r}(10570)}$ & $-m_{T(9460)}$ & $m$ & & & & \\
\hline
\end{tabular}

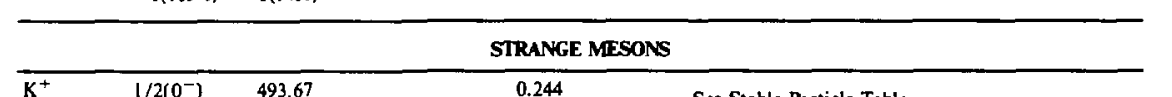

\begin{tabular}{|c|c|c|c|c|c|c|}
\hline $\begin{array}{l}\mathbf{K}^{+} \\
\mathbf{K}^{0}\end{array}$ & $1 / 2\left(0^{-}\right)$ & $\begin{array}{l}493.67 \\
497.67\end{array}$ & & $\begin{array}{l}0.244 \\
0.248\end{array}$ & & cle Table \\
\hline$K^{*}(392)$ & $1 / 2\left(1^{-}\right)$ & $\begin{array}{c}891.8 \\
\pm 0.4 \\
S=1.2^{*}\end{array}$ & $\begin{array}{r}50.8 \\
\pm 0.9\end{array}$ & $\begin{array}{r}0.795 \\
\pm 0.045\end{array}$ & $\begin{array}{l}K_{\pi} \\
K_{\gamma} \\
K_{\pi \pi}\end{array}$ & $\begin{aligned} \approx & 100 \\
& 0.15 \pm 0.07 \\
& <0.07(\mathrm{CL}=95 \%)\end{aligned}$ \\
\hline
\end{tabular}

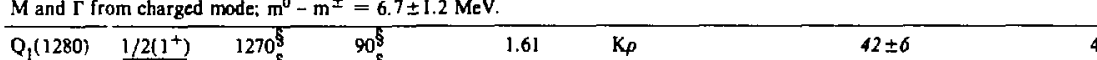

\begin{tabular}{|c|c|c|c|c|c|c|c|}
\hline $\mathrm{Q}_{1}(1280)$ & $\underline{1 / 2\left(1^{+}\right)}$ & $\begin{array}{l}1270^{5} \\
\pm 10^{8}\end{array}$ & $\begin{array}{r}90^{5} \\
+20^{8}\end{array}$ & $\begin{array}{r}1.61 \\
\pm 0.11\end{array}$ & $\begin{array}{l}\mathrm{K} \rho \\
\kappa \pi \\
\mathrm{K}^{*} \pi \\
\mathrm{K} \omega \\
\mathrm{Ke}\end{array}$ & $\begin{array}{r}42 \pm 6 \\
28 \pm 4 \\
16 \pm 5 \\
11 \pm 2 \\
3 \pm 2\end{array}$ & $\begin{array}{r}45 \\
299\end{array}$ \\
\hline$\kappa(1350)$ & $1 / 2\left(0^{+}\right)$ & $\begin{array}{l}\sim 1350 \\
\pm\end{array}$ & $\sim 250$ & $\begin{array}{r}1.82 \\
\pm 0.34\end{array}$ & $K \pi$ & seen & 574 \\
\hline
\end{tabular}

See note on $K \pi \mathrm{S}$ wave. ${ }^{\ddagger}$

\begin{tabular}{|c|c|c|c|c|c|c|c|c|}
\hline$Q_{2}(1400)$ & $\underline{1 / 2\left(1^{+}\right)}$ & $\begin{array}{l}1414 \\
\pm 13\end{array}$ & $\begin{array}{r}180 \\
\pm 10\end{array}$ & $\begin{array}{r}2.00 \\
\pm 0.25\end{array}$ & $\begin{array}{l}\mathrm{K}^{*} \pi \\
\mathrm{K}_{\rho} \\
\mathrm{K} \ell \\
\mathrm{K} \boldsymbol{\omega} \\
\end{array}$ & $\begin{array}{r}94 \pm 6 \\
3 \pm 3 \\
2 \pm 2 \\
I \pm J \\
\end{array}$ & & $\begin{array}{l}410 \\
308 \\
294 \\
\end{array}$ \\
\hline$K^{*}(1430)$ & $1 / 2\left(2^{+}\right)$ & $\begin{array}{r}1434^{\S} \\
\pm 5^{\S}\end{array}$ & $\begin{array}{l}100^{\S} \\
\pm 10^{\S}\end{array}$ & $\begin{array}{r}2.06 \\
\pm 0.14\end{array}$ & $\begin{array}{l}\mathrm{K} \pi \\
\mathrm{K}^{*} \pi \\
\mathrm{K}^{+} \pi \pi \\
\mathrm{K} p \\
\mathrm{~K} \omega \\
\mathrm{K} \eta \\
\end{array}$ & $\begin{array}{r}44.8 \pm 2.3 \\
24.6 \pm 2.0 \\
13.0 \pm 2.6 \\
8.8 \pm 1.1 \\
4.2 \pm 1.5 \\
5 \pm 5^{5}\end{array}$ & $\begin{array}{l}S=2.7^{*} \\
S=1.1^{*} \\
S=1.1^{*} \\
S=1.3^{*}\end{array}$ & $\begin{array}{l}623 \\
424 \\
374 \\
334 \\
320 \\
492 \\
\end{array}$ \\
\hline $\begin{array}{l}\text { L } 1770)^{\frac{7}{7}} \\
\text { See note on }\end{array}$ & $\begin{array}{l}\frac{1 / 2\left(2^{-}\right)}{} \\
L(1770) \neq\end{array}$ & $-1770^{\S}$ & $-200^{\S}$ & $\begin{array}{r}3.13 \\
\pm 0.35\end{array}$ & $\begin{array}{l}\mathrm{K}^{*}(1430) \pi \\
\mathrm{K}^{*}(892) \pi \\
\mathrm{Kf}\end{array}$ & $\begin{array}{l}\text { dominant } \\
\text { seen } \\
\text { seen }\end{array}$ & & $\begin{array}{l}278 \\
652\end{array}$ \\
\hline See note on & $1 / 2\left(3^{-}\right)$ & $\begin{array}{l}1775^{\S} \\
\pm 10^{\S}\end{array}$ & $\begin{array}{r}140^{\S} \\
\pm 20^{\S}\end{array}$ & $\begin{array}{r}3.15 \\
\pm 0.25\end{array}$ & $\begin{array}{c}K \pi \pi \\
+\{K \rho \\
+\left[K^{*} \pi\right. \\
K \pi\end{array}$ & $\begin{array}{l}\text { large } \\
\text { large] } \\
\text { large] } \\
17 \pm 5^{5}\end{array}$ & & $\begin{array}{l}793 \\
616 \\
654 \\
812\end{array}$ \\
\hline \multicolumn{9}{|c|}{ CHARMED, NONSTRANGE MESONS } \\
\hline $\begin{array}{l}D^{+} \\
D^{0}\end{array}$ & $1 / 2\left(0^{-}\right)$ & $\begin{array}{l}1869.4 \\
1864.7 \\
\end{array}$ & & $\begin{array}{l}3.495 \\
3.477 \\
\end{array}$ & See & Table & & \\
\hline $\mathrm{D}^{*+}(2010)$ & $\begin{array}{l}1 / 2\left(1^{-}\right) \\
\because D^{+}+n\end{array}$ & $\begin{array}{r}2010.1 \\
\pm 0.7 \\
=145 .\end{array}$ & $\begin{array}{c}<2.0 \\
0.2 \mathrm{MeV}\end{array}$ & 4.041 & $\begin{array}{l}D^{0} \pi^{+} \\
D^{+} \pi^{0} \\
D^{+} \gamma\end{array}$ & $\begin{array}{c}64 \pm 11 \\
28 \pm y \\
8 \pm 7\end{array}$ & & $\begin{array}{r}39 \\
38 \\
136\end{array}$ \\
\hline$D^{* 0}(2010)$ & $1 / 2\left(1^{-}\right)$ & $\begin{array}{r}2007.2 \\
\pm 2.1\end{array}$ & $<5$ & 4.029 & $\begin{array}{l}D^{0} \pi^{0} \\
D^{0} \gamma\end{array}$ & $\begin{array}{l}55 \pm 15 \\
45 \pm 15\end{array}$ & & $\begin{array}{r}44 \\
137\end{array}$ \\
\hline
\end{tabular}

CHARMED, STRANGE MESON

$\vec{\rightrightarrows}$\begin{tabular}{lllll}
\hline $\mathrm{F}^{+}$ & $0\left(0^{-}\right)$ & 2021 & 4.084 & See Stable Particle Table \\
\hline
\end{tabular}




\section{Meson Table (cont'd)}

Contents of Mesom Dotis Card Listines

\begin{tabular}{|c|c|c|c|c|c|c|c|c|c|}
\hline \multicolumn{8}{|c|}{ Non-strange ( $S=0 ; C, B=0$ ) } & \multicolumn{2}{|c|}{ Strange $(|S|=1 ; C, B=0)$} \\
\hline \multicolumn{2}{|r|}{ entry } & $\left.\mathbf{I}^{\mathrm{G}_{(}} \mathrm{J}^{\mathrm{P}}\right) \mathrm{C}_{\mathrm{n}}$ & entry & $\mathrm{I}^{\mathrm{G}}\left(\mathrm{J}^{\mathrm{P}}\right) \mathrm{C}_{\mathrm{n}}$ & \multicolumn{2}{|c|}{ entry } & $\left.I^{G_{(J}}{ }^{P}\right) C_{n}$ & entry & $I\left(J^{P}\right)$ \\
\hline$\pi$ & & $1^{-}\left(0^{-}\right)+$ & $f^{\prime} \quad(1515)$ & $0^{+}\left(2^{+}\right)+$ & $\rightarrow \delta$ & $(2450)$ & $1^{-}\left(6^{+}\right)+$ & $\boldsymbol{K}$ & $1 / 2\left(0^{-}\right)$ \\
\hline$\eta$ & & $0^{+}\left(0^{-}\right)+$ & $\rho^{\prime}(1600)$ & $1^{+}\left(1^{-}\right)-$ & $-e^{+} e^{-}$ & $-(1100-2200)$ & & $K^{*}(892)$ & $1 / 2\left(1^{-}\right)$ \\
\hline$\rho$ & (770) & $1^{+}\left(1^{-}\right)-$ & $\rightarrow \theta \quad(1640)$ & $0^{+}\left(2^{+}\right)+$ & $\rightarrow \overline{\mathrm{N} N}$ & $(1400-3600)$ & & $Q_{1}(1280)$ & $\left.1 / 2(]^{+}\right)$ \\
\hline$\omega$ & (783) & $0^{-}\left(1^{-}\right)-$ & $\omega(1670)$ & $0^{-}\left(3^{-}\right)-$ & $\rightarrow \mathrm{x}$ & $(1900-3600)$ & & $x \quad(1350)$ & $1 / 2\left(0^{+}\right)$ \\
\hline$\eta^{\prime}$ & (958) & $0^{+}\left(0^{-}\right)+$ & $A_{3}(1680)$ & $1^{-}\left(2^{-}\right)+$ & $\eta_{c}$ & (2980) & + & $Q_{2}(1400)$ & $1 / 2\left(1^{+}\right)$ \\
\hline $\mathbf{s}^{*}$ & (975) & $0^{+}\left(0^{+}\right)+$ & $\phi^{\prime}(1680)$ & $0^{-}\left(1^{-}\right)-$ & $\mathrm{J} / \psi$ & $(3100)$ & $0^{-}\left(1^{-}\right)-$ & $\rightarrow \mathrm{K}^{\prime}(1400)$ & $1 / 2\left(0^{-}\right)$ \\
\hline$\delta$ & (980) & $1^{-}\left(0^{+}\right)+$ & B $(1690)$ & $1^{+}\left(3^{-}\right)-$ & $x$ & (3415) & $0^{+}\left(0^{+}\right)+$ & $K^{*}(1430)$ & $1 / 2\left(2^{+}\right)$ \\
\hline$\phi$ & ( $(1020)$ & $0^{-}\left(1^{-}\right)-$ & $\rightarrow \phi(1850)$ & 0 & $P_{c}$ or & $x(3510)$ & $0^{+}\left(1^{+}\right)+$ & $\rightarrow \mathrm{L} \quad(1580)$ & $1 / 2\left(2^{-}\right)$ \\
\hline H & $(1190)$ & $0^{-}\left(1^{+}\right)-$ & $\rightarrow \times(1850)$ & $\left(2^{+}\right)$ & $x$ & (3555) & $0^{+}\left(2^{+}\right)+$ & $\rightarrow \mathrm{K}^{\bullet}(1650)$ & $1 / 2\left(1^{-}\right)$ \\
\hline B & (1235) & $1^{+}\left(1^{+}\right)-$ & $\rightarrow S \quad(1735)$ & & $\rightarrow \eta_{c}^{\prime}$ & $(3590)$ & + & $L \quad(1770)$ & $1 / 2\left(2^{-}\right)$ \\
\hline$\rightarrow \rho^{\prime}$ & $(1250)$ & $1^{+}\left(1^{-}\right)-$ & $\rightarrow \delta \quad(2030)$ & $1^{-}\left(4^{+}\right)+$ & $\psi$ & (3685) & $0^{-}\left(1^{-}\right)-$ & $K^{*}(1780)$ & $1 / 2\left(3^{-}\right)$ \\
\hline$f$ & (1270) & $0^{+}\left(2^{+}\right)+$ & h $(2040)$ & $0^{+}\left(4^{+}\right)+$ & $\psi$ & (3770) & $\left(1^{-}\right)-$ & $\rightarrow \mathrm{K}^{*}(2060)$ & $1 / 2\left(4^{+}\right)$ \\
\hline$A_{1}$ & $(1270)$ & $1^{-}\left(1^{+}\right)+$ & $\rightarrow \pi(2050)$ & $1^{-}\left(3^{+}\right)+$ & $\psi$ & (4030) & $\left(1^{-}\right)-$ & $\rightarrow K^{*}(2200)$ & \\
\hline$\rightarrow \eta$ & (1275) & $0^{+}\left(0^{-}\right)+$ & $\rightarrow \pi(2100)$ & $1^{-}\left(2^{-}\right)+$ & $\psi$ & $(4 \mid 60)$ & $\left(1^{-}\right)-$ & Charmed $(\mid \mathrm{C})$ & $=11$ \\
\hline D & $(1285)$ & $0^{+}\left(1^{+}\right)+$ & $\rightarrow \rho \quad(2150)$ & $1^{+}\left(1^{-}\right)^{-}$ & $\psi$ & $(44 ! 5)$ & $\left(1^{-}\right)-$ & D $(1870)$ & $1 / 2\left(0^{-}\right)$ \\
\hline$\epsilon$ & (1300) & $0^{+}\left(0^{+}\right)+$ & $\rightarrow \in(2150)$ & $0^{+}\left(2^{+}\right)+$ & $\Upsilon$ & $(9460)$ & $\left(1^{-}\right)-$ & $D^{*}(2010)$ & $1 / 2\left(1^{-}\right)$ \\
\hline$\pi$ & - $(1300)$ & $1^{-}\left(0^{-}\right)+$ & $\rightarrow \rho(2250)$ & $1^{+}\left(3^{-}\right)-$ & $\Upsilon$ & $(10020)$ & $\left(1^{-}\right)-$ & $F \quad(2020)$ & $0\left(0^{-}\right)$ \\
\hline$A_{2}$ & $2(1320)$ & $\mathrm{j}^{-}\left(2^{+}\right)+$ & $\rightarrow \in(2300)$ & $0^{+}\left(4^{+}\right)+$ & $\tau$ & (10350) & $\left(1^{-}\right)-$ & $\rightarrow F^{*}(2: 40)$ & \\
\hline $\mathbf{E}$ & $(1420)$ & $0^{+}\left(1^{+}\right)+$ & $\rightarrow \rho(2350)$ & $1^{+}\left(5^{-}\right)-$ & $\mathrm{T}$ & $(10570)$ & $\left(1^{-}\right)-$ & Botiom (Beau & ty) $(|\mathrm{B}|=1)$ \\
\hline & & & & & & & & - B $(5200)$ & \\
\hline & & & & & & & & $\rightarrow$ Exotics & \\
\hline
\end{tabular}

- Indicates an entry in the Meson Data Card Listings not entered in the Meson Table. We do not regard these as established resonances. All the entries in the Listings can be found in the Table of Contents of the Meson Data Card Listings immediately preceding these footnotes.

₹ See Meson Data Card Listings.

- Quoted error includes scale factor $\mathbf{S}=\sqrt{\chi^{2} /(\mathrm{N}-1)}$. See footnote to Stable Particle Table.

+ Square brackets indicate a subreaction of the previous (unbracketed) decay mode(s).

5 This is only an educated guess; the error given is larger than the error on the average of the published values. (See the Meson Data Card Listings for the latter.)

a. $\Gamma M$ is approximately the half-width of the resonance when plosted against $\mathbf{M}^{2}$.

b. For decay modes into $\geq 3$ particles. $p_{\text {max }}$ is the maximum momentum that any of the particles in the final state can have. The momenta have been calculated by using the averaged central mass valus, without taking into account the widths of the resonances.

c. From pole position $(\mathrm{M}-\mathrm{i} \Gamma / 2)$.

d. The $e^{+} e^{-}$branching fraction is from $\mathrm{e}^{+} e^{-} \rightarrow \pi^{+} \pi^{-}$experiments only. The wo interference is then due to wo mixing only, and is expected to be small. See note in the Meson Data Card Listings. The $\mu^{+} \mu^{-}$branching fraction is compiled from 3 experiments,

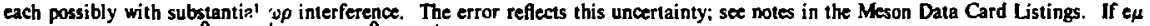
universality holds, $\Gamma\left(\rho^{0} \rightarrow \mu^{+} \mu^{-}\right)=\Gamma\left(\rho^{0} \rightarrow e^{+} e^{-}\right) \times 0.99785$. 


\section{Meson Table (cont'd)}

e. Empirical imits on fractions for other decay modes of $\rho(770)$ are $\pi^{ \pm} \eta<0.8 \%$ (LL=84\%), $\pi^{+} \pi^{+} \pi^{-} \pi^{-}<0.15 \%, \pi^{ \pm} \pi^{+} \pi^{-} \pi^{0}<$ $0.2 \%(\mathrm{CL}=84 \%)$.

f. Empirical limits on fractions for other decay modes of $\omega(783)$ ise $\pi^{+} \pi^{-} \gamma<5 \%, \pi^{0} \pi^{0} \gamma<1 \%, \eta+$ neutral(s) $<1.5 \%, \mu^{+} \mu^{-}<$ $0.02 \%$.

g. Empirical limits on fractions for other decay modes of $\eta^{\prime}(958)$ are $\pi^{+} \pi^{-}<2 \%$ (CL=B4\%), $\pi^{+} \pi^{-} \pi^{0}<5 \%$ (CL=84\%), $\pi^{+} \pi^{+} \pi^{-} \pi^{-}$ $<1 \%(C L=95 \%) \pi^{+} \pi^{+} \pi^{-} \pi^{-} \pi^{0}<1 \%(C L=84 \%), 6 \pi<1 \%, \pi^{+} \pi^{-} e^{+} e^{-}<0.6 \%, \pi^{0} e^{+} e^{-}<1.3 \%(C L=84 \%), \eta e^{+} e^{-}<1.1 \%, \pi^{0} \rho^{0}$ $<4 \%, \eta \mu^{+} \mu^{-}<1.5 \times 10^{-5}, \pi^{0} \mu^{+} \mu^{-}<6 \times 10^{-5}$.

h. The mass and width are from the $\eta \pi$ mode only. If the $\mathrm{K} \bar{K}$ channel is strongly coupled, the width may be larger.

i. Empirical limits on fractions for other decay modes of $\phi(1020)$ are $\pi^{+} \pi^{-} \gamma<0.7 \%$, cor $<5 \%(C L=84 \%), \rho \gamma<2 \%(C L=84 \%)$, $2 \pi^{+} 2 \pi^{-} \pi^{0}<1 \%(\mathrm{CL}=95 \%), 2 \pi^{+} 2 \pi^{-}<0.1 \%$.

j. Empirical limis on fractions for other decay modes of $\mathrm{B}(1235)$ are $\pi \pi<15 \%, \mathrm{~K} \overline{\mathrm{K}}<2 \%$ (CL=84\%), $4 \pi<50 \%$ (CL=84\%), $\phi \pi<$ $1.5 \%(\mathrm{CL}=84 \%), 7 \pi<25 \%$, $(\overline{\mathrm{KK}})^{ \pm} \pi^{0}<8 \%, \mathrm{~K}_{\mathrm{S}} \mathrm{K}_{\mathrm{S}} \pi^{ \pm}<2 \%, \mathrm{~K}_{\mathrm{S}} \mathrm{K}_{\mathrm{L}} \pi^{ \pm}<6 \%$.

k. Empirical limits $(\mathrm{CL}=95 \%)$ on fractions for other decay modes of $\mathrm{f}(1270)$ are $\eta \pi \pi<1 \%, \mathrm{~K}^{0} \mathrm{~K}^{-} \pi^{+}+$c.c. $<0.5 \%, \eta \eta<2 \%$.

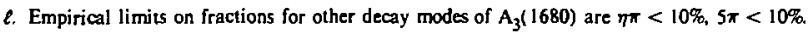

m. Includes $\overline{\mathrm{p}} \pi^{+} \pi^{-} \gamma$ and excludes $\mathrm{p} \bar{p} \eta \mathrm{p} \overline{\mathrm{p}} \boldsymbol{\omega}, \mathrm{p} \overline{\mathrm{p}} \eta^{\prime}$.

n. The ( $(1440)$ evidence is listed under $E(1420)$; see E(1420) mini-review.

o. Empirical limits on fractions for other decay modes of $\chi(3415)$ are $2 \gamma<0.17 \%$, $\mathrm{p} \overline{\mathrm{p}}<0.011 \%$.

p. Empirical limits on fractions for other decay modes of $\chi(3510)$ are $\left(\pi^{+} \pi^{-}\right.$and $\left.\mathrm{K}^{+} \mathrm{K}^{-}\right)<0.2 \%, \gamma \gamma<0.16 \%, \mathrm{p} \overline{\mathrm{p}}<0.13 \%$

q. Empirical limits on fractions for other decay modes of $\chi(3555)$ are $2 \gamma<0.06 \%, \mathrm{p} \overline{\mathrm{p}}<0.10 \%, \mathrm{~J} / 4 \pi^{+} \pi^{-} \pi^{0}<1.5 \%$.

Esrablished Nonets, and octet-singlet mixing angies $\theta$ obtained from $1 "$ the two isosinglets, the "mainly octet" one is written first, followed b. tion from ideal mixing.

\begin{tabular}{|l|l|c|c|c|c|}
\hline$\left(\mathrm{J}^{\mathrm{P}}\right) \mathrm{C}_{\mathrm{n}}$ & Nonet members & $\theta_{\text {lin. }}$ & $\theta_{\text {quadr. }}$ & $\delta_{\text {lin. }}$ & $\delta_{\text {quadr. }}$ \\
\hline$\left(0^{-}\right)+$ & $\pi, \mathrm{K}, \eta ; \eta^{\prime}$ & $-24.4 \pm 0.1^{\circ}$ & $-11.1 \pm 0.2^{\circ}$ & $-59.7 \pm 0.1^{\circ}$ & $-46.4 \pm 0.2^{\circ}$ \\
\hline$\left(1^{-}\right)-$ & $\rho, \mathrm{K}^{*}, \phi ; \omega$ & $35.9 \pm 0.5^{\circ}$ & $38.6 \pm 0.4^{\circ}$ & $0.6 \pm 0.5^{\circ}$ & $3.3 \pm 0.4^{\circ}$ \\
\hline$\left(2^{+}\right)+$ & $\mathrm{A}_{2}, \mathrm{~K}^{\circ}(1430), \mathrm{f}^{\prime} ; \mathrm{i}$ & $26 \pm 3^{\circ}$ & $28 \pm 3^{\circ}$ & $-9 \pm 3^{\circ}$ & $-7 \pm 3^{\circ}$ \\
\hline$\left(1^{+}\right)+^{+}$ & $\mathrm{A}_{1}, \mathrm{Q}_{\mathrm{A}}, \mathrm{E} ; \mathrm{D}$ & $52 \pm 13^{\circ}$ & $51 \pm 12^{\circ}$ & $16 \pm 13^{\circ}$ & $15 \pm 12^{\circ}$ \\
\hline
\end{tabular}

$+m\left(Q_{A}\right)$ is assumed to be the average of $m\left(Q_{1}\right)$ and $m\left(Q_{2}\right)$.

More generally, because of unitarity, the mixing angles are enetgy dependent and complex above the first threshold (see Appendix Il $C)$, which is important especially for the scalar and the axial mesons. Note also that the two axial strange mesnns $\left(Q_{1}\right.$ and $\left.Q_{2}\right)$ are mixtures of the exact SU(3) states: $\mathrm{Q}_{1}=\cos \phi \mathrm{Q}_{\mathrm{A}}+\sin \phi \mathrm{Q}_{\mathrm{B}}, \mathrm{Q}_{2}=-\sin \phi \mathrm{Q}_{\mathrm{A}}+\cos \phi \mathrm{Q}_{\mathrm{B}}$. Below we give the mixing angles of and $\phi$ obtained in a unitary mixing scheme using both masses and widths as input data:

\begin{tabular}{|c|c|c|c|}
\hline \multirow{2}{*}{$\frac{\left(\mathrm{J}^{\mathrm{P}}\right) \mathrm{C}_{\mathrm{n}}}{\left(\mathrm{I}^{+}\right)+}$} & \multirow{2}{*}{$\frac{\text { Nonet members }}{A_{1}, Q_{A}, E ; D}$} & \multicolumn{2}{|l|}{ Mixing angles } \\
\hline & & $\delta_{\mathrm{DE}}(1283)$ & $=14^{\circ}+i 1^{\circ}$ \\
\hline & & ${ }^{\delta} \mathrm{DE}(1418)$ & $=25^{\circ}+i 8^{\circ}$ \\
\hline \multirow[t]{4}{*}{$\left(1^{+}\right)-$} & $B, Q_{B}, H^{\dagger} ; H$ & $\delta_{\mathrm{HH}^{\prime}}(1190)$ & $=-6^{0}+i 4^{0}$ \\
\hline & & $\delta_{\mathrm{HH}^{\prime}}(1400)$ & $=-15^{\circ}+i 10^{\circ}$ \\
\hline & & $\phi_{Q_{1} Q_{2}}{ }^{(1270)}$ & $=50^{\circ}+i 3^{\circ}$ \\
\hline & & $\phi_{Q_{1} Q_{2}}{ }^{(1414)}$ & $=61^{\circ}-i 3^{\circ}$ \\
\hline \multirow[t]{2}{*}{$\left(0^{+}\right)+$} & $\delta, k, S^{*} ; \epsilon$ & $\delta_{S \cdot \varepsilon}(975)$ & $=+4^{\circ}+i 29^{\circ}$ \\
\hline & & $\delta_{\mathrm{Se} t}(1300)$ & $=-33^{\circ}+i 7^{\circ}$ \\
\hline
\end{tabular}

${ }^{\dagger}$ as yet, not seen experimentally 


\section{Baryon Table}

April 1982

The following short list gives the name, the nominal mass, the quantum numbers (where known), and the status of each of the Baryon States in the Data Card Listings. States with 3- or 4-star status are included in the Baryon Table below, the others are omitted because the evidence for the existence of the effact and/or for its interpretation as a resonance is open to question.

\begin{tabular}{|c|c|c|c|c|c|c|c|c|c|c|c|c|c|c|}
\hline$N(939)$ & Pit & $\cdots$ & $\Delta(1232)$ & P33 & *ne" & $\mathbf{M}(1115)$ & POI & $+\infty+\infty$ & $\Sigma(1193)$ & PII & $* \cdots$ & $\Xi(1317)$ & Pll & $\cdots$ \\
\hline$N(1440)$ & PII & ent* & $\Delta(1550)$ & P31 & $*$ & $\Lambda(1405)$ & SOI & $+\infty+\infty$ & $\Sigma(1385)$ & $P 13$ & na** & $\nexists(1530)$ & P13 & 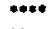 \\
\hline$N(1520)$ & $\mathrm{Dl} 3$ & 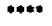 & $\Delta(1600)$ & P33 & $m$ & $\Lambda(1520)$ & D03 & nate & $\Sigma(1480)$ & & - & $\Xi(1630)$ & & "* \\
\hline$N(1535)$ & S11 & •n." & $\Delta(1620)$ & S31 & 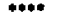 & $\Lambda(1600)$ & P0I & $*+$ & $\Sigma(1560)$ & & "* & $\Xi(1680)$ & S11 & $"$ \\
\hline$N(1540)$ & P13 & $\bullet$ & $\Delta(1700)$ & D33 & +4+" & $\Lambda(1670)$ & Sol & ninen & $\Sigma(1580)$ & D13 & $*$ & $\Xi(1820)$ & 13 & $\cdots$ \\
\hline$N(1650)$ & SII & *ne* & $\Delta(1900)$ & S31 & $\rightarrow$ & $\Lambda(1690)$ & D03 & *n+on & $\Sigma(1620)$ & Sil & *" & $\Xi(1940)$ & & $\cdots$ \\
\hline$N(1675)$ & D15 & $\operatorname{mon}$ & $\Delta(1905)$ & $F 35$ & $\min$ & $\Lambda(1800)$ & SO1 & $\cdots$ & $\Sigma(1660)$ & $P 1 \mathrm{I}$ & 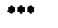 & $\Xi(2030)$ & 1 & $m$ \\
\hline$N(1680)$ & F15 & מ⿰氵 & $\Delta(1910)$ & $\mathrm{P} 31$ & ane* & $1(1800)$ & $\mathbf{P O 1}$ & m* & $\Sigma(1670)$ & D13 & $\bullet \bullet \bullet$ & $\Xi(2120)$ & & - \\
\hline$N(1700)$ & D13 & $+\infty$ & $\Delta(1920)$ & P33 & $* *$ & $\Lambda(1800)$ & G09 & Dead & $\Sigma(1670)$ & & 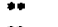 & $\Xi(2250)$ & & : \\
\hline$N(1710)$ & P11 & 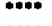 & $\Delta(1930)$ & D35 & $\cdots "$ & $\Lambda(1800)$ & & " & $\Sigma(1690)$ & & $" *$ & $\underline{\Xi}(2370)$ & 1 & $"$ \\
\hline$N(1720)$ & $\mathbf{P 1 3}$ & $\operatorname{mon}$ & $\Delta(1940)$ & D33 & $\bullet$ & $\Lambda(1820)$ & FOS & $m$ & $\Sigma(1750)$ & S11 & $\cdots$ & $\Xi(2500)$ & & $*$ \\
\hline$N(1990)$ & $F 17$ & $\cdots$ & $\Delta(1950)$ & F37 & $\infty$ & $\Lambda(1830)$ & D0S & $* * * *$ & $\Sigma(1770)$ & Pll & Dead & & & \\
\hline$N(2000)$ & F1s & $\omega$ & $\Delta(2150)$ & S3I & * & $\Lambda(1890)$ & $\mathrm{P} 03$ & $+\infty+\infty$ & $\Sigma(1775)$ & D1s & $\bullet$ & $\Omega(1672)$ & $\mathrm{P03}$ & $* * *$ \\
\hline$N(2080)$ & D13 & $* * 0$ & $\Delta(2160)$ & & * & $A(2000)$ & & * & $\Sigma(1840)$ & P13 & - & & & \\
\hline$N(2100)$ & S11. & * & $\Delta(2200)$ & G37 & $"$ & $\Lambda(2020)$ & F07 & - & $\Sigma(1880)$ & PII & $" *$ & $\Lambda_{c}(2282)$ & & w" \\
\hline$N(2100)$ & PII & * & $\Delta(2300)$ & $\mathrm{H} 39$ & 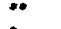 & $\Lambda(2100)$ & G07 & $*$ & $\Sigma(1915)$ & F15 & $* *$ & & & \\
\hline$N(2190)$ & G17 & $\cdots$ & $\Delta(2350)$ & D35 & - & $\Lambda(2110)$ & F0S & $\infty$ & $\Sigma(1940)$ & D13 & $\rightarrow *$ & $\Sigma_{c}(2450)$ & & 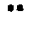 \\
\hline$N(2200)$ & Dis & $\cdots$ & $\Delta(2400)$ & F37 & - & $\Lambda(2325)$ & D03 & - & $\Sigma(2000)$ & S11 & - & & & \\
\hline$N(2220)$ & $\mathrm{H} 19$ & $\cdots$ & $\Delta(2400)$ & G39 & - & $\Lambda(2350)$ & & $* \cdots$ & $\Sigma(2030)$ & F17 & $\cdots$ & $\Lambda_{b}(5500)$ & & - \\
\hline$N(2250)$ & G19 & $\bullet \bullet "$ & $\Delta(2420)$ & $\mathrm{H} 311$ & $\cdots$ & $\Lambda(2585)$ & & $\cdots$ & $\Sigma(2070)$ & F15 & " & & & \\
\hline$N(2600)$ & I]11 & "*" & $\Delta(2500)$ & & : & & & & $\Sigma(2080)$ & P13 & *" & Dibaryons & & \\
\hline$N(2700)$ & $K 113$ & - & $\Delta(2750)$ & 1313 & - & & & & $\Sigma(2100)$ & G17 & * & $\mathrm{NN}(2170)$ & In2 & $\cdots$ \\
\hline$N(2800)$ & G19 & - & $\Delta(2850)$ & & $\cdots$ & & & & $\Sigma(2250)$ & & *n+. & $N N(2250)$ & $3 F 3$ & $\cdots$ \\
\hline$N(3000)$ & & - & $\Delta(2950)$ & $\mathrm{K} 315$ & - & & & & $\Sigma(2455)$ & & 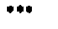 & $N N(?)$ & & - \\
\hline$N(3030)$ & & $\cdots$ & $\Delta(3230)$ & & $\cdots$ & & & & $\Sigma(2620)$ & & $* *$ & $\Delta N(2 ; 30)$ & $3 \mathrm{~S} 1$ & $\cdots$ \\
\hline$N(3245)$ & & • & & & & & & & $\Sigma(3000)$ & & $*$ & $\Xi N(?)$ & & 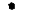 \\
\hline$N(3690)$ & & . & $Z O(1780)$ & P01 & $:$ & & & & $\Sigma(3170)$ & & * & & & \\
\hline \multirow[t]{8}{*}{$N(3755)$} & & " & $Z 0(1865)$ & DO3 & : & & & & & & & & & \\
\hline & & & $Z 1(1900)$ & $\mathrm{P} 13$ & - & & & & & & & & & \\
\hline & & & $\begin{array}{l}Z I(2150) \\
Z 1(2500)\end{array}$ & & 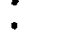 & & & & & & & & & \\
\hline & & & & & 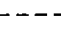 & 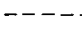 & & 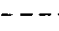 & 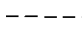 & & -- & 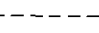 & 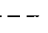 & \\
\hline & . & & & & & & & & & & & & & \\
\hline & 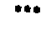 & & & & & & & & & & & & & \\
\hline & $\because 1$ & & a & & & & & & & & & & & \\
\hline & & & & & & & & & & & & & & \\
\hline
\end{tabular}

\begin{tabular}{|c|c|c|c|c|c|c|c|c|}
\hline \multirow[b]{2}{*}{ Particle $^{a}$} & \multirow[b]{2}{*}{${ }_{1(\mathrm{~J}} \mathrm{P}_{1} \mathrm{~L}_{2}^{b} \cdot 2 \mathrm{~J}$} & \multirow[b]{2}{*}{$\begin{array}{l}P_{b e a n}^{c}(G \mathrm{eV} / \mathrm{c}) \\
\sigma=4 \pi \AA^{2}(\mathrm{mt})\end{array}$} & \multirow{2}{*}{$\begin{array}{c}\text { Mass }^{d} \\
\text { M } \\
(\mathrm{MeV})\end{array}$} & \multirow{2}{*}{$\begin{array}{l}\text { Fulle } \\
\text { width } \Gamma \\
\text { (MeV) }\end{array}$} & \multirow{2}{*}{ 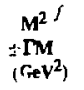 } & \multicolumn{3}{|c|}{ Partiel decay mode } \\
\hline & & & & & & Mode: & $\begin{array}{c}\text { Fraction } \\
\text { (\%) }\end{array}$ & $\stackrel{\mathbf{p}^{i}}{\mathbf{M e V} / \mathrm{c})}$ \\
\hline \multicolumn{9}{|c|}{$S=0 \quad I=1 / 2$ NUCLEON RESONANCES $(N)$} \\
\hline $\begin{array}{l}\mathrm{p} \\
\mathrm{n}\end{array}$ & $1 / 2\left(1 / 2^{+}\right)$ & & $\begin{array}{l}938.3 \\
939.6\end{array}$ & & $\begin{array}{l}0.880 \\
0.883\end{array}$ & \multicolumn{3}{|c|}{ See Stable Particle Table } \\
\hline$\overline{N(1440)}$ & $1 / 2\left(1 / 2^{+}\right) \mathrm{P}_{11}^{\prime}$ & $\begin{array}{l}\mathrm{p}=0.61 \\
\sigma=31.0\end{array}$ & $\begin{array}{r}1400 \text { to } \\
1480\end{array}$ & $\begin{array}{r}12010 \\
350 \\
(200)\end{array}$ & $\begin{array}{r}2.07 \\
\pm 0.29\end{array}$ & $\begin{array}{l}N_{\pi} \\
N \eta \\
N \pi \pi \\
{\left[\begin{array}{l}\Delta \pi \\
N \rho \\
N E\end{array}\right.}\end{array}$ & $\begin{array}{c}50-70 \\
8-18 \\
\sim 30 \\
12-28 \\
\sim 7 \\
\sim 5\end{array}$ & $\begin{array}{r}397 \\
+ \\
342 \\
143 \\
+ \\
+\end{array}$ \\
\hline$\overline{N(1520)}$ & $1 / 2\left(3 / 2^{-}\right) \mathrm{D}_{13}^{\prime}$ & $\begin{array}{l}p=0.74 \\
\sigma=23.5\end{array}$ & $\begin{array}{l}1510 \text { to } \\
1530\end{array}$ & $\begin{array}{r}100 \text { to } \\
140 \\
(125)\end{array}$ & $\begin{array}{r}2.31 \\
\pm 0.19\end{array}$ & $\begin{array}{c}\bar{N} \pi \\
N_{\eta} \\
N_{\pi \pi} \pi \\
{\left[\begin{array}{l}\Delta \pi \\
N \rho \\
N E\end{array}\right.}\end{array}$ & $\left.\begin{array}{c}50-60 \\
<1 \\
35-50 \\
15-25 \\
15-25 \\
<5\end{array}\right]$ & $\begin{array}{r}456 \\
149 \\
410 \\
228 \\
+ \\
+\end{array}$ \\
\hline$\overline{N(1535)}$ & $1 / 2\left(1 / 2^{-}\right) \mathrm{S}_{11}^{\prime}$ & $\begin{array}{l}p=0.76 \\
\sigma=22.4\end{array}$ & $\begin{array}{r}1520 \text { to } \\
1560\end{array}$ & $\begin{array}{r}100 \text { to } \\
250 \\
(150)\end{array}$ & $\begin{array}{r}2.36 \\
\pm 0.23\end{array}$ & $\begin{array}{c}\mathrm{N} \pi \\
N \eta \\
N \pi \pi \\
{\left[\begin{array}{l}\Delta \pi \\
N \rho \\
N \epsilon\end{array}\right.} \\
\end{array}$ & $\left.\begin{array}{c}35-50 \\
40-65 \\
\sim 5 \\
\sim 1 \\
\sim 3 \\
\sim 2\end{array}\right]$ & $\begin{array}{r}467 \\
182 \\
422 \\
242 \\
\dagger \\
+\end{array}$ \\
\hline
\end{tabular}




\section{Baryon Table (cont'd)}

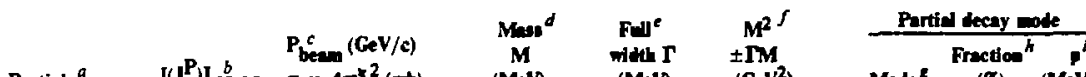

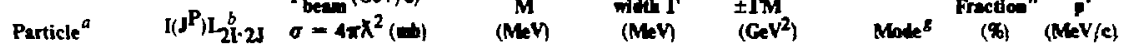
\begin{tabular}{lrlrrrrrr}
\hline$N(1650)$ & $l / 2\left(1 / 2^{-}\right) S_{11}^{\prime \prime}$ & $P=0.96$ & 1620 to & 100 to & 2.72 & $N \pi$ & $55-65$ & 547 \\
& & $\sigma=16.4$ & 1680 & 200 & \pm 0.25 & $N \pi$ & -1
\end{tabular} $\begin{array}{llr}\text { SK } & 5-10 & 161 \\ \text { SK } & 3-10 & +\end{array}$

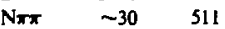
$\left[\begin{array}{lll}N \pi \pi & \sim 30 & 4-15 \\ N \rho & \sim 20 \\ N \epsilon & <5 & 344 \\ + & +1\end{array}\right]$

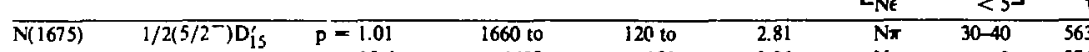

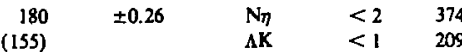

\begin{tabular}{|c|c|c|c|c|c|c|c|}
\hline & & & & & & $\begin{array}{l}N \pi \pi \\
{\left[\begin{array}{l}\Delta \pi \\
N \rho\end{array}\right.}\end{array}$ & $\left.\begin{array}{l}55-70 \\
50-65 \\
\sim 5\end{array}\right]$ \\
\hline$N(1680)$ & $1 / 2\left(5 / 2^{+}\right) \mathbf{F}_{15}^{\prime}$ & $\begin{array}{l}\mathrm{p}=1.01 \\
\sigma=15.2\end{array}$ & $\begin{array}{r}1670 \text { 10 } \\
1690\end{array}$ & $\begin{array}{r}110 \text { to } \\
140 \\
(125)\end{array}$ & $\begin{array}{r}2.82 \\
\pm 0.21\end{array}$ & $\begin{array}{l}N \pi \\
N \pi \\
N \pi \pi \\
{\left[\begin{array}{l}\Delta \pi \\
N \rho \\
N E\end{array}\right.}\end{array}$ & $\begin{array}{c}55-65 \\
<1 \\
\sim 40 \\
\sim 12 \\
\sim 10 \\
\sim 20\end{array}$ \\
\hline
\end{tabular}

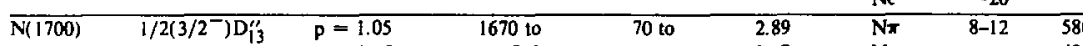

$\sigma=14.5 \quad 1730 \quad 120 \quad \pm 0.17 \quad \mathrm{~N} \pi \quad \sim 4 \quad 400$

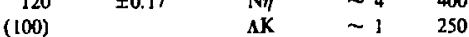

$\begin{array}{lll}N \pi \pi & \sim 85 & 547\end{array}$

$\left[\begin{array}{lll}\Delta \pi & & 15-40 \\ N \rho & \sim 5 \\ N \epsilon & <40\end{array}\right]+\begin{array}{r}385 \\ + \\ +\end{array}$

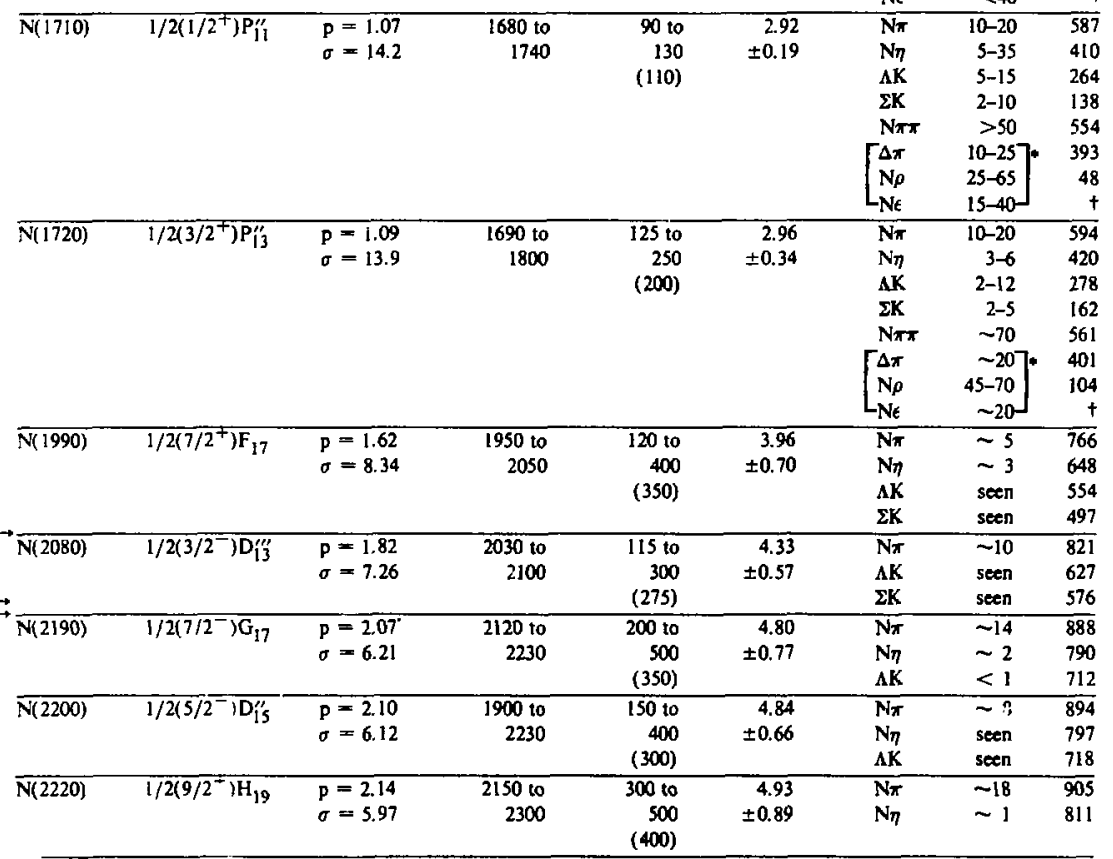


Baryon Table (cont'd)

\begin{tabular}{|c|c|c|c|c|c|c|c|c|}
\hline \multirow[b]{2}{*}{ Particle $^{a}$} & \multirow[b]{2}{*}{$\mathbf{I}\left(\mathrm{J}^{\mathbf{P}}\right) \mathbf{L}_{\mathbf{2}}^{b} \cdot \mathbf{2 J}$} & \multirow[b]{2}{*}{$\begin{array}{l}P_{\text {beam }}^{c}(G e V / c) \\
\sigma=4 \pi X^{2}(m)\end{array}$} & \multirow{2}{*}{$\underset{(\mathbf{M} V)}{\mathbf{M} \mathbf{m}^{d}}$} & \multirow{2}{*}{$\begin{array}{l}\text { Fure } \\
\text { rin } \Gamma \\
\text { (MeV) }\end{array}$} & \multirow{2}{*}{$\begin{array}{c}\mathbf{M}^{2} f \\
\pm \mathbf{M} \\
\left(\mathbf{G e} \mathbf{V}^{2}\right)\end{array}$} & \multicolumn{3}{|c|}{ Purtial decay rode } \\
\hline & & & & & & Model & $\begin{array}{c}\text { Fraction } \\
\text { (\%) }\end{array}$ & $(\mathrm{MeV} / \mathrm{c})$ \\
\hline$\overline{N(2250)}$ & $1 / 2(9 / 2) G_{19}^{\prime}$ & $\begin{array}{l}\mathrm{p}=2.21 \\
\sigma=5.74\end{array}$ & $\begin{array}{r}2130 \text { to } \\
2270\end{array}$ & $\begin{array}{r}200 \text { to } \\
500 \\
(300)\end{array}$ & $\begin{array}{r}5.06 \\
\pm 0.68\end{array}$ & $\begin{array}{l}\mathrm{N} \pi \\
\mathrm{N} \pi\end{array}$ & $\begin{array}{l}\sim 10 \\
\sim 2\end{array}$ & $\begin{array}{l}\overline{923} \\
831\end{array}$ \\
\hline$\overline{N(2600)}$ & $1 / 2\left(11 / 2^{-}\right) I_{111}$ & $\begin{array}{l}\rho=3.12 \\
\sigma=3.86\end{array}$ & $\begin{array}{r}2580 \text { to } \\
2700\end{array}$ & $\begin{array}{l}>300 \\
(400)\end{array}$ & $\begin{array}{r}6.76 \\
+1.04 \\
\end{array}$ & $\mathbf{N} \pi$ & $\sim$ & 1126 \\
\hline $\begin{array}{l}\rightarrow \mathrm{N}(3030) \\
\rightarrow\end{array}$ & $1 / 2(?)$ & $\begin{array}{l}p=4.41 \\
\sigma=2.62\end{array}$ & -3030 & $\begin{array}{r}-400 \\
(400)\end{array}$ & $\begin{array}{r}9.18 \\
\pm 1.21 \\
\end{array}$ & $N \pi$ & $\begin{array}{r}(\mathrm{J}+1 / 2) \mathrm{x} \\
<0.1\end{array}$ & 1366 \\
\hline \multicolumn{9}{|c|}{$S=0 \quad I=3 / 2$ DELTA RESONANCES $(\Delta)$} \\
\hline$\Delta(1232)$ & $3 / 2\left(3 / 2^{+}\right) \mathrm{P}_{33}^{\prime}$ & $\begin{array}{l}p=0.30 \\
\sigma=95.0\end{array}$ & $\begin{array}{r}1230 \text { to } \\
1234\end{array}$ & $\begin{array}{r}110 \text { to } \\
120 \\
(115)\end{array}$ & $\begin{array}{r}1.52 \\
\pm 0.14\end{array}$ & $\begin{array}{l}N_{\pi} \\
N_{\gamma}\end{array}$ & $\begin{array}{r}99.4 \\
0.6\end{array}$ & $\begin{array}{l}227 \\
259\end{array}$ \\
\hline \multicolumn{9}{|c|}{$\begin{array}{ll}\Delta(++) \text { pole position: } & M-i \Gamma / 2=(1210.6 \pm 0.5)-i(49.7 \pm 0.3) \\
\Delta(0) \text { pole position: } & M-i \Gamma / 2=(1210.3 \pm 1.0)-i(53.0 \pm 1.0)\end{array}$} \\
\hline$\Delta(1600)$ & $3 / 2\left(3 / 2^{+}\right) \mathrm{P}_{33}^{\prime \prime}$ & $\begin{array}{l}\mathrm{p}=0.87 \\
\sigma=18.7\end{array}$ & $\begin{array}{r}1500 \text { to } \\
1900\end{array}$ & $\begin{array}{r}150 \text { to } \\
350 \\
(250)\end{array}$ & $\begin{array}{r}2.56 \\
\pm 0.40\end{array}$ & $\begin{array}{l}N_{\pi} \\
N \pi \pi \\
{\left[\begin{array}{l}\Delta \pi \\
N \rho\end{array}\right.}\end{array}$ & $\left.\begin{array}{r}15-25 \\
\sim 80 \\
20-65 \\
<10\end{array}\right]$ & $\begin{array}{r}512 \\
473 \\
301 \\
+\end{array}$ \\
\hline$\overline{\Delta(1620)}$ & $3 / 2\left(1 / 2^{-}\right) \mathrm{S}_{31}^{\prime}$ & $\begin{array}{l}p=0.91 \\
\sigma=17.7\end{array}$ & $\begin{array}{r}16 \times 0 \text { to } \\
1650\end{array}$ & $\begin{array}{r}120 \text { to } \\
160 \\
(140)\end{array}$ & $\begin{array}{r}2.62 \\
\pm 0.23\end{array}$ & $\begin{array}{l}\mathrm{N \pi} \\
\mathrm{N} \pi \pi \\
{\left[\begin{array}{l}\Delta \pi \\
\mathrm{N} \rho\end{array}\right.}\end{array}$ & $\left.\begin{array}{c}25-35 \\
\sim 70 \\
35-50 \\
<40\end{array}\right]$ & $\begin{array}{r}526 \\
488 \\
318 \\
+\end{array}$ \\
\hline$\overline{\Delta(1700)}$ & $3 / 2\left(3 / 2^{-}\right) D_{33}^{\prime}$ & $\begin{array}{l}\mathrm{p}=1.05 \\
\sigma=14.5\end{array}$ & $\begin{array}{r}1630 \text { to } \\
1740\end{array}$ & $\begin{array}{r}190 \text { to } \\
300 \\
(250)\end{array}$ & $\begin{array}{r}2.89 \\
\pm 0.43\end{array}$ & $\begin{array}{c}N_{\pi} \\
N_{\pi \pi} \\
{\left[\begin{array}{l}\Delta \pi \\
N \rho\end{array}\right.}\end{array}$ & $\left.\begin{array}{c}10-20 \\
\sim 85 \\
<50 \\
\sim 40\end{array}\right]$ & $\begin{array}{r}580 \\
547 \\
385 \\
+\end{array}$ \\
\hline$\Delta(1900)$ & $3 / 2\left(1 / 2^{-}\right) S_{31}^{\prime \prime}$ & $\begin{array}{l}\mathrm{p}=1.44 \\
\sigma=9.71\end{array}$ & $\begin{array}{r}1850 \text { to } \\
2000\end{array}$ & $\begin{array}{r}130 \text { to } \\
300 \\
(150)\end{array}$ & $\begin{array}{r}3.61 \\
\pm 0.24\end{array}$ & $\begin{array}{l}\mathbf{N \pi} \\
\mathbf{2 K}\end{array}$ & $\begin{array}{l}6-12 \\
\sim 10\end{array}$ & $\begin{array}{l}710 \\
410\end{array}$ \\
\hline$\overline{\Delta(1905)}$ & $3 / 2\left(5 / 2^{+}\right) F_{35}$ & $\begin{array}{l}\mathrm{p}=1.45 \\
\sigma=9.63\end{array}$ & $\begin{array}{r}1890 \text { to } \\
1920\end{array}$ & $\begin{array}{r}250 \text { to } \\
400 \\
(300)\end{array}$ & $\begin{array}{r}3.63 \\
\pm 0.57\end{array}$ & $\begin{array}{l}N_{\pi} \\
\Sigma K \\
N \pi \pi \\
{\left[\begin{array}{l}\Delta \pi \\
N \rho\end{array}\right.} \\
\end{array}$ & $\left.\begin{array}{c}8-15 \\
<3 \\
\sim 80 \\
10-307 \\
\sim 60\end{array}\right]$ & $\begin{array}{l}713 \\
415 \\
687 \\
542 \\
421 \\
\end{array}$ \\
\hline$\overline{\Delta(1910)}$ & $3 / 2\left(1 / 2^{+}\right) P_{31}^{\prime \prime}$ & $\begin{array}{l}p=1.46 \\
\sigma=9.54\end{array}$ & $\begin{array}{r}1850 \text { to } \\
1950\end{array}$ & $\begin{array}{r}200 \text { to } \\
330 \\
(220)\end{array}$ & $\begin{array}{r}3.65 \\
\pm 0.42\end{array}$ & $\begin{array}{c}N \pi \\
2 K \\
N \pi \pi \\
{\left[\begin{array}{l}\Delta \pi \\
N \rho\end{array}\right.} \\
\end{array}$ & $\left.\begin{array}{c}20-25 \\
2-20 \\
>40 \\
\text { smal } \\
<40\end{array}\right]$ & $\begin{array}{l}716 \\
421 \\
691 \\
545 \\
426 \\
\end{array}$ \\
\hline$\overline{\Delta(1920)}$ & $3 / 2\left(3 / 2^{+}\right) P_{33}^{\prime \prime \prime}$ & $\begin{array}{l}p=1.48 \\
\sigma=9.39\end{array}$ & $\begin{array}{r}1860 \text { to } \\
2160\end{array}$ & $\begin{array}{r}190 \text { to } \\
300 \\
(250) \\
\end{array}$ & $\begin{array}{r}3.69 \\
\pm 0.48 \\
\end{array}$ & $\begin{array}{l}\mathbf{N} \pi \\
\mathbf{I K}\end{array}$ & $\begin{array}{r}14-20 \\
\sim z\end{array}$ & $\begin{array}{l}722 \\
431\end{array}$ \\
\hline$\Delta(1930)$ & $\overline{3} / 2(5 / 2=) D_{35}^{\prime}$ & $\begin{array}{l}\mathrm{p}=\lfloor .50 \\
\sigma=9.21\end{array}$ & $\begin{array}{r}1890 \text { to } \\
1960\end{array}$ & $\begin{array}{r}150 \text { to } \\
350 \\
(250)\end{array}$ & $\begin{array}{r}3.72 \\
\pm 0.48\end{array}$ & $\begin{array}{l}\text { Na } \\
\text { IK }\end{array}$ & 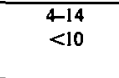 & $\begin{array}{l}729 \\
441\end{array}$ \\
\hline$\overline{\Delta(1950)}$ & $3 / 2\left(7 / 2^{+}\right) F_{37}^{\prime}$ & $\begin{array}{l}\mathrm{P}=1.54 \\
\sigma=8.9 i\end{array}$ & $\begin{array}{r}1910 \text { to } \\
1960\end{array}$ & $\begin{array}{r}200 \text { to } \\
340 \\
(240)\end{array}$ & $\begin{array}{r}3.80 \\
\pm 0.47\end{array}$ & $\begin{array}{c}N \pi \\
\Sigma K \\
N \pi \pi \\
{\left[\begin{array}{l}\Delta \pi \\
N \rho\end{array}\right.} \\
\end{array}$ & $\left.\begin{array}{l}35-45 \\
<1 \\
\sim 10 \\
\sim 40 \\
-20\end{array}\right]$ & $\begin{array}{l}741 \\
460 \\
716 \\
574 \\
469 \\
\end{array}$ \\
\hline$\overline{\Delta(2420)}$ & $3 / 2\left(11 / 2^{+}\right) \mathrm{H}_{3 ! 1}$ & $\begin{array}{l}P=2.64 \\
\sigma=4.68\end{array}$ & $\begin{array}{r}2380 \text { to } \\
2450\end{array}$ & $\begin{array}{r}300 \text { to } \\
500 \\
(300) \\
\end{array}$ & $\begin{array}{r}5.86 \\
\pm 0.73\end{array}$ & $\mathrm{N \pi}$ & $5-15$ & $\overline{1023}$ \\
\hline$\overline{\Delta(2850)}$ & $3 / 2\left(?^{+}\right)$ & $\begin{array}{l}P=3.6 \\
\sigma=3.05\end{array}$ & $\begin{array}{r}2800 \text { to } \\
2900 \\
\end{array}$ & $\begin{array}{l}-400 \\
(400)\end{array}$ & $\begin{array}{r}8.12 \\
\pm 1.14\end{array}$ & $\mathbf{N} \pi$ & $\begin{array}{r}(\mathrm{J}+1 / 2) \mathrm{x} \\
\sim 0.25 j\end{array}$ & 1266 \\
\hline$\Delta(3230)$ & $3 / 2(?)$ & $\begin{array}{l}p=5.08 \\
\sigma=2.25\end{array}$ & $\begin{array}{r}3200 \text { to } \\
3350\end{array}$ & $\begin{array}{l}\sim 440 \\
(440)\end{array}$ & $\begin{array}{r}10.43 \\
\pm 1.42\end{array}$ & $N_{\pi}$ & $\begin{array}{c}(\mathrm{J}+1 / 2) \mathrm{x} \\
\sim 0.05\end{array}$ & $\overline{1475}$ \\
\hline
\end{tabular}




\section{Baryon Table (cont'd)}

\begin{tabular}{|c|c|c|c|c|c|c|c|c|}
\hline \multirow[b]{2}{*}{ Particle $^{a}$} & \multirow[b]{2}{*}{$\mathbf{I}\left(\mathrm{J}^{\mathbf{P}}\right) \mathbf{L}_{\mathbf{1} \cdot \mathbf{2}}^{b}$} & \multirow[b]{2}{*}{$\begin{array}{l}P_{b e a n}^{f}(G e V / c) \\
\sigma=4 \pi X^{2}(n b)\end{array}$} & \multirow{2}{*}{$\begin{array}{c}\text { Mass }^{d} \\
\text { Mev) }\end{array}$} & \multirow{2}{*}{$\begin{array}{l}\text { Fulle } \\
\text { ridth } \Gamma \\
\text { (MeV) }\end{array}$} & \multirow{2}{*}{$\begin{aligned} & M^{i f} \\
& \pm M^{f} \\
&\left(G e V^{2}\right)\end{aligned}$} & \multicolumn{3}{|c|}{ Purtid decay nole } \\
\hline & & & & & & Made & $\begin{array}{c}\text { Fraction } \\
\text { (क) }\end{array}$ & $(\mathbf{M e V} / \mathbf{c}$ \\
\hline \multicolumn{9}{|c|}{$S=-1 \quad$ I=0 LAMBDA RESONANCES $(\Lambda)$} \\
\hline $\bar{\Lambda}$ & $0\left(1 / 2^{+}\right)$ & & 1115.6 & & 1.245 & \multicolumn{3}{|c|}{ See Stable Panticle Table } \\
\hline$\overline{\Lambda(1405)}$ & $\alpha\left(1 / 2^{-}\right) S_{01}^{\prime}$ & $\begin{array}{l}\text { Below } \\
K^{-} p \\
\text { threshold }\end{array}$ & $\begin{array}{l}1405 \\
\pm 5^{\ell}\end{array}$ & $40 \pm 10^{2}$ & $\begin{array}{r}1.97 \\
\pm 0.06\end{array}$ & $\Sigma \pi$ & 100 & 152 \\
\hline$\overline{\Lambda(1520)}$ & $\alpha\left(3 / 2^{-}\right) D_{03}^{\prime}$ & $\begin{array}{l}\mathrm{p}=0.395 \\
\sigma=82.2\end{array}$ & $\begin{array}{l}1519.4 \\
\pm 1.0^{\ell}\end{array}$ & $15.6 \pm 1.0^{l}$ & $\begin{array}{r}2.31 \\
\pm 0.02 \\
\end{array}$ & $\begin{array}{l}N \overline{\bar{K}} \\
\Sigma \pi \\
\Lambda \pi \pi \\
\sum \pi \pi\end{array}$ & $\begin{array}{c}45 \pm 1 \\
42 \pm 1 \\
10 \pm 1 \\
0.9 \pm 0.1\end{array}$ & $\begin{array}{l}244 \\
267 \\
252 \\
152 \\
\end{array}$ \\
\hline$A(1600)$ & $\mathrm{O}\left(1 / 2^{+}\right) \mathrm{P}_{01}^{\prime}$ & $\begin{array}{l}p=0.58 \\
\sigma=41.6\end{array}$ & $\begin{array}{r}1560 \text { to } \\
1700\end{array}$ & $\begin{array}{r}50 \text { to } \\
250 \\
(150)\end{array}$ & $\begin{array}{r}2.56 \\
\pm 0.24\end{array}$ & $\begin{array}{l}\text { NKK } \\
\Sigma \pi\end{array}$ & $\begin{array}{l}15-30 \\
10-60\end{array}$ & $\begin{array}{l}343 \\
336\end{array}$ \\
\hline$\Lambda(1670)$ & $O\left(1 / 2^{-}\right) S_{01}^{\prime \prime}$ & $\begin{array}{l}p=0.74 \\
\sigma=28.5\end{array}$ & $\begin{array}{r}1660 \text { to } \\
1680\end{array}$ & $\begin{array}{r}25 \text { to } \\
50 \\
(35) \\
\end{array}$ & $\begin{array}{r}2.79 \\
\pm 0.06\end{array}$ & $\begin{array}{l}\mathrm{N \overline {K }} \\
\Sigma \pi \\
\Lambda \eta \\
\end{array}$ & $\begin{array}{l}15-25 \\
20-60 \\
15-35\end{array}$ & $\begin{array}{r}414 \\
393 \\
64 \\
\end{array}$ \\
\hline$A(1690)$ & $O\left(3 / 2^{-}\right) D_{03}^{\prime \prime}$ & $\begin{array}{l}p=0.78 \\
\sigma=26.1\end{array}$ & $\begin{array}{r}1685 \text { to } \\
1695\end{array}$ & $\begin{array}{r}50 \text { to } \\
70 \\
(60)\end{array}$ & $\begin{array}{r}2.86 \\
\pm 0.10\end{array}$ & $\begin{array}{l}\mathrm{N \overline {K }} \\
\sum \pi \\
\Lambda \pi \pi \\
\sum \pi \pi \\
\end{array}$ & $\begin{array}{r}20-30 \\
20-40 \\
\sim 25 \\
\sim 20 \\
\end{array}$ & $\begin{array}{l}433 \\
409 \\
415 \\
350 \\
\end{array}$ \\
\hline$\overline{A(1800)}$ & $a\left(1 / 2^{-}\right) S_{0 !}^{\prime \prime \prime}$ & $\begin{array}{l}p=1.01 \\
\sigma=17.6\end{array}$ & $\begin{array}{r}1720 \text { to } \\
1850\end{array}$ & $\begin{array}{r}200 \text { to } \\
400 \\
(300)\end{array}$ & $\begin{array}{r}3.24 \\
\pm 0.54\end{array}$ & $\begin{array}{c}N \bar{K} \\
\Sigma \pi \\
\Sigma(1385) \pi \\
N^{*}(892) \\
\end{array}$ & $\begin{array}{r}25-40 \\
\text { seen } \\
\text { seen } \\
\text { seen } \\
\end{array}$ & $\begin{array}{r}528 \\
493 \\
345 \\
+ \\
\end{array}$ \\
\hline$\overline{\Lambda(1800)}$ & $\alpha\left(1 / 2^{+}\right) \mathrm{P}_{01}^{\prime \prime}$ & $\begin{array}{l}\mathrm{p}=1.01 \\
\sigma=17.6\end{array}$ & $\begin{array}{r}1750 \text { to } \\
1850\end{array}$ & $\begin{array}{r}50 \text { to } \\
250 \\
(150)\end{array}$ & $\begin{array}{r}3.24 \\
\pm 0.27\end{array}$ & $\begin{array}{c}\mathrm{N} \overline{\mathrm{K}} \\
\mathbf{\Sigma} \pi \\
\mathbb{Z}(1385) \pi \\
\mathrm{N}^{*}(892) \\
\end{array}$ & $\begin{array}{r}20-50 \\
10-40 \\
\text { seen } \\
30-60 \\
\end{array}$ & $\begin{array}{r}528 \\
493 \\
345 \\
+ \\
\end{array}$ \\
\hline$\Lambda(1820)$ & $O\left(5 / 2^{+}\right) F_{0 s}^{\prime}$ & $\begin{array}{l}\mathrm{p}=1.06 \\
\sigma=16.5\end{array}$ & $\begin{array}{r}1815 \text { to } \\
1825\end{array}$ & $\begin{array}{r}70 \text { to } \\
90 \\
(80) \\
\end{array}$ & $\begin{array}{r}3.29 \\
\pm 0.15\end{array}$ & $\begin{array}{c}\mathrm{NK} \\
\sum \pi \\
\Sigma(1385) \pi\end{array}$ & $\begin{array}{r}55-65 \\
8-14 \\
5-10 \\
\end{array}$ & $\begin{array}{l}545 \\
508 \\
362 \\
\end{array}$ \\
\hline$\overline{\Lambda(1830)}$ & $\mathrm{O}\left(5 / 2^{-}\right) \mathrm{D}_{05}$ & $\begin{array}{l}\mathrm{p}=1.08 \\
\sigma=16.0\end{array}$ & $\begin{array}{r}1810 \text { to } \\
1830\end{array}$ & $\begin{array}{l}60 \text { to } \\
110 \\
(95) \\
\end{array}$ & $\begin{array}{r}3.35 \\
\pm 0.17\end{array}$ & $\begin{array}{c}\mathbf{N K} \\
\Sigma \pi \\
\Sigma(1385) \pi \\
\end{array}$ & $\begin{array}{r}3-10 \\
35-75 \\
>15\end{array}$ & $\begin{array}{l}553 \\
515 \\
371 \\
\end{array}$ \\
\hline$\Lambda(1890)$ & $0\left(3 / 2^{+}\right) \mathbf{P}_{03}^{\prime}$ & $\begin{array}{l}\mathrm{p}=1.2 \mathrm{i} \\
a=13.6\end{array}$ & $\begin{array}{r}1850 \text { to } \\
1910\end{array}$ & $\begin{array}{r}60 \text { to } \\
200 \\
(100)\end{array}$ & $\begin{array}{r}3.57 \\
\pm 0.19\end{array}$ & $\begin{array}{c}\mathbf{N \overline { K }} \\
\Sigma \pi \\
\Sigma(385) \pi \\
N^{\prime} \cdot(892)\end{array}$ & $\begin{array}{r}20-35 \\
3-10 \\
\text { seen } \\
\text { seen }\end{array}$ & $\begin{array}{l}599 \\
559 \\
420 \\
233\end{array}$ \\
\hline$\Lambda(2 ! 00)$ & $O\left(7 / 2^{-}\right) G_{07}$ & $\begin{array}{l}p=1.68 \\
\sigma=8.68\end{array}$ & $\begin{array}{r}2090 \text { to } \\
2110\end{array}$ & $\begin{array}{r}100 \text { to } \\
250 \\
(200)\end{array}$ & $\begin{array}{r}4.41 \\
\pm 0.42\end{array}$ & $\begin{array}{c}\mathbf{N K} \\
\Sigma \pi \\
\Lambda \pi \\
\Xi \mathrm{K} \\
\mathbf{A} \boldsymbol{\omega} \\
\mathbf{N K}^{\star}(892) \\
\end{array}$ & $\begin{array}{r}25-35 \\
\sim 5 \\
<3 \\
<3 \\
<8 \\
10-20 \\
\end{array}$ & $\begin{array}{l}751 \\
704 \\
617 \\
483 \\
443 \\
514 \\
\end{array}$ \\
\hline$\overline{\Lambda(2110)}$ & $O\left(5 / 2^{+}\right) F_{05}^{\prime \prime}$ & $\begin{array}{l}p=1,70 \\
\sigma=8.54\end{array}$ & $\begin{array}{r}2090 \text { to } \\
2140\end{array}$ & $\begin{array}{r}150 \text { to } \\
250 \\
(200)\end{array}$ & $\begin{array}{r}4.45 \\
\pm 0.42\end{array}$ & $\begin{array}{c}\mathrm{NK} \\
\Sigma \pi \\
\Lambda \omega \\
\Sigma(1385) \pi \\
N^{*} \bar{K}^{*}(892) \\
\end{array}$ & $\begin{array}{r}5-25 \\
10-40 \\
\text { seen } \\
\text { seen } \\
10-60 \\
\end{array}$ & $\begin{array}{l}757 \\
711 \\
455 \\
589 \\
524 \\
\end{array}$ \\
\hline$\Lambda(2350)$ & $0\left(9 / 2^{+}\right)$ & $\begin{array}{l}p=2.29 \\
\sigma=5.84\end{array}$ & $\begin{array}{r}2340 \text { to } \\
2370\end{array}$ & $\begin{array}{r}10040 \\
250 \\
(150) \\
\end{array}$ & $\begin{array}{r}5.52 \\
\pm 0.35\end{array}$ & $\begin{array}{l}\mathrm{N \overline {K }} \\
\Sigma_{\pi}\end{array}$ & $\begin{array}{l}\sim 12 \\
\sim 10\end{array}$ & $\begin{array}{l}915 \\
867\end{array}$ \\
\hline$\Lambda(2585)$ & $\alpha(?)$ & $\begin{array}{l}p=2.92 \\
\sigma=4.35\end{array}$ & -2585 & $\begin{array}{r}\sim 300 \\
(300) \\
\end{array}$ & $\begin{array}{r}6.68 \\
\pm 0.78 \\
\end{array}$ & $\mathbf{N \overline { K }}$ & $\begin{array}{r}\mathrm{J}+1 / 2) \mathrm{x} \\
\sim 1.0 j\end{array}$ & 1060 \\
\hline
\end{tabular}


Baryon Table (cont'd)

\begin{tabular}{|c|c|c|c|c|c|c|}
\hline & & & $\operatorname{sing} d$ & & $M^{2}$ & Partial decay mode \\
\hline article ${ }^{a}$ & $\left.\mathbf{I}\left(\mathrm{J}^{\mathbf{P}}\right) \mathbf{L}_{\mathbf{F} \cdot \mathbf{b}}\right)$ & $\begin{array}{l}P_{\text {beam }}^{c}(G e V / c) \\
\sigma=4 \pi X^{2}(a)\end{array}$ & $\underset{\mathbf{M}}{\mathbf{M} V}$ & $\underset{(\mathrm{MeV})}{\operatorname{with} \Gamma}$ & $\underset{\left(\mathbf{G} \in \mathbf{V}^{2}\right)}{\min }$ & $\begin{array}{c}\text { Fraction } \\
(\mathscr{O}) \quad \mathrm{p}^{h} \\
(\mathrm{M} V \mathrm{~V} / \mathrm{e})\end{array}$ \\
\hline
\end{tabular}

\begin{tabular}{|c|c|c|c|c|c|c|c|c|}
\hline \multicolumn{9}{|c|}{ S=-1 I=I SIGMK RLSONANCES (2) } \\
\hline$\overline{\mathbf{\Sigma}}$ & $1\left(1 / 2^{+}\right)$ & & $\begin{array}{r}(+) 1189.4 \\
(0) 1192.5 \\
(-) 1197.3\end{array}$ & & $\begin{array}{l}1.415 \\
1.422 \\
1.434\end{array}$ & See Sta & ble Particle & $\overline{\text { Tab!e }}$ \\
\hline$\overline{\mathbf{Z}}(1385)$ & $\overline{\mathrm{I}\left(3 / \mathbf{2}^{+}\right) \mathrm{P}_{13}^{\prime}}$ & $\begin{array}{l}\text { Below } \\
\mathbf{K}^{-} \mathbf{p} \\
\text { threshold }\end{array}$ & $\begin{array}{c}(+) 1382.3 \pm 0.4 \\
S=1.6^{m} \\
(0) 1382.0 \pm 2.5 \\
S=1.6^{m} \\
(-) 1387.4 \pm 0.6 \\
S=2.2^{m}\end{array}$ & $\begin{array}{c}35 \pm 1 \\
s=1.0^{m} \\
\sim 35 \\
40 \pm 2 \\
s=1.9^{m} \\
\end{array}$ & $\begin{array}{r}1.92 \\
\pm 0.05\end{array}$ & $\begin{array}{l}\Delta \pi \\
\Sigma \pi\end{array}$ & $\begin{array}{l}88 \pm 2 \\
12 \pm 2\end{array}$ & $\begin{array}{l}208 \\
127\end{array}$ \\
\hline$\overline{\Sigma(1660)}$ & $1\left(1 / 2^{+}\right) P_{11}^{\prime}$ & $\begin{array}{l}\mathrm{p}=0.72 \\
\sigma=29.8\end{array}$ & $\begin{array}{r}1630 \text { to } \\
1690\end{array}$ & $\begin{array}{r}40 \text { to } \\
200 \\
(100) \\
\end{array}$ & $\begin{array}{r}2.76 \\
\pm 0.17\end{array}$ & $\begin{array}{l}\mathrm{N} \overline{\mathrm{K}} \\
\mathrm{A} \pi \\
\mathrm{\Sigma} \pi \\
\end{array}$ & $\begin{array}{r}10-30 \\
\text { seen } \\
\text { seen }\end{array}$ & $\begin{array}{l}405 \\
439 \\
385 \\
\end{array}$ \\
\hline$\overline{\Sigma(1670)}$ & $\mathrm{l}\left(3 / 2^{-}\right) \mathrm{D}_{13}^{\prime \prime}$ & $\begin{array}{l}p=0.74 \\
\sigma=28.5\end{array}$ & $\begin{array}{r}1665 \text { to } \\
1685\end{array}$ & $\begin{array}{r}40 \text { to } \\
80 \\
(60) \\
\end{array}$ & $\begin{array}{r}2.79 \\
\pm 0.10\end{array}$ & $\begin{array}{l}\text { N⿳亠口冋 } \\
4 \pi \\
2 \pi \\
\end{array}$ & $\begin{array}{r}7-13 \\
5-15 \\
30-60 \\
\end{array}$ & $\begin{array}{l}414 \\
447 \\
393 \\
\end{array}$ \\
\hline$\Sigma(1750)$ & $\|\left(1 / 2^{-}\right) S_{11}^{\prime \prime}$ & $\begin{array}{l}p=0.91 \\
\sigma=20.7\end{array}$ & $\begin{array}{r}1730 \text { to } \\
1800\end{array}$ & $\begin{array}{l}60 \text { to } \\
160 \\
(90)\end{array}$ & $\begin{array}{r}3.06 \\
\pm 0.16\end{array}$ & $\begin{array}{l}\mathrm{NK} \\
\Delta \pi \\
\Sigma \pi \\
\Sigma \pi\end{array}$ & $\begin{array}{r}10-40 \\
\text { soen } \\
<8 \\
15-55\end{array}$ & $\begin{array}{r}486 \\
507 \\
455 \\
81 \\
\end{array}$ \\
\hline$\overline{\Sigma(1775)}$ & $1\left(5 / 2^{-}\right) \overline{D_{15}}$ & $\begin{array}{l}p=0.96 \\
\sigma=19.0\end{array}$ & $\begin{array}{r}1770 \text { to } \\
1780\end{array}$ & $\begin{array}{r}105 \text { to } \\
135 \\
(120)\end{array}$ & $\begin{array}{r}3.15 \\
\pm 0.21\end{array}$ & $\begin{array}{c}N \bar{K} \\
\Lambda \pi \\
\Sigma \pi \\
\Sigma(1385) \pi \\
\Lambda(1520) \pi\end{array}$ & $\begin{array}{r}37-43 \\
14-20 \\
2-5 \\
8-12 \\
17-23 \\
\end{array}$ & $\begin{array}{l}508 \\
525 \\
474 \\
324 \\
198 \\
\end{array}$ \\
\hline$\Sigma(1915)$ & $1\left(5 / 2^{+}\right) F_{15}^{\prime}$ & $\begin{array}{l}p=1.26 \\
\sigma=12.8\end{array}$ & $\begin{array}{l}1900 \text { to } \\
1935\end{array}$ & $\begin{array}{r}80 \text { to } \\
160 \\
(120)\end{array}$ & $\begin{array}{r}3.67 \\
\pm 0.23\end{array}$ & $\begin{array}{c}N \bar{K} \\
\Lambda \pi \\
\Sigma \pi \\
\Sigma(1385) \pi\end{array}$ & $\begin{array}{l}5-15 \\
\text { seen } \\
\text { soen } \\
<5\end{array}$ & $\begin{array}{l}618 \\
622 \\
577 \\
440 \\
\end{array}$ \\
\hline $\bar{\Sigma}(1940)$ & $1\left(3 / 2^{-}\right) \mathrm{D}_{13}^{\prime \prime \prime}$ & $\begin{array}{l}p=1.32 \\
\sigma=12.1\end{array}$ & $\begin{array}{r}1900 \text { to } \\
1950\end{array}$ & $\begin{array}{r}150 \text { to } \\
300 \\
(220)\end{array}$ & $\begin{array}{r}3.76 \\
\pm 0.43\end{array}$ & $\begin{array}{c}N \bar{K} \\
\Lambda \pi \\
\Sigma \pi \\
\Sigma(1385) \pi \\
\Lambda(1520) \pi \\
\Delta(1232) \bar{K} \\
N^{\prime} \cdot(B 92)\end{array}$ & $\begin{array}{l}<20 \\
\text { seen } \\
\text { seen } \\
\text { seen } \\
\text { seen } \\
\text { seen } \\
\text { seen }\end{array}$ & $\begin{array}{l}637 \\
639 \\
594 \\
460 \\
354 \\
410 \\
320 \\
\end{array}$ \\
\hline$\overline{2}(2030)$ & $\mathrm{I}\left(7 / 2^{+}\right) \mathrm{F}_{17}$ & $\begin{array}{l}\mathrm{p}=1.52 \\
\sigma=9.93\end{array}$ & $\begin{array}{r}2025 \text { to } \\
2040\end{array}$ & $\begin{array}{r}15010 \\
200 \\
(180)\end{array}$ & $\begin{array}{r}4.12 \\
\pm 0.37\end{array}$ & $\begin{array}{c}N \bar{K} \\
\Lambda \pi \\
\Sigma \pi \\
\Xi K \\
\Sigma(1385) \pi \\
\Lambda(1520) \pi \\
\Delta(1232) \bar{K} \\
N \bar{K}^{*}(892) \\
\end{array}$ & $\begin{array}{r}17-23 \\
17-23 \\
5-10 \\
<2 \\
5-15 \\
10-20 \\
10-20 \\
<5 \\
\end{array}$ & $\begin{array}{l}702 \\
700 \\
657 \\
412 \\
529 \\
430 \\
498 \\
438 \\
\end{array}$ \\
\hline$\overline{3(2250)}$ & l(?) & $\begin{array}{l}p=2.04 \\
\sigma=6.76\end{array}$ & $\begin{array}{r}2210 \text { to } \\
2280\end{array}$ & $\begin{array}{r}60 \text { to } \\
150 \\
(100) \\
\end{array}$ & $\begin{array}{r}5.06 \\
\pm 0.23\end{array}$ & $\begin{array}{l}N \bar{K} \\
\Delta \pi \\
\Sigma \pi\end{array}$ & $\begin{array}{l}<10 \\
\text { seen } \\
\text { seen }\end{array}$ & $\begin{array}{l}851 \\
842 \\
803\end{array}$ \\
\hline$\overline{\Sigma(2455)}$ & I(?) & $\begin{array}{l}p=2.57 \\
\sigma=5.08\end{array}$ & -2455 & $\begin{array}{l}-120 \\
(120) \\
\end{array}$ & $\begin{array}{r}6.03 \\
\pm 0.29 \\
\end{array}$ & $\mathbf{N \mathbf { K }}$ & $\begin{array}{c}(\mathrm{J}+1 / 2) \mathrm{x} \\
-0.2 \mathrm{~J}\end{array}$ & $\overline{981}$ \\
\hline$\overline{\Sigma(2620)}$ & l(? ?) & $\begin{array}{l}p=3.02 \\
\sigma=4.19\end{array}$ & $\sim 2600$ & $\begin{array}{l}-200 \\
(200) \\
\end{array}$ & $\begin{array}{r}6.86 \\
\pm 0.52 \\
\end{array}$ & $N \overline{\mathbf{K}}$ & $\begin{array}{r}(J+1 / 2) \mathrm{x} \\
\quad 0.3 j\end{array}$ & 1081 \\
\hline
\end{tabular}




\section{Baryon Table (cont'd)}

\begin{tabular}{|c|c|c|c|c|c|c|c|c|}
\hline \multirow[b]{2}{*}{ Particle ${ }^{a}$} & \multirow[b]{2}{*}{$\mathrm{I}\left(\mathbf{J}^{\mathbf{P}}\right) \mathbf{L}_{2 \mathrm{I} \cdot 2 \mathbf{J}}^{\boldsymbol{b}}$} & \multirow[b]{2}{*}{$\begin{array}{l}P_{\text {beam }}^{c}(\mathrm{GeV} / \mathrm{c}) \\
\sigma=4 \pi X^{2}(\mathrm{mb})\end{array}$} & \multirow{2}{*}{$\underset{\text { Mev) }}{\text { Mess }^{d}}$} & \multirow{2}{*}{$\begin{array}{l}\text { Fulle }^{e} \\
\text { width } \Gamma \\
\text { (MeV) }\end{array}$} & \multirow{2}{*}{$\begin{aligned} & M^{2} f \\
& \pm \Gamma M \\
&\left(\mathrm{GeV}^{2}\right)\end{aligned}$} & \multicolumn{3}{|c|}{ Partial decay node } \\
\hline & & & & & & Mode & $\begin{array}{c}\text { Fraction } \\
\text { (\$) }\end{array}$ & $(\mathbf{M e V} /$ \\
\hline \multicolumn{9}{|c|}{$S=-2 \quad I=1 / 2$ CASCADE RESONANCES $(\bar{z})$} \\
\hline$\Xi$ & $1 / 2\left(1 / 2^{+}\right)$ & \multicolumn{3}{|c|}{$\begin{array}{l}(0) 1314.9 \\
(-) 1321.3\end{array}$} & $\begin{array}{l}1.729 \\
1.746\end{array}$ & \multicolumn{3}{|c|}{ See Stable Particle Table } \\
\hline$\Xi(1530)$ & $1 / 2\left(3 / 2^{+}\right) P_{13}$ & \multicolumn{2}{|r|}{$\begin{array}{c}(0) 1531.8 \pm 0.3 \\
S=1.3^{m} \\
(-) 1535.0 \pm 0.6\end{array}$} & $\begin{array}{r}9.1 \pm 0.5 \\
10.1 \pm 1.9 \\
\end{array}$ & $\begin{array}{r}2.34 \\
\pm 0.02\end{array}$ & $\Xi \pi$ & 100 & 148 \\
\hline$\Xi(1820)$ & $1 / 2(3 / 2)$ & \multicolumn{2}{|r|}{$\begin{array}{c}1823 \\
\pm 6^{\ell}\end{array}$} & $20_{-10}^{+15^{l}}$ & $\begin{array}{r}3.31 \\
\pm 0.04\end{array}$ & $\begin{array}{c}\Delta \bar{K} \\
\Sigma \bar{K} \\
\Xi \pi \\
\Xi(1530) \pi\end{array}$ & $\begin{array}{l}\sim 45 \\
\sim 10 \\
\text { small } \\
\sim 45\end{array}$ & $\begin{array}{l}396 \\
306 \\
413 \\
231\end{array}$ \\
\hline$\Xi(2030)$ & $1 / 2(?)$ & & $\begin{array}{c}2024 \\
\pm 6^{l}\end{array}$ & $16_{-5}^{+15^{2}}$ & $\begin{array}{r}4.12 \\
\pm 0.03\end{array}$ & $\begin{array}{c}\Lambda \bar{K} \\
\Sigma \bar{K} \\
\Xi \pi \\
\Xi(1530) \pi\end{array}$ & $\begin{array}{l}\sim 20 \\
\sim 80 \\
\text { smal! } \\
\text { small }\end{array}$ & $\begin{array}{l}587 \\
524 \\
573 \\
418 \\
\end{array}$ \\
\hline $\mathrm{\Omega}^{-}$ & $0\left(3 / 2^{+}\right)$ & \multicolumn{3}{|c|}{$1672.4 \pm 0.3$} & 2.797 & \multicolumn{3}{|c|}{ See Stable Particle Table } \\
\hline $\mathrm{A}_{c}^{+}$ & $0\left(1 / 2^{+}\right)$ & \multicolumn{3}{|c|}{$2282 \pm 3$} & 5.21 & \multirow{2}{*}{\multicolumn{3}{|c|}{$\begin{array}{c}\text { See Stable Particle Table } \\
\text { Partial decay node } \\
\end{array}$}} \\
\hline & & \multirow[b]{2}{*}{$\begin{array}{l}P_{\text {beam }}^{c}(G e V / c) \\
\sigma=4 \pi K^{2}(m a b)\end{array}$} & \multirow{2}{*}{$\underset{(\mathrm{MeV})}{\text { Muss }^{d}}$} & \multirow{2}{*}{$\begin{array}{l}\text { Full }^{e} \\
\text { midtb } \Gamma \\
(\mathrm{MeV})\end{array}$} & \multirow{2}{*}{$\begin{array}{c}\mathrm{M}^{2 f} \\
\pm \mathbf{M M} \\
\left(\mathrm{GeV}^{2}\right)\end{array}$} & & & \\
\hline Particle $^{a}$ & $\left(\mathbf{J}^{\mathrm{P}}\right)^{2 S+1_{\mathrm{L}_{\mathrm{J}}}}$ & & & & & Mode & $\begin{array}{c}\text { Fraction } \\
\text { (\%) }\end{array}$ & $\langle\mathbf{M e V} /$ \\
\hline \multicolumn{9}{|c|}{$S=0$ DKBARYONS } \\
\hline$d$ & $0\left(1^{+}\right)$ & & 1875.6 & & 3.518 & & & \\
\hline$N N(2 \mid 70)$ & $1\left(2^{+}\right)^{I} D_{2}$ & $\begin{array}{l}p=1.26 \\
\sigma=16.5\end{array}$ & $\begin{array}{r}2140 \text { to } \\
2190\end{array}$ & $\begin{array}{r}50 \text { 10 } \\
125 \\
(90) \\
\end{array}$ & $\begin{array}{r}4.71 \\
\pm 0.20\end{array}$ & $\begin{array}{l}\mathrm{NN} \\
\pi \mathrm{d}\end{array}$ & $\begin{array}{r}10-20 \\
\text { seen }\end{array}$ & $\begin{array}{l}545 \\
241\end{array}$ \\
\hline$N N(2250)$ & $1\left(3^{-}\right)^{3} \mathrm{~F}_{3}$ & $\begin{array}{l}p=1.49 \\
\sigma=12.7\end{array}$ & $\begin{array}{r}2200 \text { to } \\
2400\end{array}$ & $\begin{array}{r}75 \text { 10 } \\
225 \\
(150)\end{array}$ & $\begin{array}{r}5.06 \\
\pm 0.34\end{array}$ & $\begin{array}{l}\mathrm{NN} \\
\pi \mathrm{d}\end{array}$ & $\begin{array}{r}20-30 \\
\text { seen }\end{array}$ & $\begin{array}{l}621 \\
318\end{array}$ \\
\hline \multicolumn{9}{|c|}{$S=-1$ DIBARYONS } \\
\hline $\ln (2130)$ & $1 / 2\left(1^{+}\right)^{3} s_{1}$ & $\begin{array}{l}p=0.64 \\
\sigma=61.5\end{array}$ & $\begin{array}{r}2100 \text { to } \\
2200\end{array}$ & $\begin{array}{r}5 \text { to } \\
25 \\
(15)\end{array}$ & $\begin{array}{r}4.54 \\
\pm 0.03\end{array}$ & $\Delta N$ & $\operatorname{seen}$ & 282 \\
\hline
\end{tabular}




\section{Baryon Table (cont'd)}

$\rightarrow$ Each arrow in the left-hand margin indicates there is an entry in the Data Card Listings lor a baryon that is not well enough established (status less than 3 stars) to be incieded here. There is a short list of all the baryons in the Listings, whatever their status, at the front of this Table.

$t$. This mode is energetically forbidden when the nominal mass of the decaying resonance (and of any resonance in the final state) is used, but is in fact allowed due to the finite width of the resonance(s).

* The modes in braskets are sub-reactions of the first preceding unbracketed mode.

a. The nominal mass here (in MeV) is used for identification. See column 4 for the actual mass.

b. When there is more than one baryon with the same quantum numbers, one prime is attached to the spectroscopic symbol for the first of them (e.g.. $S_{j}^{\prime}$ ), two primes to the second, etc.

c. The quantities here are calculated using the nominal mass of column 1 .

d. Usually a conservatively large range of masses rather than a statistical average of various determinations of the mass is given. In these cases, the mass determinations are nearly entirely from various phase-shift analyses of more or less the same da1a. It is thus not appropriate to treat the determinations as independent measurements or to average them together. The masses, widths, and branching fractions in this Table are Breit-Wigner parameters. The Data Card Listings also include pole parameters where they are available.

e. Usually a conservatively large range of widths rather than a statistical average of various determinations of the width is given (see note $d$ for the reason). The nominal value in parentheses is then simply a best guess.

f. The quantities here are calculated using the nominal mass of column 1 and the rominal width of column 5 .

g. For information on the $\mathrm{N} \gamma$ decay modes of the $\mathrm{N}$ and $\Delta$ baryons, see the mini-review on these states in the Listings.

h. Most of the inelastic branching fractions come from partial-wave analyses, and these determine $\sqrt{\mathrm{xx}^{\prime}}$, where $x$ and $x^{\prime}$ are the elastic and inelastic branching fractions, not $x^{\prime}$ directly. Thus any uncertainty (and it is often considerable) in $x$ carries over into $x^{\prime}$. When $x^{\prime}$ so determined is really poorly known, we here simply note that the mode is seen. The values of $\sqrt{\boldsymbol{x}^{\prime}}$ are given in the Data Card Listings.

i. For a 2-body decay mode, this is the momentum of the decay products in the rest frane of the decaying particle. For a mode with more than two decay products, this is the maximum momentum any of the products can have in this frame. The nominal mass of column $I$ is used, as is the nominal mass of any resonance in the final state.

$j$. The size of the bump in the total eross section gives $(J+1 / 2) x$, where $x$ is the elastic branching fraction, but the value of $J$ is not known.

k. These pole positions are from fits to phase shifts (without Coulomb corrections). The Data Card Listings now inciude pole positions and residues for most of the $N$ and $\Delta$ resonances. See Sect. I of the $N$ and $\Delta$ minj-review in the Listings for a brief discussion of the advantages of pole parameters over the usual Breit- Wizner parameters.

$\ell$. The error given here is only an educated guess. It is larger than the error on the weighted average of the published values (the ersor on this average is given in the Listings).

m. The error given here has been scaled up by the "S factor" (see the * footnote to the Stable Particle Table for how $S$ is defined) because the various measurements disagree more seriously than one would expect from statistics. 


\section{PHYSICAL AND NUMERICAL CONSTANTS*}

\section{PHYSICAL CONSTANIS}

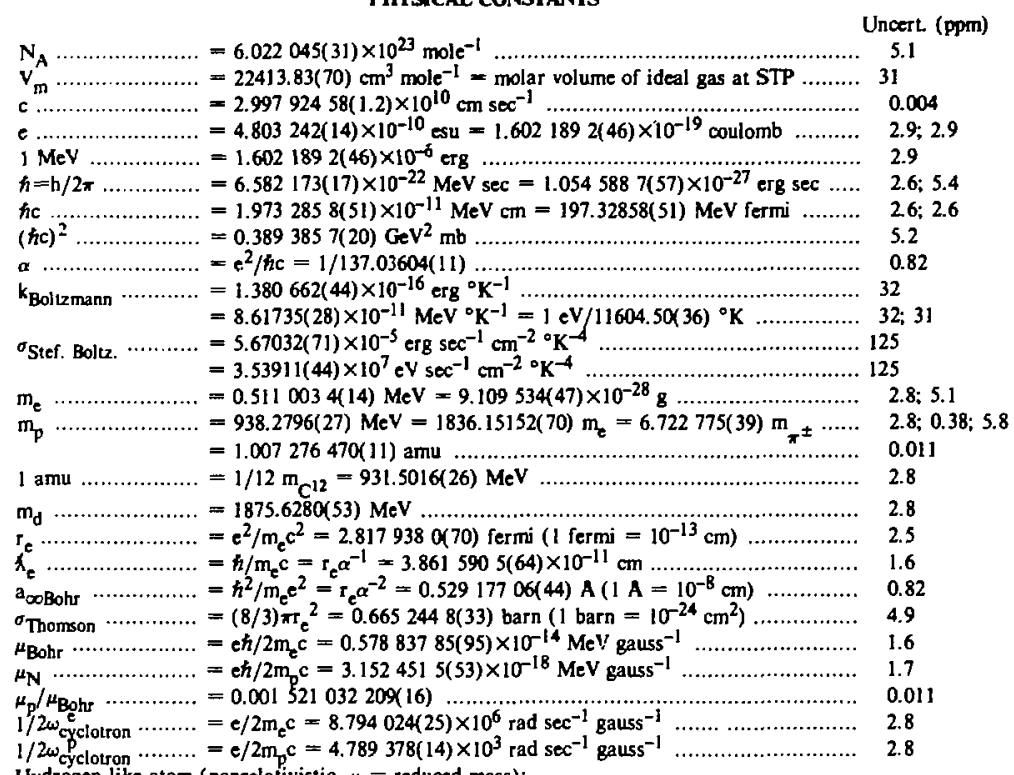

Hydrogen-like atom (nonrelativistic, $\mu=$ reduced mass):

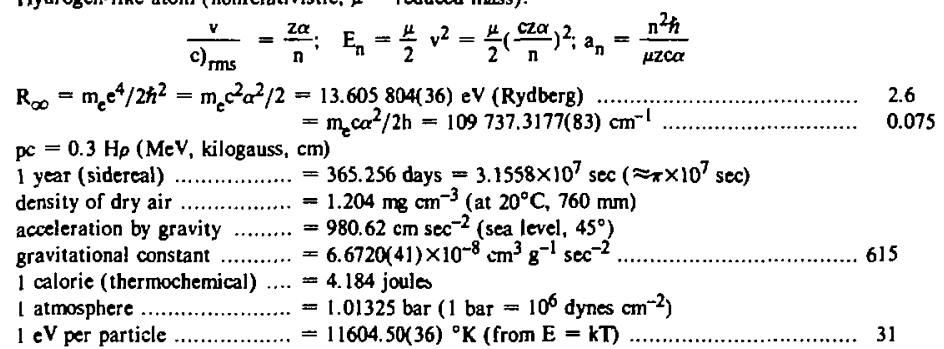

\section{NUMERICAL CONSTANIS}

$\begin{array}{ll}\pi & =3.1415927 \\ \mathrm{e} & =2.7182818 \\ \ln 2 & =0.6931472 \\ \log _{10} 2 & =0.3010300\end{array}$

$1 \mathrm{rad}=57.2957795 \mathrm{deg}$
$1 / \mathrm{e}=0.3678794$
$\ln 10=2.3025851$
$\log _{10}=0.4342945$

$\sqrt{\pi}=1.77245385$
$\sqrt{2}=1.4142136$
$\sqrt{3}=1.7320508$
$\sqrt{10}=3.1622777$

\footnotetext{
"Revised 1982 by Barry N. Taylor. Originally prepared by Stanley J. Brodsky, based mainly on the "1973 Least-Squares Adjusiment of the Fundamental Constants," by E. R. Cohen and B. N. Taylor, J. Phys. Chem. Ref. Data 2, 663 (1973). The figures in parentheses correspond to the one-standard-deviation uncertainty in the last digits of the main number. The equivalent uncertainty in parts per million (ppm) is given in the last column. Note that the uncertainties of the output values of a least-squares adjustment are in general correlated, and the general law of error propagation must be used in calculating additional quantities.

The set of constants resulting from the 1973 adjustment of Cohen and Taylor has been recommended for international use by CODATA (Committee on Data for Science and Technology), and is the most up-to-date, generally accepted set currently available. However, since the pub-

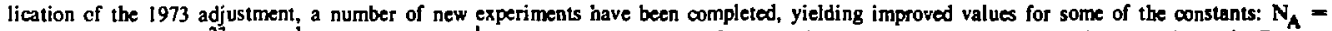
$6.0220978(63) \times 10^{23} \mathrm{~mole}^{-1}(1.04 \mathrm{ppm}) ; \alpha^{-1}=137.035963(15)(0.11 \mathrm{ppm}) ; \mathrm{nl}_{\mathrm{o}} / \mathrm{m}_{\mathrm{e}}=1863.15300(25)(0.14 \mathrm{ppm}) ; \mathrm{and} \mathbf{R}_{\infty}=$ $109737.31521(11) \mathrm{cm}^{-1}(0.001 \mathrm{ppm})$. But it must be realized that, since the output values of a least-squares adjustment are related in a complex way and a change in the measured value of one constant usually leads to corresponding changes in the adjusted values of others, one must be caytious in carrying out calculations using both the output values from the 1973 adjustment and the results of more recent experiments. A new adjustment is planned for compietion by mid 1982.
} 


\section{CLEBSCH-GORDAN COEFFICIENTS, SPHERICAL HARMONICS, AND \& FUNCTIONS}

Note: $A, T$ is to be understood over every coefificient; c. g., for $-8 / 15$ read $-\sqrt{8 / 15}$.

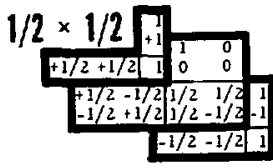

$$
\begin{aligned}
& Y_{1}^{0}=\sqrt{\frac{3}{4 \pi}} \cos \theta \\
& Y_{1}^{1}=-\sqrt{\frac{3}{8 \pi}} \sin \theta e^{10} \\
& Y_{2}^{0}=\sqrt{\frac{5}{4 \pi}} \frac{3}{2} \cos ^{2} \theta-\frac{1}{2}
\end{aligned}
$$

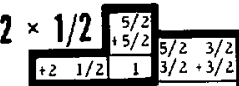
$1+2-1 / 2 \quad 1 / 5 \quad 4 / 5 \quad 5 / 2 \quad 3 / 2$

$+1+1 / 2] 4 / 5-1 / 5+1 / 2+1 / 2$

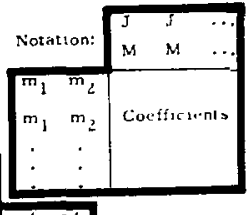

$1 \times 1 / 2 \longdiv { 3 / 2 }$

$+1+1 / 2-1+1 / 2+1 / 2$

$Y_{2}^{1}=-\sqrt{\frac{15}{87}} \sin \theta \cos \theta c^{i \phi}$

\begin{tabular}{rr|rr|rr}
+1 & $-1 / 2$ & $2 / 5$ & $3 / 5$ & $5 / 2$ & $3 / 2$ \\
$0+1 / 2$ & $3 / 5$ & $-2 / 5$ & $-1 / 2$ & $-1 / 2$ \\
\hline
\end{tabular}

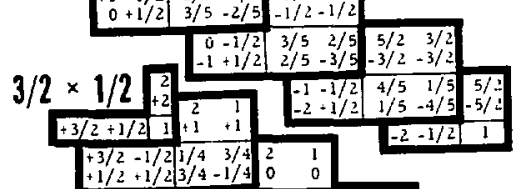

$2 \times 1$ 3 \begin{tabular}{rr|rrr}
0 & $-1 / 2$ & $2 / 3$ & $1 / 3$ & $3 / 2$ \\
$-1+1 / 2$ & $1 / 3$ & $-2 / 3$ & $-3 / 2$ \\
\hline & -1 & $-1 / 2$ & 1
\end{tabular}

$$
Y_{2}^{2}=\frac{1}{4} \sqrt{\frac{15}{2 \pi}} \sin ^{2} \theta e^{210}
$$

\section{$3 / 2 \times 1 \longdiv { 5 / 2 }$}

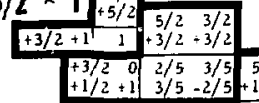

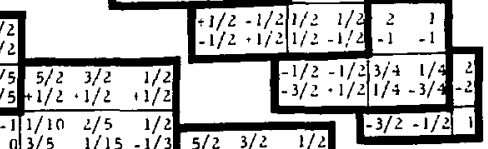

$1 \times 1+2$

$+2+1: 1+2+2$

\begin{tabular}{rr|rrrr}
+2 & 0 & $1 / 3$ & $2 / 3$ \\
+1 & 1 & 3 & 2 & 1 \\
$2 / 3$ & $-1 / 3$ & +1 & +1 & +1
\end{tabular}

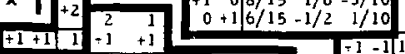

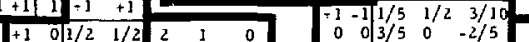

\begin{tabular}{ll|llllll}
$+3 / 2$ & $-1 / 10$ & $2 / 5$ & $1 / 2$ & & & & \\
$+1 / 2$ & 0 & $3 / 5$ & $1 / 15$ & $-1 / 3$ & $5 / 2$ & $3 / 2$ & $1 / 2$
\end{tabular}

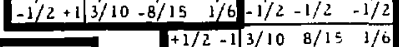

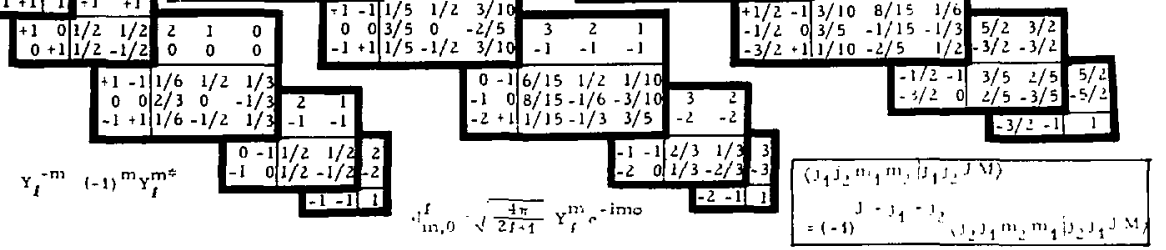

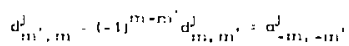

$2 \times 3 / 2 \sqrt{7 / 2}$

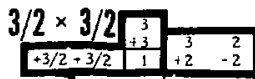

$d_{1 / 2,1 / 2}^{1 / 2} \cos \frac{\pi}{2} \quad d / \frac{1}{2}-1 \frac{1}{2}=-\sin \frac{t}{2}$

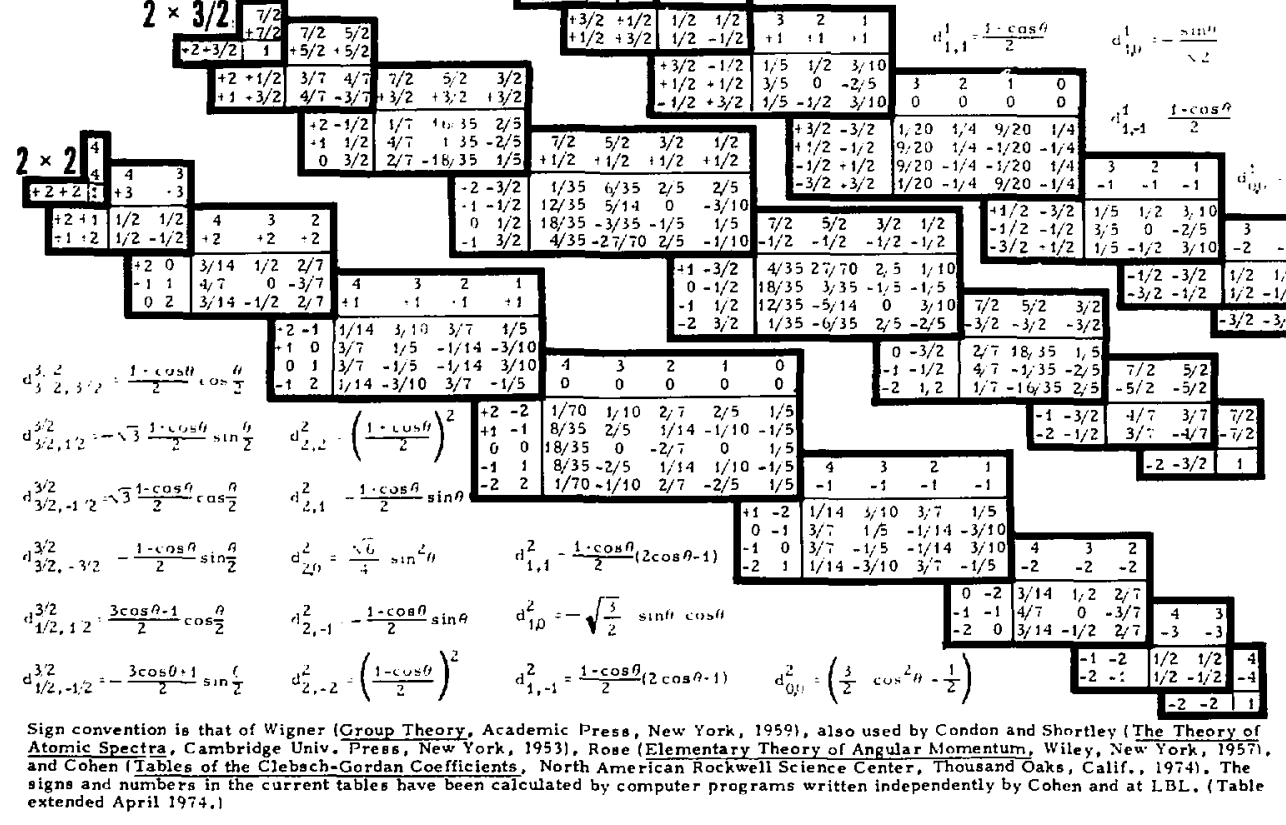




\section{SU(3) ISOSCALAR FACTORS, SU(N) MULTIPLICITIES, AND PROPERTIES OF QUARKS}

The most common $2 y$ used isoscalar factors, corresponding to the singlet, octet, and decuplet content of $8 \otimes 8$ and $10 \otimes 8$, are displayed at the right. The notation uses particles names to identify the coefficients, so that the pattern of relative couplings can be seen at a glance. We illustrate the use of the coefticients by example; see J. J. de Swart, Rev. Mod. Phys. 35, 916 (1963) for detailed explanation and piase conventions.

$A \sqrt{ }$ is understood over every integer in the matrices; the exponent $\frac{1}{2}$ is a reminder of this. For example, in de Swat's notation the $\equiv+\Omega \mathrm{K}$ elemert of our $10+10 \mathrm{e}$ matrix reads

$$
\left(\begin{array}{cc|cc}
10 & & 8 & 10 \\
0 & -2 & 5 & 1 \\
2 & -1
\end{array}\right)=\frac{-\sqrt{6}}{\sqrt{24}} \text {. }
$$

Intra-multiplet relative decay strengths can be read directly from our matrices. Thus, the partial widths for $\Delta^{*} \rightarrow(N \pi) I=3 / 2$ and $\Omega^{*} \rightarrow(\equiv \bar{K})_{1=0}$ are in the ratio

$$
\left.\frac{\Gamma\left(S^{*}+(E \bar{K})_{I=0}\right)}{\Gamma\left(\Lambda^{*}-(N \pi)_{I=3 / 2}\right)}=\frac{12}{6} \times \text { (threshold factors }\right) \text {. }
$$

Supplying isospin Clebsch-Gordan coefficients one obtains, e.g.,

$$
\frac{\Gamma\left(\Omega^{*}+E^{0} K^{-}\right)}{\Gamma\left(\Delta^{*}+2^{0}\right)}=\frac{1 / 2}{2 / 3} \times \frac{12}{6} \times \text { tf }=\frac{3}{2} \times \text { tf } .
$$

Partial widths for $8+8 \odot 8$ involve a linear superposition of $8_{1}$ (symetric) and $8_{2}$ (anti-symetric) couplings. For example,

$$
\left[\left(E^{*} \rightarrow \bar{I}\right)-\left(-\sqrt{\frac{9}{20}} g_{1}+\sqrt{\frac{3}{12}} g_{2}\right)^{2}\right. \text {. }
$$

The relation between $g_{1}, g_{2}$ (with de Swart's normilization) and the standard $D, F$ couplings appearing in the interaction Laqrangian,

25

$$
\mathcal{L}=-\sqrt{2} D \operatorname{Tr}\left([\bar{B}, B]_{+} M\right)+\sqrt{2} \operatorname{F} \operatorname{Tr}([\bar{B}, B]-M),
$$

$$
D=\frac{\sqrt{30}}{40} g_{1}, \quad F=\frac{\sqrt{6}}{24} g_{2}
$$

Thus, $\quad \because\left(E^{*}+\Xi \pi\right) \sim(1-2 a)^{2}$

wher: $a \equiv 0,(D+F)$.

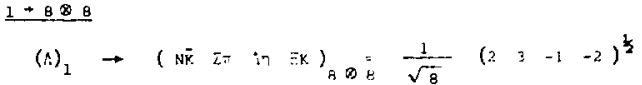

$\underline{8}+808$

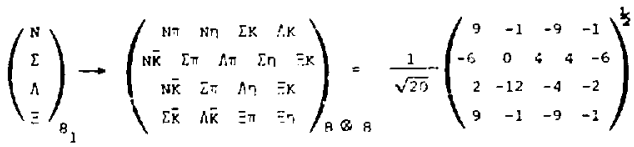

$\underline{8}_{2}+\mathrm{g} \otimes \mathrm{a}$

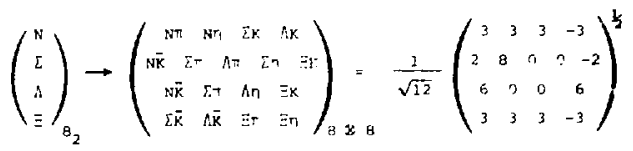

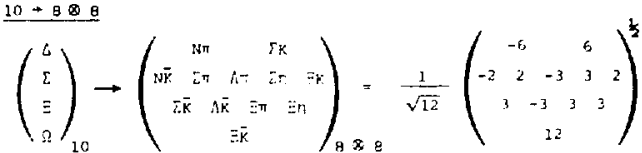

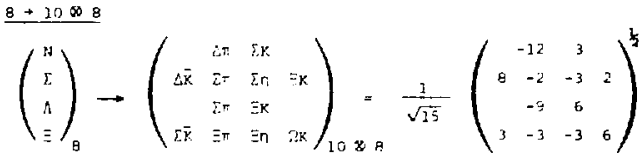

$10+108$

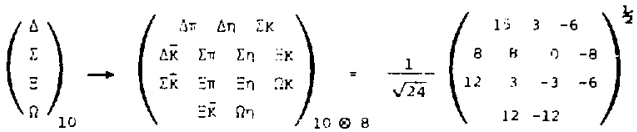

\section{SU(n) Multiplicities}

The table below gives the multiplicities of the multiplets that occur in $q q, q \bar{q}$, and qqq systeng in various $\mathrm{su}(\mathrm{n})$. Normal mesons are qū systems, and normal baryons are qqq systems. Also given are the multiplets that occur in meson-baryon scattering when the meson multiplet is the one to which the pion belongs and the

\begin{tabular}{|c|c|c|}
\hline 99 & & \\
\hline $\begin{array}{l}\text { SU }(2): \\
\text { st }(3): \\
\text { S(T(4): } \\
\text { sU }(n):\end{array}$ & $\begin{array}{l}202 \\
303 \\
404 \\
100\end{array}$ & 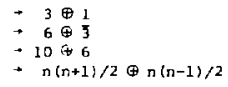 \\
\hline वर & Meson & \\
\hline $\begin{array}{l}\operatorname{su}(2): \\
\operatorname{su}(3): \\
\operatorname{su}(4): \\
\operatorname{su}(n):\end{array}$ & $\begin{array}{lll}2 & 0 & 2 \\
3 & 0 & 3 \\
4 & 9 & \overline{4} \\
n & 0 & \bar{n}\end{array}$ & $\begin{array}{l}+3 \oplus 1 \\
+891 \\
+15 \oplus 1 \\
+\left(n^{2}-1\right) \oplus 1\end{array}$ \\
\hline
\end{tabular}

baryon multiplet is the one to which the proton belongs. Complexconjugate representations are indicated by a bar. The two 20-dimensional representations of su(4) are indicated as 20 (which contains the su(3) decuplet) and 20' (which contains the su(3) octet). The $C(N, H)$ 's are the binomial coefficients $N ! /[M !(N-M) !]$.

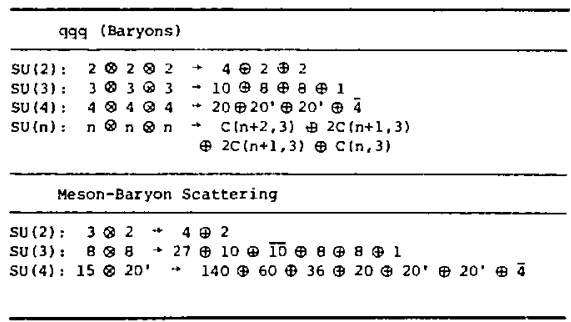


Propertieg of Quarkg

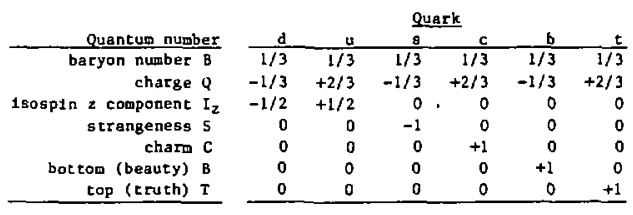

\section{WEAK INTERACTIONS OF QUARKS AND LEPTONS}

The "standard" $\operatorname{su}(2 \downarrow) U(1)$ mode $]^{1,2}$ is described here for six quarks and six leptons in left-handed doublets of sU(2) weak and right-handed singlets of sU(2) weak $\left(T_{3}=\right.$ third component of weak 1sospin)

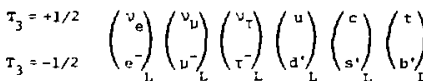

$$
\begin{aligned}
& T=T_{3}=0 \quad \mathbf{e}_{R}^{-} \quad U_{R}^{-} \quad \bar{z}_{R}^{-} \quad u_{R} \quad d_{R} \quad c_{R} \quad s_{R} \quad t_{R} \quad t_{R} .
\end{aligned}
$$

Mixing occurs between quarks $d, 5$, b of charge $-1 / 3$ (by convention the charge $2 / 3$ guarks, $u, c, t$, are unmixed and can be witzen

$$
\left(\begin{array}{c}
d^{\prime} \\
s^{\prime} \\
u^{\prime}
\end{array}\right)=\left(\begin{array}{ccc}
v_{u d} & v_{u s} & v_{u b} \\
v_{c d} & v_{c s} & v_{c b} \\
v_{t d d} & v_{t s} & v_{t b}
\end{array}\right)\left(\begin{array}{l}
d \\
s \\
b
\end{array}\right)
$$

where, for example, the mixing matrix element $v$ modulates the strength of the udw vertex. In the Kobayashi-maskawa parametrization ${ }^{2}$ this matrix is expressed

$$
\left(\begin{array}{l}
a^{\prime} \\
s^{*} \\
b^{\prime}
\end{array}\right)=\left(\begin{array}{ccc}
c_{1} & s_{1} c_{3} & s_{1} s_{3} \\
-s_{1} c_{2} & c_{1} c_{2} c_{3}+s_{2} s_{3} \mathrm{e}^{i \delta} & c_{1} c_{2} s_{3}-s_{2} c_{3} \mathrm{e}^{\mathrm{i} \delta} \\
-s_{1} s_{2} & c_{1} s_{2} c_{3}-c_{2} s_{3} \mathrm{e}^{i \delta} & c_{1} s_{2} s_{3}+c_{2} c_{3} \mathrm{e}^{\mathrm{i} \delta}
\end{array}\right)\left(\begin{array}{l}
d \\
s \\
b
\end{array}\right)
$$

where $c_{i}=\cos \theta_{j}, s_{i}=\sin \theta_{i}, i=1,2,3$. In the Liait $\theta_{2}=\theta_{3}=\delta=0$, this reduces to the usual cabibbo mixing with $\theta_{1}$ th. Cabibbo angle.

The interaction Lagrangian is

$$
\mathscr{L}_{1 n t}=e\left[a^{\alpha} J_{a}^{e m}+\frac{1}{\sin \theta_{w} \cos \theta_{w}} z^{a} j_{\alpha}^{N}+\frac{1}{\sqrt{2} \sin \theta_{w}}\left(w^{+a} J_{\alpha}^{c}+w^{-a} J_{\alpha}^{C+}\right)\right] \text {. }
$$

Here $E_{W}$ is the weak gixing angle in the relations

$$
\begin{aligned}
& w^{*}=2 \cos \theta_{W}+A \sin \theta_{W} \\
& B=-2 \sin \theta_{W}+A \cos \theta_{W}
\end{aligned}
$$

which relate the physical fields $A$ (photonl and $z$ (neutral weak gauge boson) to $w^{6}$ (su(2) gauge field). The chargea eurrent is written

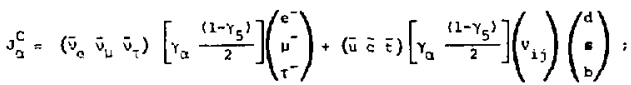

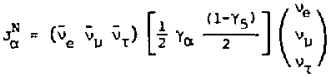

$$
\begin{aligned}
& +(\overline{\bar{\alpha}} \bar{\tau})\left[-\frac{1}{2} \gamma_{a} \frac{\left(t-\gamma_{s}\right)}{2}+\sin ^{2} \theta_{w} \gamma_{a}\right]\left(\begin{array}{l}
e \\
u \\
t
\end{array}\right) \\
& +(\bar{u} \bar{c} \bar{c})\left[\frac{1}{2} \gamma_{a} \frac{\left(1-\gamma_{S}\right)}{\overline{2}}-\frac{2}{3} \sin ^{2} \theta_{W} \gamma_{a}\right]\left(\begin{array}{l}
u \\
c \\
E
\end{array}\right) \\
& +(\bar{d} \bar{s} \bar{E})\left[-\frac{1}{2} \gamma_{\alpha} \frac{\left(1-\gamma_{5}\right)}{2}+\frac{1}{3} \sin ^{2} g_{w} \gamma_{\alpha}\right]\left(\begin{array}{l}
d \\
s \\
b
\end{array}\right) \text {. }
\end{aligned}
$$

where for fermion $f$ the coupling $\left[\tau_{\alpha}^{f}\right]$ has a V-A term deperding on $T_{3}^{f}$ and a vector term depending on charge $Q_{f}$ :

$$
\left[r_{\alpha}^{f}\right]=\left[T_{3}^{t} Y_{\alpha} \frac{\left(1-Y_{5}\right)}{2}-Q_{f} \sin ^{2} \theta_{w} Y_{a}\right] \text {. }
$$

The effective Lagrangian for exchange of $w^{4}$ and $z$ between two currents reduces at low $q^{2}$ to

$$
L_{\text {weak }}=\frac{G}{\sqrt{2}} 4\left(\mathrm{~J}^{\mathrm{Ca}} \mathrm{J}_{\alpha}^{+}+203^{\mathrm{Na}} \mathrm{J}_{\alpha}^{\mathrm{N}}\right)
$$

with $G / \sqrt{2}=\pi \alpha /\left(2 M_{W}^{2} \sin ^{2} \theta_{W}\right), \quad a=e^{2} /(4 \pi)$, and $D=M_{h}^{2} /\left(M_{z}^{2} \cos ^{2} \theta_{W}\right)$. Assuning the simplest Higgs structure, $D=k$, and the $W$ and 2 masses are relaced by $M_{z}=M_{w} / \cos \theta_{W}$. Currently reported values of the weak isteraction parame ers are

$$
\begin{aligned}
& \left.\begin{array}{l}
\left|v_{u d}\right|=\left|\cos \theta_{1}\right|=0.9737 \pm 0.0025 \\
\left|u_{u s}\right|=\left|\sin \theta_{1} \cos \theta_{3}\right|=0.229 \pm 0.012
\end{array}\right\} \text { Ref. } 3 \\
& \left.\left|v_{u b}\right|=\left|\sin _{1} \sin _{3}\right|=0.06 \pm 0.06\right\} \text { Fef } 5.4 ; \\
& \left.\left|v_{c b}\right|^{2} \gg\left|v_{u b}\right|^{2} \quad\right\} \text { ReE } 5 \\
& \left.G=G_{\nu}=(1.16612 \pm 0.00002) \times 10^{-5} \mathrm{Gev}^{-2}\right\} \text { Refs. 3, A ; } \\
& \sin ^{2} \theta_{\omega}=0.224 \pm 0.019, \quad 0=0.992 \pm 0.020 \\
& \sin ^{2} \theta_{H}=0.229 \pm 0.010,0 \equiv 1 \text { (fixed) ReF. } y \text {. }
\end{aligned}
$$

The resulting mass estimates for $w^{ \pm}$and $z$ are $H_{W}=37.3 \mathrm{GeV} / \sin \theta_{w}$ $=77.9 \pm 1.7 \mathrm{GeV}$, and $\mathrm{A}_{2}=88.8 \pm 1.4 \mathrm{GeV}$, where che numerical values are obtained using the simplest Higgs strueture $(0 \equiv 1)$. Electroweak radiative corrections to these estimates may be as large as 5 s. 10

\section{Lepton-Nuclean Inclusive Scatsering}

For reactions $\ell+N+q^{\prime}+X$, differential eross sections can be written using several choices of independent variables. These are related by

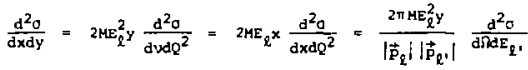

$$
\cong \frac{2 \pi M E_{\ell^{y}}}{E_{\ell !}} \frac{d^{2} \sigma}{d \Omega E_{\ell !}}
$$

where $v, Q^{2}, x$, and $y$ are defined in the Relativistic Kinematics section IV(C), $E_{\ell}, \vec{P}_{Q}$ and $E_{Q^{\prime}}, \vec{p}_{Q^{\prime}}$ are the incident and outgoing lepton lab energies and momenta, and $M$ is the target nucleon mass. 


\section{WEAK INTERACTIONS OF QUARKS AND LEPTONS (Cont'd)}

\section{Structure Funceions ${ }^{31,12}$}

For charyed-current $t C . \Omega$, and neutral-current (N.C.) reactions, we have

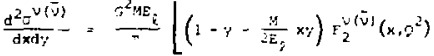

$$
\begin{aligned}
& \left.-\frac{y^{2}}{2} 2 \times F_{1}{ }^{\prime(\bar{v})}\left(x, Q^{2}\right) \pm\left(y-\frac{y^{2}}{2}\right) \times F_{1}^{\nu(\bar{v})}\left(x, r^{2}\right)\right] \text {. }
\end{aligned}
$$

where tho upper and lower slgns refer to $v$ and $\bar{T}$ scattering. respectivel $\%$, and $F_{3} 25$ defined is a positive quantity. ${ }^{13}$ The otrer cistem structure functions $w_{1}$ are related by $\mathrm{HH}_{2}=\mathrm{F}_{2}$, whe $=\mathrm{F}_{2}$, ane $W \mathrm{~W}_{3}=\mathrm{F}_{3}$. For electron ard muan scatterinq, $r_{y}=0$, asd $r^{2}$ is replaced by $8 \pi^{2} a^{2} /\left(Q^{2}\right)^{2}$ The ratio of the langitudinally to transversely polarized photon absorption cross section is

$$
R=\frac{2}{a_{T}}=\frac{1}{2 x F_{1}\left(x, Q^{2}\right)}\left[F_{2}\left(x, Q^{2}\right)-2 x E_{1}\left(x, Q^{2}\right)+\frac{4 M^{2} x^{2}}{Q^{2}} F_{2}\left(x, Q^{2}\right)\right] \text {. }
$$

To compare with the parton-model predictions kxlow, we write for $E_{2} \rightarrow x$

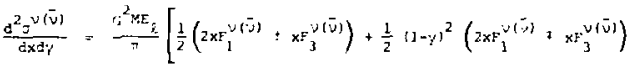

$$
\begin{aligned}
& \left.+(1-y)\left(F_{2}^{v(\bar{v})}-2 x F_{1}^{v(\bar{v})}\right)\right] \text {, }
\end{aligned}
$$

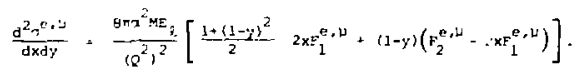

\section{The Fitee-Quark-Parton-Model Pridictions ${ }^{14}$}

For tils madel in the gjorken liatt $\left(Q^{2}, v \rightarrow \infty\right.$ with $x$ fixed $)$ $F_{i}\left(x, \sigma^{2}\right) \rightarrow F_{i}(x), 1,12$ for spin-t quark partons, we have $2 x F_{1}(x)=F_{2}(x)$, the Callan-6ross relation. Thus, in this appl ximation, $R=0)$ and ihere is no $(1-y)$ term in the cross section.

$$
\text { - }(c . c .) \frac{d^{2} g^{v H}+\mu^{2} x}{d x d y}=\frac{a^{2} M E_{g}}{\pi} 2 x \sum_{q}\left[f_{q}(x)+f_{q}(x)(1-y)^{2}\right] \text {. }
$$

For $\bar{v}_{i j} \cdot u^{+} x$, interchange $f_{g}(x)$ and $f_{\bar{n}}(x)$ in the formula. Here $f_{g}(x) d x$ is the number of quarks $g$ in the target rueleon with momentum $f$ raction $x$ to $x+d x$. he include $f_{f}(x)$ and $f_{\bar{g}}(x)$ in the sum only for neqative (positive) charged quarks and antiquarks in vivi reactions.

$$
\begin{aligned}
& \text { - }(M, C .) \frac{d^{2} J N \cdot w x}{d x d Y}=\frac{G^{2} M E_{q}}{\pi} 2 p^{2} \times \sum_{g}\left\{\left(\varepsilon_{L}^{q}\right)^{2}\left[f_{q}(x)+f_{q}(x)(1-y)^{2}\right]\right. \\
& \left.+\left(\varepsilon_{R}^{q}\right)^{2}\left[f_{q}(x)(1-y)^{2}+f_{\tilde{q}}(x)\right]\right\} \text {. }
\end{aligned}
$$

and the sum surs pore all quarks. Here the neutral-current coupling is decomposed according to

$$
\Gamma_{a}^{j}=c_{L}^{q} \gamma_{a} \frac{\left(1-r_{5}\right)}{2}+c_{x}^{T} r_{a} \frac{\left(1+r_{5}\right)}{2}
$$

with left- and right-handed coupling constancs $c_{L}^{q}$ and $c_{R}^{q}{ }^{15}$ In the "scandard" su (2) $\varnothing \nu(1)$ model

$$
E_{I}^{q}=T_{3}^{q}-Q_{q} \sin ^{2} \theta_{W}, \quad E_{R}^{q}=-Q_{q} \sin ^{2} \theta_{w} .
$$

Eor $\bar{y} \cdot \bar{\nu} x$, interchange $c_{L}^{q}$ and $c_{R}^{q}$ in the cross-section formula.

$$
\text { - (E.M.) } \frac{d^{2} o^{e, H}}{d x d y}=\frac{B \pi \alpha^{2} H E_{q}}{\left(Q^{2}\right)^{2}} \times \sum_{q} Q_{q}^{2}\left[f_{q}(x)+E_{q}(x)\right] \frac{1+(1-y)^{2}}{2} \text {. }
$$

Comparison with earlier structure function formulas gives:

$$
\text { (c.c.) } \begin{aligned}
F_{2}(x) & =2 \times \sum_{q}\left[f_{q}(x)+E_{q}(x)\right], \\
x^{F_{F}}(x) & =2 \times \sum_{\bar{q}}\left[f_{q}(x)-f_{\bar{q}}(x)\right] ;
\end{aligned}
$$

$$
\begin{aligned}
& \text { (N.C.) } F_{2}(x)=20^{2} \times \sum_{q}\left[\left(\varepsilon_{L}^{q}\right)^{2}+\left\{c_{P}^{q}\right)^{2}\right]\left[f_{q}(x)+f(x)\right] \text {. } \\
& x F_{3}(x)=20^{2} \times \sum_{q}\left[\left(\varepsilon_{L}^{3}\right)^{2}-\left(\epsilon_{p}^{q}\right)^{2}\right]\left[f_{1}(x)-\varepsilon_{\frac{-}{4}}(x)\right] \text {. } \\
& F_{j}^{y}(x)=F_{i}^{\bar{v}}(x) \quad \\
& \text { (E.M.) } \quad F_{2}(x)=x \sum_{q} Q_{q}^{2}\left[f_{r_{i}}(x)+f_{q_{i}}(x)\right] .
\end{aligned}
$$

In the examples low, $u(x), \vec{u}(x), d(x), \vec{a}(x)$, etc, near. $f_{G}$ ' $f_{\bar{q}}$ ' for the ind $i v i d u a l$ auark (antiquark) in the protos (for
neutron, jnterchange $u(x)$ and $d(x)$ ). Charm production is eaken into account.

$$
\begin{aligned}
& F_{2}^{y_{p}} \cdot u^{-} k=2 x[d(x)+s(x)+\bar{u}(x)+\bar{c}(x)] \\
& \dot{r}_{2}^{p p} \cdot \mu^{+} x=2 x[u(x)+c(x)+\bar{d}(x)+\bar{g}(x)] \\
& x_{3}^{u F}+u^{-} x=2 x[d(x)+s(x)-\bar{u}(x)-\bar{c}(x)] \\
& x_{3}^{\bar{u} p} \cdot u^{+} x=2 x[u(x)+c(x)-\bar{d}(x)-\bar{s}(x)]
\end{aligned}
$$

Hereafter we neqlect small contributions of the $s, \bar{s}, c, \bar{c}$ guarks in the sea.

- For charge-symmetric nucles weh $4(x)=y(x)+d(x)$. $\bar{f}(x)=\bar{i}(x)+\bar{d}(x)$.

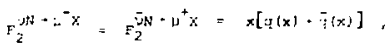

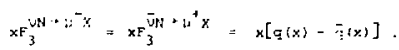

$$
\begin{aligned}
& \left.r_{2}^{e p, u F}(x)=x\left[\frac{4}{9}(u t x)+\vec{u}(x)\right)+\frac{1}{9}(d(x)+\vec{d}(x))\right] \\
& \bar{F}_{2}^{n d}(x) \geqslant \frac{5}{18} \quad F_{2}^{v(\bar{v}) d} . \mathrm{C} . \\
& \text { ( } \frac{5}{18} \text { : average squared charqe of u,d quarks). }
\end{aligned}
$$

J. S. Glashow, Nucl. Phys, 22, $579(1961)$; 5. We1sberq, Phys. Rev. Lett. $19,1264(196 \overline{7})$; $\bar{\lambda}$. Salam, in Elementary Particle Theary, edited by $N$. Svartholm (Almqvist wiksell, stockholm. 1968). 367 . For inclusion of hatrons, see 5. L. Glashow. J. Iliopoulos, and L. Maiani, Phys, Rev. D2, $1285(1970)$.

2. M. Kobayashi and K. Maskawa. Prog. Theor. Phys. 49, 652 (1973).

3. R. E. shrock and L.-L. wang, thys, Rev. Lete. 41, 1692 (1978)

4. R. E. Shrock, 5. B. Treiman, and L. $-L$. Wang, phys. Rev. Lett. 42,1589 t1979).

5. Bounds on $\operatorname{sinfl}_{2}$ and sint can be obtained from analyses of $k^{0}-\bar{K}^{0}$ mixing and $k_{L}+h^{+} j^{-}$. Modulo quadrant ambiquities, the matrix $V$ is then determined. These anal $\gamma$ ses $^{6}$ and later direct

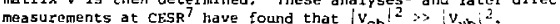

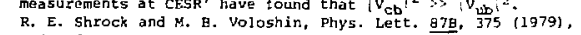
and Ref. 4

7. I. J. Spencer et al., Phys. Rev. Lett. 47, 771 (1981); C. Bebek et al.. Phys. Rev. Lett. 46, 84 (1981); A. Silverman. - Proc. of 20th Intl. Symp. on iepton and $\mathrm{y}$ 'hoton Int., Bonn. 1981. P. 138.

9. Evaluated using the $u$ mean life from this adition of the Review of particle properties. The error quot:d zepresents the experimental uncertainty. There is an additional theoretical uncertainty arising from the order $x^{2}$ radiative correction to the $u$ decay rate which has not been estimated. A conservative estidecay rate which has not been estimated. A
mate of this uncertainty was made in Ref. 3 .

9. J. E. Kim, P. Langacker, M. Levine, and H. H. Willians, Rev Mod. Phys. 53, 211 (1981).

10. A. Sirlin, Phys. Rev, D22, 971 (1980); w. J. Marciar. and A. Sirlin, Phys. Rev. D22, 2695 (1980); C. H. Llewellyn Smith and J. F. Wheater, Phys. Lett. 105日, 486 (1981).

11. J. D. Bjorken, Phys. Hev, $\frac{1059}{179}$ (1969).

12. J. D. Bjorken and E. A. Paschos, Phys. Rev. 195. 1975 (1969)

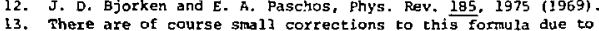
There are of course small corrections to chis formula due to
finite $w$ and $z$ masses, non-zero quark masses, and radiative processes.

14. R. P. Feynan, Photon-Hadron Interactions (W. A. Benjamin, Reading, Mass., 19721.

15. E.g., H. Quinn, Proc. Summer Inst. on Particle Physics, SLAC-215 [1978\}, P. 167. 


\section{RELATIVISTIC KINEMATICS}

\section{BASICS}

(a) Lorentz transformations -- Let $E$ and $\vec{p}$ be the energy and 3-momenturn of a particle or system as seen from a cerlain inertial frame, and tet $E^{*}$ and $\overrightarrow{p^{*}}$ be the same quantilies as seen from a second inertial frame that moves with velocity $\vec{\beta}$ relative to the first. Then starred and unstarred quantities are related by

$$
\left(\begin{array}{l}
E^{*} \\
P_{1}^{*}
\end{array}\right)=\left(\begin{array}{cc}
\gamma & -\gamma \beta \\
-\gamma \beta & \gamma
\end{array}\right)\left(\begin{array}{l}
E \\
P_{1}
\end{array}\right), \quad P_{1}^{*}=P_{1} .
$$

Here $\gamma=\left(1-\beta^{2}\right)^{-1 / 2}$, and subscripts $\|$ and 1 indicale components of $\vec{p}$ or $\vec{p}$ that are parallel or perpendicular to $\vec{\beta}$ (often $\eta$ is used for $\gamma \beta$ ). The inverse transformation is given by changing $\beta$ to $-\beta$. A particle of mass $m$ at rest in the second frame, so that it is moving at velocity $\vec{\beta}$ relative to the first, has $E^{*}=m$ and $\vec{p}^{*}=0$, so here

$$
E=r m, \vec{p}=\gamma \vec{\beta} m .
$$

In any frame, the energy, momentum, and mass are related by

$$
E^{2}=p^{2}+m^{2} \text {. }
$$

(b) Four momenta; scalar products -- The 4-momenlum vector of a particle or system having energy $E$ and 3 -momentum $\vec{p}$ is

$$
q=(E, \vec{p})=\left(E, p_{x}, p_{y}, p_{z}\right) \text {. }
$$

Conservation of energy and the components of 3 -momentum for any process $a+b+\ldots+1+2+\ldots$ may then be written as

$$
q_{a}+q_{b}+\ldots * q_{1}+q_{2}+\ldots
$$

Although the components of a 4-momentum are different in different frames, the scalar product of any two 4-momenta $q$ and $\mathrm{q}^{\circ}$, defined as

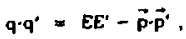

is an invariant: i.e., in numerical calculations the same result is obtained in any frame, and in algebraic calculations results obtained in different frames may be equated. For a particle of mass $m$. the scalar product $q \cdot q$ is

$$
q \cdot q=q^{2}=E^{2}-\vec{p}^{2}=m^{2} \text {. }
$$

The invariant mass $M$ (or total c.m. eneray) of an $n$-partlcle system is given by

$$
\mathbf{M}^{2}=\left(\sum_{i=1}^{n} q_{l}\right)^{2}=\left(\sum_{i} E_{l}\right)^{2}-\left(\sum_{l} \vec{p}_{l}\right)^{2} .
$$

where $q_{i}=\left(E_{j}, \vec{p}_{j}\right)$ is the 4 -momentum of the $j^{\text {th }}$ particle.

(c) Electric and marnetic forces -- In Gaussian cgs units, the force on a particle with charge $q$ moving with velocity $\vec{v}$ in electric and magnetic fields $\vec{E}$ and $\vec{B}$ is

$$
\vec{f}=q \vec{E}+q \vec{B} \times \vec{B} \text {. }
$$

where $\vec{\beta}=\vec{\gamma} / c$. The units are $\vec{F}$ in dynes, $q$ in esu, $\vec{E}$ in stat volts/cm, and $\vec{B}$ in gauss. In mksa units, the force is

$$
\overrightarrow{\mathbf{F}}=q \overrightarrow{\mathrm{E}}+q \vec{v} \times \vec{B} .
$$

where the unils are $\vec{F}$ in newtons $\left\{1 N=10^{5}\right.$ dynes $\}, q$ in coulombs $\left(1 \mathrm{C} \approx 3 \times 10^{9}\right.$ esu; each 3 in this section is really $2.9979 \ldots$ ), $\vec{E}$ in volts $/ m$ ( $t V=t / 300$ statvolt), and $\vec{B}$ in tesla (t $T$ $=10^{4} \mathrm{G}$ ). The force is zero if $\vec{E}$ and $\vec{B}$ ore at right angles, $\vec{\beta}$ (or $\vec{v}$ ) is in the direction $\vec{E} \times \vec{B}$, and $\beta=E / B$ (cess) or $\vee=E / B$ (mkso).

In a uniform, static magnetic field, the path of a charged particle is a helix of constant radius $R$ and constant pitch angle $\lambda$, with the axis of the helix being along $\vec{B}$. The moment um is related to the other quantities by

$$
p \cos \lambda=3 \times 10^{-4} Q B R \text {. }
$$

where the unils (very mixed!) are $p$ in GeV/c, $q$ in multiples of the electronic charge $e, B$ in $X G$, and $R$ in $\mathrm{cm}$. The angular velocity about the axis of the helix is

$$
\omega=3 \times 10^{-4} \mathrm{qB} / \mathrm{rm} \text {. }
$$

where the units are $w$ in $\mathrm{rad} / \mathrm{sec}, q$ in multiples of the electronic charke e, $B$ in $\mathrm{kG}$, and the energy $r m$ in GeV.

\section{DECAYS}

(a) Survival probabilities -- Let a particle have mass $m$ and proper mean tife $t_{0}$. In a frame in which its 4 -moment um is (E. $\vec{p})$, the probability that it survives a time grealer than $t$ before decaying is

$$
\text { Prob. }(>t)=\exp \left(-t / \gamma_{0}\right)=\exp \left(-m t / E_{\tau_{0}}\right) .
$$

The probability that it goes a distance greater than $x$ before decaying is

$$
\text { Prob. }(>x)=\exp \left(-x / \gamma \beta c \tau_{0}\right)=\exp \left(-m x / p c T_{0}\right) ;
$$

values of $\mathrm{cr}_{0}$ (in $\mathrm{cm}$ ) are given in the Stable Particle Table. If the particle has charge $\pm e$ and is in a uniform magnetic field $\vec{B}$ [see I(c)], then the probability that the projection of its helical path on the plane perpendicular to $\vec{B}$ turns through an angle greater than $\theta$ before decaying is

$$
\text { Prob. }(>\theta)=\exp \left(-\operatorname{Cm} \theta / B \tau_{0}\right) \text {. }
$$

where, if $m$ is in GeV, $\theta$ in $\operatorname{deg}, B$ in $k G$, and $T_{0}$ in sec, then $C$ is numerically $1.942 \times 10^{-9}$. This last distribution is independent of $p$ or the helical pitch angte $\lambda_{\text {; }}$ its only dependence is gecmetrical.

(b) Iwo-body decays - A particle of mass $m$ decays into $t$ wo particles, masses $m_{1}$ and $m_{2}$. In the rest frame of $m_{1} t$ he energies of $m_{1}$ and $m_{2}$ are

$$
\begin{aligned}
& \epsilon_{1}=\left(m^{2}+m_{1}^{2}-m_{2}^{2}\right) / 2 m \\
& \epsilon_{2}=\left(m^{2}+m_{2}^{2}-m_{1}^{2}\right) / 2 m .
\end{aligned}
$$

In this frame, the 3 -momenta of $m_{1}$ and $m_{2}$ are equal and opposite and of magnitude

$$
\begin{aligned}
k & =\left(\epsilon_{1}^{2}-m_{1}^{2}\right)^{1 / 2}=\left(\epsilon_{2}^{2}-m_{2}^{2}\right)^{1 / 2} \\
& =\left\{\left[m^{2}-\left(m_{1}+m_{2}\right)^{2}\right]\left(m^{2}-\left(m_{1}-m_{2}\right)^{2}\right]\right\}^{1 / 2} / 2 m .
\end{aligned}
$$

See also the third paragraph of IIt(b).

(c) Three-body decays -- A particle of mass $m$ decays into three particles, masses $m_{1}, m_{2}$, and $m_{3}$. The invariant masses $\mathrm{m}_{i j}$ of the 2-particle systems, where $\mathrm{m}_{i j}^{2}=\left(q_{i}+q_{j}\right)^{2}$, satisfy the relation

$$
m_{12}^{2}+m_{13}^{2}+m_{23}^{2}=m^{2}+m_{1}^{2}+m_{2}^{2}+m_{3}^{2} \text {. }
$$

so that only two of the three $m_{i j}$ 's are independent. In a rectangular Dalitz plot, $m_{13}^{2}$ (say) is plotted against $m_{12}^{2}$. The kinematic boundaries may be calculated as follows: (i) The lower and upper limits on $m_{12}^{2}$ are $\left(m_{1}+m_{2}\right)^{2}$ and $\left(m-m_{3}\right)^{2}$. (ii) For any $m_{12}^{2}$ bet ween these limits, the lower and upper limits on $m_{13}^{2}$ are given by taking the + and - signs in

where

$$
m_{13}^{2}=\left(E_{1}+E_{3}\right)^{2}-\left(p_{1} \neq p_{3}\right\rangle^{2} \text {. }
$$

$$
\begin{aligned}
& E_{1}=\left(m_{12}^{2}+m_{1}^{2}-m_{2}^{2}\right) / 2 m_{12} \\
& E_{3}=\left(m^{2}-m_{12}^{2}-m_{3}^{2}\right) / 2 m_{12} \\
& p_{1}=\left(E_{1}^{2}-m_{1}^{2}\right)^{1 / 2} \\
& P_{3}=\left(E_{3}^{2}-m_{3}^{2}\right)^{1 / 2} .
\end{aligned}
$$

(These aire the energies and momenta of particles 1 and 3 in the rest frame of $m_{12}$.) The rhase-space density is uniform over the areas of both the above and the following form of the Dalit $x$ plot.

In a triangular Datitz plot, the kinetic entergies $T_{1}, T_{2}$, and $T_{3}$ of the final-slate particles in the rest frame of $m$ are plotted as the distances inward from the sides of an equilateral triangle whose altitude is the energy $\theta$ released by the decay:

$$
Q=T_{1}+T_{2}+T_{3}=m-m_{1}-m_{2}-m_{3} \text {. }
$$

The kinetic energies are related to the $z$-particle invariant masses by

$$
2 m T_{1}=\left(m-m_{1}\right)^{2}-m_{23}^{2}=\left(m \frac{m a x}{23}-m_{23}^{2} .\right.
$$

etc. 


\section{RELATIVISTIC KINEMATICS (Cont'd)}

(d) Four-body decays -- A particle of mass $m$ decays into four particles, masses $m_{1}, m_{2}, m_{3}$, and $m_{4}$. In a trianqle (or Goldhaber) plot, the invariant mass of two of the particles is plotted against that of the other $\left(w_{0}\right.$, say $m_{34}$ versus $m_{12}$, where $m_{j j}^{2}=\left(q_{j}+q_{j}\right)^{2}$. The kinemalic houndaries of this plot are the sides of the triangle whose vertices are at the poin $t s\left(m_{12}, m_{34}\right)$ $=\left(m_{1}+m_{2}, m_{3}+m_{4}\right),\left(m_{1}+m_{2}, m-m_{1}-m_{2}\right)$, and $\left(m-m_{1}-m_{4}\right.$, $\left.m_{3}+m_{4}\right)$. The phase-space density is not uniform over the enclosed area.

\section{II. REACTIONS (MAINLY 2-BODY)}

(a) Initial state -- Two particles, masses $m_{1}$ and $m_{2}$, interact. In the lab frame, where particle 2 is at rest, the 4 -momenta are $\left(E_{1}, \vec{p}_{1}\right)$ and $\left(m_{2}, 0\right)$. In the $\mathrm{c} . \mathrm{m}$. frame, where the 3 -momenta are equal and opposite, the 4 -momente are $\left(E_{1}, \vec{k}\right)$ and

$\left(\epsilon_{2},-\vec{k}\right)$. Then the total $c . m$. energy $E$ is given by

$$
E^{2}=\left(E_{1}+\epsilon_{2}\right)^{2}=m_{1}^{2}+m_{2}^{2}+2 E_{1} m_{2} .
$$

The c.m. energies of particles 1 and 2 are

$$
\begin{aligned}
& \epsilon_{1}=\left(m_{1}^{2}+E_{1} m_{2}\right) / E=\left(E^{2}+m_{1}^{2}-m_{2}^{2}\right) / 2 E \\
& \epsilon_{2}=\left(m_{2}^{2}+E_{1} m_{2}\right) / E=\left(E^{2}+m_{2}^{2}-m_{1}^{2}\right) / 2 E .
\end{aligned}
$$

The c.m. momentum $k$ is

$$
k=p_{1} m_{2} / E .
$$

See also the expression in $I J(b)$ for $k$, in which replace $m$ with $E$

The velocity of the $\mathrm{c} . \mathrm{m}$. relative to the lab is

$$
\beta=P_{1} /\left(E_{1}+m_{2}\right) \text {. }
$$

The parameters for the Lorentz transformation between these frames [see $I(a)]$ are

and

$$
r=\left(E_{1}+m_{2}\right) / E
$$

$$
\gamma \beta=\mathrm{P}_{1} / E .
$$

(b) Two-body final states -- In the reaction $1+2 \rightarrow 3+4$, let the masses be $m_{i}$ and the final-state $\mathrm{c} . \mathrm{m}$. 4 -moment a be $\left(\epsilon_{3}, \vec{k}^{\prime}\right)$ and $\left(\epsilon_{4},-\vec{k}^{\prime}\right)$. Then

$$
\begin{aligned}
& \epsilon_{3}=\left(E^{2}+m_{3}^{2}-m_{4}^{2}\right) / 2 E \\
& \epsilon_{4}=\left(E^{2}+m_{4}^{2}-m_{3}^{2}\right) / 2 E ;
\end{aligned}
$$

and

$$
\begin{aligned}
k^{\prime} & =\left(\epsilon_{3}^{2}-m_{3}^{2}\right)^{1 / 2}=\left(\epsilon_{4}^{2}-m_{4}^{2}\right)^{1 / 2} \\
& =\left\{\left(E^{2}-\left(m_{3}+m_{4}\right)^{2}\right]\left[E^{2}-\left(m_{3}-m_{4}\right)^{2}\right]\right\}^{1 / 2} / 2 E .
\end{aligned}
$$

Let $\theta_{3}$ be the lab production angle of particle 3 (the angle between $\vec{p}_{3}$ and $\vec{p}_{1}$ ), and let $\theta_{3}$ be the c.m. production anglc (the angle between $\vec{k}^{\prime}$ and $\vec{k}$ ). These angles are related by

$$
\tan \theta_{3}=\frac{P_{31}}{P_{34}}=\frac{\sin \theta_{3}}{\gamma\left(\cos \theta_{3}+\beta / \beta_{3}\right)} .
$$

where $P_{31}$ and $P_{34}$ are the components of $\vec{P}_{3}$ perpendicular and parallel to $\vec{p}_{1}$, and $\beta_{3} \times k / \epsilon_{3}$ is the c.m. velocity of particle 3 . [See III(a) for $\gamma$ and $\beta$.] If $\beta>\beta_{3}$, then particle 3 can only go forward in the lab, the maximum $\theta_{3}$ being given by

$$
\tan \theta_{3}^{\max }=\beta_{3}\left(\frac{1-\beta^{2}}{\beta^{2}-\beta_{3}^{2}}\right)^{1 / 2} \text {. }
$$

The components of $\overrightarrow{\mathrm{p}}_{3}$ satisfy

$$
\left(\frac{p_{31}-\gamma \beta \epsilon_{3}}{\gamma^{\prime}}\right)^{2}+\left(\frac{p_{3 k}}{k^{\prime}}\right)^{2}=1 \text {, }
$$

which is the equation of an ellipse with semi-major axis $r k^{\prime}$ and semi-minor axis $k^{\prime}$. Thus the possible lab momenta of particle 3 are the vectors to the ellipse from the point a distance $\gamma \beta \epsilon_{3}$ back along the major axis from the center of the ellipse.
The results of the preceding paragraph also apply to 2 -body decay. Just set $m_{2}=0$, in which case $E=m_{1}$. (The decayproduct masses are here $m_{1}$ and $m_{4}$, not $m_{1}$ and $m_{2}$ as in II(b).I

The Mandelstam variables $s, t$, and $u$ are the Lorentz scalars defined in terms of the particle 4-momenta $q_{i}$ as

$$
\begin{aligned}
& s=\left(q_{1}+q_{2}\right)^{2}=\left(q_{3}+q_{4}\right)^{2} \\
& t=\left(q_{1}-q_{3}\right)^{2}=\left(q_{2}-q_{4}\right)^{2} \\
& u=\left(q_{1}-q_{4}\right)^{2}=\left(q_{2}-q_{3}\right)^{2}
\end{aligned}
$$

They satisfy the relation

$$
s+1+u=m_{1}^{2}+m_{2}^{2}+m_{3}^{2}+m_{4}^{2},
$$

so that only two of the three are independent. Evaluating $s$ in the c.m. frame gives

$$
s=\left(\epsilon_{1}+\epsilon_{2}\right)^{2}=E^{2} .
$$

and evaluating $\mathrm{t}$ and $u$, the 4-momentum-transfer-squared variables, in this ?rame gives

$$
\begin{aligned}
\mathrm{l} & =\mathrm{m}_{1}^{2}+\mathrm{m}_{3}^{2}-2 \epsilon_{1} \epsilon_{3}+2 k k^{\prime} \cos \theta_{3} \\
& =\mathrm{l}_{0}-4 \mathrm{k}^{\prime} \sin ^{2}\left(\theta_{3} / 2\right) \\
\mathrm{u} & =\mathrm{m}_{1}^{2}+\mathrm{m}_{4}^{2}-2 \epsilon_{1} \epsilon_{4}+2 k k^{\prime} \cos \theta_{4} \\
& =u_{0}-4 k k^{\prime} \sin ^{2}\left(\theta_{4} / 2\right),
\end{aligned}
$$

where $\theta_{4}$ is the c.m. production angle of particle $4\left(\theta_{3}+\theta_{4}=\pi\right)$. and

$$
\begin{aligned}
& t_{0}=t\left(\theta_{3}=0\right)=\left(\epsilon_{1}-\epsilon_{3}\right)^{2}-\left(k-k^{\prime}\right)^{2} \\
& u_{0}=u\left(\theta_{4}=0\right)=\left(\epsilon_{1}-\epsilon_{4}\right)^{2}-\left(k-k^{\prime}\right)^{2} .
\end{aligned}
$$

The dirlerences $\Delta t=t_{0}-t_{n}$ and $\Delta u=u_{0}-u_{n}$, where $t_{n}=t\left(\theta_{3}=\right.$ $\pi)$ and $u_{\pi}=u\left(\theta_{4}=\pi\right)$, are

$$
\Delta t=\Delta u=4 k^{\prime} \text {. }
$$

For elastic scaltering, where $m_{1}=m_{3}=m$ and $m_{2}=m_{4}=M$, $t_{a}$ is zero and

And now

$$
t=-2 k^{2}\left(1-\cos \theta_{3}\right)=-4 k^{2} \sin ^{2}\left(\theta_{3} / 2\right) \text {. }
$$

$$
u_{0}=\left(m^{2}-M^{2}\right)^{2} / 5 .
$$

Evaluating $t$ in the lab frame gives

$$
t=-2 \mathbf{H T}_{4} \text {. }
$$

where $T_{4}=E_{4}-M$ is the lab kinetic energy of particle 4 . For small-angle elastic scattering.

$$
(-t)^{1 / 2} \approx k \theta_{3} \approx p_{1} \theta_{3} \approx p_{4} \text {. }
$$

where $p_{1}, \theta_{3}$, and $F_{4}$ are lab quanlities.

IV. OTHER VARIABLES

(a) Rapidity - For a system of energy $\mathbf{E}$ and moment um $\overrightarrow{\mathrm{p}}$, the rapidity $y$ is given by

$$
y=\frac{1}{2} \ln \left(\frac{E+P_{1}}{E-P_{1}}\right)=\tanh ^{-1}\left(\frac{P_{\mathbf{l}}}{E}\right)=\ln \left(\frac{E+P_{1}}{m_{l}}\right) .
$$

where $p_{n}$ is the component of $\vec{p}$ along a particular axis (the "rapidity axis", chosen, for example, parallel to the direction of an incoming beam), and $m_{1}=\left(m^{2}+p_{1}^{4}\right)^{1 / 2}$. Inverting (hese equations, we find

$$
\begin{aligned}
& E=m_{1} \cosh y \\
& P_{t}=m_{1} \sinh y .
\end{aligned}
$$

The shape of a rapidity distribution is invariant under a Lorentz Iransformation between inertial frames with relative motion parallel to the rapidily axis. Such a transformation is given by

$$
y^{\star}=y-\ln [\gamma(1+\beta)]=y-\frac{1}{2} \ln \left(\frac{1+\beta}{1-\beta}\right) .
$$

where the sign of $\boldsymbol{\beta}$ is positive in the di:ection of increasing rapidity and $\mathrm{p}_{\mathrm{l}}$. 


\section{RELATIVISTIC KINEMATICS (Cont'd)}

(b) Scaline variable, hadron reactions -- In the inclusive reaction $h+2+3+X$, with $h$ any hadron, Feynman's $x$ for particle 3 is defined as

$$
x=k^{\prime} / k_{\text {max }}^{*} \text {. }
$$

where $k^{\prime}$ is the c.m. momentum of particle 3. $k^{\prime}$ max is obtained [see Sec. III(b)] using the smallest mas' $m_{X}$ [called $m_{4}$ in III(b)] consistent with quantum conservation, laws. At high energies, $k_{\text {max }}^{\prime}=\sqrt{s} / 2$. Rapidity and $x$ are related at large $\sqrt{s}$ by

$$
x=\frac{2 m_{1}}{\sqrt{s}} \sinh y^{*} \text {. }
$$

where $y^{*}$ is eval'sated in the c.m.

(c) Scaling variables, lepton reactions -- For the inclusive reaction $l+2 \rightarrow l^{\prime}+X$, with particles $l$ and $l^{\prime}$ leptons, we define the 4-vector

so that

$$
q=\left(p_{t}-p_{l}\right)
$$$$
Q^{2} \equiv-q^{2}=2 \varepsilon_{l} \varepsilon_{q^{\prime}}-2\left|\vec{p}_{l} \| \vec{p}_{t}\right| \cos \theta-\mathbf{m}_{l^{\prime}}^{2}-\mathbf{m}_{l}^{2} 2^{\dagger} 0
$$

where $\theta$ is the $t \rightarrow t$ scattering ongle, and the preceding relation is valid in any frame. Aso useful are

and
$Q^{2}, \nu$, and $W$ ore Lorentz invariants, and the notation "LAB" refers to the reference frame with porticle 2 at rest. (Note; $v$ is sometimes written $v=\mathrm{P}_{2} \bullet$, leading to the replacement of $m_{2} \nu$ with $\nu$ throughout.)

Scaling variables in common use include $x \equiv \omega^{-1}=\theta^{2} / 2 m_{2} \nu, 0 s^{\dagger} \times s^{\dagger}$,

and

$$
y=m_{2^{\nu}} / p_{t} * P_{2}=\left(\left(E_{l}-E_{l}\right) / E_{l}\right]_{L N B}, 0 s^{\dagger} y \leq 1 .
$$

Both $x$ and $y$ are dimensionless.

Cross sections for inclusive reactions in the energy region where masses are negligible can be written in terms of $\varepsilon_{4}$ and certain pairs of these variables, usually $Q^{2}$ and $\nu_{1} x$ and $y$, or $Q^{2}$ end $x$. If, in any frame. $\left|\vec{p}, \| \vec{p}_{\ell}\right|=E_{l} E_{l}$, and

$E_{\ell} E_{l} \sin ^{2}(\theta / 2) \gg m_{l}^{2}$ and $m_{l}^{2}$ (i.e., $m_{\ell}, m_{l}$ small), then

$$
Q^{2}=4 E_{t} E_{t} \cdot \sin ^{2}(\theta / 2)
$$

and

$$
x=\frac{2 E_{t} E_{d} \sin ^{2}(\theta / 2)}{m_{2}{ }^{1}} .
$$

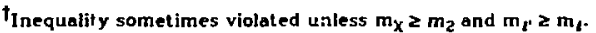

\section{LORENTZ INVARIANT PHASE SPACE FORMULAE}

For a system of $n$ particles with owerall four-mumentum p and final faur munenta $p_{1} \cdots \cdots, \Gamma_{n}\left[p_{i}=\left\{E_{i}, \vec{P}_{i} \mid\right\}\right.$. Lorentz Invariant Phase Space is given by

$$
\begin{aligned}
& \therefore \operatorname{LIPS}\left(s ; p_{1}, \cdots, p_{n}\right)=(2 \pi)^{4} \delta^{4}\left(p-\sum_{i} p_{i}\right) \frac{1}{(2-)^{3}} \prod_{i=1}^{n} \frac{d^{3} \vec{F}_{i}}{2 E_{i}} \text {. } \\
& \text { For 2-budy: d LIPS }\left(s, p_{1}, p_{2}\right)=\frac{1}{(2 \pi)^{2}} 5^{4}\left(p-p_{1}-p_{2}\right) d^{4} p \frac{i \vec{p} c m !}{4 \sqrt{5}} d s_{1}^{c m} \text {. } \\
& \text { For } 3 \text {-body: } d \operatorname{LIFS}\left(s, p_{1}, p_{2}, p_{3}\right)=\frac{1}{(2 \pi)^{5}} \delta^{4}\left(p-p_{1}-p_{2}-p_{3}\right) d^{4} p \frac{1}{32 s} d s_{12} d s_{23} d o d \cos i k d y \text {. }
\end{aligned}
$$

wherco. 0 . and $\gamma$ ar. Euler angles.

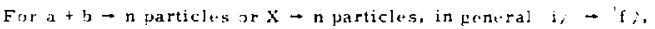

$$
\sigma_{i \bar{i}}=\frac{1}{A F} \int A_{i f} \cdot 2 \text { d LIPS }\left(s: p_{1}, \cdots, p_{n}\right)
$$

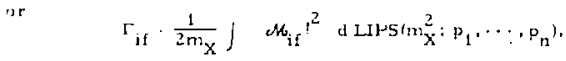

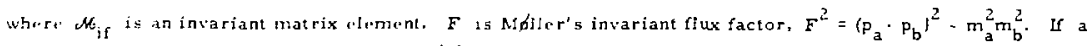
is beam, b, target $\left(\vec{P}_{b}^{l a t}=0\right)$, then $F=\vec{P}_{a}^{l a b} m_{b}=\cdot \vec{P}_{a}^{c m} \mid \sqrt{5}$.

For lastic scattering in c. $m ., \vec{p}_{3}^{c m},=\vec{p}_{1}^{c m}$, and (2) ant (4) yield

$$
\frac{d \sigma}{d \Omega}=\frac{M^{2}}{(8 \pi)^{2} s} \text { or } \frac{d \sigma}{d t}=\frac{d t^{2}}{64 \pi \cdot \vec{P}_{a}^{\mathrm{cm} \cdot{ }^{2}} \mathrm{~s}} \text {. }
$$

The nurmalization is such that the optical theorem reads

$$
\operatorname{Im} \mathcal{M}_{\mathrm{t}=0}=2 \mathrm{P}_{\mathrm{a}}^{\mathrm{cm}} \mid \sqrt{\mathrm{s}} \sigma_{\mathrm{tot}} .
$$

The choice of Eq. (1) implies a particular normalization of any spinors that may occur in chi. The advantage of this normalization is that it ereatly simplifies the structure of oho by putting factors such as $\frac{1}{(2 \pi)^{3}} \frac{1}{2 E}$ into the phasc space where they really belong. In addition, the labels, $i, f$, refer to specific spin (helicity) states, so that the usual "average and sum" rule is implicit. 


\section{C.M. ENERGY AND MOMENTUM VS. BEAM MOMENTUM}

$$
E_{c m}{ }^{d E_{c m}}=m_{p}{ }_{p} T_{\text {bear }}=m_{p} v_{\text {beam }}{ }^{d P_{\text {beam }}} \approx m_{p} \mathrm{dP}_{\text {beam }}
$$

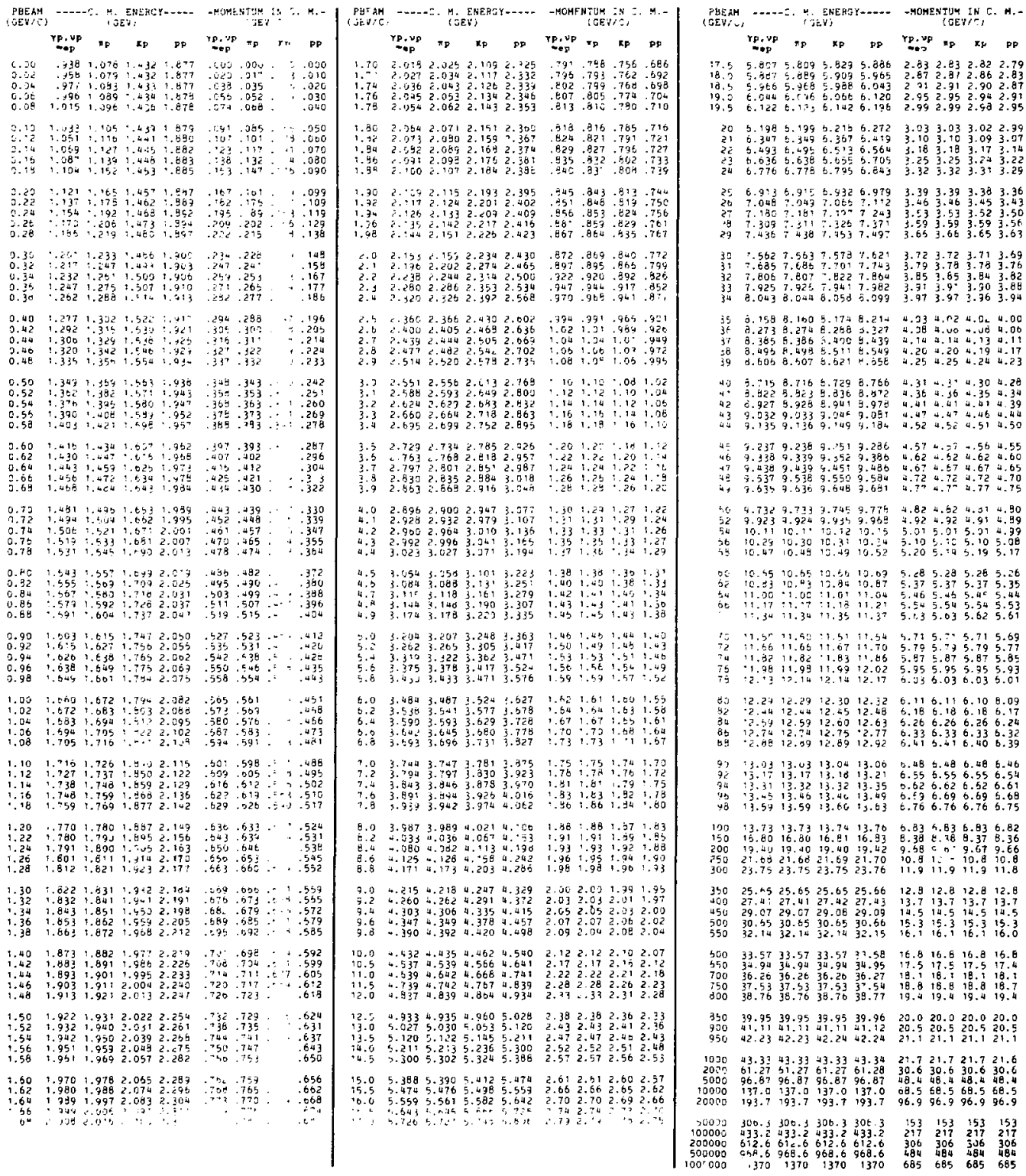




\section{PROBABILITY AND STATISTICS}

\section{A. PROBABILITY DISTRIBLTIONS AND CONFIDENCE IEVEL.}

We give here properties of the thren probability distributions most communly used in high encrpy physics: Normal for

Gaussian), Chi-squared, and Joissun. We Marn the rrader that the re is no universal convention for the lernt "ernfiritence level" as used by phrsadist thise, isplicat definitions are given for -ach distribution, and that cortespend to ammon itsage. It is explained below how

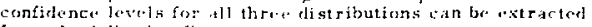
from the follewing figur..

\section{$\chi^{2}$ Confidence Level vs. $\chi^{2}$ for , Degrees of Freedom}

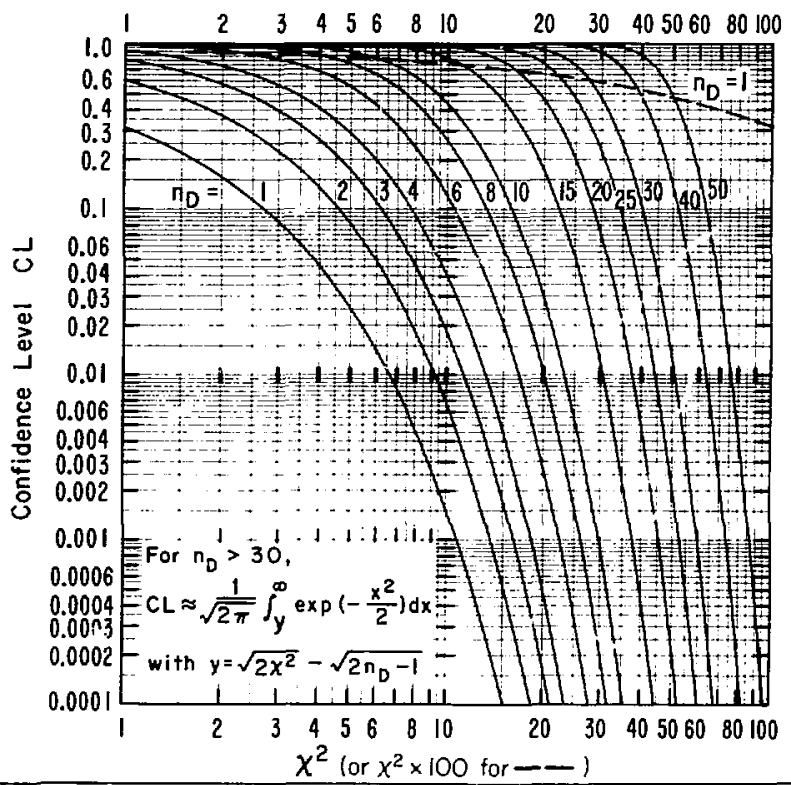

\section{A.4. Xormal Distribution}

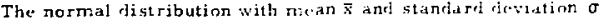
(variance $\left.\sigma^{2}\right)+s$ :

$$
P(x) d x=\frac{1}{\sqrt{2 \pi} \sigma} \cdot x^{-(x-x)^{3} / 2 \sigma^{2}} d x .
$$

The confidence Jued assoriater with an ubserverd duviation from the mean, 5, is the probability that $x \cdot x>b, i, \ldots$.

$$
C L=2 i_{\bar{x}+6}^{x} \text { ix } \mathrm{I}^{\prime}(x)
$$

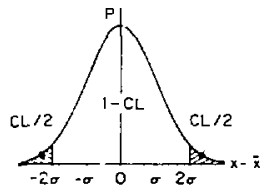

[ The spall figure in Eq. (2) is drawn with $s=2 \sigma$.$] CL is civn$ The small fipure in Eq. (2) is drawn whe figure at $\mathrm{x}^{2}=\left\{f_{1}, \sigma^{2}\right.$.

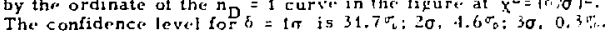
The ceneral eonfidence interval, $1-\mathrm{Cl}$., (which is also some. times called confidence levell for ${ }^{\prime \prime}=1 \sigma$ is $68.30,20,05.4^{\prime \prime}$

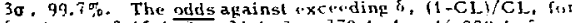
$\delta=1 \sigma$ are $2.15: 1, \overline{2 \sigma}, 21: 1: 3 \sigma, 370: 1: 40,16,900: 1: 5 \sigma$.

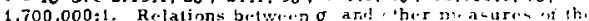

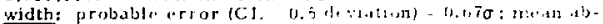
solute deviation $=0.80 \mathrm{\sigma}:$ PMS feriation $=\sigma$; half $*$ idth at halt maximum $=1.18 \sigma$

\section{A.2. Chi-squared Distritution}

The chi-squared distribution for $\mathrm{n}_{\mathrm{D}}$ Heprees of frectent is:

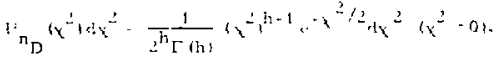

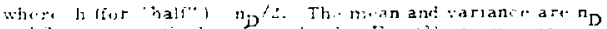

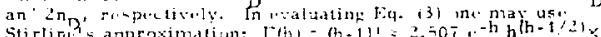

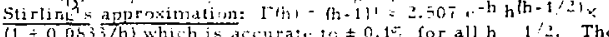

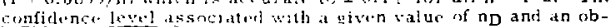

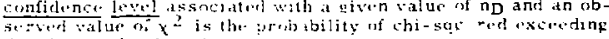
the abserved value, i. '.,

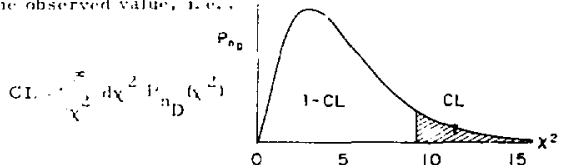

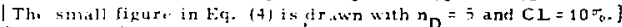
CL. is ploltert as a function of $x^{2}$ far soveral Values of $n_{0}$ in the abexe fipure. Fur larke $n_{\mathrm{D}}, x^{2}$ beculars normally distributed about $\mathrm{n}_{\mathrm{D}}$. Thus,

$$
y_{1}=t x^{2}-\pi_{0} \mid x \sqrt{2 n_{D}}
$$

berenies normally distributed with unit standard dreviation. A betice apprisximation, due to Fisher, 1 is that $x$. not $x^{2}$. becomes

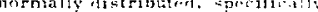

$$
y \quad \sqrt{2 x^{2}}-\sqrt{2 n D^{-1}}
$$

approuches normality wish unit standart devation. For small CL's in particuler, ye is much more accurate than $y_{1}$. Thus, $f \circ n_{D}=50$ anel $x^{2}=80$, th\%true CI. $=0.45 \sigma^{\circ}$, but $y_{1}$ is 3.0 cor responding to d C l w $0.13{ }^{\circ}$. while $y_{2}$ is 2.7 corresponding to a CL of $0.35 \sigma_{i}$. 


\section{PROBABILITY AND STATISTICS (Cont'd)}

A.3. Poisson Distribution

The Poisson distribution with menn ñs:

$$
v_{\bar{n}}(n)=\frac{\left.0^{-(n)}(n)\right)^{n}}{n !}(n=0,1,2, \ldots) \text {. }
$$

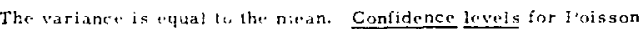
distributions ar" us u.tly defined in terny 4 of quantities called "upper limits" an follnws: The e.onfiringere level associaterl with a given upper limit $X$ and an observed walue $n_{0}$ of $n$ is the proba. bility that $n \cdot n_{0}$ if $\bar{n}=\therefore, \ldots$....

$$
\begin{aligned}
C 1 . & \underbrace{\infty}_{n=r_{0}+1} P^{(n)} \\
=1 & \sum_{n=0}^{n_{0}} N^{(n)}
\end{aligned}
$$

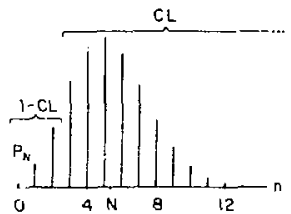

[ The amall figure in Fq. $|8|$ is trawn wath $n_{0}=2$ an CI. $\left.=40 \%.\right]$

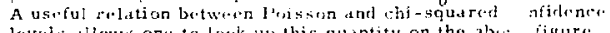
levels allows nne to lock up this quintity on the alo, ifgur.. Specifically, the quantity 1 -CL. is piwen by the ardin "of the

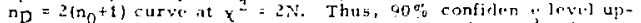
per linits for $n_{0}=0$, 1 . and 2 are given by half the value cor

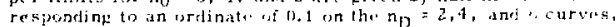
respertively: the walues ar, $\therefore=1.5$. 3.9. and 5,3 .

Tables of confiedunce levels for doll threw of these dis butions, the retation between proisson and thi-squared confirst. F levels. and nun i-rous other useful tables anil relations niny b. founel in R.f. $\therefore$.

\section{B. STATISTICS}

We conseder herr the situation in which one is pres nt with $\lambda$ indepe necent data, $y_{7} \pm \sigma_{n}$, and it is resireil to make sor. inference aloout the "true" value of the quantity represe aterl by these datat. Fur this purpose we interprit cach rlatum i as at single sample point drawn randomly land independently if the other data) from a distribution having meant $\bar{y}_{p}$ (which wr wish to "stimate) and variance $\sigma^{2}$. Felentification of the true $\sigma_{n}$ with the $\sigma_{n}$ datum is an approximation which may become suriou:ly inaccurate when $\sigma_{n}$ is an appreciable fraction of $y_{n}$-) Some s.ethods of estimation commoniy used in high unergy physics are ...en below: ser Ref. 3 for numrrous appications. Section $B . \therefore$ deals

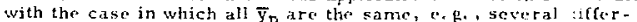
ent measurements of the same quantity: Sec. B.2, deals with the case in which $\bar{Y}_{n}=T\left(\mathrm{x}_{n}\right)$, where $*_{n}$ ripresents some set of : nde'pendent variables, $a$. g., cross-sectinn measurentents it i irous values of energy and anple, $x_{n}=\left\{E_{n},{ }_{n}\right\}$.

\section{B.1. Singl, Mran an: Variance Estimates}

(1) If the $y_{n}$ represent a set of values all supposedly drawn : rom a single distribution with mean $\bar{y}$ and $y$ ariance ${ }^{2}$ (i. $c$. . the $c .$, are all the same, but the. common value is unknown) then

$$
\begin{aligned}
& \eta_{e}=\frac{1}{N} \sum_{n} y_{n} \text { an } 1 \\
& \sigma_{e}^{2}=\frac{1}{N-1} \Sigma_{n}\left(y_{n}-\bar{y}_{c}\right)^{2}=\because[(\vec{z})-\because]
\end{aligned}
$$

are unbidsed estimates of $\bar{y}$ and $\sigma^{2}$. The varianer of $\bar{y}$ is $\sigma^{2} ;$. If the parent distribution is normat and $X$ is larfe, the varianci. of $\sigma_{i}^{2}$ is $2 \sigma^{4} / x$.

(2) If the $\bar{y}_{n}$ all have the commion val!n. $\bar{y}$ and the $\sigma_{n}$ are known, then the werghted average

$$
\bar{y}_{t^{\prime}}=\frac{1}{w} \bar{n} w_{n} y_{n} \text {. }
$$

wher $w_{0}=1 / \sigma^{2}$ and $w=2 w_{n}$ is an appropriate unbiased estimate of $F^{n}$. This choice of weighting factors in Eq. (11) minimizes the variance of the estimate; the variance is $1 / \mathrm{w}$.

\section{B.2. Linear Least Squares Fit}

A least squares fit of the function $y(x)=\Sigma_{i} a_{i} f_{i}(x)$ to independent data $y_{n} \pm \sigma_{n}$ al points $x_{n}\left(1, \Omega .\right.$. a Legentice fit in which the $f_{i}$ are Legendre polynomials and the $a_{\mathrm{f}}$ are Legendre coefficients) gives the following estimales of the parameters $d_{i}$ :

$$
a_{e, j}=\sum_{j, n} v_{i j} f_{j}\left(x_{n}\right\} y_{n} / o_{n}^{2} .
$$

Hise $V$ is the covarianes natrix of the fitted parameters

$$
v_{i j}=\overline{\left(a_{1, i}-\bar{a}_{c, i}\right)\left(a, \ldots j-\bar{a}_{t, j, j}\right.} \text {. }
$$

which is given by

$$
\left(v^{-1}\right)_{i j}=\sum_{n} f_{i}\left(x_{n}\right) f_{i}\left(x_{n}\right) / \sigma_{n}^{2} \text {. }
$$

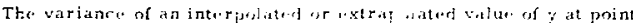
$x, y_{1}=\Sigma a_{n, i} f_{i}(x)$. is:

$$
\overline{\left(y_{e}-\bar{y}_{e}\right)^{j}}=\underset{i j}{*} v_{i j} t_{i}(x) f_{1}(x) \text {. }
$$

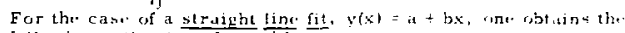
following $\cdot$ atimates of at anit b,

$$
\begin{aligned}
& a_{y}=\left\langle s_{y} s_{x x}-s_{x} s_{x y} 1 / 0,\right. \\
& b_{1}=\left\{s_{1} s_{x y}-s_{x} s_{y} 1 / D,\right.
\end{aligned}
$$

where

$$
\begin{aligned}
& s_{1}, s_{x}, s_{y} \cdot s_{x x}, s_{x y}= \pm\left(1, x_{n}, y_{n}, x_{n}^{2} \cdot x_{n} y_{n}\right) / a_{n}^{2} . \\
& D=s_{1}, s_{x x}-s_{x}^{2} .
\end{aligned}
$$

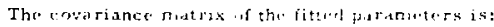

$$
\left(\begin{array}{cc}
v_{a a h} v_{a b} \\
v_{a b b} v_{b b}
\end{array}\right)=\frac{1}{D} \cdot s_{x} \quad s_{1 /}
$$

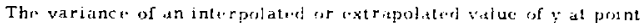
$x$ is :

$$
\overline{\left(s_{1}-\overline{Y_{1}}\right)^{2}}=\frac{1}{s_{1}}+\frac{s_{1}}{D} x-\frac{s_{x}}{s_{1}} .
$$

\section{ERROR PROIAGATION}

Wo consider here the situation in which sune wishes to calculate the value and reror ref a function of simm isther quantitio's with

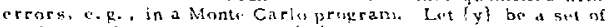
random variable with means ' $y$ ' and covariance matrix $V$. Then the mean and varienes of a function of the se variables are approximalely (10 sucont orrer in $\{y-\bar{y}\}$ ):

$$
\begin{aligned}
& \bar{r}=\left\{(\{y\})+\frac{1}{2} \sum_{\bar{m} n} v_{n \in n} \frac{\partial^{2} f}{\partial y_{m} \partial y_{n}}\{y\}=\{\bar{y}\}\right.
\end{aligned}
$$

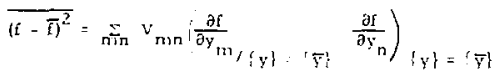

E. g., the mean and variancir of a function of a single varidble with mean $\bar{y}$ and variance $g^{2}$ ar

$$
\begin{aligned}
& \bar{\Gamma} \approx f(\bar{y}) \\
& \frac{(f-x)^{2}}{2}=\sigma^{2} f^{\prime}(\bar{y})^{2} .
\end{aligned}
$$

Note that these equations will usually be applied by substituting some measured quantities, $\{\tilde{y}\}$ say, for the true means, $\{\bar{y}\}$. If as is often the case, $\tilde{y}_{n}-\bar{y}_{n}$ is of urder $\sqrt{y_{n n}}$, then there is no point in heeping the second order terms in Eq. $(20)$ or (22) since the sulastitution itsclf intrarduces first orrer crrars.

1. R. A. Fisher, Statistical Methods for Research Workers (Oliver and Boyd Edinburgh and Loudon, 1958)

2. M. Abramovitz ancl I. Stegun, erds.. Handbook of Mathematical Functions (National Burcau of Standards, Applied Matirematics Series, Vol. 55, Washington, 1964).

3. W. T. Eadie, D. Drijard, F. E. James, M. Roos, and B. Sadoulet, Statistical Methoxis in Experimental Physics (North-Holland, Amsterclam and London, 1971). 


\section{PARTICLE DETECTORS, ABSORBERS, AND RANGES*}

\section{A. DETECTOR PARAMETERS}

In this section we give various parameters for common detectors. The quoted numbers represent at best an order of magnitude, and are useful only for preliminary design. A more detailed introduction to detectors can be found in "A Consumer's Guide to Particle Detectors," by D.J. Miller, Rutherford Lab Report RL-76-072, July 1976.

A.1 Scintillators: Photon yield $\approx 1 \gamma / 100 \mathrm{eV}$ in plastic scintillator ${ }^{1}$ and $\approx I \gamma / 25 \mathrm{eV}$ is. Nal. 1.2

A. 2 Cerenkov: ${ }^{3}$ Half-angle $\theta_{c}$ of cone aperture in terms of velocity $\beta$ and index of refraction $n$ :

$$
\theta_{\mathrm{f}}=\arccos \left(\frac{1}{\beta \mathrm{n}}\right)-\left[2\left(1-\frac{1}{\beta \mathrm{n}}\right)\right]^{1 / 2} .
$$

Threshold velocity: $\beta_{\mathrm{t}}=1 / \mathrm{n} ; \quad \gamma_{\mathrm{t}}=1 / \sqrt{1-\beta_{\mathrm{t}}{ }^{2}}$.

Therefore, $\beta_{t} \gamma_{t}=1 / \sqrt{2 \delta+\delta^{2}}$, where $\delta=n-1$. Values of $\delta$ for yarjous commonly used gases are given as a function of pressure and waveleng:h in Ref. 4; for values at atmospheric pressure, see the Table of Atomic and Nuclear Properties, following.

Number of photons, $\mathrm{N}$, per $\mathrm{cm}$ :

$$
\begin{aligned}
& N=\frac{\alpha}{c} \int\left(1-\frac{1}{\beta^{2} n^{2}}\right) 2 \pi \mathrm{d} \nu=\frac{\alpha}{c} \beta_{\mathrm{t}}{ }^{2} \int\left(\frac{1}{\beta_{\mathrm{I}}{ }^{2} \gamma_{1}{ }^{2}}-\frac{1}{\beta^{2} \gamma^{2}}\right) 2 \pi d y \\
& \approx 500 \sin ^{2} \theta_{\mathrm{c}} / \mathrm{cm} \text { (visible spectrum). }
\end{aligned}
$$

A.3 Photon Collection: In addition to the photon yield, one should take into account the light collection efficiency ( $\leqslant 10 \%$ for typical $1 \cdot \mathrm{cm}$-thick sciatillator), attenuation length $\left(\approx 1\right.$ to $4 \mathrm{~m}$ for typical scintillators $\left.{ }^{5}\right)$.

\begin{tabular}{|c|c|c|c|}
\hline Detector Type & Accuracy (rms) & $\begin{array}{l}\text { Resolution } \\
\text { Time }\end{array}$ & $\begin{array}{l}\text { Dead } \\
\text { Time }\end{array}$ \\
\hline Bubble chamber & $\approx \pm 10$ to $\approx \pm 150 \mu$ & $\approx 1 \mathrm{~ms}$ & $\approx 1 / 20 \mathrm{~s}$ \\
\hline Streamer chamber & $\pm 300_{\mu}$ & $\approx 2 \mu \mathrm{s}$ & $\approx 100 \mathrm{~ms}$ \\
\hline Oplical spark chambe & $\pm 200 \mu^{b}$ & $\approx 2 \mu \mathrm{s}$ & $\approx 10 \mathrm{~ms}$ \\
\hline \multicolumn{4}{|l|}{ Magnetostrictive } \\
\hline spark chamber & $\pm 500_{\mu}$ & $\approx 2 \mu \mathrm{s}$ & $\approx 10 \mathrm{~ms}$ \\
\hline Proportional chamber & $\geq \pm 300 \mu^{c, d}$ & $\approx 50 \mathrm{~ns}$ & $\approx 200 \mathrm{~ns}$ \\
\hline Drifi chamber & \pm 50 to $300 \mu$ & $\approx 2 \mathrm{~ns}^{e}$ & $e \approx 100 \mathrm{~ns}$ \\
\hline Scintillator & .- & $\approx 150 \mathrm{ps}$ & $\approx 10 \mathrm{~ns}$ \\
\hline Emulsion & $\pm 1 \mu$ & .- & .. \\
\hline
\end{tabular}
and qu ?ntum efficiency of the photomultiplier cathode $(\leqslant 25 \%)$.

\section{A.4 Typical Detector Characteristics:}

\footnotetext{
a Multiple pulsing time.

${ }^{b} 60 \mu$ for high pressurc.

C $300_{\mu}$ is for $1 \mathrm{~mm}$ pitch.

d Delay line cathode readout can give $\pm 150 \mu$ parallel to anode wire.

e For two chambers.
}

A.5 Shower Detectors: Typical energy res utions (FWHM) for incident electron in the $1 \mathrm{GeV}$ range. $\mathrm{E}$ in $\mathrm{GeV}$. For a fixed number of radiation lengths, FWHM in the last three detectors would be expected to be proportional to $V_{\mathrm{t}}$ for $\mathrm{t}\left(=\right.$ piate thickness) $\geq 0.2$ radiation lengths. ${ }^{6}$

NaI ( 20 rad. lengths): $\frac{2 \%}{E^{1 / 4}}$

Lead Glass (14 rad. lengths): $B \frac{10-12 \%}{\sqrt{\mathrm{E}}}$

Lead-Liquid Argon (15.75 rad. lengths): ${ }^{6}$

(42 cells: $1.1 \mathrm{~mm}$ lead, $2 \mathrm{~mm}$ liquid argon, $\frac{16 \%}{\sqrt{E}}$

$2.3 \mathrm{~mm}$ lead-G10, $2 \mathrm{~mm}$ liquid argon)

Lead-Scintiliator Sandwich ( 12.5 rad. lengths):
(66 cells: $1 \mathrm{~mm}$ lead, 5 mm scintillator)

Proportional Wire Shower Chamber (17 rad. lengths): ${ }^{10} \frac{40 \%}{\sqrt{E}}$

( 36 cells: $0.474 \mathrm{rad}$. length type-metal $+\mathrm{Al}$, $9.5 \mathrm{~mm} 80 \% \mathrm{Ar} \cdot 20 \% \mathrm{CH}_{4}$ gas)
A.6. Proportiomal Chamber Wire Instablility: The limit on the voltage $V$ for a wire tension $T$, due to mechanical effects when the electrostatic repulsion of adjacent wires exceeds the restoring force of wire tension, is given by 11

$$
v \leq \frac{s T^{1 / 2}}{8 C}
$$

where s, $\ell$, and $C$ are the wire spacing. length, and capacitance per unit length. An approximation to $C$ for chamber half-gap $t$ and wire diameter $d($ good for $s \leqslant t)$ gives $^{12}$

$$
v \leqslant 59 T^{1 / 2}\left[\frac{t}{\ell}+\frac{s}{\pi \ell} \text { in }\left(\frac{s}{\pi d}\right)\right] \text {. }
$$

where $V$ is in $k V$, and $T$ is in grams.

A.7 Proportional and Drift Chember Potentials: Potential distributions and fields for an array of parallel line charges $q(\operatorname{coul} . / \mathrm{m})$ along $z$ and located at $y=0, x=0 . \pm a . \pm 2 a \ldots .$. can usually be calculated with good accuracy from (MKSA):

$V(x, y)=-\frac{a}{4 \pi \epsilon_{0}}$ \&n $\left\{4\left[\sin ^{2}\left(\frac{\pi x}{a}\right)+\sinh ^{2}\left(\frac{\pi y}{a}\right)\right]\right\}$.

\section{B. COSMIC RAY FLUXES}

The fluxes of particles of different types depend on the latitude, their energy, and the conditions of measurement. Some typical sea-level values ${ }^{i}$ for charged particles are given below:

I flux per unit solid angle about vertical direction crossing unit horizontal area

$J_{1}$ perpendicular component of total flux crossing unit horizontal area from above

$\mathrm{J}_{2} \quad$ total flux crossing unit horizontal area Total Hard Sort Intensity Component Component

l. $^{1.1 \times 10^{-2}} \quad \overline{0.8 \times 10^{-2}} \quad \overline{0.3 \times 10^{-2}} \mathrm{~cm}^{-2} \mathrm{sec}^{-1} \mathrm{sterad}^{-1}$

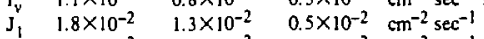

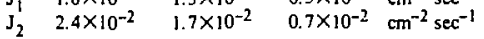

Very approximately, about $75 \%$ of all particles at sea-level are penetrating. and are muons. The absolute lux of protons at sea-level, in a momentum range $700-1100 \mathrm{MeV} / \mathrm{c}$. is $1.5 \times 10^{-5} \mathrm{~cm}^{-2} \mathrm{sec}^{-1}$ sterad ${ }^{-1}$, or $\sim 0.1 \%$ of all particles.

The muon flux at sea-level has a mean energy of $2 \mathrm{GeV}$ and a differential spectrum falling as $E^{-2}$. steepening smoothly to $E^{-3.6}$ above a few $T e V$. The angular distribution is $\cos ^{2} \theta$, changing to sect at energies above a $\mathrm{TeV}$, where $\theta$ is the zenith angle at production. The $+/-$ charge ratio is 1.25-1.30. The mean energy of muons originating in the atmosphere is roughly $300 \mathrm{GeV}$ at slant depths $\gtrsim$ a few hundred meters. Beyond slant depths of $\sim 10 \mathrm{~km}$ water-equivalent, the muons are due primarily to inthe-earth neutrino interactions (roughly $1 / 8$ interaction ton $^{-1}$ year $^{-1}$ for $E_{v}>300 \mathrm{MeV}$, - constant throughout the earth). ${ }^{14}$ Muons from this source arrive with a mean energy of $20 \mathrm{GeV}$, and have a flux of $2 \times 10^{-13}$ $\mathrm{cm}^{-2} \mathrm{sec}^{-1}$ sterad $^{-1}$ in the vertical direction and about twice that in the horizontal. ${ }^{15}$ down at least as far as the deepest mines.

\section{PASSAGE OF PARTICLES THROUGH MATTER}

C.1 Energy Loss Rates for Heavy Charged Projectiles: A heavy projectile (much more massive than an electron) of charge $Z_{\text {ince, incident at }}$ speed $\beta c$ ( $\beta \gg 1 / 137)$ through a slowing medium dissipates energy principally via interactions with the electrons of the medium. The mean rate of such energy loss per unit path length $x$ may be written as: ${ }^{16}$

$$
\begin{aligned}
& \left(\frac{\mathrm{dE}}{\mathrm{dx}}\right)_{\text {inc }}=\frac{\mathrm{D} \mathrm{Z}_{\text {med }} \rho_{\mathrm{med}}}{A_{\text {med }}}\left(\frac{\mathrm{Z}_{\mathrm{inc}}}{\beta}\right)^{2} \\
& \times\left[\ell \mathrm{en}\left[\frac{2 \mathrm{~m}_{\mathrm{c}} \gamma^{2} \beta^{2} \mathrm{c}^{2}}{l}\right)-\beta^{2}-\frac{\delta}{2}-\frac{\mathrm{c}}{\mathrm{Z}_{\text {med }}}\right]\{1+\nu\},
\end{aligned}
$$




\section{PARTICLE DETECTORS, ABSORBERS, AND RANGES (Cont'd)}

where $D=4 \pi \mathrm{N}_{A_{e}}{ }^{2} m_{e} c^{2}=0.3070 \mathrm{MeV} \mathrm{cm} / 8$ (see Physical and Numerical Constants Table)

Here $Z_{\text {med }}$ and $A_{\text {med }}$ are the charge and mass numbers of the medium and $\rho_{\text {med }}$ is the mass dens: $: y$ of the medium; $l, \delta, C$, and $\nu$ are phenomenological functions. Frequently, the values of $\delta_{1} \mathrm{C}$, and $y$ are negligibly small; the parameter $/$ characterizes the binding of the electrons of the medium. As a rule of thumb, we may estimate $I$ for an idealized medium as $I=16\left(Z_{\text {med }}\right)^{0.9} \mathrm{eV}$ when $Z_{\text {med }}>1$. For realistic media the value of $/$ will vary at the $10 \%$ level from this estimate; for $\mathrm{H}_{2}, I=20.0 \mathrm{eV}$. We may approximately treat media which are chemical mixtures or compounds by computing

$$
\frac{d E}{d x} \approx \sum_{n=1}^{N}\left(\frac{d E}{d x}\right)_{n}
$$

with $(d E / d x)$ appropriate to the $n^{\text {th }}$ chemical constituent (using $\rho_{\text {med }}^{(n)}$ as the partial density in the formula for $\mathrm{dE} / \mathrm{dx}) .^{17}$

The function $\delta$ represents the density effect upon the energy loss rate; it is non-negligible only for highly relativistic projectiles in denser media. ${ }^{18}$ For ultra-relativistic projectiles, $\delta$ approaches $2 \ell n \gamma+\infty n-$ stant, where the value of the constant depends upon the density of the medium as well as its chemical composition.

The function $C$ represents shell corrections to the energy loss rate. ${ }^{16}$ These effects are non-negligible only for projectiles with speeds not much faster than the speeds of the fastest electrons bound in the medium.

The function $v$ represents corrections due to higher-order electrodynamics. ${ }^{19}$ These effects become important when $\left|Z_{\text {inc }} / \beta\right|$ is comparable 1. 137. For relativistic unit-charge projectiles, $|\nu|$ is of the order of $1 \%$; positively charged projectiles lose energy more rapidly than do their charge conjugates. ${ }^{19.20}$

For non-relativistic projectiles, our formulae above are inapplicable. At the very slowest speeds, total energy loss rates are believed to be proportional to $\beta$, rising through a peak at projectile speeds comparable to atomic speeds, after having passed hrough a smaller peak (due to elastic Coulomb collisions with the nuclei of the slowing medium ${ }^{21}$ ) at intermediatc speeds. In some cases. energy loss rates depend significantly upon the relation of the projectile trajectory to the crystalline structure of the slowing medium. ${ }^{22}$

For relativistic projeatiles, $(\mathrm{dE} / \mathrm{d} x)_{\text {inc }}$ falls rapidly with increasing $B$ until reaching a minimum around $\beta=0.96$ (almost independent of medium). followed by a slow rise. Because of the density effect, the quantity in square brackets approaches $\ell n \gamma+$ constant for large $\gamma$.

The quantity $(\mathrm{dE} / \mathrm{dx})_{\mathrm{jnc}} \delta \mathrm{x}$ is the meon total energy loss via interactions with electrons of the medium in a layer of thickness $\delta x$. For any finite $\delta x$. Poisson fluctuations cañ cause the actual energy loss to deviate from the mean. For thin lavers, the distribution is broad and skewed, being peaked below $(\mathrm{dE} / \mathrm{dx}) \delta \mathrm{x}$. and having a long tail toward large energy losses. ${ }^{23}$ Only for a very thick layer $\left[(d E / d x) \delta x \gg 2 m_{e} \beta^{2} \gamma^{2} c^{2}\right]$ will the distribution of energy losses become nearly Gaussian. The large fluctuations of the total energy loss rate from the mean are due to a small number of collisions involving large energy transfers. The fluctuations are greatly reduced for the so-called restricted energy loss rate, described in Section C. 4.

C.2 Ionization Yields: Physicists frequently relate total energy loss to the number of ion pairs produced in the stopping medium. This relation becomes complicated for relativistic projectiles due to the wandering of energetic knock-on electrons whose ranges exceed the dimensions of the fiducial volume. For a qualitative appraisal of the non-locality of energy deposition by such modestly energetic knock-on electrons in various media, see Ref. 24. The mean energy loss per ion pair produced, $W$, is essentially constant for relativistic projectiles, but increases at slow projectile speeds. ${ }^{25}$ The numerical value of $W$ for gases can be surprisingly sensitive to trace amounts of various contaminants. ${ }^{25}$ of course. in addition to the preceding effects, practical ionization yields may be greatly influenced by subsequent recombinations, etc. ${ }^{26}$
C.3 Energetic Knock-On Electrons: For a relativistic spinless pointcharge projectile. the production of high energy (kinetic energy $T \gg n$ electrons is given by (neglecting the spin of the electron):

$$
\frac{d^{2} N}{d T d x}=\frac{1}{2} D\left(\frac{Z_{\text {med }}}{A_{\text {med }}}\right)\left(\frac{Z_{\text {inc }}}{\beta}\right)^{2} p_{\text {med }} \frac{1}{T^{2}} \text {. }
$$

for $I \ll \mathrm{T} \leq \mathrm{T}_{\max }$, where

$$
\mathrm{T}_{\max }=\frac{2 \mathrm{~m}_{\mathrm{c}} \beta^{2} \gamma^{2} \mathrm{c}^{2}}{1+2 \gamma \frac{\mathrm{m}_{\mathrm{e}}}{\mathrm{M}_{\mathrm{inc}}}+\left(\frac{\mathrm{m}_{\mathrm{e}}}{\mathrm{M}_{\mathrm{inc}}}\right)^{2}} .
$$

$\mathrm{M}_{\mathrm{inc}}$ is the mass of the incident projectile, and all other quantities are as in Section C.1. This formula does not differ significantly from the precise result. incorporating spin effects, for any projectile (including $\mathrm{e}^{ \pm}$) in the restricted range $l \ll T \ll T$ T available for various projectiles. ${ }^{27,28}$ Our formula is inaccurate for $T$ close to $l$; for $2 l \leqq \mathrm{~T} \leqq 10 l$, the $1 / \mathrm{T}^{2}$ dependence above becomes $\simeq \mathrm{T}^{-m}$ with $3 \leq \eta \leq 5$. ${ }^{29}$

C.4 Rates of Restricted Energy Loss for Reintivistic Charged Projectiles: The variability of energy loss for heavy projectiles is due primarily to the variability in the production of energetic knock-on electrons.

Bremsstrahlung and pair-production processes make this variability even greater for electrons than for heavy particles as projectiles (see, e.g. the figure "Fractional Energy Loss for $\mathrm{e}^{+}$and $\mathrm{e}^{-}$in Lead," following). If an instrument, such as a bubble chamber, is capable of isolating these high-energy-loss interactions, then it is appropriate to oonsider the rate of energy loss excluding them, i.e.. a restricted energy loss rate. The mean energy loss rate via all collisions which have energy transfer $T$ such that $T \leq E_{\max } \ll T_{\max }$ is: 16

$$
\begin{aligned}
\left(\frac{\mathrm{dE}}{\mathrm{dx}}\right)_{\leq \mathrm{E}_{\max }}=\frac{1}{2} \mathrm{D} \frac{Z_{\text {med }} \rho_{\text {med }}}{A_{\text {med }}}\left(\frac{Z_{\text {inc }}}{\delta}\right)^{2} \\
\times\left[\operatorname{m}\left(\frac{E_{\max } T_{\max }}{I^{2}}\right)-\beta^{2}-\delta-\frac{2 C}{Z_{\text {med }}}\right] .
\end{aligned}
$$

Notice the overall factor of $1 / 2$.

The density effect causes the restricted energy loss rate to approach a constant. the Fermi plateau value. for the fastest projectiles.

C.5 Multiple Scattering through Small Angles: As a charged particle traverses a medium it is defiected by many small-angle elastic seatterings. The bulk of this deflection is due to elastic Coulomb scattering from the nuclei within the medium, hence the usual identification as multiple Coulomb scattering (note. however, that strong interactions do contribute to the total multiple scattering for hadronic projectiles). For both Coulomb and strong interactions, the Central Limit Theorem provides little useful guidance in establishing the precise nature of the distribution of the total deflections resulting from multiple scattering. The true distribution is roughly Gaussian only for small defiection angles while it shows much greater probability for large-angle scatterings $\geq$ a few $\theta_{0}$, see below, depending on absorber) than the Gaussian would suggest. These tails on the distribution (a few \% of peak height in the region where the Gaussian part becomes negligible) are more pronounced for hadrons than for muons as projectiles. The large-angle behavior of these distributions is best estimated by computing the exact distribution for the vectorial sum of the largest deflections based upon the true elastic scattering cross section of the projectile against the medium ${ }^{30}$ or, when applicable, by interpolation from tabular data, ${ }^{31}$ An casier alternative which may suffice for non-critical applications would be to use a Gaussian approximation with the following width: ${ }^{32}$

$$
\theta_{0}=\frac{14.1 \mathrm{MeV} / \mathrm{c}}{\mathrm{p} \theta} \mathrm{z}_{\mathrm{inc}} \sqrt{\mathrm{L} / \mathrm{L}_{\mathrm{R}}}\left[1+\frac{1}{9} \log _{10}\left(\mathrm{~L} / \mathrm{L}_{\mathrm{R}}\right]\right] \text { (radians). }
$$




\section{PARTICLE DETECTORS, ABSORBERS, AND RANGES (Cont'd)}

where $\mathrm{p}, \boldsymbol{\beta}$, and $\mathrm{Z}_{\mathrm{inc}}$ are the momentum (in $\mathrm{MeV} / \mathrm{c}$ ), velocity, and charge number of the incident particle, and $L / L_{R}$ is the thicioness, in radiation lengths, of the scattering medium. $L_{R}$ for certain materials is given in the Table of Alomic and Nuclear Properties of Materials (following). The angle, $\theta_{0}$ is a fit to Moliere ${ }^{30}$ theory, accurate to about $5 \%$ for $10^{-3}$ $<\mathrm{L} / \mathrm{L}_{\mathrm{R}}<10$ except for very light elements or low velocity where the error is about 10 to 20\%. In this Gaussian approximation, $\theta_{0}$ has the meaning

$$
\theta_{0}=\theta_{\mathrm{plane}}^{\mathrm{rms}}=\frac{1}{\sqrt{2}} \theta_{\mathrm{space}}^{\mathrm{mms}} .
$$

The non-projected (space) and projected (plane) angular distributions are given approximately ${ }^{30}$ by the Gaussian forms:

$$
\begin{aligned}
& \frac{1}{2 \pi \theta_{0}^{2}} \exp \left(-\frac{\theta_{\text {space }}^{2}}{2 \theta_{0}^{2}}\right) d \Omega \\
& \frac{1}{\sqrt{2 \pi} \theta_{0}} \exp \left(-\frac{\theta_{\text {plane }}^{2}}{2 \theta_{0}^{2}}\right) d \theta_{\text {plane }}
\end{aligned}
$$

where $\theta$ is the deflection angle.

Other quantities are sometimes used to describe the amount of multiple Coulomb scattering: the auxiliary quantities $\psi_{\text {plane, }} \mathrm{y}_{\text {plane, }}$ and $s_{\text {plane }}$ (see the figure) obey:

and

$$
\begin{aligned}
& y_{\text {plane }}^{\mathrm{rmx}}=\frac{1}{\sqrt{3}} L \theta_{\text {płane }}^{\mathrm{rmm}}=\frac{1}{\sqrt{3}} \mathrm{~L} \theta_{0} . \\
& \psi_{\text {piane }}^{\text {rms }}=\frac{1}{\sqrt{3}} \theta_{\text {plane }}^{\max }=\frac{1}{\sqrt{3}} \theta_{0} \text {. } \\
& \mathrm{s}_{\mathrm{planc}}^{\mathrm{rms}}=\frac{1}{4 \sqrt{3}} \mathrm{~L} \theta_{\mathrm{phanc}}^{\operatorname{mins}}=\frac{1}{4 \sqrt{3}} L \theta_{0} .
\end{aligned}
$$

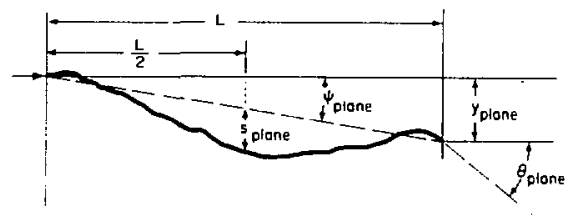

All the quantitative estimates in this section apply only in the limit of small $\theta_{\text {plane }}^{\text {rma }}$ and in the absence of large-angle scatters.

C.6 Longitulinel Distribution of Electronugnetic Stowers: A photon of energy $E(\mathrm{GeV}) \geq 0.1 \mathrm{GeV}$ converting in a semi-infinite medium produces an electromagnetic cascade whose intensity initially increases with depth and then falls off. The average number of $e^{ \pm}$with kinetic energy above $1.5 \mathrm{MeV}$, crossing a plane at a depth of $\mathrm{t}$ radiation lengths from the beginning of the medium, in a material of atomic number $\mathbf{Z}$, calculated using the Monte Carlo program EGS, ${ }^{33}$ can be fit by the empirical formula ${ }^{34}$

$$
N=N_{0} t^{*} c^{-b t} \text {. }
$$

where $N_{0}=5.51 \mathrm{E} \sqrt{Z} \mathrm{~b}^{a+1} / \Gamma(\mathrm{a}+1)$ and $\mathrm{b}=0.634-0.0021 \mathrm{Z}$. For $\mathrm{Z}$ $\geq 26, a=2.0-Z / 340+(0.664-Z / 340)$ in $E$. For $Z=13, a=1.77$ -0.52 en $\mathrm{E}$ The maximum intersity, $\mathrm{N}_{\max }$, occurs at the depth $\mathrm{t}=$ $a / b$. The maximum error of the fit occuis in the vicinity of this depth and is less than $0.15 \mathrm{~N}_{\max }$. The integral of the tail, $\int_{1.5}^{\infty} \mathrm{N} d \mathrm{~d}$ is fit to better than $2.5 \%$. The total longitudinally-projected $\mathrm{e}^{ \pm}$path length. $\int_{0}^{\infty} \mathrm{Ndt}=5.51 \mathrm{E} \sqrt{\mathrm{Z}}$, is less than the total $\mathrm{e}^{ \pm}$path length due primarily to multiple Coulomb scattering.
'Prepared April 1974 by Sherwood Parker and Bernard Sadoulet. Revised April 1982 by Sherwood Parker. Ray Hagstrom. Didier Besset, and John Learned.

1. Methods of Experimental Physics, L.C.L. Yuan and C.-S. Wu, editors, Academic Press, 1961, Vol. SA. p. 127.

2. R.K. Swank. Ann. Rev. Nucl. Sci. 4, 137 (1954), and G.T. Wright, Proc. Phys Soc. B68, 929 (I955).

3. Methods of Experimental Physics, L.C.L. Yuan and C.-S. Wu, editors, Academic Press, 1961, Vol. 5A p.163.

4. E.R. Hayes, R.A. Schluter, and A. Tamosaitis, "Index and Dispersion of Some Cerenkov Counter Gases," ANL-6916 (1964).

5. Nuclear Enterprises Catalogue.

6. D. Hitlin et al., Nucl. Instr. and Meth. 137, 225 (1976). See also W.J. Willis and V. Radeka, Nucl. Instr. and Meth. 120. 221 (1974), for a more detailed discussion.

7. E.B. Hughes et al., IEEE Transactions on Nuciear Science NS-19, No. 3. 126 (1972).

8. M. Holder et al., Phys. Letters 40B, 141 (1972), and J.S. Beale et al., "A Lead-Glass Cerenkov Detector for Electrons and Photons," CERN Writeup, Intl. Conf. on Instrumentation in H.E.P., Frascati (1973).

9. W. Hofmann et al., DESY 81/045 (July [981). See also S. L. Stone et al., Nucl. Instr, and Meth. 151, 387 ( (1978).

10. R.L. Arderson et al., "Tests of Proportional Wire Shower Counter and Hadron Calorimeter Modules," SLAC-PUB-2039 (1977).

11. T. Trippe, CERN NP Internal Report 69-18 (1969).

12. S. Parker and R. Jones, LBL-797 (1972), and A Morse and B. Feshbach, Methods of Theorerical Physics, McGraw-Hill, New York, 1953, p. 1236

13. B. Rossi, Rev. Mod. Phys. 20, 537 (1948).

14. J.G. Learned, F. Reines, and A. Soni, Phys. Rev. Lett. 43, 907 (1979).

15 M.F. Crouch et al., Phys. Rev. D18, 2239 (1978)

16. U. Fano, Aлn. Rev. Nucl. Sci. 13, I (1963).

17. H.A. Bethe and J. Ashkin, Experimental Nuclear Physics, Vol. 1, E. Segre, editor, John Wiley. New York, 1959.

18. A Crispin and G.N. Fowler, Rev. Mod. Phys. 42. 290 (1970).

19. For $Z^{3}$ calcti ntions with $Z=1$, see J.D. Jackson and R.L McCarthy, Phys. Rev. B6, 4131 (1972).

20. For an approximate treatment of high-Z projectiles, see P.B. Eby and S.H. Morgan, Phys. Rev. A5, 2536 (1972).

21. See, for instance, G. Sidenius, Det Kong. Janske Viden. Selskab Mat.-Fysk. Med. 39, No. 4 (1974).

22. See, for instance, S. Datz, "Atomic Collisions in Solids" in "Structure and Collisions of Ions and Atoms," Springer Verlag, Berlin, 1978. p. 309.

23. See. for instance, KA. Ispiriar, A.T. Margarian, and A.M. Zverev, Nucl. Instr. and Meth. 117, 125 (1974).

24. L.V. Spencer "Energy Deposition by Fast Electrons." Nat'! Bureau of Slandards Monograph No. 1 (1959).

25. "Average Energy Required to Produce an Ion Pair," ICRU Report No. 31 (1979)

26. N. Hadley et. al, "List of Poisoning Times for Materials," TPC. LBL-79-8 (1981).

27. For unit-charge projectiles, see E.A. Uehling, Ann. Rev. Nucl. Sci. 4. 315 (1954)

28. For highly charged projectiles. see J.A. Doggett and LV. Spencer, Phys. Rev. 103, 1597 (1956). A Lorentz transformation is needed to convert these center-of-mass data to knock-on energy spectra.

29. N.F. Mott and H.S.W. Massey, The Theory of Alomic Collisions, Oxford Press, London, 1965.

30. For a thorough discussion of simple formulae for single scatters and methods of compounding these into multiple-scattering formulae, see W.T. Soott, Rev. Mod. Phys. 35, 231 (1963). For detailed sumr maries of formulae for computing single scaters, see J.W. Motz, $H$. Olsen, and H.W. Koch, Rev. Mod. Phys. 36, 881 (1964).

31. E.V. Hungerford and B.W. Mayes, Atomic Data and Nuclear Data Tables 15, 477 (1975).

32. V.L. Highland, Nucl. Inst. and Meth. 129, 497 (1975) and important modification Nucl. Inst. and Meth. 161, 171 (1979).

33. R. Ford and W, Nelson, SLAC-210 (1978).

34. A similar form has been used by E. Longo and I. Sestili, Nucl. Inst. and Meth. 128. 283 (1975), and J. Sass and M. Spiro. CERN p p Tech. Note 78.32 (1978). 


\section{PARTICLE DETECTORS, ABSORBERS, AND RANGES (Cont'd)}

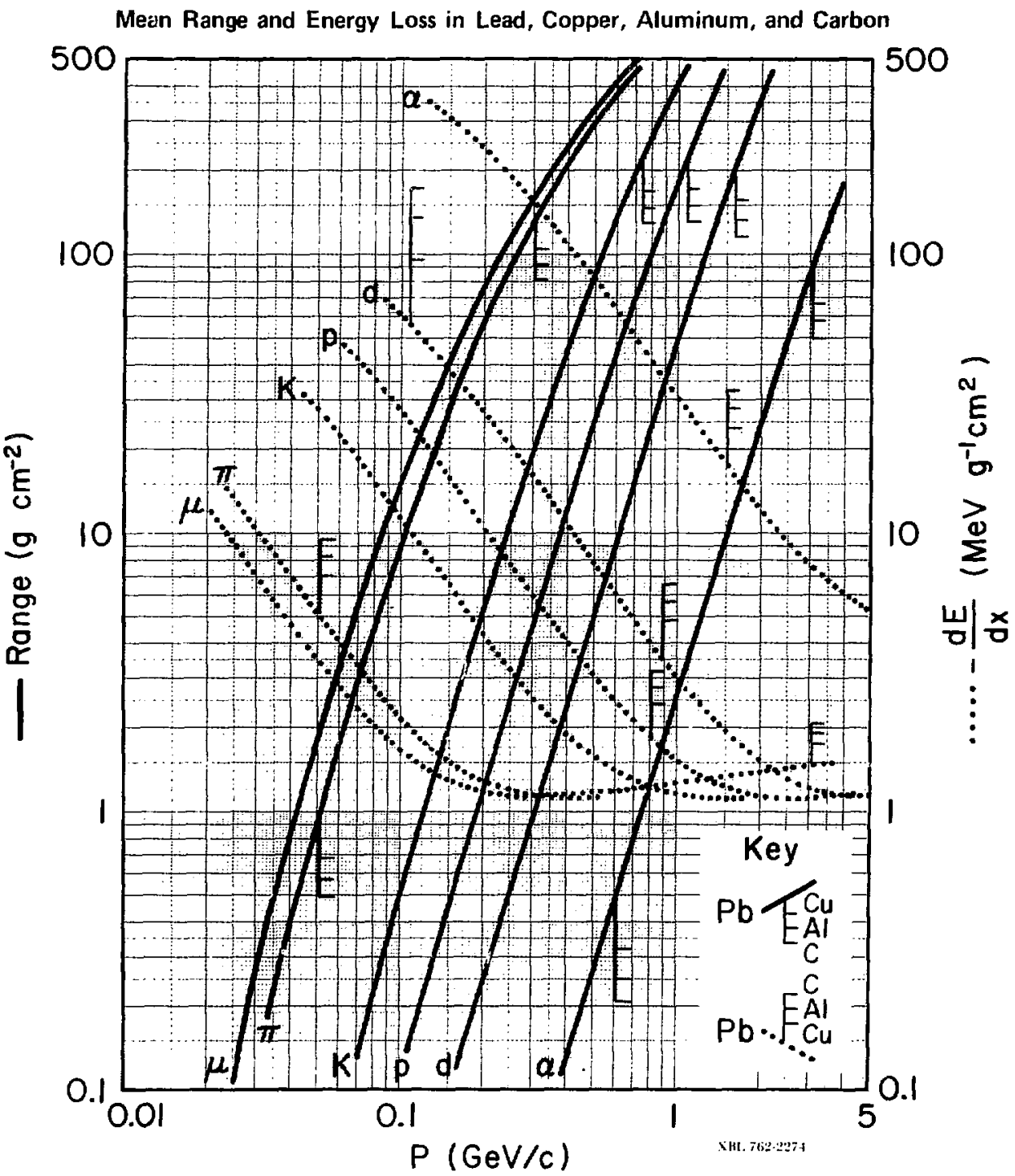

Mean range and energy loss due to ionization for the indicated particles in Pb, with scaling to Cu, Al, and $C$ indicated, using Bethe-Bloch equation (Section C. 1 above) witl corrections. Calculated using program of Hans Bichsel (UCRL-17538), with density correction zdded (Hans Bichsel, private conmunication). See also Joseph F. Janni [Air Force Weapons Laboratory Technical Report No. AFWL-TR-65-150 (1966)]. The average ionization potentials (I) assumed were: $P b(820$ eV), $C u(320$ eV), Al (166 eV), and C(T7.5 eV). Figure indicates total path length; observed range may be smaller (by $\sim 1 \%-2$ in heavy elements) due to multiple scattering, primarily from small energy-loss collisions with nuclei. The lunctional forms have not been experimentally verified to better than roughly $\pm 1 \%$. For higher energies refer to cliscussion by Cobb [1A Study of Some Electromagnetic Interactions of High Velocity Particles with Matter, "University of Oxford Report HEP/T/55 (1973/ and by Turner ["Penetration of Charged Particles in Matter: A Synuosium", National Academy of Sciences, Washington D.C. (1970), p. 48). Scaling to other beam particles is, to a good approxination, described by the expression on the next page. 
PARTICLE DETECTORS, ABSORBERS, AND RANGES (Cont'd)

Mean Range and Energy Loss in Liquid Hydrogen

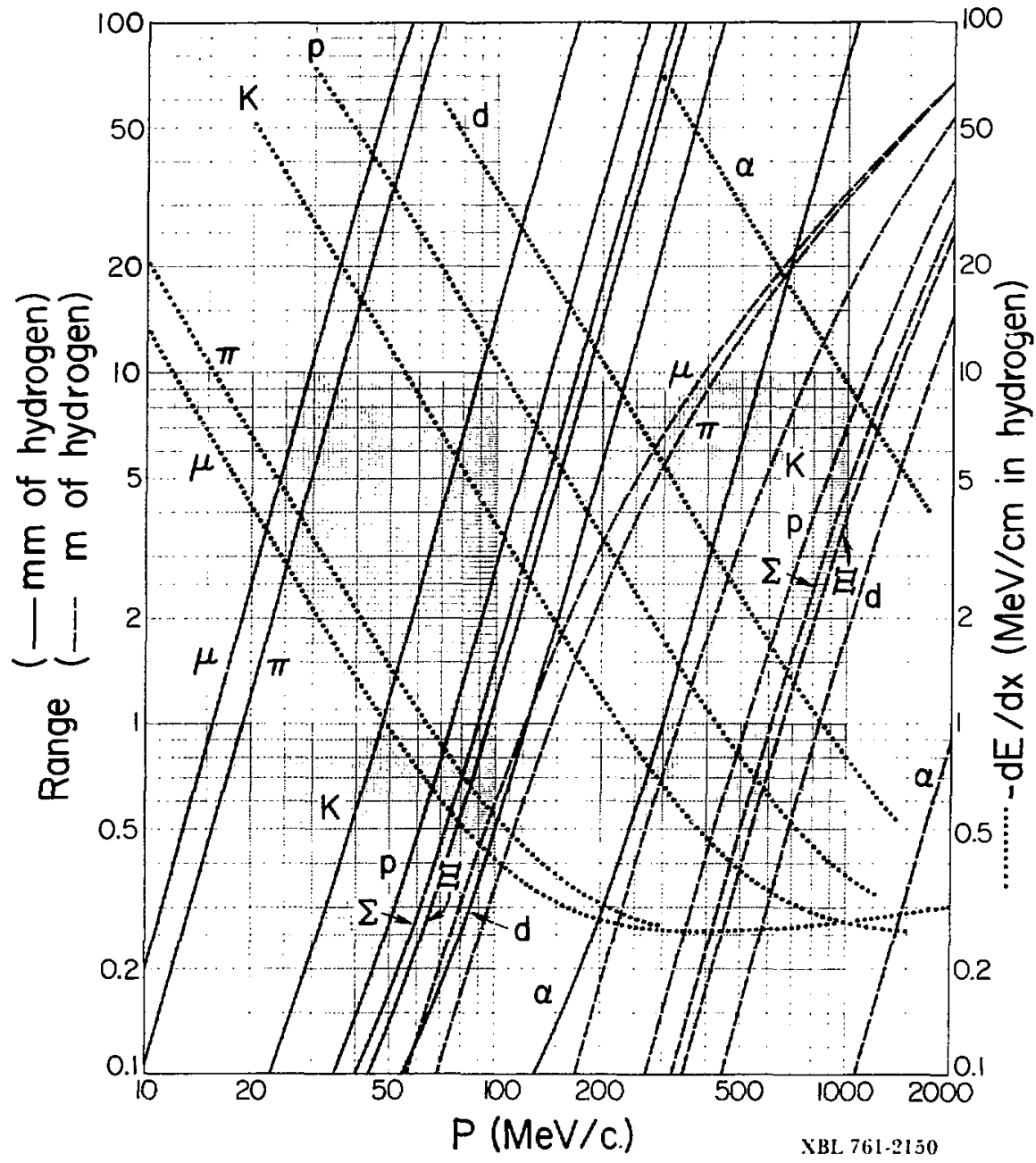

Range and energy loss in liquid hydrogen bubble chamber, based on Bethe-Bloch equation (Section $c .1$ above), using an average ionization potential for $\mathrm{H}_{2}$ of $\mathrm{I}=20.0 \mathrm{eV}$, which is an approximate average of the experimental result of Garbincius and Hyman [Phys. Rov. A2, 1834 (1970)] and the theoretical result of Ford and Browre [Phys. Rev. A7, 41a (1973)]. Bubble chamber conditions are chosen to be those of Garbincius and Hyman: parahydrogen of density $=0.0625 \mathrm{~g} / \mathrm{cm}^{3}$ (note) range a $1 /$ density), with vapor-pressure 60.8 lb/in (absolute) and temperature $26.2^{\circ} \mathrm{K}$. The functional dependence of the Bethe-3loch equation is not experimentally verified to better than about \pm 1 over large momentum ranges. It should be noted that the number of bubbles fer cm of a track in a bubble chamber is nearly proportional to $1 / \mathrm{B}^{2}$, not $\mathrm{de} / \mathrm{dx}$. For the 1 inear portions of the range curves, $R \times p^{3.6}$. Scaling law for particles of other mass or charge (except electrons): for a given medium, the range $R_{b}$ of any beam particle with mass $M_{b}$, charge $z_{b}$ and momentum $p_{b}$ is given in terms of the range $R_{a}$ of any other particle with mass $M_{a}$, charge $z_{a}$, and momentum $P_{a}=P_{b} M_{a} / M_{b}$ ( $i . e$., having the same velocityl by the expression:

$$
R_{b}\left(M_{b}, z_{b}, P_{b}\right)=\left[\frac{M_{b} / M_{a}}{z_{b}^{2} / z_{a}^{2}}\right] R_{a}\left(M_{a^{\prime}} z_{a}, P_{a}=p_{b} M_{a} / M_{b}\right) .
$$




\section{PARTICLE DETECTORS, ABSORBERS, AND RANGES (Cont'd)}

\section{Mean Electron Range in Lead, Copper, Carbon, and Liquid Hydrogen}

Hean range of electrons in the continuous-slowing-down approximation, taking into account energy loss by collisions with atomis electrons and by bremsstrahlung: strong fluctuations are to be expected for individual tracks. This range is the cotal : ith length; the "fractical iange" - a comnon measure of straight-line penetration distance - is shorter because of multiple coulonb scatcering, which becomes increasingly important as the electron slows down. E.g., for a fast electron the ris projected angle due to multiple coulomb scattering reaches 1 radian by the time the electron has slowed to $0.4 \mathrm{MeV}$ in hydrogen, 1.5 MeV in carbon. $9 \mathrm{MeV}$ in copper, and $24 \mathrm{Mov}$ (off scale) in lead. Electron energy defosition and penetration probability vis. range are discussed by 1.. $v$. Spencer. "Energy Dissipation by Fast Electrons," NBS Monograph $\$ 1$, 1959 , and S. M. Seltzer.

"Transmission of Electrons through Foils," NBSIR 74, 457 (1974) - Electrons which have energy less than 0.2 MeV in $\mathrm{Ar}$, $T(\mathrm{keV})$

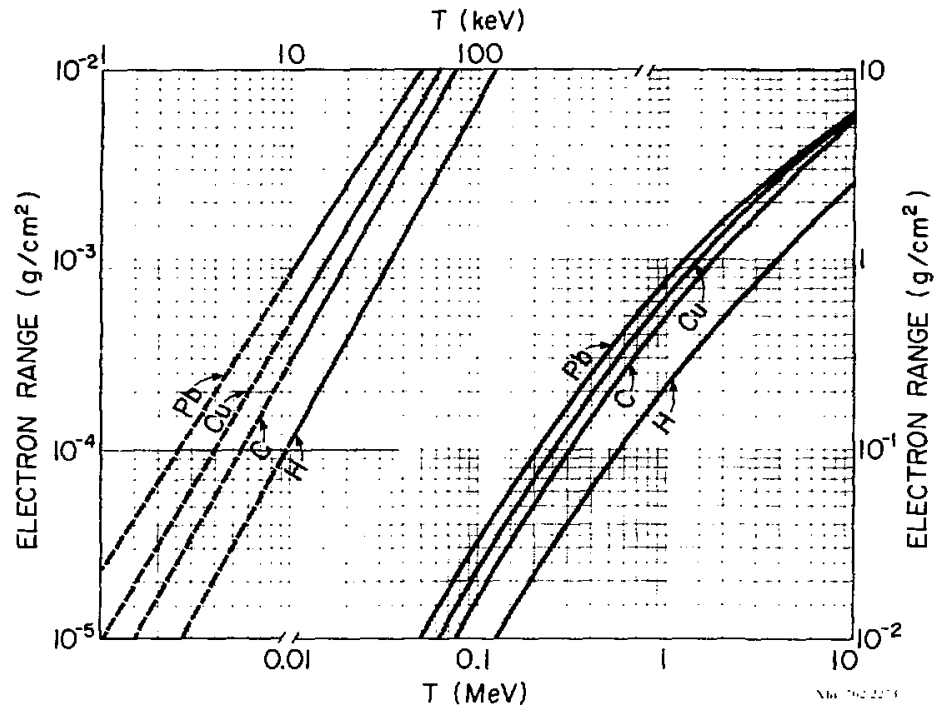

$1.5 \mathrm{MeV}$ ir $\mathrm{Cu}, 3.5 \mathrm{MeV}$ in $\mathrm{Sn}$, and $5 \mathrm{MeV}$ in $\mathrm{Pb}$ are likely to deposit los of their energy behind theje startinq plane. The practical fanqe, R, is defined as that absorber thickness obtained by extrapoloting to zero the linearly decreasing part of the curve of penetration probability vs. absorber thickness. Data for $A]$ in the tinge of the $f i g i r e$ are available, and $f$ it $(t o-108) R_{p}=A T[1-B /(1+C T)] \mathrm{mg} \mathrm{cm}^{-2}$ [a form sugqested by K.-H. Weber. Nucl. Inst. Aeth. 25, 26I (1964)], with $A=0.55 \mathrm{mg} \mathrm{cm} \mathrm{cm}^{-2} \mathrm{kev}^{-1}, B=0.9841$, and $\mathrm{C}=0.0030 \mathrm{kev}$. At this panetration depti, $90-958$ of the incident aectrans have stopped. Data for other elements are sketchy, but suggest that bigner-z $(-50)$ elements have $1<R_{p} / R_{1}(A l)<1.4$ below $-10 \mathrm{keV}$, and $0.6 \div \mathrm{R}_{\mathrm{p}} / \mathrm{R}_{\mathrm{p}}(\mathrm{Al}) \leq 1$ above $\sim 100 \mathrm{keV}$. The "critical energy" (above which the energy loss jue to tremsstrahlung exceeds that due to ionization, and shawering becomes important) is 400 Mov far hydrogen, 100 MeV far carbon. 25 MeV for coprer, and $10 \mathrm{MeV}$ for lead. The mean posityon range may differ from the mean electron range by several percent. sec Berqer and Seltzer, NASA 5P-30L2 (1964) and SP-3036, and P. Trower, UCPL-2426, Vol. 111, Rev. (1956). 1- J0 keV range was obcained by Iincar extrapolation; in this region the true range may actually lie above the curyes.

\section{Fractional Energy Loss for $\mathrm{e}^{+}$and $\mathrm{e}^{-}$in Lead}

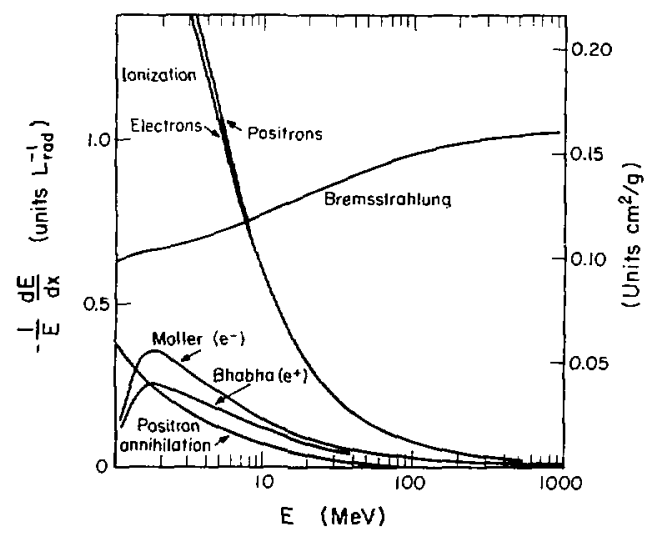

Fractional energy loss per radiation length in lead as a function of electron or positron energy. Electron (positron) scattering is considered as ionization when the energy loss per collision is below $0.255 \mathrm{MeV}$, and as Moller (Bhabha) scattering when it is above. Adapted from Fig. 3.2 from Messel and Crawford, Electron-Photon Shower vistribution Function Tables for Lead, Copper and Air Absorbers, Pergamon Press, 1970. Messel and Crawford use $L_{r}(\mathrm{~Pb})=5.82 \mathrm{~g} / \mathrm{cm}^{2}$, but we have modified the figures to reflect the value given in the Table of Atomic and Nuclear Properties of $\mathrm{Ma}$ terials (following), namely $L_{r}(\mathrm{~Pb})=6.4 \mathrm{~g} / \mathrm{cm}^{2}$. The development of electron-photon cascades is afproximately independent of absorber when the results are expressed in terms of inverse radiation lengths (i.e. scale on left of plot). 


\section{PARTICLE DETECTORS, ABSORBERS, AND RANGES (Cont'd)}

\section{Photon Mass Attenuation Coefficients, Energy Deposition}

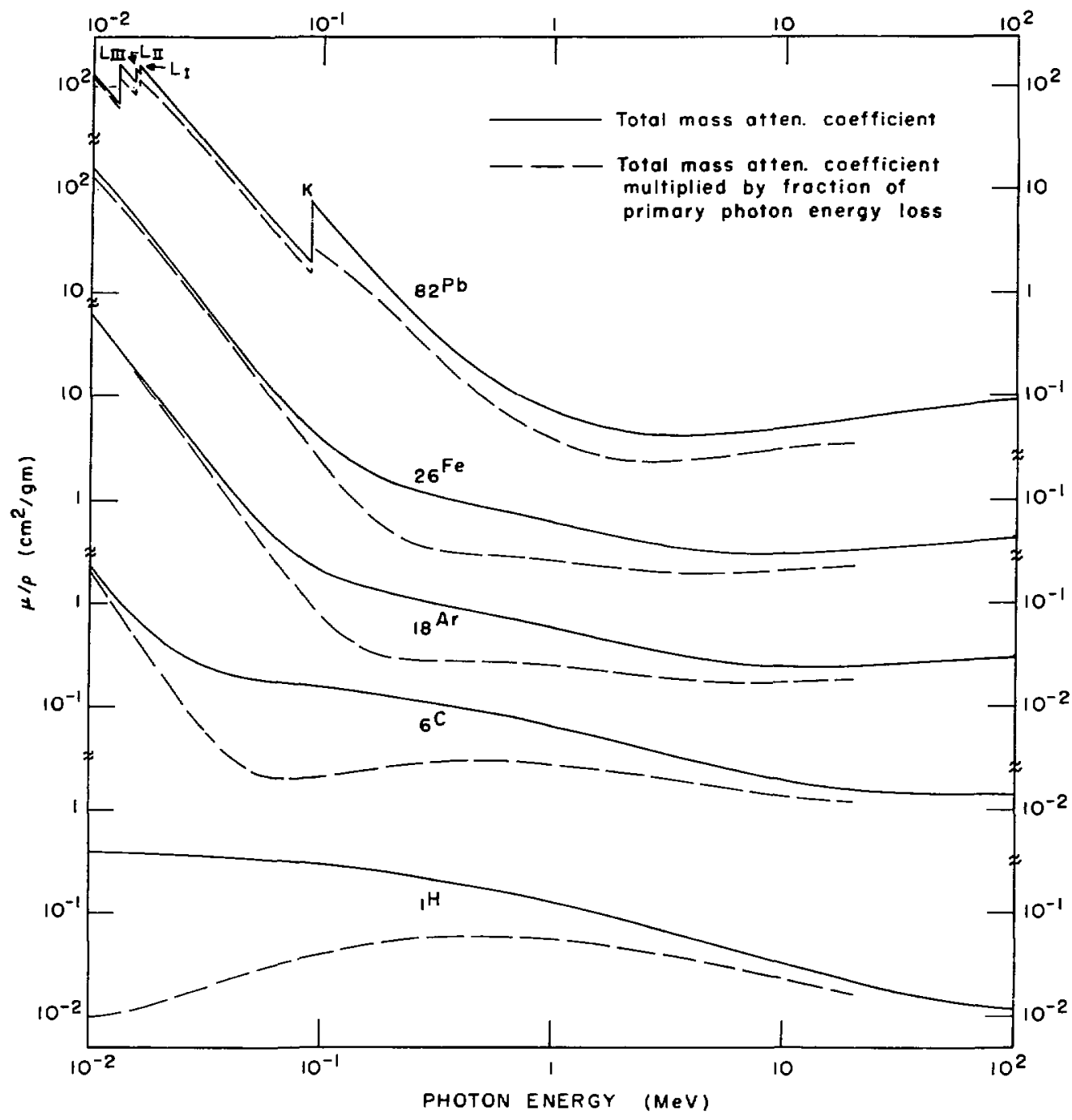

The pinoton mass attenuation coefficient for various absorbers as a function of photon energy (solid curves). For a homogeneous mediun of density $p$, the intensity $I$ remaining after traversal of thickness $t$ is given by $I=I_{0} \exp (-L t)$. The accuracy is a few percent. Interpolation to other $z$ should be done in the cross section $\mathrm{J}=(\mu / \rho) \mathrm{M} / \mathrm{N}_{\mathrm{A}} \mathrm{cm}^{2} /$ atom, where $\mathrm{M}$ is the atomic weight of the absorber material and $\mathrm{N}_{\mathrm{A}}$ is Avogadro's number. For a chemical compound or mixture, use $(\mu / \rho)_{\text {eff }} \cong \sum_{i} w_{i}(\mu / \rho)_{i}$, accurate to a few percent, where wi is the proportion by weight of the $i^{\text {th }}$ constituent. 1 The dashed curve is the mass energyabsorption coefficient, giving $\mu / 0$ multiplied by the fraction of photon energy deposited in a small volume (assumed large enough to contain the ranges of most secondary electrons) about the interaction. This fraction is smaller than 1.0 because such processes as compton scattering and electron bremsstrahlung imply radiation of some of the energy away from the imnediate area. The absorption coefficient is an approximation to the energy available for chemical, biological, and ather effects associated with exposure to ionizing radiation. See next page for high energy range. From Hubbell, Gimn, and фverbl, J. Phys. Chem. Ref. Data 9, $1023(1980)$. Figures courtesy J. H. Hubbell. 
PARTICLE DETECTORS, ABSORBERS, AND RANGES (Cont'd)

Photon Mass Attenuation Coefficients (High Energy)

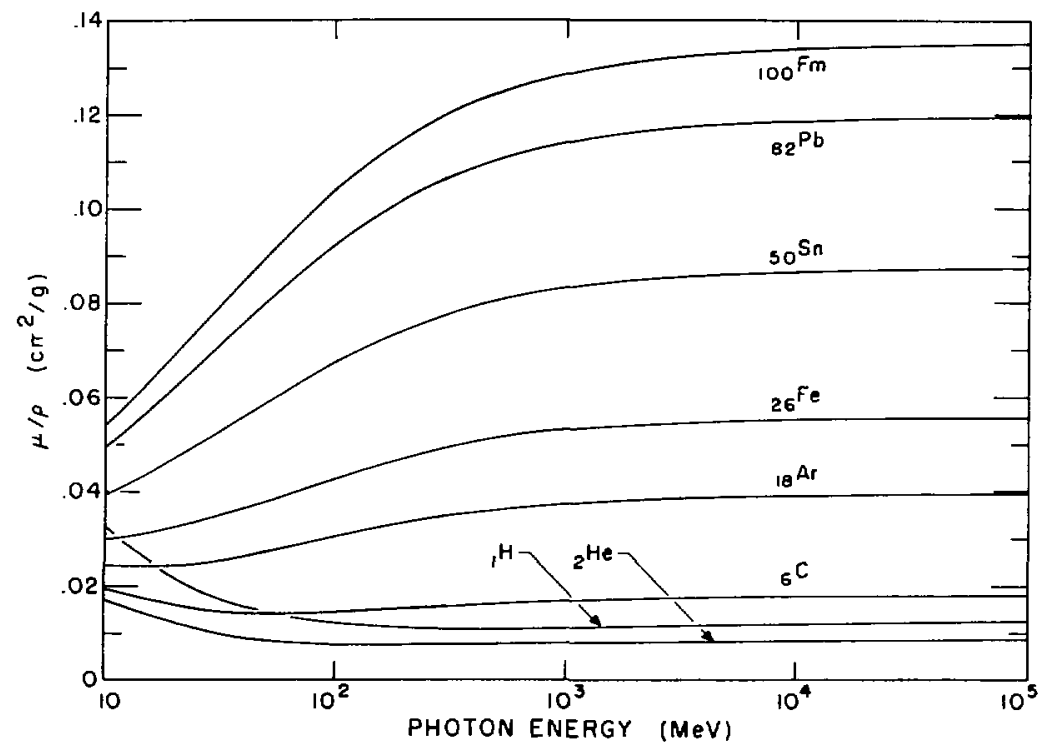

The photon mass attenuation coefficient, high energy range (note that crdinate is linear scale). See zaption on previous page for details.

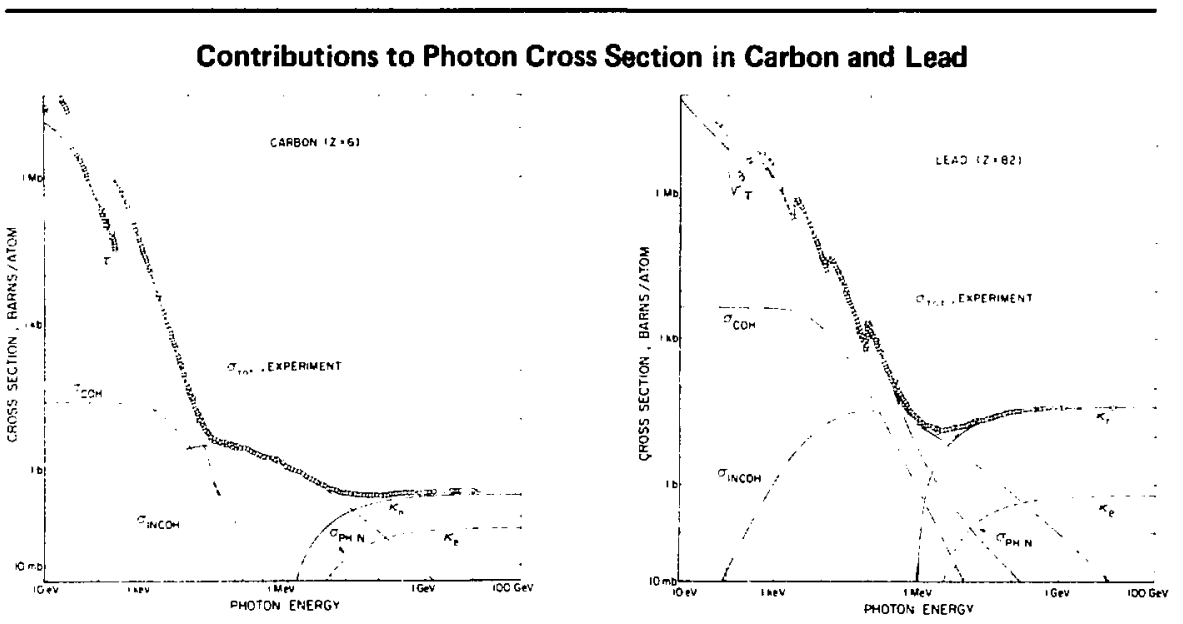

Photon total cross sections as a function of energy in carbon and lead, showing the contributions of different processes.

$$
\begin{array}{ll}
\tau & =\text { Atomic photo-effect (electron ejection, photon absorption) } \\
\sigma_{\mathrm{CUH}}= & \text { Coherent scattering (Rayleigh scattering - atom neither ionized nor excited) } \\
\sigma_{\mathrm{INCOH}}= & \text { Incoherent scattering (Compton scattering off an electron) } \\
\kappa_{\mathrm{n}} & \text { Pair production, nuclear field } \\
\kappa_{\mathrm{e}}= & \text { Pair production, electron Eield } \\
\sigma_{\mathrm{PH}, \mathrm{N} .}= & \text { photonuclear absorption (nuclear absorption, usually followed by emission of } \\
& \text { a neutron or other particle) }
\end{array}
$$

From Hubbell, Ginst, and øverbø, J. Phys. Chen. Ref. Data 9, 1023 (1980). Figures courtesy J. H. Hubbell. 


\section{PARTICLE DETECTORS, ABSORBERS, AND RANGES (Cont'd)}

\section{Atomic and Nuclear Properties of Materials*}

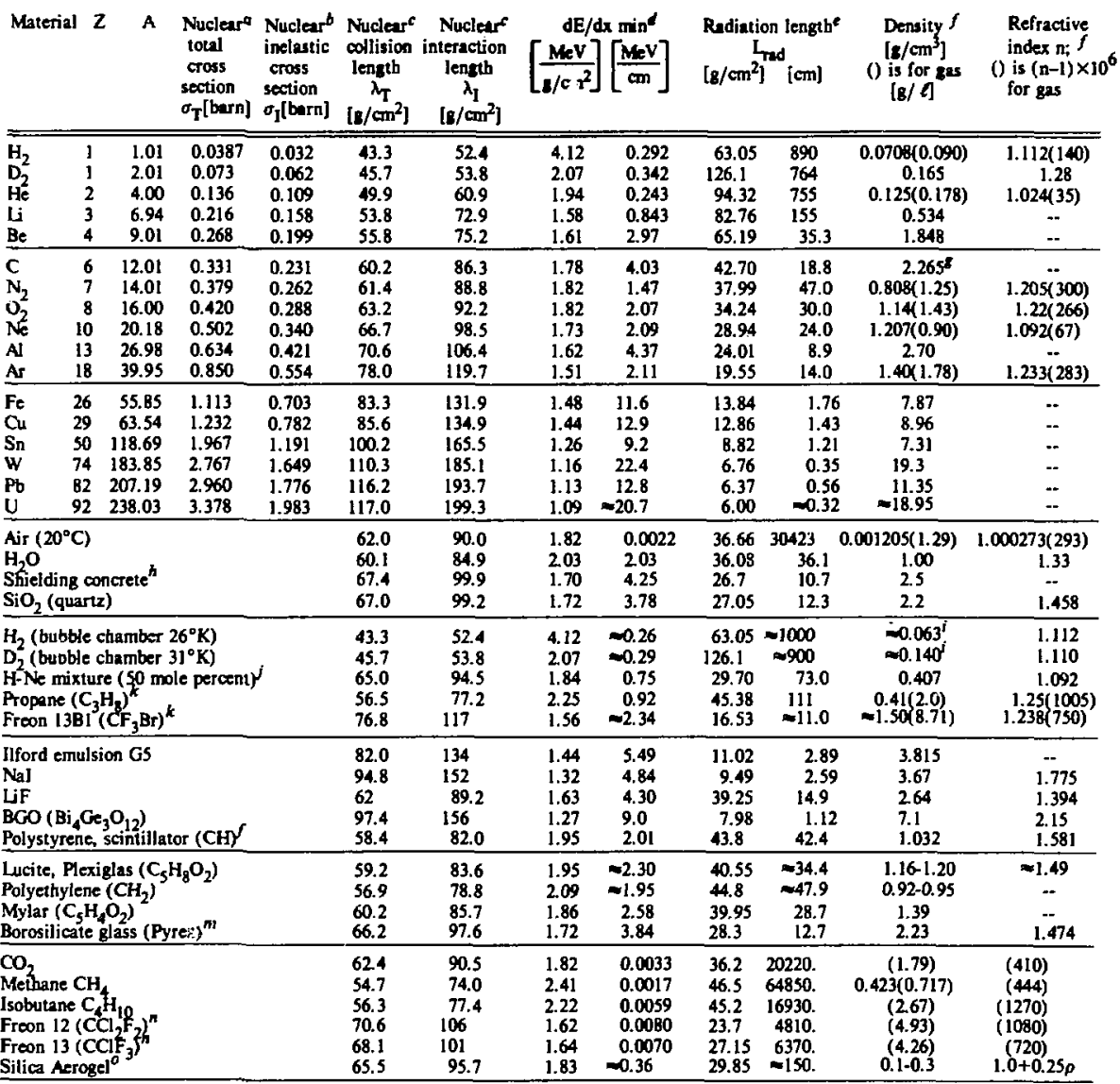

\begin{tabular}{lllllll}
\hline Spark or proportional chamber & $0.028 \%$ & $0.020 \%$ & $\ldots$ & 0.034 & $0.067 \%$ & 0.019 \\
\hline
\end{tabular}

- Table revised March 1982 by J. Engler. For details, see Report KfK BODO, Kernforschungzentrum D 75 Karlsruhe, P.O. Box 3640, Federal Republic of Germany.

a. $\sigma$ at $80-240 \mathrm{GeV}$ for neutrons ( $\sim \sigma$ for protons) from Murhy et ai., Nucl. Phys. B32, 269 (1975).

b. $\sigma_{\text {jpelatic }}=\sigma_{\text {totel }}-\sigma_{\text {elagtic }}-\sigma_{\text {funsielentic }}$ for neutrons at $60-375 \mathrm{GeV}$ from Roberts et al., Nucl. Phys., B159, 56 (1979). For protons and other particles, see Carroll et al., Phys. Lett. as, 319 (1979); note that $\sigma_{\text {f }}(p) \sim \sigma_{[}(n)$.

c. Mean free path between collision ( $\lambda T)$ or inelastic interaction $(\lambda I)$, calculated $\lambda=A /(N \times \sigma)$.

d. For minimum-ionizing protons and pions from Burkes and Berger, Tables of Energy Lasses and Ronges of Heavy Charged Particles, NASA-SP. 3013 (1964). For electrons see: Penetration of Charged Particles in Matter, NAS-NS39 (1964).

e. From Y.S. Tsai, Rev. Mod. Phys, 46, 815 (1974).

$f$. Values for solids, or the liquid phuse at boiling point. Values in parentbeses for puseous phuse STP ( $0^{\circ} \mathrm{C}, 1$ atm), except where noted. Refrac tive index given for sodium D line.

g. For pure graphite, industrial graphite density my vary $2.1-2.3 \mathrm{~s} / \mathrm{cm}^{3}$.

h. Standard shielding blocks, typical composition $\mathrm{O}, 52 \%$ Si $32.5 \% \mathrm{Ca} 6 \%$, $1.5 \%, \mathrm{Fe} 2 \%$, $4 \%$ plus reinforcing iron bars. Attenuation length $\ell=115 \pm 5 \mathrm{~B} / \mathrm{cm}^{2}$, also valid for carth (typicaf $\rho=2.15$ ) from CERN-LRL-RHEL Shielding exp., UCRL-17841 (1968).

$i$. Density may vary about $\pm 3 \%$ dependins on operating conditions.

j. Values for typical workine conditions witb $\mathrm{H}_{2}$ terget: 50 mole percent, $29^{\circ} \mathrm{K}, 7$ atm

$k$. Values for typical chumber working conditions: Propane $\sim 57^{\circ} \mathrm{C}, 8-10 \mathrm{~atm}$ Freon $13 \mathrm{BI} \sim 28^{\circ} \mathrm{C}, 8-10 \mathrm{~atm}$

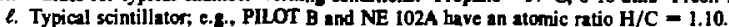

$m$. Main components: $80 \% \mathrm{SiO}_{2}+12 \% \mathrm{~B}_{2} \mathrm{O}_{3}+5 \% \mathrm{Nm}_{2} \mathrm{O}$.

n. Used in Cerenkow counters. Values at $26^{3} \mathrm{C}$ and 1 atm. Indices of refraction from E.R. Hayes et al., ANL-6916 (1964).

o. $n\left(\mathrm{SiO}_{2}\right)+2 n\left(\mathrm{H}_{2} \mathrm{O}\right)$ used in Cerenkov counters, $\rho=$ density in $/ \mathrm{cm}^{3}$. From $\mathrm{M}$. Centin et al., Nucl. Instr. Meth. 118, 177 (1974).

p. Values for typicil construction: 2 layers $50 \mu \mathrm{m} C \mathrm{Cu} / \mathrm{Be}$ wires, $8 \mathrm{~mm}$ gep, $60 \%$ argon, $40 \%$ isobutane or CO; 2 layers $50 \mathrm{\mu m} \mathrm{Mylur} / \mathrm{Aclar}$ foils. 


\section{ELECTROMAGNETIC RELATIONS}

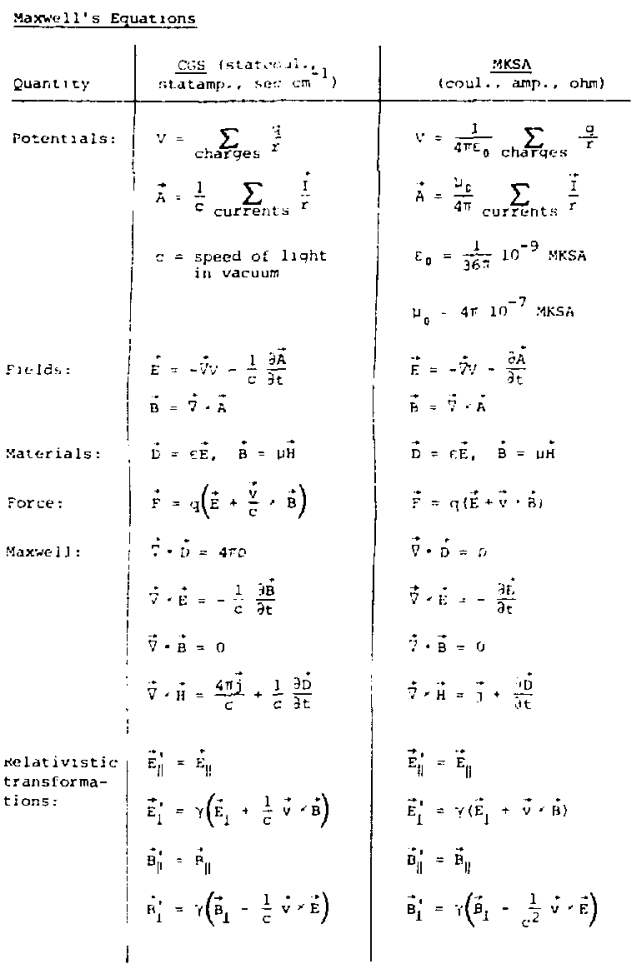

\section{Impedances: Alternating Currents (MKSA)}

Ohm's law: $v=z T, \quad v=v_{0} e^{i \omega t}$

1. impedance of self-inductance $1: \quad z=i \omega L$.

2. Impedarce of a capacitor of capacitance $c=z=\frac{1}{i \omega C}$.

3. Impedance of a flat conductor of width $w$ at high frequency: $z=\frac{11+i 11}{x}$

$$
\begin{aligned}
& \text { c. = resistivicy in } 30^{-\mathrm{k}} \mathrm{ma} \\
& \sim 1.7 \text { for cu } \\
& -2.4 \text { for ru } \\
& -2.8 \text { for } A 1 \\
& \text { (AI alloys may have up } \\
& \text { to dcubja ihis value. } \\
& \{=\text { effective skift dejiti. }
\end{aligned}
$$

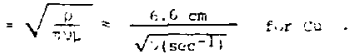

4. Im:tedance of fret spaze: $z=\sqrt{w, / t},=47,7 \ldots$

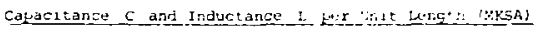

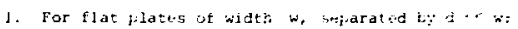

$$
c=\frac{E}{d} ; \quad i=-\frac{a}{i}
$$

2. For coax cakle of intertor and axteyse sath $r_{1}$ and $z_{2}$ :

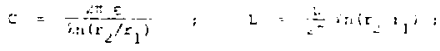

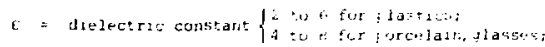

$$
\begin{aligned}
& \text { - = malnetac susceptibl! } 1 \text { \%. }
\end{aligned}
$$

Transmissio: Lintis (No Lons) (Mrsin)

Velocity $=1 / \sqrt{i . K}=1 / 2 . . .5$.

Impedance $=\sqrt{\mathrm{L} / \mathrm{C}}$.

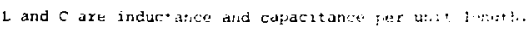

Synchrotrom Raduaring: (C:S).

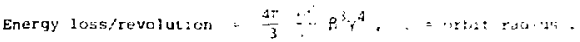

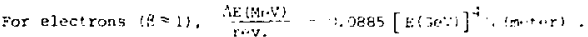

Critical frequency: $\omega_{c} \quad 3 r^{3}:$.

Frequency spectrun (for if $\because 1)$ :

$$
\begin{aligned}
& I(\omega) \equiv 3.3 \frac{E^{2}}{c}\left(\frac{\alpha b}{6}\right)^{1 / 3}, \omega \cdots \omega_{c}:
\end{aligned}
$$

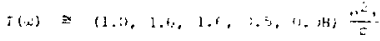

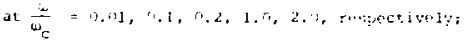

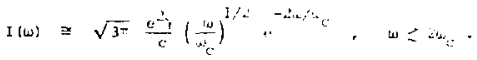

$$
\begin{aligned}
& \text { The radiation } 15 \text { confined to anyles } \& \text { Lh mlatuve to } \\
& \text { the instantaneous direction of motion. }
\end{aligned}
$$

See J. D. Jackson, Classical Electrodynamics, atd ickituon (John wiley s Sons, New York, 1975 ) for mori formular and detalls. (Prepared April 1974; revised April 1990.)

\section{RADIOACTIVITY AND RADIATION PROTECTION}

Unit of activity = Curie:

$1 \mathrm{Ci}=3.7 \times 10^{10}$ disintegrations $/ \mathrm{sec}$

Linit of exposure dose for $x$ and $y$ radiation = Roentgen: $1 \mathrm{R}=1 \mathrm{esu} / \mathrm{cm}^{3}=87.8 \mathrm{erg} / \mathrm{g}\left(5.49 \times 10^{7} \mathrm{MeV} / \mathrm{g}\right)$ of air Unit of absorbed dose $=$ rad

i rad $=100 \mathrm{erg} / \mathrm{g}\left\langle 6.25 \cdot 10^{7} \mathrm{MeV} / \mathrm{g}\right\}$ in any material Unit of dose equivalent (for protection) $=5 \mathrm{em}$ : rems (Roenteen equivalents for man) = rads $\times \mathrm{QF}$, where QF (quality factor) depends upon the type of radiation and other factors. For y rays and $\mathrm{HE}$ protons, $Q F=1$; for thermal neutrons, $Q F=3$; for fast neutrons, $Q F$ ranges uP to 10; and for a particles and heavy ions, $Q F$ ranges up to 20.
Maxinumpermissible occupational dose fur the whole body: 5 rem/year (maximum 3 rem/calerdar quarter)

Fluxes (per $\mathrm{cm}^{2}$ ) to liberate 1 rad in carbon:

$3.5 \times 10^{7}$ ninimuin ionizing singly charged particles $1.0 \times 109$ photons of $1 \mathrm{MeV}$ ene $\mathrm{fgy}$

(These fluxes are correct to within a factor of 2 for all materials. 1

Natural background: 120 tu 130 millirem, year cosmic radiation (ciarged particles + neutronut -251 cosmic radiation (Y rays)

costiation from cock and air (y ray $-2512$ 
PERIODIC TABLE OF THE ELEMENTS

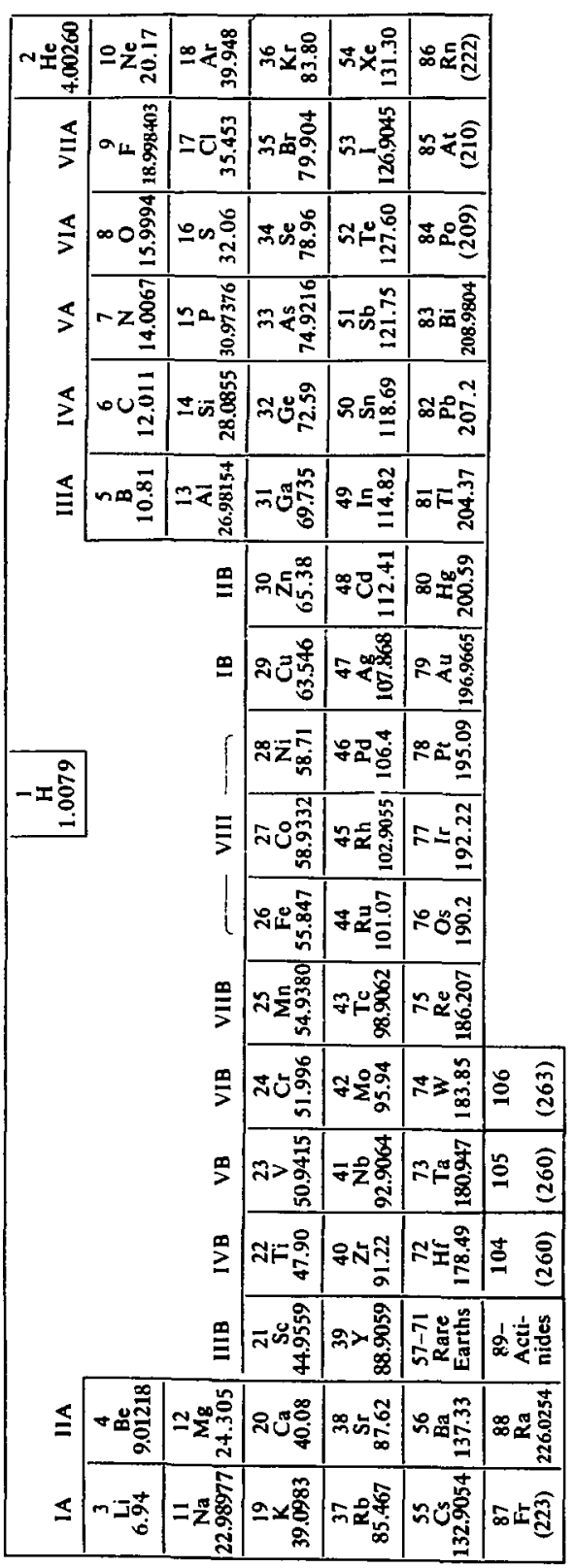

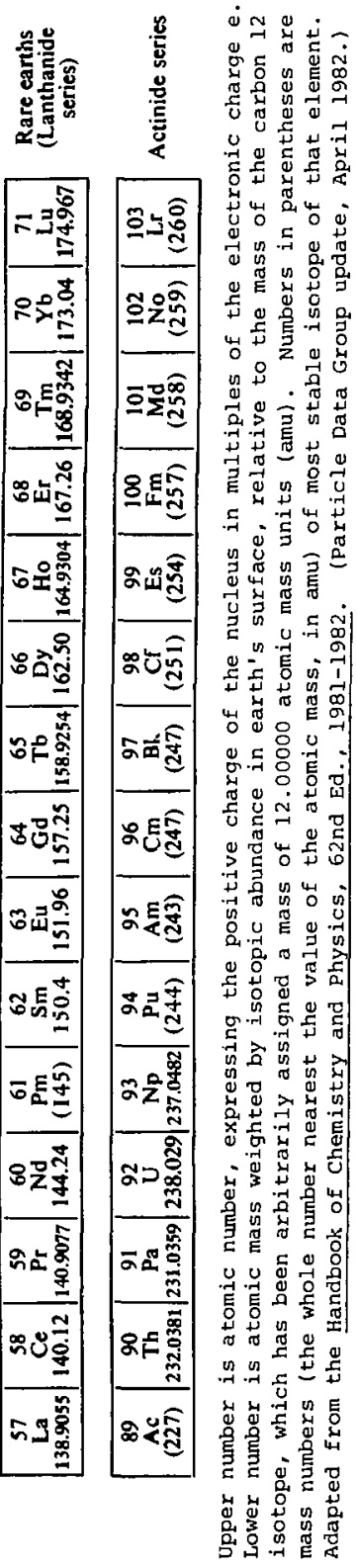




\section{PLOTS OF CROSS SECTIONS AND RELATED QUANTITIES}

NOTF: THE FIGURES IN THIS SECTION ARE INTENDED TO SHOW THE "BEST" OR "MOST REPRESENTATIVE" DATA IN THE OPIHION OF THE COMPILER. THEY ARE NOT MECESSARILY COMPLETE COMPILATIONS OF ALL THE WORLD'S RELIR.BLE DATA.
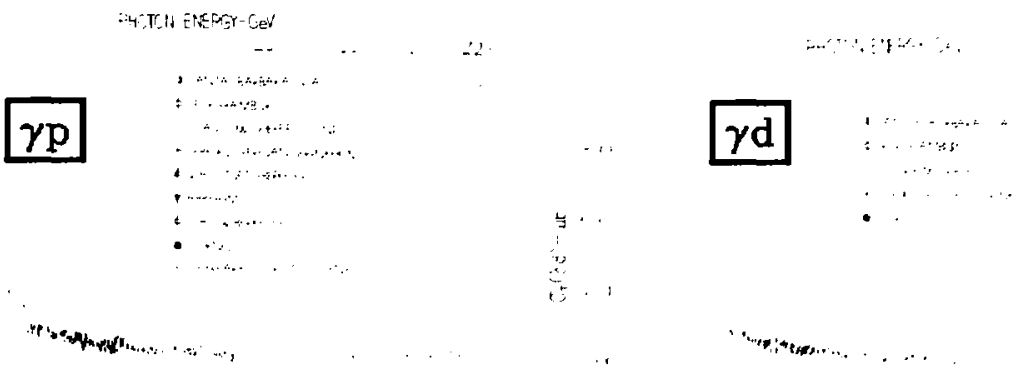

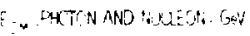

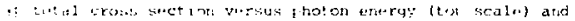

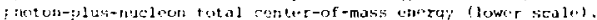

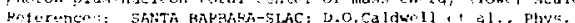

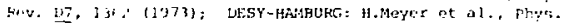

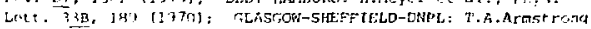

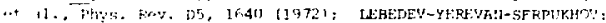

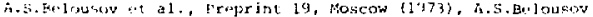
..t al.

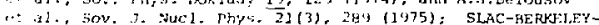

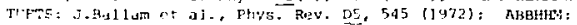

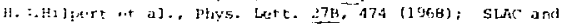

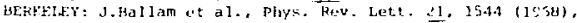

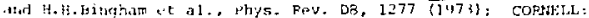

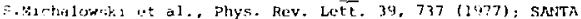

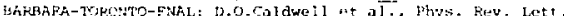

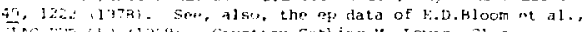

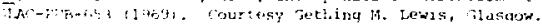

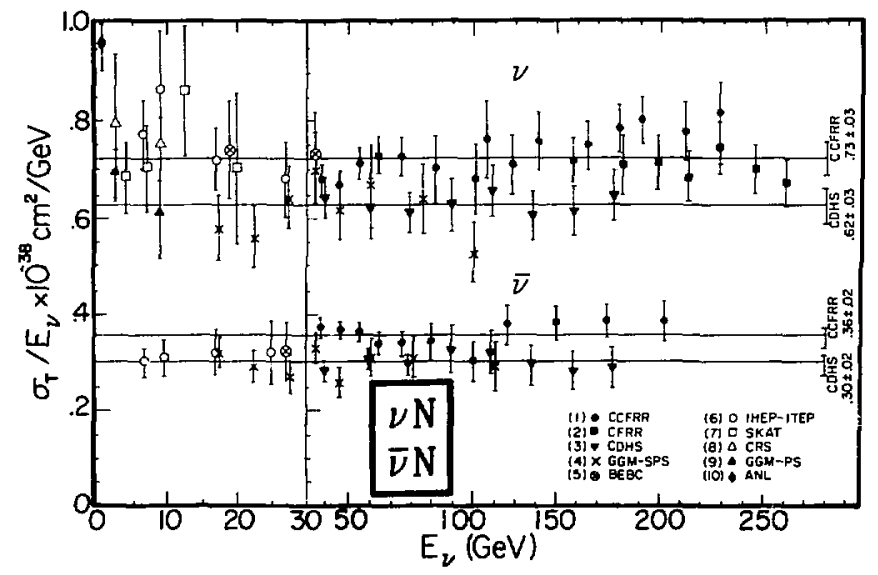

${ }_{T} / E$ for the muon neutrino and antineutrino charged-curtent total crass section as a function of neutrino energy. The irror bars inclure both statistical and systematic errors. The straight lines are averages for the CCrRR and CDHS m.casurements. References: (1) R. Blair et al., in Froc. of Neucrino 'B1, univ. = Hawais (1981): (2) James Roy Lee, Ph.D. Thesis, Caltech (1981), "Measurements of WN Chargei Current Cross $5 e c+$ :.s from Eu=2s GeV to Eh= $260 \mathrm{GuV}, "$ (3) J. G. H. le Groot et al., Zeit. fur Physik C - Particles and Fields 1. 143 (1979): (d) J. Morfin ec al., Ptys. Lett. 104B, 2 ?5 (1981); (5) D. C. Colley et al., Zeit. fur Physik C - particles and Fields 2 , 187 11979); (G) A. s. Vovenko et al., Sov. J. Nuc1. Phys. 30, 527 (1979); (7) D. s. Baranovet al., Phys. Lett. 81日. 255 (19791: (A) C. Baltay et a1., phys. Rev. Lett. 44, 916 (1980): (9) $\mathrm{S}$. Ciampolillo et al.. Phys. Lett. g4B. 281 (1979); a 21 5. J. Barish et al., Phys. Rev. D19, 2521 (1979). Courtesy M. Shaevitz, sevis Labozatory. 
PLOTS OF CROSS SECTIONS AND RELATED QUANTITIES (Cont'd)

\section{Structure Functions}
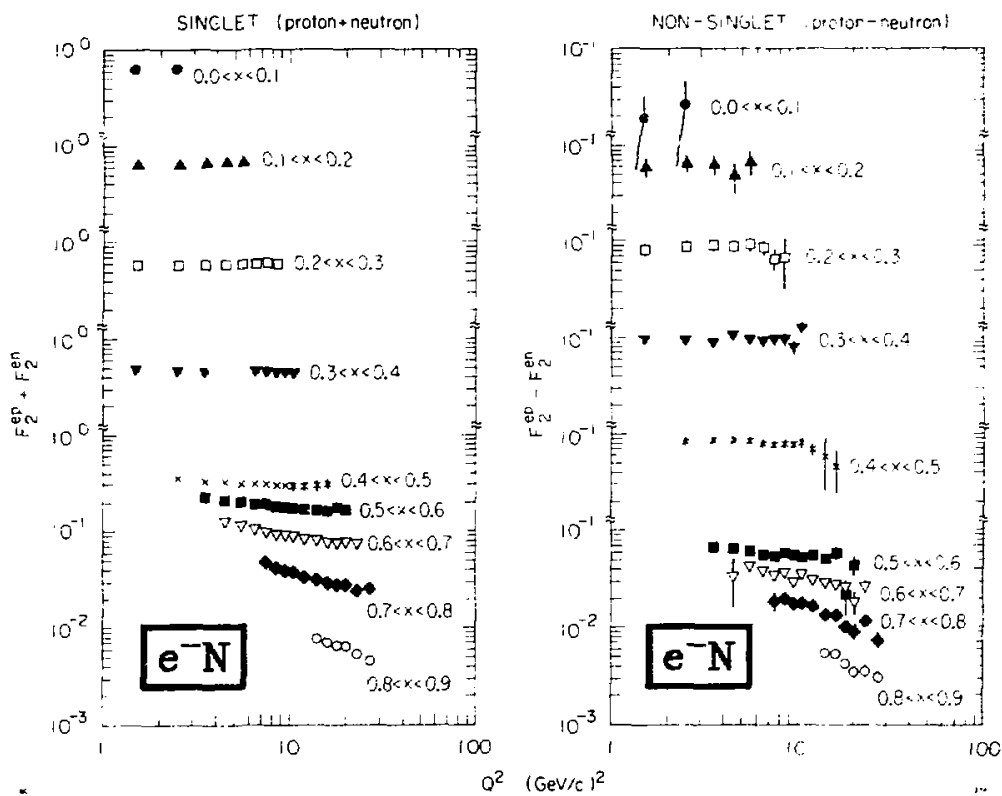

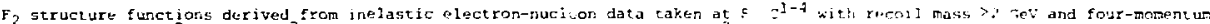
transfer squared $Q^{2}>1$ (Ge:/c) ${ }^{2}$ are show. Fot detinitions of $F_{2}$, $x_{1}$ and $Q^{2}$. see the "Pelat $1 v i s t i r$ Finematzcs" section vid the "Weak Interactions of Quarks and Leptons" section. $R \equiv \sigma_{\mathrm{V}} / \sigma_{\mathrm{T}}=0.21$ was assumed. Si'stemetic errors are compirable in size to the data point 5 mbols. Cotrections for nucleon merion in deuterium have been made. afese corrections are small except for $x>$ a.7.

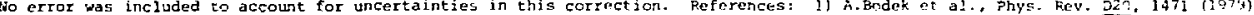
2) w.B.Atwood, SLAC Report No. I8S (1975); 3) M.D.Hestayer, sLAC Report No. 214 (1978); 4) 5.5tein et al., Fhys. Rev. D]2, 186; 1975). Courtesy w. B. Atwood, SLAC.
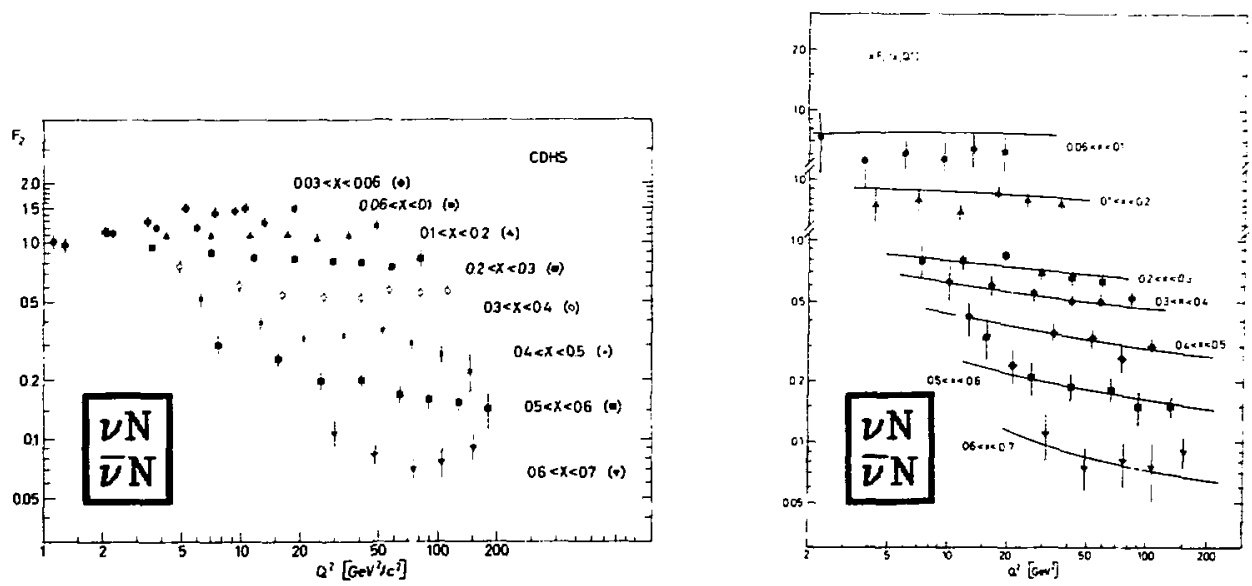

Nucleon structure functions as measurud by the ct.ys collaboration in high energy (30 200 iev) charged-current neutrino- and antinev-rino-nucleon scattering [J.G.H.de Groot et al., Z. Physik C - Patticles and Fields 1, 143 1]979); reproduced by permission]. Definitions, and a discussion of the significance of these structure functions, may be found in the above reference, and also in the "Weak Interactiong of Quarks and leptons" sectlan of the present work. See ce Groot et al. . [or a discussion of experimental detalla, including corrections, etc. Curves are bared on a oCD parametrization of Buras and Gaemers [:tucl. Phys. B132. 249 (1978)]. 


\section{PLOTS OF CROSS SECTIONS AND RELATED QUANTITIES (Cont'd)}
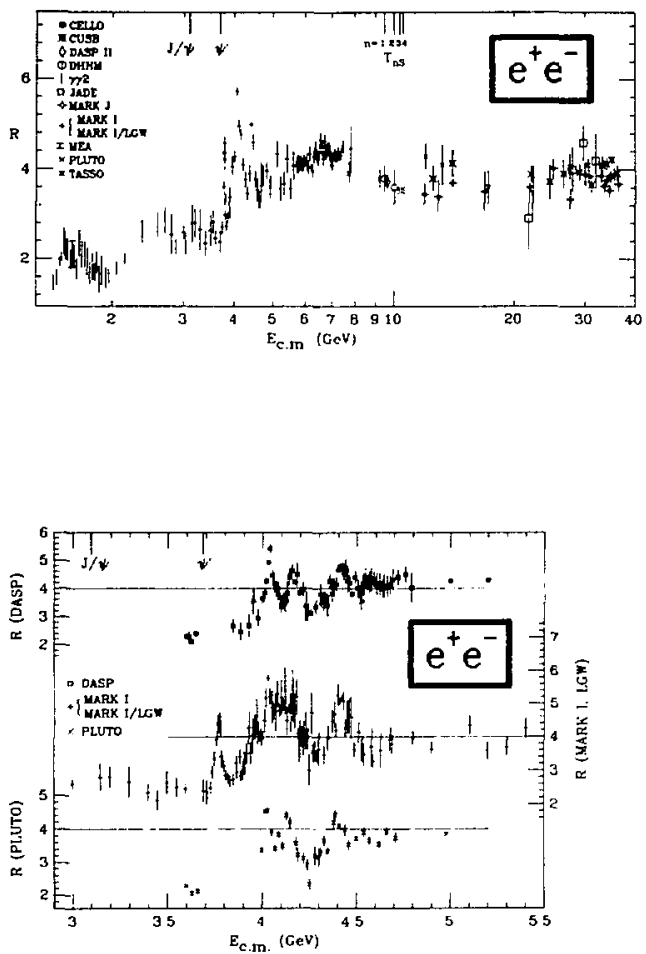

Heasu: ment of dnnth:ation proseseds via on ptotom. The denominator is a calculdesd quantary:

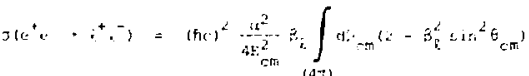

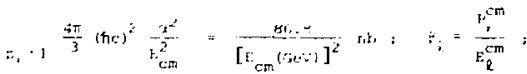

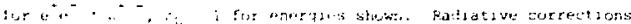

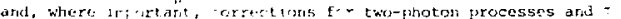

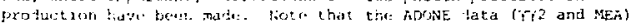

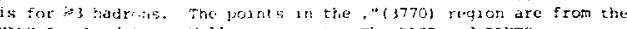

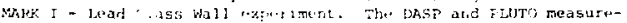

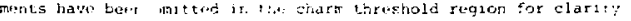

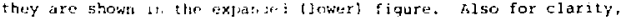

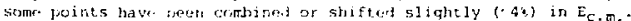
systematac normalization corros are thot illoluded; they rango from

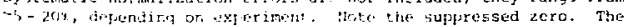

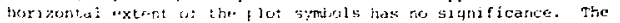

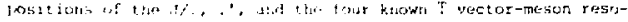

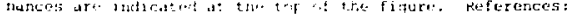

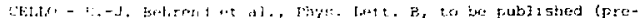

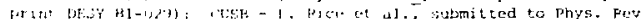

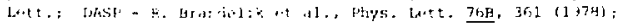

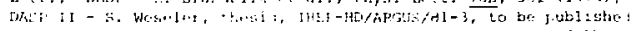

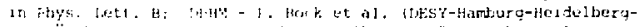

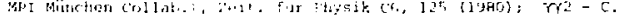

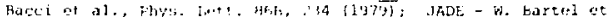

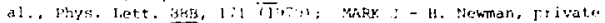

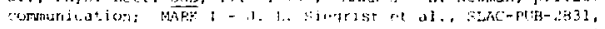

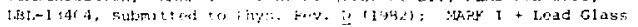

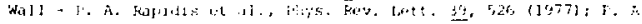

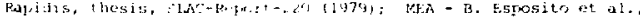

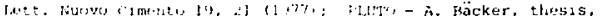

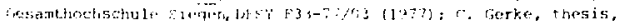

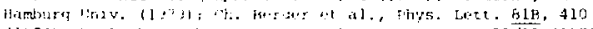

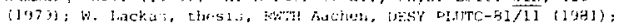

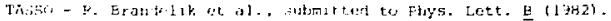

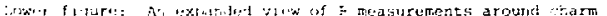

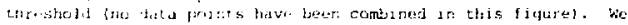

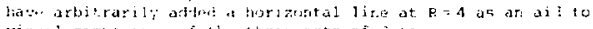

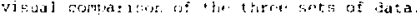

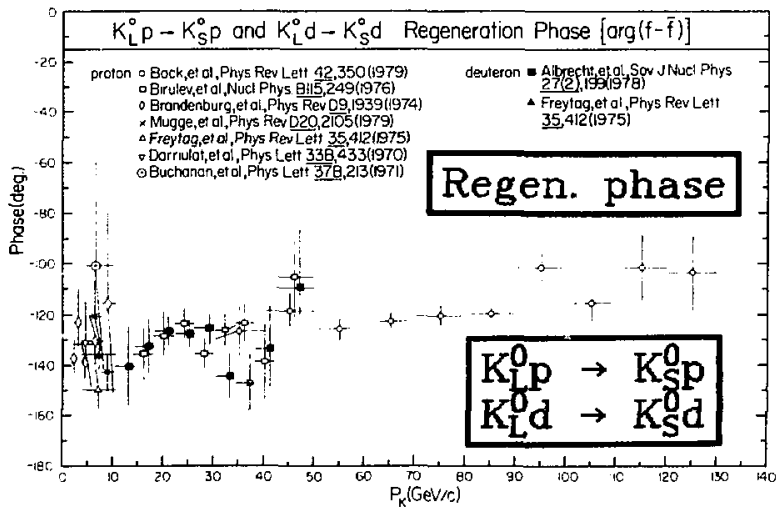

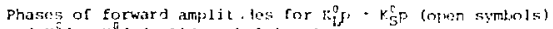

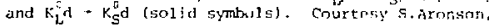
Brookhuven Nitional Laboratory. 
PLOTS OF CROSS SECTIONS AND RELATED QUANTITIES (Cont'd)

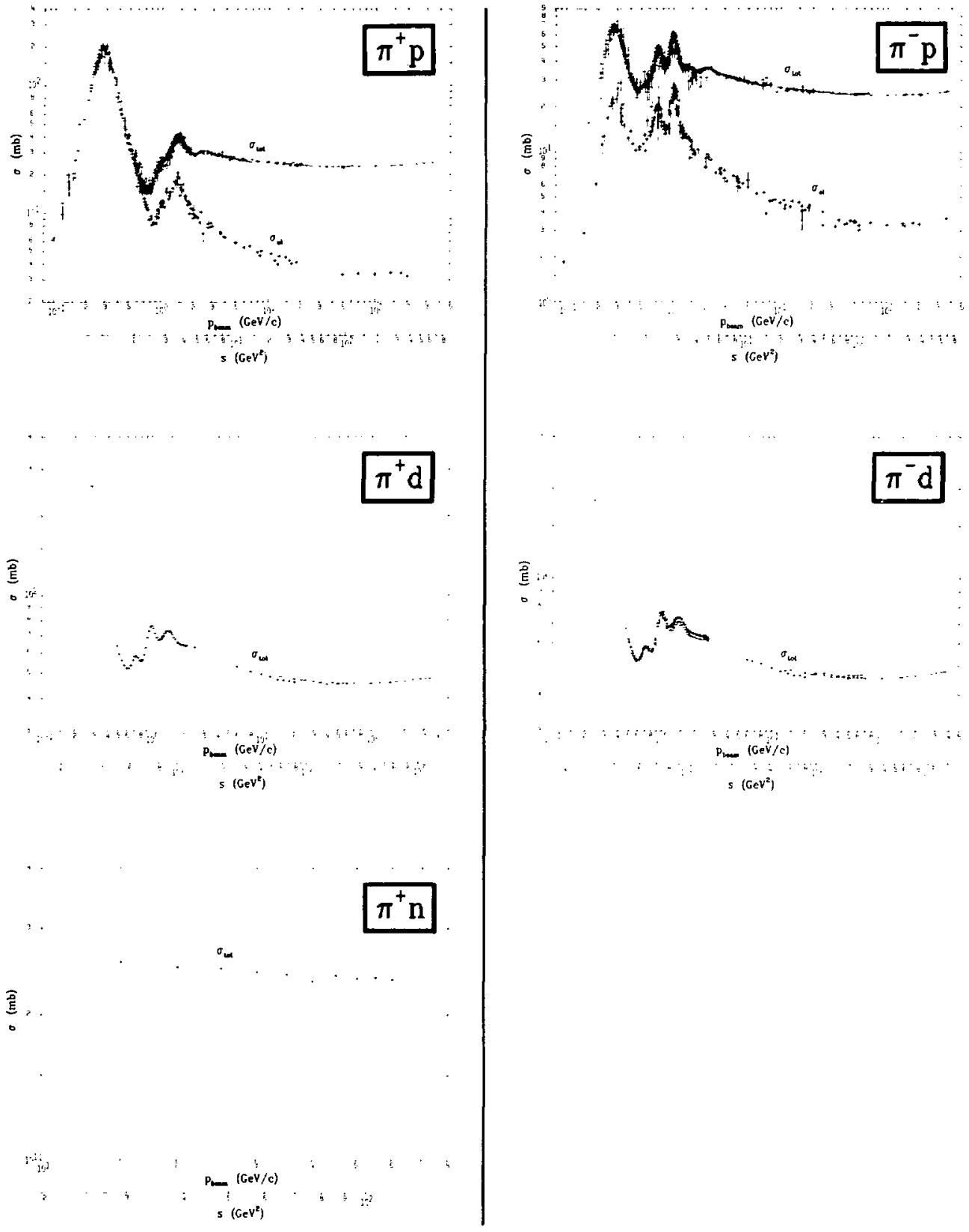


PLOTS OF CROSS SECTIONS AND RELATED QUANTITIES (Cont'd)

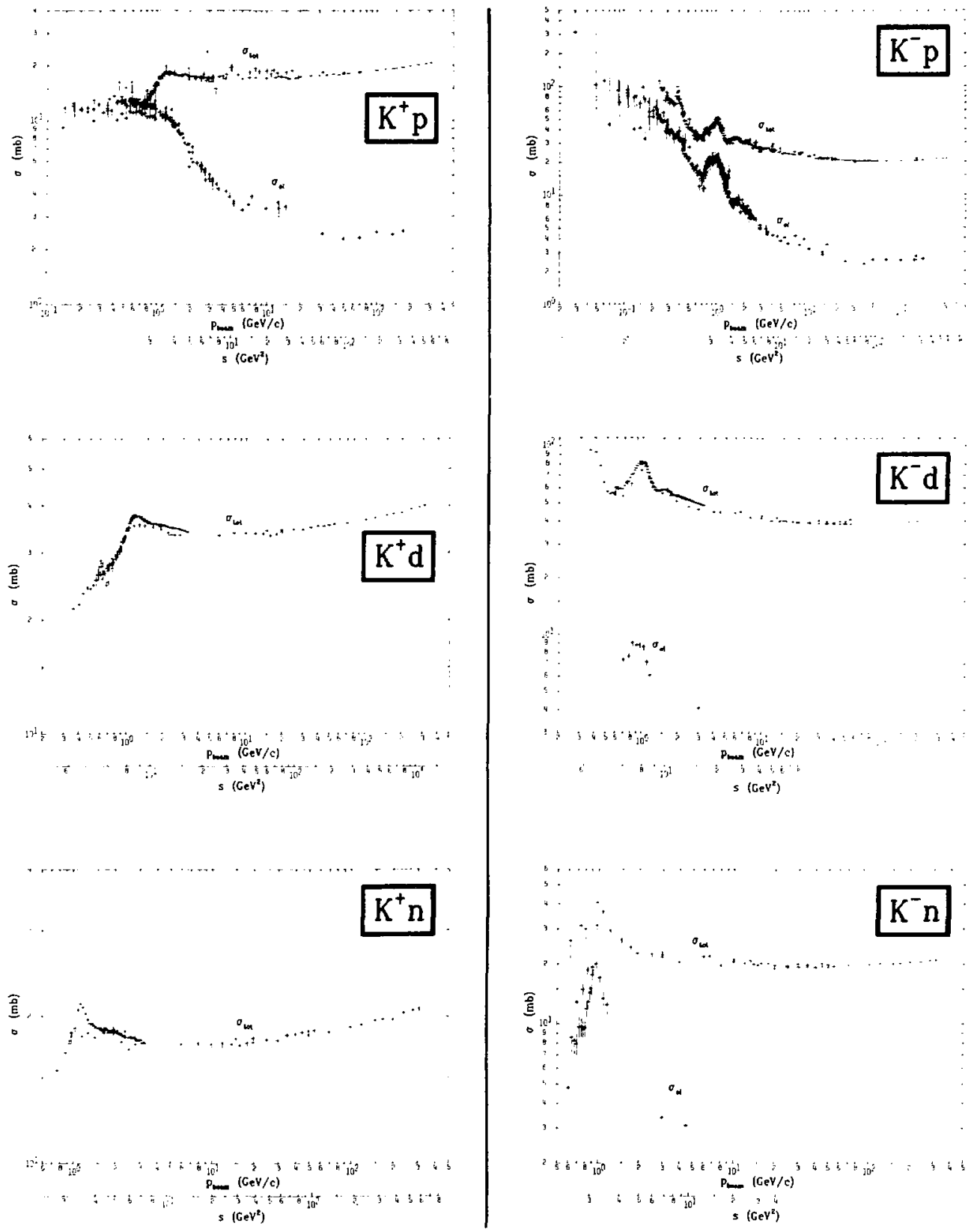


PLOTS OF CROSS SECTIONS AND RELATED QUANTITIES (Cont'd)

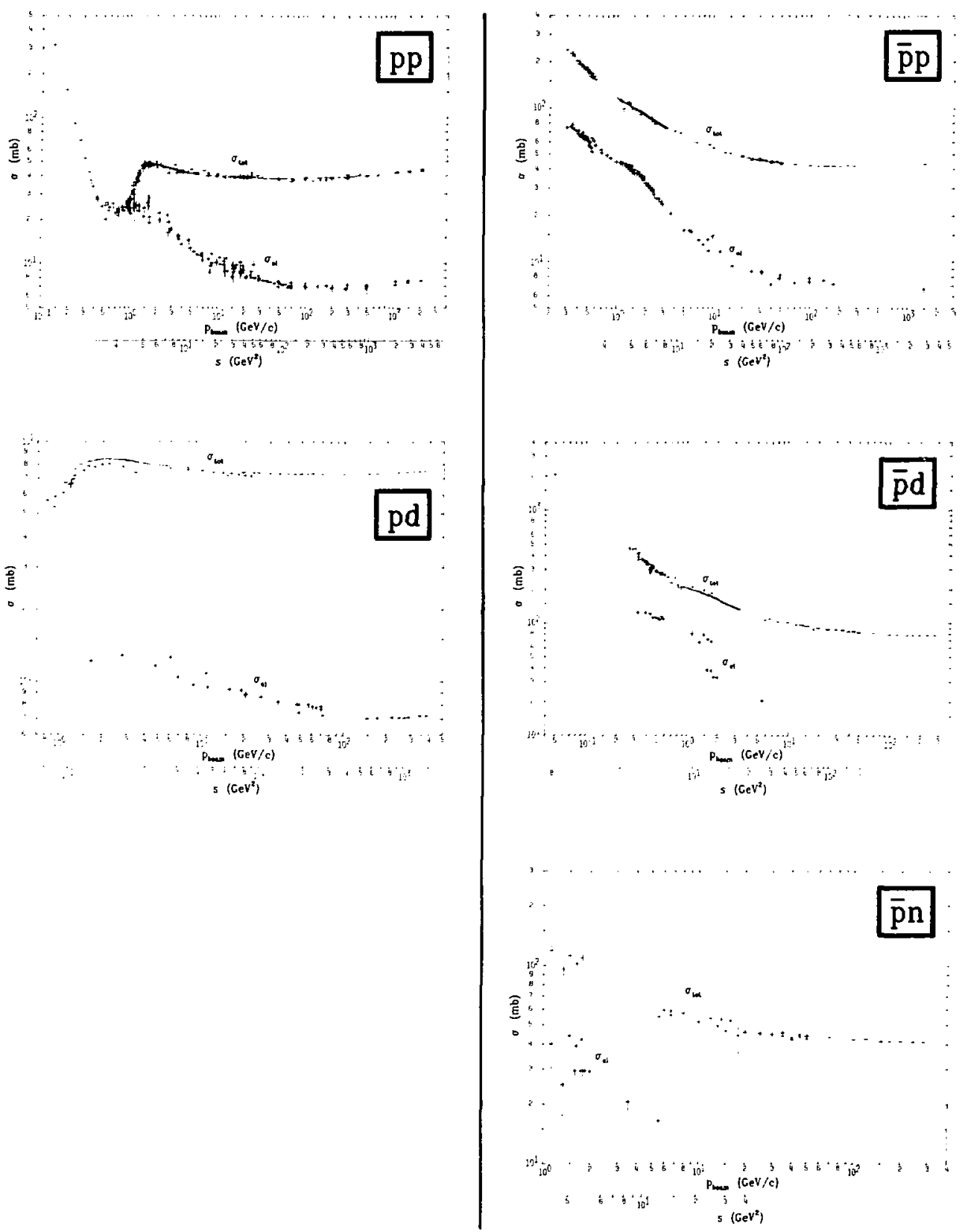

Hadranic total and glastic cross sections vs. laboratory bean momentum, Pbeam, and center-of-mass enerqy squared, s. Figures courtesy v. Flaminio, W. G. Moorhead, and D. B. O. Morrison, CERN. 


\section{DATA CARD LISTINGS}

\section{Illustrative Key}

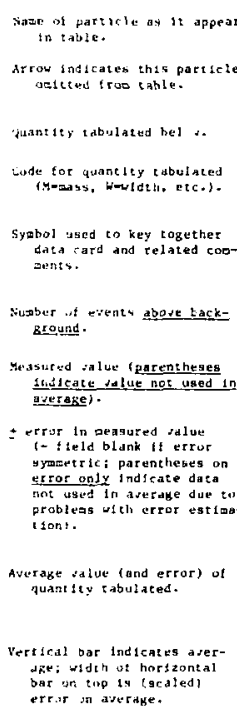

Vhlue and errar tor each experteerat.

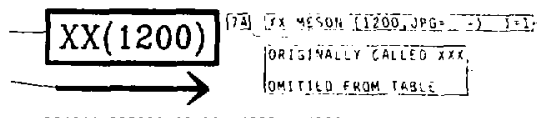

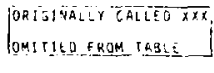

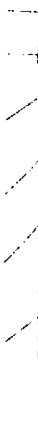

些

9 [xrg1200i MASS ME V]

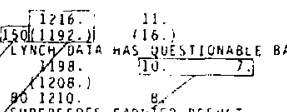

IMERRIi

LYACH - 60

CKGROUNO SUETHACITUH

OIEREE

$O A A S P E-2.1<-D$

$69 \div \mathrm{C}$

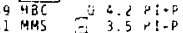

SOPERSEES EARETER HESUI:

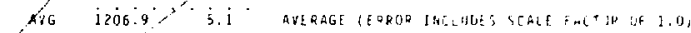

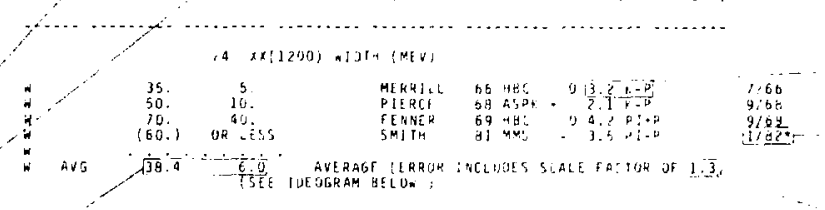

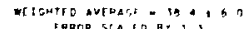

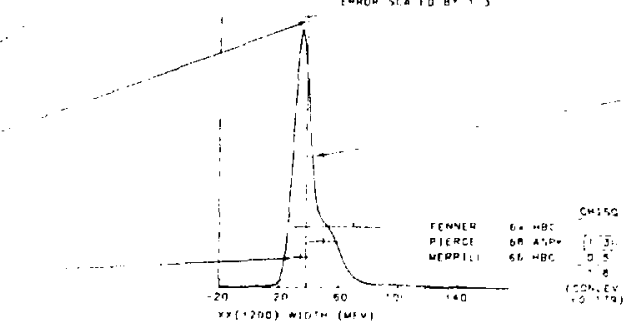

ia $x \times(1 \% 00)$ PARTLAL UECAR MUDE:

Partial decay mode thateled by
$P_{1}$ i.

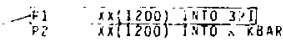

$\cdots+$
Particle aetar, and quantio nuebers lif knowit.

Parucle code ifor listerial use ontyl.

General extousts on particie.

Abbrejated xoterence for thas result; fuld reference diten belins.

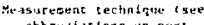
abbreilat tors un next Fafe).

Changutsh of patrherers derected.

Nedrt tan producting particle, ut conernty,

bat this regnit enlered latewelsk indicates cesult adqed of changed since preit aus Edition!

Sonle tartar a lindlates toronsistent fala.

Ideagram to displey inerss19tets: data: curta 1s xus of ciaussians, one for each experiseth farea of thusstan - Mespari uldeth Gausslati - \pm error'.
Branchlng rat io (2abeled by $\left.R_{3}\right)$.

Value land exrorl of quantity cabulated, as determined tene constraiaed tit (uf lno a1
rarlog for for this particles)

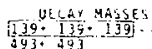

74 (XIIZUO) BRANCHING RATLOS
Hapresencatice masyes of docay produlte lused for Edlcu-

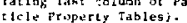

Canczibueturn at expertwent co $t^{2}$ (11 hir entey present, culatink $x^{2}$ or araje tactor oecative ai serg latge
Branching catio H in terma of plons $p_{1}$ aboje.
Refurences, itsted by year,

Abbrevlated taterence fort uurnat, report. preprint. ecc. (see tbbrefitions next peges.

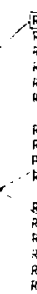

nuttior(s).

Quincum number deterbinotions in this reference

Institutionisy of author(s) (see abbrevtations an nex: page).

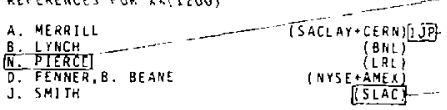

SHI TH 69 BL 618 B 372

J. $5 M] I H$

Linacy

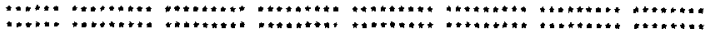




\section{Illustrative Key (cont'd)}

\section{Abbreviations}

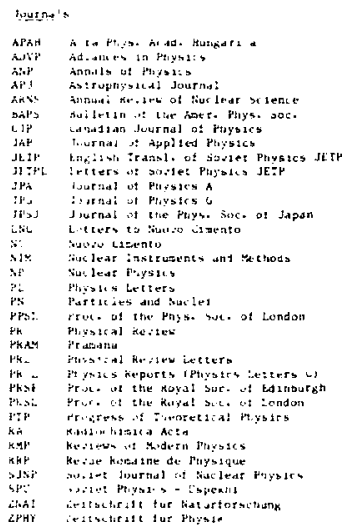

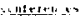

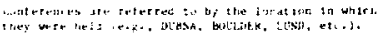

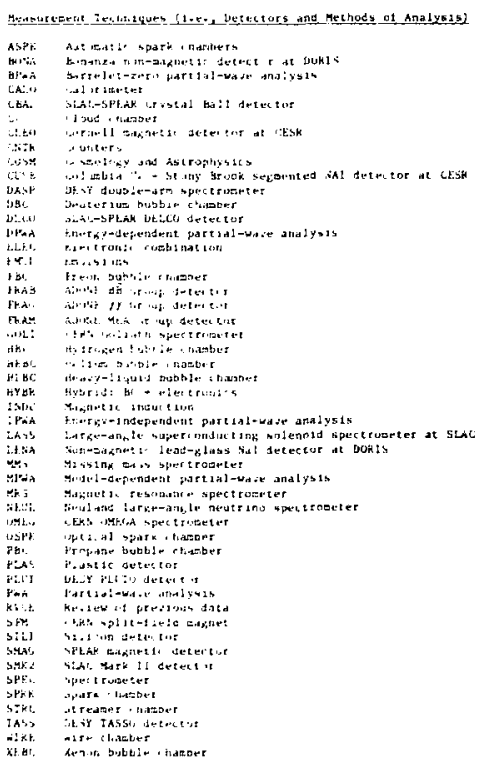

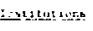

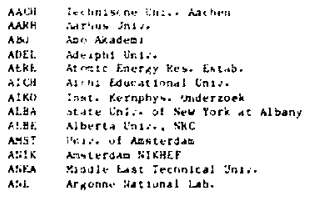

Aschen, Gerenay

Abo, Finl and

Garden C1Ey. H. Y.. USh

Harue11, Batks., Eng 1 and

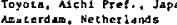

Alus Lerd an. We ther 1

Albany, N. Y.. U5A

Anstersas. Hetherlands

Aesterd um. Metheriands

Ankars, Turkey
Axpuane, ill., USA
Inazizue Lons [Cant"d]

Al12 Un1we of Aritona
AhLS Arizutas State binis.

ATER Sucleat Rey. Centeri berohritos

ATHU Unt 2 of Achun

Auct Un1\% ot Auckiand

bakc Un1s. Et Barcelon

Baik1 Inti. de bart

GART dortol de-sed
BASL base: Unte

EEDF bedord collene

ise 11 hubs:
unt? of bergen

last. Hachenergieptys. Daw

Un1 2 sern

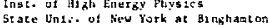

Ht minghar Uni

Hrooktiages Natiunat Lab.

Yisels boir lnst.

3ol he State

intu. de mordealux

hess ton tina...

brandets Uisi.

Unti. of Britisit Collutibis at Histol H. H. Hilis

Uani:. Libre de ar uke:tes

Avicharest State Vint.

Lettral Hevedreh Inst. D! Physich

lnat. des Hautes Etutes Sc 1 .

Lub. de Pliza. Corpusculatre

liarack le Inar. of Woshington

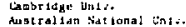

corlezwn bind:

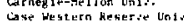

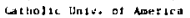

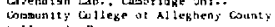

willege de France

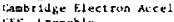

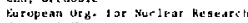

Jris. of disidgo

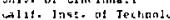

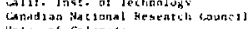

intu of bolurto

is luebra Unin.

wlerdo be ate lint.

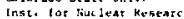

cecy lins... at Nev rotk

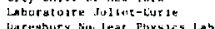

baresbury tiuclear Playses Lab.

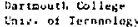

thas of Iernn

Wiutscher biektrunen-bynchiratiron

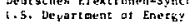

Un 1 .. vue taund

Duke Uni:
Un 1 .. of Durnat

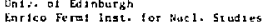

El miture int leke

Eeole Polytechna que

U:1: Eriangent-kiurnbers

suise Federal inst. of teinnol."ige

Univ. di Firenze

Fisk thill

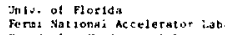

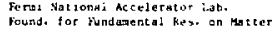
Found. Far hondarental Res.

Unit. of Frelburs

Hortad state ton

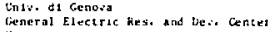
Unive de Gernede

Unise of cilasikou

Una.. Gtaz Ceolinglcal suriey of cinats

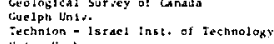

Univ. He thetury

intire of Hawall

thas. Hetdelbets

Helsingin Yilopitato

Hiroshled Unly.

Lini? of Houstun
inst. for ad ranced St wy

inst. for ad ranced st wy
intersiat 1 linal bue breas wachiciea

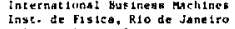

Iilinets inot. of Tect.

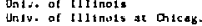

Inat. Lave-Langevin

Unty. of teidana
thys, Inst. Da1v. Innebruck

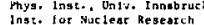

lnst. lot Nuedear kesertch

Uniw. of tewa

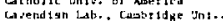

mintersicy collek

Eebrew th1se

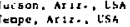

At hene, Grecere

Aurzland, Niece zeol and

Ra reciona. Spath

Swart limure, , pa., UbA

Gabel, Svicterland

Lenditi, Enx linns

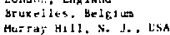

Eareen, koruaj, oDk

ceithen sertin, ob

bo iub: sut it italy

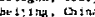

Ba righaton, S. Y. U.A

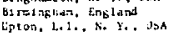

wiptenhogent, Jermar

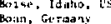

thom, Greas ny

Hoinzen, Miss... UT 5 A

bristri; tirnland
providence. H. I., lisa

Hruxelles, gelglus

buenarest, humanta

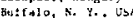

butes-gut-Yintie, Fratre

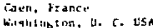

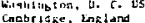

Conbriage, kasbland

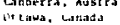

Patesburgh. Pr... USA

cisio

enszidge. England

patckburgh, Penti. USA

Parise Prance

Ganoble, Fianc:

uetera, Sultertand

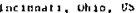

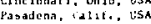

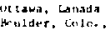

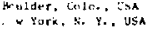

itlider, in Y. Y. USA

Fort coiltrk, Lolo. USA

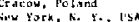

aris, France

Cin reabury, Fnpiand

that rave t. K. H. . LSA

De: $: 1$. Nir theriand

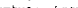

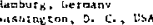

Bertmund, terermany

Gurtata, s. C., cisa

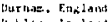

Dubitr, irelant

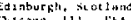

Mizcogia. III.. L'sA

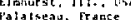

bz laneen, feerangy

zurteh, Swaterrland

Firenze, leaty

Sastivalie, terno. lest

Gatnadile, Fla.. L'SA

sactran. Tlit. ubs

Freacali, itaiy

Frelburk, wermany

schenec iady. s. Y. W USA

Genczed, Suz Lxerland

Graz, Aestria

Gurava, Comada

halla, torael

Hanbuq h. Cermuny

Lobaridge, Moss.., USA

Honolulu. Hasalt. JaA

Heidelberk. Geranty

Heisinik1, Finland

Hirush1ma, Japhn

Houstun, Texab, USA

Princetor, N. J., USA
Palo Alto, Calit. USA

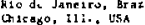

Urbara, :11., USA

Chreabo, 111., USA

Grenoble, France

bl ooningtan, Ind., USA

thasbruck, Austry

tokyo. Japon

Tokyo, Japan
Loua CLty. Loua, USA

IOWA 


\section{Illustrative Key (cont'd)}

\section{Abbreviations (cont'd)}

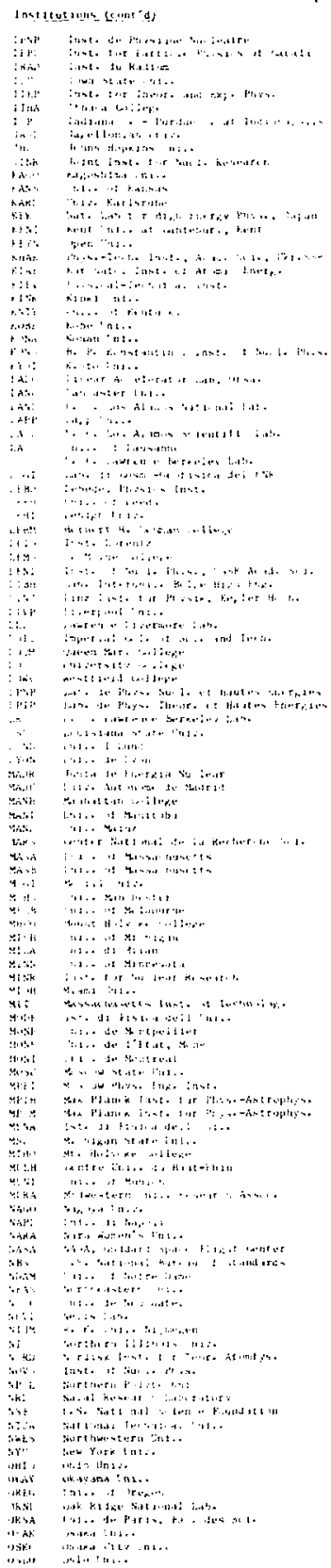

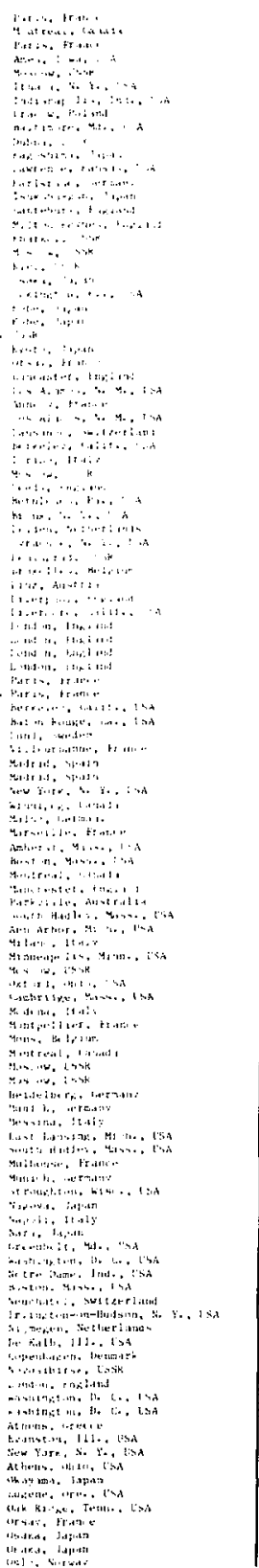

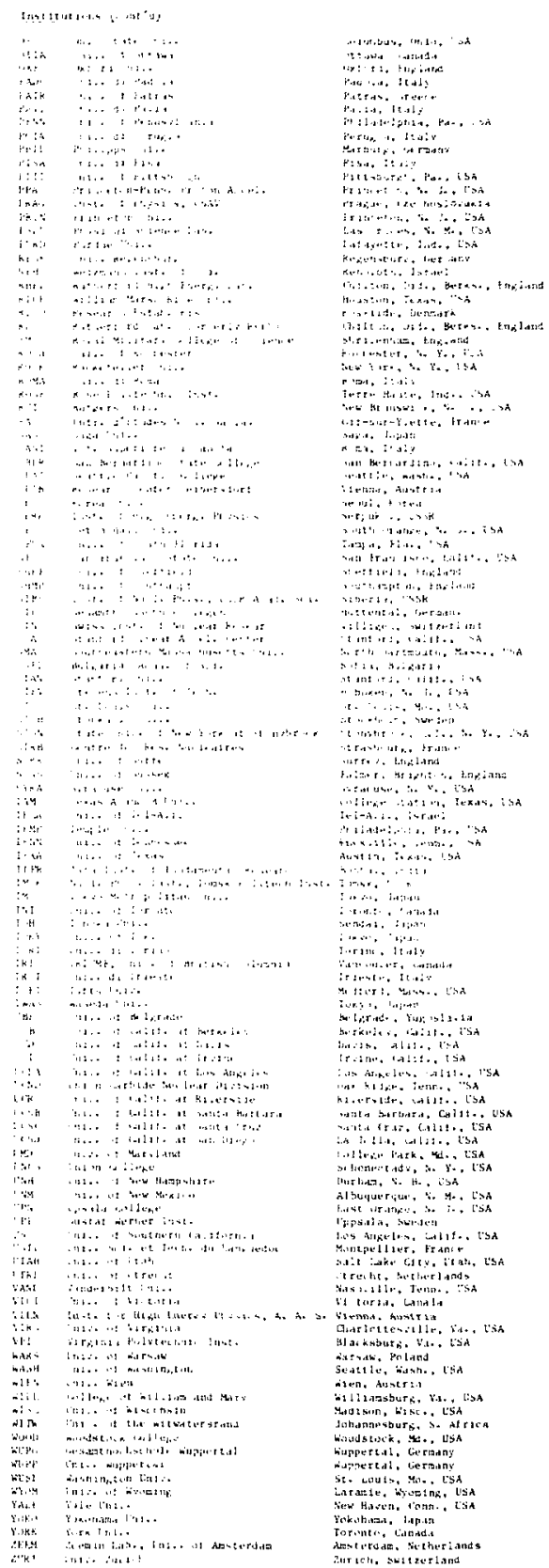




\section{Stable Particles}

$\gamma, \nu$

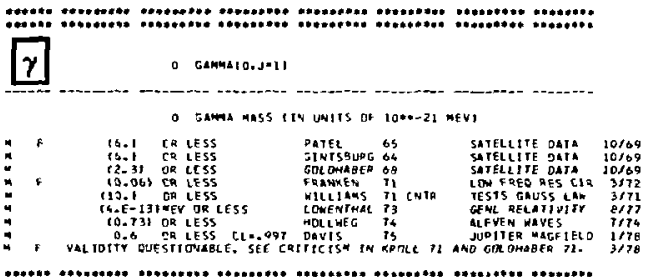

REFERENCES FOP GAMMA

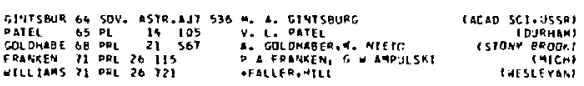

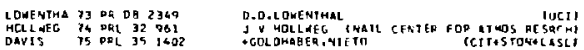

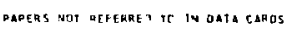

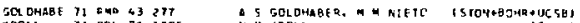

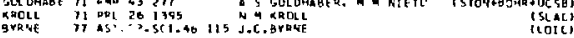

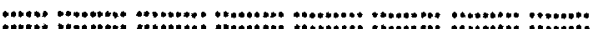

\section{Neutrinos}

(by R. E. Shrock, State Univ. of New York, Stony Brook)

With this issue the section on neutrino properties has been expanded and reorganized. As before, there are listings which deal specifically with $v_{e}, v_{\mu}$, and $v_{T}$. In addition, in the category of searches near the end of the Stable Particle Listings, we include sections which deal with correlated bounds on neutrino masses and lepton mixing but which do not pertain to any one weak eigenstate individually. Furthermore, we include constraints from cosmological and astrophysical data. (Since this Review is a compendium of particle properties, traditionally derived more or less directly from particle and nuclear physics, we treat astrophysical data on a different footing from particle physics data and have been somewhat less comprehensive in our coverage of the former,

In contrast to the other particles in this Review, the neutrinos $v_{e}, v_{H}$, and $v_{T}$ are defined as weak eigenstates (that is, states which couple weakly with unit strength to $e, U$, and $T$ ) and are not, in general, states of definite mass. In the conventional case, where all neutrinos were assumed to be massless and hence degenerate, it was possible to define the weak eigenstates to be simultaneously mass eigenstates. However, in the

\section{Data Card Listings}

For notation, see key at front of Listings. general case of massive (non-degenerate) neutrinos, the weak eigenstates have no well-defined masses, but instead are 1 inear combinations of mass eigenstates. Thus, if one considers this general case, as, of course, one does in quoting mass limits, it is inconsistent to assume that the weak and mass eigenstates coincide. Let us denote the charged leptons as the set $\left[l_{a}\right\}, a=$ $1, \ldots, n$, whece $n \geq 3$ is the number of generations, with $l_{1} \equiv e, \ell_{2} \equiv \mu$, and $l_{3} \equiv \tau$. In the standard $S u(2){ }_{[} \times U(1)$ electroweak theory ${ }^{1}$ the mixing of the left-handed components of the mass eigenstates $\left(u_{j}\right)_{L}$ to form the weak gauge-group eigenstates ( $\left.v_{Z_{a}}\right)_{L}$ is specified by the transformation

$$
\left(v_{\ell_{a}}\right)_{L}=\sum_{j=1}^{n} u_{a j}\left(v_{j}\right)_{L}
$$

where $U^{+}=U^{-1}$. IIn the case of Dirac neutrinos there are $r$ ight-handed components of the $v_{j}$, but they are singlets under the gauge group; in the case of Majorana neutrinos in the standara theory there are no right-handed components.) The ordering of the mass eigenbasis is defined such that $U$ is as nearly diagonal as possible, i.e. $\left|u_{j j}\right|$ (no sum on $\left.j\right\rangle \geq\left|u_{j k}\right|, k \neq j$. This does not imply that $m\left\{v_{j}\right\rangle>m\left(v_{k}\right)$ if $j>k$, although this ordering might be regarded as natural in view of the similar one that obtains in the quark sector. The virtue of this convention is that a mass limit on "m( $\left.\nu_{i}\right)$ " can be used as a definite limit on $v_{j}, j^{a}=a$, the dominantly coupled mass eigenstate in $\mathrm{V}_{l}$.

Thus, in this general case of $n$ massive ( $D i-$ rac or Majorana) neutrinos, decays such as $H^{3} \rightarrow$ $\mathrm{He}^{3}+\mathrm{e}^{-}+\bar{v}_{\mathrm{e}}$ and $\pi^{+}+\mu^{+} \nu_{\mu}$, which have been used to set the best bounds on the respective neutrino masses, really consist of incoherent sums of the separate decay modes $\mathrm{H}^{3} \rightarrow \mathrm{He}^{3}+\mathrm{e}^{-}+\bar{v}_{j}$ and $\pi^{+}+\mu^{+}+v_{k}$, where the $v_{j}, v_{k}$ are mass eigenstates, and the indices $j$ and $k$ range over the subset $\{1, \ldots, n\}$ allowed by phase space in these two respective decays. 2 The coupling strengths for the $j$ 'th modes are given for the two decays by the factors $\left|\mathrm{u}_{1 j}\right|^{2}$ and $\left.\mid \mathrm{u}_{2 j}\right\}^{2}$, respectively. There are, in addition, certain kinematic factors depending on the $m\left(v_{j}\right)$ which 


\section{Data Card Listings}

For notation, see key at front of Listings.

\section{Stable Particles}

enter in determining the branching ratio for the f'th decay mode. Assuming that the off-diagonal elements of the lepton mixing matrix $u$ are small relative to the diagonal elements, the dominantly coupled decays are the ones with coupling strength $\left|u_{a j}\right|^{2}, a=j, i . e \cdot \mathrm{H}^{3} \rightarrow \mathrm{He}^{3}+\mathrm{e}^{-}+\bar{v}_{1}$ and $7^{+} \rightarrow u^{+} v_{2}$.

It follows that the old neutrino mass 1 imits quoted in the literature for $m\left(v_{e}\right) n, m\left(v_{\nu}\right) n$, and " $\left(v_{T}\right)$ " are meaningful only insofar as they are reinterpreted as 1 imits on the corresponding mass eigenstates. Specifically, a bound such as the Bergkvist 1 imit, ${ }^{3}$ "m( $\left.v_{e}\right) "<60 \mathrm{ev}$ (90\% cL). really constitutes a weighted 1 imit on each of the mass aigenstates $y_{j}$ in the weak eigenstate $v_{e}$ which are kinematically allowed to occur in tritium decay and which are coupled with strength $\left|\mathrm{u}_{1 j}\right|^{2}$ sufficiently large to make a significant contribution to the observed spectrum. It is thus certainly a limit on $v_{1}$. If leptonic mixing is hiecarchical as quark mixing is known to be (as least for the first three generations), i.e. $\left|u_{j j}\right|^{2}>\left|u_{j k}\right|^{2}, k \neq j$, then $v_{1}$ is the only mass eigenstate significantly constrained by a bound on "m( $\left.v_{e}\right)$." Furthermore, a neutrino mass 1 imit cannot be stated in isolation; it always contains some implicit dependence on the relevant lepton mixing angles. Fortunately, this dependence is relatively unimportant for the dominantly coupled decay modes, i.e. e $\vec{v}_{1}, \mu \bar{v}_{2}$, and $\tau \bar{v}_{3}$. Since these modes were the ones responsible for the mass $1 \mathrm{im}$ its given previously, the latter can be reinterpreted without significant complication as proper limits on $m\left(v_{j}\right), j=1,2$, and 3 , respectively.

In addition to mass and lifetime limits, we have added data on neutrino magnetic dipole moments. These are of interest because a massless, purely chiral (empirically, left-handed) Dirac neutrino cannot have a magnetic (or electric) dipole moment. The same is true for a Hajorana neutrino, whether massless or massive, because of its defining property of being self-conjugate.

If one considers the possibility of nonzero masses for neutrinos, for consistency one must also consider the leptonic mixing which would in general occur concomitantly. Accordingly we have devored one category in the searches section to correlated bounds on neutrino masses and lepton mixing angles. These can be divided into two types. First, there are those due to decays involving neutrinos in the final state, which must be recognized to have the general multi-mode structure pointed out above. In the two most sensitive cases suggested as tests for neutrino masses and mixing, 2 one obtains a 1 imit on $m\left(v_{j}\right)$ and $\left|u_{a j}\right|^{2}$ individually for each $j$. Second, there are those due to processes involving the propagation and subsequent interaction of neutrinos. The latter are often called neutrino "oscillation" 3 limits, although this term is correct only if the differences in neutrino masses are sufficiently small relative to their monenta that the propagation is effectively coherent in a quantum nechanical sense; otherwise, the individual $v_{j}$ from a given decay such as $\pi_{\mu 2}$ or $k_{\lrcorner 2}$ propagate in a measurably incoherent manner and there is no "oscillation". Experimentalists usually present the ir results in terms of a simplifying model in which mixing is assumed to occur only between two neutrino species. Then the transformation equation becomes

$$
\left(\begin{array}{c}
v_{l a} \\
v_{p_{b}}
\end{array}\right)=\left(\begin{array}{c}
\cos \theta \sin \theta \\
-\sin \theta \cos \theta
\end{array}\right)\left(\begin{array}{l}
v_{i} \\
v_{j}
\end{array}\right) .
$$

Let the distance between the source of the neutrinos and their point of interaction be labeled as $x$, and their energy as $E$. Assume furthermore that the m( $\left.\nu_{j}\right)$ are such that the coherence assumption is valid. Then, the probability of an initial $v_{l_{a}}$ being equal to $v_{l_{b}}$ at time $t$ or equivalently (given the above assumption) at distance $x=t$, is

$\left|\left\langle v_{d_{b}}(0) \mid v_{l_{a}}(t)\right\rangle\right|^{2}=\sin ^{2} 2 \theta \sin ^{2}\left(\frac{\Delta m^{2} x}{4 E}\right)$.

where

$$
\Delta m^{2}=m\left(v_{i}\right)^{2}-m\left(v_{j}\right)^{2} .
$$

Thus, neutrino oscillation experiments cannot measure individual neutrino masses, but only differences of masses squared, and indeed these ale generally weighted in a more complicated way by mixing-matrix coefficients than in the two-specits 
Stable Particles

$\nu, \nu_{e}$

model. Experimental results are presented as allowed regions on a plot, the axes of which are $\left|\Delta m^{2}\right|$ and $\sin ^{2} 2 \theta$. These are often sumarized in terms of the asymptotic 1 imits $\left|\Delta \mathrm{m}^{2}\right|_{\max }$ for $\sin ^{2} 2 \theta=1$, and $\sin ^{2} 2 \theta$ for "large" $\left|\Delta x^{2}\right|$, i.e., sufficiently large $\left|\Delta \mathrm{m}^{2}\right|$ that the detector averages over many cycles of oscillation for there ceases to be any coherence). We refer the reader to the original papers for the two-dimensional plots; for the purpose of these Listirgs we shall give only the asymptotic limits.

An important question has to do with whether neutrinos are Dirac or Mjorana (sele-conjugate) particles. In the former case neutrinoless double beta decay, $(Z, A)+(2+2, A)+e^{-}+e^{-}$, is forbidden from occurring. In the Majorana case it may occur, if (a) neutrinos ace massive and/or (b) there are right-handed leptonic currents. In the 1 ight-neutrino case an upper limit on neutrinoless double beta decay yields a correlated upper bound on the quantity

$$
\vec{m} \equiv\left|\sum_{j=1}^{n} v_{1 j}^{2} m\left(v_{j}\right)\right|
$$

and $\eta$, the fractional admixture of right-handed leptonic current.

Further explanatory notes are included in the Listings.

\section{References}

1. S. Heinberg, Phys. Rev. Lett. 19, 1264 (1967); A. Salam, In Elementary Particle Theory: Relativistic Groups and Analyticity, edited by N. Svartholm (Alquigt and Wiksell, Stockholm, 1968), p. 367. See also S. Glaahow, Nucl. Phys. 22, 579 (1961): S. Glashow, J. I1 iopoulos, and L. Maiani, Phys. Rev. D2, 1285 (1970); and, for the $n=3$ case, $M$. Kobayashi, and T. Maskawa, Prog. Theor. Pliys. 49, 652 (1973).

2. R. E. Shrock, Phys. Lett. 968, 159 (1980); Phys. Rev. D24, 1232 (1981); Phys. Rev. D24, 1275 (1981).

3. 2. Maki, M. Nakagawa, and S. Sakata, Prog. Theor. Phys. 28, 870 (1962); B, Pontecorvo, Sov. Phys. JETP 6, 429 (1957); I, 172 (1958); 2h. Ek. Theor. Fiz. 53, 1717 (1967) Sov. Phys. JETP $\underline{26}, 984$ (1968) ; v. Gríbov and B. pontecorvo, Phys. Lett. $\underline{28 \mathrm{~B}}, 493$ (1969).

\section{Data Card Listings}

For notation, see key at front of Listings.

4. For recent studies of neutrinoless double beta decay see r. Primakoff and S, P. Rosen, Ann. Rev. Nucl. Sci, 31, 145 (1981); S. P. Rosen, Proc, of 1981 Intl. Conf. on Heutrino Physics and Astrophysics (Maui, Hawaii), eds. R. J. Sens et al., v.2, p. 76; W. C. Haxton, G. L. Stephenson, Jr., and D. Strottman, PhYs. Rev. Lett. 47, 153 (1981); M. Doi, T. Kotani, k. Nishiura, K. Okuda, and E. Takasugi, Phys. Lett. 103B, 219 (1981), and Prog. Theor. Phys. 66, 1739 and 1765 (1981).

$\nu_{e}$

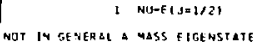
1 NUTE "MASS" IEVI

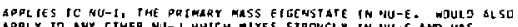

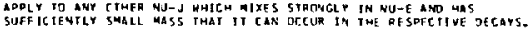
vare - THE ABQPEVIATION AHA IS USED BELOA FOR ANTINU

\begin{tabular}{|c|c|c|c|c|c|}
\hline 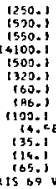 & 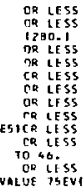 & 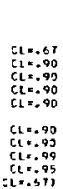 & 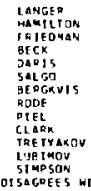 & 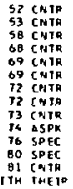 & 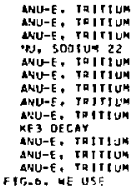 \\
\hline
\end{tabular}

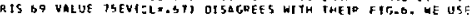

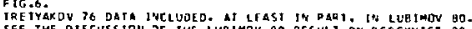

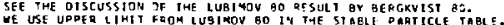
IHE IR LJUER LIMIT MEEDS CONF IQMATION.

1 INU-11 - IRNU+1| MaSS OtFF. IFU

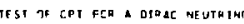

14.5ESTCD LESS CL.

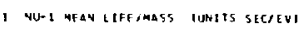

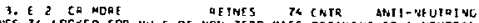

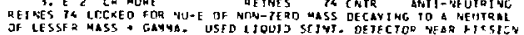

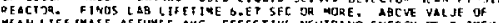

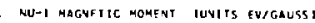
MUST VAN1SM FTR MAJORANA NEUTRTNO OR DUAELT CHIRAL MLSSLTSS

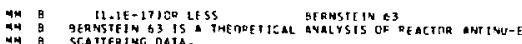

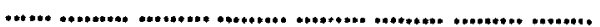

LANGEY 52 PR 8 B 699 FAM

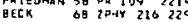
BEANSTE

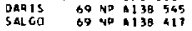
GEXGKVIS 72 पP 430317

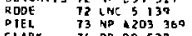

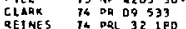

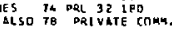

TRE TYAXO 76 aAsup as 10-1 AER ALSO TO RU CONF. AACHEY

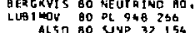
4
SINPSOA

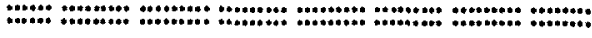

REFERENCES FDR NiJ-E

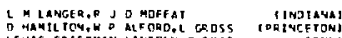

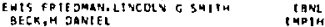

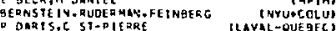

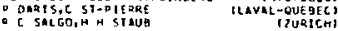

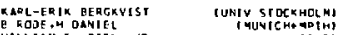
EL JOFF

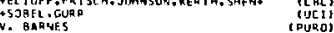

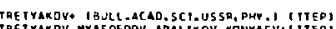

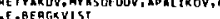

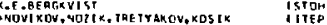

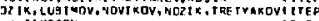


๒

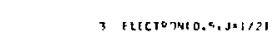

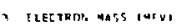

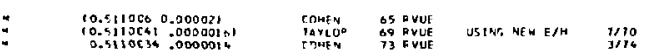

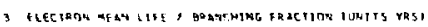
TEST DO CHAGG CONSEDVATIOY

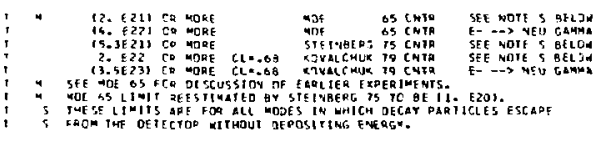

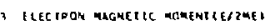

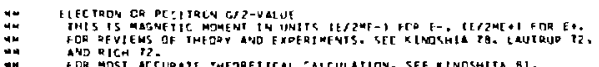

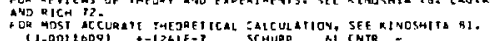

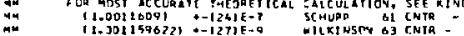

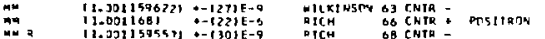

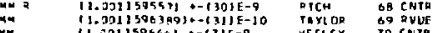

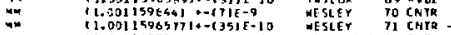

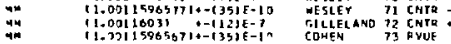

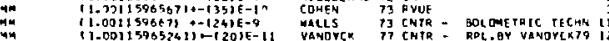

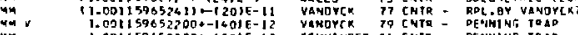

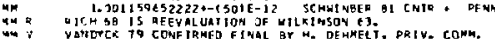

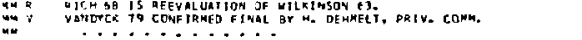

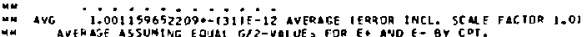

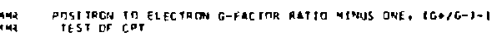

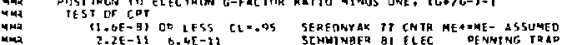

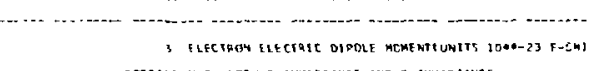

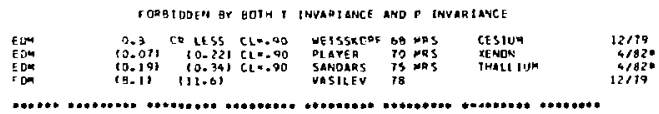

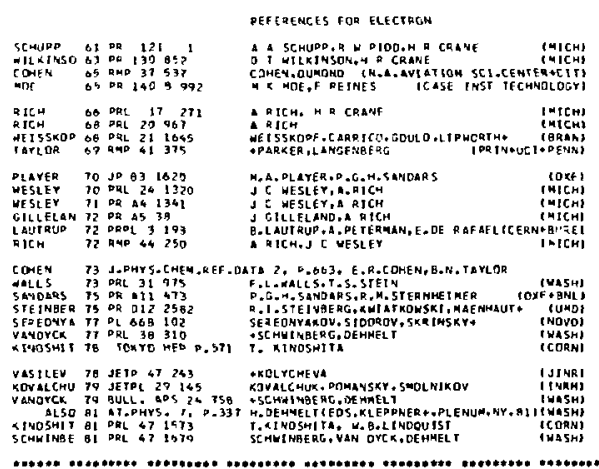

$\because \ldots+\ldots$.

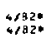

$\nu_{n}$

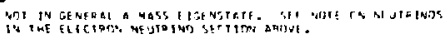

$$
\begin{aligned}
& \text { - }
\end{aligned}
$$

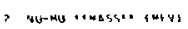

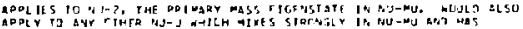

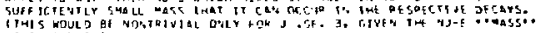
ITHIS WOUL?

\subsection{1 ghe LeSS
14.01 ro if 55

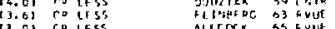

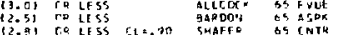

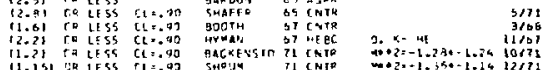

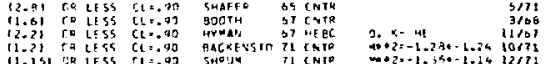

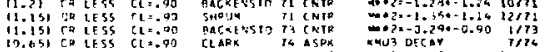

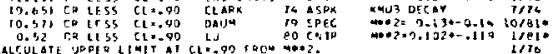

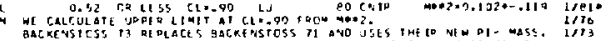

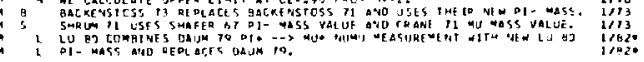

C.

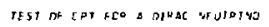

ON

10.651 10 ITS CL:.09 LLAR

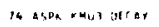

$11 / 15$

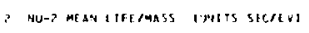

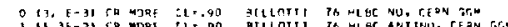

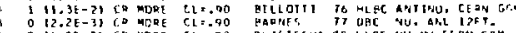

$0(2.2 E-3)$
J $(1.00-21$
0

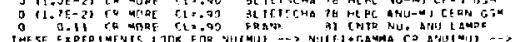
AME
AME

(......................

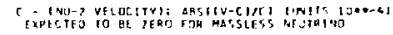

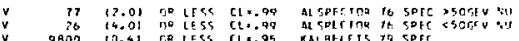

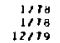

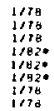

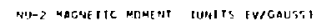

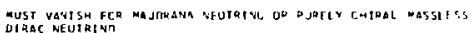

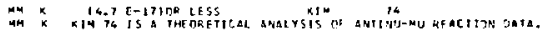

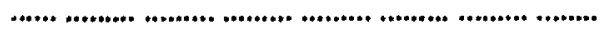

$\underset{1 ; 0820}{1,8020}$

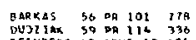

FEINGEX, O3 ARE 113,331

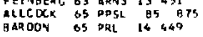

REFERENCES FOA N.J-WJ

SHAFER GS DRL IS 923

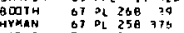

BUEKEHST 71 of 368407

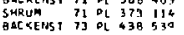

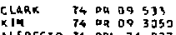

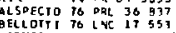

ARRES TI PRL 381049

RAL QFLEE I TQ OPL 431361

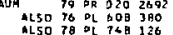

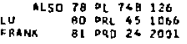

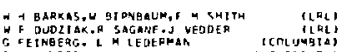

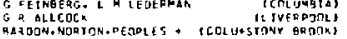

Q F SHAFER, COONE, JENKINS

BOD1H, JOHKSOH, LIFL)

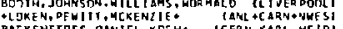

GULKENSTOSS, DAVICL, KCE H. ICERN, KARL, HEIDI

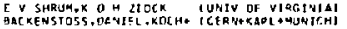

FELIOFF, FRISCH. JOHNSOR, KERTH, SHEN, ILPLJ

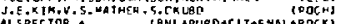

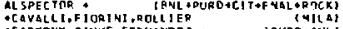

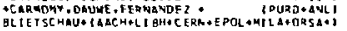

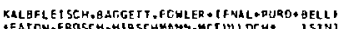
DAUM, OUBAL EATOH, FROSCH, WLCUL LOCH+ ISI I E TH,

DAUM, EATON, FRDSCH, HIOSC, maYN, O IIIN

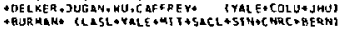

… 
Stable Particles

$\mu$

$\mu$

$440 \times 1100 \cdot \mathrm{J}=1 / 21$

$$
\text { - Muan mass TMevi }
$$

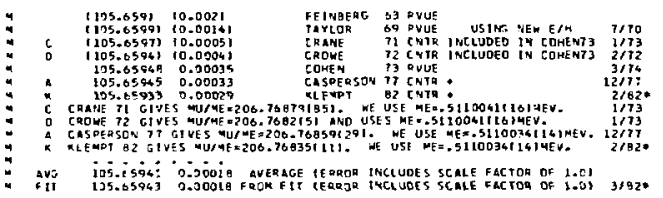

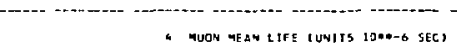

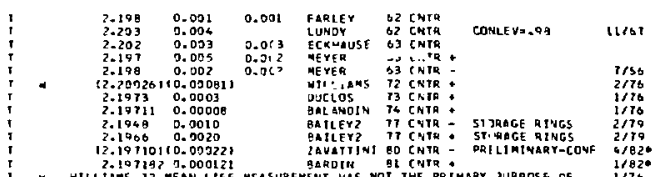

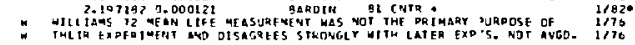

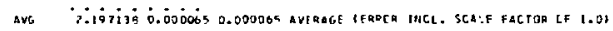

\begin{tabular}{|c|c|c|c|c|c|}
\hline \multicolumn{6}{|c|}{ - Me/MU- Mean Liff QAtio } \\
\hline $\begin{array}{l}\text { or } \\
\text { of } \\
\text { of }\end{array}$ & $\begin{array}{l}: 000 \\
10003 \\
1.72004\end{array}$ & $\begin{array}{l}0.301 \\
0.0010 \\
0.20011\end{array}$ & 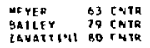 & 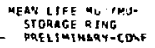 & 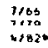 \\
\hline aV: & $i .00040$ & volomin & Ifragna ircludes S & EFACTOA of 1.01 & \\
\hline
\end{tabular}

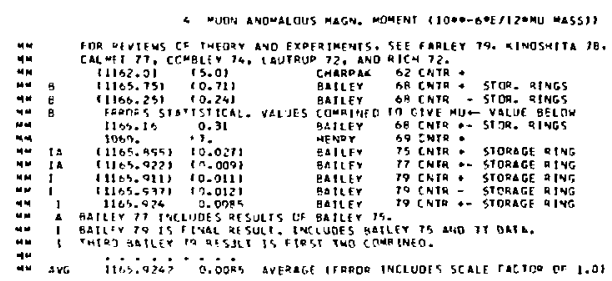

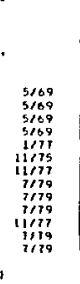

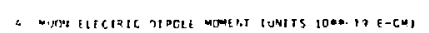

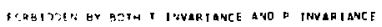

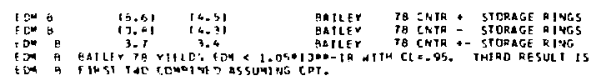

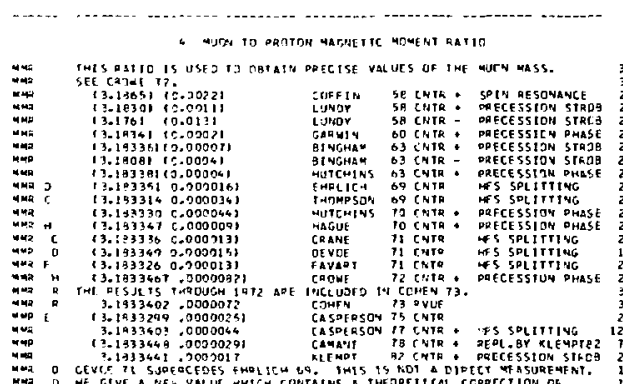

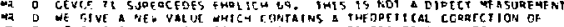

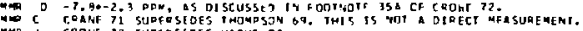

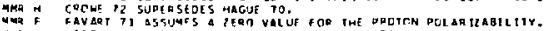

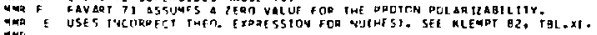

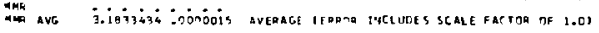

$3 / 72$ 2172

2772
$2 \pi 72$
$2 \pi 72$
$2 / 12$

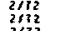

Data Card Listings

For notation, see key at front of Listings.

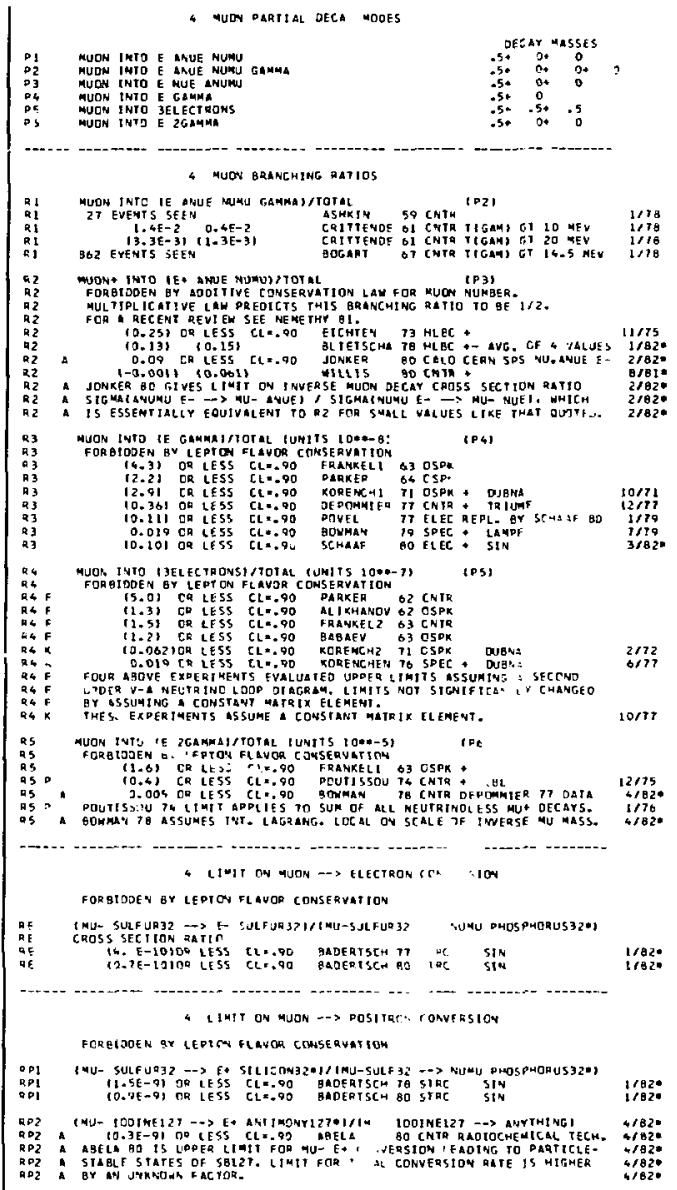

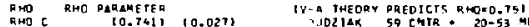

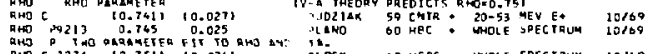

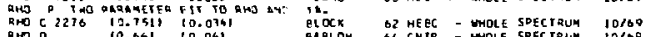

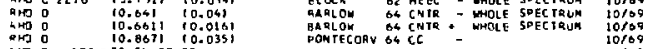

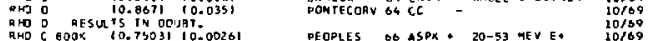

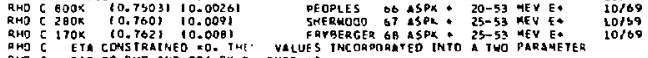

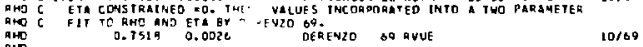

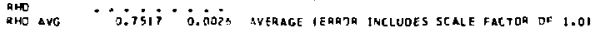

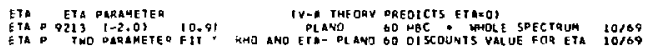

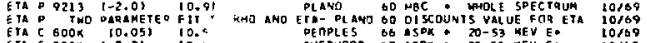

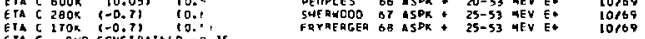

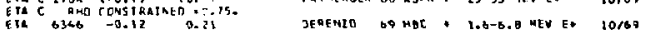




\section{Data Card Listings}

For notation, see key at front of Listings.

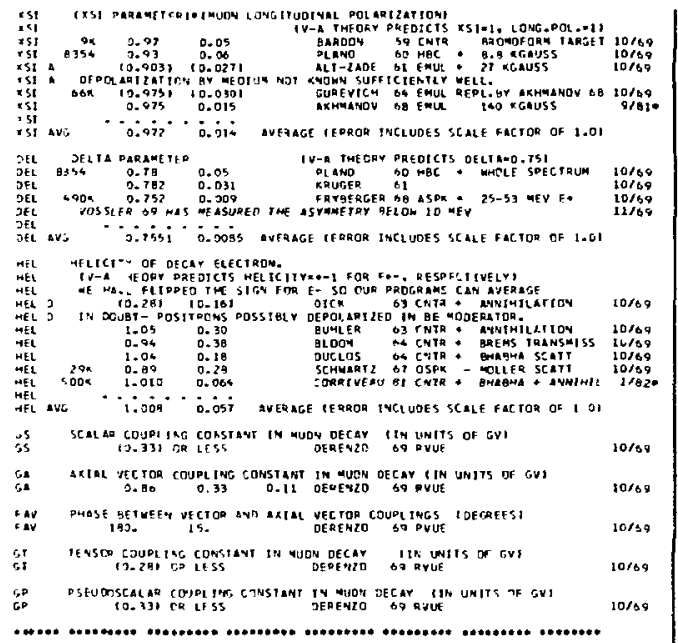

\begin{tabular}{|c|c|c|c|}
\hline 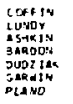 & 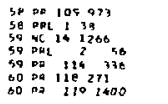 & 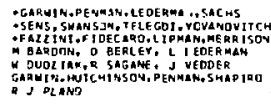 & 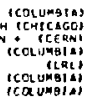 \\
\hline $\begin{array}{l}\text { ALI-LADE } \\
\text { CAITEEND } \\
\text { \& HUCER } \\
\text { ALIKHAYO } \\
\text { SLOCK } \\
\text { CMARGAK } \\
\text { FALAR }\end{array}$ & 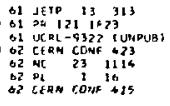 & 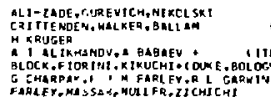 & 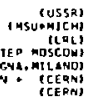 \\
\hline 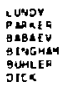 & 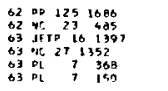 & 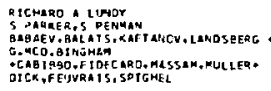 & 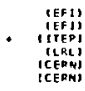 \\
\hline 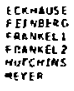 & 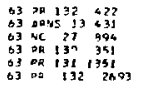 & 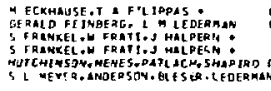 & 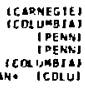 \\
\hline $\begin{array}{l}\text { GLRLDU } \\
\text { SLOOM } \\
\text { JUCLOS } \\
\text { GUREVICH } \\
\text { POMTECJR } \\
\text { PARKER }\end{array}$ & 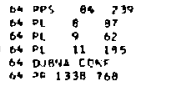 & 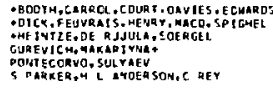 & 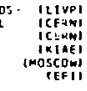 \\
\hline 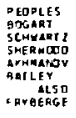 & 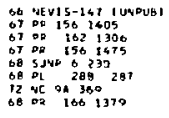 & 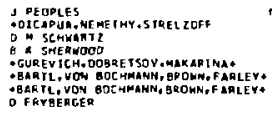 & 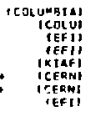 \\
\hline 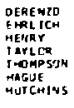 & 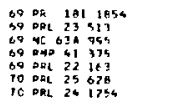 & 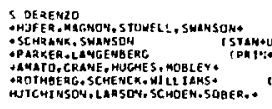 & 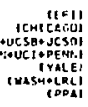 \\
\hline $\begin{array}{l}\text { ALSO } \\
\text { AVART } \\
\text { CAEACHI } \\
\text { DRE MCHE }\end{array}$ & 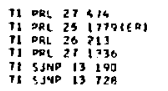 & 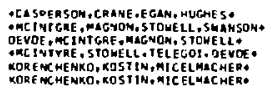 & $\begin{array}{r}\text { IVALEI } \\
\text { ICHICAGDI } \\
\text { \{CHICAGOI } \\
\text { ICMICAGOI } \\
\text { IJTNRI } \\
\text { IJINRI }\end{array}$ \\
\hline
\end{tabular}

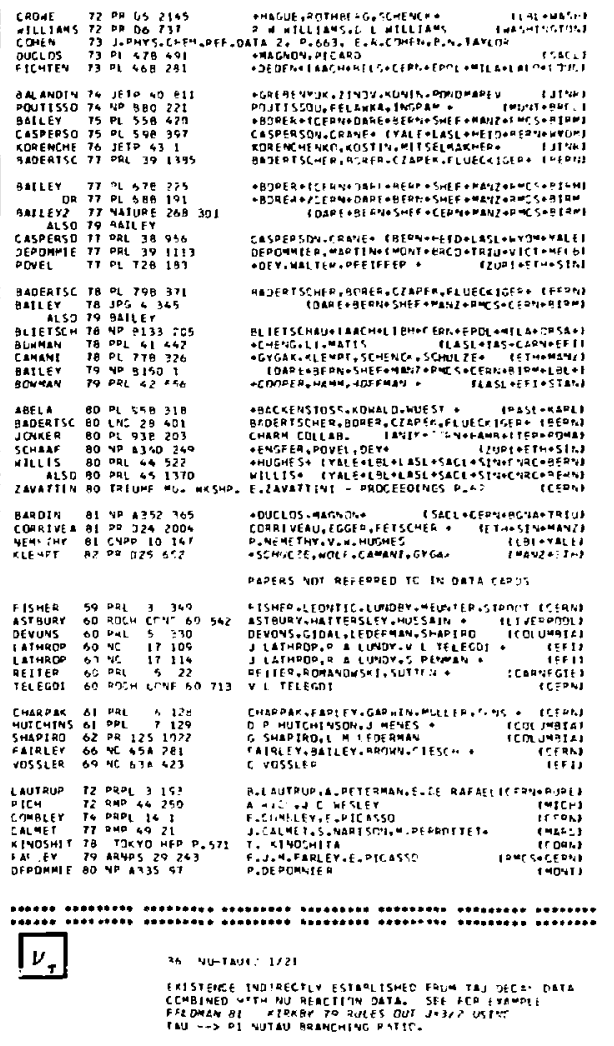

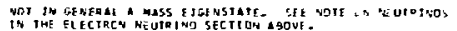

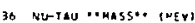

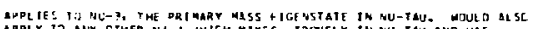

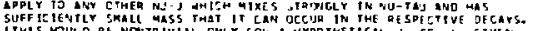

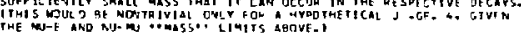

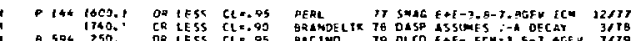

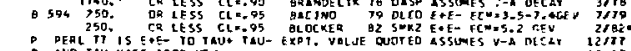

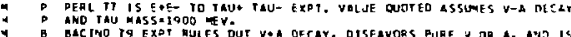

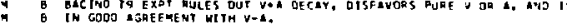

\section{QEFERENCES FOR NU-T AU}

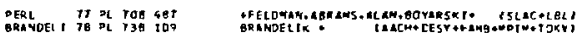

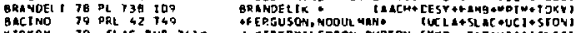

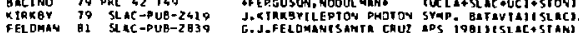

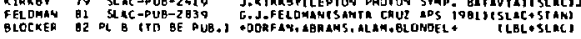


Stable Particles

$\tau^{*}$

$\tau^{7}$
35 TAU+-11705.JA1/21 MEAVY LEPTON;

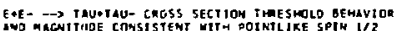

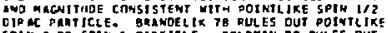

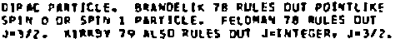

35 TAU MASS IAEVI

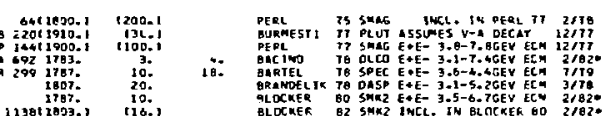

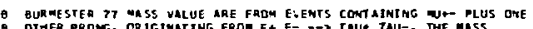

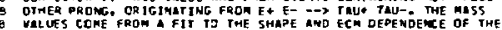

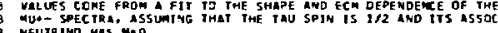

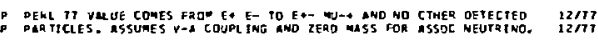

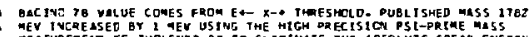

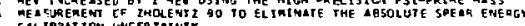

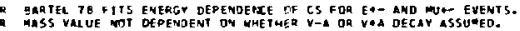

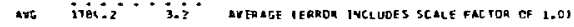

12177

\section{ISU MEAN LIFE TUNITS TONO-13 SECI}

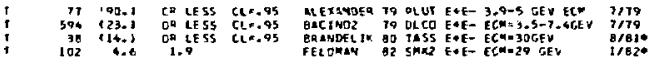

35 TN PARTIRL JFCAY mages

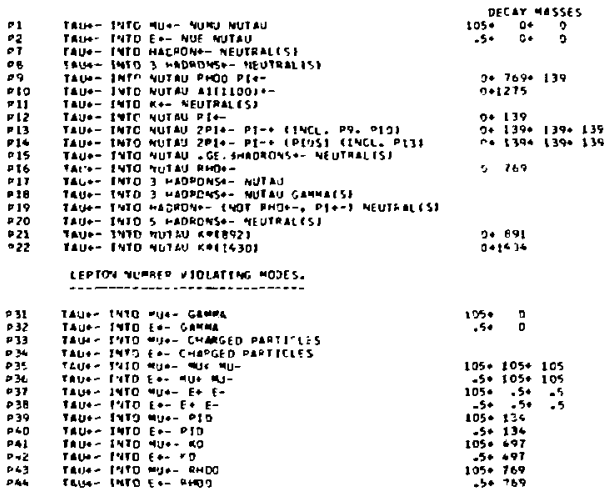

\section{EIT IED PAHTUL DECA: MODE BHANCHING HMACTIONS}

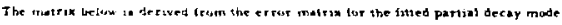

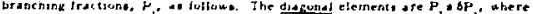

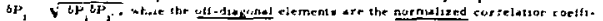

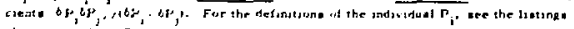

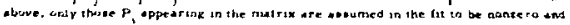

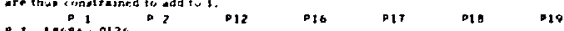

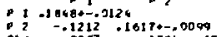

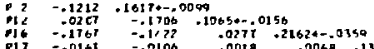

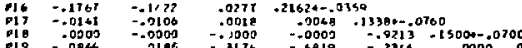

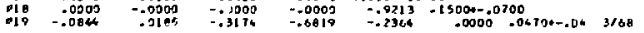

Data Card Listings

For notation, see key at front of Listings.

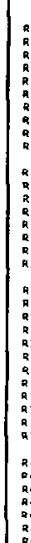

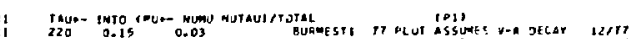

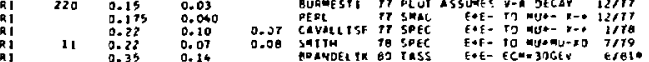

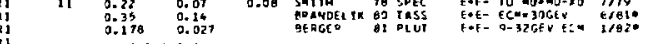

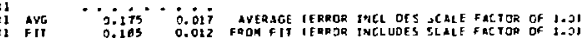

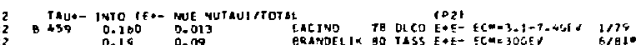

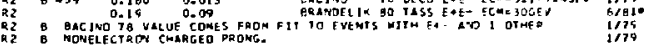

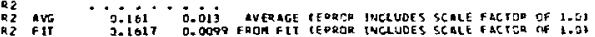

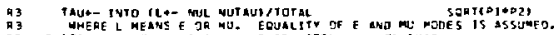

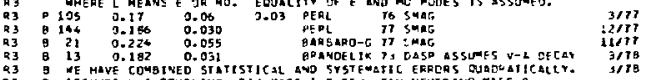

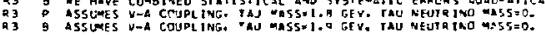

A3
R 3 AVG
Q 3 FiT

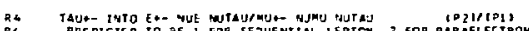

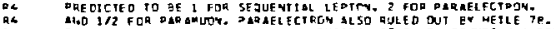

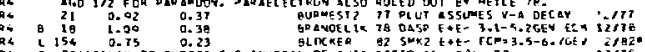

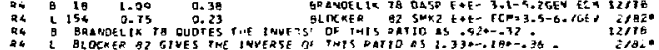

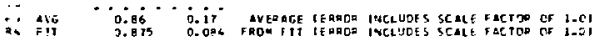

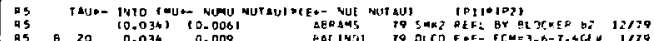

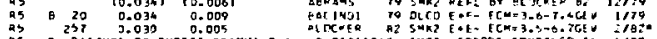

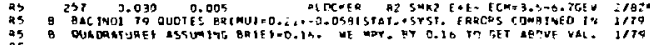

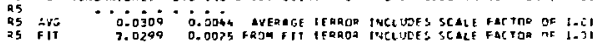

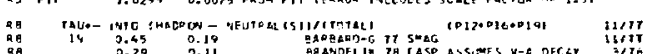

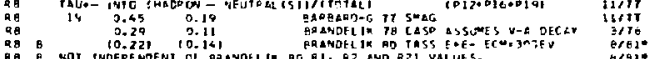

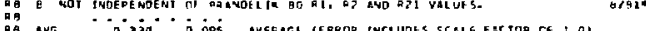

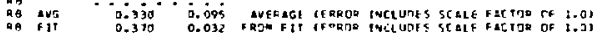

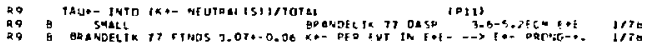

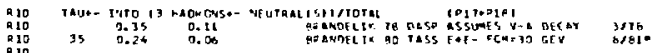

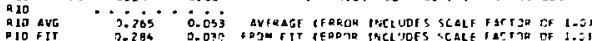

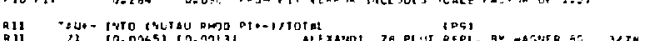

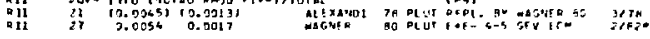

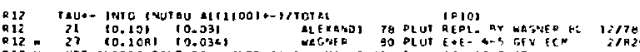

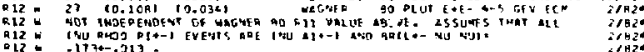

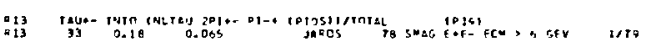

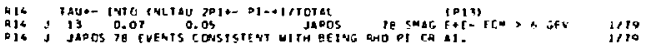

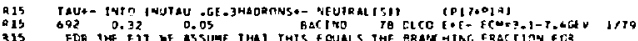

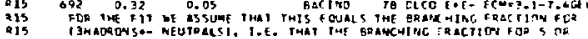

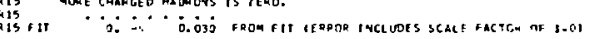

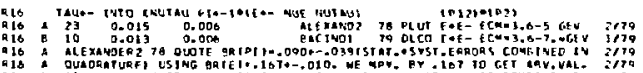

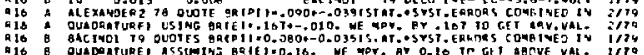

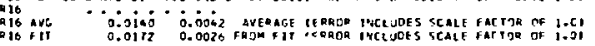

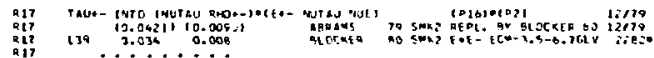

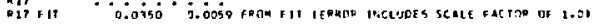

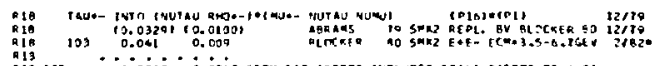

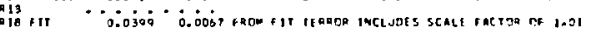




\section{Data Card Listings}

For notation, see key at front of Listings.

\section{Stable Particles}

$\tau^{ \pm}, \pi^{ \pm}$

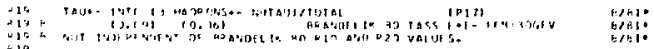

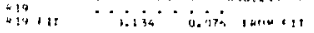

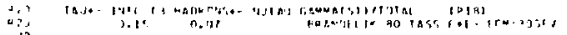

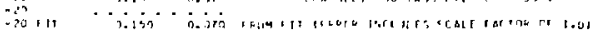

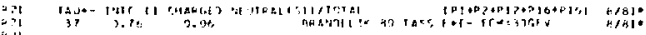

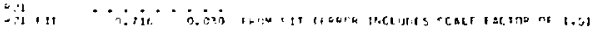

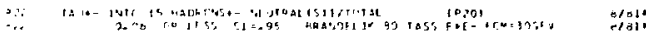

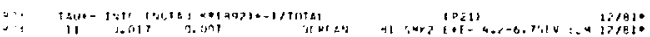

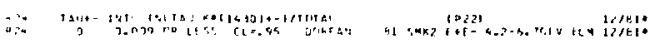

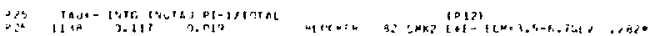

3.

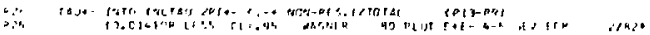

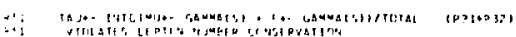

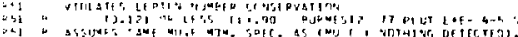

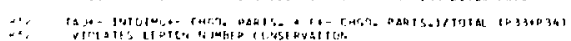

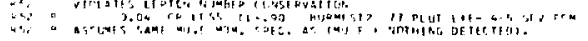

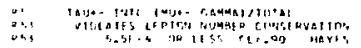

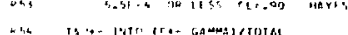

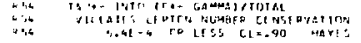

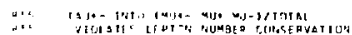

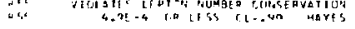

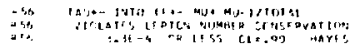

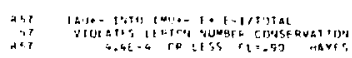

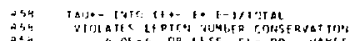

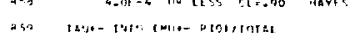

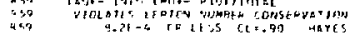

460
$2 t 0$
$x \rightarrow 0$

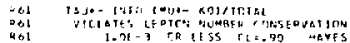

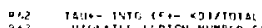

Roz
ow

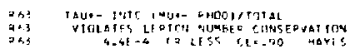

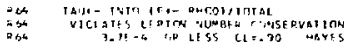

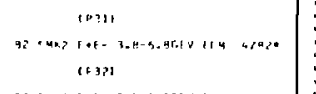

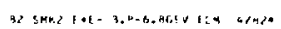
(1035)

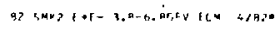

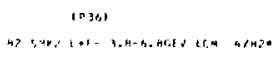
[D? 1$]$

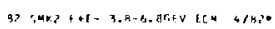
(2) 3 e)

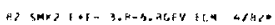
(a)

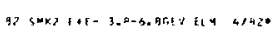
|pac|

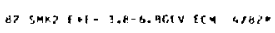
(Da1)

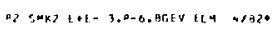
[a4?

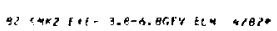
(ans?

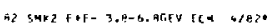
10441

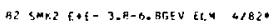

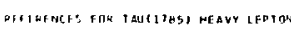

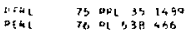

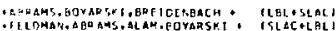

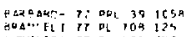

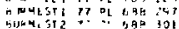
Cavitis it, ot HAXBAPO-TSAL TIEOT.

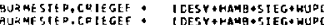

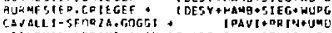

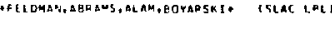

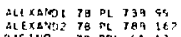

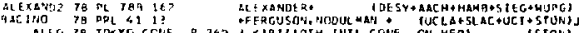

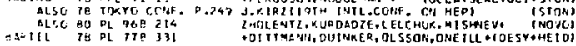

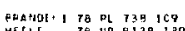

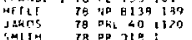

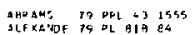

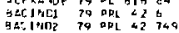

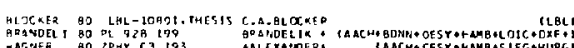

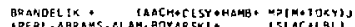
(a)

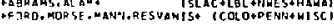

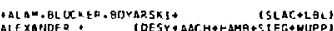

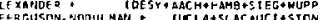

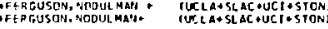

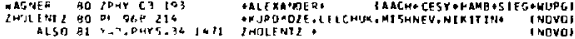

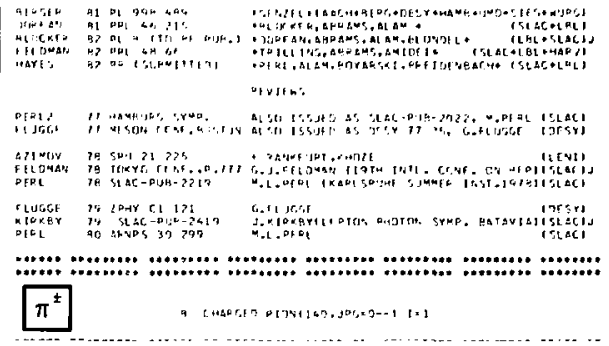

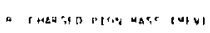

139.17 a.j?

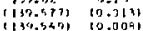

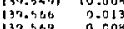

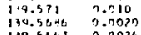

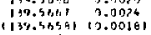

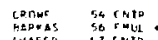

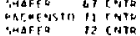

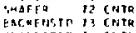

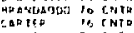

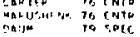

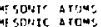

MEs

Desolite atouts

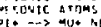

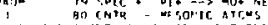

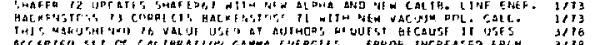

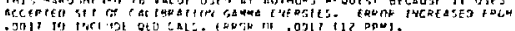
ony

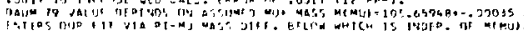

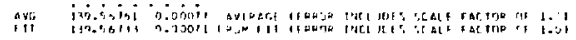
: $3 / 6=$

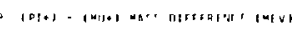

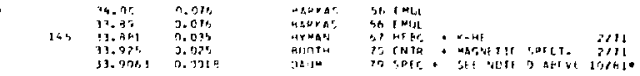

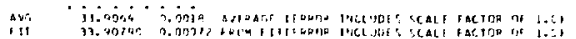

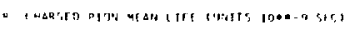

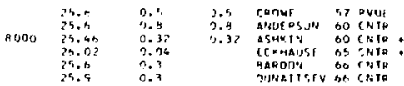

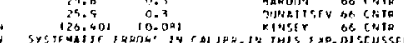

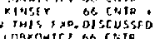

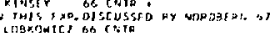

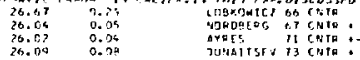

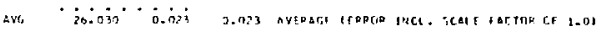

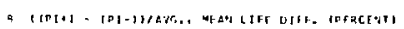

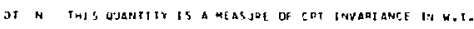

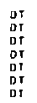

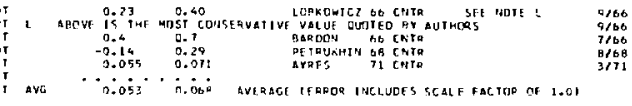

a GHaPGED pion paptial oecar yaces

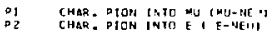

P3 CHAR. PIOH FNTO DJ TMU-NEUI GaNYA

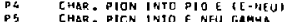

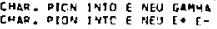

\begin{tabular}{|c|c|c|c|}
\hline & Car & $\triangle S$ SES & \\
\hline $\begin{array}{c}105 \\
.5+\end{array}$ & 0 & & \\
\hline 105 & $a$ & $a$ & \\
\hline 134 & $.5 *$ & $a$ & \\
\hline $\begin{array}{l}.54 \\
.54\end{array}$ & o** & . & .5 \\
\hline
\end{tabular}

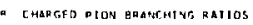

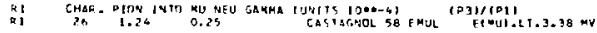


Stable Particles

$\pi^{*}, \pi^{0}$

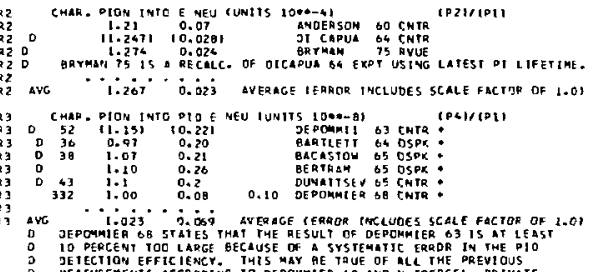

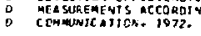

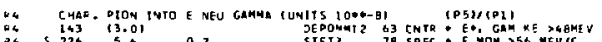

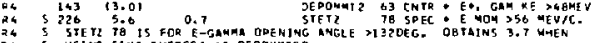

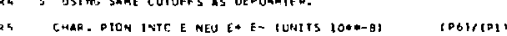

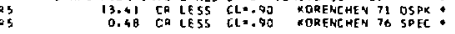

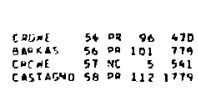

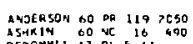

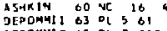

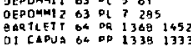

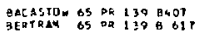

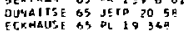

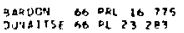

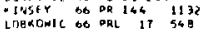

rraA

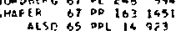

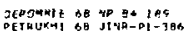

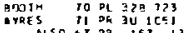

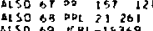

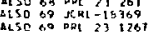

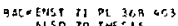

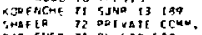

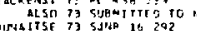

GRYMAN 75 DO 01113937

gRationOD

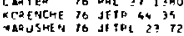

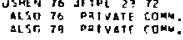

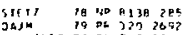

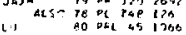

\section{peferences fub chatge pion}

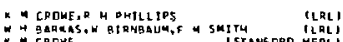

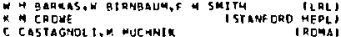

H 1 ANDEASOH. FUSTI, R H HILLER . IFFII ASHKINFALZINL, FTOECABO,LSPMAN" TCERHI

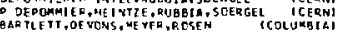

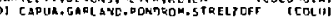

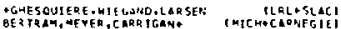

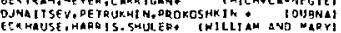

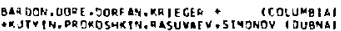

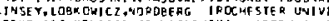

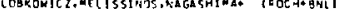

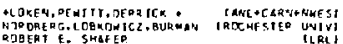

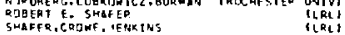

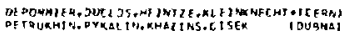
Itive

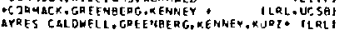

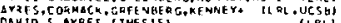

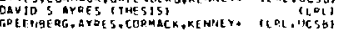

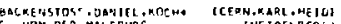

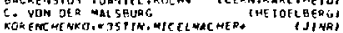

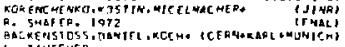

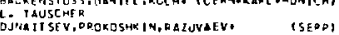

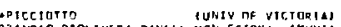

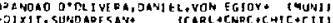

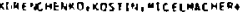

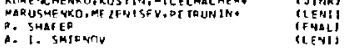

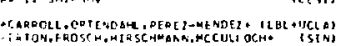

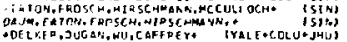

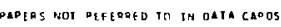

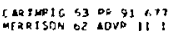

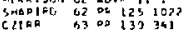
SoOICAN OA No the os?

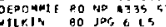

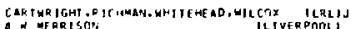

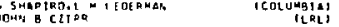

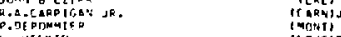

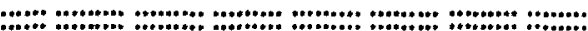

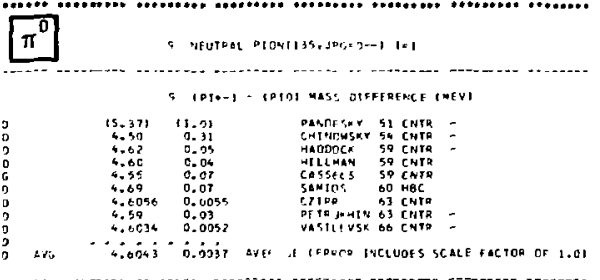

Data Card Listings For notation, see key at front of Listings.

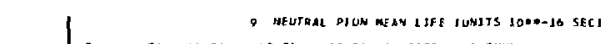

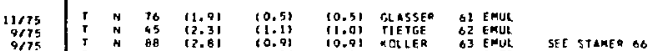

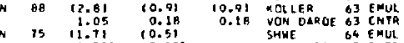

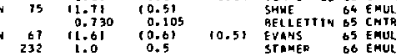

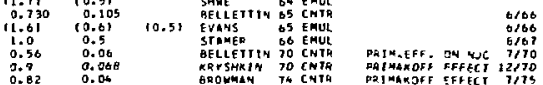

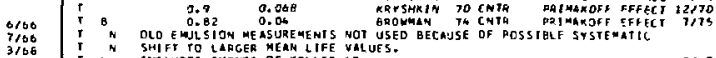

$2 \pi 72$
2,72
2,72
2,72

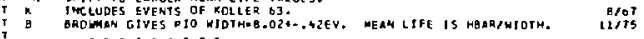

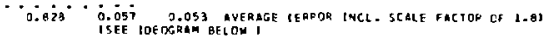
WE IGHIED AYERAGE $=1.207 \pm 0.080$

32820
123790
$12 \pi 90$ $1 / 18$

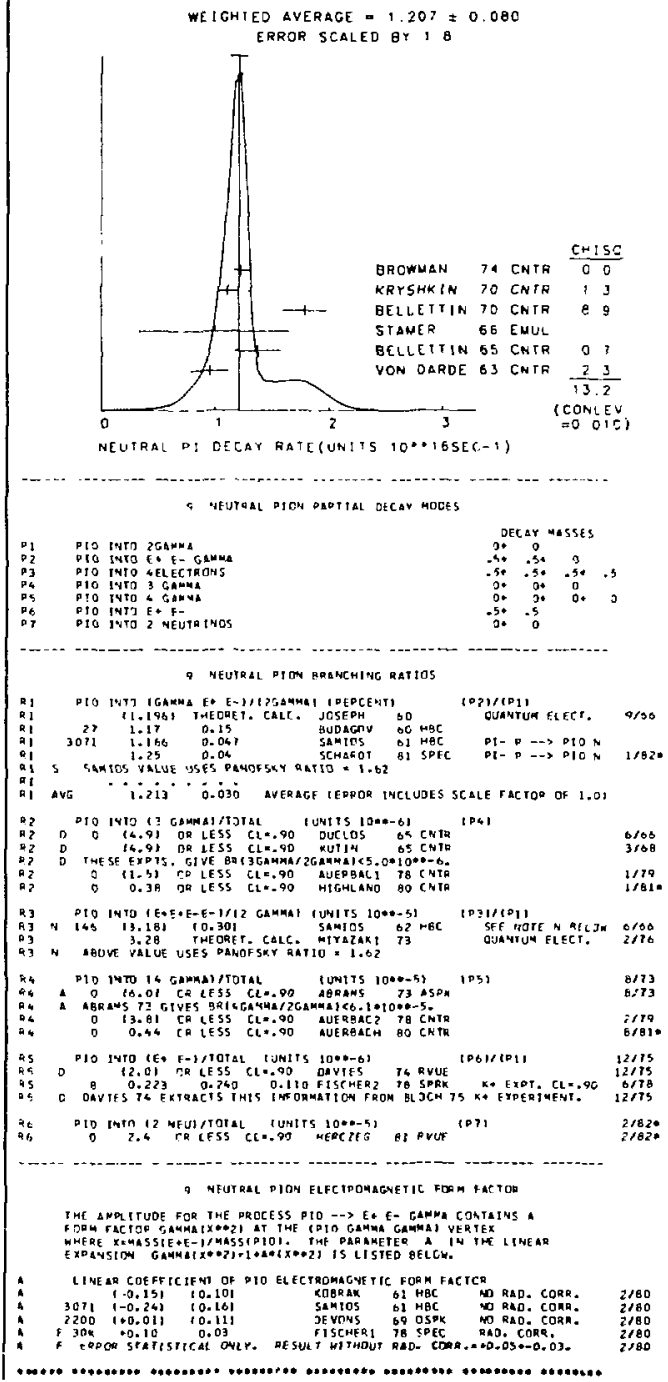
ERROR SCALED $B \div 18$ 
Data Card Listings

For notation, see key at front of Listings.
Stable Particles

$\pi^{0}, \eta$

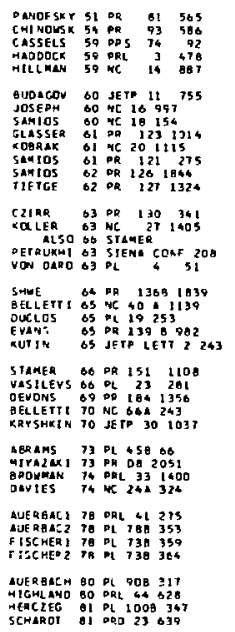

REFERENCES FOR NEUTHCL MTOU

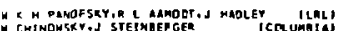

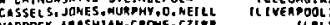

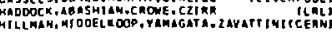

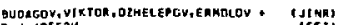
TCOLUMB: TEF

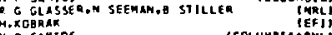

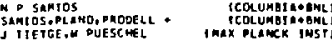

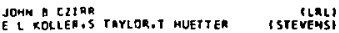

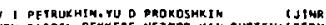

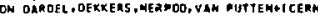

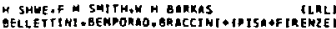

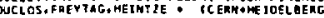

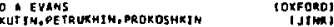
STAMER, TAYY OA, ROLLER, MUETTER: ISTEUENS

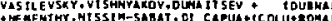
DEA

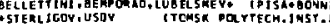

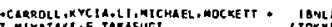

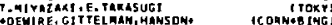

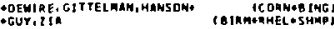

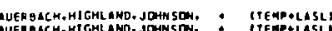

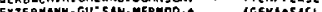

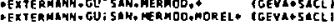

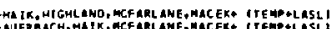

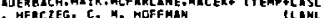

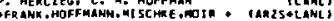

$$
\text { 14 EIA Mass Lmevi }
$$

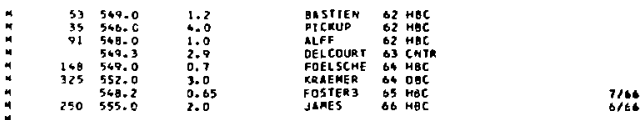

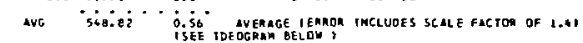

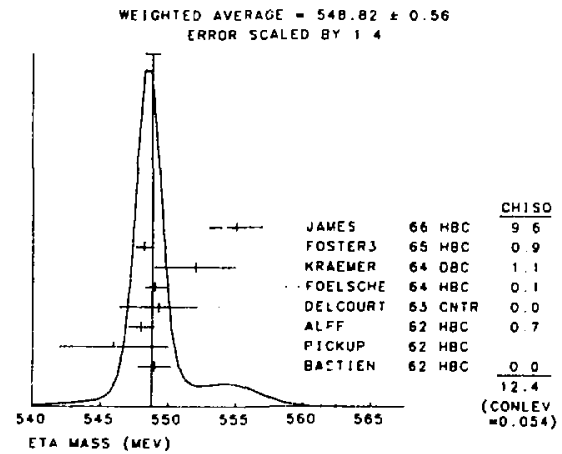

$$
\text { IS ETS MIOTH }
$$

EIS WTOTH DETERM MMED FROA MASS SPECTRUM TUMIYS WEY,

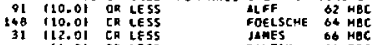

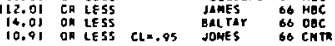

E1A HIOTH DETERK IMEO FROM OECAY MATE I UMITS KEVI

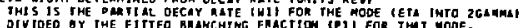

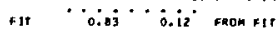

14 ETh DARTIAL DECAY MODES

PL ETA INTO 2GAMM"

ET:

E TA INTO EO E- Dito

ETA $1470 \mathrm{E} \cdot E-P 1+P 1$

Exh

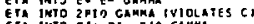

Era lNT0 :1* PI- plo catar

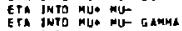

ErA

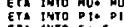

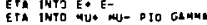

OELCAY MASSES

1340
1390
1390 ${ }_{130}^{1340}$

1390.1390 .134

$3340.554-5.50$.

$3.5+.540$

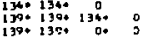

$205+105$

105. 105. 130

. $5+.5$

\section{FITTED PARTLAL DECAY MODE URANCHING FRACTIONS}

The matrie below so derived from the erenr matrix for the itted partiel decaly mude

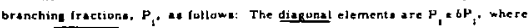

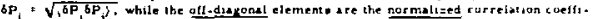

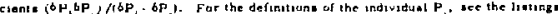
above: only thoue $P$, appearing in the matrix are alsumed in the fit to be nonzero end are thue canet ramed to add ta 1 . 3 ,

P : $3505+1$.0014, 2

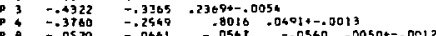

ERTED PAR CIOL DECAY MODE RATES

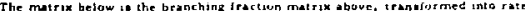

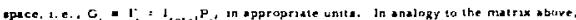

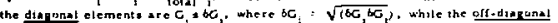

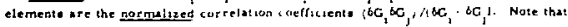

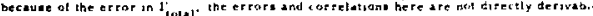
from thate sbove.

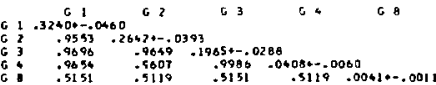

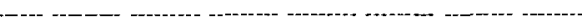

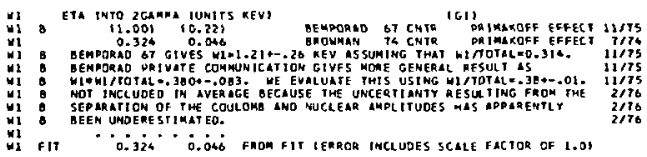

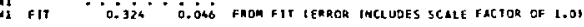

\begin{tabular}{|c|c|c|c|c|c|c|c|c|c|}
\hline & \multirow[t]{2}{*}{ 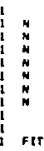 } & 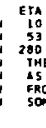 & \multicolumn{2}{|c|}{ 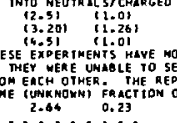 } & \multicolumn{2}{|c|}{$\begin{array}{l}\text { PICKUP } \\
\text { QASTIEN } \\
\text { JAMES } \\
\text { T DEEN USED II } \\
\text { OARTE CLEARLY } \\
\text { ORTED VALUES } \\
\text { F MOOE THYS } \\
\text { BALTAYZ }\end{array}$} & \multirow[t]{2}{*}{ 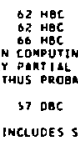 } & 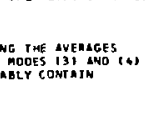 & \multirow[t]{2}{*}{$\begin{array}{l}6 / 06 \\
11 / 67\end{array}$} \\
\hline & & $\begin{array}{r}10 \\
10 \\
53 \\
200 \\
1 \\
15 \\
6 R \\
50 \\
\end{array}$ & 2.436 & D. $076^{\circ}$ & FROM FIT & TERRoR & & SCALE EACTOH OF 1.0 S & \\
\hline \multirow{2}{*}{$\begin{array}{l}2 \\
k 2 \\
2 \\
2 \\
2 \\
2 \\
2 \\
2\end{array}$} & \multirow{2}{*}{\multicolumn{2}{|c|}{$\begin{array}{r}\text { E14 } \\
75 \\
\text { AVG } \\
\text { FIT }\end{array}$}} & 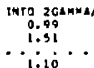 & \multicolumn{2}{|c|}{$\begin{array}{l}\text { A/CHARGED } \\
0.40 \\
0.43 \\
\because \cdots\end{array}$} & $\begin{array}{l}\text { CALAFONO } \\
\text { KE HOLLT }\end{array}$ & \multicolumn{2}{|r|}{$|P 1| /\left|D_{3}+P_{4}+D_{0}\right|$} & \multirow[t]{2}{*}{$12 / 75$} \\
\hline & & & $\begin{array}{l}1.10 \\
1.342\end{array}$ & $\begin{array}{l}0.03 \\
0.050 \quad F\end{array}$ & $\begin{array}{l}\text { ANEALGE } \\
\text { FROM FIT }\end{array}$ & $\begin{array}{l}\text { CERROR } \\
\text { TERROR }\end{array}$ & $\begin{array}{l}\text { INCLUDES S } \\
\text { INCLUOES S }\end{array}$ & $\begin{array}{l}\text { SCALE FACTOR OF } 1.0) \\
\text { SCALE FACTOR OF } 1.03\end{array}$ & \\
\hline
\end{tabular}

I4 EIL ORLNCHING RETIOS

(by A. V. Barnes, Lawrence Berkeley Laboratory)

For several years the measucements of the branching ratio $\left(\eta+\pi{ }^{0} \mathrm{r}\right) /(\eta \rightarrow$ neutrals) have been in disagreement. The recent upper linit masurement reported in DAYYDOV 81 is far below the previous politive reaults. The earlier 


\section{Stable Particles}

$\eta$

positive results are each suspect. The earliest report (DIGIUGNO 66) was a photon spectrum measurement. It depends on a Monte Carlo to fit the observed spectrum with that expected from a combination of photons from the decays $\eta+\gamma$, $\eta \rightarrow \pi^{0} \gamma$, and $\eta+\pi^{0} \pi^{0} \pi^{0}$. Their result is of course sensitive to the assumptions of this Monte carlo. FELDMAN 67 is an optical spark chamber experiment. The scanning efficiency for the 5- and 6-photon events is hard to measure and critical to their result. STRUGALSK 71 does not address the problem of contamination from the $r_{1} \rightarrow T^{0} \pi^{0} T^{0}$ decay mode. Assuming that the $\pi^{0} \gamma$ mode is absent and that the decay ratio $(n+r) /\left(n+\pi^{6} \pi^{0} \pi^{0}\right)$ is approximucely 0.9 implies there are $\approx 700 \quad 37^{\circ}$ events in the $\leqslant 4$; sample of their data. The $40 \pm 10 \eta+r^{2} \%$ decays observed could easily be misidentified $\eta+\pi^{0} \pi^{0} \pi^{0}$ decays. DAVYDON 81 accounts for the $\eta+\pi^{0} \pi^{0} \pi^{0}$ decays properly and is much more sensitive than previous measurements.

The $\pi^{0} \mathrm{rr}$ branching fraction is now assumed to be zero in our branching ratio fit. As a result, the fitted $r i$ and $3 \pi^{\circ}$ branching fractions have increased by 1.18 and 1.98 , respectively.

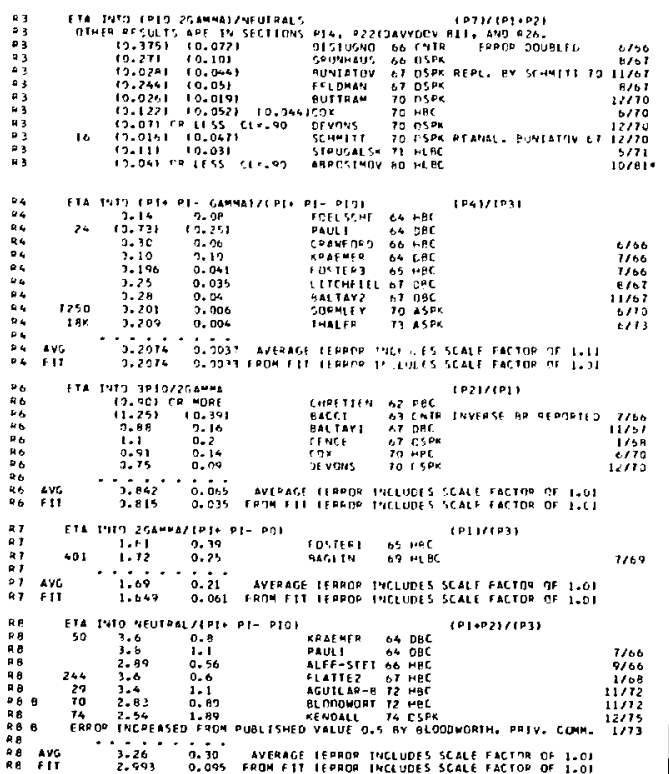

Data Card Listings

For notation, see key at front of Listings.

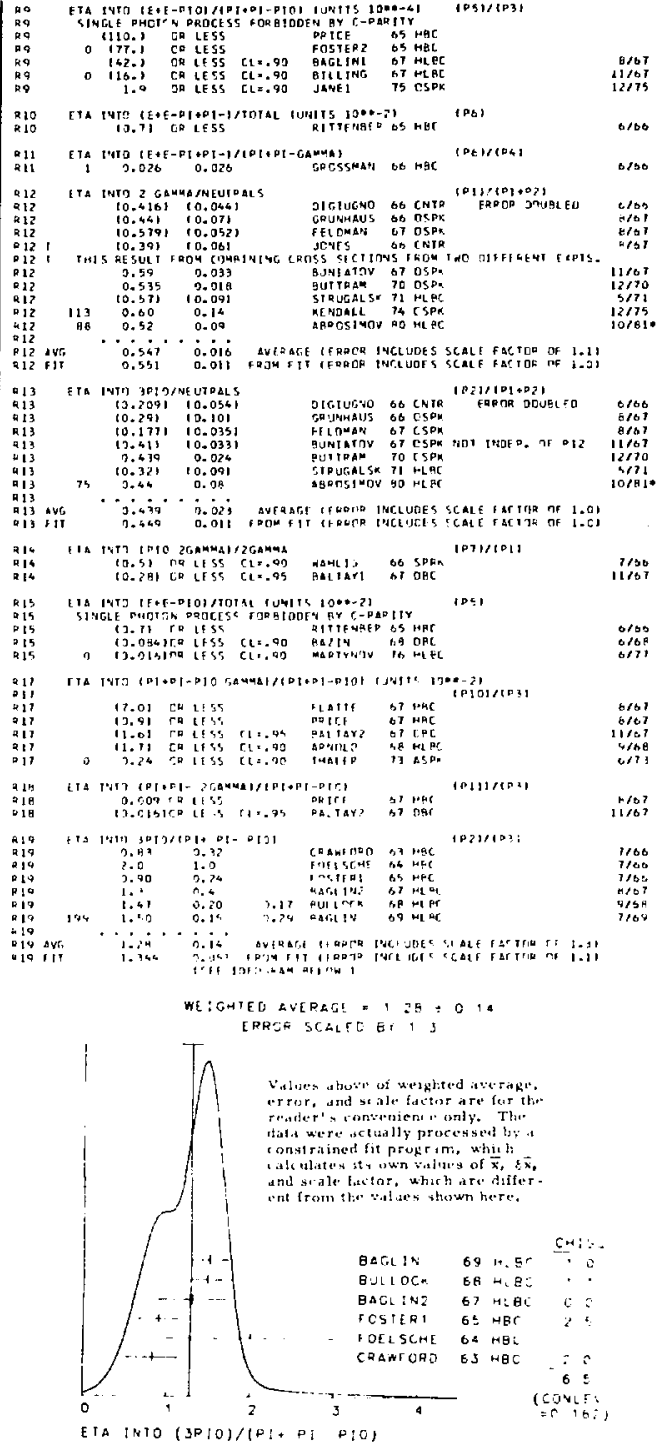

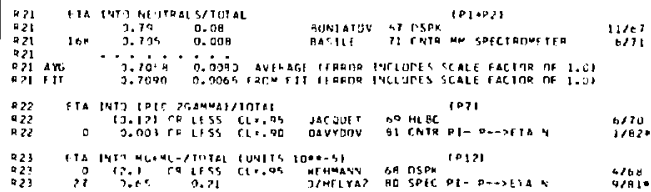


Data Card Listings

For notation, see key at front of Listings.

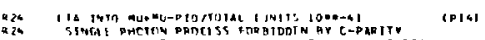

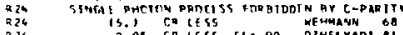

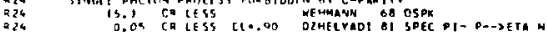

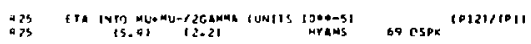

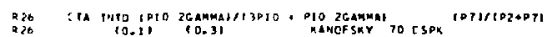

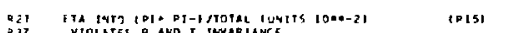

227
25

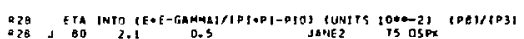

$2 / 76$
828
828

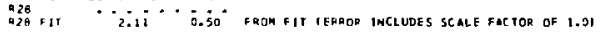

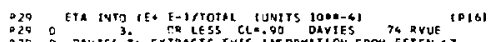

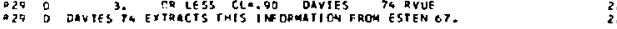

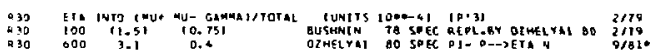

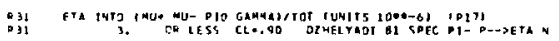

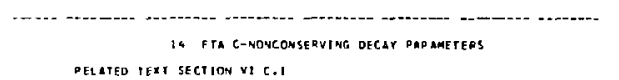

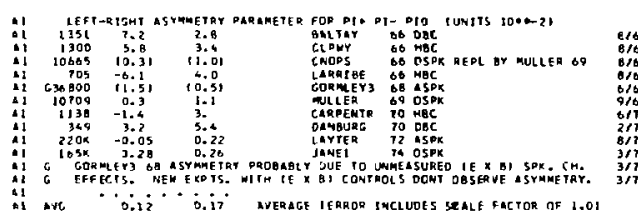

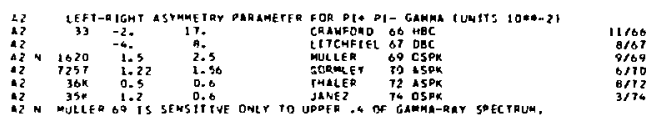

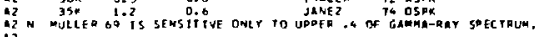

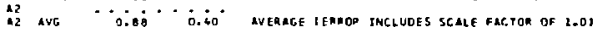

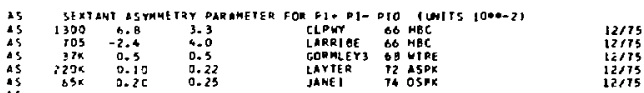

as
as
as
AVG

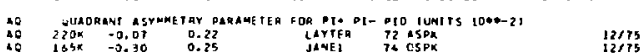

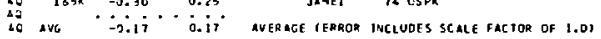

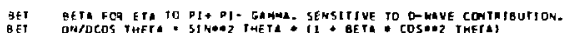

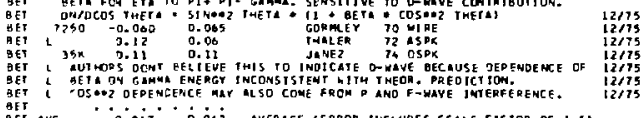

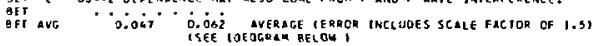

WeIGHTEO average - $0.047 \neq 0.062$

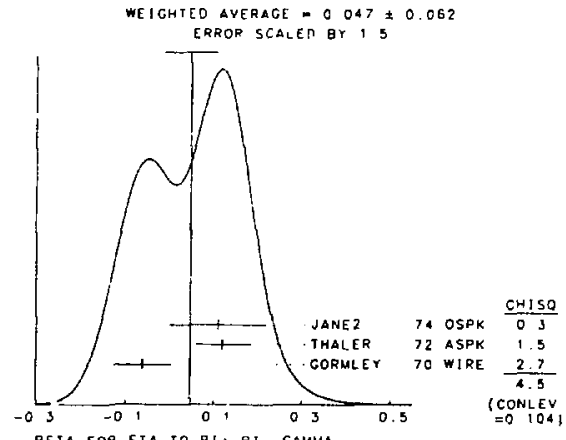

BETA TOR ETA TOPI+PI- GAMMA

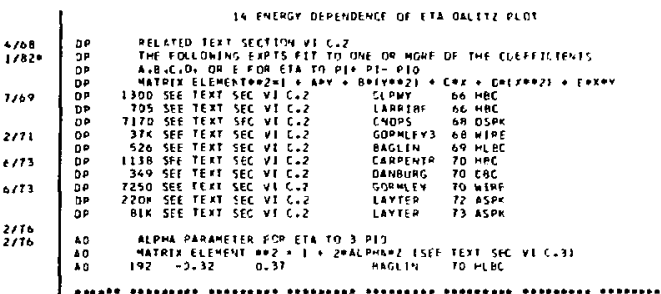

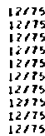

12175
22175
PEYSNEA OI PRL 7 421

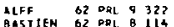

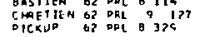

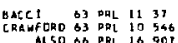

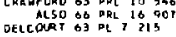

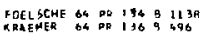

KRAEMER
PAUL

FOSTER OS PR 130 B DSZ

FESTEMS BS THEST

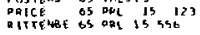

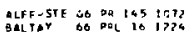

CLPuY bo of 149,1164

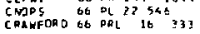

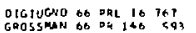

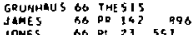

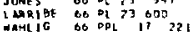

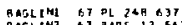

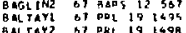

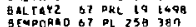

BILt the of pl 25045

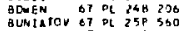

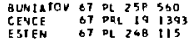

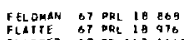

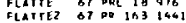

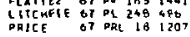

$A R \times D D O$
$B A D I N$

BULCOCK 68 PL 278632

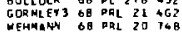

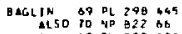

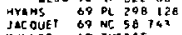

MULLEP 69 THESTS

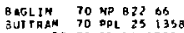

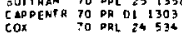

DANBURC 70 DR 0252584
DEVONS

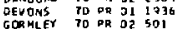

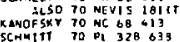

OASTLE
STRUGALS

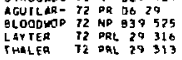

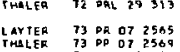

6 ROUHAN 74 PHL 321007

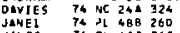

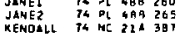

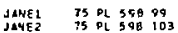

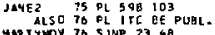

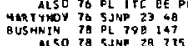

PAFEPENCES FDE ITA

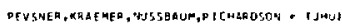

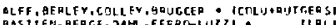

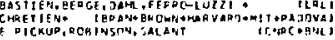

SACCT, PENSCL-SALVTHI.

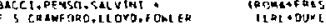

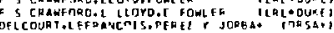

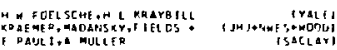

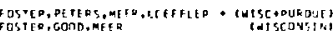

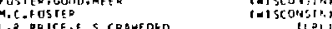

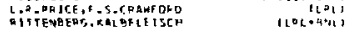

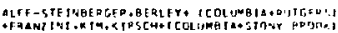

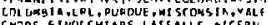

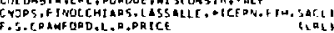

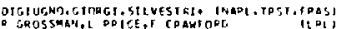

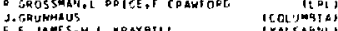

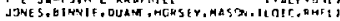

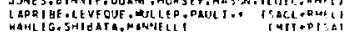

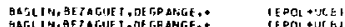

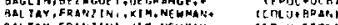

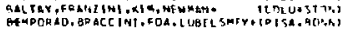

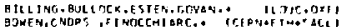

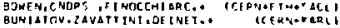

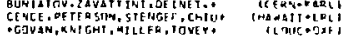

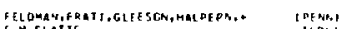

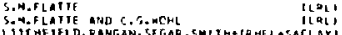

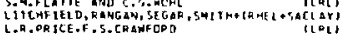

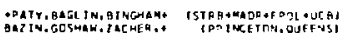

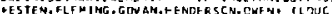

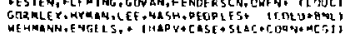

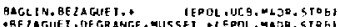

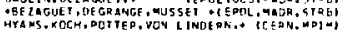

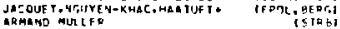

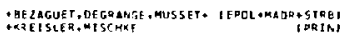

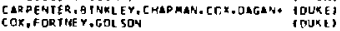

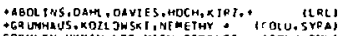

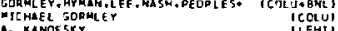

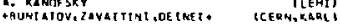
:BOLLINI, OALP LAZ, FRABEIIIt ICER W, BGAA, STRBI

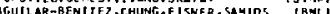

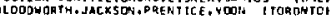

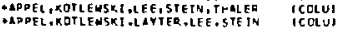

AAP PEL , KOILEHSKI,LEE, STEIN, T TALER TCOLUY

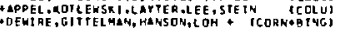
- GUY, Lia

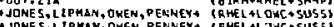

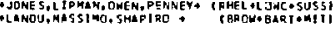

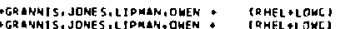

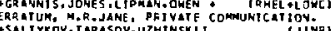

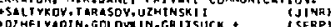

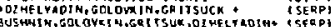


Stable Particles

$\eta, K^{*}$

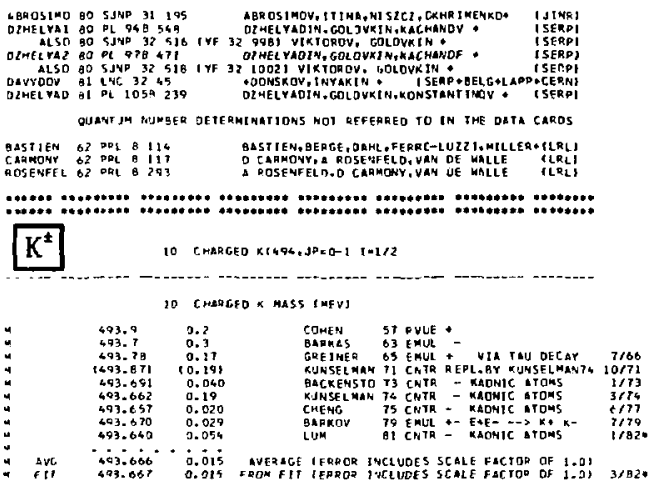

AVG
fil

17 $1 k+1 \cdots 1,-4$, MASS OIFFFRENCF IMEVI

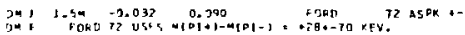
$i_{173}^{\infty}$

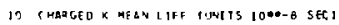
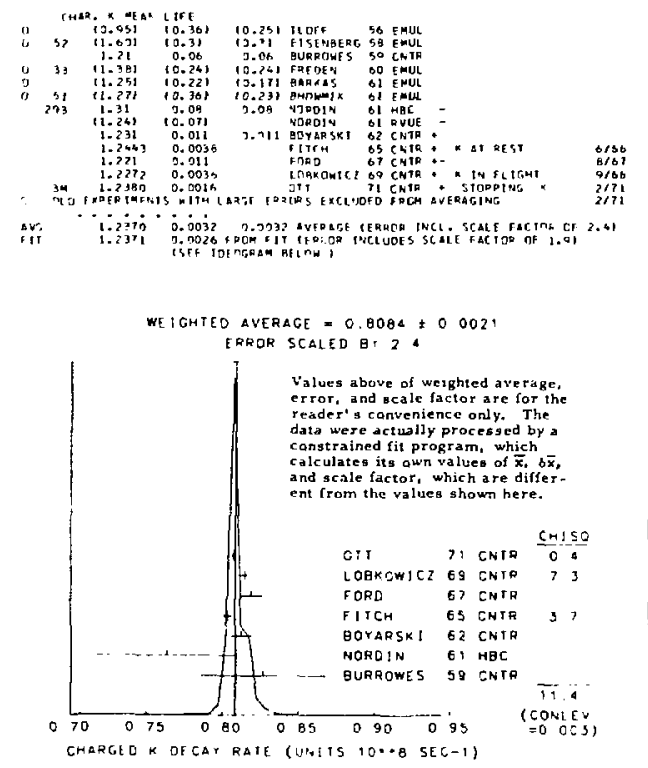

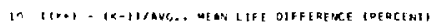

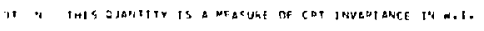

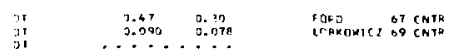

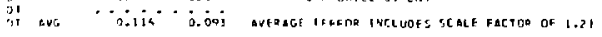

\section{Data Card Listings}

For notation, see key at front of Listings.

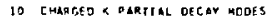

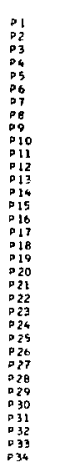
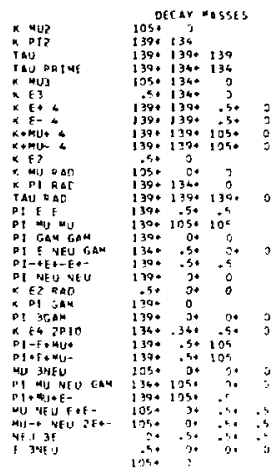

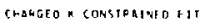

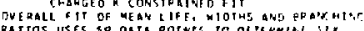

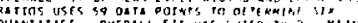

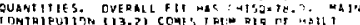

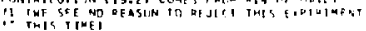

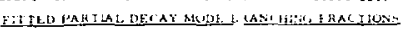

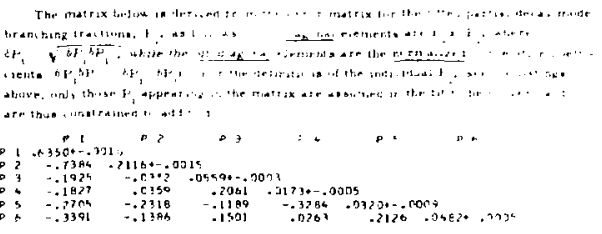

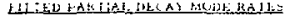

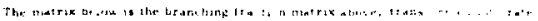

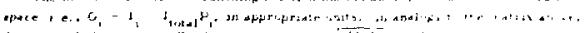

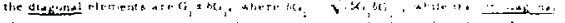

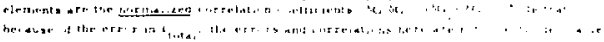

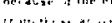

.

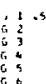

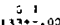

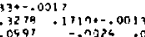

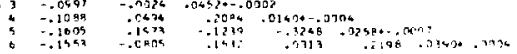

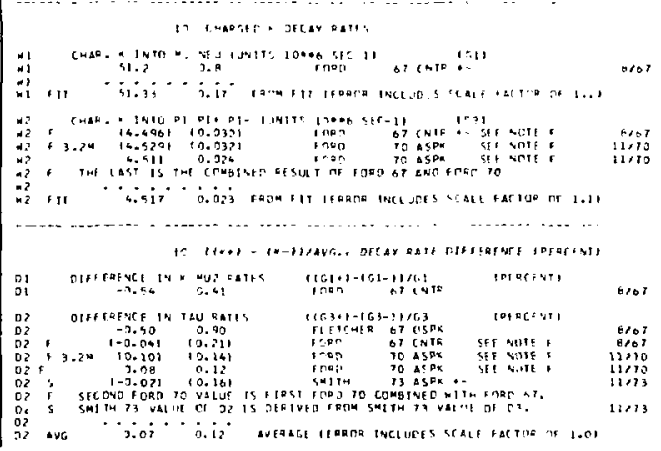



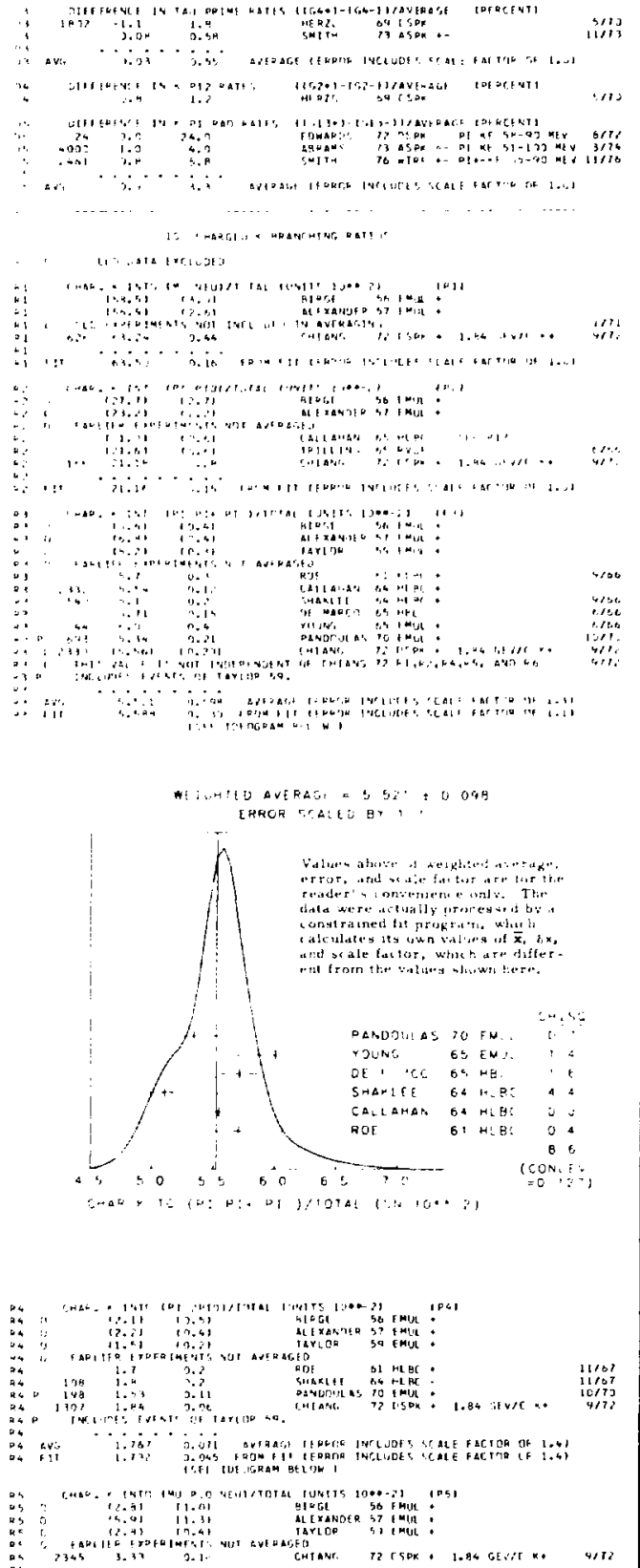

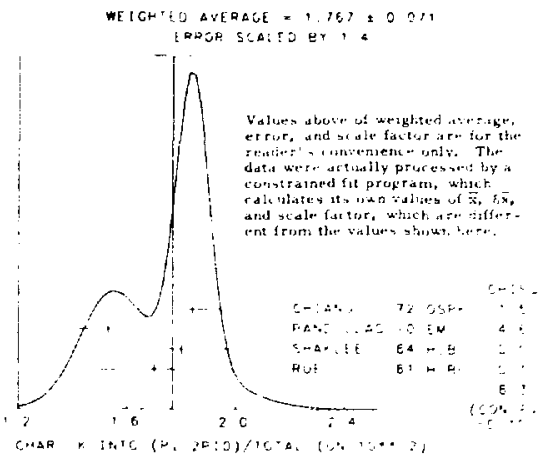

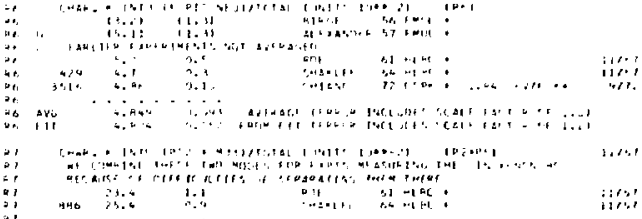

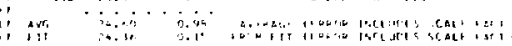

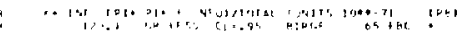

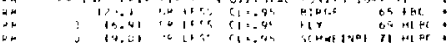

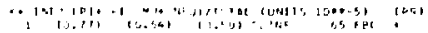

$\because$ r

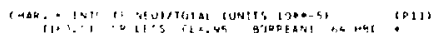

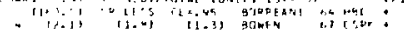

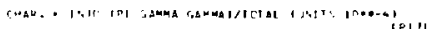

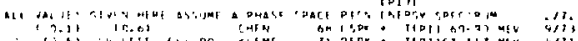

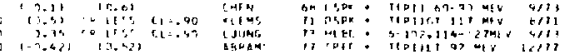

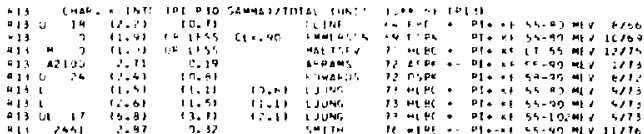

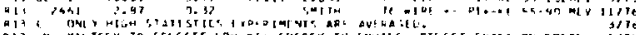

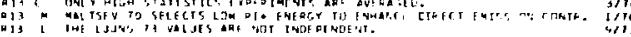
$R 13$
$Q 13$

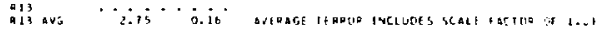

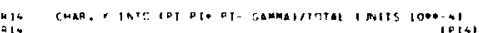

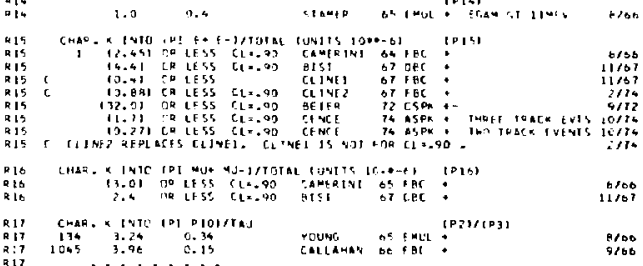

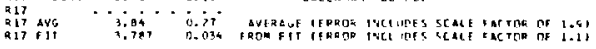


Stable Particles

$\mathrm{K}^{*}$

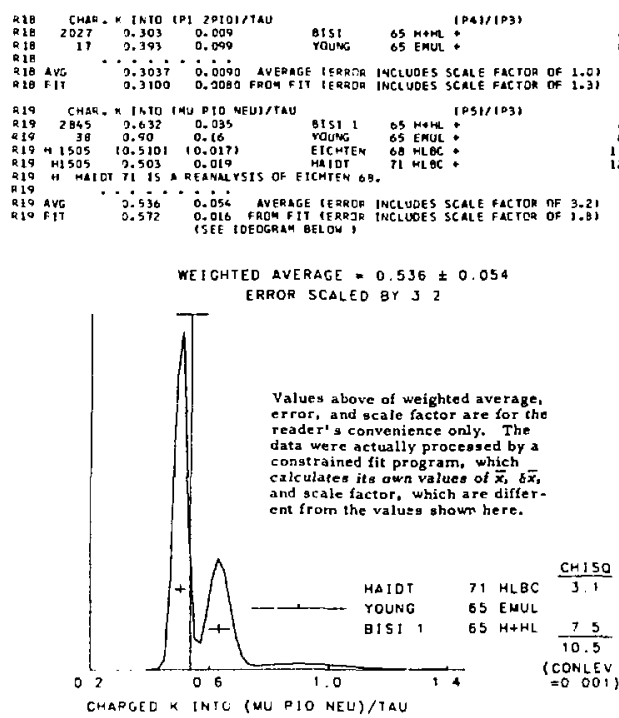

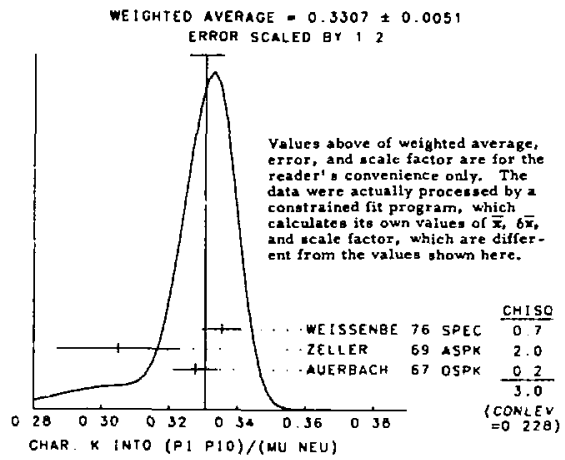

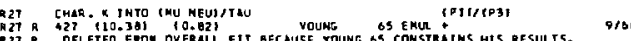

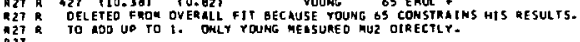

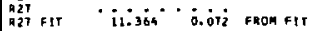

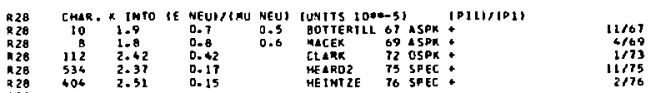

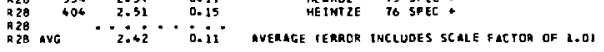

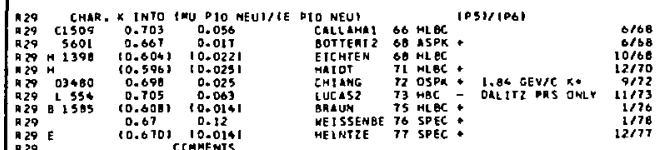

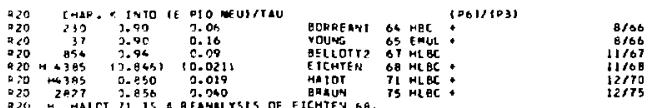

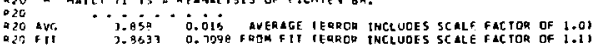

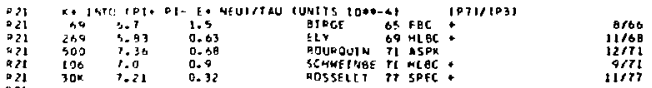

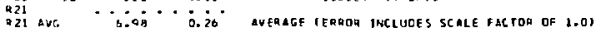

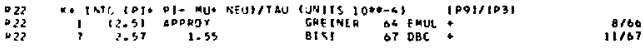

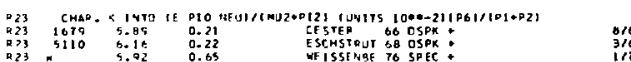

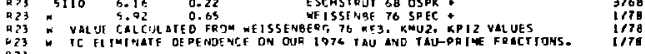

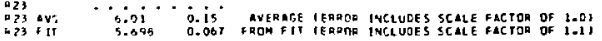

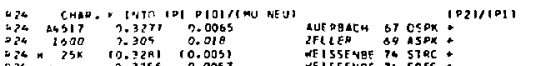

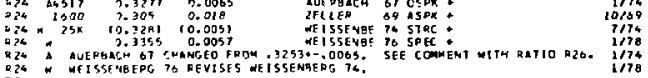

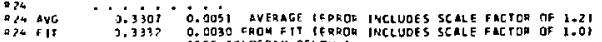

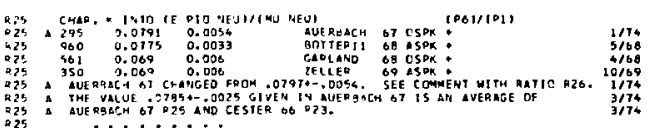

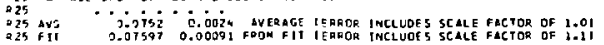

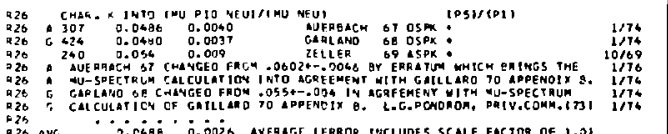

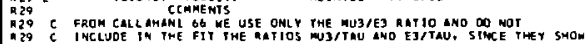

R2 $C$ LARGE OISACAEEMENTS HITH THE REST OF THE ORTA.

R29 H ONLY INOIVIOUAL RATIOS INCLUOEO IN FIT ISEE 19 ANC R2OI.

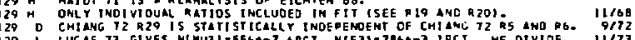

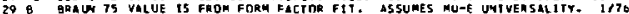

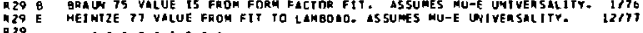

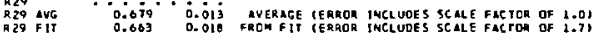

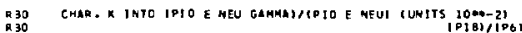

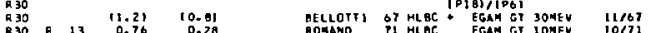

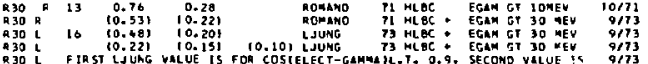

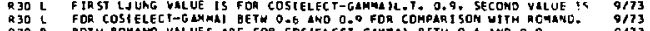

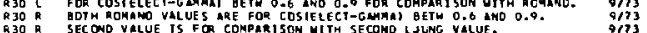

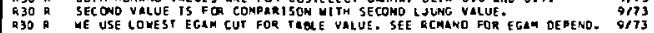

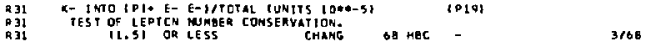

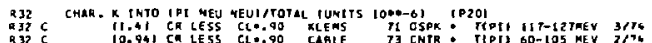

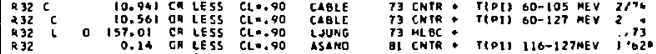

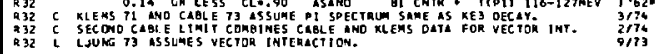

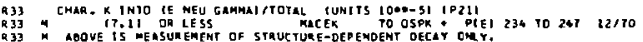

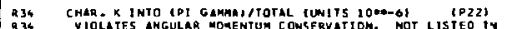

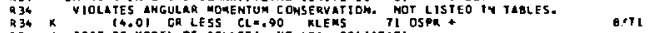

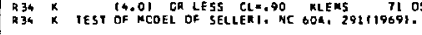

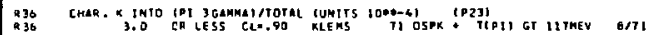

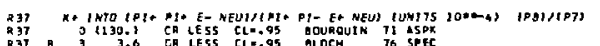

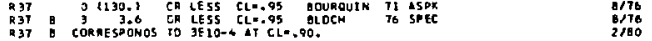

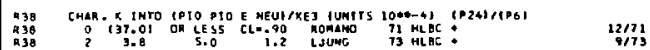

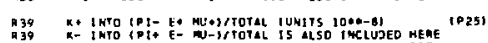

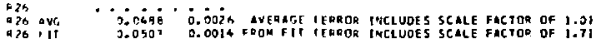




\section{Data Card Listings}

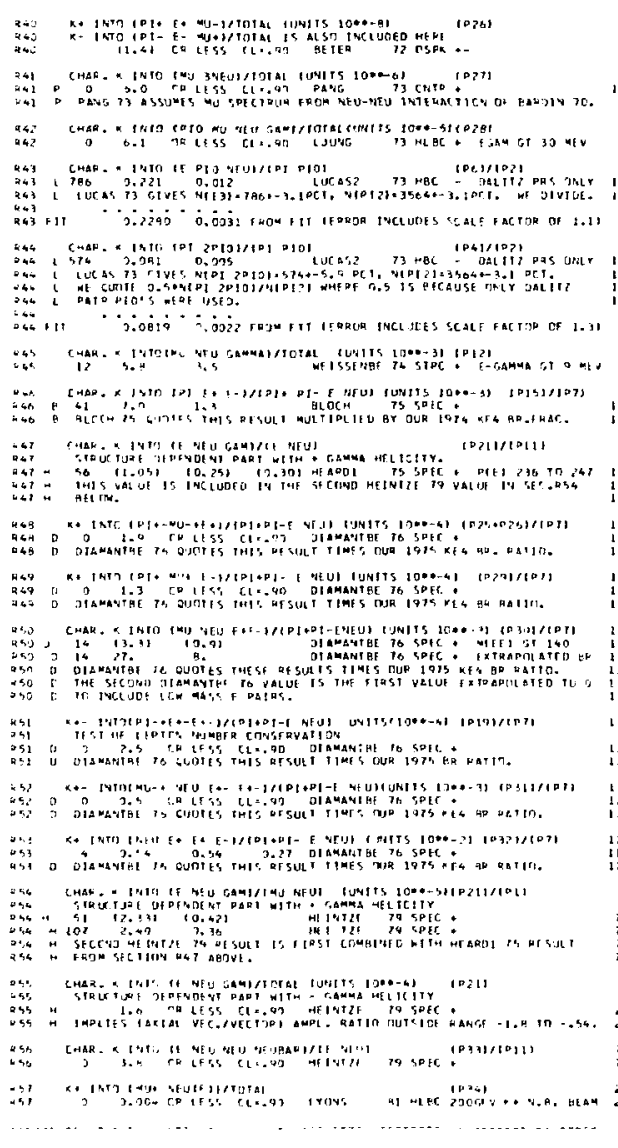

Note on Slope Parametex for $K+3 \pi$ Decays

As was discussfd in Section VI B.l of the text, for the $3 \pi$ decays of the $k$ mesons we list the slope parameter "g" which is defined, as in that section, by

$$
\begin{aligned}
|M|^{2} \times & 1+g \frac{\left(s_{3}-s_{0}\right)}{m_{T_{1}}^{2}}+h\left(\frac{s_{3}-s_{0}}{m_{T+}^{2}}\right)^{2} \\
& +j \frac{\left(s_{2}-s_{1}\right)}{m_{T+}^{2}+}+k\left(\frac{s_{2}-s_{1}}{m_{T+}^{2}}\right)^{2}+\ldots .
\end{aligned}
$$

where

$$
\begin{aligned}
& s_{i}=\left(i_{-K}-\underline{p}_{i}\right)^{2}=\left(m_{K}-m_{i}\right)^{2}-2 m_{K}{ }^{T} \\
& s_{0}=\frac{1}{3}\left[s_{i}=\frac{1}{3}\left(m_{K}^{2}+m_{1}^{2}+m_{2}^{2}+m_{3}^{2}\right)\right.
\end{aligned}
$$

$\mathrm{F}^{\prime} \underline{P}_{i}$ are the four-vectors for the $\mathrm{K}$ and the $i^{\text {th }}$ yion, and the index 3 refers to the odd yion, i.e., the third pion in the decays listed below.

$\prod_{i / m}$

iins

$$
\text { (1) }
$$

We refer to the three possible charged decays as $\tau, T^{\prime}$, and $\tau^{\prime \prime}$ :

$$
\begin{aligned}
& \tau^{+} \quad k^{+}+i^{*}+t^{+} \\
& \tau^{ \pm \pm} k^{ \pm}+\pi \pi^{0} \pi^{0} \pm \\
& \tau^{0} \quad k_{L}^{0} \rightarrow \pi^{+} T^{-} \pi^{0}
\end{aligned}
$$

The measurements of $y$ vary considerably beyond the authors' quoted errors as can be seen in the ideograms associated with the GT+, GT-, and GTP subsections of the $K^{+}$Data Card Listings and the aTo subsection of the $K_{L}^{0}$ Listings.

There is no indication of a CP-violating asymmetry in $K_{L}^{0}$ decay as measured by the coefficient $j$ given in subsection JTO of the $K_{L}^{0}$ Listings.

The nigh-statistics $t^{2}$-decay experiment of MESSNER 74 firds sicnizicant non-zero yuadratic coefflcients $h$ and $k$. CHO 77 , a lower-stalistics $t^{0}$ experiment, obtains results in agrecment with MESSNER 74 but can also obtain good fits with a linear term (g) only. The correlation bet' $3 \mathrm{~cm}^{\mathrm{m}}$ the linear and guadratic coefficients chances the CHO $77 \mathrm{~g}_{\tau}$, from $0.629 \pm 0.017$ (linear fit) to $0.681 \pm$ 0.024 (quadratic fit). nnother experiment,

PEACH 77 , does not observe this correlation and is in ayreement only with the linear fit of CHO 77 .

There is some evidence for a non-zero $k$ coefficient from $i^{+}$experiments. FORD 72 ( $1.5 \mathrm{~m}$ events) have studied $K^{ \pm} \cdot \pi^{ \pm} \pi^{ \pm} \pi^{\ddagger}$ and find that the $x^{2} / D F$ goes from 1.38 to 1.20 for $D F=150$ when the second order and CP-violation terms are addea. 
Stable Particles

\section{$\mathrm{K}^{ \pm}$}

However, the authors state that since their Coulomb correction is larger than the experimental errors and is not well known, it is difficult to interpret these results. DEVAUX 77 also finds a non-zero $k$. Because of the above evidence for quadratic terms, and for consistency in our treatment of $\tau^{\circ}$ and $\tau^{ \pm}$decay, we now include in our averages only those $\tau^{0}$ and $\tau^{ \pm}$experiments for which we have information on the three coefficients $g, h$, and k. Correlations prevent us from comparing fits which do not include these three parameters. For $\tau^{\prime \pm}$ decays we compile $g$ and $h$ only since no experiments measure $k$.

\section{Parametrizations}

In the literature other definitions of slope parameters have appeared. We have converted to the definitions of $g, h, j$ and $k$ in $\mathrm{Eg}$. (1) from whatever experimental quantity has been reported. We give the conversion to the definition (1) for the most widely used parametrications and tabulate the conversion factors for the reader's convenience.

a) For analysis of charged $K^{\prime} s$ and some $K^{0}$ experiments, the expression often used is:

$$
|M|^{2}=1+a_{y} y+b_{y} y^{2}+a_{y} x+e_{y} x^{2}
$$

with

$$
\begin{aligned}
& x=\frac{3 T_{3}-Q}{Q}, \\
& x=\frac{\sqrt{3}\left(\mathrm{~T}_{1}-\mathrm{T}_{2}\right)}{Q}, \\
& Q=\mathrm{m}_{\mathrm{K}}-\sum \mathrm{m}_{\mathbf{i}},
\end{aligned}
$$

The relevant formulae are:

$$
Y=-\frac{3}{2} \frac{s_{3}-s_{0}}{m_{K} Q}+\Delta, \quad x=\frac{\sqrt{3}}{2} \frac{s_{2}-s_{1}}{m_{K}}
$$

with

$$
\Delta=\frac{\pi-m_{3}}{\mathrm{~g}}\left(2-\frac{\mathrm{m}_{3}+\mathrm{m}_{1}}{\mathrm{~m}_{\mathrm{K}}}\right)
$$

and
Data Card Listings

For notation, see key at front of Listings.

$$
\begin{aligned}
& g=\frac{-c_{y}\left(a_{y}+2 b_{y} \Delta\right)}{1+a_{y} \Delta+b_{y} \Delta^{2}}, \\
& h=\frac{c_{y}{ }^{2} b_{y}}{1+a_{y} \Delta+b_{y} \Delta^{2}}, \\
& j=\frac{c_{y} d_{y}}{\sqrt{3}\left(1+a_{y} \Delta+b_{y} \Delta^{2}\right)}, \\
& k=\frac{c_{y}{ }^{2} e_{y}}{3\left(1+a_{y} \Delta+b_{y} \Delta^{2}\right)},
\end{aligned}
$$

with

$$
c_{y}=\frac{3}{2} \frac{m_{\pi^{+}}^{2}}{m_{K^{2}}^{2}}
$$

b) For the analysis of some $k^{0}$ experiments the expression used is

$$
\begin{aligned}
|M|^{2} & =1+2 a_{t} \frac{m_{K}}{m_{\pi^{+}}^{2}}\left(2 T_{3}-T_{3 \max }\right) \\
& +b_{t}\left(\frac{m_{K}}{m_{\pi^{+}}^{2}}\right)^{2}\left(2 T_{3}-T_{3 \max }\right)^{2} .
\end{aligned}
$$

with

$$
\mathrm{T}_{3 \max }=\frac{\left(m_{k}-m_{3}\right)^{2}-\left(m_{1}+m_{2}\right)^{2}}{2 m_{k}}
$$

The ralevant transformations are

$$
T_{3}=-\frac{s_{3}-s_{0}}{2 m_{K}}+\frac{Q}{3}(1+\Delta)
$$

and

$$
\begin{aligned}
& g=\frac{-2 a_{t}-b_{t} c_{t}}{1+a_{t} c_{t}+\frac{b_{t} c_{t}^{2}}{4}} \\
& h=\frac{b_{t}}{1+a_{t} c_{t}+\frac{b_{t} c_{t}^{2}}{4}}, \\
& c_{t}=\frac{2 m_{K}}{m_{\pi^{+}}^{2}}\left[\frac{2}{3} Q(1+\Delta)-T_{3 \max }\right] .
\end{aligned}
$$




\section{Data Card Listings}

c) Other $K^{0}$ authors use the same form of matrix element as given in b) above with a linear terr. only, but define

$$
T_{\max }=\frac{2}{3} Q .
$$

The relevant transformation is then

$$
\underline{\underline{u}}=\frac{-2 a_{u}}{1+a_{u} c_{u}}
$$

with

$$
c_{L}=\frac{4 m_{K}}{3 m_{\pi^{+}}^{2}} Q \Delta
$$

d) older $k^{0}$ analyses were done using

$$
|M|^{2}=1+a_{v} \frac{T_{3}}{m_{k}} \text {. }
$$

The Eelevant transformatior. is then

$$
a=\frac{-c_{v}^{a} v}{1+d_{v} v^{a}}
$$

witr.

$$
c_{v}=\frac{m_{\pi^{2}}^{2}}{2 m_{K}^{2}}
$$

ard

$$
d_{v}=\frac{Q}{3 m_{K}}(1+\Delta)
$$

c) The CP-violating term in $\mid \mathrm{M}^{2}$ for $\mathrm{r}_{\mathrm{L}}^{0} \rightarrow$ $\pi^{+} \pi^{-} \pi^{0}$ experiments has been parametrized in suveral ways. BLANPIED 68 and SCRIBANO 70 use the parametrization given in (b) above with no quadratic term and with an additional CP violating term. BLANPIED 58 parametrizes the cP-violatina term as

$$
20_{B} \frac{m_{k}}{\pi_{\pi^{+}}^{2}}\left(T_{1}-T_{2}\right)
$$

The rolurant transformation is then

$$
j=\frac{\sigma_{E}}{1+c_{t}{ }^{a} t}
$$

with $c_{t}$ as defired in (b) above. SCRIBANO 70 parametrizes the CP-violating term as

$$
\frac{2}{\sqrt{3}} v_{5} \frac{T_{1}-T_{2}}{T_{12 \max }}
$$

where $\mathrm{T}_{12 \max }$ is the maximum kinetic energy of particle 1 or 2 , the charged $\pi^{\prime} 5$, given by

$$
T_{12 \max }=\frac{\left(m_{K}-m_{1}\right)^{2}-\left(m_{2}+m_{3}\right)^{2}}{2 m_{K}} \text {. }
$$

The resulting transformation is then

$$
j=\frac{m_{\pi^{+}}^{2}}{\sqrt{3} m_{K} T_{12 \max }} \frac{\sigma_{s}}{\left(1+c_{t}{ }^{2} t^{\prime}\right.} .
$$

SMITH 70 gives the asymetry

$$
a=\frac{N_{+}-N_{-}}{N_{+}+N_{-}}
$$

where $\mathrm{N}_{+}$is the number of events with $\mathrm{T}_{1}>\mathrm{T}_{2}$ and $N_{-}$is the converse. BLANPIED 68 gives the relation $\sigma_{B}=\alpha / 1.16$ wh. 1 ch allows $\omega_{s}$ to use the transformation to $j$ given above for BLANPIED 68 .

For the reader's convenience we give a table of numerical values for $Q,{ }^{2}{ }_{3 \max }, T_{12 \max }, \Delta, c_{y}, c_{t}$, $c_{u}, c_{v}$, and $d_{v}$, obtained using the masses from the current edition.

\begin{tabular}{llll} 
& $\mathrm{T}^{ \pm}$ & $\tau^{\prime \pm}$ & $\tau^{0}$ \\
\hline $\mathrm{Q}$ & 74.97 & 84.17 & 83.57 \\
$\mathrm{~T}_{3 \max }$ & 48.08 & 53.20 & 53.85 \\
$\mathrm{~T}_{12 \mathrm{max}}$ & 48.08 & 53.99 & 53.12 \\
$\Delta$ & 0.0000 & -0.0790 & 0.0798 \\
$c_{\mathrm{Y}}$ & 0.7895 & 0.7032 & 0.7025 \\
$c_{\mathrm{t}}$ & 0.0962 & -0.0769 & 0.3204 \\
$c_{\mathrm{u}}$ & 0.0000 & -0.2246 & 0.2272 \\
$c_{\mathrm{v}}$ & 0.0400 & 0.0400 & 0.0393 \\
$\mathrm{~d}_{\mathrm{v}}$ & 0.0506 & 0.0523 & 0.0604 \\
\hline
\end{tabular}

\section{References}

see the reference sections of the $K^{ \pm}$and $K_{L}^{0}$ Data Card Listings.

See also the review of T.J. Devilin and J.O. Dickey, Rev. Nod. Phys. 51, 237 (1979), which contains an ralysis of $K \rightarrow 2 \pi$ and $K \rightarrow 3 \pi$ data in terms of traisition amplitudes with appropriate energy dependence. 
Stable Particles

$K^{*}$

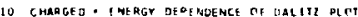

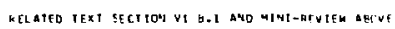

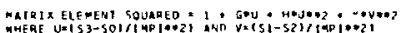

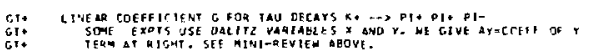

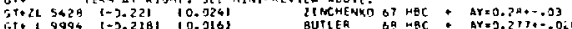

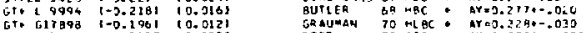

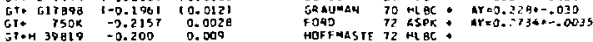

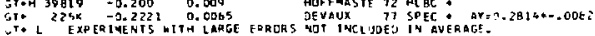

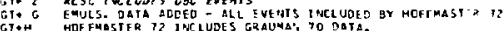

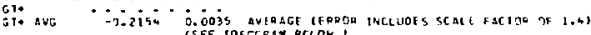

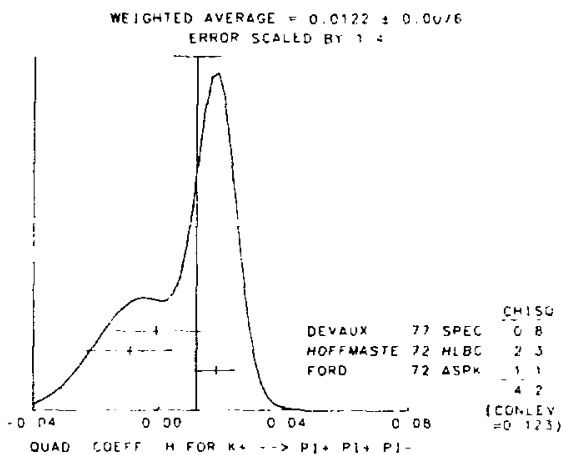

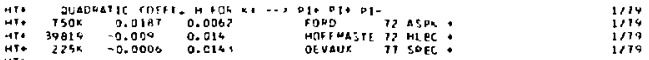

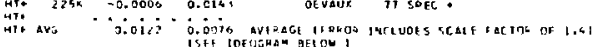
WIGHTED SVEPAGE $=-0.2154 \pm 0.0035$

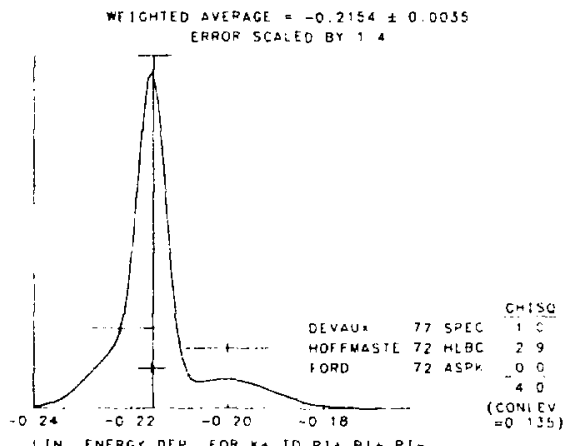

LIN ENERGR DEN FOA $K+$ TOPI+ $A 1+P I-$

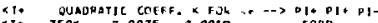

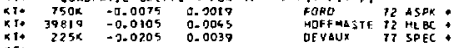

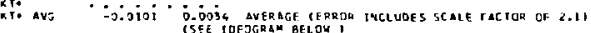

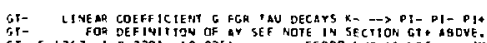

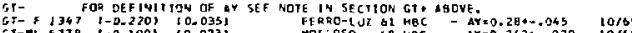

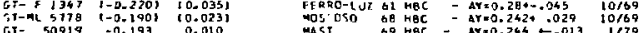

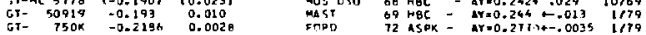

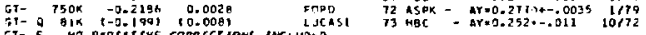

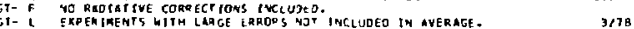

$3 / 78$

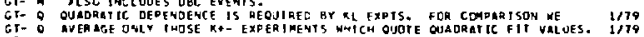

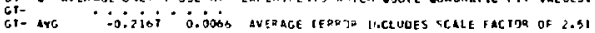

Data Card Listings

For notation, see key at front of Listings.

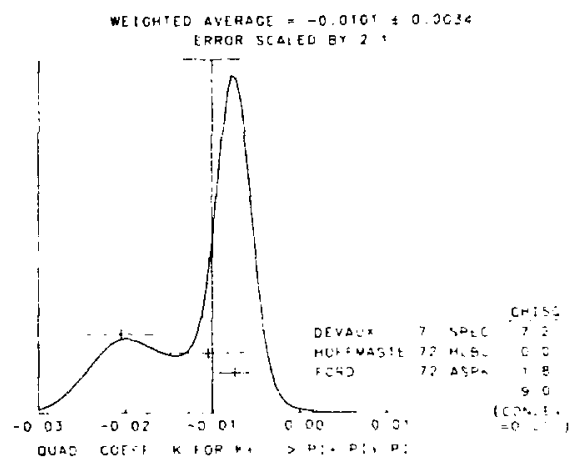

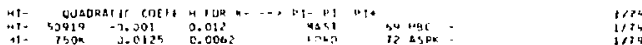

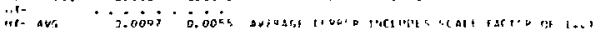

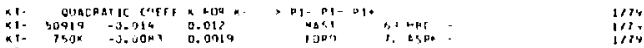

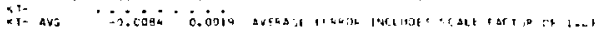

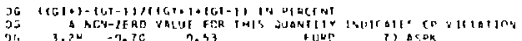

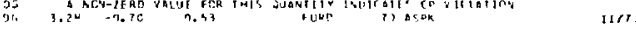

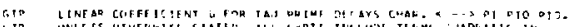

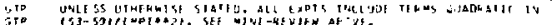

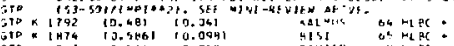

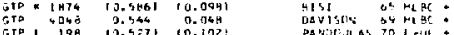

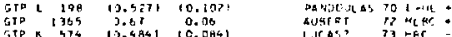

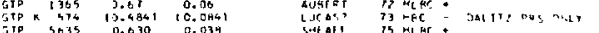

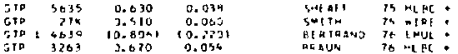

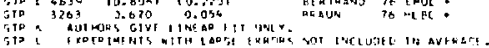

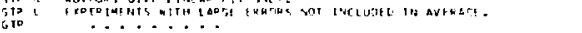

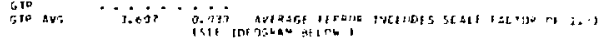

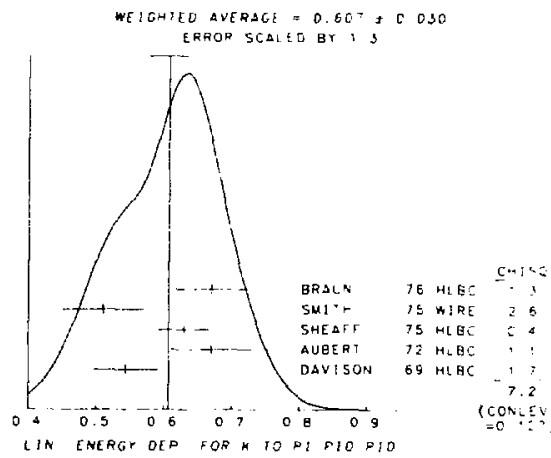

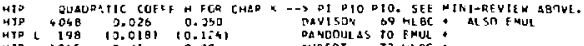

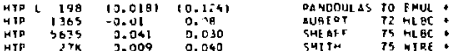

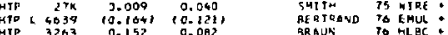

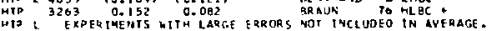

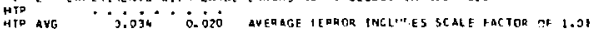




\section{Data Card Listings}

\section{For notation, see key at front of Listings.}

\section{Stable Particles}

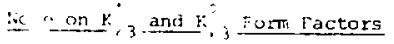 \\ W.finitions of the rarameters $\lambda_{+}, \ell, 01, \lambda_{0}$,
} $f_{S}^{\prime},{ }^{\prime}$ and $\mathrm{H}^{\mathrm{T}} \mathrm{F}_{+}$and a general discussion of the mitrocis of analysis are given in Section VI B.2 of the text.

This note describus the contents of the Lata card isstings for the two $k_{j 3}$ parametrizations, $1{ }^{\prime}, '(\mu)$ and $\left({ }^{+}, \cdot\right)$, which were discussed in the texh. Problems related tc our data entries for atcividual experaments àre discussed and a comparisor: of results i: given.

\section{K. Experiments}

The matrix olement for $\mathbf{k}_{1,3}$ decay, assuming a ! we vector current, is gives by Eq. (2) in section VI B.2 of the text. Yost experiments appear to be compatibis ritl. the assumption that $f_{+}$depends lisearly cet $t$ and that $f_{\text {- }}$ is constant. Only DaLLY $7:\left(k_{.3}\right)$ aprears to require - $\neq 0$ by about three standard deviations). A single data bin at luw $\mathrm{y}^{\text {? }}$ :x.ums ic be remponsible. The effect is not observed if. tis ingh-statistics experiment of DoNALDSON2 74

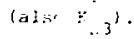

\section{$\therefore+$ (O) farametrization: Y data from $\mathrm{k}_{\text {in } 3}$} deca: are coteredines the $K^{+}$and $K_{L}^{*}$ sections of

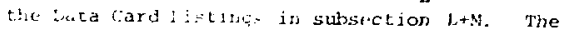
corresconding $:(0)$ values are entered in subsection $X i h, X i B$, or $\because 16$, deferiding on whether Hethod $A, B$, or $C$, discussed belon and in the text, was used. The datu cart. contain the valucs, one-standarddeviation $:$ rrorm $\therefore$, and $\because(d)$, as well as the correlatic. d: $(r) / d{ }$, all indicated on the $e^{-i / 2}$ litelifoid cortour below. The zorrelations are giver on the rizhe side of the $5(0)$ data cards.

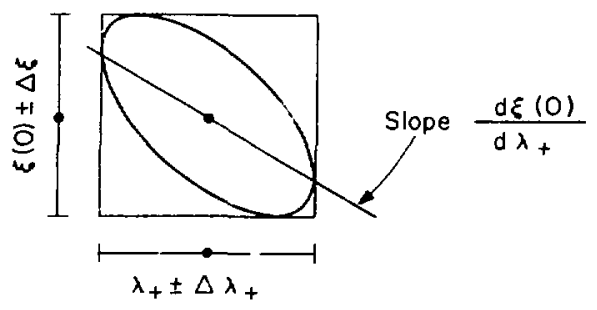

$\times B L 743 \cdot 2682$
- + Paranetrization: This parametrization is used in recent $k_{i 3}$ aralyses. To facilitate comparison between expeximents, we convext earlier experiments from the $\left(\lambda_{+}, i(0)\right)$ parametrization to $\left({ }+{ }^{\prime}{ }_{0}\right)$ whenever possible (i.e., when', and $;(0)$ values, errors, and correlations are giver). The transformation between these parametrizations is:

$$
\begin{aligned}
& y_{y}=x_{+}+a^{2}(0), \\
& \therefore a_{0}^{2}=\left(1+2 a \frac{d z(c)}{a+}\right) c^{2}+a^{2}: z^{2}, \\
& \frac{d i}{d i+}=1+a \frac{d i(a)}{d i+} .
\end{aligned}
$$

where $a=m_{m}^{2} i\left(m_{K}^{2}-m^{2} ;\right.$. The: value, the oncstandard-deviation error $\because ;$, and the correlation $d{ }_{0} / d{ }^{2}$ are given in subsection 1.0 of the data cards.

We also convert $1,{ }_{+}$, results into the : ${ }_{+}$, 5(0)) parsatrizat jor whenever isojele so that subsection LO is ersentially equivalcut to tho three subsections XIA, XIB, and YIC.

Inoividual analyses have ascex a variot $\because$ of parametrizations. Problems arlse when triny to express their results in terms of the :arimetrizations used iere. The discussion of these problems Is divided into three sections correstording $L_{1}$, the three methods of a:alyses discussed in the rext.

Method A: Lalitz plot analyses and :2:0n spectrum analyses usually decermine ' + and ' f'! (or ' ${ }_{0}^{\prime}$ values, errors, and correlation. Such measurements are enterod in the $L+::$, XIA, and $L i$ subsections. They give rise to the crror cilifsec shown in Figs. 1 and 2 . These are approximatiors to likelihoou contours.

Some analyses of this type $f i x+$ and setermite $5(0), * 9 .$, CARPEMTER 66 and PEACi 73 (both $x_{j}^{*}$ ). We enter $\bar{f}(0)$ and $a f(0) / d t_{+}$in tion xis section a.xd quve the fixed ? value in the data card footiote. The $\xi(0)$ error is parenthesized because it icos not include the ancretainty in the vulue of " + . Thes, resulcs, uransformed to : measurements, aive riss to brnas in Fic. 2. These ban!s are also approximations tr tie likeliliood, ontours. The actua

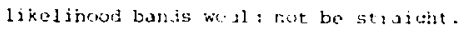


Stable Particles

$\mathrm{K}^{*}$

In some cases, we alter on error from its published value in order to obtain an error ellipse with a width which matches the error in $\zeta(0)$ for fixed ' ${ }_{+}$. Thase adjustments are noted in the $f(0)$ data card footnotes, e.g., for CALLAHAN 166 and HAIDT $71\left(K^{+}\right.$subsection XIA), where the published errors and correlation violate the constraint $\left|c_{j}\right| \therefore 1$ on the normalized correlation coefficient $c_{i c}$ given by

$$
c_{y z}=\frac{i j+}{i j} \frac{\mathrm{d} j(0)}{\mathrm{d} \eta_{+}} .
$$

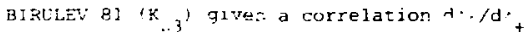
whici, while not unphysical, is so large that the resulting crror ellifse is unreasonably narrox. As a result, in the fit to be discussed, BIRLLEV H] Would dominate even DFHALDSON2 74 whech has 10 times the Blplutiv 81 statistirs. To prevent this, wh make the assumption $d \cdot / d_{+}=0$, giving the broat errug elligse showr in Fig. 2.

In some cases, e.g., BRÃuN 1 73, the paranetri-

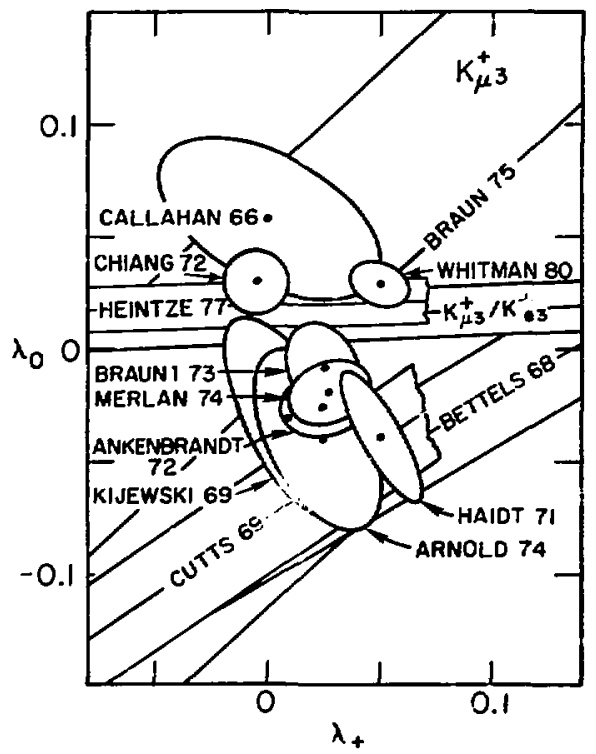

Fig. 1. One-standard-deviation $\left(e^{-1 / 2}\right)$ jikelihoud cortours in the $\left({ },{ }^{\prime}{ }_{0}\right)$ plane for $\mathrm{K}_{\mathrm{U}_{3}}^{+}$.
Data Card Listings

For notation, see key at front of Listings.

zation used is ${ }^{2}, \varepsilon, \varepsilon(0), \varepsilon\left(t^{*}\right)$, where $t^{*}$ is the weighted average of $t$ with heighting according to the sensicivity to $:$. In this case we do not use

$\xi(0)$. It is a badiy determined parameter comparable to $\lambda$ or the slope of $i(t)$. Instead, we use

$$
\begin{aligned}
& E(0)=E\left(t^{*}\right)\left(1+t_{+} t^{*}\right) \\
& \frac{d \xi(0)}{d \lambda_{+}}=\frac{d_{3}\left(t^{*}\right)}{d_{+}^{*}}\left(1+t_{+} t^{*}\right)+i\left(t^{*}\right) t^{*}
\end{aligned}
$$

with the BRALN 173 values, $+=0.027, ;(6.5)=$ $-0.34 \pm 0.20$, and $\mathrm{d} \equiv(6.6) / \mathrm{d}_{+}=-14$, we cbtain

$$
E(n)=(-0.40 \pm 0.24)-15\left(y_{+}-0.027\right):
$$

or for their fitted ${ }_{+}=0.025=0.017$, we get $5(0)=-0.36=0.40$.

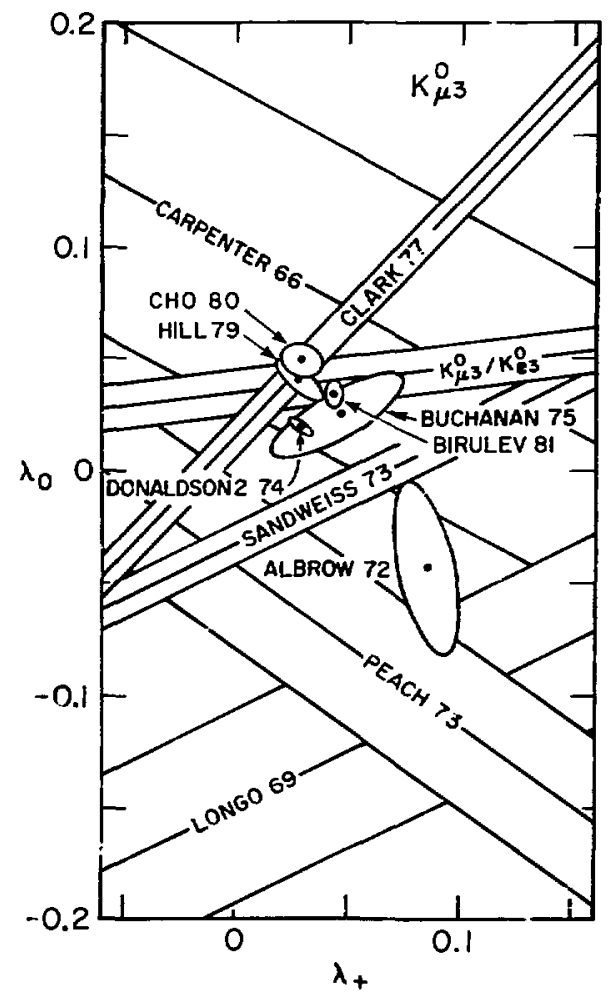

Fig. 2. One-standard-deviation $\left(e^{-1 / 2}\right.$, Jikelihood contours in the $\left(A_{+}, A_{0}\right)$ plane for $x_{j 3}^{*}$. 


\section{Data Card Listings}

For notation, see key at front of Listings.

Method R: Branching ratio experiments cannot determine $\lambda_{+}$and $\xi(0)$ simultaneously, but simpiy fix a relationship between them, given in section VI B. 2 of the text. Results are usually quoted as values of $\xi(0)$ at fixed $\lambda_{+}$. We list these results in subsection XIB, but we do not average them because the $\lambda_{+}$values differ. Instead, we compute a combined result by using the relations in the text and our fitted values of $\Gamma\left(K_{\mu_{3}}^{ \pm}\right) / \Gamma\left(K_{e_{3}}^{ \pm}\right)$ars $\Gamma\left(\mathrm{K}_{\mathrm{H} 3}^{0}\right) / \Gamma\left(\mathrm{K}_{\mathrm{e} 3}^{0}\right)$, which include the branching ratios from these experiments. The branching ratios from our 1980 edition and the results for $\zeta(0)$ and $\lambda_{0}$ evaluated at $\lambda_{+}=0.030$ are

\begin{tabular}{lcc} 
& $\mathrm{K}^{ \pm}$ & $\mathrm{K}_{\mathrm{L}}^{0}$ \\
\hline$\Gamma\left(\mathrm{K}_{\mu 3}\right) / \Gamma\left(\mathrm{K}_{\mathrm{e} 3}\right)$ & $0.663 \pm .018(\mathrm{~s}=1.7)$ & $0.695 \pm .017$ \\
$\xi(0)$ & $-0.20 \pm .15(\mathrm{~s}=1.7)$ & $+0.08 \pm .13$ \\
$\mathrm{~d} \xi_{(0) / \mathrm{d} \lambda_{+}}$ & -11.9 & -10.3 \\
$\lambda_{0}$ & $+0.014 \pm .012(\mathrm{~S}=1.7)$ & $+0.037 \pm .011$ \\
$\mathrm{~d} \lambda_{0} / \mathrm{d} \lambda_{+}$ & +0.04 & +0.12 \\
\hline
\end{tabular}

The current (1982 edition) values differ only in the addition of the CHO $80 \mathrm{~K}_{\mathrm{L}}^{0}$ branching ratio measurement. We include their Dalitz-plot-analysis results in our overall form factor fit, and since their branching ratio measurement is not an ir. Jependent result, we do not include it here. The scale factor $s$ in the above table is the amount by which the error has been multiplied in order to compensate for discrepancies in the branching ratios. These $\lambda_{0}$ results give rise to the $\mathrm{K}_{\mathrm{H}_{3}} / \mathrm{K}_{\mathrm{e} 3}$ bands in Figs. 1 and 2 .

Method C: Polarization experiments measure $\langle\xi\{t\}\rangle$, the weighted average of $\xi(t)$ over the $t$ range of the experiment, where the weighting accounts for the variation with $t$ of the sensitivity to $\xi_{3}(t)$. Such measurements are entered in subsection XIC.
To reinterpret these results in the $\left(\lambda_{+}, E(0)\right)$ parametrization, we recognize that ${ }_{+}=0$ corresponds to $\xi(t)$ constant (always assuming $\lambda_{-}=0$ ) so that

$$
\left.\xi(0)\right|_{\lambda_{+}=0} \equiv\langle\xi(t)\rangle
$$

The correlation with $\lambda_{+}$is given by the following relations (valid for small $\lambda_{t}$ ):

$$
\begin{gathered}
\xi(0)=\langle\xi(t)\rangle\left(1+\lambda+\left(\frac{t}{m_{\pi}^{2}}\right)\right\rangle, \\
\frac{d \xi(0)}{d !+} \approx\left\langle\xi_{1}(t)\right)\left(\frac{t}{m_{T !}^{2}}\right),
\end{gathered}
$$

where $\left(t / m_{\pi}^{2}\right)$ is the average value of $t$ weighted by the sensitivity to $\xi(t)$. These results, transformed to $\lambda_{0}$ and $\mathrm{d} \lambda_{0} / \mathrm{d} \lambda_{+}$values, are entered in subsection LO and give rise to bands in Figs, 1 and 2 . In Pigs. 1 and 2, we do not include those polarization measurements for whic $d \varepsilon(0) / d\rangle_{+}$ is not ontainable. Also we do not include the MERLAN $74\left(\mathrm{~K}_{\mu 3}^{+}\right)$polarization resul because the signs of $\xi,(0)$ and $d \xi(0) / d \lambda_{+}$are ofrosite, w! ereas the above equation requires them to be the same (since $t: 0$ ).

Comparison of $k$ experinents: Figures 1 and 2 show the likelihood contours in the $\left\langle\lambda+{ }^{\prime}{ }_{a}\right\}$ plane for $k_{\mu 3}^{+}$and $k_{\mu j}^{3}$ respectively.

Wost $\mathrm{K}_{\mu 3}^{+}$Dalitz plot results (ellipses) shown are fairly consistent and appear to cluster between the $k_{1.3} / k_{e 3}$ result and the polarization results of BETTELS 68 and CUTTS 69 . However, the latest experiment, WHITMAN 80 , finds larger values of ${ }_{0}$ and $\lambda_{+}$than most others.

The $k_{\mu 3}^{\prime \prime}$ results are even less consistent. The latest results, HILL 79, CHO 80, and BIRULEV 81, are not in very good agreement with the earlier highprecision experiment of DONALDSON2 74 .

$x^{2}$ fits to the results show in Fig. 1 and Fig. 2 yield the following values for $\lambda_{+}$and $\lambda_{0}$. The corresponding values of $\xi(0)$ are also given. 
Stable Particles

$\mathrm{K}^{ \pm}$

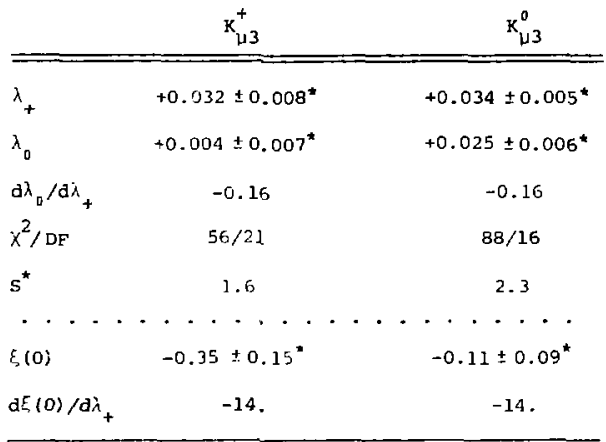

"All errors have been increased $b_{i}^{*}$ the scale factor $S=\left(X^{2} / D F\right)^{1 / 2}$ to take into account the discrepancies between measurements.

In view of the large $x^{2} / \mathrm{DF}$, the fit results should be taken with a grain of salt. The largest contributors to $x^{2}$ in the $k_{133}^{+}$case are WHITMAN 80 with 12, CHIANG 72 with 8.3 , the polarization results, BETTELS 68 with 7,4 and CUTTS 69 with 5.9, and the " spectrum result of ANKENBRANDT 72 with 5.3. In the $k_{\nu 3}^{u}$ case the largest contributors are the polarization results of SANDWEISS 73 with 21, LONGO 69 with 14, and CLARK 77 with 8.4, and the Dalitz plot results of ALBROW 72 with 12 , CHO 80 with 10, DONALDSON2 74 with 6.0 , BfRULEV 81 with 5.8, and PEACH 73 with 5.8. All other $x^{2}$ values were less than 5 .

The DONALDSON2 74 result

$$
\begin{aligned}
& \lambda_{+}=0.030 \pm 0.003 \\
& \lambda_{0}=0.019 \pm 0.004
\end{aligned}
$$

clearly dominates the statistics in the $\mathrm{K}_{\mathrm{\mu} 3}^{\mathrm{o}}$ case. The + value is consistent with the $k_{e 3}$ value of ${ }^{\prime}{ }^{\prime}$, and with the pole approximation

$$
f_{+}(t)=f_{+}(0) \frac{m_{K^{*}}^{2}}{m_{K^{*}}^{2}-t} .
$$

Their $f_{0}(t)$ extrapolates linearly to the callanTreiman point. It is less than two standard deviations from the $k_{j 13} / K_{e 3}$ result.

$$
\text { Ke3-Experiments }
$$

The $f_{\text {_ }}$ term of the matrix element [Eq. (2) text

\section{Data Card Listings}

For notation, see key at front of Listings.

Section VI B.2] can be neglected for $\mathrm{K}_{\mathrm{e}}$ because it is proportional to the lepton mass. The $E_{f}$ term is usually assumed to be linear in $t=q^{2}=\left(P_{K}-P_{\pi}\right)^{2}$, the square of the four-momentum transfer, i.e.. the effective mass of the lepton pair. We quote the linear coefficient $\lambda_{+}^{e}\left(L_{+}+E\right.$ on the data cards). There has been some suggestion of departure from linearity [CHIEN $71\left(\mathrm{~K}_{\mathrm{e}}^{0}\right)$ and ' sunet, Gaillard, and Gaillard ${ }^{l}$ - Review] but no mpelling evidence. The $\lambda_{+}$results are fairly consis $\cdots n t$ and the average values are

$$
\begin{array}{ll}
\mathbf{k}_{\mathrm{e} 3}^{+}: & \lambda_{+}=0.0285 \pm 0.00 \\
\mathrm{k}_{\mathrm{e} 3}^{0}: & \lambda_{+}=0.0300 \pm 0.0016 \quad(5=1.2)
\end{array}
$$

where the $\mathrm{K}_{\mathrm{e} 3}^{0}$ error has been multiplied by the scale factor 1.2 to compensate for in-onsistencies (see ideogram in $K_{L}^{0}$ section $L+E$ ).

See also the excellent reviews of . :illard and Chounet, ${ }^{1}$ Chounet, Gaillard, and Gailk : , ${ }^{2}$ and Pondrom, 3

\section{References}

1. M. K. Gaillard and L. M. Chounet, "K ${ }_{23}$ Form Factors," CERN 70-14 (May 1970), and Phys. Letters 32B, 505 (1970).

2. L. M. Chounet, J. M. Gaillard, and M. K. Gaillard, Physics Reports 4C, 199 (1972).

3. L. G. Pondrom, "Weak Decay Processes," Proc. Particles and Fields 1976, BNL, Oct. 6-8, 1976.

$$
10 \text { CHARGEO K FORH FACTDRS }
$$

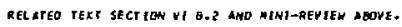

IN THE FOAM TACTOK COMAEATS, THE FOLLONING ABEREVIATIONS ARE USED. F+ WO F- ANE FORW FACTORS FOR THE VECTOR MA TAIX EL EMEMT

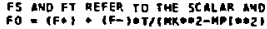

L+. L- AHO LO ARE THE LTHEAR EXDAWS ION COEFFS. DF FO. F- ANO FO.

L+ REFERS TO THE KNVI YALUE EXEEPT IT THE KES SECTIONS.

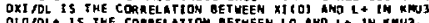

OTOFOL. IS THE CORRELATION BETMEEN LO AHO LP IN KMU3.

on = DOA ITZ DLQT AYALYSIS

P1 = Ol SPETTRUM ANALYSIS

MU = MU SPECTRUM ANALYS IS

DDL = MU DCLAIZAAT TOM ANALYSIS

to inazsts

$E$ = POS ITRON OR ELECTRON SPECTMUN ANALYSIS

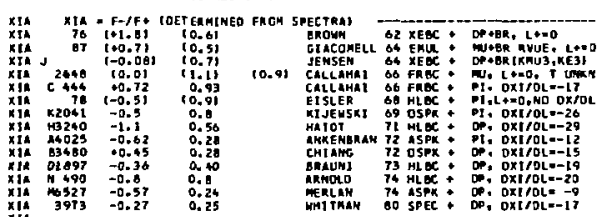

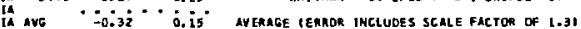

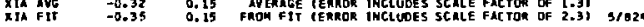

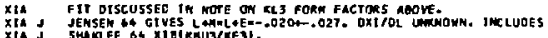

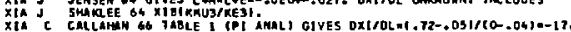




\section{Data Card Listings}

\section{Stable Particles}

For notation, see key at front of Listings.

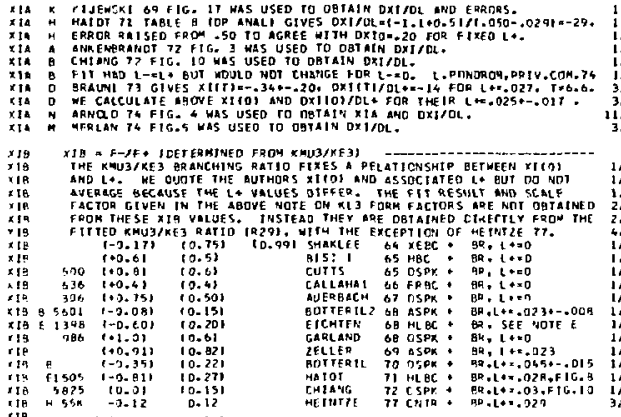

IP Fit - O.

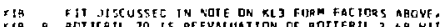

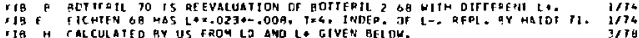

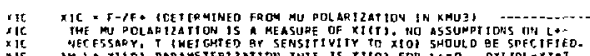

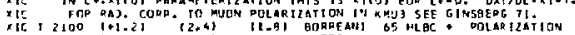

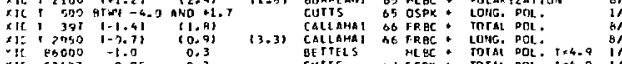

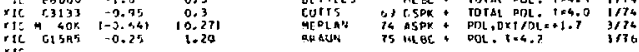

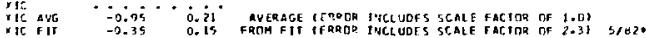

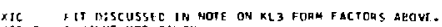

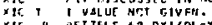

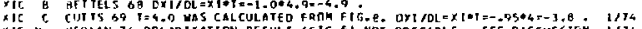

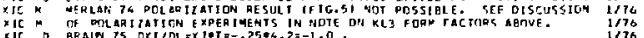

\begin{tabular}{|c|c|c|c|c|c|c|c|}
\hline $\begin{array}{l}2648 \\
308 \\
2950 \\
6000 \\
3133 \\
709\end{array}$ & 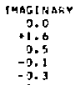 & $\begin{array}{l}\text { PAg1 } \\
1.0^{\text {IF }} \times \\
1.3 \\
1.4 \\
0.3\end{array}$ & $\begin{array}{l}0.5 \\
0.4\end{array}$ & 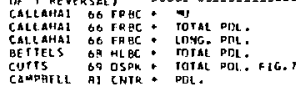 & 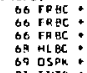 & 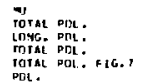 & $\begin{array}{l}1 / 74 \\
1 / 78 \\
1 / 74 \\
1 / 74 \\
1 / 74 \\
1 / 42\end{array}$ \\
\hline
\end{tabular}

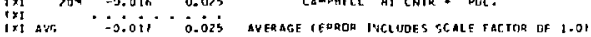

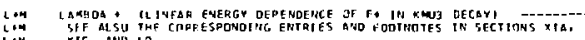

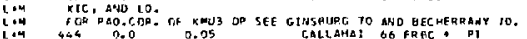

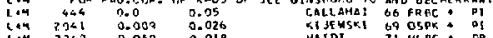

i.4 $32430.053 \quad 0.019$

$\begin{array}{rrr}1.4 & 3490 & -0.006 \\ 1.4 & 1097 & 3.023\end{array}$

$\begin{array}{rrr}1.4 & 890 & 0.025 \\ 1.4 & 5527 & 0.027 \\ 104 & 3974 & 0.059\end{array}$

$\lim _{i \rightarrow 4}$ avi

0.019
0.015
0.017

0.017

AMTET

CHFANE 72 ESTPK:

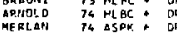

0.013

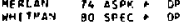

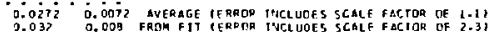

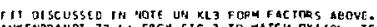

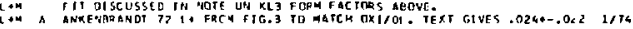

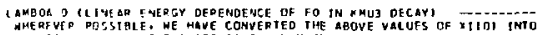

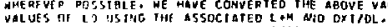

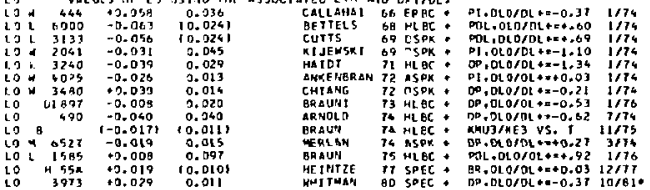

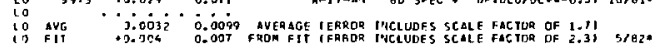

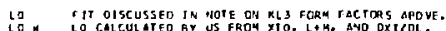

IO

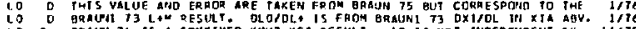

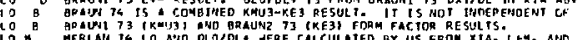

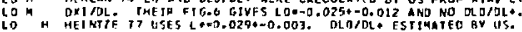

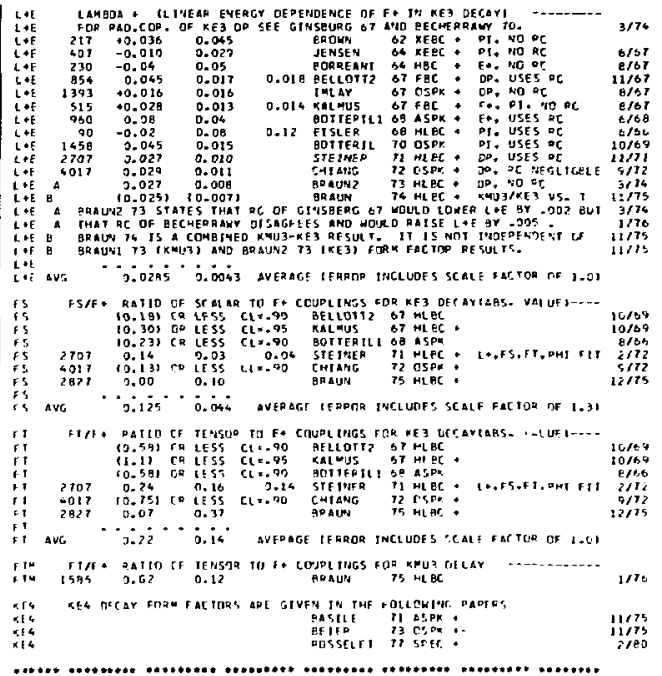

RETEDFNCES FDU CHADCED

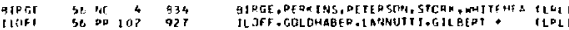

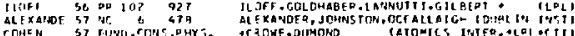

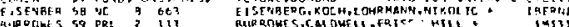

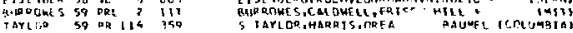

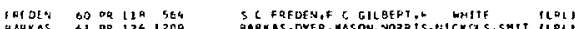

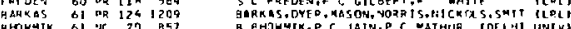

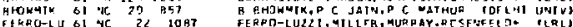

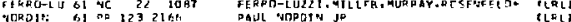
प⿴囗十

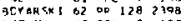

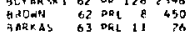

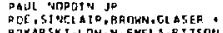

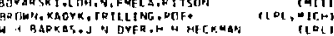
BCRREANI OS DL 12 123

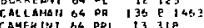
CCIME

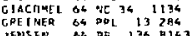

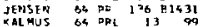

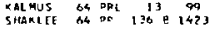

GIRG OS OR 139 a 1600

AISI, 65 HE IS 760

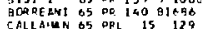

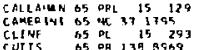

OF aAFCO GS DQ 240 E 1430

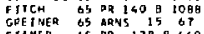

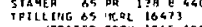

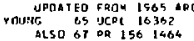

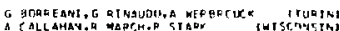

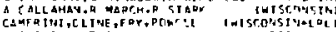

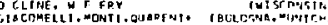

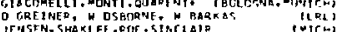

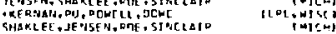

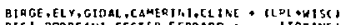

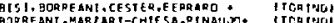

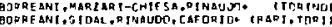
a CALLAHAitD CiINE

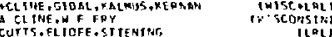

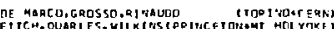

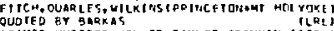

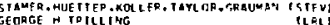

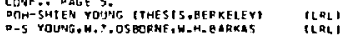

CALLAHA OS OR 1501153

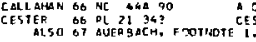

ADERGACH OT PE 155 15OS

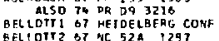

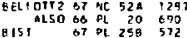

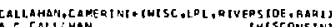

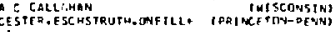

ROT TERI

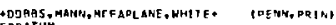
ERRATUM

geliftTl.pulita

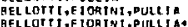

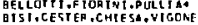

M IILANS

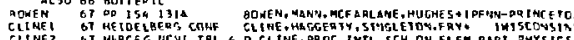

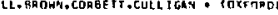

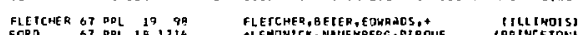

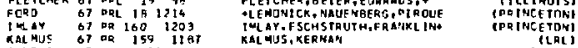

LINCHENK B? RUTERSITHESISI 
Stable Particles

$\mathrm{K}^{*}, \mathrm{~K}^{0}$

BETTELS 6Q NC SOA 1106

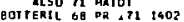
ODITERI GQ DQ 174 2601

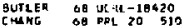
CAEN OE PRT 20 P3 EISTER OQ DR 1691000

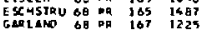
MAScose ab TMESTS CUTTS 69 OH 1041390 DAvisOn to Da 180 133 ELY
EMEASON 69 PRL 23393

HER 20 O9 AR 1861493 DJJENSKT O9 UCALC MLEX ALS OQ OAL 17 PQL 22

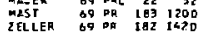
EOTTEAIL $70 \mathrm{PL} 318325$
FOAD GRAUMAN TO PO DI 1277 YAC EK 4AC EK TO PH DI 1249 MAL TSEV 70 5.J4P 10 ST BASILE T1 PL 368 BI9

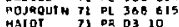

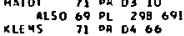

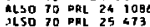
KUNAELAA 71 PQ $34 \mathrm{P}$ GeS OTI
ROMANO 11 PR D3 52
31
PLE 525

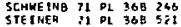
$\triangle$ DA AMS 72 PQL 291114

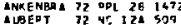

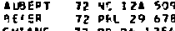

Clark 72 Do 101274

EOWLROS 72 PR O5 2720

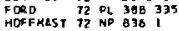

AERAMS 73 PRE $305 C$ BACKEAST 73 OL 436431 BPAUNL I3 of a BRAUNZ 73 OL 478185 BRAUNZ 73 DL 478185

LJUNG T3 DR DB 1301

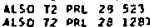
ALSO O9 DR: 23326 LUCAS1 73 OR OQ 715

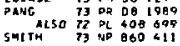

ARHOLD 74 OA DOL2Z1 BRAUN 74 FL 519 353 ALSO T3 THESIS TUNDUAL." KUNSEL TA T4 OR CQ 2469
WERLAN T6 PR D9 107 UETSSENB 74 PL 489476 $\begin{array}{lll}B L O C H & 75 & 560 \\ B R A U N & 201\end{array}$ ERAUN 75 NP BS4 210

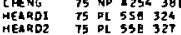
SHEAFF 75 DH $31225 T^{\circ}$ 5 जITH 55 HD 09145

OERTALNO 76 NP 9114387

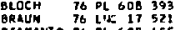

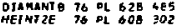
SHI TH TO NP Q109 173

A BRAMS 71 DR OLS 2? OEVAUX 71 PlO B126 11

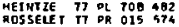

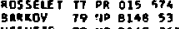

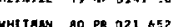

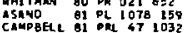

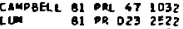

(VDUS A1 $20 \mathrm{HY}$ C10 215

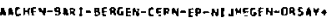
GOT FERILL. BRRAN, CLEGC, CCREETT : COXFQRO

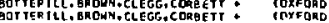

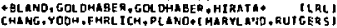

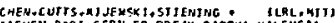
AACHEN-BLP I-CEAN-EP-ORS AY-PADOVA-VALEHC TH

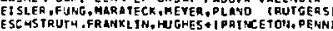

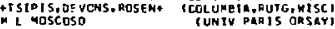
-STIENIJT, VIECAND, DEUTSCH CLAL.MIJ) CUITS,STIENING, HIEGAHO. CFUTSCH ILRL, MITI HEACESTOW, GARKAS, E VANS, FUNG. POPTERE CUCA

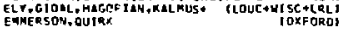

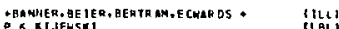

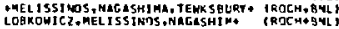
MECEK, MAN, MC FARL INE, ROBEAT S + PEAN, TEMPLE

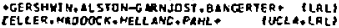

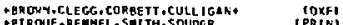

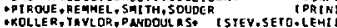

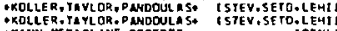

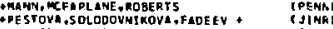

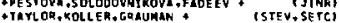
4BREHIN, DI LNANT-BER CER, RUNZ * ISACL\&GEVAI

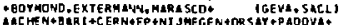

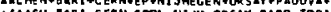
\& AACH, BAR I, C ENN, EDOL, NT JH, OR SAY, DADD, TORL

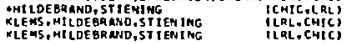
P. OUNSELMAN
OTT.PPITCHLRD
(KYONING)
(LOOM)

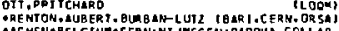

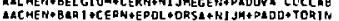

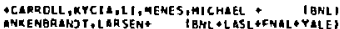

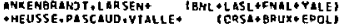

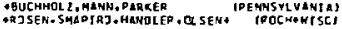

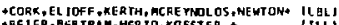
- BE IFR. EERTRAH, HERZ O. KGESTEA + IILL)

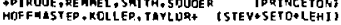
ACAR2CLL, KYCRA, LI, MENES, NICHAEL P 19NL)

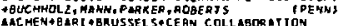

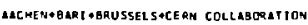

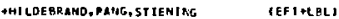
O.L JUNG. D.CLINE O.L JUNG D.LJUNG (UISC) CAMER IHI, L JUNG, SHEAFF, CL INE PYISC) LUEAS.TAFT.WILL'S
LUCAS.TAFT,WILIS

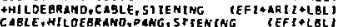

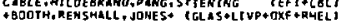
C L ARNOLO.B P ROE.D SIMCLAIR IMICHI

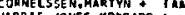

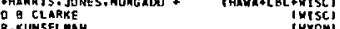
R. KUNSELMAN (YYOM)

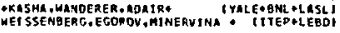
- GREMIN, GUHCE, DEVAUX, IS ACL+GEVL,

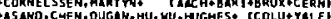

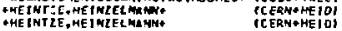
M. SHEAFF
*BOOTM, AE NSHALL, SONES+ ICLASALIVP+OXFORMELI

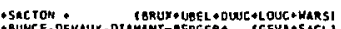
-BUACE, DE VAUX, DIAMANT-BERGERA TCEVAESACLI DJ IMANT-BERGER, BLDCH, DEVAUX + ISACL + GEYA]

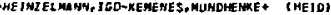

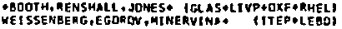

- CARROLL, KrCIR+LI, MICHAEL, mOCKETT + CBNLI

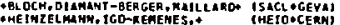

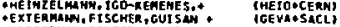
IMOVDOKIAES

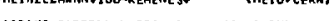

ABRAMS . CAREOLL, KYCIE.LIO I1LLC+ENL+ILLI

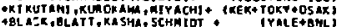

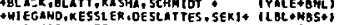
+RLORJAR, WYATT TOXFI MINATIOAS NOT MEFEREED TO IN THE OATR CMMOS

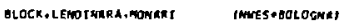

Data Card Listings

For notation, see key at front of Listings.

\begin{tabular}{|c|c|}
\hline 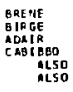 & 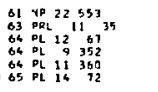 \\
\hline 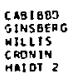 & 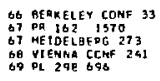 \\
\hline $\begin{array}{l}\text { BARDIN } \\
\text { BECHERA } \\
\text { GEARIAG } \\
\text { GEILLAD } \\
\text { GINSEERG }\end{array}$ & 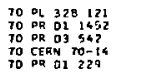 \\
\hline $\begin{array}{l}\text { INS BERG } \\
\text { HOUNET }\end{array}$ & 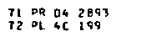 \\
\hline
\end{tabular}

Dapers not referred to in oets cards

APENE, EGAROT, QN IST

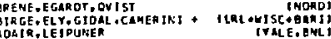
CAB19gO, MAK SFMOWIC 2 CABIBEO, MAK SY MUHICZ

CABIBBO

EOMRRD 5 GINSBERG TU. MASS BOSTONI

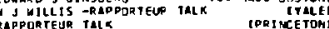

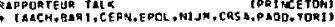
GERDIN,gILETIKT, PONTECER VD T. BECTERASWT

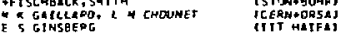
F 5 THNSERE $\left(\begin{array}{lll}1 \\ 111\end{array}\right.$

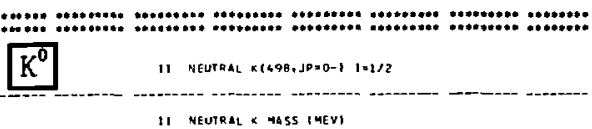

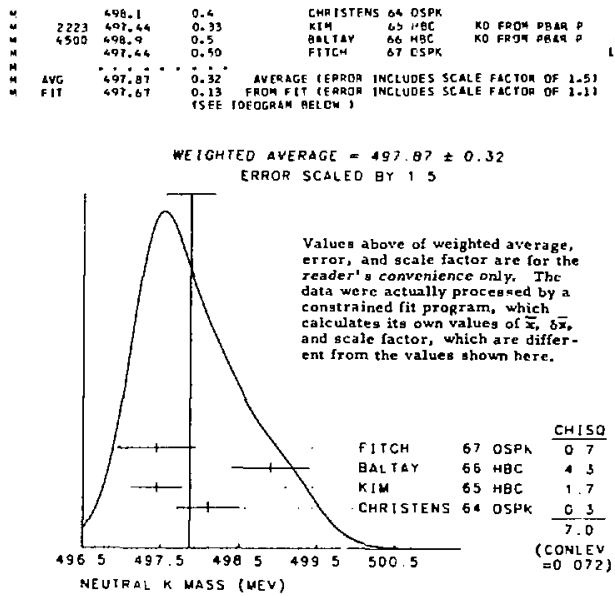

11 |KO\} - $\{K+-\}$ MaSS OIFRER ENCE |MEY

\begin{tabular}{|c|c|c|c|c|c|c|c|c|}
\hline $\begin{array}{r}8 \\
717\end{array}$ & $\begin{array}{l}3.9 \\
5.4 \\
3.50 \\
3.71 \\
3.65\end{array}$ & $\begin{array}{l}0.6 \\
1.1 \\
0.25 \\
0.35 \\
0.21\end{array}$ & & $\begin{array}{l}\text { ROSENFEL } \\
\text { CARHFORD } \\
\text { BURNSTEIM } \\
\text { KIA } \\
\text { HIL }\end{array}$ & 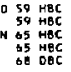 & 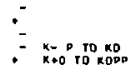 & & $\begin{array}{l}0 / 68 \\
3168\end{array}$ \\
\hline $\operatorname{avg}_{i+1 \mathrm{~T}}^{\operatorname{Nvg}}$ & 3.92 & 0.14 & $\begin{array}{l}\text { AVERAGE } \\
\text { FROM FIT }\end{array}$ & $\begin{array}{l}\text { E IERNOR } \\
\text { T IERROR }\end{array}$ & $\begin{array}{l}\text { INCLUDES } \\
\text { INCLUDES }\end{array}$ & $\begin{array}{l}\text { SCALE FACTOA OF } \\
\text { SCALE FAC TOA OF }\end{array}$ & $\begin{array}{l}1.03 \\
1.11\end{array}$ & $3 / 820$ \\
\hline
\end{tabular}

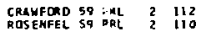
RASENEEL SA DPL 2 TI 110

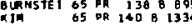
BALTAY BO PQ $142 \quad 532$

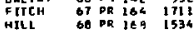
REFERENCES FOR NEUTRAL * CRAMFORD, CREST1, GOOD.STEVENSCN, TICHO CLACI

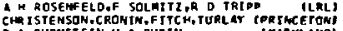

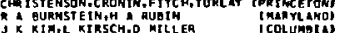
BaL TAY, SANOME ISS, S rONEHTLL - IYALE BMLI

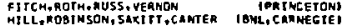


Data Card Listings

For notation, see key at front of Listings.

$K_{s}^{0}$

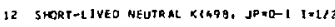

12 KOS AEAN LTFE TUNTS $300 \%-10$ SET

MOS MEAN LTFE IPQE - 1971 EXPEPIMENTS

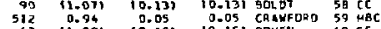

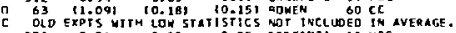

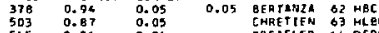

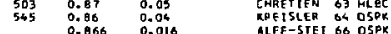

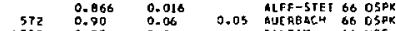

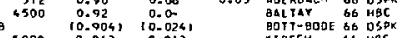

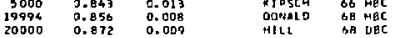

H $20000 \quad 0.872 \quad 0.009$

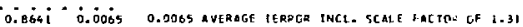

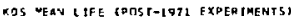

THESE JAL UES ARE USEO TO DETEPHINE THF STAB1E PARTICLF TABLF

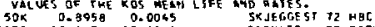

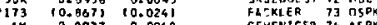

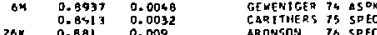

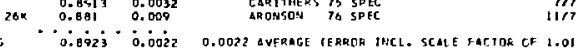

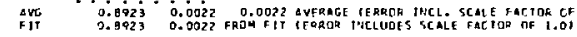
CT RMENTS

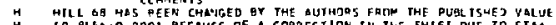
10. eno

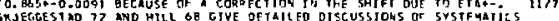
ENCONATERED IN TM15 TTPE TE EXPE DTHEHT.

KOS MEAN LIFF MOT THE PQI IAARY GUANTITY YEASURED IN THIS EXPT.

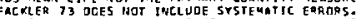

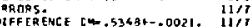

THE DW DEPENCENCE OF THE TOTA DECAY QATE ITNYERSE MFAN LIFE' IS

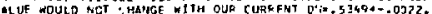

12 KoS patyat decar mots

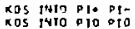

kas ivia une rum

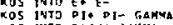

$\operatorname{xos} 205050$

17 os arancheng patios

cos inta cort

$\begin{array}{lll}3.64 & 0.04 \\ 0.70 & 0.09\end{array}$

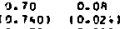

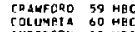

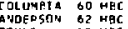

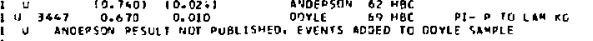

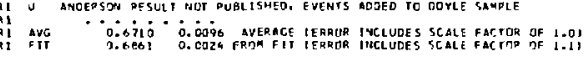

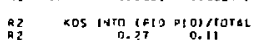

CDAMFORD 58 Hac 1021

$\begin{array}{ll}0.77 & 0.11 \\ 0.36 & 0.06 \\ 0.30 & 0.035\end{array}$

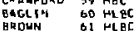

$1066 \quad 0.335 \quad 0.01$

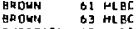

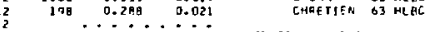

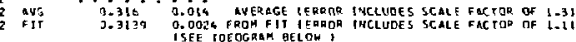

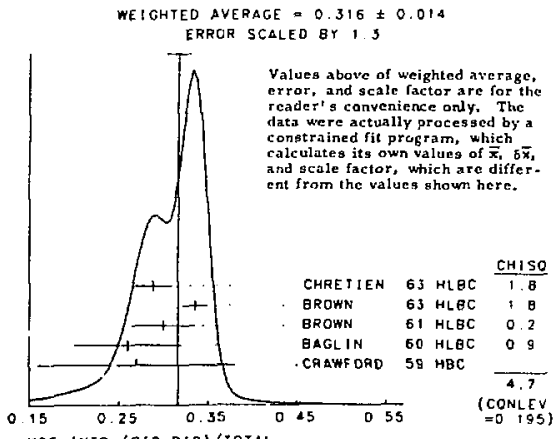

$139+139$

.5. .5

13. 100
$9 / 60$

a/to

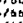

(1)

03
$p 3$

R3 $63016 \quad 17.2851 \quad 0.055$

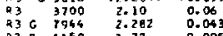

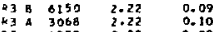

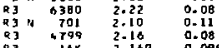

$\begin{array}{llll}83 & 114 & 2.16 & 0.08 \\ 83 & 2=169 & 0.094 \\ 03 & 1315 & 2=11 & 0.09\end{array}$

$(101) / 102)$

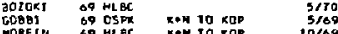

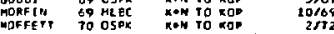

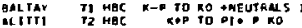

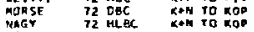

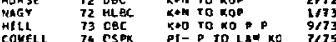

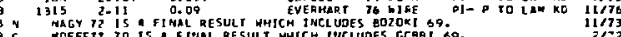

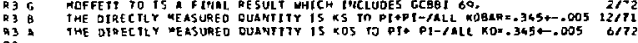

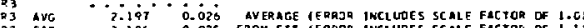

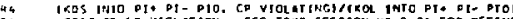

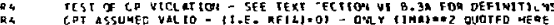

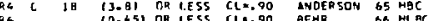

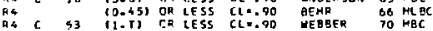

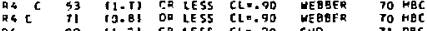

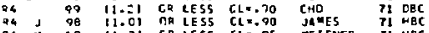

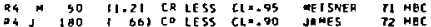

ind

24,19

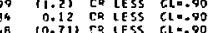

JONES
METLALF
MALLOY

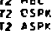

a C 5 EC CNO

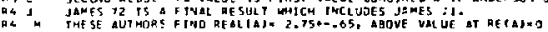

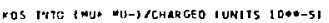

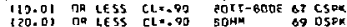

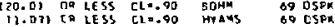

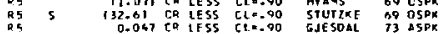

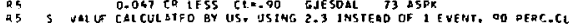

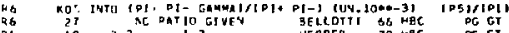

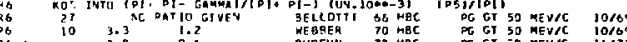

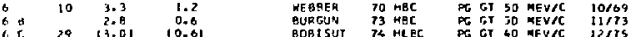

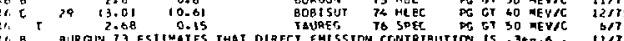

Da

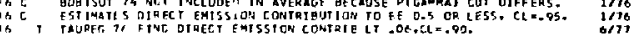

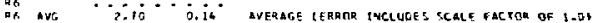

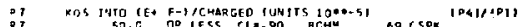

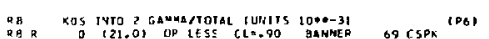

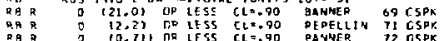

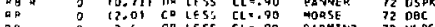

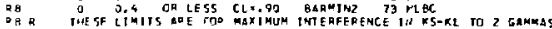

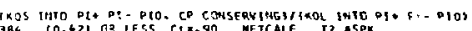

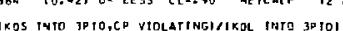

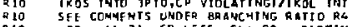

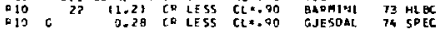

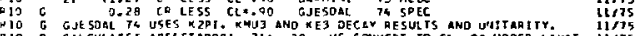

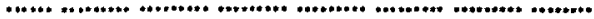

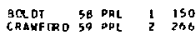

AAGLIN 60 NC 131043

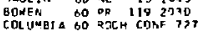

BAONN Of NC 191155

ANDERSON GE CERA CONF A3b

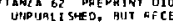

CMENIEN O3 PR 1312200

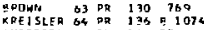

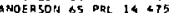

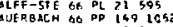

B AL TALO 65 AUEFAACH 932

PEFA

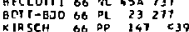

acri-9nD or Pl $248 \quad 158$

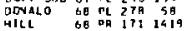

RANYER GQ PR 1 AD 2033

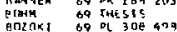

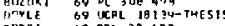

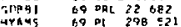

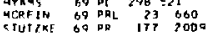

RFFEDENCES FDo ros

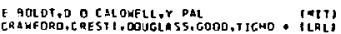

SAGL IN, BLOCH, ERTSSON. HENESSY + TFPOLS

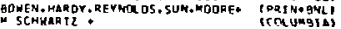

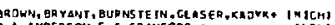

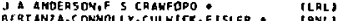
JHCD5. BNGUST OS.

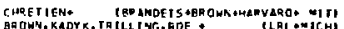

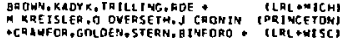

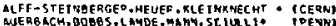

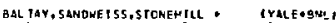

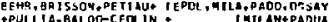

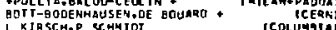
BOTT-BODE rhaUSEN, OE gCUARO, CASSEL, TCERM]

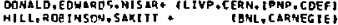

*CROYT N. L IH.DILCHER

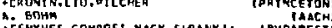

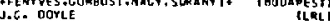

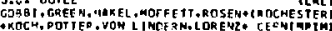

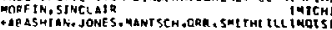


Stable Particles

$K_{S}^{0}, K_{L}^{0}$

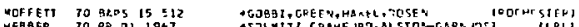
gaL TAR il OEL 27 LQ7Q

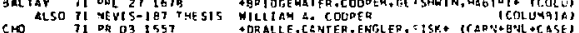

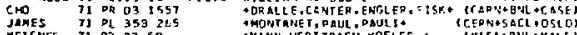
MEISHEF 71 MO 0350

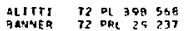

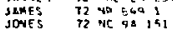

METCALF 72 व $40 \mathrm{P} 763$

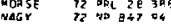

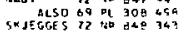

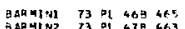

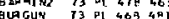

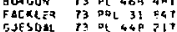
hile is os not 1200 yalcar is do jo 1753 BOEjSUT 74 Lix II sat

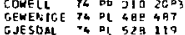
A AL COCEO $75 \times 2$ SA $6 E$ ? CLA ITHE 75 DTL 341240

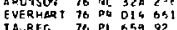

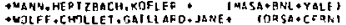

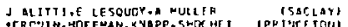

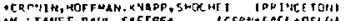

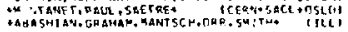
- NE UHOFER NICBERGALL+ TCER+ IDN+HTERI

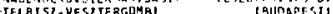

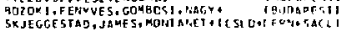

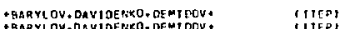

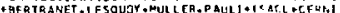

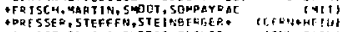

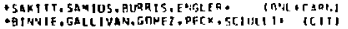

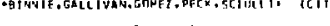

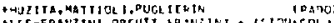

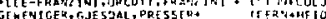

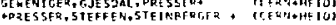

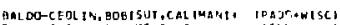
CAR ITHERS, MODIS, JYGR FN. PUS

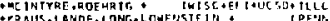

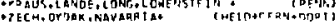

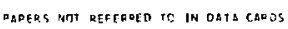

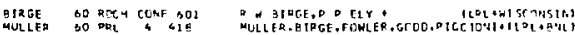

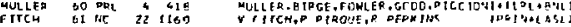

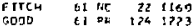

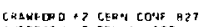
AUE RBACH O5 OWL 16192

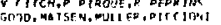

IS CRAHTORz

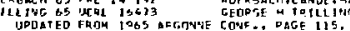

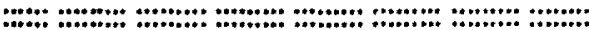

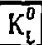

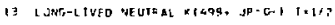

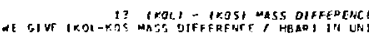

(2.26) $\quad(0.351$

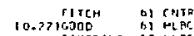

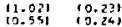

(3.). $340, \quad 10.341$

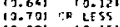

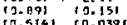

CAHEPIMI B2 HLEC
AURFT

10. 2019ALDO-CE' ES MLC
CHRISTEDS ES OSDK

CHRISTEMS ES OSDK
MITC

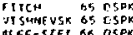

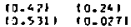

$17.3 B 1$ 9AIDOCED 6. WLAC

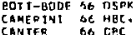

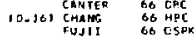

FUJII CO ESFK

ME ISNER: CO HAC

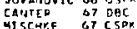

$\begin{array}{ll}\text { YS SCHYE } & \text { QT CSPK } \\ \text { BALAT } & \text { OQ OSPF }\end{array}$

HFLAGP GA OSPK

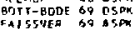

CULLLN 70 CN1R

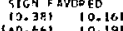

$\begin{array}{ll}10.651 & 10.111 \\ 10.591 & 10.131\end{array}$

$\begin{array}{rr}(0.520) & 10.064) \\ 100.4971 & 10.046)\end{array}$

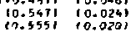

$\begin{array}{cc}0.542 & 0.006 \\ 10.5421 & 10.0061\end{array}$

$\begin{array}{ll}(0.5421) & (0.008) \\ (10.481) & (0.052) \\ (0.536) & (0.007)\end{array}$

$\begin{array}{ll}10.671 & 10.16)\end{array}$

10.7151040

AAOFSON POA PK

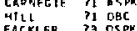

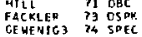

GEMENTC3 34 SOEC CAO NETHOD

$\begin{array}{ll}0.5360 & 0.0030 \\ 0.5334 & 0.0040\end{array}$

arsunes co rens.

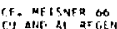

KJ+1] TIYT HYPEF

REGE,

SD SCATTED IHP D. $11 / 6$

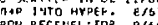

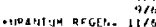

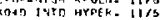

aL DECEDT DATCE

GLO METHOO

CREGEN

GAO WFTMOOO

T,AS RE THCO

CHO NETHOD
CWO ASYMAETRY

01600 7
7
7
7
7
7
7

$3,1,68$
3,60

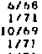

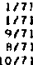

9171
$10 / 72$
11073
1107

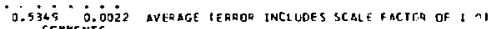

$x$ IO AT TE QPT OS OE EM MADE TO CORAECT OL DEA EXPER:ATHTS WITH LARGE

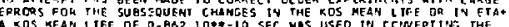

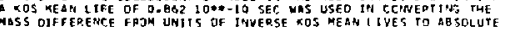

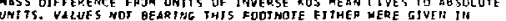

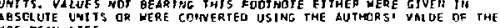
KOS TEAN IIf $E$.

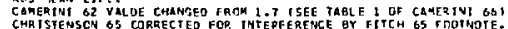
CHR ISFE NSCH OS CORGECTED FOP. TNTEPFERENCE BY FITCH GS TMOHWOTE.

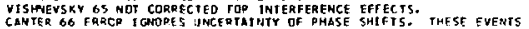

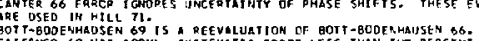

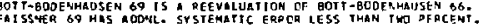

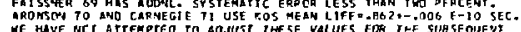

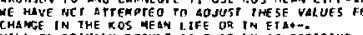

HILL 71 PRIWBGY RESULY IS THAT DU IS DOSIIIVE.

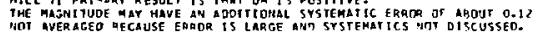

Data Card Listings

For notation, see key at front of Listings.

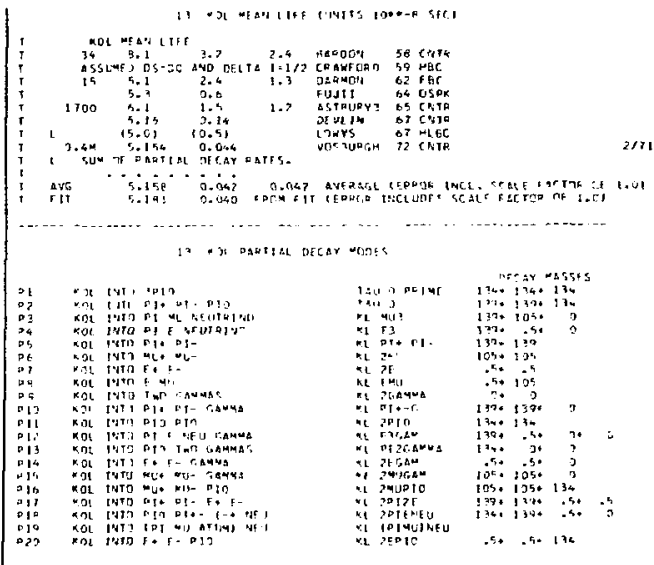

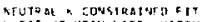

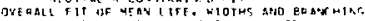

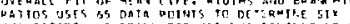

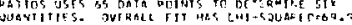

318
$3 / 76$

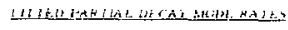

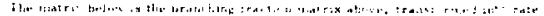

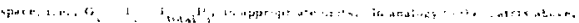

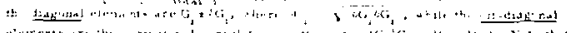

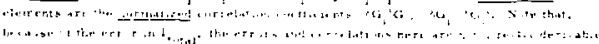

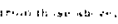

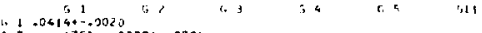

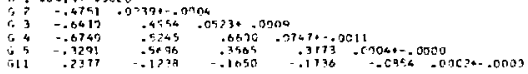

$$
\begin{aligned}
& 13 \text { KOI DECAY RATFS }
\end{aligned}
$$

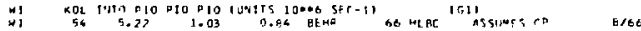

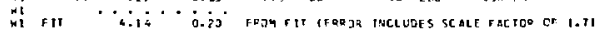

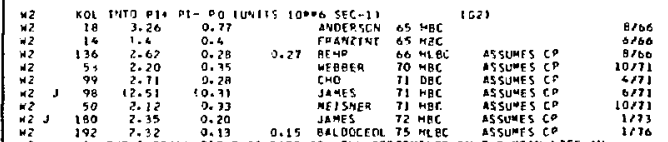

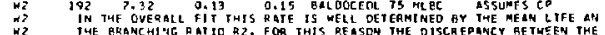

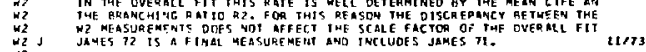

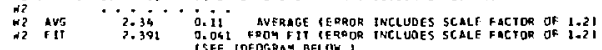




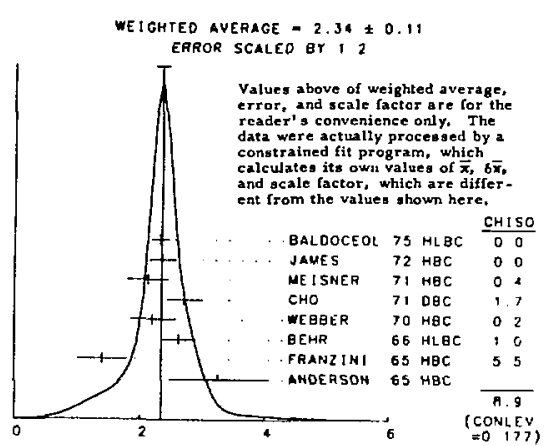

KOL RATE INTO PI+PI-PIO (10\%6 SEC-1)
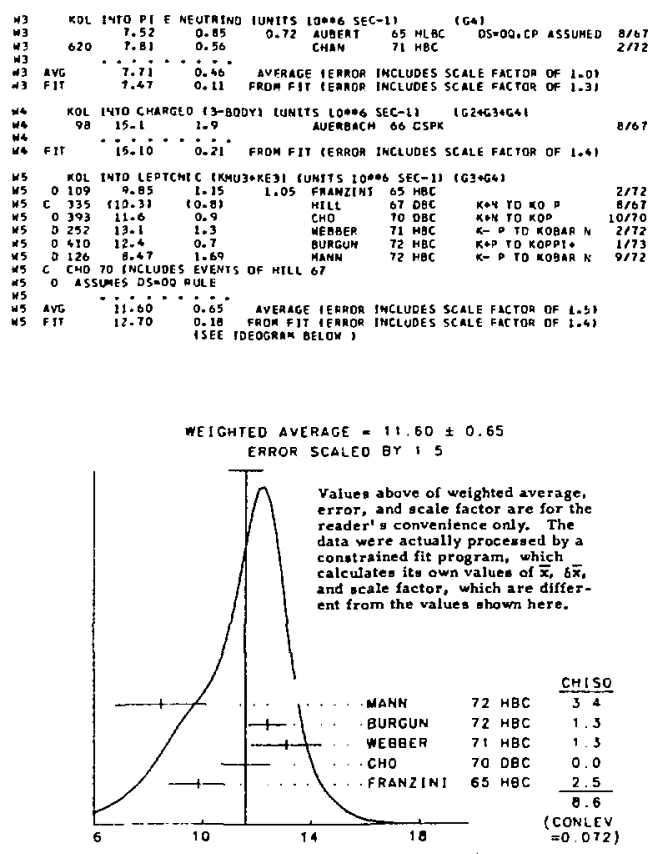

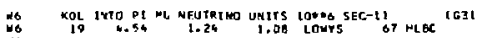

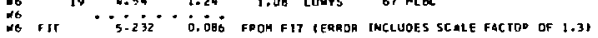

13 KOL GRANCHING MATIOS

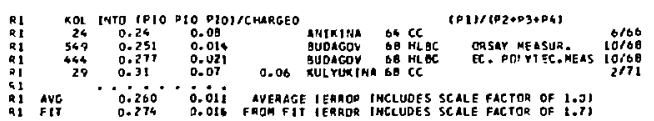

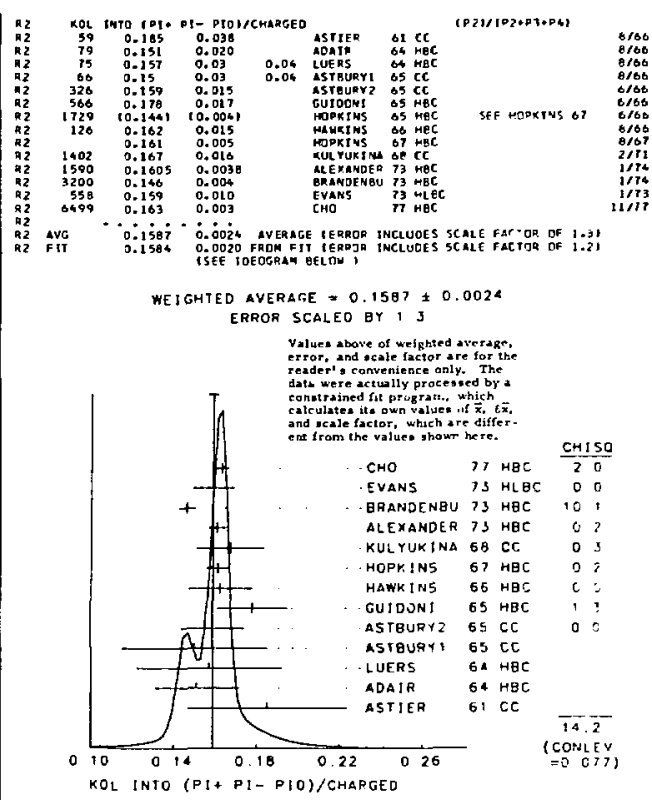

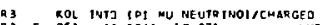

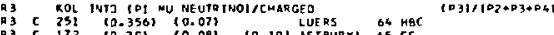

R C 172 (0.35) $10.081 \quad 10.101$ \&STBURYI BS CC

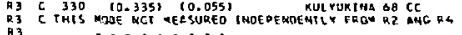

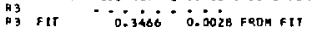

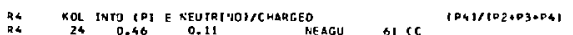

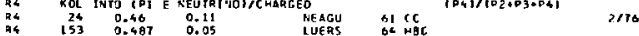

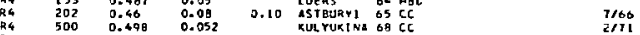

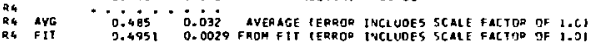

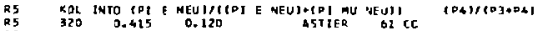

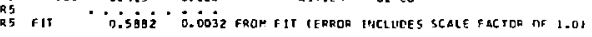

R6 KaL INTD IPT +PI-PLOIJTOTAL IPZ]

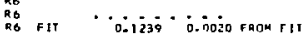

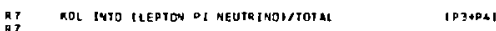

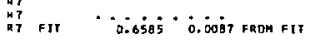

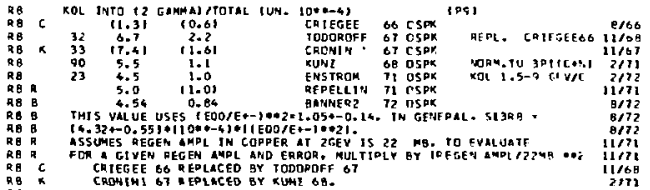

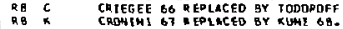

DQ
$R B$
AVE

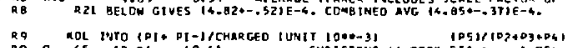

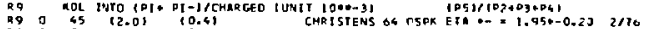

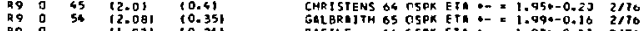

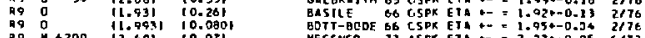

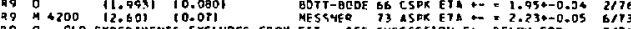
9 O OLO EXPER INENTS EXCLUDE, FRON FIT: SEE SUESECTION EQ RELOW FAR

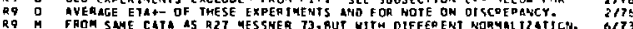

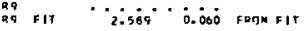


Stable Particles

$K_{L}^{0}$

\begin{tabular}{|c|c|c|c|c|}
\hline a & $\begin{array}{l}1 N 10101 \\
0.81 \\
0.92 \\
0.7 \\
0.81 \\
0.71 \\
10.671 \\
10.711 \\
10.6481 \\
0.68 \\
0.761 \\
0.862 \\
0.662 \\
0.702\end{array}$ & $\begin{array}{l}\{0.131 \\
0.041 \\
0.0901 \\
0.08 \\
0.044 \\
0.040 \\
0.037\end{array}$ & 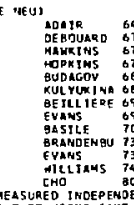 & 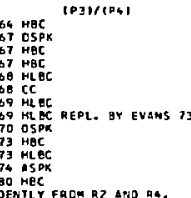 \\
\hline
\end{tabular}

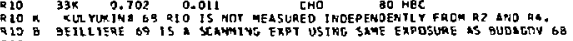

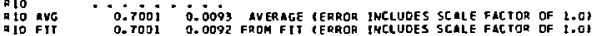

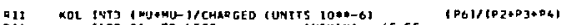

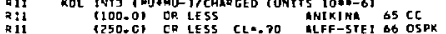

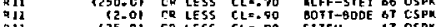

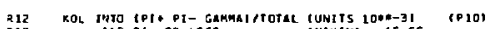

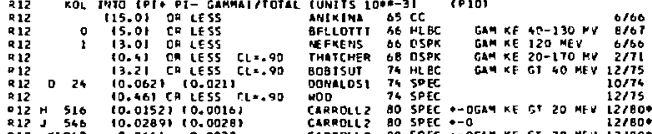

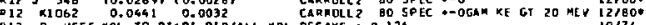

Q12 D USES KOL TO DTHPI-PIDKALL KOL DECAYS 0.126

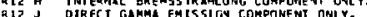

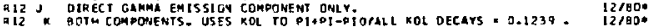

RI3 KOL INTO IC. E-1/CMARGEO IUNTTS 10*0-6) $107 /$ /P2*D3*D4\}

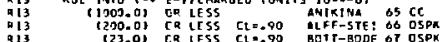

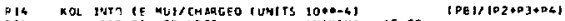

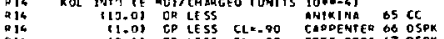

(J.11) CR LESS CL=.90 BOTI-BJDE BT OSPK

RES

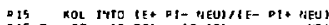

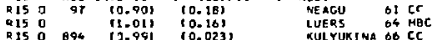

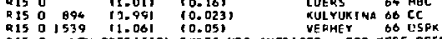

R15 O LOW PRECISICA FXPTS NDT MVERAGTO. FOR MDRE PRECISE VALUE.

ION GELOK.

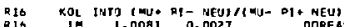

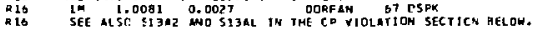

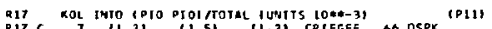

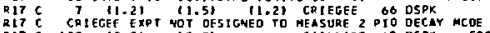

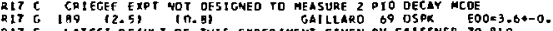

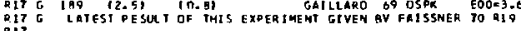

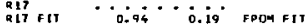

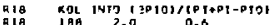

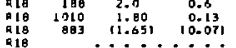

LLESANYA OA FBC (P13/102)

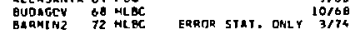

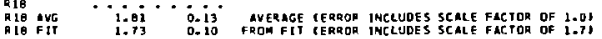

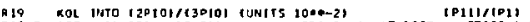

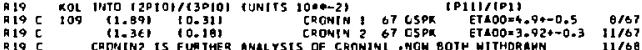

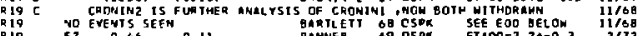

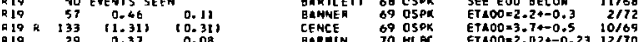

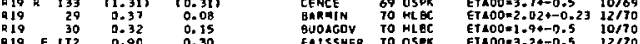

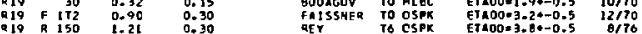

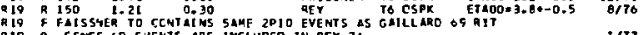

Ris a CENCE 59 EVENTS ARE INCLUDEO IN REY 70 .

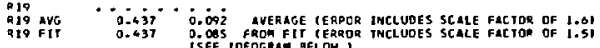

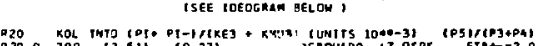

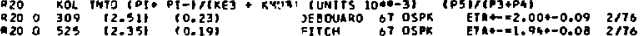

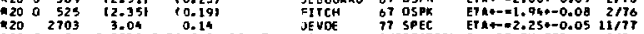

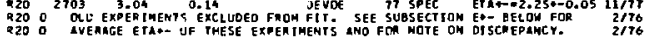
P20 fit $3.070^{\circ}$ O. $075^{\circ}$ FROM FII IERROQ INCLUDES SCALE FACTOR OF 1.01 606

$9 / 60$
$9 / 60$
9167

$1 / 12$

$5 / 69$
Data Card Listings

For.notation, see key at front of Listings.

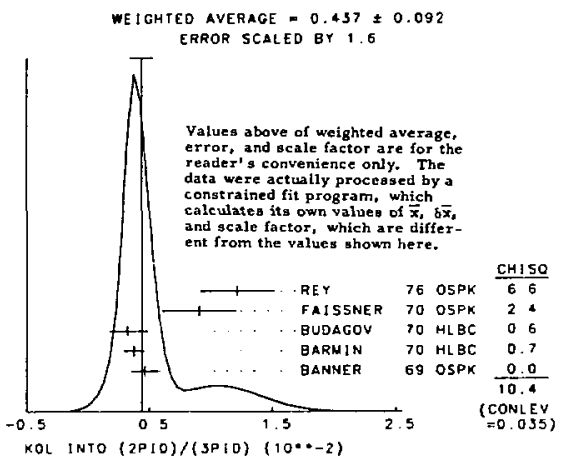

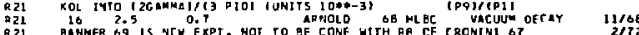

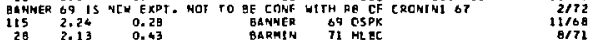

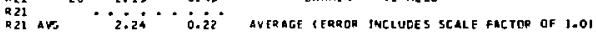

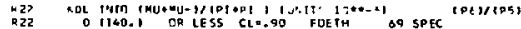

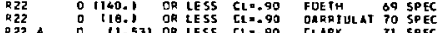

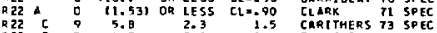

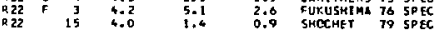

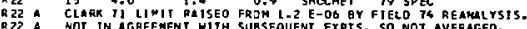

Q22 F FUKUSHIMA 76 ERRORS ARE AT CLAOD PERCENT.

a 22 ave $4.47^{*} \cdot 0.95^{*}$ average terrok theluoes scale factó of 1.01

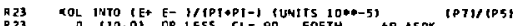

R23 D 110.01 DR LESS CL=.90 FOETH OQASPK 5

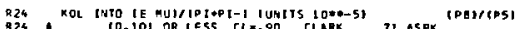

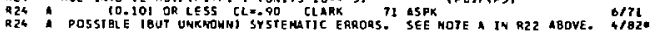

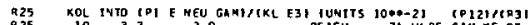

R26

2.0

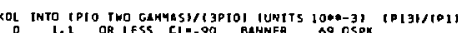

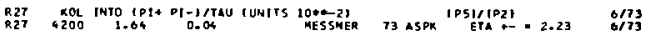

R27 F1T - 1.635. - 0.035 FROM FIT (ERRDR INCLUDES SCALE FACTON DF 1.0)

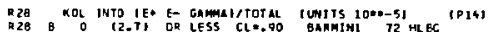

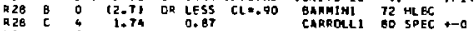

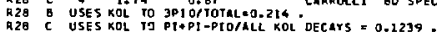

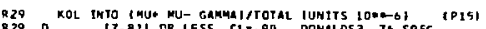

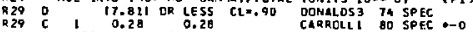

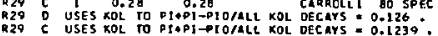

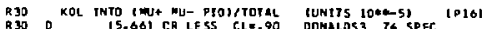

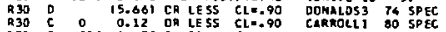

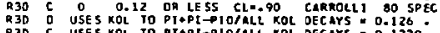

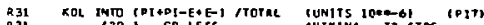

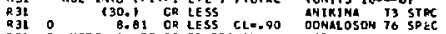

R 31 U USES KOL TO PI+PI-PIOKALL KOL DECAYS O.128.

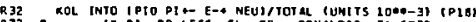

Q 32 D $10 \quad\{2,21$ DR 2 ESS $C L=.90$ DOMALOS $\}$ IA SPEC

R32 D DOHALOSON3 14 USESKOL TO P14P1-P1OKLLL KOL DECAYS $=0.126$.

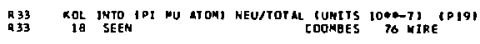

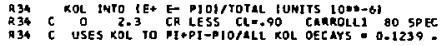

3174

$12100 \%$,

$12 / 73$

22771

$12 / 75$

(2)

(1)

$12 / 75$

6rm

$12 / 195$

b/77.

om

12700: 
13 SDL ENERGP DEPEHDENCE OF ORL:TI PLOT REL ATEO TEXT SECTION YI B.1 AND MINI-REVJEW ON SLOPE PAKAME TERS TON OF THE OATK CARO LISTINGS A

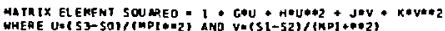

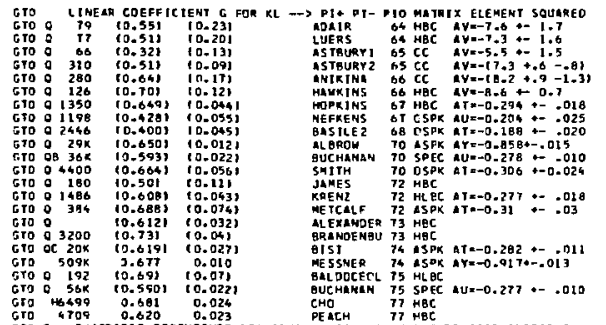
GTO "TOG 0.020 O. O23 GTO O HTO ANO NTO QEL DWH. CORRELATIONS PRE VENT US FROM AVEGACTNS RESULIS

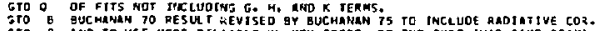

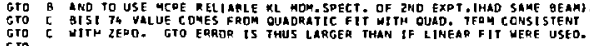

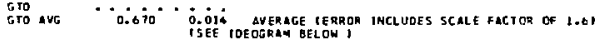

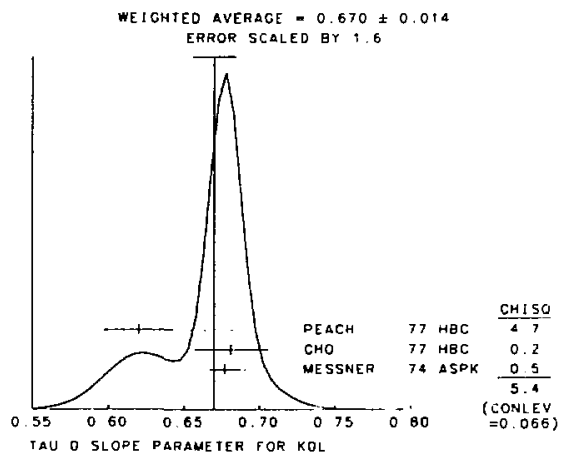

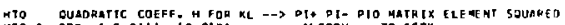

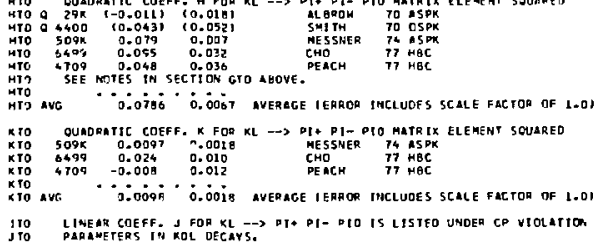

\section{$13 \times$ OL FORY FACTORS}

KELATED TEXT SECT IOY VI 0.2 AND MINT-PEVIEH DK FORH FACTORS
IT THE CHARGEC K SECT TON OF THE OATA CARO LISTINGS AROVE.

TN TME FORM FACTOQ COMNENTS. THE FOLLOUING ABEREVIATIONS ARE USEO. F+ WHT F- ARE FOQH FACTORS FDR THE VECTOP MATRIX
FS ANO FT REFER TO FHE SCALAR AND TENSOR TER

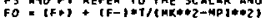

L+, L- AHO LO SRE TME LIHEAR EXPAHSIOY COEFFS, OF FA, F- AND FO,

L4 QRFERS TO THE KWUZ VALUE EXCEDT IN THE KE3 SECTIOWN.

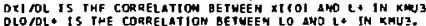

DLO DLLA IS THE CORRELATIOH BETHEEN LO NNO LA IN KMUZ.

DP = OALITI PLOT ANBLYSIS

PI - PI SPECTRUM MNALYSIS

POL: WU POLARILAT ION ANALYSIS

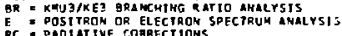

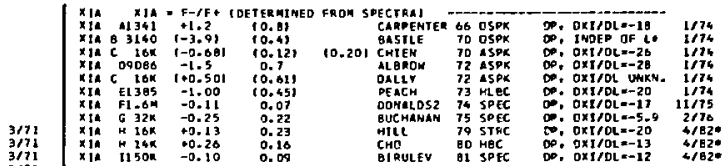

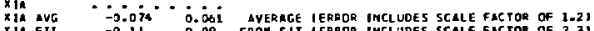
5/Ez"

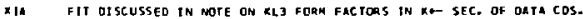

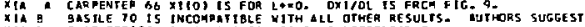
XIA THAT FFFICTEMCY ESTIMATES NGMT GE RESMONSIELE.

XIA C CMIEN 7J EQRORS ARE STATISTICAL OHLY. OXI

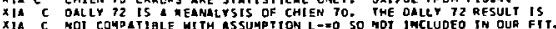

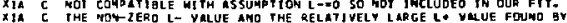

$x$ IA 5 OALLY 72 COME MAINLY FROM SINGLE LOH BIN IFICS.1+2).

IA $C$ TWE (FE, XI) CORRELATIOM WAS TGMORED.

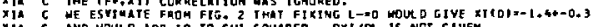
X IA C AHO WDULD AOE TO TO CHI SQUAPES. DXI/O 15 NOT CIVEM.

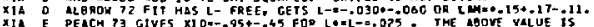

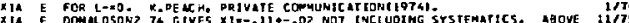

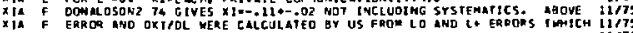

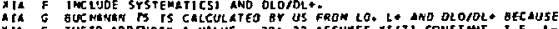

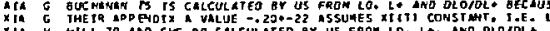

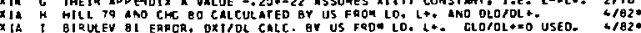

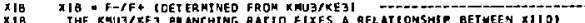

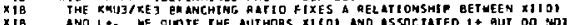

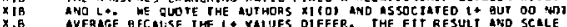

EACTORS IN THE XO SECTIOY OF

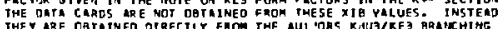

THE YRE OBTH TNEO OI RECTLY FHOM THE AUI IORS

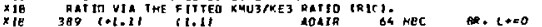

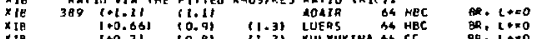

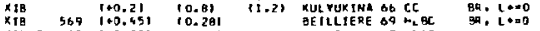

$\times 19$ E $13091-0.221 \quad(0.301$

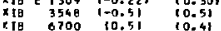

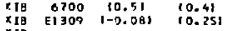

EVAFS SO HLE
QASTLE TO OSPK

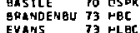

Q2. $1+=.02$

1173

KIB FIT OISCUSSEC IN NOTE ON KL3 FORH FACTDRS IN KG SEC. OF OATA COS.

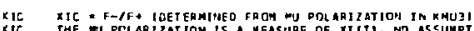

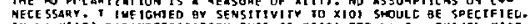

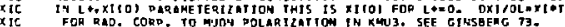

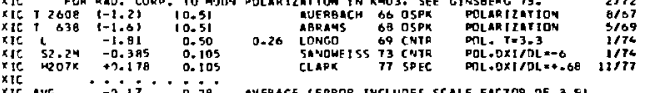

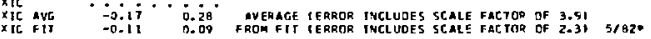

XIC FIT OTSCUSSEO IN NOTE ON KL3 FOOM FACTOAS IN K*- SEC- DF OATA COS.

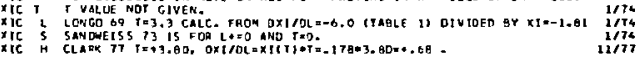

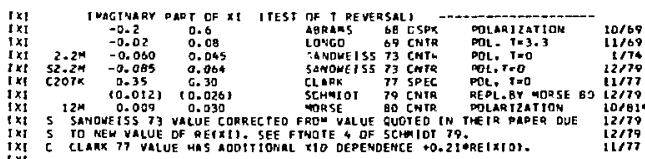

$3 / 78$
$3 / 78$
$3 / 78$

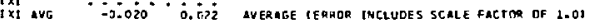

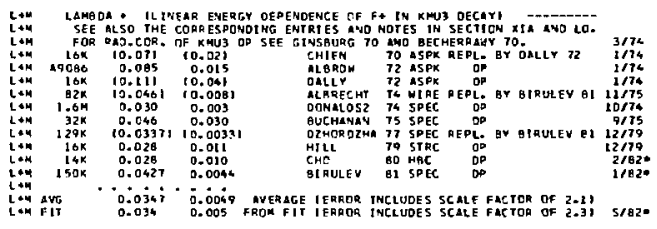

L*4 FIT DISTUSSEO IN ROTE ON KLI FORA FACTORS IN Y.- SEC. JF OATA COS. 
Stable Particles

$\mathbf{K}_{\mathbf{L}}^{0}$

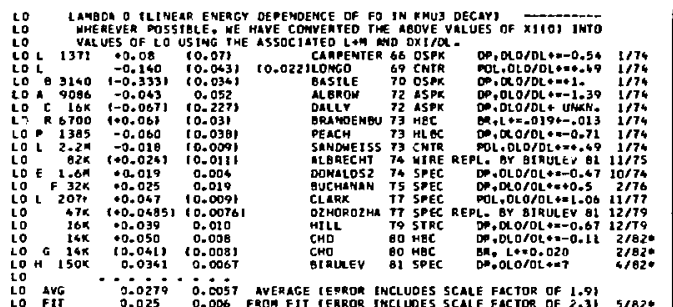

LO FIT OISCUSSEO IN NOTE ON KL3 FONM FACTORS IN KO SEC. OF DATA COS. LO L LO VHLUE TS FOA L*0.03 CALCULATED BY US FHOH X10 BNO OXI/OL.

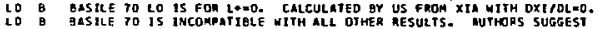
LO B THAT EFFICIENCY ESTIMRTES MIGHT OE GESPONS MELE

LO ALBROH 72 LO IS CALCULATEO EY US FROM KIA,L+ ANO DXIJOL. THEY GIVE

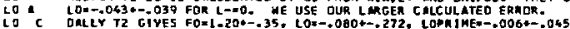
BUT WITH A OIFFEREMT OEFINTI LON OF LO. OUR OUOTED LO IS M1S LO/FO. LO I WE CANOT CALCULATE TRUE LO ERROR WITIOUT HIS ILO.FOI CORMELATIONS.

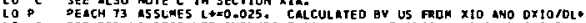
LO R FIT FOT LO DOES YOT INELUDE THTS VALUE BUT T'ISTE $\triangle D$ IHELUDES THE

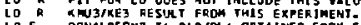

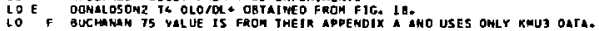

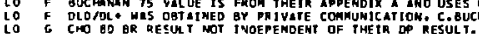

LO 4 BIRUEV BI GIVES OLO

LaMEOA - IL INEAR ENERGY DEPEMOEMCE OF F. IN KO EJ DECRY, --

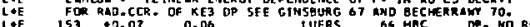

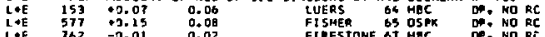

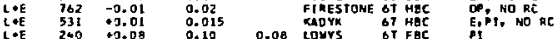

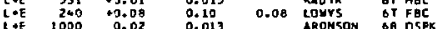

$\begin{array}{llll}C+E & 1000 & 0.02 & 0.013 \\ 1+E & 6000 & +0.023 & 0.012\end{array}$

$\begin{array}{llll}\text { LEE } & 42 k & 0.023 & 0.005\end{array}$

$\begin{array}{llll}\text { CEE } & 161 & 0.05 & 0.01 \\ \text { LAE } & 1910 & 0.022 & 0.014 \\ \text { L.E } & \text { S600 } & 0.045 & 0.014\end{array}$

C.E 1671 $0.015 \quad 0.013$

$\begin{array}{cccc}\text { LEE } & 2171 & 0.040 & 0.012 \\ \text { LEE } & 25 \mathrm{~K} & 0.0210 & 0.002 \mathrm{O}\end{array}$

$\begin{array}{lrrr}l & 24 K & 0.044 & 0.006 \\ l+E & 48 K & 10.0321 & 10.00421\end{array}$

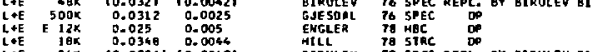

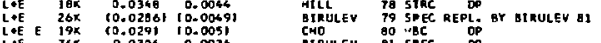

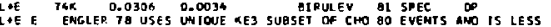
LEE E ENGLER TA USES UAIOUE KE3 SUES

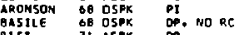

CIST 7I ASPK DE

MEUHOFER 72 ASWK

GTANOENBU 73 ASPK

GLANGETt

os

op

op

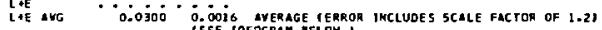

WEIGHTED AVERAGE $=0.0300 \pm 0.0016$ ERROR SCALEO BY 1,2

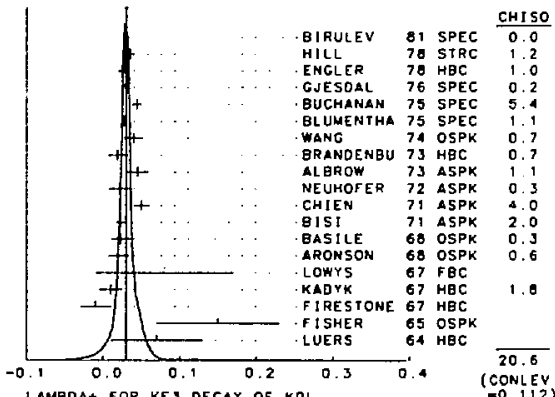
LAMBDAT FOR KEJ DECAY OF KOL

RAT TO OF SCALAF TD KE COUPLIHGS FOR KES

5800 10.191 CR LESS CL $=.95$ ALGROW 73 ASPX

25K 50.041 OR LESS GL-.60 GLUMENTHA TS SPEC

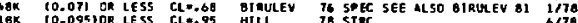

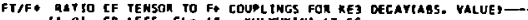

5000 (1.01 CR LESS CL.68 KULTHEIHA 67 CC

25K (0.23) OR LESS CLF.80 OLUMEHTHA 75 SPEC

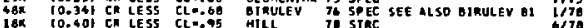

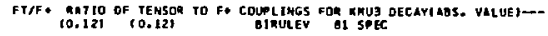

Data Card Listings

For notation, see key at front of Listings.

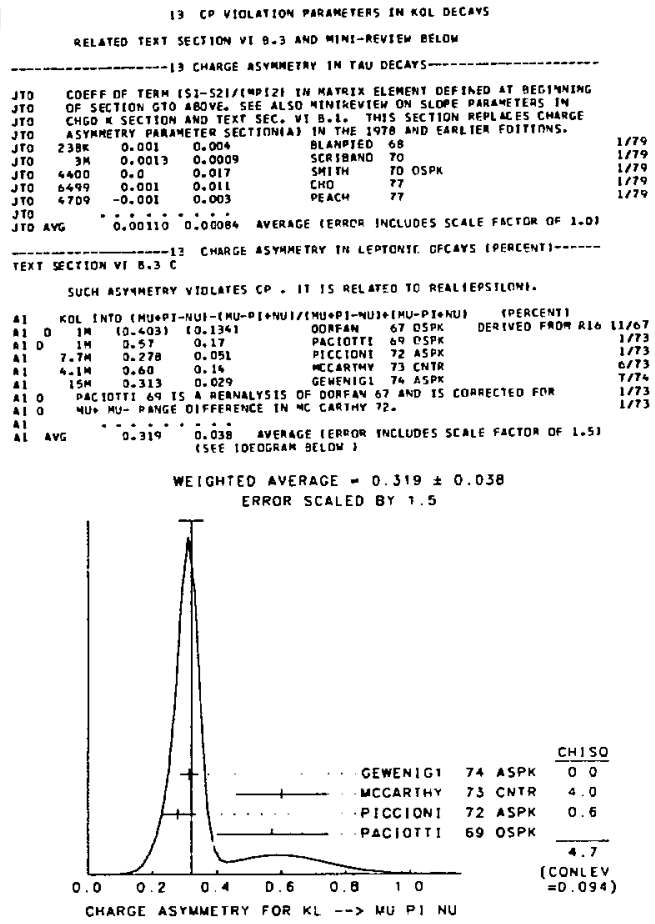

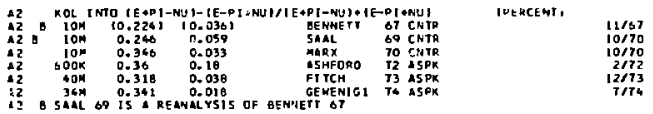

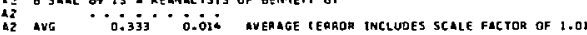

AL KOL $1 N T O(t L+1-1 L-1)(1 L+1+(L-1)$ CCOROINEO AL AND A2) IPERCENTI

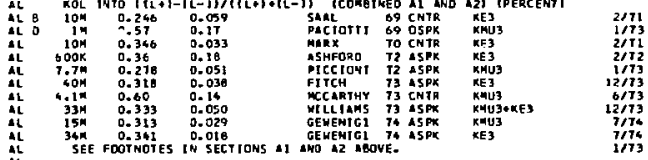

al avo $0.330^{\circ}$ D. oilz average terror thcluoes scale factor of 1.01

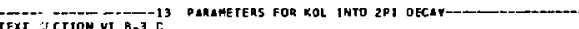

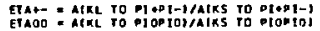

THE FITTEO VRLUES OF ETL+- AND ETAOO GIVEN EELON ARE ITE RESUL TS

OF A FIT TO EJA4-, ETADO AWO ETADO/ETAO- RESULTS D TKE VALUES LISTEO

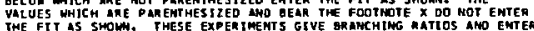

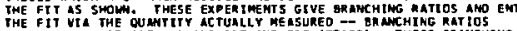

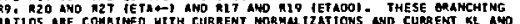

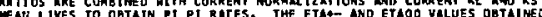

FhO THESE MTES ARE ENTERED BELOW WITH THE MAME TGKL/GKS". 


\section{Data Card Listings}

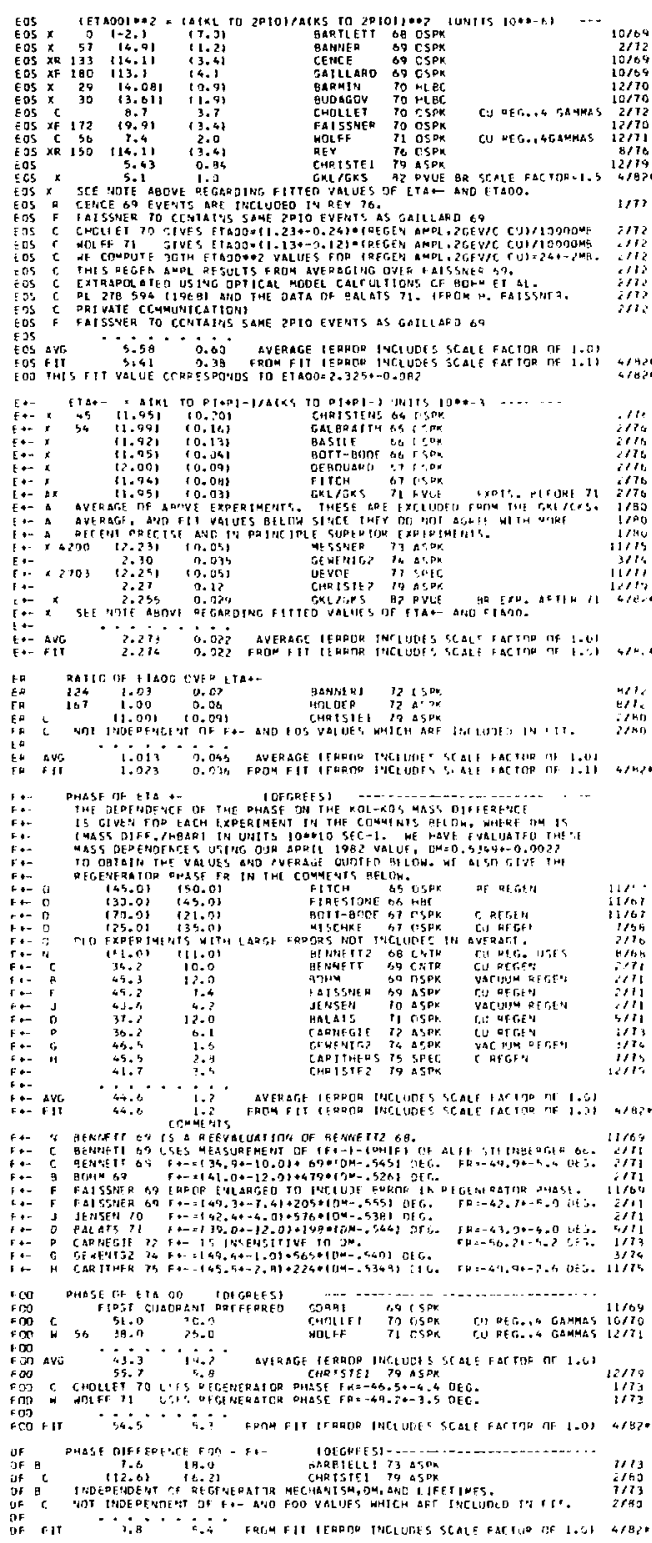

Superweak Model predictions for $\mid \eta_{0} / n_{t-} ! 4_{+-}$and Ref

In terms of the parameters eefined in the text,
Sec. VI R(d), the superweak model ${ }^{1}$ predicts that ${ }^{2}$

$$
\begin{aligned}
& \left|n_{00} / r_{+-}\right|=1 \text {. } \\
& t_{t-}=\dot{n}_{0}=\tan ^{-1}\left(\frac{2 \pi \pi_{T} s}{\hbar}\right) \text {. } \\
& R_{0}=r_{+-}\left[I+\left(\frac{2 i \sin s}{\hbar_{1}}\right)^{2}\right]^{-1 / 2}
\end{aligned}
$$

and

The Jatter two expressions and the values of the $K_{\mathrm{I}}^{0}-\mathrm{r}_{\mathrm{S}}^{0}$ Hass difference $i \mathrm{~m}=(0.5349 \pm 0.0022) \times 10^{10}$ n $\sec ^{\mathrm{I}}{ }^{-1}$, the $\mathrm{K}_{\mathrm{S}}^{0}$ mean life ${ }_{\mathrm{S}}=(0.8923 \pm 0.002 ?) \times 10^{-10}$ ser, and the magnitude of the $K_{T^{n}}^{n}, \pi^{+} \pi^{-} / K_{5}^{r}, \pi^{+} \pi^{-}$ amplitude ratio $! n_{+-} !=(2.274 * 0.022) \times 10^{-3}$, all from the :urrent alition, result in the predictions the

$$
\therefore 1-\quad \therefore,-\left(1+2.63^{2}+1.14\right)^{\circ}
$$

atsut

$$
\text { Res }=11.41 ; \cdot 9.016) \cdot 10^{-3} .
$$

The ahove peudirtions can be comparea with the

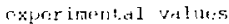

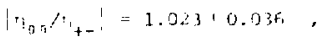

$$
\begin{aligned}
& !_{+-}-(44.6 ! 1.2)^{\circ} \\
& \therefore 0=(5,4.5,5.3)^{\circ} . \\
& \text { Fo: }=(1.621+0.083) \div 10^{-3}
\end{aligned}
$$

where Pe: has been computed using the relation

$$
\mathrm{F} x+\frac{5}{2}\left(\frac{|1-x|^{2}}{1-|x|^{2}}\right),
$$

and our current values of the charge asymmetry parameter for leptonic $k_{L}^{0}$ decay $\delta=(0.330 \pm 0.012) \&$ and the is $=-h g$ ampliture $($ Rex, $\operatorname{Imx})=(0.009 \pm 0.020$, $-0.004 \div 0.026)$.

The superweak predictions are in agreement with tine data except for the measured value of poo, which is two stancard deviations above the prediction. This results primarily from the CHRISTENSON 79 moasurement t $_{0}=(55.7+5.8)^{\circ}$.

\section{References}

1. I. Wolfenatein, Phys. Lett. 13, 562 (1964).

2. T. D. I.ce and $\mathrm{L}$, Wolfenstei:., Phys. Rev. 138B, 1490 (1965). 
Stable Particles

$K_{L}^{0}$

$17 x=1050-00$ AMPL I TUDE $1 / 105=00$ AMPL TTUDE

PELATLO TEXI SECTION VI 8.4

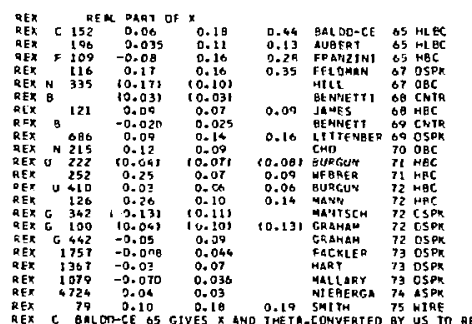

K+ CHARGF EKCHNG IITO? K+ CHARGE EXCTSG II/S? OBAR D NO LMADA IISSO

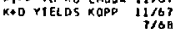
PEAR D 5169

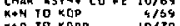
$K+0$ to KOPP $10 \%$ ro

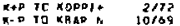

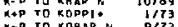

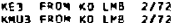
PI $\rightarrow$ TO nO LMBOA ZMT

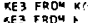

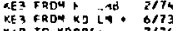

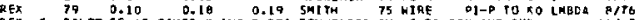

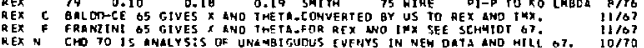

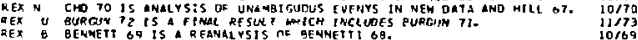

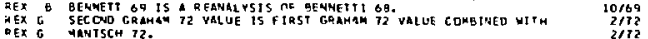

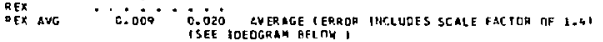

WEIGHTED AVERAGE $=0.009 \pm 0.020$

ERROR SCALED BY 1.4

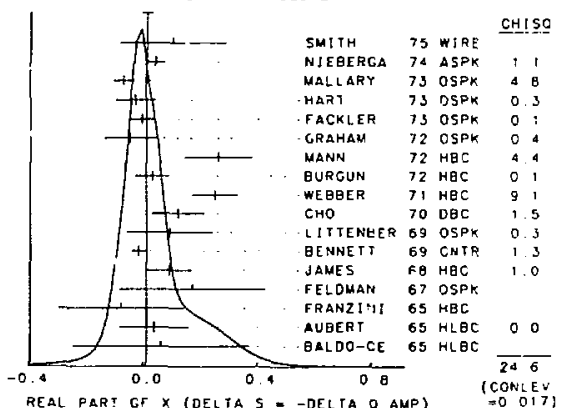

REAL PARI GF $\times$ (DELIA $5=-D E L T A$ O AMP) (CONLEV )

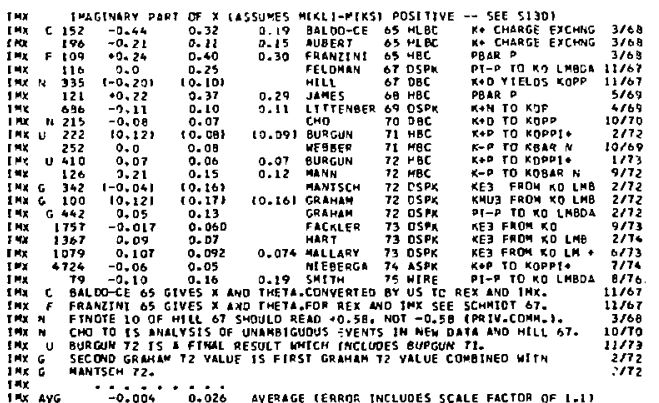

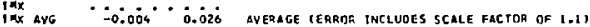

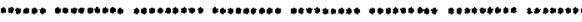

meferences fot col $\begin{array}{llll}\text { EDOO OI PR } 124 & 1223 \\ \text { NEICU OI TRL } & 552\end{array}$

CAMER JNI G2 PR 12 B 3*2

ALEIR of DL 12 o3

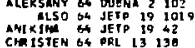

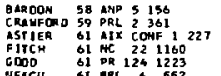

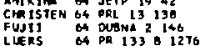

M BARDON-K LANDE IL LEDERMAN ICCLUMBTA+BNL!

CRAMFOAO,CREST1, DOUGLASS,GODQ + ILRL)

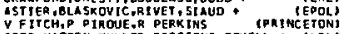

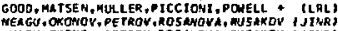

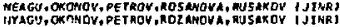

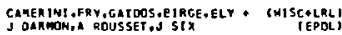
R COAIA.L B LEIPUNER TYOLEBALI

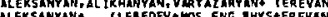

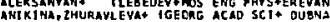
CHP ISTENSON, CRONIN,FITCH, TURLAY IPRIHCETOH

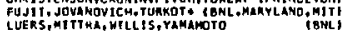

Data Card Listings

For notation, see sey at front of Listings.

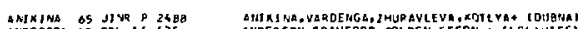

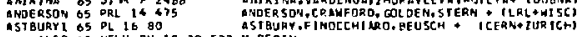

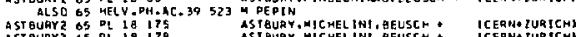

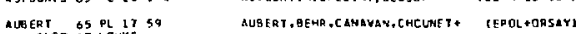
PAL KLOCO OP LOHYS

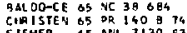
FISHER
FITCH

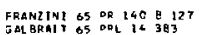

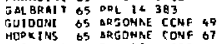

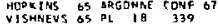

ALFF-5IE of PL 21,595 ANIKINE At SJMP 23390

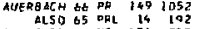

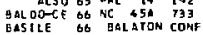

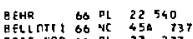

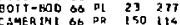

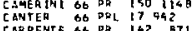

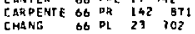
CRIEGEE of PRL 11,150

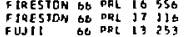

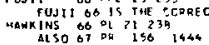

JOVANON! BE DPL ITICTS

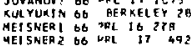

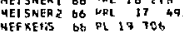

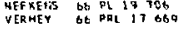

BEHNETT G7 PRL $19 \quad 993$

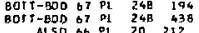

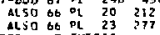

CANTER D7 THESIS

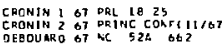

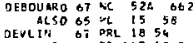
aEVLII

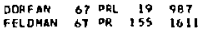

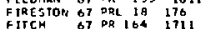
$\begin{array}{lllll}\text { FIICH } & 67 & \text { PR } & 164 & 1711 \\ \text { HANKINS } & 67 & 08 & 196 & 1446\end{array}$ HEPKINS OT DDL I9 195

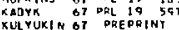
KULYUKI IN OT PREPFINT
L OWYS OT PL 248 I5

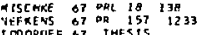
AGAAAS 60 PR 27,1603

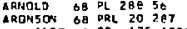
BAD ALSO BS OQ 1751700 BARTLET T 68 OQL 215.5 BAS ILE 68 PL 208 S42

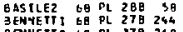

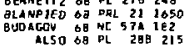

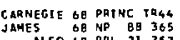

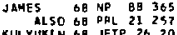

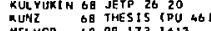

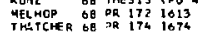
G ANNER 69 PR 1802053 ALSO OB PRL 21 : 103

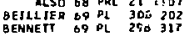

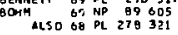
BOTT- 300 G9 CERM 69-7 329

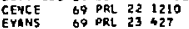
EANSIER G9 PL 308204
FCETH GAT LLLE 64 HC 592453

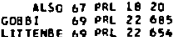

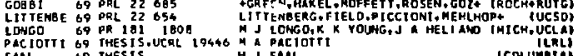

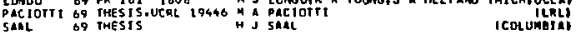

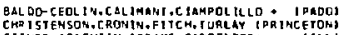

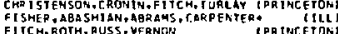
FRLNTINI,KIRSCH, PLARO I ICOLUME IA +RUTGERS

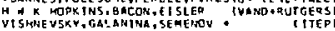
ALFF- STE TNBERGEP, HEUER +RUBB IA * TGERN

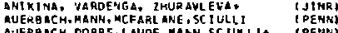

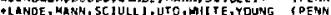

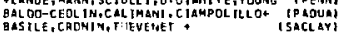
-QR ISSOY,QALOO-CEOL IM, AUBERTA IPADD,EPOLI

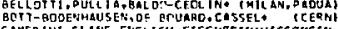

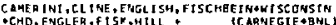
CARPENIER,ABSSHRN, ABRAMS, FISHER IILLINOIS GEOT, FRQUE NFELOEQ FIRESTOAE,KI M, LALH, SANDLES SST IYALE, GNL

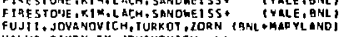

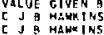

(YYLLE) JOYANOYICH,FUJTI, TURKOT IOAN HIANL+UMO+KIT

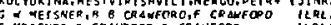

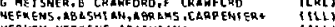

BENHETT, NYGREN, SAAL, STE INBER GER + I COL UMG TAS

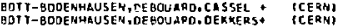

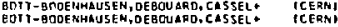
J. M. CANEF
ICARAECTE]

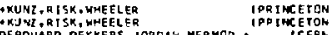

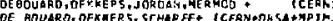

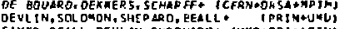

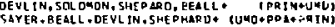
GORFAN, ENSTROM, RAYMONO, SCMMRRTL OISLAC LLRLI FIA STOHE YKIM,LACH, SANOUEISS + TYALE, JNL, FO TORTHCETON
TYALI

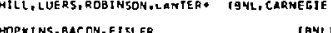

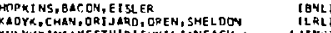

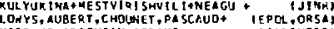

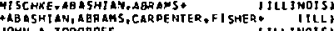
+ABAS,IAN, MISCHKE, NEFK ENS, SMITH* (ILLL INOISI

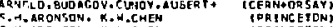

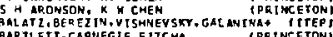
QARTLETT, CARTEGTE.FITCH* OACANTETON, BASHLE, CROM IN, I HEVENET, TURLAY. ISACLAY, +ERONIN. THEVENE T, TUFLAY, ZYL BERAJCH+ ISACLAY,

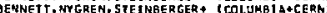

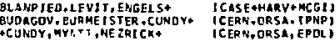
R.X.CARNEGTE TPRINCETON,

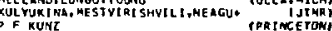

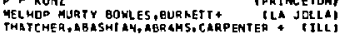
TEROMI R.LLUU, PILCHER GREINCETOMI BAYNER CRONIN.LIU.PTLCHER IPRINCETONI

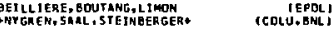
G0 - BDOENHAUS EN, OE GOUARO, CASSEL. ICEQNI C. E. JOHES, TETERSDH. STENGERT (HAYAII, LRL)

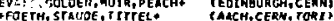

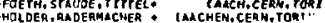

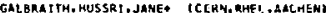

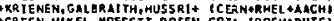
CAL GRAIIM, MANYING. JONES S IAERE+ RR TS+ RMEL JOMN A TOOOHOFF

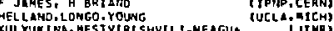


Data Card Listings

For notation, see key at front of Listings.
Stable Particles

$\mathrm{K}_{\mathrm{L}}^{0}, \mathrm{D}^{ \pm}$

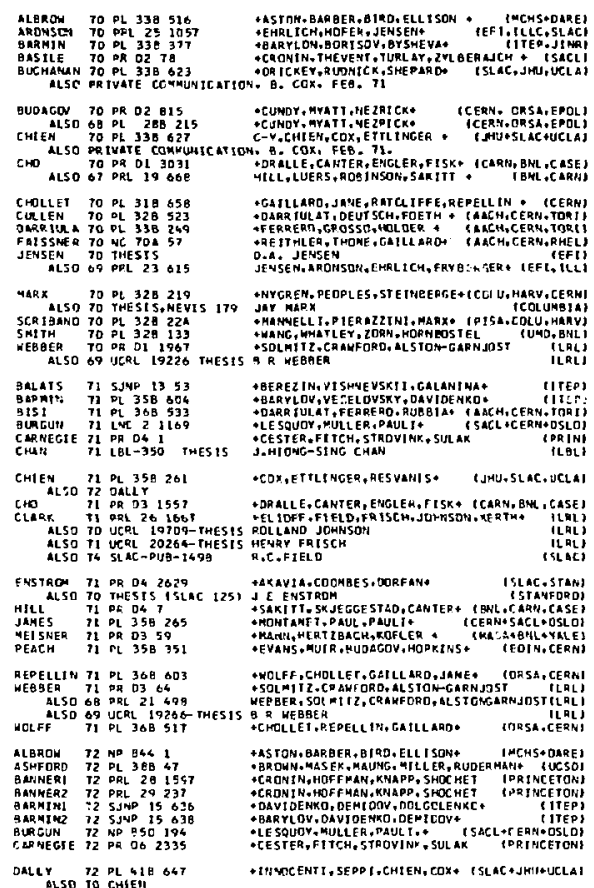
ALSO IO CHSEN

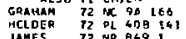

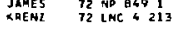

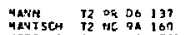

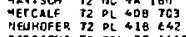

DICC JOW 72 DQL $291 \leqslant 12$

ALSO 74 PR 092939
VOSGURC 72 PR DO 1936
ALSO 71 DRL 26 BGE

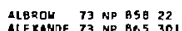

ALERANE 73 ND BE5 301

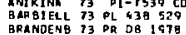

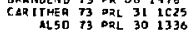

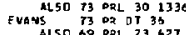

FACRLER 73 OFL 31 Q44

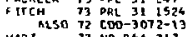

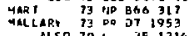

بCC ARTHY 73 OF 0768 , 68 ,

ALSO 72 PL 428 201
PLSO 71 THESS L8L-550

MESSALR 73 PQL 30 B 876

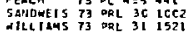

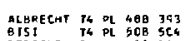

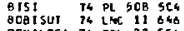

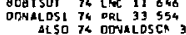

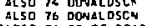

DONALS 74 POR 092960

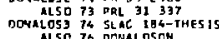

CEHERTEI IS OL $698<03$

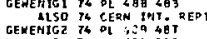

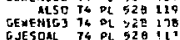

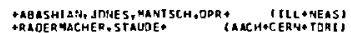

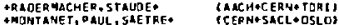

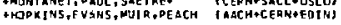

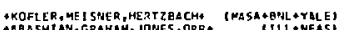

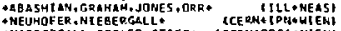

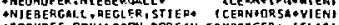

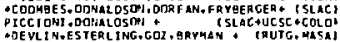

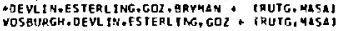

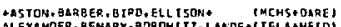

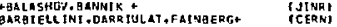

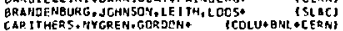

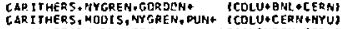

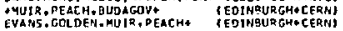

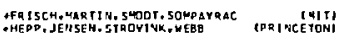

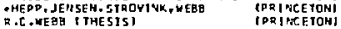

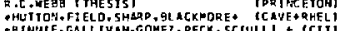

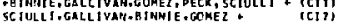

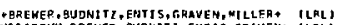

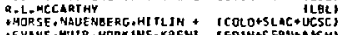

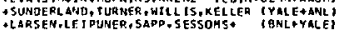
DUGMA+BEPL 1 F. Q BUDAPE ST + PRAGUE + SEF PUKH* SDF1A

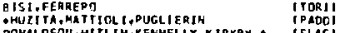

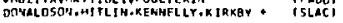

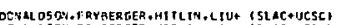

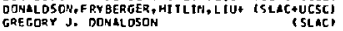

GEHEVIGER, G,JES DAL, KLMAE, PAE SSER \& IC ERM+ HETOI

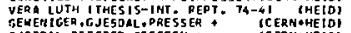

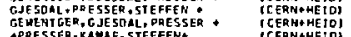

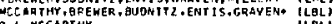

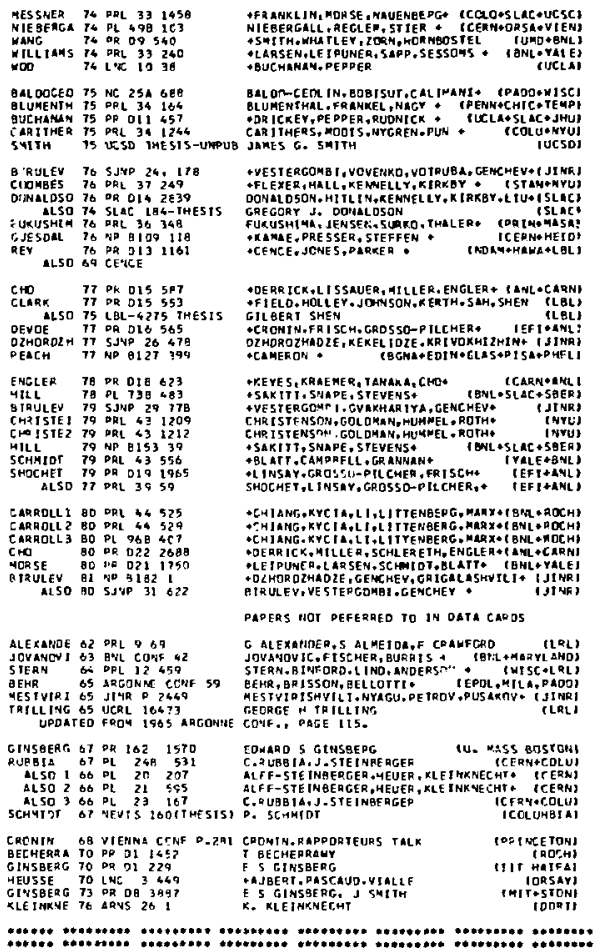

$D^{t}$ 31 CHARGE OII BQ9030=0- $I=1 / 2$

31 THARGEO D MASS (WEV)

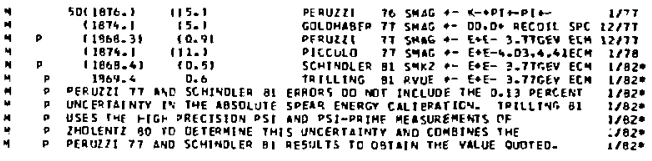

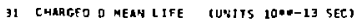

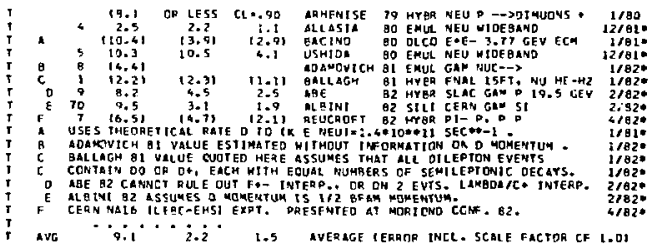


31 CMARGEO D PARTIAL DECAY MODES

D. JNIO K- DI+ PI

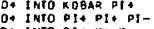

O* INTO Plt K+ K-

* INTO E: NUE

D* IRTD E: ANYTHING

D* INTO K- ENYTHTHG

0. TMTO K. ANY THING

D+ INTO KOEAR PI: PIO

D* INTD KOARA P1 P PIP Ot-

De 1 NTS PIt PIO

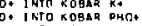

INTO ETL ANYIHING

D. mcoes afe conage conjugates of the apoue mooes
OECAP MLSSES

$493+1390130$

1394. 139. 139

1394.393 .473
$.53+139.139$

891* 139

$497 * \quad 394+136,1394$

$47+\quad 394$
1394 390.139

139.134
697.493

4910768

\section{*1 CHARGED D BRANCHING OATIOS}

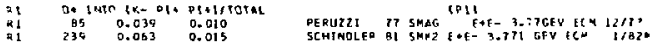

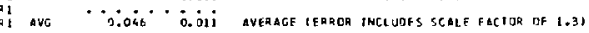

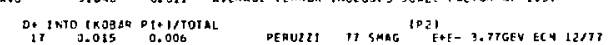

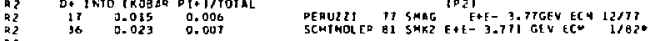

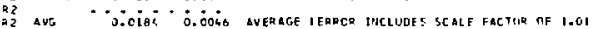

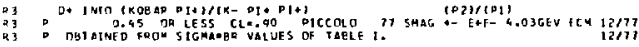

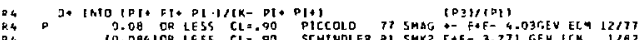

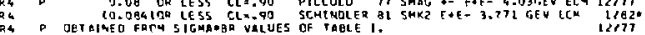

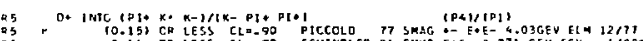

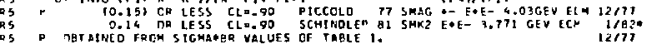

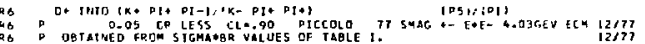

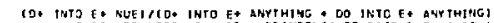

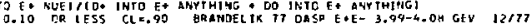

D. $\triangle N D$ dO TNTO IE+ ANYTHINGIJITOTAL D* ANO DON

THIS GIVES Q WEIGHTE D Q VERAGE OF

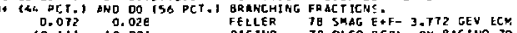
13.111 10.021 BACTNO TE OLCO HEPL. GY BACTNO I9

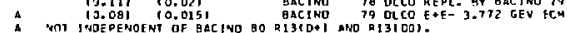

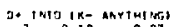

$\begin{array}{lll}3 & 0.10 & 0.07 \\ 26 & 0.29 & 0.05\end{array}$

coes

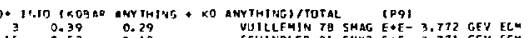

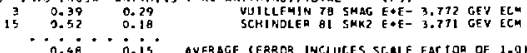

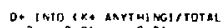

$\begin{array}{ccc}2 & 0.06 & 0.06 \\ 12 & 0.00 & 0.06\end{array}$

VULLLEII 79 SMAG $E \bullet E-3.772$ GEV ECK

SCHINDLER GI SMRE E+E- 3,T7L GFV ECM

ail ave - o.060 0.033 average terror lir

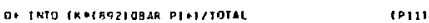

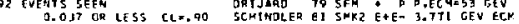

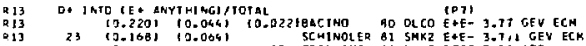

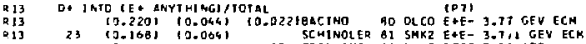

R.

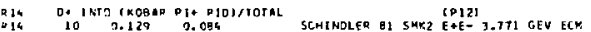
DA INTJ IKOB 21 ONTJ IKOBLR PT+ P1" P1-1/TOTAL

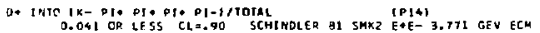
J* IRT IKOBAR QRDAITTOTAL

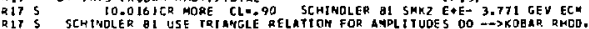

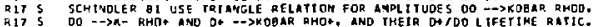

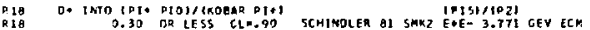

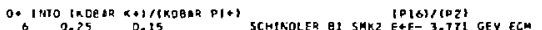

R.19

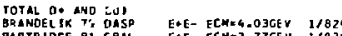

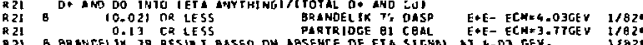

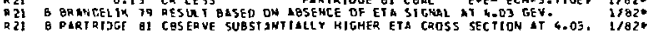

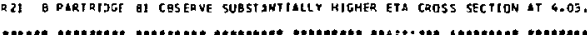

RETERENCES FOR CHADCEC $C$

GOL DnAat to Det 37255 DER Jizl 76 PQL 37569

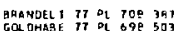

GOL OHABE 77 PL 698503

PERJIZI 77 ORL 391301
PICCCLO T7 DL TOS 260

FACTNE TE DPL 40 O

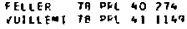

BAMENISE 79 DQ HOP 115

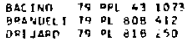

ALLASIA RO WP HITB 13
BATINO

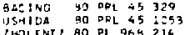

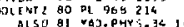

SDM YCVIC $\theta 1$ De 989 271

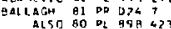

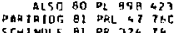

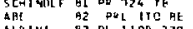

ALEINI 82 PL 11 DO 319

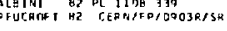

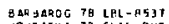

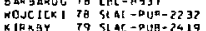

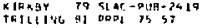

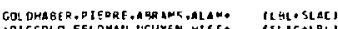

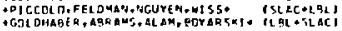

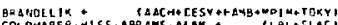
COL OMABER, NISS, APRAMS, ALEN E (LAL FLOC)

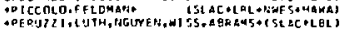

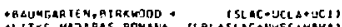

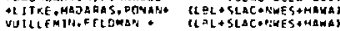

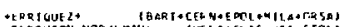

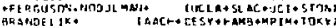

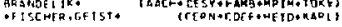

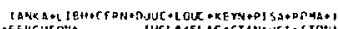

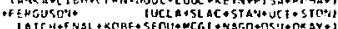

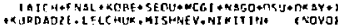

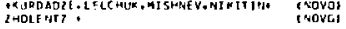

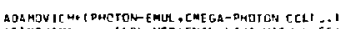

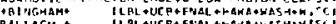

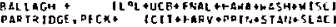

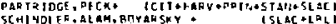

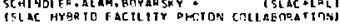

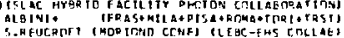
EEVIFWS

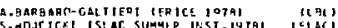

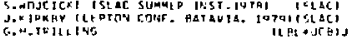

(1,

$\mathrm{D}^{0}$

$\Rightarrow$ MEUTRAi DIIABS, JP=0-1 $\quad t=1 / 2$

IDF A RECINT RIVIEW SFE TMILLTHG MI

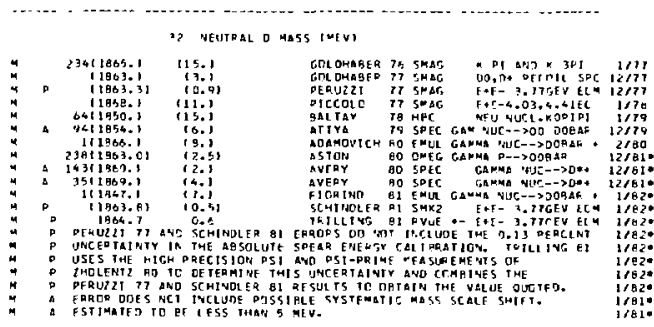

$\lim _{1 / 79}$

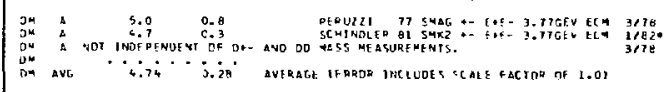

Note on the $\mathrm{D}^{\mathrm{D}}$ Lifetipe

The $D^{0}$ lifetime is a subject of uncertaincy, with the problems of inconsistent experimental results not yet resolved. Earller results, primarlly using emulsion targets, reported lifetimes of the order of $1 \times 10^{-13} \mathrm{sec}$ or less. The $\mathrm{e}^{+} \mathrm{e}^{-}$ colliding-beams results indicate a charged/neutral D lifetime ratto of 3 or posstbly much larger (subject to some theoretical uncertalnties). Many of these results are characterized by relatively large quoted errors, and the most recent experiments suggest that the $D^{3}$ lifetime lies in the range $2-7 \times 10^{-13} \mathrm{sec}$. The observation of individual events with proper lifetimes of the order of 


\section{Data Card Listings}

\section{For notation, see key at front of Listings.}

$20 \times 10^{-13} \mathrm{sec}$ suggests that the mean life may Indeed be longer than first thought, perhaps comparable to the $\mathrm{D}^{ \pm}$iffetime. The possibility of there being 2 lifetimes in the manner of the $K_{S}^{0}-K_{2}^{0}$, or of there being more than one type of massive weakly-decaying neutral particle, has been discussed, but there is no solid evidence.

$$
32 \text { NEUTRAL O MEAN LIFE CUNITS 10*0-13 SEEI }
$$

\section{(B.0) CR LESS CL-.70 ARMENTSE 19 HYER NEU P $\rightarrow$ OTHUCHS +}

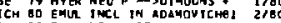
$10.531 \quad(0.57) \quad 10.251$ ALLASTA BO EMU NEE WDOEAANO

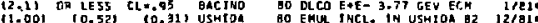

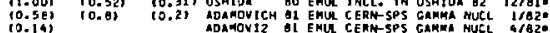

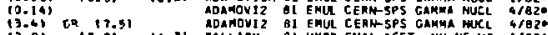

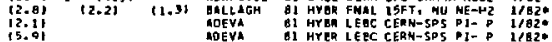

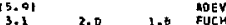

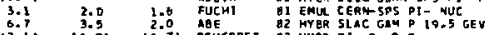

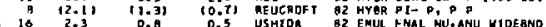

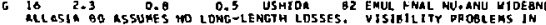

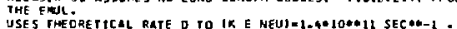

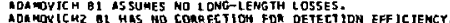
ADAFON LC KZ OL HAS NO COARECTION FD OETECT IDN EFF IC IENCY. CONTAIN DO OR D4. EACH HITH EOUAL MUMBERS OF SEMITEDTOWIC DEC AYS

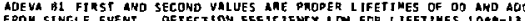
SEC aR LESS-

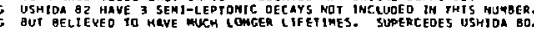

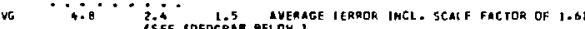
WEIGHTED AVERAGE $=0.210 \pm 0.000$ ERROR SCALED BY 1.6
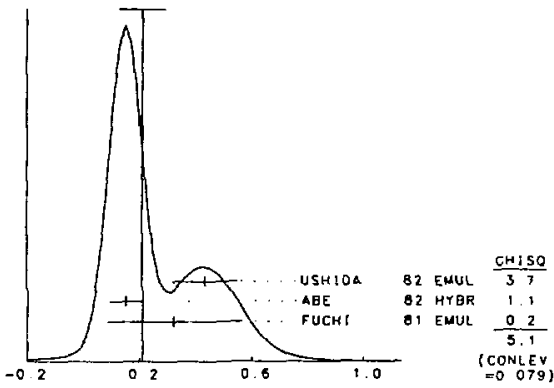

NEUTRAL D DELAY RATE (UNITS $10 \cdots 135 E C-1$ )

32 I CHaAge OI/CMEUTRal OI MEAN LIFE Fat TO

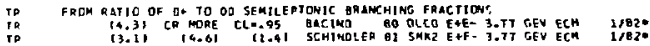
32 neuthat D pagtial oecar modes

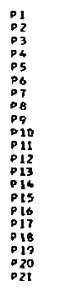

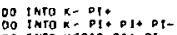
DO INIO KOBAR PI: DI-

DO THTO PTOPI

DO INTD $K+$ PI- IYIA DOBARY

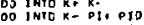

DO INTG KDAR PIO

DO INTG KOART PIO

DO INYO $k$ - ANYTHING

DO INTO KOBAG AHYTHIME + KO ANVTHING

Do ivis $x=18921-010$

of INTO $k+169210$

OO TITO KOBAR RHOO

09 inTo $P\{-P Y-P I+P S+$

DO INTO UHF ITTEO MODES \{KEEPS FIT OCM. HAPOY,

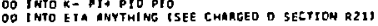

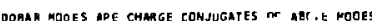

DECAY MASSES

$493+134+139+139+139$

4976 1394 1390.139

$130 \% 139$

$493+110$

$493+493$
$493+1390$
6974

91. 139

$891+134$

1974 769. $1394+139+139$

491+ $139+134+136$

ETTED PARIAL DECAY MODE BXANCHINC EAACJION:5

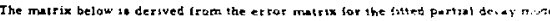

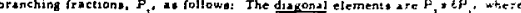

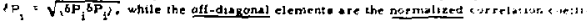

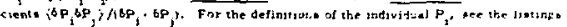

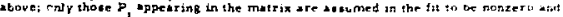
are thus cositrajed to add to 1.

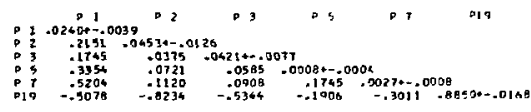

32 NEUTRAL O gRATKHIWG RATIOS

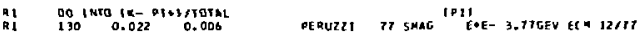

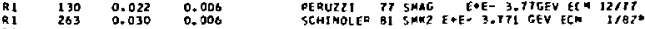

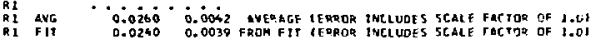

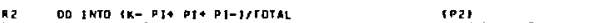

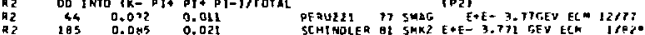

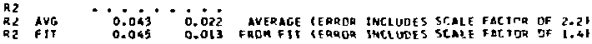

OO INTO IKOAAP PI+ PI-IITOTRL

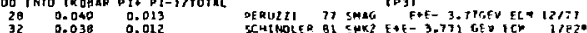

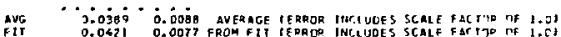

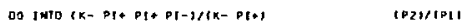

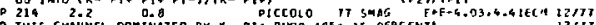

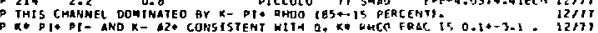

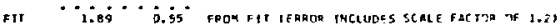

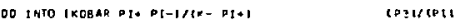

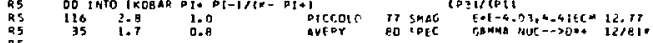

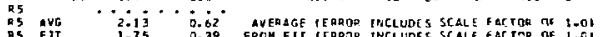

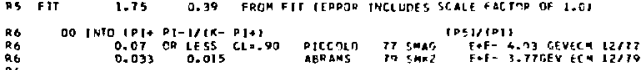

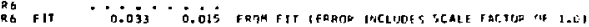

De $\{$ NTo $\{k+*-1\} \mid K-p\}\}$ 
Stable Particles

$D^{0}, F^{\mathbf{E}}, \mathbf{B}, \mathbf{P}$
Data Card Listings

For notation, see key at front of Listings.
COCDHAE 76 ORL 37255

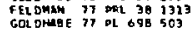

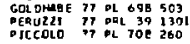
AQA TAY TA PRL ti 73

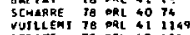

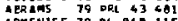
AEYENISE 79 PL PLG 115

acomovic 80 of 89842 ?

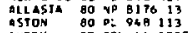

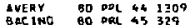
USHIOA
$I$ HOL EOT $Z$ BD
BO

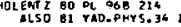

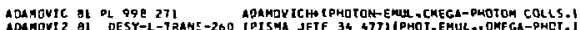
4150,90 sambiter

ADEVA BI OL 2028285 grelach of or 024 7

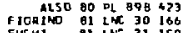
FUCH1 of LKe 31150

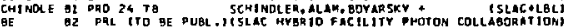
AGE

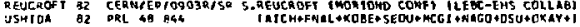

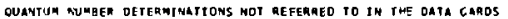

VGUYYN II PRL 39262

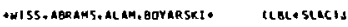
REVIEWS

+AGUILAR-BENITEZ + LLEQC-EHS COLLABDARTIONI

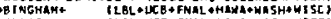
BELLAGH + RL

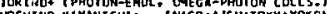
GMLOMABER, OI ERRE, ABRAMS, MLAMA TLBLOSLACI

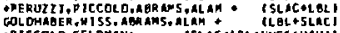

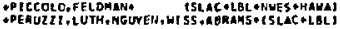

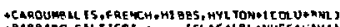

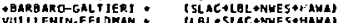

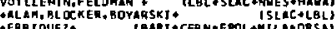

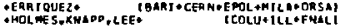
ADAMOYTCHA CPHOTOH, EMUL, CMEGA-PMOTON COLLS.I

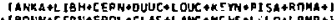

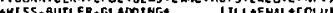
ERG USP

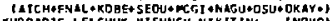

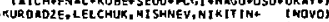
2HOLFTIZ -

BA8Baroc 78 L8L-853

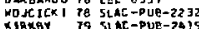

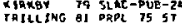

A-BARBIAD-GALTIERT IEKTCE 1978) ILBL,

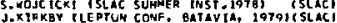

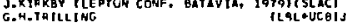

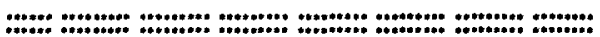
$\mathbf{F}^{ \pm}$

24 F+12020.5P= ,

34 F+-120801 mass (MEVI

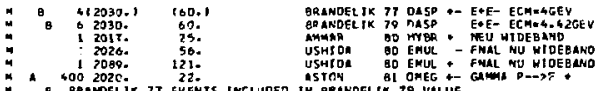

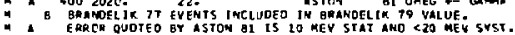

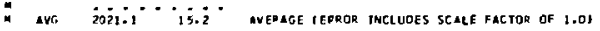
36 F+-120201 MEAM LIEE IUYISS LOAN-13 SEC,

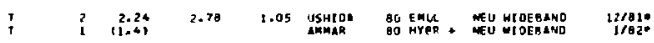

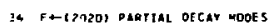

Q1 F+- NTO ETA PID-

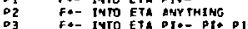

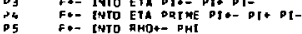

DECAY maSSES

$548 * 239$

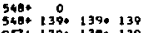

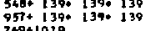

36 F+-(2020) BRATCHING DATIOS

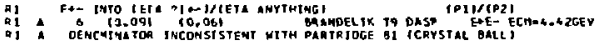

$R_{2}$
$R 2$
FE- INTO ETA DTE- DI. PI- ASTOH

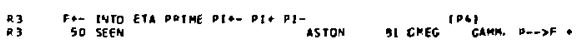

Q4 FE- INTO QHO+- PHI

asionz

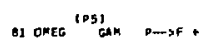

REFEQENCES FOR FT-12020,

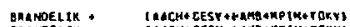

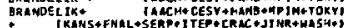

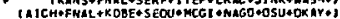

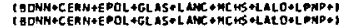

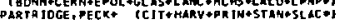
REviEus

G.H.TR HLLING

[LBL * UCE)

:

$12 / 77$ Tis

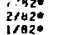
1/80:

iz/a1:

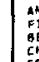

\section{Bes
Sho
Sho}

(1)

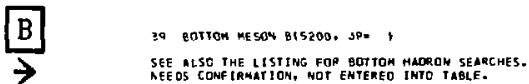

39 B MASS IMEV

S140 TO 5290
5275 OR LESS

39 C PARTLA DECAY aDOES

D1 B INTO ELECTRON AHYTHING

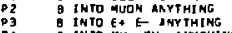

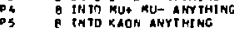

39 B BRAMCHING FRACTIONS

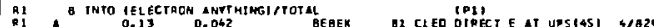

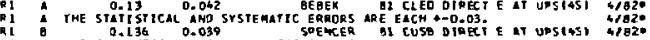

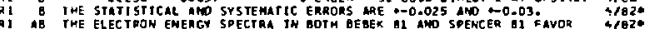

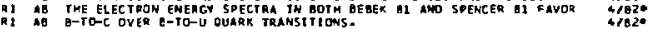

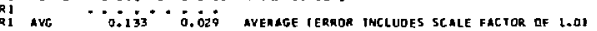

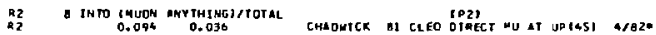
R3 B INTO LFE E- ANYTHING)/TOTAL

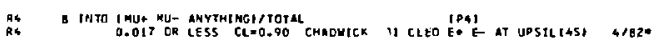

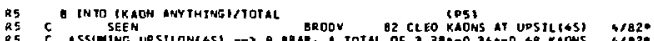

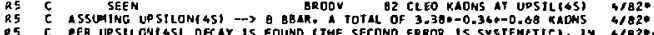

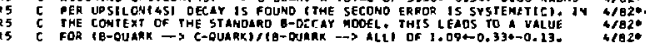

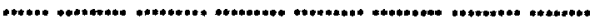
REFERENCES FOR SOTTOM MESON OLIS 2001

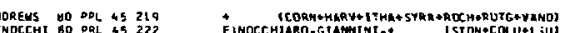

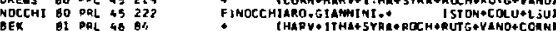

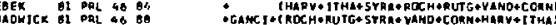

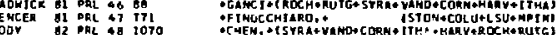
1

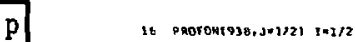

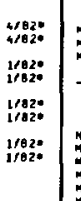

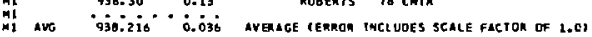

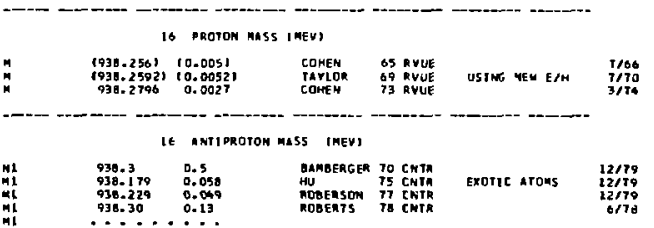




\section{Data Card Listings}

\section{For notation, see key at front of Listings.}

\section{Stable Particles}

16 RROTON MERN LIFE, GRAMCHING GRACTIOY TUNTSS YEARS BARYCN CONSERVATIDU NOULO OE VIDLATEO BY A FINITE DHOTON MEAN IIFE. TSEE ALSO NEUTROH ANTINEUTKON OSCILLATIONS IA HEUTRON SECTION BELJU FOR ANOTHER TEST OF GAQYON CONSERYAT ION-: SEE ML SO THE MEV IE WS OY REIMES AND SCMURTZ, SURVEYS TH HIGH EMERGV

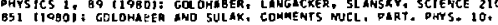
215 (1981):

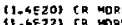

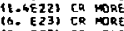

(3. ह233)

19.

(1..2630? $\mathrm{CP}$ Pont

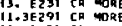

(1.) 5301 Ch

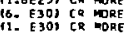

II. GE 303 CQ TORE CLE.90

$211.23 \in 30,158$ 40R

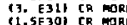

2. E 3 C I $\mathrm{C}$ MAR

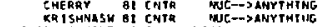
AEFEAENCE ALSO GIVES LIMITS FOR DTHER MDDES. WE GIYE HIGHESI LIMIT. FTRST LEARNEC 79 VALUE SUPERCEOES ME1NES 74 . SECONO LEARNED 79

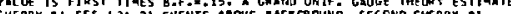
VALUE IS FIRST TIMES H.F.=. OS, A GMAHD UNIF. CAUGE THEOPY ESTIMATE.

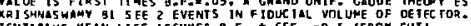

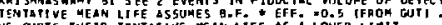

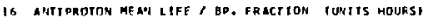
LIMITS SMIUM ADE PARTILL MEAN LIFE FOD MODE SHOWH AT RTGHT.

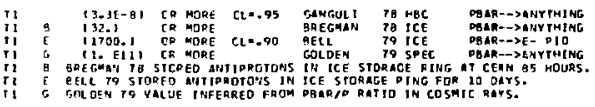

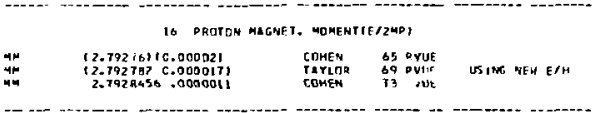

16 ANITPROTON MAGNFTIC MOMEHT IE/ZMP,

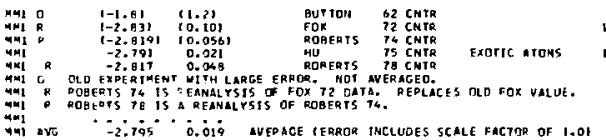

16 PHOTDN ELECTRIC DIPOLE MOKENI IUNITS TOEE-23 E [M]

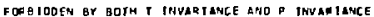

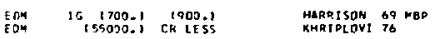
$10 / 69$
$1 / 18$

IS DROTON ELECTRDN CHARGE DIFFERENCE CUNTTS EI

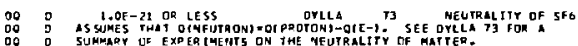

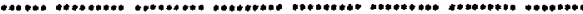

PEFERENCES FOH PROTON

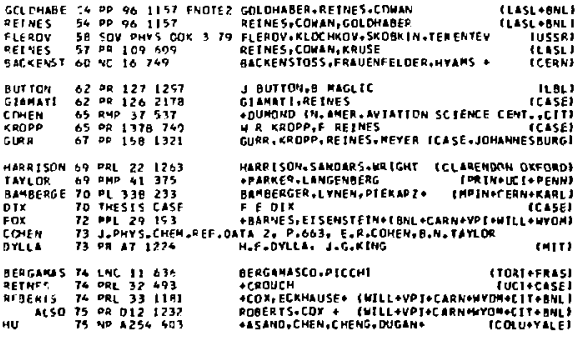

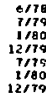

$\prod_{3 / 70}^{7 / 70}$ n

inge

2/832: $1 / 82 \circ$

\section{BREGMAN 78 PL 780174

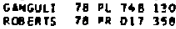 BELL $79 \mathrm{PL}$ BS 215 GOCDEN 79 PRL 431196

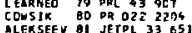 ALEKSEEV BI JETPL 33 ES

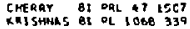

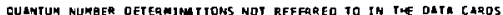 KMLOEEO TO PQL 331037
FRANKLIN 77 PQ O16 910

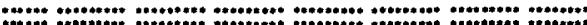

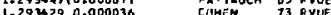

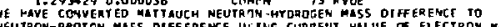 AHO I HYCRDGEN BINOT MC ENERGY DF I3.S EY, TWE MELSUREMENT OF THE MEUTRON MERN LIFE BY SOSNCVSXIT S9 MAS RECENT RESULT OF CMRISTENSEN G7. 20. THE VALUE CF CMIGV DE-

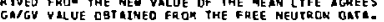
Ime.pHYs onstrogstogras (T) MEUT LON1539, J-1/21 $\{* 1 / 2$

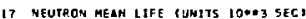
0.0130 .020
SOSMOYSKI ST PILE \\ $\begin{array}{cc}10.9351 & 10.0161 \\ 0.918 & 0.016\end{array}$ \\ $0.0771 \quad 10.009$ \\ $\begin{array}{ll}0.937 & 0.018 \\ 0.675 & 0.095\end{array}$ \\ CHRTSTENS OT DILF RE \\ CHR ISTENS 72 PTLE \\ BRRNE BO DILE \\ KOSVINTSE DO OILE \\ INFERRE VALUE

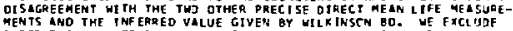 THE SE TMO RESUL TS FRDM THE AVERAGE. AS IS RE CCMPEMDED GT IL KINSON BO-

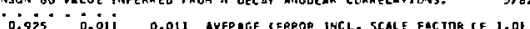

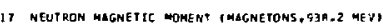

$12>0$

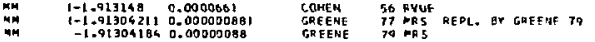
3186
3178

17 NEUTRON ELECTRIC OL POLE MONENT (UNITS JON-23 F [N] FCREIOOEM BY GOTH T INURRIAYCE AND D INYARIANCE

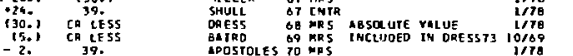

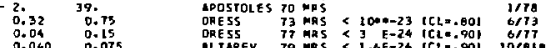

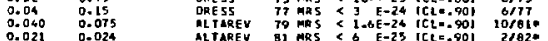

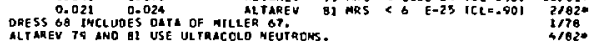

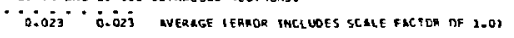

17 NEUTRON CHARCE

SEE SEGTION OC IN THE PROTON OMTA CARD LISTINGS ABOVE

17 MEUTROM DARTIML OECAT WJOES

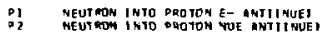
DECAY WASSES

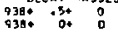

\section{NEUTRON ORLHCHINE RAT TOS}

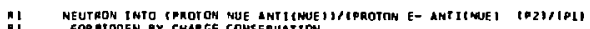
RI EOR BTOOEN GX CHARGE CONSERVATTON

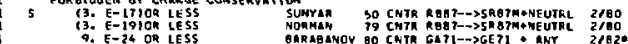

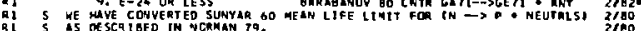




\section{Stable Particles}

n. $\Lambda$

17 LimIt ON MFUTRON-ANTIMEUTRON OSCLLLAJ JONS

NAN MERN TIME FOA N-RNTIH TRAMSITION IN VACUNG TUHITS SEC.I

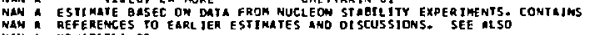

WAN MOMAPATKA BR.

17 NeUtadin getM decay parameters

RELATEU IENT SECTICH VI D.I

AV C GA/GV ISEE TFXI FOA SIGN CONUEMTIONI

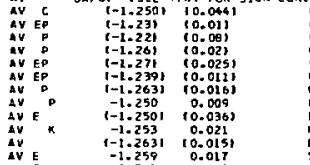

DT RYUE SEE MDTE C BELOU CHR ]STENS GT CNIR M DECAY FT VALUE

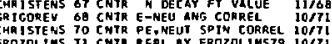
FRO2OL I WS TI CMTh REPL. BV EROZOL IMS79 10/71 ROPTTENS 72 CNTH N DEC.+ FT VALUE $1 / 73$
ROPF 73 RYUE H DEC.+ FT VALUE $1 / 73$ OOGROZEWS 75 CHIR REPL, OY SIRATOHA 7812175 RROHW 75 CNIR PE.NEUT SPIN COPREL LFT7 EROZOLIMS 77 CKTR REPL.BY ERDZOLTMSTO $1 / 78$

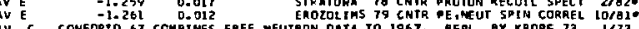
LY C CONFORTO 67 CCMBIMES FRE NEUTRON DHTA TO 1967. HEPL. OY KROPF 73 . 1/73

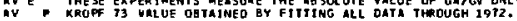

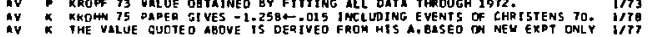

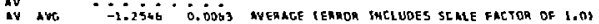

phase hale cF ga felative to oV coereess

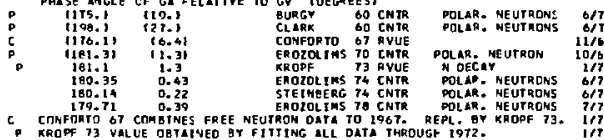

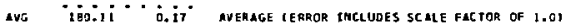
THRE E-VEC Y OA CCAMELATION COEFFICTENT OI MEASURE S CCMPONENT DF NEUTROM SPIN PERPFMOTCULAR DO LANE IN BETI OECAY. SHDULD BE

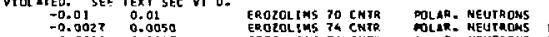
$\begin{array}{llll}-0.0011 & 0.0017 & \text { STEINBERG 74 CNTR TOLLE. NEUTRDNS } \\ 0.0022 & 0.0030 & \text { EROZOLIHS TO CNTR POLAR. NEUTRONS }\end{array}$

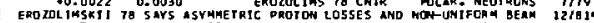

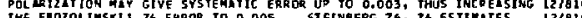
THE SYSTEMATIC ERRORS TO BE INSICNIFICANT IM THEIR EXPEQIMENT.

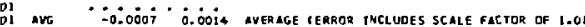

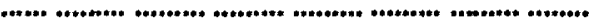

REF EREMCES FOR NEUTMON

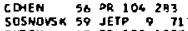

BUAGY OO OR 1201829

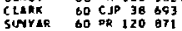

Y Y COHEN, CORMGQD, RAMSEF SBML +HARYMROS SOSMOYSMII, SBIVAK, PROKOFEY - IIAE ROSCOK

|BNL)

MLT TAUXH OS NP OT I

CHA ISTEM G7 PL 2 OEB 21

MILLER O7 PRL 19 3EI

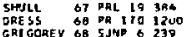

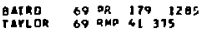

AMOSTRE TO RRP is 343

CHRISTEN TO OQ C1 169?

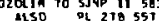

ERTZOLIA 71 JETPL 13252

CMISTEN 72 OR JS 1620

KROPF 73 PR DT 3147 PUAL

EROLOLI' 74 JETPL $20 \quad 345$

STEIMEER T4 PRL 33 H1

DOAORE 75 MR O13 2460

DRESS 7 PR DIS O

ENDEOLI II JETPL 23663

GREEMF TT 70718297

STRATOWA 78 PR D18 39700

ALTAREY 79 JETPL 29730

EROLOLIM 79 SJMP 30356

GREEME 79 DA 020213

OMABANO gO JETPR 32350

KOSYINTS dO JETPL 31236

CLTREY H $102 \mathrm{a} 13$

Creirres of OL 991 350

TSTA TAX PLANCK INST-CHEM,

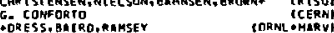

OQRESS, BATRO, RAMSEY

(1) $\mathrm{T}+\mathrm{B}$ ite

UE:KKO, MJLLER, MAMSEY TORNL MARY

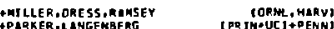

TPLOKER, I LNGEKBERG THEUCI +PENN

POSTOLESCU, IDNESCU, IOHESCU-BUJOA - I GUCH,

GROZOL IMSTI, BONOLREHKO. + TKRAE

TKTLE

EROZOL ]HSXI1.BOHDAREAKO + TKIAEI

CHP I STENSEN, NIELSON, BAHWEM, SAONNW CRISO

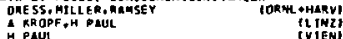

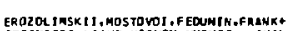

STEINBERG.LIAUO, VICHOW, HUGHES TYALE+GKEN STEIHEERG, LI AUO, VIGHON, RUGHES CYALE+GMEMI DOGROLEMSKY.KER SCHEAUM. MOMAW,PAUL - ISEIE
KROHN, RTHEO

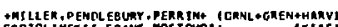

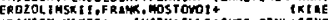

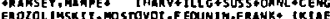

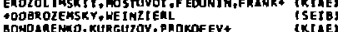

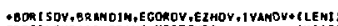

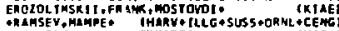

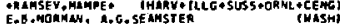

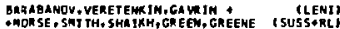

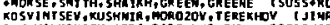
ROSVINISEV, KUSHNIR, MOMO2OV, TEREKHOV I J IMA

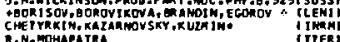

(6)

Data Card Listings

For notation, see key at front of Listings.

2

JACX5ON 57 PR 10651 ?

COHEN 65 MMP 37537
BHLLL OS PL 19 651

FAPERS YOT REFERRED TD IN DAYA GAPDS

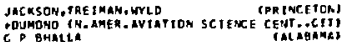

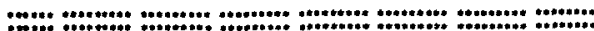

$\Lambda$

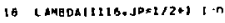

18 LAMGDA MASS (MEV)

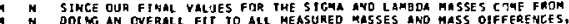
DOI NG AN OVERALL FIT TO ALL MEASURED RASSES AND MASS O!FFERENCES,

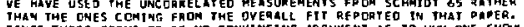
SINCE THERE SEEMS TD QE NO CDNVINCTHE ARGUAEAT 25 TO WHY ONE SHEDV

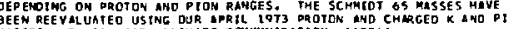
MASSE. D. SCHET JT. PRIVATE COMMUNICATION, 119761 .

$1115.44 \quad 0.12$ OHOWM1K 63 9YUE, SEE NDTE 1 GEL JW ABOVE LAHBOA WSS HAS BEEH ACISED 35 KEY TD ACCOUMT FOA GS KEV

\begin{tabular}{|c|c|c|c|c|c|c|c|}
\hline 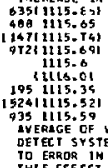 & $\begin{array}{l}0.07 \\
0.041 \\
0.0 .1 \\
0.8 \\
0.21 \\
0.12 \\
10.031 \\
0.08\end{array}$ & 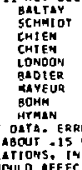 & $\begin{array}{l}85 \\
65 \\
66 \\
66 \\
66 \\
67 \\
67 \\
10 \\
12\end{array}$ & $\begin{array}{l}\text { MAC } \\
\text { HBC } \\
\text { MEC } \\
H \in C \\
H \in C \\
\text { HAC } \\
\text { EMUL } \\
\text { EMUL } \\
\text { HEAC }\end{array}$ & $\begin{array}{r}\text { SEE NOTE N } \\
\text { O.Q PUAR } \\
\text { G.Q PGAR } \\
\text { Z.4 PBUR }\end{array}$ & $\begin{array}{l}\text { DTDTIS. } \\
\text { DANIIL } \\
\text { DALAAT }\end{array}$ & $\begin{array}{l}6 / 66 \\
3 / 74 \\
4 / 67 \\
9 / 67 \\
6 / 66 \\
0 / 67 \\
11 / 67 \\
3 / 72 \\
11 / 71 \\
3 / 72 \\
3 / 72 \\
3 / 72 \\
3 / 72\end{array}$ \\
\hline
\end{tabular}

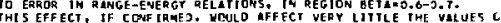
BHOWHICK B3 INO MAYEUR OT.
ERROR DURELY STATISTICAL.

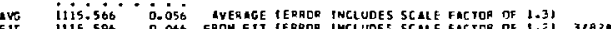
ISEF IOEDGRAM QELOM ? WE TGHTEO AYERAGE $=1115.566 \pm 0.056$ ERRDR SCALED BY:

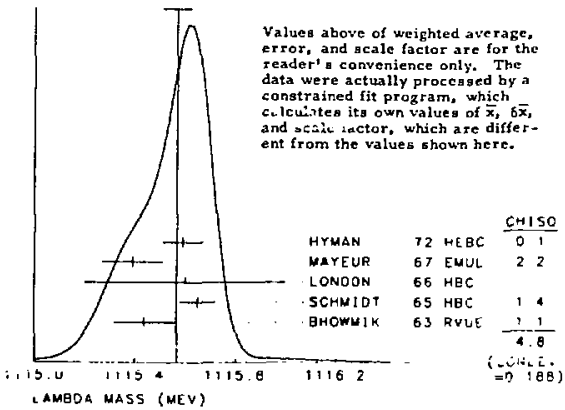

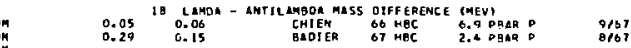

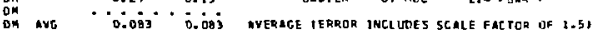

10 LAMBOE MEN LIFE (UMITS 100\%- JO SEC)

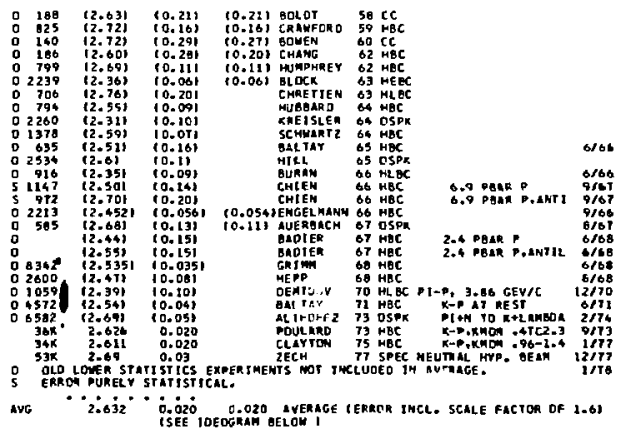


Data Card Listings

For notation, see key at front of Listings.

Stable Particles

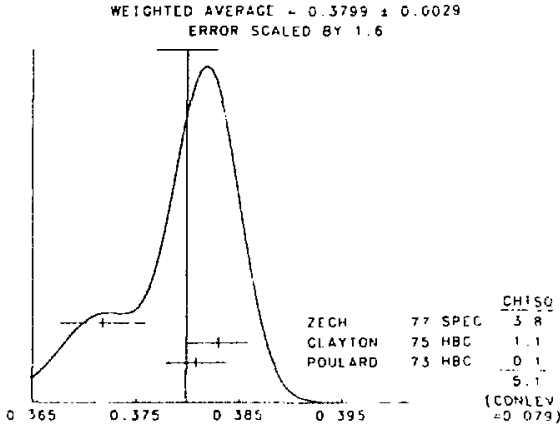

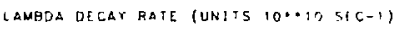

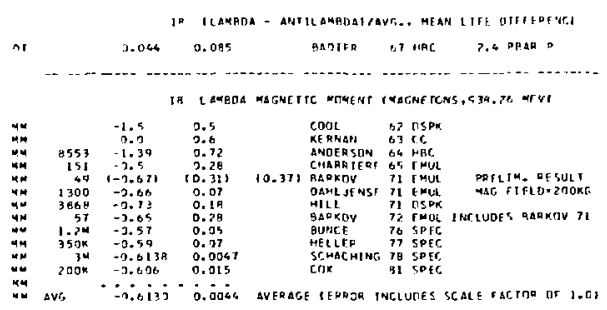

ser

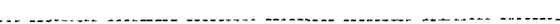

16. LAMBOA ELECTAIC DIPDLE MIMENT IUNTIS 190 F-14 + (M)

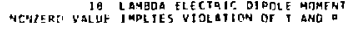

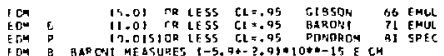

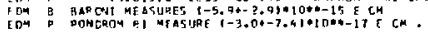

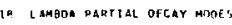

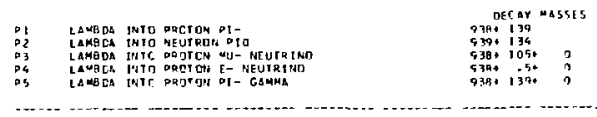

IA LAMBOL ARENCHINT, RATIOS

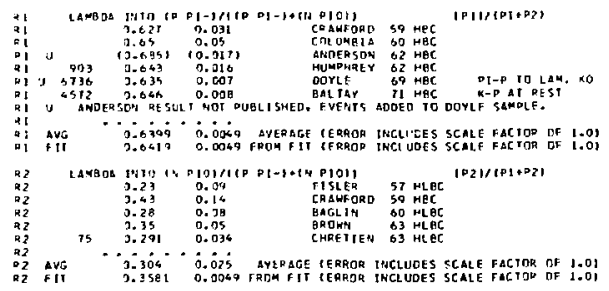

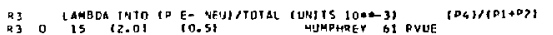

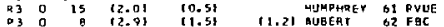

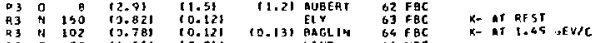

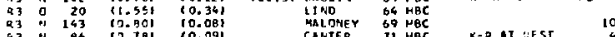

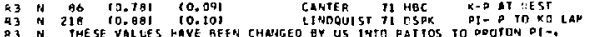

23
23

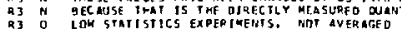

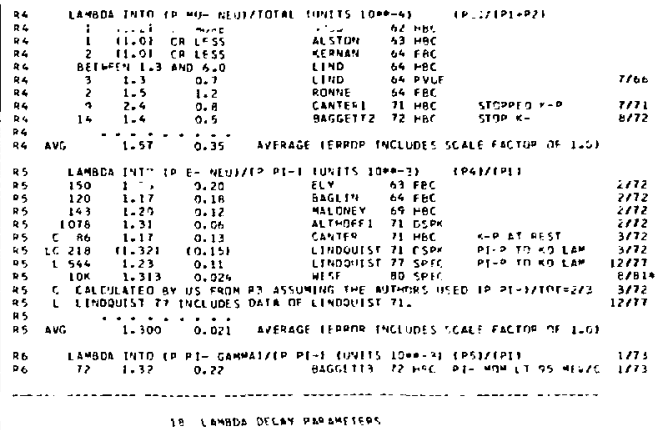

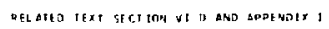

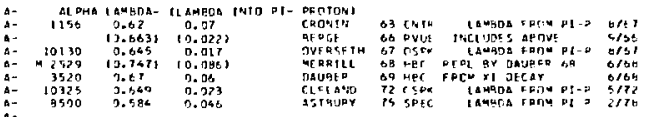

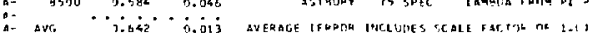

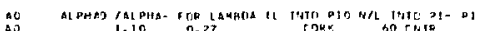

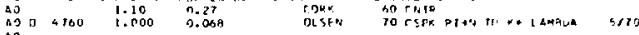

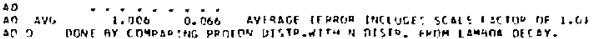

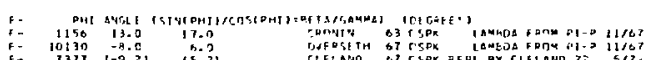

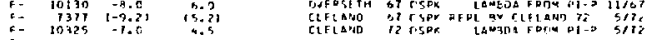

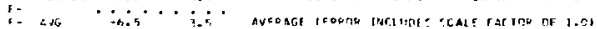

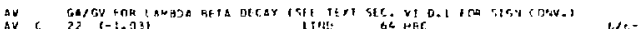

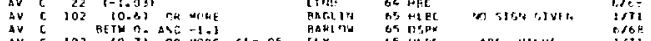

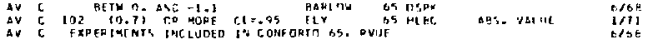

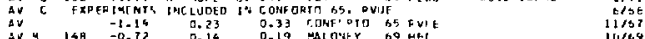

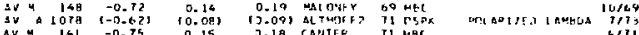

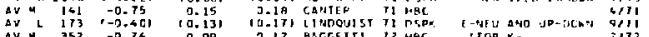

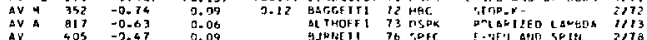

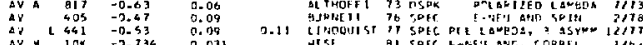

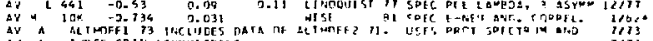

AV A THPEF SPIN PiYMUT TKIIS.

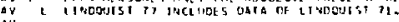

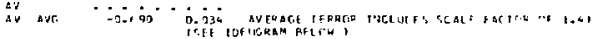

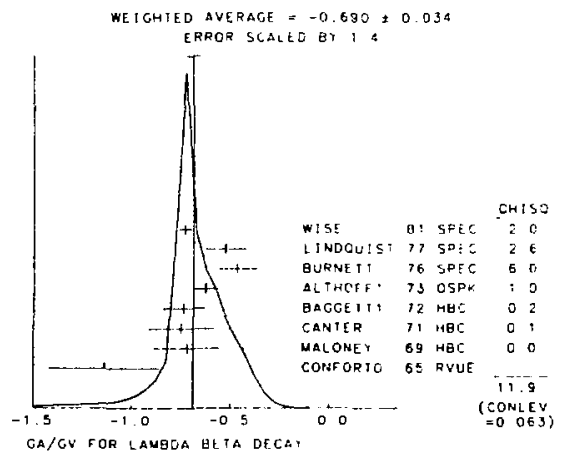


Stable Particles

$\Lambda, \Sigma^{*}$

EISLER $5 T$ TC 5 TPD

CRAKFOR $D 9$ DRL 2 266

BAEL IN SO

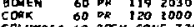

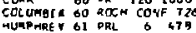

ANDEASON GZ CERY CDHF B32

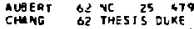

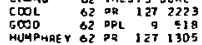

ALSTOH A9 UCRL 10426

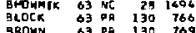

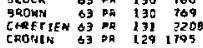

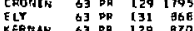

ANDERSON 64 DEI 13 167

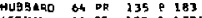

MEQNAN O4 DR 133 B 1271

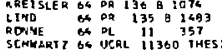

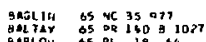

BAFLOW 65 of 18 of

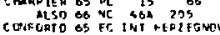

ELY of Dil 137 B B 30 ?

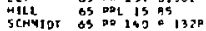

BEA SE OS EEDKELFY OC

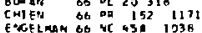

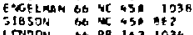

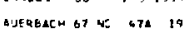

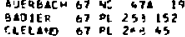

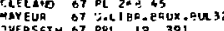

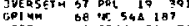

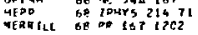

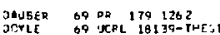

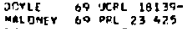

ACre 70 Ve 70 ? 394

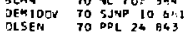

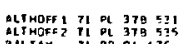

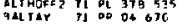

3expy 71 METPL 1460

CANTEA 71 DRL 256960

CANTERi

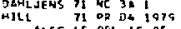

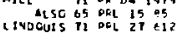

MAGGETTL 12 120HY 249279

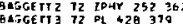

3A2ROY 72 JETPL 1610

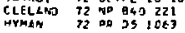

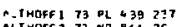

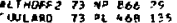

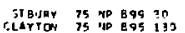

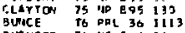

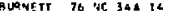

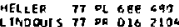

L INDOUIS 7700016210

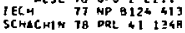

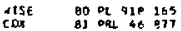

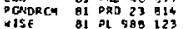

REFERENCS FOR LAUBOA

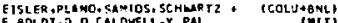

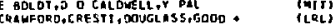
BAGLIN,ELOCH, ERISSON, HENAESSY + TEPOLI

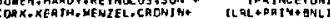

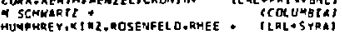
ANOERSDM, CAMMFORD, GOLOEN,LLOVD * ILALI

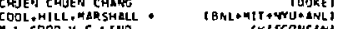

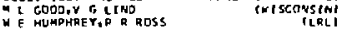
ALSTON, KIRZ MEUFELO, SCL HITZ, WDHEMUT TLELI

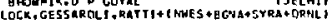
OQ JWN, KAOYK, TR!LLING, ROE * B (LRL+MICH] CWE TIEM.CRDUCH+ REFAN+BROWN+HAR VARD+NITI

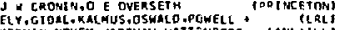

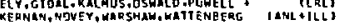

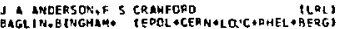
HUBBAPD. BEPGE, KALBFLEI SCH, SHAFEP + (LOL)

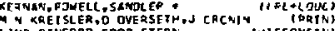

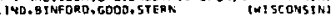
RONPE:
JTSF OH AOAM SCHMLRT?

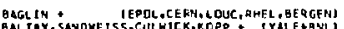

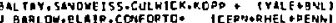

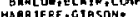

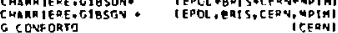

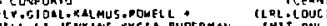

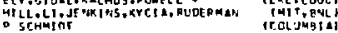

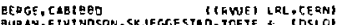

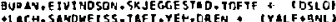

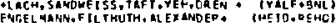

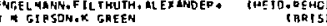

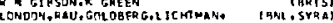

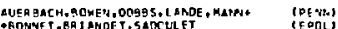

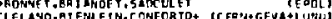

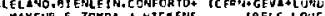

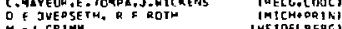
H. - J.GPIMH IHETOELAEDCS

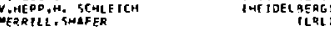

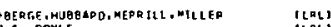

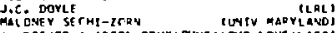

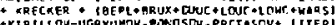

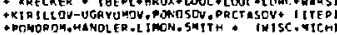

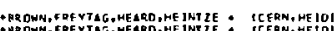
Po DSTER

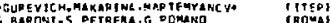

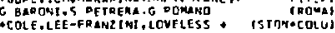

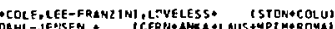

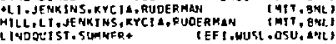

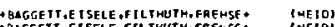
BHGGEYT, EISELE.FIL THUT H, RREHSE TH THELD

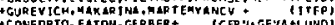

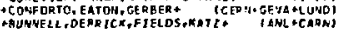

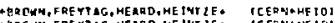

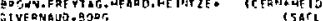

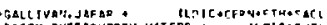

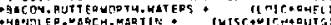

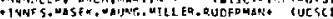

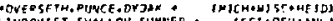

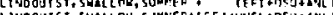

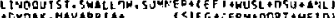

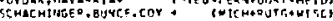

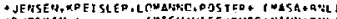

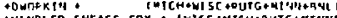

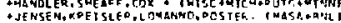

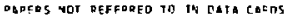

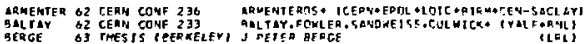

"evect
Data Card Listings

For notation, see key at front of Listings.
$\Sigma^{+}$

$1951641+11189.50=1 / 2+1,1=1$

19 STOMA. HLSS IMEVI

7. SEE NCTE PAFCFCING LAHBOE MASS LISTINGS

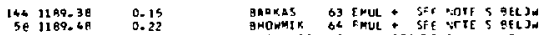

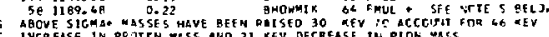

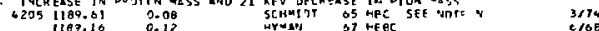

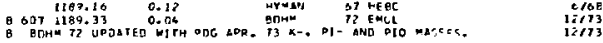

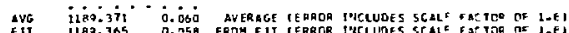

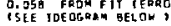

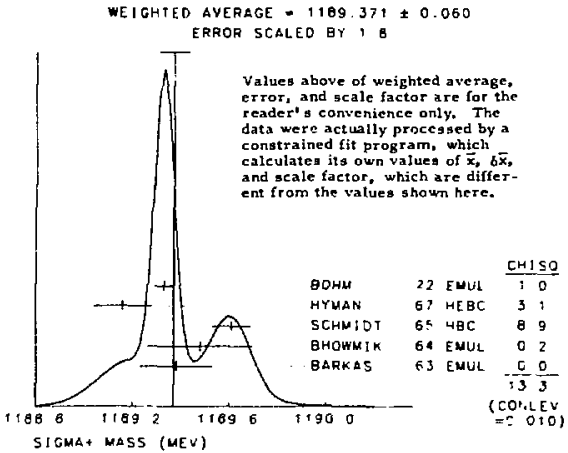

10 SIGUE, MEAN LITF ILWISS $10 * *-10$ SEC

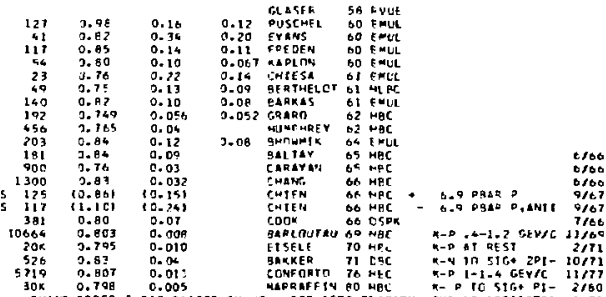

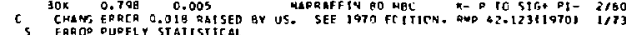

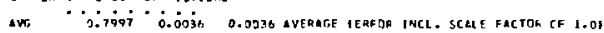

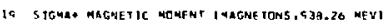

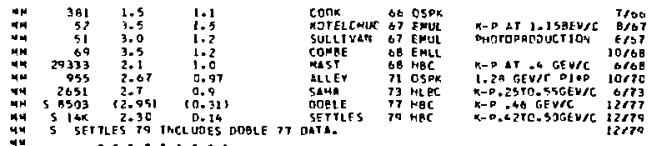

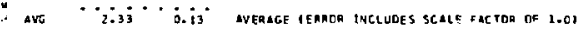

in SICMLA PENTILL OECAY MODES

PI SIGMA* TNTC PLCTCN PIO

STGMA. INTO NEUTROA PIt

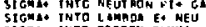

SIGAL: INTO L WHDN E+ MEU

STGMA. IMTO NEUTMON MUA NEUTHINO

SIGWA: IMTO NEUT REN EQ NEUTR ThO

$930+139$

$1130+1390: 0$

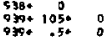

$938.5 \% .5 \%$ 

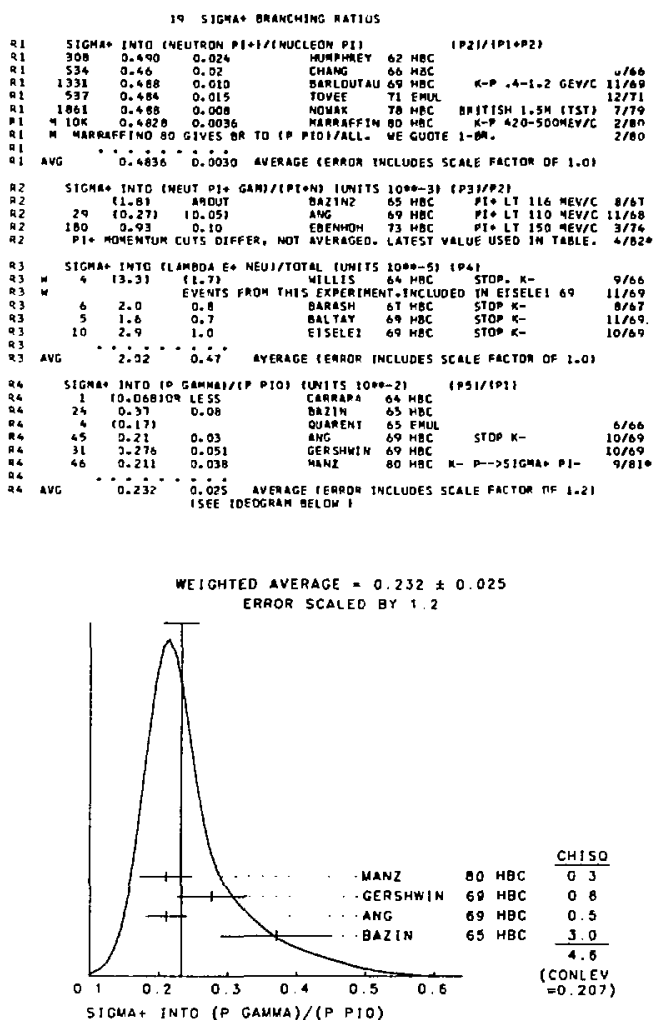

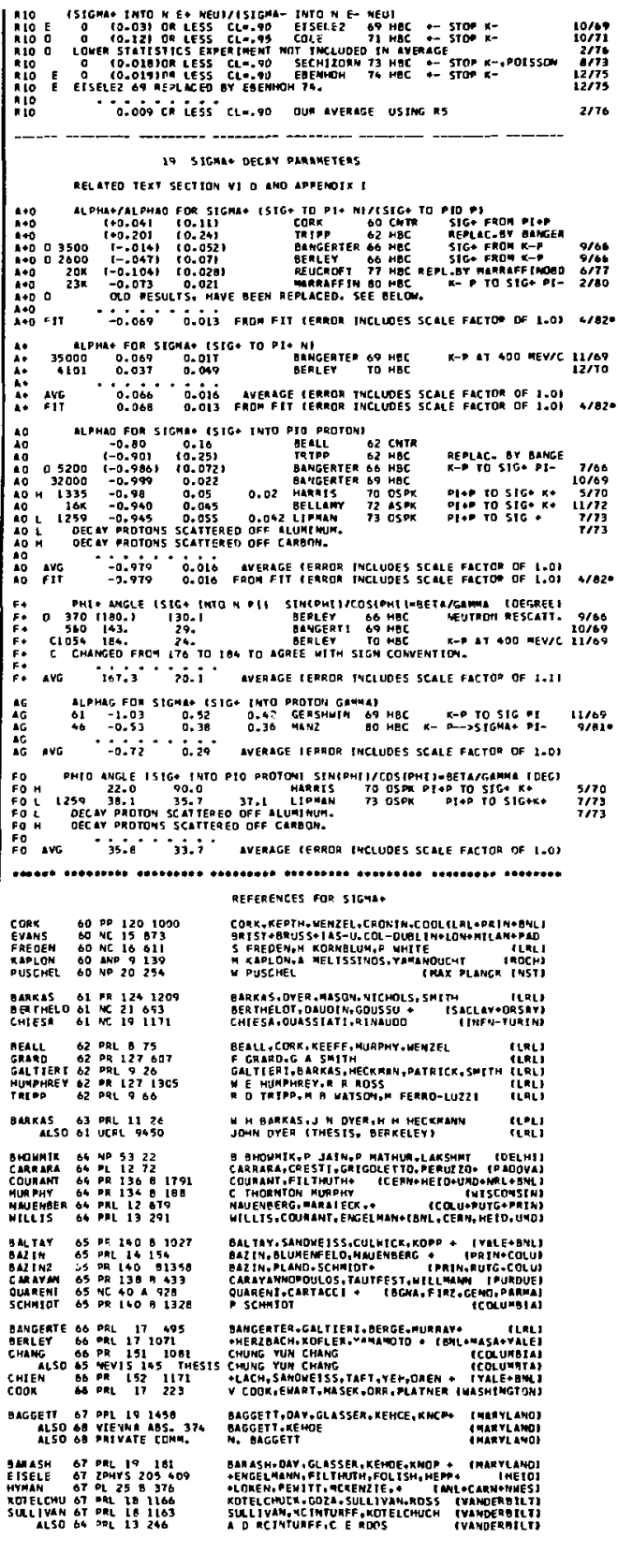


Stable Particles

$\Sigma^{+}, \Sigma^{-}$

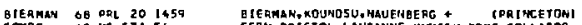

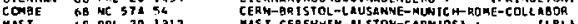

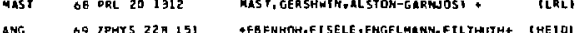

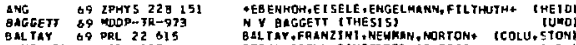

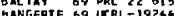

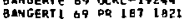
BARLOUTS 69 ND 914,153

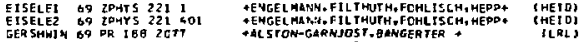
LRL
ILRLI

BERLEY TO DR 912015 TYAMIN,HERTZBACH,KOFLER D IGML, MASA, YALEI

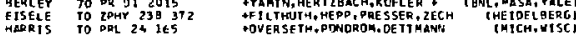

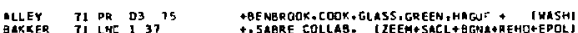

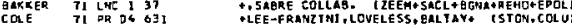

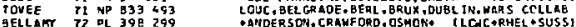
getLAT 72 DL 398299 ALSO 73 \$I 13 HE-73.2 NOW

EBENHOH 73 2PAY 204413

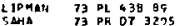
SECHILOR 73 OP OB 12 EAEVHOH 76 2PHY 200357 COAFDRT 76

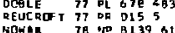
SETLES 19 on 0202154

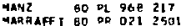

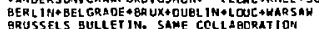
-E1 SEL E. FIL THUTH, HEPP.LEITNER,THOUY, THEIOI

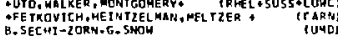
DETSELF.ENGFLMANM. FILTHUTH. HEPP + THET D? - GOPAL, KAL YUS,LITCHF TELD, D DSS + IRHEL + LOTC

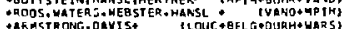

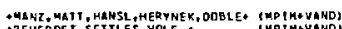

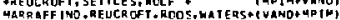
papers NOT pefeamed io ill data chros

TLASEP SA CERN CONF 270 GLASER, GDUO, MORQISON \{ UICHOLRA I OUANTJM RUMEE DETERHINATIRNS NOT REFERREO TO IN THE DRTA CARDS

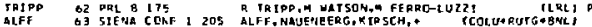

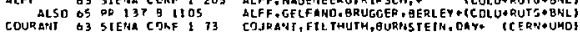

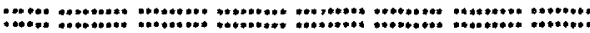

$20 \quad 5164 A-11197, J P=1 / 201 \quad 1=1$

20 STTMA- MASS TMEVI

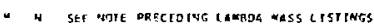

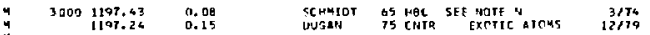

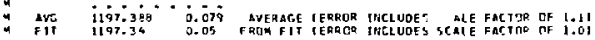
20. (SIGMA-1 - ISIGMAT) MaSS CIFFERENCE I -FVI

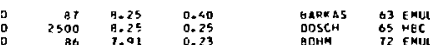

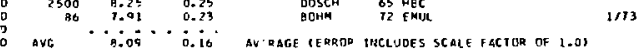

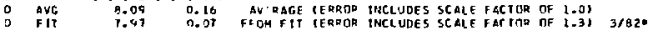

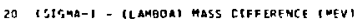

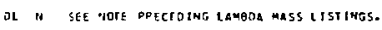

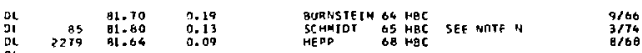

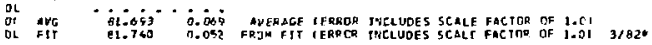
is SIGMA- MEAH LIFE IUNITS I0E- 10 SECI

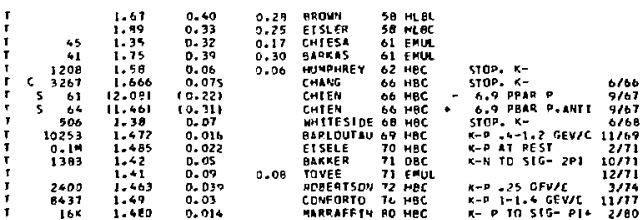

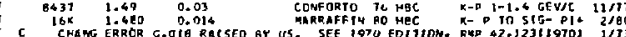

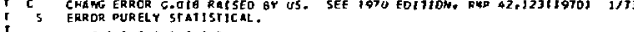

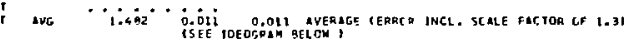

Data Card Listings

For notation, see key at front of Listings.

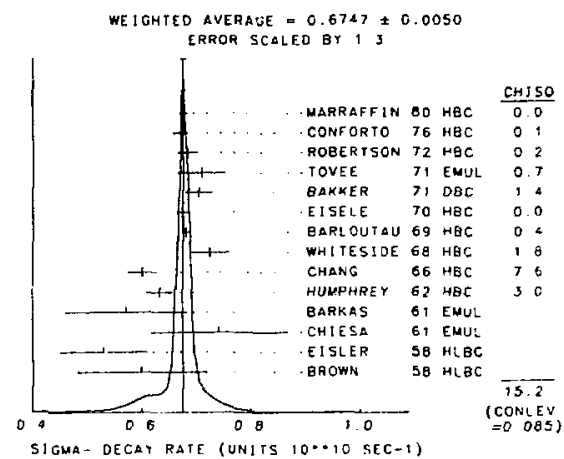

iD SIGMA- MAGNETIC MOMENT IMAGNFTONS,930.20 MEVI

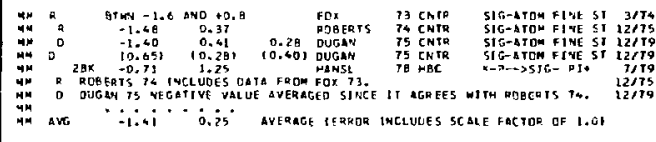

IC SIGMA- PARTIAL OECAY MDOES

P1 SIGMA- TNTE NEUTRON D1-

SI SIGA- INTO NEUT OSN DI- GSHMA

SIGME- IVTO MEUTRCN MU- NEUTPIHO
STGMA- J NTG NEUTRDN E- NEUTRINO

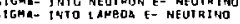

Detar massés

$939 * 139$.

$\begin{array}{rl}939+130 * & 9 \\ 839 * 105 * & 0 \\ 1115, \text { :5* } & 9 \\ 115 & 0\end{array}$

LV STCMA- ERRALHING PATIOS

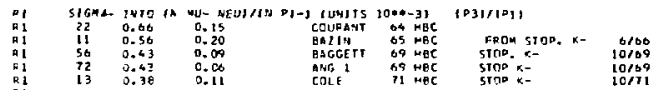

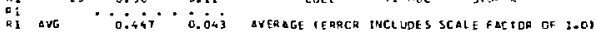

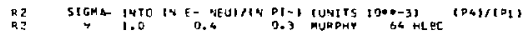

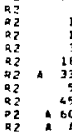

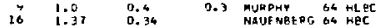

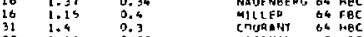

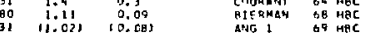

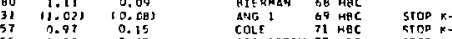

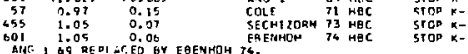

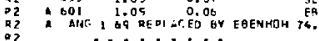

6108
10709
10711
6173
$1 / 76$
1176

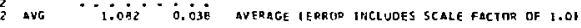

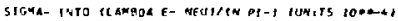

$\begin{array}{llll}B_{3} & 11 & 0.75 \quad 0.28 & 0\end{array}$

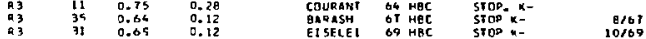

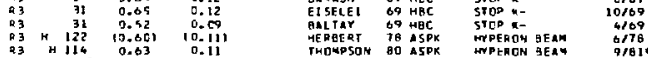

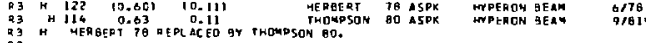

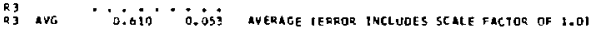

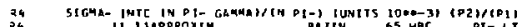

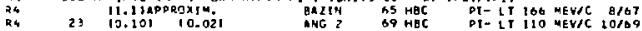

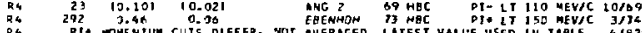

20 STGMA- DECAY PADRMETERS

FELATFO TEXT SEGTION VI D AMO APDEROSX I

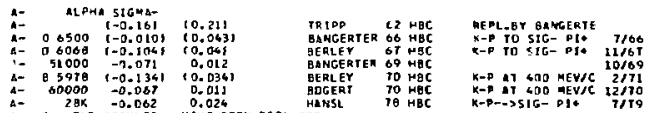

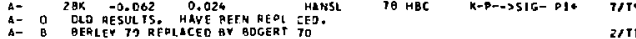

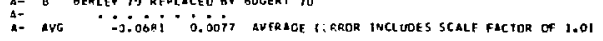




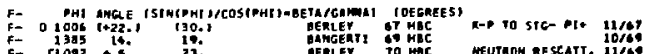

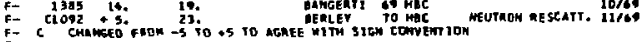

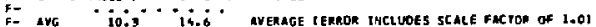

Note on $\Sigma^{-}+h e^{-v}$

(by J. A. Thoupson, University of pittaburgh)

The decay $\Sigma^{-}+\Lambda \mathrm{e}^{-} \mathrm{v}$ ig of apecial intereat because its forw is predicted by the strong form of CVC and is not sensitive to the ourcent octet assumptions or $\mathrm{SU}_{3}$ etructure constants which encer into cabibbo's predictions for the other hyperon decays. For $\Delta s$ - 0 tranaitions, the weak interaction vector curcent is related to the electroagnetic curcent through a nultiplicative constant, set by neutron beta decay, and an isospin rotation.

The decay $\Sigma^{0} \rightarrow h \gamma$ (the isospin-rotation analogue of $\left.\Sigma^{-} \rightarrow \Lambda \mathrm{e}^{-} \mathrm{V}\right)$ is mediated predominantly through the magnetic interaction, assuning there are no inhomogeneities in the $\Sigma^{0}, \Lambda$ charge distributions. Thus we expect the $9_{\mathrm{WH}}$ tera $\left[g_{W H} \sim \frac{\mu_{\Sigma A}}{\sqrt{2}} \sim-\frac{\sqrt{3}}{2} \mu_{n}\right.$ (by $\mathrm{su}_{3}$ )] to dominate the vector part of the weak current. The strong CVC predictions are thus: $g_{V} / g_{R}=0$ and $g_{\mathrm{FM}}-1.6$.
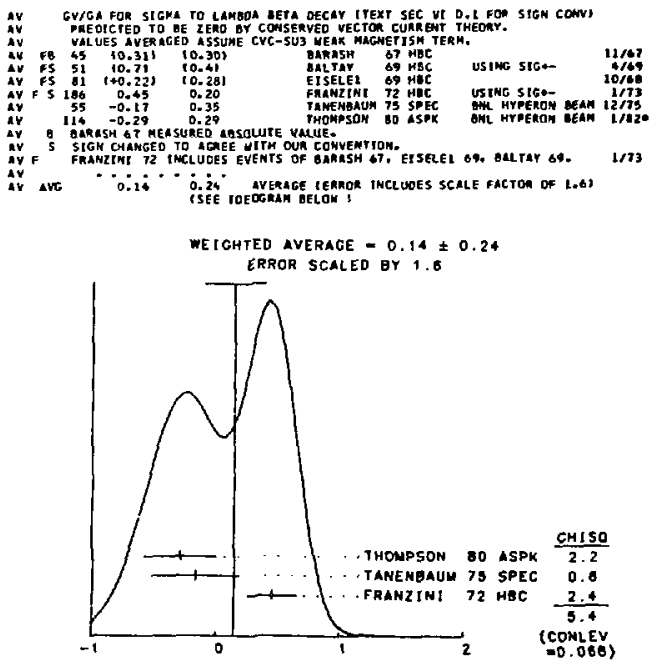

GV/GA FOR SIGMA- TO LAMBDA BETA deCAY
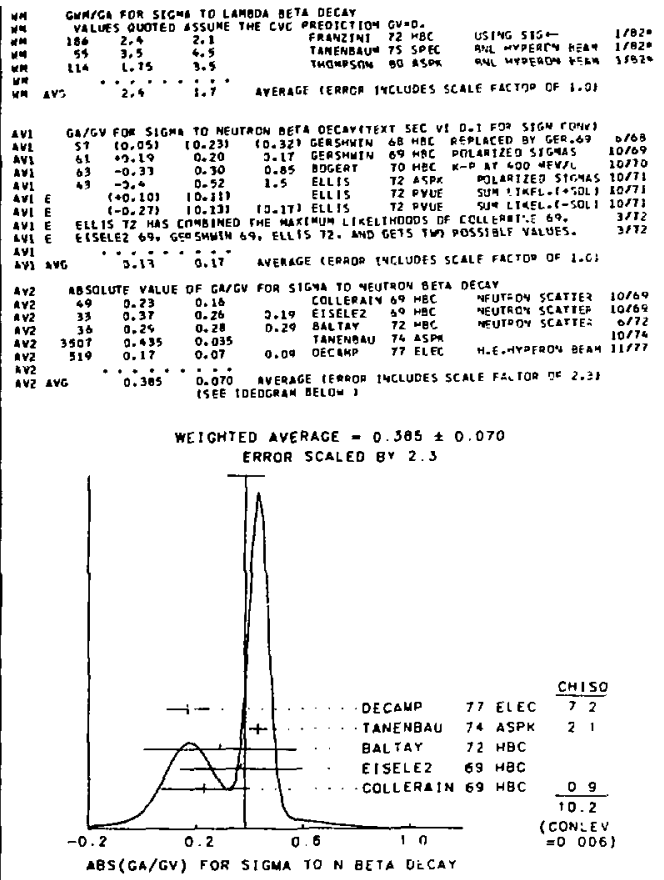

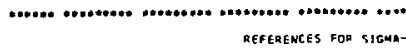

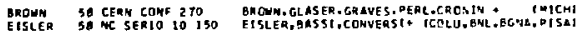

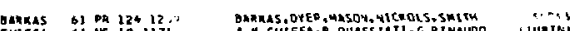

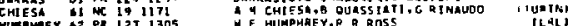

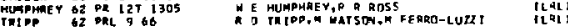

Barkas Go MIL 1220

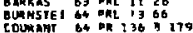

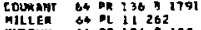

MILLER S4 PL 112262

QATIN 45 OR 14001350

DOSEA क5 OL it 215

SCHNitso 65 of 151 10月1

SCMLERTE 60 ONL 17 495

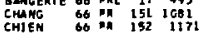

BMASH O7 OKL 19 IHI

MELEF 67 ORL 1957

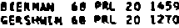

W HARKAS, J DPER.H H HECKMAN ILPL BURNSTEIN, DAY,KEFEE, SECMI TORN, SNON TUNG

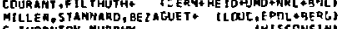

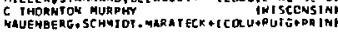

BAZIN, PLAMD, SCHM LOT * GOAINAPUTGOCOLUI

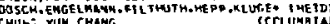
CHUNS VUA CHANG

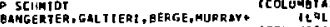
CHUHG YUK CHAHE

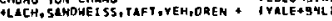
BAHASH, OAY, GLASSER, K EFOE, KMOP * THAOYLANDI

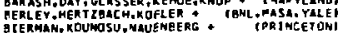

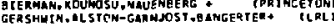

HEDP do $20 M Y 21471$,

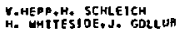
(HE TOELBEOG]
iOBERT INI

ANE I 49 RPHY 223103

AMG 2 CO IPHY 228152

ONGTAY 67 PRL 22 6L5

GALERTE OP UCRL-19Z6t

QMLOUTA 69 FE B14 153

TOLLEAS W 19273198

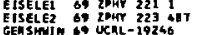

AenLET 70 on ol zols

OOGERT 70 MA 02 : 610

AMG,EISELE, ENGELMAMN, F TLTHUTH . THE1DI

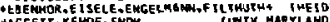
BAECET, KEHOE, SHDW ROGER ODELL GANGERTER TYHES IS)

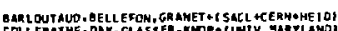
CEL

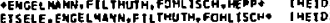
ESTELE, EMGELLAYN, FILTHUTH, FOHL TSCH, THEIOI

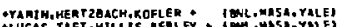

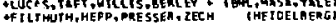
DAKKER PI LAE 137
CAE

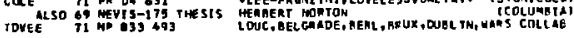


Stable Particles

$\Sigma^{-}, \Sigma^{0}, \Xi^{-}$

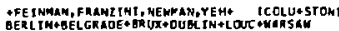
OEx

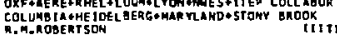

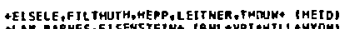

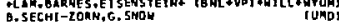

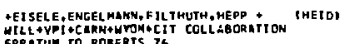
ERRATUA TO ROBERTS 74 .

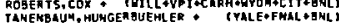

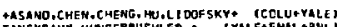

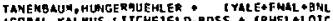

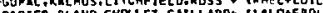

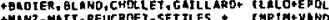
TELETAMO, COOPER, DQ I5, ENGELS - TPITTHAMI

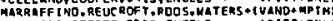

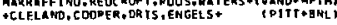

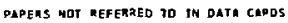

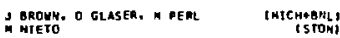

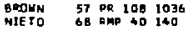

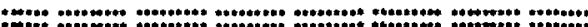
$\Sigma^{0}$

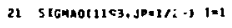

"1 ISIGMA-1 - ISTGMAOT MASS OIFf EREHCE IWEY)

DI N SEE NOTE PRELEDING LAMBOA MASS LISTINGS.

D1 II 4 it

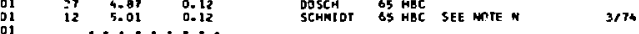

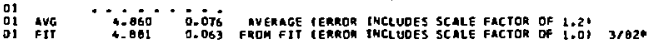

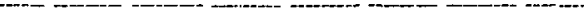

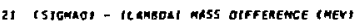

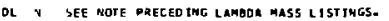

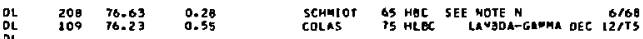

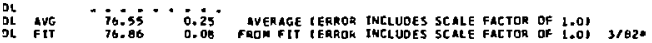

- - - - - - - - - - -

21 SIGMAD MEAN LIFE SUNITS 10 MN-19 SEC

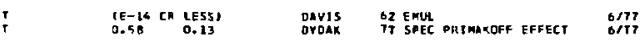

a) STGKaO PaAtial oecar modes

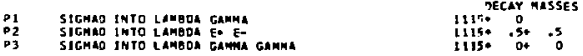

and 0

21 SIGKAO GRANCHING RATIOS

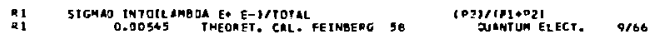
R2
R2

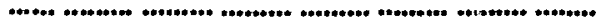
REFEREMCES FOR SIGMAO

FEIMEEG 50 PA 1091019

DAVIS

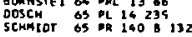

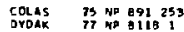

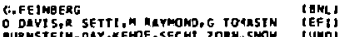

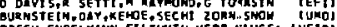

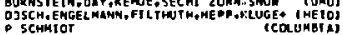

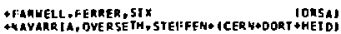

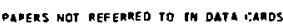

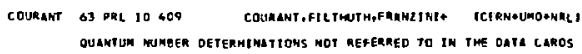

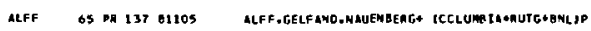
:
Data Card Listings

For notation, see key at front of Listings.

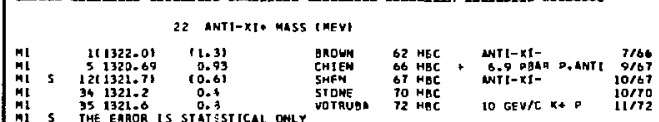

Al 5 留

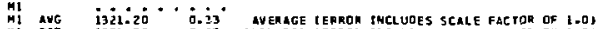

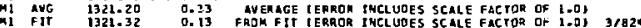
THE FIT ASSUMES $x I$ ANO ANY I-X! MASSES EDUAL.

\begin{tabular}{|c|c|c|c|c|c|}
\hline OM & 1.0 & $\begin{array}{l}\text { 2. } 1 \times: \\
1.1\end{array}$ & $\begin{array}{l}I-X \mid 0) \\
\text { CHIEM }\end{array}$ & $\begin{array}{l}5 \text { DIFF ERE } \\
\text { AB HaE }\end{array}$ & $\begin{array}{l}\text { IMEVI } \\
\text { t.P PQAR }\end{array}$ \\
\hline
\end{tabular}

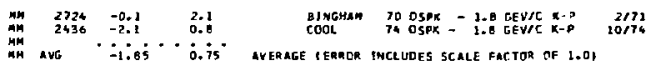

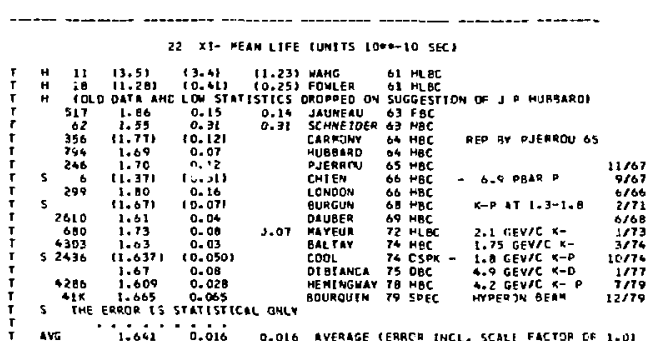

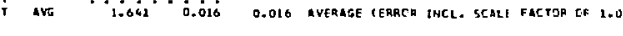

22 ANTI-KI - MEAN LIFE (UNTIS LONO 10 SEC?

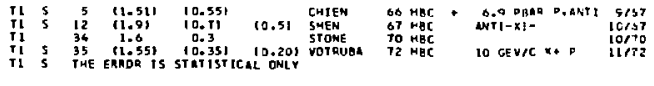

iz xi- paatial oecar hooes

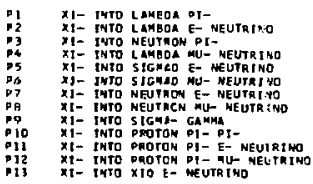

DECAT MASSES

1115: 139 . 0

1115: 130 105.

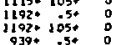

$939 \% 105 \%$

$939 * 105 \%$
$1197+100$
$930+130 * 139$

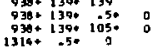




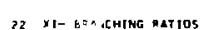

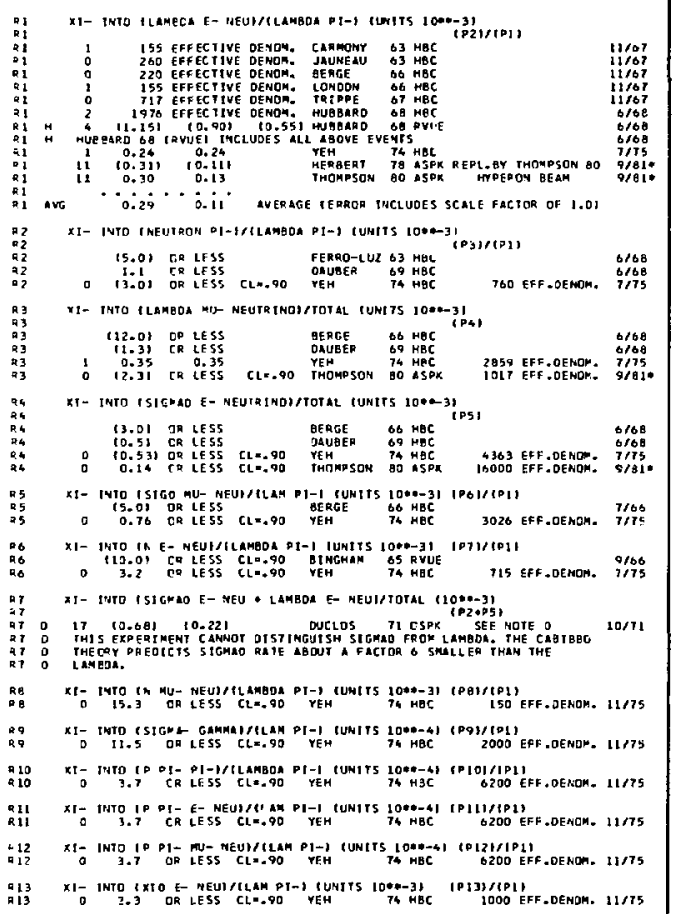

\section{XI- OECAY PARAME TERS}

relateo texi SEction Vi D ano appenolX :

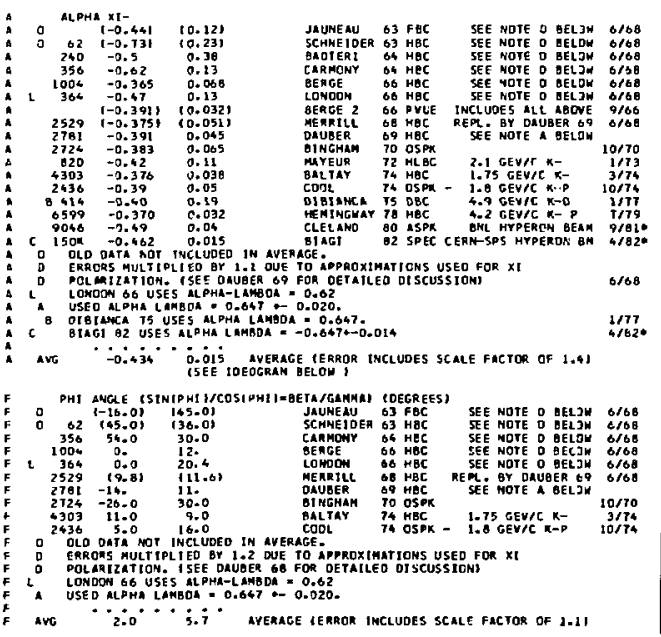

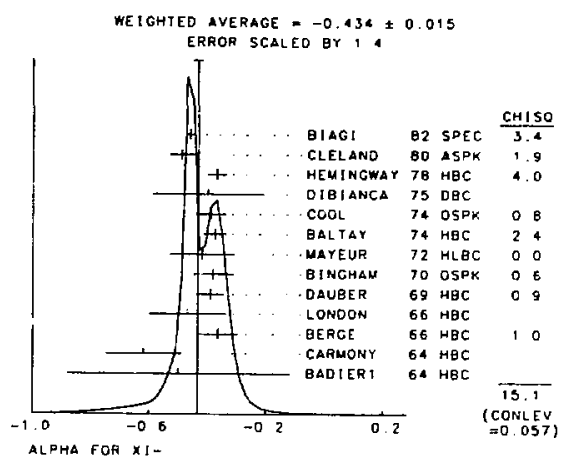

REFERENCES FUR $X:-$

$\begin{array}{ll}\text { FONLER } & \text { O1 DRL } 6134 \\ \text { NANC } & \text { \&1 JEID } 13512 \\ \text { BNDWN } & \text { G2 DRL E } 245\end{array}$

CAKMONY 63 DRL 10381 JAUNERU G3 SIENA CCAF 4 JAUNERU
ALSO 62 ? $\mathrm{PL}: 561$
SCHNEJOE 63 PL 360

CARHONT 64 PRL 12492

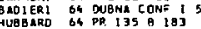

BINGHAN 65 PRSL 265 202

PJNARON O5 PRL 14275
PJEAROU 65 THESTS

BERGE, OS PR IAT SL5

EERGE ${ }^{2}$ S6 PERKELEY CONF
LCNDDN
CHIEN

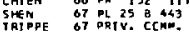

BUPGUN 68 NPBS $B$ THT HUBBARO
OB PRL 20405
MERRILL $\triangle B$ DR IBT 1202

DWUAER $69 \mathrm{PR} 1791262$

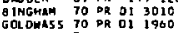

5 TOKE TO PL 320515

OUCLOS 11 MP 832493

$\begin{array}{lllll}\text { MRYEUR } & 12 & \text { NP } & \text { OAT } & 333 \\ \text { VOFRUBA } & 72 & 4 P & B 45 & 71\end{array}$

HILUET $72 \mathrm{PL} 420372$

BALTAY 74 PR O9 49
COOL 74 PR DLO 192

ALSO 72 PAL 291630

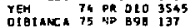

HEMINGA TS HP BL42 205

HER GERT 73 PRL 401230
GOUROUTH

CLELAND SO Oa O21 12

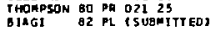

F OHLER, BIREE, EBERHARD, ELY, GOCD, PONFLL+ILQLL

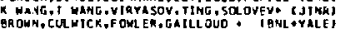

CARMONY, PJERROU
FERRO-LUZZI, RLSTON, ROSE MFELD, WOJCTCKE HULLEL

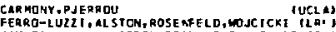

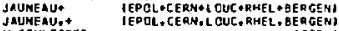

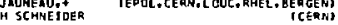

CARMONY, PJERROU, SCHLEIN, SLATER, STORK + UUCLAJ J

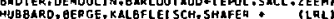

H. HINGHA
SCHLEIN,SLAFE,SHITH,STORK.TICMO IEERMI

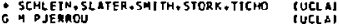

BERGE, EBERHARO, MUBQARD, PERR TLL D LLRLI

LONDON, RAU. GOL DBERG. I ICHTM ANA IBNL \& S TRACUSE)

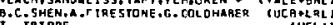

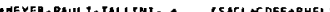

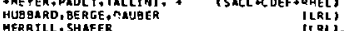

TGERGE, HUGOARD, MERR TLL, MIL LER ILRL, OCOO. HOUAPHAEY, SAHOER, W ILL TANS IUC SD, WASHI

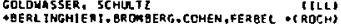

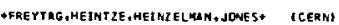
-VAN OINST, UILEUET + (BRUX+CEAN+TUFT+LOUCI

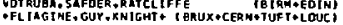

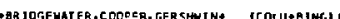

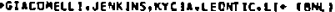

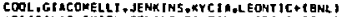

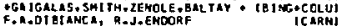

HEM INGMAY, MAMENTER OS* ICERN+IEEM+NIJN+OXFI

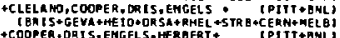
+COOPER, DAIS, EMGELS, HERGERT: IPIJT+BNL,

(1)

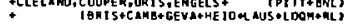

$\Xi^{0}$

$23 \times 1011315$, JPe1/2 । $1-1 / 2$

$23 \times$ xtO MASS (HEV)

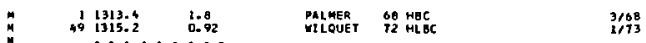

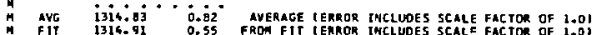
3/82"

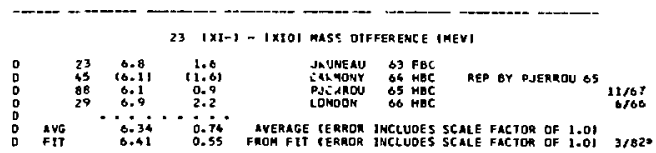




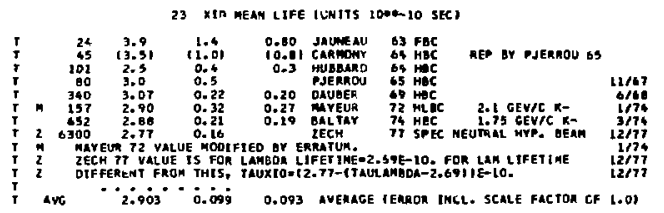

23 XIO MAGNETIC NOMEMT IMAGMETONS, $93 \% .26$ ๓EV

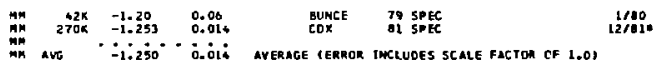

Z: $X 10$ PARTIAL DECAY NODES
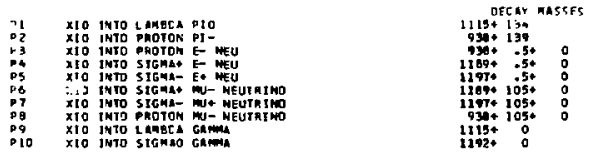

$23 \times 10$ GRAMCHING RATIOS

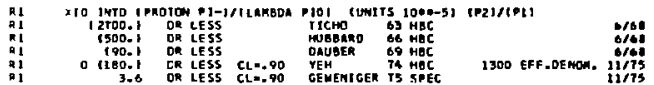

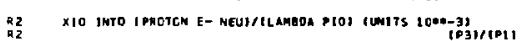

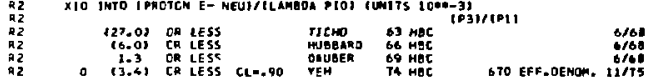

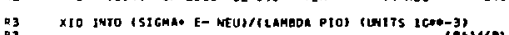

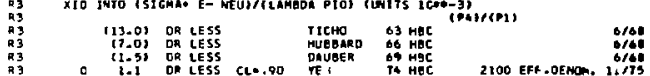

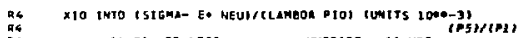

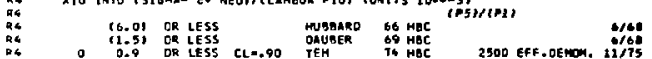

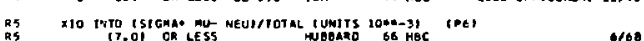

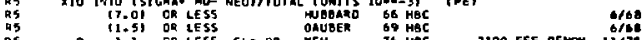

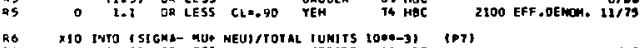

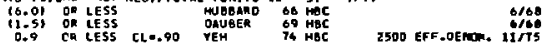

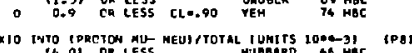

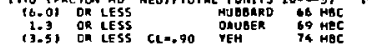

竞

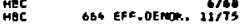

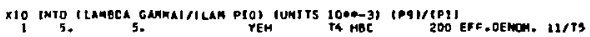

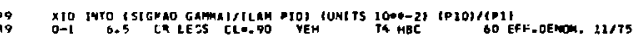

23 x 10 OECAY PAKAMETER

REL ATED TEXT SECTIOH YI D AHO apdemolX I

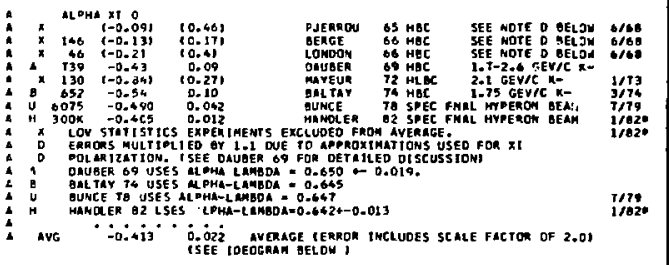

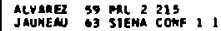

I ICHD 63 OL COAF 410

CARNOW 64 PRL 12 482

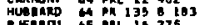

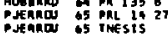

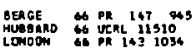

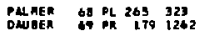

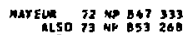

VILQUET 72 NL 428372

BALTAT 74 PH OQ 49

YEM 74 DA DID 3545

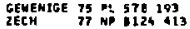

BUNE TH ON DLA $\triangle 33$

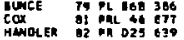

FEIGHTED AVERAGE $=-0.413 \notin 0.022$

ERROA SCALED OY 2.0

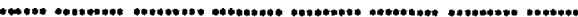

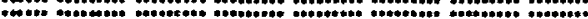

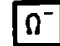

24 ONECA-12672, JPE-3/2: $1=0$

OURTUM MUMaELS ASSIGREO FROM SU3

24 OMEGA- MASS IMEV?

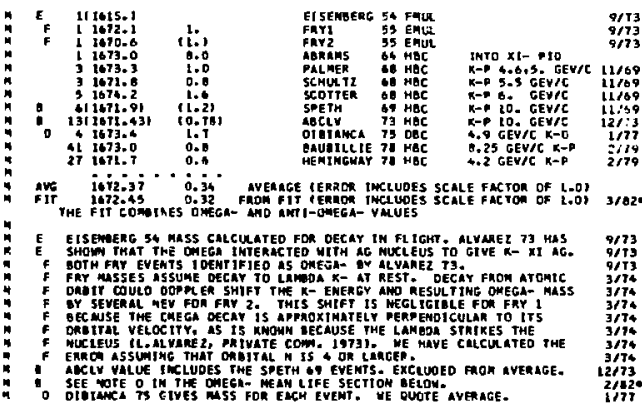




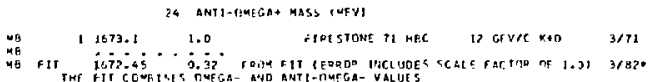
36 OWEGA- MEAM LIFE IUNATS ION-10 SEC

\begin{tabular}{|c|c|c|c|c|c|c|c|c|c|}
\hline & i & 10.71 & & & PARNES I & 64 & $\operatorname{tiBC}$ & & $2 / 60$ \\
\hline & I & $i+4 i$ & & & BARHES ? & 36 & wac & & 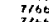 \\
\hline & 1 & $\mid 1.851$ & & & $\begin{array}{l}\text { COLLEY } \\
\text { RICHARDSO }\end{array}$ & 65 & toc & & \\
\hline & 1 & $(1.20)$ & & & $\begin{array}{l}\text { RICHARQSU } \\
\text { SCHULTL }\end{array}$ & & a & & $\begin{aligned} 7186 \\
11 / 67\end{aligned}$ \\
\hline & $i$ & 10.001 & & & SERUT T2 & a & HEC & & 11167 \\
\hline & $i$ & 10.635 & & & SEIVULT & $6 \theta$ & tac & & $11 / 67$ \\
\hline & i & 10.251 & & & SCOTIER & $B \rightarrow$ & HeC & & tobo \\
\hline & i & $(0.30)$ & & & SCOTIER & $6 \mathrm{~B}$ & MBE & & $t / 68$ \\
\hline & i & (3) 71 & & & SCOUIER & $B \mathrm{~B}$ & & & $4 / 68$ \\
\hline & $i$ & 10.091 & & & SeOtifa & $O B$ & $\mathrm{HaC}$ & & $4 / 6 \theta$ \\
\hline & i & $(1.04)$ & & & SCOTTLA & $\theta \mathrm{B}$ & & & \\
\hline & & $12.3 \pm 1$ & & & Scottek & & & & $\operatorname{cose}$ \\
\hline 0 & 16 & 11.391 & 10.451 & 10.311 & AGCLY & 73 & $\begin{array}{ll}116 C \\
\mathrm{DBC}\end{array}$ & x-1 $10 . G F+/ t$ & 12173 \\
\hline & & $\begin{array}{l}0.1131 \\
0.4 \times 21\end{array}$ & & & $\begin{array}{l}\text { DIMIAicA } \\
\text { DE QIANCA }\end{array}$ & $\begin{array}{l}75 \\
75\end{array}$ & $\begin{array}{l}\text { DHC } \\
\text { DBC }\end{array}$ & 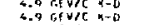 & int \\
\hline & $i$ & 10.7021 & & & OIBIANCA & 75 & Dec & 4.7 refy/c $n-6$ & 1177 \\
\hline & 1 & 10.2281 & & & Dlerantca & 5 & & $-9 \cos v / c k=0$ & 1177 \\
\hline & 40 & 0.00 & 0.16 & 0.12 & nAUATLLIE & Pa & HAC & 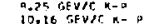 & $\begin{array}{l}2 / 79 \\
e>78\end{array}$ \\
\hline$D$ & $\begin{array}{r}108 \\
30\end{array}$ & $\begin{array}{l}11.411 \\
0.75\end{array}$ & $\begin{array}{l}10.151 \\
0.14\end{array}$ & $\begin{array}{c}0.241 \\
0.11\end{array}$ & 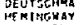 & 78 & $\begin{array}{l}\text { MES } \\
\text { HAE }\end{array}$ & 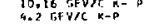 & 219 \\
\hline & & 0.922 & 0.028 & & gourouth & 19 & setc $c$ & CESH SAS 4 YOE ROM GU & 11279 \\
\hline & & & & Fous & 5 of auclV & & & & \\
\hline $\mathbf{0}$ & $4 \mathrm{EC}$ & of & am & $015+2+3$ & EEVENT W & & & IT FXPfHIMFNIS. & $\begin{array}{l}2 / 80 \\
2 / 820\end{array}$ \\
\hline & & 0.719 &.$\overline{229}$ & 0.02 & & & & - SCALE TACTOR UF & .01 \\
\hline
\end{tabular}

74 THEGa- pantial dFCAY UDDES
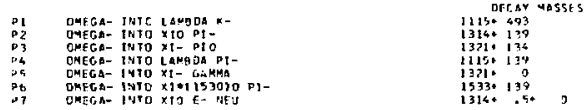

24 DUEGA- PRAHCHIHG RATIOS

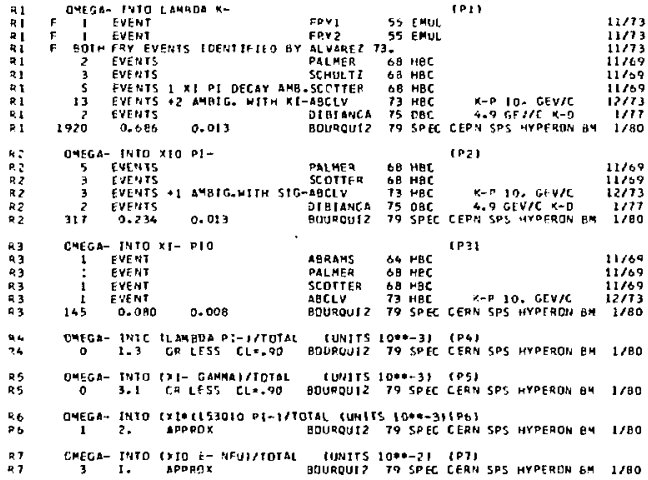

24 OMEGA- DEGAY PARAMETERS

- elated section vi D in text

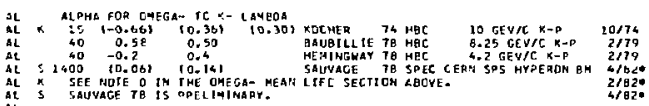

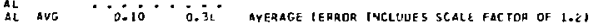

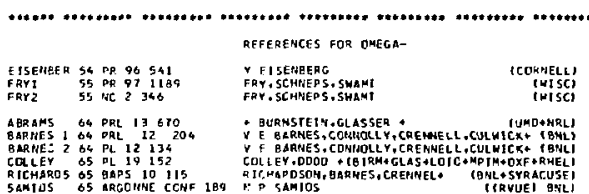

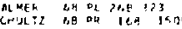

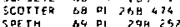

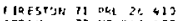

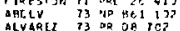

0
$X C H E Q$

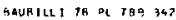

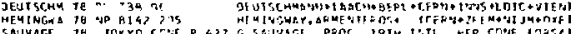

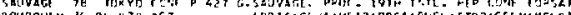

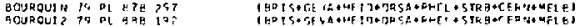

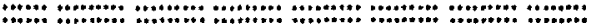

$\Lambda_{c}^{+}$

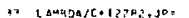

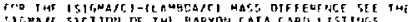

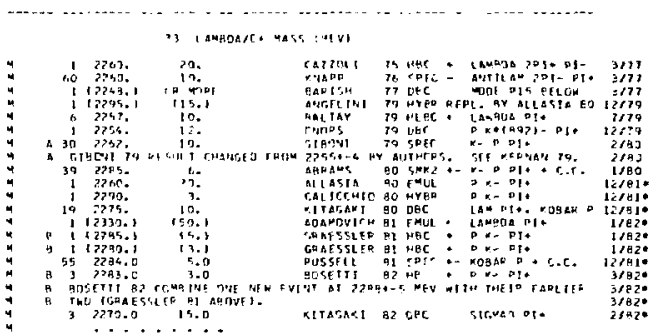

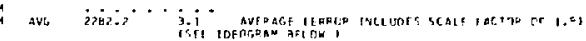

WEIGHIEO AVERAGE $=2287.2+3$.

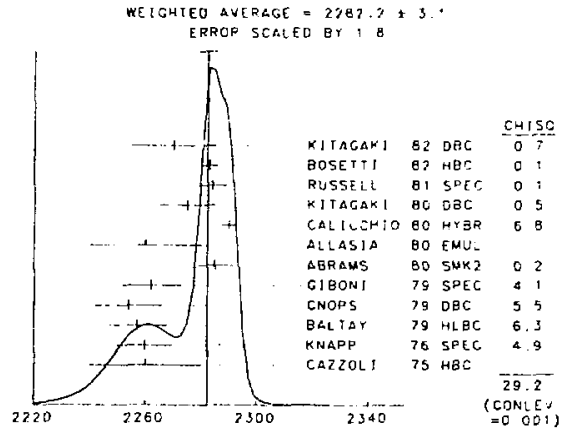

LAMBDAFC, MASS

CDNLED

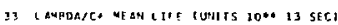

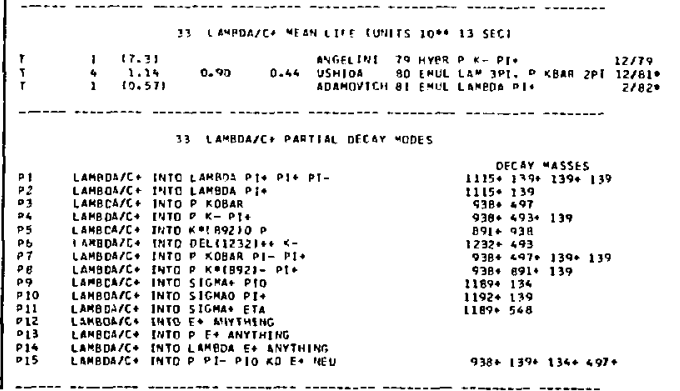


Stable Particles

$\Lambda_{c}^{+}, \Lambda_{b}^{0}$, MASSIVE $\nu^{\prime} S$ AND LEPTON MIXING
Data Card Listings

For notation, see key at front of Listings.

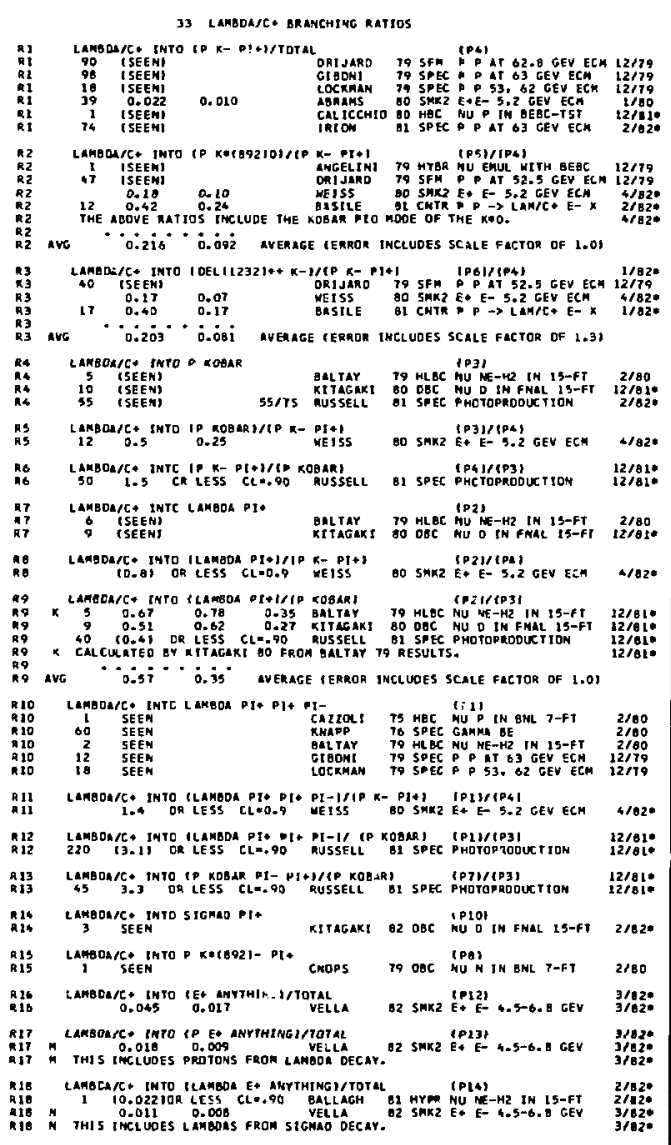

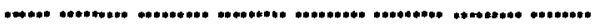

REFERENCES FOR LAMBDAJC*

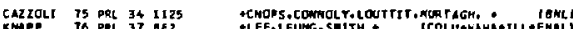

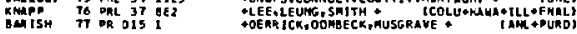

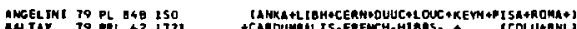

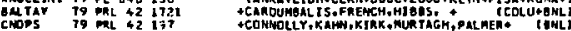

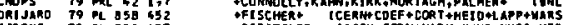

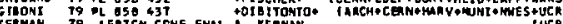

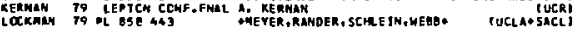

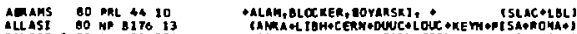

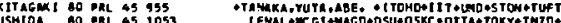

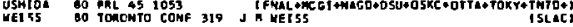

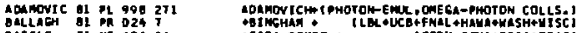

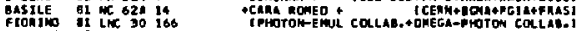

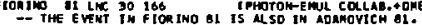

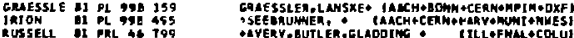

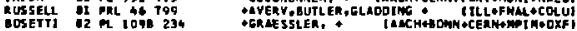

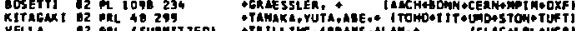

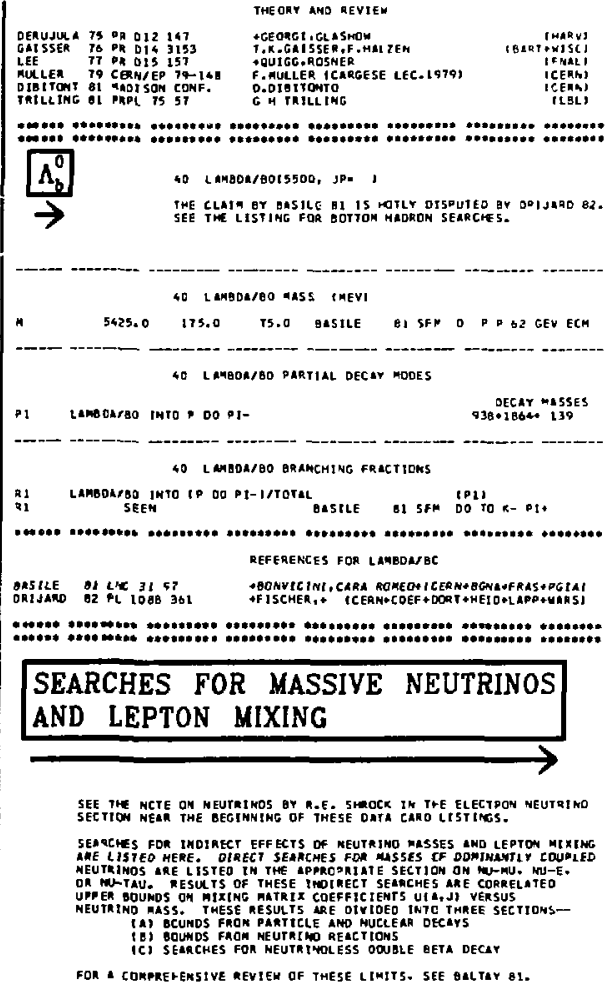

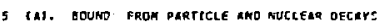

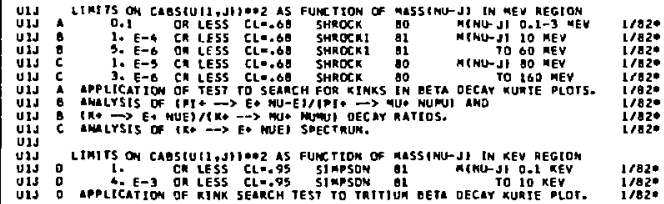

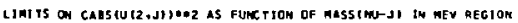

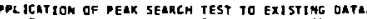

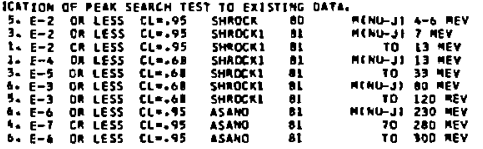

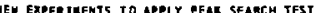

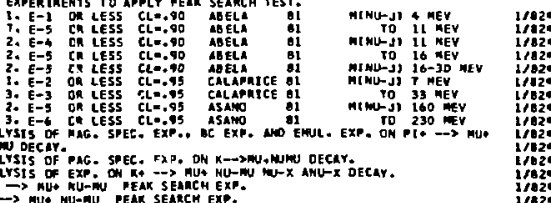


Data Card Listings

For notation, see key at front of Listings.
Stable Particles MASSIVE $\nu$ 'S AND LEPTON MIXING

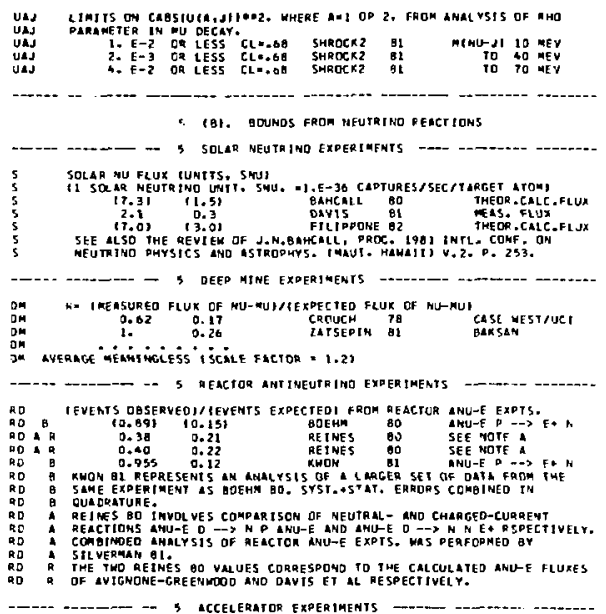

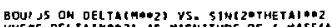

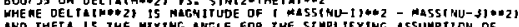

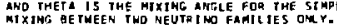

EACM EXPERTHENTAL RESULT IS A PLOT GIVING ALLOUEO ANO EXELUDED

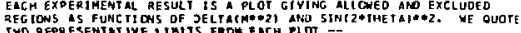

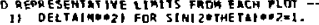

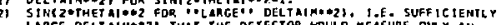

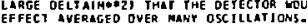

EXDEA IMENTS ARE DF THO GEMERAL TYPES -

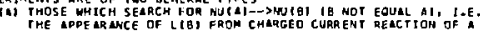
NuTAl GEaA

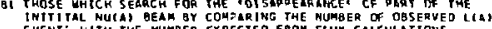

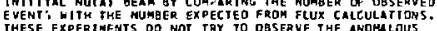

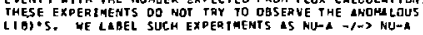

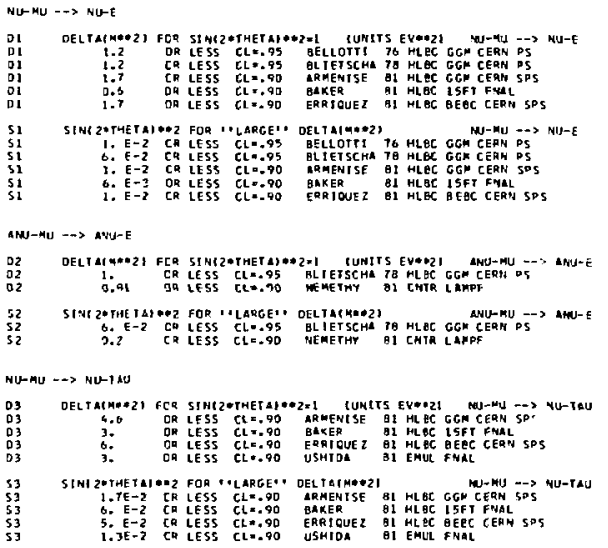

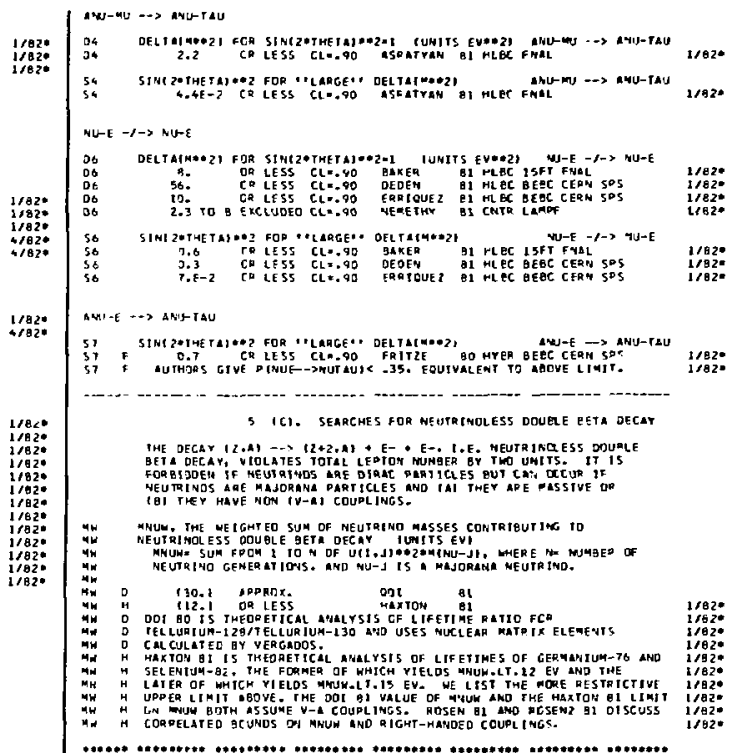

EELCOTI To LNC 17 is 9L.1ETSL,

BAHCALL AO DRL 45 945

BOENES 46 SCL ENGE 191264

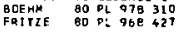

REI HES $B 0$ ORL 45 1207

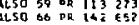

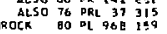

$\triangle Q L L A$ BL DL 1059203

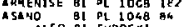

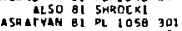

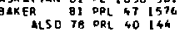

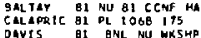

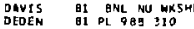
Dot 1910 ALSO BI PTP 661785 ERR DOUE 2 BL O 102872

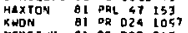
NEMETHY OI PQ $0232 E 2$ ROSEN O1 ARQS "1 145 Sheocsi 78 R4P 50 il 11

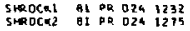

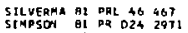
USAIDA B1 pरL 471694

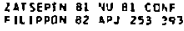

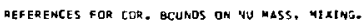
TCAVALL L, F IOR INI, ROLLIEP intas

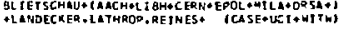

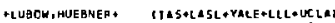

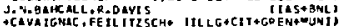

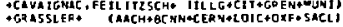
F.RE TNES.H.W. SABEL, E.DASIEPB F. ORFINIS, C.L.COHAN

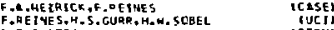
mace! ODAUA, EATON, F ROSEH, JOS T,XETTLE, STEIHEOI SIN]

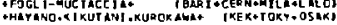

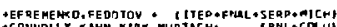

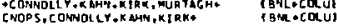

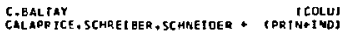

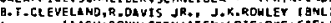

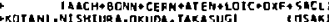

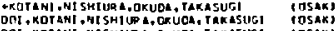

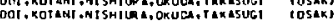

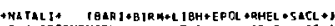

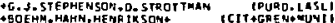

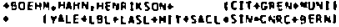
S.P.ROSEN
S. P, ROSEN

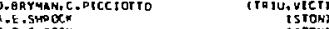
1/82: 1/82* 1/82: 
Stable Particles

$\nu$ BOUNDS, HEAVY LEPTON SEARCHES

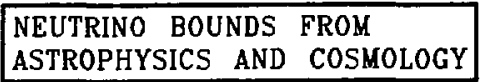

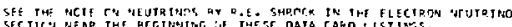

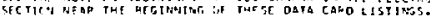

\section{Note on $v$ Mass Limits}

There 1 imits apply to $m_{\text {tot }} g$ iven by

$$
m_{\text {tot }}=\sum_{j=1, n}\left(\frac{g_{v_{j}}}{2}\right) m_{v_{j}}
$$

where $n$ is the number of neutrino species and $g_{v}$ is the number of independent components in the $j$ neutrino field; $9_{y}=4$ for Dirac neutrinos; $g_{y_{j}}=2$ for chiral Ma jorana neutrinos.

\section{- ifu wass tevi}

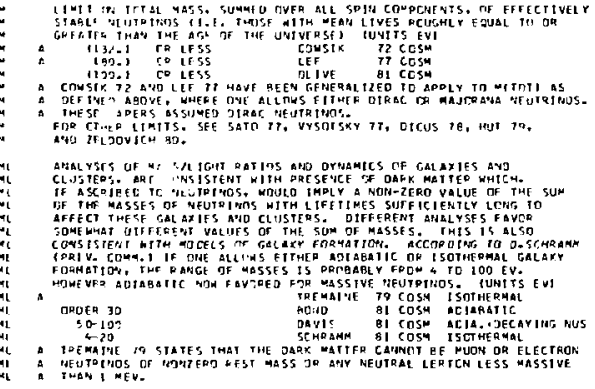

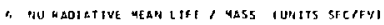

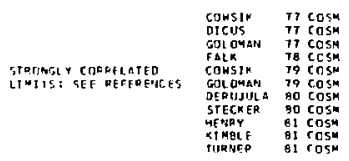

- POSSIFt L LIHITS ON RUYBEF DF LJGHT LE ABOUT 1 MEVI

WUMEA COUPLIH, HT TH FULL WIAK STRENGTH

(7) OB LFSS SHYAR ISMA 50 COSA

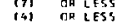

CRITITISAR OF ROUIOD

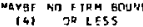

coitic isa chess

141 D० LES

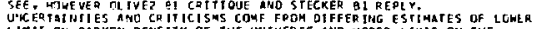

LIMIT JV BARYCA DEQSITY OF THE UNIVERSE AND UPPER LIMIT ON FUE

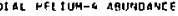

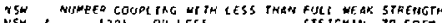

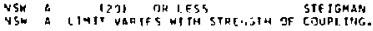

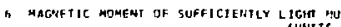

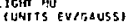

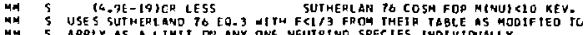
MM 5 APPLY AS A LIMIT DF ANY ONE NEUTRIND SOECIES IMOTVIDUSLLY.
Data Card Listings

For notation, see key at front of Listings.

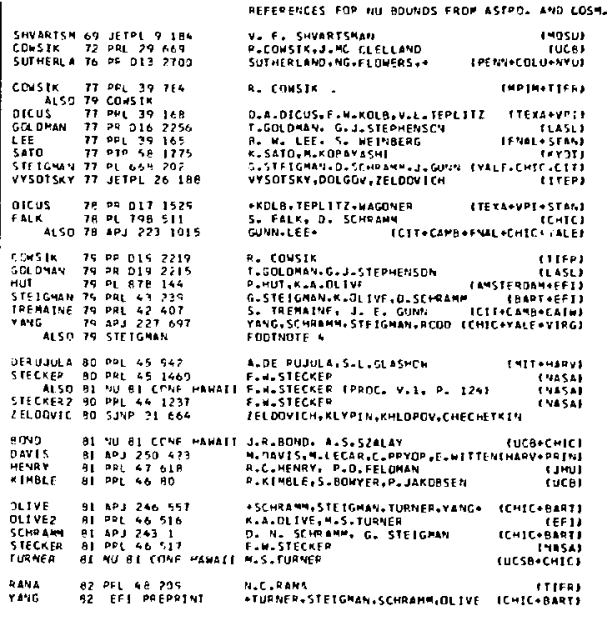

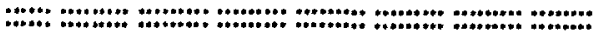

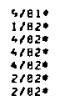

HEAVY LEPTON SEARCHES

Data on the $\tau^{ \pm}$(1785) heavy lepton are listed in a separate section above, following the $e$ and 1 listings.

The following section contains information on searches for heavy leptons of other types and searches for the $\tau^{ \pm}$in collisions other than $\mathrm{e}^{+} \mathrm{e}^{-}$.

Several types of heavy leptons (that is, non-strongly-interacting fermions other than $e$ and $\mu$ ) have been proposed. In the Data Card Listings we distinguish four types, ${ }^{1,2}$ Each has a corresponding antiparticle with opposite charge and lepton number. For convenience we onit writing the antiparticles in the following descriptions. The four types are:

Sequential Leptons $\left(L^{-}, V_{L}\right)$. Such a pair is assumed to have its own separately strictly conserved lepton number $n_{I}=+1$. This means that the radiative decays

$$
\left.\begin{array}{l}
L^{-}+e^{-} Y \\
L^{-}+\mu^{-} Y
\end{array}\right\} \text { are forbidden, }
$$

while the weak decays (assuming $\mathrm{mn}_{L^{-}}$sufficiently massive) 
Data Card Listings

For notation, see key at front of Listings.
Stable Particles HEAVY LEPTON SEARCHES

$$
\left.\begin{array}{l}
L^{-}+v_{L} e^{-} \bar{v}_{e} \\
L^{-}+v_{L} u^{-} \bar{v}_{H} \\
L^{-}+v_{L} \text { hadrons }
\end{array}\right\} \text { are allowed. }
$$

There could be an increasing mass sequence of such pairs. It is frequently assumed that the neutrinos are massless.

Decay rates are assumed calculable from conventional weak interactions theory. For $\mathrm{L}^{-}$mass between 1 and $3 \mathrm{GeV}$, the branching fraction to each of the two leptonic modes should be roughly 108 to 208. For $\mathrm{L}^{-}$mass above $1 \mathrm{GeV}$, the mean life should be $\leqslant 10^{-12} \mathrm{sec}$, too short to be observed in a track chamber. 1

Paraleptons $\left(E^{+}, E^{0}\right)$ and $\left(M^{+}, M^{0}\right)$. These pairs have the same lepton numbers as the opposite-charge ordinary leptons, i.e., $\mathrm{e}^{-}$and $\mu^{-}$, respectively. Radiatire decays are again forbidden and decays similar to those allowed for $\mathrm{L}^{-}$are allowed here, e.g. .

$$
\begin{aligned}
M^{+} & \rightarrow v_{\mu} e^{+} v_{e} \\
\text { or } \quad M^{+} & \rightarrow v_{\mu} \mu^{+} v_{\mu} \text {. }
\end{aligned}
$$

However, the lightest member is not stable as is the case for sequential leptons, so that bizarre decay schemes such as lassuming $m_{E^{0}}<m_{E^{+}}$)

$$
\begin{gathered}
E^{+} \rightarrow E^{0} \mu^{+} v_{\mu} \\
\longrightarrow e^{-} e^{+} v_{e}
\end{gathered}
$$

are allowed.

Heavy leptons of this type (and/or a neutral intermediate boson $z^{0}$ ) are desired in unified gauge theories of weak and electromagnetic interactions to cancel unphysical high energy behavior in such processes as $e^{+} e^{-}+W^{+} W^{-} .3$

ortholeptons $\left(\mathrm{F}^{-}\right.$and $\mathrm{N}^{-}$). These have the same lepton numbers as $\mathrm{e}^{-}$and $\mu^{-}$, respectively. They may or may not have associated neutral leptons. Radiative decays are allowed in addition to weak modes similar to those of sequential leptons. The radiative mode can dominate or can be relatively unimportant depending on the model. ${ }^{4}$ Decays such as

$$
\mathrm{F}^{-}+\mathrm{e}^{-}+\text {hadrons }
$$

are also allowed.
Long-Lived Penetrating rarticles. Heavy leptons could have long mean lives undex certain circumstances. For example, if $\mathrm{m}_{\mathrm{v}_{\mathrm{L}}}>\mathrm{m}_{\mathrm{L}^{-}}$, then $\mathrm{L}^{-}$. the sequential lepton, is completely stable since its lepton number is conserved.

Experimental results. The results are summarized in the Data Card Listings below. Mass limits for sequential leptons are listed in subsection MS, while all other types are listed together in subsection $M$.

The Listings also contain cross-section upper limits reported as results of unsuccessful searches. we no longer list cross sections for anomalous ep events in $\mathrm{e}^{+} \mathrm{e}^{-}$collisions. These cross sections are consistent with coming from $\mathrm{e}^{+} \mathrm{e}^{-}+\tau^{+} \tau^{-}$where the $\tau^{ \pm}(1785)$ is assumed to be a spin-1/2 Dirac point particle with a mass about $1785 \mathrm{MeV}$.

\section{References}

1. M. L. Ferl and P. Rapidis, SLAC-PUB-1496 (October 1974).

2. C. H. Llewellyn Smith, Invited paper presented at the Royal 5ociety Meeting on "'ew particles and New Quantum Numbers, 11 March 1976, Oxford Ref. 33/76.

3. J. D. Bjorken and C. H. Llewellyn Smith, Phys. Rev. D7, 887 (1973).

4. F. Wilczek and A. Zee, Nucl. Phys. B106, 461 (1976).

SEE DEDL, GI FTR A REVIEM

PROPERTIES OF THE TAUT IITGSI HEAUY LEDTON AND ITS A5SOCIATED

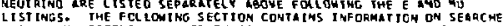
FOR HEAVT LEDTCNS OF OTHER TYPES AND SEARCHES FOA TOUU- IN UPPER CINITS REPDTTED AS NEGATIVE SEAFCH RESULTS. WE NO IONGER LIST CAOSS SECTIONS FOR THE ESTABLISMEO ORECESS E* E- $\rightarrow$ TAU* TAU

$$
75 \text { MEAVY LEOTON MASS LIMITS }
$$

LIMITS ADOLP CVLY TO HEAYY LFPTON TYPE GIVEN IN COMHENT AT MIGHT ON 3/T DAT COARO SS QEE DU:

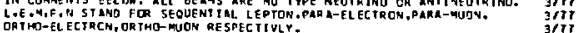

SEOUEUTIAL HEAVY LEPTON MaSS UIMITS IGEVI

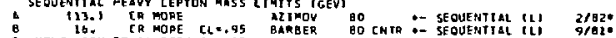

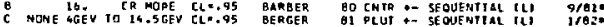

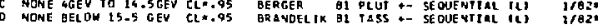

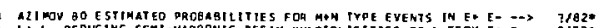

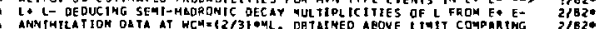

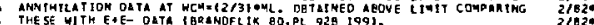

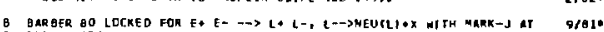

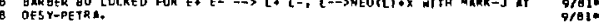

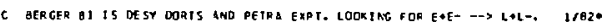

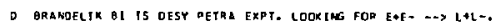


Stable Particles

\section{HEAVY LEPTON SEARCHES}

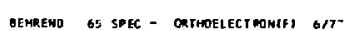

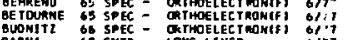
gAFMA IO CMTE : LOWG-LIVED

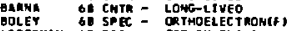
LIOERMAN OT OSPE - ORTHOMUONCMI RDTHE GS AVUE - ORTHOELECTROMIFI

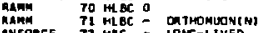
ANSORGE T3 HAC : LONC-LIVED AACCI T3 ELEC

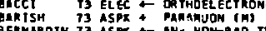

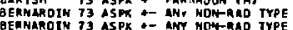

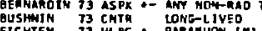
EICHTEM 73 HLQC. PARAMUON (N)

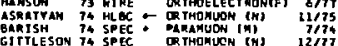
GITLESOM 74 SPEC . OR THOMUCN (T) $12 / 7$

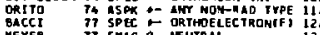

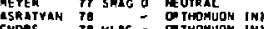

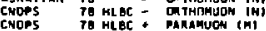
ERtout 270 MLEC ERHLTUE
MOLDER

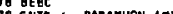
78 CNTh + pananuon (a) 1 SAEC D PARRMUONIMOBARI CLARX
MAYS
MAYS MOAE I GEV TO O GEV GEV CLE-90 NOHE BETMEEN 0.6 AND 3.3
TOAE BETUEN 0.5 RND 3.3

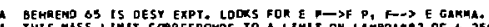

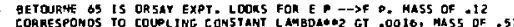
COAMESPOMOS TO COUPL

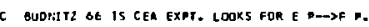

- ona og is slac photomoduction expt.

gOL EY OQ IS CEA EXPT. COOKS FOR E P-PF P. MASS OF -1 CORRESPONDS E LAMBOACOE GT OL OL.

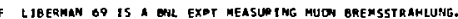

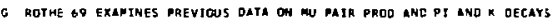

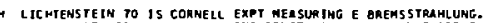

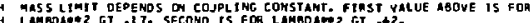

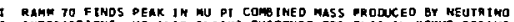
INTEAACTICAS. HE ALSO CLA IMS EYIDENCE FOR FHIS IN KOMUZ DECAYS IN

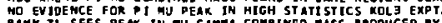

RAMK 71 SEES PE MX IN MU CANMA COMBIMED MASS PRODUCED BY MEUTRI MOS.

1 ANSCRGE 33 LODKS FOM ELECTRON PAJR PROD AHO ELECTRCALLIKE grEMSS.

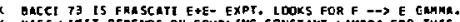

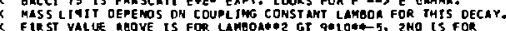

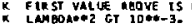

GARI ISH 73 I5 FNAL 50,145 GEV MEU EXPT. LOOKS FOR IMEU NUCLEON $\rightarrow$

WEV HEVI WITH BRa.3.

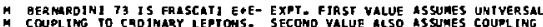
M CONPLING TO
TO MADAONS.

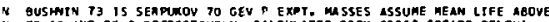
N TE-10 ANO 3E-g RESPECTIVELY. CALCMUATED FROM CROSS SECTOC GELOWI

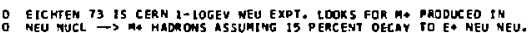

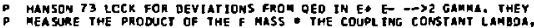
MEASMEE THE PRODUCT OF THE F MAS

- isnatran to uses eighren t3 data on meu nucl $\rightarrow-$ hadgons and

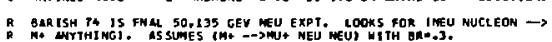

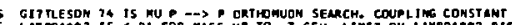

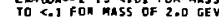

T ORI TO 7 L LCEKED FOR HEH- PAINS GIVIMG HN-E PAIRS. MASS LJHIT REFERS

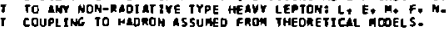

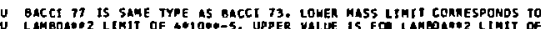
$4 \lim _{1,50100.30}$

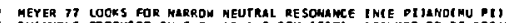

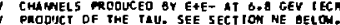

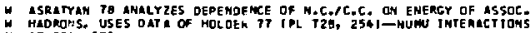
- IT CERM-SPS.

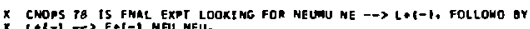

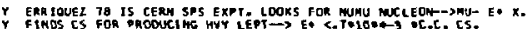

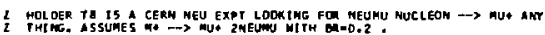

Data Card Listings

For notation, see key at front of Listings.

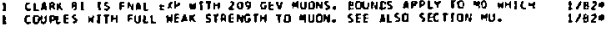

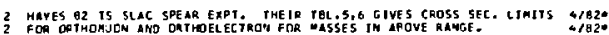

HEAW LEPTCM LIMI TS TNEUTA TKO NULLEONI

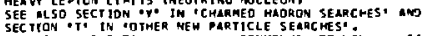

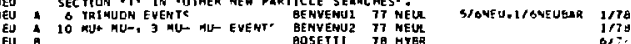

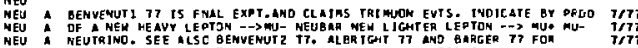

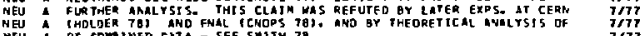

NEU I OF CDMATHED CDTR - SEE SMITM 78.

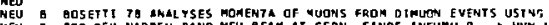

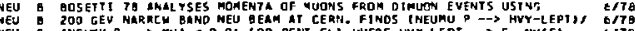

MEU E INEWUU P $\rightarrow$ MUI
NEU B

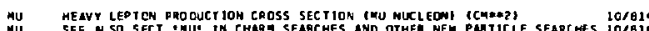

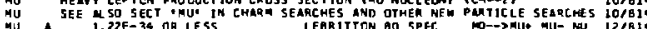

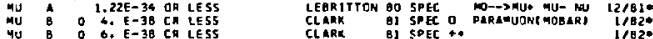

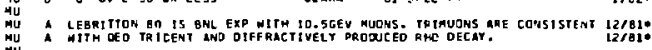

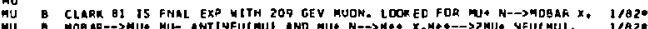

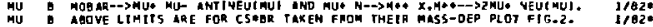

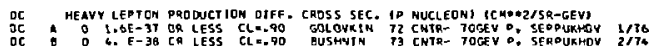

:mi

(x)?

$6 / 77$

477

or77

ont7

orm

$6 / 77$

$1 / 16$

$1 / 18$

$3 / 77$

$2 / 74$

$2 / 74$

$2 / 74$

$\underset{2 / 76}{2 / 76}$

orm

2176

$1 / 74$

12777

12177

$3 / 74$
$3 / 76$
$3 / 76$

3277

$12 / 71$

12,7

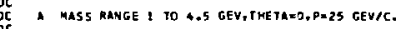

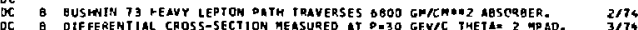

$1 / 76$

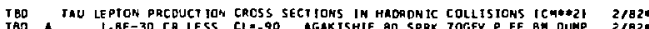
TBD 1.8E-30 CA LESS CL..90 RGAKISHIE QD SPRK TOGEY O FE BW DUMP

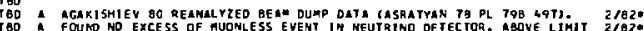

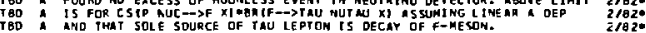

IC INYARIAYY hEAVY LE PTON PRDD. CROSS SEC. IP NUCLEONI ICMAN2/CEVEN3I

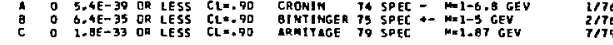

a CROMIN TA 15 AM FMAL 300 GEV P CU EXPT. LOOKEO FOR LOME LIVEO 2770

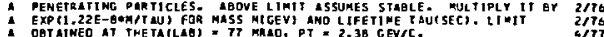

- aintinger 75 is a 30-300 ceV P C expt. LoOkec for lang lived 2370

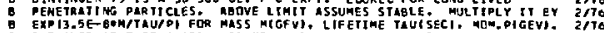

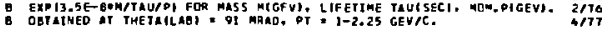

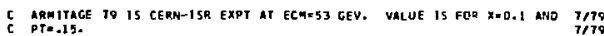

RP

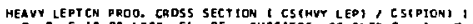
12/910:

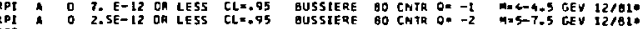

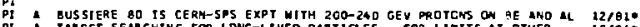

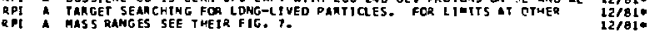

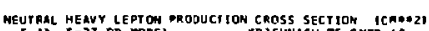

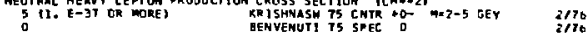

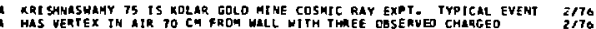

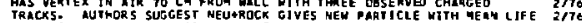

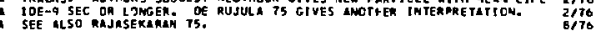

- denvenut 1 is is an fall experiment hh:ch rougrly similates the

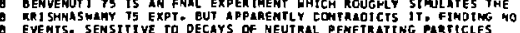

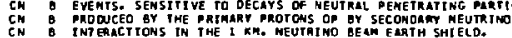

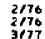

$3 / m$

1779
1779
$1 / 79$

$8 / 77$

$i_{i n 3}$

om:

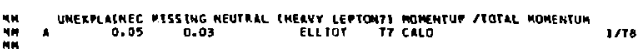

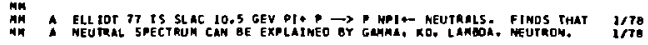


Data Card Listings

Stable Particles

For notation, see key at front of Listings. WEAK GAUGE BOSON, FREE QUARK SEARCHES

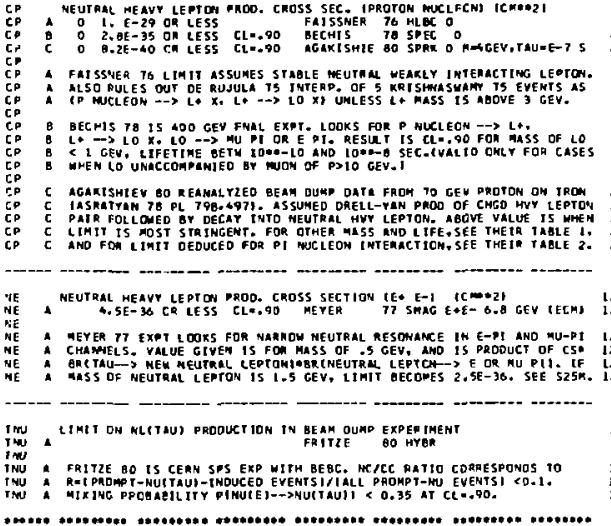

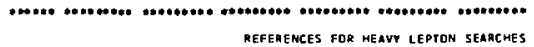

BEMREND
BET OS DRA

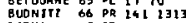

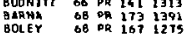
LIBERAAN GQ PAL 22 G69 ROTHE
LICHTENS
70

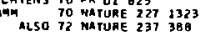
BAMH $n$ NAT.PH.SC.230 145 GAONGIN 72 D D 428136 $B A C R C$
$B A R I S H$
$B 3$ RERN AROOI 30 NC 1719383 BUSHNTS $701 \mathrm{NA}$ A 1158

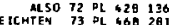

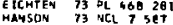
ASRATYAN 74 DL 49848 B

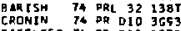

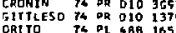
BENYENUT TS PRL 3E 16 BS

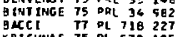
SHNAS 75 DL 570 1C5
ALSO 75 PAL 35620 Fats SMER Te $P_{2}$ g08 402 BARENOV TI PL 708269 BENVEWU1 T7 PRL 36,57 ALSa 77 PRL 38 il 1187 BENVEWI 77 PRL 38 IIt:3 ELLOT 71 PR 015 LES MEYER TI PL TOQ 609 ASRATYA 7E PL TOE 237
gLLTAY TE TOKYC CGAF. BECH1S 78 PRL 40 GGZ

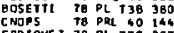

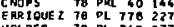

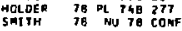
LRMTTAGE 79 NP B150 97

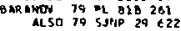
AGaK1SHI go SNMP 32345 ALIMDV 80 JETPL 32 OS

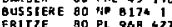
FRITZE
LEB
LETITO

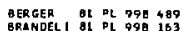

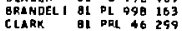
CLARK OI PRL 46299
HEYES OL

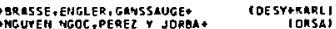

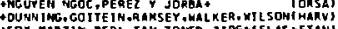

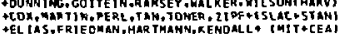

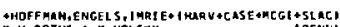

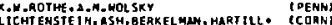

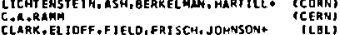

$$
\begin{aligned}
& \text { C.A.rama } \\
& \text { [CERN? }
\end{aligned}
$$

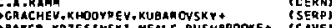

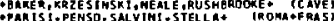

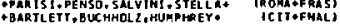

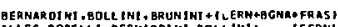

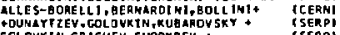

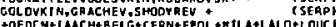

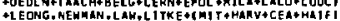
+CERSHT EJN- KAF FANOV, RUQ ANT ZEV, LAP TH* I SERPI

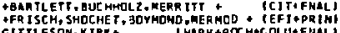

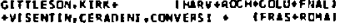

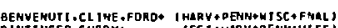

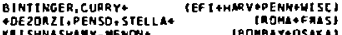

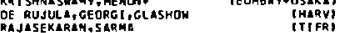

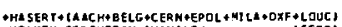

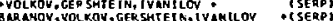

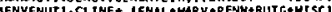

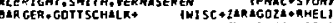

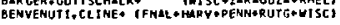

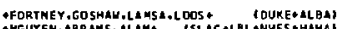

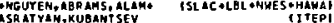

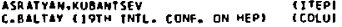
+CHAMG. DOME EC K. ELL SHOR TH, GL ASSEF A, LAU t TUMOI

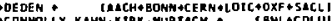

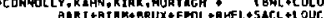

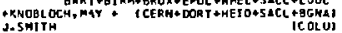

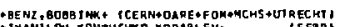

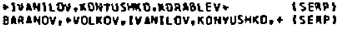

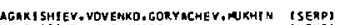

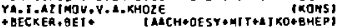

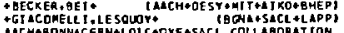

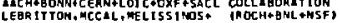

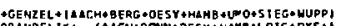

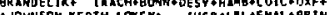

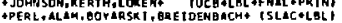
REVIENS

PERL al SLAC-PUL-z75z Mal.PERL, PHYS. In COLL. CONF, V.p.1. ISLACI :

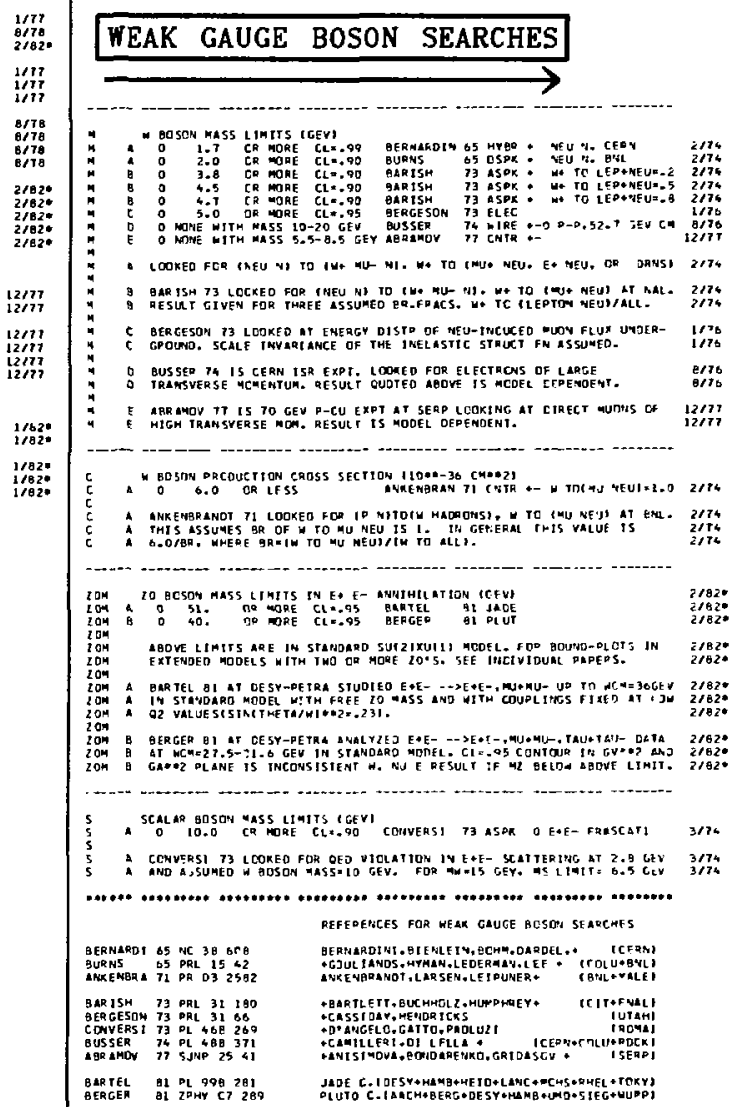

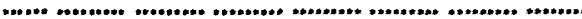

\section{FREE QUARK SEARCHES}

SEARCHES FOR TRT EGRALLY CHARGEO OUARKS APPEAR ALONG WITH CTMIH

bote on Free guark Searches

Five and nine instances of charge $-1 / 3$ and $+1 / 3$ respectively have been reported by Fairbank's group at Stanford (LARUE 77, 79, and 81) using magnetic levitation of superconducting niobium balls. These events are shown in Fig. 1 . There has as yet been no independent confirmation of the existence of free quarks.

The best searches for quarks in cosmic rays $y$ ield upper linits on the flux of quarks of about 


\section{Stable Particles}

\section{FREE QUARK SEARCHES}

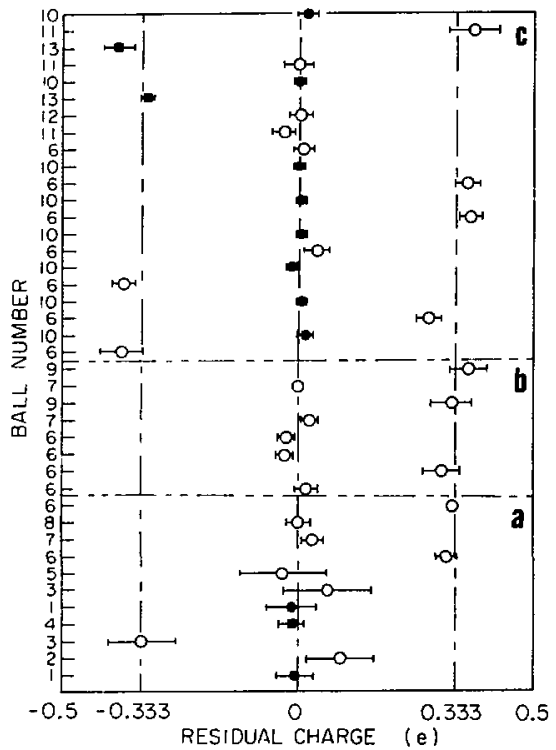

Fig. 1. Residual charge measurements in chronological order from LARUE 77 (a), 79 (b), and 81 (c). Ball radius 140 (open circles), 116 (solid squares), and $98 \mu \pi$ (solid circles). Figure adapted from LARUE 81: La Rue, Phillips, and Fairbank, Phys. Rev. Lett. 46,767 (1981).

$10^{-11} \mathrm{~cm}^{-2} \mathrm{gter}^{-1} \mathrm{sec}^{-1}$. Cross-section upper limits established from proton accelerator experiments and calculations based on production models ${ }^{l}$ imply that free quarks, if they exist, have a mass greater than about $5 \mathrm{GeV}$. Hass 1 imits from photon and electron beam searches are slightly lower, but more reliable, depending only on the QED calculations for quark pair production. Limits on free quark concentrations in stable matter vary enormously depending on the source of matter and the technique.

The largely negative result of guark searches does not prove that free quarks do not exist, but indicates that they are hard to $f$ ind. De Rujula, Giles, and $J a f f e^{2}$ have considered the question of

\section{Data Card Listings}

For notation, see key at front of Listings.

unconfined quarks in a franework of a renormalizable, spontaneously broken versio. of QCD, and conclude that: (1) production cross sections are sma11, (2) interaction cross sections with nucleons are very large, and (3) the physical masses of quarks are probably very large. On this basis, prinordial quarks would be expected to be nonintegrally charged, superheavy nucleon complexes.

We group quark searches by experimental technique - proton beams, photon beans, neutrino bearis, electron beams, $\mathrm{e}^{+} \mathrm{e}^{-}$annihilations, cosmic rays, and stable matter. Proton beam experiments generally measure quark production cross sections (we quote these in section $\mathrm{C}$ ), differential crosgsection ratios (sections $A F$ and RPI), or differential cross gections (sections $I C$ and $D$ ). The photon beam experinent measures cross section per equivalent quanta (aection DG), and the neutrino experiment measureg the ratio of quark events over total events (section NEU). Searches with electron beans may measure differential cross sections (section G) and set limits on the quark mass (section $W$ ). Searches in $\mathrm{e}^{+} \mathrm{e}^{-}$annihilation present the ratio to the $\mu$-pair cross section (sec$t$ ion $\mathrm{EE})$. Oosmic ray experiments measure quark flux (section F), and searches in stable matter measure quark concentration (section RHO) . Wost of the accelerator and cusnic ray experiments have searched for fractionally charged particles, but some have searched for massive otable particles which would have low velocity. The latter searches are usually sensitive to a range of charges and fuay appear in the section below on Other wew particle searches.

We have relied heavily on the review of $L$. $w$. Jo nes ${ }^{3}$ for data prior to April 1977.

\section{References}

1. T. K. Gaisser and F. Falzen, Phys. Rev. Dll, 3157 (3.975).

2. A. de Rujula, R. C. Giles, and R. L. Jaffe, Phy s. Rev. Dl7, 285 (1978).

3. ᄂ. W. Jones, Res. Mod. Phys. 69, 717 (1977). 
Data Card Listings

For notation, see key at front of Listings.
Stable Particles FREE QUARK SEARCHES

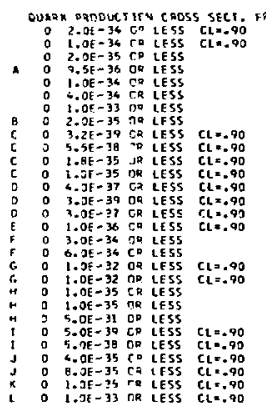

FROM PROTON DEAM EXPIS. (CUE+2)

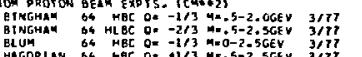

HAGOPIRN BQ MAC O* $01 / 3$ ME.5-2.5CEV $3 / 7 T$

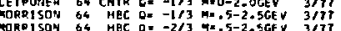

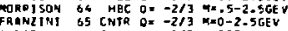

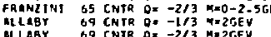

ML LABY $O Q$ CNYR $O=-2 / 3$ Mo 2 GEV

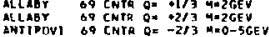

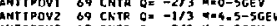

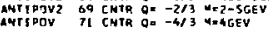

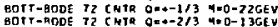

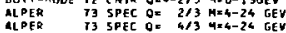

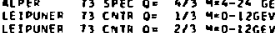

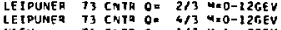

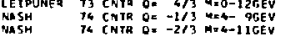

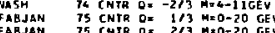

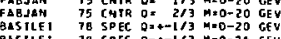

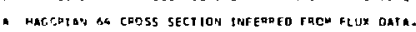

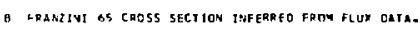

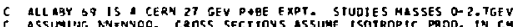

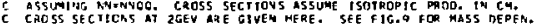

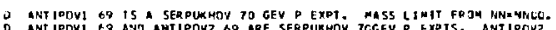

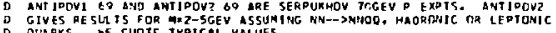

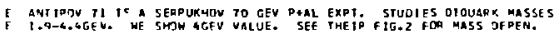

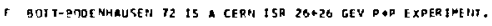

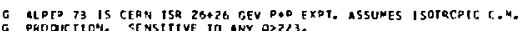

M LET PUIER 73 IS AM NAL 300 GEV P EXPERIMENT.

1 NASH 74 IS FAAL EXPT USTNG 200 AHD 300 GEV PRGIONS. SEE FIT 2 , PGGE 1

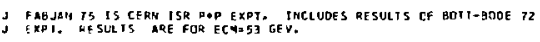

K 345ILES 78 IS CERH I5A EXPT HISH EC

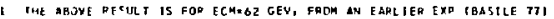

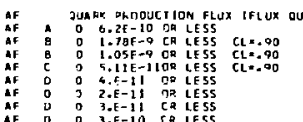

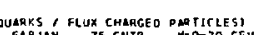

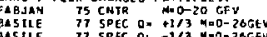

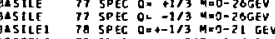

gozzoL:

8027001
9027041
gozzoLi

79 (Nita

79
79 CNTA
79 CNTI

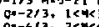

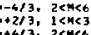

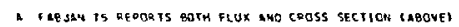

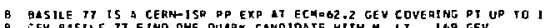

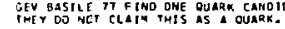

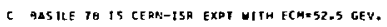

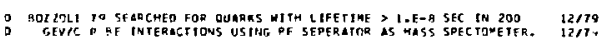

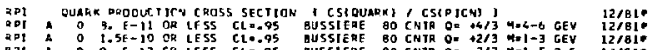

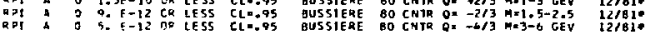
QDI
20

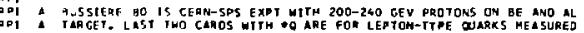

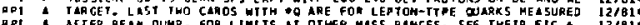

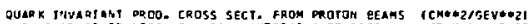

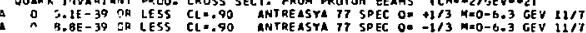

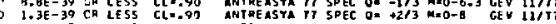

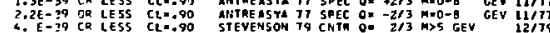

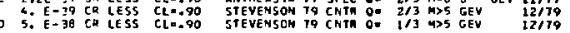

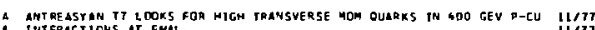

a toreractions at frat.

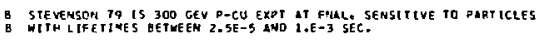

$12 / 79$
$12 / 79$

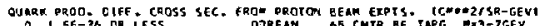

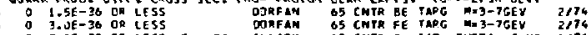

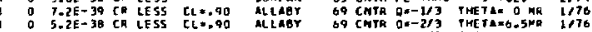

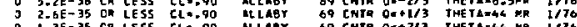

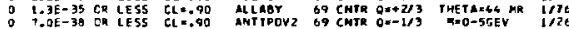

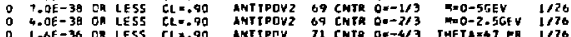

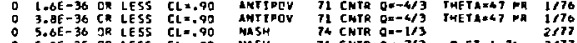

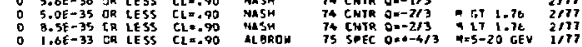
$1.06-33 \mathrm{CA}$
0
0

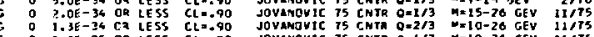

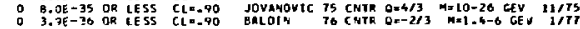

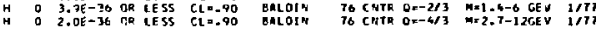

A DORFAN O5 is A 30 GEV/C P EXPEAIMENT LT BML. Y=.18-.995

a SEe foot note $C$ in SUBSection $C$ above.

C SEE GROTHOTE U IN SUasection C ABove.

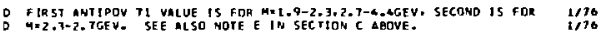

E MASH 74 IS FARL EXPZ USING 200 ANO 309 GEV PPOTONS- VARUES ARE FUR $2 / 15$

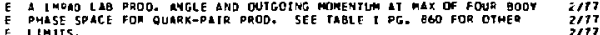
E Limits.

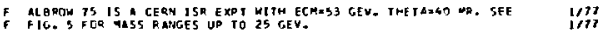

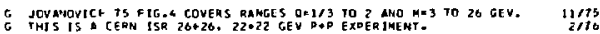

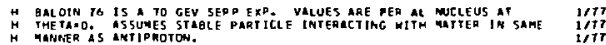

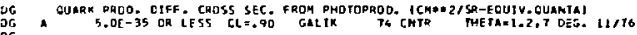

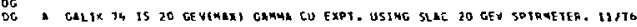

$2 / 74$

2777

MEU OUAFK PQOOLCTICN IN NEUTRIAO gEAHS GQUARK FLUX MUU EYENTI

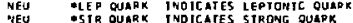

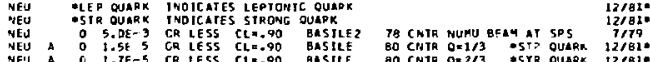

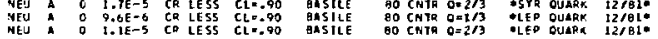

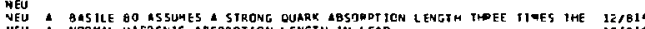

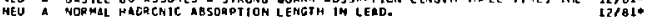

LYMT ON GUARK MASS FAOM ELECTRON BEAMS

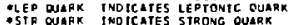

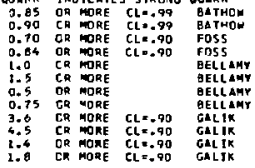

[GEV/C**2)

$2 / 19$
$2 / 79$
$2 / 79$

i 1.8 CK MORE CL=-90 GaLjK

6) CNTR QNI/3

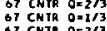

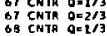
SO CNTR $O=2 / 3$
$6 B$ CNTR $Q=1 / 3$
OB CNTR $O=213$

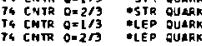
TLEP OULRE TLEP GUAR *Lep quaRk TLEp OUABa TLEP DUARK
TST OUAFK asta gusak STT guara
STR guak

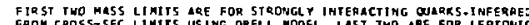
FROM CROSS-SEC LLMITS USIMG ORELL ROOEL. LAST
DUARKS. EXPT USES PHOTOPROOUCTIDN ON COPPER.

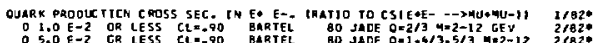

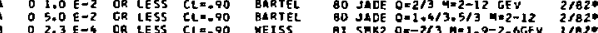

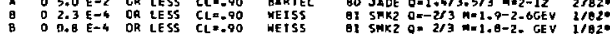

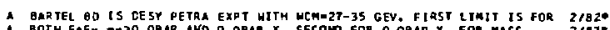

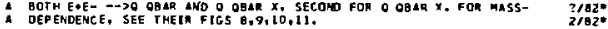

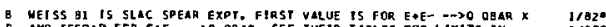

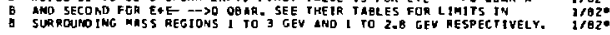


Stable Particles FREE QUARK SEARCHES
Data Card Listings

For notation, see key at front of Listings.
GUAR FLUX FRCM COSMIC RAY EXPERIMENTS TOUHAERCMOH2-SR-SECI

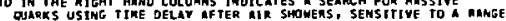

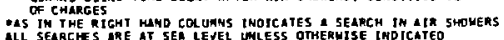
AIL SEARCHES IAE AT SER TEVEL UHLESS OTHERHISE THD TCATED

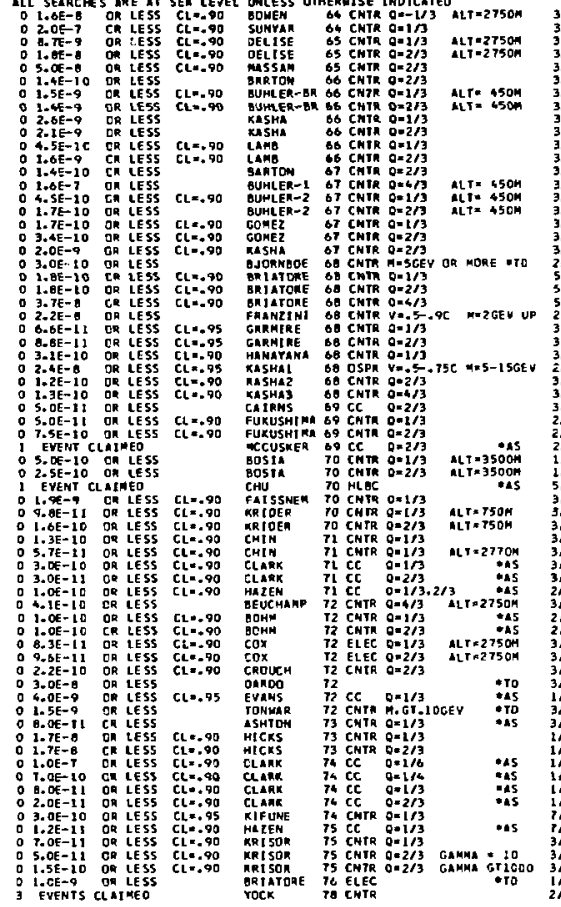

- BARTOH 60 HAC 220000 G/CMOAZ EXTRA SHIELDING

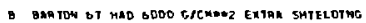

C BUHLER-1 GT AMO BUHEEM-2 OT HAD TSO G/CMED EXTMA SMIELDING

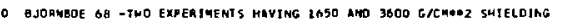

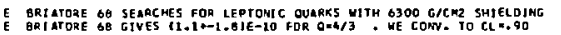

F FRANZJNI SE MEASURES VELOCITY OIRECTLY OY TDF

G CAIPNS GQ OQSERYED \& POSSTBLE DUARK CAMOTDLTES

H FUE tUSHIM 69 DOES MOT RIM.

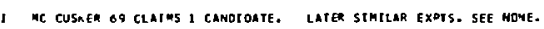

$4 \quad g=2 / 3$ IF MASS LT b.S GEV. $9=1 / 3$ IF MASS E GEV.

a DARDJ 72 HAO 7000 G/GMOEZ EXIRA SHIELOING

$L$ HICKS T3 LOCKE AT LARCE ZEMIJH AHGLES, THUS USING THE AT HOS PHEAE

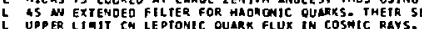

H. KIFUNE 74 LCCKED AT LARGE TENITH AHGLES. FROM THE TA FLUX LIMIT. THEY

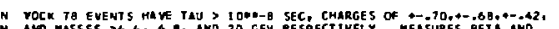

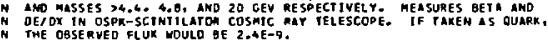

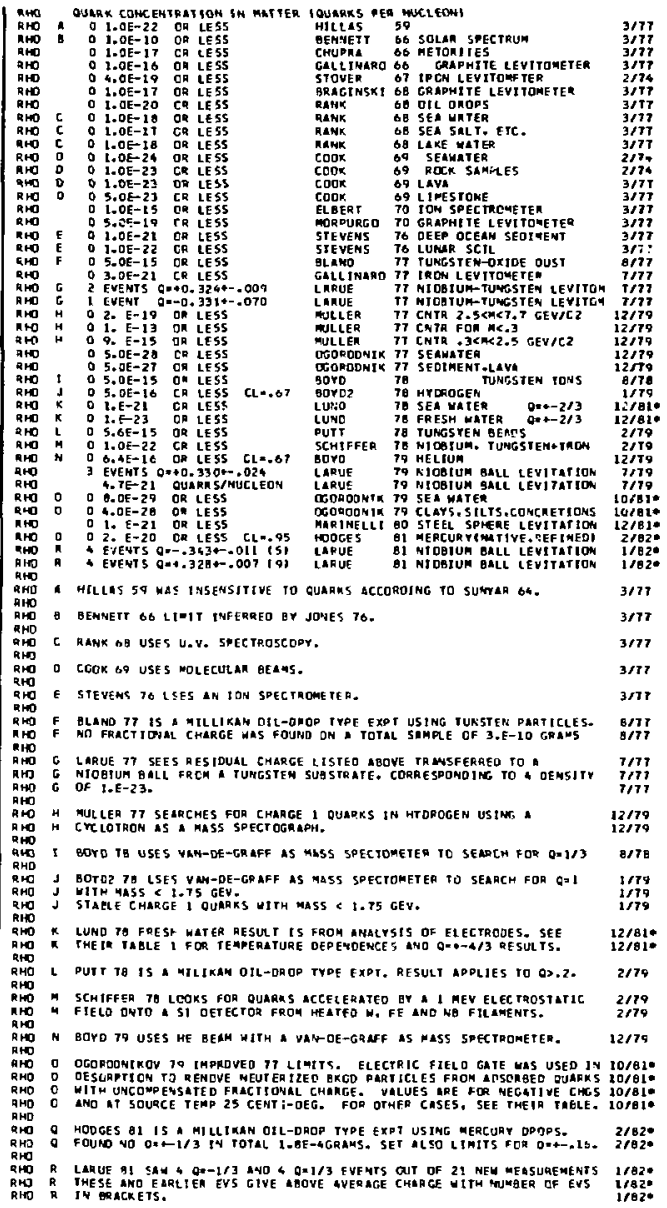

HILLAS 57 MATURE IES 892

REFEREHCES FOR OUARK SEARCHES

HILLAS, CRAMSHAM IAEREI

DOICK INSON, OIEBOLO KCCH,LETTHE TCERNAEPOL!

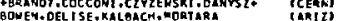

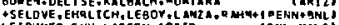

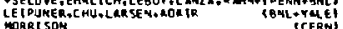

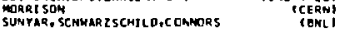
OEL I SE, BONE N

Aarizi

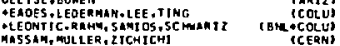

GARTOH, STOCAEL

(TMOL)

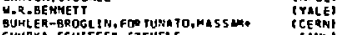
CHUPKA, SCHIFFER, STEVERS

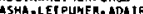

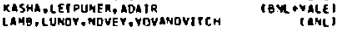




\begin{tabular}{|c|c|c|}
\hline 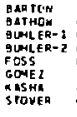 & 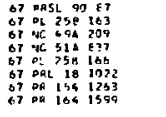 & 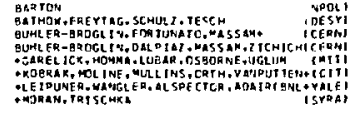 \\
\hline 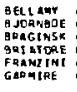 & $\begin{array}{l}1391 \\
241 \\
51 \\
5>0 \\
1013 \\
1200\end{array}$ & 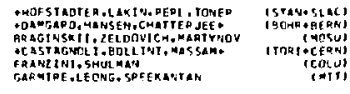 \\
\hline 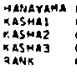 & 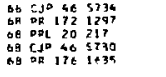 & 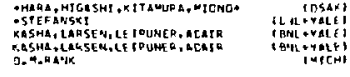 \\
\hline 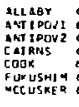 & 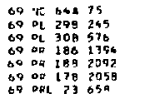 & 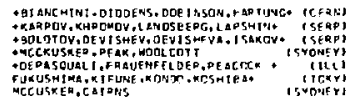 \\
\hline 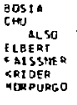 & 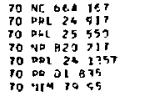 & 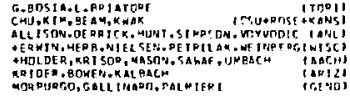 \\
\hline 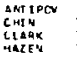 & 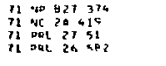 & 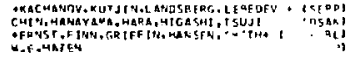 \\
\hline 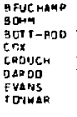 & 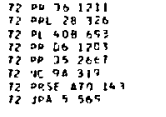 & 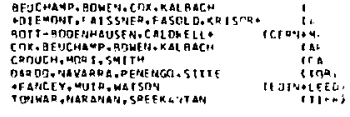 \\
\hline $\begin{array}{l}\text { S } \\
\text { S }\end{array}$ & 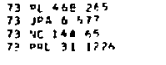 & 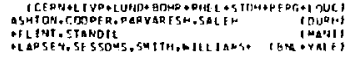 \\
\hline 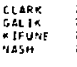 & $\begin{array}{l}2721 \\
1046 \\
1020 \\
20 A\end{array}$ & 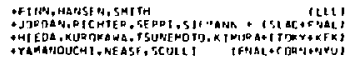 \\
\hline 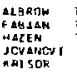 & 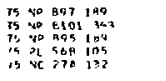 & 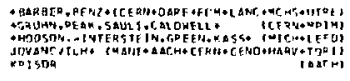 \\
\hline 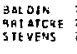 & 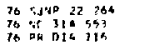 & 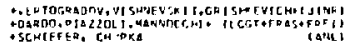 \\
\hline 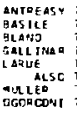 & 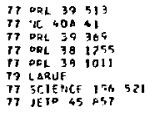 & 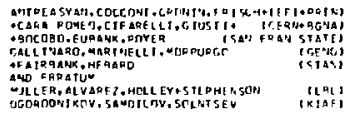 \\
\hline 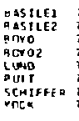 & 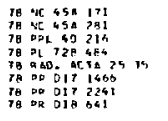 & 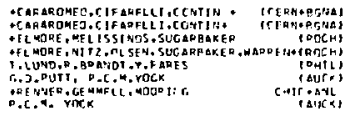 \\
\hline 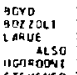 & $\begin{array}{l}70 \% \\
79: \\
790 \\
790 \\
790\end{array}$ & 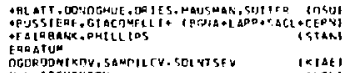 \\
\hline 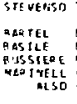 & & 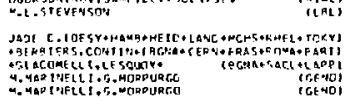 \\
\hline 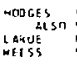 & 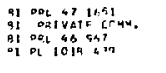 & 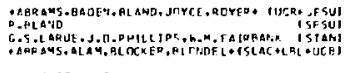 \\
\hline & & WEVTFW ARTITLLFS \\
\hline , & 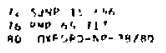 & 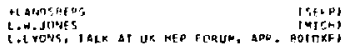 \\
\hline
\end{tabular}

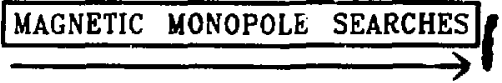

Wote on Magnet ic Nonopole Searches

(by W. P. Trower, virginia Polytechnic Institute and State University)

Al though the idea that magnetic monopoles might exist is suggested by the usual formulation of Maxisl's equations, no observed phenomenon requires them for its explanation. ${ }^{1}$ Nonopoles first became intereating with the assertion that a single monopole anywhere in the universe would result in electric charge quantization everywhere. ${ }^{2}$ The sole predicted property of this monopole was the magnituōe of its least magnetic charge $e / 2 a$, the Dirac charge. A monopole of two Dirac charges has been suggested, ${ }^{3}$ and an electrically charged monopole, a dyon, proposed. 4 Oserved pure multi-photon showers were attributed to virtual monopole pair-production/-annihilation. 5 Monopoles have become indispensable to many gauge theories, and most grand unification theories require a monopole of mass $>5 \times 10^{15} \mathrm{GeV}$. Estimates of the monopole number density at the earth have been made. ${ }^{6}$

Experiments to detect monopoles have essentially been based on either ionization or magnetic induction. Ionization experiments have relied on the fact that an elementary relativistic magnetic charge would produce more ionization than a relativistic electrical charge. Massive monopoles would have, however, lower velocities, $\beta \sim 10^{-3}$, and would thus reduce the prospects for ionization measurements. The theory of monopole energy loss at these velocities is currently confused, but progress is being made. ${ }^{7}$ It is however likely that prospects for ionization identification of massive monopoles will remain diminished.

Induction experiments measure the value of the monopole magnetic charge by detecting changes in magnetic flux induced when a monopole passes through a superconductor (CABRERA 75). These measurements are independent of monopole electric charge, mass, and velocity. 
Stable Particles

\section{MAGNETIC MONOPOLE SEARCHES}

With the recent attribution of large mass to the monopole, searches in matter are less appea 1ing as the ferromagnetic trapping energies become comparable to terrestrial gravitet onal binding. Accelerators do not possess sufficient energy to produce real monopoles. Cosmic ray searches hold increased promise. possibly monopole evidence will be obtained from direct astrophysical observation.

The following compilation with the indicative experimental limits should be used as a directory to the 1 iterature.

\section{References}

1. J. D. Jackson, CERN-77-17 (1977) .

2. P. A. M. Dirac, Proc, Royal Soc, of London Al33, 60 (1931).

3. J. Schwinger, Phys. Rev. 144, 1087 (1966).

4. J. Schwinger, Science 165,757 (1969).

5. M. A. Rudierman and D. Zwanziger, PhYs. Rev. Lett. 22, 146 (1969).

6. G. Lazarides, Q. Shafi, and T. F. Walsh, Phys. Lett. 100B, 21 (1981).

7. See, e.g., S. E. Ahlen and K. Kinoshita, to be published in Phys. Rev, D (1982).

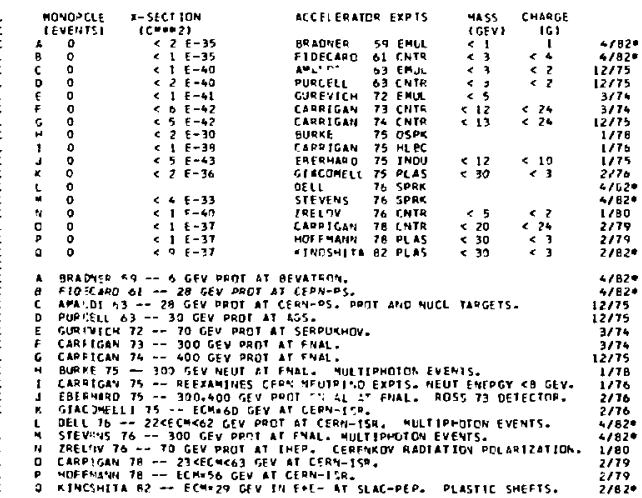

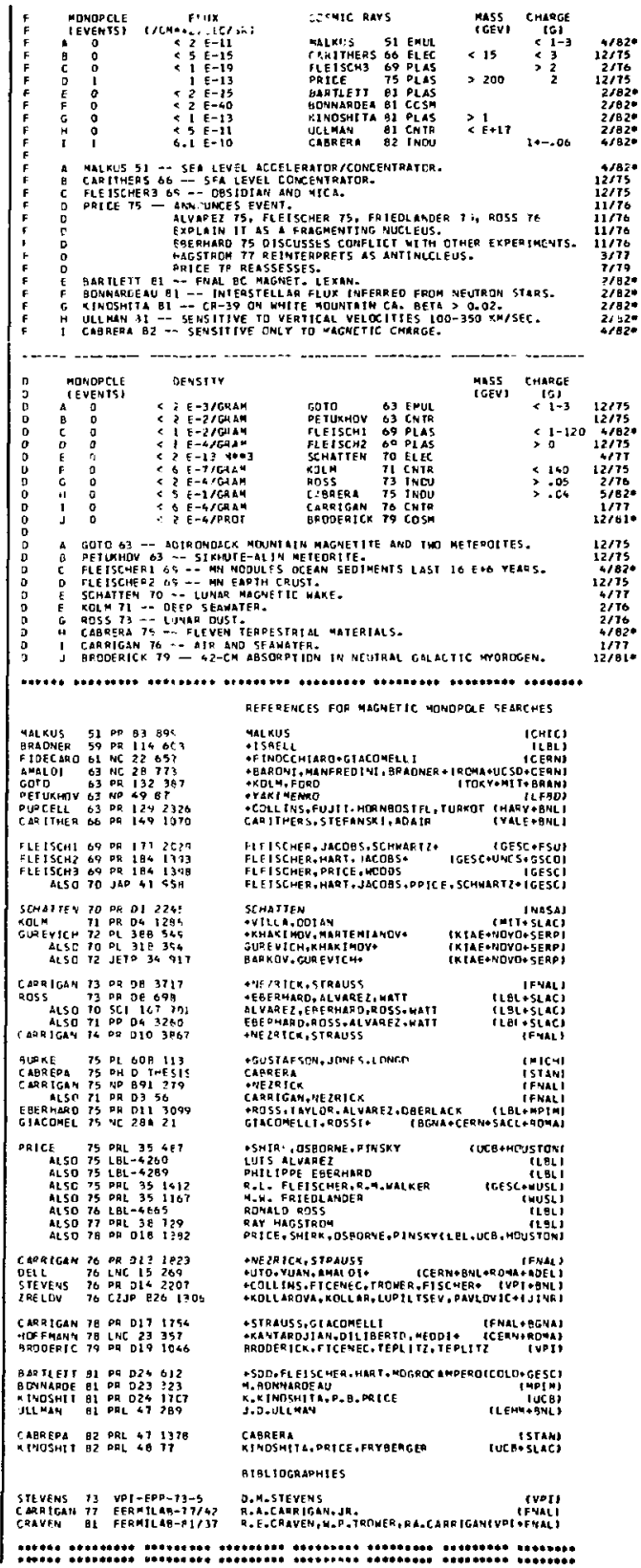

Data Card Listings

For notation, see key at front of Listings. 


\section{CHARM SEARCHES AND EVIDENCE}

Data on specific charmed states are listed in separate sections in the appropriate places in the Data Card Listings: $D, F$, and $\Lambda_{c}$ - stable particles; $D^{*}, F^{*}$ - Mesons; $\Sigma_{c}$ - Baryons.

Evidence for charin not directly relatable to a given state is listed in this section. Neutrinoinduced dilepton events are sumnarized. Short-lived tracks in emulsions are also dealt with.

Tri-muon production in neutrino interactions is summarized in the other New Particle Searchea section.

\section{Neutrino-induced Dilepton Events}

Many neutrino experiments have now observed dilepton events. These data are summarized in subsections $Y, V_{0}, V_{A}$, and VAP. Bubble chamber experiments have observed neutrino-induced $\mathrm{H}^{-} \mathrm{e}^{+}$events associated with strange particle production in the reaction

$$
U N \rightarrow,-e^{+} K^{0}(\text { or } \Lambda)+\text { anything. }
$$

Production of charmed particles (c) in neutrino interactions would be expected to give rise to such events via the mechanism

$$
\nu_{\mu} \mathbf{N}+\mu_{-}^{-} \mathrm{C}+\text { hadrons }
$$

where the Cabibbo-favored transition would predict a strange particle among the hadrons. Thus the appearance of neutrino-induced opposite-sign dimuon events, $\mu^{-} \mathrm{e}^{+}$events, and associated strange particles can be understood via the charm mechanism. (For another potential explanation see the Heavy Lepton Searches section above.)

Recent experiments show that like-sign dileptons, on the other hand, are produced much in excess of theoretical expectations. See section $Y$ below.

\section{Short-Lived Tracks in Enulsions}

The mean life of a weakly decaying charmed meson or baryon of mass $M$ (in GeV) is expected to be in the rangel

$$
\tau=\left(10^{-11} \text { to } 10^{-13} \mathrm{gec}\right) \times 1 / \mathrm{M}^{5}
$$

with a corresponding mean path length for lab mo- mentur $\mathrm{P}$ (in $\mathrm{GeV} / \mathrm{c}$ ) of

$$
l=\frac{\mathrm{ECT}}{M}=\left(30 \mu \text { to } 3000_{\mu}\right) \times \mathrm{p} / \mathrm{M}^{6} .
$$

Thus even at Ferrilab energies, the decays of such particles are hard to observe directly in rost bubble, streamer, or other chambers, so emulsion is often used. We list data for these experiments in subsections $\mathrm{CC}$ and $\mathrm{EM}$ below.

Recent experiments using special bubble chanbers, emulsions, and silicon detectors have been able to identify the particular charm state with some degree of confidence and to estimate the mean life. These measurenents are ligted in the separate sections associated with the particular states.

\section{Charm Searches}

Bxperimental evidence for charm production has now been accumulated in various reactions. sections $C P$ and CPI include several types of evidence for associated charm production in $\mathrm{pN}$ and $\pi N$ collisions: the prompt $l_{\mu}$ and le sigrals; low-mass $\nu^{+}{ }_{\mu}-$ pairs with missing energy: oppositesign $\mu$ e events; observations of $D$ and $\Lambda_{c}$ in the hadronic final states; as well as hadronic chacm decay in association with a lepton trigger. observations of prompt muons in beam dump experiments are 1 isted in section $B D$.

Sections $C G$, MU, and $D$ include evidence in photon, muon, and neutrino beam experiments. Charmed baryon production in $\mathrm{e}^{+} \mathrm{e}^{-}$reactions is listed in section $\mathrm{CE}$; further information can be obtained from the Listings.

\section{References}

1. M. K. Gaillard, B. W. Lee, and J. L. Rosner, Rev. Mod. Phys. 47, 277 (1975).

PROPERTIES OF IHE CHARMED D. OE, F, FE, LAMBDA/GA, AND SIGMA/C+ THE FOLLOWING SECT ION CONTAINS INFORMLTION ON SEARCHES FCR GTHEQ CHAP MED PART TCLE STATES AND SEARCHES FCR THE ABOVE STATES IN NEW CDLLISTICN PAOCESSES.

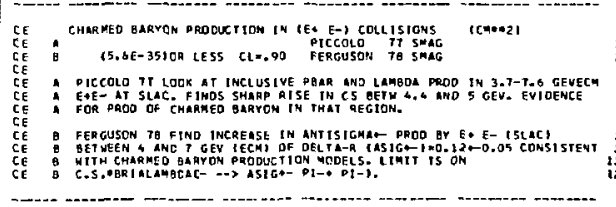


Stable Particles CHARU SEARCHES
Data Card Listings

For notation, see key at front of Listings. $c G$
$c G$
$c G$
$c G$
$c G$
$c G$
$c G$
$c G$
$c c$
$c c$
$c G$
$c G$
$c G$
$c G$
$c G$
$c G$
$c G$
$c G$
$c G$
$c G$
$c G$
$c G$
$c G$
$c G$
$c G$
$c G$
$c G$
$c G$
$c G$
$c G$
$c G$
$c G$
$t G$
$6 G$
$G G$

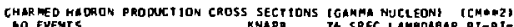

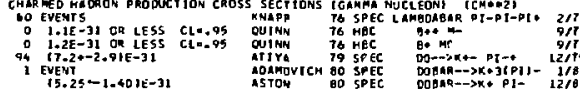

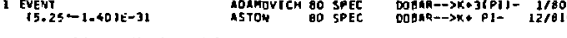

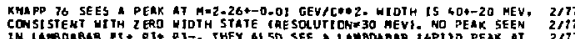

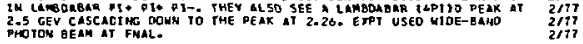

QUIW 76 USED a 9.3 GEV PHOTON gEAY AT SLAC. SEE TABEES 1 ANO 3 FOR IMO TVIDUAL CMANMELS.
NO WISSING MEUTRALS.

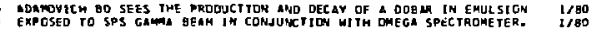
ASTON BO IS CERN-SPS OMEG A-SPECTADMETEQ EXP NITH 20-70 CEY PHOTONS. 12/B1:

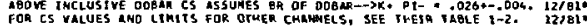

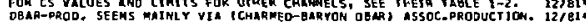

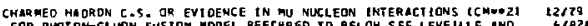

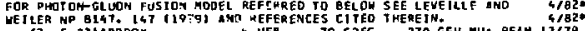

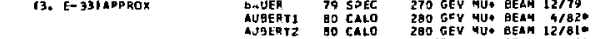

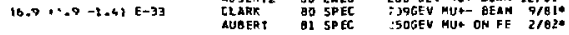
QALER 79 SEES 449 DIMUDYS, GA TR ! MUONS. TME MaSCP ITY OF OJMUONS

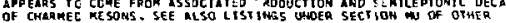
OF CHAMMEC RESOML, SEE RLSO
STAELE PARTICLE SEARHES.

12279 12179
$12 / 79$
12179

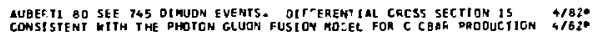

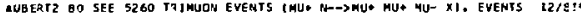

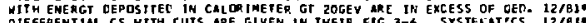

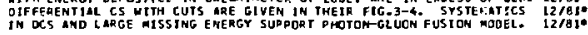

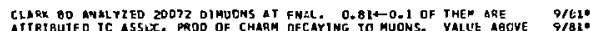
is OIFTRACTIVE CHAR MUOPROO C.S. EXTRAPOLAT EO PHOTON C.5.

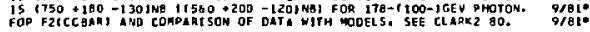

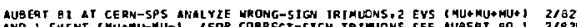

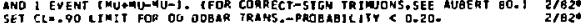

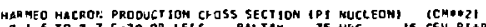

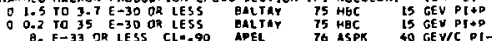

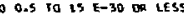

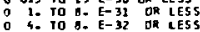

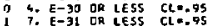

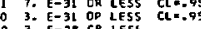
0.029 CA LESS CLE.9D 0
0 CL $=.90$

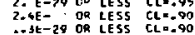

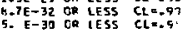
12 40. $\mathrm{E}-30$

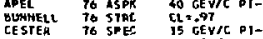
GHIOINT T6 SPEC 19 GEV'C DISACRT LIVEO 2-SGEY 2 ím HACOPIAN 76 OEC LOKG IIVEOT-1.9,2-5 HACOPSAN 70 OEC LOWC LTVEDT-1-9.2-S
KLEMS

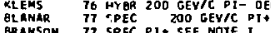
REANSON 77 SPEC OY SEE NOOE I
BRANSOH SPEC PI- SEE MOTE I CODDARO 77 MQC DOBAR C.. JOHCXNEER 7 STRC 225 GEY/C PI

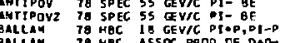

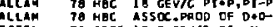

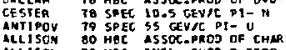

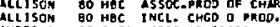

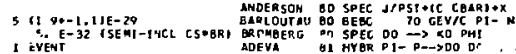

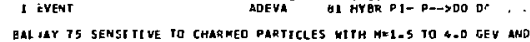

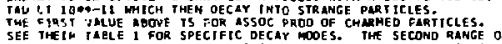

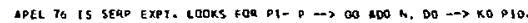

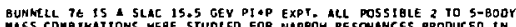

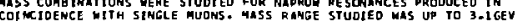

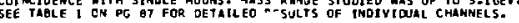

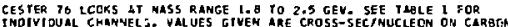

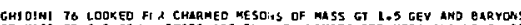

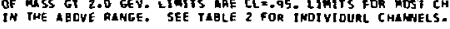

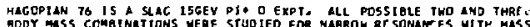

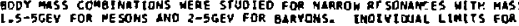
TWO AHO THAEE $900 Y$ DECAY FAOH MANY RE ACTIDNE ARE GIVEN.

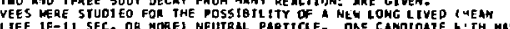

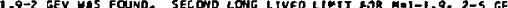

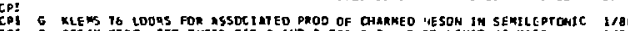

章,

intin

$\operatorname{lin}_{1 / 2}$

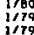

i,tio

络:

7176

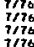

776

if

$i_{1 / 7}$

in?

2,77

$2 / 77$ $2 / 77$

$227 \%$ 2176 $2 / 76$
ATIYA 79 is FNal EXPF USING 50-200 geV Protoms. C. S. Assumes 3. $0 E-31$ OR LESS CLE. 2.0610
$2.68-30$
$0 R$

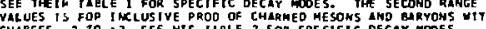

CPI H BLAMAG IT IS FNAL EXPT. LIMII IS FOR CSABP iC RUONS. ASSUMES

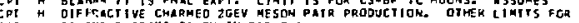
con

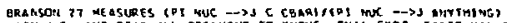

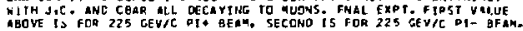

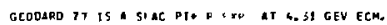

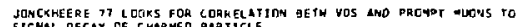
SIGNAL Decar of CHAB YEO PABTicle.

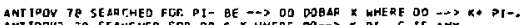

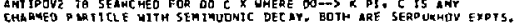

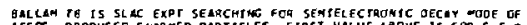

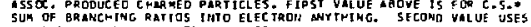

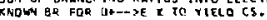

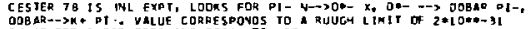
CMOOZ FOR DOEAR PAOD ANO DECAY TO KPT.

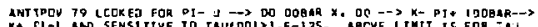

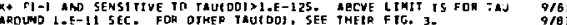

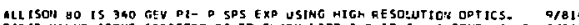

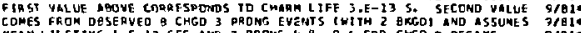

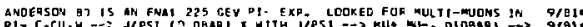

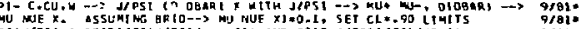

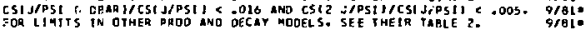

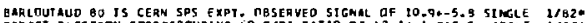

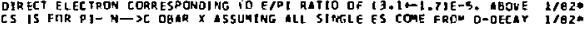

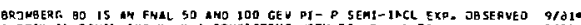

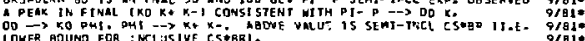

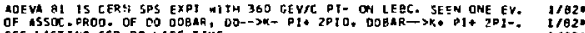

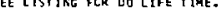

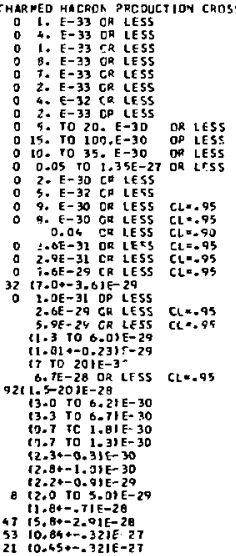

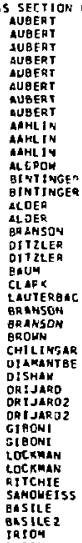

\section{TS SPEC

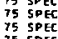 \\ 75 SPEC
75 SPEC
75 SPEP \\ $\begin{array}{ll}75 \\ 75 \\ 75 & \text { SPEP } \\ \text { SPEC } & \end{array}$ \\ $70 \mathrm{HACC}$
76
$\mathrm{HgC}$}

$70 \mathrm{FGC}$

VIINGES 76 SPEC

THTHGE 76 STEC

77
SPEC
LDER

ITZLER 71 SPEC $2 / 76$

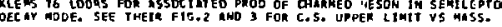


Data Card Listings

For notation, see key at front of Listings.
Stable Particles CHARM SEARCHES

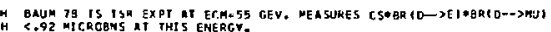

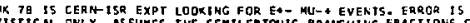

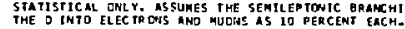

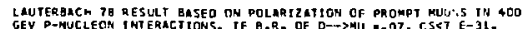

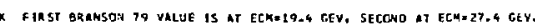

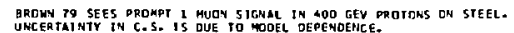

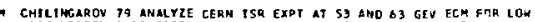
MASS LEPTOH PATR P POE

oramantberger 79 uses too gey paotans to re aho locks for his sing

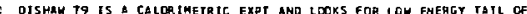

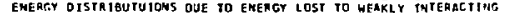
- PARTICLES. ASSUHES SEMTLEPTORIC B.R $=0.11$.

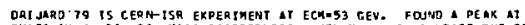

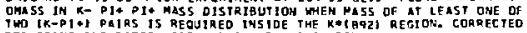

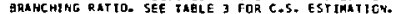

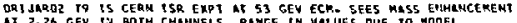

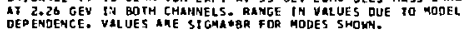

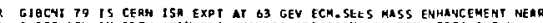

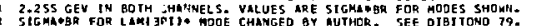

LOCXAAN 79 TS A CERN ISR ERPI AT 53 LND G2 GEV ECH. SEEF, A MASS

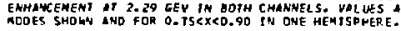

RITCH1E 80 15 AN FNAL 350 GEV PRCTON FE EXP. MEASUREO PROMPT WUDNS OR

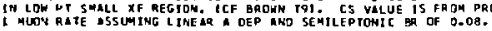

SANONEISS DO IS AN FNAL EXP NITH 350 GEV PROTONS ON NE STRC.

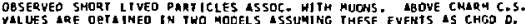

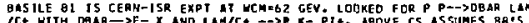

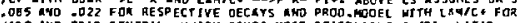

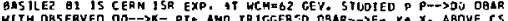

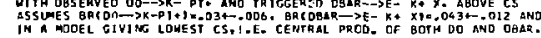

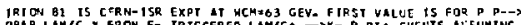
We

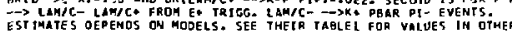
MODELS IND C.S. UPPER LIHITS FOP DQAR D*.D OGAR* LAMIC DEART PROOS.

CHAR MEO HADRON PROOUCTION CHOSS
$\begin{aligned} & 0 \\ & 0 \\ & 0\end{aligned} 1.9 E-31$ CR LESS
1.OE-31 CR LESS
2.SE-29 OR LESS Cl..975
?.: $\hat{E}=32$ OR LES
BLESER
BLESER
OLESER
ULESER
IN MUCLEONI ICHEOZI
75 SPEC $K+P 1-$ M
75 SPEC $K+P 1-1.8$ GEV
75 SOEC $K-5$ GEV
75 SPEC K- P. HA2.5 GEV
ABOL IHS 76 SPEC
ABOL INS 76 SNEC
O. E-3Z OS LESS
$0-2$ SEE CONMENT O GELO
1. E-31 OR LESS CL=.90

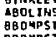

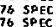
76 SPEC
$x-\infty$
O 2. E-31 OR LESS CLE.,99
LIDron
C. To L L mimos po

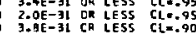
$3.2 E-31$
$0 R$ LESS CL:.90
$0.3 E-30$ CR LESS CLF.90
D $1.1 \mathrm{E}-30$ LR LESS CI $=.90$
SPELARING PO SPEC K.. P1-t, MA1. AGGE

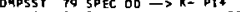

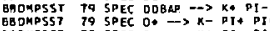 EBDAPST 79 SPEC 00 Z) K- PI+ PI,

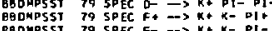

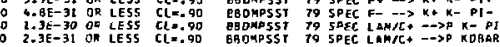

gleser 75 uses negurons up to 300 cevic, be tareet. examines mass

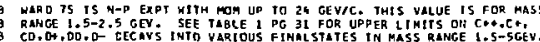

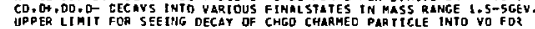

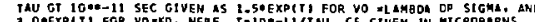

C ABOLINS 76 IS FNAL EXPT. USES MEUTRGHS UP TO 300 GEV/C ON BE TAAG.

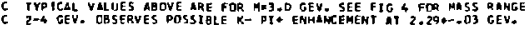

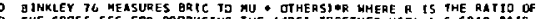

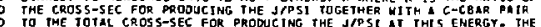

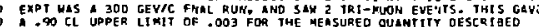

D ABVE.

E $\triangle 80 L I N S 78$ IS 4250 GEVIC N-BE EXPT LOOXING FOR HARROH ENHANCEMENTS

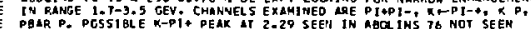

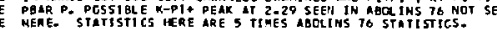

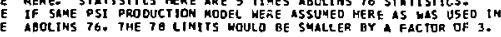

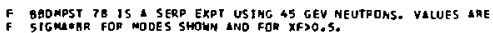

G LIFTON 70 IS 300 GEV N-BE EXPT AT ENAL. VALUE DUOTEO IS FOA

ASSOC CMAPM PPOO

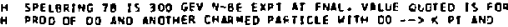

H PROD OF OO AND ANOTHER CHLAED PAFT!CLE WITH DO - $\rightarrow K$ PI LND

1 aBDMPST 19 IS SAME EXPERIMENT AS BQDMSST 78.

$8 / 78$
$6 / 78$
0,18

$6 / 76$

$12 / r 9$

1239

12159
12579

2279
12174

$12 \pi 78$

1277

12779
12779
12778

12779

12779

12119

12779

12179

12719

$12 \pi 79$

$9 / 810$

9/910

$9 / 810$

9.81:

$1 / 32:$

i 1820

2/32*

2182\%

11820

$1182:$

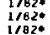

$\sum_{2 m 71}^{2 m 7 n}$

im7

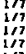

1,77
6770
$7 m 9$
3

7779
8778
8778
12779

8718
12179
12879
$12 / 78$

$12 / 270$

12779
12779
122790

$12 / \pi$

$32 \pi$

in

$1 / 77$

1/77

im

$\prod_{1 \rightarrow 1}$

你

隽

$6 / 78$
678
8778

8778
6178
0178

orra

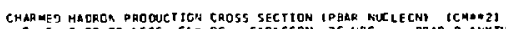

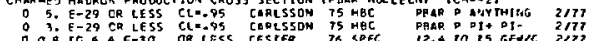

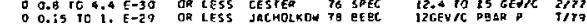

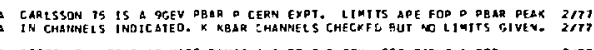

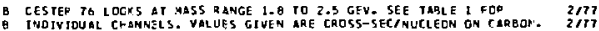

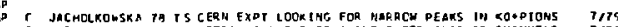

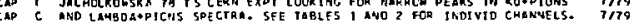

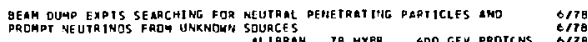

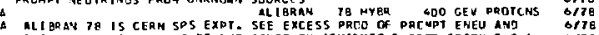

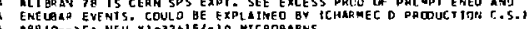

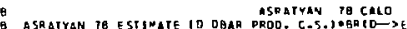

OF $10.5+-0.51$ STh

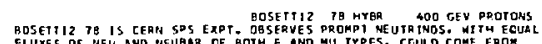

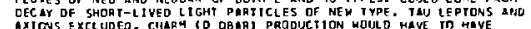

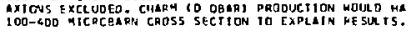

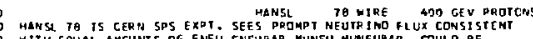

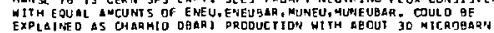
EXPLAISED DS OST
CROSS SECTICH.

$$
\operatorname{cotens} 70 \operatorname{cspo}
$$

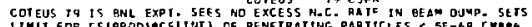
ICl $=.901$.

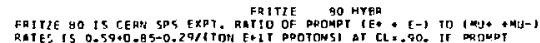

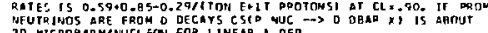

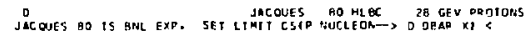

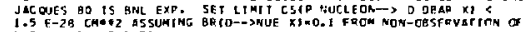
NUTCINOUCEO EYENTS.

SDUKAS OPO CBLD 28 GEY PDOTOR:S

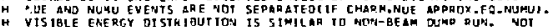
HIS IBLE EAERGY DI STHIAGT

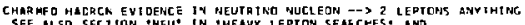

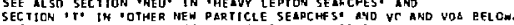

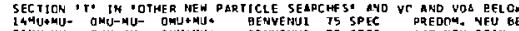

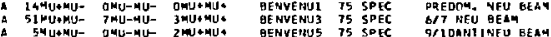

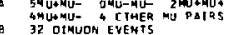

c 4 MU+E- 64U-E*

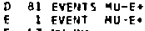

F. 20 Mid

H 17 EVENTS MU-E*

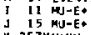

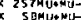

$x$
$t$

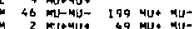

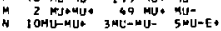

N $2 m$ undu- inueE-

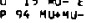

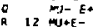

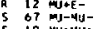

I 21 HU+HU-

I1 11 my+4

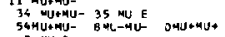

$x+\frac{9}{3}+40-$

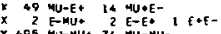

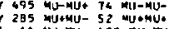

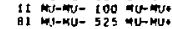

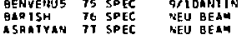

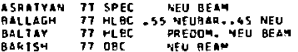

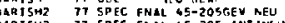

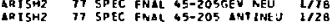

QL FT2 ACKE 77 QYUUE

GOSFTYI $\gg 7$ MYER

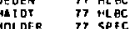

77 SPEC INCDENT REU
SPEC

MOLOEP
MOLDER

促

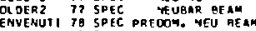

GE HVENUII TG SPEC PREDOW: WEUEAR EEAM

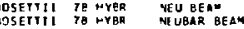

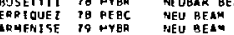

nosanovi to mas

AERGE 79 rat

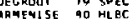

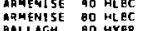

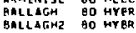

MEU BEAY

NEUALR EEAM

NEUBRR BEAM

MEU MNDUCED

AMMOSOV

BALLEH 91 HY RY RUMU, NUMUALD JND.

JDNEFR AI CALO NEU INDUCED
JONKER OI CALU NEUBLR IKOUCEO

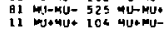

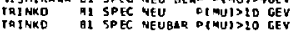

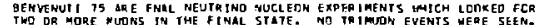

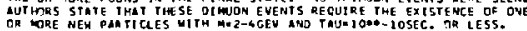

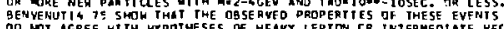

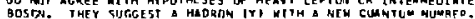

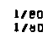

$2 / 78$

$6 / 70$
$6 / 78$
$6 / 78$

67

$6 / 78$
$6 / 78$
$6 / 78$

379

7179

$1,82=$

$1 / 920$

9/81:

9rest:

4/9t: 9/m1*

(1)

$\frac{2 \pi 76}{2 / 170}$ (lis (2)

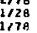
I2xit 12,77

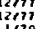
$1 / 79$
1979
$6 / 76$
orro 0178
$1 \% 90$
$1 / 80$

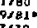
1880 12\%at: $2 \pi 0^{2}$

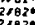
 182. 76 2716 2176 
Stable Particles

CHARM SEARCHES
Data Card Listings

For notation, see key at front of Listings.

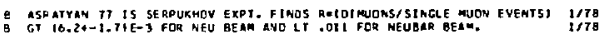

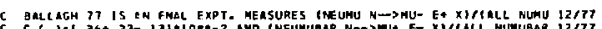

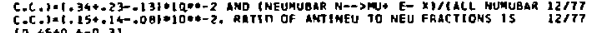
C $10.45+0.6-0.31$.

- Bel taY Ti IS FNal EXPT In NeON-Hz mixtuat.

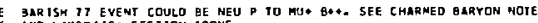

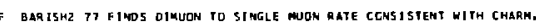

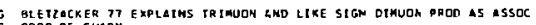

4 goserti 17 is FaAL IS-Ft CHamber Expt.

DEDEN 7 IS CERN MTDE GAND FYDT, EHAX:Z GEV.11+-5 EVENTS ABQUE a BackGR TUNO OF G EVENTS.

J HAjOT 72 IS SAME EXPT AS VONKROEH 76 LISTEO UNOER SZOVO. MEASURES

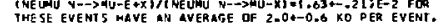

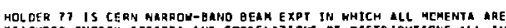

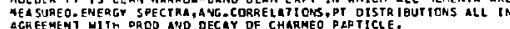

$8 \pi t$

$3 / 77$

$2 / 78$

$12 \pi 77$

$m$

12277

ims

$1 / 1 \%$

$12 / 77$

HOL DEFZ $" 15$ 200GEV MARROW DANO EXPT, AFYEA OACKCFOUND SUBTRACTION 2237

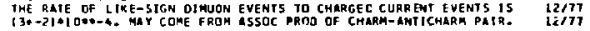

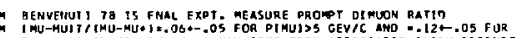

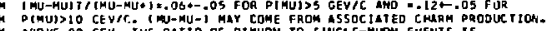

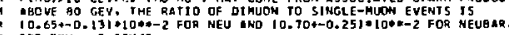

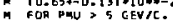

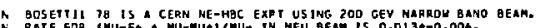

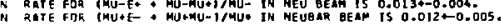

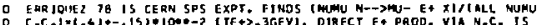

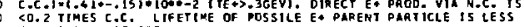

C. THAS 30100-12 SEC.

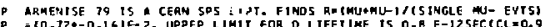

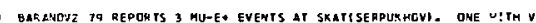

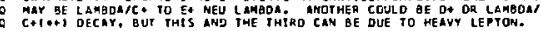

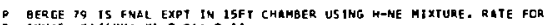

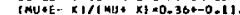

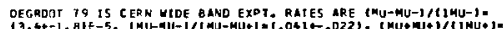

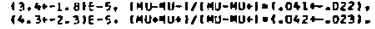

1 apmenise oo is cean-sps exp hith bebc exroseo to hroe-Band me UaAR

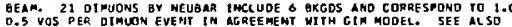

SEC TICN Y IN GOTTCM SEARCHES.

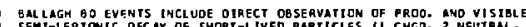

SEMT-LEP OONIC OEC AY OF SHOQT TES

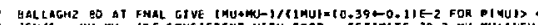

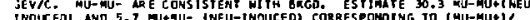

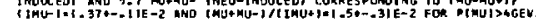

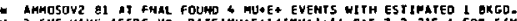

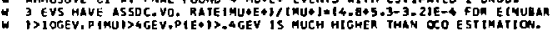

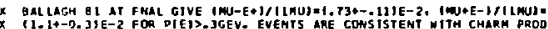

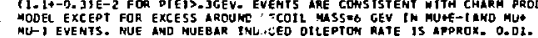

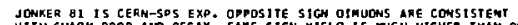

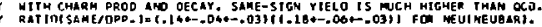

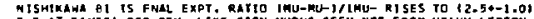

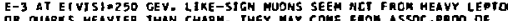

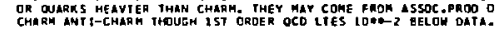

TRINAO B1 IS FNAL EXPT. (DROMPT MU-MU-1/21MU- =10.340-0.091E-3 ANO

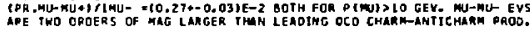
- SMSTEMatic OESCRTPTION COMMENT

ATE

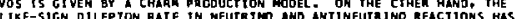
BEEN MEASURED TO BE MUCH HICMER THAN THEDRETICAL EXPECTAT JON.

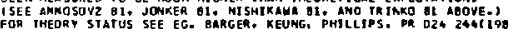

139

${ }_{136} 1379$

orre

9/a10

$9 / 910$

$\underset{i / B 0}{1 / 80}$

(2)

$12 / 810$

$1 / 60$

$1 / 180$

9/8 j* ,

22030

$\frac{1}{2, \pi r^{2}}$

2/az:

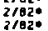

年, 2820

${ }_{2 / 82^{\circ}}$

11022

西,

$1 / 820$

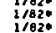

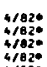

,

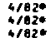

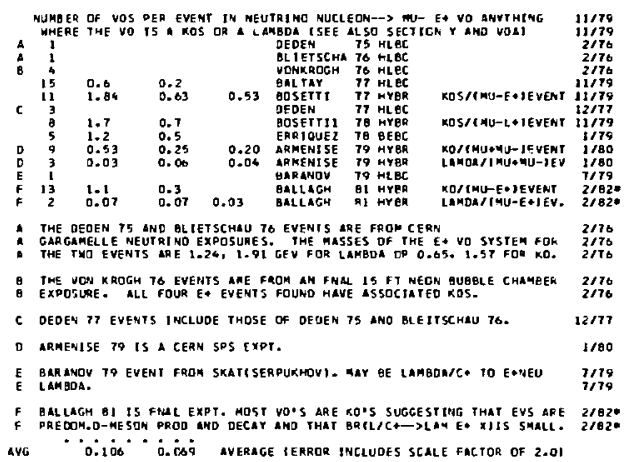

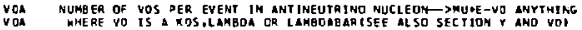

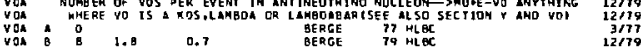

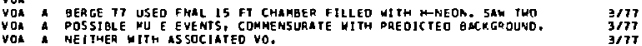

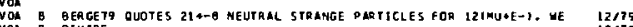

VOA B DIV 10E.
VoA

VOA C BMLLAGH BI IS FRAL EXPT.

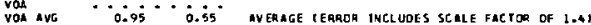

YAP NUMBER DF VOS PER EVENT IN AMT T-NEUTRINO MUCLECN-DSSAME-S 5 IOS

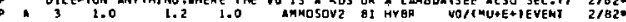

a ahosavz ol is fanal Expt.

2/82*

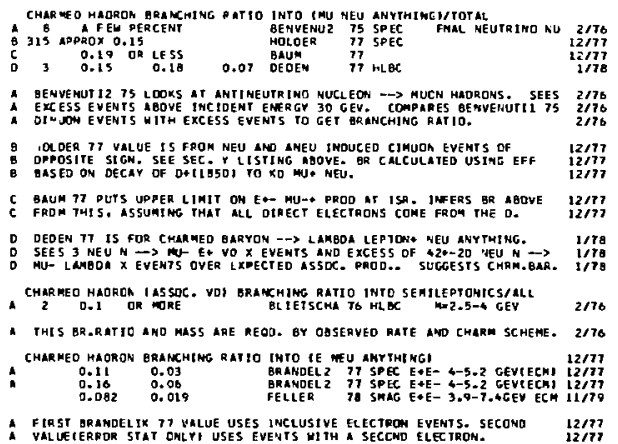

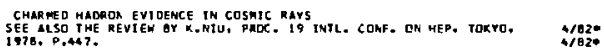

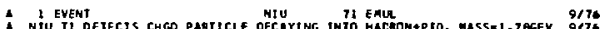

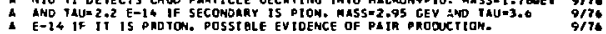

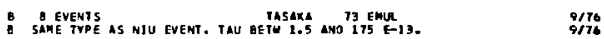

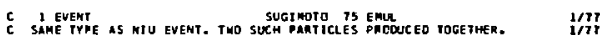

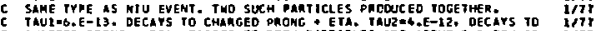

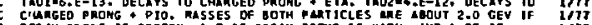

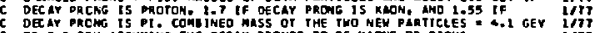

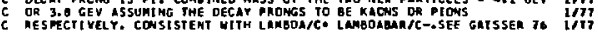

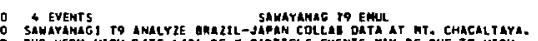

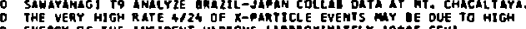

$1 / 10$
$1 / 10$
$1 / 100$
1 


\section{Data Card Listings}

For notation, see key at front of Listings.

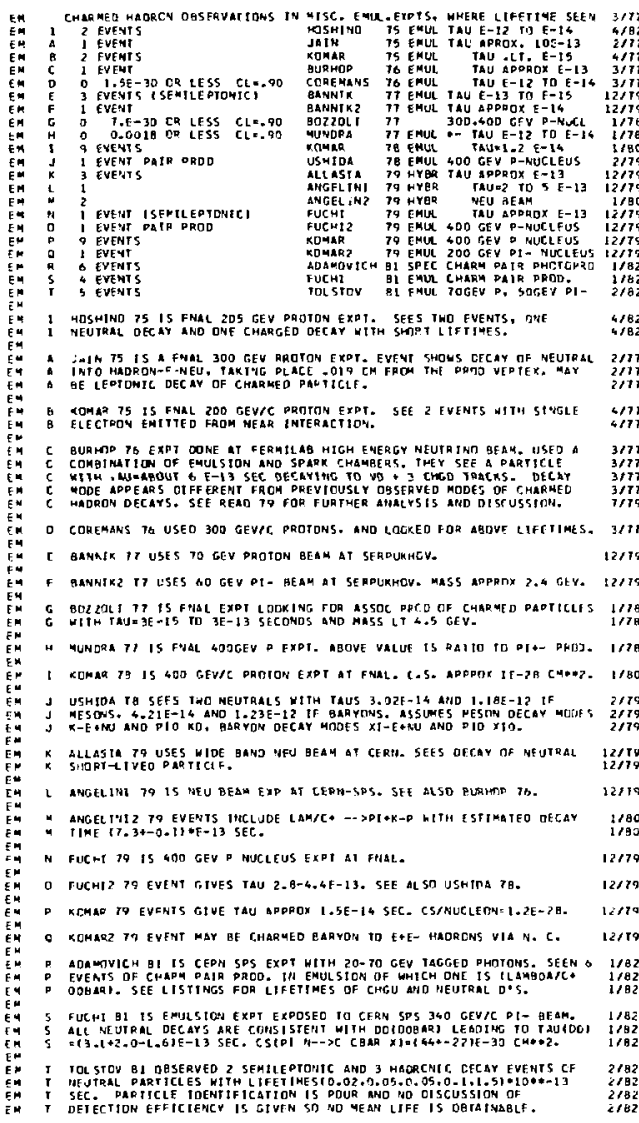

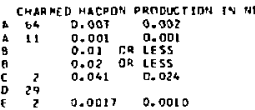

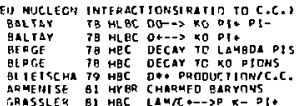

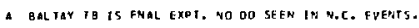

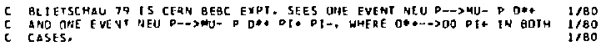

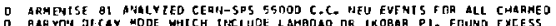

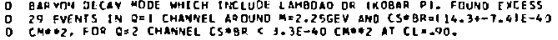

E GRISSLER BI IS CERM BEAG EXOT. SEEN 2 EVENTS INTERPQETFD AK

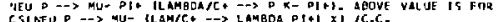

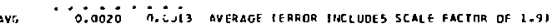

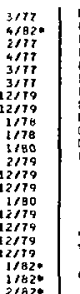

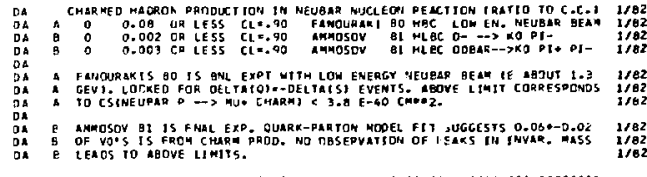

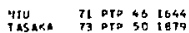

avbert of pat 35416

9ALI ALY

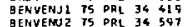

$\begin{array}{llll}\text { ALSO } 74 \text { PRL } & 34 & 597 \\ 984 & 984\end{array}$

AENUENO TS PAL 351129

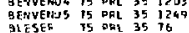

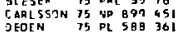

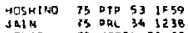

SOMAAR IS JETPL 21230

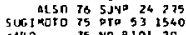

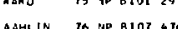

ABDL

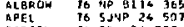

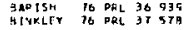

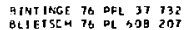

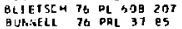

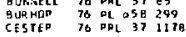

COAFHANS Po PL 650,400

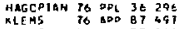

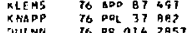

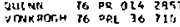

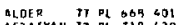

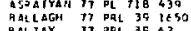

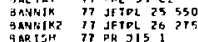

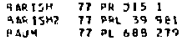

REAGE 77 PpL 39766

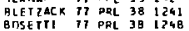

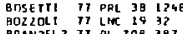

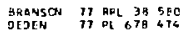

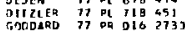

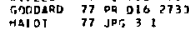

4CLDER 77 PL 0.79377

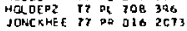

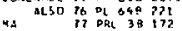

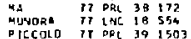

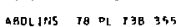

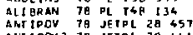

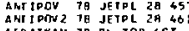

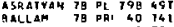

Alt

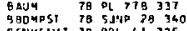

At) Ans

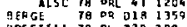

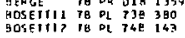

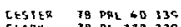

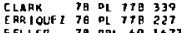

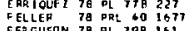

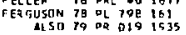

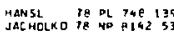

KDMAR TE JETOL 2B 45

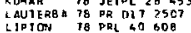

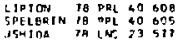

REFEFENCES FOR CHAMED HADAON SEATCHES

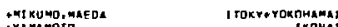

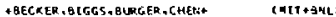

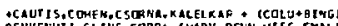
GE HVERUT I, CLINE, FORO* M MAPV, PEWN, UISC,FMLL

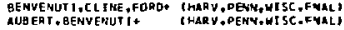

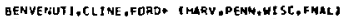
QENEENUT I,CLINE,FORD THARY ,PENN, UISC,FNAE

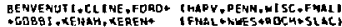

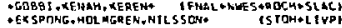

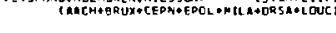

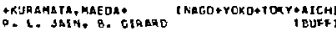
+ORLOVA, TRE TYAXOVA.CHER MYAYSKIt TLEDI

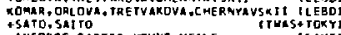

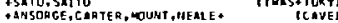

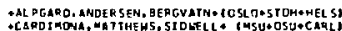

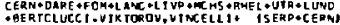

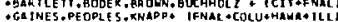

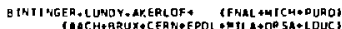

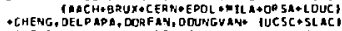

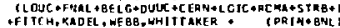

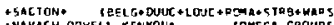
AMAVACH, ODWELL, KENYOHA TOMEGA GROUPS

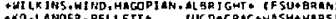

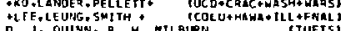
OFKY, TA MERINT, CLIEIE* THISC +LBL + EERHQHAWA

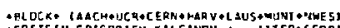
- EPSTE IH, CRIGJPIEV, RAL GAHIDV * IITEPASERP

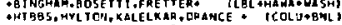

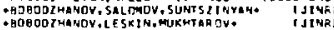

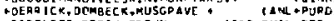

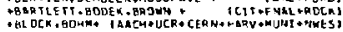

ODI H TANCA. EMANS + TFHAL SERD+TTEP, MICHA A gOYTF, FAI SSLER, GARELICK, GETIMER ISTONAL AS

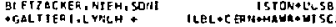

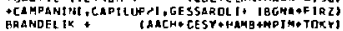

- SANOERS, SHITH, THALER, ARDER SCY TPQTNAEF I : I IACH\&BELG-CERN+EPDL + ILACLLLO+LOUC

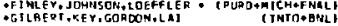
TBERKELE +CERN + HAUAI T T I SC ONS TH

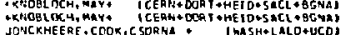

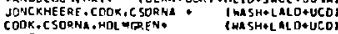

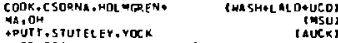

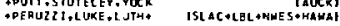
- MA TTHEWS SI DNELL - IMSU.CARL +F MaL + OSUI

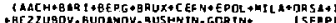

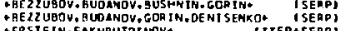

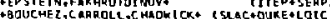

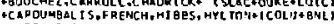

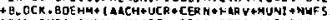

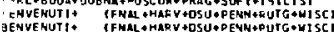

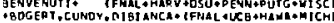

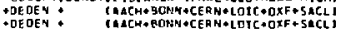

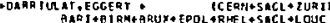

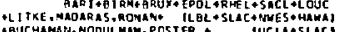

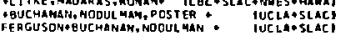

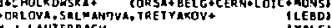

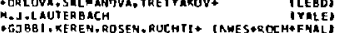

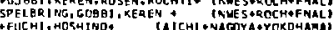

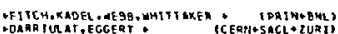

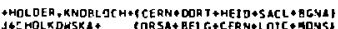

站:


Stable Particles

\section{BOTTOM HADRON, TOP HADRON SEARCHES}

Qunsit 79 मf 878287 ANEELNI 79 PL 800 S28 AFENTSE 79 PQ

BMAMUV 79 PL 810261 ARANOV $795 \mathrm{JNP} 20,622$ MAUER 79 ORL 431551 AGECE $19 \mathrm{DL} B 1 \mathrm{O}$ OS

BLIETSCH T9 DL 808108 BROUN 79 OR2 33410

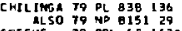
ccreus

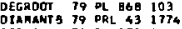
DISHAN 79 PL 858142

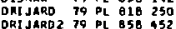

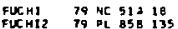
KOHARO 79 THES STS 2940 KOMARz 70 SJMP 2950

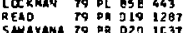
andmovic of $P(898427$

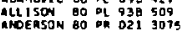

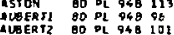
BALLACH BO DL 998423 BALLAGH2 80 PR 02156

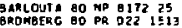

CLARK 80 PRA 45682

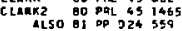

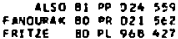
RACDUES OD OR 0211206

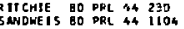
SDJKAS 80 PRL 44364

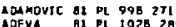
ANesor

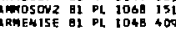

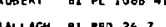

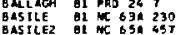

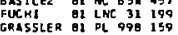
IRION BI PL 999455 SONKER \&l PL 1078264

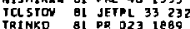

GAISSER TB OR 014 3153 ANT IPOY 99 JETPL 30 30 GIBONI in PL GSG 437 RREEAISE BO PL 948 S27
CANKA+BRUX+CERN+DUUC+LOUC+KENA+PISA+ROMA+1

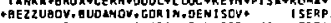

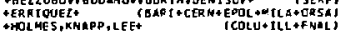
-iVANIL OV, KONYUSHKO, KOR ABLEY. ISEap)

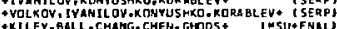

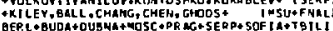

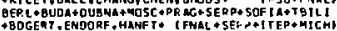
BL IETSCHAUt CACCH+BONA+CERN+MPI M+OXF!

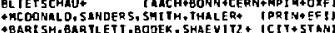
EMILIHEAROY,C LARRE

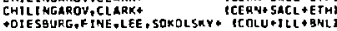

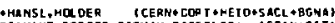

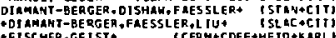

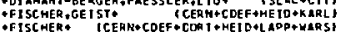

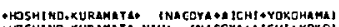

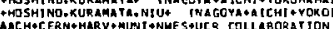

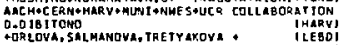

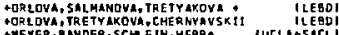

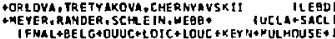
K. SAMALEEAGGI AOAMOVICH+ T PHOTOH-EMUL, OMEGA-PHOTCN COLLS.I

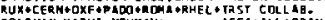
CIBDYNatGR

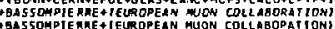
aI NGHAH: TUCB. LBL, FHAL, HAHA, HASH, WI SC,

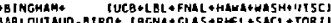

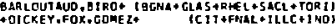
- JOHHSDH, KERTH, LOKE H* IUCB+LOL * FNAL, PA TMJ

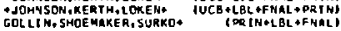

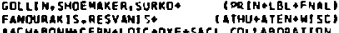
TKALELKAR, MJLLER, PLANO+ IRUIGESTEVUCCOLUI

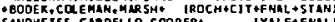

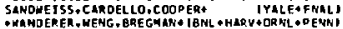

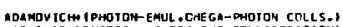

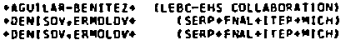

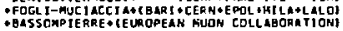

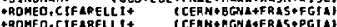

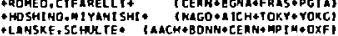

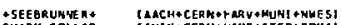

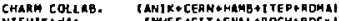

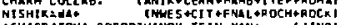

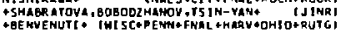
REVIEUS REFERAED TO IN DRT CARDS

T.K.GAISSER,F,HALL FN

(EART 4HISC)

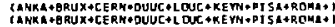

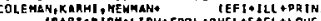

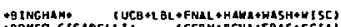

\section{BOTTOM HADRON SEARCHES}

SEE ALSD THE LISTIMGS FOR THE BtSZOO, ANO THE

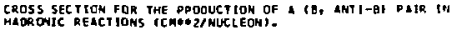

- B. E-39 OR LESS CL..90 COLEHAH EO SPEC 225 GEVPC PR- NUCA

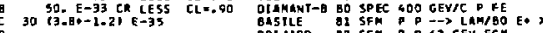
- coleman oo loci for pi- hucleon $\rightarrow$ a ebrr ahything, folloned ay

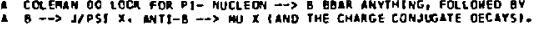

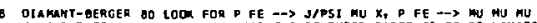

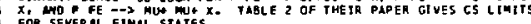
C OSTLE I ISP AT GZ GEY ECM AT THE CERN-ISR. SP GSTANDAROC DEVIATIOH PEKK IS SEEM IN THE K- PT+ OF- MASS SPECTRUTH WHEH

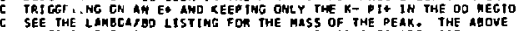

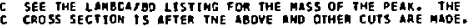

O OAI JMAO B2, WITH SAME ACCELERATOR, ENEATE, AHO OETECTOR AS BASILE

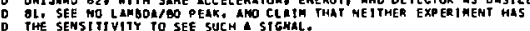

SETIVITY TO SEE SUCh A STGKAL.
Data Card Listings

For notation, see key at front of Listings.

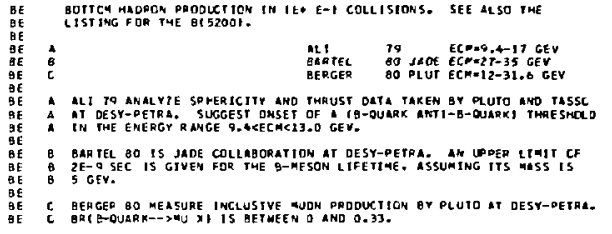

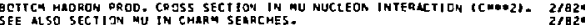

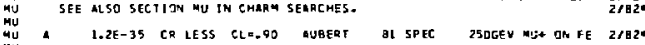

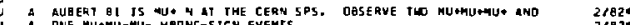

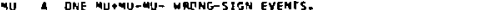

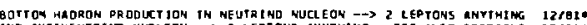
ANO RNTIYEUTRT MO MUCLEON $-\rightarrow$ 2 LEPTONS ANYTMING. SEE ALSO SECTIONS

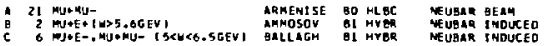

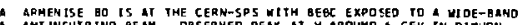

A ANTHEUTRINO BE AM- DBSERVED DEAK AT RROUHO B GEV IN DIAUDN

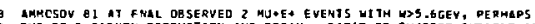
DUe Ta a-Barvon dor (2.03.3-1.6)E-3.

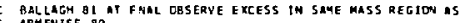

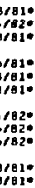
8020 $12<010$ $2 / 82^{\circ}$ $12 / 81 *$ $2 \pi z^{2}$ $2 / 320$ 21020

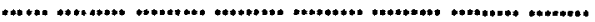
REFERENCES FOR BOTtOM HADRON SEARCHES

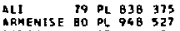

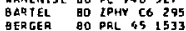

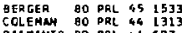

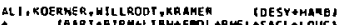

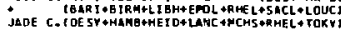

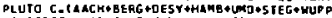

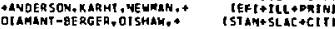

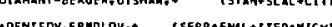

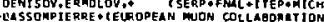

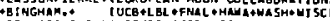

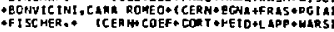

LMAOSOU BI PL 1069151

BRLACH BI PRO 24 ?

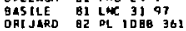

.

\section{TOP HADRON SEARCHES}

TOP HADQCN PRCDUCTION IN IE* E-I COLLISONS

DOP HADRON PRCDUCT ION
HOHE ECM=22-31.6 GEV
MONE ECME

NONE

MOWE ECM-28-31.6 GEV

F NONE ECM-12-31.6 GEV

BARTELI
BARTELL
QARAER
GERGER
GARQER
BERGER
GLATEL

19 JAOE A

0
6 pLUT

GD PLUT MU

MLL ABOVE REASUREMENTS ARE DONE AT OEST-PETRA. THE LAST COUMM

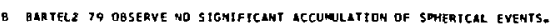

D BERGER 70 FINO RE3.8000.22 UHITH ALONG WITH SPAERICITY AMD THEUST

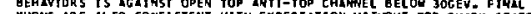

E BAPBER 80 FIND NO EYIOENCE FOR AN OPEN TDP AHZI-TOP THRESHOLO INR. G/OI* THQLST OIST. AHO INCLUST VE WUONS. ENERCY SCAN IN THE QRATE 29-94EC ANT I-TOP-CUARKI DOUMO STLTE.

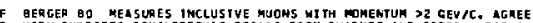

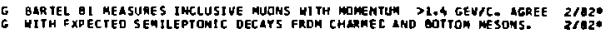

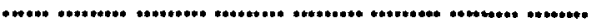
REFERENCES FOR TOP HADECH SEAMCHES

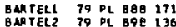

GAMBER TQ DL BSB 46

BERGER TO PL BGE 413

BERGER BO PRL is 1753

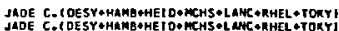

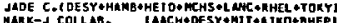

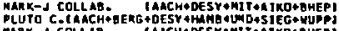

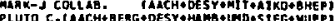

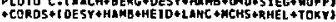

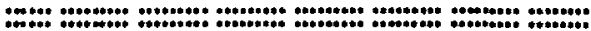

sas: \&/91: 61: 6ro1:

:/81: 
Data Card Listings

For notation, see key at front of Listings.
Stable Particles OTHER STABLE PARTICLE SEARCHES

\section{OTHER STABLE PARTICLE SEARCHES}

We collect here those searches which do not fit neatly Into one of the above search categories. These include axion searches (section $A X$ ), supersymmetric partner searches (SYM), trimuon and four-lepton production in neutrino and anti-neutrino reactions ( $T$, $F L$ ), and heavy particle searches in accelerator experiments (EE, $\mathrm{CH}, \mathrm{CS}$, D, ICH, RPII, BD) and in cosmic rays (F).

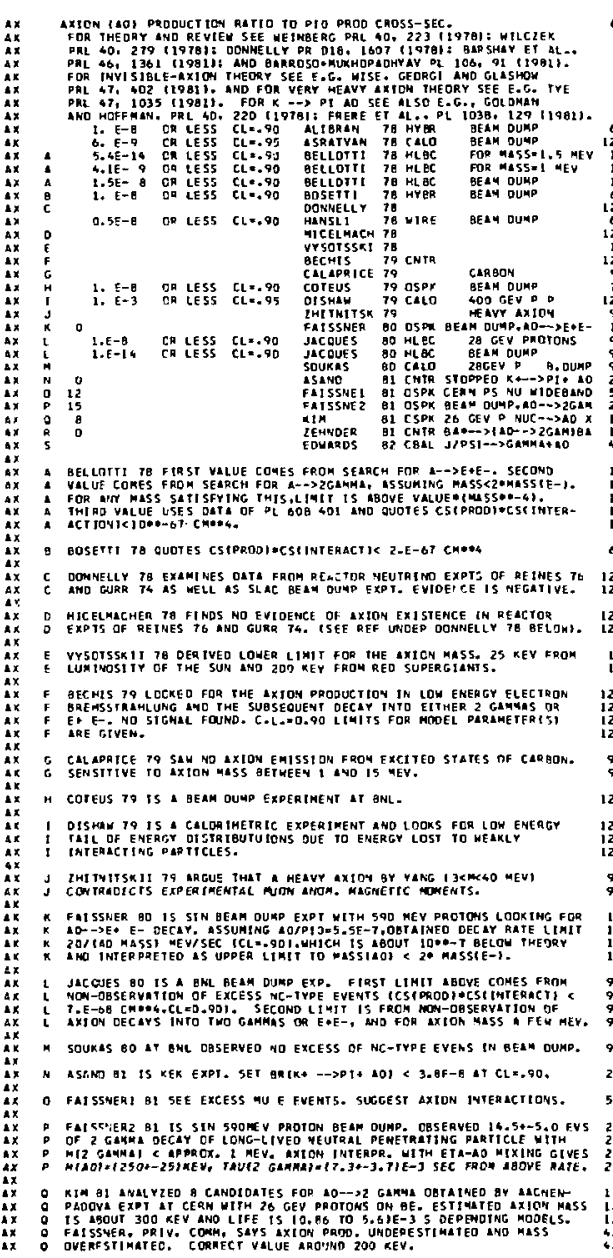

\section{$1 / 820$ \\ 11828: \\ T/82* \\ $4 / 020$ \\ $4 / 82$.}

TRIMLON FRCDUCTION IN MEUTRYNO MUCLEON THERAC MONS

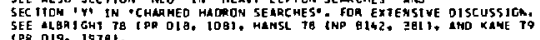

A 2 EVENTS MU- MU MU

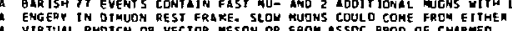

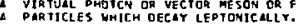

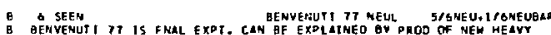

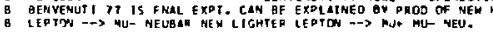

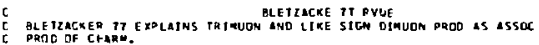

$0 \quad 3$ SEEN

RGE OER "ISPEC

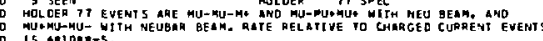

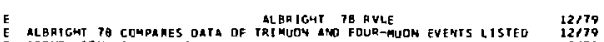

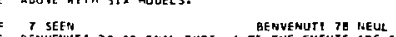

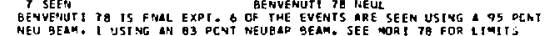

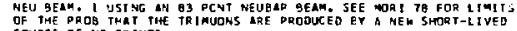

SOURCF Of MEUTRIMOS.

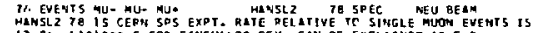

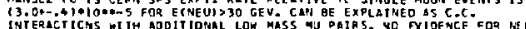
INTERACTICGS
HEAUT LEDTON.

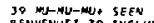

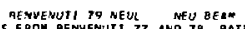

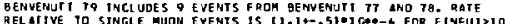

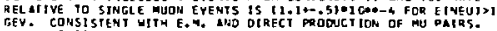

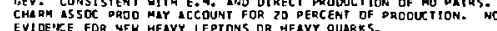

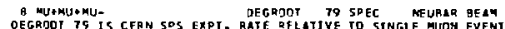

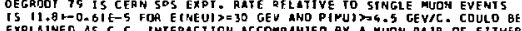

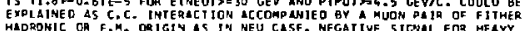
MADRONIC

FOUA-LEPTCK PR SOUCTIOH IN NEUTRIRO-NUCLEON INTERACTIGNS

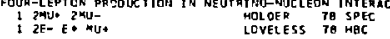

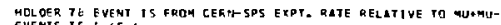

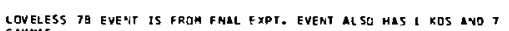
GMMAS.

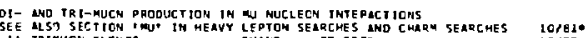
II TRIMUON EEEN TS

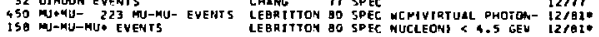

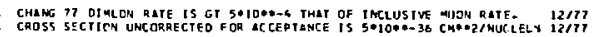

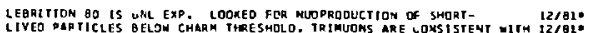

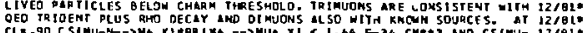

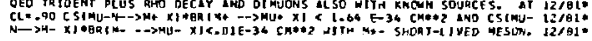

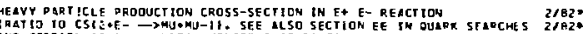

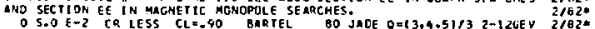

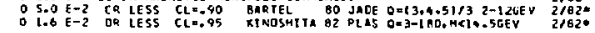

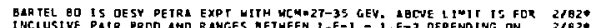

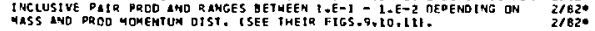

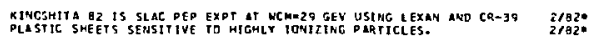

heavy particle panduction ckoss section ICMa.zi

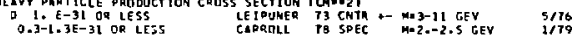

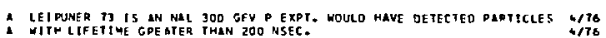

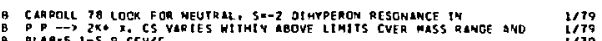


Stable Particles

\section{OTHER STABLE PARTICLE SEARCHES}

CS MEAVY PARTICLE DROOUCTION CROSS-SECTION TCHAZZMUCLEOU,

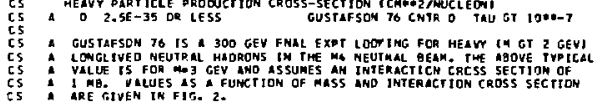

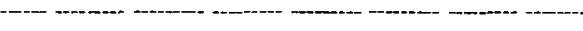

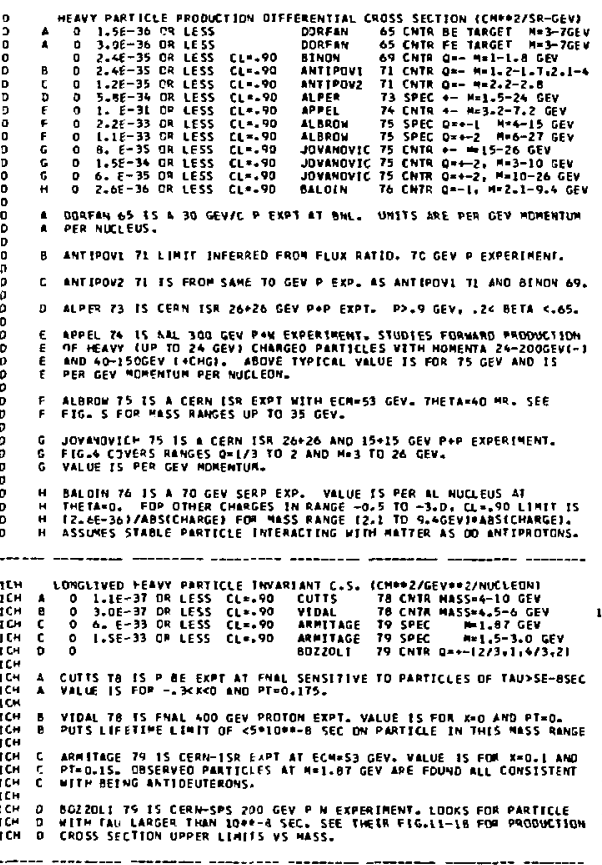

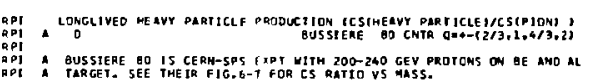

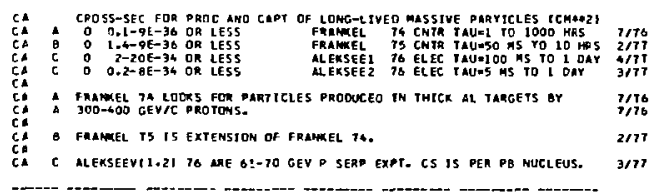

Data Card Listings

For.notation, see key at front of Listings.

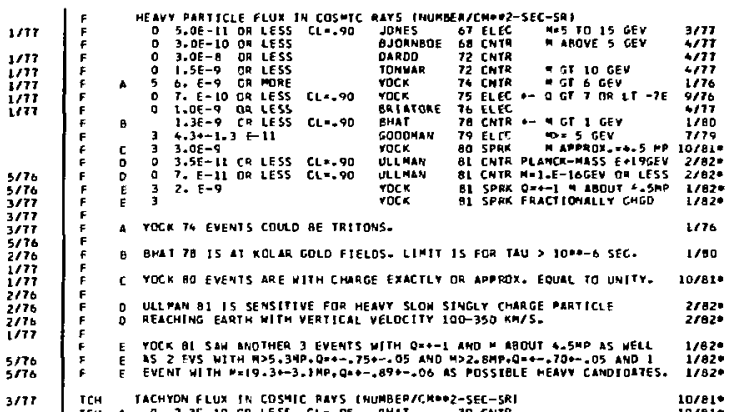

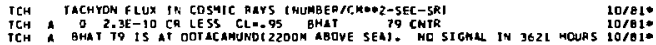

$3 / 77$

$5 / 76$

$2 / 176$ $5 / 76$

III7

$2 / 76$
$2 / 76$
$5 / 76$

$5 / 76$

$1 / 77$
$1 / 77$

$1 / 79$ 2179
$2 / 79$ 7179
7779
7199 $1 / 80$ 1180

$12 / 810$
$12 / 810$ 122820 Iow

LHEHY 18ETHEEA MU ANO E MESSESI PARTICLE MASSTUNITS-ELECTRON MASSESS

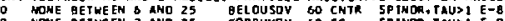

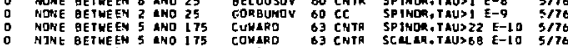

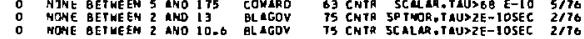

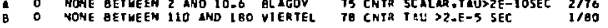

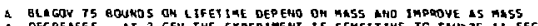
- DEC QEASES. PT 2 GEV THE EXPEEIMENT IS SEMSTTIVE TC TAUP $3 E-11$ SEC

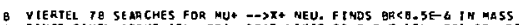

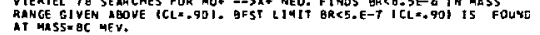

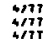
$1 / 80$ $1 / 80$
$1 / 300$
$1 / 80$

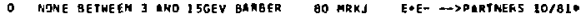

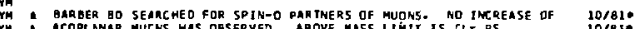

CON CDNEEHTRAT ION OF HEAYY TCHARGE+MII STABLE PAOTILLES IN MATTEA

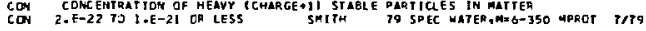

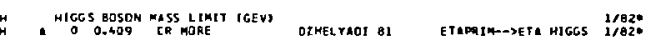

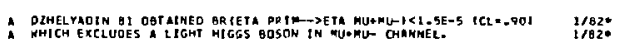

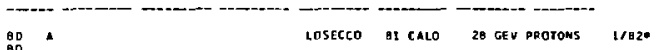
BD
$B 0$
BD

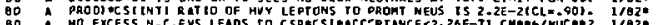
$B 0$
$B O$

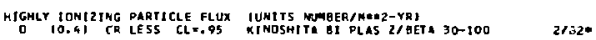

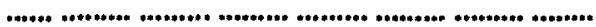




\section{Data Card Listings \\ For notation, see key at front of Listings.}

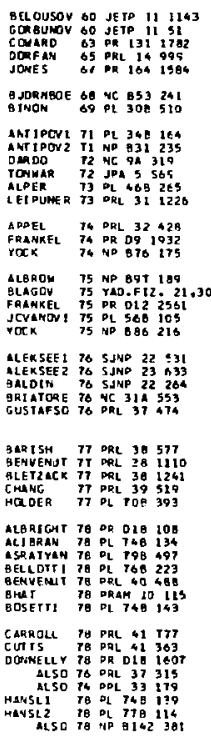

mefeaences for dTHER heM Particle searches

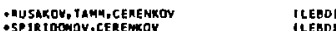

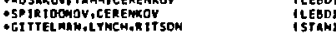

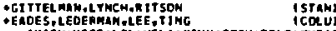

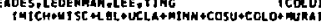

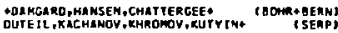

-OENISOY,ODNSKOV, GON IN, KACHAHOY. TSEAPI

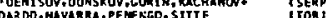

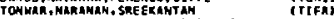

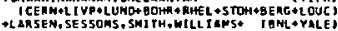

+BOUHOU1M, GAINES, LEOEMAA, MAAR+ ICOLU+FMAL)

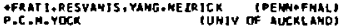

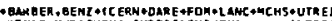
TKOMAR. HURA SHOVA, STREI SHCHIEOVA. TLEBO

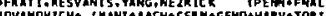

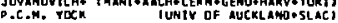

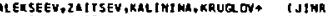

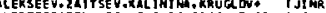

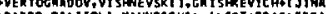

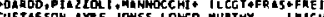

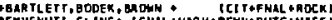
OENVE WUT, , CL INE + TFHAL + HARV + PENHORUTG+WISE OLET2ACKER, NIEH. SON? (STON+UCSO

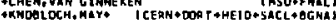

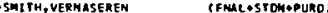
AACH+BAR L+BERC+BRUX+CEAN+EPOL +MILA+OASA. -EPSIEIN,FAXHRUTOINON* TITEPASERPI

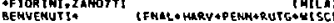

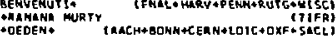

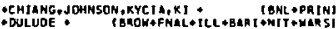

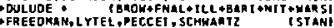
REIMES, GURR, SOBEL RETMES, GURR, SOBEL
GURR +REINES, SOQEEL

+HOL DER , KNOBLOCH E C CERA + COR T \& HE TO OS ACL + B GWA +HOLDER, KNOEL OCH ( CERN+ DORT + HE IO*S ACL + EGNA)
HANSL, HOLOE Q
HAC DER 70 iL 730105 TOOELESS 76 PL 7 B日 505 Mont 78 oRL 40432

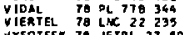
VTSOTSSK 7A JETPL 27302

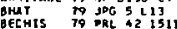

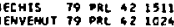

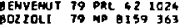

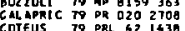
DECROOT 79 PC OSB 231 DISHAU 70 DL 858142

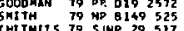

\begin{tabular}{l} 
gMBSER \\
OMRTEL PRL \\
\hline 0
\end{tabular} GUSTEL 80 ZPHA CE $2 S$

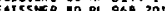

JACOUES AD PR D21 1206

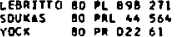

asano o1 PL 1070150

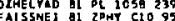

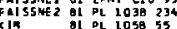
K1NOSMIT BI DK 024 . $17 \mathrm{CT}$ COSECCO BI PL 1028200

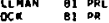
ZEHHDER OI OL 1040,94 Eculans 82 Dat 49903

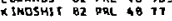

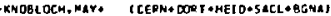

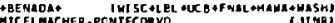

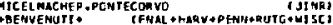
(FNAL + HARV +PENI+RUTG+ WISCI tHAHN, SCMACHER WYSOTSSXI + TINST .APPL. MATH. .USSR ACR. SCT -)

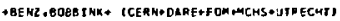
BHAT, COPALARF ISHHAH, EUPTA, TCKHAR TTIFK

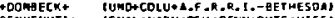
GENVEWUTI + IF NAL +HARV +OSU TPENN+PUTG+ UISCS

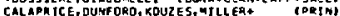

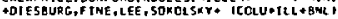

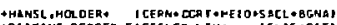

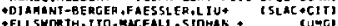

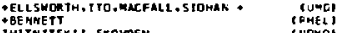
2 HI TA I TSK I T. SKO VPEN

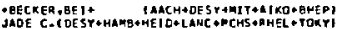

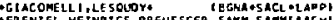

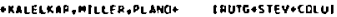
LEBR 1 TTCH. MCCELL, DELTSSTMOS* IROCH* BML OMSFI

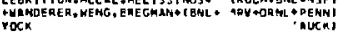

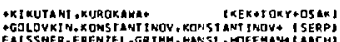

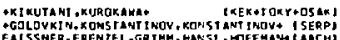

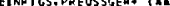
P. Q.KTM.CH.STAMM

K.XINOSHITA,D. B. DR ICE IULe?

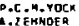

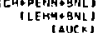

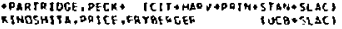


Mesons

$\pi^{ \pm}, \pi^{0}, \eta, \rho(770)$

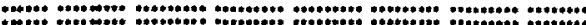

$$
\mathrm{S}=0, \quad \mathrm{C}=0 \text { MESON STATES }
$$

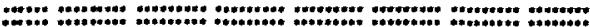
$\pi^{2}$

Q CHARCEO PIDNI140,JPG=O-I I=L

SEE STABLE PARTICLE DATA CARD LISTINGS

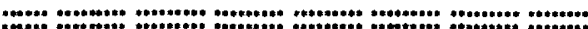
T0

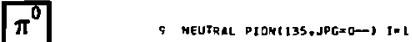

SEE STAGLE PARTICLE DATA CARD LISITINGS

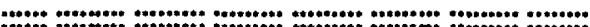

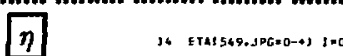

SEE STRGLE PARTICLE DATR CARD LISTIMGS

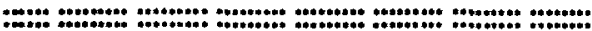

$\rho(770) \quad \therefore$ Mationo.seg $=1-+1$ t=1

\section{Bte on the $\rho^{\circ}$ Mass and Width}

Because of the broadness of the $\rho$ meson, its shape is not very well described by a Breit-wigner formula, which is a narrow-resonance approximation. Although nost experimental distributions can be well described by a celativistic Breitwigner formula with $a$ p-wave width and an additional shape parameter (PISUT 68), the resulting resonance parameters will clearly depend on this model. A consistent set of such determinations (PISUT 68, ESTABROOKS 74, BARTACUCCI 76, WTCKLUND 78, HEYN 80) yields $\pi_{\rho^{0}}=(769 \pm 3)$ MeV, $\Gamma_{\rho^{0}}=$ $(154 \pm 3)$ HeV.

At tempts have been made to determine the $\rho$ pole position in a more model-independent way (LANG 79, BCAACIK BO). It is comforting that these determinations agcee perfectly with the above nass average (LANG 79, however, finds a sonewhat smaller width).

Independent support cones from an $5 U(4)$-genecalization of the Gell-kann-Okubo mass formula, which in the 1 imit of exactly ideal mixing can be written (MONTONEN 75)

$$
\rho=\frac{2\left(\psi_{K}^{*}-\phi D^{\star}\right)+\omega\left(D^{\star}-K^{\star}\right)}{2(\psi-\phi)-\left(D^{*}-K^{*}\right)} .
$$

Since the masges of the vector mesons on the right-hand $\mathrm{side}$ have $\mathbf{a l l}$ been determined to a nuch better precision than that of the $p$, they can be
Data Card Listings

For notation, see key at front of Listings.

used to determine that the mass of the $\rho=1768 \pm$

2) Mev. The theoretical error due to non-ideal

mixing $1 \mathrm{~s}$ only of the order of $\pm 1.5 \mathrm{MeV}$.

Thus we conclude that the nass of the $\rho$ is

(769 \pm 3$) \mathrm{MeV}$, somewhat lower than quoted in the 1976-80 editions.

\section{- RHO MASS IMEYI}

WE NO LCNGE LIST S-MAYE BAEIT-NBGNEA FITS. PGAR DATA YTTH HIGH COMETNATRRIAL BACKCAOUND, MND INS IGNIFICANT DR DOUBTFUL DATA.

SEF ALSO THE MINI REVIEM ABDVE.

C CHARGEO OULV

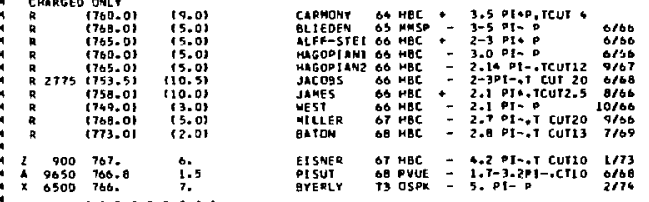

4 hug Tob.0.

No MEUTRAL CNLY

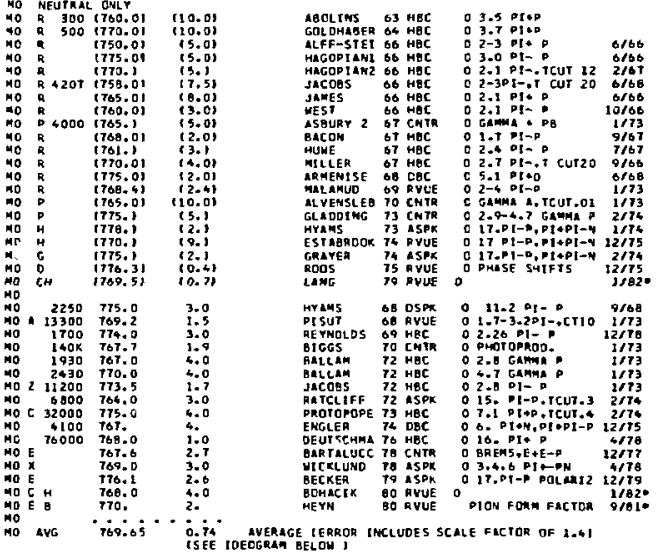

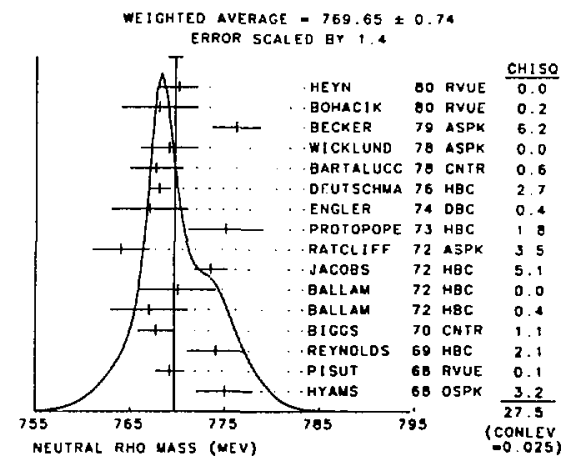




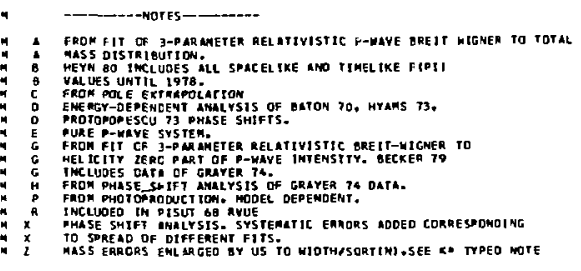
- (RHOOS - [RHOO-) MASS OTFFEREMCE INEYI

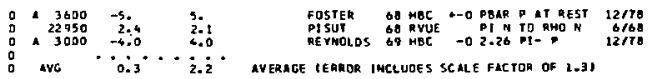

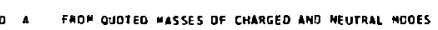

8 RHO HITH (MEV)

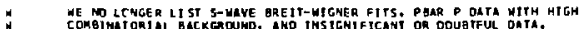
SEE EURTHER MTHI-REYTEW AQOVE.

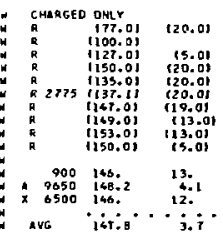

ChRMONY G6 HAC $3.5 \mathrm{Mt}+\mathrm{P}_{\text {, TCUT }}$

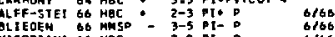
MAGOPAAN1 OS HEC = 3.0 PI- D

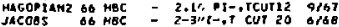

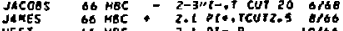

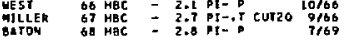
E1SMER 67 HEC - 4.2 P1-.I CUTIO $9 / 67$

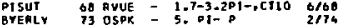

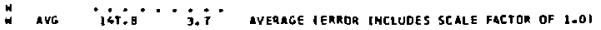
NO NEUTRAL ONLY

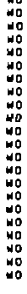

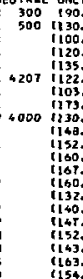

(10.01

110.04
120.01
115.01
133.01

(1) 3.01

(13.0)

(1.5.)

\{1.5.)

(16.0)

(10.0)

(5.0)

ril:)

t163.) (t.t)

$\begin{array}{rrr}20250 & 145.0 \\ 00 & 13300 & 169.0 \\ 1700 & 143-0\end{array}$

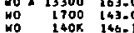

wo 22430155.0

$\begin{array}{lll}1 & 1930 & 145.0 \\ 00 & 6800 & 157.0\end{array}$

No 632000160.

4100
40 $76000 \quad 154.0$

$\begin{array}{ll}\text { WO E } & 150.9 \\ \text { NO } & 152.0 \\ \text { NO } & 162.0\end{array}$

wO $C \mathrm{CH} \quad 161.8$

$\begin{array}{lll}C H & 1+9.01 & 11.31\end{array}$

MO AVG

155. 1.0

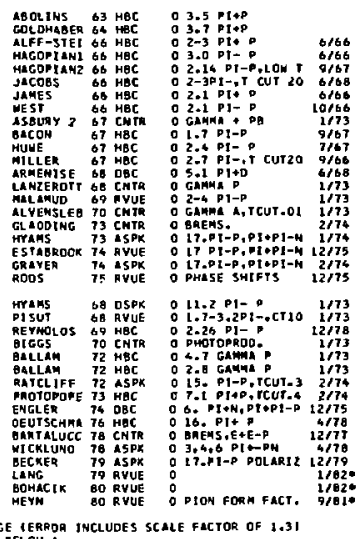

ANERACE IERRDA INCLUDES SCALE FACTOR OF 1.3 TSEe IOEOGRaM BeIOU i

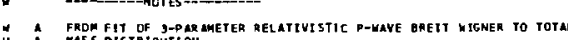

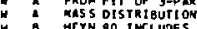

a heYh go tMCLUDES ill SPACELIKE AHO TIMELIKE FIPI

- G VALUES UNTIL 1978 .

- D ENERGY-OEPENDENT AMHLYSTS OF BAJDN 7O. HYANS 73 ,

* DOTOPOPESCU 73 PHASE SHIFTS.

- F PUAE P-WAVE SYSTEH.

W G FROM FIT OF 3-PARAHETER RELATIVISTIC BREJT-WI GNER TO

* G WELTCiTY ZERC PAHT DF - WAY

H FRCM PHASE SHIFT MALYSIS OF GMAYER 74 OAIA.

- F FOH PHOTOPRODUCTIOH. MODEZ DEMEHOENT.

- R THCLUDEO IN PITUR GQ RVUE

* PHASE SHIFT SMALYSIS. SYSTEMATIC ERRORS aDdEO CORRESDOMDING

* TO SPREAO OF OIFFERENT FTTS.
} 
Mesons

$\rho(770), \omega(783)$
Data Card Listings

For notation, see key at front of Listings.

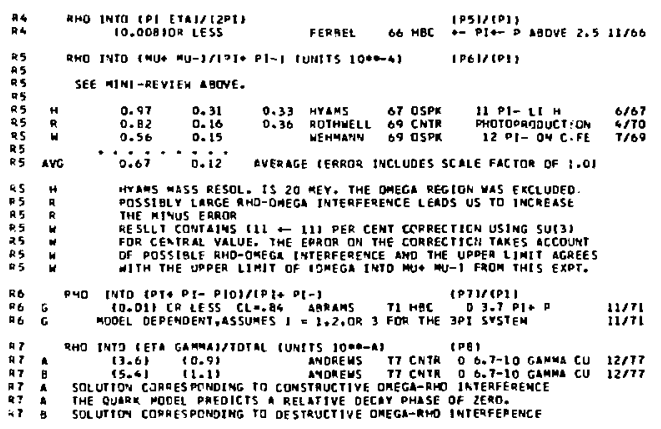

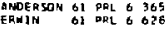

SENhEY 62 OR $126 \quad 736$

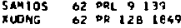

Hept IKS 63 PEL 1138 :

CHADUIC 63 OC 29515

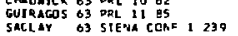

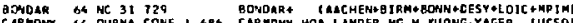

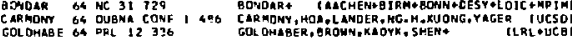
ALY 65 PL 15 A2

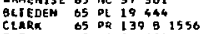
GuTar 65 NC $3930 \mathrm{~L}$ LANZEROT 65 PQL 15210 ACCENS1 of Pt 20557 ALFF-STE B6 DQ 1451072 OLIEOEH OL NC 4371 CAMRR TOC OB DR 1 166 994 CAUTSCHM $66 \mathrm{PR} 20 \mathrm{B2}$ FERSEL O6 PL 21111 FTOECARO O6 PL 23163 HAGDPIANIBS PR 145 1128 MUSON $66 \mathrm{PL} 2091$
JACOBS 66 UCRL-1687?

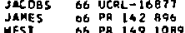
ALLES-80 OT NC 50 a 776

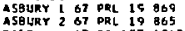
ANON OP PR L5T 1263 GMNER GT PC $25 \% 30$ ONTOH BT PL 250419 CLEAR OT OT NA B 492399 OANYSE 67 MC 51 A 901 FRENCH OT MC 52.442
HERTIEC OT PR 155 IAb HUWE 67 PL 249252

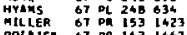
ABC COL GO NP B4 501 ARMENISE 68 NC 542999 ASTVACA $\mathrm{OB}$ PL $27 \mathrm{~B}$ 45 OATDH OA PR 174 1574

CHUNG $69 \mathrm{PQ}$ 165 1491

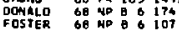
HUSON 60 DL 208200 JONES 60 PR 166 i 1405

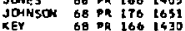
LAASA GB PR 166 1355

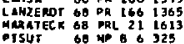

ALGUSTIL 69 OL 28 \& 508 AUSLEHDE 69 SAMP 9 O ERAF 80 D 108 2060 JUHALA G9 OR 186 140 1 1 WMALAO 69 PR LOE 146 ADTT 69 OA $176{ }_{1566}^{2061}$ REYMOLOS 69 OR 184 1424
RDOS
REFERENCES FOR RHO

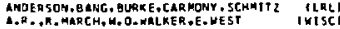

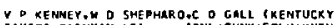
SAYI DS, GACHMAH, LEA. TBNL + CUNY +COLUTKNTY ABCL INS, L ANDER, MEKLHOP, NGUYEN, YAGER IUCSO

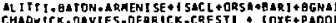
CHLDFICK. DAVIES. DEAKICK. CRESTI + TOXF+PADO

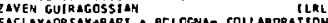
ALYER,CRITTENDEN, HARTIN, RHDDE + TINOTANAY CERM MISSING MASS SPECTROMETER GROUP ICENN

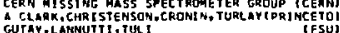
GU RAY. LARNUTTI, TUL I

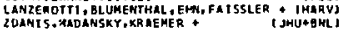
ACCENS 1, ALLES- GOAELL L, FRENCH, FRISK + ICERNI ALFF - S JE 1MEERGER, A ER LEY BRUCGER O C C OL UT RUT G OFR AMZ INI, LUT JENS, SE VER INS, T TCKO+CCOUUMGIA CER MISSIMG MASS SPECTROMETEA GADUD ICERM

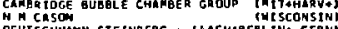
OEUT SC HHANN, STEINBEPG - IAACH4BERLIN+ CERH FEREEL
GeH fIOECARO, POIRIEF ,P SCHI AYON (ADCHE STER] MAGOPI AN. SELOVE , ALITTI, OAT ON* I DENH SACLAY

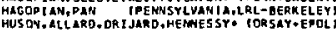

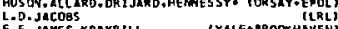

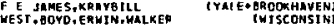
LLLES-BDAELLL, FRENCH,FRISK.+ [CENH+ GOHN] - BECKEP + BERTRMM+JDOS + JORDAN* COESY+COLU

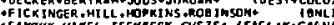

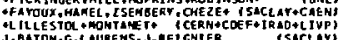

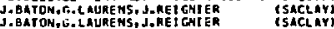

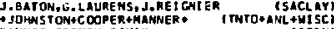

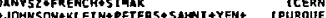

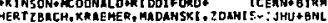

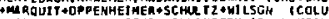

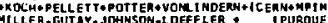

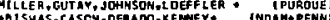
ACHEH+ DERLLH+CERN COLLHORATIONO

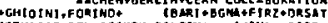

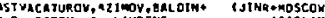

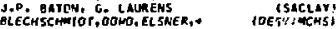

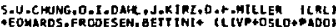

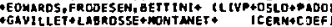

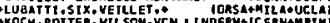
-POIRIER, B ISMAS,GUTAV. INOAMOPURDeSLAC - CASON+B]SMAS+DERADDOGROVESA TMOTKEOANE

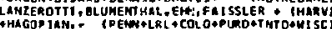

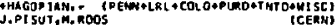
- BI ZOT + BUONAHAISSINSK + L LALANEE+ CORSN-1

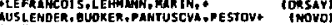

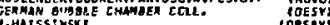

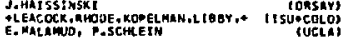

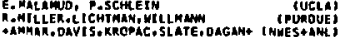

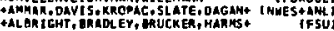

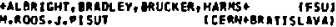

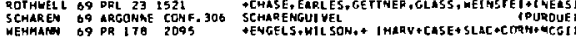
ALVENSLE TO PAL 24786 BATON TO $D$ D 335528

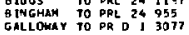
ABRAMS IJ OR O 4653

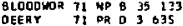
AnTLLOH T2 PL 30.555

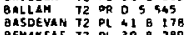
ORIVER TZ No a 3 a $^{2}$ EISENBER 72 PQ OD 515 GRAYER T2 PHIL+CONF.

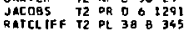
TAK AHASH T2 PQ OO 1260 BYERLY T3 DR D 7637 CHARLESW T3 NP B 65253 HYAHS 73 MP 9 O4 134

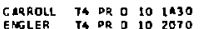

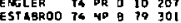
COLOI 74 PRL 331450

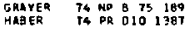

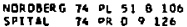

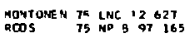
DEUTSCHA TO YP B 103426 anOREWS TT PRL 32 lse

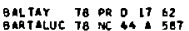

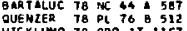

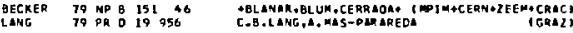

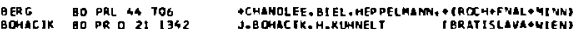
BOHACIK
MEYH
BO 2 PHY 211342

Con $\omega(783)$ I DMEGAT 7B3,JPGEY--1 $1=0$

$$
1 \text { OMEGH MASS IMEV) }
$$

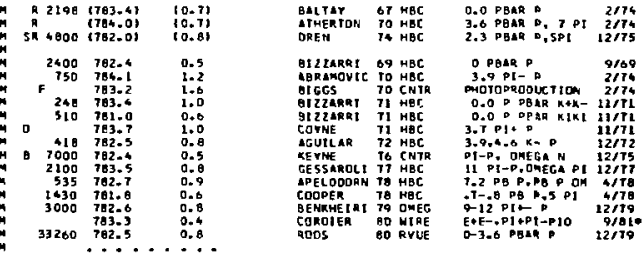

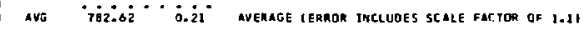

H O DQSERVE OY THRESHOLO-CROSS1NG TECHNI OUE. MLSS RESCL.. 4. B MEV FUHE

- O FRD BEST-RESOLUT IOW SAMPLE OF COTHE TI

- F ASSLATHG checa HITUH 12.6 MEV.

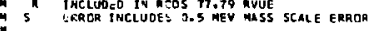

\section{OAEGa FULL WIOin tMEV}

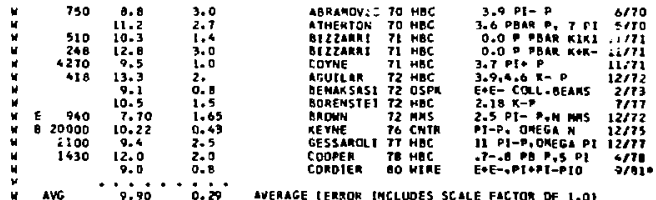

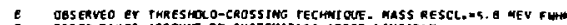

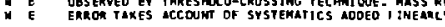


I DUEGa PARTIAL dECAY wODES

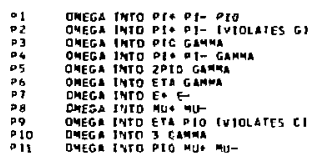

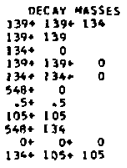

\section{EITTED PARTLAL DECAY MODE BRANCHIIG FRACTIONS}

Thic matra below in deriwed from the ex ror matrux for the filled partial decal muete bianchurg fractions. P, as follows: The diagonal etements are $F$, 6P, where

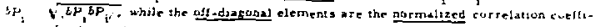

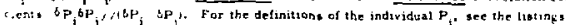
thate. orty those $P$, appearing in the matrix are ancumed in the fit to be nanzera and are this constrained io add iot.

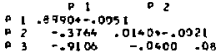

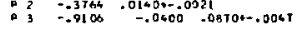

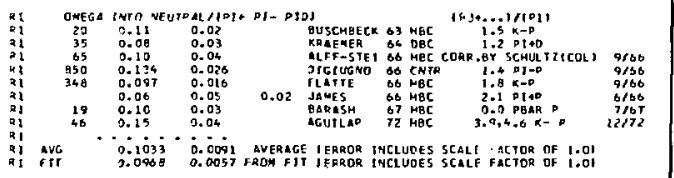

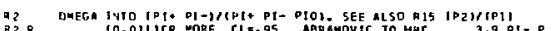

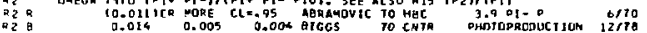

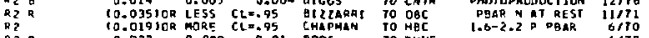

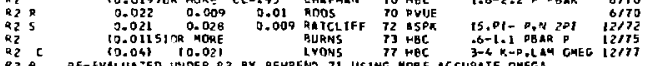

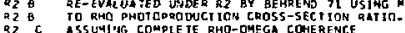

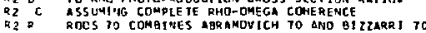

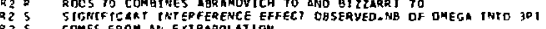

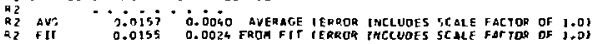

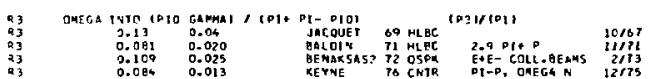

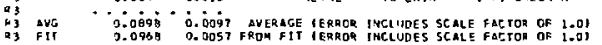

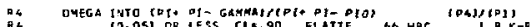

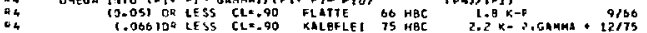

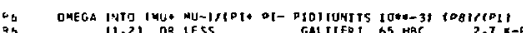

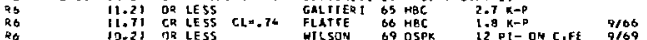

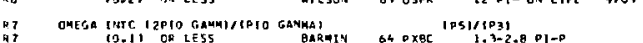

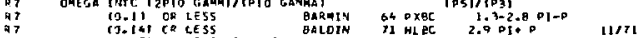

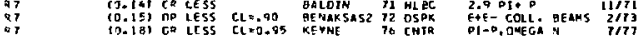

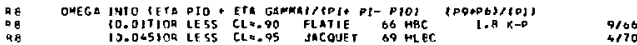

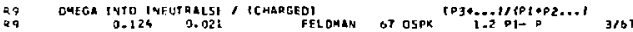

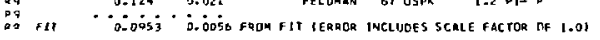

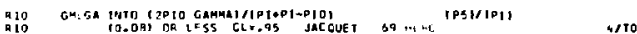

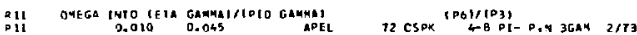

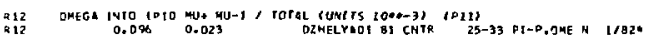

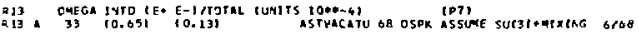

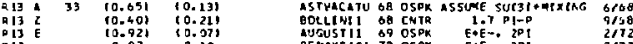

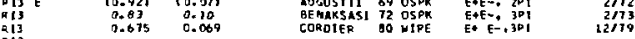

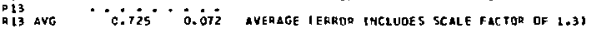

R21 A NOS RESCLVEO FROM RHO DECAY. ERROP STATISTICAL OHL."

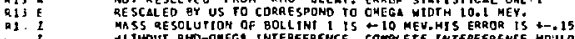

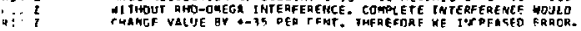

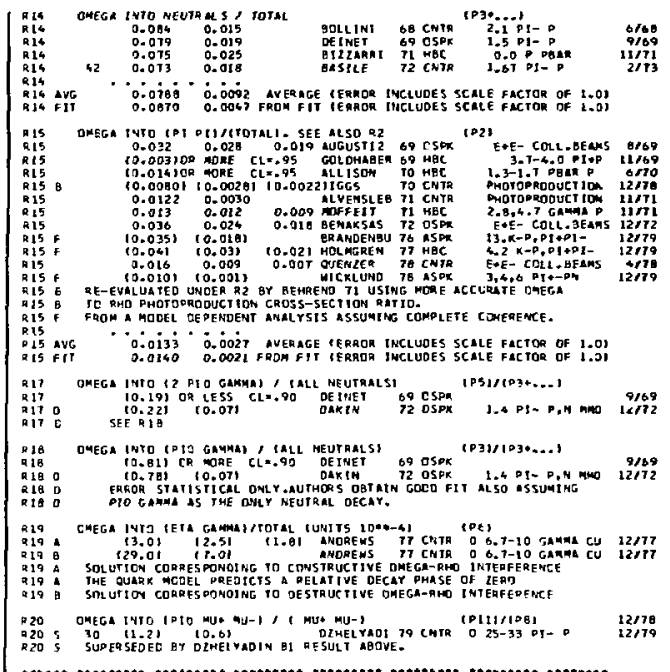

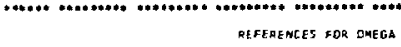

Mat52 IC D1 PQL $; 179$

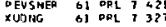

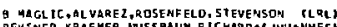

MIFF 62 PRT 9325

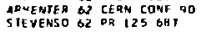

NGGTEA HUS KUOAG, GER RLO R L WNCH RLRL

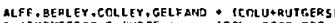

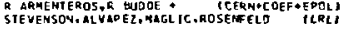

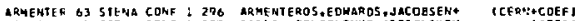

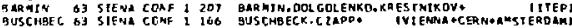

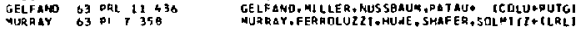

BAR IIN BL JETD $18 \quad 1299$

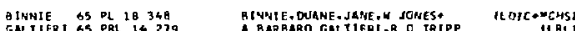

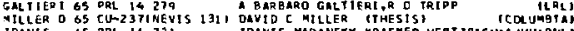

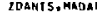

ALFF-5TE of PR 1651672

DIGTUTEO
FLATE
JWES

ALF F-STE TNBERGEQ. BERLEY. gPUGGER+ICOL U+ RUTGS

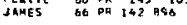

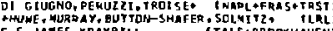

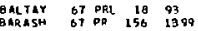

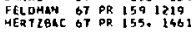

ASTIVACAT 68 PL 27 B 45

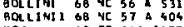

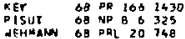

AuECSYIL 69 PL 26 \& 2013

AETL2ARB I O9 4P \& 14169

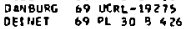

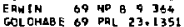

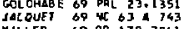

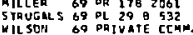

ABRAMOUI 70 NP $\mathrm{B} 20 \quad 209$

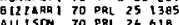

A THERTON 70 NP $\mathrm{E} 248221$

B $16 G 5$
CASON 70

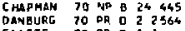

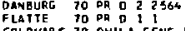

GOLOHABE TO PMILA,CCNE. P.

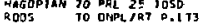

MGALAS 71 PR O 4653

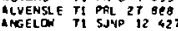

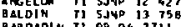

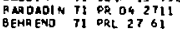

BIZZARR: 71 NA B 27 IAO

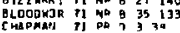

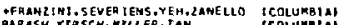

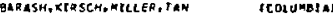

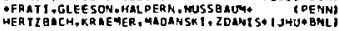

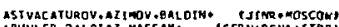

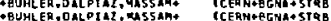

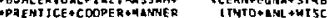

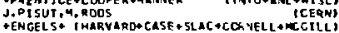

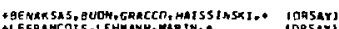

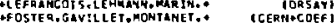

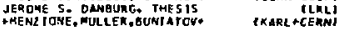
- MALKER, GOSHAM, MEI NBEFC IMTSE PRRINGYAYOI

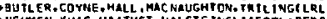
$R_{0}$ MILLER L L ICHYKAN, HILLYANN BICHARO WISSON ISEE aLSO PR 179 20951 (HIRVI

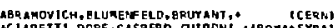

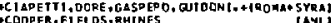
-BCATR, CELNIKER, DCAINED, TRENCH* IECRHE IDN

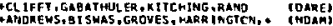

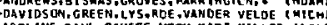

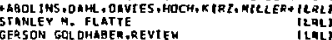

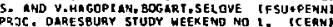

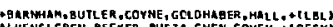

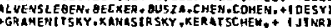
TVERGAKOW, TREGUKHOYSKY, SHI SHEY "ITEPI

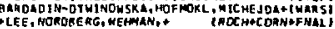

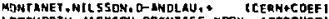

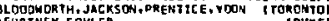


Mesons

$\omega(783), \eta(958)$
Data Card Listings

For notation, see key at front of Listings.

\begin{tabular}{|c|c|}
\hline $\begin{array}{l}\text { COYME } \\
\text { FIELOS } \\
\text { MAITHENS } \\
\text { MOFFEIT }\end{array}$ & 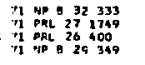 \\
\hline 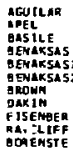 & 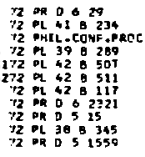 \\
\hline ILANG & 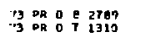 \\
\hline 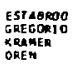 & 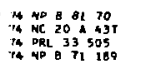 \\
\hline 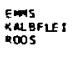 & 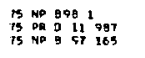 \\
\hline $\begin{array}{l}\text { GRANDEWB } \\
\text { KEYRE } \\
\text { ALSO }\end{array}$ & 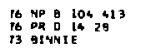 \\
\hline 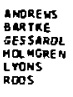 & 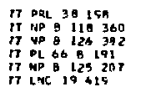 \\
\hline 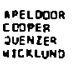 & 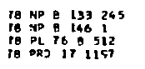 \\
\hline $\begin{array}{l}\text { BENKMEIR } \\
\text { OZHELVLD }\end{array}$ & $\begin{array}{lllll}79 & \text { NP } & 9 & 250 & 268 \\
19 & 01 & \text { on } & 8 & 143\end{array}$ \\
\hline $\begin{array}{l}\text { COPD:ER } \\
0005\end{array}$ & $\begin{array}{llll}50 & N P & 172 & 13 \\
30 & L^{14 C} & 27 & 321\end{array}$ \\
\hline
\end{tabular}

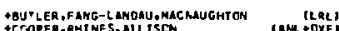

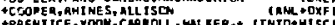

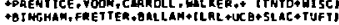

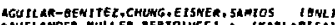

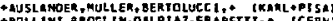

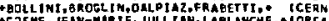
TSA

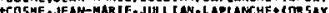

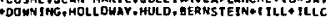
- HA USER, KRE TSLEK, MI SCHKE TPR HCETON

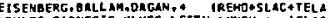

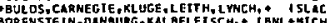

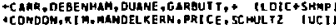

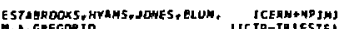

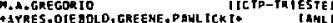

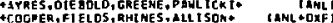

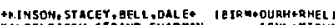

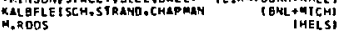

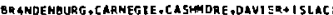

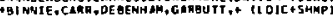

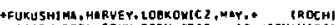

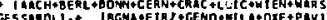
- J S

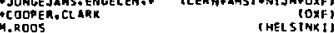

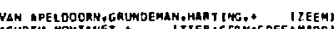
AGUR TU. MOHTANET.

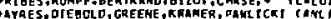
GENKHE IRI + EI SEMSTEIH, + EPOL +CER N+CDEF FLALOI

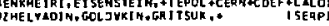
tDELCEURT, ESCHSTRUTH,FULDA., ILALOI

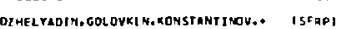

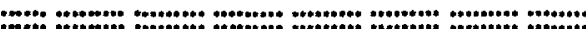
$\eta(958) \quad 2$ EN PRIAf $1958.905=0-+1$ 100

Note on the $\mathrm{J}^{\mathrm{P}}$ Assignment of $\mathrm{n}^{\prime}(958)$

From the Dalitz plot analyses of the $n^{\prime}+\pi \pi n$ and $\eta^{\prime} \rightarrow \pi^{+} \pi^{-} \gamma$ decays and from the observation of an $n^{\prime}+\gamma \gamma$ decay mode, all assignments except $\mathrm{J}^{\mathrm{PC}}=0^{-+}$and $2^{-+}$are excluded. The Dalitz plot: analyses favor spin 0 , but cannot rule out spin 2 . The indication of anisotropy in the decay of very forward-produced $n^{\prime}$ (KALBFIEISCH 73) has not been confirmed by BALTAY 74, thus again favoring spin 0 , but still not ruling out spin 2 (LEDNICKY 77).

Two recent analyses, however, seem to have

finally established the spin 0 assignment of the ' $\mathrm{l}$ '.

CERRADA 77 perform a partial-wave analysis of the $\Pi \pi \pi$ system produced in the reaction $\mathrm{K}_{\mathrm{p}}^{-} \rightarrow \mathrm{n}^{\prime} \Lambda$, taking into account the $\eta^{\prime}$ and $\Lambda$ joint decay angular correlations. They conclude that $\mathrm{J}^{\mathrm{P}}$ is unambigh ously $0^{-}$(see also DElaguila 77).

hoUSSARIE 77 analyze a large sample of events from the reaction $\pi^{-} p+\eta^{\prime} n$ at beam momenta just above threshold. They verify that the $n^{\prime}$ is produced in a relative s-wave state, and thus the Adair condition is satisfied by their total sample of some 1800 events. The decay angular distribution of the $n^{\prime}$ is consistent with isotropy, and thus ROUSSARIE 77 conclude that the spin cannot be 2 .

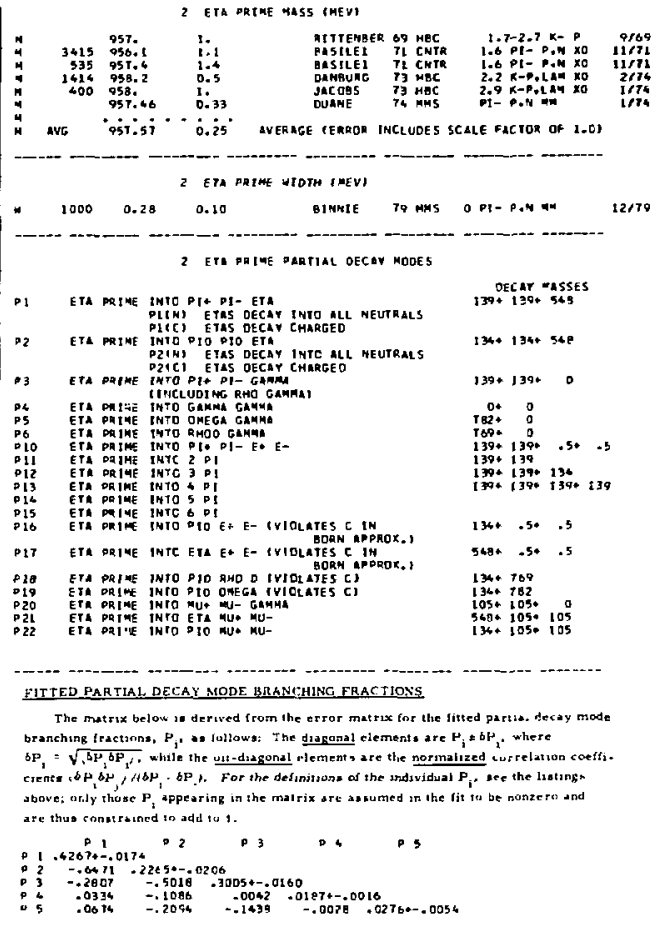

Note on $n^{\prime}(958)$ Branching Fractions

In our calculation of the branching frartions of the $\eta^{\prime}(958)$, we use the decay modes $\eta \pi \pi$ (including $\eta \pi^{0} \pi^{\circ}$ ), $\rho^{0} Y, \omega Y$, and $\gamma Y$. It is assumed that the rate $\eta+$ neutrals is 71.08 .

In the fit we do not use the constraint

$$
R=\frac{\Gamma\left(\eta^{1}+\pi \pi^{+} \pi^{-}\right)}{\Gamma\left(n^{1}+n \pi^{0} \pi^{0}\right)}=2
$$

from I-spin conservation. The result of the fit is in agreement with it: $R=1.8 \pm 0.2$.

$$
5 \text { ETL PRTME PARTILL WIOTHS IREVI }
$$

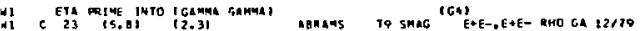

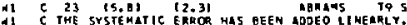

\section{ETA PRIME BRANCHISG OATIOS}

SEE MIMI-REVIEM hEOVE.

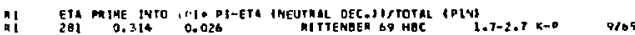
Ri
aI
AI FIT 


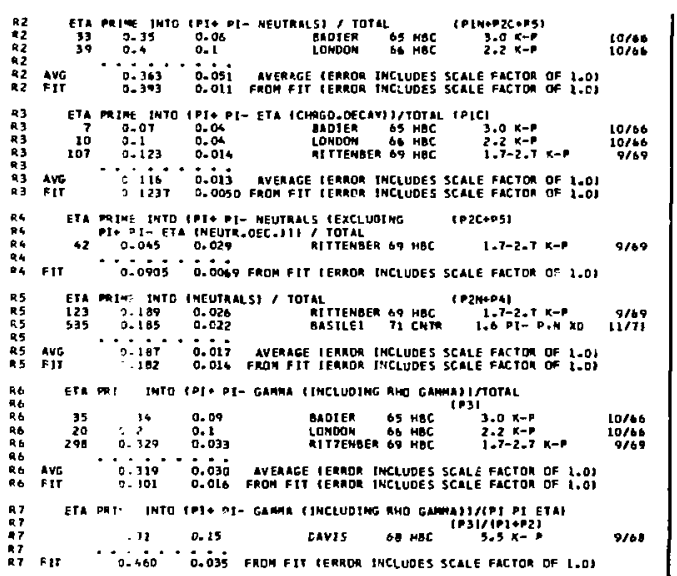

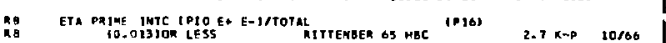

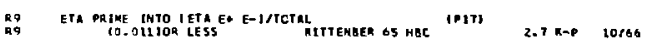

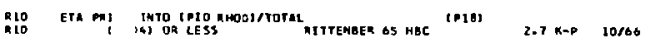

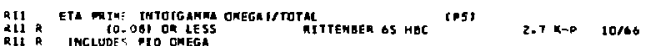
R11 R IACLUDES MIO CMEGA

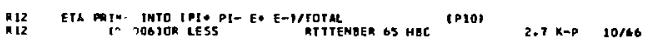

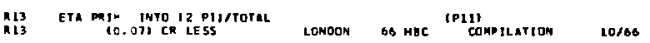

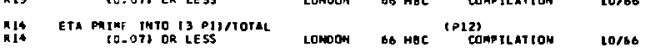

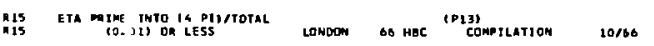

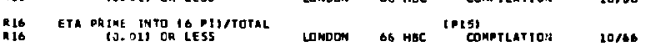

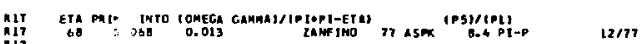

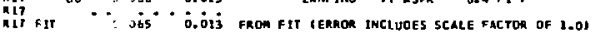

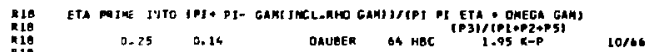

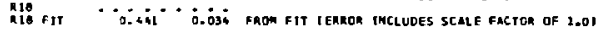

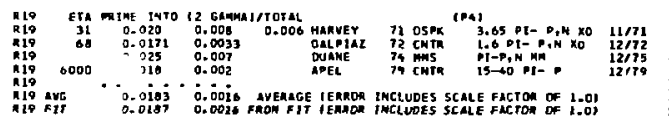

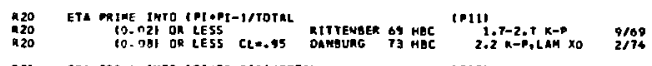

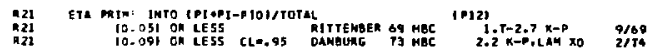

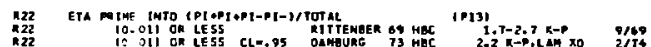

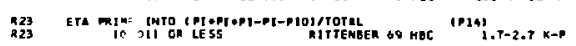

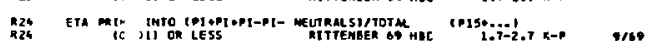

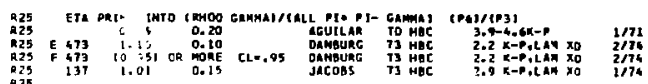

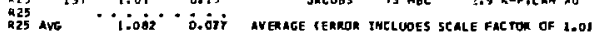
Q25 E EOUIULENT STRTENENTS

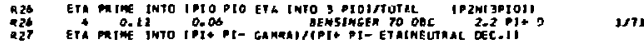

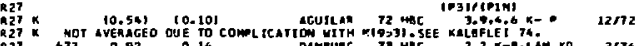

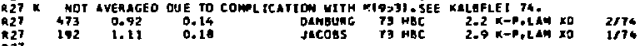

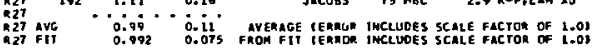

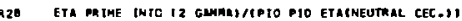

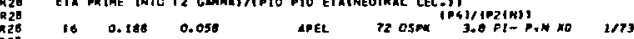

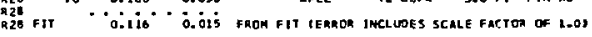

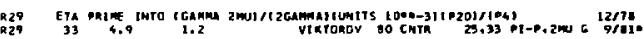

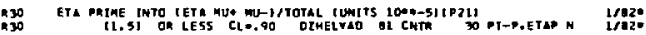

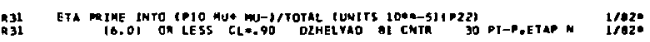

2 ETA PRIME C-MOHCOYSERVIHG DEC EEL UTEO TEXT SECTION $\mathrm{VIC}$

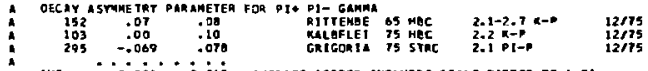

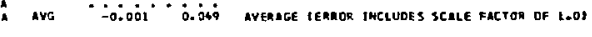

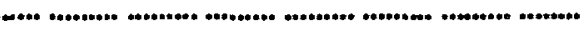

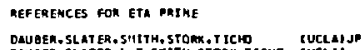

DANBER GH PRL 13 ats

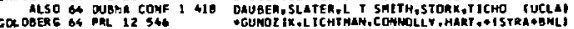

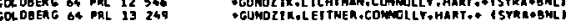

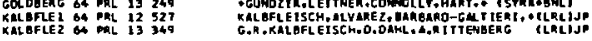
BaDier 65 Q 217337 RIEHLLE 65 PL $19,439$. RTTERAE 65 PRL 13536
TALLLIHC O5 PL 19427

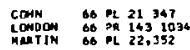
3 MREAMO- 68 PRL 20369

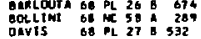

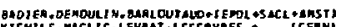

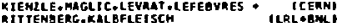

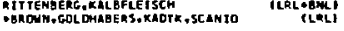

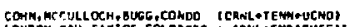

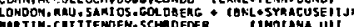

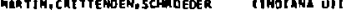

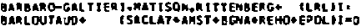

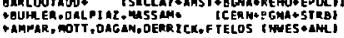

OUFEY 69 PL 29.005

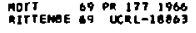

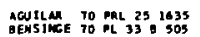
BMOAOIN 71 PA D4 2711 OASILEI 71 NC: 3 A 371

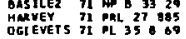
ADILAR 72 pm Do6 29

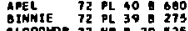

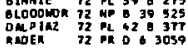
OWBunc 73 PR O 3744 JACOIS 73 FE D 10

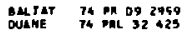
OUAHE 74 VAL 32425 GAIGonta is me 091232

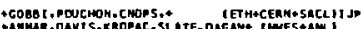

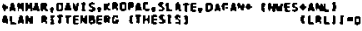

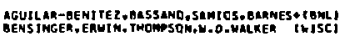

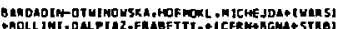
OLL

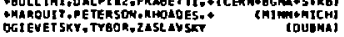
GGUTLAR-GEMI TEZ.CHWHE, EISHER , SANIOS TOHLI

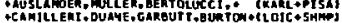

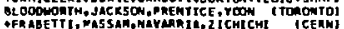

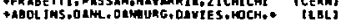
WALAFLEISCH.BORESTEIM,CHAOHAM,OCBML+NTCHJ JP

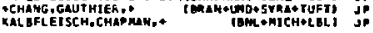

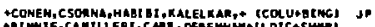

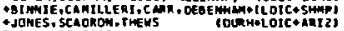

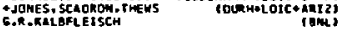
CERAR $n$ me $12611 \%$ DELAGUIL I7 Th al6 2033 , tEontCEY in EZ10521.22.23

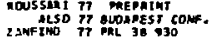

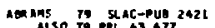

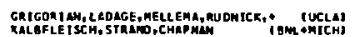

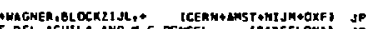
F.OEL AGUILA AND N.G.OCWCEL IBAMCELOMRI IO

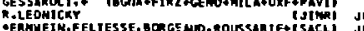

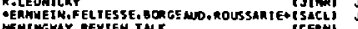

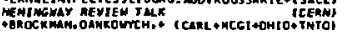

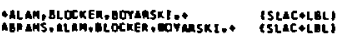

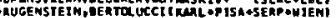

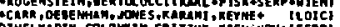

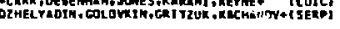

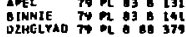
VTK TOMOV LO SJME $32 \leqslant 20$

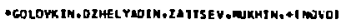
OZHEL YAOTH, GOLOVMIN, MONSTANTINOY," ISERPI 
$S^{\prime}(975)$

3 S*1875.JPG=04+1 $1=0$

UMOER THIS ENTRY ME ITS PARANETERS OF THE POLE IN THE
ISOSC MAA S MAVE. FOR A MINI-REYTEM SEE UNDER EPSTLON. FOA EARLY WOAK USYHG BREIT-WIGHER OR SCATTER 1H'

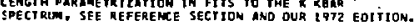

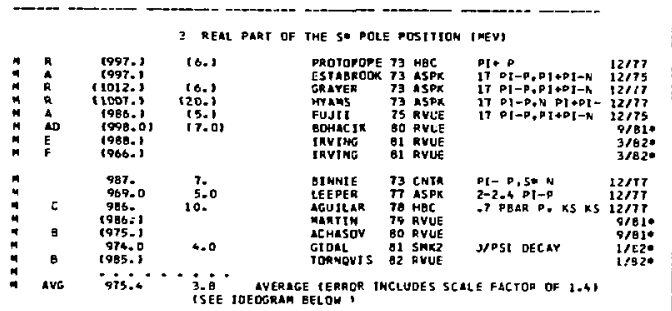

WEIGHTED AVERAGE $=9: 5.4 \pm 3.8$ ERROR SCALEO BY 14

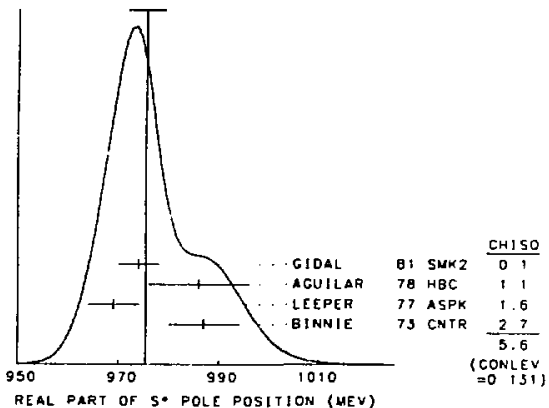

M I FRON STHGLE CHAWEL FIT TO HYAMS 73 DATA.

COUPLE CHEMMEL AMAL YSIS WITH FINITE W1OTH CORRECTIJONS. SEE INIAEV POLE DOSI TIORS FHOM ALMOS TODEL-INOE PENOENT PARAMETR IZATION FRON CODLEO CHANHEL FIT TO HYAMS 33 AND PROTOFODESCO

TNELASTICTTY WND TO THE KS KS INYARIANT HASS

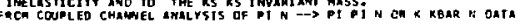
FRON GRAYER 73 AH COHEN DO

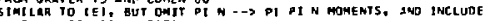
Inction 3 NEGATIVE JMAG. PAAT OF THE SA POLE POSITION IMEVI
10.1

121.1

(15.) 15.1

(19.0)

15.0 50.0

70.100

14.00
1260.1

i6.3 - i,

ACR SE MOTES UNDER AEAL PALT

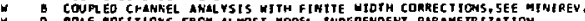

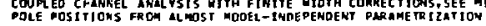

(19.1 19.1
3 So DARTIAL JeCAY moOES

PI SE INTO K NGLA

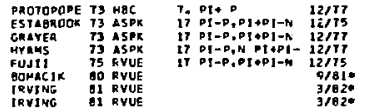

BINATE 73 CNTR PI- P.S* id $12 / 7$

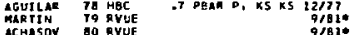

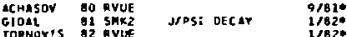

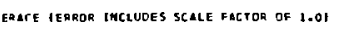

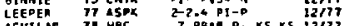

T) Basuratig atrios

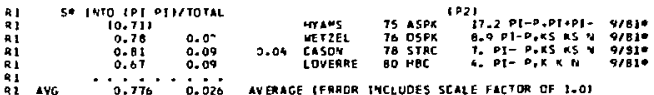

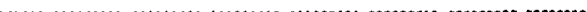

HANG BI JETP 13323 HANG TSU-TSENG, VETSLLER, VRANA.* IINRI

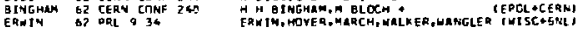

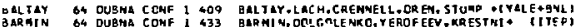

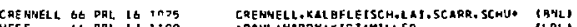

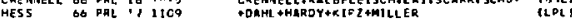

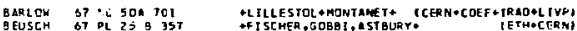

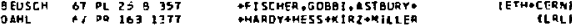

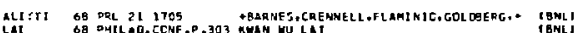

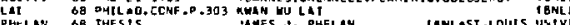

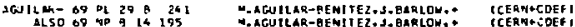

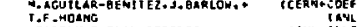

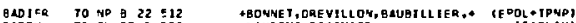

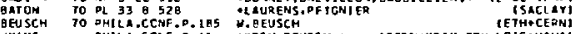

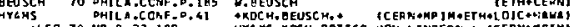

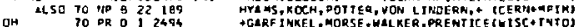

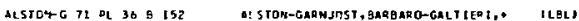

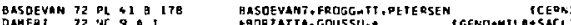

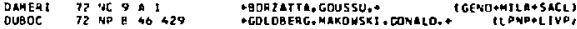

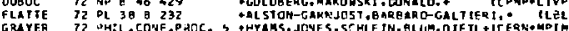

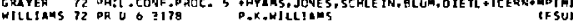

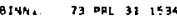

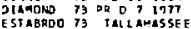

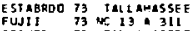

GRATER T3 TALLAHISSEE

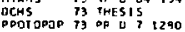

GREYER 7C IP E 75189

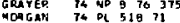

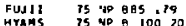

MORGAN T5 ARGONSE CON
PARLICKI 75 OR DLE E?I

aAANOEB 76 NP 8106413

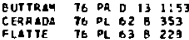

FLATFE 76 PL B3 8229

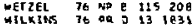

FROGAIT 77 NO $\mathrm{B} 129 \mathrm{99}$

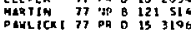

agilan 7g NPE 140 3

BALAND 79 NP ${ }^{2}$ ?4D 22

ACHASON 79 PL 980307

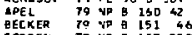

$\begin{array}{llllll}\text { AECKER } 7 \% & \text { ND } & 151 & 46 \\ \text { COROEN } 79 & \text { ND } & 157 & 250\end{array}$

ESTABROD 79 PQ D 19267

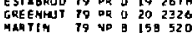

MARTIM 79 VP
DOL Y 158520
79

ACHASOV BO SJVP 32560

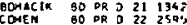

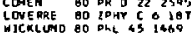

ACHASOY 61 DL 102 a 196

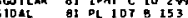

TEVING BI TPHY C 1045

ROUSSAR I 91 DL LOS F 35

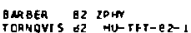

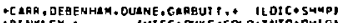

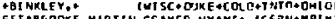

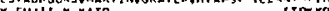
Y.FUSIT, N.KATn

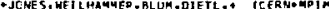
H. OCHS
PPOTOPOPESCU, GARHOST, GALT : EH B, FLATTEO (LIML) HHYAMS, JONES BLUN,DIETL, KOCH* ICEPNAMPINI AHYAMS. JONES.Q. JW.JTEL

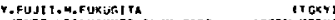

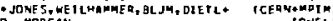

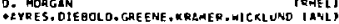

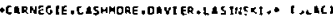
-CRAMLEY, DUKE,LAUB,LEEPR. PETERSTI II

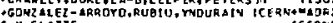

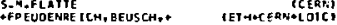
AFP EUDENRE ICH, BEUSCH, TET HOCEAHOL OIC

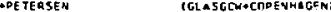

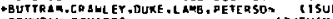

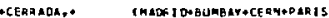

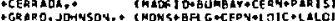

oE viranin. Shestanoy I rovo TAUSL . TER, MULLER, AEHAX. TKARL +PISA

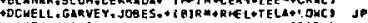
P. Estagrotexs - ivtemanis

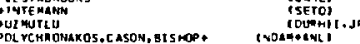
- OEYTANTN. SHESTAKOY [NOJO]

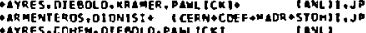

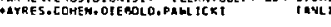

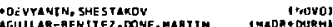

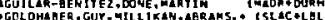
- MLR TIM.JDNe

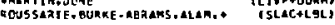
-OLINTOK, QROOBE CK, HROOKES. C TOMAE+LANC OSHEE! M. A.TORNGYIST
9IGT OZ CERN CONF 247 AICITS BRANDT R CARRARA + ICETR,

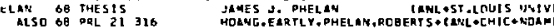

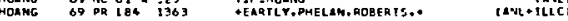

HYAMS 75 ND 8000205

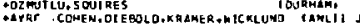

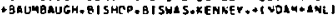

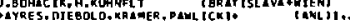


$\delta(980)$ 3S DeLTa(990,JPG-0+-1: :-1

The quantum numbers of the $\delta(980)$ resonance are: $I^{G}=I^{-}$from its production in $D^{0}+\delta \pi$, from its $n \pi$ decay, and from the absence of a $\pi \pi$ decay; and $J^{P}=0^{+}$from the absence of a $3 \pi$ or pr decay (LIFKIN 69, GRASSLER 77) and from the decay distributions of the $\eta \pi$ decay. With these guantur numbers the $\delta(980)$ is expected to couple to the $I=1 \mathrm{KK}$ system, too, and to explain the nearby $\overline{K K}$ threshold enhancement (ASTIER 67).

In the scalar SU(3) nones, the $\delta(980)$ has been a cause of problens. The near degeneracy with the $S^{*}(975)$ has been difficult to understand: if the $s^{*}$ is malnly an $s_{\bar{s}}$ systen and the $\delta$ malnly a ud system, naive mass rules predict $m\left(s^{\star}\right)-m(\delta)$ $\approx 200 \mathrm{MeV}$. Simllarly, naive mass rules predict $m(k)-m(\delta) \simeq 100 \mathrm{MeV}$, whereas the $k$ tends to appear near $1350 \mathrm{MeV}$.

Another problem has been the ratio of the widths: $\Gamma(K) / \Gamma(\delta)$ is experimentally about 6 , whereas su(3) symetry presicts 1.4. A solution to this problem came from coupled-channel analyses of the $\eta \pi$ and the $\bar{K} \bar{K}$ channels (MORGAN 75 , FLATTE 76). Above the $k \bar{K}$ threshold strong absorption makes the apparent width shrink, whereas below threshold strong analyticlty effects reduce the apparent width.

Recent multi-channel analyges (ACHASON 79,80, BRANON 80, TORNQVIST 82) confirm this picture, taking also the $\eta^{\prime} n$ channel into account. Horeover the rost conplete analysis (TORNOVIST 82) solves all the above problems at tine same time.

Thus the picture of the $\delta(980)$ that energes is a guark-antiguark state with large gqg components in the form of virtual two-neson states. The physical masses of all the scalar mesons are strongly influenced by the number of nearby 2-imegon thresholds. Thus the $\delta(980)$ feels strongly the $\eta \pi, k \bar{k}$, and $n^{\prime} \pi$ thresholds, whereas the $k(1350)$ Is far away from the $\mathrm{K} \pi$ and $\mathrm{K} \eta^{\prime}$ thresholds and couples very weakly to the $\mathrm{K} n$ threshold. This is the reason for the observed large $\kappa-\delta$ mass difference. The small $s *-\delta$ mass difference, on the other hand, is due to the isoscalar meson feeling the $k \bar{K}$ threshold a factor of 2 times stronger than the isovector meson does.
Thus the conventional g $\bar{g}$ model is sufficient, and no $q \bar{q} q \bar{q}$ multiplet is necessary to explain the observed scalar mesons (TORNgVist 82). See also the mint-reviews under $\varepsilon(1300)$ and $\kappa(1350)$ and Appendix IIC.

36 DELTALOBOI MASS (MEVI

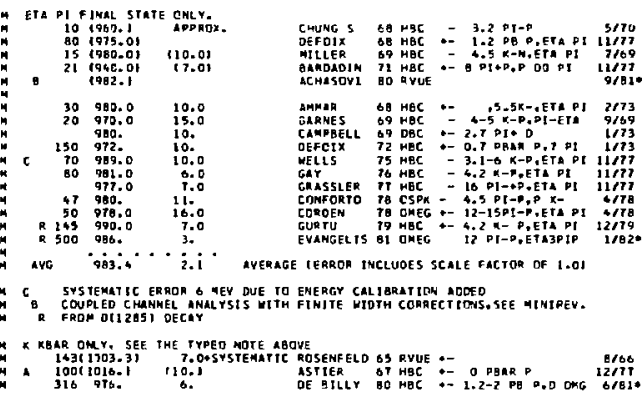

A ASTIER OP INCLUDES OAYA OF BARLON G7, CONFOFTO GT, ARAENTEROS O5.

36 DELTME 9OO) WIOTH (HEV)

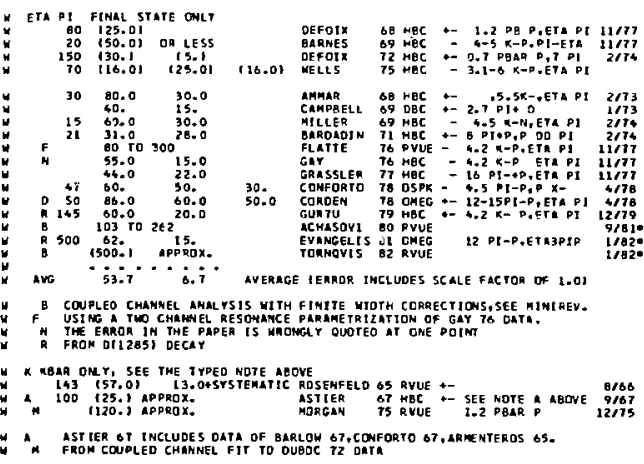

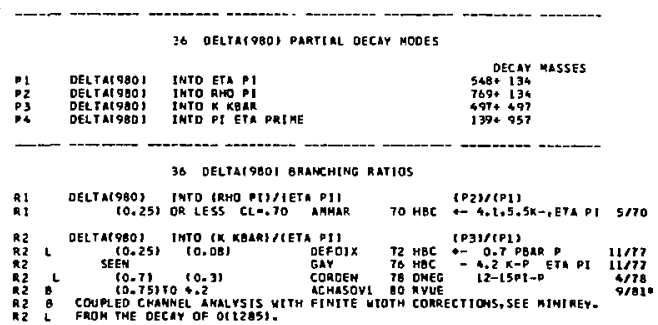

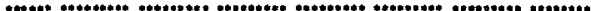
REFERENCES FOR DELTATQSOI

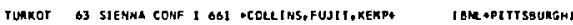

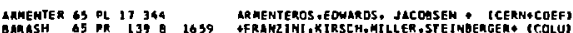

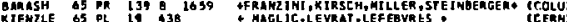

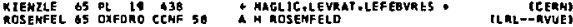


Mesons

$\delta(980), \phi(1020)$
Data Card Listings

For notation, see key at front of Listings.
ALLEN D \&S PL 225453

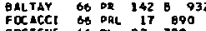
ALL1SON 6T PL 258619
ASIER OI PL 2589294
ASTIEA GT INCLUES DiTA OF BAILLOW, BT AC 502393 ARNAER 2 BI OL 258300

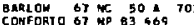

Awar 60 ORL 21183 CHAMG S OB PR 165140 GRTIEMI GB DPL 20349 JUHAA 69 OPL 27 B $25 \%$ BARNES OS PRL 23010 CAMPAELL OS PRL 22120 JUHALA OS PR 22134 1461

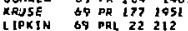
ATLLER $89 \mathrm{PL} 29 \mathrm{~B} 255$

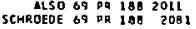

ABDLINS 70 PQL 25460

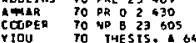

MNOERSON 71 PRL 26 L 108

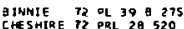
DefOIX 12 40 844125 DUBOC T2 No 84029 A thertan 73 pl 43 9 249 QINNIE TS DRL 372392

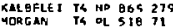

BUT TRAM TS PRL 35970 MORGAN TS ARGONWE CCNF.

$\begin{array}{llllll}\text { CaA } & 70 & \mathrm{DL} & 63 & \mathrm{~B} & 220 \\ \text { FLATTE TO } & \mathrm{PL}_{2} & 63 & \mathrm{~B} & 226\end{array}$

GRASSLER II YO B 121199 IRING 77 PL TO B 217
MARTIN
MAY
MAP COAFOPTO 78 LNC 23419

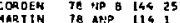
$\triangle C H A S C Y$ TO PL OE 8 BET

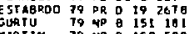

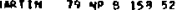

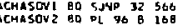

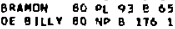
EVAMGELI BI NP 8 ITE 197 TCRNOVI 5 B? HIJTFI-9Z-1

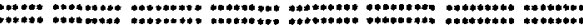
$\phi(1020)$

(4) PNI $11020,3 P G=1,1, t=0$

4 : T MASS TMEV

+GP FISHER, G GODOEA,L MaRSHAL, SEARS YCOLOJ G. HLACH, SANOWEETSS, TAFT, YEN, STOMEHILL TYALE)

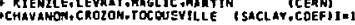

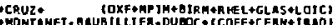

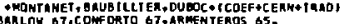

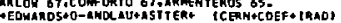

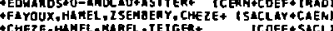
MDPTAHEL D-a

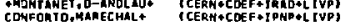

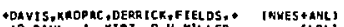

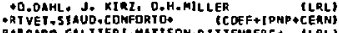
GAMBARQ-GALTIEAL, MATISON,RI TEHBEAG+ ILRLI

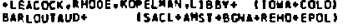

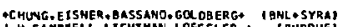
J-H.CAMPBELL, LICHTMAN, LOEFFLER, (PUROUE)

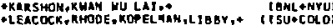

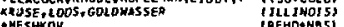

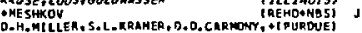

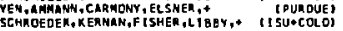

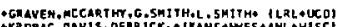

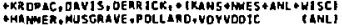
TEHIUPPUNG YIDU

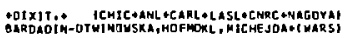

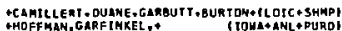

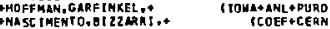

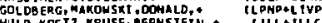
TFRENEK, FRENCH, GHI DIM1, HILPERT, * ICERNI

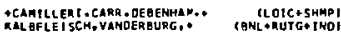

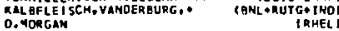

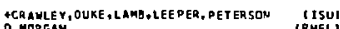

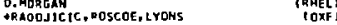

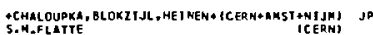
C CAACM+BERL+ BONH+CERN +CRRC +MEIO+WARS

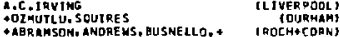

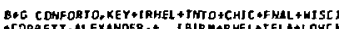

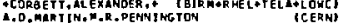
+oe vyanin. 5Me stakov thovol

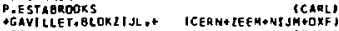
Dozarlu Toura

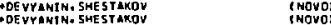

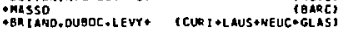
EVAMGELISTA+CBARI+BOMN*CER +DARE+LIVGOMILAS m.a.torneVt 5 t (HELS)

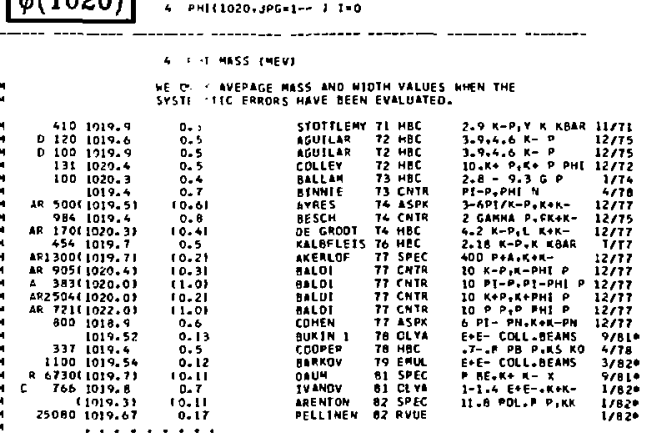

A SYSTEHITC EPROPS HOT EVALUATED.

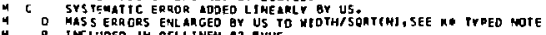

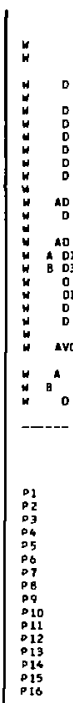

\author{
- Amt HOTH (MEV) \\ HE OMLY LA ERAGE MASS AHD HOTH VRLUES WHEM THE
}

\begin{tabular}{|c|c|c|c|c|}
\hline $\begin{array}{l}1.4 \\
8.29 \\
0.12 \\
1.7 \\
1.9 \\
1.0 \\
0.7 \\
1.5 \\
1.1 \\
10.81 \\
0.6 \\
0.37 \\
11.31 \\
0.50 \\
0.20 \\
0.8 \\
0.55 \\
0.6\end{array}$ & 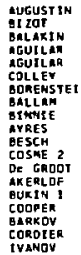 & 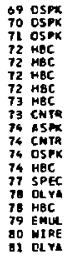 & 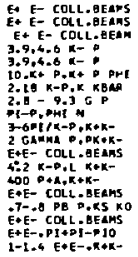 & 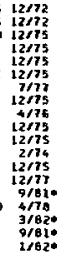 \\
\hline
\end{tabular}

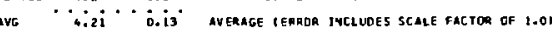

sustematic eragrs mot evaluhteo.

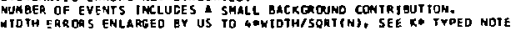

- phi pantial oecar modes

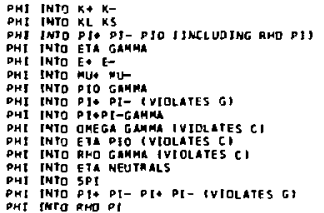

OECAY MASSES

493.4934

$139+139+134$

$348+0$

$105+105$

134.100

$139+139$

$782 *$
$769+134$
$769+\quad 0$

PHI INIO Pit PI- PIt PI- IVIOLATES GI

$139+139+139+1390$

$100+130$

\section{FITTED PARTIAL DECAY MODE BRANCHING FRACTIONS}

The matrix below in derived from the error matrax for the fited partial decay frode oranching fractions, $P_{i}$, as bollows: The diegonal elemenis are $P_{j} \neq 6 P_{i}$, whete

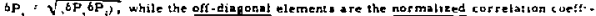

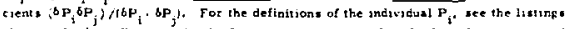

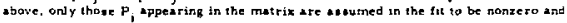
are thut congtrained to add to 1 .

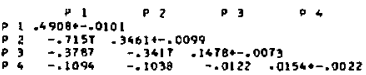

4 PHI SRLNCHikg RLTIOS

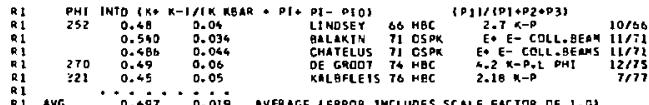

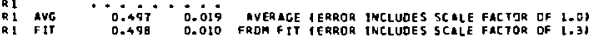

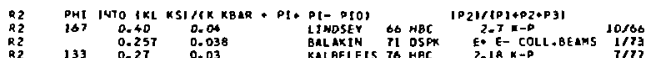

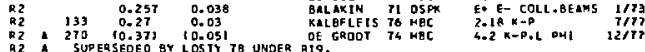

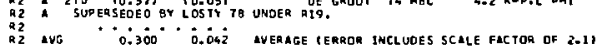

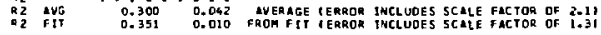

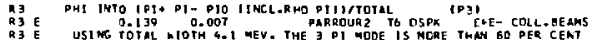
Q 33 USI W

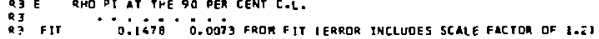

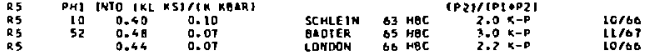

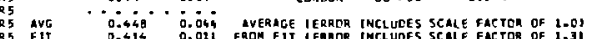

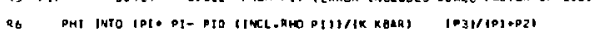

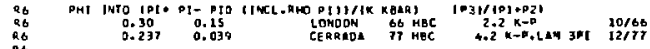

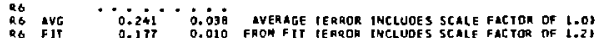

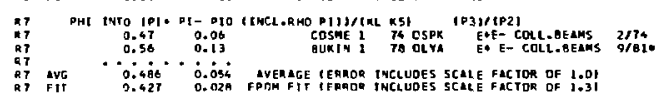




\section{Data Card Listings}

For notation, see key at front of Listings.

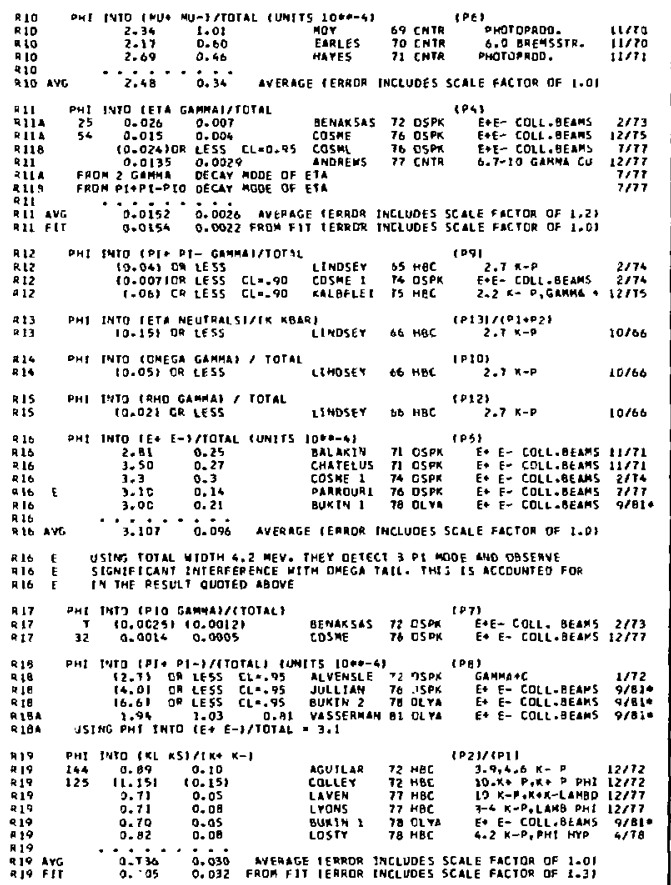

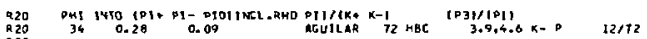

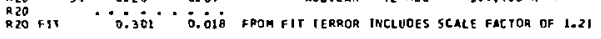

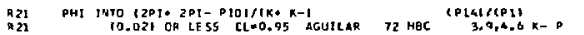

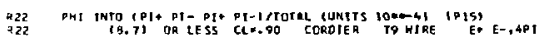

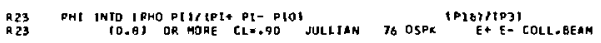

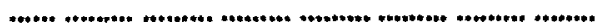

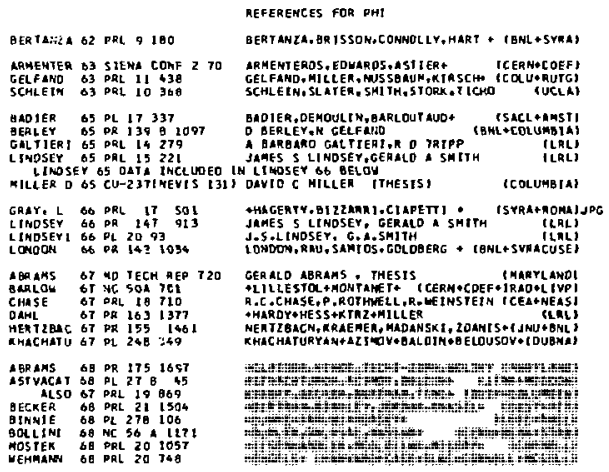

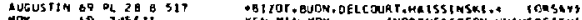

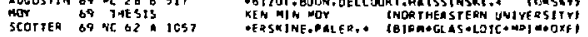

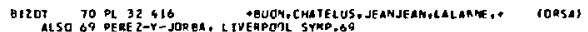

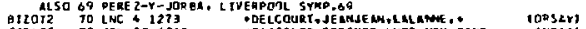

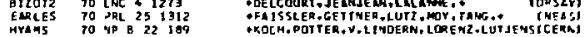

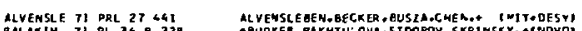

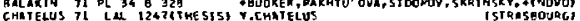

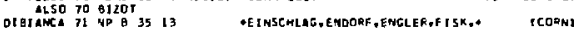

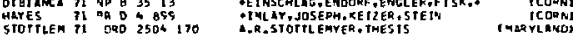
SCUILAR 12 PR D \& 29 ALVESSLE 72 PRL 2860

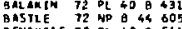
BEAKSAS 72 PL 62 B 511 SORENSTE 72 PR
COL LEY 72,1558

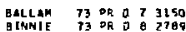

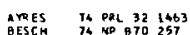

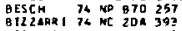

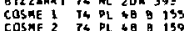
COS GE

KMLEFLEI IS PR DII 997

COSME 76 PL 63 B 952

JKLLLAN 78 THLISI YCL.2 R29

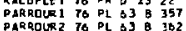

AKERLOF T7 PRL 39861

ANOAEES 77 PFL 38198

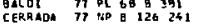

COOEN TIT DRL 33269

EVAHGE TI NP B 127 384

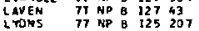

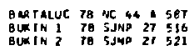

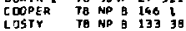

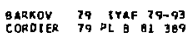

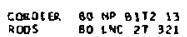

QNe* 81 PL 300 \& 439

I MANOV O1 PL 1078297

VAS SELSOA B2 OI OL 99 B 62

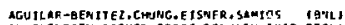

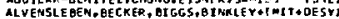

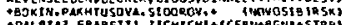

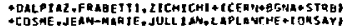

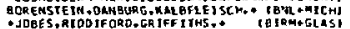

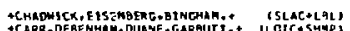

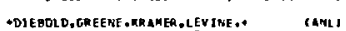
THART HANY, ROSE KRAGUT SCHAE IDEG, PAUT, , IBOVWI

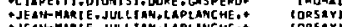

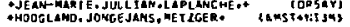

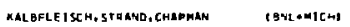

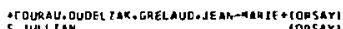

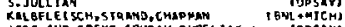

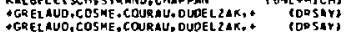

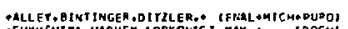

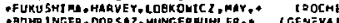

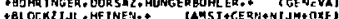

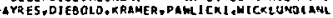

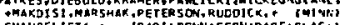

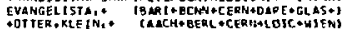

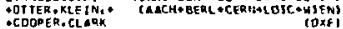

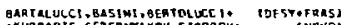

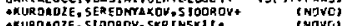

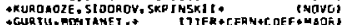

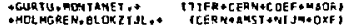

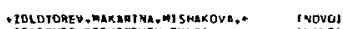

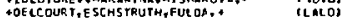
,DELCDURT, ESEHSTRUTH. FULCA. TOESAY) DDELI INEN THELS

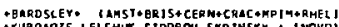

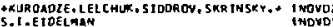

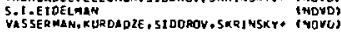
ARENTOW
PELL INE
B2 " $\mathrm{H}(1190)$ 30 HA1190.JPG: $1 *-1$ t=0 30 H(1190) MASS (mEV)

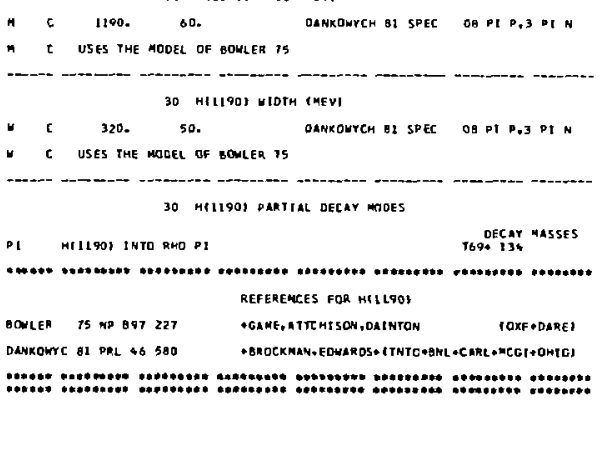

Drol* ore1* 
Mesons

$B(1235), \rho(1250)$
Data Card Listings

For notation, see key at front of Listings.

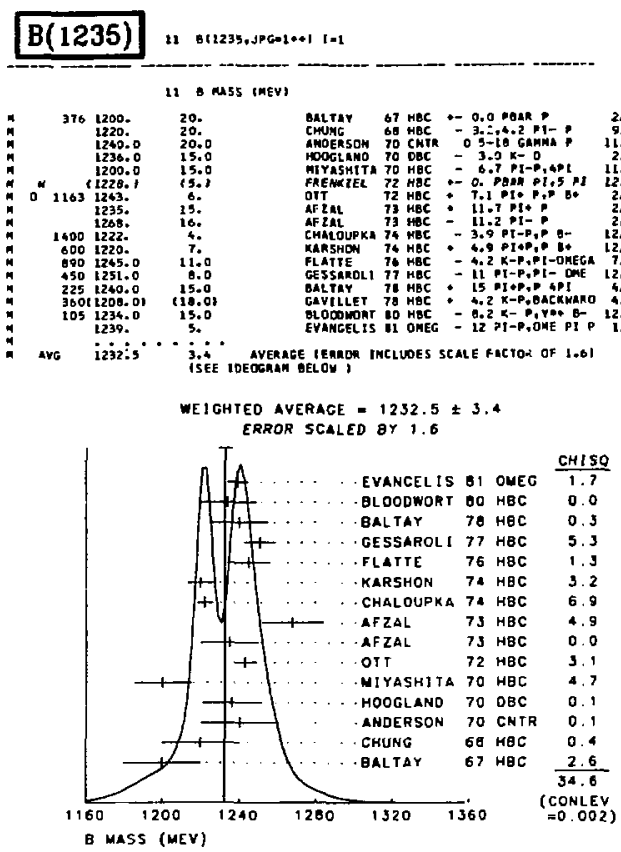

DO FROM FIT OF THE MASS SPEC TRUM
FIT REQUTHES AN ADOTTYOMAL JPR- RESOHAHCE

$$
11 \text { Utath (MEV) }
$$

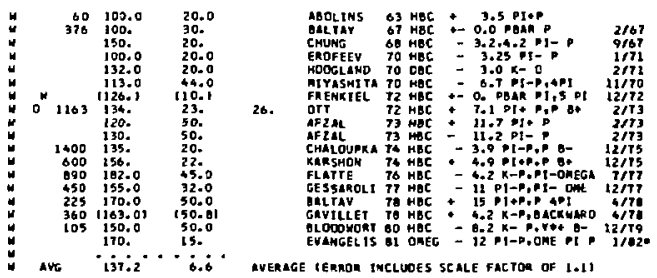

- O FROF FIT OF THE MASS SPECTRUM

11 D PARTIAL DECAY HOOES

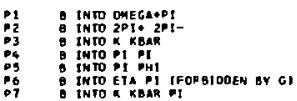

\section{B gRAnCHIHC aAtTos}

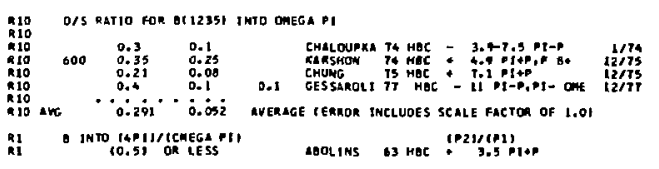

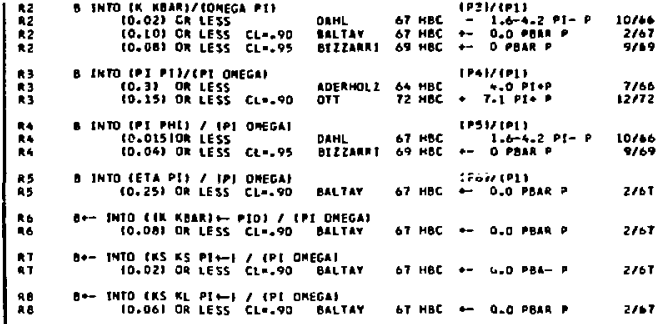

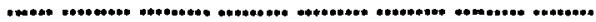

REFGAEMCES FOR :

ABOL ITS 63 PRL 21136

ADENHA 2 on OL 10240

ADENHac 2 bo 10240

GOL OHME GS PRL 15118

oul tar of onl 2093

LEE

ASCOLE 68 PRL 201411

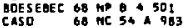

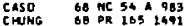

DTzZANR 1 OQ HP O 14169

ANoEkSON TO Pa O 127

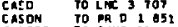

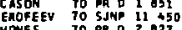

MOOGLMO 70 PL $3{ }^{2}$ O31

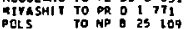

WEREAOUE TO LAC 1267

DEVDNS T1 OEL 27 16I6

FMEMKIEL 72 MP $\$ 7761$

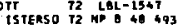

AFIAL T3 \%LL 13 A

AMEHISE T3 ALCT

AMAOLO T3 LAC 6707

CASON 173 ME : 7197

CHENG $73 \mathrm{PL} 47 \mathrm{~B} 320$

GLLLAM 74 He 076375

CHALOUPK 76 PL $\$ 16$ 407
CARSHOX Th PR 010 360

CCUAHC IS PR O11 2420

FLATIE T6 PL B4 1225

GESSAROL IT WD 126382

BATAY $7 E$ PR D 1762

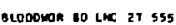

EVANGEL 1 O1 $\mathrm{NP}$ a $11819 \mathrm{~T}$

(1)
AOOL IMS, LAMDER, MEML MOP, NUOMG, YAGEN IUCSDI

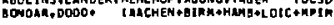

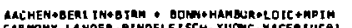

C GOLOHABER,S GOLOHABER, MADYK, SHEN ILRLI

- SEVER IENSA YEH+ZAMEL LO TCOLUOONLI

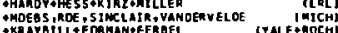

+CRAYLEY, WONTARA.SHAPIRO ILLI JP

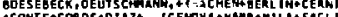

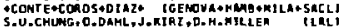

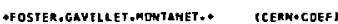

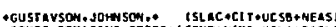
+ CONTE , TOHA SINI , COKDS+ I GEHD+HAMO OHILA+SACL

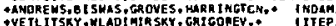

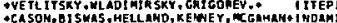

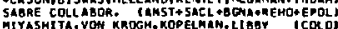

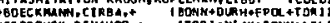

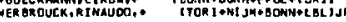

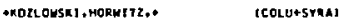

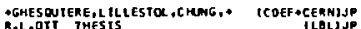
SISTEASDOH, HALR I SOH, HEYOA. JOHASOM, O ( HARVARD)

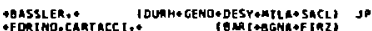

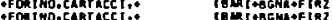

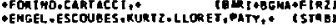

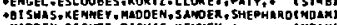

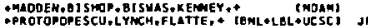

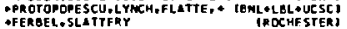

-CHAOUTCK, OIMGHAM, FRETTER ISLAC+LBL • MHIMI

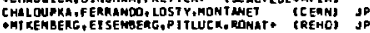
+PROTOROPESCU. LMCH,FLATTE,+ 18ML+LBL +UCSC) JP M.S.OUG DVIKOV.T.A. EROFEEV (ITEP) JO

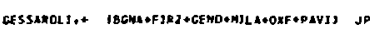

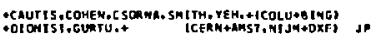

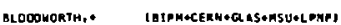
EVIMGEL ISTACIBAR [4 BOH+ CEANO OMAEOL IVPOMILA] GVEY TSKE

'

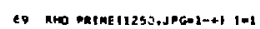

EVIOETCE MDI COMPELING. OMITIEO FROA TABLE.

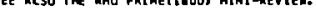

69 NRO ONIMEC12501 MASS TMEVI

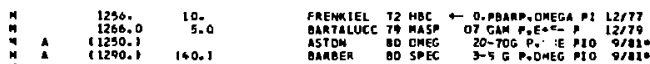

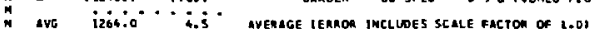

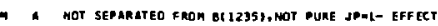

65 AHO PAIMECL2SOI WTOTH [MEV]

\begin{tabular}{|c|c|c|c|c|c|c|}
\hline 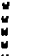 & $\begin{array}{l}130.0 \\
110.9 \\
1900.1 \\
1320.1\end{array}$ & $\begin{array}{r}20 . \\
35.0 \\
1100.1\end{array}$ & $\begin{array}{l}\text { FREMKIEL } \\
\text { BARTALUCS } \\
\text { SSTOW } \\
\text { BAREER }\end{array}$ & $\begin{array}{l}72 \text { MBC } \\
7 \% \text { MASP } \\
\text { o0 OMEG } \\
\text { 80 SPEC }\end{array}$ & 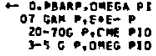 & $\begin{array}{l}12 / 77 \\
12 / 79 \\
9 / 60 \\
8 / 10\end{array}$ \\
\hline
\end{tabular}

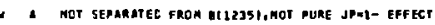


Data Card Listings

For notation, see key at front of Listings.
Mesons

$\rho(1250), f(1270)$

\begin{tabular}{|c|c|c|}
\hline & & ES FAR RWD PEINE \\
\hline ANOERSCH & $\begin{array}{llllllll}70 & 02 & 0 & 1 & 27\end{array}$ & 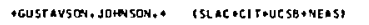 \\
\hline $\operatorname{coc} \operatorname{sen} x$ & II UCAL 20128 & H.J.POODLSKY,PH. O. THESIS \\
\hline $\begin{array}{l}\text { REPIKIE L } \\
\text { RTLF }\end{array}$ & 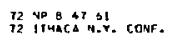 & 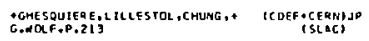 \\
\hline $\begin{array}{l}\text { CHUNG } \\
\text { BAMMON: }\end{array}$ & 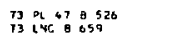 & 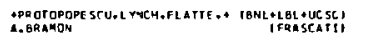 \\
\hline 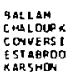 & 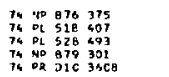 & 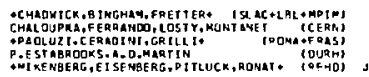 \\
\hline 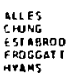 & 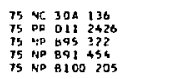 & 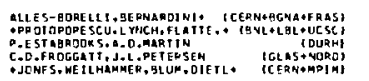 \\
\hline Bassoup I & TA PL OS 8397 & 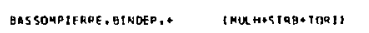 \\
\hline 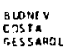 & 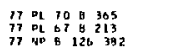 & 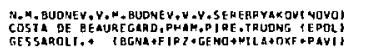 \\
\hline Buxin & 7901739226 & 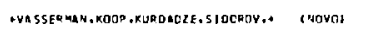 \\
\hline 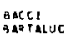 & $\begin{array}{ll}79 \\
79\end{array}$ & 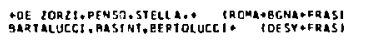 \\
\hline $\begin{array}{l}45104 \\
B A R B E R\end{array}$ & 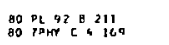 & 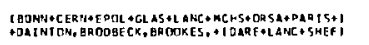 \\
\hline
\end{tabular}

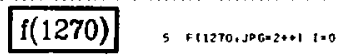

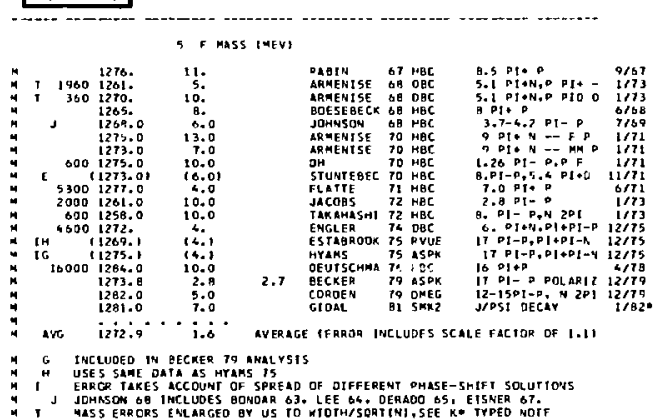

$$
5 \text { F NIOTH IMEN }
$$

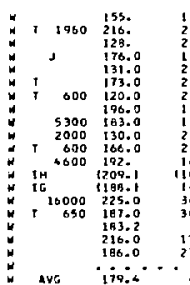

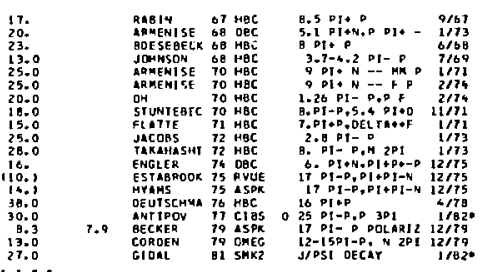

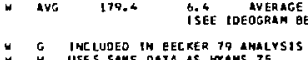

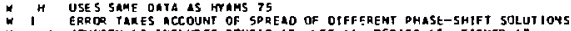

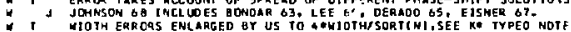

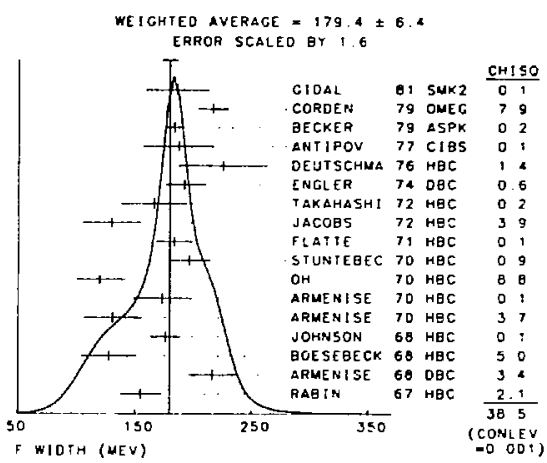

F PARTial decar mudes

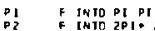

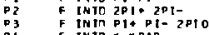

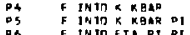

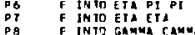

DECAY Masses

${ }_{390}^{1390} \begin{aligned} & 139 \\ & 139+139+139\end{aligned}$

$1390.1396 .136 . \quad 136$ 497.497 .1139 $548+$
$548+$
048

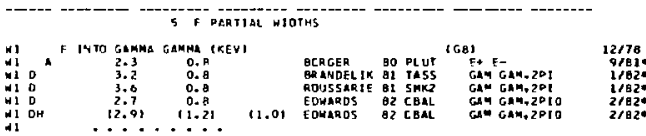

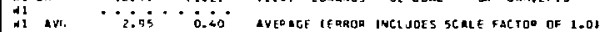

al a USING MASS. WIOTH AND GRTF FO 2011 FROM DOG 1970

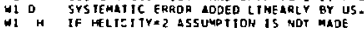
5 F BRANCKING Ratios

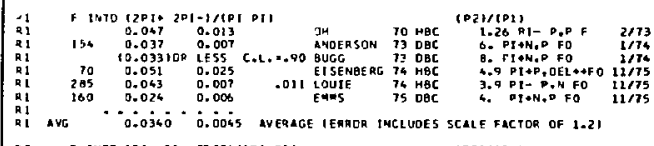

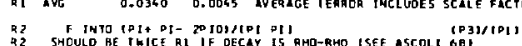

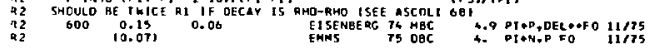

R3 F INTO IK KALRICLPY PI)

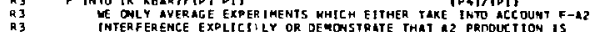

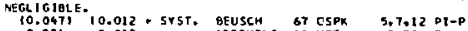

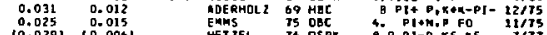

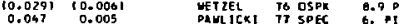

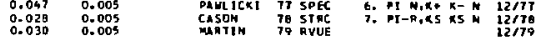

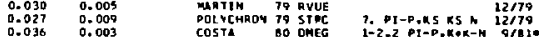

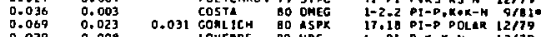

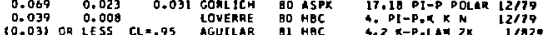

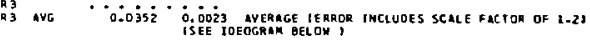

Ra C TMIS OE TERHIMAT ION MAS OUAMT ITATIVELY ACCOUNTED FOR EOTH F-PRIME

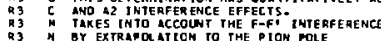

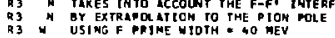


Mesons

I(1270), $A_{1}(1270)$

WEIGHIED AYERAGE $=0.0552 \pm 0.0025$ ERROR SCALED GY 1.2

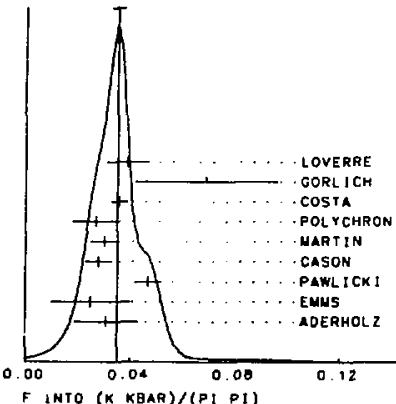

80 hac $\frac{C H I S O}{0.2}$

80 ASPK

80 OMEG

0.1

0.0

1. 1

2.1

5.6

0.5

$\frac{0.1}{10.1}$

(CONLEY

F INTO (K KBAR)/(PI PI)

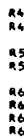

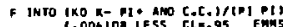

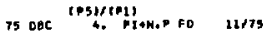

F INTO TETA T' PI'PCPI MII

75 (POI)LAL)

4. Mtom.p Fo Hits

F THTO (ET: ETA: /CPY PI)

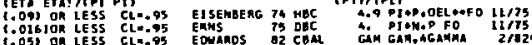

16

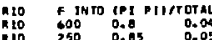

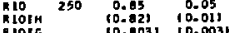

$\begin{array}{lll}R 1016 & 10.0031 & 10.0031 \\ 710 & 0.867 & 0.016 \\ 010 & 0.75 & 0.04\end{array}$

n10

P. - .

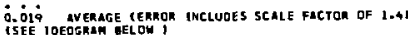

M10 G ITCLUDED TH OEC KER T9 ANALTSIS

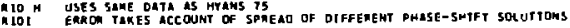

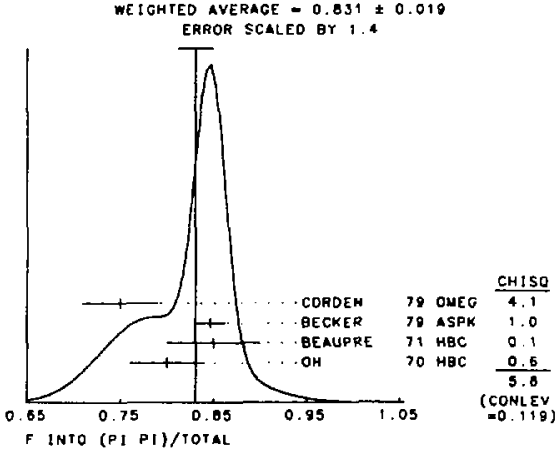

\begin{tabular}{|c|c|}
\hline 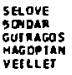 & 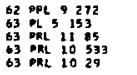 \\
\hline 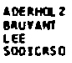 & 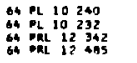 \\
\hline 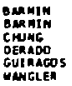 & 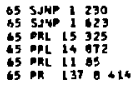 \\
\hline $\begin{array}{l}\text { ICCENSI } \\
\text { JAtots } \\
\text { detLIG }\end{array}$ & 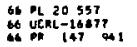 \\
\hline
\end{tabular}

MEFERENCES FOR F

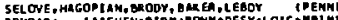

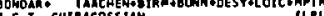
2-G. T. Gurkancossian

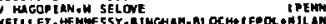

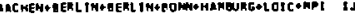
GRUTAMT, GOL DAEMG,HOLDER,FLEUMY - CCERM +EPQLL I EE,ROE. STHCLAMR.VWDER VELDE (MICH) odickson, HamLic, DOL COLEMKO, EL ENSRV, EROFE EY, ITTEP MOSCDUI JP

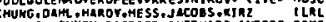
QE3ADO, KEMMEY, POIRIER, S MEPHA RO INOTRE ORME I. T GUfRLCOSST HA aCCENST,ALLES- GORELLI, \& RENCH, MTISK * ICERM. CO.JACOAS, THESIS
Data Card Listings

For notation, see key at front of Listings.

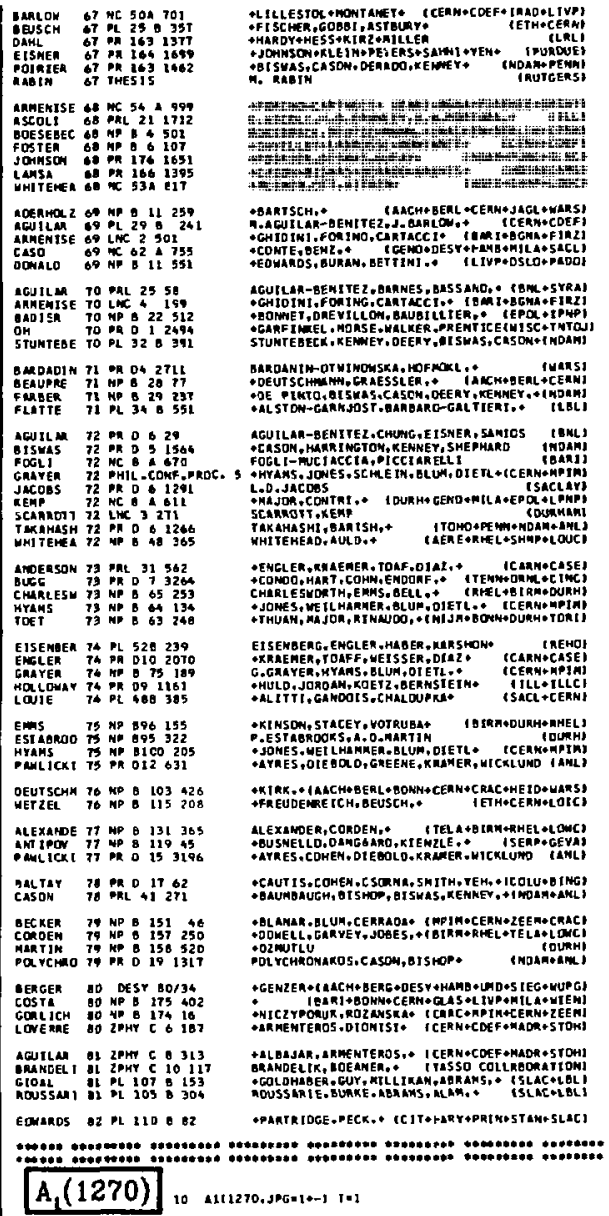

The long-standing question concerning the resonance interpretation of the $h_{1}$ has been considerably clarified since the last edition of this Review. The results of the partial-wave analyaes obtained In two recent high-gtatistics experinents dealing with the diffractive (DAuy 80,81) and charge-exchange (DANKOwYCH 81) production of the $3 \pi$ system in $\pi p$ interactions, clearly show that the behavior of the $1^{+} \mathrm{so}^{+}$intensity with the $3 \pi$ mase and the phase variation of the $1^{+} \mathrm{SO}^{+}$(pr) anplitude with repect to other waves lalready reported In a atudy of diffractive production from nuclei [PBREgR 7B)] require the presence of 
Data Card Listings

For notation, see key at front of Listings.
Mesons

$A_{1}(1270)$ both Deck background and a resonance.

The resonance parameters of the $A_{1}$ resonance are obtained by fitting the data (intensity and relative phases) to a phenomenological amplitude containing direct resonance production and a coherent Deck background which is rescattered through the resonance (BOWLER 75, BASDEVANT 77). In the context of this model-dependent analysis, the Deck background is responsible for making the peak of the $1^{+} 50^{+}$intensity occur some $110 \mathrm{MeV}$ below the most likely resonance mass.

We take the mass values for the $A_{1}$ from these reactions $(1240 \pm 80 \mathrm{MeV}$, DANKOWYCH B1; $1280 \pm 30$ Hev, Dhum 81). Note, however, the result regorted in a study of a backwardly produced $3 \pi$ system in the reaction $\mathrm{K}_{\mathrm{p}}^{-}+\Sigma^{-} \pi^{+} \pi^{+} \pi^{-}(1041 \pm 13 \mathrm{MeV}$, GavitLET 77). Based on a rather snall statistical sample, GAVILLET 37 fitted the $1^{+} s$ partial-wave intensity in terms of a single Breitwigner function disregarding a possible background component in the $I^{+} 5$ wave.

10 A1 MaSS IMEYI

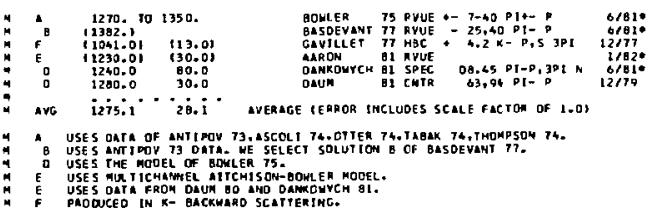

10 AI HOTH (4EV)

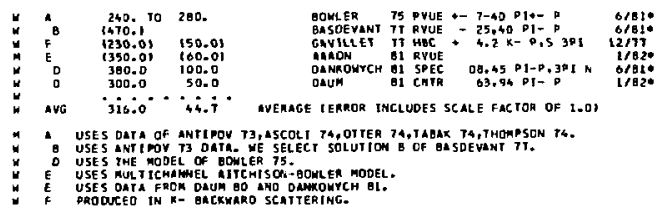

10 AL OARTIAL OECAY MODES

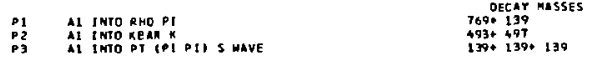

I0 al branehimg natios

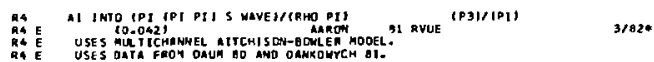

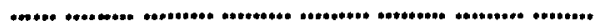
MEFEREHCES FOR A1

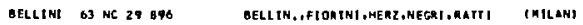

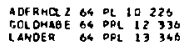

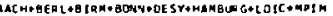

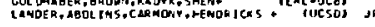

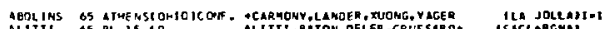
ALLARO

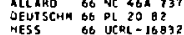
Bllison of Pl 258619

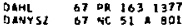

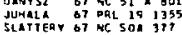

ARMENISE ob ol 200 O30 $45 C 013$ OB PRL 28113 A

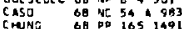

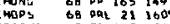

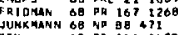
SB PR 1861430

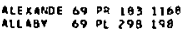
ALLABY 69 Pt 298 198

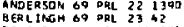

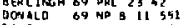

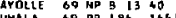

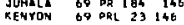

ARMENISE 70 LINC 4 199 45001 TO DRL 25562 $6 A 50$ TO

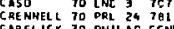

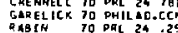

ASCOL: 71 PRL 24929

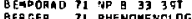

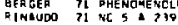
BeaENYT 72 NF 837 (2) BLOODOR 72 NDO

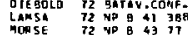

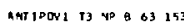

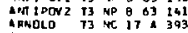

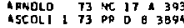
ASCOLI 273 PRT 31 795

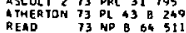
ASCOLL 74 DQ 091983 BOULE 74 NP $B 74493$

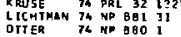

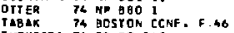

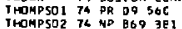
LaASHIAK 75 PRL 34 G91

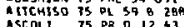

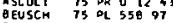
OOSETII IS MP 3 BDi 304 OONLER 75 HO 897227 EMAS I T5 ND 8931 EEYS 2 IS PL $00 \mathrm{~B} 109$ HONE 75 PRR OI1 996

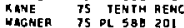

BavBILL1 70 NP B $115 \quad 237$

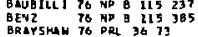

BaLTAY TY DRL 30591

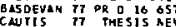
CANTIS II FHESIS AEVIS 222

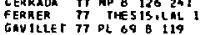
TLEER 71 PLOB 129420

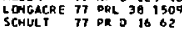

ALEXANDE 78 PL 73 B 99

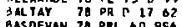
BDSDEVAN 78 PRL 40994 CONDEA 79 ME 8.1367

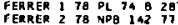
JAR OS 78 PR4 $\$ 01120$

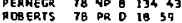
COROTER 18 PL B OI 399

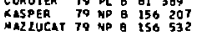

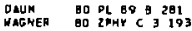
AMON BI PR D $8+120$ P BELLINI BI PRL

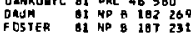

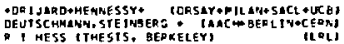

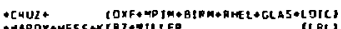
OAMPOY ICERMI

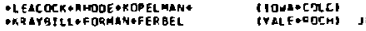

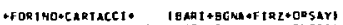

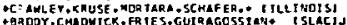

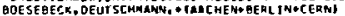

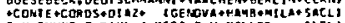

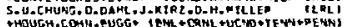

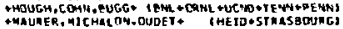

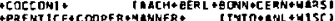
G.ALFXLMJE R.A.F TRE STOME, G. COL OHABER ILALI

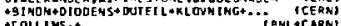

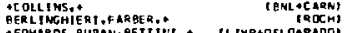

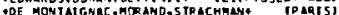

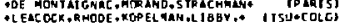

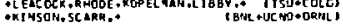

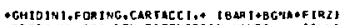

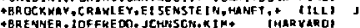

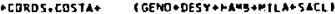
OKARSHON LA1 SC A AR, STMS

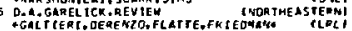

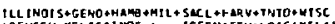

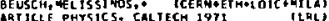

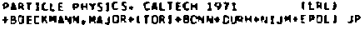

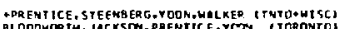

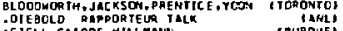

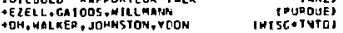

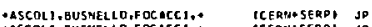
TASCOL T, BUSNELLO,F OCACCI, ICERN+SERP)

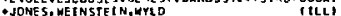

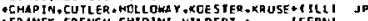

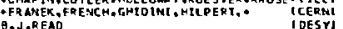
*CUTLER. JONES, KRUS E, ROBERTS, WE: ASTE INAT TLLI R DBERTS. EOELSTEMt

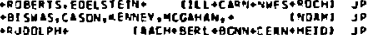

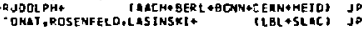

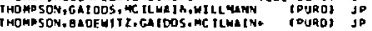

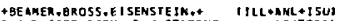
T.S.Q.ABIIIHISOM, Q. J.GOLDING TOXFORD?

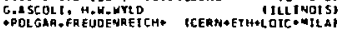

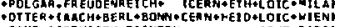

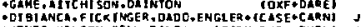

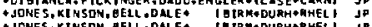

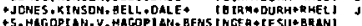
T5. MAG OPRAN, V. HACSP IAR, BENS INGER R TF SU+BEANI

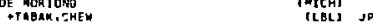

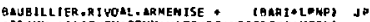

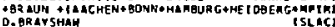

tCautis, Kalezkar

BASOEVANT, BERG
C.V.CAUT'S

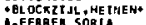

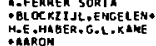
:AAROH

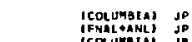
IANS T OCE RN+MI JMEOXXY) JP CAMST, CERH+MIJM*OKKF, JO

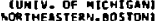
I ROR TMESTTERM,OOSTON) JP

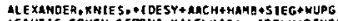
TCAUT IS, COHEN, C SORHA, KALEL KAR* ICOLUEBTNG) DOHELL, GARYEY, JOBES+ (BIRM+REELTTELA+LOMCI JP TREILLE, RIVET * IORSAY CERMOCOEF+LPAP a

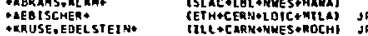

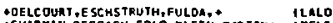

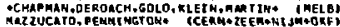

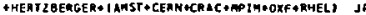

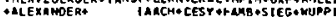
PLAGGACRE

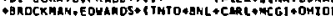

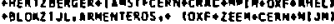
" 
Mesons

$\eta(1275), D(1285)$
37 ETALI275. JPG $=0-11=0$

SEEN IK PHASE SHIFTS AMALYSTS DF THE ETA PJA PJ-

WHIT COW LRMRTIOH. OMTITED FROM TABLE.

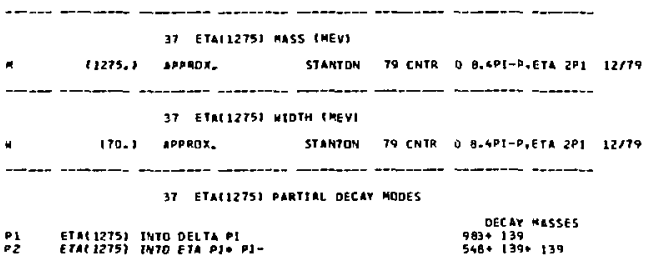

37 ETAILRTSI GRAMEHING RATIUS.

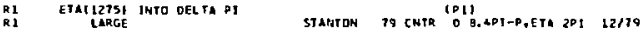

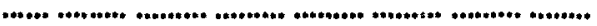

REFERENCES FOR ETATI275,

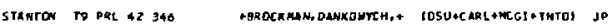

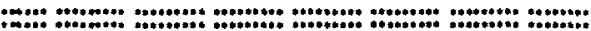

$D(1285), 01205 . J P G=10.11=0$

- D AASS (MEV)

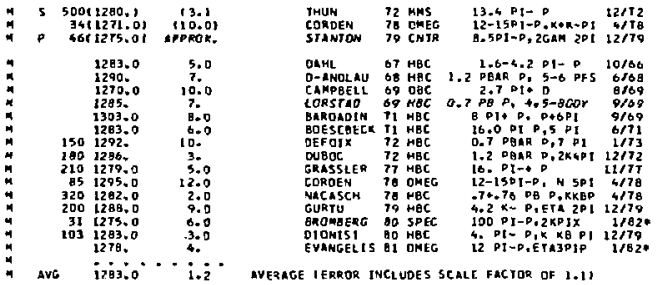

M PROM DMASE SHIFT MNATYSIS OF EIA PITPI- SYSTEM.

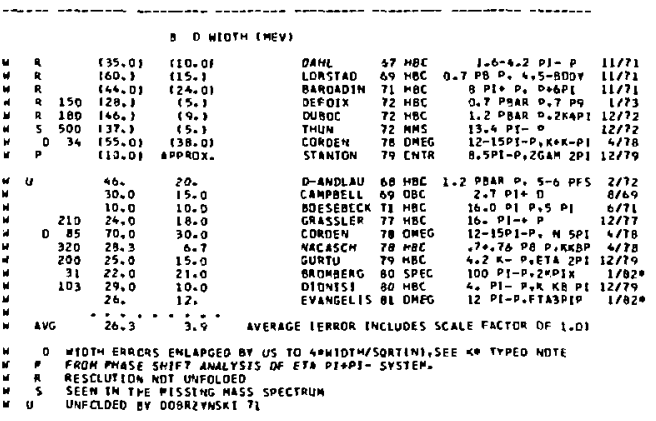

G D DAGTIAL DECAY MOJES

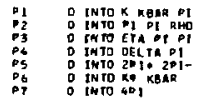

DECAY MS5ES

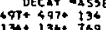

$134+1340 * 769$

$139+134.139 .139$

$139+1990139.139$

\section{FITIED PARTIAL DECAY MODE BRANCHWG FRACTIONS}

The matrix belau is derived tram the error matrix for the finted partial fiecal mote Eranching fractiona, $P_{i}$, as follows: The diegomal etemeritn are $P_{i}$ \& $8 P_{j}$, where

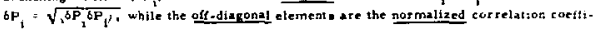

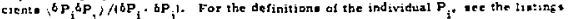
above; only those $P_{i}$ appearing in the mayrix are alsumed in the fit to be nonacro one ze the contrained 10 add to 1.

P t $11117+10256$ P 3 P 7

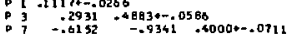

a D GRMNCHING RATLOS

THE D BRANCHTRG RATIDS FIT IS MAOE WITH THE ASSUMPTIOM THLT THE O INTO \&PI OECAY IS ALWAYS VIA OECAY THTO TH I PI PI

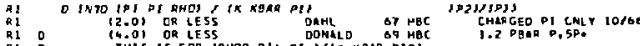
41 D THIS IS FOP IRHOO PJ+ DT-13IK K.QBR DIOI

$R 2$
$R 2$

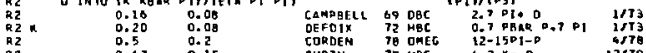

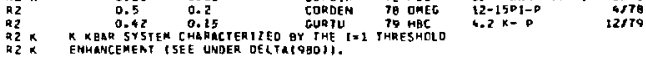
R2 2 EMHANGERERT ISEE UNDET DRC

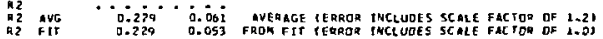

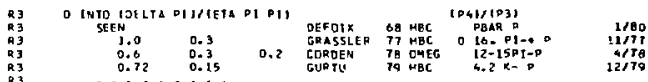

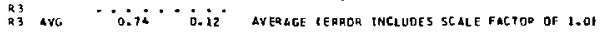

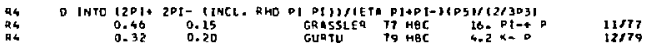

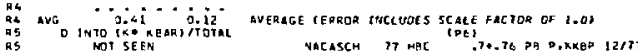

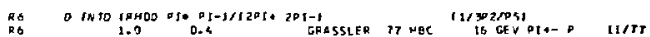

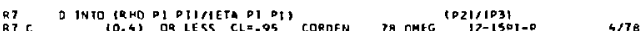

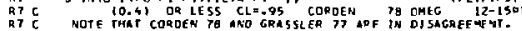

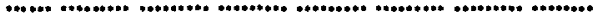

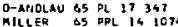

BRALOH or We 50 \&

D-LNOLAU 6 A NP B $5 \quad 693$

DEFOIX QB Pl 28 E 353

CAMPBELL 60 ORL 221274 ODNALD 89 YP 811551

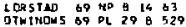

imar po pr dz a jo

BSMOAOIN II aR OS 271 BDE SEAEC II PL 34 B o5? GOLDEER 71 LNC 1 627

BERENYI 72 ND 637621 CHADMAY I2 ND

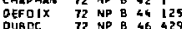

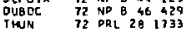
VUILLEI IS LWE 14105

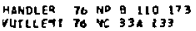
GRASSLEA $7 T$ YP $B \quad 121 \quad 189$ $\begin{array}{llllll}\text { CCRDEN } 78 & \text { NP } & 144 & 253 \\ \text { IRYING } & 78 & 4 P & 8 & 239 & 327\end{array}$ HACASCH 7O UP B 135203 GURTU $79 \mathrm{KP} B$ 151 181

GROMEET BO PA D 22 1513

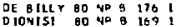

EYAMGELT BI MD B ITE $19 T$

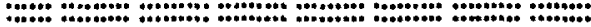

PEF ERENCES TOP D

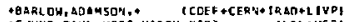
TCFUVG, OAHE, HES S, HAROY , KITZ, . ILQL +UCA

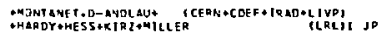

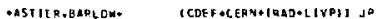

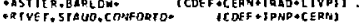

PL iCHTMAN, *

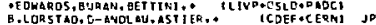

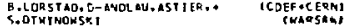

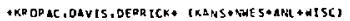

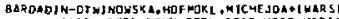

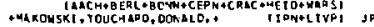

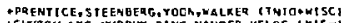

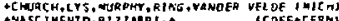

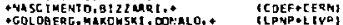

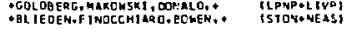

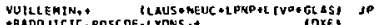

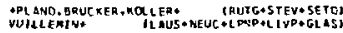

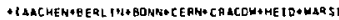

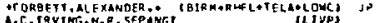

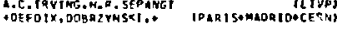

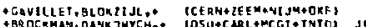

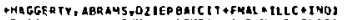

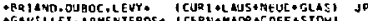

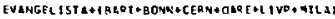

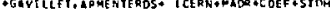




\section{Data Card Listings}

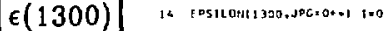

\section{S-Wave $\pi \pi$ and $k \overline{\mathbf{k}}$ Interactions}

In this note we discuss information on the non-strange $I^{G} P C \times 0^{+} 0^{++}$partial wave (S wave) coupled to the $\pi \pi$ and $K \bar{K}$ systems.

Near the $\pi \pi$ threshold the $S$ wave shows no resonant behavior. For a discussion of the relevant scattering lengths and various resonancelike kinematic effects, see.our 1978 edition.

up to the $\rho$ meson mass region, the phase shift $f_{0}^{0}$ is (qualitatively) uniquely determined; it $r$ ises monotonically and reaches $60^{\circ}$ to $70^{\circ}$ neat $700 \mathrm{MeV}$ (SONDEREGGER 69, BATON 70, BAILLON 72, CARROL 72, ERENKIEL 72, GAIDOS 72, PROTOPOPESCU 73, HYAMS 73, OCHS 73, ENGL ER 74, ESTABROOKS 74,75. GRAYZR 74).

In the early phase-shift analyses two solutions for $\delta_{0}^{0}$ were found (the "up-down ambiguity") in the 700 to $900 \mathrm{MeV}$ region. The "up" solution corresponds to an $\epsilon$ resonance under the $\rho$ meson with mass and width similar to the $\rho$ meson, the $\varepsilon(800)$. The "down" solution is characterized by an approximately energy-independent phase shift of almost $90^{\circ}$, showing no resonant behavior. This ambiguity was consiciered rssolved in favor of the "down" solution by the observa$t i o n$ of a very rapid decrease in the rodulus of the s-wave amplitude between $900 \mathrm{MeV}$ and the $\overline{K R}$ threshold, followed by a sharp drop in the elasticity. $\delta_{0}^{0}$ is $\sim 90^{\circ}$ at about $900 \mathrm{MeV}$ and reachus $\sim 180^{\circ}$ around $990 \mathrm{MeV}$ (FLATTE 72, GAIDOS 72, hYAMS 73, BINNIE 73, ENGLER 74). Howe ser, the region is complicated by the simultaneous presence of the $S^{\star}$ resonance and the opening of the $\bar{k} \overline{\mathrm{K}}$ channel, permitting almost discontinuous jumps from ore solution to another.

Without polarization information, the reaction $\pi N$ - $\pi N$ cannot be analyzed unambiguously due to the fact that there are more helicity anplitudes than observables (see, e.g., Doworue 35). Thus one is obliged to make some supplementary assunptions.

An amplitude analysis \{E9TABROOKS 74\} of the largest $\pi^{-} \mathrm{p}$ (unpolarized) $\rightarrow \pi^{+} \pi^{-} \mathrm{n}$ experiment (HYAMS 73, GRAYER 74) still finds both the "up" and "down" solutions. This analysis assumes both spin coherence (the unnatural-parity-exchange, schannel helicity amplitudes are nucleon spin-flip. i.e., no $A_{1}-1$ ike exchange) and phase coherence (the $s-$ wave anplitude and the unnatural-parityexchange, meson helicity-zero P-wave anplitude have the same phase). These assumptions may tend to bias the results (MORGAN 74, DONOHUE 75,79).

The advent of $\pi^{-} p$ (polarized) $\rightarrow \pi^{+} \pi^{-} n$ data (BECKER 79) has made both the spin coherence and phase coherence assumptions unnecessary. Analyzing their data in a model-independent way, BECKER2 79 also find both the "up" and the "down" solutions.

The reaction $\pi^{+} p+\pi^{+} \pi^{-} \Delta^{++}$has been analyzed in the region 660 to $860 \mathrm{MeV}$ (OHENS 76, DONOHUE 79) and in the region 600 to $920 \mathrm{MeV}$ (GELFAND 78), using all the information carried by the $\Delta^{+t}$ decay. The conclusion from both analyses is that the $\varepsilon(800)$ of the "up" solution cannot be ruled out.

The only way to rule out a narrow $\varepsilon$ under the $\rho$ meson (the "up" solutionl is to study the $\pi^{\circ} \pi^{\circ}$ system. With the exception of one experiment (BISWAS 81), all the $\pi^{\circ} \pi^{\circ}$ experiments agree that no such narrow resonance is present and that the "down" solution describes the data well (DEINET 69, SONDEREGGER 69, SHIBATA 70, BENSINGER 71, AFEL 72,79, BRAUN 73, SKUJA 73, RIESTER 75, GRIVAZ 76, DAVID 77, BORREANI 79,81). The phase shifts of BISwAS 81 lie much lower than all others in the 300-700 MeV region, thus requiring a sudden phase motion in che $\rho$ region to match the "down" solution above the $\rho$.

The region of elastic $\pi \pi$ scattering is known to extend to about $990 \mathrm{NeV}$, near the $k \bar{K}$ threshold (BATON 70, CARROLL 72, PROTOPOPESCU 73, BYAMS 73 , ocas 73). Beyond $1 \mathrm{GeV}$ we therefore have to consider the two channels $\pi \pi$ and $k \bar{k}$, and beyond 1100 MeV the $\eta$ nharnel also opens up. In addition, the solutions have inherent ambiguities related to the Barrelet zeros of the anplitudes. Thus HYAMS 75 find four solutions in the region 1.0 to $1.8 \mathrm{GeV}$, ESTABROOKs 74 find eight solutions, and CORDEN 79, extending the $\pi \pi$ analysis to $2.08 \mathrm{GeV}$, find another eight solutions. 
Mesons

$\epsilon(1300)$

In the past mapy of these solutions have been ruled out by imposing continuity in vario: $s$ ways, as well as analyticity and unitarity (FROGGATT 75,77, COMMON 76, MARTIN 78) .

Unfortunately, the polarization information (BECKER 79) has not yet been fuily analyzed. One notes that a model-independent partial-wave analysis (BECKER2 79) agrees qualitatively with solutions $\beta$ and $\beta$, (OE MARTIN 78).

The $B$ and $\beta^{\prime}$ anplitudes describe the experimental moments in each bin without any explicit smoothing; they are analytic in $s$ and approximately analytic in $\cos \theta$. They take into account all waves up to $\ell=4$. The $B$ solution has a highly elastic $S$ wave, whereas the $S$ wave of solution $B^{\prime}$ is somewhat inelastic (MARTIN 7 a). The unique solution of FroGGaTT 77, which has explicit smoothness built in and which takes account only of $\ell \leqslant 3$ waves, is rather similar to $\beta$. However, it has problens with unitarity, apparently because of the neglected $G$ wave (MARTIN 78).

The $S$ wave is clearly resonant in the data of BECKER2 79. In the 1150 to $1400 \mathrm{HeV}$ region both the S-P and S-D phase differences show the presence of a broad resonance, and the intensity of the $S$ wave confiras this by exhibiting a peak at about $1300 \mathrm{MeV}$ with a width of about $300 \mathrm{MeV}$; see Fig. 1 (a).

The amplitude analysis of the $\pi^{-} p \rightarrow \pi^{+} \pi^{-} n$ experiment of CORDEN 79 has two preferred solutions which are close to $B$ and $B^{\prime}$, giving some support for an $E(1300)$. Also the $S$ wave in the $\pi^{0} \pi^{0}$ system tends to confirm the $E(1300)$ by staying near its unitarity 1 imit around $1200 \mathrm{MeV}$ (APEL 79).

Independent evidence for the $\varepsilon(1300)$ comes from studies of the $k \bar{k}$ systems. In the reaction $\pi^{-} p-k_{s}^{0} k_{s}^{0} n$, the $s$ wave exhibits a large intensity in the $1300 \mathrm{MeV}$ region (WETZEL 76, LOVERRE 80), with some evidence for a bump. Moreover, the $\mathrm{Y}_{0}^{2}$ moment shows a large negative excursion indicating S-D interference (CASON 76, WETZEL 76, POLYCHRONAKOS 79, GOTTESMAN 80, LOVRRRE 801. The rain problem is the isospin of the burp: if OPE were the only mechanisn, $I=0$ would be assured. However, an $I=1$ non-OPE contribution in the same region cannot be excluded. Hoceover, the $I=1$
Data Card Listings

For notation, see key at front of Listings.

$\mathrm{K}^{-} \mathrm{K}^{0}$ system doeg show some peaking (MARTIN 79). so one will posalbly have to disentangle two resonances in the $\mathrm{R}_{\mathrm{S}}^{0} \mathrm{~K}_{\mathrm{S}}^{0}$ bump.

In agreenent with this, the $\mathrm{K}^{+} \mathrm{K}^{-}$system produced in $\pi^{-} p, \pi^{+} i$, and $\pi^{-} p$ (prisarized) scattering clearly shows the 5 wave peaking at $1300 \mathrm{CeV}$ Isee Fig. 1 (b), (c) 1 ; again, both $I=0$ and $I=l$ may be present (PAWLICKI 77, MARTIN 79, COHEN 80, COSAn 80, GORLICH 80, WICKLUND 80).

To get from phase shifts to' resonance parameter $s$ and $q \bar{q}$-composition one has to make coupledchannel analyses. It has become evident that it is not enough to consider the channels $\pi \pi$ and $\overline{k K}$ only; n must also be included. Two such fully unitarized analyses (ACHASON 79,80,81, TORNQVTST 82) finally appear to bring order to the scalar su(3) nonet. TORNovisT 82 is more general in considering simultaneously all the pseudoscalarpseudoscalar meson pairs coupling to the full scalar multiplet, but confirms well the carlier results of ACHASON 79,80 .

The picture amerging (TORNQVIST 82) is that of a dominantly $q \bar{q}$-system with large $q \bar{q} q \bar{q}$ components in the form of virtual two-meson bound
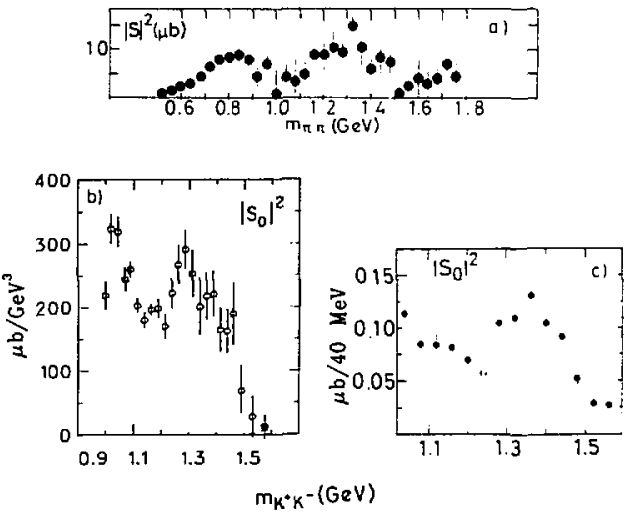

Fig. 1. (a) The absclute intensity (in $\mu b$ ) of the $\pi^{+} \pi^{-} S$ wave in $40 \mathrm{MF} \cdot \mathrm{V}$ bins (without dividing by the bin size), as given by the "down" solution of BECKER2 79. (b) Absolute intensity (in $\mu b / \mathrm{Gev}^{3}$ ) of the $\mathrm{K}^{+} \mathrm{K}^{-} \mathrm{S}$ wave, as given by the favored solution of COHEN 80 , for $|t|<0.08 \mathrm{GeV}^{2}$. (c) The absolute intensity (in $\mu \mathrm{b} / 40 \mathrm{MeV}$ bin) of the $\mathrm{K}^{+} \mathrm{K}^{-} \mathrm{S}$ wave, as given by the favored solution of GORLICH 80 . 


\section{Data Card Listings

states. At the Su(3) level, two isoscalar resonances are needed, a narrow $s^{*}$ superimposed on a broad $\varepsilon$. The position and width of the $s^{*}$ are well determined by its interference with the $E$, visible as a dip in the $\pi \pi+\pi \pi$ cross section. The $5^{\star}$ appears particularly clearly as a $\pi^{+} \pi^{-}$peak in $J / \psi$ decay (GIDAL 81 ) and as a 2-prong peak in $\gamma$ interactions (BRANDELIK 81).

The mass and width of the $\varepsilon$, however, are difficult to define in any simple way, its Breltwigner shape being completely distorted by hadronic mass renormalization effects (cusps) from the $\pi \pi, k \bar{K}$, and $\eta \pi$ channels (MORGan 74, ACHASOV 79,80,81, IRVING B1, TORNOVIST B2).

The long-standing contradiction between the small coupling of the $s$ to $\pi \pi$ - evidence for a pure $\mathbf{s} \overline{\mathbf{S}}$ system - and the Gell-Mann-okubo mass formula prediction of the $s^{*}$ being an su(3) singlet, is also now resolved. The mixing angle turns out to be a very strong function of energy, nearly ideal below the $K \bar{K}$ threshold, but rapidly approaching the configuration $s^{\star}$-octet/ $\varepsilon-s i n g l e t$ at about $1400 \mathrm{MeV}$ (TORkQVIST 82). This also lends support to the simpler analyses which find an average $\overline{K K}$ branching ratio in the 1300-1400 $\mathrm{MeV}$ region of the order of 108 (MARTIN 79, ESTABROOKS 79. GREENHUT 79, COSTA 80, GORLICH BO, LOVERRE 80).

For further discussion of the scalar nonet, see the mini-reviews under $\delta(980)$ and $k(1350)$ and Appendix IIC.

16 EPSILON MASS TKEYI

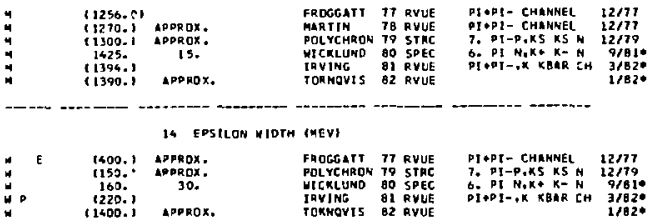

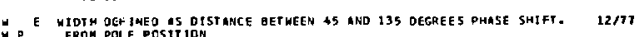

14 EASTLOM PARTIAL DeECAY mODES

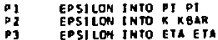
OECAY MASSES $139 * 139$ 497.597
540.540

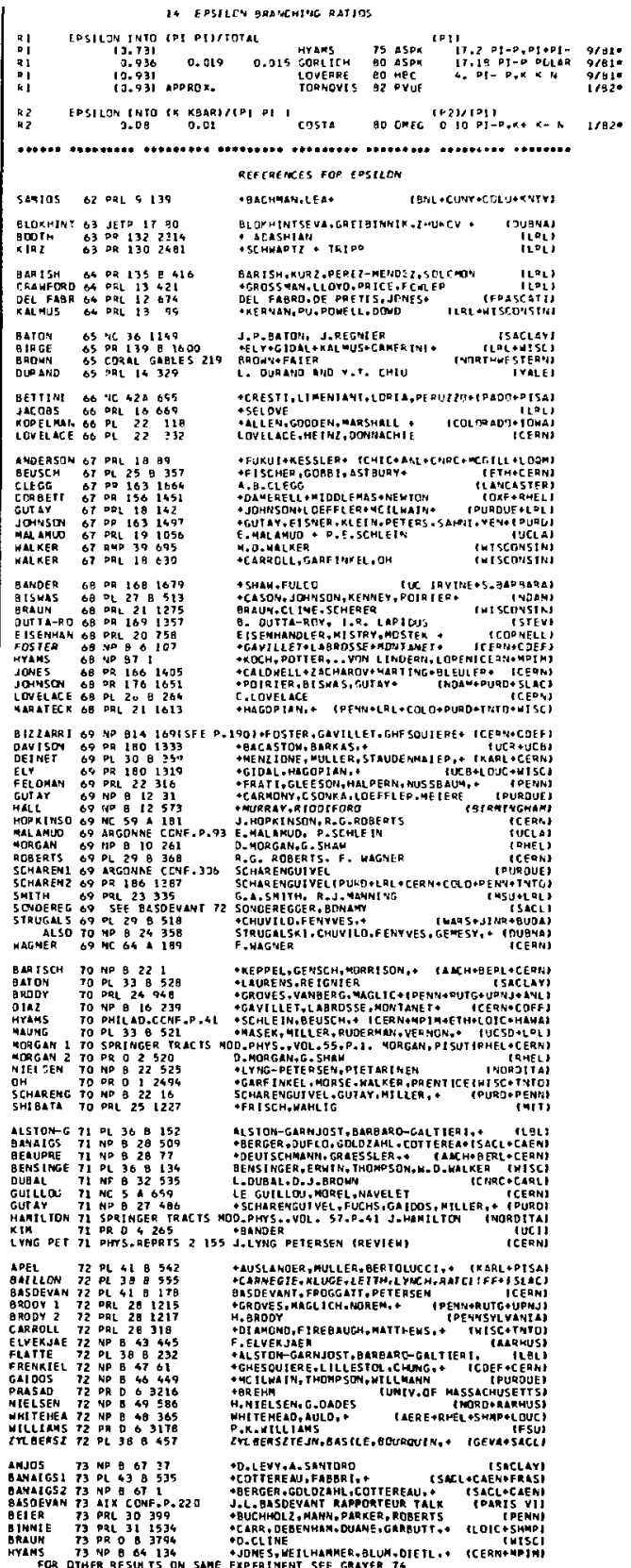


Mesons

$\epsilon(1300), \pi(1300), A_{2}(1320)$
Data Card Listings

For notation, see key at front of Listings.

DCHS THESIS

PILKUHN PB

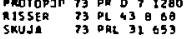

BASOEYAN 74 $7 F$

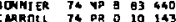

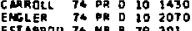

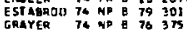

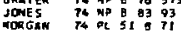

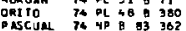

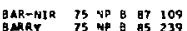

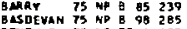

DDNOHLE 75 KC 25 A 400

FRDGGAT 75 NP B O 15.54

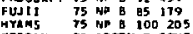

MOAGAN 75 ARTOMAE CONF.

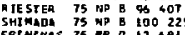

BANAIGS Th HP E 10552

CASOA 76 PRL 361485

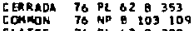

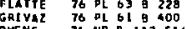

Garvar 76 ND 8112514

WEIZEL 76 NP 8115208

FRDGEAT II HP Q 12989

OAVIO 77 PD O 1262027

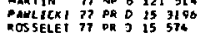

coutlar 78 MP \& 14073

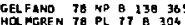

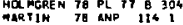

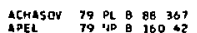

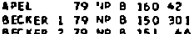

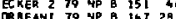
COAOEN 70 4O 157250

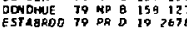
GREENHW T9 PR O 202328

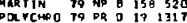

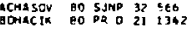

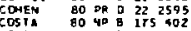
COS BILY 80 40 g 176 1 Gon

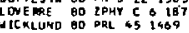

tCMASON O1 PL 102 \& 190 AGullar al zPHY $C$ to 290 OORE ANS gI

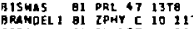

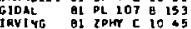

THESIS

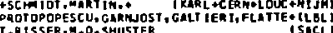

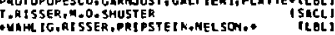

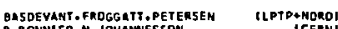

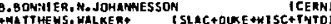

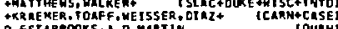

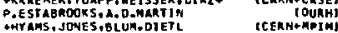

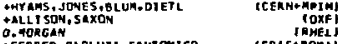

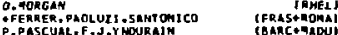

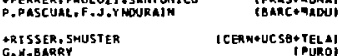

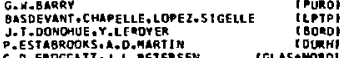

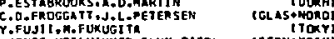

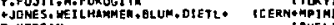

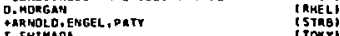

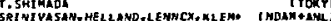

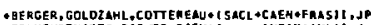

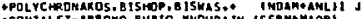

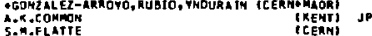

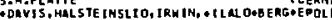

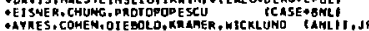

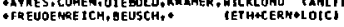

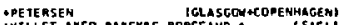

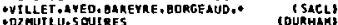

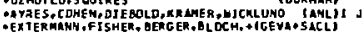

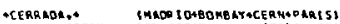

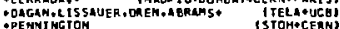

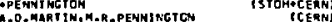
OOE VYAYIN, SHEET AKON -aU SLAMOER, MULLER, ReHakt TKarlopisal

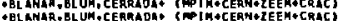

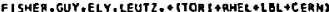

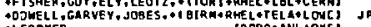
-LEROYF
TESTEOANL +OXXI) P.ESTABRonxs INTEMANA

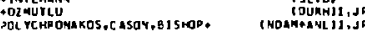

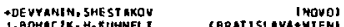

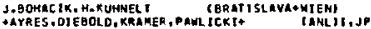

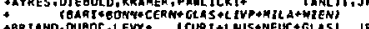

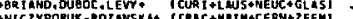
GOT TE SNAN, JaCOBS

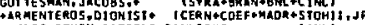

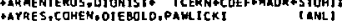

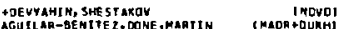

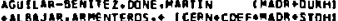

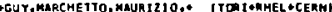

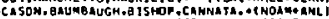

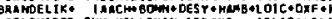

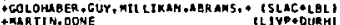

N-R. TORngV ISt

IMELSI

* on

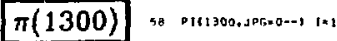
SEEN IM PARTSAL MAVE GRALYSIS OF THE
OIFFP ACTIVELY DROOUEE 3 PI SYSTEF.

58 DIII3001 MASS (MEU)

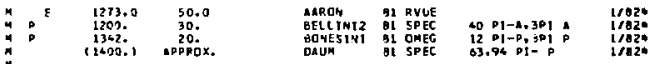

4. ayerage méayingiess iscile factor = z-6)

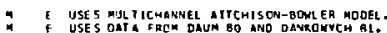

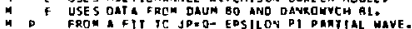

\section{SA PIILJODI MTOTH IMEVI}

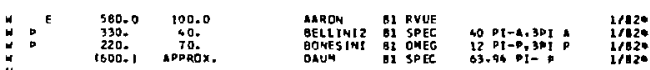

" average méailitigiess" iscile factor = 2.11

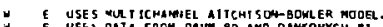

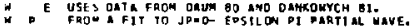

SQ PISI300I PARTt A DECAY MODES

P1 PIC13001 Into \&HO DI

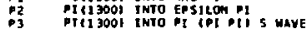

OECAY mASSES

$1300+139$

$1390+139+139$

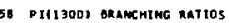

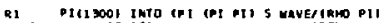

3/82*

R1 E USES RU TTCMAHEL AITCHISOH-BONLER MOOEL.

:

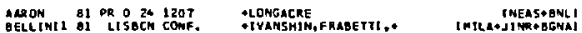

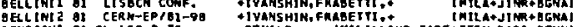

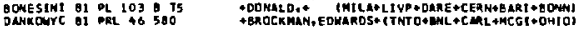

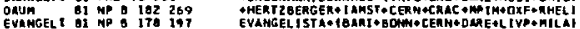

-

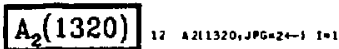

CML EXPENIHENTS GIYJME HASS E AROR LESS THAN $15 \mathrm{MEV}$

12 I2 MASS IMEQI, BPI HODE

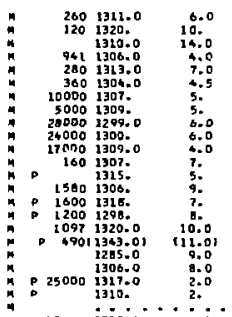

ARMENISE 60 DEC $0.5 .1 P 100 \quad 9 / 67$

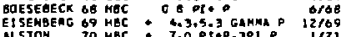
DOEKRANH TO FiBC 05, Pl OP BARNHAM 71 HEC + 3.7PI+ P. [3PII. 11/71 BINHIEI 71 WMS = DT-0 MEAR A2 TMR MIMI BOWEN 71 AMS - 5. P1- P gONEN TI AMS - T. PI- D P

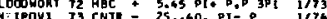

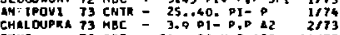
EMAS 75 OBC

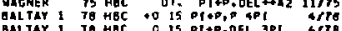

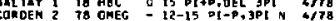

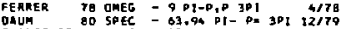

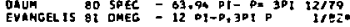

a u

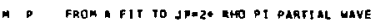

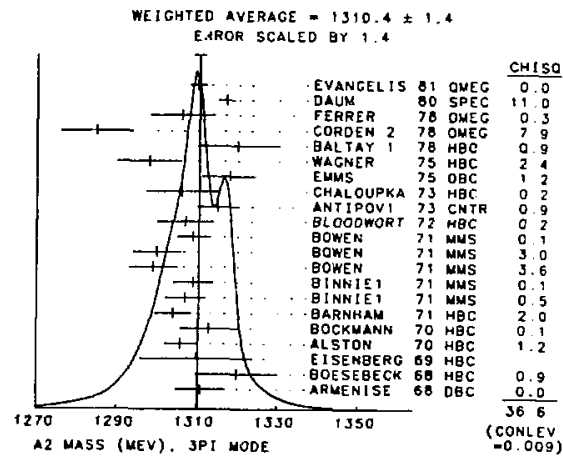

12 AZ MASS IMEVI. CHARGE Q KBAR DOOE

$\int_{n}$

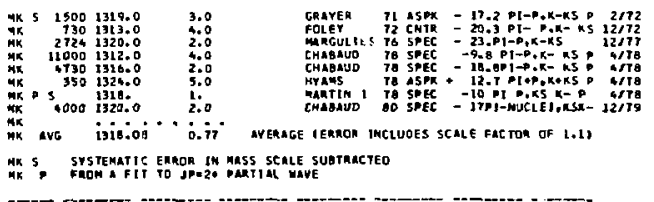




\section{Data Card Listings}

For notation, see key at front of Listings.

12 A2 MASS IMEYI, IIN OI MODF

\begin{tabular}{|c|c|c|c|c|c|c|}
\hline $\begin{array}{ll}\mathrm{E} & 1000 \\
\mathrm{EM} & 200 \\
2561 \\
1653\end{array}$ & $\begin{array}{l}1123 . \\
11324.1 \\
1136.2 \\
1330.7\end{array}$ & $\begin{array}{r}9 . \\
8.9 \\
1.7 \\
\therefore .6\end{array}$ & $\begin{array}{l}\text { MEV } \\
\text { COMFGNTO } \\
\text { DEEFOSSE } \\
\text { DELFOSSE }\end{array}$ & $\begin{array}{l}\text { 13 CSPK - } \\
73 \text { OSPK : } \\
\text { B1 SPEC: } \\
81 \text { SPEC : }\end{array}$ & 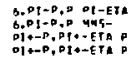 & $\begin{array}{l}1 / 76 \\
1 / 76 \\
1 / 82 \\
1 / 820\end{array}$ \\
\hline syô & injo: & i.i & WVERACE IE RRDA & IHCtutes S & ALE RACTOD OF 2.93 & \\
\hline
\end{tabular}

12.2 UtOTM (MEV). 301 MODf

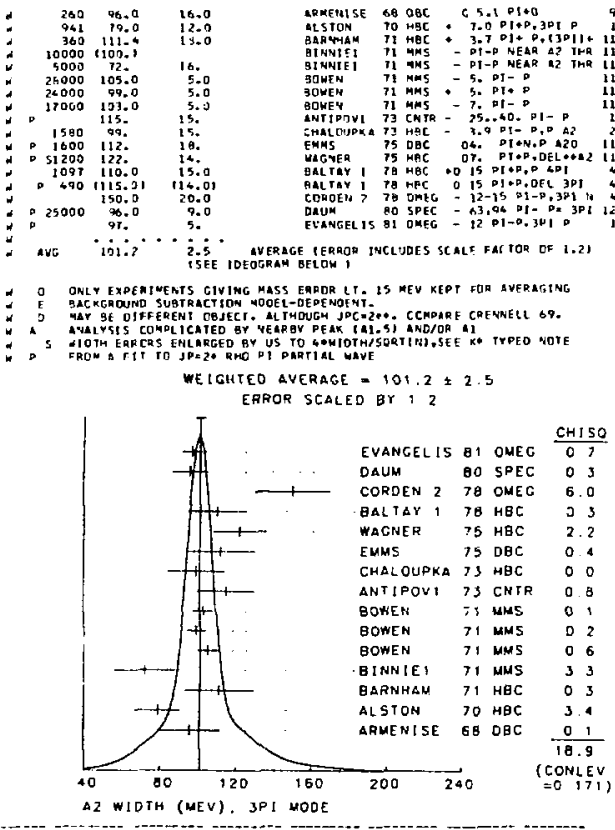

12 Iz MTOTH IMEVI, CMRREEO $\times$ KEAR MUOE

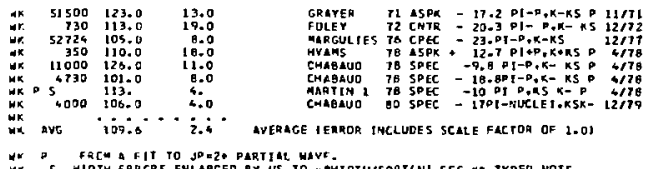

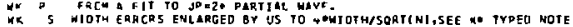

12 A2 WIOTH (NEV), ETA OI MODE

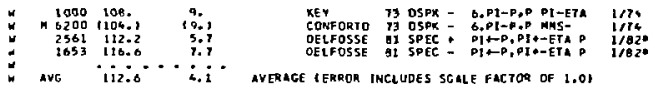

AVG II2.0 AVRAGE TERROR INCLUDES SGLLE FACTOR OF 1.0

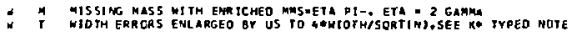

12 Az pabYial oEcay moties

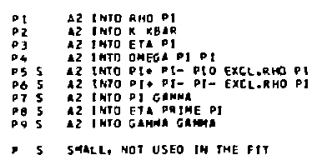

DECAY MASSES

709+ 139

$593+197$

$139+339 * 102$

139* 139. 134

139. 130

5 SThY L, NOT USEO IH THE FT1
F1T TED PAHTML DECAY MUDE BRANCHINC FRACTIONS

The matrix below is derived from the esror matrix for the fitted partial decay mose

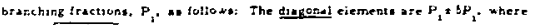

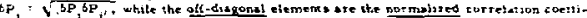

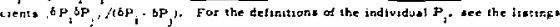
above, only those $r_{1}$ appearing an the metrix are assumed in the $f_{11}$ to be nonzera ans ate this constrained to atid to t.

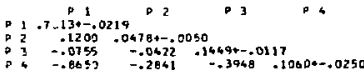

12 A2 PARTIAL WLOTHS

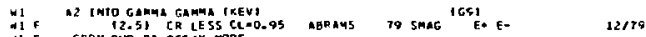

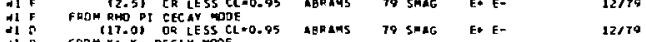

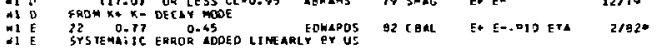

\section{Iz GRAMHIWE RATIOS}

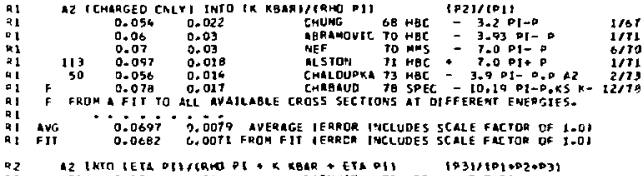

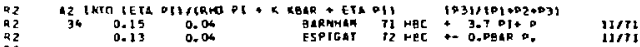

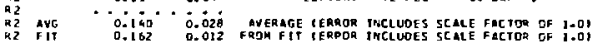

Q 3 A2 INTO IETA DII, / (FHO PI)

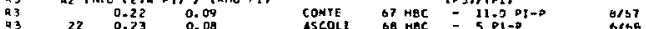

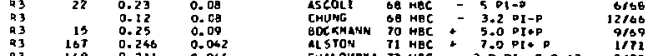

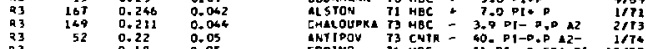

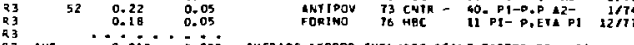

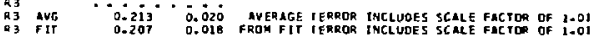

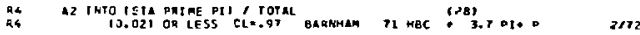

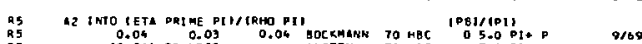

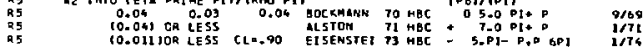

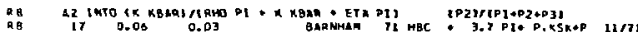

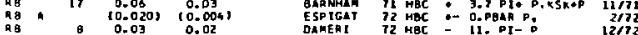

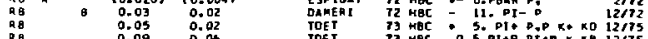

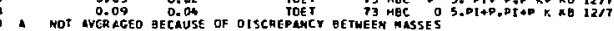

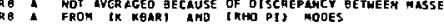

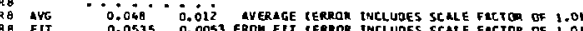

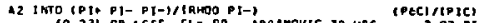

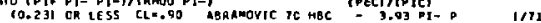

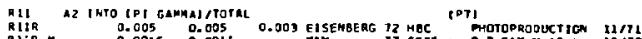

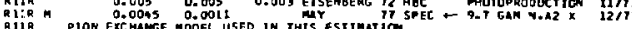

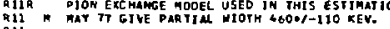

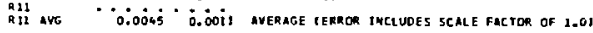

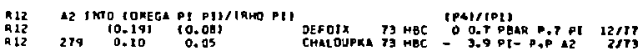

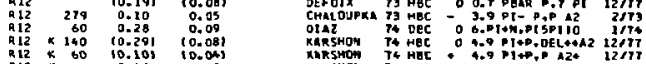

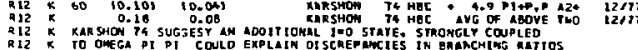

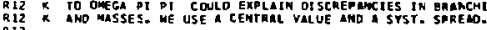

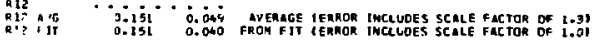

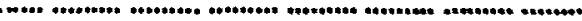
MEFEREMCES FOH az

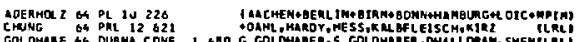

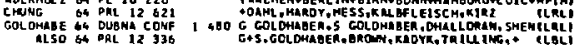

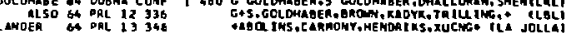


Mesons

$A_{2}(1320), E(1420)$
Data Card Listings

For notation, see key at front of Listings.

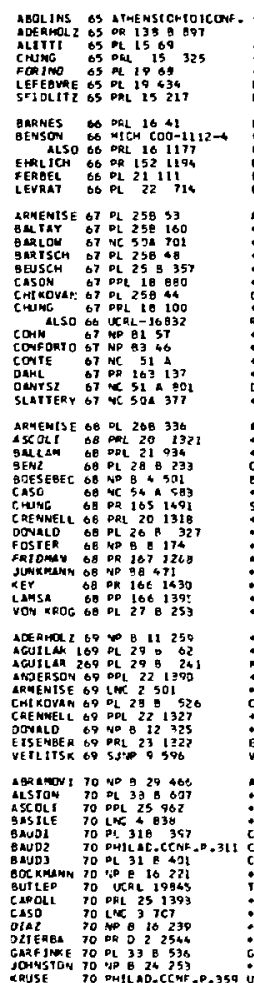

crume 05 ped 15 , 325 LFIOLIT GS PAL 1521 21

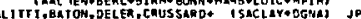

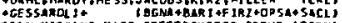
CERN MISS 'NG MASS SFECTROMEJEA GAOUP ICERN BRREES FOWLER,LAI, ORENS TELH - IBNL +CUNYH

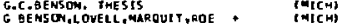

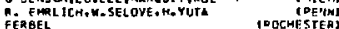
FEREEL

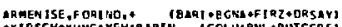

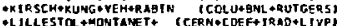

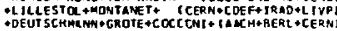

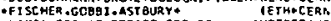
- LAHSA, BISHAS, DERADO,GROVES, CNOT DEEANES

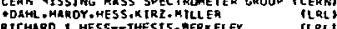

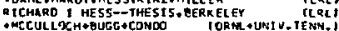

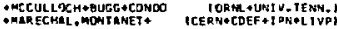

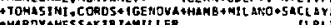

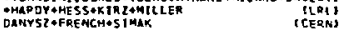
TKRAYE ILL+FORMAN+FEREIL IYALE*RDCHI] JP

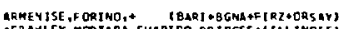

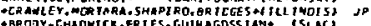

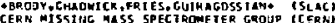
DOE SEOSTE DeUrs

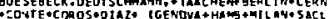

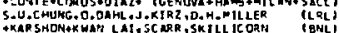

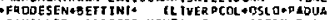

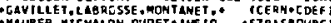

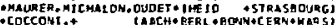

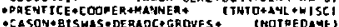

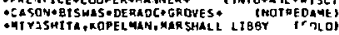

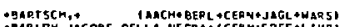

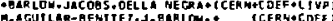

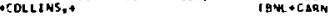

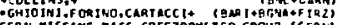
CERA HISSTAC MASS SPECTROMETE SROUP (LEQSi) JP

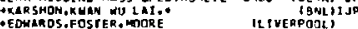

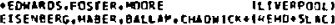
VEILIYSKY. Go. IGOREYEY, GR ISHIN,

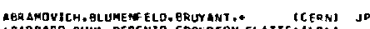

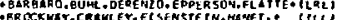

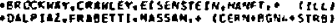

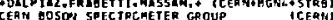
CEAN BOSON SPECTI, SAETER GROUP

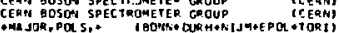

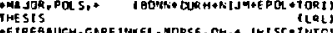

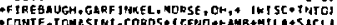

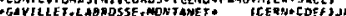

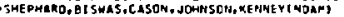

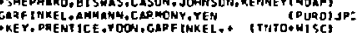

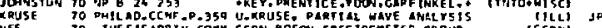

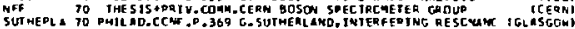

\begin{tabular}{|c|c|c|}
\hline 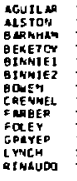 & 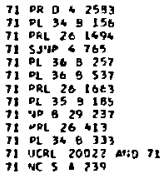 & 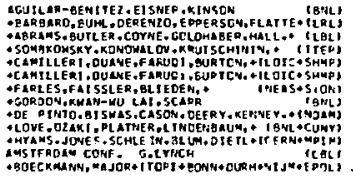 \\
\hline 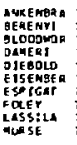 & 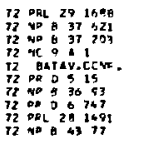 & 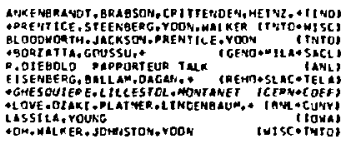 \\
\hline 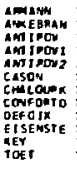 & 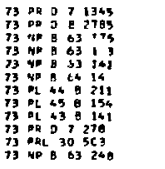 & 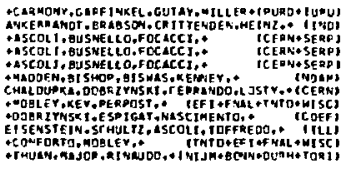 \\
\hline 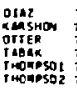 & 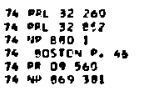 & 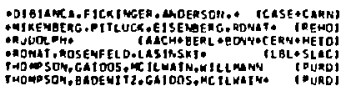 \\
\hline
\end{tabular}

ABasmian 75 PCAL 34691 EMHS 15 PL

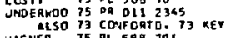
GAGNER 75 ML $58 E$ EDT FOM 1 Ne 70 NC 135 to5 HAROLER TO NP B 11617 CERR LOR 77 YP PAT ICK I 72 PQ

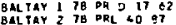
CHAACOD 78 \%O B 14534

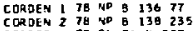

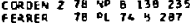

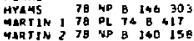
AER AMS 19 SLAC-PUS ?\$2I CHABSUV 80 DQ 9175199

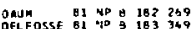

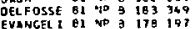

EDWARDS EZ PL 120 Q

......... : :.......................... $E(1420)$

The $\mathrm{E}(1420)$ has $\mathrm{J}^{\mathrm{PC}}=1^{++}$(DIONISI 80) just like the isoscalar companion $D(1285)$. Thus the $D$ and $E$ normally both appear in the same experiments.

Recently a new resonance, the $r(1440)$, has been claimed in the decay $\mathrm{J} / \psi+\gamma \mathrm{K}^{+} \mathrm{K}^{-} \nabla^{-1}$ (Scharfe $80,81)$, On top of the $E$. It differs fror the $E$ in three respects:

(a) No D (1285) is produced.

(b) It has $\mathrm{J}^{\mathrm{PC}}=\mathrm{0}^{-+}$.

(c) Its decay is dominantly $\delta(980) \pi$, unlike the $E(1420)$.

We note that the early $\bar{F} p$ annihilation experiment at rest (BAILLON 67) agrees with (a) and (b).

We do not open a new entry for the $2(1440)$ for this edition, but we group BAILLON 67 and SCHARRE 80,81 separately under the $E(1420)$.

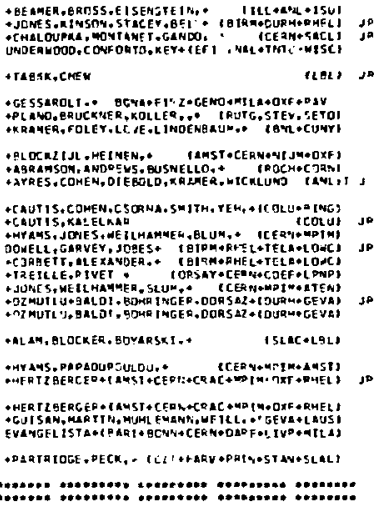

EJAMES. 


\section{Data Card Listings}

For notation, see key at front of Listings.

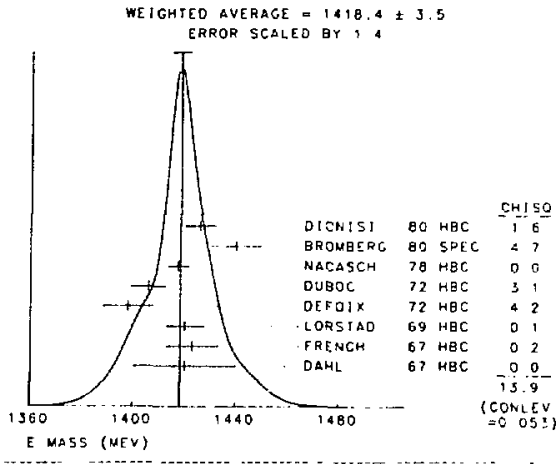

a E MIOTM (meV)

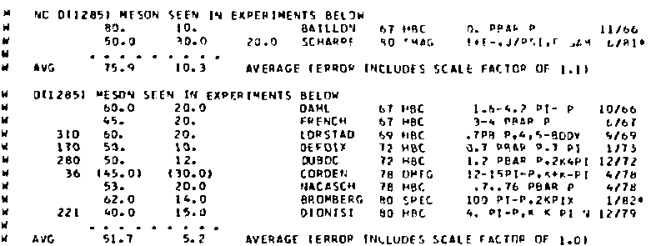

- f phptal ofcay modes

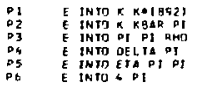

Defse masses

4976.891 .139

1390.1970 .139

$563,139.139$

$1394.1394 .139+130$

$$
\text { - e grahenthg ratios }
$$

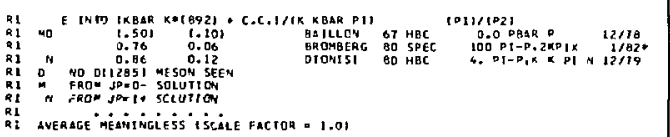

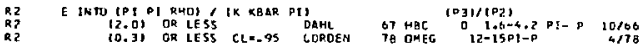

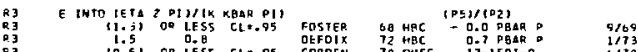

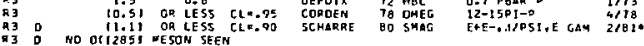

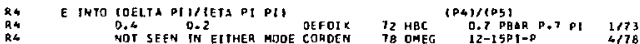

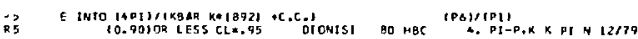

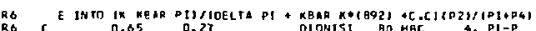

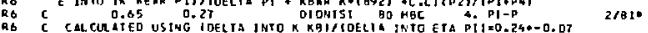

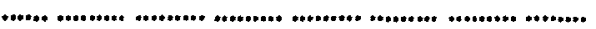

BAILLON DT NC SOA 393

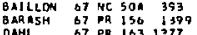

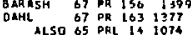
FRENCH GT OF PRL 524 A 1074

FOSTER GQ MP 3 G 114

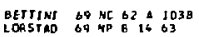

OEVONS 71 PAL 273614

$\begin{array}{llllll}\text { CMAPNAN } & 72 & \text { NP } & 42 & 4 \\ \text { OEFOIX } & 72 & \text { NP } & \text { B } & 44 & 125 \\ \text { OUEOC } & 72 & \text { NP } & \text { B } & 46 & 429\end{array}$

VUILLEN 75 LNC $1+165$

\section{REFERENCES FOR E}

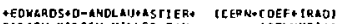
TARASH,KIA SCH,HILLER, TAN TC TCOLUMBIA] MILLER,CHUMG. DAHL, HESS, MAROY, KIRZ + ILQL OUCGI

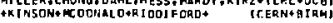

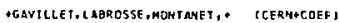

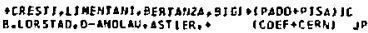

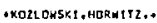
(COLL+SYRa)

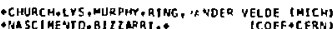

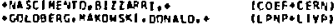

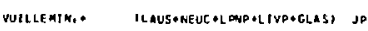

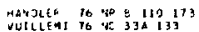

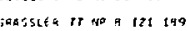

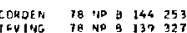

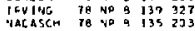

STONION 79 PRL 42346

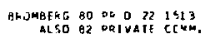

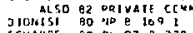

CHANCWT HI Pal 40 GE

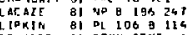

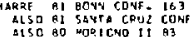

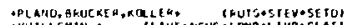

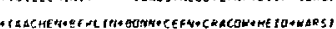

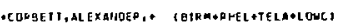

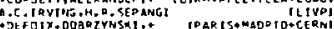

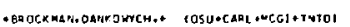

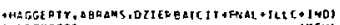

C. HAOARER

: Gavila,

CHASOMITI

+NAVELTT

LIDKIN

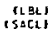

(FNAL)

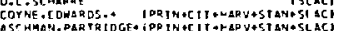

$=$

\section{i(1515)}

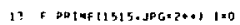

$$
17 \text { F PRIME 4A55 RMFVI }
$$

$$
\begin{array}{ll}
0 \\
p \\
0 \\
4 \\
4 \\
4 \\
4 \\
4 \\
4 \\
4
\end{array}
$$

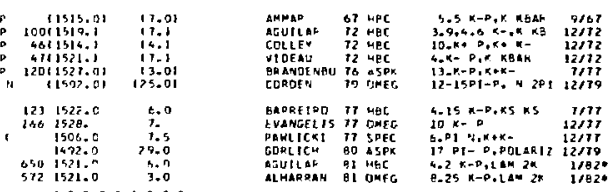

ave is $200^{\circ} \cdot 3.5^{*}$ avehage tepaga theludes scal f ractop or 1.11

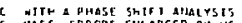

MASS EDFCES ENI AHGED BY US OT FACT7O 1.5.

FPOA AN AMPL T TUOE APRLYSIS KHERE THE FPR TNE WIOTH AFIO

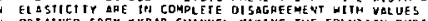

\begin{tabular}{|c|c|c|c|c|c|c|c|c|c|}
\hline 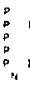 & $\begin{array}{r}150 \\
4 t \\
47 \\
120\end{array}$ & $\begin{array}{l}135.01 \\
169.1 \\
129.1 \\
143.1 \\
151.01 \\
1105.01 \\
150.01\end{array}$ & $\begin{array}{l}175.01 \\
122.1 \\
115.1 \\
130.1 \\
18.01 \\
422.01 \\
103.01\end{array}$ & $(50.0)$ & $\begin{array}{l}\text { AHKAP } \\
\text { ACUILER } \\
\text { COLLEY } \\
\text { VIOEAU } \\
\text { GPANDE'IBU } \\
\text { COPOEN } \\
\text { GOMLICH }\end{array}$ & $\begin{array}{l}37 \\
72 \\
72 \\
72 \\
76 \\
79 \\
60\end{array}$ & $\begin{array}{l}\text { HAC } \\
\text { HAC } \\
\text { HAC } \\
\text { NBC } \\
A S P X \\
\text { OMCG } \\
\text { ESOK }\end{array}$ & 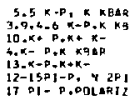 & $\begin{array}{r}9 / 51 \\
12172 \\
12172 \\
12172 \\
7177 \\
12179 \\
12179\end{array}$ \\
\hline c & $\begin{array}{l}123 \\
1 \in \Delta\end{array}$ & $\begin{array}{l}52.0 \\
72.0 \\
36.0 \\
92.0 \\
98.0 \\
90.0\end{array}$ & $\begin{array}{l}19.0 \\
25.0 \\
15.0 \\
39.0 \\
16.0 \\
14=0\end{array}$ & 0 & 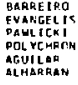 & $\begin{array}{l}37 \\
77 \\
77 \\
79 \\
81 \\
91\end{array}$ & $\begin{array}{l}\text { PEC } \\
\text { GMEG } \\
\text { SPEC } \\
\text { SIRC } \\
\text { HEC } \\
\text { CMEC }\end{array}$ & 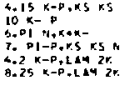 & $\begin{aligned} 7277 \\
12177 \\
12177 \\
12179 \\
11820 \\
1 / 820\end{aligned}$ \\
\hline
\end{tabular}

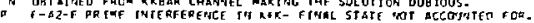

$$
13 \text { F PRTME HIDTH IMEYI }
$$

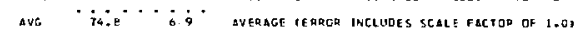

C WTH A PMASE SHTFT ANALYSIS

C WIDTH EFPCAS ENLEQGED HY US EY FACTOR 1.S.

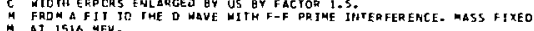

N SEE VDTE U UNOE MASS.

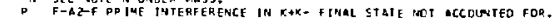

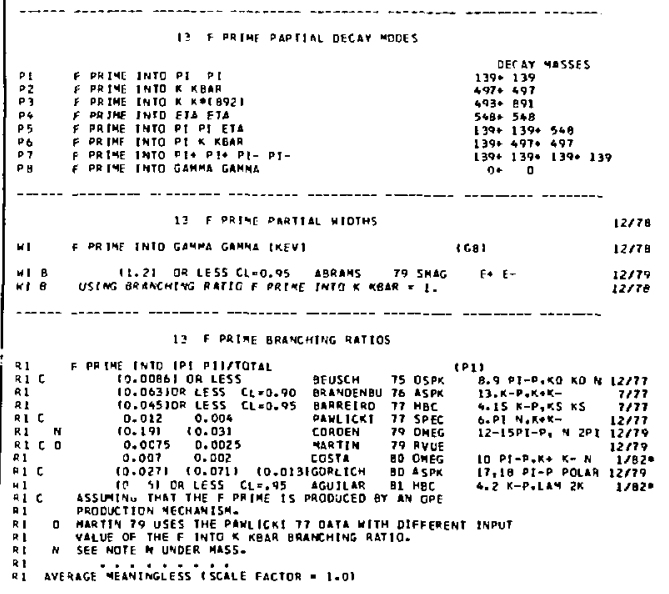


Mesons

$f^{\prime}(1515), \rho(1600)$
Data Card Listings

For notation, see key at front of Listings.

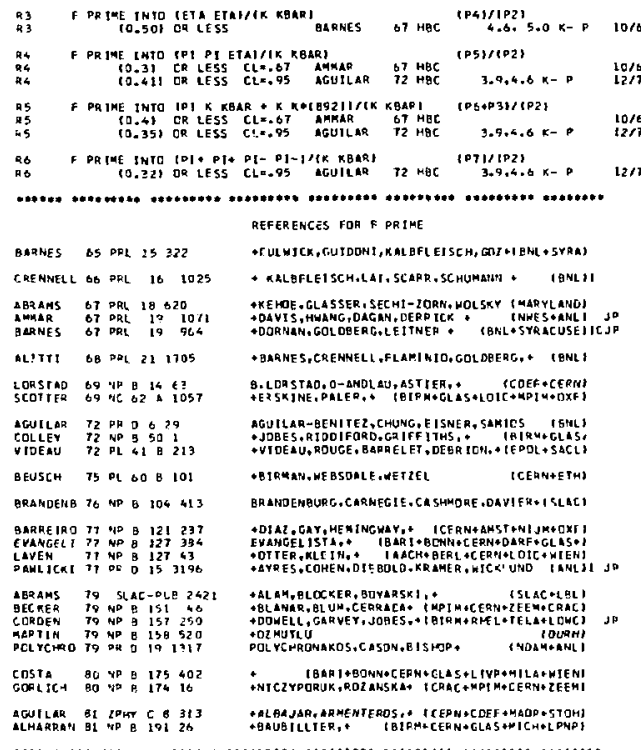

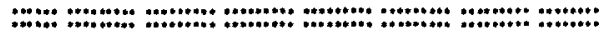

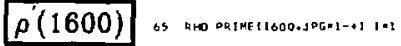

The $\rho^{\prime}(1600)$ has been seen in the $\rho^{0} \pi^{+} \pi^{-}$final state, in photoproduction (BINGHAM 72, DAVIER 73, SCHACHT 74, ALEXANDER 75, LEE 75, ATIYA 79, RICHARD i9, BARBER 80, ASTON 81), in $\mathrm{e}^{+} \mathrm{e}^{-}$annihilation (BARBARINO 72, CONVERSI 74, CORDIER 79, COSME 79, BACCI 80, DELOOURT 81), in electroproduction. (KILLIAN 80), and in a $\pi^{+} d$ experiment (DIBIARCA 81). If the $\pi^{+} \pi^{-}$subsystem were in an $S$ wave, as has often been assumed, one would also expect to see the $p^{\prime}$ decaying into $\rho^{0} \pi^{0} \pi^{0}$. This has, however, not been seen (ATKINSON 82), Thus the most likely decay chain is $\rho^{\prime}+A_{1} \pi+\rho \pi r \rightarrow 4 \pi$.

For the determination of the $\rho^{\prime}(1600)$ parameters we turn to its relatively rare $\pi^{+} \pi^{-}$decays, which do not have the problems of the above decay chain. The $\pi^{+} \pi^{-}$final state has been produced in $\pi$ " $p$ interactions (HYAMs 73, BECKER 79), in photoproduction (ATIYA 79, ASTON 80), and with weaker evidence in $\mathrm{e}^{+} \mathrm{e}^{-}$annihilation (reviewed by GENSINI 78, HEYN 80). The mass and width in these experiments are consistently $1600 \mathrm{MeV}$ and $300 \mathrm{MeV}, \mathrm{re}-$ spectively. Note, however, that these paraneters are the results of very simplified analyses which are not adequate for such a broad resonance. An atterapt to determine the $\rho^{\prime}$ pole position in a more mode1-indepe- Jent way (LANG 79) from the Hyams 73 data $Y$ ields a mass at 1660 MeV.

The elusive $\rho^{\prime}(1250)$ has recently been reclaimed in the diffractively photoproduced $w^{\circ}$ system (ASTCN 80, BARBER 80). However, the $\mathbf{J}^{\mathbf{P}}$ determinations are complicated by the simultaneously present $B$ (1235) resonance. In addition, other dynamical effects obscure the interpretation of the $\rho^{\prime \prime}(1250)$ as a resonance.

AS PHO ORIMF MASS INEV

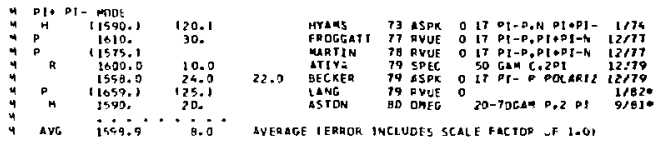
zret* $p 1-1$ Hot

$450 \quad 1430$.

1601550

50.

D 851550.1 150.1 160.1

Ax 1160t.1 139.1

$\theta \quad 1411700.1$

1520.020.

20.
60.
25.

1570

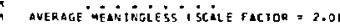

BINGHAM 72 HAC

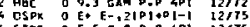

SCHACHT 74 STPC 0 5.5-9 C P.P 4P1 12775

ALEXANOE 75 HAC 07.5 GAMP. API $12 / 75$

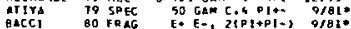

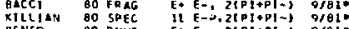

20-70,4 $P, 4$ pI $9 / 910$

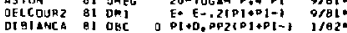

- S STMDE RELATTY. BREITAIGNER FII MITH MUOEL DEPENDENT HIOTH ASSIMING PHD+EPSILON DECAY MDOE INTERFERES WITH AITPI BACKGRTUUT

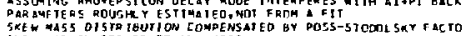

STMPLE RECATI.

FACM PHASE SHIFT AHALYSIS DF HYAMS TZ OATA

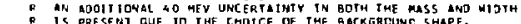
1S PRESEHT DUE IDTHE CHOTCE OF THE BACKGRCUNE 5 HAPE.

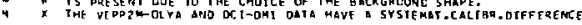

$$
\text { BS PHO PRIME WTOTH TMEVI }
$$

\begin{tabular}{|c|c|c|c|c|c|c|c|c|}
\hline 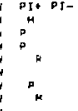 & 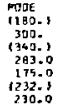 & $\begin{array}{l}150.1 \\
100 . \\
14.0 \\
98.0 \\
136.1 \\
80.0\end{array}$ & $\$ 3.0$ & $\begin{array}{l}\text { HYAHS } \\
\text { FROGGATT } \\
\text { MARTII } \\
\text { ATYYL } \\
\text { BECKER } \\
\text { LANE } \\
\text { ASTON }\end{array}$ & $\begin{array}{l}73 \\
77 \\
78 \\
79 \\
79 \\
79 \\
80\end{array}$ & $\begin{array}{l}\text { ASPK } \\
\text { DVUE } \\
\text { DYUE } \\
\text { SPEC } \\
\text { ASPK } \\
\text { PYUE } \\
\text { DUEG }\end{array}$ & 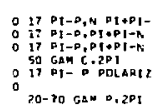 & $\begin{array}{l}12179 \\
12177 \\
12177 \\
12179 \\
12574 \\
11820 \\
9 / 624\end{array}$ \\
\hline AvG & 278. & 14.6 & JER & If RRCD & $\mathrm{NCt}$ & s. & TOA OF 1.11 & \\
\hline 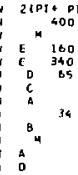 & $\begin{array}{l}1-1400 t \\
650 . \\
367 . \\
400 . \\
553 . \\
1340.1 \\
1000.3 \\
1700.3 \\
110.3 \\
1230.1 \\
200 . \\
510 . \\
400 .\end{array}$ & $\begin{array}{l}100 . \\
100 . \\
120 . \\
700 . \\
1110.1 \\
1100.1\end{array}$ & & 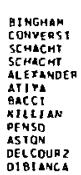 & $\begin{array}{l}72 \\
74 \\
74 \\
74 \\
75 \\
70 \\
80 \\
80 \\
80 \\
81 \\
81 \\
81\end{array}$ & 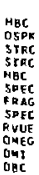 & 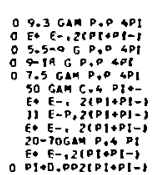 & $\begin{array}{l}12 / 72 \\
12 / 75 \\
12 / 75 \\
12 / 75 \\
12 / 75 \\
91810 \\
9 / 610 \\
9 / 810 \\
9 / 810 \\
9 / 810 \\
9 / 810 \\
1 / 1820\end{array}$ \\
\hline
\end{tabular}

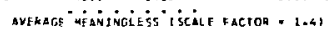

SIMPLF RELATIY. BREITMIGMER FIT HITH MODEL OEFENOERT MIOTH

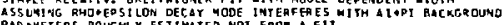
DAQAMETERS ROUGH Y ESTTMATED, NO1 FROE A FII

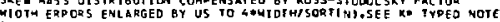
INC. LUJEO IN EECKER 79 AYACYSIS ST MPLE RELATTY. BREIT

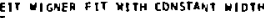

FRDH DHASE SMIFT AYALYSIS OF HYAMS 73 DAIA

THE MASS AND WTOIH

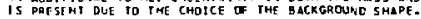




\section{Data Card Listings}

For notation, see key at front of Listings.
Mesons $\rho(1600), \theta(1640)$

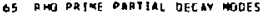

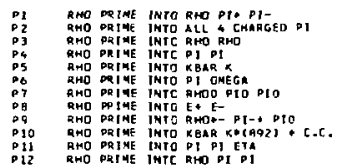

G5 RHO PRIME PAFTIAL WIOTHS IKCY

42 RHO PRINE THTE E* E-

(6)

$\times 120 \quad 17.51 \quad(1,5)$

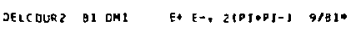

H: D MOOEL DE PENAENT, NOT IMOEAEMOENT OF DELCOUA 2 EI WICTM TIMES EOE-

\section{G5 RHO PRIME GRANCHTNG RATITS}

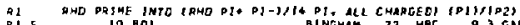

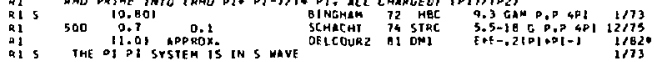

R3 RHO PRTME IHTE (PI, PT-1/14 PI, MLL CHAOCEO) (PL)/1P?)

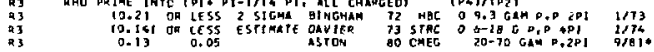

R4 RHO PAIME INTC (PBAR NOJI4 PI, ALL CHARGEO) HEC TPSIPTPZI

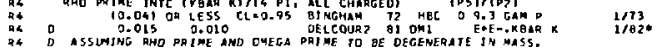

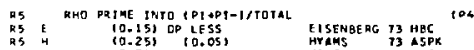

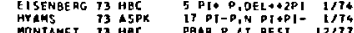

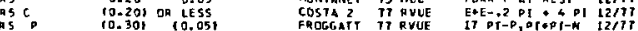

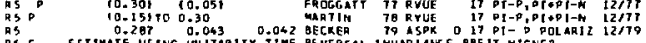

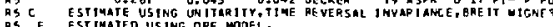

AS F EST IMATED USING OPE MODEL.

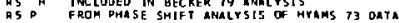

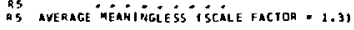

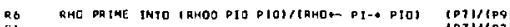

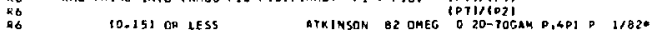

RT RHO PRIAE INTO IPJ OD- MEUTRALSITIGPT, ALL CHARGEDI

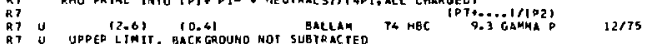

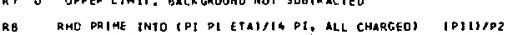

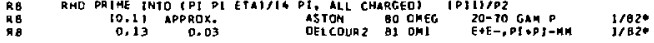

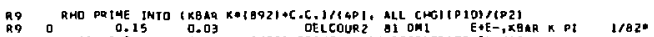

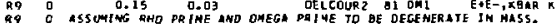

65 RHO PRINE GIITHGIE EE-I/GTTOTALI IKEVI

IHIS COMBIFATION OF A PARTSL WIOTH WITH THE PARTIAL MTOTH INID

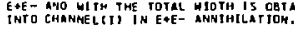

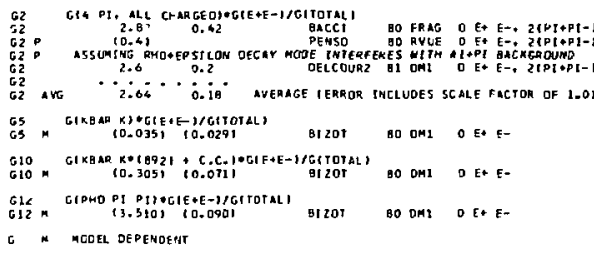

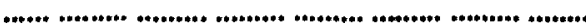
Deferences for rwo prime

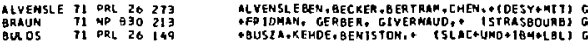

$3 / 82 *$

$3 / 824$

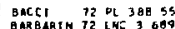

BARBATIN 72 LNE 3609

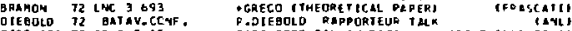

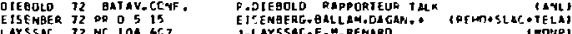

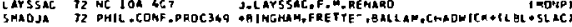

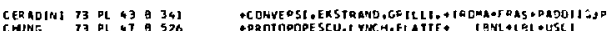

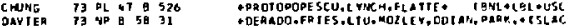

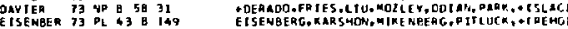

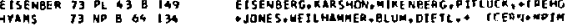

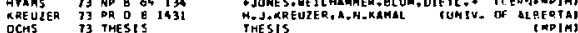

OCHS TH THESIS

DONTANET T3 ERICE SCPOOL

Aallam 74 MP E 70375

GERAAGE T 74 LNE $\$ 11251$

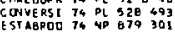

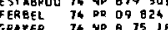

GRAYES T4 NP B 75 LBO

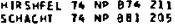

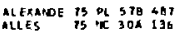

ALLES 75 PC 304136

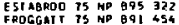

HVEST T5 4P 8100205

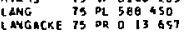

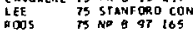

THESIS

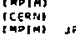

J.C. H. DARK

DD. ANGELD.SPILLAVIINI, VALENTE DOUATFRAS

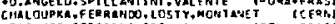

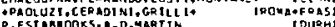

P. ESTMEMOQKS.A-D.MARTIN

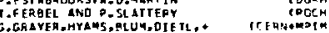

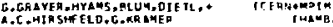

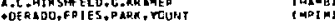

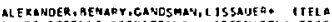

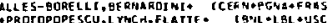

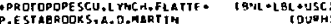

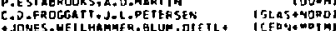

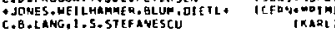

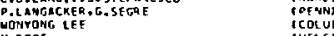

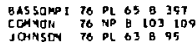

BAS SOKPIERRE , GINDED,*

IMUA H+5TEBTIRI)

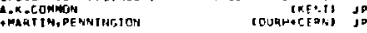

BLONEY TT PL 7S 0305

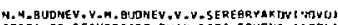
COSTA OE BERUREGARD. PHAM,PIPE,IRJCHE IEPJU tr,lase aguth $\cos r A 277 \mathrm{Pl}^{2} 710345$

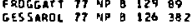
CENSTNT 70 DR D 57 1368

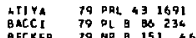

BECKEA IO NP B 151 4D

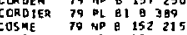

COSHE 79 NO E 152 219

RICHAHD 79 FERMILAS SYMP.

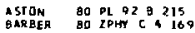

BACE1 BO PL OS E 139

HEYH

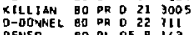

P-DNANEL 60 PL 95 B 143
ALSO 82 NE 1 TO

cesschosita toch

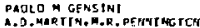

I tiaci

HOL ME S,KNAOP, LEE, SETO,* TCOLUE TLL + TMALI

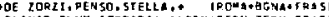

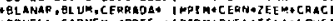

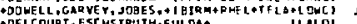

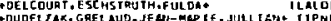

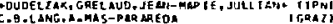
F. TiCHARa

ifreatio

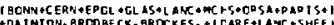

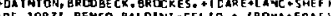

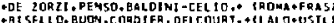

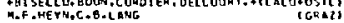

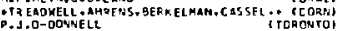

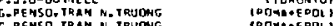

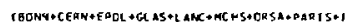

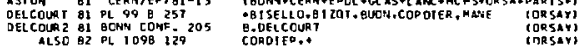
ALSO B2 PL $1098 \quad 1$ ZO

CorDIFo.:

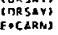

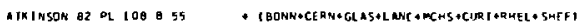

*

$\theta(1640)$ 68 THETAL1640, JPG $=2+0 .+1 \% 0$

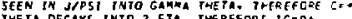
THE TA OECAYS INFO 2 ETh. THE REFORE TCEOT.

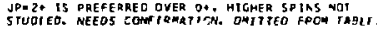
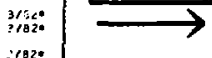

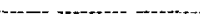

"

GO THETAIIOLOI HASS (MEYI

EOWAFOS B2 CEAL O JTPST, T,AM IETA

"

6 THETAS164D) WIOTH (MEY)

220. 100. 70. EOMAADS B2 CBAL O JMOSI.CAM $2 E$ TS

2PE 20

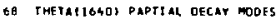

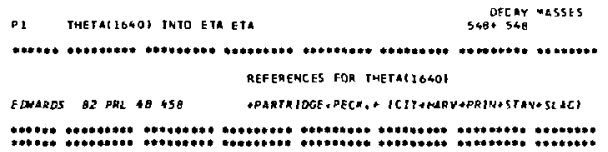


Mesons

$\omega(1670), A_{3}(1680)$

$\omega(1670)$ as OAEGAC1670, JPG=3-- I=0.

45 OMEGR(1670) MLSS TMEYI

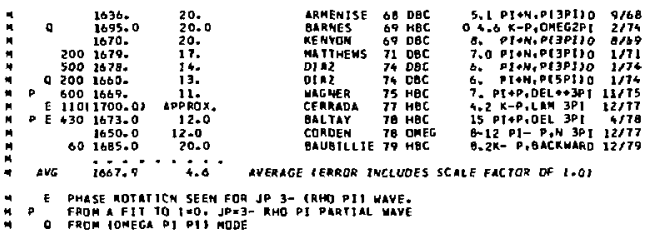

- O FROM IOMECA D1 P1i MODE

45 OAEGASSOTOS WIOTM IMEVI

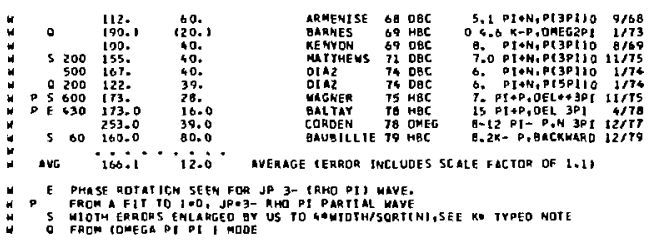

45 DMEGAI26TOY DARTIAL DECAY MCDES

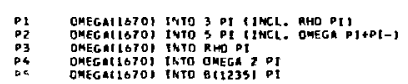

DECAY MASSES

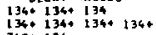

$769+136+134$

$1233 * 136$

45 OMEGACLOTOS BRATCHING FATIOS

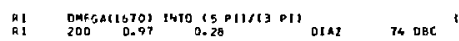

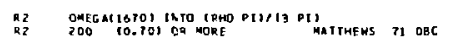

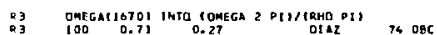

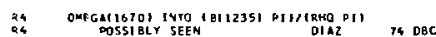

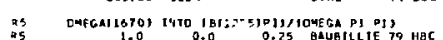

[P2I:TPII

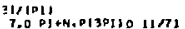

(04) $/ 103]$

PFIIO 12/76

05 Ifro3i

the Pith, Pt5PtIO $1 / 14$

(D5)/ 1040

B.5K- P, QACKMAFO 15179

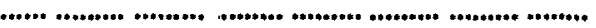

ARMENTSE SQ DL 260336

BLRNES 69 PRL 23162

WENYON 39 DDL 73160

A AMENISE TO LNC \& 190

MATTHEHS 71 OP O 32561

DIAL 14 DQL 32260

WAGTER 75 DL 590261

CERAMCE TI TOP A 126241

Batiay 78 OAL 40 E

BaverLLI 79 OL B BQ 131

+cosen
REFEREMCES FOP DMFGAC1670

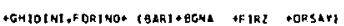

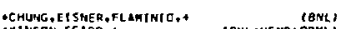

EXINSON.SCARR.* TENL AUCMO DRRE]

+PRENT ICE, YOOH, CARAOLL, * ITATO+WISCI

oDRENTICE, YDON, CARROLL: : TIMTO+HISC)

+DLETAMCA, FICKINGER, ANDERSOH, * ICASE+CMRNI

TABAK,CHEY ILELJ JP

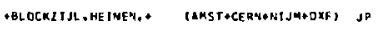

-CAUTIS.KaLELKar

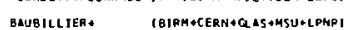

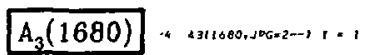

Evidence for the existence of the $A_{3}$ meson was previously confused due to its appearance near the f $\pi$ threshold in the diffractive-like process $\pi N \rightarrow$ $\pi \pi \pi$, much like the $A_{1}$ meson. Thile everybody agreed that there was a $\sim 300$ Mev wide f $\pi$ enhancement in the $J^{P}$ IH $=2^{-}$So partial wave at about 1650
Data Card Listings

For notation, see key at front of Listings.

MeV, some claimed non-resonant states (ANTIPOVl

73, ASCOLIl 73, BALTAY 77), while others saw evidence for a resonance in the phase variation with respect to other partial waves (OTTER 74, THOMPSON 74).

In the non-diffractive charge-exchange reaction $\pi^{+} p+\pi^{+} \pi^{-} \pi^{0} \Delta^{++}$(WAGNER 75, BALTAY 77 , CAUTIS 77) and in the bypercharge-exchanged reaction $\mathrm{x}^{-} \mathrm{p} \rightarrow \pi^{+} \pi^{-} \pi^{0} \Lambda$ at $4.2 \mathrm{GeV} / \mathrm{C}$ (CERRADA 77 ), there is no evidence for $A_{3}$ production.

Definitive proof for the resonant nature of the $A_{3}$ has been given by PERNEGR 78 ( $3 \pi$ system diffractively produced on nuclei) and DAUM 80,81 and EVANGELISTA al $(3 \pi$ diferaction on proton target). In all these experiments the $2^{-} \mathrm{So}^{+}(f \pi)$ partial-wave anplitude exhibits resonance-like phase variation.

In a simultaneous fit to the four $2^{-}$waves $(\varepsilon \pi, \rho \pi$, twice $f \pi)$, DAUM BI needs a heavier companion to the $A_{3}$ in addition to the Deck background. This fit probably gives the most reliable estimates of the $A_{3}$ and its heavier companion, which we name $\pi(2100)$.

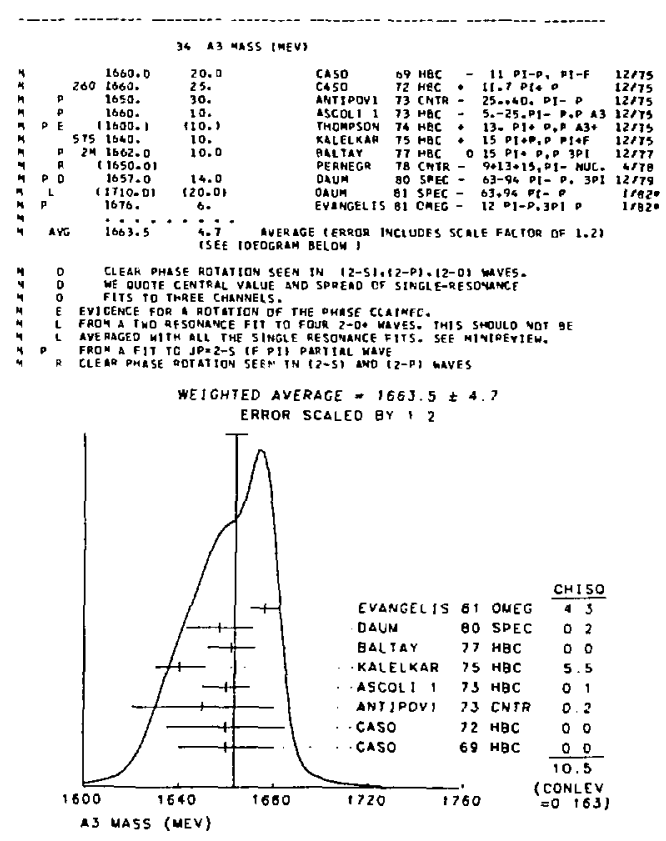


Data Card Listings

For notation, see key at front of Listings.
Mesons $A_{3}(1680) . \phi(1680)$

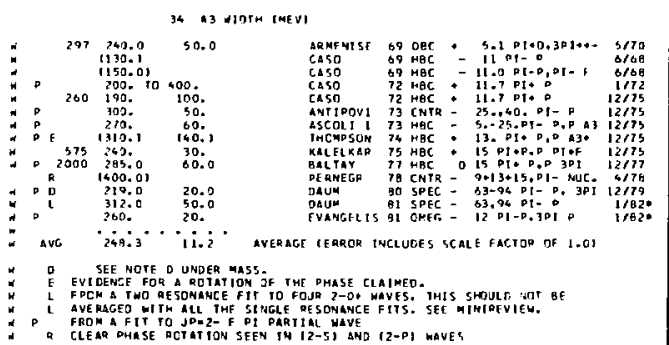

36. A3 DARTIAL DECSY MODE:

\begin{tabular}{|c|c|}
\hline 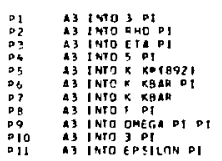 & 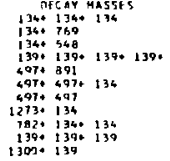 \\
\hline
\end{tabular}

34 A 3 GRAMCHING RATIOS

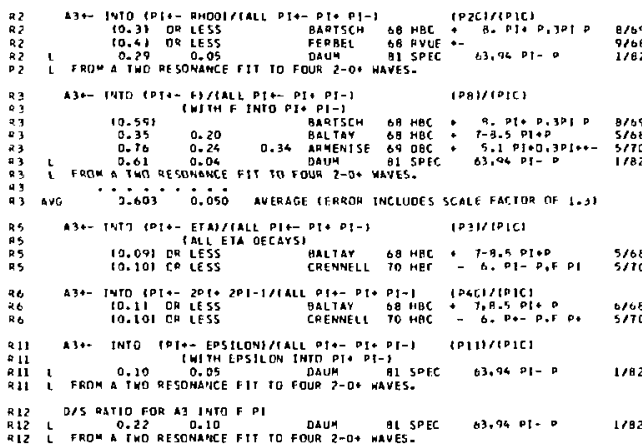

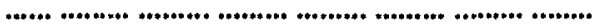
REFEREAT ES FDR A3

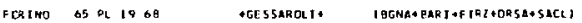
FOCACC1 Bo OOL 17 gaO LEVAT 60 DL 25 TR 74

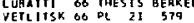
OANYSZ 67 YC 514001

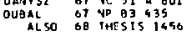

BAL TAY BA PRL 20 BA?

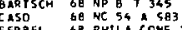
FER GEL OB PHTLA.CONF.

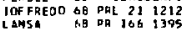

ARHENISE GQ LAT 2501

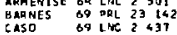
GRAMOENB TO HP BIS 369 CAENHELLL 70 PPL CHIEN TO PHILAO. CTHF

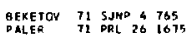
ALEXANDE 72 NP 34529

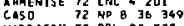

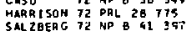

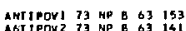

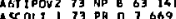
$A \times C L Y$
$A S C O L I$ CERH MISSING MASS SPCETPOHETEQ GRTUP (CERN)

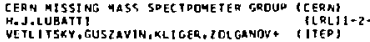

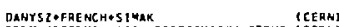
CERA MISSINC MASS SPECTROMETER GRTUD ICEANAI
I GENEVEI

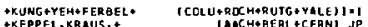
TKEP EL, KRAUS,

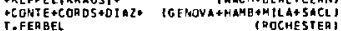

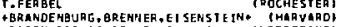

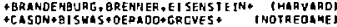

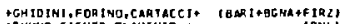

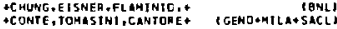

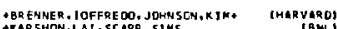

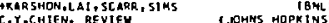

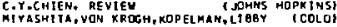
A SOMGKOUSKY, KOMOHALOV, KRUT SCHININ, + IITEPI JP

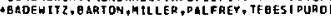

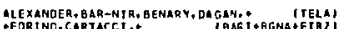

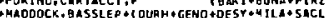

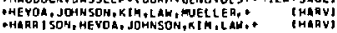

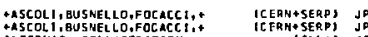

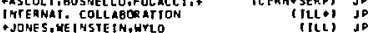

\begin{tabular}{|c|c|c|}
\hline 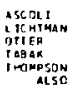 & 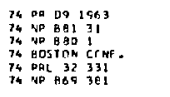 & 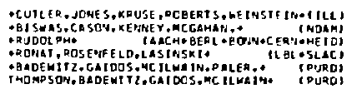 \\
\hline 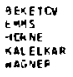 & 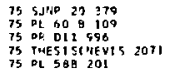 & 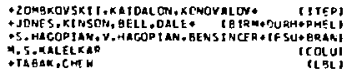 \\
\hline $\begin{array}{l}\text { BAL IAY } \\
\text { CAIIS } \\
\text { CEPDADA }\end{array}$ & 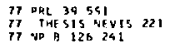 & 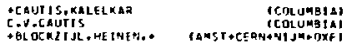 \\
\hline 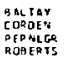 & 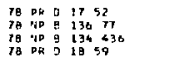 & 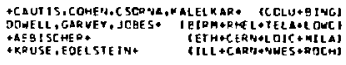 \\
\hline OAUH & 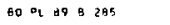 & 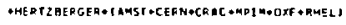 \\
\hline 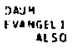 & 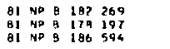 & 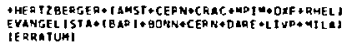 \\
\hline
\end{tabular}

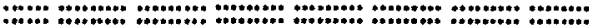

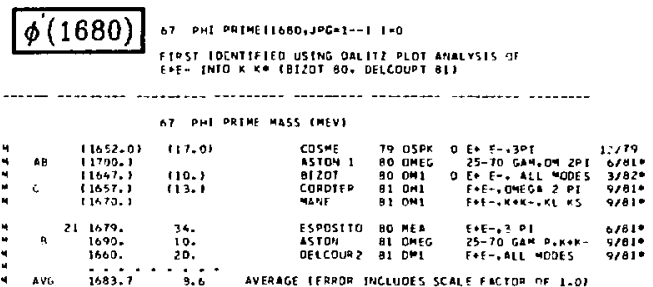

4 PESCHANCE HOT DISTINGUISHEO FHOM THAESHOL O ENHLYCE MENT

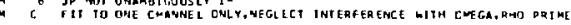
or PMI DQTME HIDTH INEVI

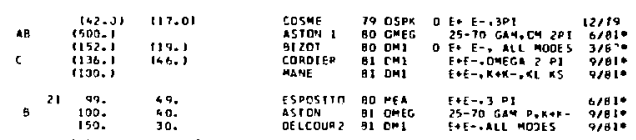

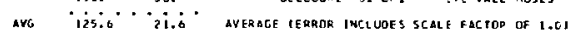

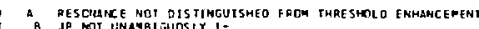

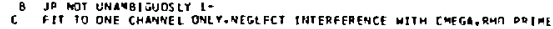

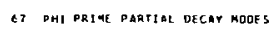

PI PHI DQIML INTO DMEGA 2 O

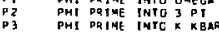

DECAY MASSES

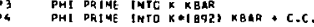

$3820+130 \%+130$

$493 *$
$792+493$

b) PHI PRIME granchinc patlos

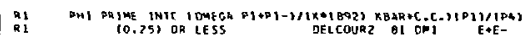

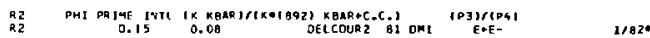

$1 / 82 \cdot$

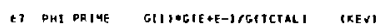

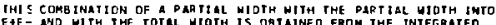

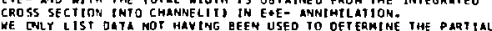

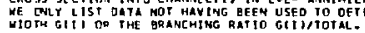

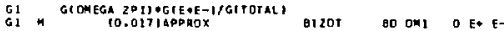

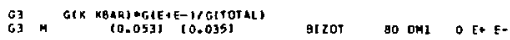

3/82*

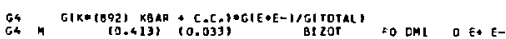

c. M MODEL DEP EMdENT

3/82" 
Mesons

$\phi(1680), g(1690)$
Data Card Listings

For notation, see key at front of Listings.

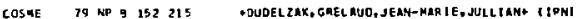

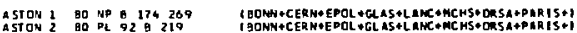

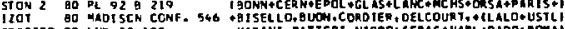

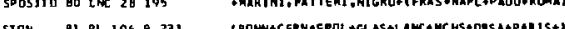

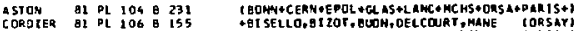

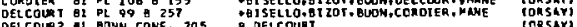
DELCOLA 2 GI BQNA CONF. 205 \#.DELCOURT
MAME

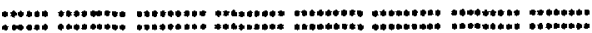
$g(1690)$ is $511690, \mathrm{JPG}, 3-+1=1$

15 G MASS INEVI

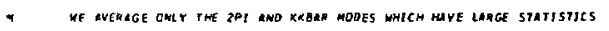
- 2 DI $M D E$

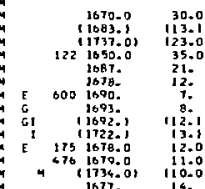

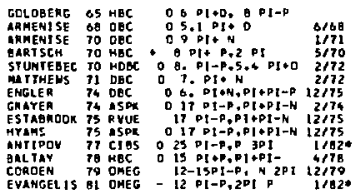

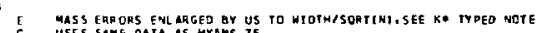

7 O USES SREE OATA AS HTANS IS

I ERRCO TAXES ACCOUNT OF SPREAO OF OIFFEDENT PHASE-SHIFT SOLUTIONS

- EROM A PMASE SMIFI SORUTION COMTAIMING A F PRIME RIIOH H

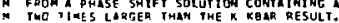

r KAAR K KBAR OI MODE

$$
1590.0 \quad 16.0
$$

P 5 ox 1692. 125

$\begin{array}{ll}1849.0 & 5.0 \\ 1694.01 & 18.01\end{array}$

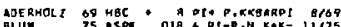

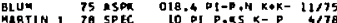

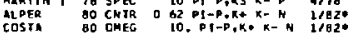

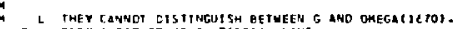

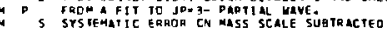

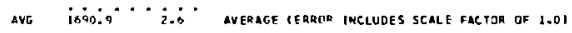
$14011+4006$

1441200.015 .0

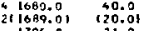

1705.0
1630.0

21.0

16085.1 119.119 .1

1370.

10.1
15.0
10.1

19:1

list3:

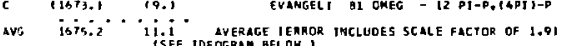

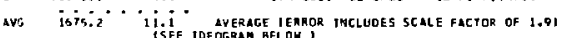

Bactay OB HBC + T, 0.5 PI+ P

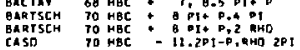

CASDES 70 HBC

$72 \mathrm{HBC}+10,-12, \mathrm{KAD}$

CASON $13 \mathrm{HBC}$

Th $-4,5$ PITD,D ADI

IHOMPSON $74 \mathrm{HBC}$ - $13 \mathrm{PI} P$

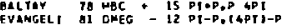

EVANGELI GI DHEC - 12 PI-P. (ADI)-

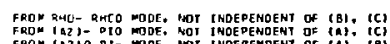

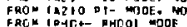

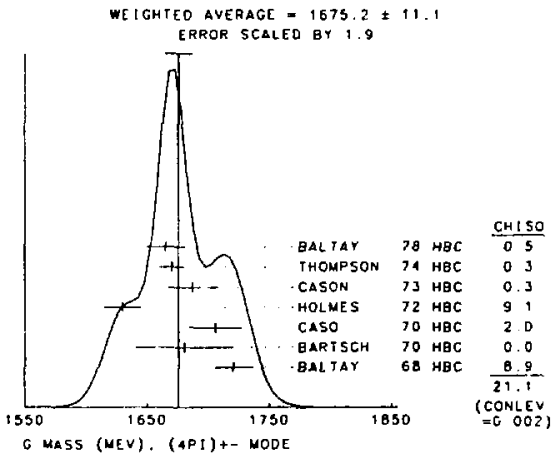

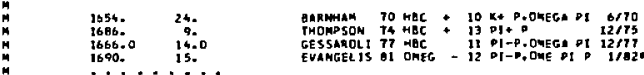

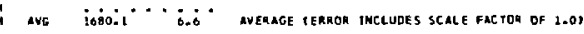

15 G MIOTH (MEV)

WE AVERAGE OALY THE ZPI AHO KKBAR MDDES WITH HAVE GRGE STATISTICS

P NODE

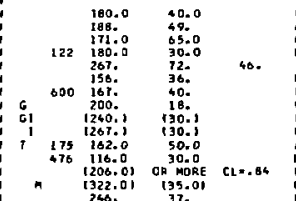

GOLOBERG 65 HAC 0 OP

ARAOAERG 65 HEC

ARAENISE

GARTSCH TO MBC. PI+ P.2 DI

STUNTEBEC 70 HOEC D $3.01-0+5,4$ DI*0 $2 / 72$

74 OBC 002

GRAYER 74 ASPK 017 Pt-P.PIOPI-M $2 / 74$

ESTABROOK 75 RVUE 17 PI-P.PTAPI-K $12 / 75$

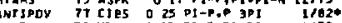

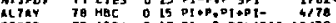

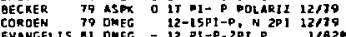

- I FROM PHASESHTFT ANALYSIS

C USES SAME Java as hams

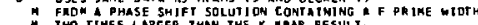

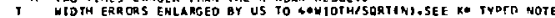

K KMEN + K KBAR FI MOOE

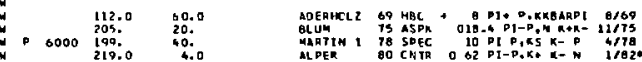

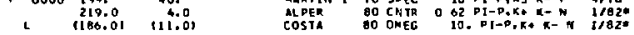

W L THEY GaNHOT OISTIHEUISH GETWEEN G ANO OMEGAl16701.

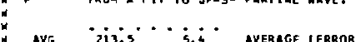

SSEF IOEOGRAH BELOW

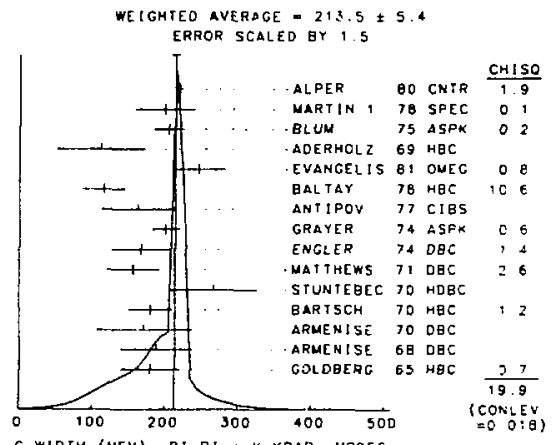

G WIOTM (MEV). PI PI + K KBAR MOOES

(4)

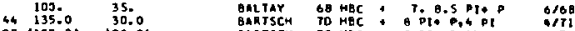

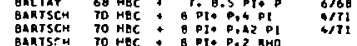

BAMTSCH TO HEC: B PTA D, 2 RHO

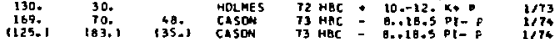

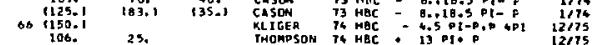

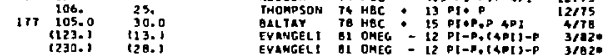

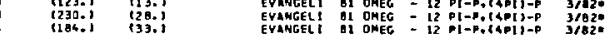

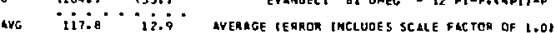

- FRON QMD- ANCO MOOE. NOT INDEPENDENT OF [B], ICI

FROM MLZ) PIO WOOE. NOT INOEPENOENT OF IAI: ICI

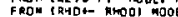

DMEGA DI MCOE

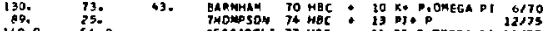

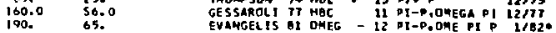

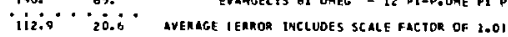


Data Card Listings

For notation, see key at front of Listings.
Mesons

$g(1690), \phi(1850)$

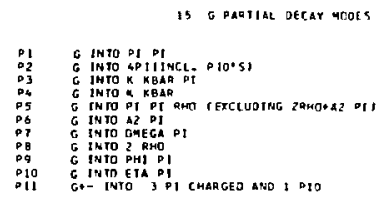

FIT TED FARTSL DECAY MODE BRANCHLNG FHACTIONS

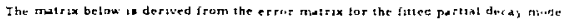

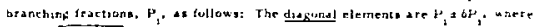

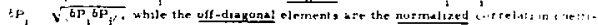

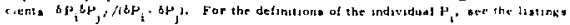

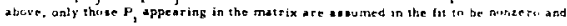
are thue constraned to add 109.

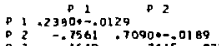

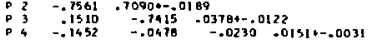

15. G BRLNCHINC OATIOS

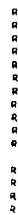

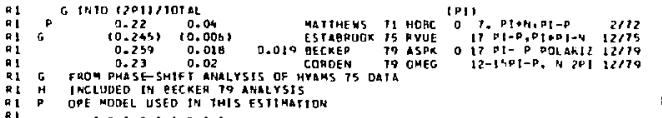

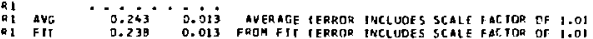

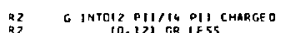

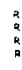

o.

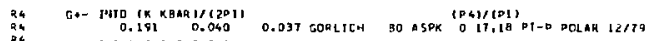

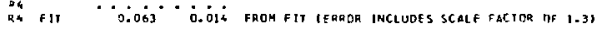

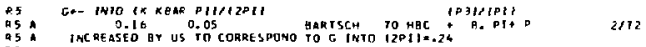

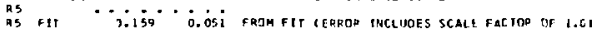

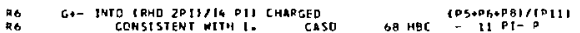

$R 6$
$R 6$
$R 6$

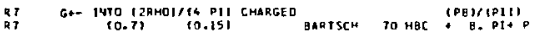

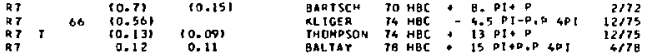

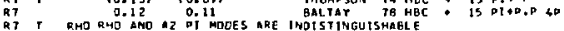

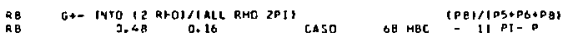

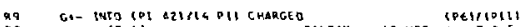

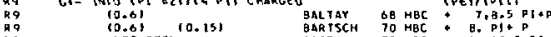

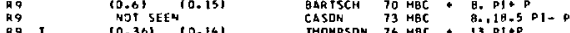

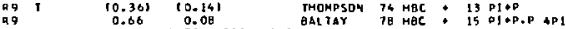

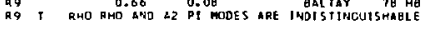

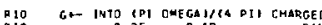

810
810
210
210
210
910
810
810
810
810

R 10 A

$\begin{array}{lll}R & 1 \\ a & 1\end{array}$

Riz

R13
$R 13$

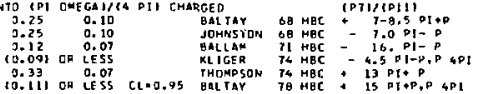

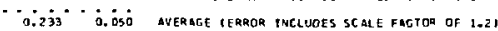

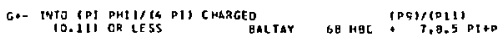

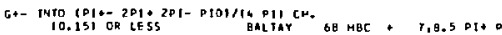

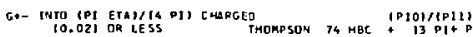

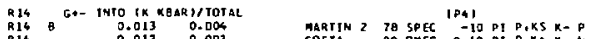

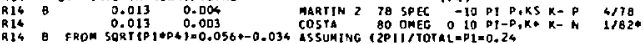

Rit $F$

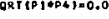

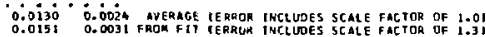

$5 / 68$
$6 / 68$
2172
12775
12775
$4 / 78$ a16
16

$$
\text { (2) }
$$

DESTSCHN OS Pi it 351

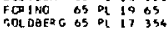

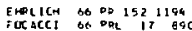

TOCACC1 60 PRL 179890

ABRAMS 87 PPL 19620

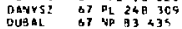

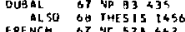

AHMEVISE OQ NE S4 A 5 F9

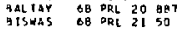

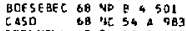

CASD BB DE 54 A 983

ADF RHAC 260 YND B II 250

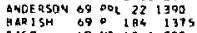

CAST
VFTLISK

ADUENISE IO 2 LNC 4199

9ARHALA TO ORL 74 ig93

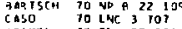

SAAMEA 0 ORL 25396

MAUREH 70 THES 15 AC. 58

BALLAM 7120032006

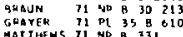

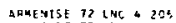

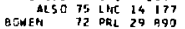

CAAION 72 ND 4 C 81

MCL WES 72 PR DO 1336

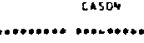

REFEAENCES Fó a

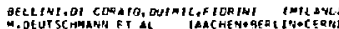

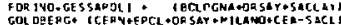

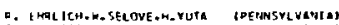
CERY WISST HG MASS SPFCTADME TER GRTUP ICERE

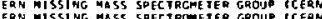

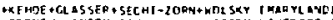

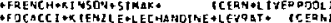

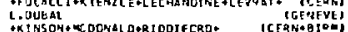

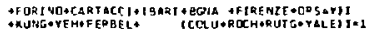

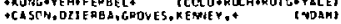

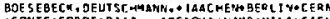

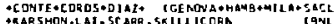

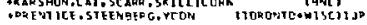

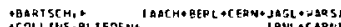
CCOLL QH5, RL IEDENA

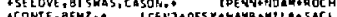

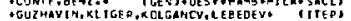

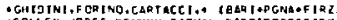

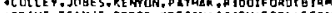

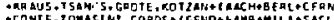

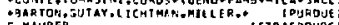

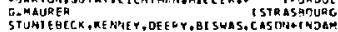

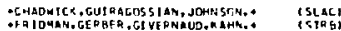

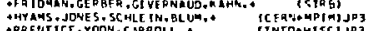

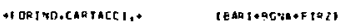

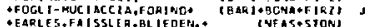

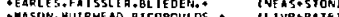

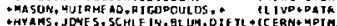

ARNOLD
CASON

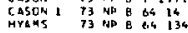

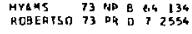

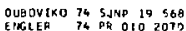

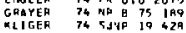

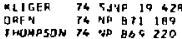

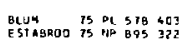

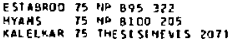
AMTIPOV II NQ tFERTEL SL STIERY, UERNE O

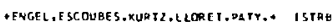

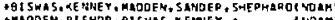

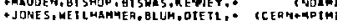

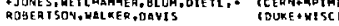

OJgCVIKOV, maISTUR, NILDV , SOKCLCY IITEPY

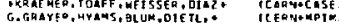

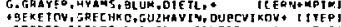

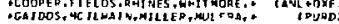

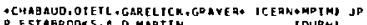

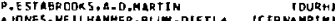
M. S. Kalflakar

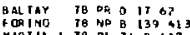
MARITH 2 TO MARTIN 278 NPO 14015

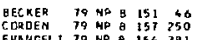
EVAMGEL T 70 ND 15439

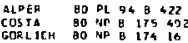

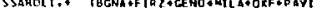

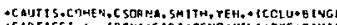

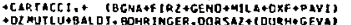

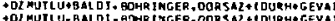

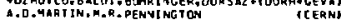

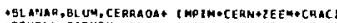

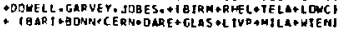

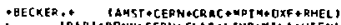

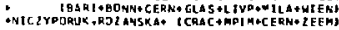

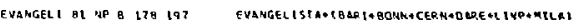

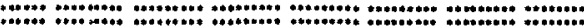

\section{$\phi(1850)$}

54 PHIC1850, JPG $13=0$

SEFN IN THE K KBAT ANO K MAR DI MASS

CEE OS CONT TRMATION. OMITTEO FACM TABLE. 
Mesons

$\phi(1850), X(1850), S(1935)$

\section{It PHIt1E50I BRAMEHIMG RATIOS}

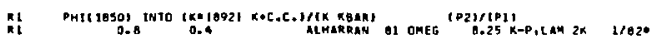

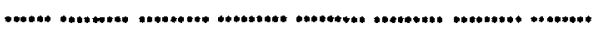
MEFERENCES FOR PHITIOSO,

A5IOM DO DL 92 \& 219 18ONN+CERN+EPOL+GT AS+LAKC+MCHS+ORSA+PARIS+1

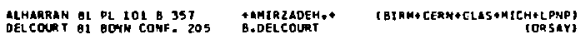

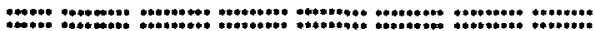
$X(1850)$ 36 $x(1250, . J P G=2 \circ, 1=$

FMOM MY AMPLITUDE ANALYSIS OF THE K+K- SYSTEM SEEN IN

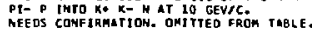

\section{$38 \times 11850)$ MASS IAEVI}

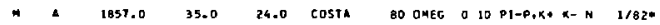

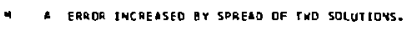

\section{$39 \times 13503$ NTOTH (MEVI}

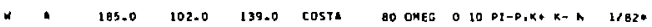

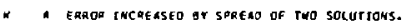

29 XetesOi paptial DEGAY mODES

D) x(lesa) INID $k+k-$

DECAT 4ASSES

$38 \times(1850)$ BRANCHING RATTOS

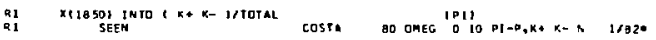

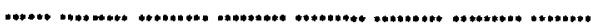
REFEQENCES FOR XI1950)

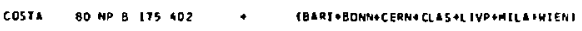
$\mathrm{S}(1935)$ 31 st1935.JPG ,

A narrow enhancement called the $S$ (1935) has been observed in the antiproton-proton total cross section ICARROLL 74, CaALOIJRA 76, BRUCKNER 77, SAKAYOTO 791.

This observation is not confirmed by more recent experinents (ALLEN 80, KAHAE 80, JASTRZEHBSKI 81, LOWENSTEIN 81), or the effect is found to be smaller in nagnitude and larger in width (BAMILTON 80).

No significant signal is observed for a narrow $S$ (1935) in backward antiproton-proton elastic scattering (GARNJOST 79), nor in the charge-exchange cross section (GARRJOST 75, CHALOUEKA 76, HAMILTON 80).

The first positive observations of the $\mathbf{S}(1935)$ in hadro-production experiments (DAUM 80) are not confirmed by more recent ones (DALM 81). In view of this situation, the only observation which remains unchallenged is the one of RICHARD 79 in a photoproduction experiment. One should wait for conficnation before taking the $S(1935)$ as a wellestablished narrow resonance.
31 S MASS IMEV!
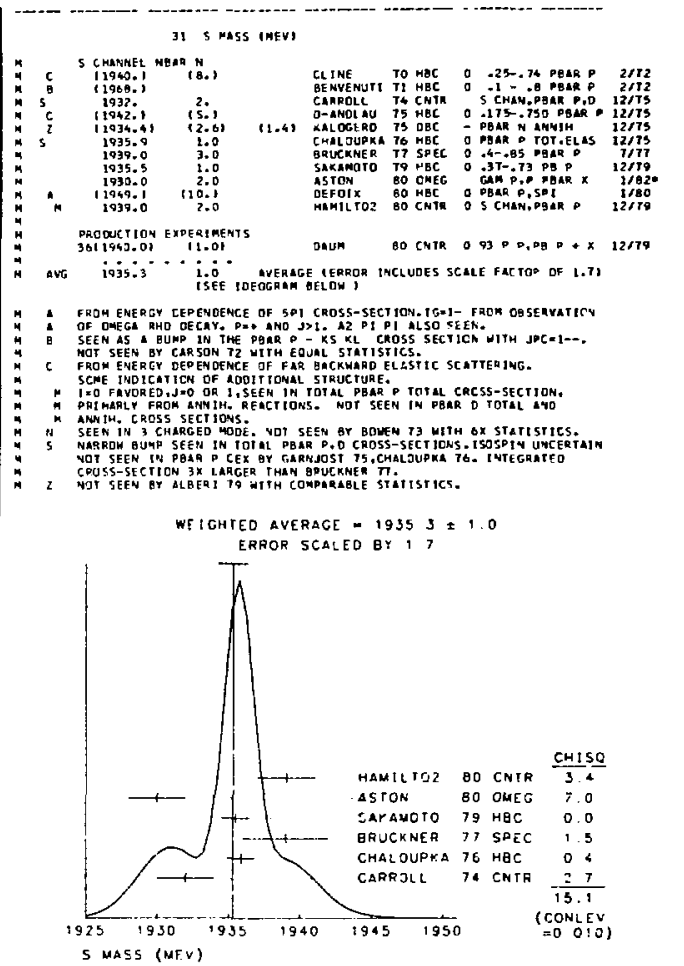

II $S$ MICTH I WEVI

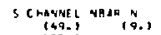

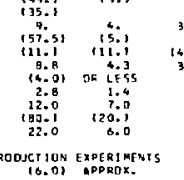

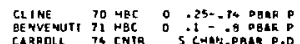

3. CARPOLL T4 CNTA S CHAM,PBLA P.D

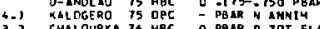

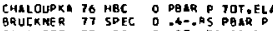

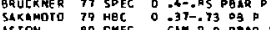

GAM P,P PDA 12179

OEFOIX BO MBC 0 PAAR D,SDI $\times 1 / 182^{\circ}$

DAUM

BO CNTR

93 D.08D, $\times 12170$

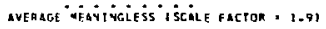

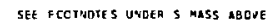

I1 S PRATIAL DECAY modes

PI

51470 PaSA D

DECAY WASSES

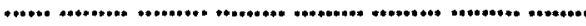

CLINE SE DEL 211268

REFERENCES FOR S(1835)

CLINE TO PREPRINT

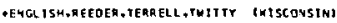

BENVENT 71 OXL 27283

D.CLINE.J.EHGLISM. O. D. REEDER RTISC].

GENVENUT I, CLINE,RUTZ, REEOER, SCHERER FH: SC,

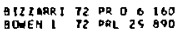

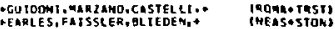

gOMEN 13 PRL 30332

GUR HS 13 PQ O 81286

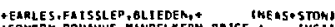
CCONOOH, DONAHUE, MLNDELKERH, PHICE, + IUC II

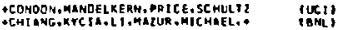




\section{Data Card Listings}

\section{For notation, see key at front of Listings.}

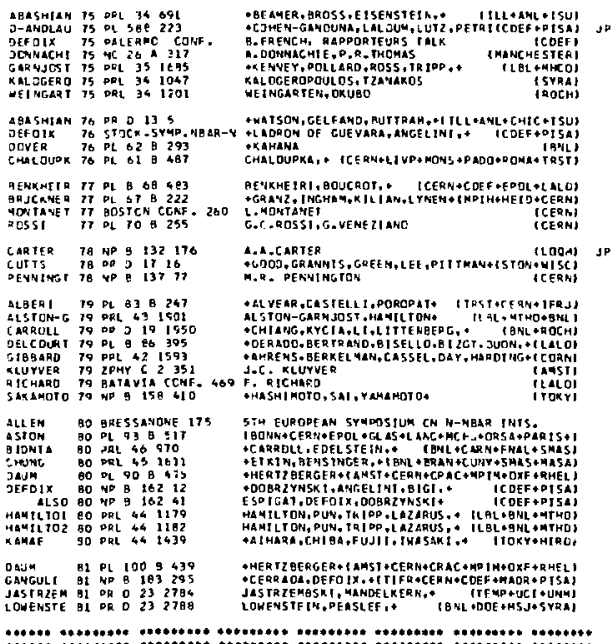

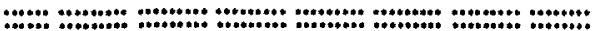

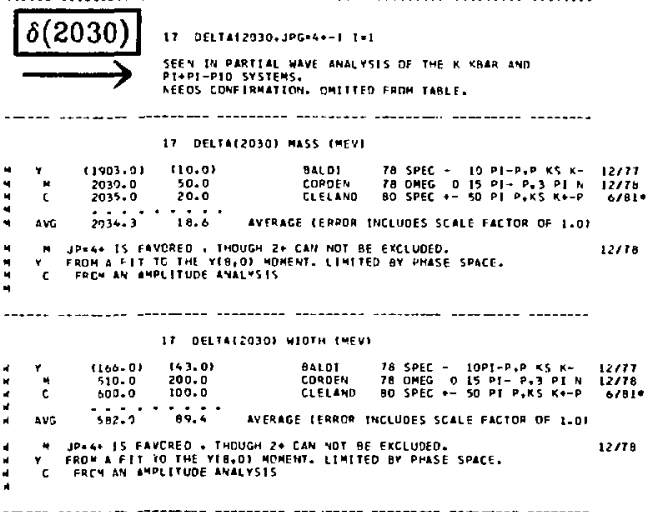

17 DELTAL 2030I TAATILL OECAY MOOES

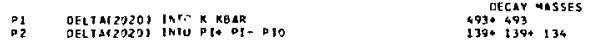

If DELTAL2030J BRaHeHING gatios

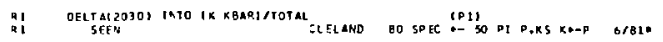
$R z$
$R 2$

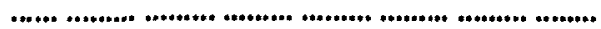

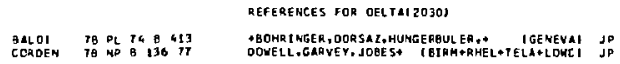

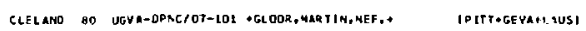

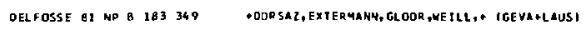
.0.

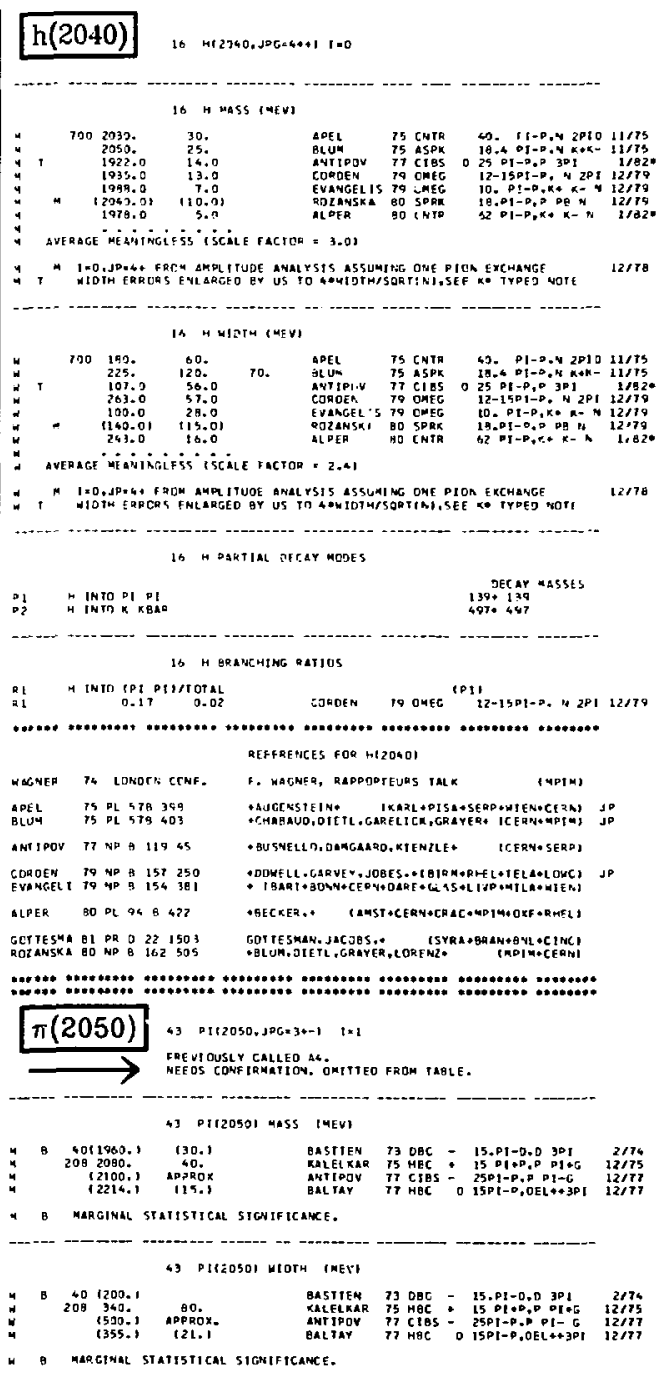

43 PIRZO50I PARTHE DECAY MCOES

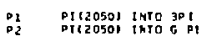

DECAY MASSES
$139+139+139$

$169+139+139$

4 DItzOSO) gRANCHING RATIOS

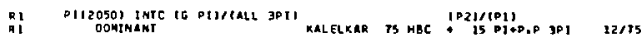




\begin{tabular}{|c|c|c|}
\hline & 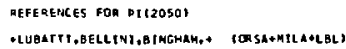 \\
\hline GaAo & 7140833357 & ICERM ETHAL OTCOYILAT \\
\hline non & 12 Yo $84 \mathrm{~T}$ an & AMASON, MUI RHE AD,R IGOPOULOS,* \\
\hline $\mathrm{JEN}$ & 13 JPASALE CENF. 13 & 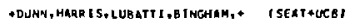 \\
\hline & I4 NP 871 19\% & -COOPER, FIELDS, RHETHE, HHLT MORE, + CAHL HOXF I \\
\hline TELARA & IEvts & $\begin{array}{r}\text { IABACC HW COLLABOALTION) } \\
\text { (COLU) }\end{array}$ \\
\hline $\begin{array}{l}\text { AMTIPOY } \\
\text { BLIAY } \\
\text { CMUTIS }\end{array}$ & $\begin{array}{l}77 \text { ND } 11945 \\
77 \text { PRL } 395919 \\
\text { THESIS NEYIS }\end{array}$ & 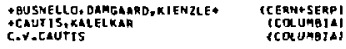 \\
\hline & & tcauris \\
\hline
\end{tabular}

.0.

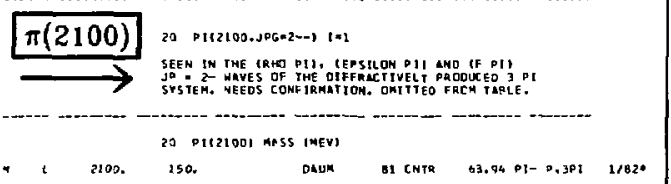

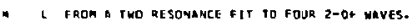

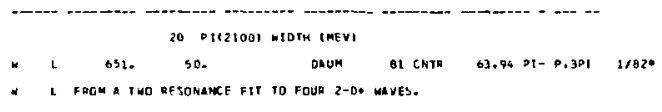

20 P(IZ1OO) DARTIAL DFtAY MCDES

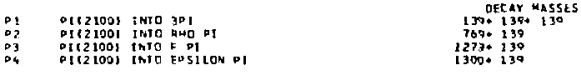

20 DIRZJOAR BRAMCHING AATLS

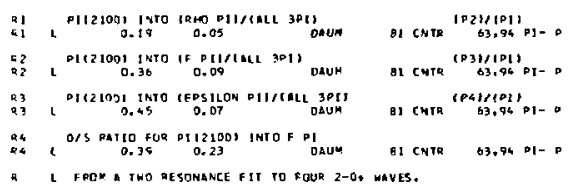

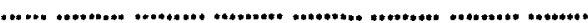
REFEA NCES FOR PI(2100)

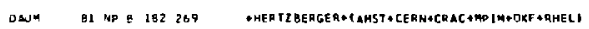

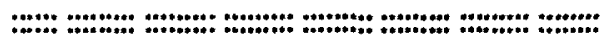

\section{Note on $T$ and $\mathrm{U}$ Regions}

The observation of broad enhancements at 2190 and 2350 MeV comes from pp total cross-section measurements (ABRAMS 67), "pp annihilation reasurements (ALSPECTOR 73), pp elastic cross-seçtion measurements (COUPLAND 77), and $\bar{p} p$ charge-exchange cross-section measurements (CUTTS 78). The mass regions centered around $2190 \mathrm{HeV}$ and $2350 \mathrm{MeV}$ have been called $T$ and $U$ regions, regpectively.

Searches for resonances in exclusive $\overrightarrow{p P}$ annihilation channels which could be coupled to the enhancements observed in the pp total cross section and is pp elastlc scattering have been unsuccessful, except for the two-body annihilation

channels $\pi^{+} \pi^{-}$and $\pi^{0} \pi^{\circ}$, where partial-wave analyses have shown that resonances are formed in the 2100-2500 MeV hase region (CARTER 77, DULUDE 78, MARIIN A 80, MARTIN 8 801. We have listed the results on these analyses under the headings $E(2150) / T_{0}(2150)$ for the $I=0, J P=2+$ wave; $\rho(2150) / T_{1}(2190)$ for the $I=1, J P=1$ - wave; $\rho(2250)$ for the $I=1, J P=3$ - wave; $E(2300) / J_{0}(2300)$ for the $I=0, J P=4+$ wave; and $\rho(2350) / U_{1}(2400)$ for the $I=1, J P=5-$ wave.

Various structures coupied to $\bar{p} p$ and observed in production experiments are listed under the

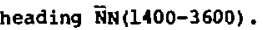

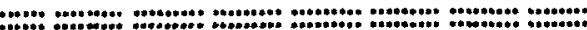

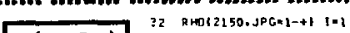

$\rho(2150)$ TMIS ENTaY WaS PRE,

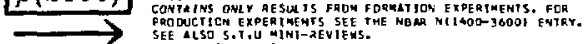
TMITEO EROM TABIE.

35 RHOT2]5CI MLSS (MEVI

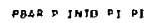

D 12100-01 AOPROX.

TABTIN 4 Go PVUE

1/92:

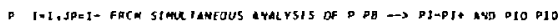
5 CHaNHeL NUCLF TN ANTINUCLEON

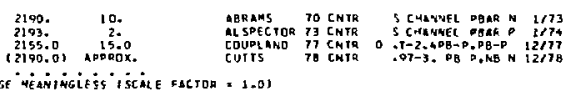

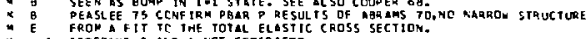
ISOSPIYSO AND I NOT SEPARETEO

$$
\text { P) PHDt } 21502 \text { WIOTH IMEVI }
$$

Dgare D INTD DI DI

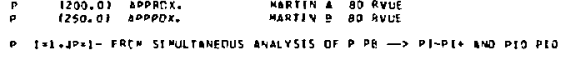$$
5 \text { CHANAEL MULLE ON MATIKUSLEON }
$$

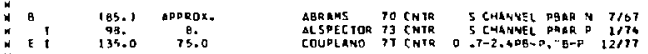$$
\text { * average meanivirisss iscale factor - z-ol }
$$

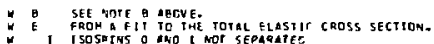

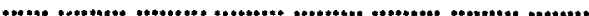

ABRAMS BT ORL $28 \quad 1239$

COJPER GO PRL 201659

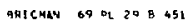

agmans to do 0 I Is,

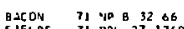

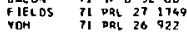

ALFEAHE 72 YP B 4529 $\begin{array}{llllll}\text { ALERAHOE } & 72 & 48 & 8 & 65 & 29 \\ \text { DONALO } & 72 & \text { DL } & 40 & 3 & 586\end{array}$ ALSPECTO 73 PAL 30511

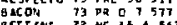
BET INI 73 W

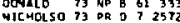
BERTANIA TH YC 23A 209 HYAMS I4 NP E 13200 gONACH! $75 * 202317$

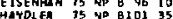

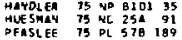

\section{REFERENCES FOR RHOCziso)}

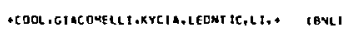

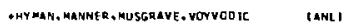

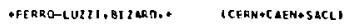

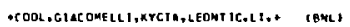

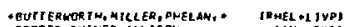

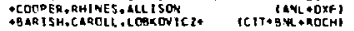

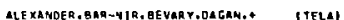

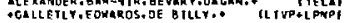

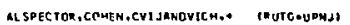

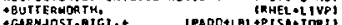

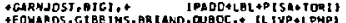

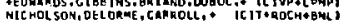

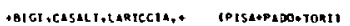

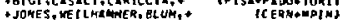

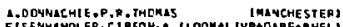

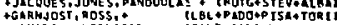

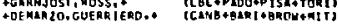


Data Card Listings

For notatinn, see key at front of Listings.

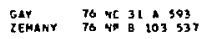

CARTER: TI OL ST: IIT

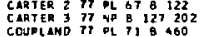

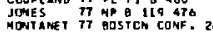

CAATER 78 MP a 132176

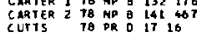

MARTin TO PL AO $B$ Q 93

MARTIN A BO ND B 160216

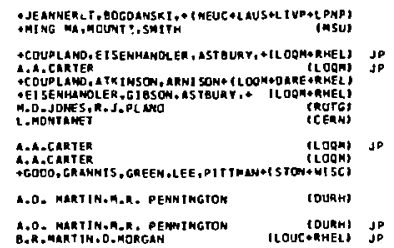

:...0.

$\epsilon(2150)$ t2 epstlontziso.JPGe 2n+1 1-0

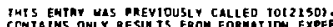

CCNTAINS ONLY QESULTS FROM FORMATIDO EXPERIMENTS, FOR

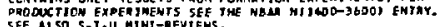
SEE ALSO SET, HINTH

42 EDSILON(ZISO) MASS IMEV)

- DGA D SATO DI DI

\section{L $\quad$ 2130.0) Poprox.}

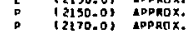

\section{DLLUEE 78 OSPK}

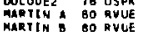

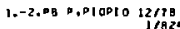

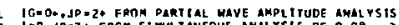

5 CMRMHEL DaLR D DE MBar

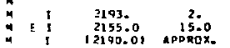

- average vemunimeiess iscile factor - to

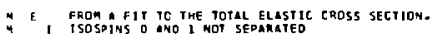

az EDSILONIzISOI MIDTH (MEV)

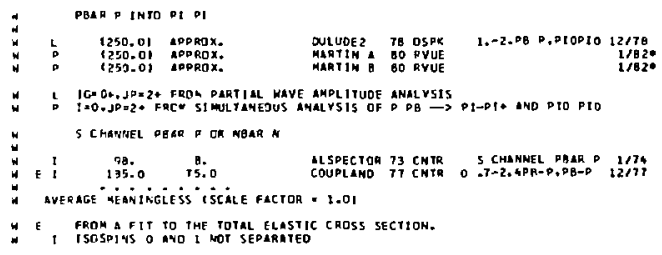

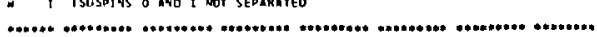

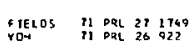

DDNALO DOL 10 O 586

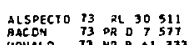

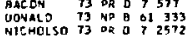

Gar 78 ve 314593

CQNBL AmO 77 OL 71 a 460

cutrs to 020118

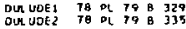

TARTIN 19 OL 86 B 93

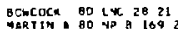

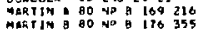

\section{Ref ERENCES for epstlontz150I}

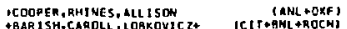

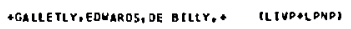

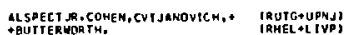
TEUTERHORTM:

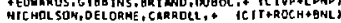

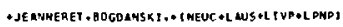

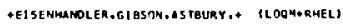

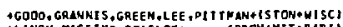

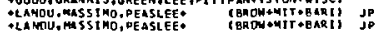

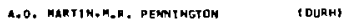

J.E. BOWCOCK, D.C. HODGSON

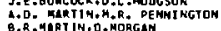

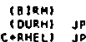

.......

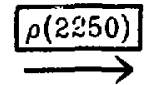

44 RHol 2250, $50 t-3-+1$ i=1

CONTA INS OKLY RESUL TS FROM FORMAT IOM EXPERTMENTS. FGR

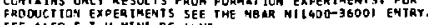
SEE ALSO S, T,U MINT-

44 phol 2250 ) mass imeVr

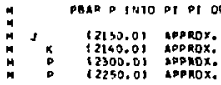

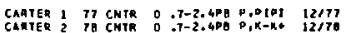

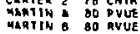

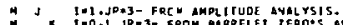

M.
4 5 CMUHNEL NGCLE ON AMTINUCLLE DN

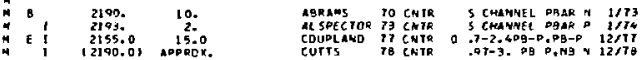
average meanitigiess iscale factor - 1.01

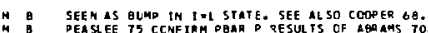

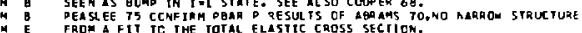

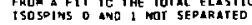

44 RMOLZ2501 NIOTI (MEN)

DBA $P$ INTO PI PI OR $K$ KB

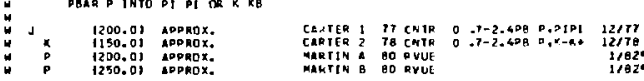

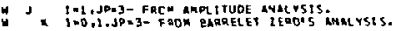

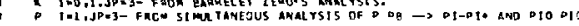
5 CHAHEE WUCLEON ANIINUELEON

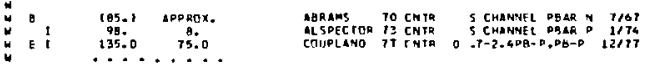

- average menuintiejs iscal e factor - 1.01

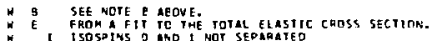

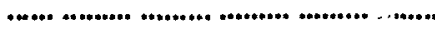

ABA ANS GP DQL 181200 DEFEAFNCES FOR RHOL 22301

COOPEA OB DRL 2015159

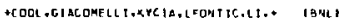

AQR AMS 70 DD O 1 L51? -hrman, maritea, muscakave, vorvodic laves

Flet OS 71 PRL 27 DOL

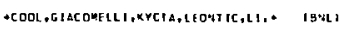

ALSPECTO 73 ORL 30511

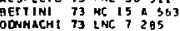

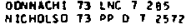

BERTLNIA 74 YC 234509

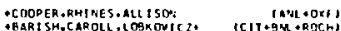
aL SPECTOR, COHEN, CVI JAROVIC H. TRUTG. JPAJT :GARNJOST, BIST,

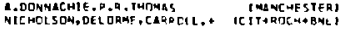
zemany To No a 103537

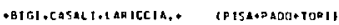

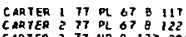

CARTER 2 TT P 6780122

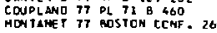
- Ming he Mounti.smitu insul

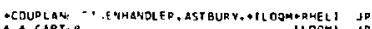

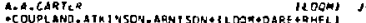
TEISEMMAMOLER, GI RSDU ASTHURY, "IDOM RUEL)

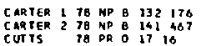

C.MON TANET

(150), JD

Matis 79 PL Bo 9 a

A.4.ctarter ingents

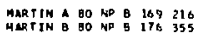

A.D. MARTIN.M.R. DE WWIMGTON

inบzh!

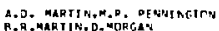

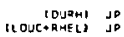

.......

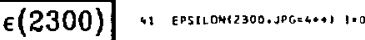

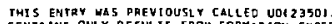

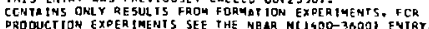

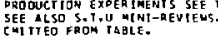

$\longrightarrow$

41 EPSILONIZ3001 MaSS IMEV) DBAR P INTO DI PI DR KE K

(2310.0) 4PPROX.

(230.0) SPPRTX:

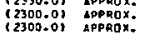

CARTER: 77 CNTR O .7-2.4PB P.P[P] $12 / 31$

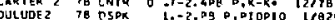

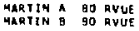

iraz:

$1=0, J P=4$ FACM MPLLTUDE MNATYSIS.

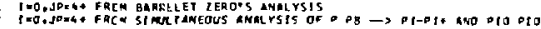
5 CHANYEL DBAR D OR RBAR A

lio.

$23451,115,01$
2380.01
2000.

ABPAMS TO CNIP
AL SDECTOR I3

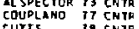

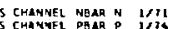

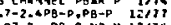

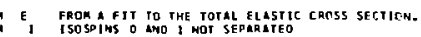


Mesons

$\epsilon(2300), \rho(2350), \delta(2450), \mathrm{e}^{+} \mathrm{e}^{-}(1100-2200)$
Data Card Listings

For notation, see key at front of Listings.

\author{
4] EOSILONIZZOOI NIOTH IMEV, \\ PBA P TNTO FI PI OA KBK \\ 1210.01 ANPROX \\ (150.0) APPROX. \\ CARTER I I7 CHTR D -7-2. $40 B$ P.PIPI 12577 \\ CAATER 2 PO CHTR O -7T-2.4FB P.K-KO 12/78

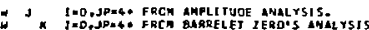

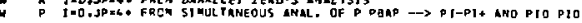 \\ 5 CHANMEL PASR O OR MBAR N

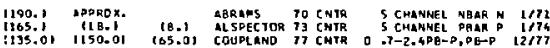

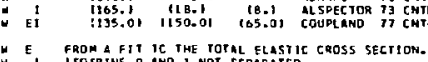 \\ W E FROM A FIT IC THE TOR LL ELASTIS
}

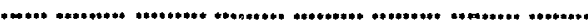

DEF ERENEES FOR EPSILONT2300)
BRTCNAN 69 PL 29.951

DERAMS TO DR D 1 15IT $\begin{array}{lll}\text { FJELO: } 71 \text { PRL } 27 & 1769 \\ \text { YDH } & \text { TI PAL } 20 & 972\end{array}$

EASINAN 12 ND G S1 20 ALSPECTO 13 PRL 30511

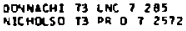
Hrams 74 No 373202 OCNNACH1 $75 \times 26 \div 317$ EISENHAN T5 NP 9 SO 108 CAETER I TO $D L$ O7 B 117 CARTER 2 T7 PL OT E 122

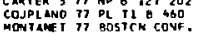
CaRteR I TE NP B 137 17O

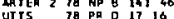
HARTIN 79 DL BO B 53 SCACOCK aO $\operatorname{LNC} 2821$

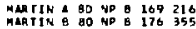

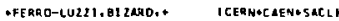

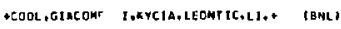
-CODPER, RHINES, RLLISON IANL +OXFI -NING ML, OH, PRRKER, SMITH, SPRAFKA TMSUI ALSPECTOR, COHEN, CVIJAHOUTCH, PRUTC,UPYJI R.OONHACHE,PA, THOMAS NICHOL SON, OELLRME. CARAOLL \& TCTT+ROCH+BNL

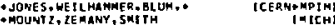
A. OONNACHIE, D, R. PHOMAS CMANCHESTER EI SE NHANOLE - COUPLAMO, ERSEMHANOLER, ASTBUAY, +CLOOH +RHELI JP ACA.CARTER

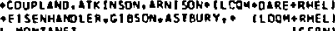
L. HONTANE I
ICERA A.A.CARTEF
A.A.CARTER COOMR JP A.A.CART ER

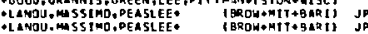
*LANOU.MASSTMO,DEASLEE GROH+MTT+GARI\} A.D. NARTIN, M.R. DEHYIAGTOH (OURH) J.E. 9OWCOCK, O-C. HOUGSOH D.0. MAGTIN, (RIRM)

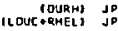

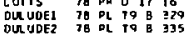

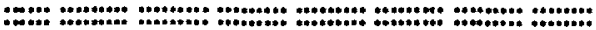

$\rho(2350)$

33 a hot 2350, JPGan 5- $)$ t $t=1$

THIS ENTAY WLS PREVIOUSLY CALLEO UZ12400)

CCNTA INS OHLY AESUL IS FROM FORMAT ION EXPERIAENTS. FOR PRDOUCTION EXPERIMENTS SEE THE NBAR NI1400-3600I ENTRY. SEE ALLO S,T,U MINI

33 FHOL 2350 ) WASS (WEN)

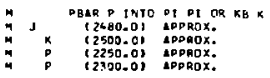

CARTEA I T7 CNTR O .TO-2.4UG D.PTOL

CARTER 27 CNIR $0.7-7-4 P A D, X-K=1277$

MARTIN A BO RVUE

1/62*

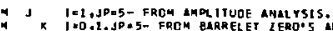

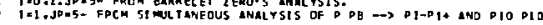

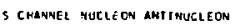

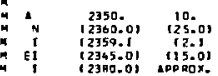

LBREMS TO CNTR S CHANAEL WB AR N $1 / 73$

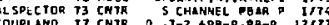

COUPLATO TI CNTR a $.7-2.4 P Q-P, P B-D$
CUTTS

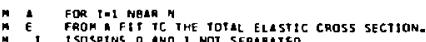

ISOSPINS O THO THE TOTAL ELASTIC

EVIOENCE FOR THIS SUMP SEFN ZH THE PBLR P OATA CF CMLPMAN 11

33 RHOLZ3SO) WIOTH (MEVI

:

If of at $k B \times$

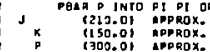

1250.01 IPPAOX.

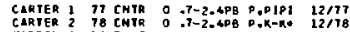
MARTIN BO PVUE

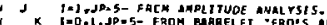

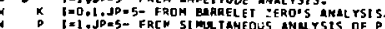

5 G HAYMEL NUCLEON ANT JTUCLEON

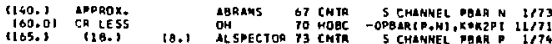

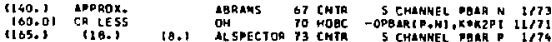

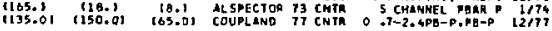

- E FRO a FIT to THE TOTAL ELASTIC CMOSS SETtIOn.

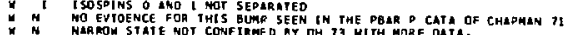

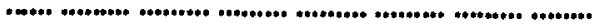

REFERENCES FGA RHOL 23503

ABRAMS BT PFL 101209

BRJCHAN 69 PL 29 B 451
CASO

$\begin{array}{llllll}\text { LBRAMS TO PR O } & 1917 \\ \text { OH } & \text { TO } & \text { ORL } & 24 & 1257\end{array}$

CHAPHAN 71 PR D4 1275

$\begin{array}{lllll}\text { CHAPHWN } & 71 & \text { PR } & 04 & 1775 \\ \text { FIELDS } & 71 & \text { DRL } 27 & 1749 \\ \text { YOH } & 71 & \text { PRL } 26 & 922\end{array}$

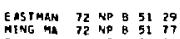

$\begin{array}{llllll}\text { HING HA } 72 & \text { NP } & 5 & 51 & 77 \\ \text { OH } & 72 & \text { NP } & \text { B } & 51 & 57\end{array}$

ALSPECTO 73 PAL 30511
HIC HOLSO 79 DR D 72572

HYALS 74 NP 73202

DOANACH1 75 Ne 20 a!t?

EISENMAN 75 ND B 96 109

CARTER I 77 DL BT Q 117

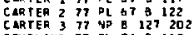

COUPLANO IT PL T1 480

CAATER 170 NP 132176

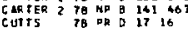

matia 79 D 80353

BONCOCK 80 LNA 2021

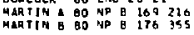

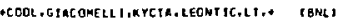

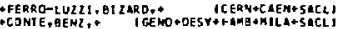

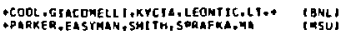

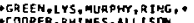

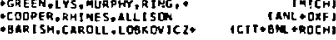

- YNEG MA.DH, DAFKER -SMITH,SPRAFKA INSUI

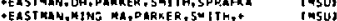

AL SDEC TOR, COMEN, CVI JAHOVICH, D (RUTG FURNJ) AIE HOL SON, DEL DRHE, CARPDLL ,* ICIT+ROCH+BYLJ

-JONES, ME IL HAMMER, BL UM.. TCERM+MPIM)

[4[CH]

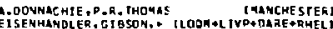

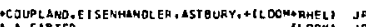

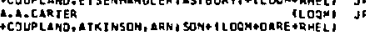

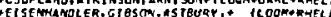

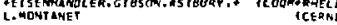

A.A.CETEP ILOMJ JP AGOCORTEE

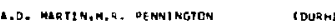

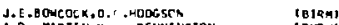

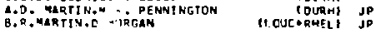

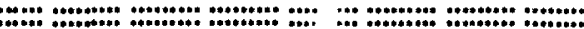

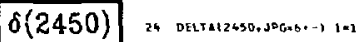
SEEN TY PAHTILL WEYE ANALYSTS OF THE K KGAN

24 OELTHI2450) - 155 in. ',

C Z45O. 20.
C FRCM AN LMPLITUDE ANALYSI

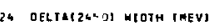

320. 40.

CLELANO 80 SPEC +- SO PI D,KE Q*-P OLEL

6 FACM IN LMPLITUDE AN IIS

26 DEL Z6SOI DARTILL DECAY MOOES

DI DELTRI245OI TNTO K RAAP OEECY MASSES

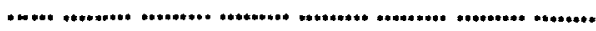

REFERENCES FOR OELTAT; - 50!

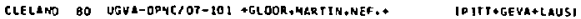

:

$\mathrm{e}^{+} \mathrm{e}^{-(1100-3200)}$

TE+E-11100-2200.JPG-1- 1 1=

TH1S ENTRY CONTAINS NOH STRANGE VEC TOR HESONS COUPLEO TO E* E-TPHDTONI OET WEEN DHI ANO JPDS I MASS REGION.
SEE AL SO RHO PAI IHET12SOI AND RHO PRTWEC1600T NENT-REVIEN. SMT TTED FROY TABLE.

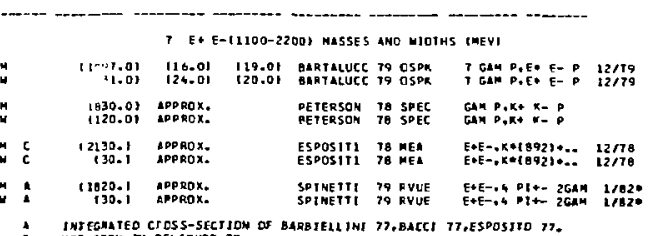

INJEGMATEO CROSS-SECTJOM OF BARBIEALINI M,BACCI TT,ESPOSIEO 7

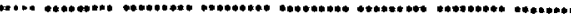

EAECt 75 PL SO 6 48:

BACCI TO DL O4 B 356

BACC! II PL 6 S6 393

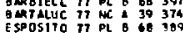

REFEREMCES FOR E+ E-11100-23000

+ HIDOL I PERSO, STRLA, BLLDINT, * ROHLFFASI

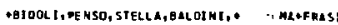

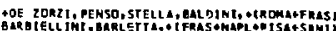
BAR TRLUCCI OERTOUCCI GRO

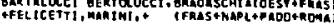


Data Card Listings

For notation, see key at front of Listings.

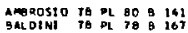

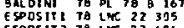

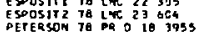

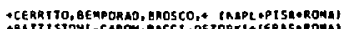

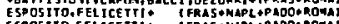

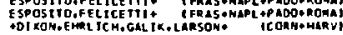

Anatalue $70 \times 49$ a 207

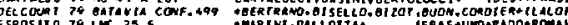

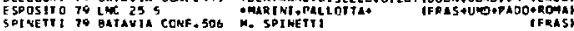

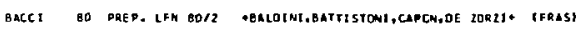

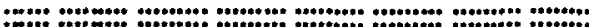

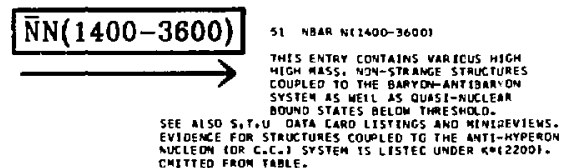

51 MgAR NT1600-3GODS MASSES AND WIOTHS TMEYI

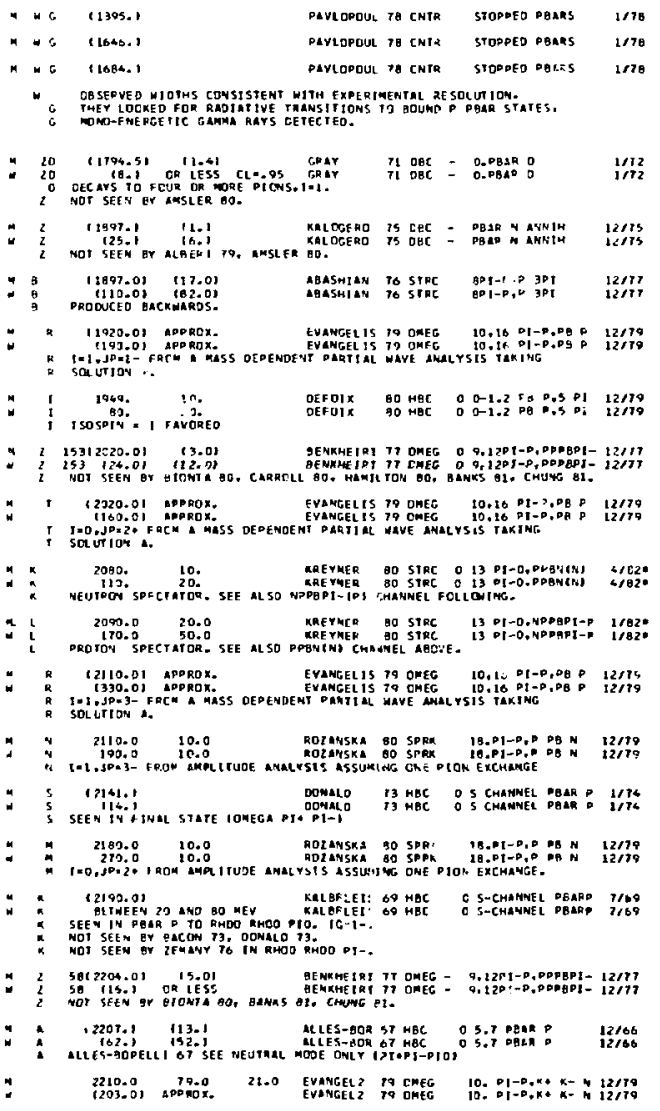

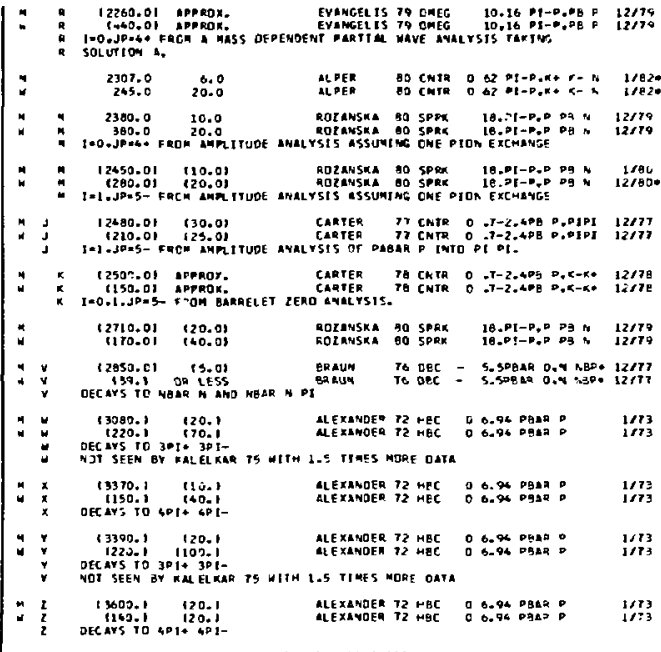

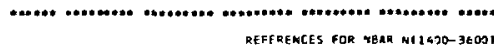

ALLE5-80 67 95501776 alexingen

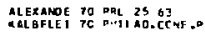
GRAY $D$ PRL 261491 AIEXANOE 12 MP B 4529

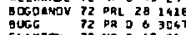
CLArIO 12 NP B

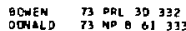

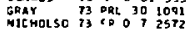
HYAMS THO 9202 DCANACHI T NC 26 \& 31 ?

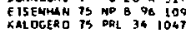

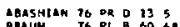

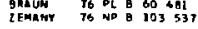

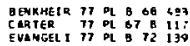

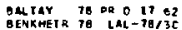
CMOTER 70 NOB PAVLDODO TE OL 728,415 ALBEA 79 IL B3 247

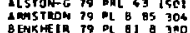

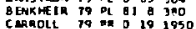
CARAOLE, 79 $=0.191750$ EYLNGEY TO EYANGEL 79 HP 9154381

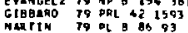
ALPER OO DL 949,22 ATSLER 80 PRL 84 A53

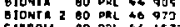
GAMNou 80 oxt 461572

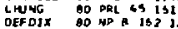

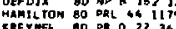

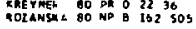

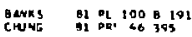

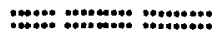

\section{REFF RENCES FOR TEAR N(14);0-3E00}

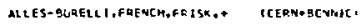

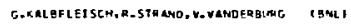

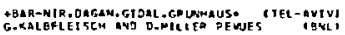
THACERT, KALOCERROPOUN

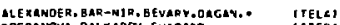

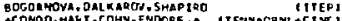

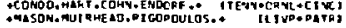

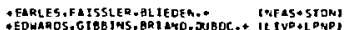

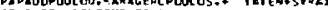
(t)

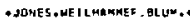

15 ERnaAPT=

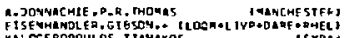

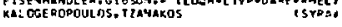

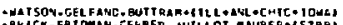

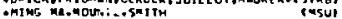

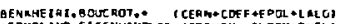

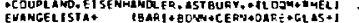

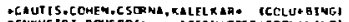

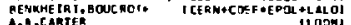

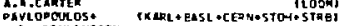

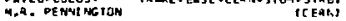

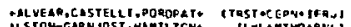

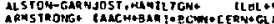

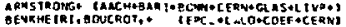

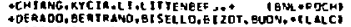

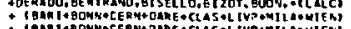

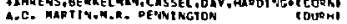

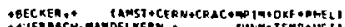

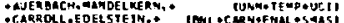

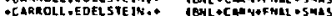

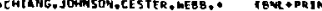

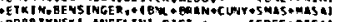
ODEBR Z

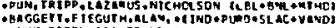

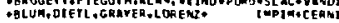

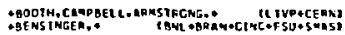
(1) 
Mesons

$\mathrm{X}(1900-3600)$, CHARMONIUM, $\eta_{c}(2980)$

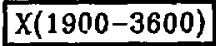

46 $x+1900-36001$

TrAS ENTAY CONTAIMS WAR IOUS HIGH WLSS

The high mass region is covered nearly continuously by evidence for peaks of various widths and decay mudes. As a satisfactory grouping into paxticles is not yet possible, we list all the $\mathbf{Y}=0$ bumps coupled neither to $\mathbf{N}$ nor to $\mathrm{e}^{+} \mathrm{e}^{-}$, and having $M>1900 \mathrm{MeV}$, together, ordered by increasina mass. Note that ANTIPOV $72\left(\pi^{-} p+\mathrm{p} \mathrm{MM}\right.$ at 25 and $40 \mathrm{Cs} / \mathrm{C})$ see no narrow bumps.

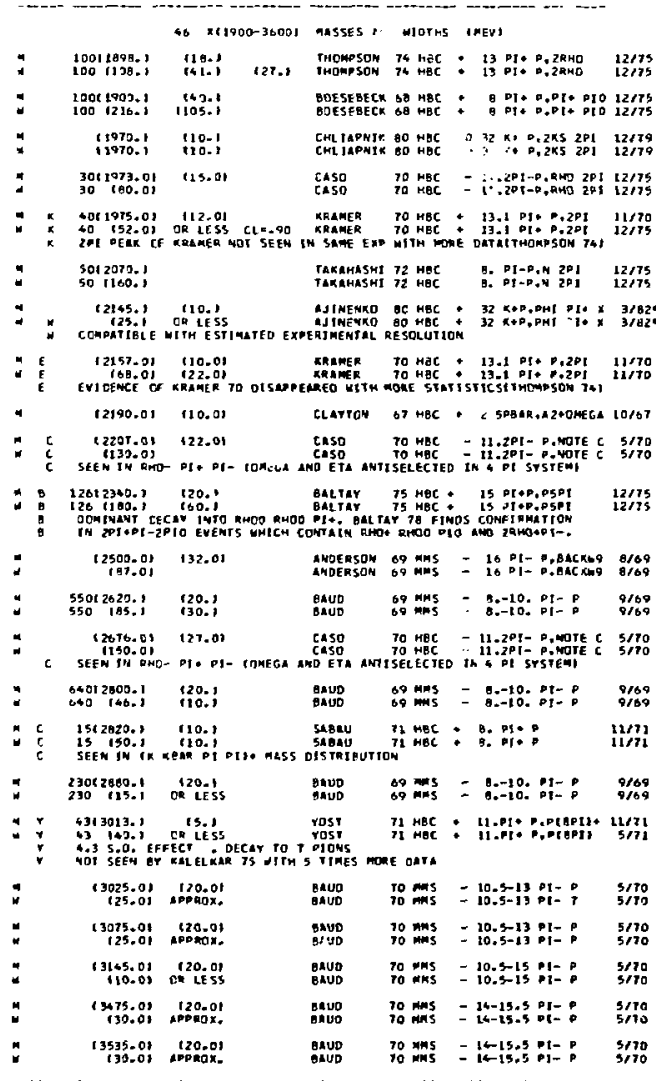

QEFEAREMCES FOA X/1900-38001

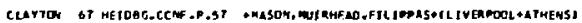
odesebec be $n=3,501$ ANDEASOM 69 OLL 271390

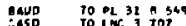

MSTO TO IAC 3 TOF BOESEBECS, OEUTSCHAMN, $\odot$ TALCHEM BERLIMEC ERMI tCoelins.e IAHL : CARH] CERA BDSOW SPECTROMETER CODUF ICERN

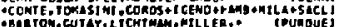

Data Card Listings

For notation, see key at front of Listings.

\begin{abstract}
SAgav 71 W
IAMAIASH 72 DO D 01206

thampson 74 asp 250

OLLTAY IS Del 35 EQT KALELARA 75 TMESISIMEVIS

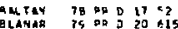

CLINE 79 ORL 43177 I

A JINEAXO $00 \mathrm{PL} 95 \mathrm{~B} 45 \mathrm{i}$

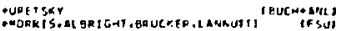

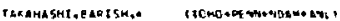

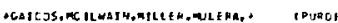

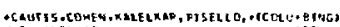

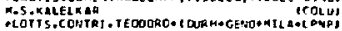

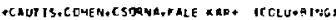

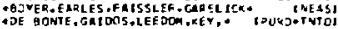

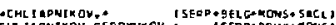
CHL ISTOM1KOV

(1)

\section{The Charmonium System}

We group into this systen those meson states commonly believed to consist of charmed-cuarkcharmed-antiquark gairs. Since the discovery of the $J / U(3100)$ (AUBERT 74, AUGUSTIN 1 i 74) this family has increased to at least 14, of which we tabulate 10 as well-established particles. Figure 1 shows the stares of charmonium below the $\psi(3585)$, interpreted by the chamoniun model, as of January 198?.

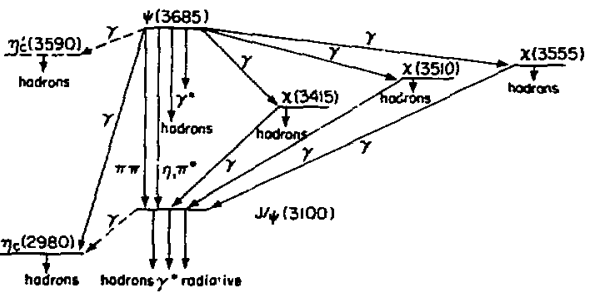

$$
\begin{array}{lllllll}
\mathrm{J}^{\mathrm{pC}} & \mathrm{O}^{-+} & \mathrm{1}^{-} & \mathrm{O}^{++} & \mathrm{1}^{++} & 2^{*+}
\end{array}
$$

Fig. 1. The current state of knowleoge of the charmonium system and transitions, as interpreted by the charnonium model. Uncertain states and transitions are indicated by dashed lines. JPC quantum number assignments are in sone cases tentative, but all are at least consistent with experiment; see individual particle listings for discussion. The notation $\gamma^{*}$ refers to decay processes involving intermediate virtual photons, including decays to $\mathrm{e}^{+} \mathrm{e}^{-}$and $\mu^{+} \mu^{-}$.

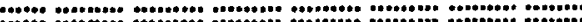

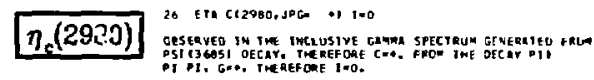

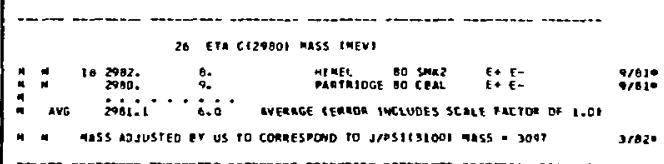




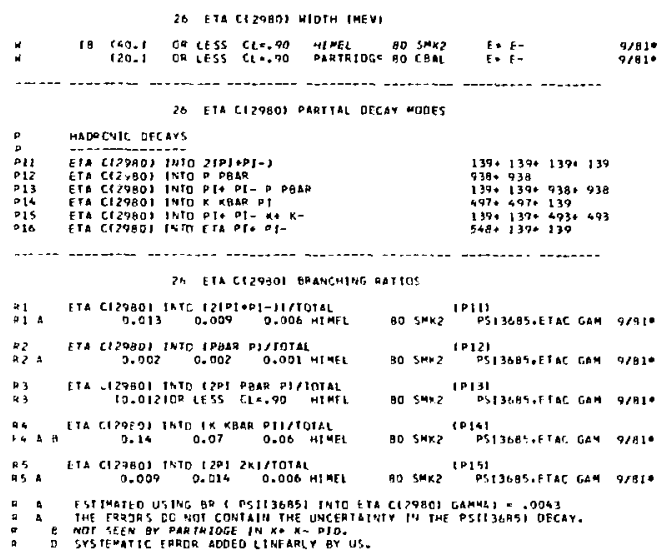

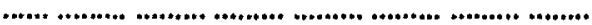

REFEREMTES fTR FTA CTZTEOT

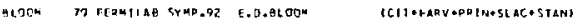

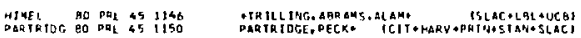

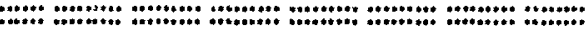

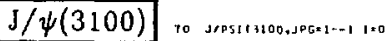

$7 C$ JPSTI310OI MASS \{MEY\}

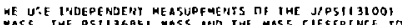
WASS, TAE PSIIJ6ASI MASS AHID IMF MASS CIFFEDINCE IO

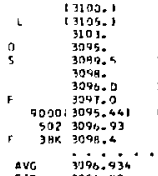

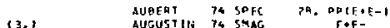

13.

4i.

30.0

3.061
0.09

0.09
2.2

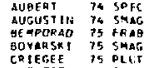

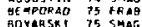

CRIEGE
PREPTI TS TS SPEC

$\mathrm{E} \bullet \mathrm{F}-$
$\mathrm{E}+\mathrm{E}-$
$\mathrm{E}+\mathrm{E}-$
$\mathrm{E}+\mathrm{E}-$

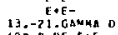

600 \& HE.T OF-

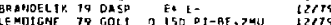

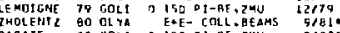

avg

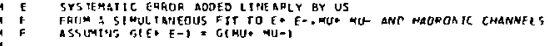

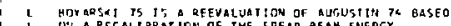

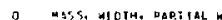

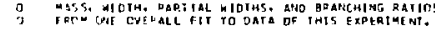

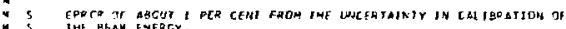

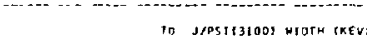

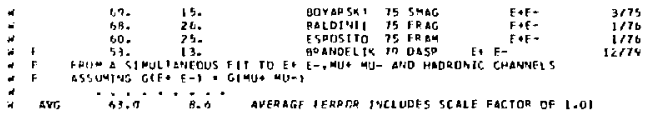

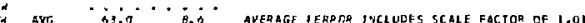

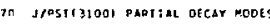

STOSICB1001 INTO EOE-

Jostlisool PRTO HaO

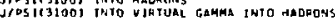

habPOIIC DEGaYS

J/P.:1310Ni INTO pl+ Di-

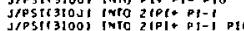

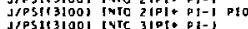

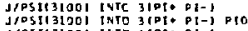

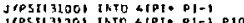

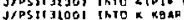

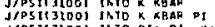

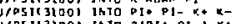

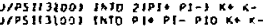

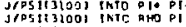

J/PSICBIDOJ JNTD RIO PI DI PI

J/PSITJOOO INTO OMEGA DI DI

J/PSIT3100 TNTO OMEGA $4{ }^{\prime}$

JPSI13100, 1NTO OMEGA K KGAR

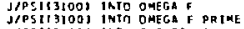

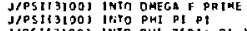

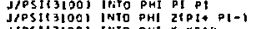

JYPSISHOOJ INIO PHI K XBAH

J/PSII3iOO) INTO PHI ETA
JPPSII3IJOI INTO DHI ETA PRIME

JePSIIHODI inTo PMI

JPSTI 330 O TMTO OHI PRTME

J'PSIT3100I TWTO A2 PI

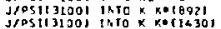

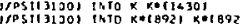

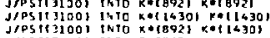

JPSIT3100 TWTO P FBAR

JRSTI3130 INTO P PHAR P10

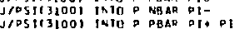

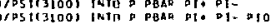

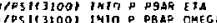

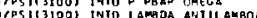

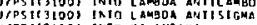

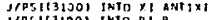

J/DEIt3inol TAto bi B

JPPSIIJ100I TNTO P PBAE EIL PRIMF

JPSTITLO PNTO SICMAO SITWABAPD

J/PSilizioo toro zint

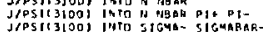

gaOTaTIVE tFCaYs

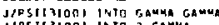

JPSIt3ionl intr 3 GaMMA

J/P5II310OI ThPO PTO GAMMA

JTPSI131001 DitO ETA PAIHE TAMMA

J/OS?131001 1WTO ETA CI29901 GA4M

J

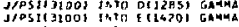

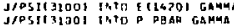

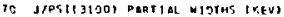

Decen MA5SES

$195+105$

139, 139

134

139. 1390: 139: 139

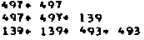

$1390139 * 1340+93$ *

$769.139+139+139$

76201990139.139

$782+1273$

$10194139 * 130$
$.019+1396139+138 *$

1019. 497.297

1019.568

$1019+1273$

$1019 \cdot 1520$

1318.160

497.891
+07.1234

Q91. 991

$1434+1414$
$191+1434$

938.938

$439 * 938 * 134$
$939 * 939+139$

938. $938+139+133$

930. $938+130+1390$

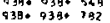

$1119+1115$

$1115 \cdot 1192$

$13140+13144$

$439+12338+957$

$1192+1192$

63* 493* $493+493$

$9360939 * 139+239$
$192+1197$

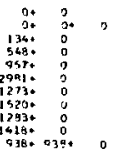

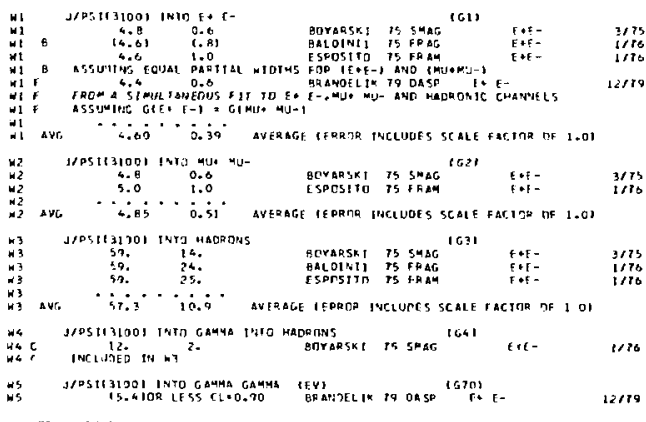


70 JIPSITHIOOI BRANCHING HAIIOS

FD THE GRANCHIME RATIOS RT - RG. SEE ML SO TME PARTLAL

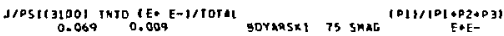

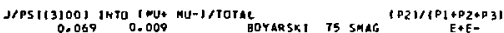

3/PSII3LOOO INTU I HEDAONSI/TOTAE

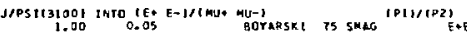

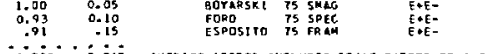

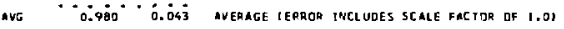

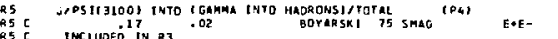

HAOACNIC OECAYS

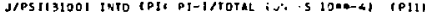

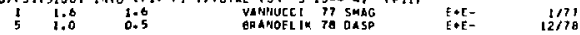

avo " T.OS - D.

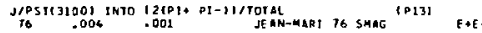

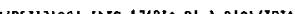

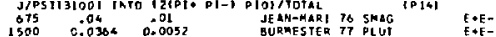

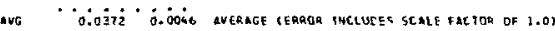

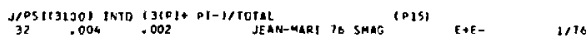

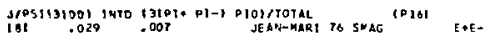

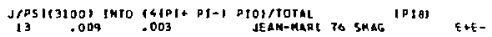

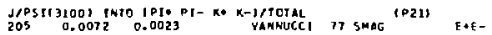

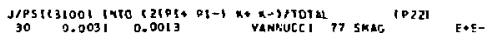

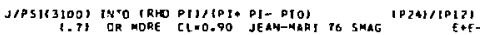

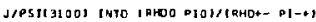

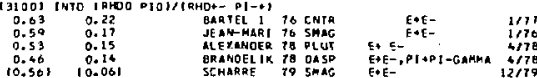

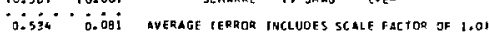

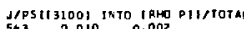

$\begin{array}{lll}543 & 0.010 & 0.002 \\ 153 & 0.013 & 0.003 \\ 193 & 0.016 & 0.006\end{array}$

$\begin{array}{cc}0.016 & 0.004 \\ 0.0133 & 0.0021 \\ 10.01321 & 0.00211\end{array}$

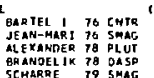

(P2a)

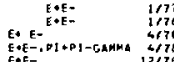

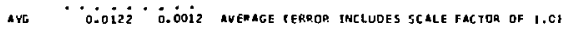

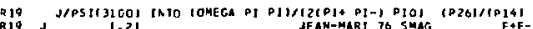

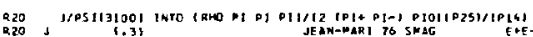

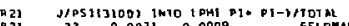

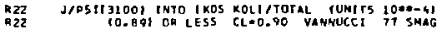

E+E -

$E \bullet E-$

$3 / 75$

$3 / 75$

irst

irs

110

$$
\begin{array}{cccc}
7 \\
2 \\
7 & 2.0 & 0.2 & 0.0
\end{array}
$$

GR ANOELIK 79 DRAG

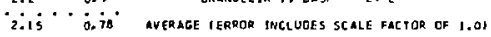

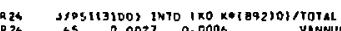

R24 450.00270 .0006 VANNUCC

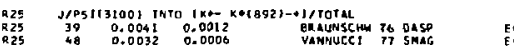

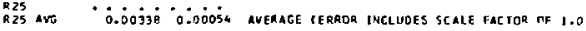

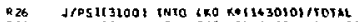

R2

a27
a27

A2

A2B
R2B

R29

230
230
T/PSII3LOOI INTO I Kr- KO11430-+1/TOTAL

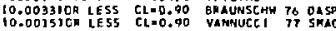

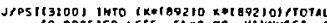

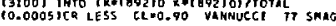

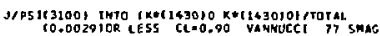

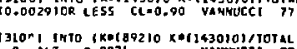

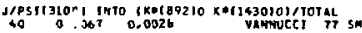

E+F-

$E+E-$
E*E-

$E+E-$

$E+E-$

$E+E-$

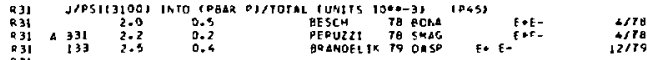

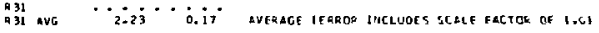

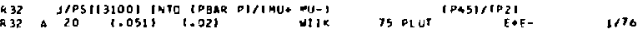

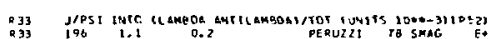

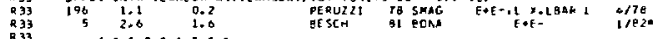

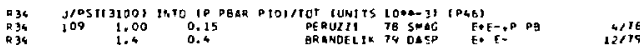

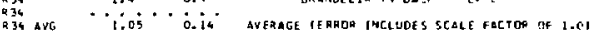

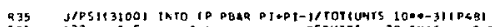

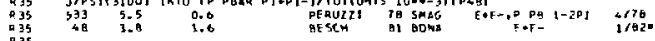

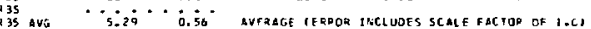

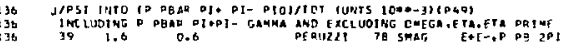

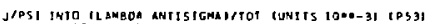

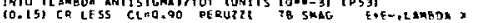

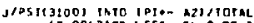

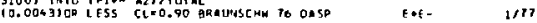

JPSTI3LOQS TPTS TOMEGA FI PIITIOTAL

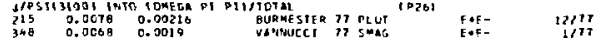

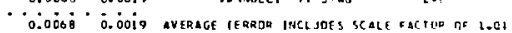

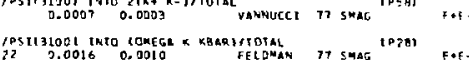

s?

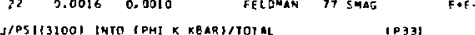

12,17

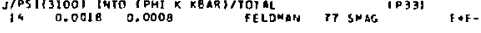

$12+1$

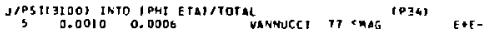

1,7

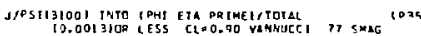

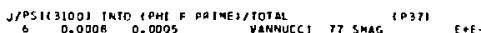

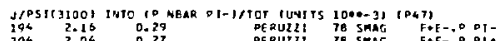

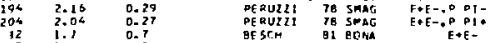

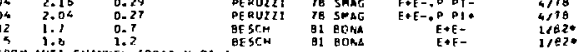

- 2.06 - i. iq anerage terror includes scale factor of 1.01

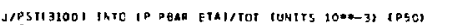

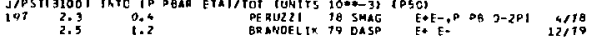

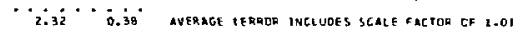

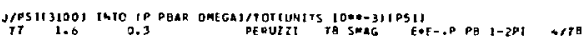

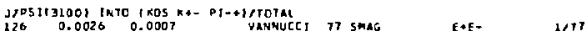

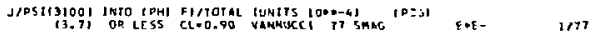

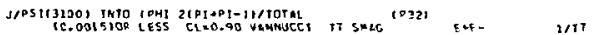

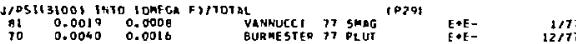

O. O. O. O

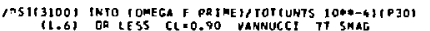

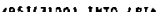

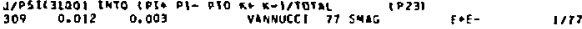

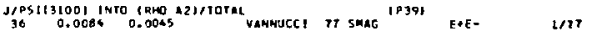

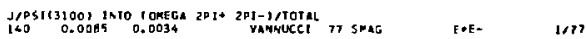

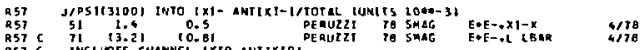

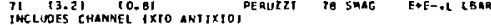

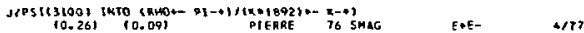

J/PSII3100) 1 16TO $18 \%-P !-+1 /$ TOTAL

870.00290 .0007 BURAESTER 77 PLUT E

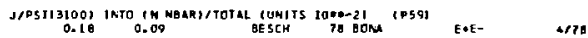

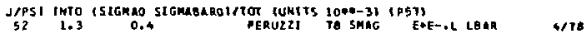




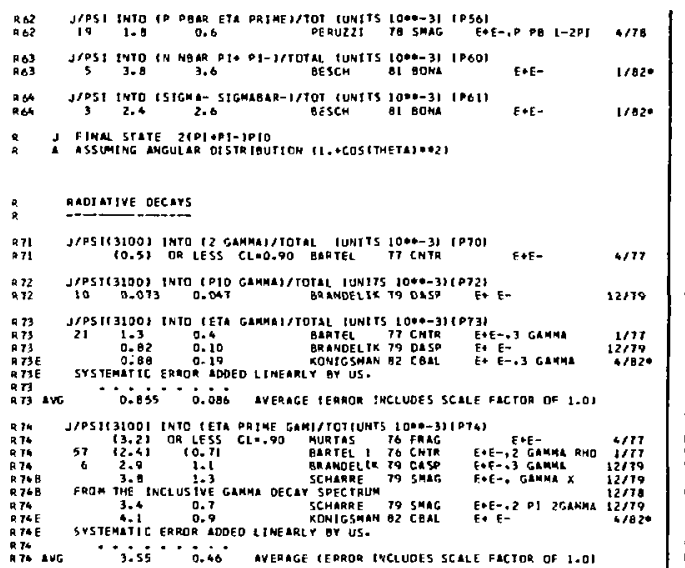

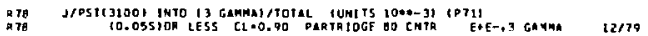

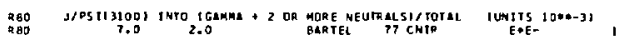

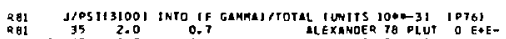

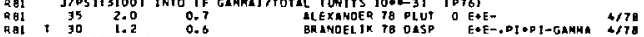

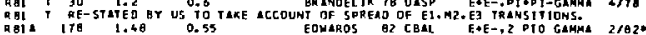

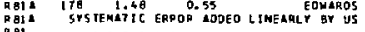

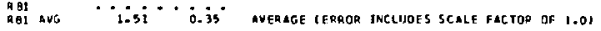

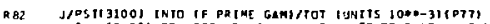

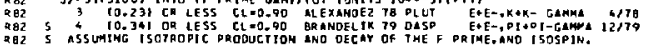

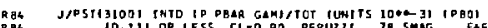

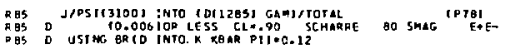

Q

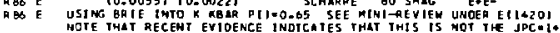
MOTE THLT RECEHT EYIDENCE INDTCATES THAT THIS IS HOT THE JPC.IA.

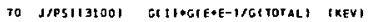

THIS COMBTNATION OF A PARTIAL WTOTH YITH THE PARTIAL MTOIH INTO EOE- ANO HTTH THE TOTAL WIOTH IS OBTA TNEB F ROM I HE INTEGRATED

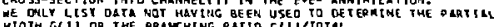

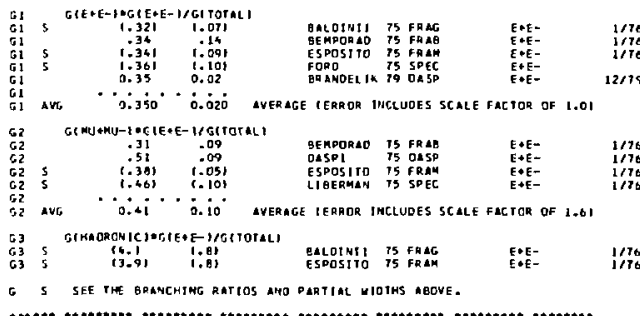

ERAISTEN TO PFL 251523 GBans 76 PRL 331453 AGH TS T4 HCL II 1 iCS MUGUSTIN 14 PRL 331406 OACG! 74 PRL 331408 BALOINI - 74 ARL 11711

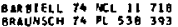
REFERENCES FOR J/P5I1312001 CHR ISTENSON + HICKS, LEDEAMAN + ICOLU* BNL + C,ERNI PBRIGGS, AUGUSTIM, BOYARS SKI+ ILRL + SLAC)

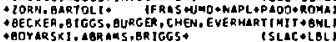

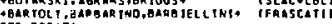
FOR ERRATH

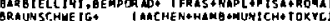

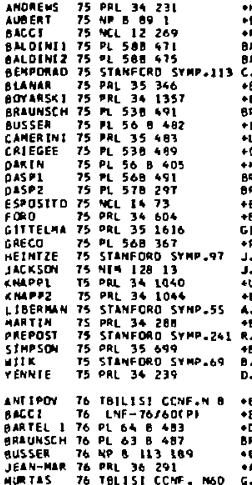

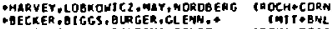

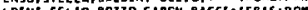
BAL N-CELIO, BOZZO.CA PGN, BACCT O TFRAS +ROMA BEmpoero

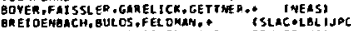

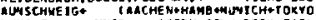
BL UMEMFFE D, BANWER; ICERN+CQ UEHDEK+ SACL

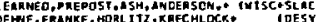
GEEISLEA, gDLOO, HEILE

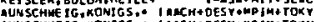
RAUHSCHUE IG.KOHIGS: TANCH+DESY TMPIMOTOKY

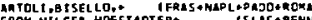

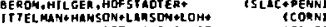
PAHCHEPT-SRIYASTAVA, SRIYASTAYA TERA J.HETNTIE
IHEIDELSERG

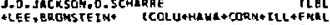
LEE, BROHSTE IN. TCOLU+HAWA CORN + TLL+FARL

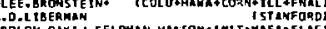

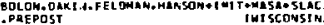

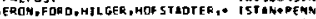

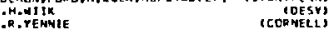

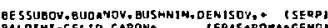

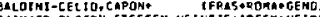
DU INKEA, OLSSON, STEFE EN, HE INT $Z E$ \& IDESY SHE IDI

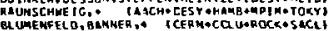

MURTAS 76 TALISt CCAf. MOO

PIERRE 76 TEIL ISI CCNF
SNYOER 76 PRL 36 I415

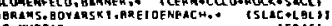

BARTEL MT PL BS B 499

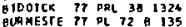

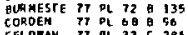
FELORAN IT OL 33 C 235 VANHUCC I IT PR O 15 1914. G.P.muhtins

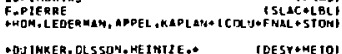

$\begin{array}{llllll}\text { MLEXANOE } & 78 \text { PL } 72 \text { B } & 493 \\ \text { BESCH } & 76 & \text { PL } 78 & 9 & 347\end{array}$

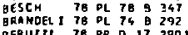

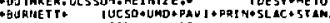

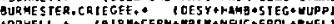

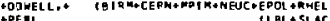
PETL $(1, B L+S L A C)$
$(S L A C+L B L)$

GRINOEL I 79 LPAT C I 233

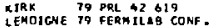
IEMOICNE TI FERMILAB CONF
SCHARRE 79 SLAC-PUE-232 TABRLAST COE $20 \div 0$ tokt

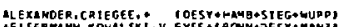

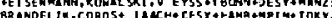
QPICCOL Co

BRANDELIK, COROS, +1AACH+ [ESY THAMB+MP] I + TOKY)

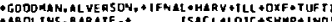

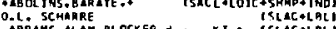

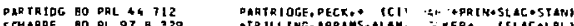

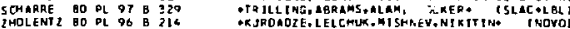

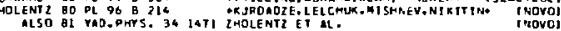

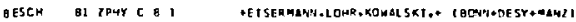

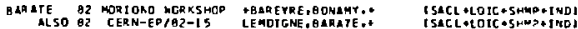

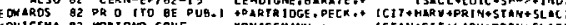

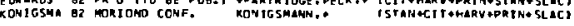
OAn $X(3415)$ 56 CHII3415.JPG=0NO) $1 \times 0$

CBSERYEO IN THE AADTATIVE DECAY OF PSII3EESI INTO

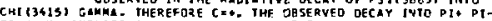

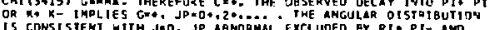

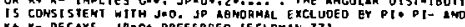

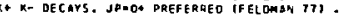

\section{se Chas36351 hass ineys}

\begin{tabular}{|c|c|c|c|c|}
\hline $\begin{array}{c}213407.01 \\
3415.0 \\
3622.0 \\
3618.0 \\
3614.0\end{array}$ & $\begin{aligned}(0.0) \\
9.0 \\
10.0 \\
4.0 \\
1.1\end{aligned}$ & $\begin{array}{l}\text { MIIK } \\
\text { QIODICK } \\
\text { GARTEL } \\
\text { TANEREAUM } \\
\text { TIMEL }\end{array}$ & $\begin{array}{l}75 \text { OASD } \\
77 \text { CNTR } \\
7 B \text { CNTR } \\
78 \text { SUAG } \\
79 \text { SMnt }\end{array}$ & 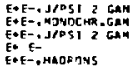 \\
\hline
\end{tabular}

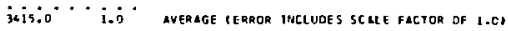

O MASS YALUE SHIFTED EY US BY AMOUNT APPROPRIATE FOR

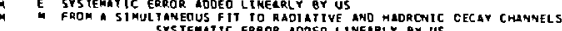
SYSTEMATIC ERROR AODEO LINEARLY GY US

SE CHII345) PARTIAL DECAY MOOFS

CHII34ISI 1 NOO PI+ PI-

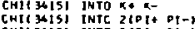

CHII3415) INTO $3(P)+$ Ot-1

CHI(3415) INTO PIE PI-KR K-

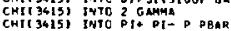

CHIC3415: INTO P TE OI- D DBAR

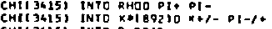

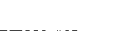

DECAP MASSES

$139+139$
$493+493$

$139+139+139+139$

1390. 13904930493

$139+1390.938 * \$ 36$

7890 1390: 139

9380.936

56 CHI 3415) ARANLHIHC RATIOS

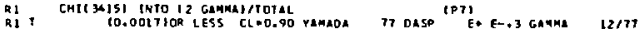

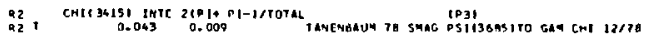


Mesons

$x(3415), P_{c}$ or $x(3510), x(3555)$

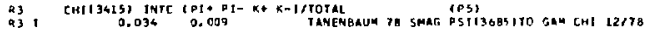

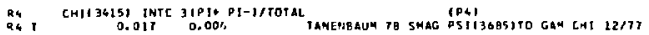

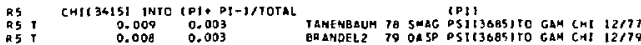

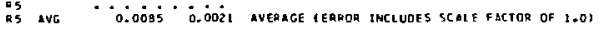

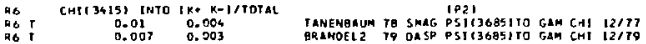

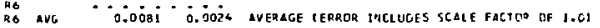

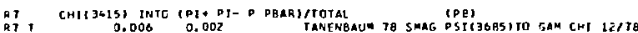

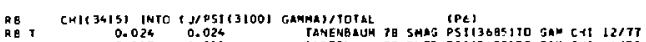

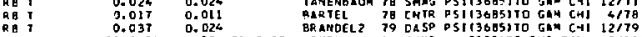

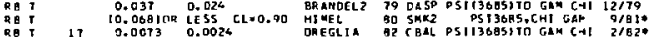

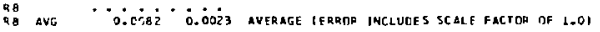

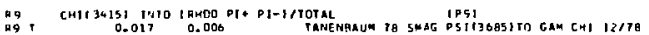

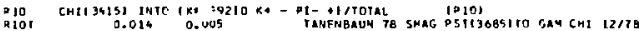

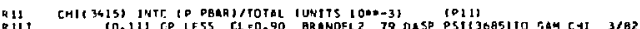

$R$ CALCJATEO USTNG P51(36BS) TO IGAMMA CHT (3415)I/TOTAL=0.082 3/820

$R$ THE ERRJA OT NGI CONTAIN THE UNCEYTATNTY IN TME PSIIBBESI DFCAY.

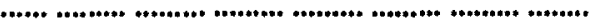
MEF ERENCES FTR CHI (13415)

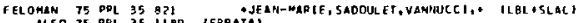

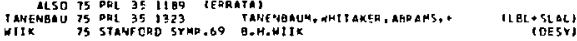

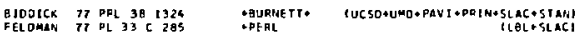

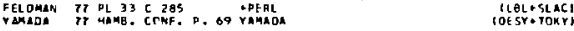

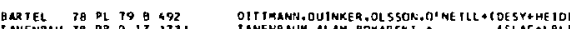

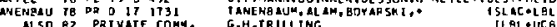

AL SO 82 DRTVIE COMA. G.H.TRILLING

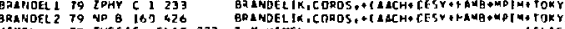

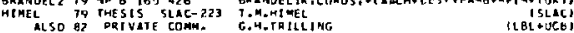

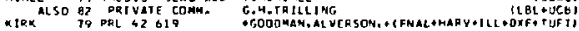

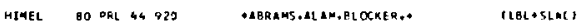

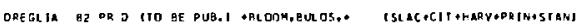

․․

$P_{c}$ or $X(3510)$ s5 oc or chitissio.jocajen tos OBSERVEO IN THE RADIATIVE SEGUENTIAL DECAY OF THE PSI ( TEST, INFD OC GAMMA, OC I

THE LACR OF OECATS IWTO DIO DI- OR MHK- IS SUGGESTIVE OF

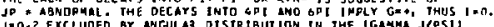

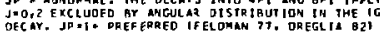

$$
\text { 5. PE 4a5s i MeVs }
$$

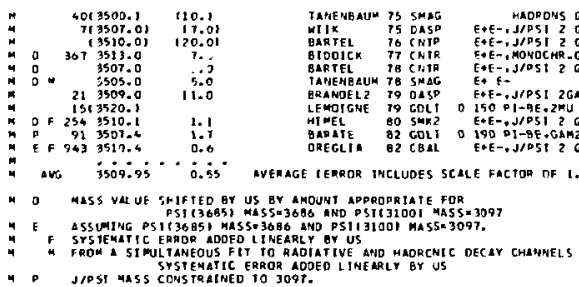

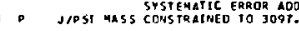

\section{DC PATITAL DECaY MOOES}

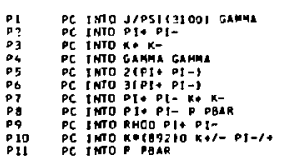

S5 PC BRANCHINC RATIOS

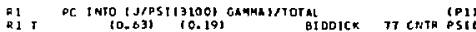

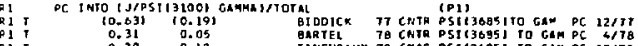

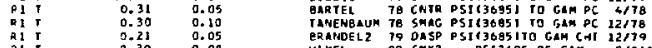

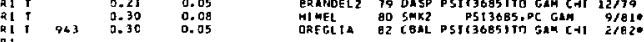

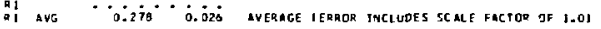

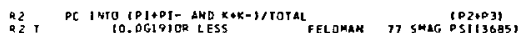
Q2 I PC TWTO DG19]OR LESS

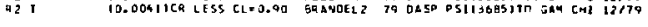

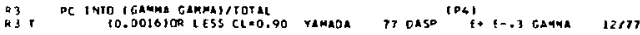

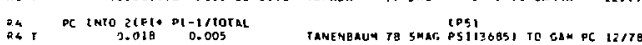
AS PC INTO

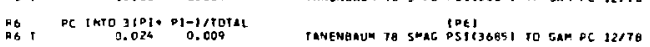

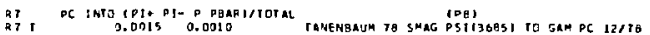

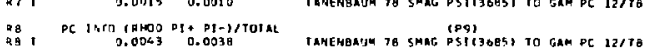

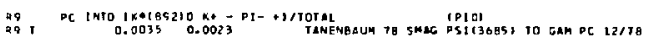

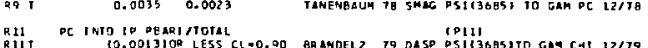

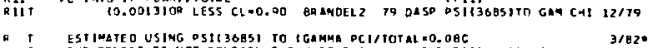

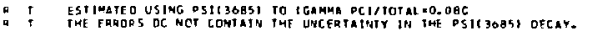

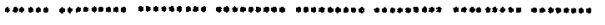

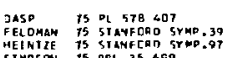
DEFEREREES FOP DE

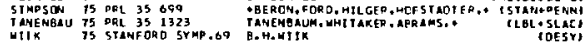

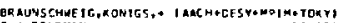
G.J.FELDHAY T.HE IYTLE

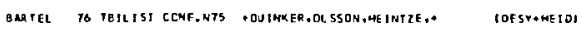

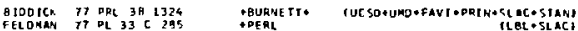

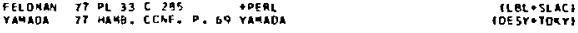

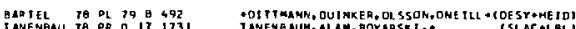

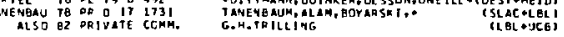

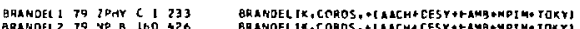

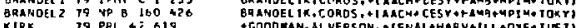

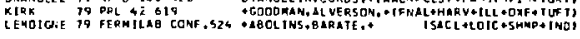

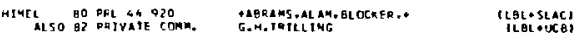

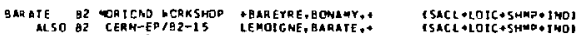

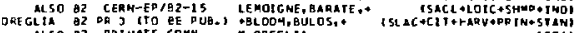
ALSO B2 PATVATE COMM. M. OAEGT IL

(2)

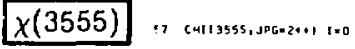

CaSERVEO IN RLOILTIYF OfCAY DF PSIITOESI IVTO CHII35551 GAMAR. THEREFDRE C*A. THE DQSERVEO OECQY SWTO API

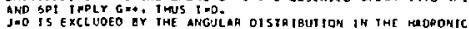
OECAYS. JD AEVORM RL EXCLUDED BY DI: P ? - AHD K. K- DECAYS.

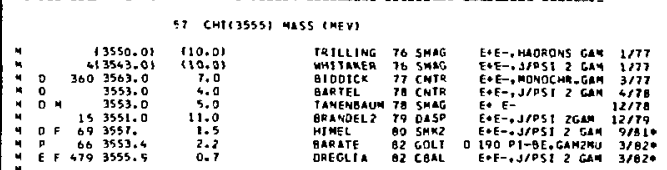

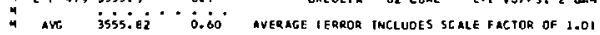

- D MASS VRLUE SHIFTEO OY US BY AMOUNT apPRDPRTATE FDR

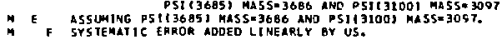

H I FYSTEMATIC ERROG ADDED LINERRLY BY US.

M J/FST MASS CCNSTERATED TO TO9T 
57 ChIJ 35551 partsal decay mades

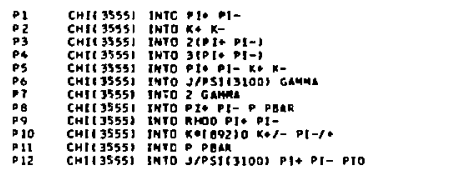

OECAY MASSES

139. 139

$139+1394+93+493$

30\%, :

1390.1390 .9340938

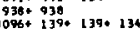

57 CHIIJS5SI BRANGHING MAINOS
$139+1340.1390139$

$769+1394 \div 139$
$691+4934 \quad 139$

\section{$\eta_{c}(3590)$}

9 ETA C13590.JPG 11

DQSERYED IN THE RAOTATIVE DECAY OF PSII36ES, JNIO

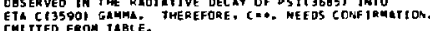

Evidence for the $r_{c}^{\prime}(3590)$ is based on the observation of a monochromatic gama 1 ine in the inclusive photon spectrum for $\psi(3685)$ decays (EDARRDS 82). No exclustve decay modes are known at this time. A signal had been reported for a state at Bimilar mass in the decay $\psi(3685)$, YYJ/W(3100) (BARTEL 7B), but this signal was not confirmed in an experiment with higher statistics (OREGLIA 82).

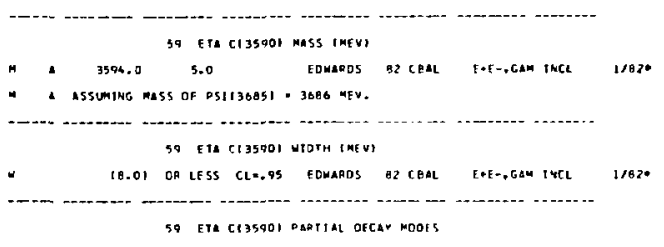

1 ETS CI35801 TATO HADETINS

TeGAY Massts

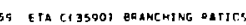

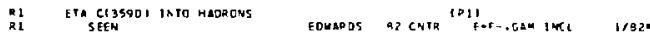

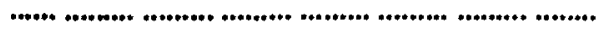
DEFEAEHCES FOR ETA CI35801

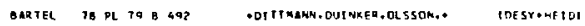

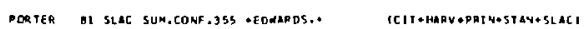

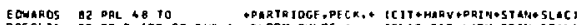

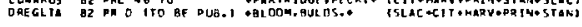

*atck $\psi(3685)$

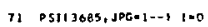

11 PSIL3Ga5) HASS IPEVI

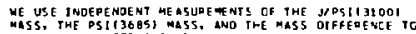

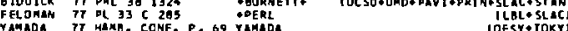

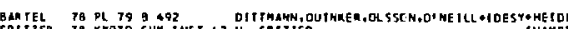
SPITEER 79 KVITO SUM.JHST.47 H. SPTTEEP

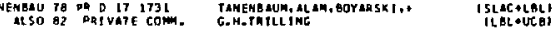

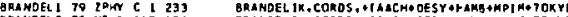
ERA NOELIR, COROS O+ IAACH+CESY OHAHE +MPINOTOKY

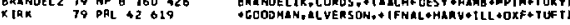

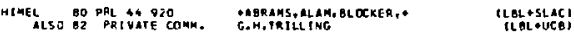

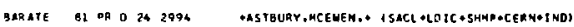

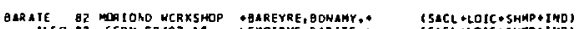

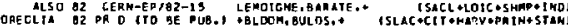

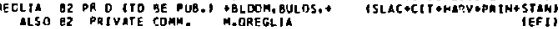

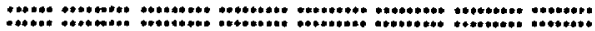
PEQFo

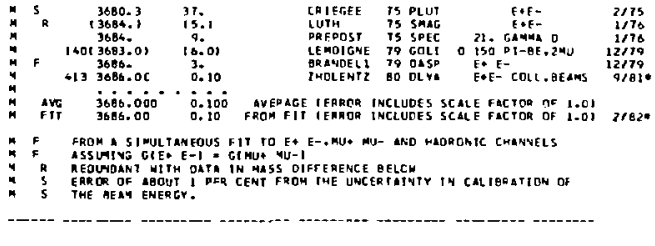


11 OS1(36851 - M/PSII3100) MASS CIFFERENCE (MEV)

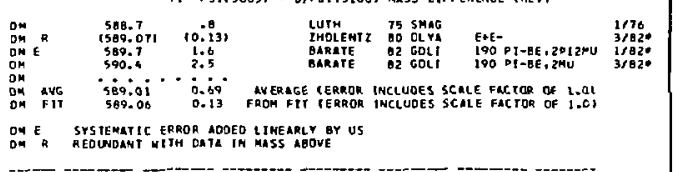

71 PSIT3685I U UDTH TKEY

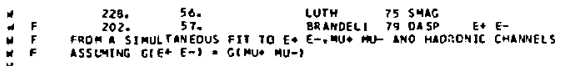

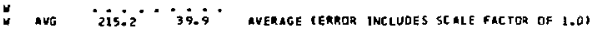

11 DSIS3685I DARTIAL DECAY HDDES

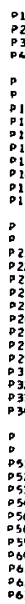

P1 PSII3685I JNTO EQ E

P2 PSI 3685) INFO HUW MU-

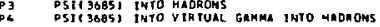

DECAY MASSES

DECAYS INTO J/PSIIJ1001 + ANYTHING

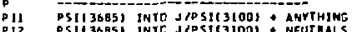

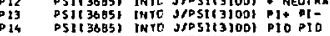

PSIS36851 TNTE JYPSIT3100I ETA

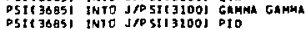

5
5

HADRONIC DECAYS

PS11 3685) 3N7O P J D?

PSII 3685 THTO R.M P

DSI 3605, INTO 2 ID T+ P1-

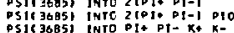

DStl 36asi INTO PAM D D

DS I 36BSI INTE LAMBOA ANTILAMEOA

D SIt 3605 I INTO X] AHrixi

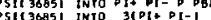

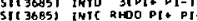

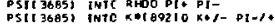

RAOTATIVE CECAYS

DSII 3685I TNYO GAHMA CaAma

P\$1 3685 , INTO PlO GaMma

PSII 36051 INTC ETA PAIME GAMAA

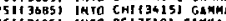

PSI 30851 INTA PCI3510) GAMAA

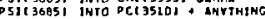

O51(3685) tNTa ETA CI2960) GLMPA

PSI 36951 INIO E (1 200 GAMMA

WED IN FIT

$30964139: 139$

30030.508

$3096 * 0.04$

1394139

$769+139$
$493+483$

$1394139+139+130$

$1390.137 \%$ 139. 1390

S구 938

$1115+1115$

$139 * 139 * 938 * 939$

ro9. $139 * 139$
$91 * 493 * 139$

DITIED PARTUL DECAY MOPE BRANCCHNG FRACTIONS

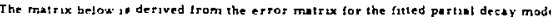

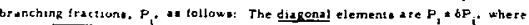

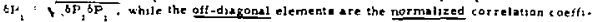

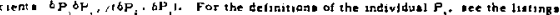

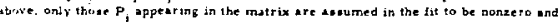
sre thut conatraned to add to 1.

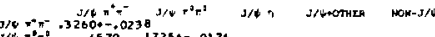

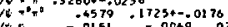

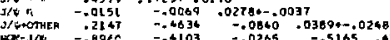

\section{DSII3OESI DARTIAL HIOTHS (KEV)}

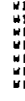

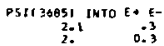

0.3 CUTH is SMAG $\mid$ TII

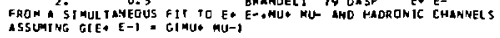

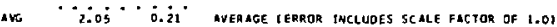

PSII3ESSI INTC REORONS
224.
S6.

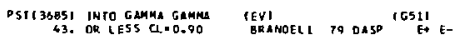

LUTH TS SMLG

71 OSIISOBSI BEANCHING PATIOS

RI
RI
II
nI
DI

DSI(3685) INTO IE* E-1/TORAL

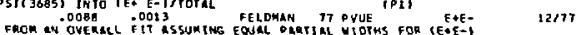

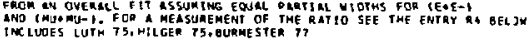

12179

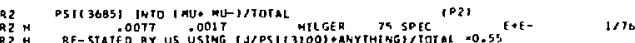

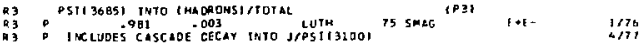

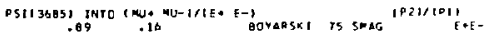

1213

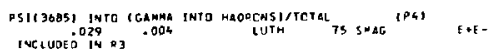

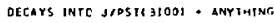

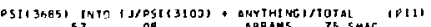

$\prod_{1,105}^{1196}$

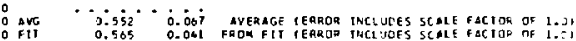

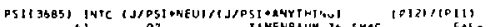

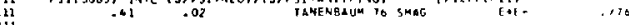

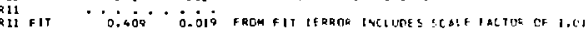

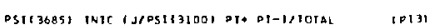

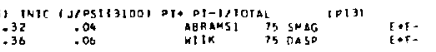

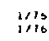

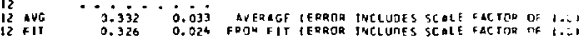

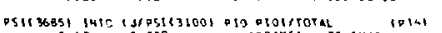

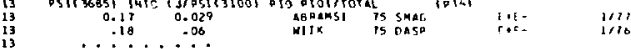

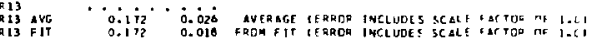

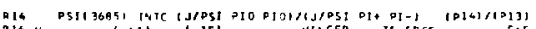

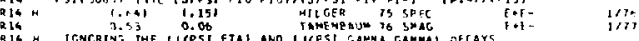

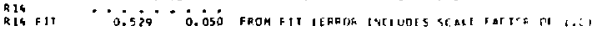

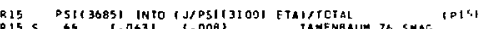

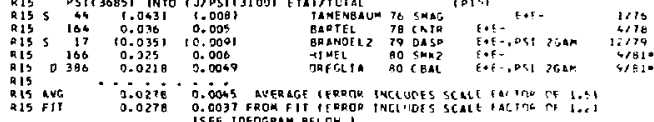

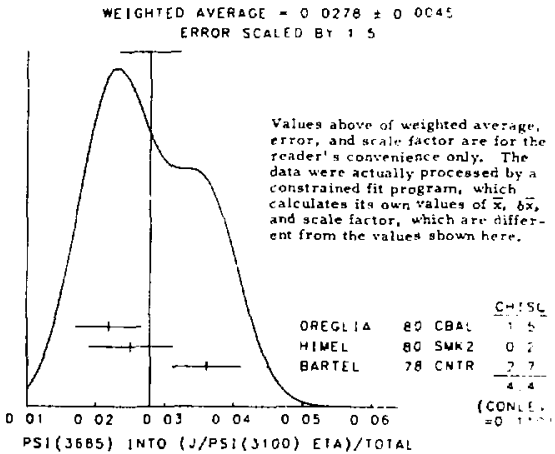

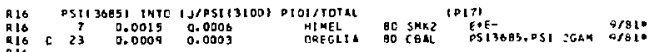

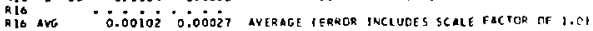

HADR DAIC DECAYS

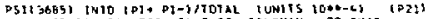

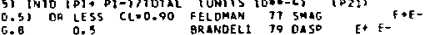

as1136851 inir 19HCO p10181010L

1-00LiOP LESS CLO

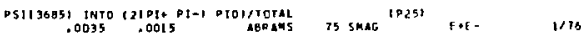

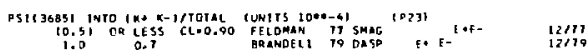




\section{Data Card Listings}

For notation, see key at front of Listings.
Mesons

$\psi(3685), \psi(3770)$

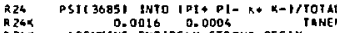$$
\begin{aligned}
& R 25 \\
& R 25 \\
& R 25 \\
& 825 \\
& 925 \\
& 925
\end{aligned}
$$

12376
12178
12177
12179
1277
12678
12177
12277

12170

$12 \% 78$

$1217 \mathrm{~B}$

$2 / 81 *$ $1 / 42$
}

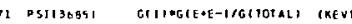

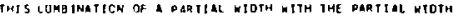

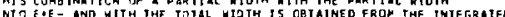

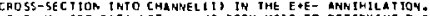

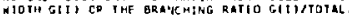

agrams TS SMAG E+E-

$1 / 76$ ME CNEY IIST DATA MOT MAVING GEFN USED TO DETEREINE THE PART! AL

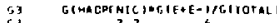

70

\section{(4)}

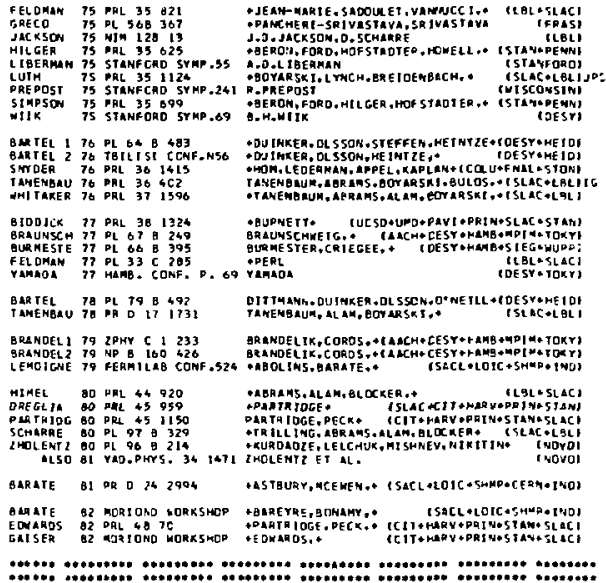

$\psi(3770)$ 93 Pst 3770. JP6.1. , i.

$$
53 \text { DSIC37701 MASS IMEYS }
$$

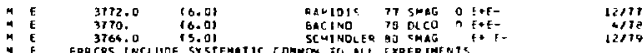
4LSS 3T69.9.

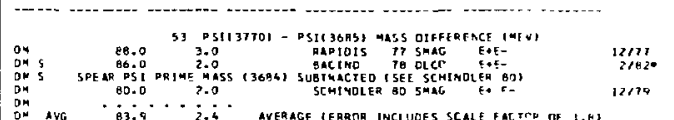

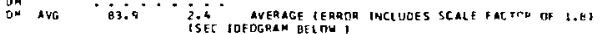
WE IGHTEC AVERAGE = $09.9 \pm 2.4$
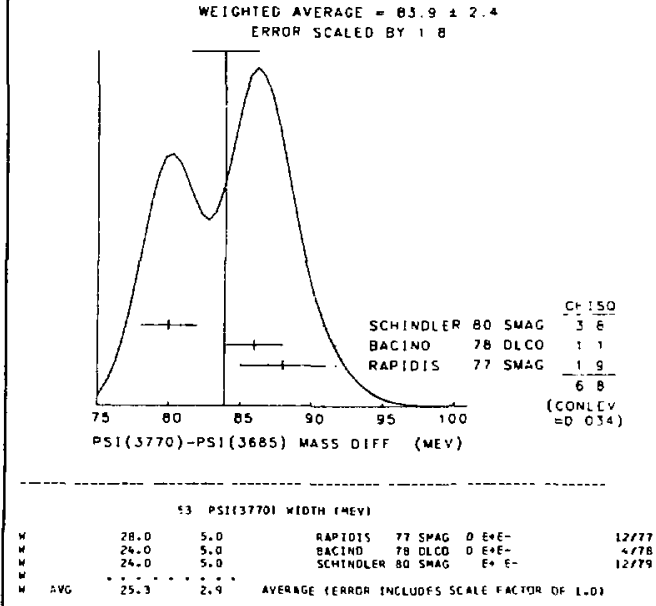

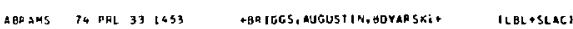

AGRAMS TS STANFORD SY AP. 29 G. S.ABPAMS

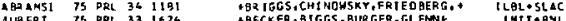

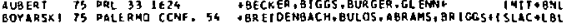

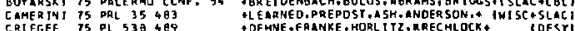

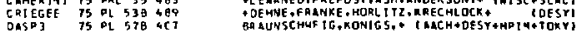


Mesons

$\psi(3770), \psi(4030), \psi(4160), \psi(4415)$

S3 OSIIJTYOI PARTIAL WIOTHS (KFV)

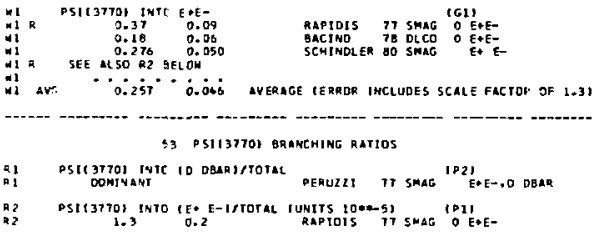

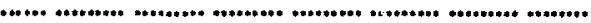

AEFEAENCES FOR DST (3TTO)

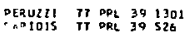

BAC IWO 78 PRL 40 OT1

ADICCOLO, FELOMAN, DERL, H I, AC, LBL, NHES + HANAI +OOBB1 oLUE + PERL +

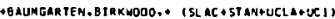

SCMINDCE 10 OA O 213716

SCMINOLER, STEGR IST, ALAMM, BOY RR SK I +I SL AC +LBL, I

coence

$\psi(4030)$

PSIC6030.JPGET- I I

SEEN CLERRLY SEPARATED FRDM THE PSIC\$1BOI

BY DASP AHD CONFIRHED WITH LESS STRTISTICS OY DLUTO SFE AR ALSD GY

72 PSII 40301 mass IAEY:

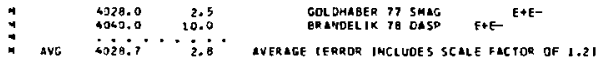

I) PSIT403OI HIDTH IMEVI

*

$52.0 \quad 10.0$

BRANOEL IT IO DASE EAE-

12 estisozol pagftal oecay hoDes

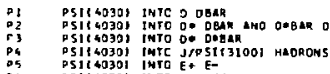

12 PSICA030) PAnTIAL WIOTMS IKEV!

11
$\because 1$

QR AMOEL IK 78 OASP IOS) EA E-

$105 * 105$

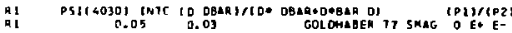

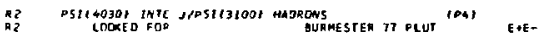

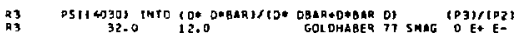

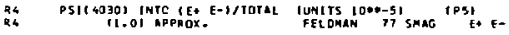

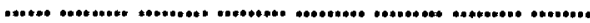
REFERENCES FOR PSI14030

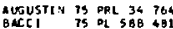
BOYASXI is PQL 34 to?

ESPOSITO 75 PC 508479

- BDYARSK1 + LERANS, BATEGS

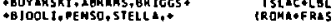

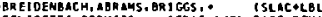

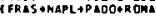

PERUZZI 76 PRL 37509 -D TCCOL O,FELOMAK.MEUTER,WTS S." ISLAC ELBLI

GUMESTE 77 PL BO 395 FELOKAN 77 oL 33 C a

LUTH 77 DL 70 G 120

+CRIEGEE, OE HWE + ICESY+F NHE+5 1EG+KUPP GOLDHABER, WISS, ABR AMS, ALLAM, LUTH, " LLBL+SLAC TPERL

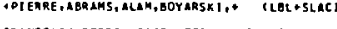

QR ANDELIK, CORDS + RAACH CEST + HAMB +MPI $R+$ TOKY

ALSD 79 TPHY C 13233

K LaKBY TO FERMILAB SYMP.10T J. KIRKEY RAPPORTEUR

(SLAC)

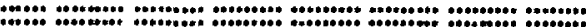

Data Card Listings

For notation, see key at front of Listings.

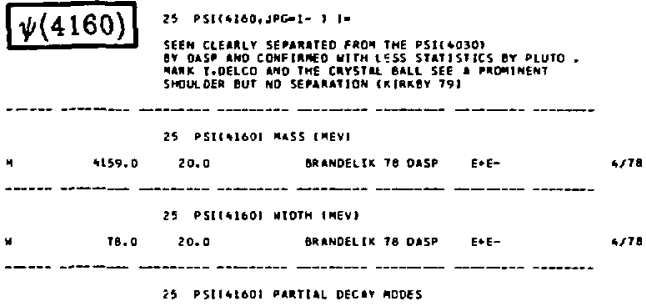

- PSIS41601 1NTOE+ E-

DEC MY wasSES

-

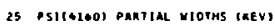

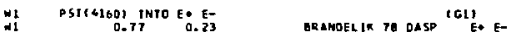

12 ,

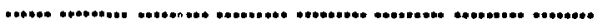
REFERENCES FOR PSIIALCO

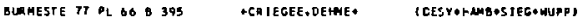

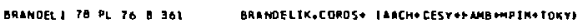

KthKer TO FERMILAG SYMP.LOT J. KJRKBY RLPPORTEUA

(stact

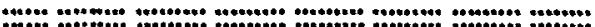

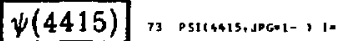

13 PSIltalsI maSS IMEVI

478

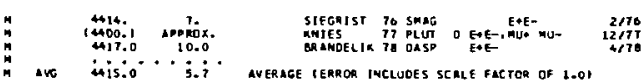

13 PSILALIS) WIOTH IMEVI

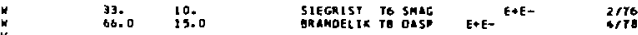

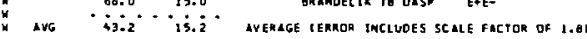

73 PSITHLIFI PARTIAL DECAY DOES

$12 / 78$

D1 PIIसISI TNTOE+E

DECAY MASSES

PI PSI 4415$) 1 N 70$ E. E-
0.49
O.

$12 / 17$ 1217

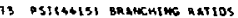

$12 \times 77$

a $121 \mathrm{TT}$

R2

REFERENCES FON PSI $1441=$ ?

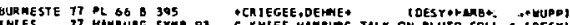

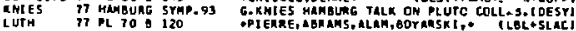

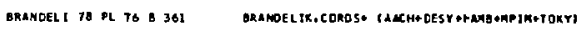

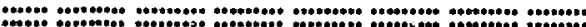




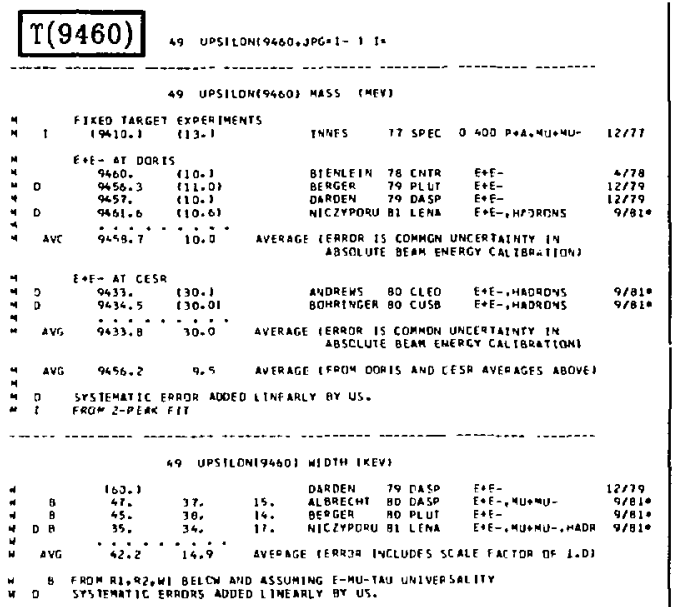

49 JPSILONI 94001 PAGIlaA. OECAY mODES

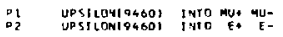

DECAT Messes $105 * 105$
$.3+\% 5$

48 UDSTLONTOMOOI DAK TIAL MIUTHS INENI

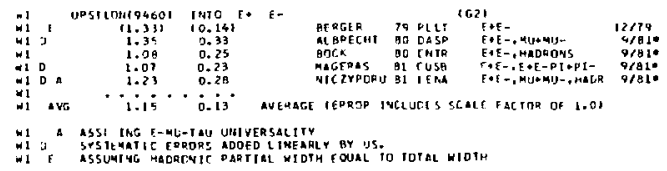

49 UDSILONI P46OI BRANCHING RAIIOS

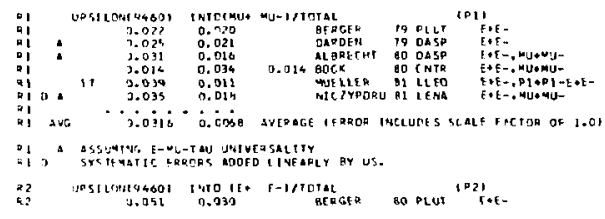

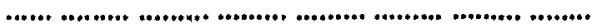

REFE \#EHCES FOR UPS ILCHT QQSOO

Crish II HL 12 \% 273

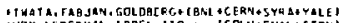

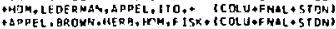

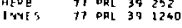

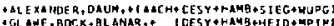

QERTER PA PI TS A 563

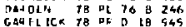

$78 \mathrm{DPL}$ \& 4 \& 35

YEA TEPRL 41 Be4

DHEELIS 19 DL 87 B $39 \mathrm{AB}$

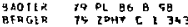

OAADER TO OL

ALBRECHT BO $x$ E 93 A 500

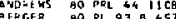

BICK

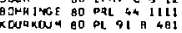

FAGERE; At FPL 46 1115

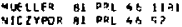

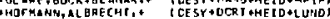

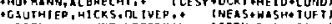
CADDEL,HERB, HOM, LEOERMAN, I ISTON, FNAL •COLU

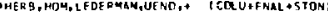

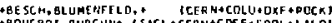

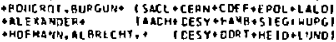

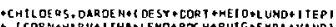

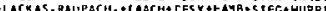

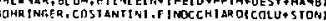
GBAL +CERHESVRA YALEI

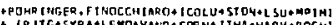

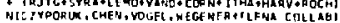

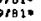
Q/81: $9 / 86$

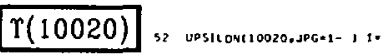

32 UPSILONLIOOZOA mASS TSEXI

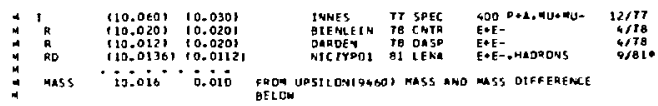

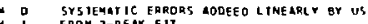

I RROM Z-PEAX FIT WATA IN WASS OIFFERENCE GELON

Fun with oara in wass oifrenence geton

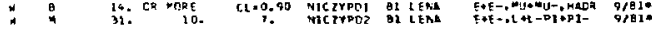

* G FRO WI AMO DI AEOVE AND ASSUMTNG E-MU-TAU UNIVERSALITY

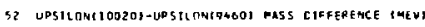

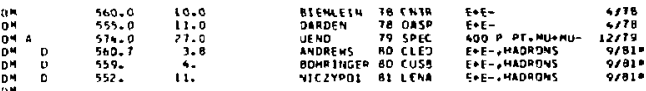

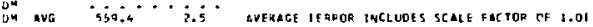

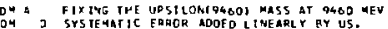

$\$ 2$ VOSHLONIIOJ20J OARTIAL OECAY MOOES

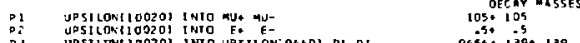

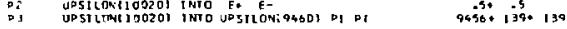

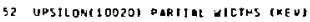

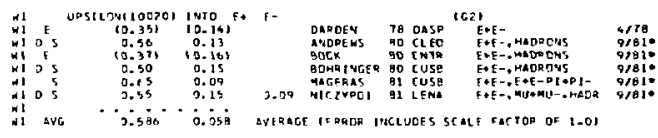

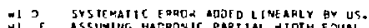

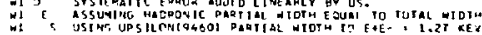

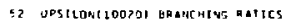

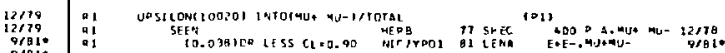

a.

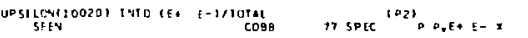

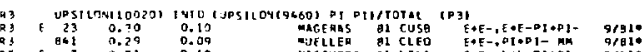

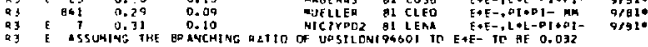

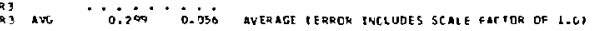

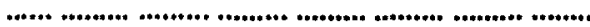

CCBa II $\mathrm{P}_{1} 72=53$

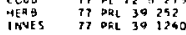

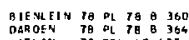

DAROEN 7O PL 78 B 364
SAPLAY 78 PRL 40435
TO

JE $70 \quad 79$ PRL 42496

ANONEWS BO PRL 441113

BDCK BO 7 PHY C 6125

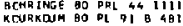

YALERAS 81 PRL 401115

MUELLER 81 PAL 461118

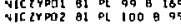

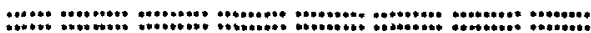

DEFEAENCES FOR UPSILONI 200201

- InATA , faB JaN . COL JgERG + BNL +C ERNa SYRA+YALE

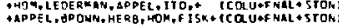

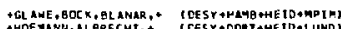

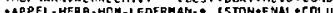

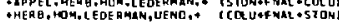

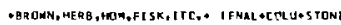

+ [COAN+HARY+ I THA+LEHO+ RDCH+RUTGESYRA + VANO)

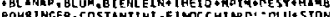

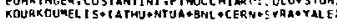

- BDHR INGER, F INDCCHITAOC+ ICOLU + STON+LSU+4DIMI

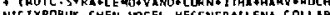

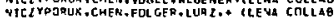

; 
Mesons

$\mathrm{T}(10350), \mathrm{T}(10570), K^{ \pm}, K^{0}, K^{*}(892)$
Data Card Listings

For notation, see key at front of Listings.
$\Upsilon(10350)$ \&s UPSILONII0350,JPG $=1-1$ i.

4a UPSILCHILOBSOI masS (GEV)

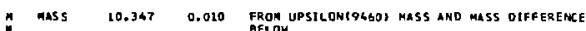

48 UPSILONILOBSOI-UPSILONI9HEO) MASS CIFFERERCE (MEV)

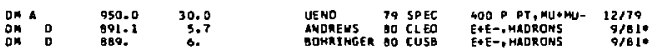

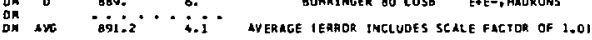
OR A F1X ING THE UDSTLONI94601 MASS AT 9460 MEV AND TME

OF A UPS TLONA 10020 )-UPSTLOHI9460) MASS DIFFERENCE AT 550 MEV.

a U UPSILONC10350i pantal oecar moOEs

P1 UESTLON1103501 iNTO nue MU-

DEEAY MASSES

44 UPSILONIIOJSDI PARTIAL MIOTHS TKEY

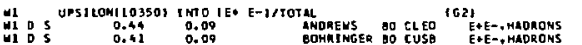

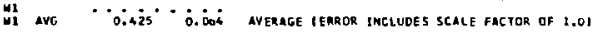

WI D SYSTEMAT tC ERTOR aDOED LIMERRLY BY US.

II S USING UPSILON(9460) PARTIAL WIOTH TO E

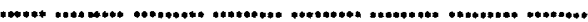

COAB $71 \mathrm{PL} 72 \mathrm{PR} 273$

MERB 77 PRL 39252

REFERENCES FOR UPSTLID I10350

$\begin{array}{lllll}\text { KAPLAN TO PRL } & 40 & 435 \\ \text { TOH } & \text { TO PRL } & 11 & 864\end{array}$

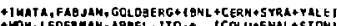

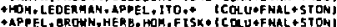

TDH TO PRL 41 BEA

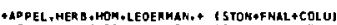
4HERg. HOR, LEDERMAN,UENO,: I COU +FNAL+STONI

HEHO TO PRL 12 to

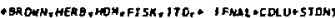

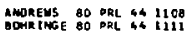

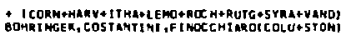

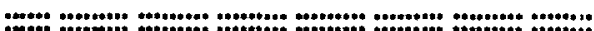

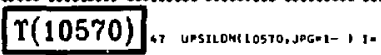

4T UPSILONILOSTOI Mass tgev:

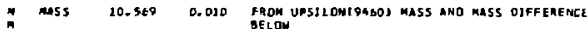

\section{UPSILOMCIOSTOS WIDTH (WEYI}

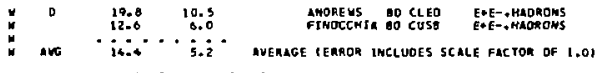
- D SYSTEMATIC ERROR aODEO LIMERALY BY US.

4 UPSILON(10S70)-UPSILOHT946O MASS EIFFEREACE IMEVI

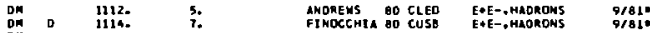

OM AVG iliz:i. :

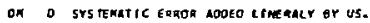

GT JPSILOMTLOSTOS RARTIAL DECAT MOOES

PI UPSILONIIOSTOS INTO MUE MUL-

OECAR MLSSES $105+105$

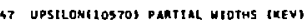

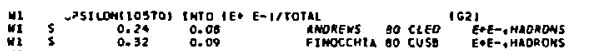

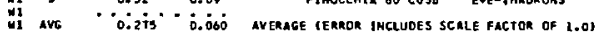

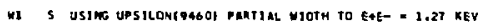

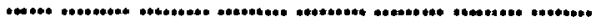
REFERE HCES FOR UPS ILONT 10STO1

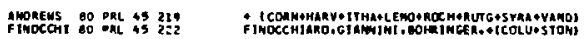

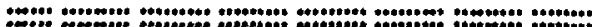

-

$$
\mathrm{S}= \pm 1, \mathrm{C}=0 \text { MESON STATES }
$$

ate

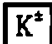

10 CHARGED $\times 1494, J P=0-1 \quad I=1 / 2$

SEE STARLE PARTICLE DATA CARO TISTTNGS

+

$\mathrm{K}^{\mathbf{0}}$

11 NEUBRAL K(498,JP=0-) $I=1 / 2$

SEE STABLE PARTICLE OATA CARD LISTINGS

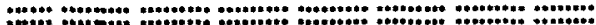

$\mathrm{K}^{\prime}(892)$

$18 \quad K * 1892 . J P=1-1 ;=1 / 2$

18 Mologal MASS INEVI

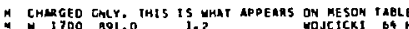

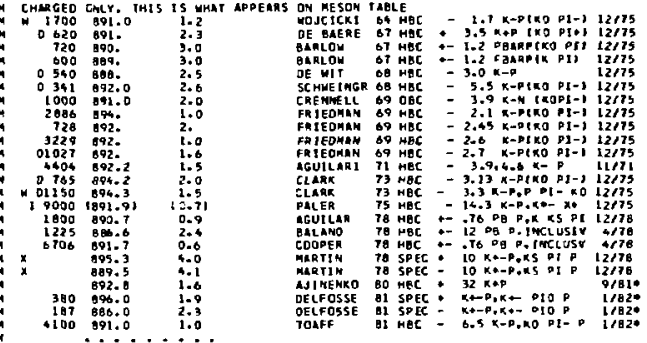

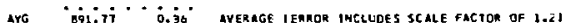

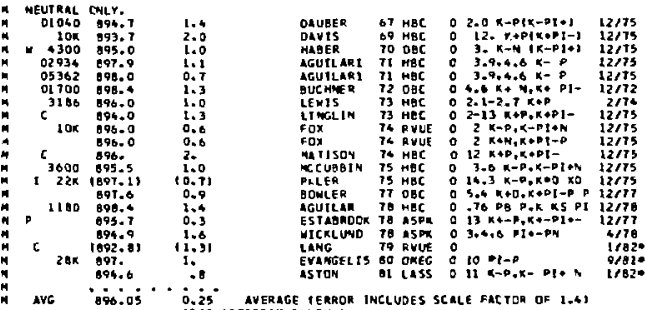
is

WEIGHTED AVERAGE $=896.03 \pm 0.25$ ERROR SCALEO BY 14

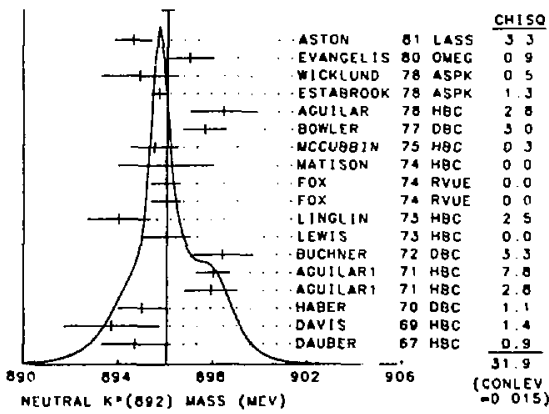




\section{Data Card Listings}

4 CROM POLE EXTRAPOLATION

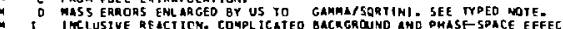
A FRO P PHSE SHIFT ANALYSTS OF 155000 EVEHT S.

SYSTEMATIC ERaD AODE

\section{Yote on $K^{*}(892)$ Masses and Mass Differences}

Unrealistically small ercors are reported by some experiments. We use simple "realistic" tests for the minimum errors on the determination of mass and width from a sample of $N$ events:

$$
\delta_{\min }(m)=\frac{\Gamma}{\sqrt{N}}, \quad \delta_{\min }(\Gamma)=4 \frac{\Gamma}{\sqrt{N}} .
$$

(For detailed discussion see the April 1971 edition of this note.) We consistently increase uinrealistic errors before averaging.

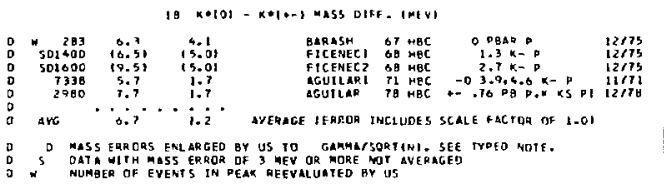

18 K*18951 HIOTH THEVI

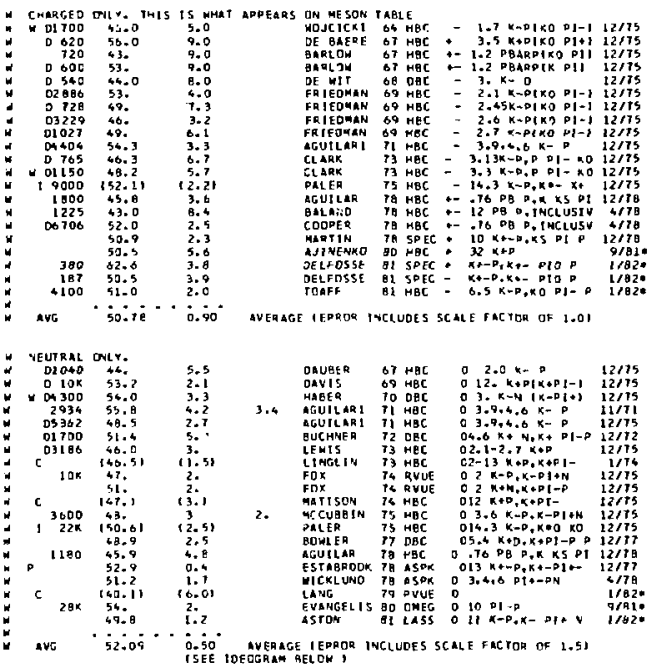

\footnotetext{
* C Fan POL E EXTRAPOLATION.

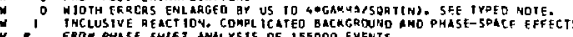

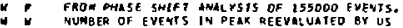

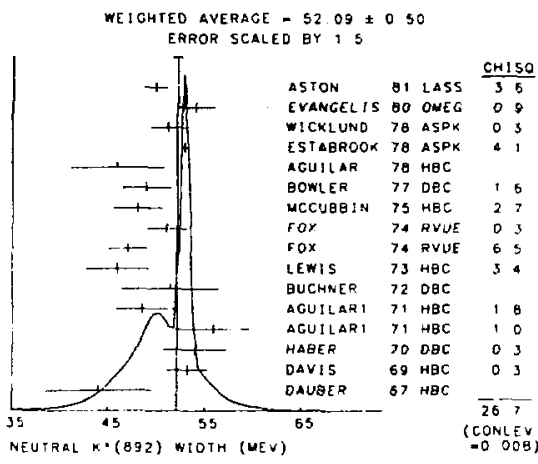

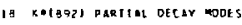

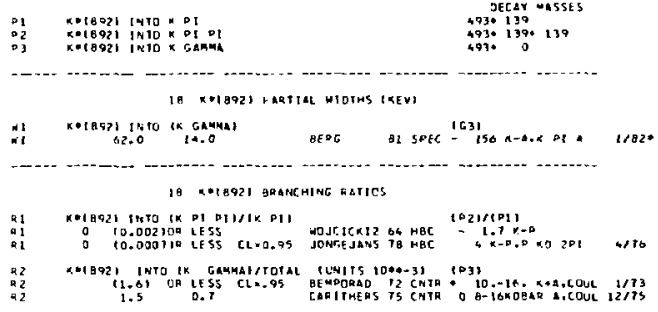

REFERENCES FOR $M+19923$

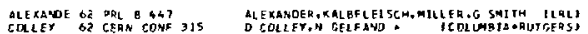

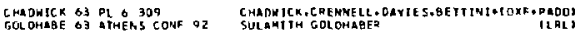
WOJCICKI GS DQ 135 Q 486 STANLEYG NDJCIEXI TEPLI SOELHAN G5 ATHENS 527 TER RO-LU 65 NC 36 LIDI FEARO-LU O5 NC 39 41

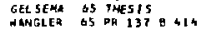

PARASH of $156 \quad 1397$

BARLOH Q7 4 N 50 A

CONFURTO OI 40 B 33469

DAUBER OI PR 153 1 1003

DE EAERE O7 NC SI A 40

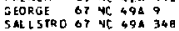

OE 1T 68 THESIS

FICENEC 1 OQ DR $10^{\circ} 1034$ FICEMEC 2 OQ PQ 1751725
KAHG

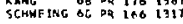
CRENMELL OQ PAL 22 ? 487 DAVIS OQ DQL 231071
OE BAERE OQ Yt OE BAERE OD It OI \$ 397 JUMALA
LIND $\begin{array}{lllll}\text { ATHERTON 70 ND } & 16 & 416 \\ \text { HABER TO NA B } 17 & 299\end{array}$ AGUIL SF 71 PRL 26 REO

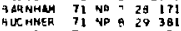
COROS II DR 04197

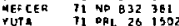

STUART LEE ADELMAY [CAVENOTSH]

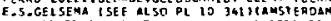
HANGLER,ERUIN,WALER ANISCONSINI

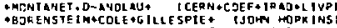
- MARECHRL, MONTANET + GERN+CDEF+ IPN+LI IVERDOOL

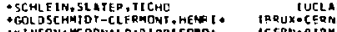

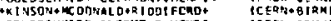

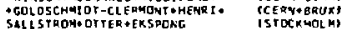
5. OE WIT IAMSTERDAMI

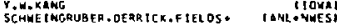
TKARSHON,LAl.ONERLL.SCARa IONL -OEREN2 O, FLATIE, AL STCH, LYHC, SOLWITL HLEL J.FAIEOAGN, PH, O. THESTS TS TRLS

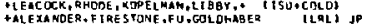

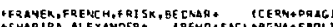
TSHAPIRA, ALEXANDER TREHOHSACL HESWA +EPOLI

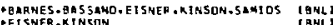

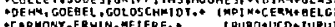
- CARMORY, ERHIN, MEIERE, I TUROAUCD+IUPU OANTICH.CAL LAHAN.EMIEM.COX.- IJOHN HOPKIVS] FERAO- LJZ2ZI, GEOPGE, HEHP T, JONGESANS ICEPNI

BARASH, NIRSCH. *ILLER,TAR TCOLUMBIAL

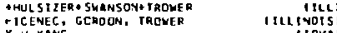

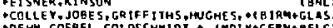


Mesons

K'(892), O REGION, Q,(1280)
Data Card Listings

For.notation, see key at front of Listings.

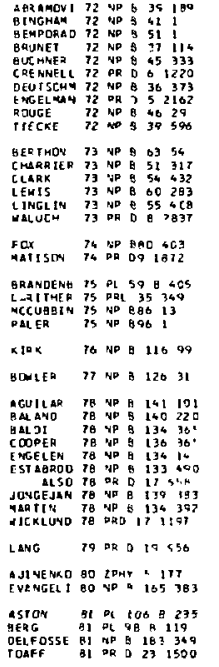

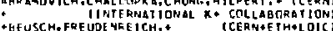

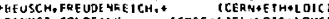

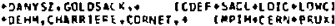

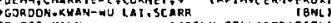

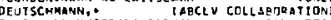

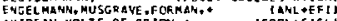

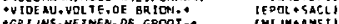
\{NIJusats

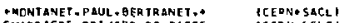

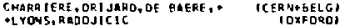

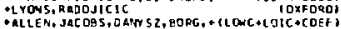
D.t INGL IN

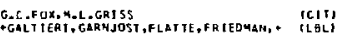

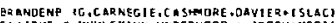

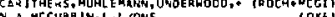

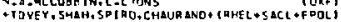

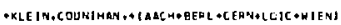

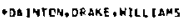
ronfocos

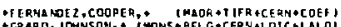
Co

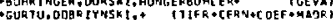

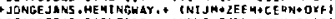

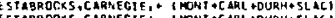

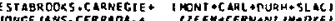
SHIHAOa

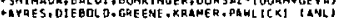

C.A.LANG,A.MAS - P QRE LREOA (arais)

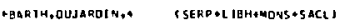
- IBAKI+BONN+CERH DARE+CLAS \& LIVR EMIIA+KIENI

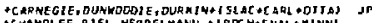

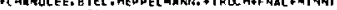

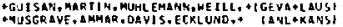

*

Note on the $Q_{1}(1280)-Q_{2}(1400)$

Since all the recent high-statistics experiments investigating the partial-wave contents of the $\mathrm{K} \pi \mathrm{T}$ system diffractively produced on protons (BRANDERBURG 76, OTTER 76, VERGEBST 79, DAUM 81) give consistent evidence for the existence of two strangeness-one axial vector mesons, we have split the " $Q$ region" entry of our last edition into two entrits: one for the $Q_{1}(1280)$ cesonance, with a mass around $1280 \mathrm{MeV}$, a width of the order of 100 MeV, and mainly coupled to the $k \rho$ channel; and another one for the $Q_{2}(1400)$ resonance, with a mass around $1400 \mathrm{MeV}$, a width of the order of 180 MeV, and mainly coupled to the $k^{*} \pi$ channel.

Ntice that, whereas both $Q_{1}(1280)$ and $Q_{2}(1400)$ are produced in diffractive processes, the non-difdiffractive reactions (ARMENTEROS 64, CRENNELL 67, 72, ASTIER 69, GAVILLET 78, ETKIN 80, RODERACK 81) select preferentially the production of one of the two states.

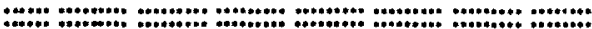

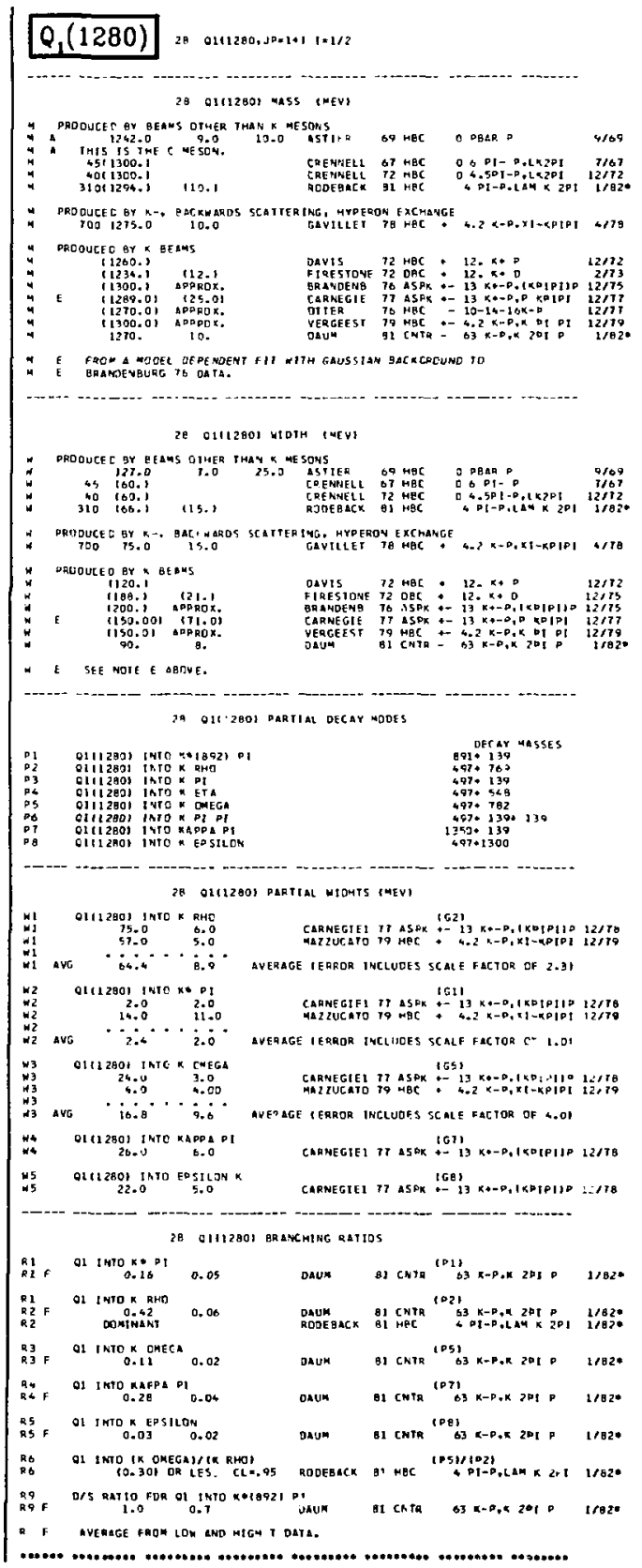


QLFCEEACES FOH OLIZZAOI

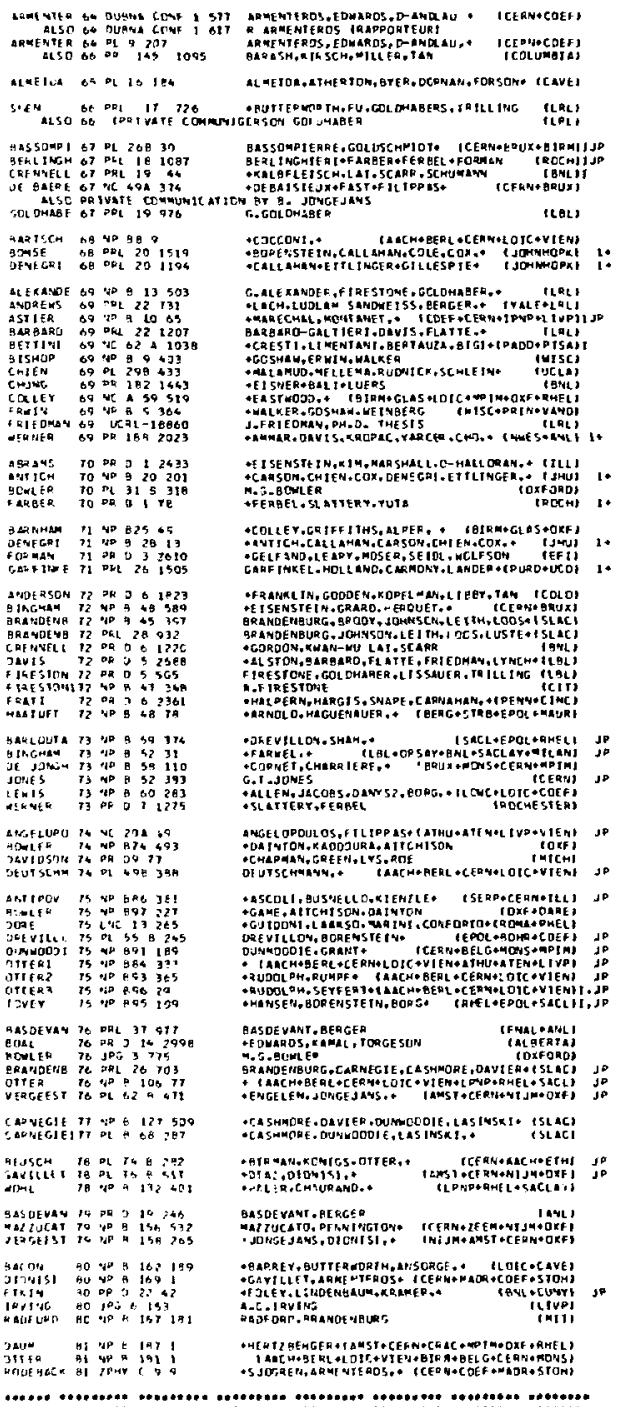

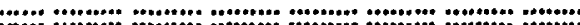

The isodoublet 5 -wave $k \pi$ phase shift $\hat{o}_{0}^{1}$ is compatible $w: t n$ elastic unitarity up to the $K r_{\eta}$ ' threshold. It grows monotonically, reaching $90^{\circ}$

at abou: $1350 \mathrm{MeV}$ (MERCER 71, BINGHM 72, RIRESTONE 71,72, KATISON 72,74, GALTIERI 73, YIMTA 73, FOX 74, BAKER 75, LAUSCHER 75, BONLER 77, ESTABROOKS 78, ASTON 81). The arbiguous "up" solution in the region of the $R^{*}(892)$ has been ruled out conclusively (MATISON 72,74, GALTIERI 73, BONLER 77. ESTABROOKS 78).

The first inelastic two-body threshold is $\mathrm{K} \pi$, which, however, is very weakly coupled to $\mathrm{r}$, in accordance with the su(3) predic:ion for the $k K$ ? coupling.

In the energy range 1350-1450 MeV the phase shift exhibits rapid notion (BOHLER 77, EST 38 FOOKS 78, MARTIN 78), which has been taken as an indication for a $k$ resonance at $1500 \mathrm{MeV}$ IESTABROOXS 79). However, the phase-shift betsavior can also be understood as a cusp effect due to the nearby $\mathrm{Kn}$ ' threshold at $1455 \mathrm{NeV}$ (TORMQVIST 82). Above this energy the inelasticity due to $R \Pi^{\prime \prime}$ is important. The pisse shift can be fitted up to about $1500 \mathrm{MeV}$ in a unitary coupled-channel analysis with proper analytic structure with one resonance, the $\mathrm{K}(1350)$, without background (TORNgVIST 82). This supports earlier interpretations (FIRESTONE 71,72, FRATI 72, ROUGE 72, CORDS 73, LAUSCHER 75, MOREAN 75, ENGELEN 78, ASTON 81 ) that the $*$ mass indeed is whete $\hat{o}_{0}^{1}$ passes through $90^{\circ}$.

At still higher energies some evidence for a second scalar resonance exists (ASMO: 81). See aiso Appendix IIC.

19 MAPPA TASS TMEVI

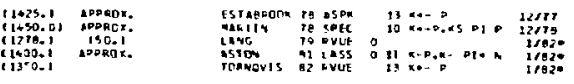

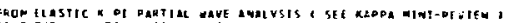

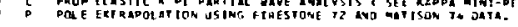
IT KAPDa WIOTH INENI

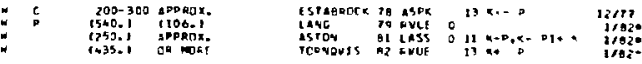

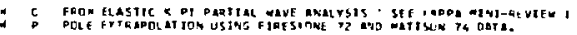
is Rapph partial oecar wCOHS

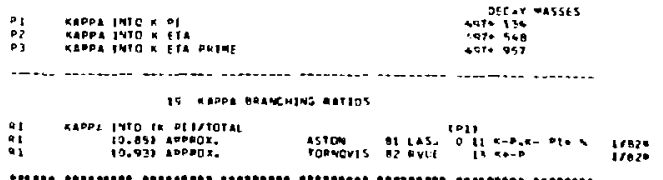


Mesons

$\kappa(1350), Q_{2}(1400)$
Data Card Listings

For notation, see key at front of Listings.
DEFEREACES FOM MAPPA

IMIPDE 60 DL 58 B 503 CAENNELL DO DPL 2246 ?

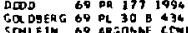
FIRESTON II PFL $261<60$ TETA
TER

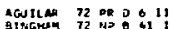
GUCWNER 72 GP

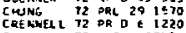

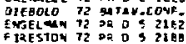

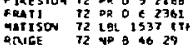

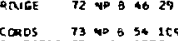

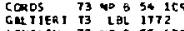
GTNGLIN 73 NDO FOx 74 No ABC \&J3

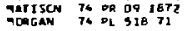

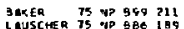

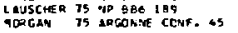
GHIEN 70 PO 10855

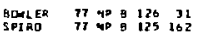
9401 78 पa 134305

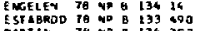

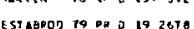
ES146007 79 pस:

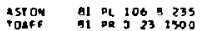

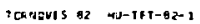

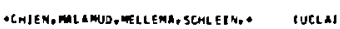

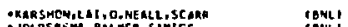

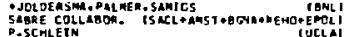
A.F TRESTONE, G- GOTDH2BER, D.L155AUER CLRL,

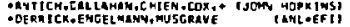

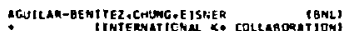

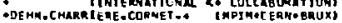

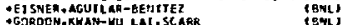

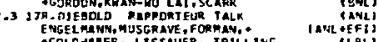

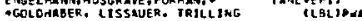

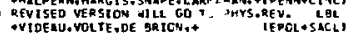

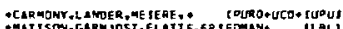

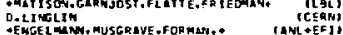
g.c.Fox,m,L-GRIss (CIII)

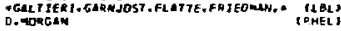

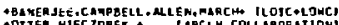

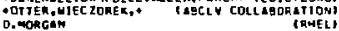

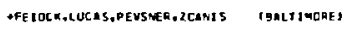

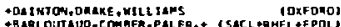

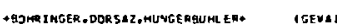

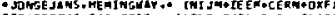

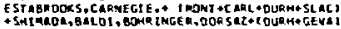
D.estagrocks

ICARLI

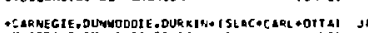

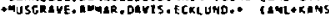

B.h.torkJVIST

INELSI

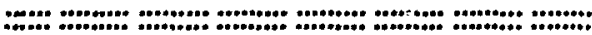

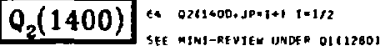

\section{Q6 Q2114008 RUSS (MEV)}

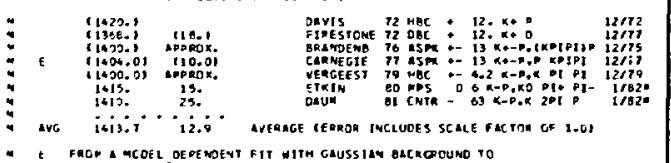

- E FONA MCOEL OE RE MOE

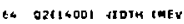

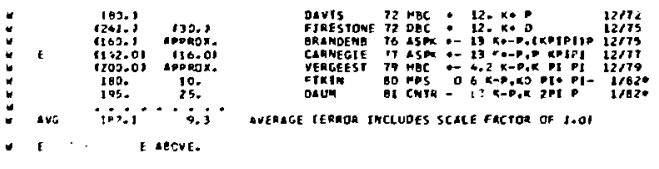

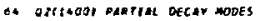

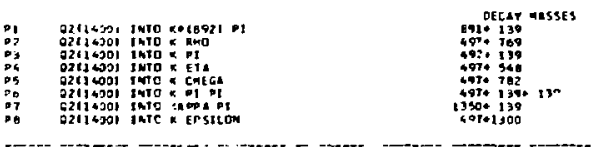

G* O2114001 OARTIAL WTDTHS IAEV,

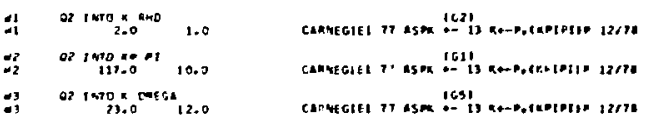

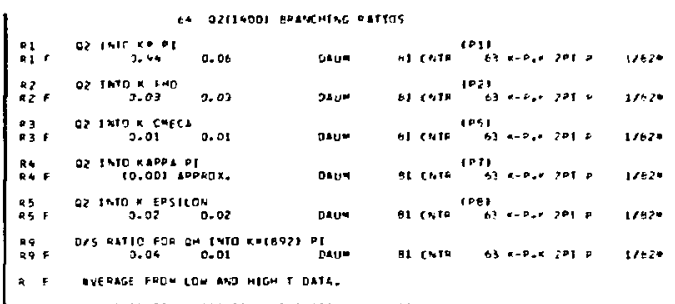

REFEREMCES FOK $3211 \mathrm{HOO}$

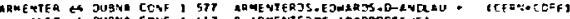

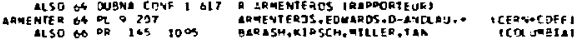

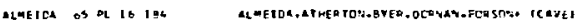

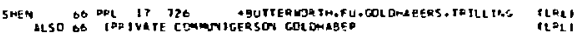

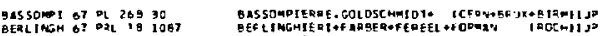

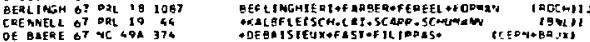

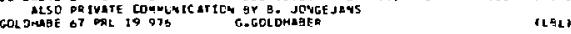
BEA TSCM 68 पD 50 O

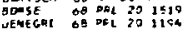
ALF XanBE OO TP 13503 ANDREMS 69 ORL $2 ?, 31$ ASI IER SO NP B ID OS BARART 69 F2L 22 1207 B ISWOP
C+1EN [HJVE 69 ON 1B2 1443

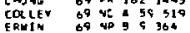

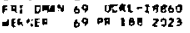
a ge ans 0 pa ? 12433

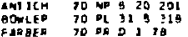
Bannum II YD B2S 45

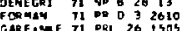
AWOERSON 72 PR 9 O 1823

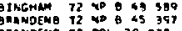
CAENAELL T2 OR O 6 1220

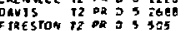

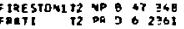

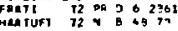

arxtoul nu vo 5037

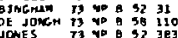

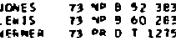
ANE LODO 14 45 204 46

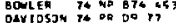
DEUTSCHA It ol 490 ing ANIIDON TS NF BAb 361 BOULER TS MP 897227 ONEYILLO iS ML 5 SQ 265 oumwow is kp por 199

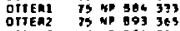
OTYER3 TO OSO 29 EASDEVAN To PRL 37917 BOLL To MO D 142900

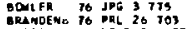

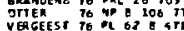

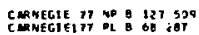
gausch 70 OL TH $2 \mathrm{Ez}$ GAVILLE 78 PL 16 18317 BASDEVAN 19 ph 019246 MLZUCAT $79=150532$

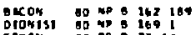
ETKin of of D 25 is

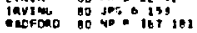

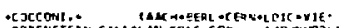

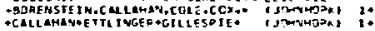

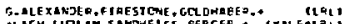

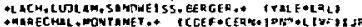

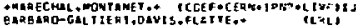

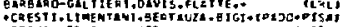

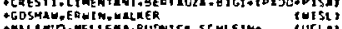

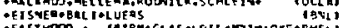

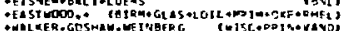

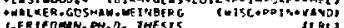

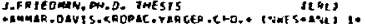

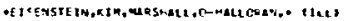

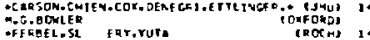

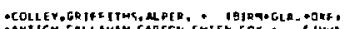

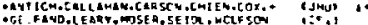

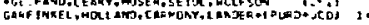

AFRAMELIW, GOODEN, KCPELFLN, LIERT, TAN TCOLC,

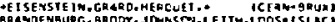
FR S Y

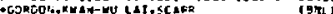

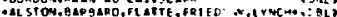

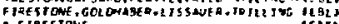
A.FIRESTOUE

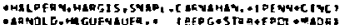

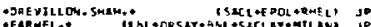

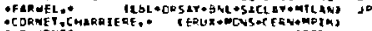
C. T.YONES

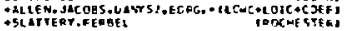

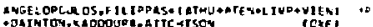

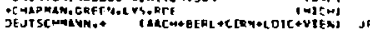

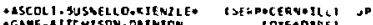

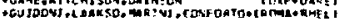

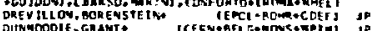

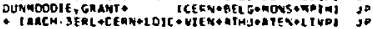

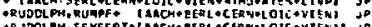

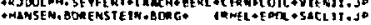
BSSOEYAWT, DERGER TFWLOML]

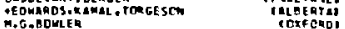

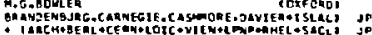

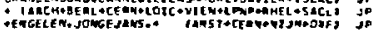

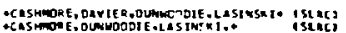

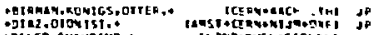

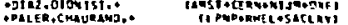

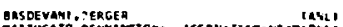

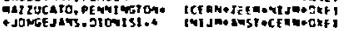

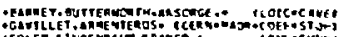

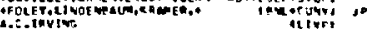
äjogrive 
Data Card Listings

For notation, see key at front of Listings.

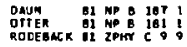

+HERT2 SERGER+ ( LMST+CERN+CRAC+HP IM+OXX+RHELI

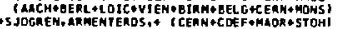

"

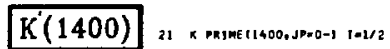

GES ERVEO IN R PI PI PAATIRL-MAVE AMALYSIS.

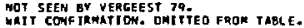

21 R PRIME MASS IMEVI

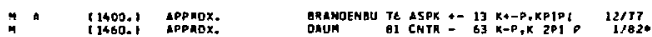

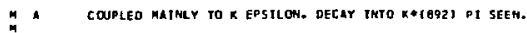

\section{2) $\times$ MMIME WIOTH IMEVI}

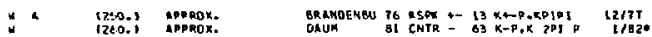

- coupled mainer to k e-stlon. decar inta katb9zi pi seen.

a) K PRTME PARTILL DECAY MDDES

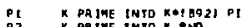

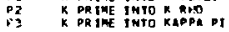

DEC AY MASSES

a914. 330

1350. 739

$21 \times$ MRIME PantTal wTOTHS TMEV

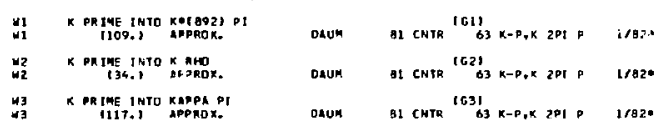

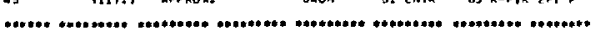

MEFEREMLES FOH X PRIME

QPAMDENB 76 PAL $36 \quad 1239$

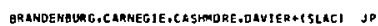

VERGEEST TO NF B 150265

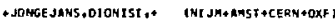

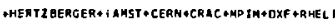

DANA 81 HP 1071

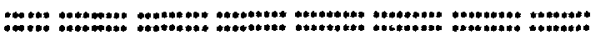

$K^{\circ}(1430)$

$22 \cdot K *(1430, J P * 2+1 \quad I=1 / 2$

WE CONSIDER THAT PHASF-SHTFT ANALYSES PROVIOE MORE QEE IABLE OETERM INLTIONS DF THE MASS AND WIOTH.

$22 \quad K \bullet 114301$ HASS (MEV)

CHAGED ONLY. HITH FINAL STATE $K$ PI

$\begin{array}{ll}0 & 39 \\ 0 & 6323 . \\ 0 & 1427.0\end{array}$

631427.0
220114.0

0 60 1414.

14001420.0

8
$8 \quad 2420.0$
0

$57911648+51$
$29211431-81$

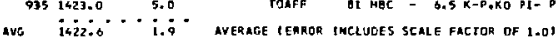

FINAL STATE K
11.0
12.0
10.0
13.0
3.1
8.0
4.0
5.6
15.01
15.81
5.0

PASSAHO OT HEC $=4-6-5-0 K-P, K O P 1-12275$ SCHWEJNGA 66 HBC $=5.5 \mathrm{~K}-\mathrm{P}$ KK DII 12778

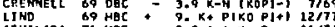
AGUILARI I1 HaC - $3+9,4.6 K-p$

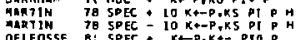

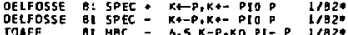

22001421.1

11001427.

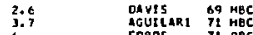

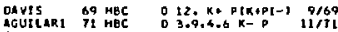

0001421.6

$300+2420.0$

6.

BURDAME 72 OEC

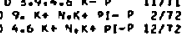
$4.6 K+N+K+P I-P \quad 12 / 72$

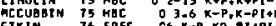

ETKIH 76 SPEC O6.K-D,KO ET+PI- $7 / 7$

1420.0

7.0
10.0
2.0

1450.: 30.

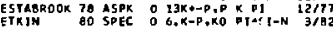

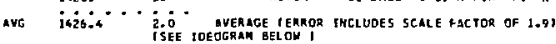

B SYS TEMATIC EMNOR RDDEO BY US.

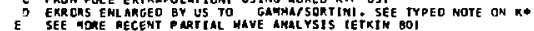

* PRO PHASE SHAFT ANaLYSTS.

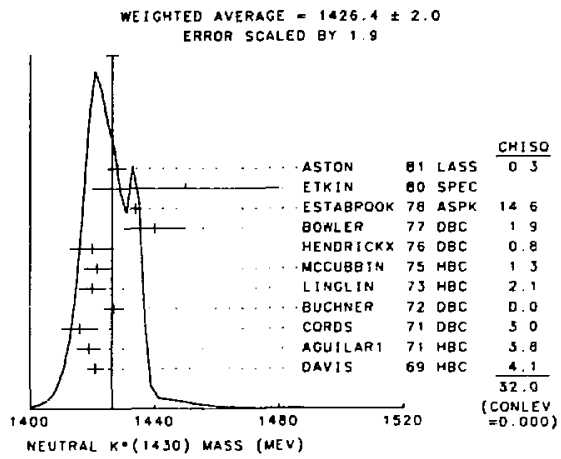

$22 K+\{1430\}$ H]DTH (MEV)

CHaRgeO OVtY, WTIH FINAL STATE K PI

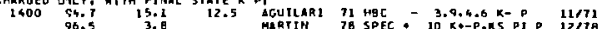

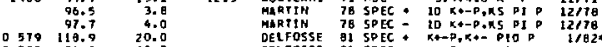

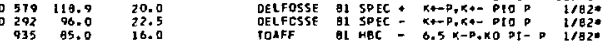

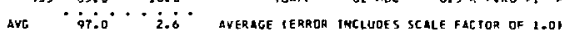

MEUTRAL OHLY

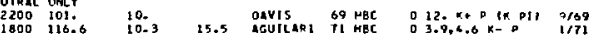

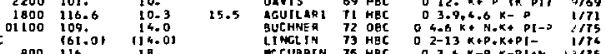

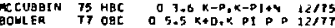

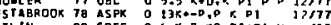

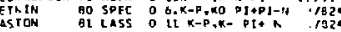

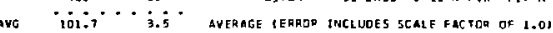

C FROM POLE EXTAAPOLATION, USING MORLO K+P OST

O EPRCAS EHLARGEO GY US TO a GAMMAISPRTIMI. SEE K* TYOED MOTE.

w helmagr of events in peak reeavaluated ar us

22 KE11430 PARTIAL OECRY MODES

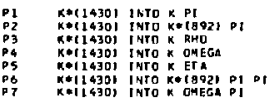

DECAY MASSE 5

$493+139$

$493+769$

$403+782$

$893+548.130+137$

\section{FITTED PARTLAL DECAY MODE BRANCHINC FAACTIONSS}

The matrix below in derived from the error matix for the fitted partial decay mode branching fractions, $P_{1}$ as follows: The diegonal elements are $P_{i}=6 P_{i}$, where

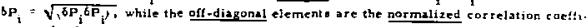

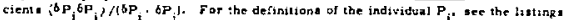

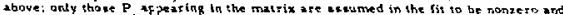
are thue conat ratied to add to 1 .

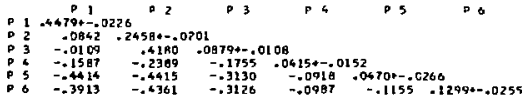

\footnotetext{
$22 K+(14301$ EMANCHING RATIOS

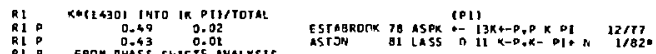

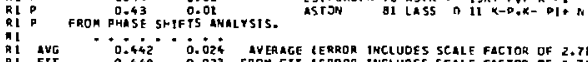

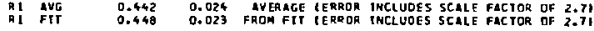

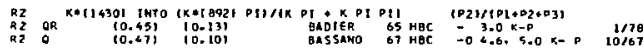


Mesons

$K^{\prime \prime}(1430), L(1580)$
Data Card Listings For.notation, see key at front of Listings.

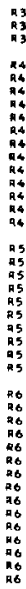

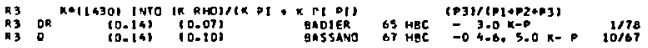

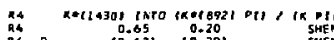

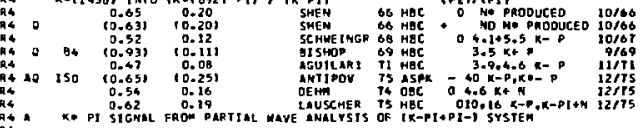

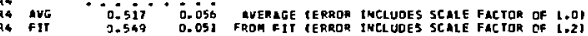
as $K * 11430$ I INTO IK OMEGAI I K PI

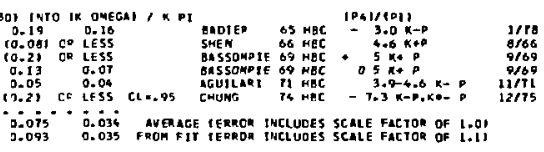

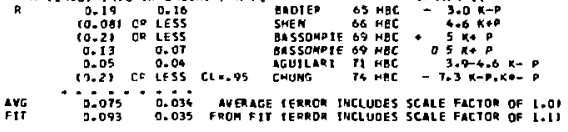

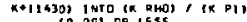
10.091 OR LESS
0.26 0.26
$(0.3)$ or LESS 10.3) CR 2555 $\begin{array}{rr}10.16 & 0.05 \\ 0.02 & 0.10 \\ 0.02 & 0.161\end{array}$

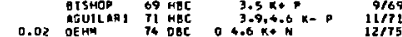

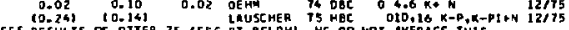

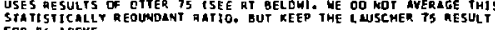

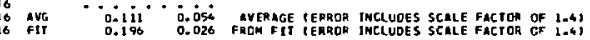

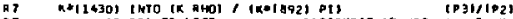

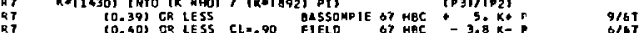

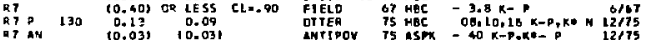

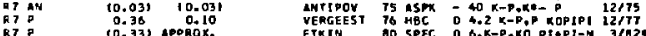

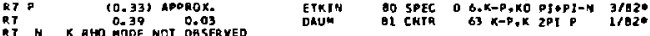

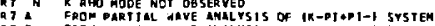

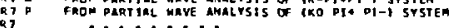

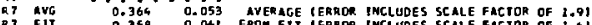

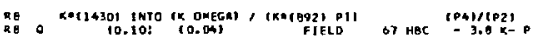

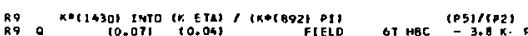

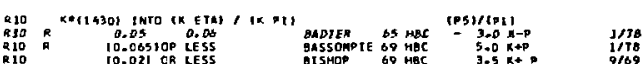

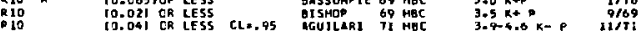

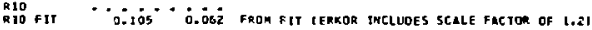

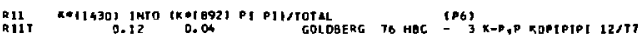

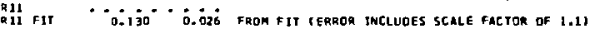

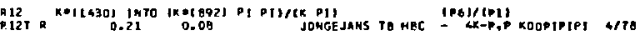

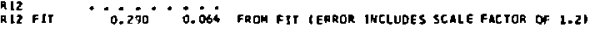

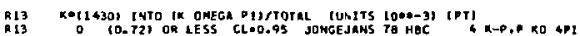

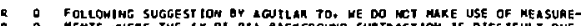

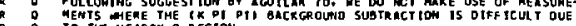
R D TO THE NEARQT Q REGION.

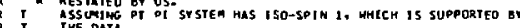

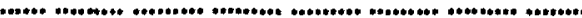

OAO1ER 65 PL 19 612

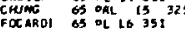

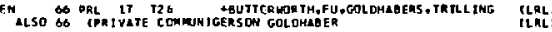

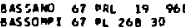

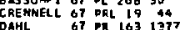
DAHL 2450 65 PK 1631377

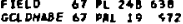

\section{REFEREHCES FOR KOI 1430}

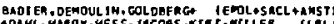

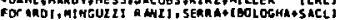

TLAL

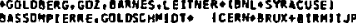

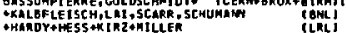

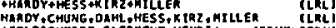

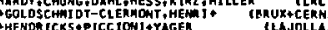
*HENONICKSA PICCION1TYAGER
GGGOLOHABER,FIRESTOHE, SMEN

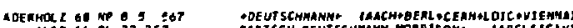
ANrICLO oubat of nests SCHME IMO

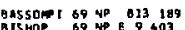

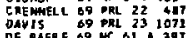

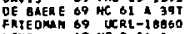
ITHO 99 MPQ 14 ?

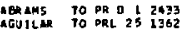
AgutLani 71 oR D 2503

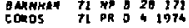
BUCANER 72 YP B 45333

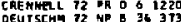
OEUTSCHA 72 NP

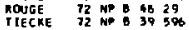
CHARMIER 73 YPE 53317 CLank 73 of 54,432

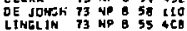
WALUCH T3 PR O O $2 E 37$ CHAMS IT PL 516413

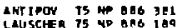

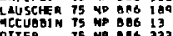
OTrER 15408433

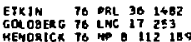
MEMORICK To To: 112189

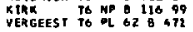
DOMLER 77 NP D 12631 Allot TO NDO 134365

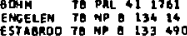
C150 78 PR 17659

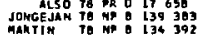
ETK IM OO PHO 02243

A5j al oL 100 B 235

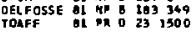

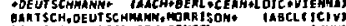

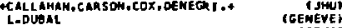

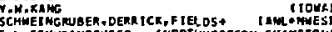

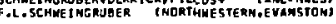
DAS SOMPRERE, GOLOSCHAC OT-CL ERP.+ IEERH+ERUXI SP

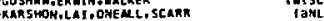

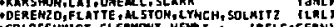
GOLOSCHA1OT-CLERMONT. HEA I.4 TELLGECERN

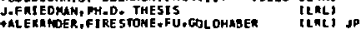

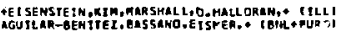
+EI SMER.KI NSON

to: W MERE,

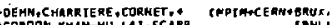

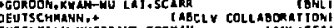

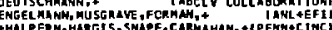

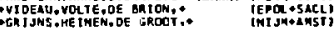

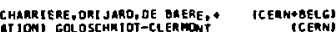
IYOWS, RAOUJICIC

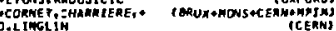

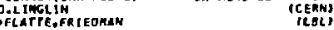

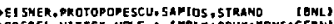

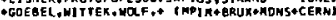
asCaLt, BU SMELLO,KI EMZLE+ I SERPaCERM+ILL?

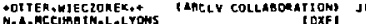

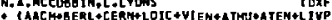

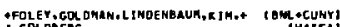
J.GOLDeERE

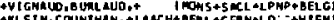

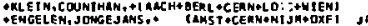

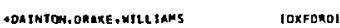

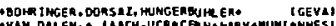

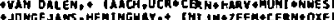

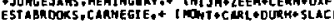

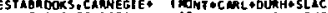

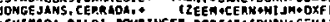

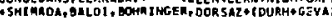

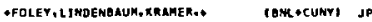

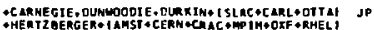

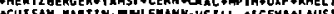
TGUI SAH, MARTIN, MHLLEAHMEUE ILL, +IGEYA +LAUS

"⿴囗十

$L(1580)$ 39 Lisso, JP=2-1 $t=1 / 2$

SEEM IN PARTIAL HaVE AMALYSTS OF THE K-PIOP1- SYSTEM ICTTER TR. SEE LIETYO) FIMIREVIEH.

39 LILSOOS MasS (MEVI

$11580.1,05000$

OSTEA $-10,14,18 \mathrm{k}-\mathrm{p}$ $12 / 79$

39 LI15801 WIOTH (MEY)

(110.) approx.

oT TE⿰

$10,14,16 \mathrm{~K}-\mathrm{P}$ $12 / 79$

39 LCBSEOI PANTIAL OECLY MOES

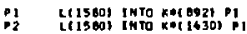

aecay masses

39 LIISBOI OARMTHITE RATLOS

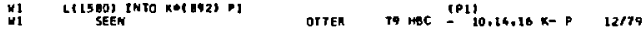

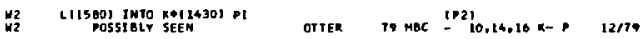

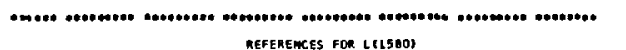

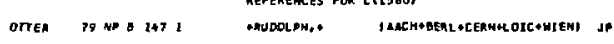

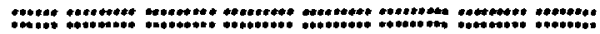


Data Card Listings

For notation, see key at front of Listings.

\section{Mesons \\ $K^{*}(1650), L(1770), K^{*}(1780)$}

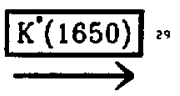

$\operatorname{s+1} 1050.9001+1,1=1 / 2$

THIS ENTKY CONTATMS VARTOUS REAKS CESERVE IN THE

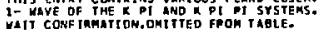

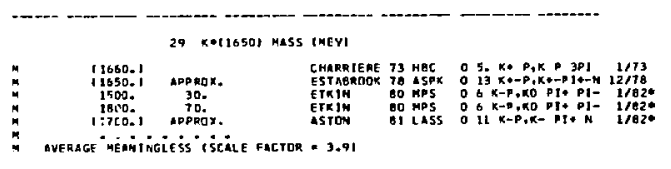

29 K\#(16SO) VIDEH (MEVI

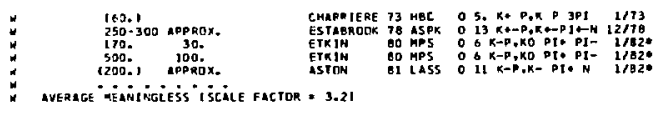

20 KMHESOR PARTIAL DECar MODES

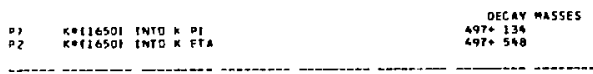

29 KDIJ65OI BR LHCHIMC RATIOS

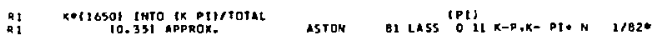

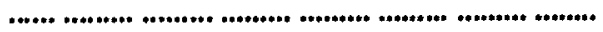

GMARAIER 13 NP B 51317 REFERENCE 5 FOR KO116501

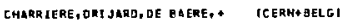

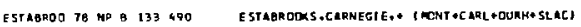
EJKIN 60 PR D 2242 TFOLEY,LJHDENBAUM, KHAMER,

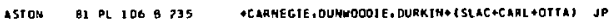

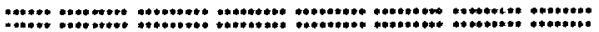
$\mathrm{L}(1770) \quad 23$ L11770, JP=2-1 1 - :22

The $L(1770)$ is seen as a bump at a mass $\sim 1.8$ GeV in the diffractive-like processes $K N \rightarrow(K \pi \pi) N$ The effect is largely dominated by the $\mathrm{J}^{\mathrm{P}}=2^{-}$'partial waves.

The long-standing questions concerning the resonant nature of the enhancement as well as its possible decay modes have been largely clarified since our last edition. A detailed partial-wave analysis based on 200,000 diffractive $K^{-} P$ $\mathrm{K}^{-} \pi^{+} \pi^{-} \mathrm{p}$ events (DAUY 81 ) establighes resonancelike phase váriations and igolates several decay modes. The behavior of the extracted $2^{-}$waves requires the existence of at least one $L$ meson, but there are indications suggesting the presence of a second state in this mass region.

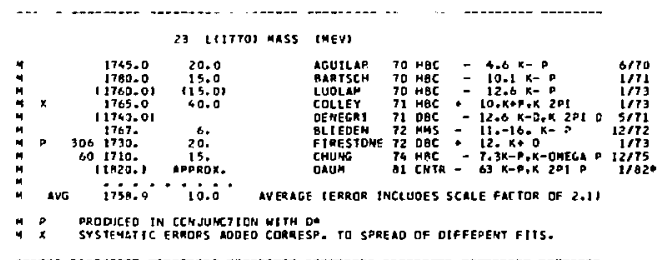

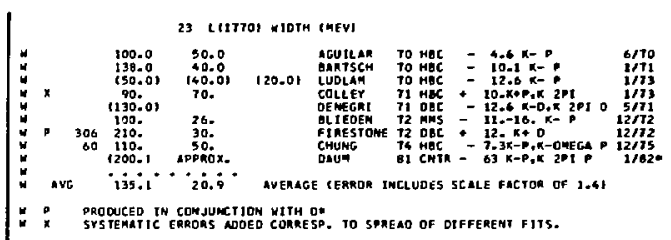

23 LCITHOF PRRTILL DECAY moES

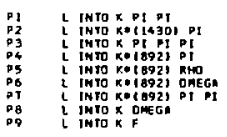

DECAT MASSES $497+1340.194$

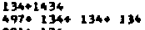
$991+136$ $991+769$
$991+782$
$991+1349$
494 $497+702$ $497 \div 1273$

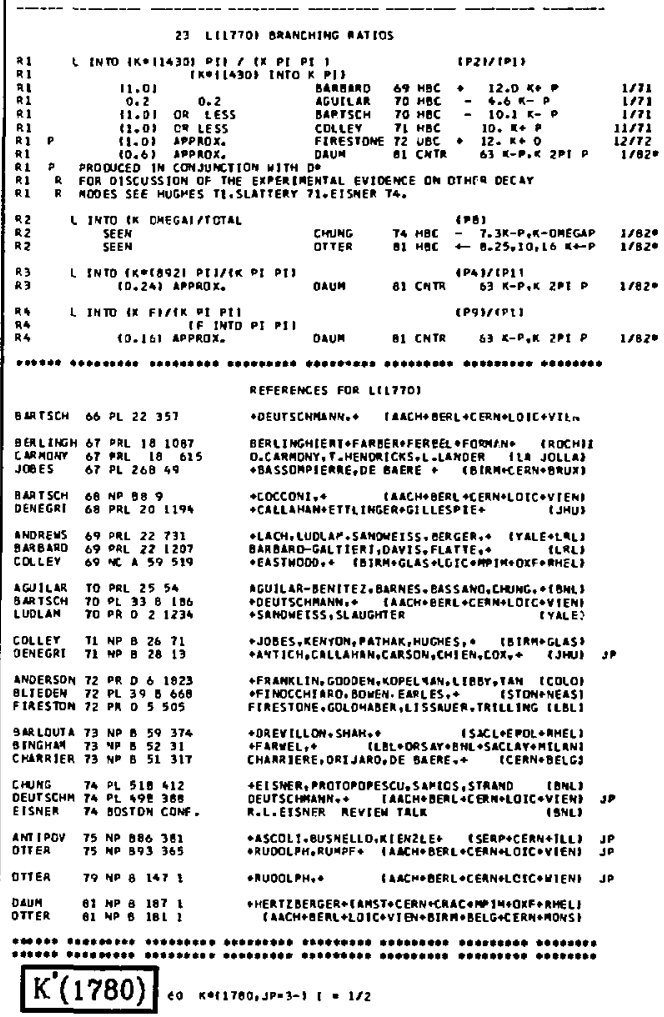

All the recent high-statistics experiments stuaying the $\mathrm{K} \pi$ syster in $\mathrm{KN} \rightarrow \mathrm{K} \pi \mathrm{N}$ interactions have shown clear evidence for the existence of a resonant effect at $\sim 1800 \mathrm{keV}$ in the $\mathrm{J}^{\mathrm{P}}=3^{-}$partial wave (BALDI 76, BRANDEHBURG 76, CHUNG 78, CLETAND 80, ASTON 81 ). The intensity of the $3^{-}$partial 
Mesons

$$
K^{\prime}(1780), K^{\prime \prime}(2060)
$$

wave of the $\mathrm{R} \pi \mathrm{\pi}$ systen produced in the charge-exchange process $\mathrm{x}^{-} \mathrm{P} \rightarrow \mathrm{x}^{0} \pi^{+} \pi^{-} \mathrm{n}$ also shows resonancelike behavior at $21800 \mathrm{MeV}$ (BEUSCa 78 , ETKIN 80). since the wass values quoted for the $\mathrm{K} \pi$ and $\mathrm{K} \pi \mathrm{\pi}$ wodes are not significantly different, it seems natural to consider them as alternative decay modes of a single resonance.

There appears to be some disagreement in the values of the width obtained using the $\mathrm{x} \pi$ channel. The measured values tend to become larger when the number of angular woments included in the fit increases. For the time being the observed disarepancies seen to orlginate frow the explicit parametrization of the experimental distributions rather than from the data themselves.

\section{DO KeHzor MASS IMEV}

م

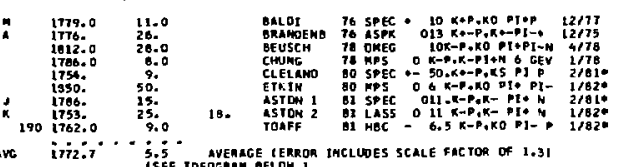
S.5

* COMFIMAED BY PHASE SHIFT ANALYStS OF ESTABRoOKS 77. YHELOS JP-3- $\quad$ F

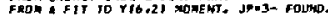

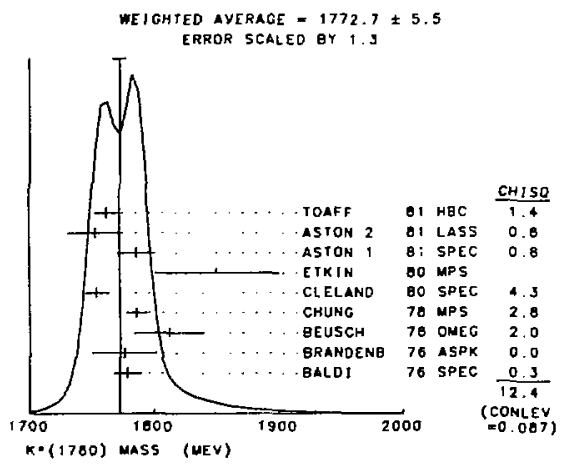

60 X0I1780) WIOTH (MEV)$$
\text { (n) }
$$

Data Card Listings

For notation, see key at front of Listings.

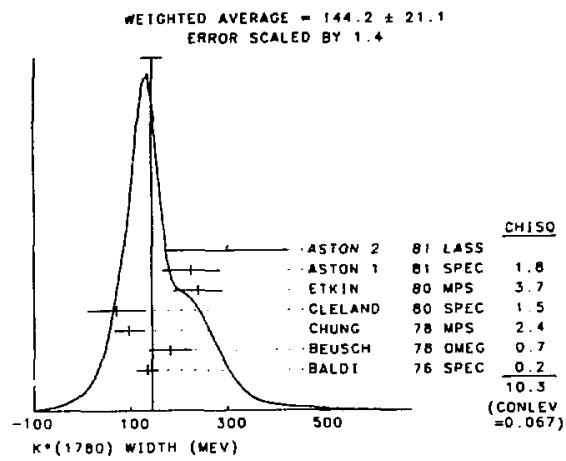

DO KetLT801 mantial decar modes

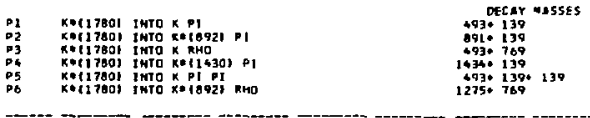

SO KOHIBOI BRANCHING RATIOS

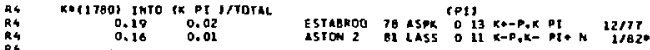

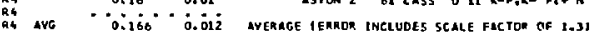

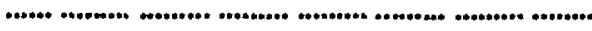

CARMONT
FIRESTON TI PRL 271160
PL 36 B 513

REFEREHCES FOR ROT1780

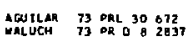

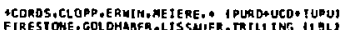

tCHUNG, EISNER, PRDTOROPESCU.SAMTOS.* I IRNL

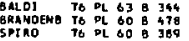

BONLER 77 NP 12831

CARMONT IT PRO 16 125

OEUSCH $78 \mathrm{PL} 748282$

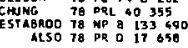

CLELAND 80 PL $\$ 78405$

ENGELEN 90 MP 8 O 167 \$1

Aston I Bu DL 998502 "FL ATIE,FRIEOMAM

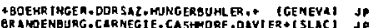

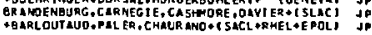

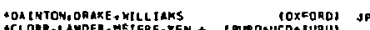

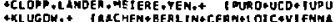

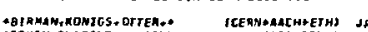
TETK[H,FLAFINO+ (BNL + BRAH+CUHY +MASA+PENH] JP

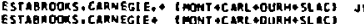

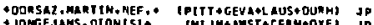

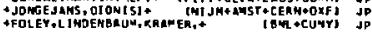

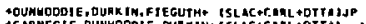

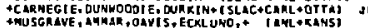

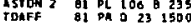

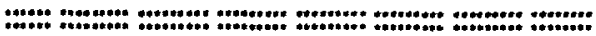

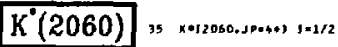

$\longrightarrow$ OMITTEO FROH TABLE.

$35 \quad K+120601$ MASS (MEVI

\begin{tabular}{|c|c|c|c|c|c|c|c|c|}
\hline $\begin{array}{l}\hat{A} \\
\mathbf{C} \\
\mathbf{D}\end{array}$ & & $\begin{array}{l}2115 . \\
2024: \\
2023: \\
2092 . \\
2070 .\end{array}$ & $\begin{array}{l}40 . \\
20 . \\
10 . \\
21 . \\
100 .\end{array}$ & 40. & $\begin{array}{l}\text { CIRMONY } \\
\text { CLELAND } \\
\text { CLELAND } \\
\text { ASTOW t } \\
\text { ASTON 2 }\end{array}$ & $\begin{array}{ll}T 7 & \text { HBC } \\
60 & \text { SPEC } \\
90 & \text { SPEC } \\
81 \text { IASS } \\
\text { B1 LASS }\end{array}$ & 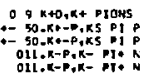 & $\begin{array}{l}1,178 \\
1 / 020 \\
1 / 62 * \\
1 / 020 \\
1 / 820\end{array}$ \\
\hline AUC & & $2035^{\circ}$ & $1 \dot{0} \dot{z}$ & AVERAC & GE CERRDR, & JMELUOES SC & CALE FAC TOR OF 2.01 & \\
\hline$\hat{b}$ & & 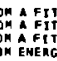 & $Y 15,01$ & iz,ol. & $\begin{array}{l}\text { Ano } 7(0,0) \\
\text { AnO } 1(8,0)\end{array}$ & $\begin{array}{l}\text { MOMENIS. } \\
\text { MOMENTS. }\end{array}$ & & \\
\hline
\end{tabular}


Data Card Listings

Mesons

For notation, see key at front of Listings. $K^{*}(2060), K^{*}(2200), D^{ \pm}, D^{0}, D^{* \pm}(2010), D^{* 0}(2010)$

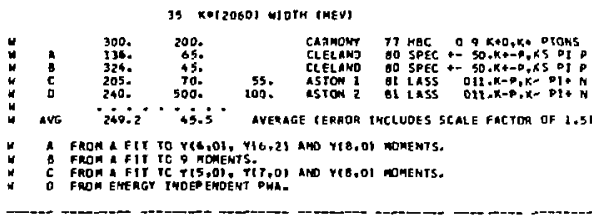

35 Kot2060) PARTILL DECAY moOrS

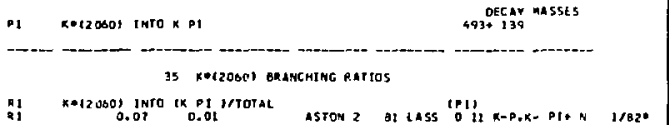

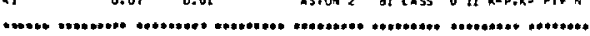
REFERENCES FOR $K O(2000)$

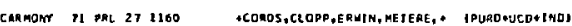

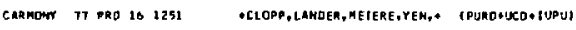

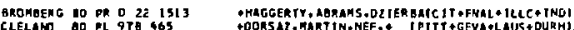

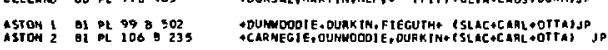

ancen $K^{0}(2200)$ a0 k012200,spa ,

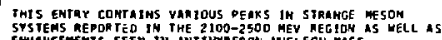

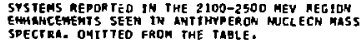

40 kotzzool mass (KEV)

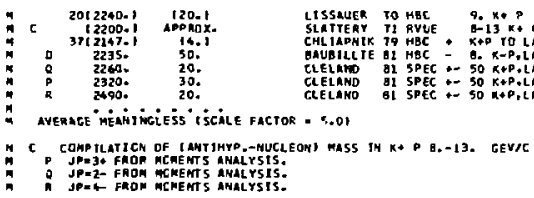

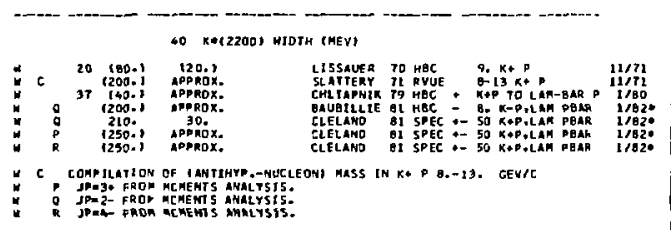

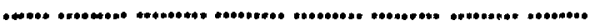
REFEREACES FOR $K *(2200)$

MLEXANDE GA PAL 20155 MLEXMNDER, FIAESTONE, GOL OHABER,SHEH RLRL

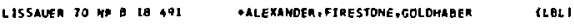

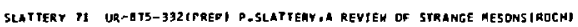

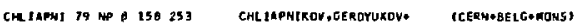

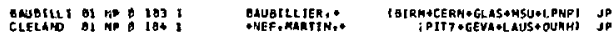

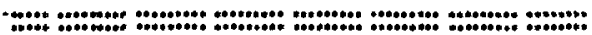

\section{$\mathrm{C}= \pm 1$ MESON STATES}

*
$D^{ \pm}$

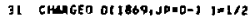
SEE STAGLE PARTICLE DATA CARD LISTINCS

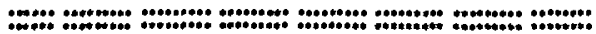

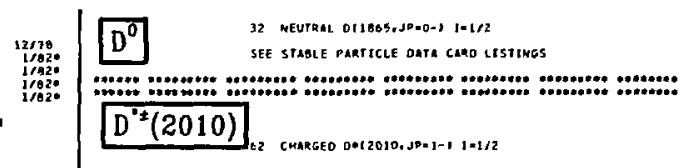

E CHARGEO D.120101 MASS IMEVI

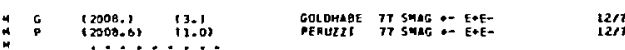

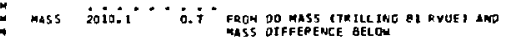

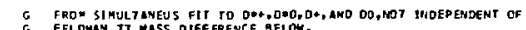

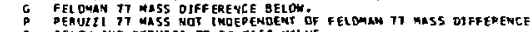

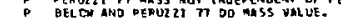

$\int \begin{array}{lll}0 \\ 0 \\ 0 \\ 0 \\ 0 \\ 0\end{array}$

12 (0\%+1 - 100) MASS DIFFEREHE IMEY!

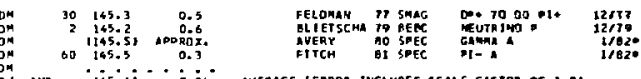

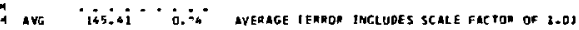

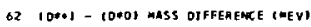

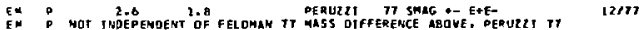

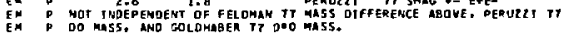

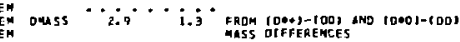

E2 CMABGE ONC2OLOI MTDTM IMEVI

$*$
$* \quad 30$
$*$ - - - - - - - - - - - - -

G2 CHARTED OA(2010) PARTJAL DECAY MODES

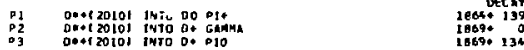

- Dokizotol imto o. DAO

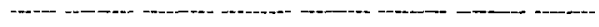

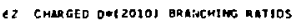

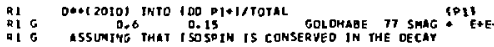

$12 / 77$

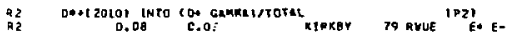

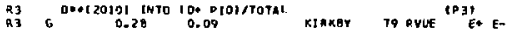

$12 / 79$

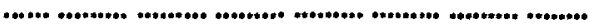
REFERE HCES FOR CYARGED DOT2010

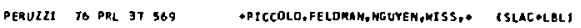

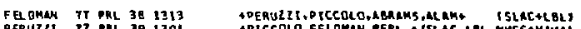
PERUZZI 77 PAL 391301
COQ OHASE 77 "PL 69 B 503

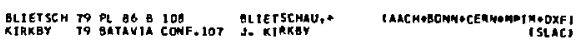

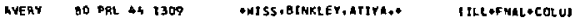

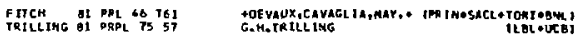

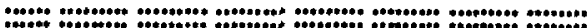

$D^{\pi /}(2010)$ a mestral ont 2010,JPE $1-1 \quad 1=1 / 2$

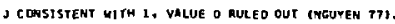

6) NEUTRAL OOI20101 MASS IMEYI

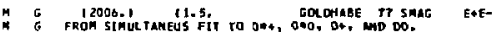

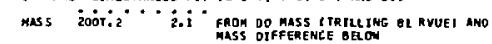

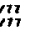

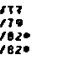




\section{Mesons}

$D^{10}(2010), F^{*}, F^{*}(2140), B$, EXOTIC MESONS

\section{Data Card Listings} For notation, see key at front of Listings.
61 10*0) - 1001 maSS OtFFenEMCE (MEV)

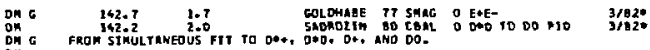

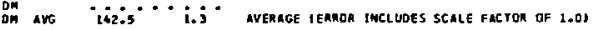

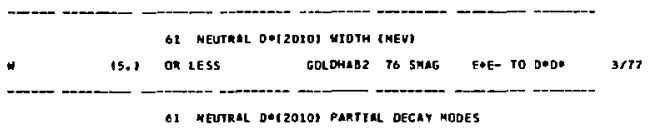

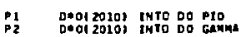
LOES: 136 MASSES

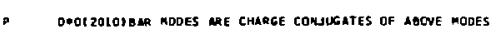

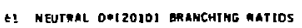

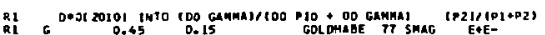

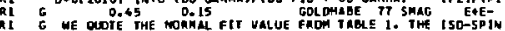

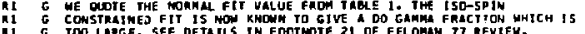

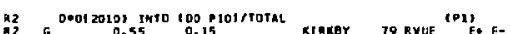

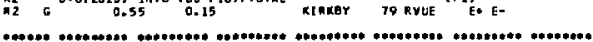

REFEMENCES FOR MEUTRAL DeI 20101

GOLDHAG 76 FRL 37255
GODDHAO

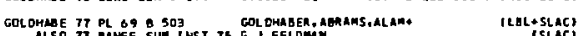

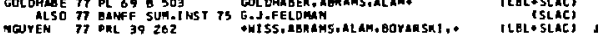

RIAKBY 79 Batayin CONF.107 J. KIRKBY

(SLAC)

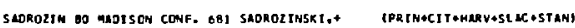

TRILLING BI ARPL 75 5T G. G.TRILLING CLELOUCBI

"ropen

$\mathrm{F}^{ \pm}$

SEE SILBLE PARTTLLE DATA CARO LISTIMGS

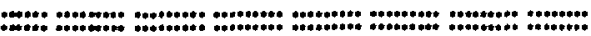

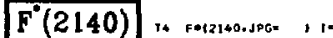

CUITIED FROM TABLE.

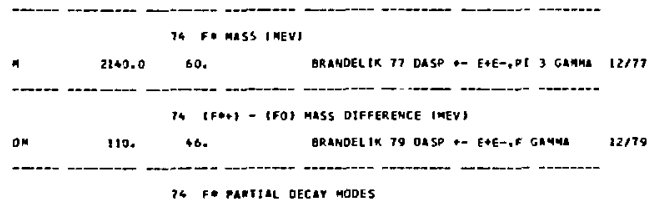

Fo INTO F GaFA

$2021+0$ DEC MASSES

14 Co gRamening astios

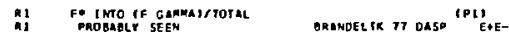

12177
BRLNDELI T7 PL TO B 132 BRAHOELI TO FL TO 361

BRAMDEL I T9 DL B 412
REFERENCES FON F*121401

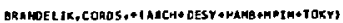

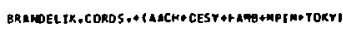

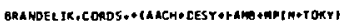

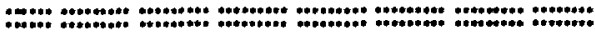

\section{$B= \pm 1$ MESON STATE}

+60

B

3

39 BOTTOA MESDN B(5700,JPE,

SEE STAGLE PARTICLE Data CARD LISTINGS

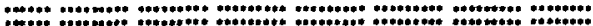

\section{EXOTIC MESON STATES}

and on

\section{EXOTICS so Exarics}

THE PURPOSE OF THIS EHTRY IS TO PRONIDE L15T OF SEC. 3 AND TABLE H, AS HELL AS THE SUGGESTIONS FOA EXPEP THENTS. NOTE THAT LPRTH I3 DAOPOSES EXPERINEWTS WHICM RRE TONCLUSIYE EVEN IF

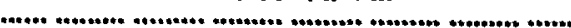
REFERENCES FOR EXOTICS RE PDRTS ON SEARCMES

ROSENFEL SQ PMILA.CCNF.P. 455 4. H. RDSENFELO $(L+1)$

DODO G9 9R 1771991 TJDLDERSMA, PLLMER. SAMIOS TSNLI

CHO
GIACOME

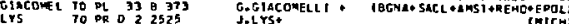

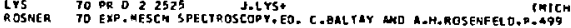

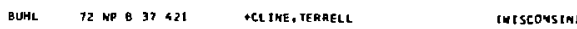

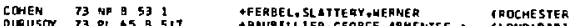

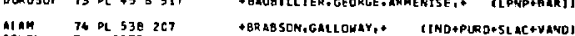
COHEH 74 SOSTON OREN 74 MP OT1 289

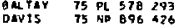
BRUMDIER 70 DL S4 Q 107 SOUCRAT T7 NA 121251 OCGLANO $77 \mathrm{MPO}$ B 126100 AOSER ALAN 78 PRL 40 1885 Lemotghe to gatavia CONF.526 KDOL JMAN SO PRG $45 \geq 16$ MEUILAR AI ZPHY CO 109 APEL B1 MP 9 , 193269 EVANGEL T G1 NP Q 178 ITT

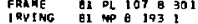

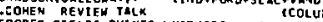

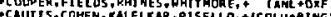

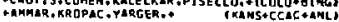
BRUMOIERS. BRUY,FLLRI, [FREIQURG +SACL •ETH] +NA VACH +RIVET'* ILALO+CERH+COEF+EPOU

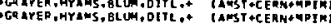

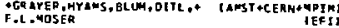

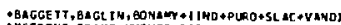

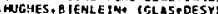
+A8QLIKS, GARATEA ISACLALBIC+SHMF+IHDS

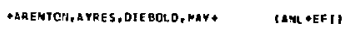

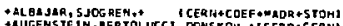

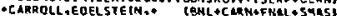

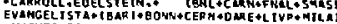
-HUCHE S- COLLEY. $2 R M S T$ SCNG + TGLAS +B IRN+CERHI

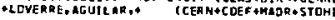
SUGGESTIONS FOR SEARCHES

DOSNER GB DQL 21 45O.246E J.L-ROSHER tTel-avivi

RDSNER TO EKD.MESOH SPECTROSCOPY,EO. C. BLLTAY AHD A.H. ROSENFELD.P.49Q

FATMAN 73 OL 43 B $3 E T$
LIPKIN 73 OR D $72 Z E 2$

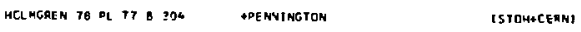
On 
Data Card Listings

For notation, see key at front of Listings.

Note on $N^{\prime} 8$ and $N^{\prime} g$

\section{Introduction}

For this edition, three $N^{\prime} s$ and one $\Delta$ have been promoted to the Baryon Table, and several new resonances that are not yet establiahed appear in the Data Card Listings. Table I.1 lists all the entries In the Listings, and gives our evaluation of the atatus of each, both overall and channel by channe1.

The masses, widths, and branching fractions of the $N$ and $\Delta$ resonances cone nainly from a few comprehensive partial-wave analyses. There are also several other recent analyses based on more linted data gets and/or energy ranges. Production and total-cross-section experiments can be valuable In establishing the existence of high mass bumps, but at lower energies these experiments have linIted statistics compared to formation experiments, and it is seldom clear which of several states having nearly the sane mass is being observed.

Even when there are good scatering data, there are two main problems in obtaining reliable resonance parameters from partial-wave analyses. F1rst, there is sometimes disagreenent anong analyses on the partial-wave anplitudes thenselves. This obviously depends on the quality and quantity of the scattering date and on the procedures used to determine the anplitudes from the data. Secondly, even if amooth curves were avallable for the anplitudes, there would st1ll be some paranetrization-dependent uncertainty about the values of the usual Breft-Wigner resonance paramecers. From a theoretical standpoint, the most unambiguously defined resonance parameter tre the pole position and residue, and tt has been found in practice that, $g$ iven sufficiently precise partialwave anplitudes, these quantities can be extracted 1n a stable and paranetrization-independent way, in spite of the fact that an extrapolation away from the physical region is required. This point has been discussed in detail with regard to the $\Delta(1232)$ in earlfer editions of this review. ${ }^{1,2}$ pole parameters have now been determined for many of the $N$ and $\Delta$ reoonances, and these are included in the Data Card Listings. In wort cased, we specify pole parameter by givirg the real and iaginary parts
TABE I.1. STATUS OF N* RESOMLACES

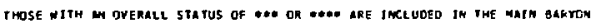

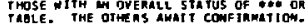

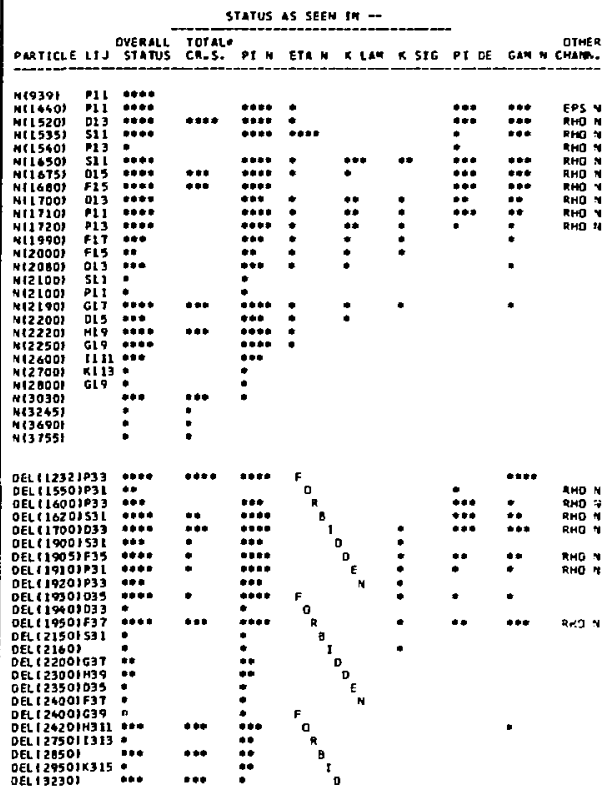

-... GODO. CLEAR, AND UAMT STAK ABLLE.

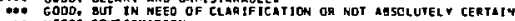
- NEEOS COF IAMATJOH.

- attriguteo to the state closest go here the cross section peaks.

of the pole position and realdue. It should be noted that these real and Imaglnary parts tend to be highly correlated. In partlcular, the absolute value of the residue 18 of ten better determined than is the phase. For further discusaion, see the relevent references, e.g., NOGOVh 73 , SPEARMAN 74 , BALL 75, LICATENBERG 75, VASAN 76, LONGACRE 77, 2IDELL 78, CUTKOSKY 79, MIROSHNICHENKO 79, 2IDELL 80 , and CUTKOSKY 80 .

The following sections discuss various recent developments In experimental $\mathrm{N}$ and $\Delta$ spectroscopy. For a discussion of earlier results, see our 1980 edition ${ }^{3}$ and the reviews of R.L. Kelly, ${ }^{4}$. Roch, 5 and A.J.G. Hey and R.L. Relly, 6

\section{References for Section I}

1. Perticle Data Group, Rev. Mod. Phys. 43, $\$ 114$ (1971). 


\section{Baryons}

$N$ 's and $\Delta$ 's

2. Particle Data Group, Phys. Lett. 39B, 103 (1972).

3. Particle Data Group, Rev. Mod. Phyo. 52, 5175 (1980).

4. R.L. Kelly, In Proceedings of the IV $v^{\text {th }}$ Internacional Conference on Baryon Resonances (Toronto, 1980), ed. H. Isgur, p. 149 .

5. R. Koch, In Proceedings of the Conference on Low and Interaediate Eneroy Reon-kucleon Physice (Rome, 198D), p. 1.

6. A.J.G. Hey and R.L. Re1ly, Physics Reports $\underline{C}$ (to be published).

\section{Two-Body Partial-Wave Analyse日 and New Resonances}

(by R.E. Hendrick, St. Bonaventure Unfversity)

Several new partial-wave analyaes have appeared and several older analyses have been extended to include new scattering data since our 1980 edition. I For $\pi N+\pi N$ reactions, we have Included results of new analyses by CUTKOSKY 80 , 2IDELL 80, KOCH 80, and CHEW 80. CUTKOSKY 80 includes new elastic cross-section and polartzation data and extends the mass range of the CUTKOSKY 79 analys 1s up to $2500 \mathrm{MeV}$. Th1s analysis reports several new baryon states and conflrms several tenrative stateg in the 2000-2400 MeV region. A brief discussion of the revised status of these $N$ and $\Delta$ states is given belov. ZIDELL 80 performs an energy-dependent partial-wave analysis from threshold to $350 \mathrm{MeV}$. Deviations from 1sospin Invariance are reported, and new masses, widths, and pole positions of the $\Delta^{0}$ and $\Delta^{++}$are given. kOCH 80 performs an energy-independent analysis over a similar low-energy range, but finds little evidence of isospla invartance violation. CHEH 80 uses a Barrelet-zero technique to analyze $\pi^{+} p$ elastic data between 155D and $2100 \mathrm{MeV}$ and reports several new S31 and P31 states, but disagrees with the parameters of the two 4-gtar $S 31$ and $P 31$ resonances established by other analyses.

Two new Inelastic analyses have been reported: an energy-dependent anslysis of $\pi^{-} p+\Lambda K^{0}$ below $1900 \mathrm{MeV}$ by MUSETTE 80, and an energy-dependent analysis of the three 1sospin-coupled reactions $\pi^{-} p^{-} \Sigma^{-} K^{+}, \pi^{-} p+\Sigma^{0} K^{0}$, and $\pi^{+} p+\Sigma^{+} K^{+}$up to 2000 MeV by LIVANOS 80 . A number of resonance masses, widhs, and $\pi N$ to $K \Sigma$ branching ratios have been included from this analyais.
For notation, see key at front of Listings.

This year, four new $N$ and $\Delta$ resonances have been pronoted fron 2-star to 3-gtar atatur and added to the Baryon Table: the D13 H(2080), D15 $N(2200), S 31 \Delta(1900)$, and $P 33 \Delta(1920)$. The D35 $\Delta(1930)$, already listed in the Baryon Table, has been pronoted from 3-star to 4-star statur.

A number of veak, higher-nass states have been added to the $N$ and $\Delta$ Listings. These include $a$ third P1I at $2100 \mathrm{MeV}$, a second D33 at $1940 \mathrm{MeV}$, a third S31 at $2150 \mathrm{MeV}$, a second D35 at $2350 \mathrm{MeV}$, and a second F37 at $240 D \mathrm{MeV}$. The $637 \Delta(2200)$ has been separated frow the $\Delta(2160)$ 1isting and has been given a separate 1 isting with 2-star status. The A39 $\Delta(2300)$ has been pronoted from 1-star to 2-star status. In addition, a number of nontnal resonance masses have been changed from our last edition to put them in better agreement with recent results.

F1gure II. I shows the partial-wave amplitudes obtained by HOEHLER 79 and by CUTKOSKY 80 .

\section{Reference for Section II}

1. Partfcle Data Group, Rev. Mod. Phys. 52, S1 (1980).

\section{The $\pi N \rightarrow \pi \pi N$ Channel}

(by R.L. Cravford, University of Glasgow)

A general $\pi N \rightarrow \pi \pi N$ event may be described by the center-of-mass energy $w$, three angles $\alpha, B$, and $\gamma$, and two sub-energies, say $w_{\pi N}$ and $w_{\pi \pi^{\circ}}$ Unlike $2+2$-body reactions, fits of $\pi N \rightarrow \pi n N$ distributions at single values of $W$ cannot be parametrized in terns of a set of constants without introducing some assumptions into the analysis. All fits to $\pi N \rightarrow \pi \pi N$ use the 1sobar mode1, which notes that almost all such events lie in quasi-2-body bands in the Dalitz plot. Thus it is assuned that any purely 3-body 1nteraction is negligible and that the reaction proceeds by the formation of quasi-2body Interuediate states.

The basic form used for the amplitudes is

$$
\begin{aligned}
& T(\pi N+\pi \pi N)-\sum\left[T_{\Delta \pi}^{J I L L^{*}}(W) \cdot B W_{\Delta}\left(w_{\pi N}\right) \cdot x_{\Delta \pi}^{J I L L^{*}}\right. \\
& +T_{N^{\star} \pi}^{J I L L^{\prime}}(W) \cdot B W_{N^{\star}}\left(w_{m N^{\prime}}\right) \cdot x_{N^{\star} T}^{J I L L^{*}} \\
& +T_{\rho S}^{J I L L^{\prime}}(W) \cdot B W_{\rho}\left(w_{\pi T}\right) \cdot X_{\rho N}^{J I L L} \\
& \left.+\mathrm{T}_{E N}^{\mathrm{JILL}^{\prime}}(\mathrm{W}) \cdot \mathrm{BW}_{E}\left(\mathrm{w}_{\pi T}\right) \cdot \mathrm{X}_{E N}^{\mathrm{JILL}}\right]
\end{aligned}
$$



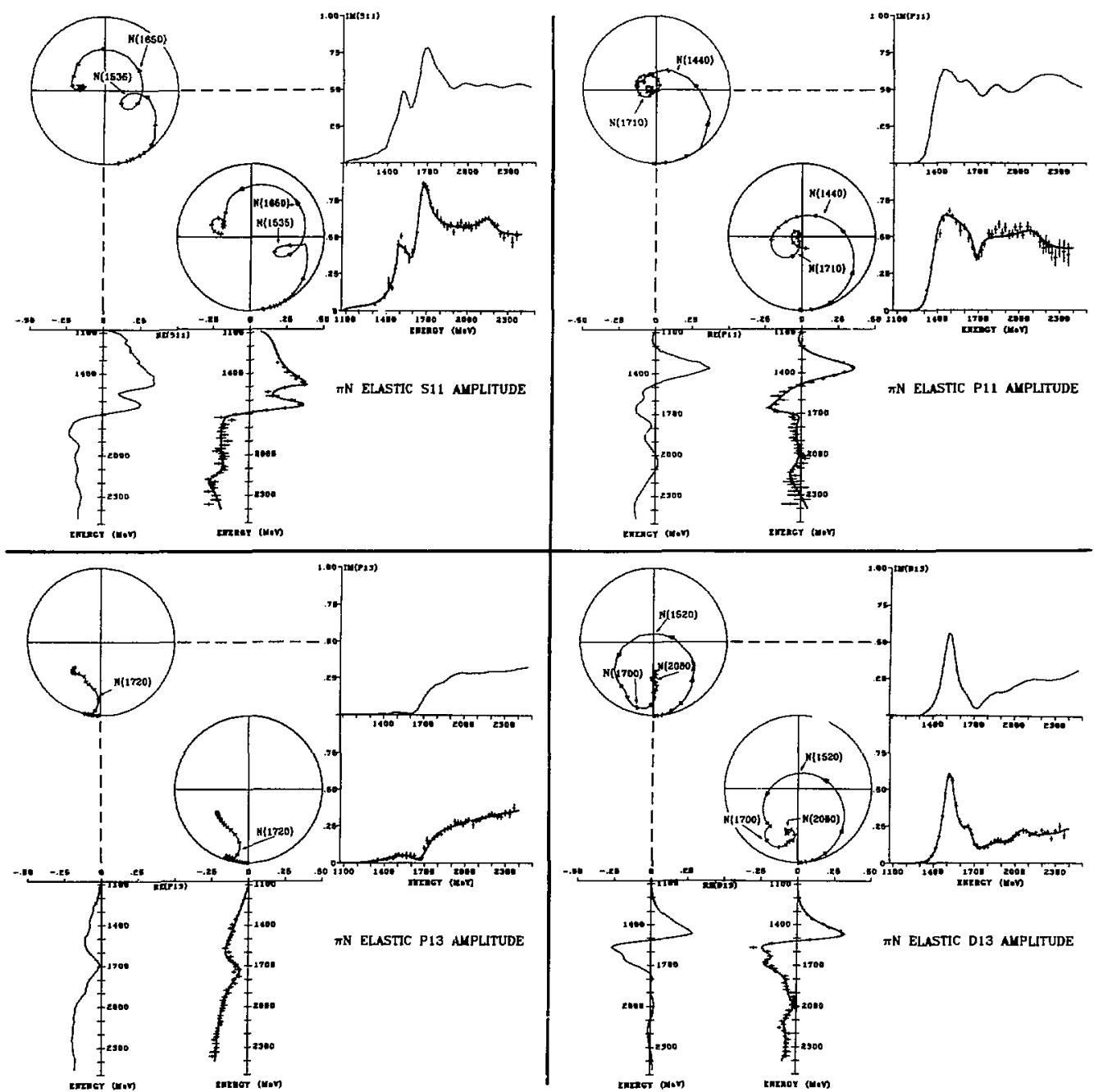

F1g. II.1(a). The L-2I-2J - S11, P11, P13, and D13 part1al-wave anplitudes for TH elagtic scatter1ng. The

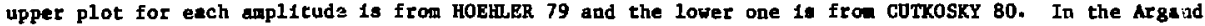
plots, the ticks are integral multiples of $50 \mathrm{MeV}$, and the established resonances are shown at their nonInal positions. The real and imaginary parts of the aplitudes as functions of energy are ahown projected in alignient with the Argand plots (In the projections of the CuTROSKY 80 anplitudes, the "data pointe" are resulte of energy-independent fits, and the curves are from an energy-dependent fit to join then). 
Baryons

$N$ 's and $\Delta$ 's
Data Card Listings

For notation, see key at front of Listings.

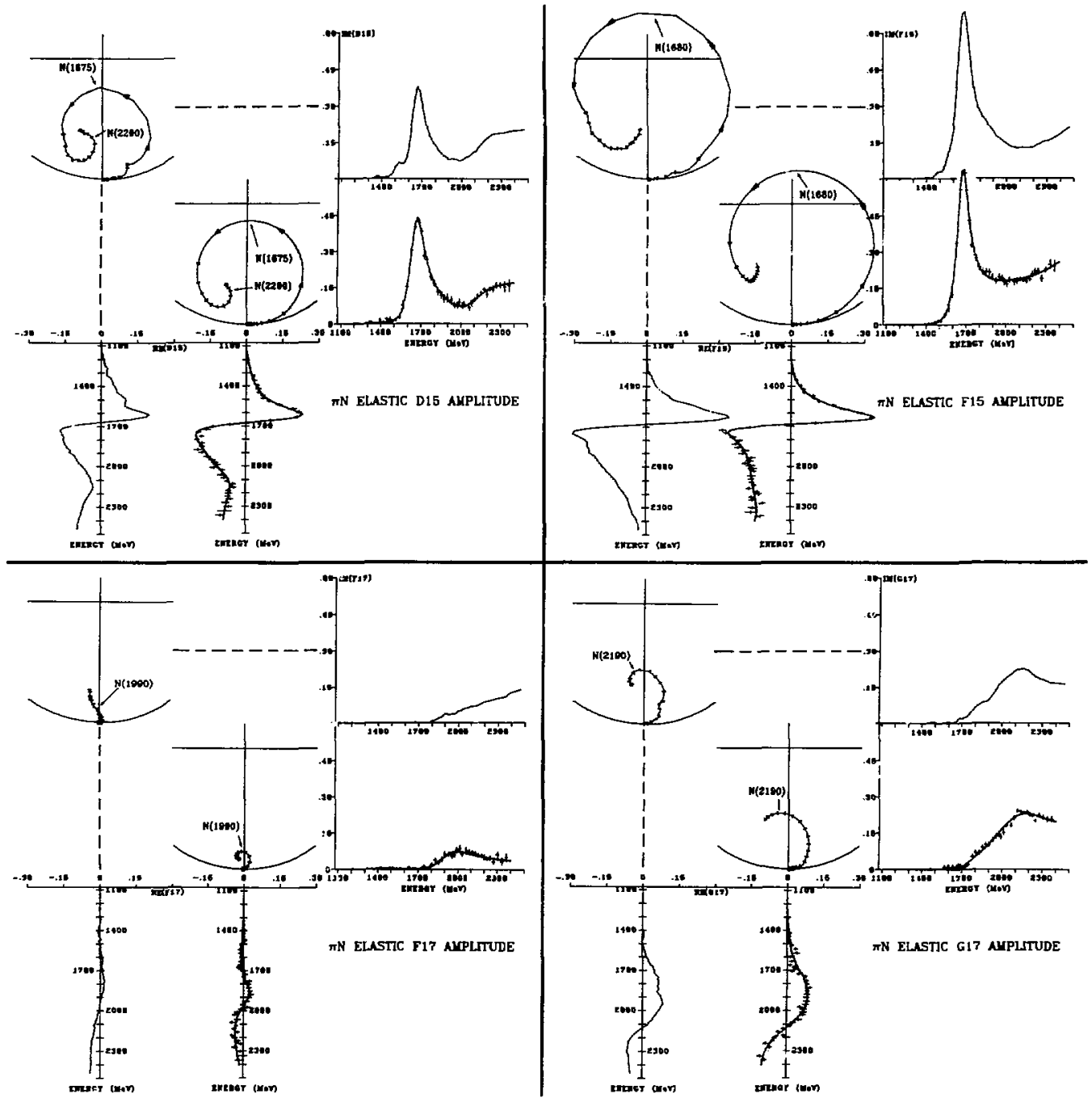

F1g. II.1(b). The L-2I-2J = D15, F15, F17, and G17 partial-wave anplitudes for $\pi$ N elastic acattering : The upper plot for each anplitude is from HOEHLER 79 and the lower one is from CUTKOSKY 80 . In the Argand plots, the ticks are at integral nultiples of $50 \mathrm{MeV}$, and the established resonances are shown at their nonInal pasitiona. The real and inginery part of the anplitudes as functions of energy are ohown projected In alignment with the Argand plote (10 the projections of the cuTKOSKY 80 aplitudes, the "data points" are results of energy-independent fits, and the curves are frow an energy-dependent fit to jolo thea). 
Data Card Listings

For notation, see key at front of Listings.
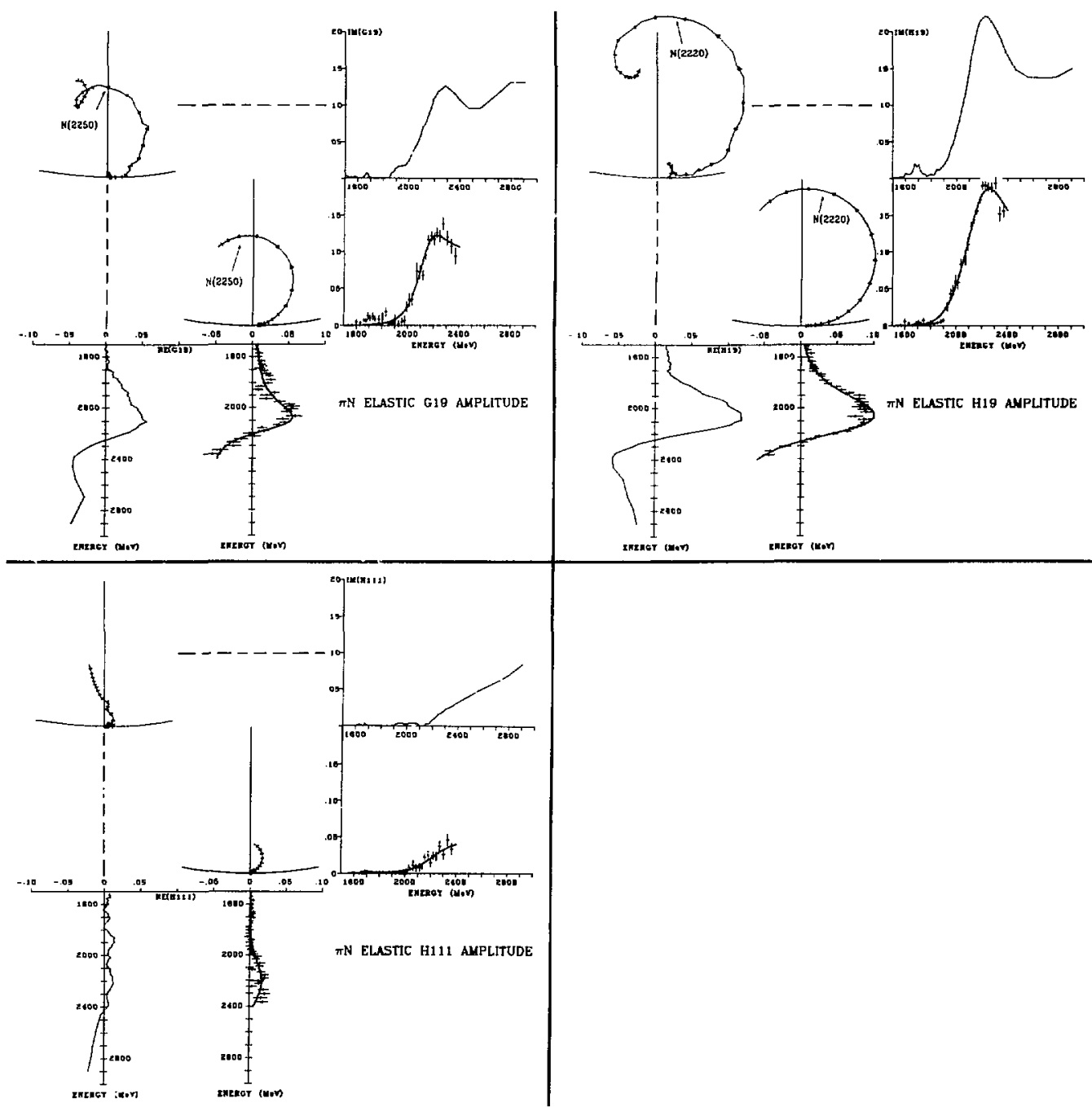

F1g. II.L(c). The L-2I.2J = G19, H19, and HI1l partial-wave amplitudes for $\pi N$ elagtic scattering. The upper plot for each amplitude 19 from HOEHLER 79 and the lower one 18 from CUTKOSKY 80 . In the Argand plots, the ticks are at Integral multiples of $50 \mathrm{MeV}$, and the established resonances are shown at their nominal positions. The real and Imaginery parts of the anplitudes as functions of energy are shown projected in alignment with the Argard plots (1n the projections of the CUTKoskY 80 amplitudes, the "data points" are results of energy-1ndependent $\mathrm{fIts}$, and the curves are from an energy-dependent fit to jo1n them). 


\section{Baryons}

$N^{\prime} s$ and $\Delta$ 's
Data Card Listings

For notation, see key at front of Listings.

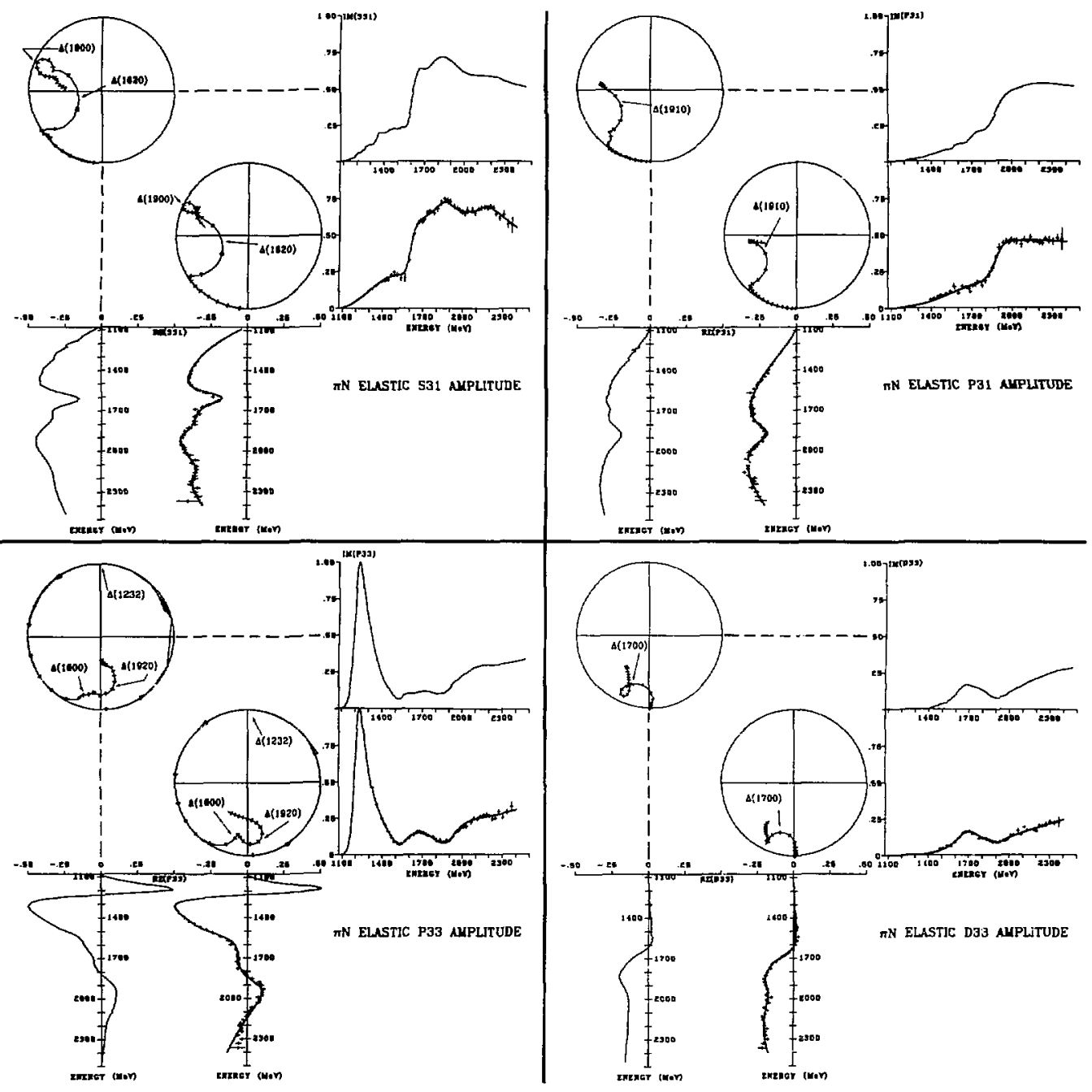

F1g. II.1(d). The $[\cdot 2 I \cdot 2 \mathrm{~J}=531, \mathrm{P} 31, \mathrm{P} 33$, and D33 pertial-wave amplitudes for $\pi \mathrm{N}$ elastic scattert.0g. The upper plot for each anplitude is fron HOEBLER 79 and the lover one is from COTKOSKY BO. In the A" and plots, the ticks are at integral multiples of $50 \mathrm{MeV}$, and the established resonances are shown at their nosinal positions. The real and imaginary parts of the anplitudes as functions of energy are shown projected In aligment with the Argand plots (1n the projectiong of the CUTKoskY 80 amplitudes, the "data points" are regults of energy-independent fits, and the curves are from an energy-dependent fit to join then). 
Data Card Listings

For notation, see key at front of Listings.

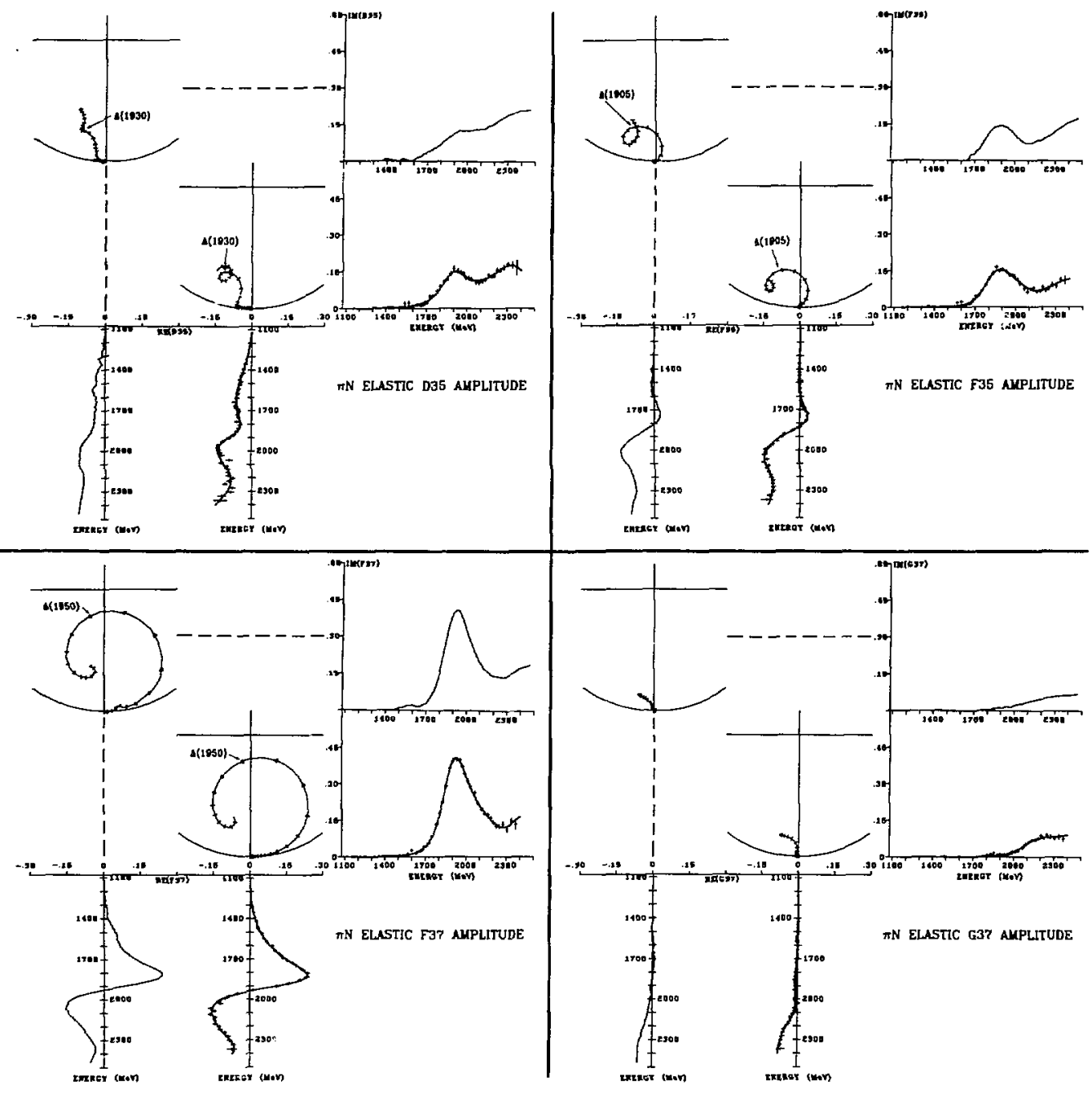

F1g. II.1(e). The L.2I-2J = D35, F35, F37, and G37 part1a1-wave amplitudes for nN elastic scatter1ng. The upper plot for each anplitude 1o from BOEHLFR 79 and the lower one 18 from CUTKOSXY 80 . In the Argand plot:, the ticks are at Integral multiples of $50 \mathrm{MeV}$, and the establiahed resonanceg are shown at their nomInel positions. The real and imaginary parts of the amplitudes as functions of energy are shown projected in alignment with the Argand plots (1n the projections of the cuTKosKY 80 amplitudes, the "data pointa" are results of energy-1ndependent $f 1 t s$, and the curves are from an energy-dependent $f 1 t$ to foin them). 
Baryons

N's and $\Delta$ 's
Data Card Listings

For notation, see key at front of Listings.

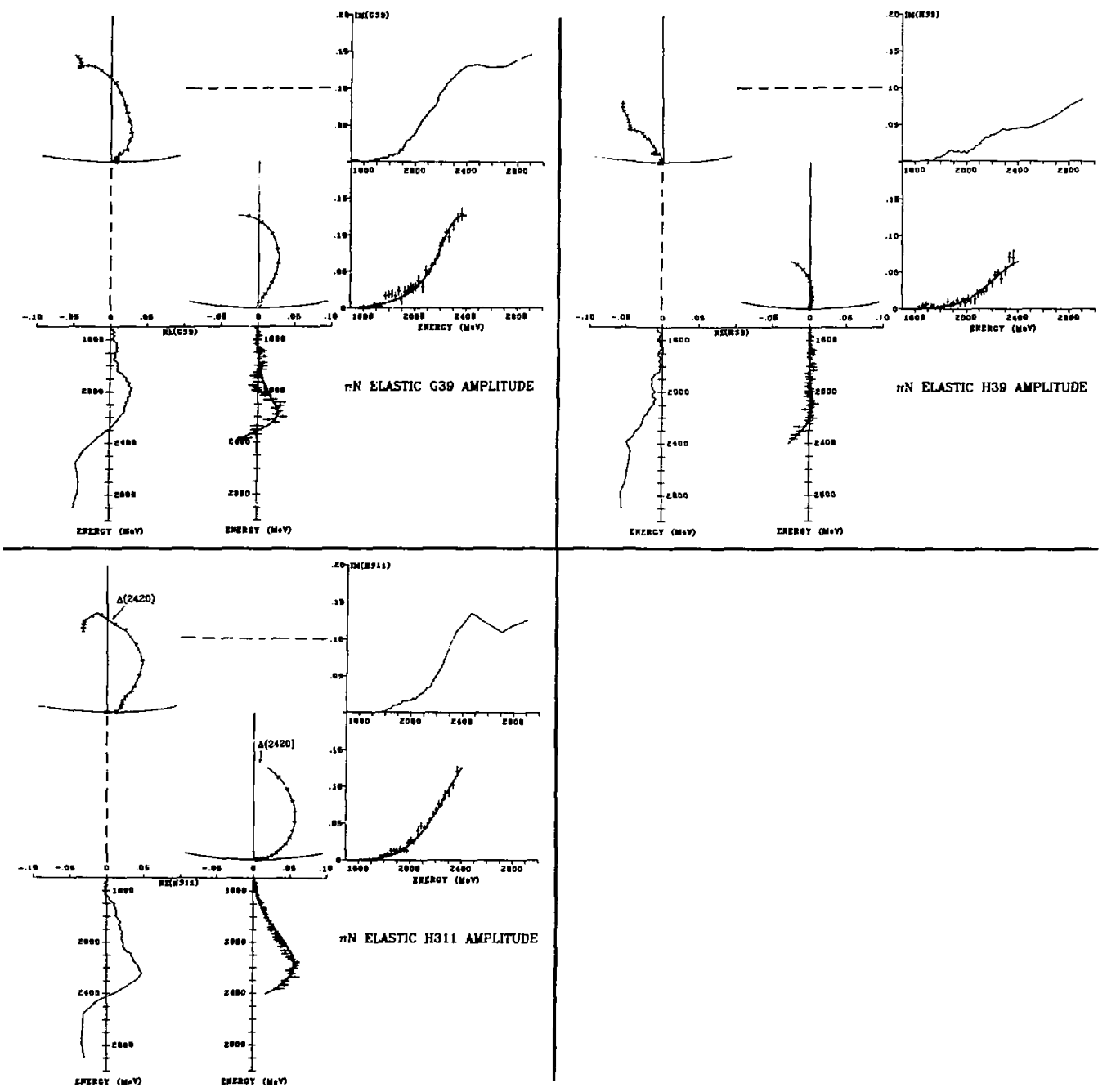

F1g. II.2(f). The L.2I·2J = G39, H39, and H311 partial-wave amplitudes for $\pi N$ elastic scattering. The upper plot for ench anplitude 1s fron bOEHLER 79 and the lower one is froe CuTKOSKY 80 . In the Argand plots, the ticks are at integral aultiples of $50 \mathrm{MeV}$, and the establiahed resonances are shown at their nomtnal positions. The real and inaginary parte of the anplitudes an functions of eaergy are ahown projected In aligrment with the Argand plots (1n the projectlong of the Currosxy 80 anplitudes, the "data points" are results of energy-1ndependent fits, and the curves are from an energy-dependent fit to join then). 


\section{Data Card Listings}

For notation, see key at front of Listings.

where in present analyses $i$ is the $\Delta(1232), N{ }^{\star}$ is the $N(1440), \rho 18$ the $\rho(770)$, and $\varepsilon 18$ the S-wave, $I=0 \pi \pi$ enhancement (not all these 1sobars are Included in every analysis). Here, BW denotes the appropriate Bre1t-W1gner or corresponding 2-body amplitude from $\pi N$ or $\pi \pi$ analyses, and $X$ is a welldefined function containing all the angular information. The decays of the resonances that are formed in the reaction are described by the partial-wave amplitudes $T_{\Delta \pi}^{J I L L^{\prime}}$, etc., where $J$ is the total angular momertum and $I$ is the total 1sospin of the state formed, and Is and I' are the orbital angular moments in the 1nitial 2-body and the EInal quasi-2-body statea. So $\vec{J} \cdot \vec{L}+\vec{S}$. $\vec{L}^{+}+\vec{S}^{\prime}$, where $S$ and $\vec{s}^{\prime}$ are the Initial and final total spins. For the on applitude, it is necessary to add the suffix $25^{\circ}$, equal to $l$ or 3 , to 1ndicate the total $\rho N$ splin. The partial-wave awplitudes are of ten denoted by $\Delta \cdot L \cdot L^{\cdot} \cdot 2 I \cdot 2 J, \rho_{2 S^{*}} \cdot L^{\prime} \cdot L^{*} \cdot 2 I \cdot 2 J$, etc.

The Ligtings give the results from four analyses.

LONGACRE 75 (LBL-SLAC) is an analysis of $200 \mathrm{~K}$ $\pi^{-} p \rightarrow \pi^{-} \pi^{0} p, \pi^{-} p \rightarrow \pi^{-} \pi^{+} n$, and $\pi^{+} p+\pi^{+} \pi^{0} p$ events for $1300 \leq W \leq 2000 \mathrm{MeV}$. Approximate unitarity constraints are fmposed using a simplifled K-matrix formal1sw that 11nks the $\pi 3 N$ channel to the $\pi N$ channel. This gives smooth solutions and elimInates the overall phase anbiguicy at each energy. The $\Delta \pi, p N$, and $\varepsilon N$ intermediate atates are Included. rouplings and T-natrix pole positiong are given for 14 resonances.

LONGACRE 77 (Saclay) 1s a coupled-channel analysis similar to LONGACRE 75 that fits $100 \mathrm{~K}$ events for $1380 \leq w \leq 1740 \mathrm{MeV}$. The couplings and fole positions are found for 16 resonances, includIng a P13 N(1540) and a P31 $\Delta(1550)$ suggested for the firat time by this analyais.

NOVOSELLER 78 (CIT) is an analysis of $\pi^{-} p \rightarrow \pi^{-} \pi^{0} p, \pi^{-} p+\pi^{-} \pi^{+} \pi$, and $\pi^{+} p-\pi^{+} \pi^{0} p$ for 1630 $\leq W \leq 1990 \mathrm{HeV}$ based on the LBL-SLAC energyindependent analysis. ${ }^{1}$ Agatn the $\Delta \pi, \rho N$, and $E N$ states are uged, but the resonances are fitted using a sople Breit-Higner amplitude rather than the K-watrix fecmalism of LOAGACRE 75. Single-pion exchange with $\pi$ rescattering is used to calcivis: $\epsilon$ the higher partial waves (taking account of a cri$t$ lc1sm made of earlier analyses), and tt 18 concluded that this 1aproves the $f(t)$ above $1800 \mathrm{MeV}$ and helps eltrinate the phase ambiguity. Another study of the 1mportance of single-plon exchange has been made by Aarou et $a 1 .{ }^{2}$ who also Eind that $1 t$ can glve 10portant corrections to the angular dependence. NOVOSELLER 78 gives two solutions, the second including the effects of pton exchange. They are given in the Listings as fits to LONGACRE 75 and NOVOSELLER 78 .

BARNHAM BO (Imperial College) is an analysis of $44 \mathrm{~K} \pi^{+} p+\pi^{+} \pi^{0} p$ and $\pi^{+} p+\pi^{+} \pi^{+} a$ events for 1440 $\leq W \leq 1700 \mathrm{MeV}$. It thus concerns only the $A$ resonances, and it uses data that were not avallable to the other analyses. The intermediate states are $\Delta \pi, \rho N$, and $\pi N(1440)$, the last belng necessary to account for the difference between the $\pi \pi^{0}{ }_{p}$ and $\pi^{+} \pi{ }^{+} n$ cross sections. Also included is the effect of single-pion exchange leading to the s-wave $\pi \pi$ state with $I=2$. The phase mbiguity is resolved by requiring that the $\pi \Delta$ amplitude for the 033 $\Delta(1700)$ have a Breit-Wigner phase. The parameters are found for four resonances, Including the $\mathrm{P} 3 \mathrm{l}$ $\Delta(1550)$, but since some of the data were also used by LONGACRE 77 it is not aleat that this resonance is being confirmed. however, there is now some evidence that it has also been seen in single-plon photoproduction. 3

There have also been analyees of $\pi^{-} p+\pi^{-} \pi^{+} n$ for $\omega \leq 1400 \mathrm{MeV},{ }^{4,5}$ a range dominated by the low energy tall of the Pll N(1440), with $E N$ as the domInant intermediate state. The energy range of these analyses is too low for them to determine decay coupling constants, and they do not appear in the listings.

It is difficult to assess the systematic uncertainties of these analyges or of the quoted couplings of the resonanceg to the Isobar states. However, those that are indicated in the listings so being well determlned by LONGACRE 77 do 1 general agtee, at least in $8 \mathrm{Ign}$, with the values from the other analyses, although some of the $D_{3}$ couplings have not been measured elsewinere. Tha Imperlal College group also claing clear measurements of the signs of $\rho_{1}$ SS31, $\rho_{1}$ DD 33, and $N^{*}$ PP33. 


\section{Baryons}

$N$ 's and $\Delta$ 's

Al1 existing 1sobar models can be criticized for neglecting possible sub-energy dependence of the partial-uave amplitudes, which makes then possibly incongistent with unitarity. ${ }^{6}$ This has been studied by Atchison and Brehm, ${ }^{7}$ who derlve an 1sobar expansion that is consistent with Bose symmetry and with sub-energy analyticticy and unitaricy. The resulting coupled integral equations are sultable for both dynamical and phenomenological studies of $\pi \mathrm{N}+\pi \pi \mathrm{N}$. They estimate the sub-energy corrections to the Isobar rodel and conclude that such corrections way not be significant for existing isobar fits but that they could become so with better data. ${ }^{B}$ A rough estiwate of these corrections has also been made by the Impertal College group, ${ }^{9}$ who also find that they are small.

\section{References for Section III}

L. D.J. Herndon, R. Longacre, L.R. Mller, A.H. Rosenfeld, G. Smadjz, P. Sod1ng, R.J. Cashmore, and D.W.C.5. Letth, Phys. Rev D11, 3183 (1975).

2. R. Aaron, R.D. Amado, R.A. Arndt, Y. Goradia, D.C. Teplitz, and V.L. Teplitz, Phys. Rev. D16, 50 (1975).

3. R.L. Crawford, in Proceedings of the $I \mathrm{~V}^{\mathrm{th}}$ International Conference on Baryon Resonances (Toronto, 1980), ed. N. Isgur, p.107.

4. R.A. Arndt, J.B. Cammarata, Y.N. Goradia, R.H. Hackman, V.L. Teplltz, D.A. Dicur, R. Aaron, and R.5. Langacre, Phys. Rev. D20, 651 (2979).

5. R. Aaron, R.A. Arndt, J.B. Cammarata, D.A. Dicus, and V.L. Teplitz, Phys. Rev. Lett. 44 , $66(1980)$.

6. R. Aaron and R.D. Amado, Phys. Rev. Lett. 31 , 1157 (1973).

7. I.J.R. Altchison and J.J. Breho, Phys. Rev. D20, 1119 (1979).

8. I.J.R. Altchison and J.J. Brehm, Phys. Rev. D20, 1131 (1979).

9. K.W.J. Barnham, in Proceedings of the Toplcal Conference on Baryon Resonances (oxford, 1976), eds. R.T. Ross and D.H. Saxon, p. 109.

\section{Photoproduct1on and Compton Scatier1ng}

(by R.L. Crawford, University of Glasgow)

The $\gamma N$ couplings of an $N$ or $\Delta$ resonance can be gtudied in any photon-induced formation process in which the coupling of the resonance to the final state is well knowa. In practice, this limits accurate sources for such information to partialwave analyses of single-pion photoproduction, where
Data Card Listings

For notation, see key at front of Listings.

there is a large amount of data and where the final state is well known from $\pi \mathrm{N}+\pi \mathrm{N}$ partial-wave ana1yees. Recently, however, some couplings have been obtalned from Compton scattering on protons. All photoproduction analyses rely heavily on $\pi N \rightarrow \pi N$ analyses for the existence, masses, and width of che resonances. In only a few photoproduction analyses are the masses and widhs treated as free parameters. However, the photoproduction results for the masses and widths are of interest since they give access to the charge +1 states.

The most 1mportant anelyses of single-plon photoproduction are revleved below. The focmalism has been described in an earlier edition of this Review, ${ }^{1}$ to wich the reader 1 s referred for additional information. There are three basic methods of analysis. All have to cope with the stab1lity problews of having four independent complex spin amplitudes at any energy and angle, tut only $s i x$ (and frequently fewer) Independent experimental measurements.

(a) SImple 1sobar model: This 1s the stmplest form of energy-dependent analysis. The partial waves are parametrized as a smooth background to which Ireft-Higner resonances are added. Usually the electric but not the magnetic Born terms are included explicitiy to reproduce the forward peak in charged pion production. This method is sufficlently flexible to glve excellent. fits to the data, but there are in principle difficulties concerning the uniqueness of the solution due to the large number of waves involved. This problem is ellownated by the form of the parametrization, but It is not clear how this may blas the solution.

The most extensive analysis of this type is METCALF 74 (for references in this form, see the Data Card Listings), which is an extension of the arller Walker analysis. ${ }^{2}$ It fits $\gamma_{p}+\pi^{+}{ }^{+}$, $v_{p} \rightarrow \pi^{0} D$, and $\gamma_{n}+\pi^{-} p$ from the first to the fourth regonance regions. FELLER 76 is a siollar analyais which does not fil $\gamma_{n} \rightarrow \pi^{-} p$, but uses data that were not avallable to METCALF 74. Other 1sobar analyses are ROSSI 73, HEMII 73, HEMII 73, BENEVENIANO 74, KRIVETS 74, TAKEDA 80, and BRATASHEVSKIJ 80; these have used relatively small and sometimes restricted data sets, usually in asgociation with a particular experiment. ISHII 80 is an 


\section{Data Card Listings}

For notation, see key at front of Listings.

1sobar analygis of proton compton scattering for laboratory photon energles from $550 \mathrm{MeV}$ to $950 \mathrm{MeV}$, covering the second resonance region.

(b) Fixed-t dispersion relations (FTDR): Th1s technique uses the apparent resonance dominance of the photoproduction amplitudes to get a relatively simple parametrization of their imaginary parts. Fixed-t dispersion relations are then used to calculate the real parts; sometimes a few but usually no additional free parameters are introduced. since there are fewer free parameters, the probability of having multiple solutions is reduced, and the requirements of analyticlty are automatically satisfled. However, the method is inflexible compared to the Isobar model and gives poorer f1ts. Also, as has been described in NOELLE 78 and elsewhere, ${ }^{3}$ the divergence of the partial-wave expansions for the dispersion integrals does not allow the use of data at all angles above the third resonance region. Some but not all analyses satisy the constraints of unttarity and time reversal Invariance as given by watson's theorem. 4

FTDR analyses have been made by groups at Berkeley (MOORHOUSE 73, KNIES 74, and MOORHOUSE 74), at Lancaster (DEVENISH 73 and DEVENISH2 74), at Glasgow (CRAWFORD 75, BARBOUR 76, BARBOUR 78, and CRAHRORD 80), at Yerevan (AZNAURYAN 77), and at Tokyo (ARAI 80 and FUJII 81). NOELLE 78 is a hybrid analysis 1ncorporating FTDR in a coupledchannel Isobar calculation.

(c) Energy 1ndependent analyges: These evaluate the partial waves by making $f 1 t s$ at a set of essentially single energies, and are thus the least blased of all analyses. It is necessary to use Watson's theorem to $f 1 x$ the complex phases of many of the partial waves and thus to get a unique solution. Due to the onset of inelasticity, this becomes difficult above the first resonance region, and only BERENDS 77 extends into the second resonance region. This analysis gets significantly different couplings from the other analyses for the D13 N(1520).

New analyses in the Data Card Listings: ARAI 80 is an FTDR analysis of $\gamma_{p}+\pi^{+} n, \gamma_{p}+\pi^{0} p$, and $\gamma_{n} \rightarrow \pi-p$ using 7768 data points for energies from 1168 to $2078 \mathrm{MeV}$ and for momentum transfers $t$ out to $-1.6(\mathrm{GeV} / \mathrm{C})^{2}$. A K-natrix formallom is used to parametrize the resonances, and a Regge formallsm 1s used for the high energy part of the dispersion Integrals.

CRAWFORD 80 is an extension of earlier Glasgow analyses to Include wore data and more resonances. The 1maginary parts of the partial waves are parametrlzed as Breit-Wigner amplitudes plus background in the $S$ and $P$ waves. The high energy parts of the dispersion integrals are parametrized using a Regge formalism, and data are fitted at all energies for $\gamma_{p} \rightarrow \pi^{+} n$, $\gamma_{p}+\pi^{0} p$, and $\gamma_{n} \rightarrow \pi^{-} p$. A total of 8838 data points are used for energies up to 2.5 GeV and for $t$ out to $-1.5(\mathrm{GeV} / \mathrm{c})^{2}$. Evidence $1 \mathrm{~s}$ found to suggest that the $P 31 \Delta(1550)$ is photoproduced, but it is not conclusive.

BRATASHEVSKIJ 80 is an Lsobar analygis of $\gamma_{p} \rightarrow \pi^{0}$ using recoll proton polarization data from Kharkov and is essentially a variation of METCALF 74.

TAKEDA 80 is similarly a variation of METCALF 74 to fit new recoll proton polarization data in $\mathrm{Y}_{\mathbf{n}}+\pi^{-} \mathrm{p}$.

FUJII $8 \mathrm{~L}$ is based on the ARAI $B O$ analysis and tncludes new neutron target data.

Resonance couplings and errors in the Data Card Listings: The listings $B$ ive the results of all recent and extensive analyses. If no error is glven, only a unique result is quoted. The Berkeley analyges and CRAWFORD $75 \mathrm{glve}$ for errors the spread of solutions around a central value. The Lancaster group gives for errors the change of value that 19 required to Increase the "best possible $x^{2} \%$ by $1 \%$. METCALE 74 , FELLER 76, AZNAURYAN 77, and ARAI 80 quote simflar errors. In BARBOUR 78 and CRAWFORD 80, the systematic differences due to the different gethods of analysis are considered to be at least as important as the purely statistical errors, and the errors quoted are obtalned by comparing with other analyses as well as from the spread of parameters over a number of fits. Thus there $1 \mathrm{~s}$ of ten a wide variation in what the errors quoted in the Listings mean.

Table IV. 1 gives a compilation of the $Y N$ decay couplings from METCALF 74, KNIES 74, HOORHOUSE 74, DEVENISH2 74, BARBOUR 76, BARBOUR 78, ARAI BO, and CRAWFORD 80 . The errors quoted are a combination 
Baryons

N's and $\Delta$ 's

Table IV.1. A compilation of tessured YN decay couplinge and of predictions of the quark model. Sources are given in the text.

\begin{tabular}{|c|c|c|c|c|c|c|c|}
\hline \multirow{3}{*}{$\frac{\text { Resonance }}{\dot{P}_{11}^{\prime} \mathrm{N}(1440)}$} & \multirow[b]{2}{*}{ Target } & \multirow{3}{*}{$\begin{array}{l}\begin{array}{l}\text { Hel1- } \\
\text { city }\end{array} \\
1 / 2 \\
1 / 2\end{array}$} & \multicolumn{5}{|c|}{ Couplinge $\left(\mathrm{GeV}^{-1 / 2} \times 10^{-3}\right)$} \\
\hline & & & \multicolumn{2}{|c|}{$\begin{array}{l}\text { Partial-Have } \\
\text { Analyses }\end{array}$} & \multicolumn{3}{|c|}{$\begin{array}{l}\text { Quark Mode1 } \\
\text { Predictions }\end{array}$} \\
\hline & $\begin{array}{l}\mathbf{p} \\
\mathbf{a}\end{array}$ & & $\begin{array}{l}-70 \pm \\
+42 \pm\end{array}$ & $\begin{array}{r}9 \\
19\end{array}$ & $\begin{array}{l}-5 \\
+4\end{array}$ & $\begin{array}{l}\text { to } \\
\text { to }\end{array}$ & $\begin{array}{l}-50 \\
+38\end{array}$ \\
\hline $3^{N(1520)}$ & $\mathbf{n}$ & $\begin{array}{l}1 / 2 \\
3 / 2 \\
1 / 2 \\
3 / 2\end{array}$ & $\begin{array}{r}-17 \pm \\
+166 \pm \\
-69 \pm \\
-136 \pm\end{array}$ & $\begin{array}{r}11 \\
7 \\
14 \\
14\end{array}$ & $\begin{array}{r}-41 \\
+95 \\
-23 \\
-102\end{array}$ & $\begin{array}{l}\text { to } \\
\text { to } \\
\text { to } \\
\text { to }\end{array}$ & $\begin{array}{r}+6 \\
+174 \\
-52 \\
-144\end{array}$ \\
\hline $11 \mathrm{~N}(1535)$ & $\begin{array}{l}\text { p } \\
\mathbf{n}\end{array}$ & $\begin{array}{l}1 / 2 \\
1 / 2\end{array}$ & $\begin{array}{l}+67 \pm \\
-78 \pm\end{array}$ & $\begin{array}{l}15 \\
29\end{array}$ & $\begin{array}{l}+68 \\
-83\end{array}$ & $\begin{array}{l}\text { to } \\
\text { to }\end{array}$ & $\begin{array}{l}+147 \\
-119\end{array}$ \\
\hline $11 \mathrm{~N}(1650)$ & $\begin{array}{l}p \\
\mathrm{q}\end{array}$ & $\begin{array}{l}1 / 2 \\
1 / 2\end{array}$ & $\begin{array}{l}+45 \pm \\
-23 \pm\end{array}$ & $\begin{array}{l}17 \\
33\end{array}$ & $\begin{array}{r}-9 \\
-45\end{array}$ & $\begin{array}{l}\text { to } \\
\text { to }\end{array}$ & $\begin{array}{r}+95 \\
+4\end{array}$ \\
\hline$N(1675)$ & $\begin{array}{l}\mathrm{p} \\
\mathrm{n}\end{array}$ & $\begin{array}{l}1 / 2 \\
3 / 2 \\
1 / 2 \\
3 / 2\end{array}$ & $\begin{array}{l}+13 \pm \\
+22 \pm \\
-37 \pm \\
-54 \pm\end{array}$ & $\begin{array}{r}8 \\
12 \\
24 \\
24\end{array}$ & $\begin{array}{r}0 \\
0 \\
-31 \\
-44\end{array}$ & $\begin{array}{l}\text { to } \\
\text { to } \\
\text { to } \\
\text { to }\end{array}$ & $\begin{array}{l}+12 \\
+16 \\
-55 \\
-78\end{array}$ \\
\hline$N(1680)$ & p & $\begin{array}{l}1 / 2 \\
3 / 2 \\
1 / 2 \\
3 / 2\end{array}$ & $\begin{array}{r}-13 \pm \\
+132 \pm \\
+29 \pm \\
-28 \pm\end{array}$ & $\begin{array}{l}10 \\
15 \\
15 \\
16\end{array}$ & $\begin{array}{r}-7 \\
+47 \\
-32 \\
-25\end{array}$ & $\begin{array}{l}\text { to } \\
\text { to } \\
\text { to } \\
\text { to }\end{array}$ & $\begin{array}{r}+24 \\
+154 \\
+27 \\
+2\end{array}$ \\
\hline$N(1700)$ & $\mathrm{p}$ & $\begin{array}{l}1 / 2 \\
3 / 2 \\
1 / 2 \\
3 / 2\end{array}$ & $\begin{aligned}-20 & \pm \\
+1 & \pm \\
0 & \pm \\
+8 & \pm\end{aligned}$ & $\begin{array}{l}12 \\
17 \\
50 \\
42\end{array}$ & $\begin{array}{l}-7 \\
-12 \\
-15 \\
-17\end{array}$ & $\begin{array}{l}\text { to } \\
\text { to } \\
\text { to } \\
\text { to }\end{array}$ & $\begin{array}{r}+9 \\
+33 \\
+25 \\
-76\end{array}$ \\
\hline$N(1710)$ & $\begin{array}{l}\mathbf{p} \\
\mathbf{n}\end{array}$ & $\begin{array}{l}1 / 2 \\
1 / 2\end{array}$ & $+3 \pm$ & $\begin{array}{l}15 \\
30\end{array}$ & $\begin{array}{r}-7 \\
-21\end{array}$ & $\begin{array}{l}\text { to } \\
\text { to }\end{array}$ & $\begin{array}{l}-37 \\
+29\end{array}$ \\
\hline $13^{N(1720)}$ & $\begin{array}{l}\mathbf{p} \\
\mathfrak{n}\end{array}$ & $\begin{array}{l}1 / 2 \\
3 / 2 \\
1 / 2 \\
3 / 2\end{array}$ & $\begin{array}{l}+60 \pm \\
-34 \pm \\
-6 \pm \\
-26 \pm\end{array}$ & $\begin{array}{l}36 \\
26 \\
25 \\
90\end{array}$ & $\begin{array}{r}-133 \\
-65 \\
-23 \\
-61\end{array}$ & $\begin{array}{l}\text { to } \\
\text { to } \\
\text { to } \\
\text { to }\end{array}$ & $\begin{array}{l}+74 \\
+46 \\
+57 \\
+12\end{array}$ \\
\hline $17 \mathrm{~N}(1990)$ & $\begin{array}{l}\mathrm{p} \\
\mathrm{n}\end{array}$ & $\begin{array}{l}1 / 2 \\
3 / 2 \\
1 / 2 \\
3 / 2\end{array}$ & $\begin{array}{r}+17 \pm \frac{ \pm}{ \pm} \\
-82 \pm \frac{ \pm}{ \pm} \\
-86 \pm\end{array}$ & $\begin{array}{l}35 \\
20 \\
25 \\
40\end{array}$ & $\begin{array}{l}-8 \\
-10 \\
-18 \\
-23\end{array}$ & $\begin{array}{l}\text { to } \\
\text { to } \\
\text { to } \\
\text { to }\end{array}$ & $\begin{array}{l}-10 \\
-13 \\
-19 \\
-25\end{array}$ \\
\hline $7^{\mathrm{N}(2}$ & $\begin{array}{l}\mathbf{p} \\
\mathbf{n}\end{array}$ & $\begin{array}{l}1 / 2 \\
3 / 2 \\
1 / 2 \\
3 / 2\end{array}$ & $\begin{array}{r}-43 \pm \\
+131 \pm 1 \\
-64 \pm \\
-60 \pm 1\end{array}$ & $\begin{array}{r}40 \\
100 \\
45 \\
130\end{array}$ & & & \\
\hline ) & $\mathbf{p}$ & $\begin{array}{l}1 / 2 \\
3 / 2\end{array}$ & $\begin{array}{l}-141 \pm \\
-258 \pm\end{array}$ & $\begin{array}{l}6 \\
8\end{array}$ & $\begin{array}{r}-94 \\
-162\end{array}$ & $\begin{array}{l}\text { to } \\
\text { to }-\end{array}$ & $\begin{array}{l}-127 \\
-220\end{array}$ \\
\hline$\Delta(1600)$ & p & $\begin{array}{l}1 / 2 \\
3 / 2\end{array}$ & $-2 \pm$ & $\begin{array}{l}30 \\
30\end{array}$ & $\begin{array}{r}-61 \\
-107\end{array}$ & $\begin{array}{l}\text { to } \\
\text { to }\end{array}$ & $\begin{array}{l}+2 \\
+4\end{array}$ \\
\hline $31 \Delta(1620)$ & $\mathbf{p}$ & $1 / 2$ & $+23 \pm$ & 38 & +43 & to & +86 \\
\hline $33 \Delta(1700)$ & $\mathbf{p}$ & $1 / 2$ & $\begin{array}{r}+109 \pm \\
+73 \pm\end{array}$ & $\begin{array}{l}31 \\
35\end{array}$ & $\begin{array}{l}+78 \\
+78\end{array}$ & $\begin{array}{l}\text { to } t \\
\text { to } t\end{array}$ & $\begin{array}{l}+106 \\
+105\end{array}$ \\
\hline${ }_{35} \Delta(1905)$ & $\mathbf{p}$ & $\begin{array}{l}1 / 2 \\
3 / 2\end{array}$ & $\begin{array}{l}+33 \pm \\
-38 \pm\end{array}$ & $\begin{array}{l}10 \\
19\end{array}$ & $\begin{array}{l}-10 \\
-41\end{array}$ & $\begin{array}{l}\text { to } \\
\text { to }\end{array}$ & $\begin{array}{l}+44 \\
+15\end{array}$ \\
\hline$P_{31} \Delta(1910)$ & $\mathbf{p}$ & $1 / 2$ & $-24 \pm$ & 15 & -16 & to & +15 \\
\hline $\mathrm{D}_{35} \Delta(1930)$ & p & $1 / 2$ & $\begin{array}{l}-46 \pm \\
+13 \pm\end{array}$ & $\begin{array}{l}\text { 50 } \\
\text { 50 }\end{array}$ & $\begin{array}{l}-17 \\
-24\end{array}$ & & \\
\hline $\mathbf{F}_{37} \Delta(1950)$ & P & $3 / 2$ & $\begin{array}{l}-73 \pm \\
-85 \pm\end{array}$ & $\begin{array}{l}12 \\
13\end{array}$ & $\begin{array}{l}-25 \\
-32\end{array}$ & $\begin{array}{l}\text { to } \\
\text { to }\end{array}$ & $\begin{array}{l}-48 \\
-69\end{array}$ \\
\hline
\end{tabular}

Data Card Listings

For notation, see key at front of Listings.

of the statistical errors from these analyses and the systematic differences between them. Also shown for comparison are the range of predictions for the couplings from recent quark model calculations. ${ }^{5-8}$ While the quantitative agreement between the quark models and the measured couplings is not yet good, there is qualitative agreement to the extent that at least one quark model gives the correct sign in the cases where the sign has been clearly measured.

\section{References for Section IV}

1. Particle Data Group, Rev . Hod. Phys. 48, S157 (1976).

2. R.L. Walker, Phys. Rev. $\underline{162}, 1729$ (1969).

3. R.C.E. Devenish, D.H. Lyth, and W.A. Rankin, Daresbury report DNPL/P109 (1972).

4. K.M. Watson, Phys. Hev. 95, 228 (1954).

5. T. Kubota and K. Ohta, Phys. Lett. 65B, 374 (1976).

6. I.M. Barbour and D.K. Pont1ng, Ze1t. Phys. $\underline{\text { C4}}$, $119(1980)$.

7. R. Konfuk and N. Lsgur, Phys. Rev, D21, 1868 (1980).

8. C.P. Forsyth, Carnegie-Mellon University preprint c00-3066-168 (1981).

\section{Electropraduction}

(by F. Foster, Univerafty of Lancaster)

Introduction: Since this review of resonance electroproduction was last revised (1978), several new data sets on virtual photoproduction of single plons hav becule avaiiuite. The vestr group has completed 1 ts detalled survey of the whole resonance region at values of $Q^{2}$ from 0.6 to $3.0 \mathrm{Gev}^{2}$. The new data on $\pi^{+}$and $\pi^{0}$ production and an analysis using the fixed-t dispersion relation methods of Devenish and Lyth are avallable in internal DESY reports, 2,3 and those on $n$ production are avallable in reports ${ }^{2,4}$ and a publication. Data at smaller $Q^{2}$ continue to appear from the Bonn group, for $n$ production ${ }^{6}$ at $Q^{2}=0.4 \mathrm{GeV}^{2}$ and for $\pi^{+}$and $\pi^{0}$ production ${ }^{7}$ at $Q^{2}=0.3 \mathrm{Gev}^{2}$. The Lancaster-Manchester group working at Daresbury has also completed new data sets at 0.5 and $1.0 \mathrm{GeV}^{2}$ using both hydrogen 8,9 and deuterlum as targets. 10-12

Analysis of the new data in terms of the approprlate resonance nultipole amplitudes has been 


\section{Data Card Listings}

For notation, see key at front of Listings.
Baryons

$N^{\prime} s$ and $\Delta^{\prime} s$ factlitated by the avallability of the DeventshLych fitting program, which uses flxed-t dispersion relation constraints. ${ }^{1}$ Ch. Gerhardt has analyzed much of the data avallable to 1978,13 while Davenport and Morris have analyzed recent Daresbury data $^{14}$ and $v$. Gerhardt has analyzed the recent DESY data. ${ }^{3}$ There are now quite consistent resultg for, in addition to the $P 33 A(1232)$ and the S11 N(1535) resonance wultipoles, the $D 13 \mathrm{~N}^{+}(1520)$ and the F15 $\mathrm{N}^{+}(1680)$ multipoles for $\mathrm{Q}^{2}$ up to $3.0 \mathrm{GeV}^{2}$, and there are some clear Ind ications of the behavior of the D33 $A^{+}(1700)$. Analys 1 of the Daresbury "neutron target" data 12 has also glven good rasults for the SII $N^{0}(1535)$ and $D 13 N^{0}(1520)$ multipoles at $Q^{2}$ $=0.5 \mathrm{GeV}^{2}$ and allowed significant tests of the generalized $\mathrm{SU}(6)_{\mathrm{W}}$ or single-quark excitation models. On the theoretical side, Alcock et al. 15 have presented ar oxcellent review of all recent quark models for resonance elec roproduction, as well as their own proposals for sultable quark models on a proper relativistic basis. They conclude that the single-quark transition model can reproduce qualitatively the observed behavior of the orb1t-f11p, spin-f11p, and spin-orbit parameters for the excication of nembers of the $[70,1]$ multiplet.

F1rst cesonance region: Excitation of the P33 $\triangle(1232)$ resonance proceeds malnly via the magnetic $M_{1+}$ aultipole; the electric $E_{1+}$ multipole is consistent with zero as 1 s expected from $S U(6)$ and the quatk motel. The scalar multipole has a small but significant negative value with $\mathrm{S}_{1+}$ belng between 5 and $10 \%$ of $\mathrm{M}_{1+}$, and as $\mathrm{Q}^{2}$ Increases the background amplitudes $E_{O_{+}}$and $M_{i-}$ account for an Increasing fraction of the single-pion cross section. The new DESY data ${ }^{2,3}$ at $Q^{2}=3.0 \mathrm{Gev}^{2}$ give $\mathrm{E}_{1+} / \mathrm{M}_{1+} \approx 0.05 \pm$ 0.05 and $\mathrm{s}_{1+} / \mathrm{M}_{1+} \approx-0.12 \pm 0.05$ at $1230 \mathrm{MeV}$, and the background ratios are $\mathrm{E}_{0+} / \mathrm{M}_{1+} \approx 0.5$ and $\mathrm{M}_{2-} / \mathrm{M}_{1+}$ $\approx-0.3$ near resonance. From 1 ts measurements of $\mathrm{M}_{1+}$, the DESY group also obtains a value for the transition form factor $\mathrm{G}_{\mathrm{M}}^{*}\left(\mathrm{Q}^{2}\right)$ at $\mathrm{Q}^{2}=3.0 \mathrm{GeV}^{2}$ that Is much less subject to systematic error than were the earlier "single arm" measurements of the total cross section. The results con $E 1$ ra that $\mathrm{G}_{\mathrm{M}}^{*}\left(\mathrm{Q}^{2}\right)$ falls more rapidly with $Q^{2}$ than does the nucleon "dipole" form factor. It is also clear that at the high values of $\mathrm{Q}^{2}$ the Devenish-Lyth program has great difficulty in fitting the shape of the $\mathrm{M}_{1+}$ varlation with mass, and also in accomodating the observed background excjtation, particularly above $1300 \mathrm{MeV}$. An unfortunate consequence is that the program cannot give unambiguous results for the excitation of the P1I N(1440) Roper resonance.

The $\left[70,1^{-}\right]$resonances: Table V.I glves the results of the various dispersion-relation fits for the low-lying negative-parity resonances $S 11$ $\mathrm{N}(1535), \mathrm{D} 13 \mathrm{~N}(1520), \mathrm{S} 1 \mathrm{~N}(1650)$, and $\mathrm{D} 33 \Delta(1700)$ from the $\left[70,1^{-}\right]$multiplet. It is difficult to

Table V.l. Results of recent fits to $\pi^{0}, \pi^{+}$, and : data for members of the $\left[70,1^{-}\right]$multiplet.

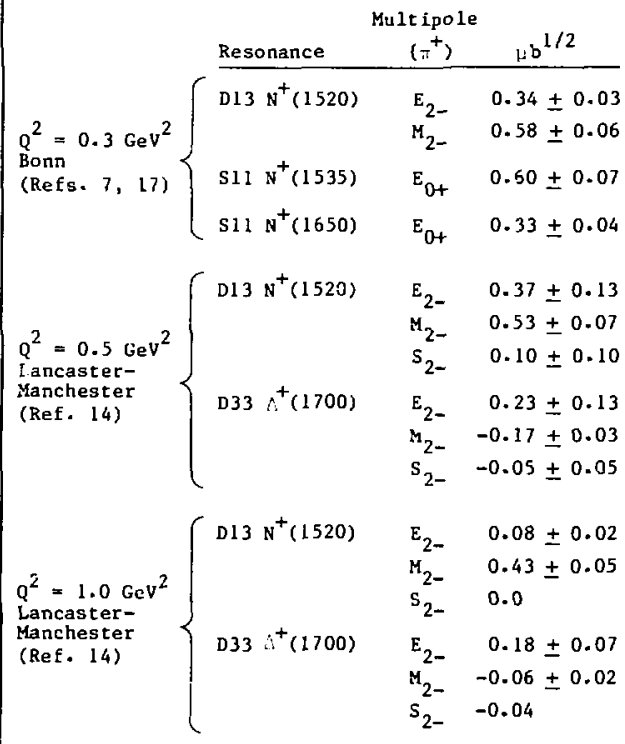

\begin{tabular}{|c|c|c|c|c|c|}
\hline \multirow[t]{2}{*}{ Resonance } & \multirow{2}{*}{$\begin{array}{c}\text { Multipole } \\
\left(\pi^{+}\right)\end{array}$} & \multicolumn{4}{|c|}{$\begin{array}{c}\text { DESY data (Ref. 3) } \\
\mu b^{L / 2}\end{array}$} \\
\hline & & $Q^{2}=0.6$ & 1.0 & 2.0 & $3.0 \mathrm{GeV}^{2}$ \\
\hline \multirow[t]{2}{*}{ Slt N(1535) } & $\mathrm{E}_{\mathrm{OH}}$ & 0.52 & 0.51 & 0.39 & 0.32 \\
\hline & $\mathrm{s}_{0+}$ & -0.30 & 0.00 & -0.11 & -0.10 \\
\hline \multirow[t]{3}{*}{$D 13 N(1520)$} & $E_{2-}$ & 0.13 & -0.04 & -0.11 & -0.08 \\
\hline & $\mathrm{M}_{2-}$ & 0.47 & 0.35 & 0.17 & 0.063 \\
\hline & $s_{2-}$ & 0.06 & 0.00 & 0.00 & 0.00 \\
\hline
\end{tabular}


Baryons

N's and $\Delta$ 's

assign uncertaintica to these results, but where this has been done it reflects the spread observed In Independent fits to the sane experimental data. Also, the 511 N(1535) aultipoles may be regarded as being very well deternined since they are extracted fron $\eta$ electroproduction data of high accuracy. The low $-Q^{2}$ data from Bonn were analyzed for the helictiy $1 / 2$ couplings only, and the multipoles here have been extracted by combining the helicity $3 / 2$ couplings from the analys is of Ch. Gerhardt. ${ }^{13}$ The trends in the data are clear: the $\mathrm{E}_{0+}$ multipole falls off very slowly so that the sil $N(1535)$ is the dowlnant component of the second resonance at $Q^{2}>1.0 \mathrm{GeV}^{2}$. Using only $\eta$ production cross sectlons and resonance peak helghts from total-cross-section measurements, the following results are obtalned: ${ }^{3}$

$$
\begin{array}{ccccc}
Q^{2}\left(\mathrm{GeV}^{2}\right) & \frac{\sigma_{\text {peak }}(\mu \mathrm{b})}{2.0} & \frac{511(\mu \mathrm{b})}{15.0 \pm 1.0} & \frac{D 13(\mu \mathrm{b})}{10.07 \pm 0.56} & \frac{5.0 \pm 1.2}{3.0} \\
7.7 \pm 1.1 & 6.85 \pm 0.37 & 0.88 \pm 1.2
\end{array}
$$

It has also been demonstrated that the effect is unlikely to be due to scalar/longltudinal photon excitation, since the following results are obtalned for $\sigma_{L} / \sigma_{T}$ using $\eta$ production cross sections at different values of the photon polarization:

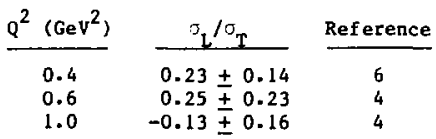

In contrast, the electric multipoles for the $\mathrm{D} 13$ and $D 33$ resonances :all rather rapidly with $Q^{2}$. This is particularl; noticeable for the D13 $N(1520)$, where $E_{2-}$ falls from 1.05 ub ${ }^{1 / 2}$ at $Q^{2}=0$ to less than $0.1 \mathrm{Hb}^{1 / 2}$ at $\mathrm{Q}^{2}=1.0 \mathrm{Gev}^{2}$, whlle the magnetic multipole $\mathrm{H}_{2-}$ falls much more slowly. The effect is associated with the well-known rapid changes in excitation of the resonances from mainly helicicy $3 / 2$ at $Q^{2}=0$ to malnly hellctty $1 / 2$ ac $Q^{2}$ $>1 \mathrm{GeV}^{2}$. More detalled discussions may be found isewhere (e.g., Ref. 17). An alternative and more Instructive point of view is to compare the $Q^{2}$ variation of the single-quark-transition-model (SQTM) parameters $A, B$, and $C$ which describe the
Data Card Listings

For notation, see key at front of Listings.

excitation of all the merbers of the $[70,1]$ wultiplet: $A$ and $B$ correspond to orbit- and spin-tilp excltation, and $C$ corresponds to simultaneous spin and orbit excitation. The SQTM works well in the photoproduction $1 \mathrm{tm}$ it where the parameters are necessary and sufficlent to describe all the exc1tacton ${ }^{16}$ and $A, B$, and $C$ are roughly $I n$ the ratio 9:4:4. The relations between $A, B$, and $C$ and the dom Inant multipole amplitude are given in Ref. 17, and it is straightforward to determine $A, B$, and $C$ as functions of $Q^{2}$ using the results outlined in Table V.l. Figure V.l shows these results, and it is certain that at $h i g h Q^{2}$ the spin-flip amplitude $B$ dotolrates. This behavior is expected from most expliclt quark models, 15,17 and FIg. V. 1 also shows the results of a recent quark model calculation 18 based on the successful photoproduction model of Kubota and Ohta. ${ }^{19}$ Two polnts may be mentioned: (1) agreement between the explicit model and the data could only be achieved by including an effective quark "form factor;" and (2) at high $Q^{2}$ the spln-orblt term $C$ seems to go significanty negative in contradiction to quark model expectatlons. 15,20

The SQTM may be further tested by comparing the predictions fir the neutron-target multipoles of $I=1 / 2$ resonances calculated from the observed values of $A, B$, and $C$ with the results of the Daresbury deuterium-target experiments. This has been done with some degree of success by the Daresbury group, $10-12,17$ and their results are given $1 \pi$ Table V.2.

The P11 N(1440): Interest in this enigmatic resonance persiats because two rather different theoretical approaches both predict that the $M_{1}$ mulcipole sign will change as $Q^{2}$ increases from zero. In photoproduction, $M_{1-}$ has the wzong sign according to the simplest quark models, but consideration of the large spin-orbit contribution to the excitation in an explicic model ${ }^{19}$ gives rough agreement with experiment. As in the $[70,1$ ] $]$ multiplet, spin-orbit excitations become less important as $\mathrm{Q}^{2}$ Increases, so the multipole should revert quickly to its norinal $81 \mathrm{gn} .{ }^{18}$ The same effect is predicted by Gavela ${ }^{21}$ using a "bag" model for electroproduction. Experimentally the situation is still far from clear, but such sign changes 


\section{Data Card Listings}

For notation, see key at front of Listings.
Baryons

$N$ 's and $\Delta$ 's are certainly not ruled out. 17

The F15 N(1680): All analyses of the data near the third resonance give congistent values for
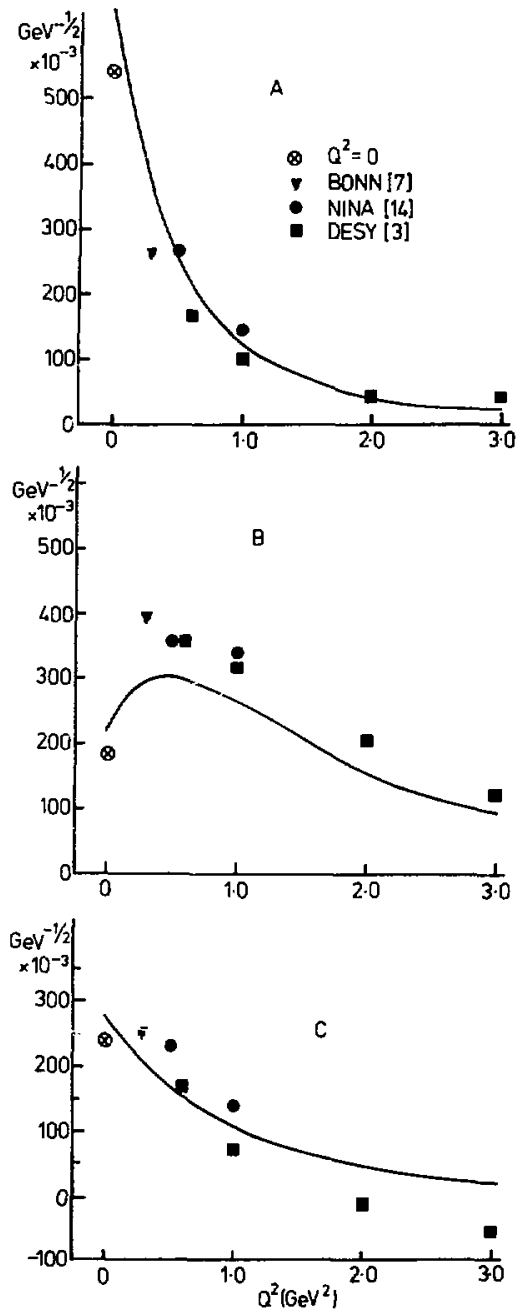

F18. V.l. The $Q^{2}$ variation of the orblt-filp, apIn-flip, and spin-orbit-flip parameters $A, B$, and $c$ for the $[70,1]$ multiplet. The curves are fron Ref. 18 . the $E_{3-}$ and $M_{3-}$ multipoles for this $156,2^{+}$w wultplet nember. Table V.3 shows the results, and again the electric multipole falls much wore rapidly than the magnetic multipole. This clearly agrees with the quark model expectation that spinflip amplitudes must dominate at h1gh $Q^{2}$.

\section{References for Section $v$}

1. R.C.E. Deven1s: and D.H. Lyth, Nucl. Phys. B93, 109 (19:5).

2. R. Haldan, DESY F21-79/03.

3. V. Gerhardt, DESY F21-79/02.

4. H. Wriedt, DESY F21-78/01.

5. F.W. Brasse et al., Nucl. Phys. B139, 37 (1978).

6. Ch. Nietzel, Bonn IR-78-15; R. Sauerve1n, Boan Ik-78-13; H. Breuke, et a1., Phys. Lett. 74B, 409 (1978).

Table V.2. A comparison of neutron-target results at $Q^{2}=0.5 \mathrm{GeV}^{2}$ (Ref.12) with $S Q T M$ predictions.

\begin{tabular}{lccc} 
Resonance & $\begin{array}{c}\text { Multipole } \\
\left(\pi^{-}\right)\end{array}$ & \multicolumn{2}{c}{$\mathrm{b}^{1 / 2}$} \\
\hline S11 $\mathrm{N}^{0}(1535)$ & $\mathrm{E}_{0-}$ & -0.11 & -0.46 \\
D13 $\mathrm{N}^{0}(1520)$ & $\mathrm{E}_{2-}$ & -0.55 & -0.43 \\
& $\mathrm{M}_{2-}$ & -0.16 & -0.07 \\
D33 $\Delta(1700)$ & $\mathrm{E}_{2-}$ & -0.13 & -0.20 \\
& $\mathrm{M}_{2-}$ & +0.029 & +0.036 \\
D13 N(1700) & $E_{2-}$ & -0.034 & -0.093 \\
& $\mathrm{M}_{2-}$ & +0.016 & -0.037 \\
S11 N(1650) & $\mathrm{E}_{0+}$ & -0.09 & -0.34
\end{tabular}

Table V.3. The FIS N(1680) resonance amplitudes.

\begin{tabular}{ccccr}
$\mathrm{Q}^{2}$ & \multicolumn{4}{c}{ Amplitudeg $(\mu \mathrm{b})^{1 / 2}$} \\
\cline { 2 - 4 }$\left(\mathrm{GeV}^{2}\right)$ & $\mathrm{E}_{3-}$ & $\mathrm{M}_{3-}$ & $S_{3-}$ & Ref. \\
\hline 0.0 & 0.47 & 0.16 & -- & \\
0.3 & $0.16 \pm 0.02$ & $0.27 \pm 0.03$ & -- & 7 \\
0.5 & $0.12 \pm 0.10$ & $0.27 \pm 0.01$ & $-0.02 \pm 0.02$ & 14 \\
0.6 & 0.13 & 0.17 & 0.01 & 3 \\
1.0 & 0.01 & $0.23 \pm 0.02$ & $0.01 \pm 0.01$ & 14 \\
1.0 & 0.07 & 0.15 & 0.00 & 3 \\
2.0 & 0.013 & 0.083 & 0.00 & 3 \\
3.0 & -0.084 & 0.038 & 0.00 & 3
\end{tabular}


Baryons

$N^{\prime} s$ and $\Delta^{\prime} s, p, n, N(1440)$

7. A. Samel, Bonn IR-79-50; H. Breuker et al., contribution to Baryon' 80 Conference (Toronto, 1980), B80-23.

8. A. Latham et al., Nucl. Phys. B156, 58 (1979).

9. A. Lathan et a1., Nucl. Phys. B189, 1 (1981).

10. J.v. Morris et al., Phys. Lett. 73B, 495 (1978).

11. J.v. Morris et al., Phys, Lett. 86B, 211 (1979).

12. J. Wright et al., Nucl. Phys. B181, 403 (1981).

13. Ch. Gerhardt, Ze1t. Eur Physik ⒋ 311 (1980).

14. M. Davenport, Thesis, University of Lancaster (1980); J.v. Morr ls, private communication.

15. J.W. Alcock et al., Annals of Physics 130, 164 (1980).

16. P.J. Litchfield et al., In Proceedings of the Topical Conference on Baryon Resonances (Oxford, 1976), ed. R.T. Ross and D.H. Saxon, p. 463 .

17. F. Foster, in Proceedlags of the IV ${ }^{\text {th }}$ International Conference on Baryon Resonances (Toronto, 1980), ed. N. Isgur, p. 79.

18. F. Foster and G. Hughes, submitted to Zelt. fur Phys $1 \mathrm{k} \underline{\mathrm{c}}$.

19. T. Kubota and K. Ohta, Phys. Lett. 65B, 374 (1976).

20. N. Cottingham, private communication.

21. M. Gavela, Phys. Rev. D21, 182 (1980).

\section{Production Experiments}

Partlal-wave analyses of cuurse separate partial waves, whereas a peak in a cross seccion or an Invariant mass distribution usually cannot be disentangled from background and analyzed for its quantum numbers; and more than one resonance may be contributing to the peak. Thus results from production experiments are kept under separate headIngs in the Listings (e.g., 1520 MEV REGION -- PRODUCTION EXPERIMENTS), and they are not used for getting numbers in the Baryon Table.

There is not much new on $N$ and $\Delta$ resonances from production experimenta in this edition, so we refer to the previous edition for a lengthy review of the diffractive production of $N \pi$ and Nmin sys$\operatorname{tens.1}$

\section{Reference for Section VI}

1. Particle Data Group, Rev. Mod. Phys. 52, S188 (1980).
Data Card Listings

For notation, see key at front of Listings.

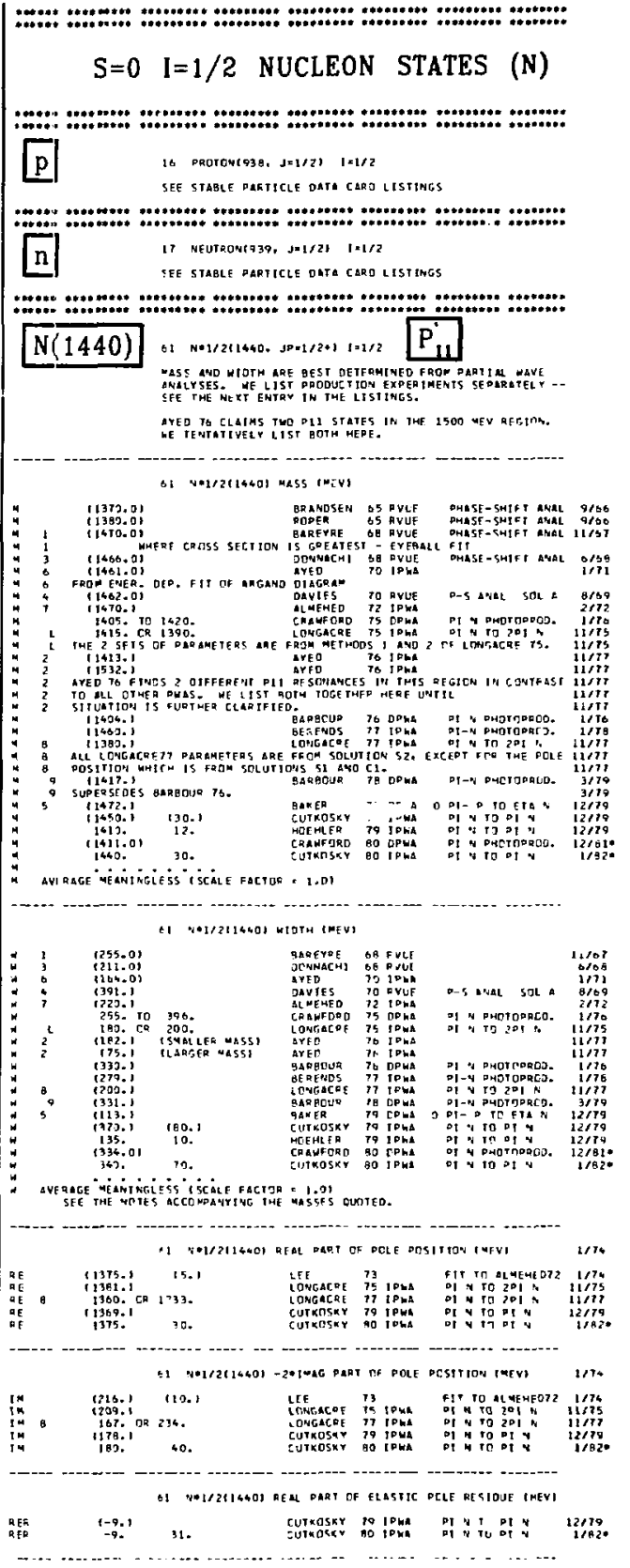


Data Card Listings

Baryons

For notation, see key at front of Listings.

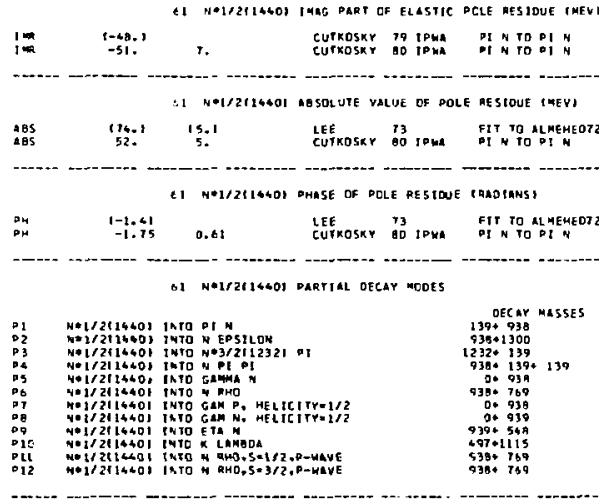

G1 NHI/2016401 GRANCHJMG DAT TOS

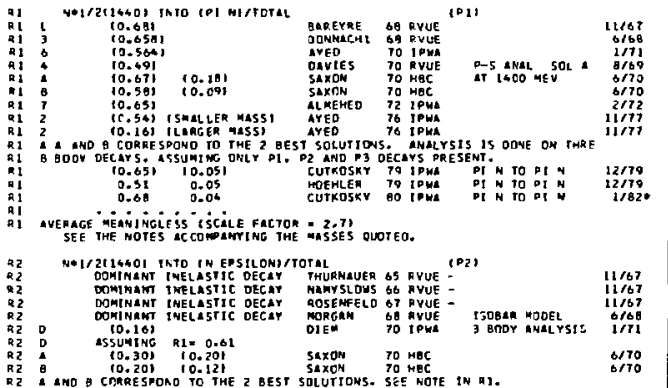

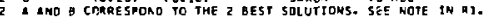

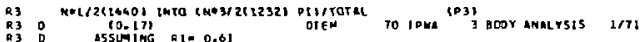

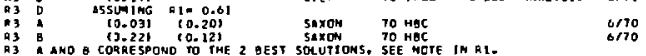

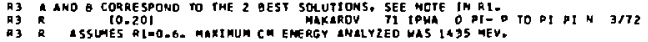

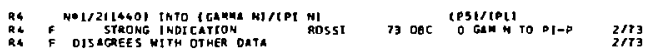

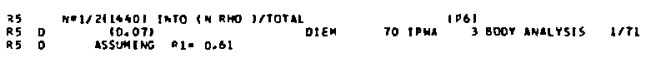

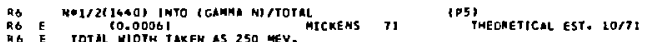

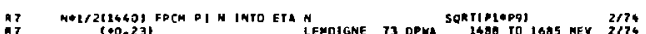

100.231
100.3201

R7: SUP ER SEDES LENOTGNE T3, USES M ARD W OF AYED TO ILARGER MASSI. AE II/73

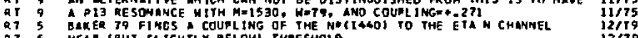

Q7 S MEACI IBUT SU SGMTLY BELOHI TKRESHOLD. 12779

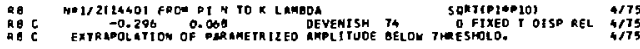

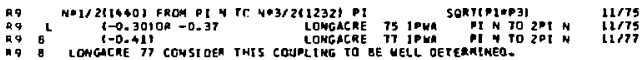

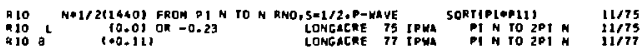

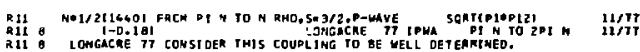

Q12

$R 12$
$R 12$
$R 12$

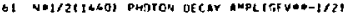

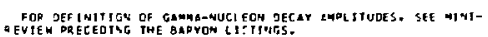

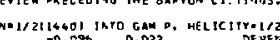

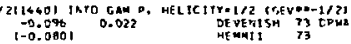

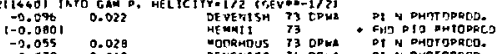

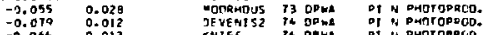

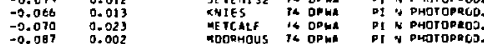

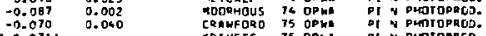

CRIVETS is DPQW OI-4 PHOTOMRCD.

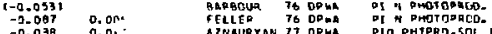

$\begin{array}{ll}-0.038 & 0.02 \\ 1-0.019 & 0.011\end{array}$

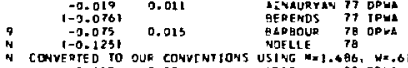

PIO OMTPRO.5C2

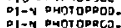

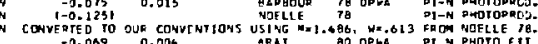

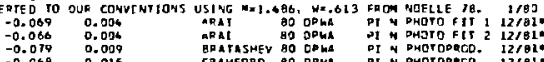

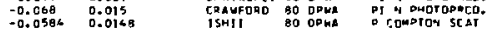

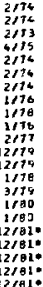

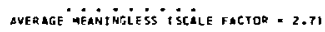

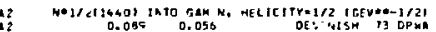

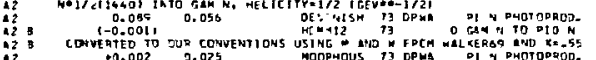

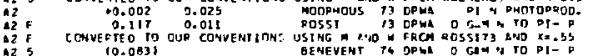

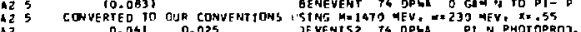

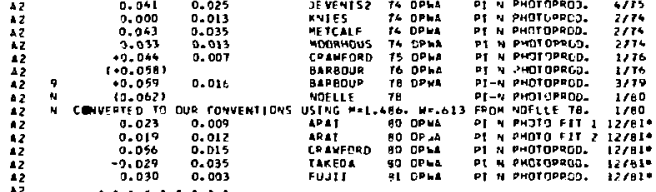

Az AVERLGE MEAMI HGLESS I SGALE FACTOR = 2.A1

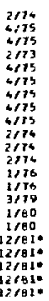

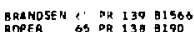

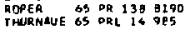
NAMYSLOK OS DR 1571329 ROSENFEL GT IRYINE SENA BAREYRE 60 OR $165 \quad 1731$

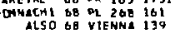
ALS 6 G YHESTS MORGEN OA DF 1662731

AREO 70 KIEV CONF

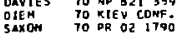

HAKKROY 71 SJNP 13 :10

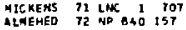
OEVENISH 79 DL 4753

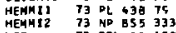
CEE

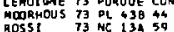
AI SO 71 L 140231163 AEMEVEAT 74 HC 192529 OEVENISH T4 NOP 891330

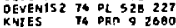

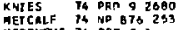

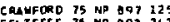
FELTESSE 75 NP 993 242 KRIVEYS 75 SSNP 20433
MLSO

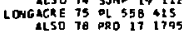

AYEP 76 CEA-N-1921

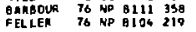
A2Manira 77 EFt-2061571-77

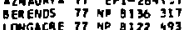

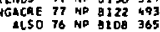

OARBOUT 70 4P B14L 253 MOELLE 70 PTP OC 78

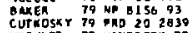
OENLER 79 MANDBOOK OF PI-
REFEPENCES FOP $4 * 1 / 2114401$

DODONNELL. MOORHOUSE

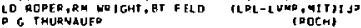

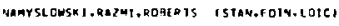
a h posenfelo, p soding (LO)!

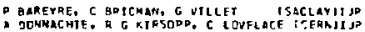

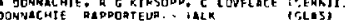

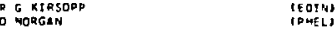

a Ared, gaAevae, o vilaet

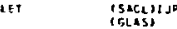

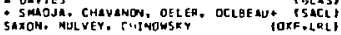

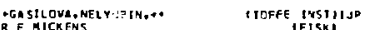

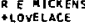

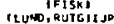

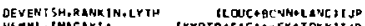

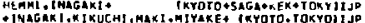

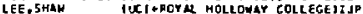

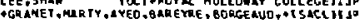
MOOAHOUSE, OAEREC ACK

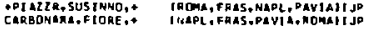
GEMEYENTAND, OAMGELO, NOT SAYS S EFANI,* IRDMAIIJP DEVENISH, FROGGATT, MAGT INTOESY, NOAD TTA, LDUCI

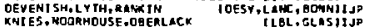

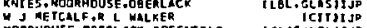
MOQRHOLSE, DBERL ACK, ROSENFELO IGLAS OLBLIJP

PL CRawsoko

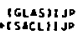

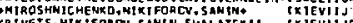

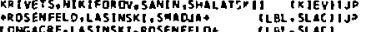
ATED ITHES ISY ISTCAY) JP

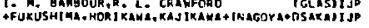

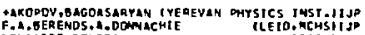

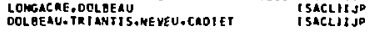

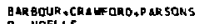
isaceitso

P. HOELLF laces

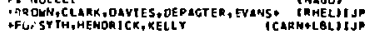

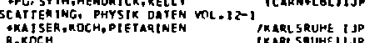


Baryons

$\mathrm{N}(1440)$

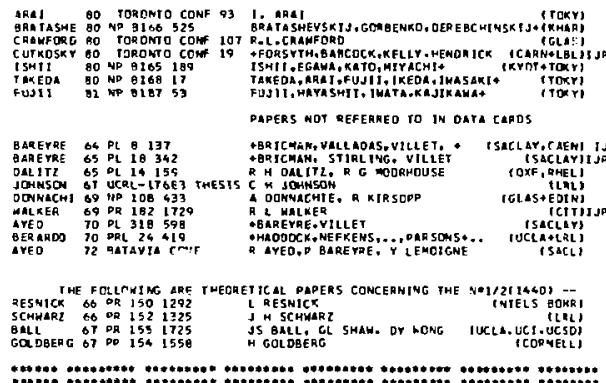

\section{MEV REGION - PRODUCTION EXPERIMENTS}

a) N*1/211440, JP. I $[-1 / 2$ DROOUCTION EXDER]MENTS

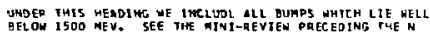
GHD OELTA LISTINGS FDR 2 OI SCUSSION OF PRODUCTION

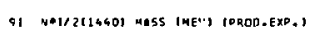

\begin{tabular}{|c|c|}
\hline 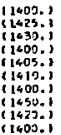 & 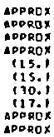 \\
\hline
\end{tabular}

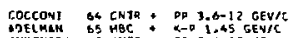

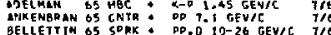

$\triangle H O L$ OSOH 66 SPRK : OD, 6-30 GEV/C TlOS

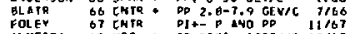

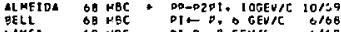

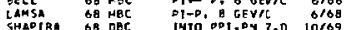

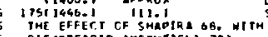

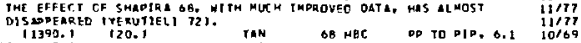

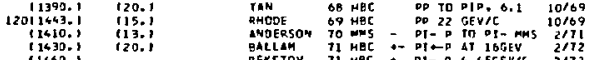

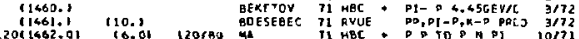

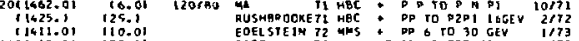

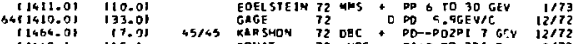

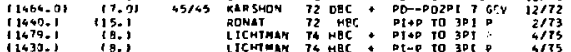

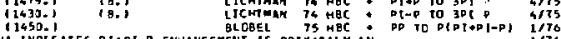

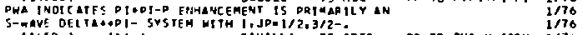

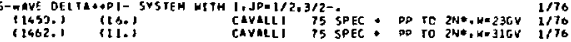

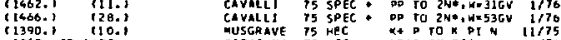

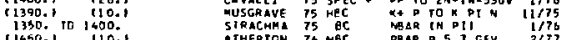

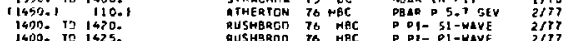

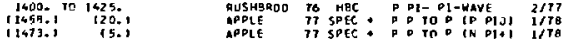

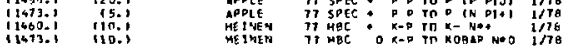

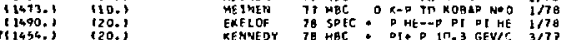

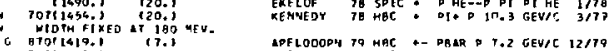

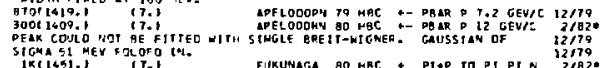

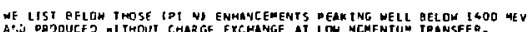

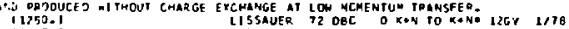

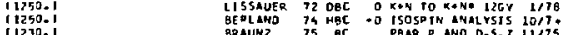

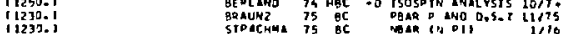

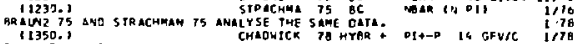

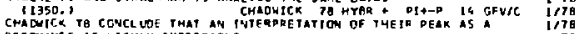

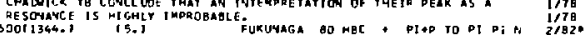

\section{CI $N+1 / 211440\}$ WIOTH INEVI TPRCO. EXP.)}

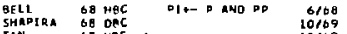

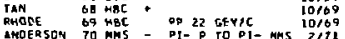

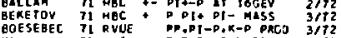

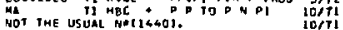
ROT THE USUAL NOLIS401. TO POP IOEEY IOTI

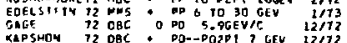

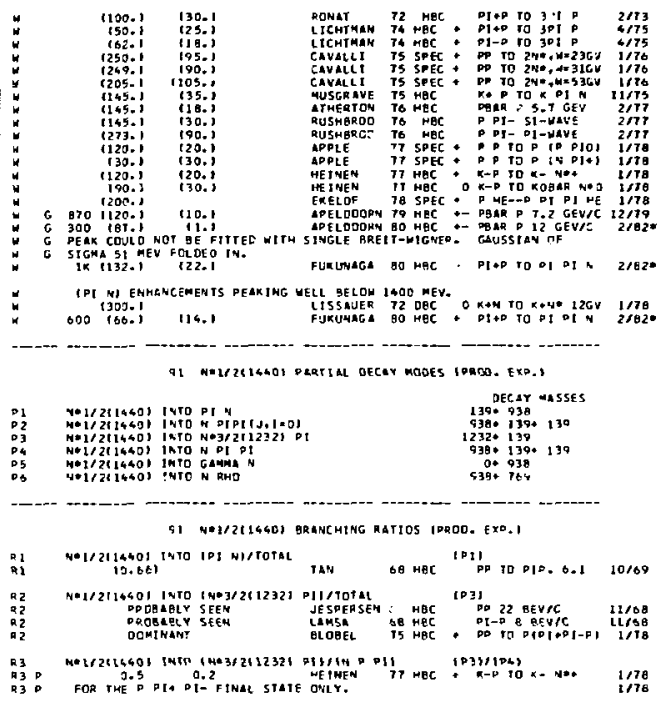

COCCONI OA OL O 13\%

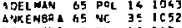
BELLETI O5 PL 18 167

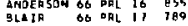

FCLEY 67 DRL $19 \quad 297$

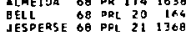

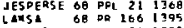

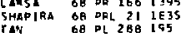
QHOOE OA DQ 187 194h
ANDERSON TO DQL 25 OQT SALLAM 71 PR O4 1046 GEKETOV 71 SJND 13 GOS

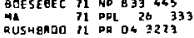
EOELSTEI 72 DR DS 1673

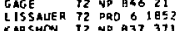

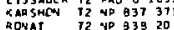

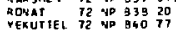
OERLAND T4 NP B75 9 "

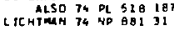
$\begin{array}{llll}\text { BLOREL TS ND } & 897 & 201 \\ \text { BRAUNE TS ND } & \text { TO5 } 503\end{array}$

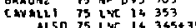

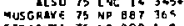

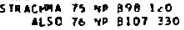

atherton 76 ND B103 301

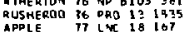

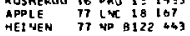
C HAOHIC 78 ODO 111713 KEELNE 78 NP 8132212

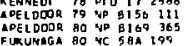
ALS 79 NC SOA 120
ALS BO

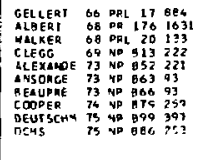

REFERENCES FOR NE1/2014SO, IDADO, ERD. IL ILLETHUY, SC AMLON. STAHLPQ ANOT, D ICERNH S L AOELMAN BFLLETIINI, COCRONI, OJOOENS + ICEPN)

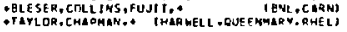

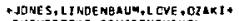
+RUSHAROOKE, SC HARENGUT YEL, ICAVE. DESYI

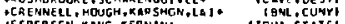

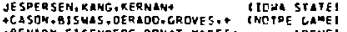

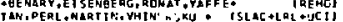

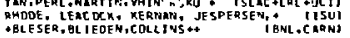

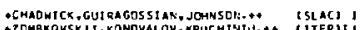
BDESEBECK, CRAESSLER+KOAUS,... [A BaCHLY]

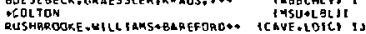

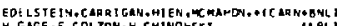

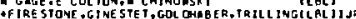
- YEKUTIELI. YAFFE. SMAPIRA,RONAT,* IREMOJ 1

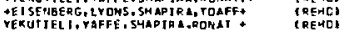
BERL ANO, HABER, HODOUS, HUL SIZER,4 (NITII

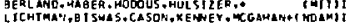

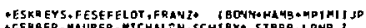

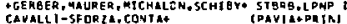

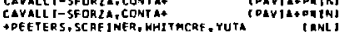

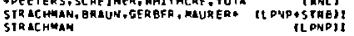
THER TON, FRENGM, SXURA, BCHE. ICE RNA PRAGF

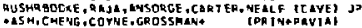

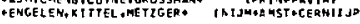

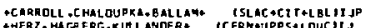

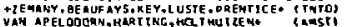

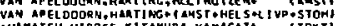

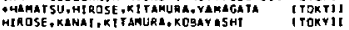

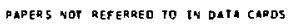
+SHITH. HOJC ICKI, CCOLION, SCHLEIN + [LRL, UELA)

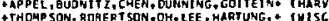
CLEGE

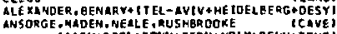

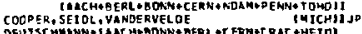

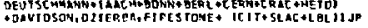




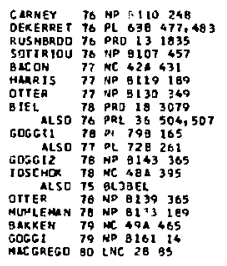

- COLCFY. JONES, KENYONe (BIRM+BRUX+C, ERNOMONS II JF RUSHBROORE, RAJR, ANSDAGE, OCARTER, NFALE (CAYE) I JP D. SOTIRTOU

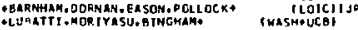

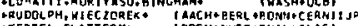

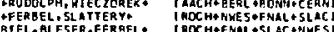
BIEL, GLESEP,FERGEL, ITOCH+FNAT, OSLAC+AMES GOGGI, MANTOVANI, CAVALLI-SFDRZA+ ICERN+PAVI

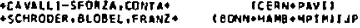

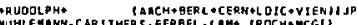

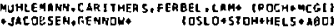

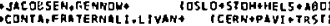
M. H, MACGHEGRP TLLLI

$\mathrm{N}(1520)$ S2 N०1/211520. JP.3/2-1 1-1/2 $\mathrm{D}_{13}^{\prime}$ THF SESTHANCE IS HELL ESTAGLISMEO.

62 Y $+1 / 2115201$ MASS INEVI

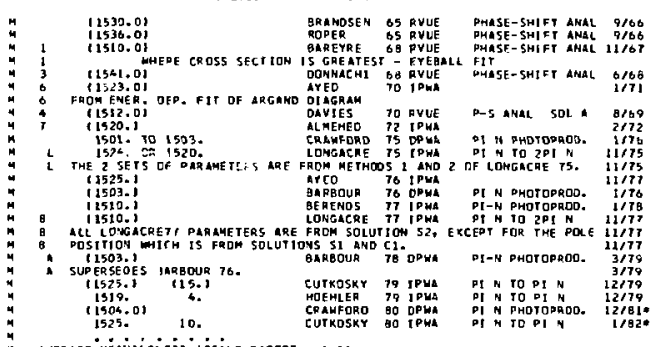
average méníngiess iscile fagtor - 1.01

G2 NO1/Z115201 WTOTH \{HEVI

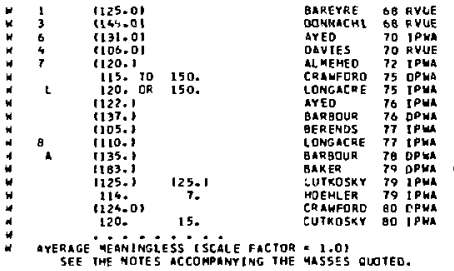

D-5 anal SOL A P1 N DHOTOPROO.

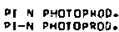

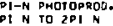

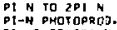

Di ${ }^{\circ}$ TO TO ETA

Di $N$ To PI Of N PHOTOPROD. $12178 \mathrm{I}$

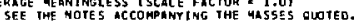

t2 N*1/2U15201 REAL PART OF POLE DOSIITON IMEVI

\begin{tabular}{|c|c|c|c|}
\hline $\begin{array}{l}R E \\
R E \\
R E \\
R E\end{array}$ & $\mathbf{a}$ & $\begin{array}{l}11514,1 \\
1908.10 R \\
1510.1 \\
1510 .\end{array}$ & 1505. \\
\hline
\end{tabular}
LONGACKE 75 IPUA LONGACRE 77 IPHA $\begin{array}{ll}\text { COTKO SKY } 79 & 7 \text { TPYA } \\ \text { CUTKOSKY } & \text { BO } 1 \text { PYA }\end{array}$

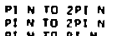
CUTKOSKY BO IPHA PI TIO PI N

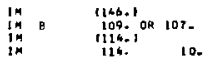
LONGACRE IS IPMA
LONGACRE TI TOMA CNIKOSKY 79 TPWA
CUTKOSKY

pI 4 io zei

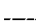

t2 N+1/211520) REAL PART OF ELASTIC PCLE RESTOUE (MEV)

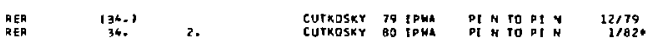

Ime $\$ 168$ 177
2772
176

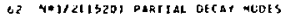

Ne1/2t15201 2570 म

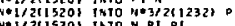

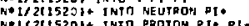

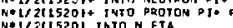

Nolizis201 thTO N ETA

No1/21 15201 INTO N RMD

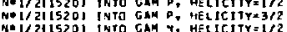

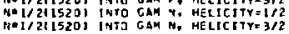

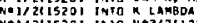

No1/2115201 iN1O N+3/2t12321 Dt.5-HAVF

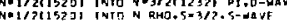

Decar assts

$\begin{array}{rl}139 \% & 938 \\ 1232 * 130 & 130\end{array}$

1232* 130.139

$439+1390.139$

$539 *+1390$
$530 *$
546

$538+1330$
5390

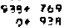

3. 939

$0+939$

$1<320139$

$232 \div 739$

\section{Q2 Nण1/21 15201 BRaNCHATG RAIIOS}

NEL 211520) INTO (DI N)/TOTAL

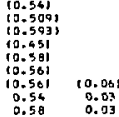

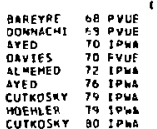

(PI)

(1)

SEE PHE NOTES ACCOMPAHYING THE MLSES OMTIED.

T-5 ANAL SOL A

liver

P 4 is

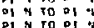

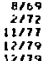

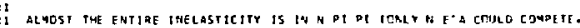

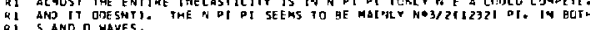

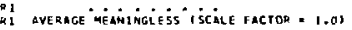

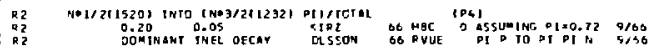

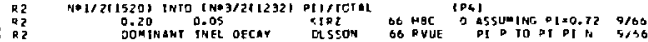

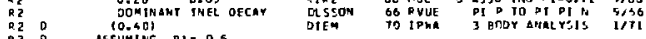

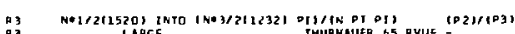

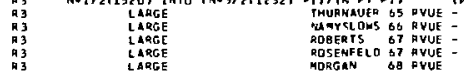
CARGE
LARGE

$11 / 6 T$
$11 / 57$ $11 / 57$
$11 / 35$
$11 / 67$
$0 / 58$

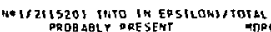
PQOB AQLT QRESEMT
10.023 ASSUजING RI= 0.5

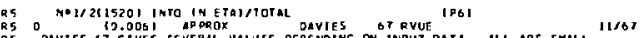

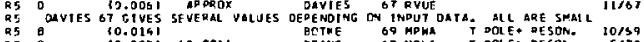

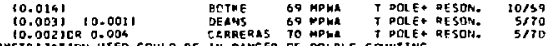

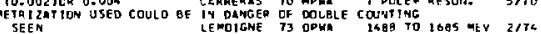
As

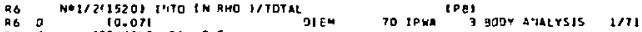
ASSUA ING $81=0.5$

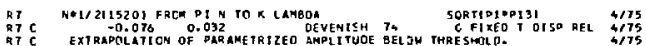

RA NEI/ZIIS2OI FRCM PI N THTO N EPSILON SORTIDINPT)

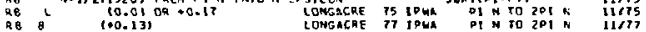

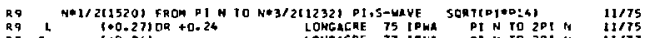

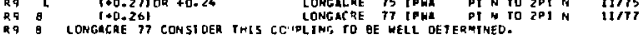

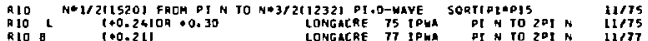

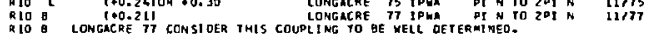

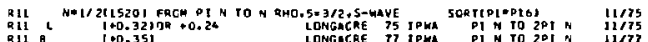

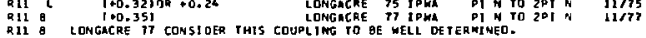

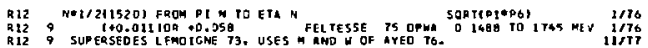

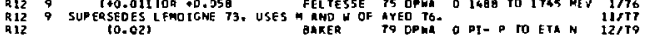
E2 N*1/2(1520) PHOTON OECAY AMPLIGEV $* *-1 / 2)$ FOR OEF IHITICN OF GAMMA- WUELEOH DECAY AMPL TtUCES, SEE MIMI
REVIEN PRECEDING THE BARYOH LISTINGS.
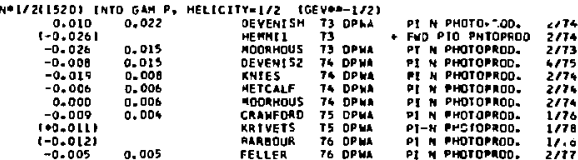


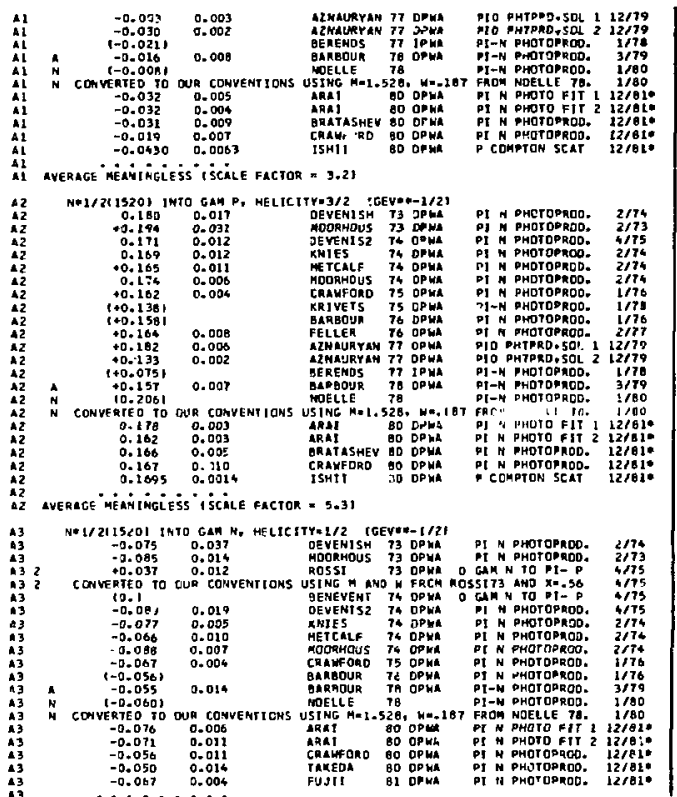

a average kejanisigiess iscile factor - 2.0

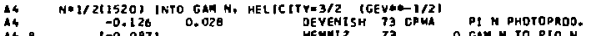

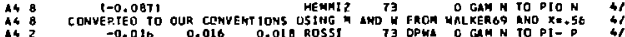

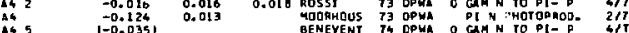

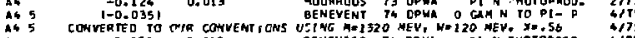

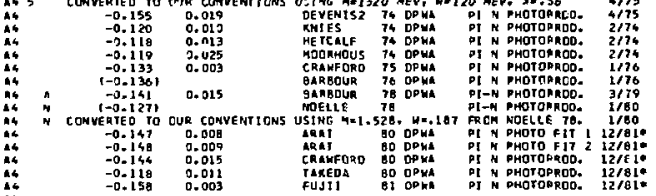

at average méntigie is i scile fagtor - 3.11

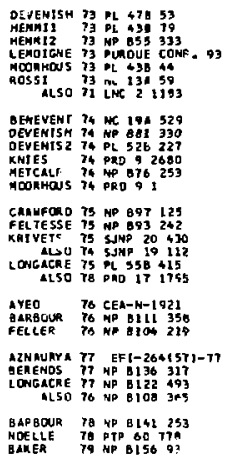

NOELLE TO PTP $8079 \%$

CUTKOSKY TO PRD 20203 ,
MOE HLER TQ MANDBCOK TF PI-N

also so TOMnTrO COHF

RRA 2 HO TSOMHO CONF 93

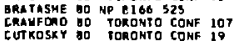

TAKEOA DO NP BLE if
FUJII OI NP BLET 53

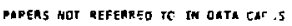

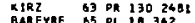

J KIRT, J SCHMART, TO OTIDP ILALI

CROJCH G5 OESY COMF II 21 : BRICMAN. STIVLLING. VILLET ISACLAYTRJP

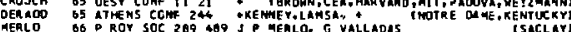

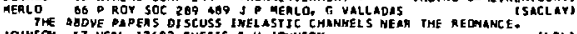

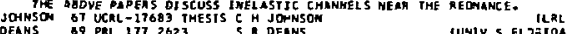

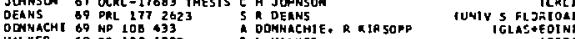

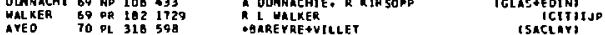

Benton

1520 MEV REGION - PRODUCTION EXPERIMENTS

- H*1/21L520, JPE 1 I*1/2 PRDOUCTION EXPERIMENTS

SEE THE MINT-REVTEN ORECEOING THE N ANO DELTM 2 SSTINGS

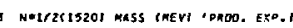

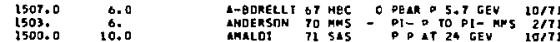

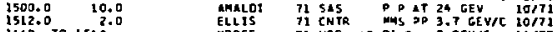

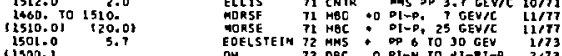

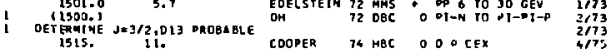

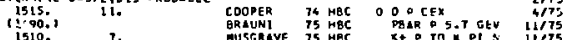

1510., 1500.1

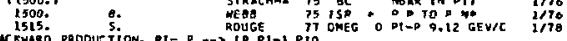

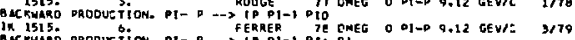

1M 1515.

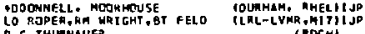

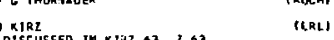

ROPER OS OR I3B 9190

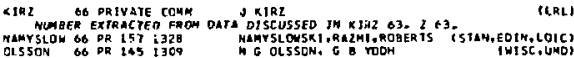

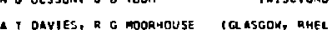

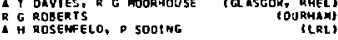

OAVIES OT NE $52 A 1112$
MOBEETS OT PREDRINF

GANENRE OB PQ 165 LT

AAEYRE OB PR 165173

aLSO BS VIENNA 130

ALSO OB THES IS
MTAGAN OQ PR 1661731

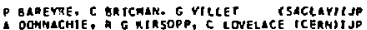

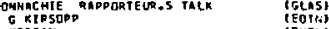

lucse:
(UNII)

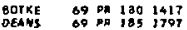

5 C BUTKE
5 DEAHS, J NOOR:

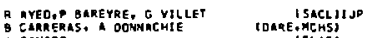

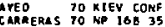

DAVIES TO NO B2I 350

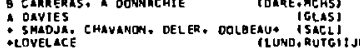

BACKMLRO PROEUETION. PI D FFEMER PR OMEE

A H=1/2U15201 HIOTH IAEV, IOROD. ERP.I

\begin{tabular}{|c|c|c|c|c|c|c|c|c|}
\hline $\begin{array}{l}55.0 \\
120.0 \\
110.0 \\
78.0 \\
100.010 \\
1100.01 \\
160.0 \\
100.0 \\
1200.1 \\
104.1 \\
150 . \\
105 . \\
105 . \\
112 .\end{array}$ & $\begin{array}{l}15.0 \\
10.0 \\
20.0 \\
2.0 \\
120.0 \\
130.01 \\
43.0 \\
39 . \\
26 . \\
30 . \\
17 . \\
10 . \\
15 .\end{array}$ & 53. & 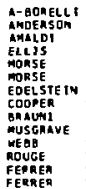 & 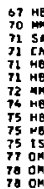 & 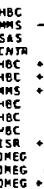 & • & 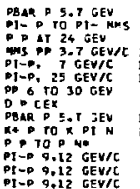 & $\begin{array}{r}10 / 71 \\
2171 \\
20 / 71 \\
10 / 717 \\
11 / 77 \\
11 / 77 \\
1 / 73 \\
4 / 75 \\
11 / 75 \\
11 / 75 \\
1176 \\
1 / 78 \\
3179 \\
3 / 70\end{array}$ \\
\hline
\end{tabular}

SEE THE NOTES AC COMANYING THE MASSE OLOTED ABOYE. 
Data Card Listings

Baryons

For notation, see key at front of Listings.

$N(1520), N(1535)$

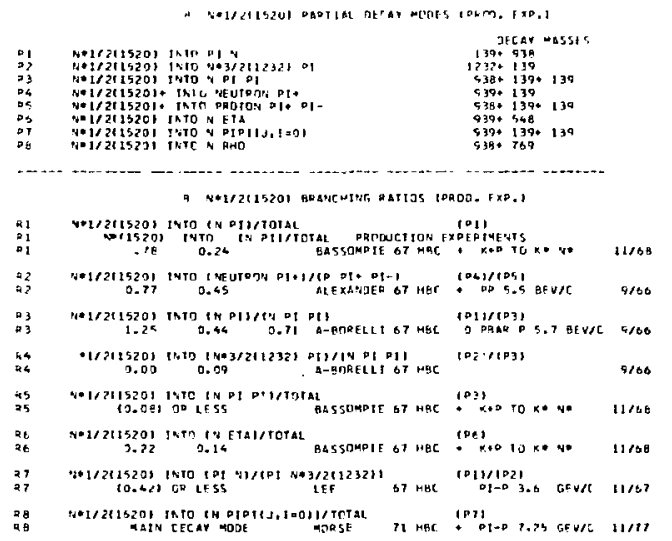

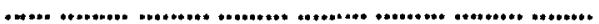

REFERENCES FOR NEL1/2015201 IPROD. EKD. I

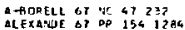

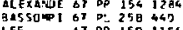

ANUERSOR TO PRL 75 G.98

AmaLOT TI PL 346435

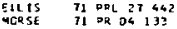

EOE:SIE: 2 PR OS 1673

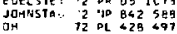

COOPER 74 TD 875290

gRAUHL 75 Y0 895 SE1

MUSGRSUE 75 TD EA7 365
STRACHE T5

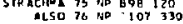

DEBA $\begin{gathered}75 \\ \text { DL }\end{gathered}$

POUGE 77 PL 699211 ;

ALLES-BORELL T - FRENCH.FPISK, MLCHE JO TCER

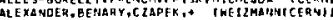

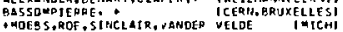

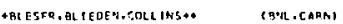

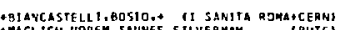

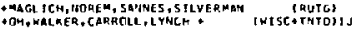

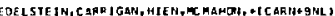
4MOLLERUD+.... JACOGSEN IBDHR, HEL S, CSLO. STOHII JP
TUCRIT LOOPEA, SEIOL. VANGERVELOE

(MICH)

4GERER, maUAER, YICHALUN, SCHIEY, ISTRE. IPNPII -PE ETEPS, SCDE INER. WHITMDRE, YUTE TS IANLS

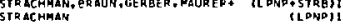

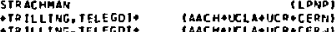

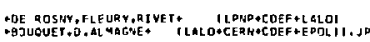

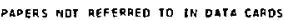

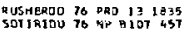

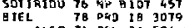
ALSO to PFL 36504,507

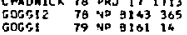

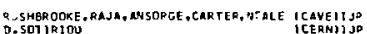

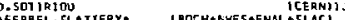
OEERBEL, SLATIERY. (MOCH+AMESAFNAL + SL AC) B IEL-BLESEA,FEREEL+ (FOCH+FMAL\& SL AC + HWESS)

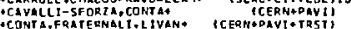

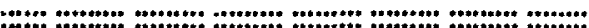

$N(1535)$ os Na1/2(1535, jes/2-) $\left[=1 / 2 \quad S_{11}\right.$

THIS RESONATLE IS WELL ESTABLISHEO

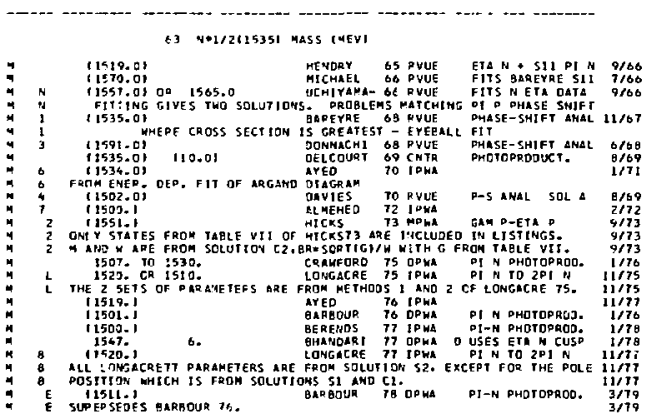

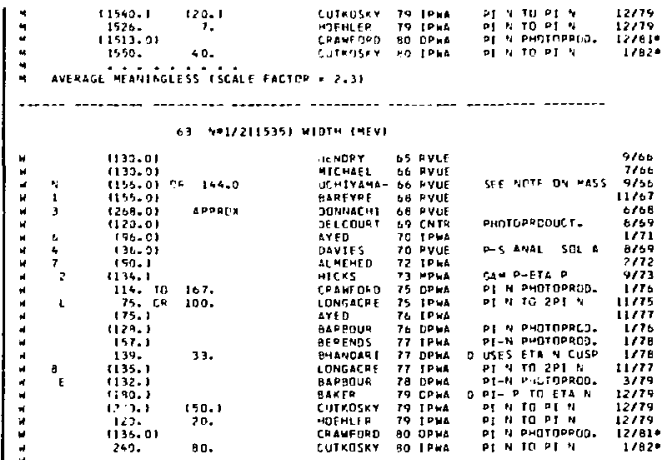

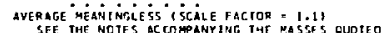

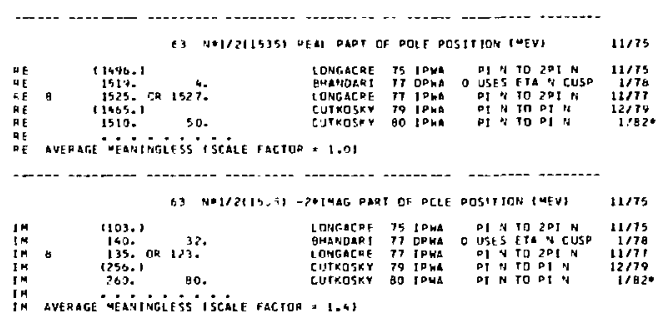

63 N.1.?IIS3SI REAL DART OF FLASIIC PCLE PESTOUE IMEVI

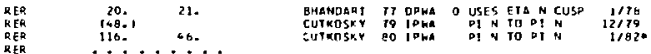

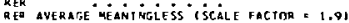

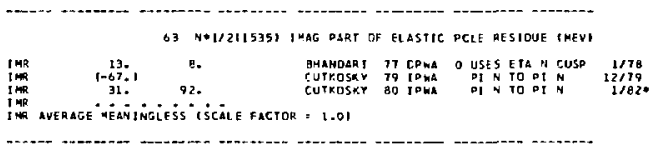

6? No1/2\{1535! PARTIAL DECAY MODES

N*1/2215351 T2170 PR

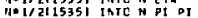

No1/211535I INTO N EPSILON

N*1/2115351 INTO N RHO

NA1/21535, INTO GAM D. HELICITYE1:

Na1)

No1/2II535) INTO $N$ RHO,S=1/2,S-WAVE

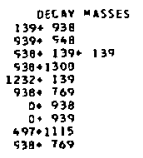

$63 \mathrm{~N}$ *L/211535I BRANCHING FATIOS

NF1/2(1535) INTS (P) N3/TOTAL

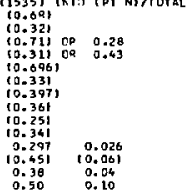

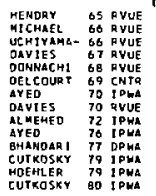

HOEHLER 79 PPWA

R1 average méningiess iscale factor = 1.7)
(PI)

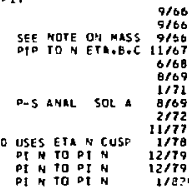


Baryons

N(1535), N(1540)
Data Card Listings

For notation, see key at front of Listings.

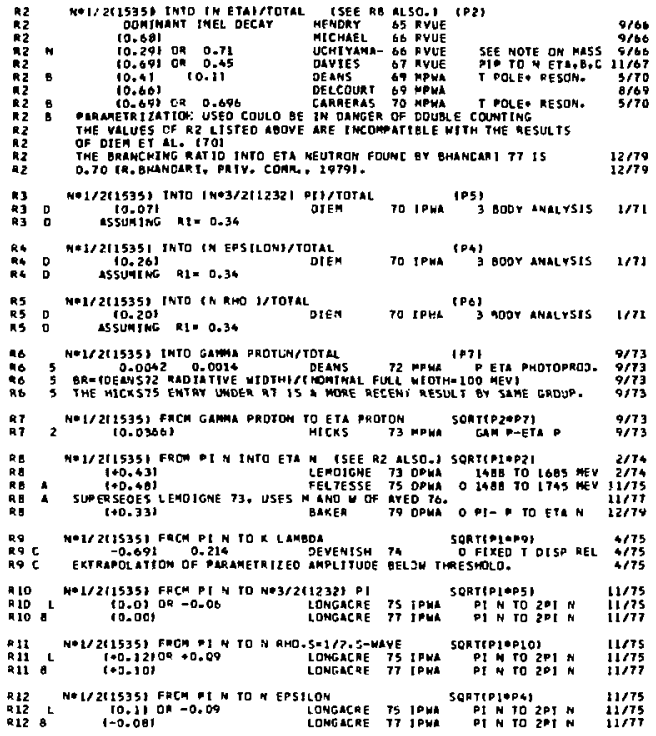

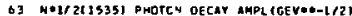

FOA OEFINITICY OF GAMMA-VUCLEON OECAY RMPLITUDES, SEE AINI-
CEVIT DRECEDING THE BAAYON LISTINGS.

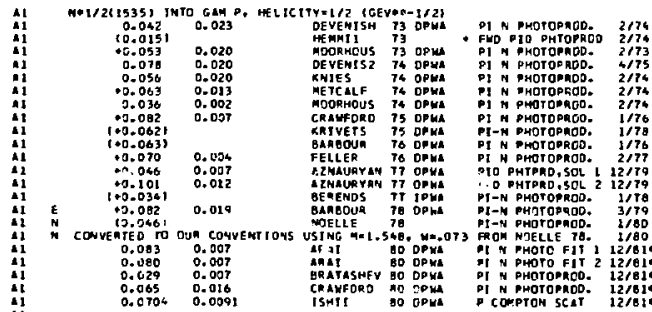

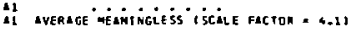

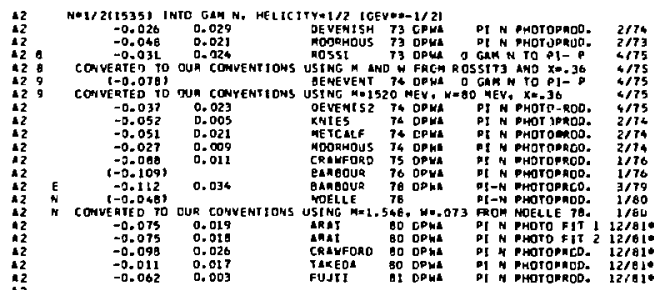

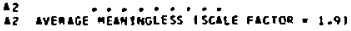

GAREYRE
ODAKACHI OA ALSO OE YIE HHA I3\%

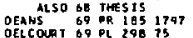

CYEE TO NLE COMF

CARMERAS TO KO LGE 35
OAVIES TO NO B21 358

DLEM
ALKEHEO 72 KP BSE 157

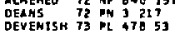

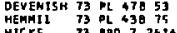

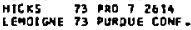

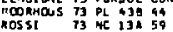

ALSO 71 IME 2 IIB3

DENEVEYT T6 NC TQA 529

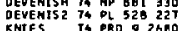

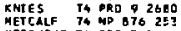

CRAYFOMO 75 HO $897 \quad 125$

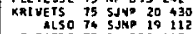

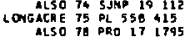

AYED 76 CEA-N-1921

FEL LEN T6 HP 8104219

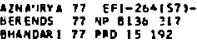

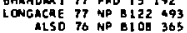

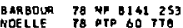

GAKER 79 NP OJSO 53

CUTKOSAY 79 MRD 202039
HOE HLEA 19 WAHDBCOK OF

also go tOMONTO CONF 3

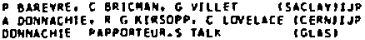

DDHAACHIE PAPPOATEUA-S TALR TOR TELS

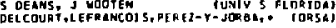

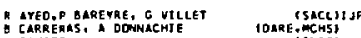

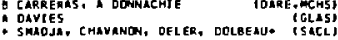

+LDVELACE

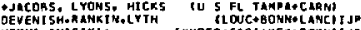

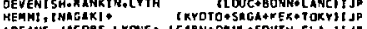

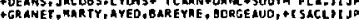

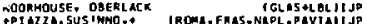

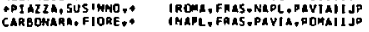

DEMEVERTANO, DANGEL d, MOTAKISTEFART . (NOMA) JP

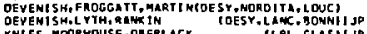
KNIES, MOCRHAUSE, ODERLACK TLOL. GLASITJP

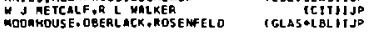

Q. LALYFGRO

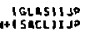

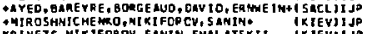

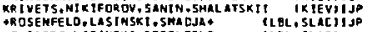

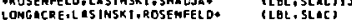

AYEO (THESIS)
T. M. BAKBOUR,R, L, CRAMFORD TSACLIIJP

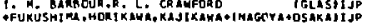

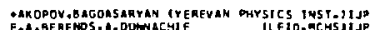

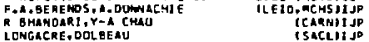

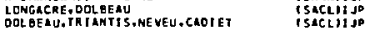

gAREOUN, GNEMFOAD, RARSONS IGLST)

P. MOELLE

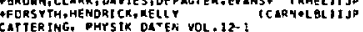

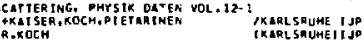

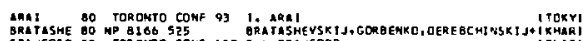

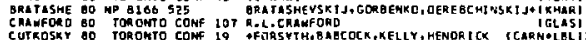

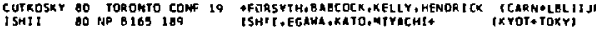

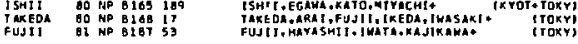

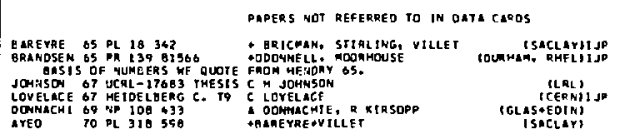

TIE FCLLONTEG ARTICLES DEAL WITH THE REACYTOHS PT- D TO ETAN

ANO GAMMA. TO ETA P NEAG THRES SWOLO. THE DATA ANO TME THEORE ICA

TUDE AS DETERMINEO IN PI

MLINLY EXOERTMENTAL -

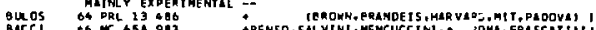

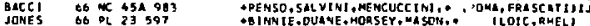

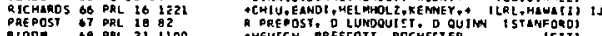

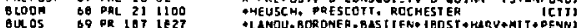

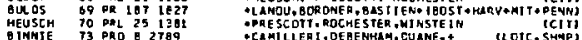

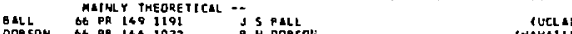

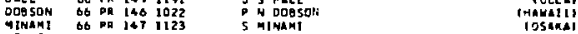

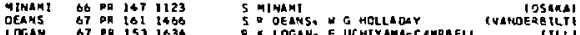

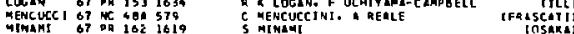

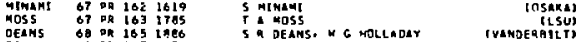

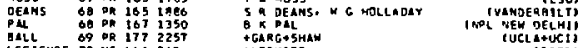

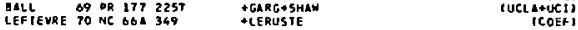

"

$N(1540)$ (ad N-1/2(1540, JP-3/20) I-1/2 $P_{13}^{\prime}$

$11 / 7$

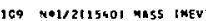
$11 / 17$

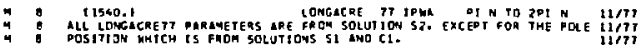

OS PL IA 17I EXPER IMEMTS. WE TAKE MUMBERS FROF ITHE SOLUTION USING IMRMOSEN OS.

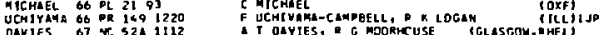

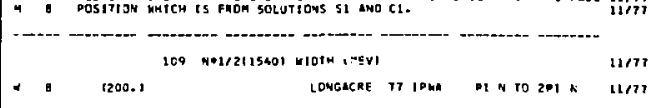




\section{Data Card Listings}

For notation, see key at front of Listings.
Baryons

$N(1540), N(1650)$

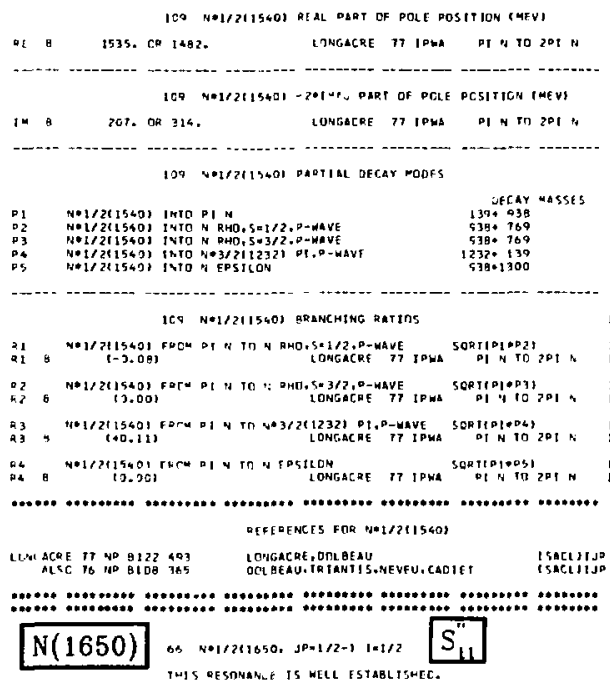

\section{T.6 N*12211650) MASS (MEV)}

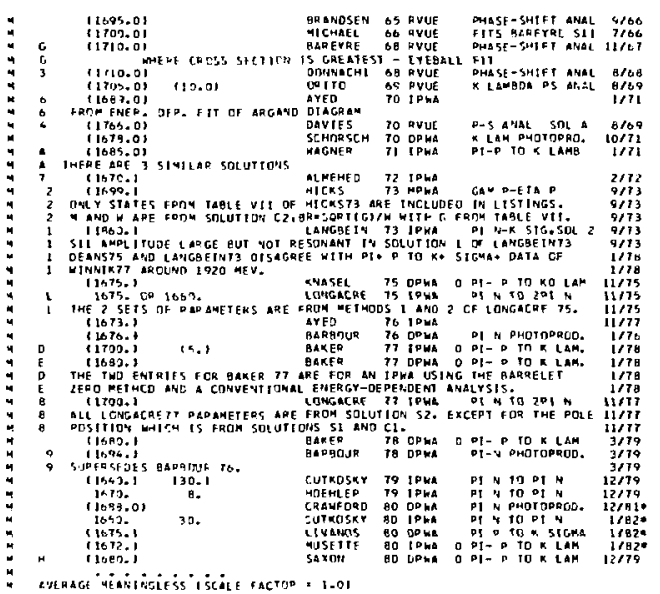

\begin{tabular}{|c|c|c|}
\hline$\xi$ & $\begin{array}{l}1543.01 \\
363.01\end{array}$ & \\
\hline & $\begin{array}{l}1300.51 \\
1106,01\end{array}$ & (15.0) \\
\hline 4 & 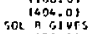 & \\
\hline${ }^{4}$, & $\begin{array}{l}179.01 \\
1120.010 \\
11200.1 \\
1195.1\end{array}$ & 21140.01 \\
\hline 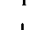 & $\begin{array}{l}1200.1 \\
1170.1 \\
150.198\end{array}$ & 31 \\
\hline & $\begin{array}{l}\text { T150.: } \\
1194.1\end{array}$ & \\
\hline E & $\begin{array}{l}1130.1 \\
199.1 \\
11701\end{array}$ & \\
\hline
\end{tabular}

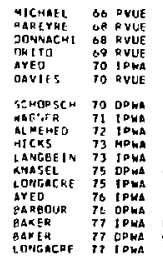

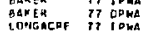

p-s afial 5042 5. LAN PHOTJPRDBI $P$ TD $R$ L LAMB

SAM P-CTA 0 PI $\mathrm{Ni-k} 5 \mathrm{Stg}, 5 \mathrm{SOL}, 9 / 77$ 0
DI DI it PMCTOPROJ.

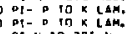

$11 / 7$
1177
$11 / 77$
$11 / 71$

$n \pi m$ iin? $11 / 377$ li1m? list

(n)

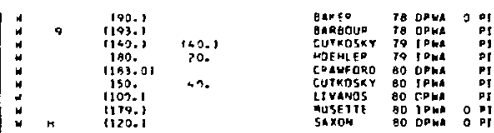

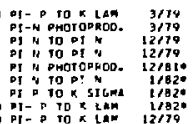

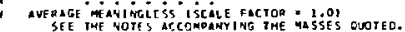

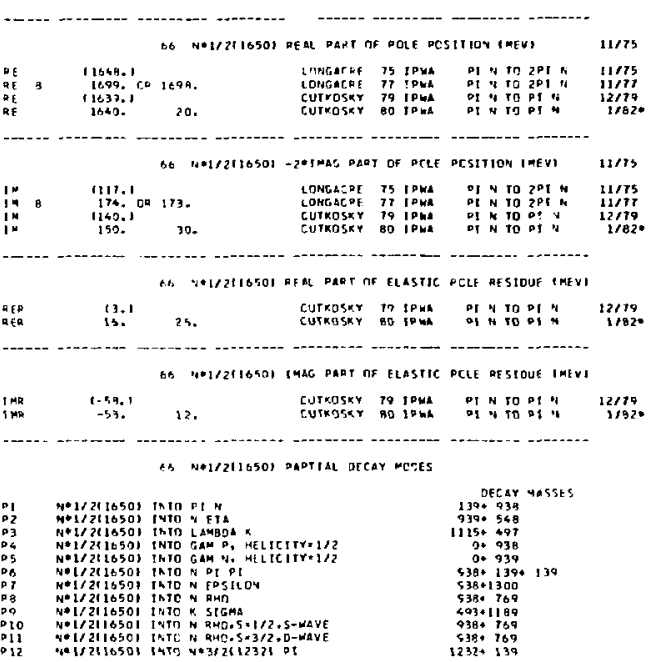

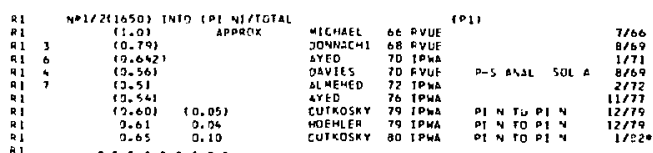

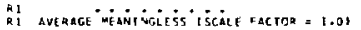

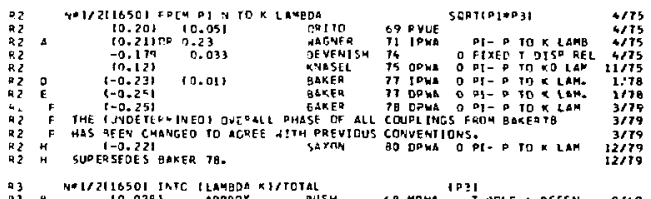

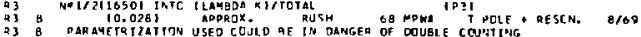

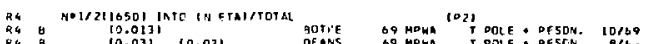

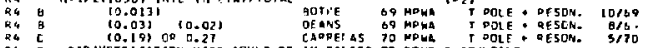

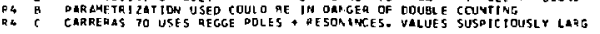

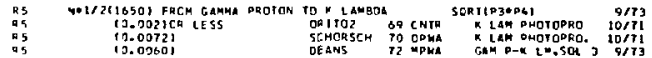

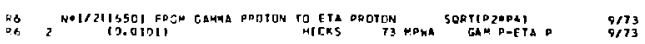

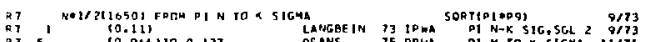

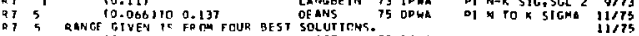

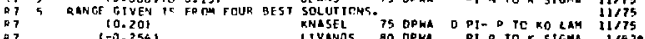

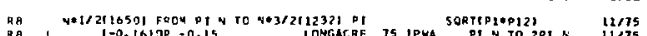

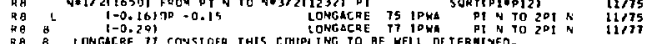


Baryons

$\mathrm{N}(1650), \mathrm{N}(1675)$
Data Card Listings

For notation, see key at front of Listings.

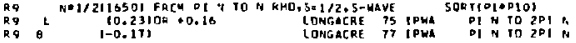

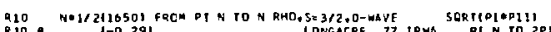

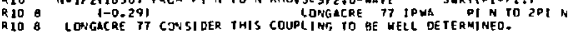

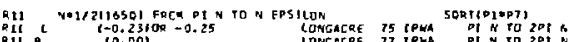

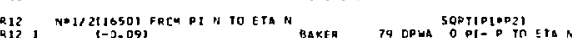

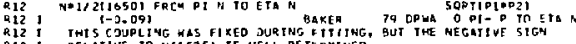
RI2 I RELLTIVE TO NIIS35I IS NELL DE TERUI WEO

BE N*1/2(16SO) PHJTCh DE CAY EHPL IGEV**-1/2)

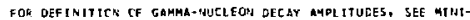
FEVIEU DXECEDIML THE BADYON IISTIMGS.

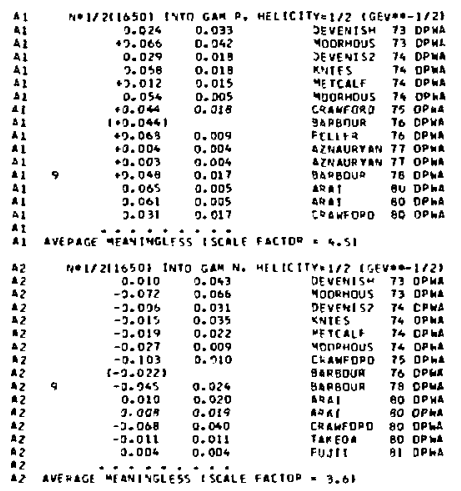

P1) $N$ PHOTapaco.

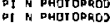

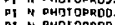
PI $A$ PHOTOPROD. P1 N PHOTOPROD. Pi 4 PHartoproo.

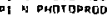

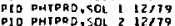
PI-s phorópaco.

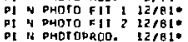

91 $N$ PHor Topego. of $N$ PHOTroprod. of $N$ PHOr OppeD. 1 i OHOTOPRCO.

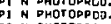

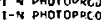

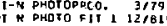
OI N phata frt 2 lizal

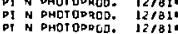

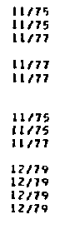

11,75
11,775
$11 / 77$

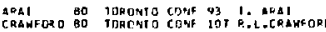

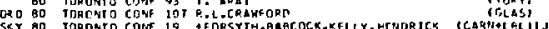
caknthatl] MUSETHE BO YC 57437

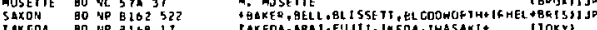

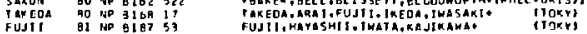

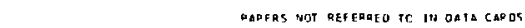

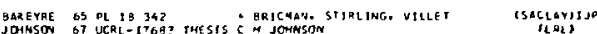

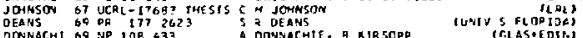

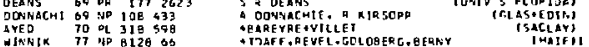

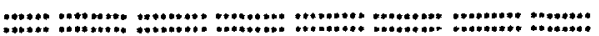
$N(1675)$ of N41/241675, Jp=5/2-1 i=1/2 D THIS QESDHAMCF IS MELL ESTAAL IEHED (4) Ne1/2116751 MASS TMEVI

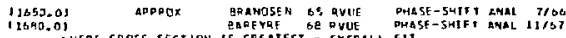
18G90. DI

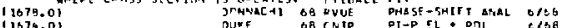

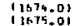

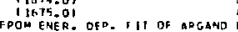
III Of apgatio dit aGRa to toma 1069.0109 1652 . 10 1607

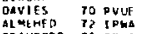

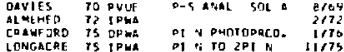
THE 360 . Ou 1060 .

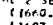

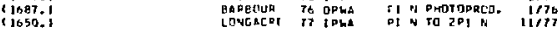

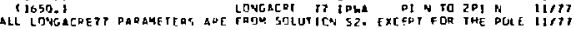

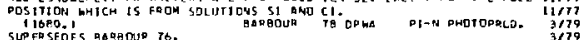

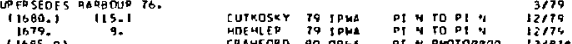

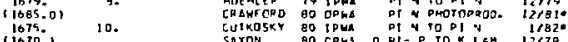
avfalge uéanivgitiss iscale factor = 1.01

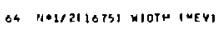

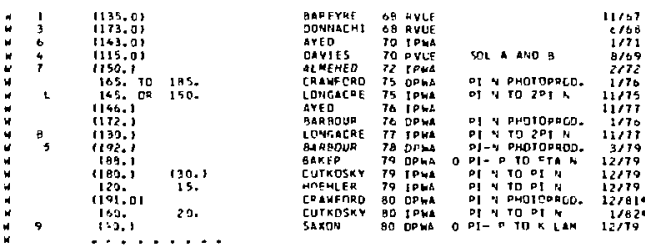

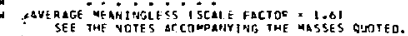

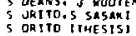

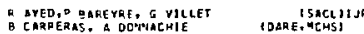
A TIETEE. HEILNBOECK

I. MACHER, C LOVELACE impini

ICEETI

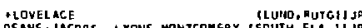
OEANS. JACOBS. L YONS, MONTCOMEAY ISDUTH FEA. IJP

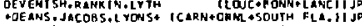

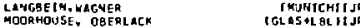
DEVEMISH, F POGGATT, MAPI SMSOE SY, NORDI Th, LOUGI

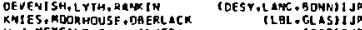

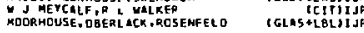
Q L CRAMFDOO

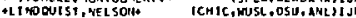

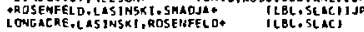

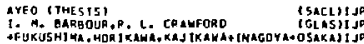

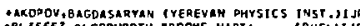

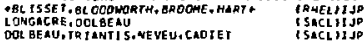
BBL ISSET, BLODONORTH, EROCHE. TRL +CAMEIIJP BAZBOUR,CRAMFORD,PANSONS

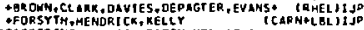

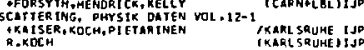

\begin{tabular}{|c|c|c|c|c|c|}
\hline \multirow[b]{2}{*}{$\begin{array}{ll}R E \\
R E \\
R E \\
R E \\
R E\end{array}$} & \multicolumn{4}{|c|}{ 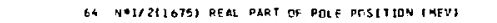 } & \multirow{2}{*}{$\begin{array}{l}11 / 73 \\
11 / 73 \\
11 / 77 \\
32 / 79 \\
1 / 820\end{array}$} \\
\hline & 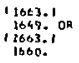 & $\begin{array}{r}1050 . \\
10 .\end{array}$ & 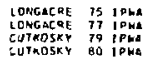 & 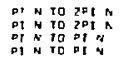 & \\
\hline & & of $N$. & 2NIMA PART of DELE & DSTIOA IMEVI & $11 / 75$ \\
\hline 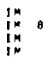 & 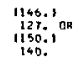 & $\begin{array}{r}127 . \\
10 .\end{array}$ & 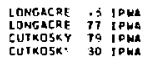 & 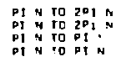 & $\begin{array}{c}11 / 75 \\
11 / 77 \\
12 / 79 \\
1 / 82 *\end{array}$ \\
\hline & & C. Ne & EAL DAFT OF ELASTHE & CLE RFSTOUE TME & \\
\hline $\begin{array}{l}\text { RER } \\
x \in R\end{array}$ & $\begin{array}{c}133.1 \\
21.1\end{array}$ & 5. & 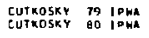 & 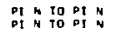 & $\begin{aligned} 12 / 79 \\
1 / 82\end{aligned}$ \\
\hline & & e4 $\mathrm{Ne}$ & F ELASVic & 10UE TKE & \\
\hline$i_{i \rightarrow R}^{i m R}$ & $\begin{array}{l}1-11.1 \\
-16.1\end{array}$ & s. & 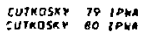 & 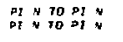 & $\begin{array}{r}12879 \\
11820\end{array}$ \\
\hline
\end{tabular}


Data Card Listings

Baryons

For notation, see key at front of Listings.

$\mathrm{N}(1675)$

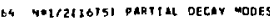

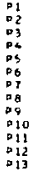

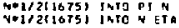

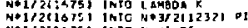

W*1/211675T INTO GAM O. HELICITYNT/

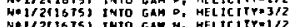

Nat12t1675) MTO GAM N. MELIC1TY-1/2

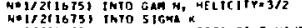

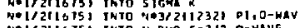

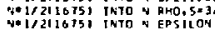

E4 V*1/241675, BRANGHING RATIOS

01
01
01
0
8
8
8
8
0
0
0
0
01

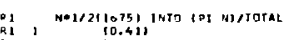
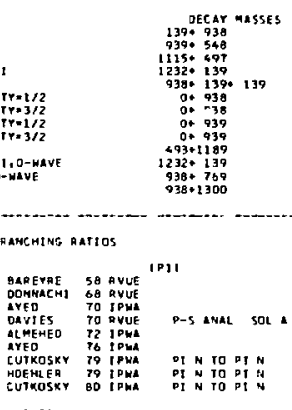

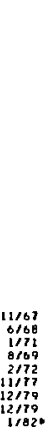

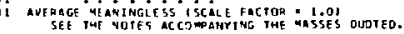

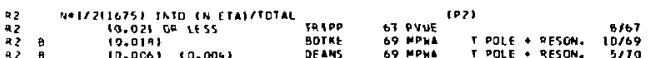

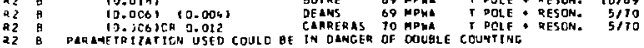

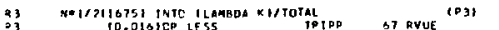

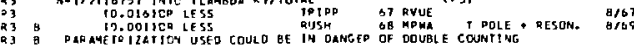

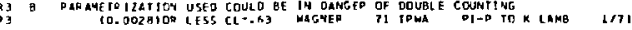

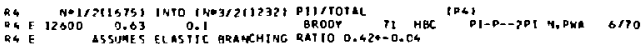

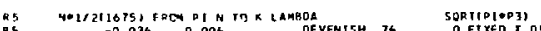

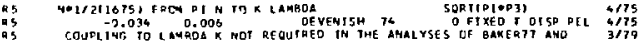
Q5 5 AAKERT. SAXON BO OPHA O PF- O ID K LAM 12179

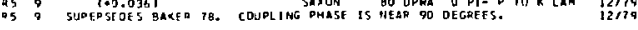

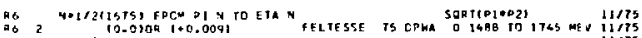

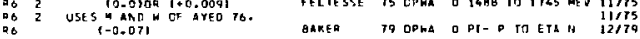

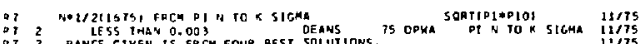

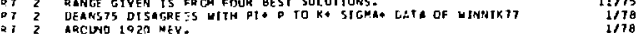
OA
OB
Q
Q

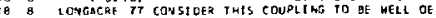

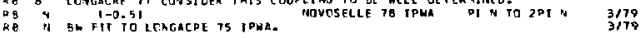

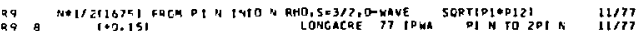

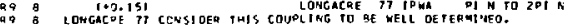

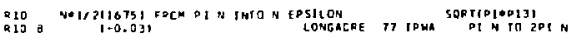

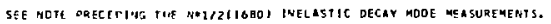

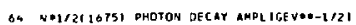

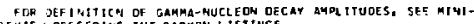

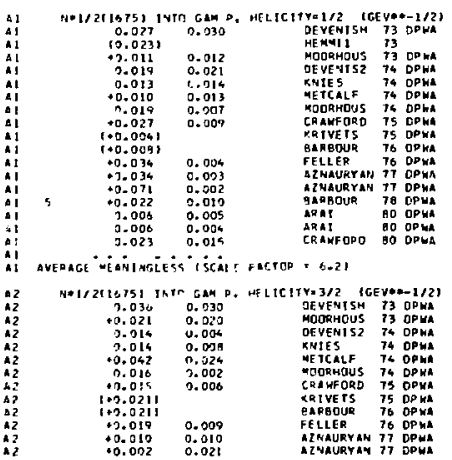

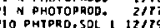

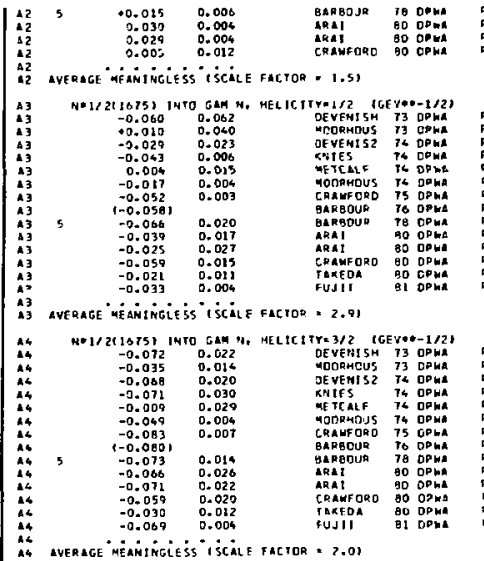

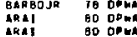
- 1.51

Dt h omatjorco.

pi a photopaco.

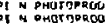

DI N PHOTOPRCD.

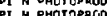

rOBRC. $3 / 78$

4 phara FJI 2 12\%ale

of N PHOTOPACD. 12/Bi*

DI N PMOTJDRCD.

PI N PHOTOPACO.

PI N PHITORROD.

Pt $\mathrm{P}$ PHOTOPDOD.

DI DADThPQOD.

Di-h PHOTOPDOO

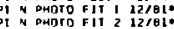

I N PHOTOPOCD. 12/B10

of $N$ P HOTOPROD. 12/AI*

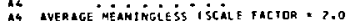

o

ORAMOSEN SS DL 10470

TATOD 67 :P0 8310

BRREYAE OB PR 1651731

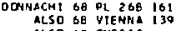

DUKE AL SO GE THESTS

QLSH INSIGH IF UR GUALITATI

$\begin{array}{llll}\text { OOTKL } & \text { OS OA } 180 & 1417 \\ \text { DEAYS } & 69 & 185 & 1797\end{array}$

ared 70 KtEV conf

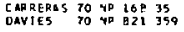

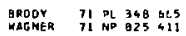

ALMEMEO 1240840157

DEVENISH 73 PL 4785 ?

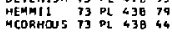

DEVEMISH 74 YO $801 \quad 330$

OEVENIS2 74 DL 528227

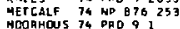

CRAWFORO 75 HE B97 125

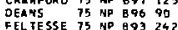

RRIVETS 75 SJNP 20430

LONGACRE 75 PJNF 195112

ALSO TO PRD IT 1795

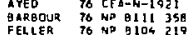

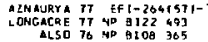

BARBDUR IA NP A $141 \quad 253$

$\begin{array}{rllll}\text { NOVOSECL } & 78 & 49 & 8137 & 509 \\ \text { ALSD } & \text { 40 } & 0137 & 445\end{array}$

PAKER 79 HP E15B 43

CUTKOSKY 79 PRO 202839
HOE HLER

aLso BO TORONTO CONF

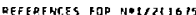

+OCOYNFLL, MOCRHDUSE

[DURHA 4 RHELIIJP

+ LEITH. + RLQL.SLAC,CEPA, HE 10, SACLAY)

P BAPEYRE. C BRTCMAN. E VILEET ISACLAYTIJP

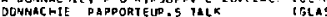

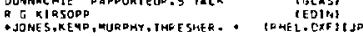

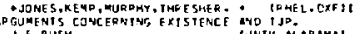

S C BOTKE

iunive clotitoal

R AYID,D SAREYRE, G YILIE

a DAVIES

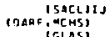

ACASAMDRE+ . PHERHDGN+..
F GAGNER, C LOVELACE

(sitaction)

+ Luvelace

RLND.OTTGHAP

OEVENISH. OARKIN.LYTH

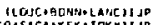

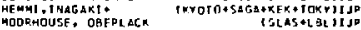

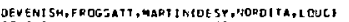
DEVENISH,LYTH,RAHKIN TOESY,LANC. DOUNOTJ QNIES. MCJQHOUSE, OSEPLACK IC FL, GLASI]

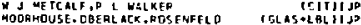

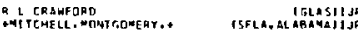

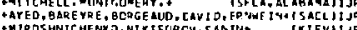

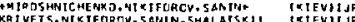
KAT I VETS, NIKIFORDV, SAHIN, SHALATSKII TKIEVIIJP LONGACAE, ASINSKT. DOSENFFL O. ZLBL-SLACIIJ

AYED (THESTSS, TSACLIIJP

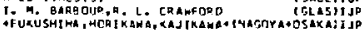

-akopov, bagoasapyap trepeyan ohysics tust ilija LORGACRE. DOR BEAU
DOLBEAU.TAIANTIS.NE VEU.CAOIET GARBOUR .CRAGFORD.PAFSCNS TGLASI $\begin{array}{ll}\text { O. E. NOVOSELLEP } & \text { ICAL TECHIIJP } \\ \text { O. E. NOVOSELLER } & \text { TCAL TECHIIJP }\end{array}$

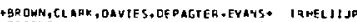
FOASYTH, HE NOPICK, KELLY CCARYLLALIIJ TOPACD. 1170 Di N PHOTODROD. DI N PHOTOPROD: 217

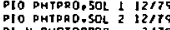

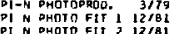

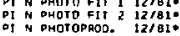

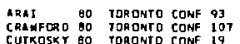

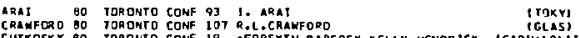

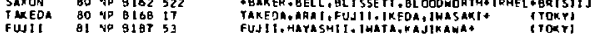

paDERS bot aEFEARED to IN OATA caros

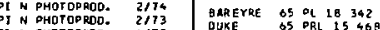

PI N PHOTOPHDD.

PI N PHOTOPEOD.

PI A PHOTOPMOD.

DI N PHDTOPRDO

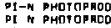

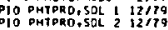

+ BRICMAH, STIALING, VILLET, TSACLAYISJP

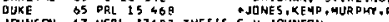

JOHNSON G7 UCRL-17OG? THESKS C 4 JOHNSON

DEANS 69 PAL 1772623
DCNNACHI 69 ND 107433

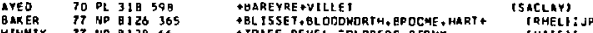

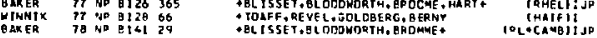


Baryons

$\mathrm{N}(1680)$
Data Card Listings

For notation, see key at front of Listings.

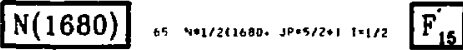

TMIS RESONANCE IS WELL ESTABLISHEO.

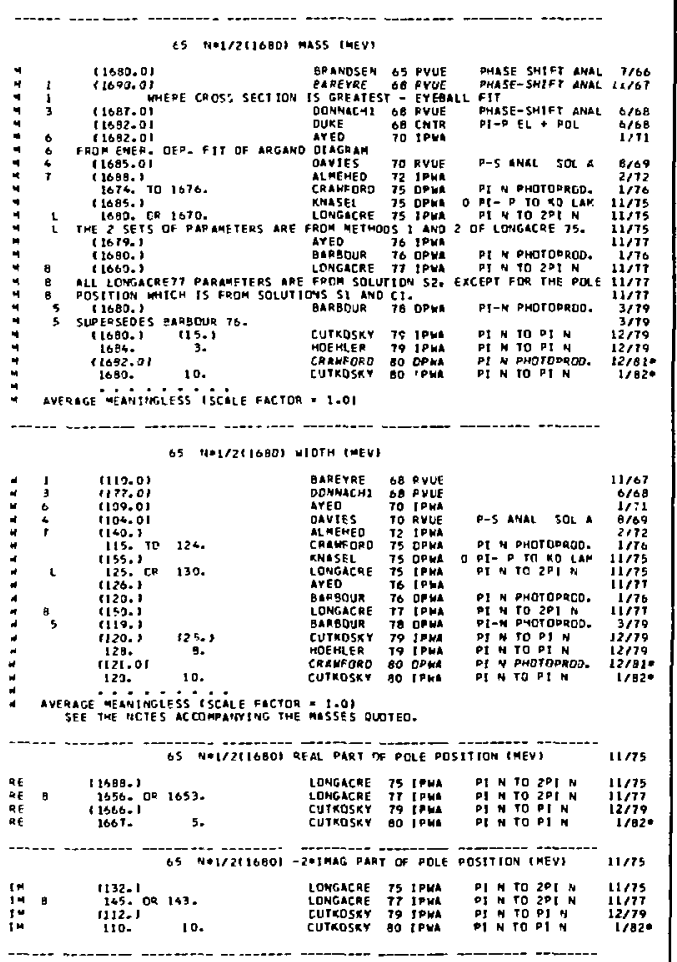

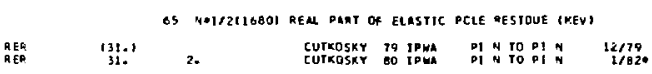

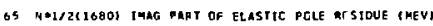

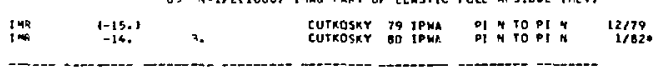

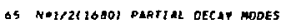

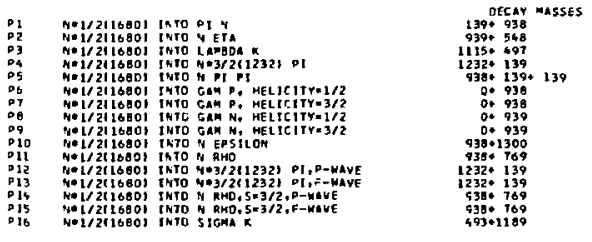

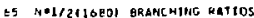

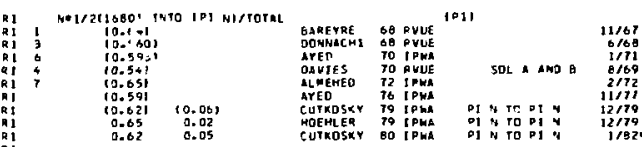

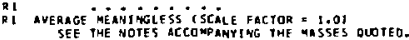

MLRE IMFRRMATIUN ON THE IMELASTIC DECAT MDOES OF THE 1090 MFU

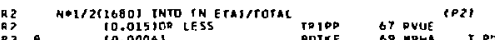

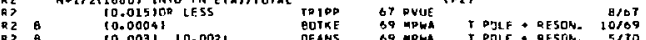

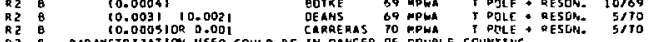
Rj n*1/zU1600

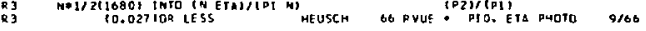

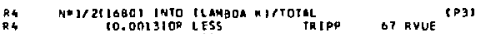

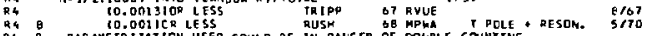

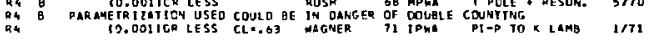

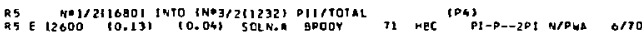

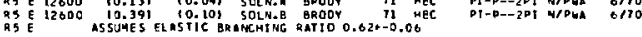

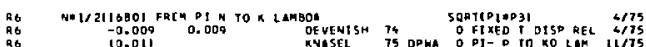

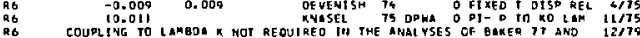
So SAXON BO.

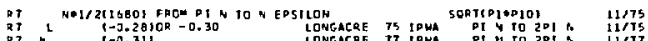
R7 8 a to.

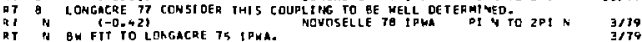

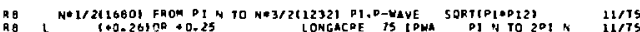

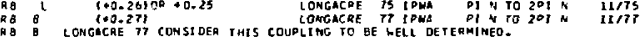

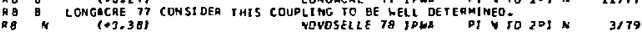
M9
Ro

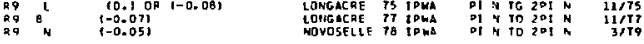

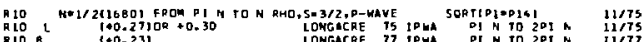

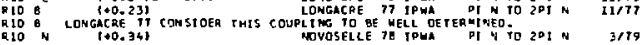

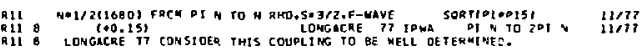

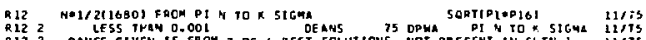

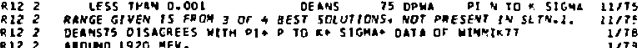

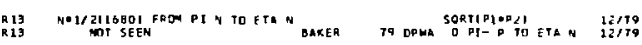
65 N*1/211680) PHOTON OECAY AMPLI GEV FOR OEFINITICN OF GAYMA-MUCLEON OECAY AMPL ITUEES. SEE MINAPEVIE PRES EDINC THE GRRYON LISTINGS

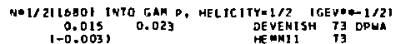
$-0.003 \quad 0.006$ $\begin{array}{rr}-0.003 & 0.004 \\ 0.027 & 0.019 \\ -0.010 & 0.014\end{array}$ $\begin{array}{ll}-0.016 & 0.014 \\ -0.008 & 0.011 \\ -0.024 & 0.003\end{array}$ $\begin{array}{lll}-0.013 & 0.002\end{array}$

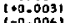
$\begin{array}{ll}10.0007 & 0.002 \\ -0.0007 & 0.002 \\ -0.010 & 0.001\end{array}$ $\begin{array}{ll}-0.010 & 0.002 \\ -0.005 & 0.015\end{array}$ $\begin{array}{ll}-0.005 & 0.015 \\ -0.028 & 0.003\end{array}$ $\begin{array}{cc}-0.026 & 0.003 \\ -0.018 & 0.014\end{array}$ Moorhous 73 poun DEVENISZ 73 DPMA HERCALF 74 DPUA POORACUS 74 OPXA CRAMFOO
KRIVETS 75 DPMA
RRA FELAER 76 DPMA AIHauRran 77 Doma AZNAURYN 77 DPMA

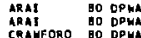
D1 in protopego. PWO P TO PHTOPROD of $N$ PHOTOPROD. Pit $n$ intolopros.

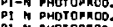
Plo PHTPRO.50 1 12/79

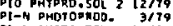

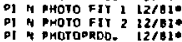

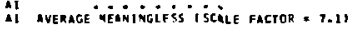


Data Card Listings

For notation, see key at front of Listings.
Baryons

$\mathrm{N}(1680), \mathrm{N}(1700)$

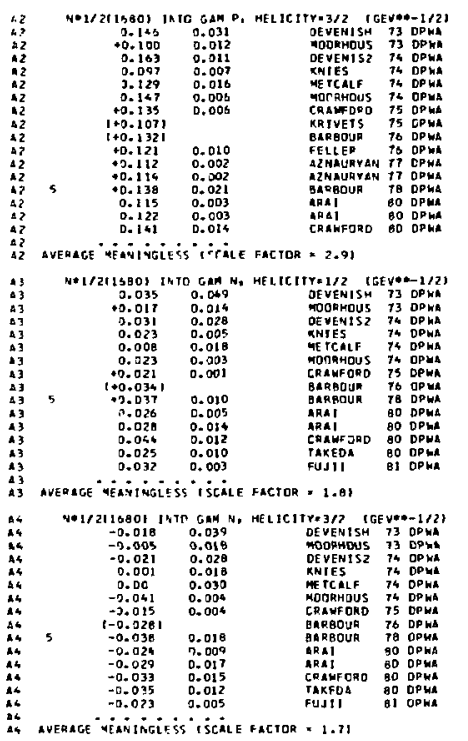

REFERERCES FOR N\%L

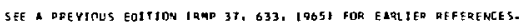
BRANOSE 1 OS DL 19420 HFIPSH 60 PRL 17,1010 ALREYGE OA DR $105 \quad 131$

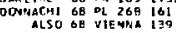
ALSO 6B VIENA
ALSO OG THESTS OUKE
RuSH

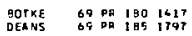
ATED 70 KIEV CONF DAVtes 70 VP 821359

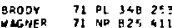
ALMEHEO 72 ND BOS 15 OEVEAISH 73 OL 47057

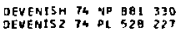
SULES TH THO

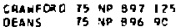
OEANS IS OPO

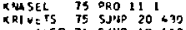

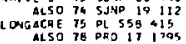

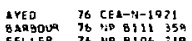

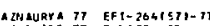

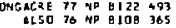
MARBOUR 7月 7D 8141253

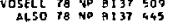

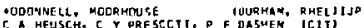

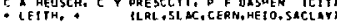
p garepae. c artcanan. g villet igaclayll Ja a JONAACHIE, R G KIRSOPP, C LONELAGE ICERY,IIJP

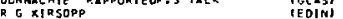

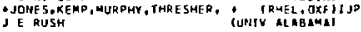

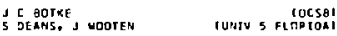

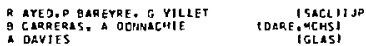

FCA SHMORE. ... HHE RNDONA. (SLACALRL)

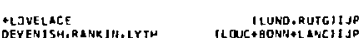

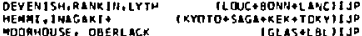

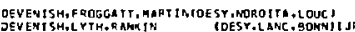

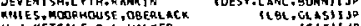

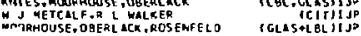

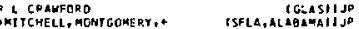

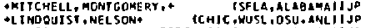

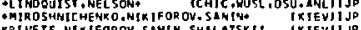
RRI VETS, NI KIFGROV. SLYMA, SHALATSKII KKIEVIIJP

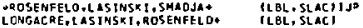

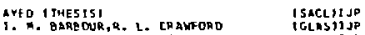

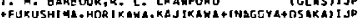

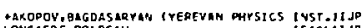

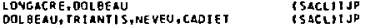
BAR MOUR . R RAKFORD, PAR SONS O. E. NDVOSELLER (rotes) BAKER 79 MP B1SO 53 PPOOHN, CLAPK, OAYIES, OE PACTER, EVANST IRHELIIJP

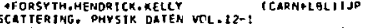

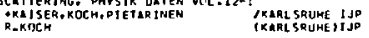

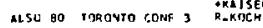
$($ rokry
(Geis)

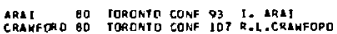

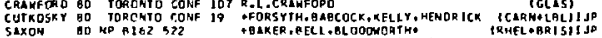

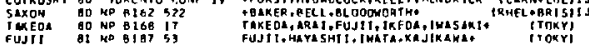

papers kot peferpeo to in onta cabos

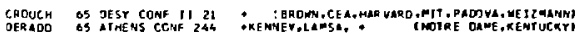

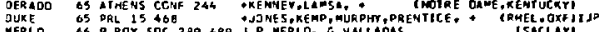

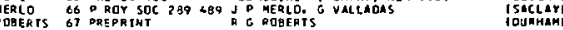

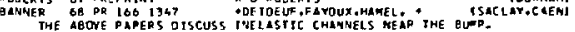

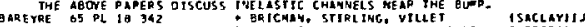
DEANS SO DRL 1772623 \$R DEANS

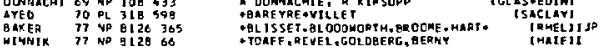

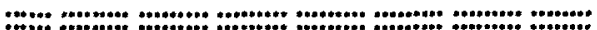
$N(1700)$ is Ne1/211700, so=3/2-1 1et/2 $D_{13}^{13}$ PMTS RE SONANCF IS HELI ESTRBLISHEC.

\section{N+1/2T1700) MASS TAF}

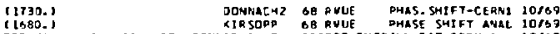

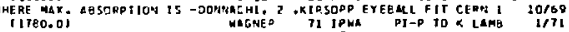
I17e0.0J 05 1670.1 CE tI790.

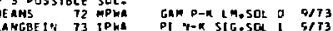

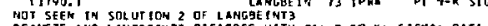

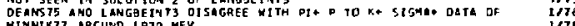

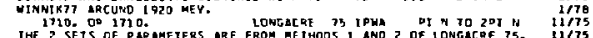

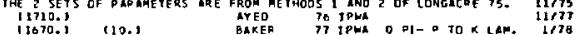

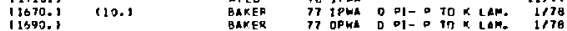

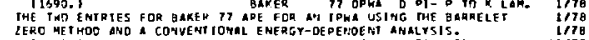

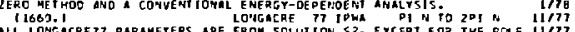

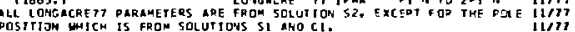
POSITIJA WIEH IS FROM SOLUTIDיIS SI ANO Cl.

$4 \quad 1718.1$ II 1880. (1670, MISS F OUMD gY BAKERT ITI- 15.

1675.025 BAKEO THO DPHA O PI- P TO K LAM

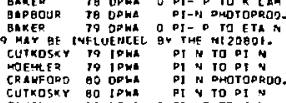
$3 / 79$
3179
12179
$12 / 19$
12179
12179 average aeningless iscale ractom = 1.91

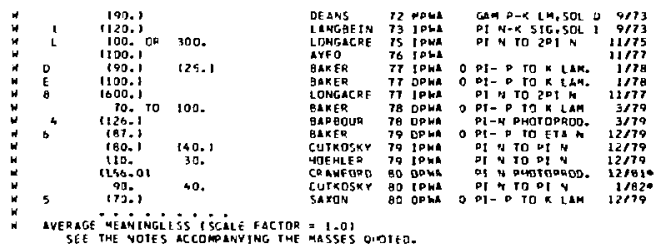

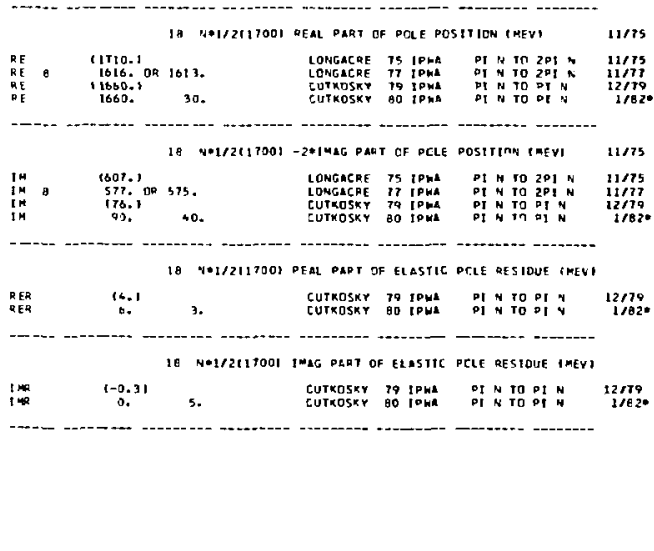


Baryons

N(1700)
Data Card Listings

For notation, see key at front of Listings.
19 Ne1/21L7001 PARIIAL OEGAY MODES

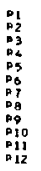

No1/217003 NTT GAM H+HELICITY=3/2

Not12tidis
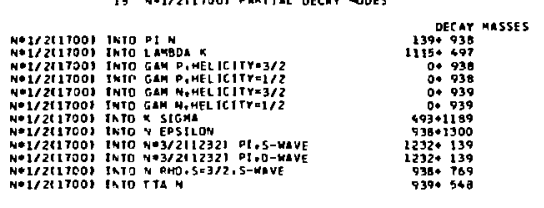

19 Y.1/211700 ERANCHING PATIOS

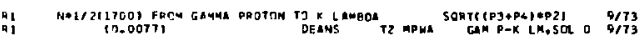

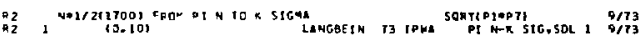

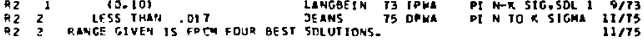

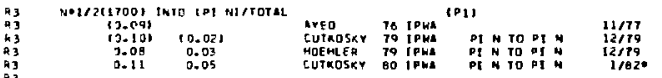

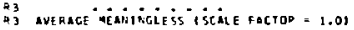

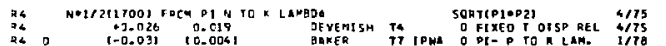

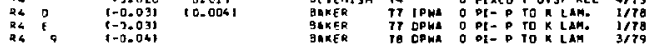

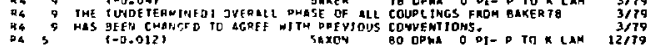

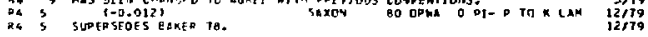
45
25
$R 5$

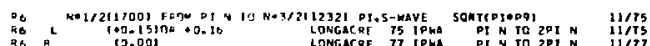

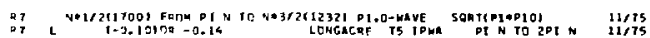

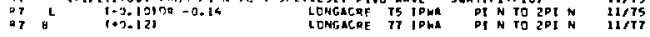

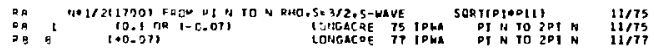

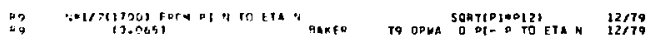

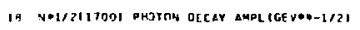

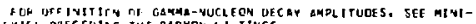

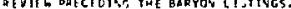

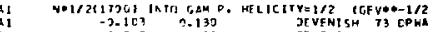

a

政

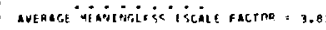

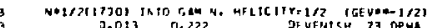

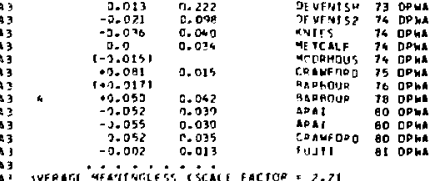

10

it

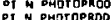

DI $N$ P P

PI N PHOTOPaOo:

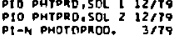

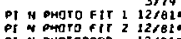

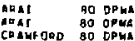

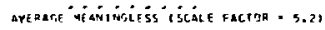

* * 1/211?

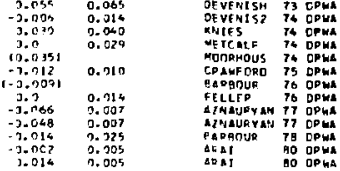

P1 "A D

of

PI 14 is

PI N PHOTOPROO

Dis

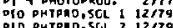

DID PHTRKD. SEL $212 / 79$

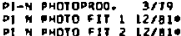

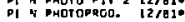

PI 4 protrop noo.

PI A PHOJORDOD.

PI N PHOTOPMOD.

iI N PHOTORED.

PI P photoproa

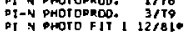

PI N PHOro $f$ II 2 12/810

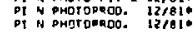
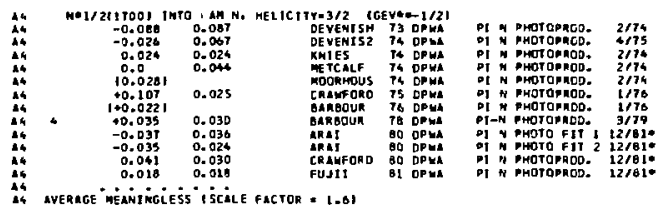

RESEREWCES FOR MEL/201 700 I

ODNASCHZ G8 VIENHA 139

KINSOPP OO TMESI5

OEANS 72 PAO 81900

OENENISH T3 ${ }_{\text {DL }}^{478} 53$

OEVEN1SH P4 NP
OE1
OEVENISZ

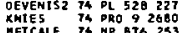

MOOAHOWS 74 PRD 91253

CRAMFDaO 75 NP AD 125

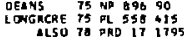

AYED 76 CEA-N-1921

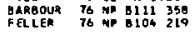

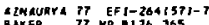

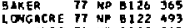

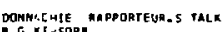

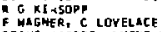

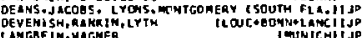
EBE IN.MAGMER

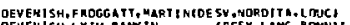

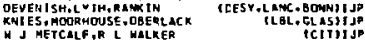

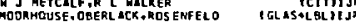

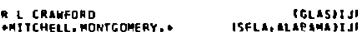
OMI TCHELL, MONTCOMERY,

SYEO (TMESIS) ISACLIJ JP

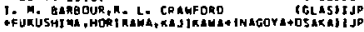

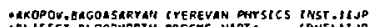

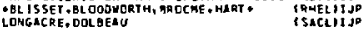

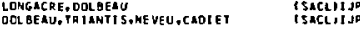

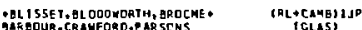

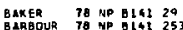

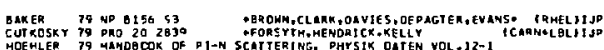

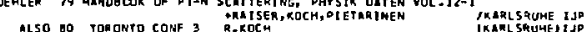

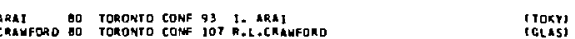

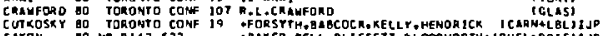

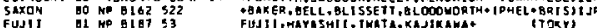

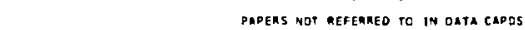

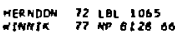

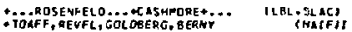

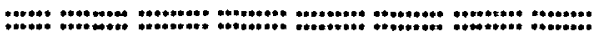

1700 MEV REGION - PRODUCTION EXPERIMENTS

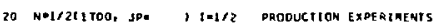

SEE THE MINI-REYIEM ORECEOIMG THE N RMO OELTA IISTINGS
FOR DISC USSIOH OF PRDDUTTIOA EXPERIMENTS.

20 MEI/2TITOO MASS EMEVI IPQDO. EKP.I

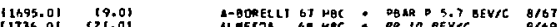

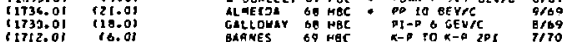

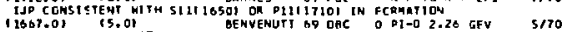

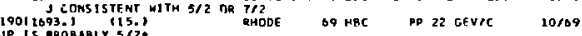

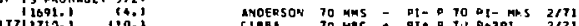
$1171691:)$ 110.2

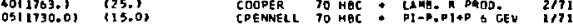

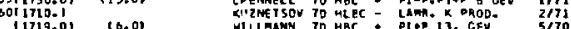

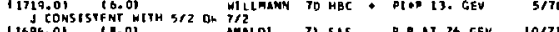

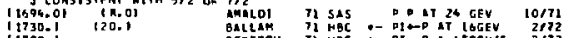

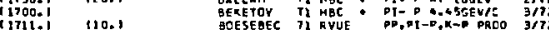

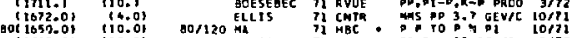

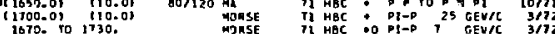

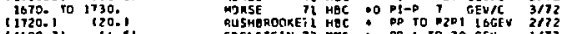

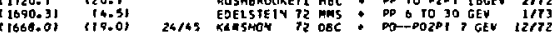

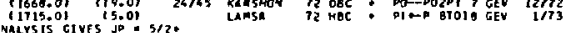

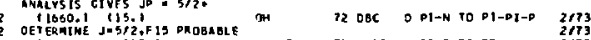

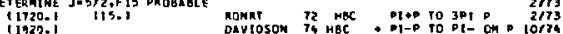
OMEGA PROTOH ENHAMCEMENT, MOMENTS GHALYSIS CONSISTENT MITH 2 JE GQ 3 IOIT

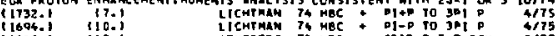

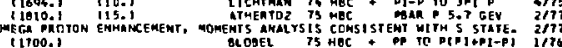

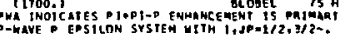


Data Card Listings

Baryons

For notation, see key at front of Listings.

$\mathrm{N}(1700)$

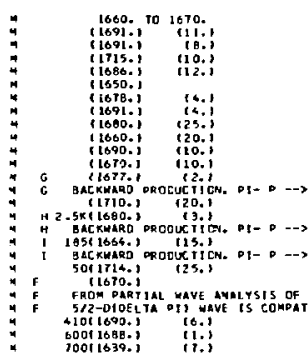

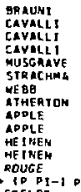

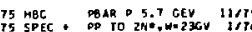

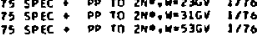

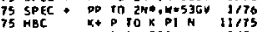

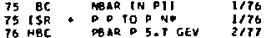

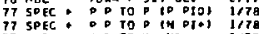

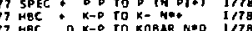

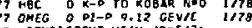

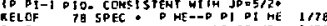

FERPER PT OMEC O D1-P $9+12$ GEW/C $3 / 78$

FERRER OR OHEG D P P1- 9.12 GEVIC $3 / 79$

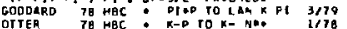

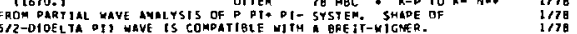

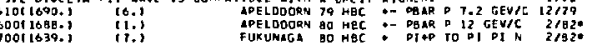

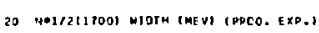

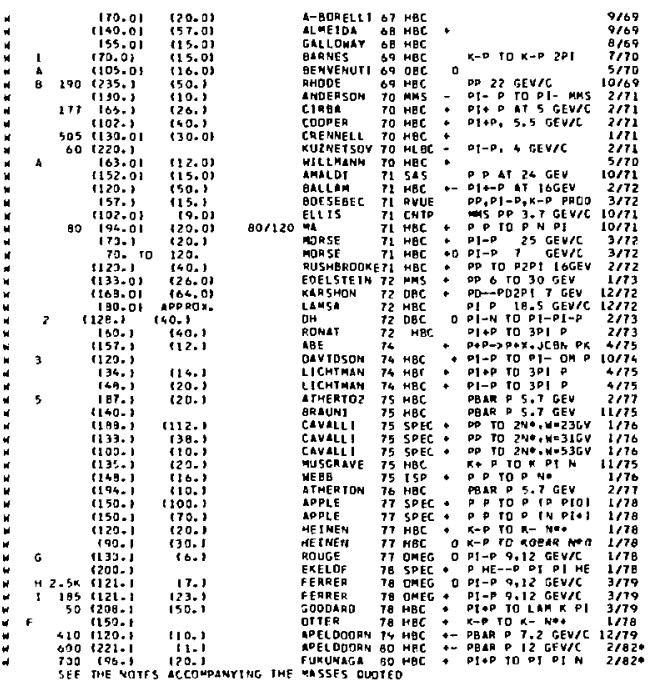

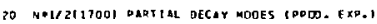

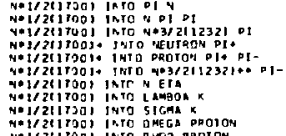

Nat)

5) nel/5t

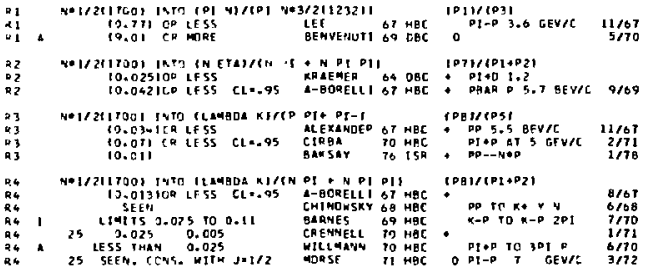

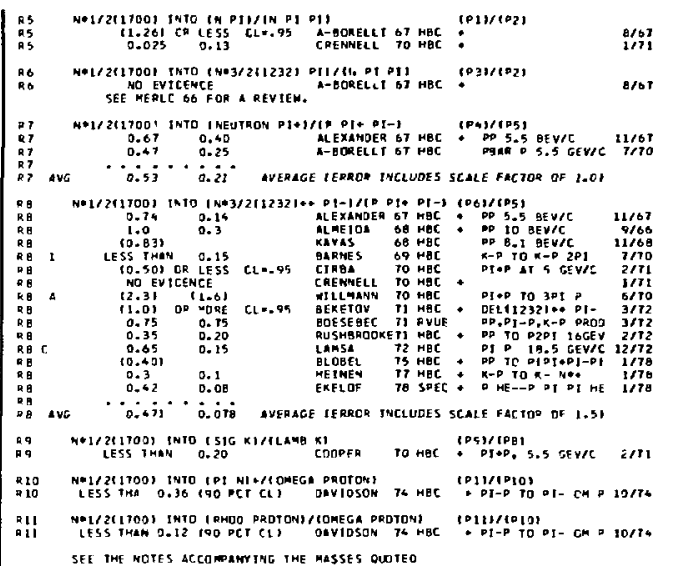

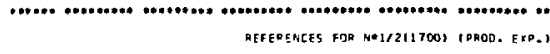

K RAEMER O4 PH 136 B644\%

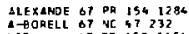
i TEA

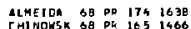
Nallowar ba pl. 278250 KAYAS OA NP BS 169

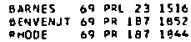
AMOEASON 7O PRL 25.699

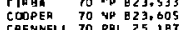
CREMELL 70 PQL 25187

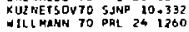

amalol 71 D 348 435

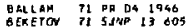

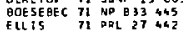

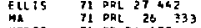

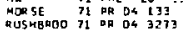
EOELSTEI 72 PR O5 1273

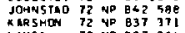

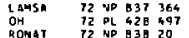
A AEE 70 PL 53 B 116 \begin{tabular}{l} 
DAVTOSON \\
DOCHTMA \\
\hline 4
\end{tabular} A THERTO2 75 ML 304505

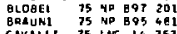
CAvalit 75 LAC 14353

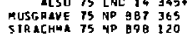

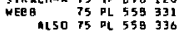

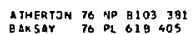
APPLE 77 LNC 18 167

ERELOF 78 NO

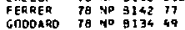

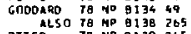

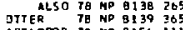

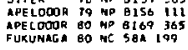

MERLO DO $P$ ROY 50 OC 280469

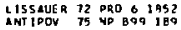

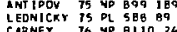

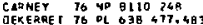

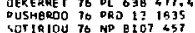

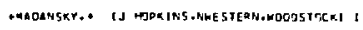
ALE XAMUER BENARY,CZLPEK.* TMEIZMANNICERNII

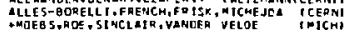
PRUSHBROOKE, * TCAVE, OE SYICFOHAI

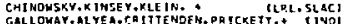

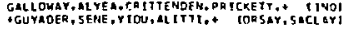

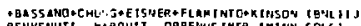

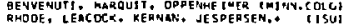

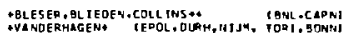

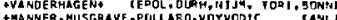

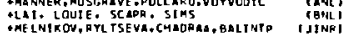
AAE LNIKON, RYL TSEVA, GHAOPAS, BALINTP I IINFI
ALAMSE, GAIDOS, EZELL

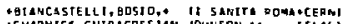

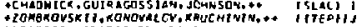

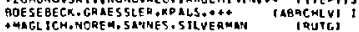

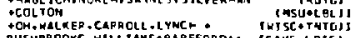
RUSHARDOKE. WILCTAM SA BAREFCRD+. TCAVE, TOTCI IJ

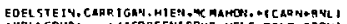

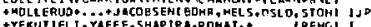

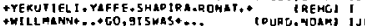

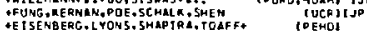
ARL SPEC IOF, EOMBFROWI12, IRUTG, UPNS.FSUS

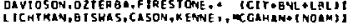

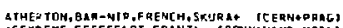

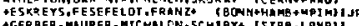

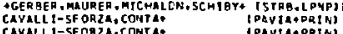

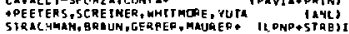

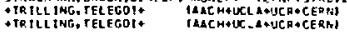

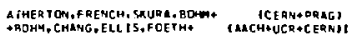
A ASH,CHENG, COYNE, GQCSSMNH+ IPRTYOPAVIAI,

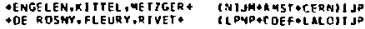

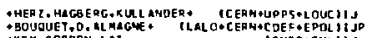

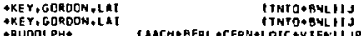
WAN APEL DODRN, HAETING

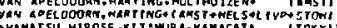

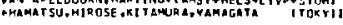
papers vot refeaned to in oata cands

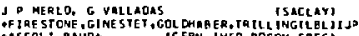

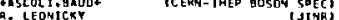

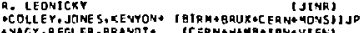

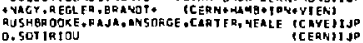


Baryons

$N(1700), N(1710)$

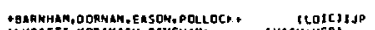

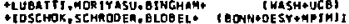

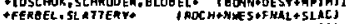

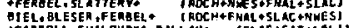

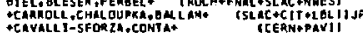

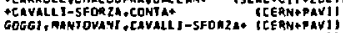

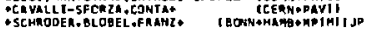

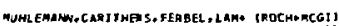

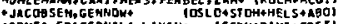

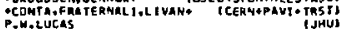

IUALERA 1600193189

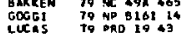

:

$N(1710)$ is No1/211710, Jeet/2+1 i=1/2 $P_{11}^{\prime \prime}$

this aesonance is WELL ESTABL ISMEC.

\section{No1/2(1710) MASS (MEY)}

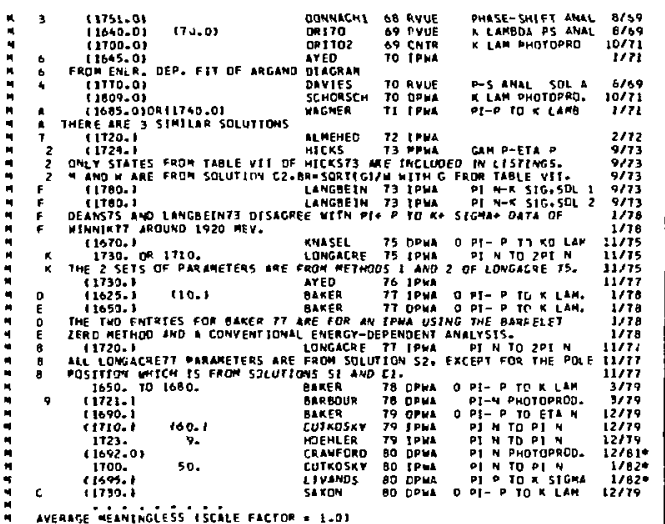
14. N*H/5417L) KIOTH (MEV)

\begin{tabular}{|c|c|c|c|c|c|c|}
\hline $\begin{array}{l}\hat{i}^{2} \\
F^{2} \\
{ }_{n}\end{array}$ & 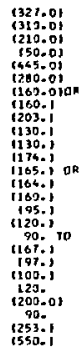 & $\begin{array}{l}150.01 \\
1220.01 \\
175.1 \\
18.1 \\
150 . \\
150.1 \\
15 . \\
30 .\end{array}$ & 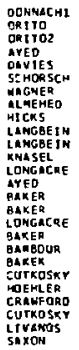 & 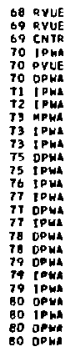 & 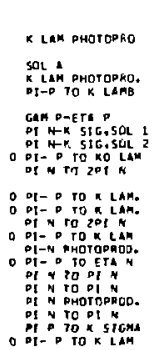 & 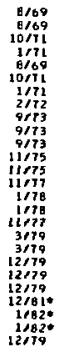 \\
\hline
\end{tabular}

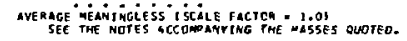

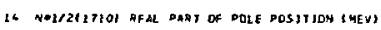

$11 / 73$

\begin{tabular}{|c|c|c|c|c|}
\hline Re & 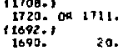 & 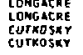 & 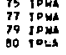 & $\begin{array}{lll} & \\
0\end{array}$ \\
\hline
\end{tabular}

16 N•1/2(1710) 20TMAG PART OF PCLE POSTTION INEVI L1/75

\begin{tabular}{|c|c|c|c|c|}
\hline & $\begin{array}{l}117.4 \\
123.4 \\
689.1 \\
80.1\end{array}$ & 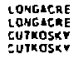 & 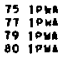 & 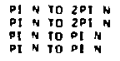 \\
\hline
\end{tabular}

Data Card Listings

For notation, see key at front of Listings.

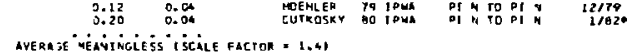

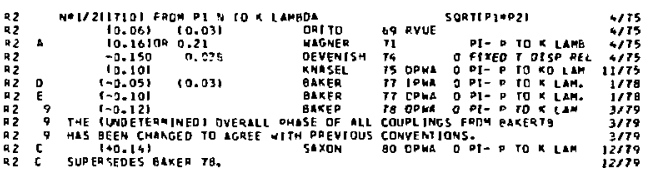

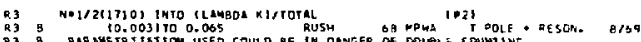

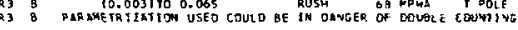

Q4 No LaITHOI IRTC IN ETHIJIOTAL

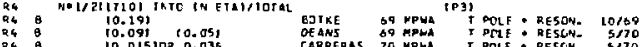

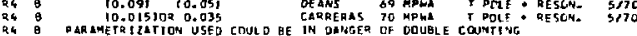

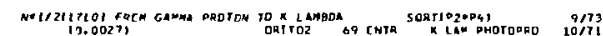

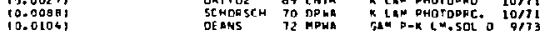

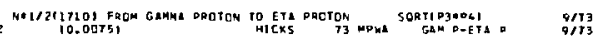

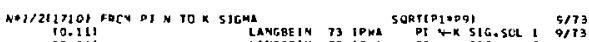

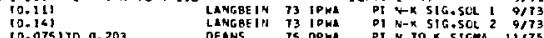

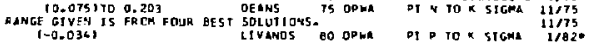

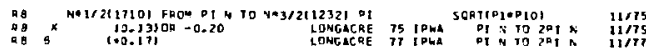

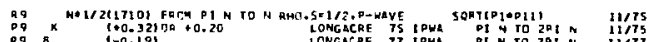

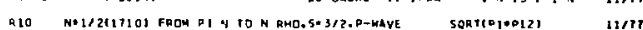

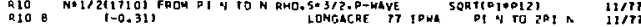

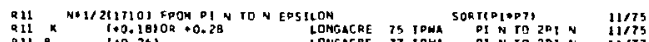

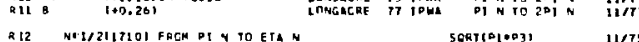

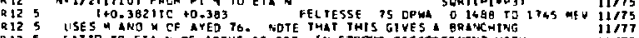

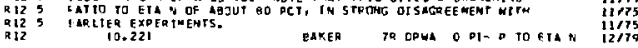

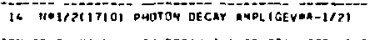

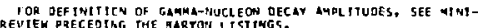

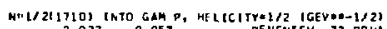

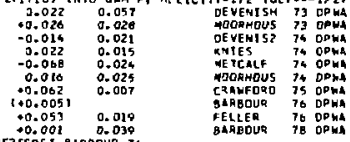

P1 N PMEThPACD. of 4 OHOTYPR DO. of $\alpha$ PHOTARDOD. Pf spmoromaco.

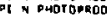
P.

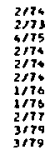




\section{Data Card Listings}

For notation, see key at front of Listings.

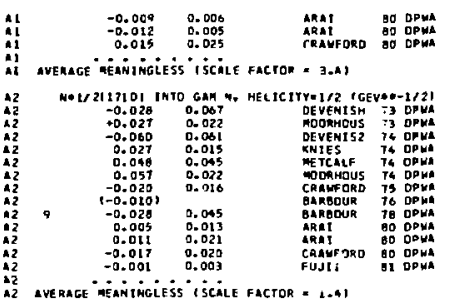

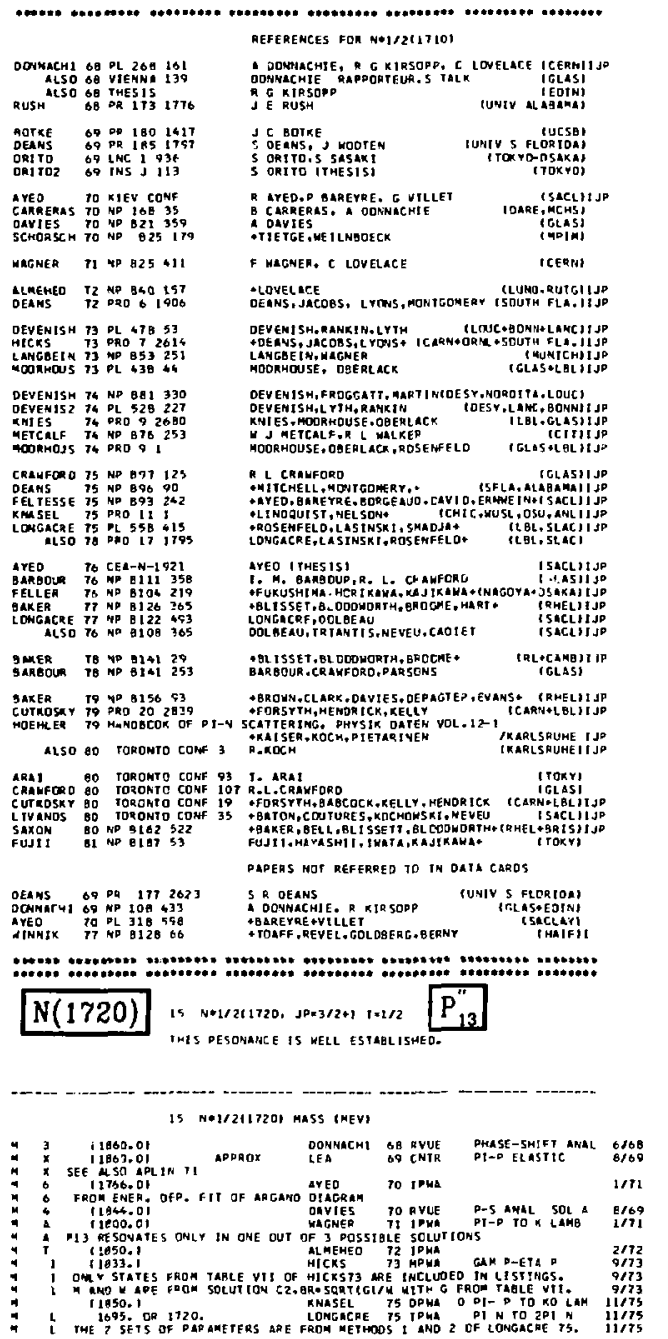

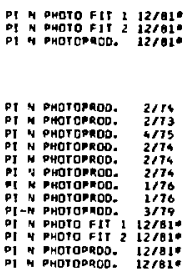

|

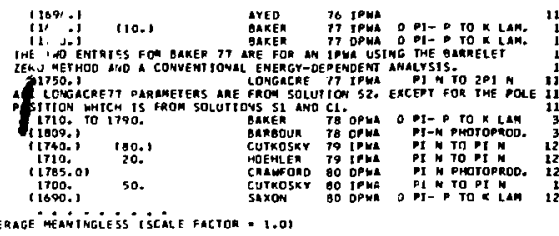

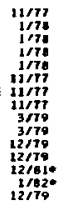

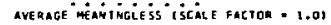

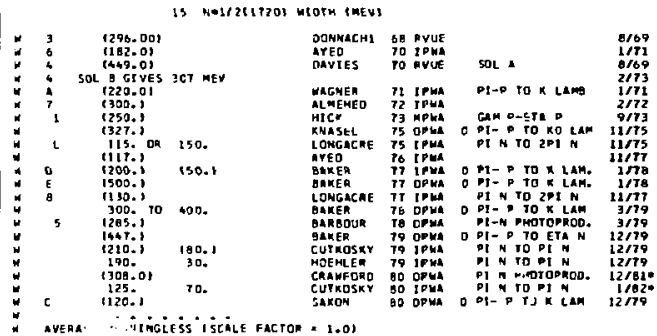

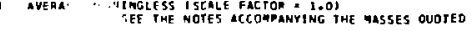

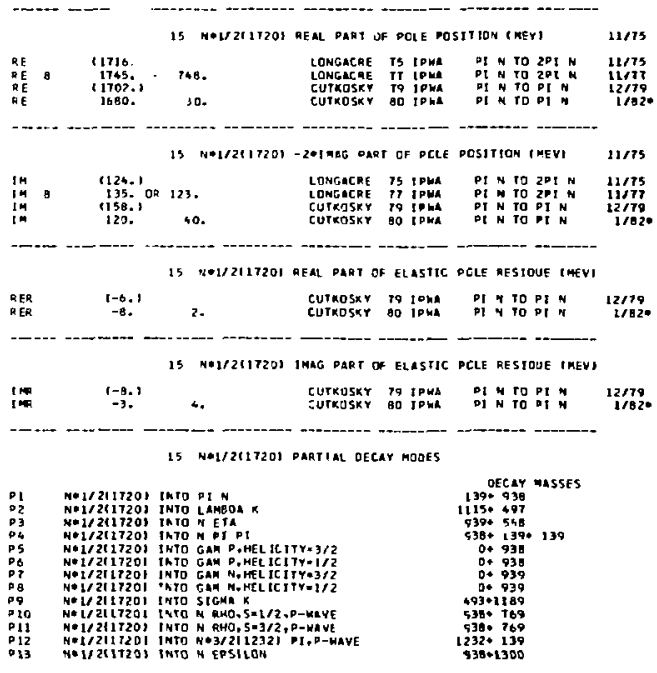

15 NEI/ZIIT20) GRANCWENG RATIOS

\begin{tabular}{|c|c|c|c|c|c|}
\hline 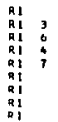 & 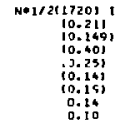 & I Dt M)/TCRAL & 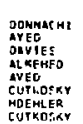 & 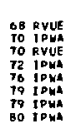 & 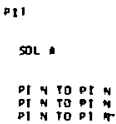 \\
\hline
\end{tabular}

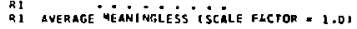

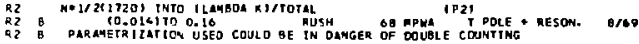

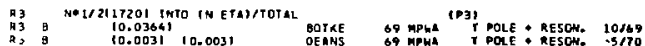

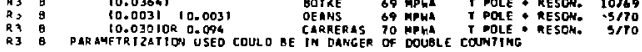


Baryons

N(1720), N(1990)
Data Card Listings

For notation, see key at front of Listings.

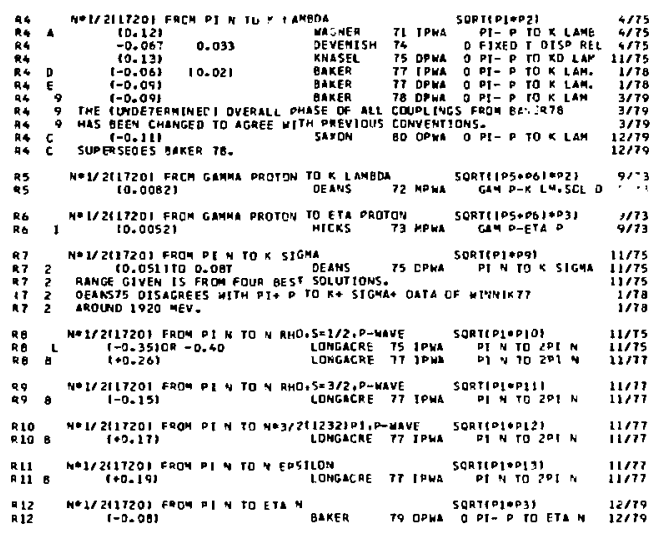

I5 N*1/2(1720) OHOTON DECAY AMPLIGEVH*-1/2)

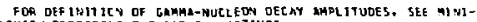
FVA PRECEDTV THE AARYON LISTINGS.

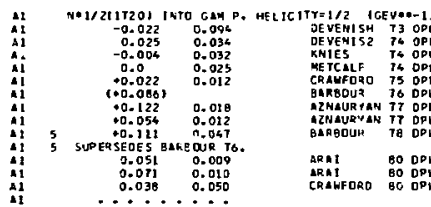

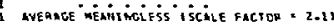

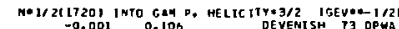

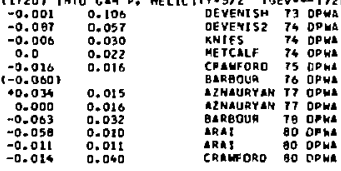

AERTINGLESS ISCRLE FACTOR = 2.01

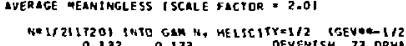

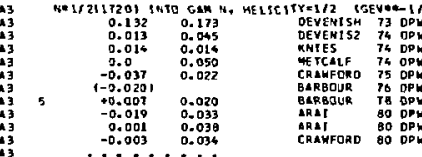

average méanlingless iscale factor - 1 .01

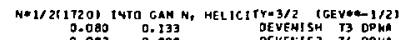
$\begin{array}{cc}0.080 & 0.133 \\ 0.0003 & 0.090 \\ -0.008 & 0.025 \\ 0.0 & 0.044 \\ -0.038 & 0.045 \\ 100.0461 & \\ 0.051 & 0.051 \\ -0.139 & 0.039 \\ -0.136 & 0.044 \\ 0.018 & 0.028\end{array}$

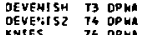

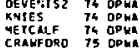

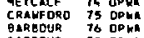

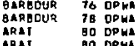

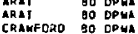

average "iesanticiess" iscile factor = 1.7)
PI N PHDTOPROO. Di N PHDTOPRUD. P1 N PHOTOPROD. OI N DMOTOPROD. PIO PHTPRD, SOL I 12,T⿱ PI-A DHOTOPROD. J/18 PI N PHDTO FII 1 12/81.

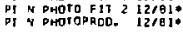

DI N DHOTOPROD.

P1 N PHOTOPRDD.
PI N PHOTOPROD.

Di N PMDTOPRDA.

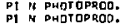
TO OHTPRD.SOL I 12/76 IO PHTPQD.SOL 2 12179

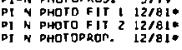

Of $N$ P POTAPRDO. OI N PHCT OPRDD DI N PHOTOPROO DI Th PMTTCPRD. PI-N PMETOPROD. $3 / 79$ PI N PHCTO FIT 2 12/81"

DI N PMOTOPROO.

PI N PMOTOPROD.

PI \& PHOTOPROO.

DI N PHOTOPRCO.

3/79

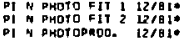

HAENEA 71 HO 25411

ALMEFEO 22 NP B $6015 \%$

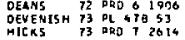
DEVENISH 74 NP OS! 330

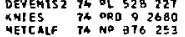
CAAHF ORD 75 ND 997125 OEANS THSE TS PQD 11 ?

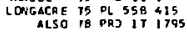

AYED
GARBOUR TE CER-N-192!
Th MP BLII 350 AZNAUARA 77 EF [-264 (57)-

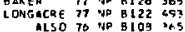

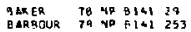

gAKER TH HP HISO 53 CUTKOSKY TS PPO 2.02879 also BO TORDNTO COMF

AQA 1 AO TORCNTO CONF 93

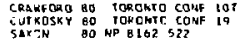

DEA

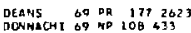

$\begin{array}{lll}\text { AYED } & 70 \mathrm{PL} & 31659 \mathrm{G} \\ \text { APLIN } 71 \text { NO } & 532253\end{array}$

MA
HINAIK TO ORD 133927
TI

men

(CEm)

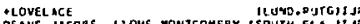

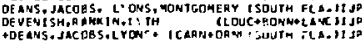

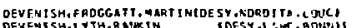

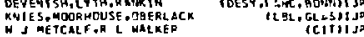
A L CRAHFQRO

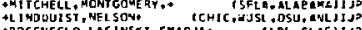

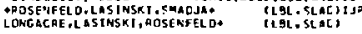

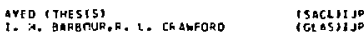

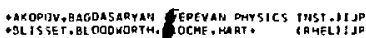

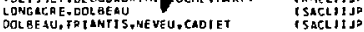

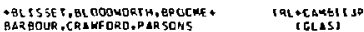
+EROWN,CLARK, OAVIES, CEPAGTER, EVUNS. IFHEL HID TFORSY TH, HENDPICK, KELLY TC L RN+ LELIIJP

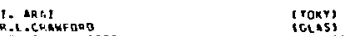

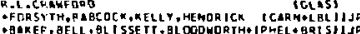

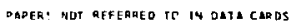

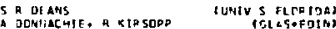

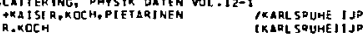

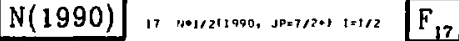

$$
17 \text { NHLL2+1970) MASS (UEX) }
$$

\$1993.01 DONMACH OQ AVUI OHASF-SHIFT ANAL

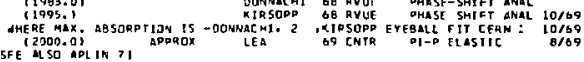

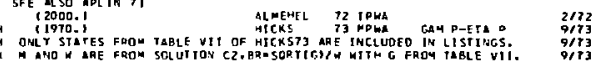

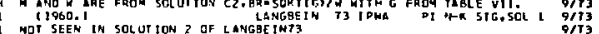

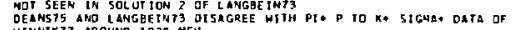
20

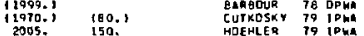

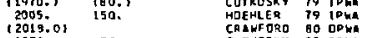
Di-n PHOTOPDOD. 1970. 50. CutKoSkY 80 TOMA

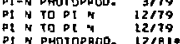

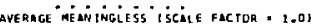
17 NOLTHLOOI WTOTH IMEVI

\begin{tabular}{|c|c|c|c|c|c|c|c|}
\hline 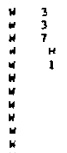 & $\begin{array}{l}1225.01 \\
1250.1 \\
1200.1 \\
1309.1 \\
1170.1 \\
1119.1 \\
1216.1 \\
6325.1 \\
350.1 \\
6295.01 \\
350 .\end{array}$ & $\begin{array}{l}1150.1 \\
100 . \\
120 .\end{array}$ & 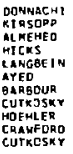 & $\begin{array}{l}68 \\
60 \\
72 \\
73 \\
73 \\
76 \\
70 \\
79 \\
79 \\
90 \\
90\end{array}$ & 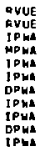 & $\begin{array}{l}\text { DHASF SHIFT AHAL } \\
\text { CAN P-ETA } \\
\text { PI N-K SIG.SOL I } \\
\text { PI-N PHOTOPNDO. } \\
\text { PI N TO PI N } \\
\text { PI N TO DI N } \\
\text { PI N PHOTOPAOD. } \\
\text { DI N TO DI N }\end{array}$ & 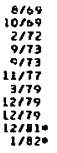 \\
\hline
\end{tabular}
average meanjigiess' iscile factor = 1.01

17 N*1/2IIT9OI REAL PART OF POLE POSITION (MEVI

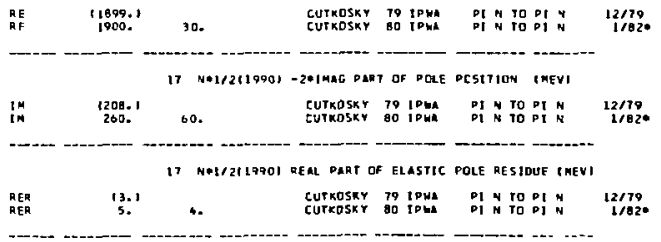

OCHNACH1 SO PL 268 O6!

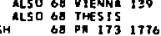
GOTKE 65 P 1001417 DEAHS OQ ON 1051791 Erro 70 XIEV CONF

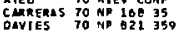

\section{REFERENCES FOR $N * 1 / 2117201$}

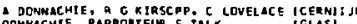

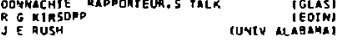

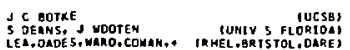

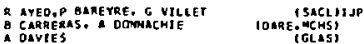


Data Card Listings

For notation, see kev at front of Listings.

Baryons

$\mathrm{N}(1990), \mathrm{N}(2000), \mathrm{N}^{\prime}(2080)$

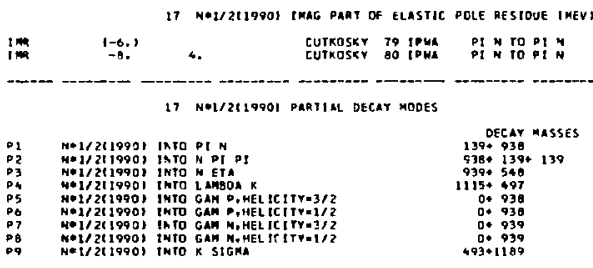

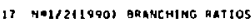

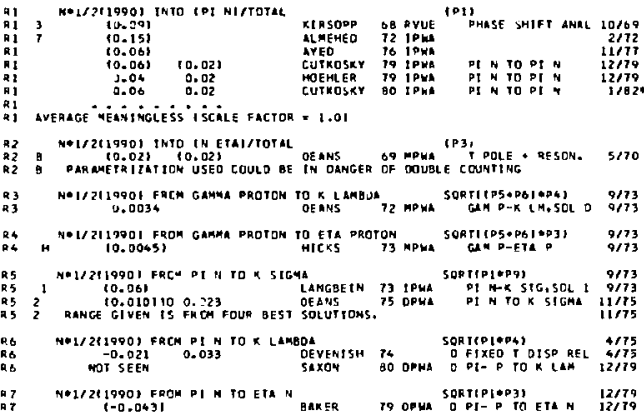

Wr

17 N*1/2119901 PHDTON OECAY AHDLICEY**-1/21

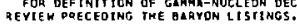

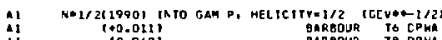

$\begin{array}{lcc}11 & \begin{array}{c}0.0401 \\ 0.001\end{array} 0.040 & \text { BARBOUR TB DPMA } \\ \text { CALHFORD BO OPWA }\end{array}$

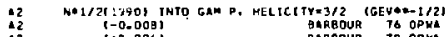

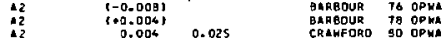

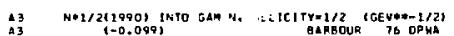

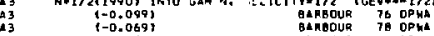
t-0.069)
-0.078
0.030

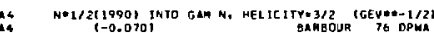

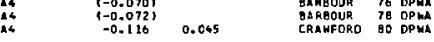

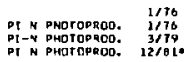

PI N PHOTOPROD. 1176

DI 4 PHOTOPACD. T/7\% PI-A PHOTAROD. $3 / 76$ P4DT0paOO.

OI N PHOTODROU. PI-A PNOTOPnaD. $3 / 3$
FOR DEFINITION OF GAHMA-NUCLEDH DECAY AMPLITUOES, SEF MINIPI-N PHOTOPHOD. $3 / 79$

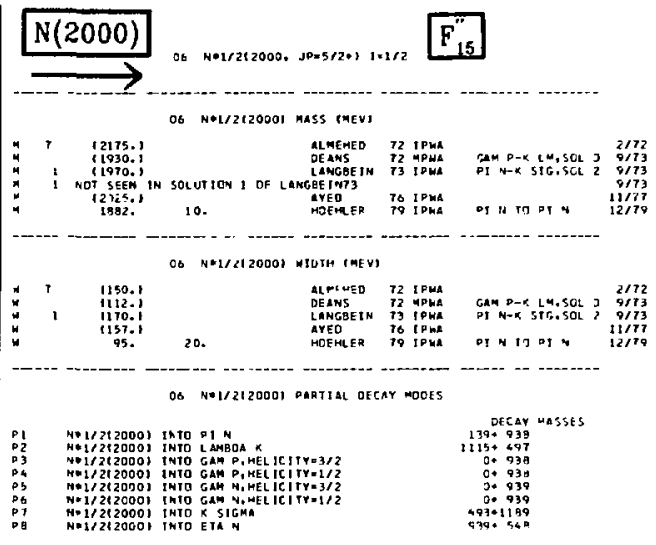

CQ NA1/5I 20003 BDANCHING OATIOS

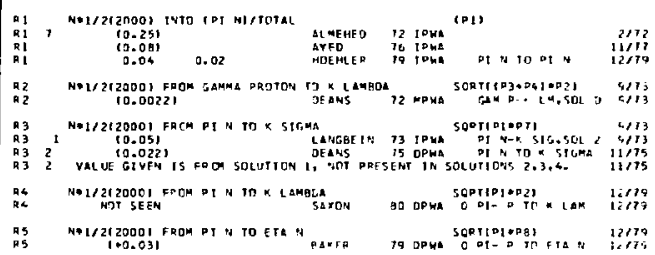

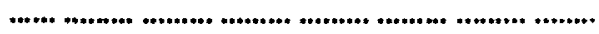

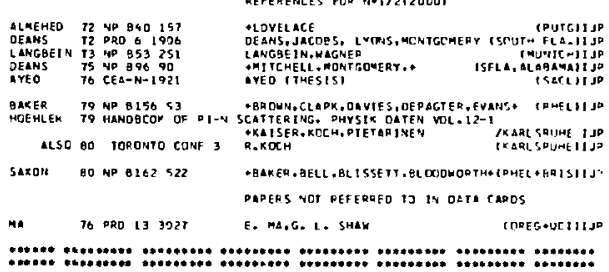

*

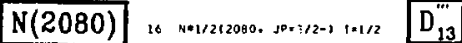

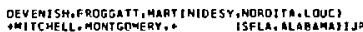

AYEO I THESISI
1. M. BRREOUR, R. L. CRAHFORO TSACLIIJP

BARGOUR, CRAMFORO, PARSONS IGLAS,

-BRONN, CLARK, OAVTES, OEQAGTER, EVANS, IRHELII JP

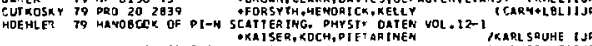
aLSO BO TORONTO CONF 3 R RAOCH

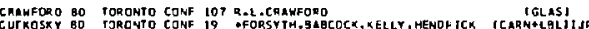
SAKDH 60 ND B1 52 S22 PADERs NOT REF ERPEO TO IN DATA CaRdS

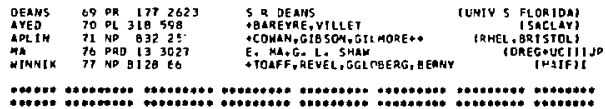

THERE IS SOAE EVIDENCF THAT THO RESONLACES EXIST IY

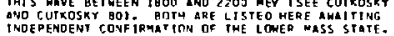

18 $N=1 / 2(2080)$ MASS (MEV)

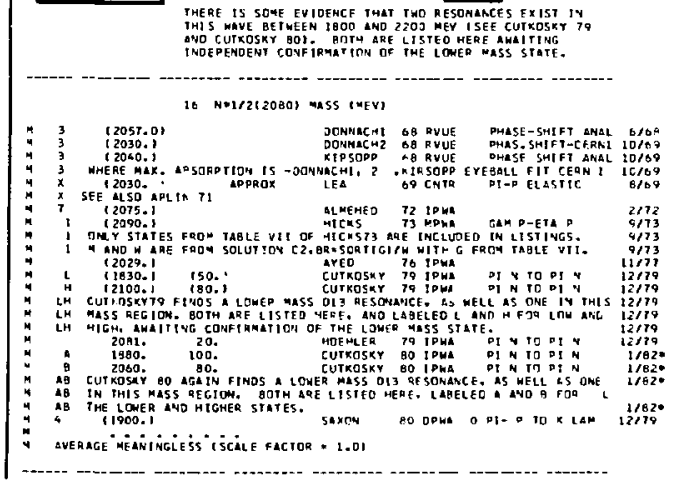


Baryons

$N(2080), N(2100)$

16 NO1/202030) W10TH IMFV

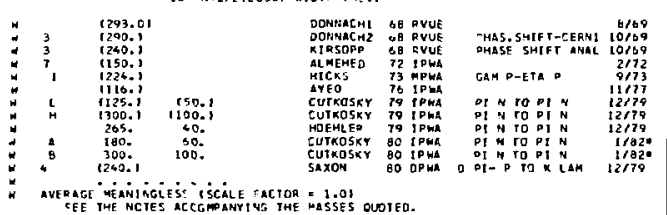

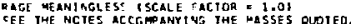

16 V+1/zIzOgOI REAL PART OF POLE POSITIOH IKEYI

\begin{tabular}{|c|c|c|c|c|c|}
\hline $\begin{array}{l}\text { RE } \\
\text { AE } \\
\text { OE } \\
\text { AE }\end{array}$ & $\begin{array}{c}11818.1 \\
1297.1 \\
: 990 . \\
20 \%\end{array}$ & $\begin{array}{c}100 . \\
7 \% .\end{array}$ & $\begin{array}{l}\text { CUTKOSKY } \\
\text { CUTKOSKY } \\
\text { CUTK0SKY } \\
\text { CuTKOSKKY }\end{array}$ & $\begin{array}{ll}70 & \text { ip } \\
79 & 1 p \\
80 & p p \\
80 & p p\end{array}$ & $\begin{array}{l}\text { IPFA } \\
\text { IP } \\
\text { IPHA } \\
\text { IPHe }\end{array}$ \\
\hline
\end{tabular}

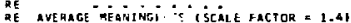

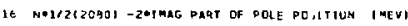

\begin{tabular}{|c|c|c|c|c|c|c|}
\hline $\lim _{14}$ & $\begin{array}{l}\$ 122.1 \\
1300.1 \\
100 . \\
200 .\end{array}$ & go. & 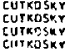 & 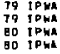 & 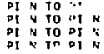 & $\begin{array}{r}12179 \\
12179 \\
1 / 820 \\
1 / 62\end{array}$ \\
\hline
\end{tabular}

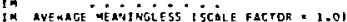

le VERTP?SOO REAL PART OF ELASTIC PCLE RESIDUE CHEYI

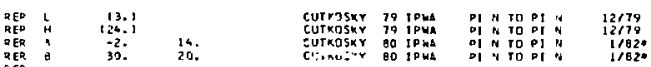

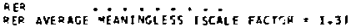

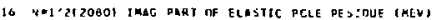

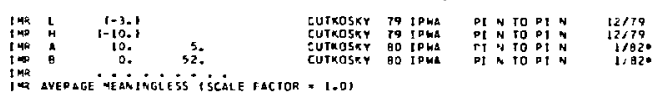

i

16 $\mathrm{H}+\mathrm{L} / 2$ T2OBOI PARTIAL DECAT MODES

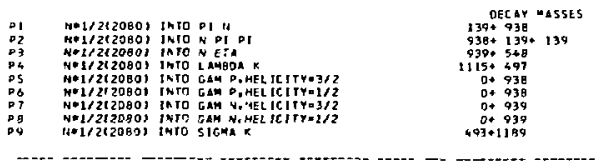

16 NO1/2I20802 BRanCHING QATIOS

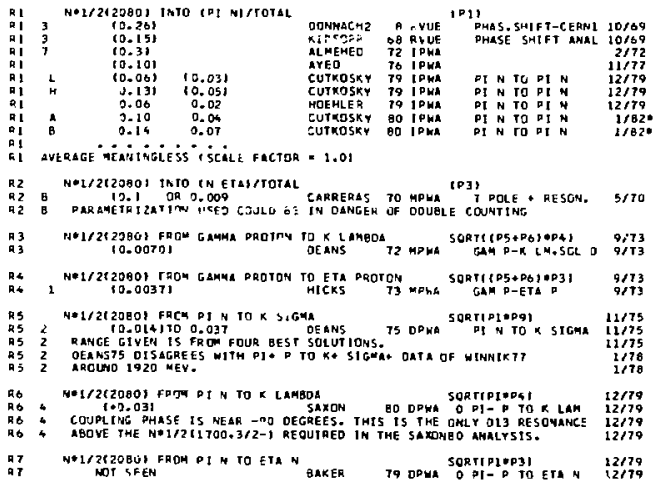

\section{Data Card Listings \\ For notation, see key at front of Listings.}


Data Card Listings

For notation, see key at front of Listings.

O4 NE1/2121001 BRANCHIVG RATITS

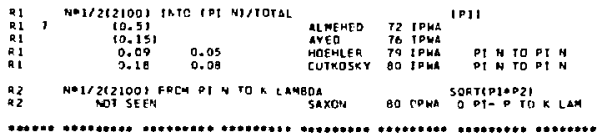

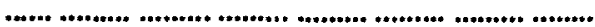

REFERENCES FOR N $11212100 \%$

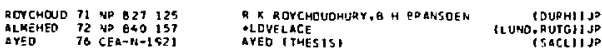

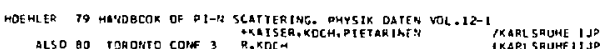

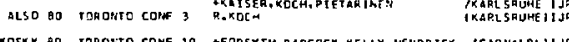

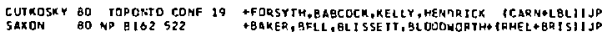
papers Not referafo to In oata caros

MA TO DQD 133027 E. PA.G. 6. 5HAW COREGOUCIIIJP * $N(2100) \quad 132 \quad N * 1 / 2 t 2100 . J P * 1 / 2+1 \quad 1=1 / 2 \quad P_{11}^{101}$

192 NO1/212100I MASS IMEN

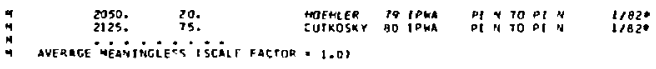

132 s.1/2t2100) W3DII (NEV)

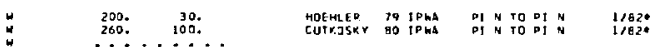

- ave gage méantigciess iscile factor a 1.01

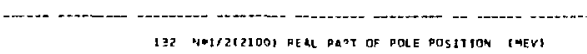

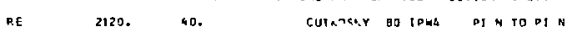

132 N"1/2121001 - 5*IMAG PART DF PCLE POSITION IMENS

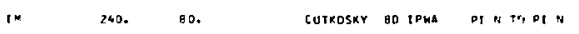

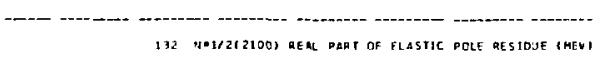

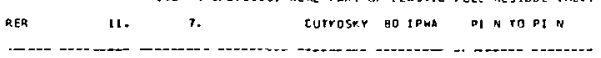

117 M*1/2(2100I THAG PART OF ELASTIC PCLE RESTOUE (HEV)

1. Be 0 b.

132 ME1/262100, PADTIAL DECAY RODES

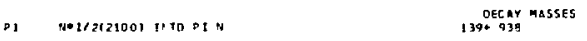

132 He1/202100) BKANCHINE. RATIOS

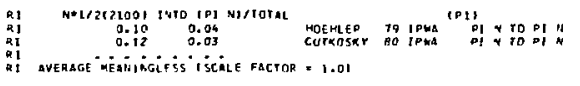

REFEREHCES FOR NO1/2121GOI

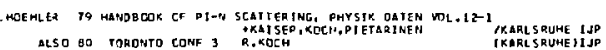

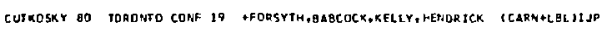
. 2100 UEV REGION - PRODUCTION EXPERIMENTS

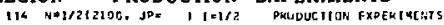
RESOHAMCE-LIKE BUMP OBSERVED IH PP TO PIN PI P AT CEEM

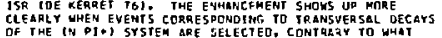

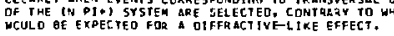

IIS N*I/2I210OI HASS IMEVI IPAOD. EXPERTMENTEI

$1 / 824$

18B2:
116 NA1/2TZ1OOI PARTIAL OECAY HODES IPRCO. FXP.

D1 $N=1 / 2(2100)$ INIO PI $\mathrm{S}$

OEC MES MES

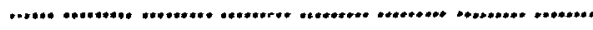

REFERENCES FOR NOH 2121001 PKCG. EXPERIMENTS

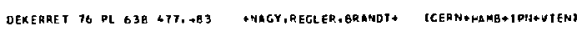

:

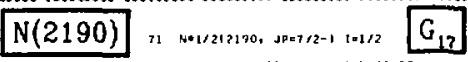

IMIS RESTHAHCE IS NELL ESTHGLISHEC.

11 Ma1f?I21901 MaSS IMEV)

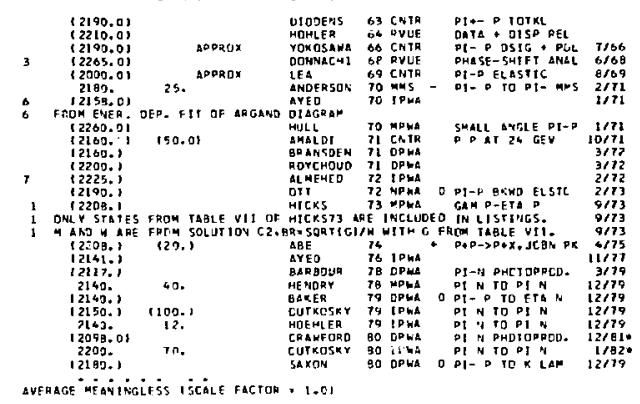

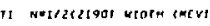

\begin{tabular}{|c|c|c|c|c|c|}
\hline 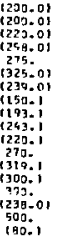 & $\begin{array}{c}50 . \\
1100 . \\
30 . \\
150 .\end{array}$ & 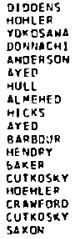 & 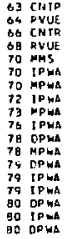 & 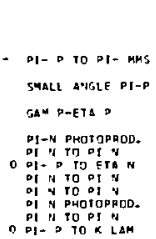 & 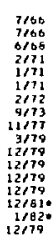 \\
\hline
\end{tabular}

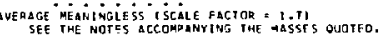

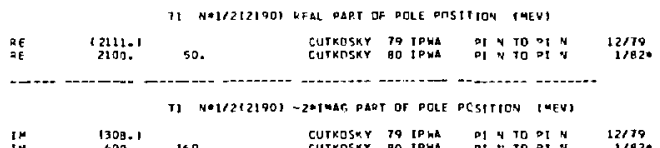

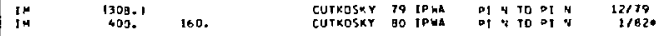

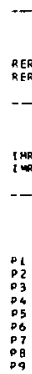

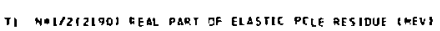

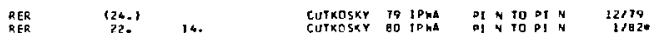

11 NO1/2121901 IMAG DART OF ELASTIC PCLF RESIOUE IMEUI

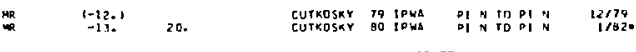

II N*IZZZITEI DARTIAL DECAY MCDES

No1/2t21901 15TO PI H

Nol12(2190) INTO LAMBOA $K$

NEL/Z121901 FNT GAM P, HELICI IYY=3/2

NOLZ2(2190), INTO GAM P,HELIC ITY=3/2

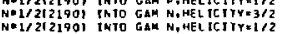

N*1/2t2170) INTO ETA N, 
Baryons

$\mathrm{N}(2190), \mathrm{N}(2200)$

JI N*1/212190I ERAMCHING RATIOS

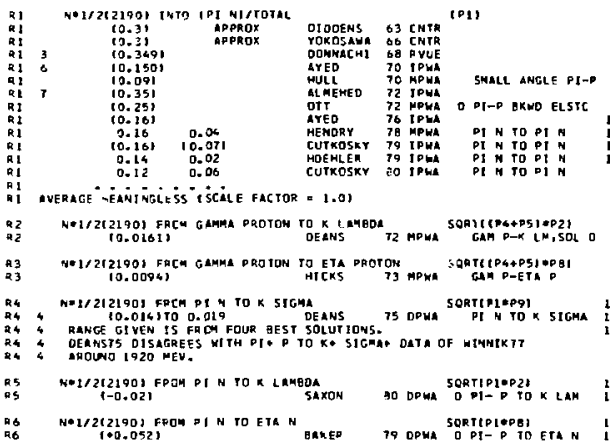

11 Ne1/zL21901 PHOTON OECAY MMPLICEVE*-l/zI

FOR DEF INIT1CN OF GAMAA-WUCLEON DECAY AMPLITUDES, SEE MINI -

REVIE PRECEDIAG THE BARYOU LISTIMCS.

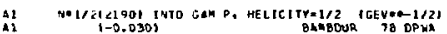

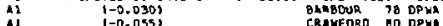

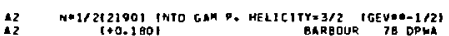

$\begin{array}{lll}12 & 1+0.1801 & 101 \\ A 2 & 10.0811 & \text { BARBOUR }\end{array}$

13 NE1/2t2190) INTO GAM N. MELIC ITY=1/2 IGEVE-1/21

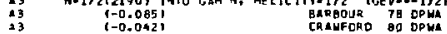

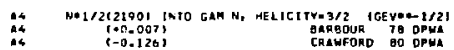

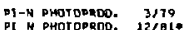

PI-n Photopado. $3 r 78$

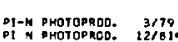

CRTWFORD

P1-N PHOT OPRED. $3 / 79$

REFEREHCES FOR $N * 1 / 2121901$

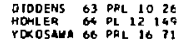

DENKJNS, KYCIA, RiLEY

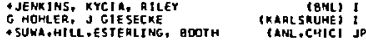

DONMACH 68 PL 26 B 161

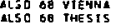

LEA BQ PL $29 B$ SEA A OONMACHIE, R G KTRSOPP. C LCVELACE (CEENIIJP DOMNACHIE ADPDORTELP - 5 TLLK TL TELS:

ANOERSJY 70 PRL 25, E99 LEA, DADES, MARD, CONAN.A TAHEL, QRTSTOL, OAREI

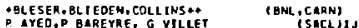
P. AYEO, P BAREYRE, G VILLET THUL, R LEACOCK
THACLII

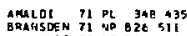

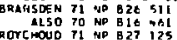

ALMEMEO 72 NP Q4O 157

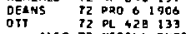
OIT ALSO 72 PL 42 LCG 133

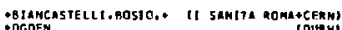

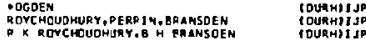
- Lovelace OEANS, J CCOBS, LYONS, MONTEONERY ISOUTH FLA., DH

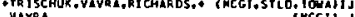

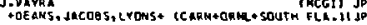

Aet $74 \mathrm{PL} 53 \mathrm{e} 116$ HAL SPEC TOR , GOMEERONIT?, CRUTG.UPAJ.FSUI WMITCHELL, MOMTGDMERY, +
ATEO (THESTS) (GLA)

IYED T6 CEM-N-192

QARBOUA TE MP B141 253 MEMDRY TS PRL 41522

CUTROSXY 79 PQO 202839 BAPBOUR, CARMFORD, DARSOHS CGLESI

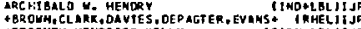
TFOASYTH, HENDR ICK, KELLY TY TARMOLBLIJJP ALso 80 Tonokito conf

SCATTERIMG. PHYSTK OATEN VOL.12-1
AKAISER, KOCH, DIETARTHEM

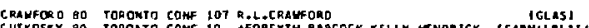

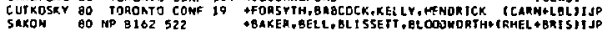
papers hot aefeaneo to th data caros

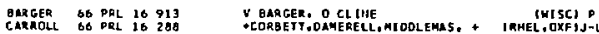

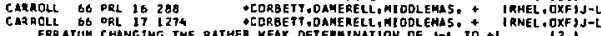

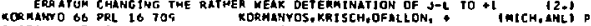

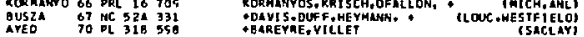

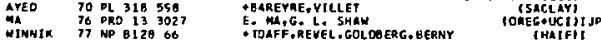

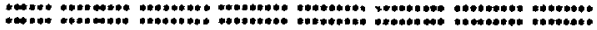

Data Card Listings

For noiation, see key at front of Listings.

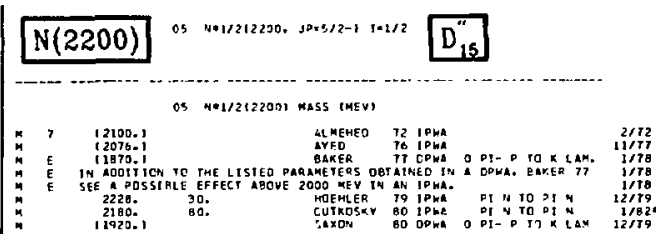

(1)

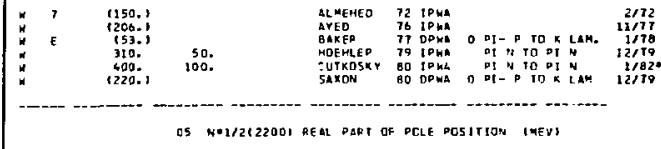

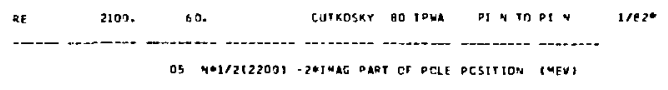

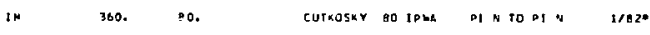

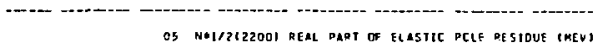

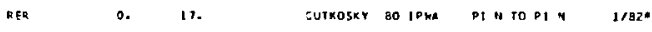

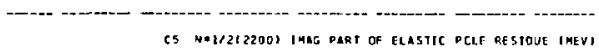

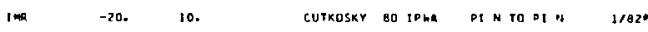

O5 H*1/2(2200) PARTICL DECAY MEOES

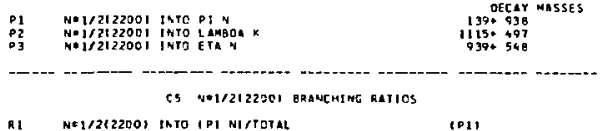

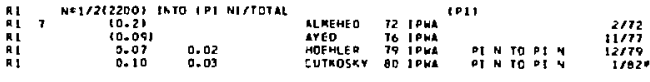

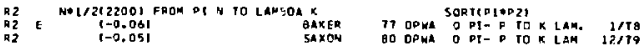

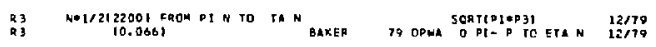

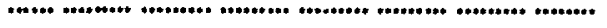

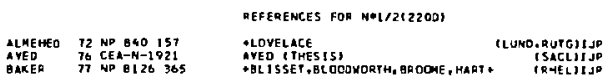

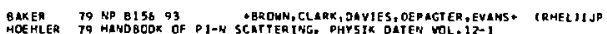

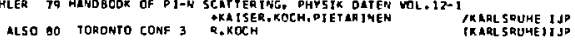

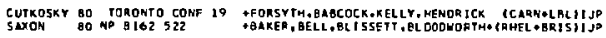
papens hot refergeo to in oata camos

MA TO PRO $13302 T$ E. MH.F. L. SHAY CDRECUCTHIJP

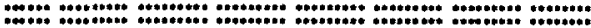

\section{MEY REGION - PRODUCTION EXPERIYENTS}

L11 NWI/2L2200, SPETI $101 / 2$ PMOOUCTION EXPERIAENTS ME LIST hete gumps OBSERved th the range 1900-2500 meV. 111 N=1S2I2200I MASS IMEV! (PROD. EXP.)

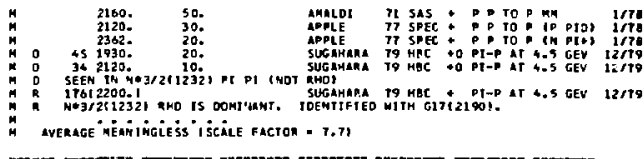




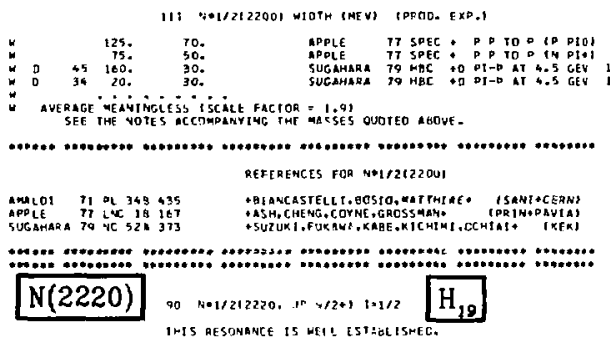

\section{प0 M-1/2t2220) MASS (MEUI}

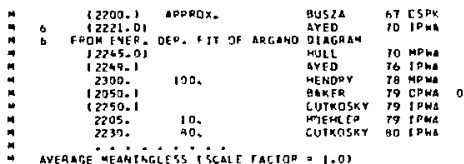

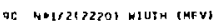

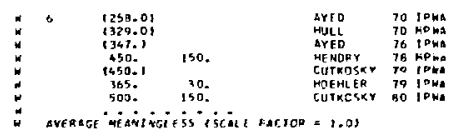

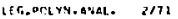
SYALL ANTLE PI-P T1/71

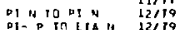

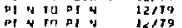
pl in of aveasige meantigless iscale tactop = 1.01

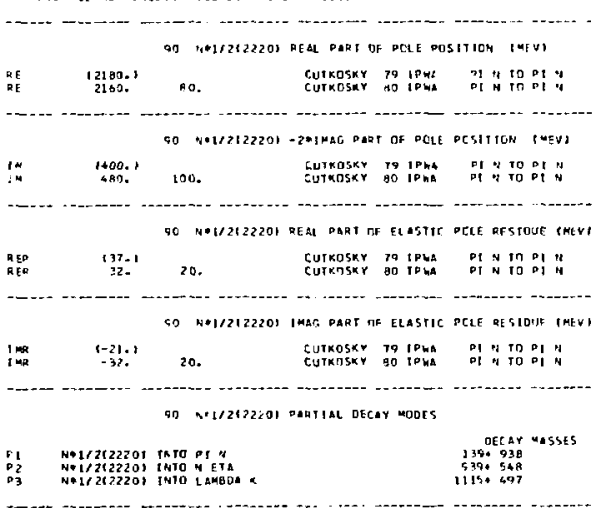

कD Nol/21?2z01 BRAHCHING RATIOS

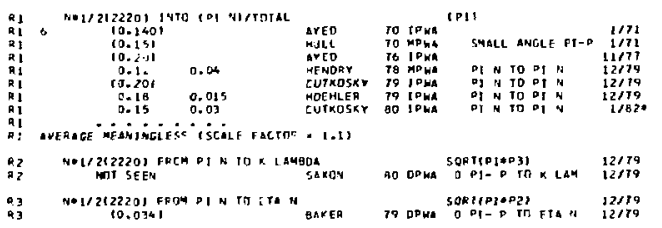

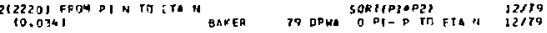

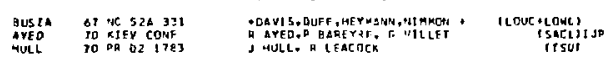

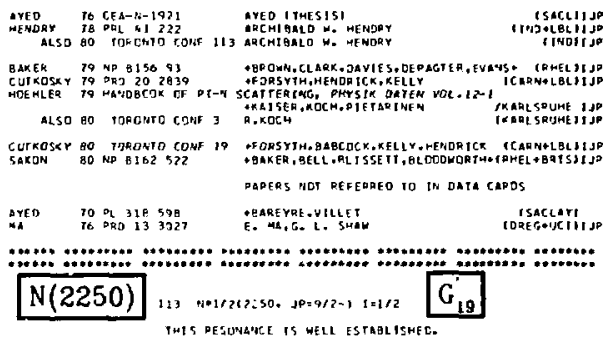

113 NO1'21225OH MASS IMEVI

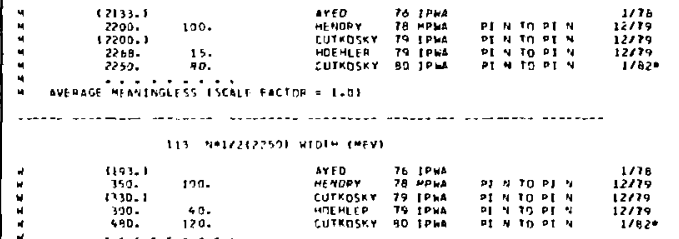

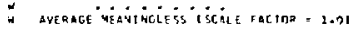

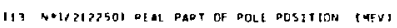

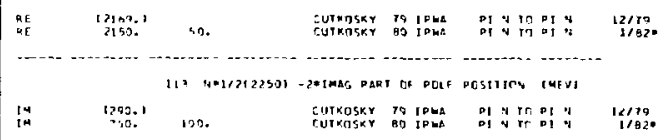

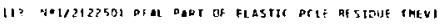

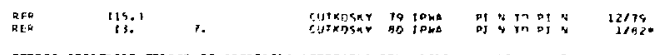

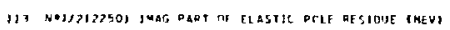

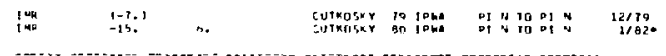

111 NeITZCZSOI DARTIAL DFCAY MODES

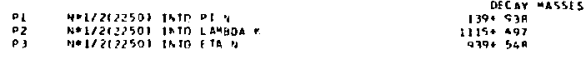

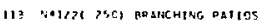

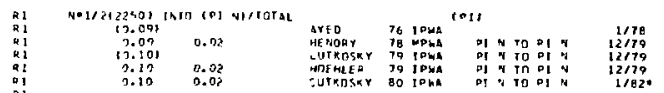

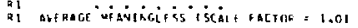

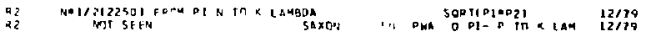

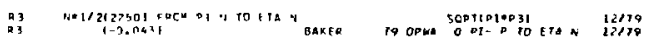

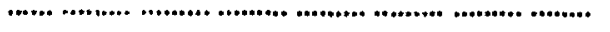

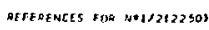

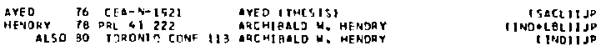

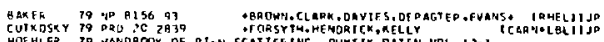

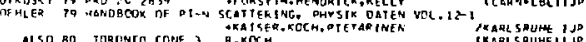

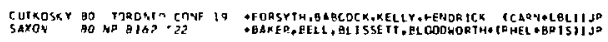
r.ten 
Baryons

$\mathrm{N}(2600), \mathrm{N}(2650), \mathrm{N}(2700), \mathrm{N}(2800)$

$\mathrm{N}(2600), 120$ Ne1/212600. ر0.21/2-1, 1-1/2 $\mathrm{I}_{111}$

120 P०1/2126001 HASS (HEYS

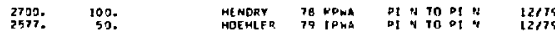
average 4éaitigiejs iscale factor = $1=11$

120 NE1/2C20001 W101h (MEV)

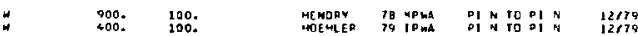
- everage menaticifis iscile factar $=3.51$

IzO N*ISZTZOOOI PARTIAL DECAY MCOFS

PI $\quad 4 \cdot 11242800) 14 T 014$

DEC AR MASES

120 N $1 / 212600$, RPANCHING DATIOS

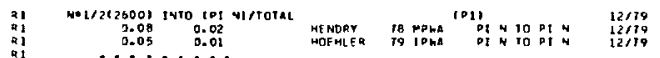
Ri average Mérit:

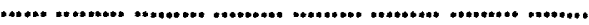
REFERENCES FOO Y*1/2t2000

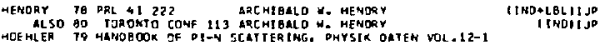
HOE HLER 19 MAMOBOOK OF DI-W SCATIERTHG. PHYSIK ONTEN VOL.12-1

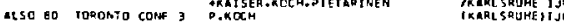

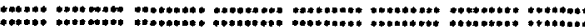

\section{HEV REGION - MISCELLANEOUS EXPERIMENTS}

72 NA 1/212650. ADYCHOUDHUAY 71 CLASH F1S124001 AHO GL9126001 TO BE DOSSIBLE FESONANCES. GRANSDEN 71 FIND THE DTSSIBLE PI N PWA S ESTRQE TSH THE EXISTENCE CF A JPA $=11 / 2$ - STATE IH IMIS REGION. EUT THE DOSSIBILITY IMAT THERE ARE ALSO OFHE STATES REMALNS. SEF THE MINI-REVIFW

12 N* $1 / 2(2650)$ HASS IMEV) (PROO. EXP.

\begin{tabular}{|c|c|c|c|c|c|}
\hline $\begin{array}{l}12700.01 \\
12600.01 \\
12600.01 \\
12633.01 \\
2649.0\end{array}$ & $10.0^{\text {ADPROX }}$ & $\begin{array}{l}\text { AL VERET } \\
\text { HOHLER } \\
\text { AHMLIG } \\
\text { GARGER } \\
\text { CITHOU }\end{array}$ & 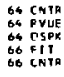 & 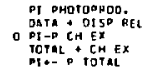 & $\begin{array}{r}11 / 67 \\
1 / 68\end{array}$ \\
\hline & $72=1 / 2126$ & WIDTH [MEV] & IPRCO. & $E \times P_{-1}$ & \\
\hline $\begin{array}{c}1100.01 \\
2200.01 \\
425.01 \\
363.0\end{array}$ & 20.0 & $\begin{array}{l}\text { AL VAREZ } \\
\text { HOHLER } \\
\text { BARERER } \\
\text { CITRON }\end{array}$ & $\begin{array}{l}\text { BS CNTR } \\
\text { O4 RVUE } \\
\text { O6 FIT } \\
\text { OG CNTR }\end{array}$ & TOTAL - CHEX & $\begin{array}{r}71660 \\
1167 \\
1106\end{array}$ \\
\hline
\end{tabular}

12 NO1/2T2S5O) DARTTAL OECAY MDOES (PROD. EXP.)

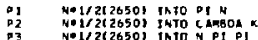

DECAY MASSES

$139 * 939$

$1115+497$
$538+139+139$

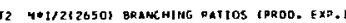

R1 NELZ212650\% THTO RP! NIFTOTAL

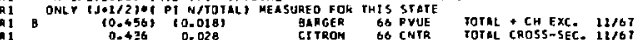

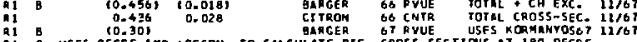

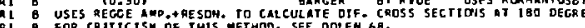

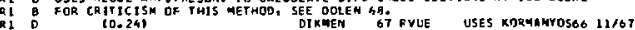

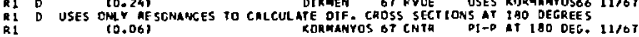

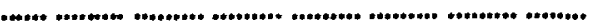

REFERENCE. OOR NA1/2126501 1\%ROR, EXP.1

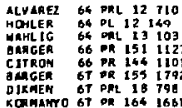

-BAR-YAM, KERA, LUCKEY, OSBOAME. - IMIT,CEA

G HOHLER, G GIESECKE

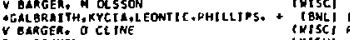

F 1 OIKMEN MRISCM, OFALLON, - (MICH, ANL)
Data Card Listings

For.notation, see key at front of Listings.

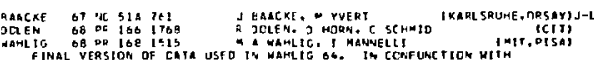

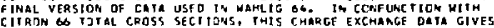

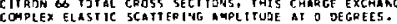

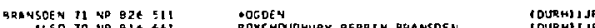

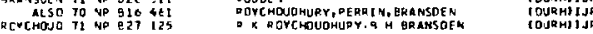

*men

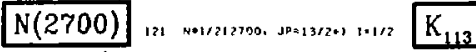

121 N"L2127001 WhSS (MEU)

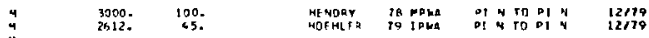

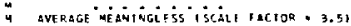

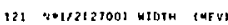

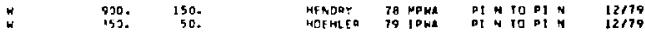

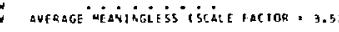

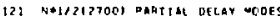

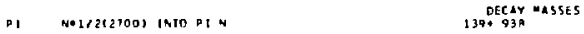

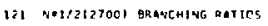

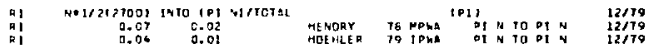

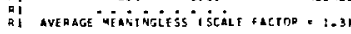

Q SEFEDENCS FOH N*I/2123T:

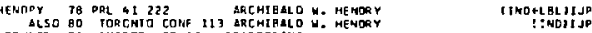

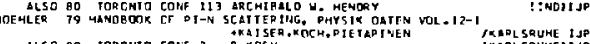

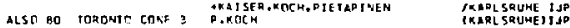

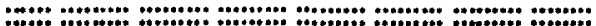

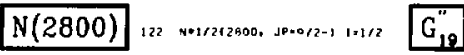

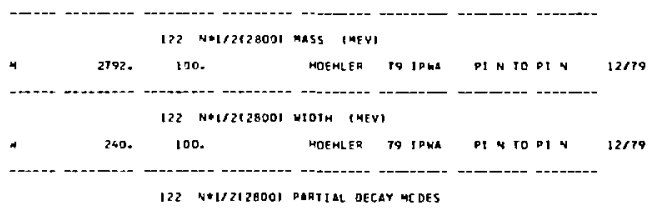

PI N*1/2128001 INTOPIN

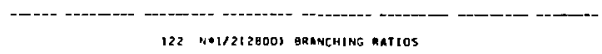

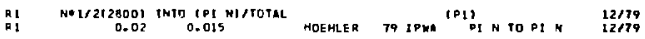

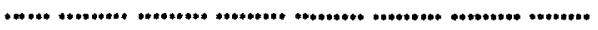

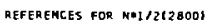

MOEHLER 79 MAYDECOK OF PI-N SCATTERING. PHYSIK DATEM VOL.12-1

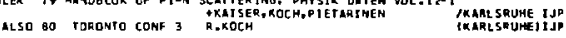
- 
Data Card Listings

For notation, see key at front of Listings.N(>3000), N(3030), $\mathrm{N}_{?}(3245), \mathrm{N}(3690), \mathrm{N}_{?}(3755)$

\section{>3000 MEV REGION - FORMATION EXPERIMENTS}

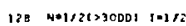

WE GST HER TO1/Z RESOIZANEES HIT MASS GREATER THAR ABOUT 3.O GEV WHICH HAYF GEEN SEEN IH A STMGLE PARTIA

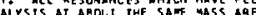
GIVEN A SFPAQATE LISTING WITH THE AOPRCORIATE OUAITUM SUMaE RS.

$128 \quad 1+1 / 2$ I $>30001$ MaSS IMEVI

\begin{tabular}{|c|c|c|c|c|c|}
\hline $\begin{array}{l}3500 . \\
3900 . \\
4190 .\end{array}$ & $\begin{array}{l}200 . \\
200 . \\
200 .\end{array}$ & $\begin{array}{l}\text { HFNDEY } \\
\text { HENDRT } \\
\text { HENDRY }\end{array}$ & $\begin{array}{l}\text { To mpwo } \\
\text { TA 4awh } \\
\text { TE mowe }\end{array}$ & $\begin{array}{ll}P \uparrow & N \\
P I & N \\
D T & N\end{array}$ & $\begin{array}{l}1115 \\
4117 \\
0119\end{array}$ \\
\hline
\end{tabular}

averace miavi intiess iscale factor = 1.51

12A N-1/2ST3000) W10Th (MEN)

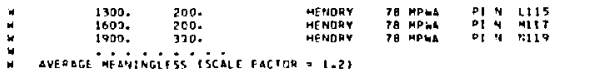

158 N*ISTIJ3ROOI PAETIAL OECAY mDEES

$\rightarrow$ No1/20330002 1NTE का

OECAY MASSES

128 N०1/21>3JONI BRANCHING PATIOS

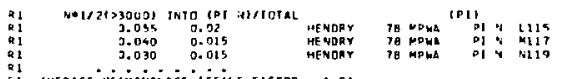

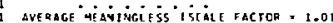

REFERENCES FOR N*1/21>3000

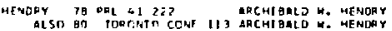

IINO+LEL II JP

A.

$\mathrm{N}(3030)$

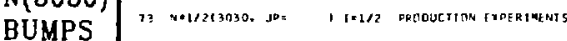

(3090. MI
(3030.01

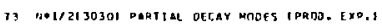

PI

139. DECAY MASTE

139. 738.139 .139

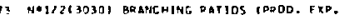

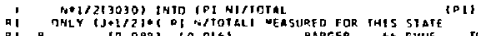

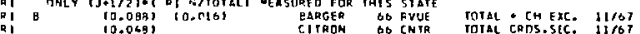

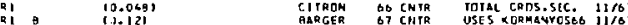

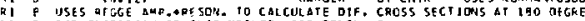

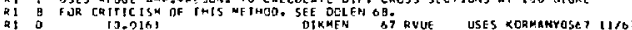

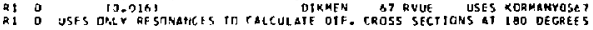
a cace

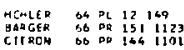

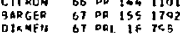

DEFEHEHCFS FOA NEI/2I303OI IPATO. EKO.

F HUHLE. I TIESECKE TKARL SRUHEI I

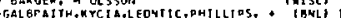

Y BARGER, D CLING
Y DIKGEN

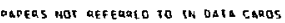

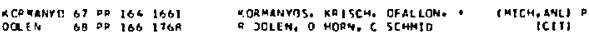

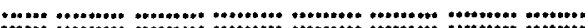

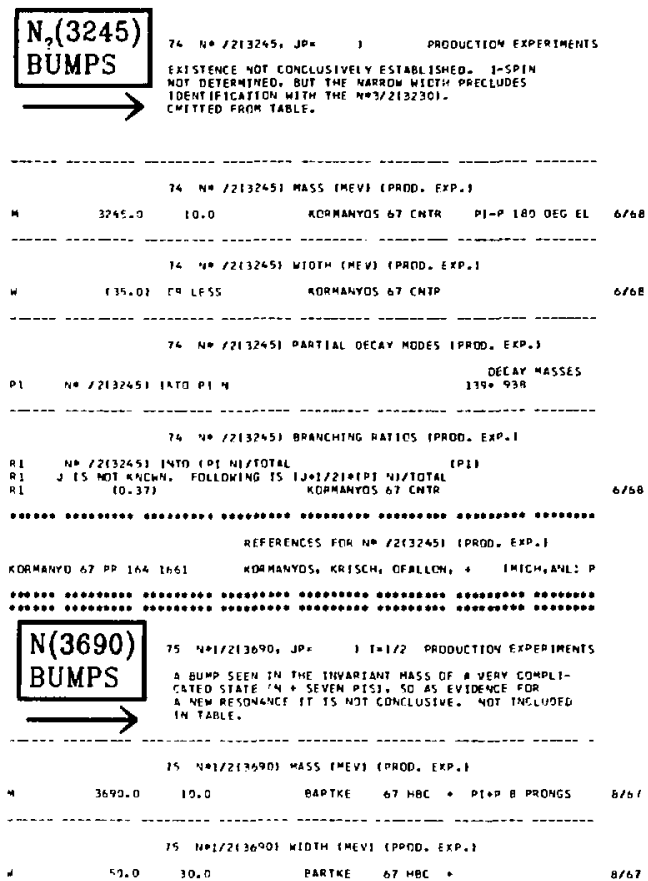

$57.0 \quad 30.0$

PARTKE BT MEC.

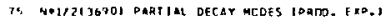

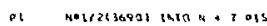

DfCAT Masses

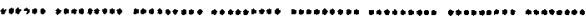

REFEAENCES FDR N"1/2130901 1PRJD. EXP.1

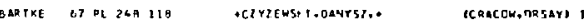

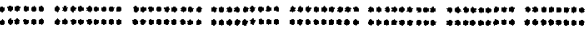

$\mathbb{N}_{n}(3755)$ TE N"1213755, JPa 1 PADSUCTICN EXPERTLENTS

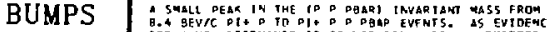

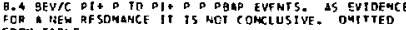
FROY TABCE.

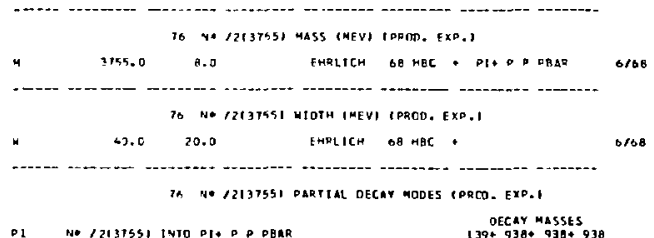

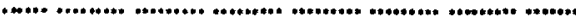
REFERENCES FOR NE 12137551 (DROD. EXD.)

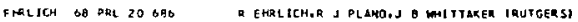

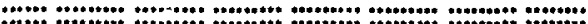




\section{Baryons \\ $\Delta(1232)$}

Data Card Listings

For notation, see key at front of Listings.
:

\section{$S=0 \quad I=3 / 2$ NUCLEON STATES ( $\Delta)$}

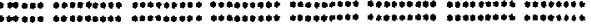

$\Delta(1232) 33$ N*2/211232, JP=3/2+1 $1=3 / 2 \quad P_{33}$

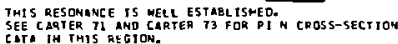

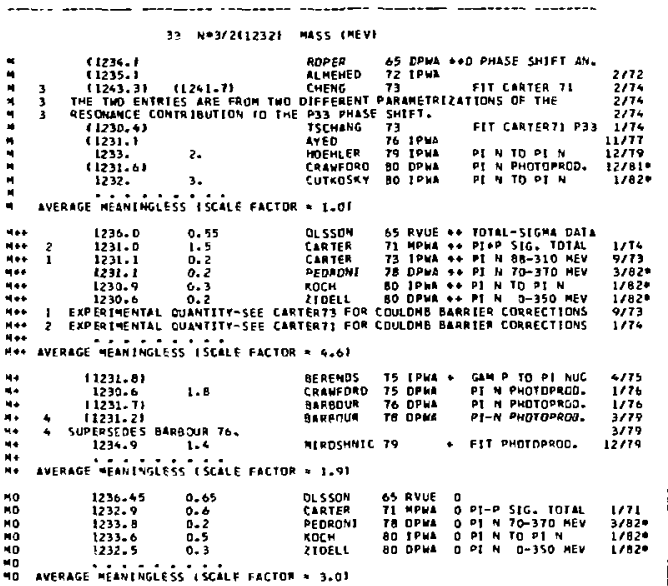

\begin{tabular}{|c|c|c|c|c|c|c|}
\hline 3 & 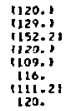 & $\begin{array}{l}1145.81 \\
5 . \\
3 .\end{array}$ & 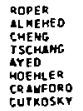 & 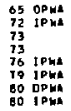 & 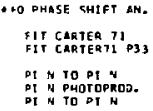 & 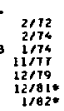 \\
\hline
\end{tabular}

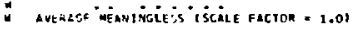

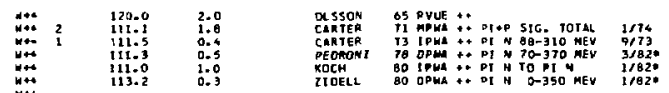

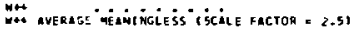

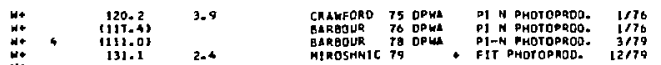

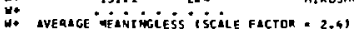

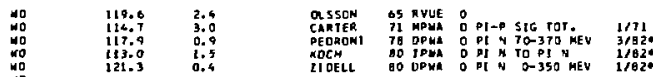

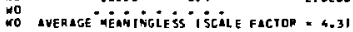

33 (N*O) - (K*++1 NASS DIFFERENCE IMEV

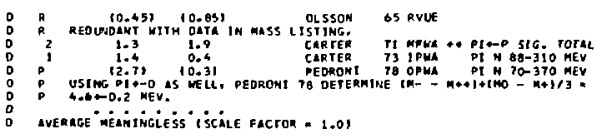

31 (ANO) - INEN+1 MIOTM OTFFEREMCE (MEVI

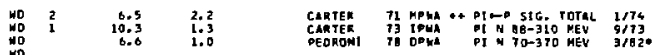

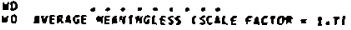

33 N.3/ZC12321 REAL DART OF DCLE DQSITION IWEVI

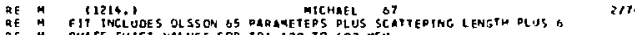

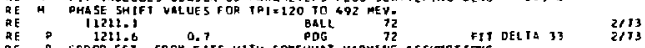

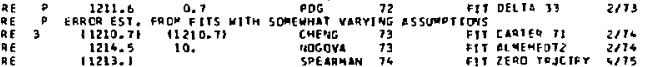

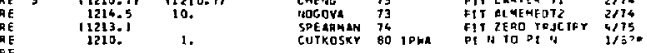

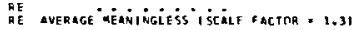

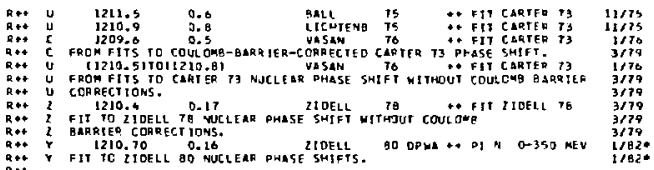

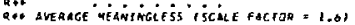

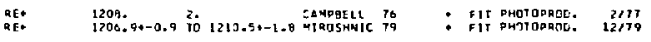

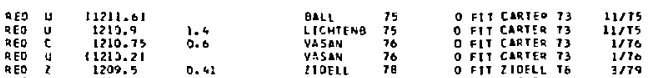

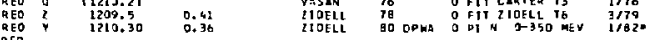

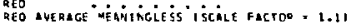

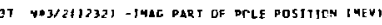

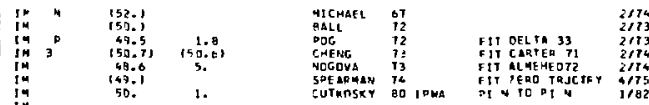

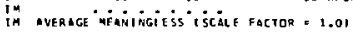

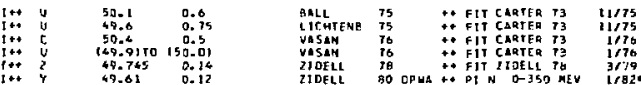

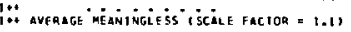

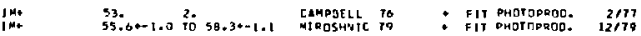

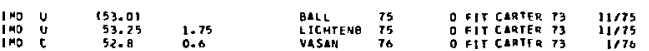

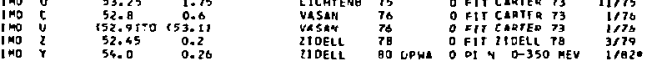

ITO

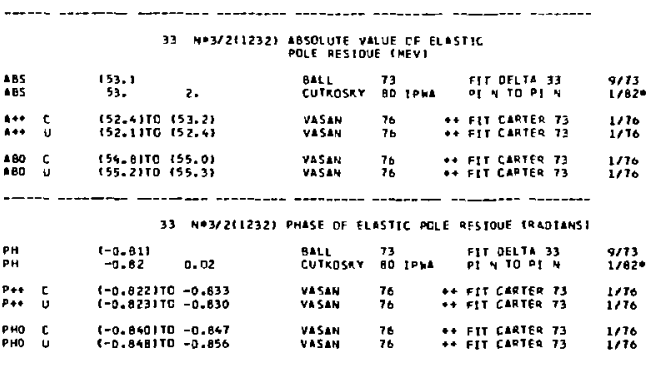

33 N*3/21L232) PHASE DF M1+(3)21 PHOTOPROONCT1ON

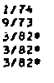

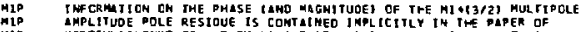

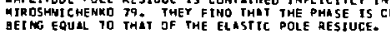

33 Na3/21123EI MLGNETIC MOMENT (NUCLEAR MGGKIONS)

1+4.TJTO 1+6.T1 MEFXENS TB

PI D TO PI P GAN 12/79

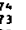

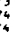
. (n) 


\section{Data Card Listings}

For notation, see key at front of Listings.

33 NE3/ZILZ32I PARTIAL OESAY MDDES

\begin{tabular}{|c|c|c|c|}
\hline $\begin{array}{l}p_{1} \\
\text { p? } \\
03\end{array}$ & 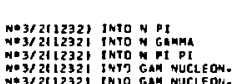 & MEL tCtTr-1/2 & $\begin{array}{l}\text { DECAY MLSSEE } \\
939+139 \\
938 * 130 \\
938 * 139+139 \\
0+838 \\
0 * 989\end{array}$ \\
\hline
\end{tabular}

3) $H+3 / 2112321$ SARCMING RATIOS

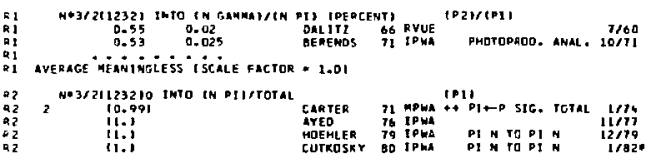

93 N०3/2112321 PHDTON OECAY ANPLIGEYMA-1/21

FOR OEFINITICN OF GaMMA- MUCLEON OECAY AMPQITUDES, SEE MIMI-

:

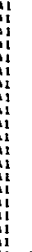

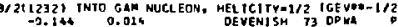

$\begin{array}{lll}-0.1+2 & 0.006 & \text { MDORHOUS } 73 \text { OPMA } \\ -0.138 & 0.004 & \text { KMIES } 76 \text { DOMA }\end{array}$

$0.140 \quad 0.006$

$-0.130 \quad 0.002$

$\mid-0.1391$

(1)

$\begin{array}{ll}-0.141 & 0.004 \\ -0.136 & 0.002\end{array}$

$4 \begin{array}{r}-0.139 \\ -0.142 \\ 1-0.1401\end{array}$

0.002
0.007

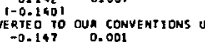

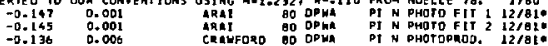

MOERHOUS 73 OPMA
KMIES
METEMLF 76 DPMA

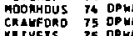

KRTVETS 75 OP
BAREOUR 74 OFWA
FELLER

PI N PHOTORRDD.

of 4 PHOTaPNoD.

PI N MOTORMOD.

P1-N Protoridoo.

OI N MDTDPRDO. I Th

TO PHTPRD, 502112170

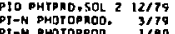

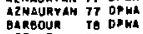

GAREOUR TO BOHA PI-N PHDTOPNOD

PI-4 PHOTOPROD. $1 /$ BOD

average ménimgléss iscile factor * 3.61

N* 3/2112321 INTO GAM HUCLEON, HELICITY-3/2 IGE YMN-1,

$\begin{array}{ll}112321 & \text { TNTO GAM NUCLEON, HEL } \\ 0.262 & 0.015 \\ -0.259 & 0.016 \\ -0.253 & 0.002 \\ -0.254 & 0.007 \\ -0.261 & 0.000 \\ -0.248 & 0.002 \\ 1-0.2531 & \\ -0.2511 & \\ -0.256 & 0.003 \\ -0.255 & 0.002 \\ -0.256 & 0.002 \\ -0.271 & 0.010\end{array}$

oI N Photophoo.

MOOHHOUS 73 OPWA
WHIES
METCAL OPW

METCALF 74 OPWA

CKAHFONO 75 DPWA
KRIVETS 75 OPWA

KRIVESS 75 OPUA
BARBDUA T6 OPWA
FELLER T6 OFWA

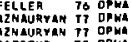

of N PHOTOPRDD.

PI N PHOTOPROD.

DI N PMOTOPROD.

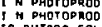

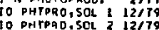

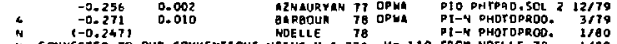

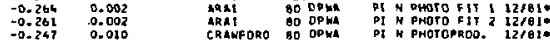

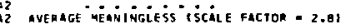

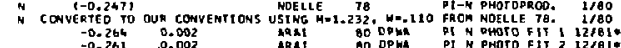

$\mid$

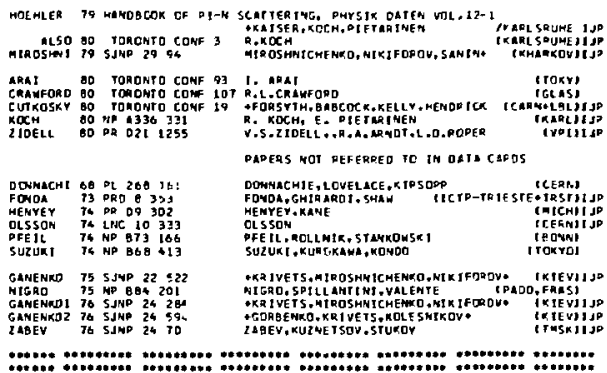

1232 YEV REGION - PRODUCTION EXPERIMENTS

OL N०3/211232. JPA3/2+1 I.3/2 PRDOUCTION EXPEA INENTS

SEE THE MINT-REVIEN DRECEDING THE N ANC DFLTA L ISTINGS
FOR A DISCUSSION OF PRDOUCFIOY EXPERIMENTS.

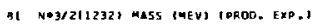

1211.

P.

ANDERSON 70 MMS
ELLIS

avg

ijizi: - is

CE IEAROD. TNCRUDES SCALF FAC TDO DF 1.01

mat

Mo: 11233.01

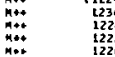

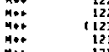

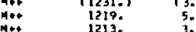

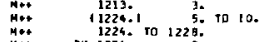

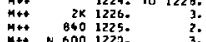

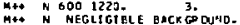

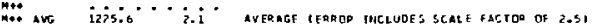

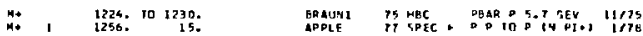

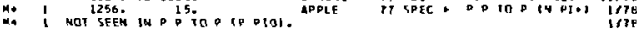

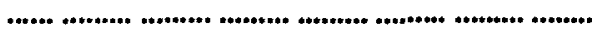

REFERENCES FON N*3/21 1232

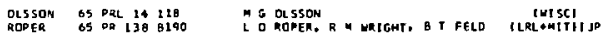

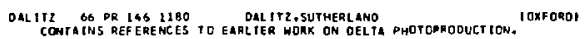

HTCHAEL 67 DR 1561677

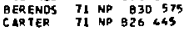

MECHAEL
IAMELII JPA

-HEAYER

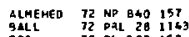

- Lovetace

[LUNO.RUTC), SO

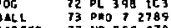

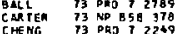

CHENG 73 PQD 72209

$400 R H D W S$
73

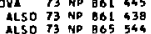

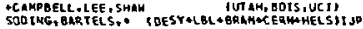

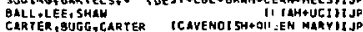

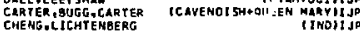

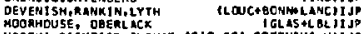

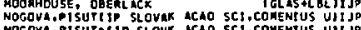

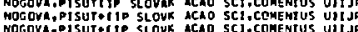

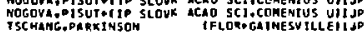

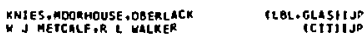

KMIES T6 PQD 92680

MDRHOSS 76 PRO 51 1

QALL 75 PKO 11117

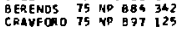

KRI VETS TS SINP 20430

LICHTEMB TS LMT 12 GTS

AYED TO CEA-N-1921

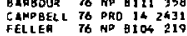

VASAN 76 NP 8106335

AINAURYA 77 EFI-2601577-T7

NEFKENS 7\% POO 18 3911

NOELLE TO

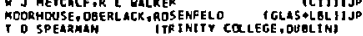

J 5 BALL,R GOLLE GOLE (UTAH)IJP

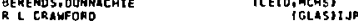

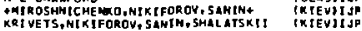

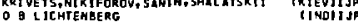

AYEO ITHESIS) R. L. CKAMFORO (SACLIEAP

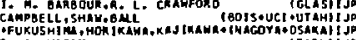
5. S. VASAN

icanis ijo

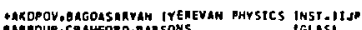

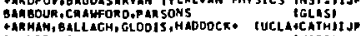

P. HOELLE

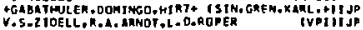

40

mo

1231.011.

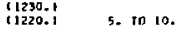

$(1261.3)$
1239.0

BRAUHI
BRAUN?

$\prod_{15}^{14} \mathrm{HBC}$

$\begin{array}{ll}\text { GIDAL } & 66 \text { OAC } \\ \text { COLTON } & 12 \text { MBC }\end{array}$

pgear p a 5.7 GeV $11 / 75$

H-

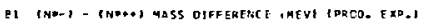

$\begin{array}{lll}0 & -1 \\ 0 \\ 0\end{array}$

GTOAL
DAKHNO

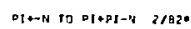

61 D०3/211235) HIOTH IMEVI (PRCO. FXO.)

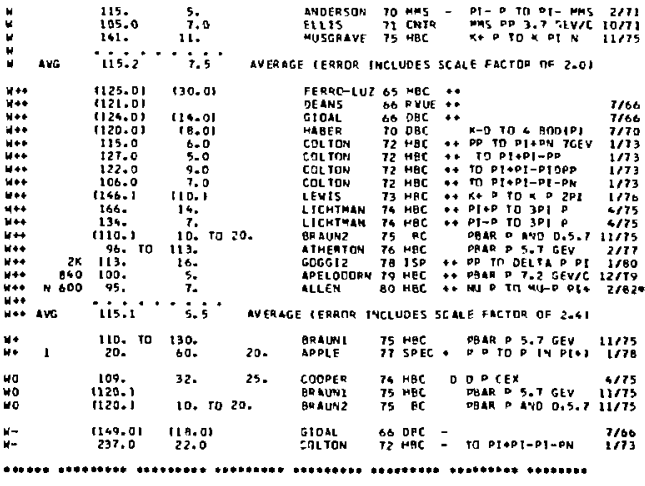

$\begin{array}{ll}7.9 & 0.8 \\ 5.5 & 3.1\end{array}$ 
Baryons

$\Delta(1232), \Delta(1550), \Delta(1600)$
Data Card Listings

For notation, see key at front of Listings.

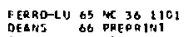

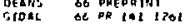

ANDERSON 70 PRL 25699

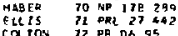

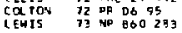

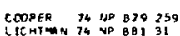

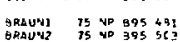

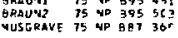

STMEIEION TE TP 51033 B

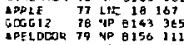

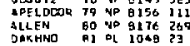

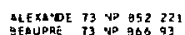

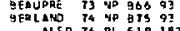

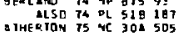
5 Thachas 75 is

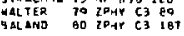

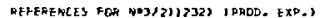

SERRO-LUZZT, GEORGE. ICERHI

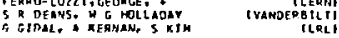

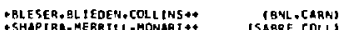

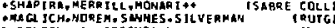
E COL TON, A KIFSCHAAUR

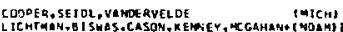

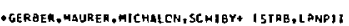

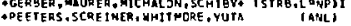

athearen.FRENCH, SKURA, BCHM. ICEAN, aqaCD,

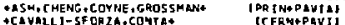

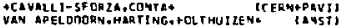

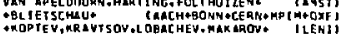

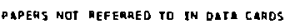

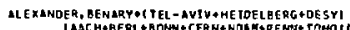

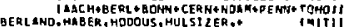

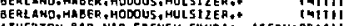

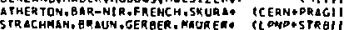

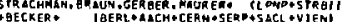

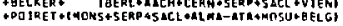

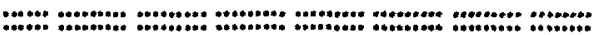
$\Delta(1550)$ 110 $4 * 3 / 211550$, sp=1/2+1 1=3/2 $\quad \mathrm{P}_{31}^{\prime} \quad 11 / 77$ 110 TH3/211550) MASS (MEVI

1177

: 5 TISSOD.

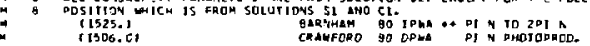

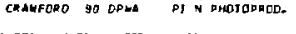

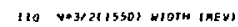

$28 \quad \begin{aligned} & 110.3 \\ & 143 ; \\ & 113 \% 0\}\end{aligned}$

LONGACRE 71 PMA P1 \& TO 2 P: N

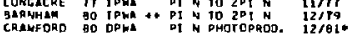

110 NE3/211550) REAL DART DF DOLE PRS ITION (MEY) $11 / 1$ at 8 1554- 39 1553.

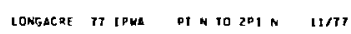

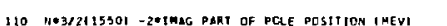

I. 8 105. CR 10.

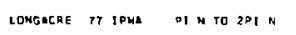

110 P.3/2E1S5OI PAOTIML DECAY MODES

D

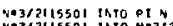

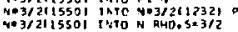

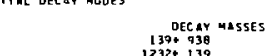

$137+938$
$1232 * 139$
$938+969$

110 Nez/2C15SO; GRanCMEHG Ratros

41,77

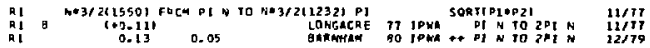

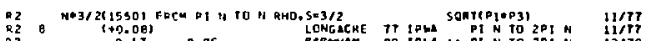

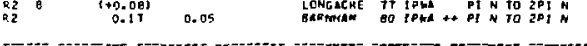

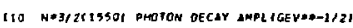

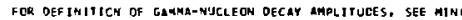
QEVIEW PRECEDIV THE GERTOH LISTINGS.

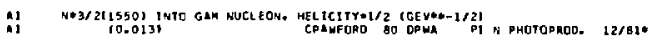

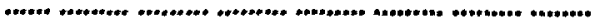

REFERENCES FOR YH3/2(1550)

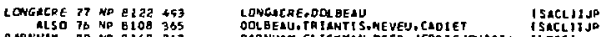

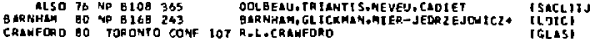
:
$\Delta(1600)$ 19 Ne3/211\%00, J0.3/2+1 1=3/2 $\mathrm{P}_{33}$

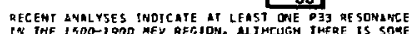

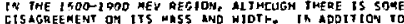
THE STATE LISTEO. THE QNALYSIS OF GAFNHAY gO IS CONSI STENT WITH SHE OAE SENEE DF ANOTHE A P33 RE SOMANC

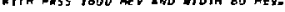
SEE ALSD THE N*3/21 Ia20, JPE3/201 LI ST ILGS.

\section{ND3/2IISOOI MASS IMEY}

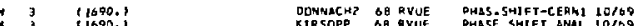

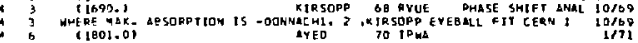

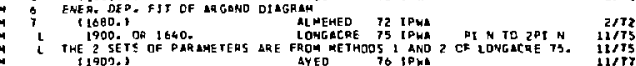

A P130.)

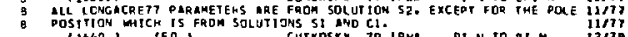

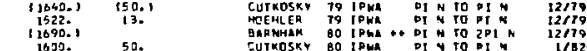
average méning ígit is "iscile factor $=1.5$

19 N*3/2110001 WTOTH IAEVI

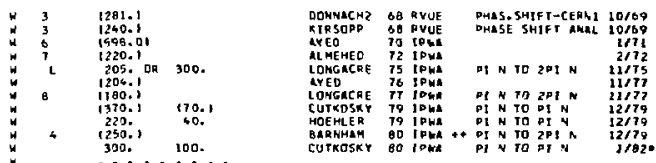

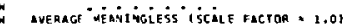
SEE THE MOTES AC COMPANY IMG MESSES QUDTED

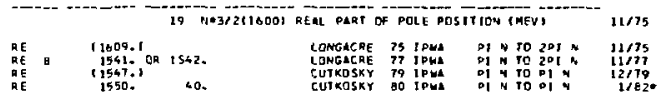

\begin{tabular}{|c|c|c|c|c|c|c|}
\hline & & $19 N * 3 / 2616004$ & -2Uthag PA & If OF PCLE & PESITION (MEU) & $11 / 15$ \\
\hline 等。 & 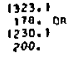 & 178. & 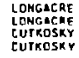 & 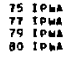 & 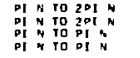 & $\begin{aligned} 21 / 75 \\
11777 \\
12,79 \\
1 / 82\end{aligned}$ \\
\hline & - & $19 \quad N=3 / 2(1800)$ & AEML Dapto & IF ELASTIC & PCLE RE SIDUE CMEVI & \\
\hline 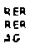 & $\begin{array}{c}1-18.1 \\
-15 .\end{array}$ & o. & $\begin{array}{l}\text { CUTKRSKY } \\
\text { CUTKOSKY }\end{array}$ & $\begin{array}{ll}19 & 19 \mathrm{pha} \\
80 & \mathrm{iphE}\end{array}$ & 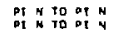 & $\begin{array}{r}12 / 79 \\
1 / 92\end{array}$ \\
\hline
\end{tabular}

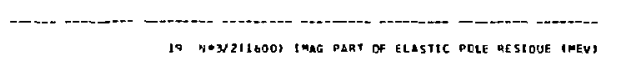

THAR
$i$ MR

IS NAY/ZL1SOOI PARTIAL DECAY MODES

\begin{tabular}{|c|c|c|c|}
\hline $\begin{array}{l}p_{1} \\
p 2\end{array}$ & 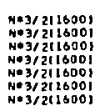 & 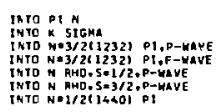 & 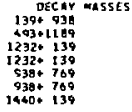 \\
\hline
\end{tabular}

19 H*3/211600\} GRANCHINC RETIOS

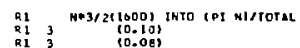
$(0.089$,

$\left(\begin{array}{lll}10.1351 \\ 10.11\end{array}\right.$

(0.20) 10.041

$\begin{array}{cc}10.203 & 10.041 \\ 0.21 & 0.06 \\ 0.18 & 0.04\end{array}$

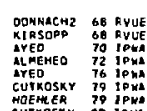

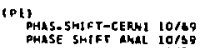

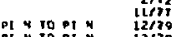
PI

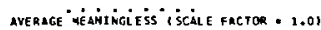

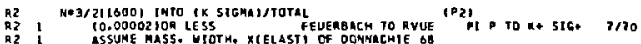

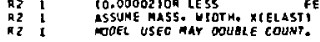




\section{Data Card Listings}

For notation, see key at front of Listings.

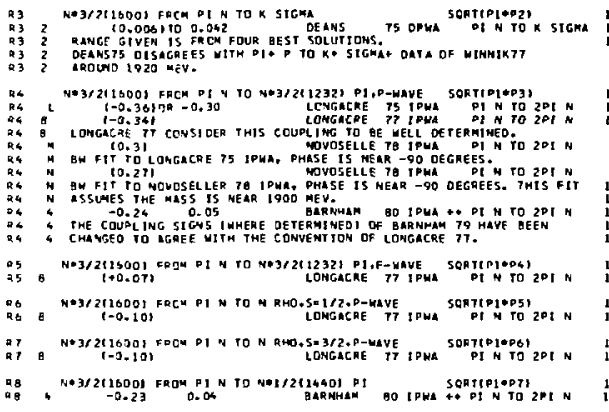

19 No3/2(1600) PHOTON DECAY AMPLTGEVEA-1/2)

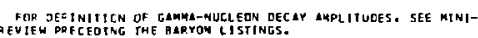

$A 1$
$A 1$
$A 1$
$A 1$
$A 1$
$A 1$
$A 1$
$A 1$
$A 1$
$A 1$
$A 1$
$A 1$
$A 2$
$A 2$
42
$A 2$
$A 2$
$A 2$
$A 2$
$A 2$
$A 2$
$A 2$
42
$A 2$

41
41
41
41
41
41
41
41
41
41
41
41
41
42
42
47
42
42
42
42
42
42
42
42

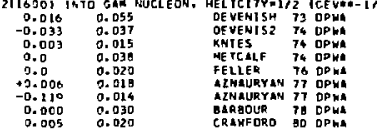

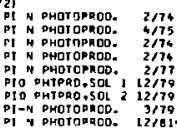

average "ueaniticie ss iscile fattor = 2.7

N*3/2I1\$0O] [NTO F,A NUCLEON. MELICTTY=3/2 (GEV*A-1/2)

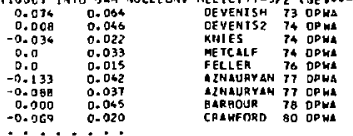

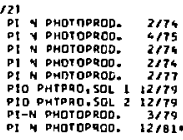

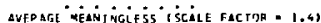

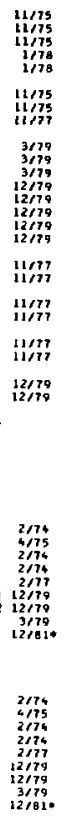

REFEPENCES FOR N*3/2118001

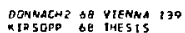

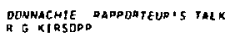

$\triangle$ YEO TO KIEV TOHF

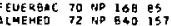

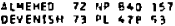
DeVEnISZ 74 OL 520227

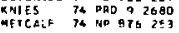

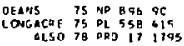

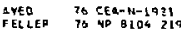

ALNAURPA 7 EFI-2641571-n

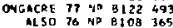

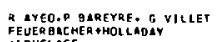
+ LOVELACE +LDVELACE
DEVENISH,RLNKIM,LYTH OEVENI SH, LYTH, RANK IN KNIE S, MODPHOUSE, OBERLACK

IM ITC HELL, MONIGOAERY .+ ISFLA, RLAGIMAIIJD (LGL, SLACJTIS
[LOL, SLAC)

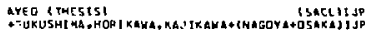

HAKOPON, BAGOLSARYAN CYEREVIN DHYSICS 1M5T MIJP

LOMGACRE.ODLEEAU
DOREAU. TRTHAIIS, HEVEU, CAOTET

(GLAs)

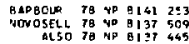

BARBOUR. CRAMEORO.PAR SONS

D. E. NOVOSELLER

ICAL TECHIJ]P

CITKOSxY 79 कR 202039

AFORSYTH, ,HENORICK, KELLY ICAAN+LBLITIP

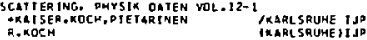

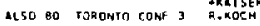

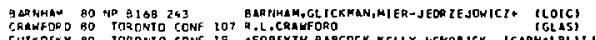

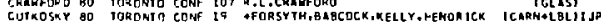
Daders nat referrego to in onte cards

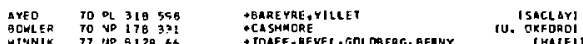

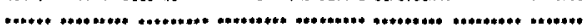

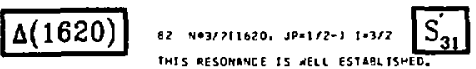

Q2 N*3/21L6201 MASS IMEV)

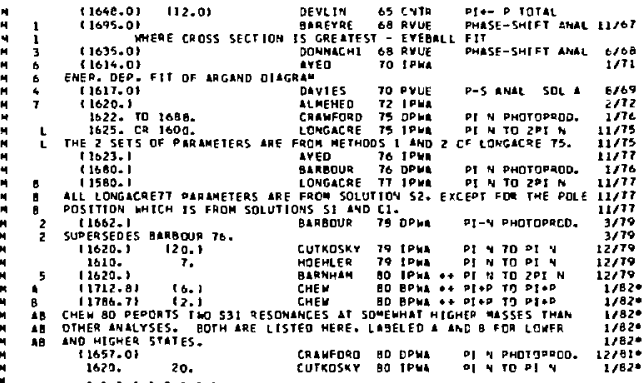

ayerage méan ingiess" iscile factor = 1.0J

A2 NH3/2C1B2OI WTOTH IMEVI

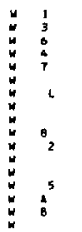

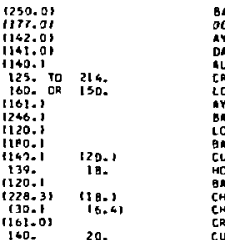

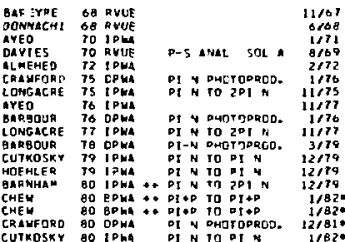

5EE TME NOTES 20 .

- auealge qeaningle ss iscale facton = 1.01

\begin{tabular}{|c|c|c|c|c|c|c|c|}
\hline \multirow{2}{*}{\multicolumn{2}{|c|}{$\begin{array}{l}R E \\
R E \\
R E \\
R E\end{array}$}} & & E $\mathrm{N} * 3 / 2$ & \multirow{2}{*}{$\begin{array}{l}\text { REAL DAPT } \\
\text { LONGACAE } \\
\text { LOWGaCRE } \\
\text { CUTKOSKY } \\
\text { CURKCSKY }\end{array}$} & \multicolumn{2}{|c|}{ OF PRLE POSITIOY (MEY) } & \multirow{2}{*}{$\begin{array}{l}11 / 15 \\
11 / 15 \\
111,17 \\
17 / 9 \\
1 / 82\end{array}$} \\
\hline & & $\begin{array}{l}\text { '1583.1 } \\
1575.2 \mathrm{CR} \\
11597.1 \\
1600 .\end{array}$ & $\begin{array}{r}1572 . \\
15 .\end{array}$ & & 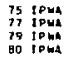 & 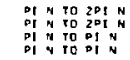 & \\
\hline & & & $B 2 N * 3 / 2(1020)$ & $-2+I M A G P \triangle Q$ & I OF DCLE & DCStition (MEV) & 11175 \\
\hline 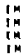 & B & $\begin{array}{l}1163.1 \\
119.10 \mathrm{Da} \\
1220.2 \\
120 .\end{array}$ & $\begin{array}{r}128 . \\
20 .\end{array}$ & $\begin{array}{l}\text { LONGACRE } \\
\text { LONGACRE } \\
\text { CUTKOSKY } \\
\text { CUTKOSKY }\end{array}$ & 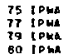 & 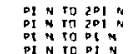 & $\begin{array}{l}11175 \\
11175 \\
12179 \\
1 / 620\end{array}$ \\
\hline
\end{tabular}

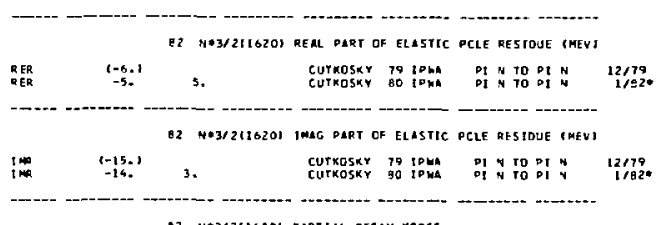

E2 N*3/2\{16201 PRQTIAL DECAY MCOES

PI N*3/2116201 INTO PI N

N*3/2t15203 1NTO GEM YUCLEON, HELICITY $=1 / 2$

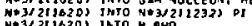

N=312t1620) INTO $N$ Al

$+139+230$

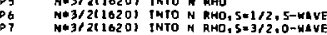

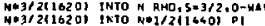

1232. 938

$938 * 769$

$938 * 759$
$938 * 769$ 
Baryons

$\Delta(1620), \Delta(1700)$
Data Card Listings

For notation, see key at front of Listings.

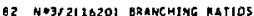

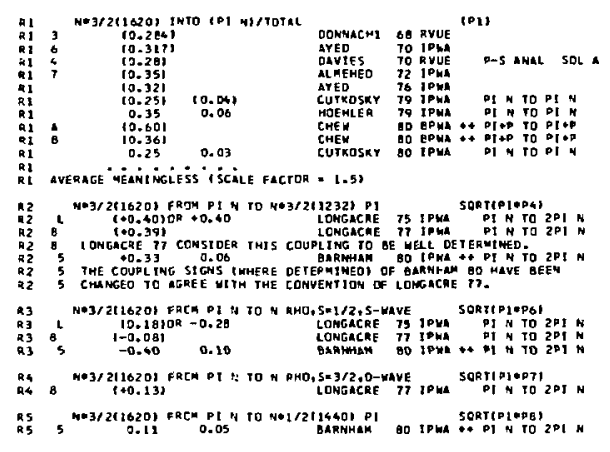

Q2 H*3/211020I RHOTON OECAY AMPLIGEYM- $-1 / 21$

FOR DEFINITICN OF GAMMA-AUCLEON DECAY MMPLITUOES, SEE MINI-

REYIEN DRECEOINC THE GLRYON LISTINCS.

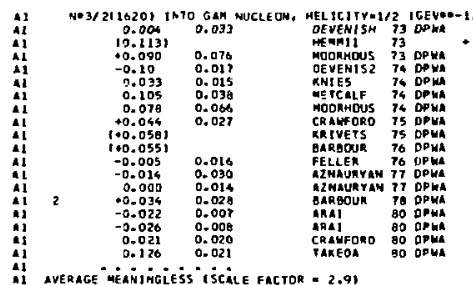

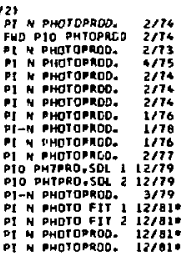

average meajicigiess iscale factor = 2.9

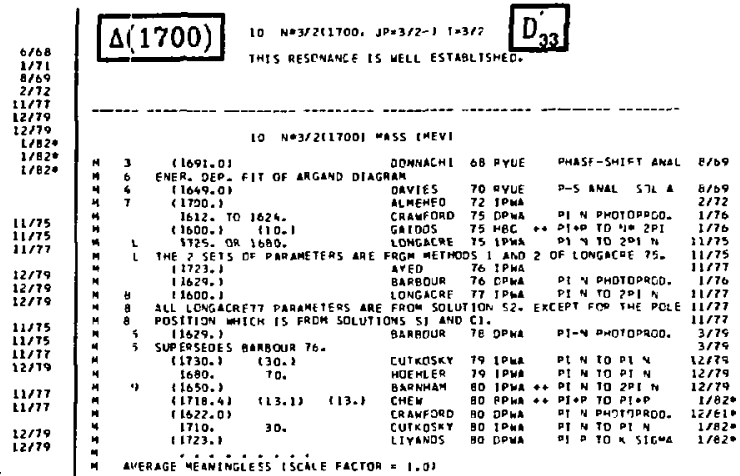

$10 \mathrm{~N} N \cdot 3 / 211$ TOOI UIOTH IMEVI

\begin{tabular}{|c|c|c|c|c|c|c|c|c|}
\hline $\begin{array}{l}3 \\
4 \\
4\end{array}$ & 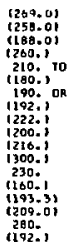 & $\begin{array}{l}268 . \\
135 ., \\
260 . \\
\\
1100.1 \\
90 . \\
126.1 \\
80 .\end{array}$ & $(20)$. & 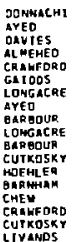 & $\begin{array}{l}80 \\
70 \\
70 \\
12 \\
75 \\
15 \\
75 \\
76 \\
76 \\
77 \\
70 \\
79 \\
79 \\
10 \\
90 \\
80 \\
80 \\
80 \\
80\end{array}$ & 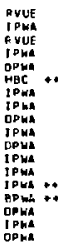 & 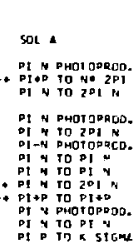 & 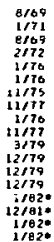 \\
\hline
\end{tabular}

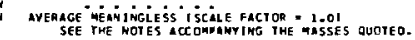

REFERENCES FOR NA3/2(1620)
T I OEYLIN.J SOLOMON, G BERTSCH (PRINCETOH) I

P ARREYRE, C GRTCMAN, G VILLET ISACLAYIIJP

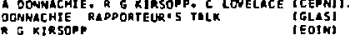

R AYED,N GLREYAE, G VILLET [SACL] IJP

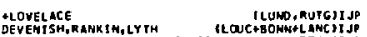

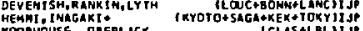

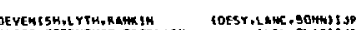

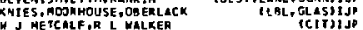

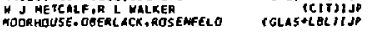

R L CRAWFORD

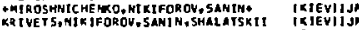

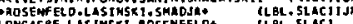

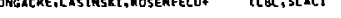
ATEO (THEXYS) TFUKUSHIAA, HDRI KAHA, KAJIKAYA+ THAGOYA+OSAKA)IJP

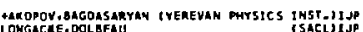

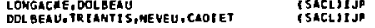

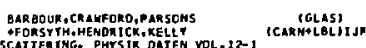

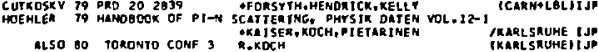

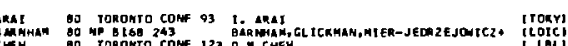
Crew 80 TOROHTO COHF 123 O.M.CHEH

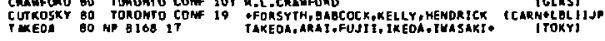
PAPERS hOt ReFEMHED tD IM OATA CAROS

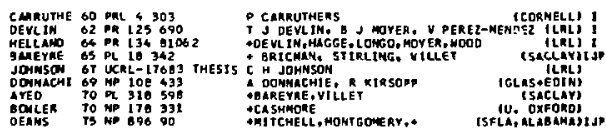

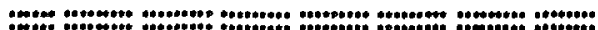

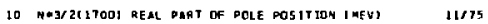

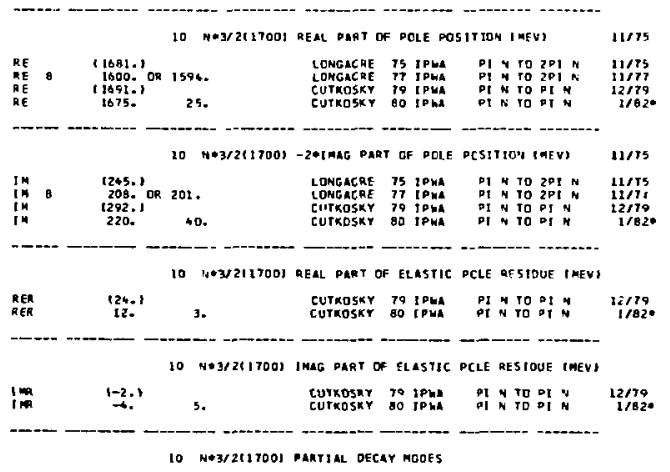

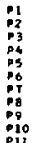

Mo3/ 217000 IMTO a SIGA

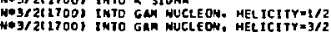

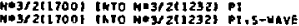

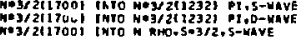

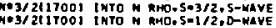

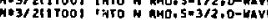

10 M*3/2HIJONS BRANEHJME RATLOS

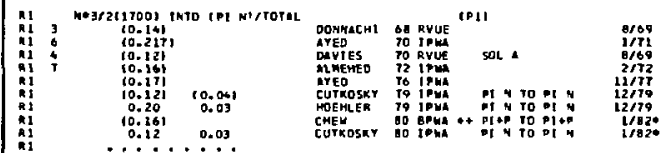

1 avenage médintrigiess is iscile facton - 1.91
39.039

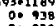

0.938

3232,1398

0

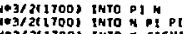

H*3/2t1700) INTO GAN HUCLEOH, HET

438. 1190.139 
Data Card Listings

Baryons

For notation, see key at front of Listings.

$\Delta(1700), \Delta(1900)$

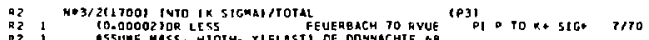

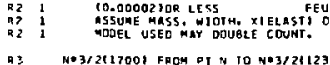

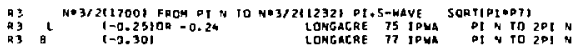

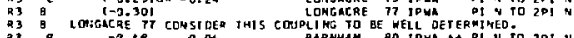

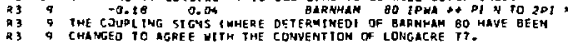

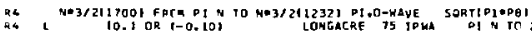

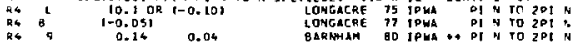

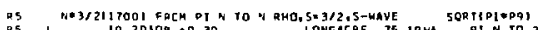

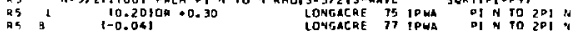

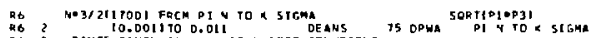

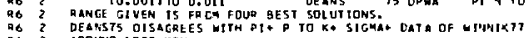

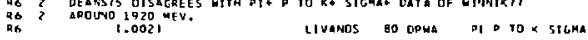

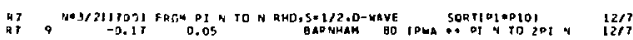

RE
Re

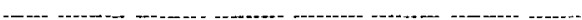

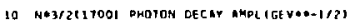

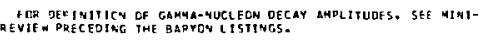
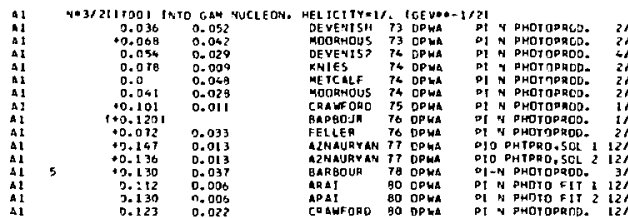

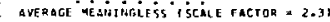

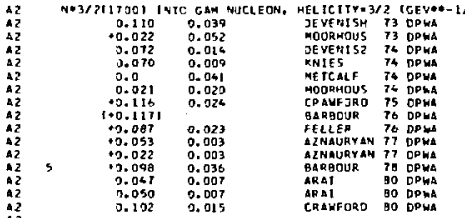

2 averade mániagiess i scale fagtor * 3.61

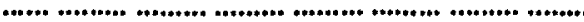

DOANACH 6 OH PL 268 161

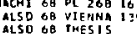

AYED 70 KIEV CONF

MLMENER 75 NP BSO 15\%

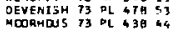

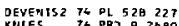

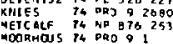

МООRHOLS IT PQD 91

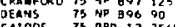

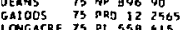

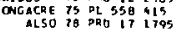

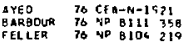
AINAUAYA IT EFt-204i57?-77

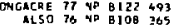

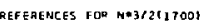

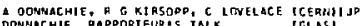

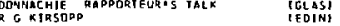

R. AYED,P QAREYRE, G VILLET TSACLIIJP

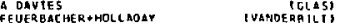

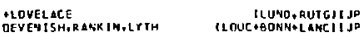

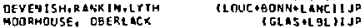

DEVENTSH, GYTH, RANKIH COESY,LAYC, BOUNIJ JP

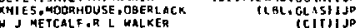

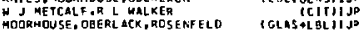
R L CRAFFORD
OHI TCHELL, MONTGCMERT,. GAS DOS. HLLLER

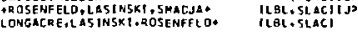

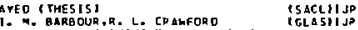

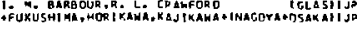
AADPDY, BAGDASAOYAM IYEREVAN DHYSTCS INST.J, JP

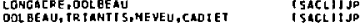

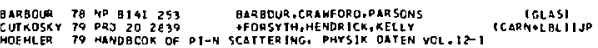

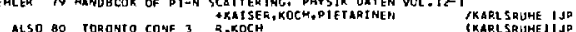

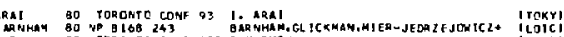

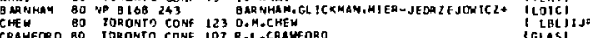

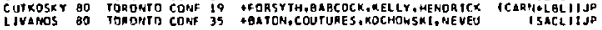

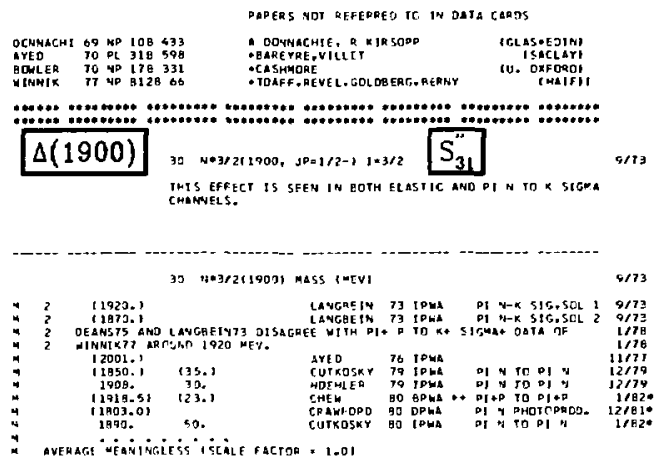

1) * TEZIITOO) WIDTM (MEV)

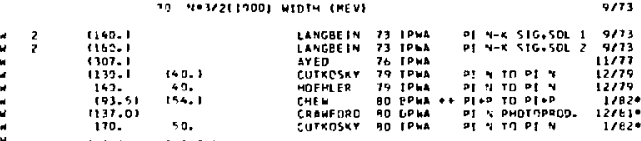

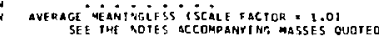

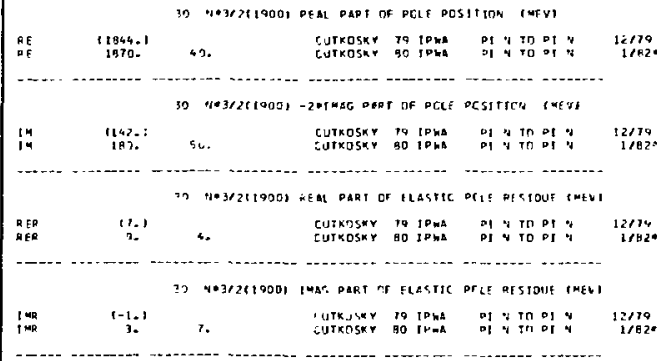

30 T*3/2L1800) PARTIAL DECAP MDDFS

$5 / 73$

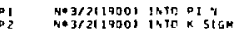

Orcar asses

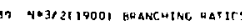

$2 \pi)^{2}$

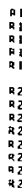

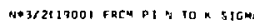

(1).1121

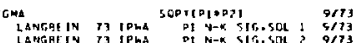
DARGBEIN 53 PPWA DI

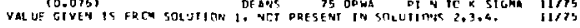

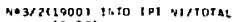

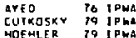

10.081 10.03

$\begin{array}{cc}0.09 \\ 10.2 \mathrm{Al}^{2} & 0.06 \\ 0.10 & 0.03\end{array}$

HOEHLER TO IPWA DI

$11 ; 7 ?$

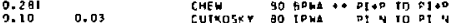

i2179

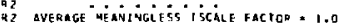

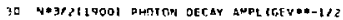

TCR OEFINITICN DF GAMYA-NUCLEON DECAT aUDLITUDES. SEE YINT-

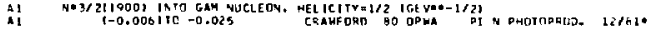

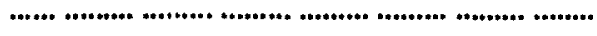

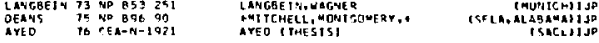


Baryons

$\Delta(1900), \Delta(1905)$
Data Card Listings

For notation, see key at front of Listings.

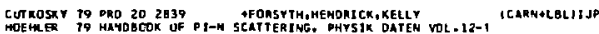

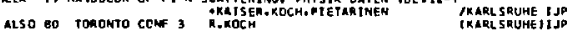
CHEW 80 TORONTO CONF L23 D. M. EHEW L LBLITJP CUTKOSKY BO TOAOHTO COHF IQ FORSTH, BABCOCK.KELLY. MENDRTCK ICARMOLBLIIJP mapers not meremmed to in oata caros

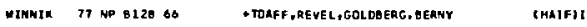
: $\Delta(1905)$ 11 N03/21/905, spes/2+1 1.3/2 $F_{35}$ THIS RESOMAYLE IS MELL ESTHELISHEC. CUTKOSKY 80 FINDS A HIGHER MASS F35 RESONANCE III

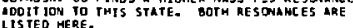

11 Ne3/2119051 MaSS IMEVI

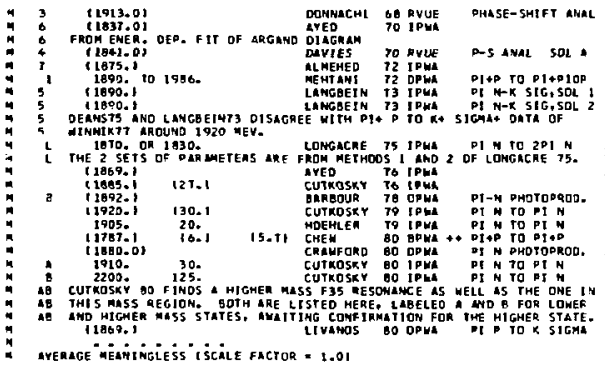

11 NE3/2419051 MIOTH ITEVI

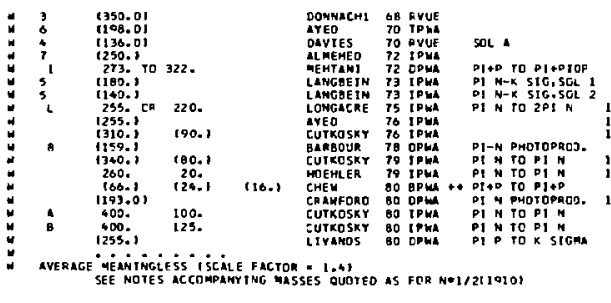

11 No3/211905I REAL DART OF POLE POSTIION (HEVI

\begin{tabular}{|c|c|c|c|c|c|}
\hline RE & $\begin{array}{l}11813.1 \\
11825.1 \\
11855: 1 \\
1830: \\
2150 .\end{array}$ & $\begin{array}{l}112.1 \\
+0 . \\
100 .\end{array}$ & $\begin{array}{l}\text { LONGACPE } \\
\text { CUTKOSKY } \\
\text { CUTKOSKY } \\
\text { EUTKOSKY } \\
\text { CUTKOSKY }\end{array}$ & 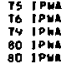 & 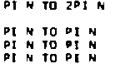 \\
\hline
\end{tabular}
ae average meninisgless iscille factior = 3.01

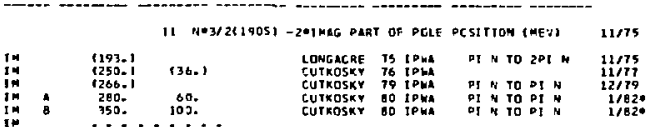
i" ayerage meationgiess iscale factor = 1.01

11 NeJ/2119051 REAL PART OF ELASTIC PCLE RESTOUE GMEVI

\begin{tabular}{|c|c|c|c|c|c|}
\hline 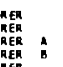 & $\begin{array}{l}111.51 \\
120.11 \\
16: \\
-14 .\end{array}$ & $\begin{array}{l}13.51 \\
63 . \\
13 .\end{array}$ & $\begin{array}{l}\text { curkoskr } \\
\text { curkoskry } \\
\text { curkosky } \\
\text { curkoskr }\end{array}$ & 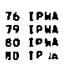 & 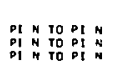 \\
\hline
\end{tabular}

11 No3/211905H 1MAG PART OF ELASTIC DCLE RESIOUE (MEY) 11 N*3/2(1909) PART TAL OECAY MOOFS

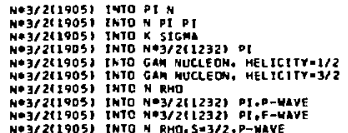

\section{1 ne3/2119053 anenchING Retios}

N*3/2119051 IWTO IPI NI rTOTLL

10.161
$(0.1271$

10.201
10.101

$(0.16)$

$(3.140) \quad 10.008$

$\begin{array}{cc}10.151 & 10.021 \\ 0.15 & 0.02\end{array}$

0.111
0.08 0.09

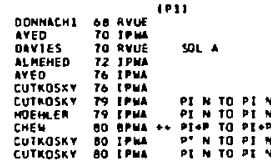

DECAY MISSES

139: 938.139

$493+1189$

:0.: 938

$938 \% 969$

12322139

936.769

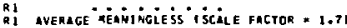

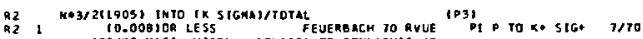
R2 1 ASSUNE MASS. NIOTH, XIELASII DF OONHACHTE OB

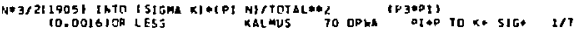

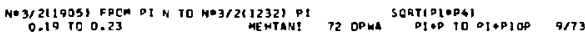

MESTLY F MAVE DECAY N*3/2119051 FRC4 PI N TO K SIGMA

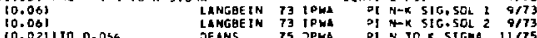
10.0211 TO 0.054
$G G$ G
$1-0.0131$

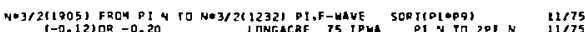

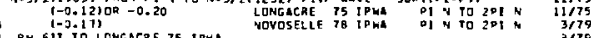

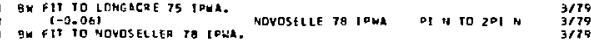

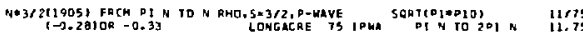

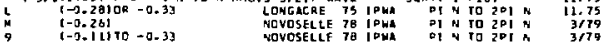

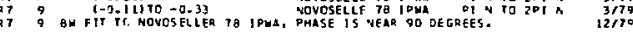

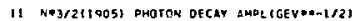

FOR OEFINTIICA OF GAMHA-NUCLEOH DECAY AMFLI TUDES. SEE MJ MIREVIEW DAECEOTHG TME gaRYON LISTIMGS.

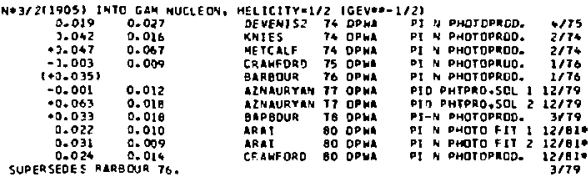

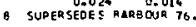

A1 average Meaving iéss iscile factop = 1.61

N*3/211905) INTO GAM MUCLEON, HELACCITY-3/2 (GEENE-1/2I

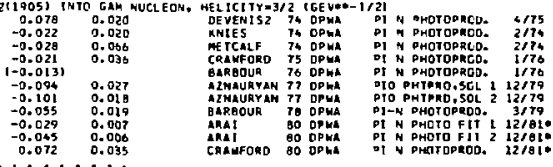

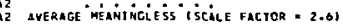

\section{7 $8 / 72$ 1820.} $2 / 79$ 0

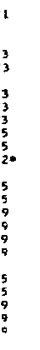


Data Card Listings

For notation, see key at front of Listings.

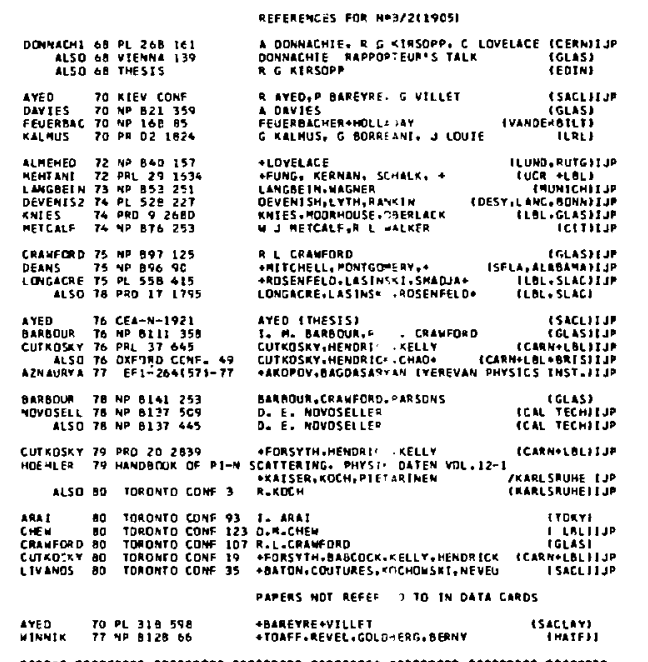

*a+*

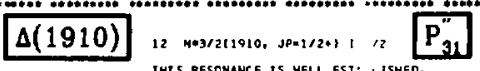
THES RESONANCE IS MELL EST: LISNED.

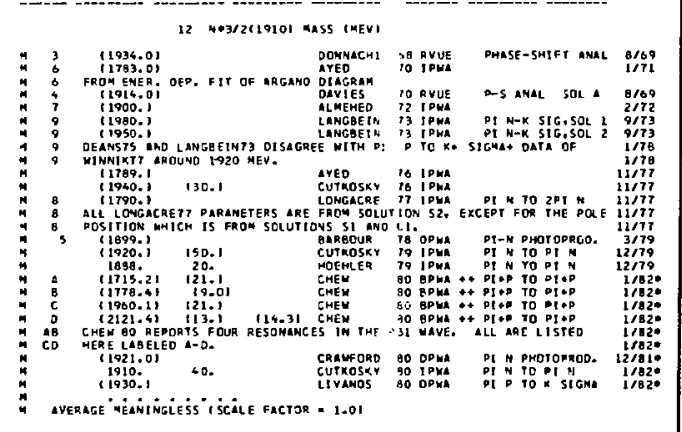

12 Na3/2(1910) WHOTH (MEV)

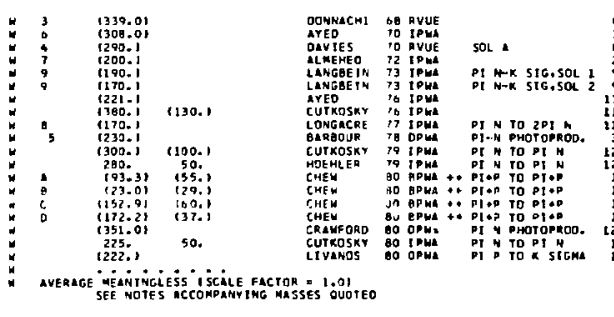

12 maz/211910\} MEAL DART OF POLE DOSITION I MEVI

\begin{tabular}{|c|c|c|c|c|}
\hline $\begin{array}{ll}R E \\
k E \\
R E \\
R E\end{array}$ & 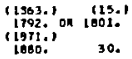 & 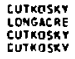 & 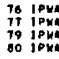 & 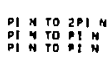 \\
\hline
\end{tabular}

\begin{tabular}{|c|c|c|c|c|c|c|}
\hline $\mathfrak{i m}_{\substack{\text { in } \\
\text { in } \\
\text { in }}} 8$ & $\begin{array}{l}1250-1 \\
172.08 \\
1200.1 \\
200 .\end{array}$ & $\begin{array}{l}12 N=3 / 2(1910) \\
134.1 \\
165 . \\
\text { 40. }\end{array}$ & 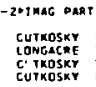 & 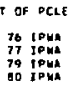 & 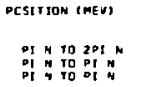 & $\begin{array}{l}11 / 77 \\
11 / 277 \\
12 / 79 \\
1 / 820\end{array}$ \\
\hline 促 & 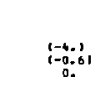 & $\begin{array}{l}120 \times 3 / 2(19010) \\
15.1 \\
10 .\end{array}$ & $\begin{array}{l}\text { REM PARY OF } \\
\text { CUTKOSKY } \\
\text { CUTKOSKYY } \\
\text { CUTKOSKY }\end{array}$ & 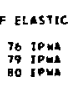 & 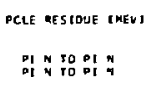 & $\begin{array}{c}1127 \\
12279 \\
1 / 92 *\end{array}$ \\
\hline ina & 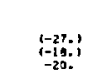 & $\begin{array}{l}12 \mathrm{~N}+3 / 2(1910) \\
15.1 \\
4 .\end{array}$ & 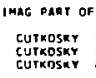 & 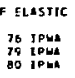 & PCLE RESTDUE (MEV) & $\begin{array}{l}11 / 71 \\
12 / 79 \\
1 / 820\end{array}$ \\
\hline 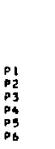 & 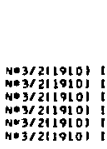 & 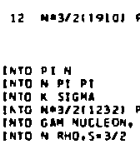 & -aatral deca & AY MODES & 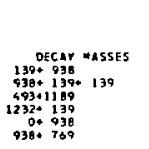 & \\
\hline 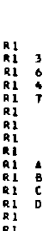 & $\begin{array}{c}\text { N*3/219101 } \\
10.301 \\
10.1281 \\
100101 \\
10.313 \\
10.161 \\
10.2251 \\
10.191 \\
0.24 \\
10.101 \\
10.201 \\
10.171 \\
10.401 \\
0.19\end{array}$ & 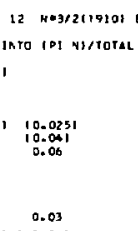 & 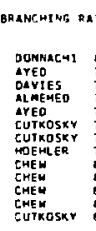 & 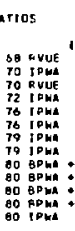 & 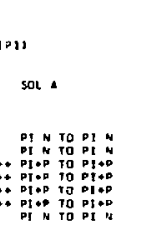 & $\begin{array}{l}0 / 39 \\
1 / 71 \\
0 / 69 \\
2 / 72 \\
11 / 77 \\
11 / 77 \\
12 / 79 \\
12 / 79 \\
1 / 182 \\
1 / 82 * \\
1 / 82 * \\
1 / 82 * \\
1 / 82 *\end{array}$ \\
\hline
\end{tabular}

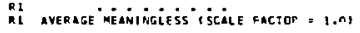

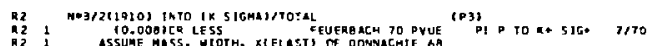

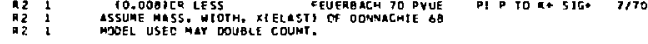

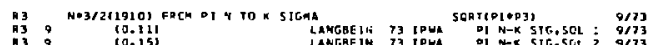

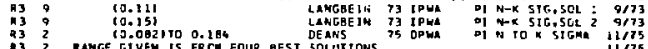

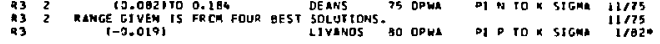

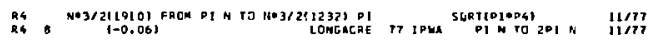
$R 5$
R5

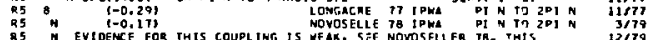

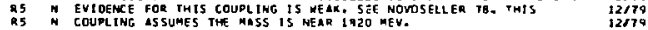

12 No3/2119101 PHOTON DECAY AMPLIGE

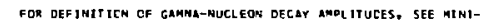
REVIEW PRECEOTNG THE BARVOY LIST1NGS.

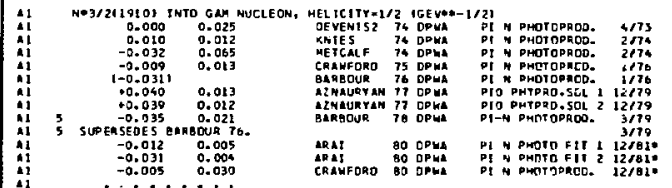

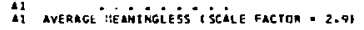

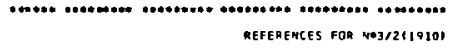

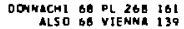
ALSO G8 YIENMA 130

AYEO 70 KIEV CONF

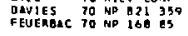

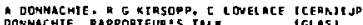

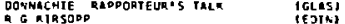

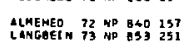
DEYENISZ 74 DL 528227 R AYEO, P Bataree, G VILLET

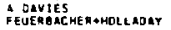
15ACLITJP IYMOERGILTI

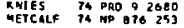

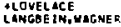
iLumperutg)isp DEYENI SH,LYTH, RIUXIN

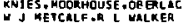

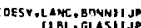
(2Bl. GLAs! ids

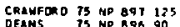
Ré CRAMForo

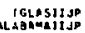

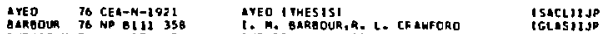

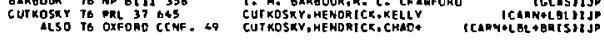


Baryons

$\Delta(1910), \Delta(1920), \Delta(1930)$
Data Card Listings

For notation, see key at front of Listings.

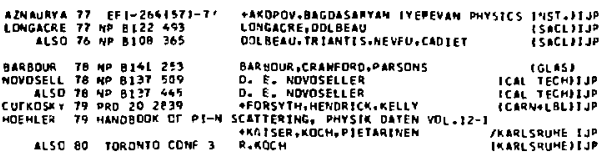

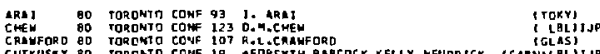

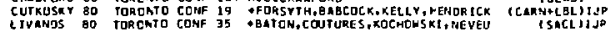
PAPERS NDT REFEPREO IC IN DATL CAPDS

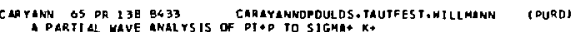

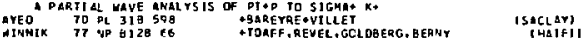

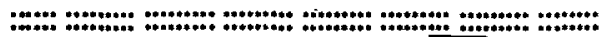

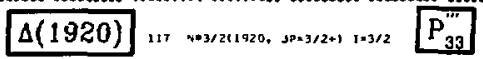

EARLY ANALYSES FOUND EYIDENCE FOR 2 P 33 DESONAHCE NEAT

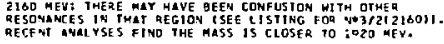

111 N*3/2(1920) MLSS IMEU1

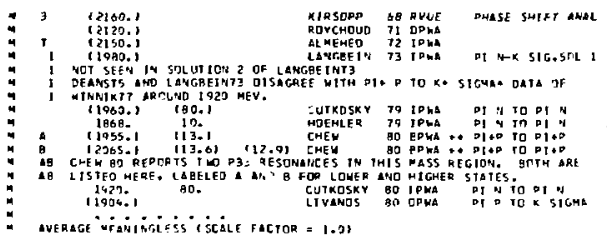

anerage uraniritiess iscile factor $=1.31$

\section{IIT N*3/211920) WIDTH IMEVI}

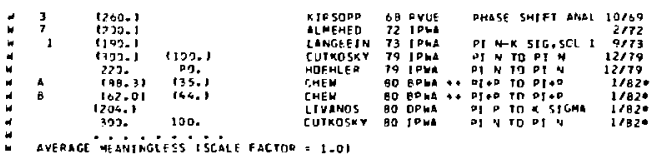

cuerace me animgifis iscale factor = 1.01

\begin{tabular}{|c|c|c|c|c|c|}
\hline $\begin{array}{l}\text { RE } \\
\text { RE }\end{array}$ & $\begin{array}{l}\text { '10,3.2 } \\
\text { 10<3. }\end{array}$ & AD. & 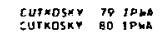 & 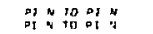 & $\begin{array}{ll}27 / 20 \\
1 / 824\end{array}$ \\
\hline & & $11, N=3 / 213020$ & -2*IMAG part of prLef & DOSITION (MEN) & \\
\hline im & $\begin{array}{l}\text { 17R0.1 } \\
300 .\end{array}$ & 100 & $\begin{array}{ll}\text { CUTKOSKY } & 79 \mathrm{TPhS} \\
\text { CUTKOSKY } & 90 \mathrm{IPhS}\end{array}$ & 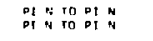 & $12 / 79$ \\
\hline & & $117 N+3 / 2019201$ & RE, AL DAPT OF ELASTIC & -CLE R(SIOUE (MEY) & \\
\hline $\begin{array}{l}\text { RER } \\
\text { DIR }\end{array}$ & $\begin{array}{l}10.1 \\
\rightarrow \rightarrow 1\end{array}$ & 1. & 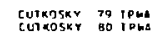 & Pl "ll & $\begin{array}{l}1217 \% \\
1 / 324\end{array}$ \\
\hline & & $112 \times 272619701$ & Tag Paet of elasitic & dCle restoue imeyi & \\
\hline$I_{\text {Ma }}$ & $\begin{array}{l}1-27,1 \\
-12+\end{array}$ & 11. & $\begin{array}{l}\text { CuTKcsky To towa } \\
\text { EUTKCSkY Bo low }\end{array}$ & 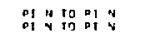 & $\begin{array}{l}12 / 79 \\
1 / 820\end{array}$ \\
\hline & & 118 & mapi al oecay rones & -2 & \\
\hline 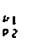 & $\begin{array}{l}N * 3 / 2119201 \\
N \cdot 3 / 2119201\end{array}$ & 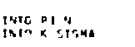 & & $\begin{array}{r}\text { DET } \triangle Y \text { AnSSES } \\
1394938 \\
403+1189\end{array}$ & \\
\hline
\end{tabular}

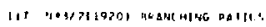

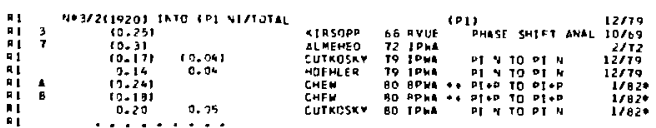

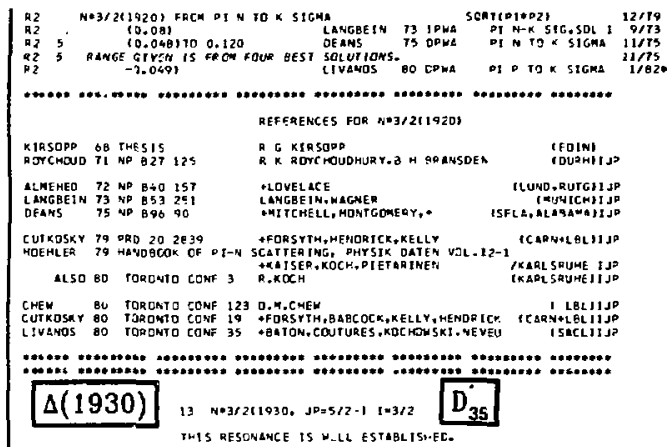

\section{NOYZIIT301 MASS (AEYI}

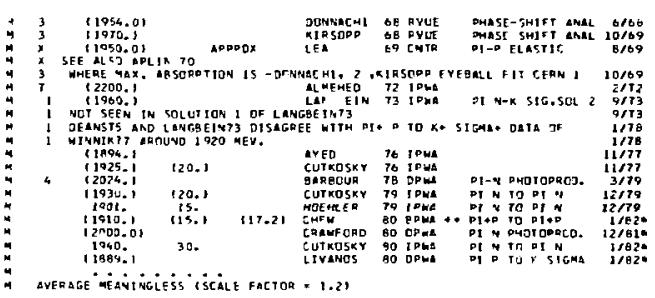

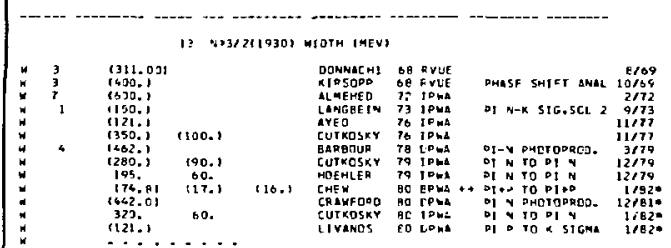

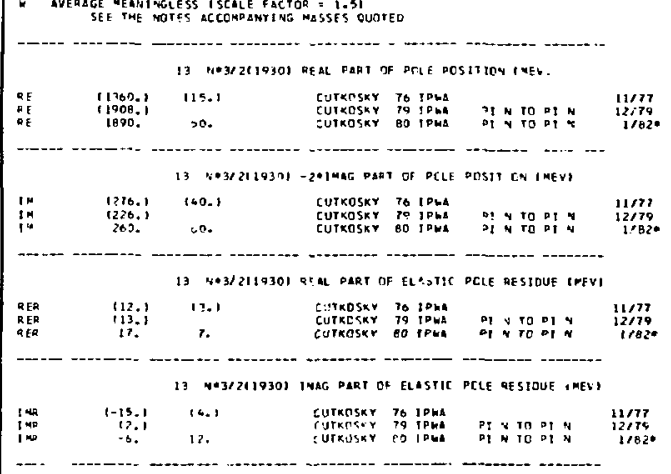

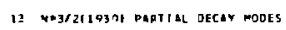

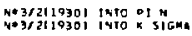

OECAY MASSES
$139+938$ $139+930$
$493+1200$ 
13 Hez/261930) BhANCMING MAYIOS
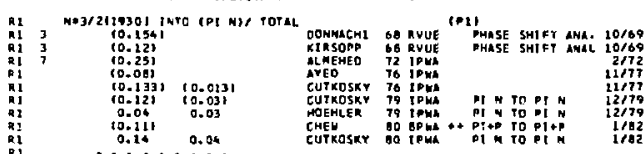

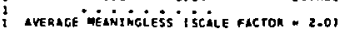

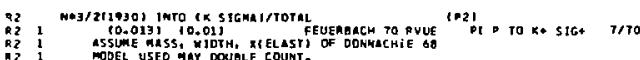

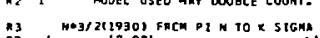

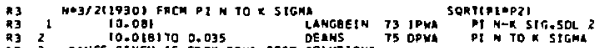

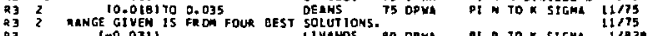

-_- - - - - - - - - - - - - - - - - - - - - - - - -

13 Na 3/Zl19301 PHOTOH DECAY AMPLIGEVE-1/2!

FOE OE: IMTTICN OF GAMMA-NUCLE ON DECAY AMELTTUDES. SEE NTNT-

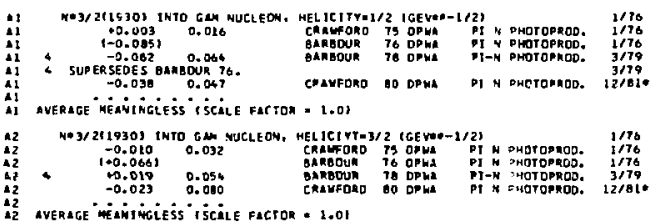

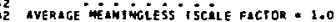

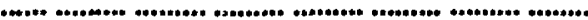
REFEREMCES FOR MOB/Z119301

OONACH: OB DL 268101 KINSODP GB THESIS

LEA $60 \mathrm{PL} 29 \mathrm{EE}$

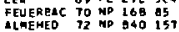

LANGEEIN TS NO OST 251

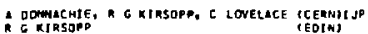

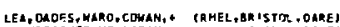

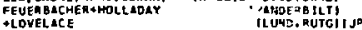
LOVELACE LAHCSE I M. VRGME

[MUNICHI] I]P

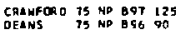

AYED T6 CEN-N-1921

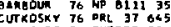

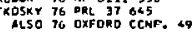
L CRLUFORD
AMI ICHELL, MONTGOMEMV.. EVED (THESIS, ISACL)?JP

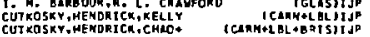

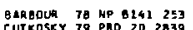

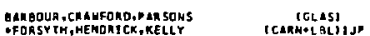

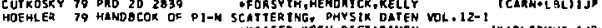
ALSO 8O TORONTO CONF 3 REKOCH

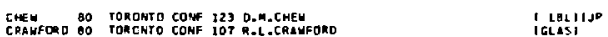

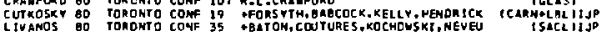
PAPERS NOT REFERED TO IN OATL GADS

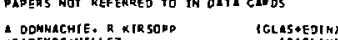

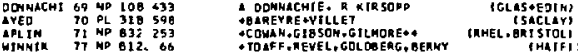

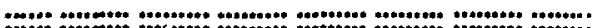
$\Delta(1940)$ i36 **3/212940, Jp=3/2-1 1-3/2 $D_{33}$

136 N०3/2119401 HA5S (MEY)

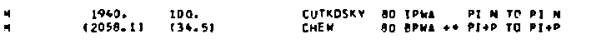

128 N\$3/21194OI WTOTH IMEVI

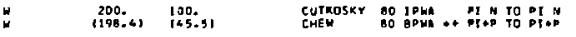

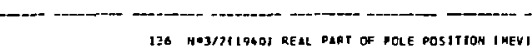

DE 1970. 100. CUTKOSKY DO IPH DI NTP TI

L36 Ke3/2(1940) 2*1MAG PANT OF DELE PCSITION IMEVI

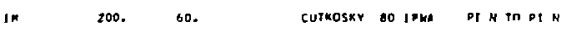

- - - - - - - -

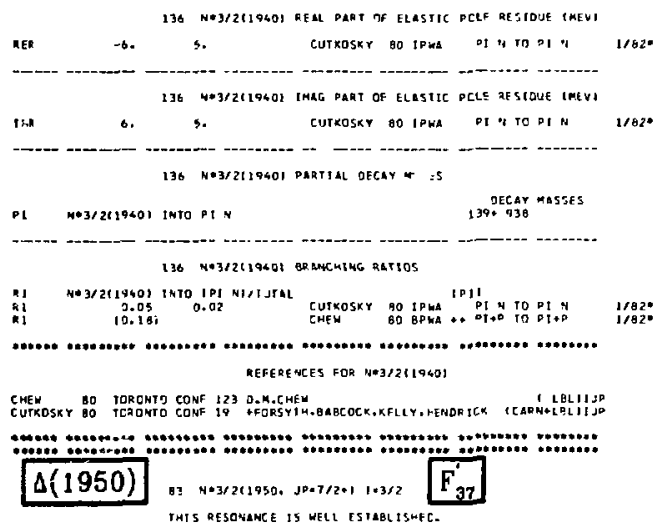

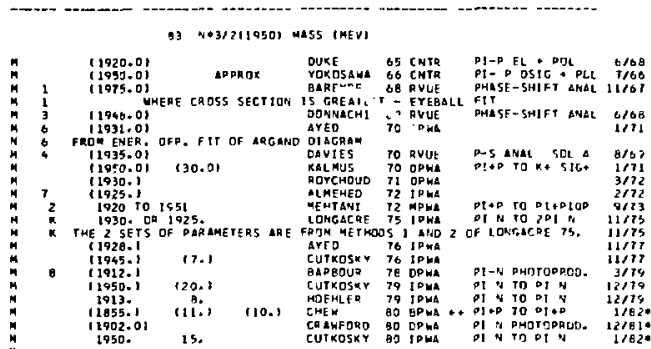

average méninigir is iscale factor = 2.21

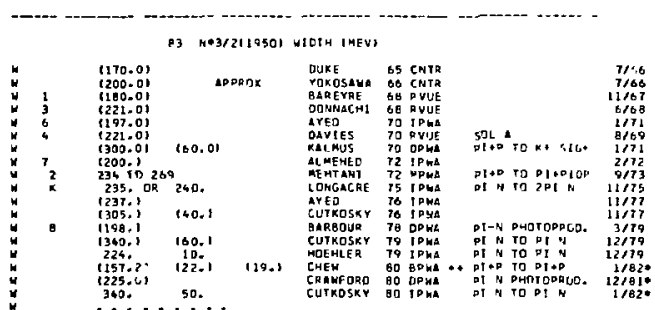

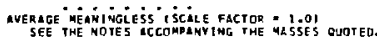

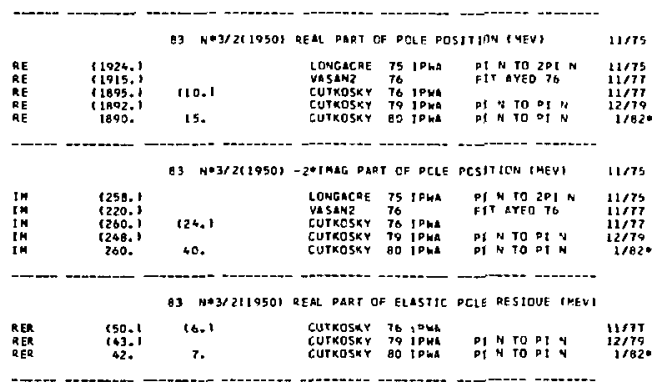


Baryons $\Delta(1950)$
Data Card Listings

For notation, see key at front of Listings.

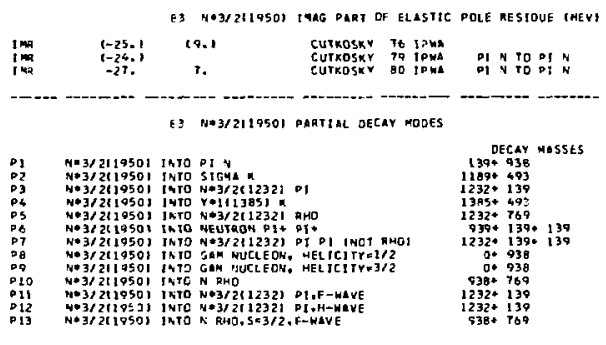

D) N*32(1950) BRANCHJNG RETIOS
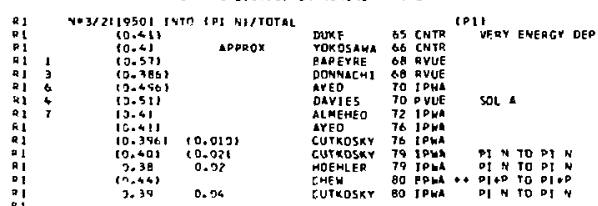

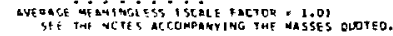

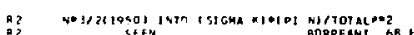

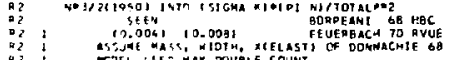

DSJAE MAKS, WDOH, XCELAS killus 70 orke

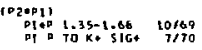

$81+b 20 x+2100$

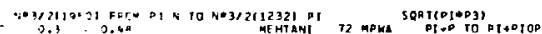

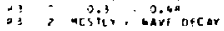

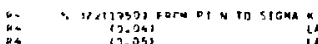

(3.05)

ian

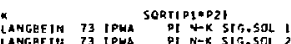

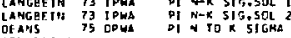

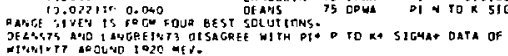

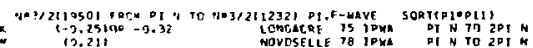

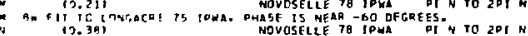

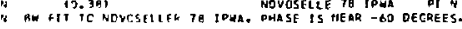

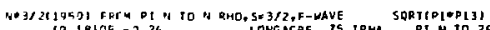

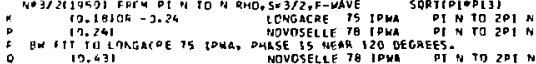

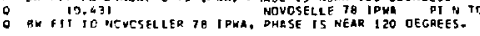

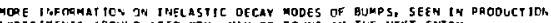
IXPEATAENSS ARTUNE 1950 UEV. ASY BE FOUNO IH THE NEXT ENTAY

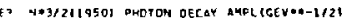

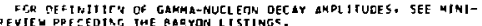

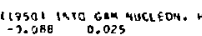

$\begin{array}{ll}1 & -0.029 \\ -3.060 & 0.015\end{array}$

$\begin{array}{rr}-0.059 & 0.012 \\ 0.029 & -0.0909\end{array}$

$\begin{array}{ll}1-0.0001 \\ -0.053 & 0.005\end{array}$

$\begin{array}{cc}1-0.076) \\ -0.078 & 0.208\end{array}$

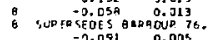

$\begin{array}{ll}-0.091 & 0.005 \\ -9.063 & 0.005 \\ -0.067 & 0.016\end{array}$

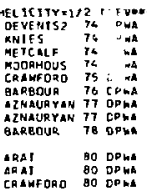

i) n photoonoD.

PI DHOTOPROD.

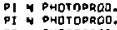

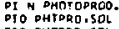

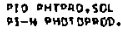

average meanivigiess iscile factona = 2.6

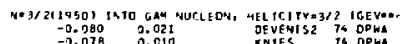
$\begin{array}{ll}-0.090 & 0.021 \\ -0.078 & 0.010 \\ 0.003 & 0.024\end{array}$

$\begin{array}{cc}0.083 & 0.02 \\ 1-0.1801 & 0.016\end{array}$

$\begin{array}{ll}1.0 .1801 \\ -9.038 \\ -0.0531 & 0.014\end{array}$

$\begin{array}{ll}-0.0651 & 0.010\end{array}$

-0.169
-0.075 $\quad 0.019$

$\begin{array}{ll}0.161 & 0.009 \\ -0.100 & 0.005 \\ -7.082 & 0.017\end{array}$

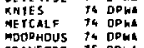

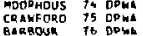

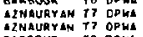

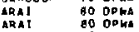

DI N PAOTO FII I $12 \angle B 10$

$7 / 60$
71160
11161
6166
1131
$8 / 69$
$2 / 72$
1177
$11 / 77$
$12 / 79$
12279
$1 / 820$
$1 / 820$

$1 / 71$

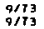

9/73 $9 / 73$

11775

11785

$3 / 79$
$3 / 77$
3
3,79
3

11775

$3 / 79$
3779
3779

3799

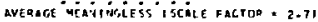

3) 4 Phoroprag. $4 / 75$

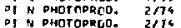
P1 PHOT OPRGO: 2174

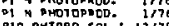

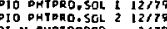

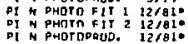

a

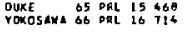

BAREYXE BO DR 1051731 BOAREAH SB UCPL 18350 ALSO OQ YIENHA 139
ILSO WO TMESIS

AYEO 70 KIEV CONF

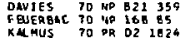

RCreHOUD 11 H० Q 27125

ALEEMED 72 HP 840257

LAMGBEIN 73 ND 8532514

JEVEH152 74 PL 528227

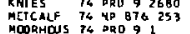

CEAHFORD IS YP 897125

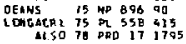

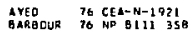

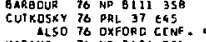

VASENE TO OXFORD CCNF.

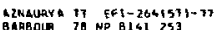

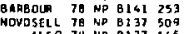

TUTKOSKY 79 PAO 202899

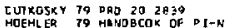

ALSO AO TORCNTO CONF

ARAS BO TORONTO CONF 93 T. 4 AAM

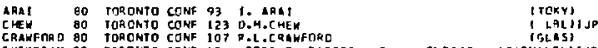

DEFERENES FOH $123 / 2119501$

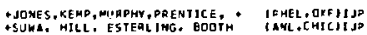

- gareyre. c eptchan. o villet loclayid de

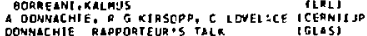

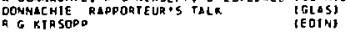

a AYED, D BAREYRE. G VILLET

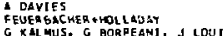

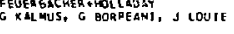

[SACL, JP

A R ROYCHOUDHUTY. $B$ H RRANSOEA

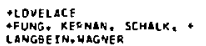

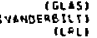

(DNat) I JP

DEVENTSH,LYTH, PANKIN IN TDESY,LAHC, GCUNHIID

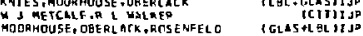

a L CAAMFORO

[GLSSIJ JP

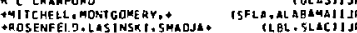

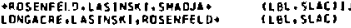

areo ITHESISI ISACLISJPP

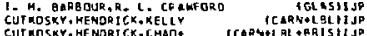

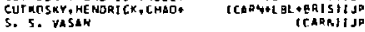

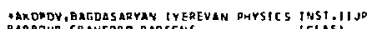

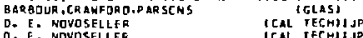

AFDRSYTh, HENORTCK, KELLYY (CARM+LBL)IJP

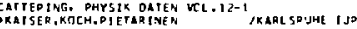

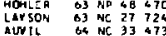

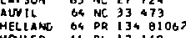

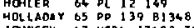

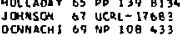

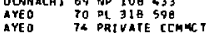

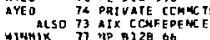

onpeas not aeferage o to jm data catos

.......

1950 MEV REGION - PRODUCTION AND $\sigma_{\text {TOTA }}$ EXP'TS

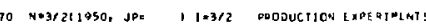

SEF THE MINT-REVIEW PRECCOLAG THE N ANO OELT LESTIRGS

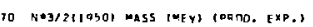

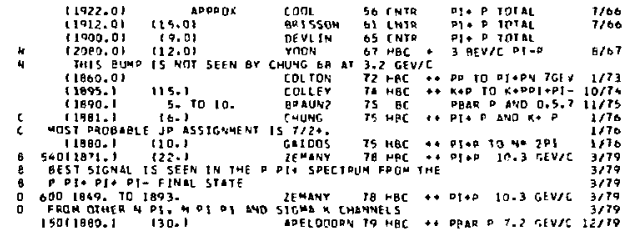

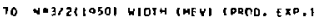

HLER, 5 EAEL

- DE VLIN, HAGGE, LONGO, MDVEP, WDO

C mollanar

DONNACHIE, A KIRSODP

GAREYDE Q VILL

AYE D, BAREYRE

TOAE REYFL, COLOEERG, OEO

$\triangle$ SA EOIn

SACLAYi
isaceitje
isacijijo

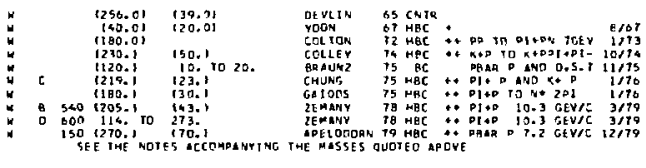


Data Card Listings

For notation, see key at front of Listings.
Baryons

$\Delta(1950), \Delta(2150), \Delta(2160), \Delta(2200)$

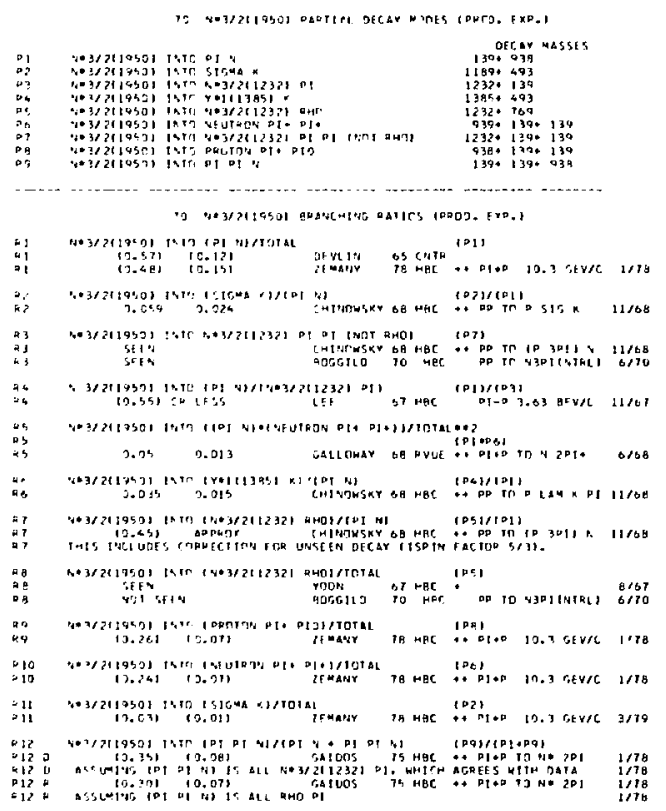

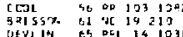

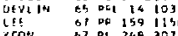

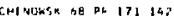

CHUMr, be DL les lis?

BGGGiti 70 ND BIS 503

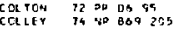

QRauiz 15 No ags Se:

CHUNG 75 at 578 304

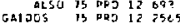

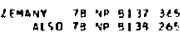

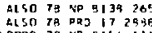

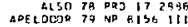

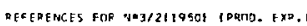

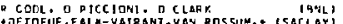

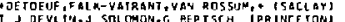

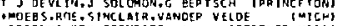
PBE QE NYI, KEY, PDENIICE, + IIORNN TC, WISL,

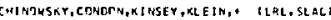

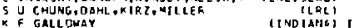

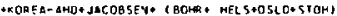

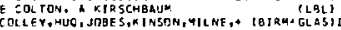
GGFRER , MAUPEA, 4TC HALON, SCHIEY, STERB, L PMP I

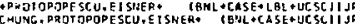
GAIDOS. MILLER

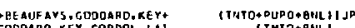

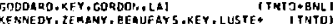
KE SNEDY, ZEMANY, GEAUFAY S,KEY, LUSTE* IINTOI

papeas mot referreo in th oate cardos

DEUTSCHA 75 MP 895367

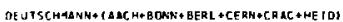

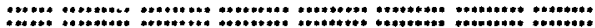

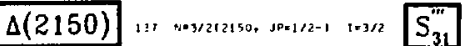

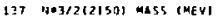

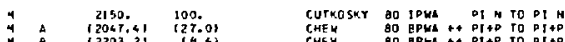

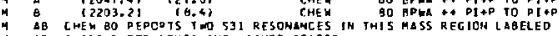

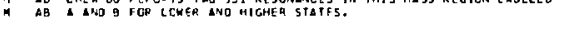

137 NE3/2(21SOL HIOTH IMEVI

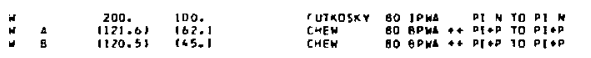

137 N*3/212150/ PEAL DAFT OF ADLE POSITION IMEYI

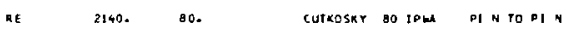
Re

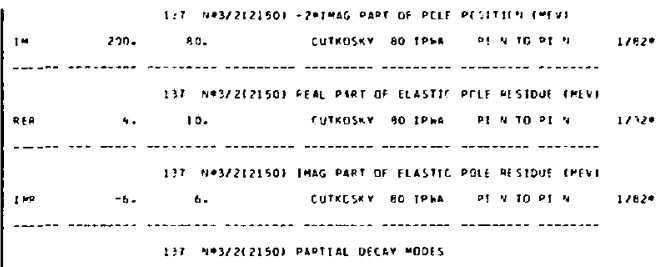

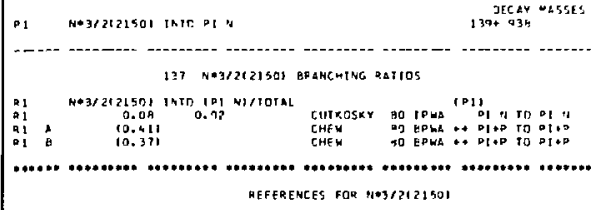

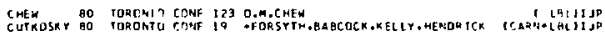

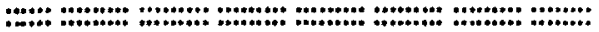

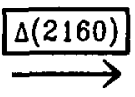

$54+3 / 215260$,

$1,1=3 / 2$

EAPIY ANAL YSES FOUNO FYTOENEE FOD A RE SOHARCE NEAR IHIS MLSS IN THE P33 PARTIAL WAYE. TWFSE Q FIND POSSIBLE EVIOENCE FOR 031 , O33, 480035

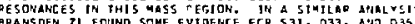

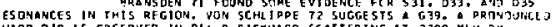
SHARP DIP IS CESEAYED IN DIS P BACKHAFO SCATTEPIHG QT 2200 MEY gY DEY T4. DUAL TYTERFEPECE MODEL AHALYSIS OF MA TE FINDS 5 IGNAL FOD

Q NAJZZ\{2ISOS RASS (MEY)

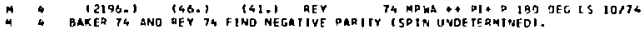

- N*3/2121601 HIDIH IMEYI

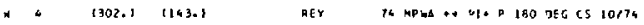

- Noz/21z1601 daptial decar modes

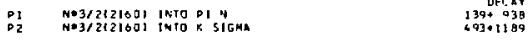
DFr.AY MASSES

9 NO3/2t2LGOI BRANCHING DatIOS

R1 No3/2121601 INTO CPI NI/TOTAL [P]

R1 \& REYTH FIMDS $1 J+1 / 21 X=.81+1-1.54 / .391$ $10 / 74$

REY HSO 74 PRL 32 PCE

REFEREHCES FOR N03/212160)

AL50 75 PRD II 2777 REY, LEHAXX, POTRTiR, DPETZZL

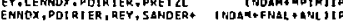
Dapers not heferaeo to in oata capos

DONACH 1 OS NP 108 B

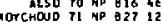

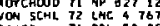

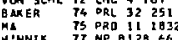

A OONMACHIE. KIRSODP IGLAS+EDIN

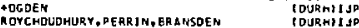
R K ROYCHOUOHURY B E ERANSOER
YOH SCKL IDPE
(DURHIIJP

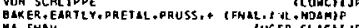
MA. 5HAW
+ TOLF, REVEL, GOLOBEAG, BERNY TUCSB, SLACBIJP
[HAIFII

*

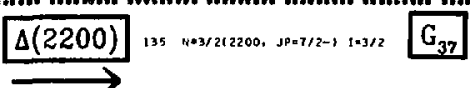

$135403 / 2122001$ MLSS (MEY)

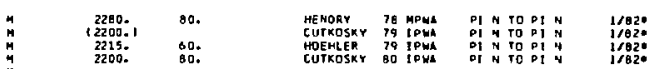
$1 / B=$

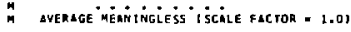


Baryons

$\Delta(2200), \Delta(2300), \Delta(2350), \Delta(2400)$

\section{Data Card Listings \\ For notation, see key at front of Listings.}

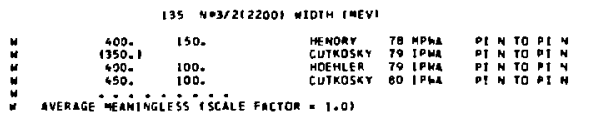

135 NO3/2122003 REAL PART OF POLE MOSITIOH (MEVS

\begin{tabular}{|c|c|c|c|c|c|}
\hline $\begin{array}{l}\text { AE } \\
\text { AE }\end{array}$ & $\begin{array}{l}12094.1 \\
2100 .\end{array}$ & 50. & $\begin{array}{l}\text { CLTKOSKY } \\
\text { SutKoskY }\end{array}$ & $\begin{array}{l}79 \text { lP IPA } \\
\text { eO jown }\end{array}$ & 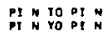 \\
\hline
\end{tabular}

135 N*3/2tz200) -201MAG PARY of PCLE PCSITSOH (MEY)

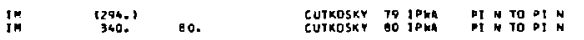

I3: NA3/2\$2200) REAL PLRT OF ELASTLC PCLE RESIOUE (MEV)

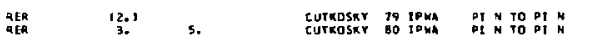

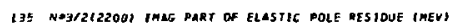

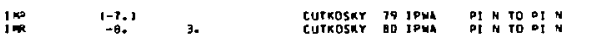

135 NE3/2t2700I PARTIRL OECAY MCDE:

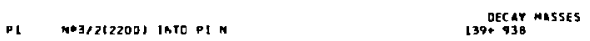

135 N*3/2T22003 GRANCHINE RATIOS

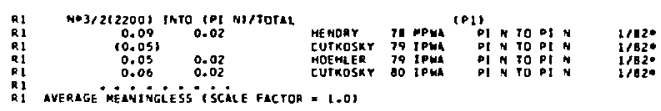

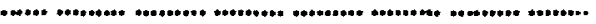

$$
\text { REFERENCES FOH ma3/21 } 22001
$$

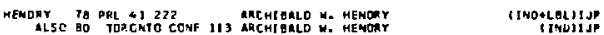

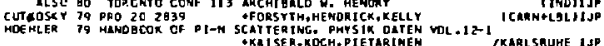

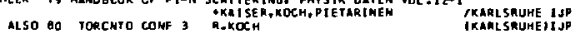

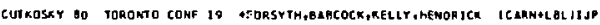
(wope

$\Delta(2300)$ 123 *43/2(2300, JP=9/2+1 1-3/2 $\mathrm{H}_{39}$

123 N+3/2I 2300 H RMSS IMEVI

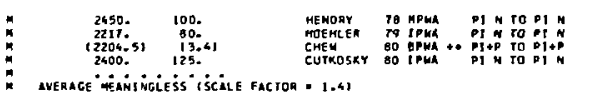

123 HO3/21230OI VIOTH IMEVI

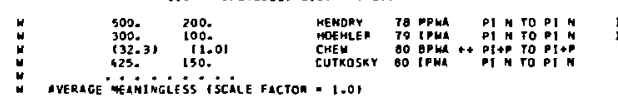

\begin{tabular}{|c|c|c|c|c|}
\hline $\mathrm{NE}$ & 2370. & $\begin{array}{l}123 \mathrm{~N}+3 / 2(2300\} \\
00 .\end{array}$ & $\begin{array}{l}\text { AE ML PART OF DOE PD } \\
\text { CUTKOSKY DO JPMA }\end{array}$ & 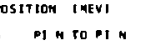 \\
\hline IN & 420. & $\begin{array}{l}123 \text { y } 103 / 2(2300) \\
160 .\end{array}$ & 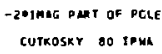 & $\begin{array}{l}\text { EOSITIOH (MEV) } \\
\text { PI h TO PI h }\end{array}$ \\
\hline RER & 9. & $\begin{array}{l}123 \quad 4 * 3 / 2123001 \\
4 .\end{array}$ & $\begin{array}{l}\text { MERL PART OF ELASTIC } \\
\text { CUTKOSKY DO IPYA }\end{array}$ & $\begin{array}{l}\text { C PCLE MESIDUE (MEV) } \\
\text { P! h TO PI N }\end{array}$ \\
\hline I m & -3. & $123 \quad 14 * 3 / 2(2300)$ & $\begin{array}{l}\text { TMAG PART OF ELASTIC } \\
\text { CUTKOSKY OO TPMA }\end{array}$ & $\begin{array}{l}\text { C PCLE MESIDUE (MEVI } \\
\text { OI h TO DE }\end{array}$ \\
\hline
\end{tabular}

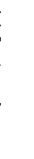

1/a9:

1/802:

1/202:

1/820:

$1 / 20$
$1 / 12:$
$1 / 72:$
$1 / 020$




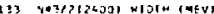

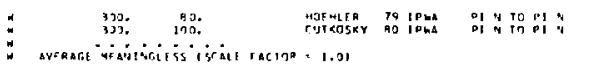

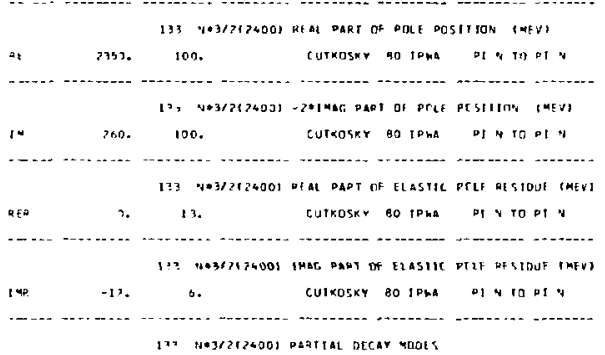

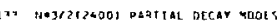

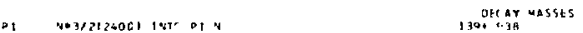

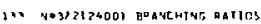

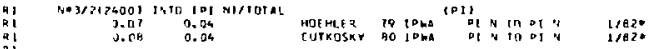

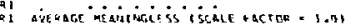

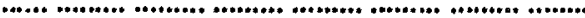
REFERETES FOR N*3/212400I

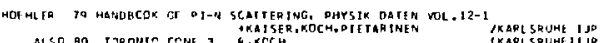

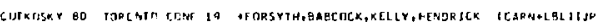

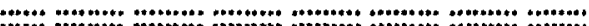

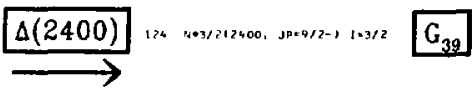

174 HE3/2124001 HASS TMEVI

\begin{tabular}{|c|c|c|c|c|c|}
\hline $\begin{array}{c}12170.4 \\
2200 . \\
7668 . \\
2300 .\end{array}$ & $\begin{array}{l}100 . \\
50 . \\
100 .\end{array}$ & $\begin{array}{l}\text { AYEO } \\
\text { HENDRY } \\
\text { GOFMLED } \\
\text { CUTKOSKY }\end{array}$ & 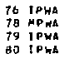 & 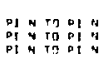 & $\begin{array}{l}11179 \\
12179 \\
12189 \\
1,320\end{array}$ \\
\hline
\end{tabular}

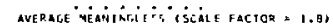

124 N*32124001 WIOTH IMEVI

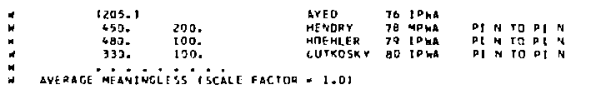

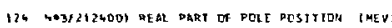

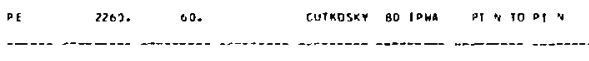

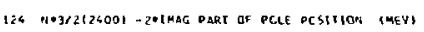
im 320. 150. CuTKeskr go tPHA Ot N TO DIN

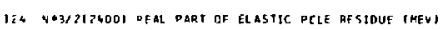
RER 1. 4. TUTKOSKY BO TPhA PI N TOPI A

124 DHzZZTZGOO TMAG PART OF ELASTIC PCLE RESTOUE (HEY)

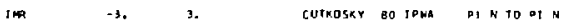

124 Pi*3/2124001 PARTIAL. OECAY MODFS

DI $N * 3 / 212400)$ INTO PI N

Decar masses

$1 / 62 *$

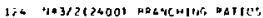

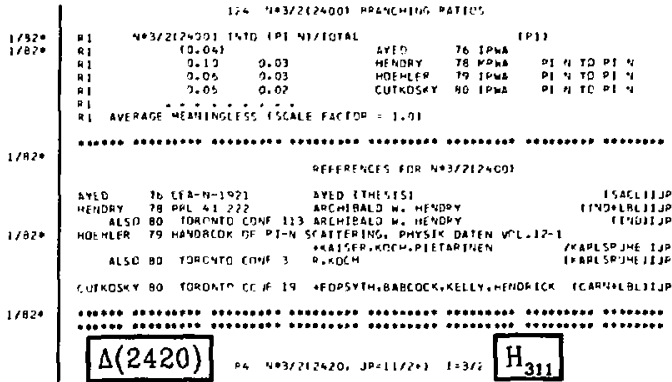

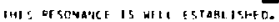

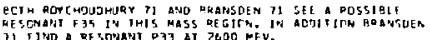

It $403 / 2124201$ 4ass (MIV)

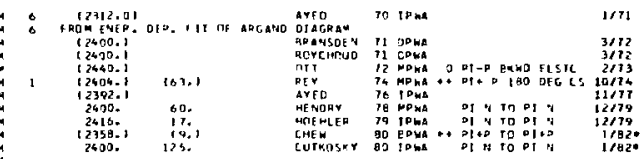

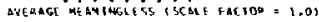

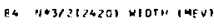

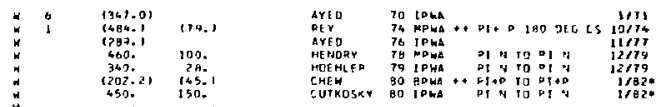

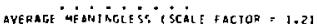

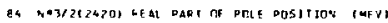

2360. 100

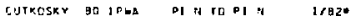

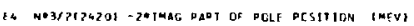

420. 100. CUTKC,KY 80 IOHK DI H IO DI A

$1 / 82 *$

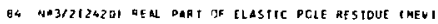

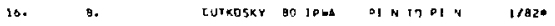

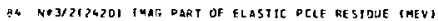

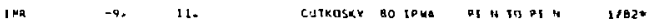

Q4 VEBZZZZZZO DARTIAL DECAY MDDES

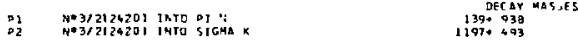

BQ NA3/2(2+20) BRANCHING RATIOS

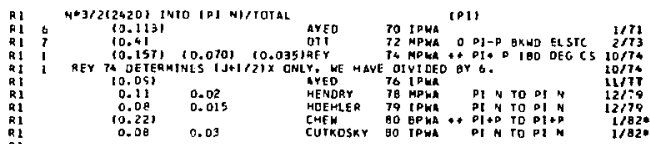

Rl average méaningifis "iscalle facton = 1.0 )

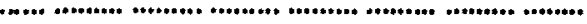


Baryons

$\Delta(2420), \Delta(>2500), \Delta(2750)$
Data Card Listings

For notation, see key at front of Listings.

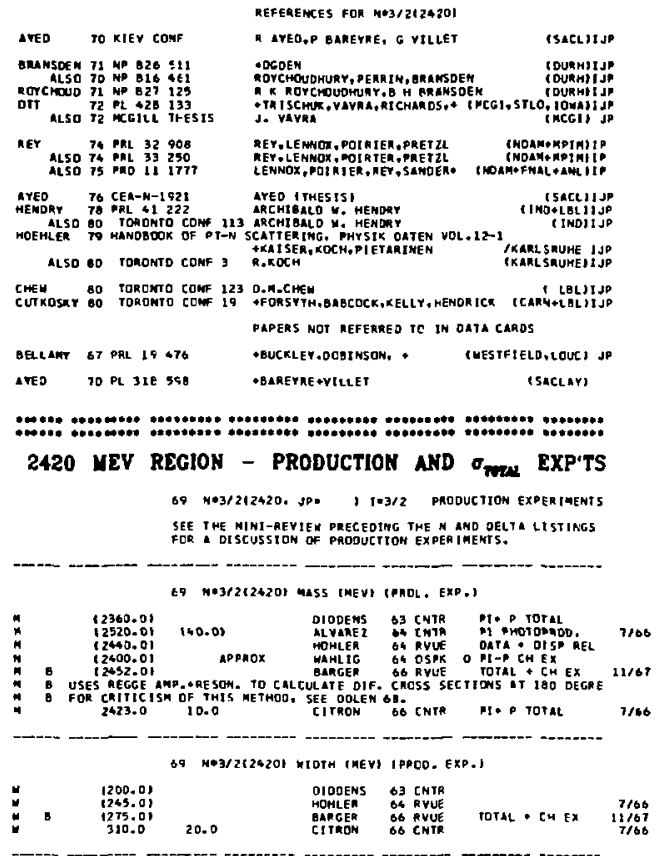

69 NO3/212420I PAPTIAL DECAY MODES IPRDD. EXP.I

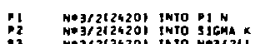

3
-4

GF NA3/212420I GRANCHIMG RATIOS (PROO. EXP.I

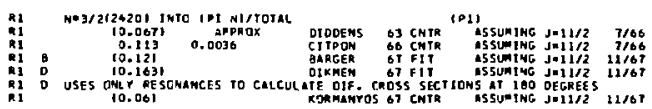

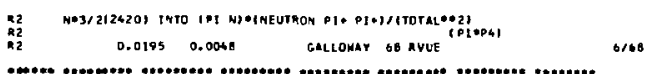

\section{MEFEREMCES ROR MOJ/2124Z201 10MOO. EXF-1}

OIDOEMS 63 PAL $202 E 2$ PJEMKINS, KYCHA RILEY

ALOACE

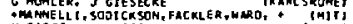

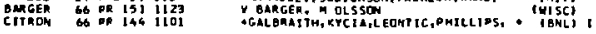

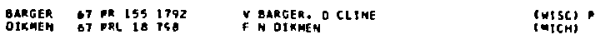

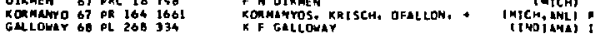

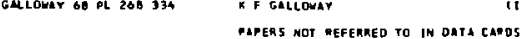

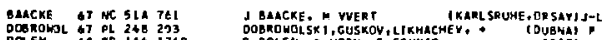

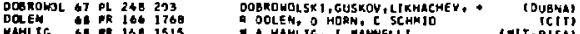

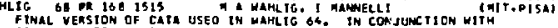

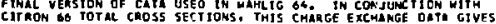

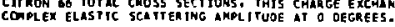

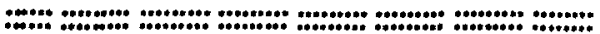

\section{>2500 MEV REGION - FORMATION EXPERIMENTS \\ $127 \mathrm{~N} * 3 / 21>2500)[0] / 2$ \\ YE LIST HERE 1-3/2 MESOMAHCES WITH KASS GREATER TWMN

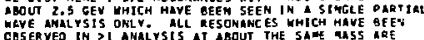

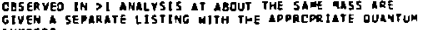 MUMBERS. \\ L27 H*3/26>2500) MESS (MEY)

\begin{tabular}{|c|c|c|c|c|c|c|}
\hline $\begin{array}{l}2650 . \\
3200 . \\
3300 . \\
3700 . \\
4100 .\end{array}$ & $\begin{array}{l}150 . \\
200 . \\
200 . \\
200 . \\
300 .\end{array}$ & $\begin{array}{l}\text { MENORY } \\
\text { MENORY } \\
\text { RENOAY } \\
\text { KENORY } \\
\text { MERDRY }\end{array}$ & 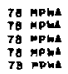 & $\begin{array}{ll}\text { PI } & N \\
\text { PI } & N \\
\text { PI } & N \\
01 & N \\
P I & N\end{array}$ & $\begin{array}{l}1311 \\
1313 \\
2317 \\
4319 \\
\text { N321 }\end{array}$ & $\begin{array}{l}12 / 79 \\
12179 \\
12 / 79 \\
12179 \\
12179\end{array}$ \\
\hline
\end{tabular} \\ average Teanimgie is iscile facton = 2.31 \\ $127 \mathrm{~N}+3 / 21>25001$ WIOTH (MEV)

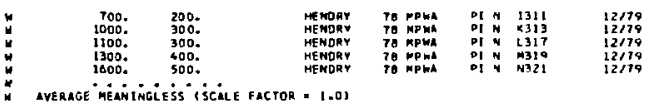

127 NO3/26 $>2500$ PAPTIAL DECAY DOCES $\underset{139+938}{\text { Decar }} \times 45565$

P1 M०3/21\%25001 INTO PI

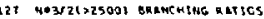

Ha3/2(1250C) INTO TP1 HI/TOTAL $\begin{array}{cc}0.06 & 10 \times 0 \\ 0.06 & 0.02 \\ 0.045 & 0.02 \\ 0.03 & 0.01 \\ 0.025 & 0.01\end{array}$ $\begin{array}{ll}0.025 & 0.01 \\ 0.010 & 0.01\end{array}$ MENDRY
HENORY
MENDRY
MENDRY
HENDRY

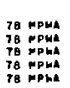
IPI) Y 1311

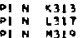
PI 1 M $12 / 79$
$12 / 199$
$12 / 79$
$12 / 19$
12179
$12 / 79$ RI average méanimgiéss iscile factor = 1.01

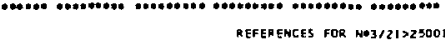

HENORY TO MRL 41222
ALSO BO TORONTO COMF 113 ARCHIBALO W. MENDRY

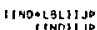

*

$\Delta(2750) \quad 12=N \cdot 3 / 212750, s_{0 \times 13 / 2-1} 1=3 / 2$ I 113

$\longrightarrow$

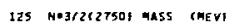

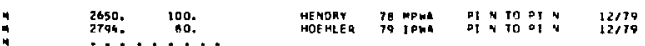
- ayenage méningiess i scale facton - 1.11

129 NE3/2IZTSOI WIOTH IMEVI

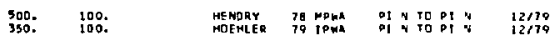

averace méningiess iscale factor = 1.11

i25 Na3/21275O) PARTIAL OECAY MODES

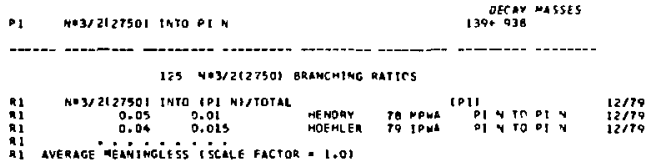

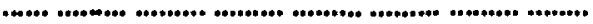
MEFERENCES FOR NO3/Z127SOI

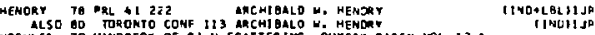

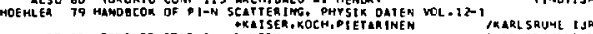
aLSO 00 TORONTO CONF 3 R,KOCH

(1) 


\section{Data Card Listings}

\section{$\Delta(2850)$
BUMPS}

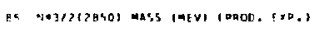

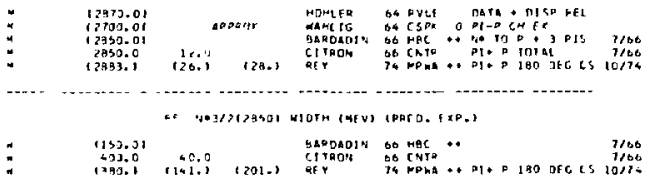

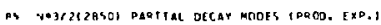

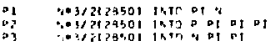
DER.AY UASSES

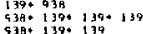

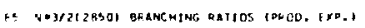

21
01
0.1

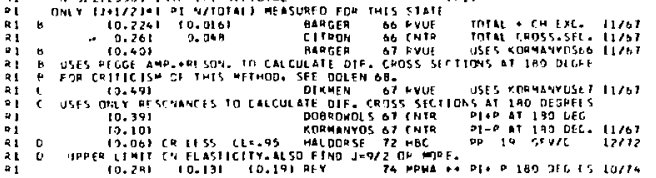

......................................................................

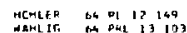

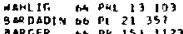

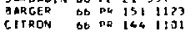

AARCE bI OF $195 \mathrm{JPa}$

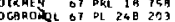

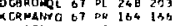

HAL DOFSE 72 "T 100 $46 \mathrm{~A}$

ALSO TH PRL 32 PC 950

REFERENCES FOA NOH/ZLZQSOI IPROD. EXP.:

G. HOHLER: J GIESECKE IKARLSPIJMES

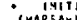

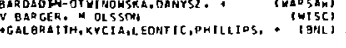

$\checkmark$ RLRGER, D CLINE

N OTKHEN GICH)

KOAMANYOS, KRISCH, DE ALLOAE, + IMICH, CNII :

HAL OOPSEN, JACOBSEN COSLOI IS

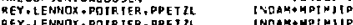

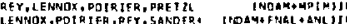

papers not aeferreo to IN oata capds

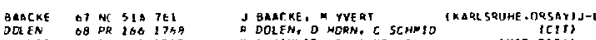
OOEN

G IMAL VERSIGN OF CA1A USEO IH WAHL IG G4. IN COHJUAC TICN WIIW

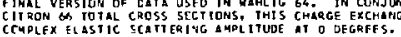

.

$\Delta(2950)$ ins $N+3 / 2\left(2950,5 P=15 / 2+1\right.$ i=3/2 $K_{315}$

\section{E No?/2129501 MASS (MEV)}

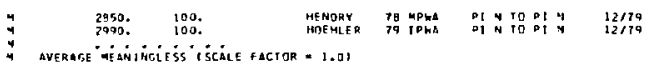

135 H*3/zizOSOL NIOTH (MEV)

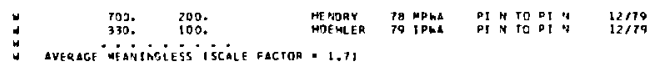

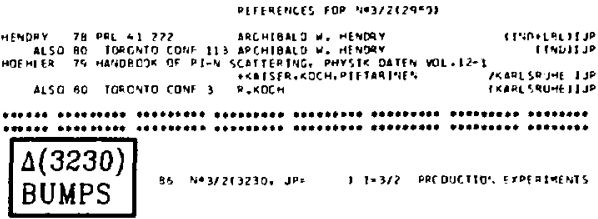

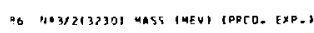

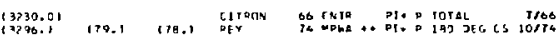

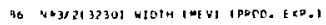

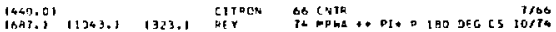

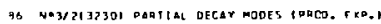

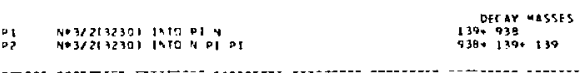

B6 N-3/2132301 BRANCMING DATIOS

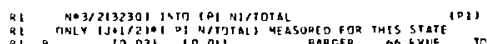

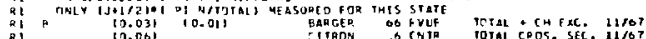

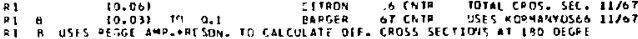

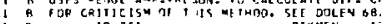

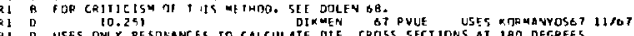

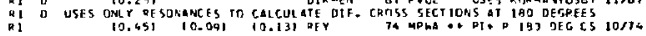

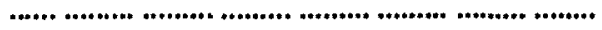

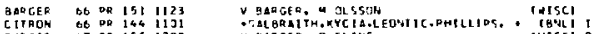

BARCER BT OD 15 S 179

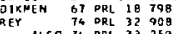

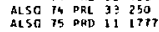

PUAGF, DCLTNE

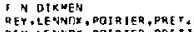

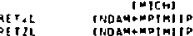

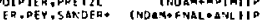

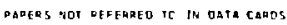

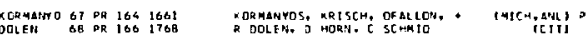

.

\section{EXOTIC NUCLEONS - 1400-1700 NEV REGION}

Exi1000-1700, Jez,, $1 \times 3 / 2$

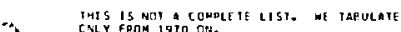

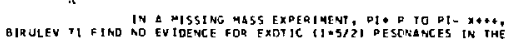

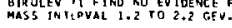

\section{EXITHOD-1 TOO) MASS RHEV)}

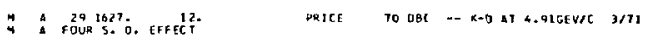

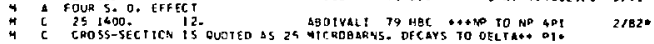
Exi1400-17001 WIOTH (MEVI

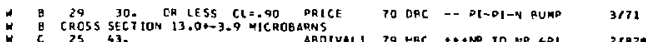

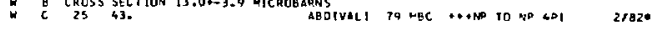

EX11600-17001 [aOSS SECTION LIMITS IMICROBARN

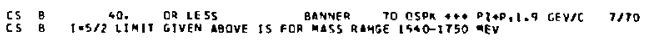

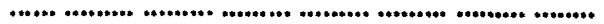
$\underset{139+938}{\text { OECAY MASSES }}$

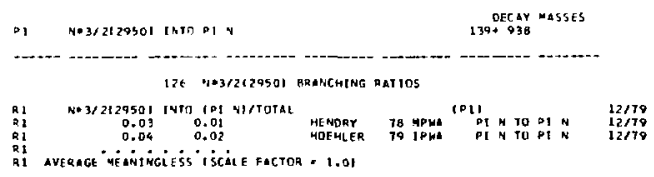

BLWNEP 70 NP 615205

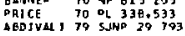

AMAHM 71 DL $34 B 53$

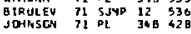
+
REFEREMCES FOR EX11400-17001

-CHeze, hamel, TElger, laccone - (saclay)

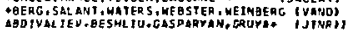

papers hot referped to in oata caros

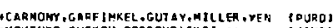

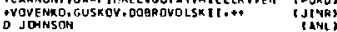


Baryons

$Z^{\prime \prime} s, Z_{0}(1780)$

\section{Note on the $S=+1$ Baryon Sygtem}

The evidence for $S=+1$ baryons was thoroughy.y reviewed In our 1976 edition, 1 and has been revlewed more recently by Kelly ${ }^{2}$ and by Oades. ${ }^{3}$

In the last few years the results of several new experiments have appeared. In particular, the $\mathrm{K}^{+} \mathrm{n}$ charge-exchange polarization data of WATTS 80 (for references in this form, see the listings that follow), the $K^{+} \mathrm{n}$ elastic polarization data of ROBERTSON 80 , and the $K_{L}^{0} p+k_{S}^{0} p$ data of CAMERON 78 , ENGLER 78, and CORDEN 79 provided bad1y needed new constraints on the $I=0 \mathrm{KN}$ amplitudes.

Three new phase-shift analyses have been reported since our last edition. EnergyIndependent analyseg were made by Martin and Dades 4 in the $I=0$ and $I=1 \mathrm{kN}$ channels, and by WATTS 80 in the $I=0$ and $I=1$ channels stmultaneously. CORDEN 82 made an energy-dependent analyals of the $I=0$ channel.

All analyses agree there ts little real ev1dence for classical Brelt-Wigner $z^{\star}$ reaonancea. The Argand diagrams for the $\mathrm{POl}$ and $\mathrm{P} 13$ amplitudes exhibit counterclockwise movement, but there are is corresponding speed maxima. However, the parametrIzations of MARTIN 75 and ARNDT 78 for the P13 amplitude do have unphysical-sheet poles. The recent low-energy $\mathrm{K}^{+} \mathrm{p}$ elastic polarization data of LOVETT 81 can be well described elther by the analysis of ARNDT 78 (which used prelimfnary data of LOVETT 81), or by other analyses not requiring structure in the PI3 amplitude.

The multi-quark states predicted by Jaffe $e^{5}$ in the MIT bag model should be most easily observed in the $\mathrm{KN}$ system. Jaffe and Low ${ }^{6}$ showed that such mult1-quark states need not appear as classical resonances in hadron-hadron scattering. Rather they would manifest themselves as poles $I_{n}$ the $P$ matrix, which connects the discrete quark states Inside the bag wich the physical scattering states outside. De Swart et al. ${ }^{7}$ have predicted the lowlying multi-quark states which might be seen in the $K N$ channel, and Rolesnel ${ }^{8}$ (using the partial-wave analysis of MARTIN 75) and CORDEN 82 have found such P-matrix poles. However, the agreement with the predictions of de Swart et al. 1s not completely compeliling.
Data Card Listings

For notation, see key at front of Listing..

\section{References}

1. Particle Data Group, Rev. Mod. Phys. 48, S188 (1976).

2. R.L. Kelly, in Proceeding of the Meeting on Exot1c Resonances (HUPD-7813) (H1roshima, 1978), ed. I. Endo et al.

3. G.C. Oades, in Low and Intermed late Energy Kaon-Nucleon Phygics (1981), ed. E. Ferrar1 and G. Violini.

4. B.R. Martin and G.C. Oades, in Proceedings of the IV Incernational Conference on Baryon Resonances (Toronto, 1980), ed. N. Isgur.

5. R.L. Jaffe, Phys. Rev. D15, 267 (1977).

6. R.L. Jaffe and F.E. Low, Phys. Rev. DI9, 2105 (1979).

7. J.J. de Swart et al,, In Proceedings of the IV ${ }^{\text {th }}$ International Conference on Baryon Resonances (Toronto, 1980), ed. N. Isgur.

8. C. Rolesnel, Phys. Rev. D20, 1646 (1979).

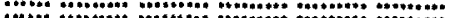

$$
S=1 \quad I=0 \text { EXOTIC STATES }\left(Z_{0}\right)
$$

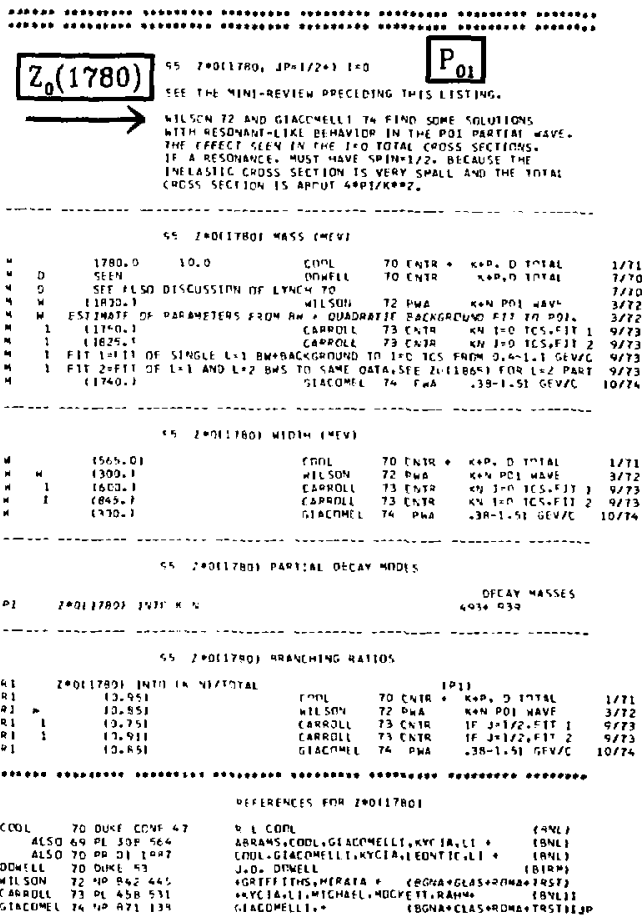


Data Card Listings

For notation, see key at front of Listings.
Baryons

$Z_{0}(1780), Z_{0}(1865), Z_{1}(1900)$

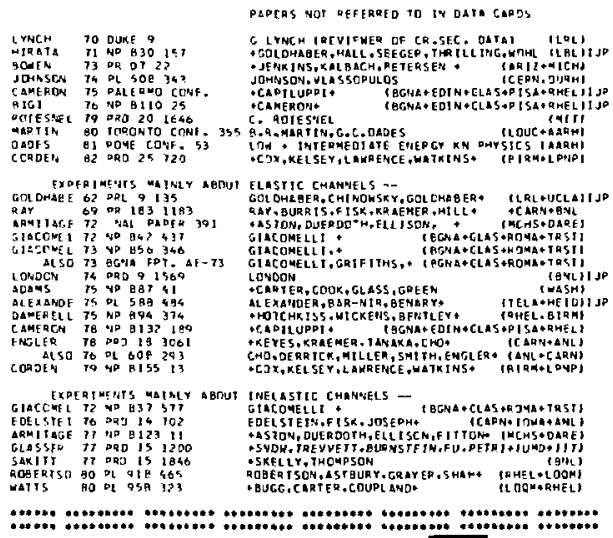

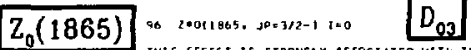

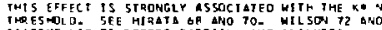
CACCOMELLIZ TS REPDH I DARTHAL WAVE AMALYSES.

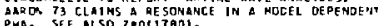

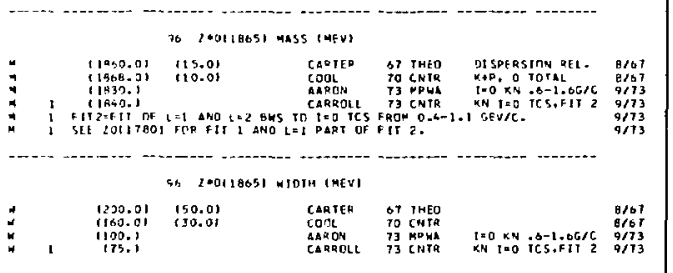

96 Z OOH LOSSI PARTIAL JECAY MODES

\begin{tabular}{|c|c|c|c|c|}
\hline & 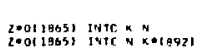 & & & 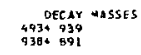 \\
\hline \multicolumn{5}{|c|}{ So TOMIIABSS BRANCNENG RATIOS } \\
\hline & 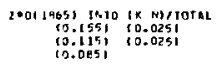 & $\begin{array}{l}\text { CARTER } \\
\text { EOOL } \\
\text { CARROLL }\end{array}$ & $\begin{array}{l}\text { OT TrEO } \\
70 \text { CKTR } \\
\text { T3 CNTR }\end{array}$ & 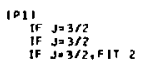 \\
\hline & 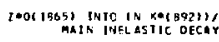 & HIRATA & $\triangle B$ HBC & $\mid 1821$ \\
\hline
\end{tabular}

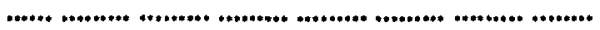

DEFEQENGES FOR 10011 BESI

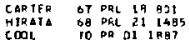

ALSO $66 \mathrm{DQL} 2.72102$

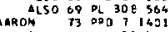

A capter

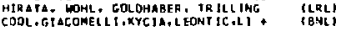

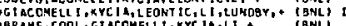

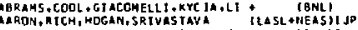

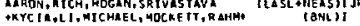

papeas not peferrea to IN Data caros

MTRATA TO DUKF 284,4

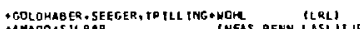

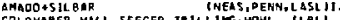

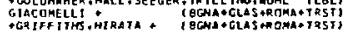

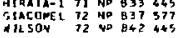

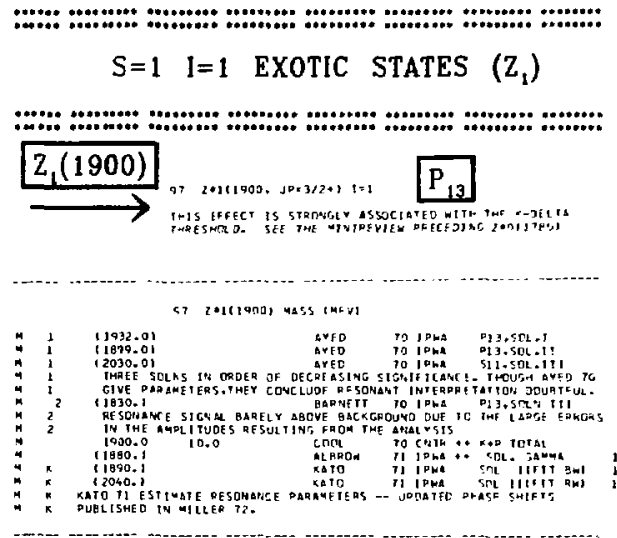

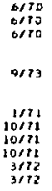

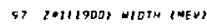

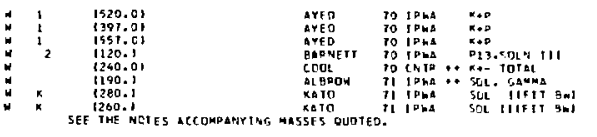

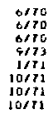

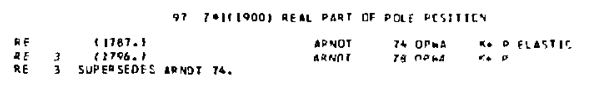

$4 r$

$4+75$
$3 \times 79$
$3 / 79$

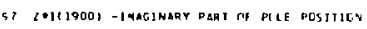

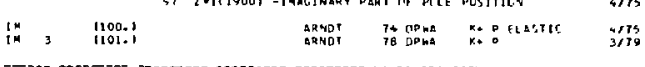

G7 T*ICIOOOI PARTLAL DECAY HODES

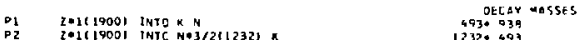

P2 $2 \cdot 161000$

97 IOI12900) RRAYCHING RATIOS

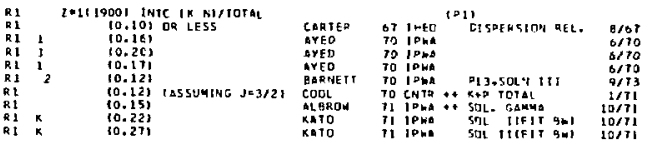
SEE MDTES ACCOMPAMYING the haSSES OVOTEO.

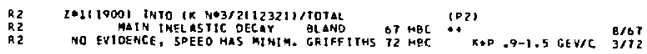

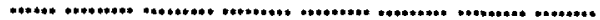

REFEREYCES Far 201119008

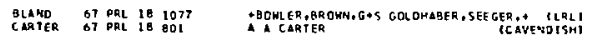

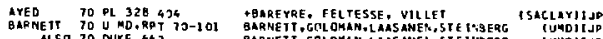

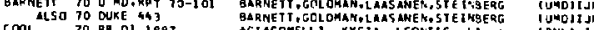

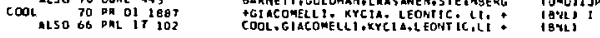

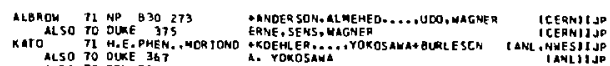

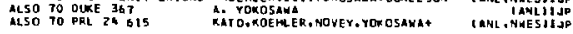

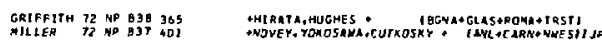

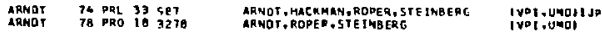


Baryons

$Z_{1}(1900), Z_{1}(2150), Z_{1}(2500)$
Data Card Listings

For notation, see key at front of Listings.

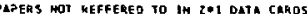

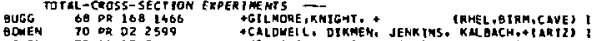

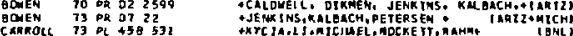
HITE K-MATAIX AMALYSTS OF SOME OF THE ERRLY KOP OATA - - G THESIS TMEORETICAL ANE WODEL OEPENOEMT AMAL YSES

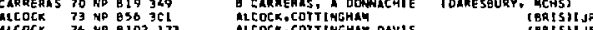

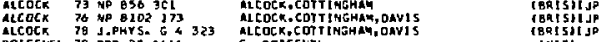
ROTESAEL 70 PRD 20 1640 323 C. ROTESNEL

BLAND EXPRIMENIS MAIMLY AgOUT IMELASTIC CHANMELS ---

BLAND G6 UCRL-19131 THESIS R W BLANO

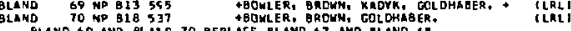

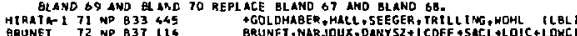

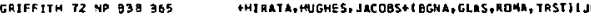

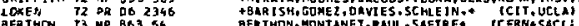
AEATHON T3 MP B63 SK

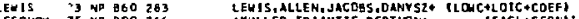

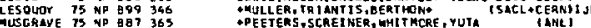

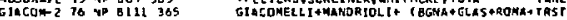

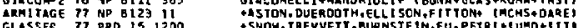
$\begin{array}{ll}\text { GLASSES } 77 \text { DRD } 15 & 1200 \\ \text { SAKITT } 77 \text { PAD } 15 & 1840\end{array}$

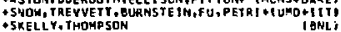

THE MA IN ELASTIC SCATTERIMG ANO POLARTLATION EXPER IMERTS -

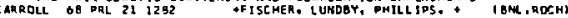

ANDEAS- 1 OO DL $29 E$ GI1

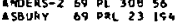

GLAND $6 \mathrm{PL} 296$ S18

IACOAE TO ND B2O 301

HEL TO DUKE 435

RDAMAS TI PRL 24160

BARNETT T1 PL $34: 055$

MHITMOAE TI OR 031092

ADA4S 72 YAL PADER 326

CHARLES 72 PL 409290 TOS

ONYSL 72 ip 14258

BAB OER 73 NP BOE IZS

BAQYETT 73 DQD E 2751 I

BISNSTEI 74 PRD 10276

CAMESON 76 ND BTB 93

ABE 75 PQD 11 1119

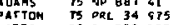

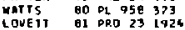

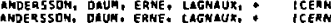
DOWELL, KATD, LUNDQUTSS, NOVEY, +1 LML, UMD

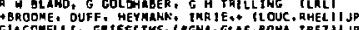
GIACOMELL I, GRTFFI THS, IEGNA, GEAS, DOMA, TRSM MJP +BLANDEGOL OHABER.TRILLIHG TLRLI

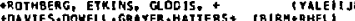
COAVIES, DOMELC, GRAYER, MAT TERS TBIRM CWHEL ETK

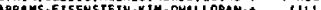
COX.DAVIES. DOHELL GRAYER + IBIQM,RHEL +COHKW, EOTAROS, CIESGN, TORIS,RHET, SHUP

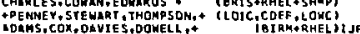

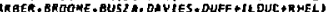
BARNETT, LAASANEN+ IUAD + MML + NWES +FNAL

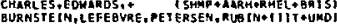

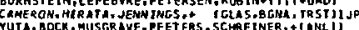
t CARTER, COOK. GLAS:. GMEEN IHASH - BARLETTA, EHALICH, ETKSN, THALE, TOKY, BNL BUGG.CAATER, COUPL ANOA CLOOM+AHELI

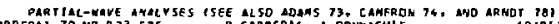

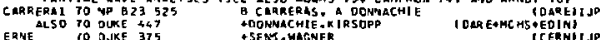
ERNE 10 OJKF 375

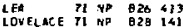
CUTKOSKY 72 NAL PAPER 210 ARTIN 72 PAFPRINT

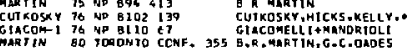

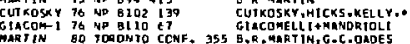

TSENT- HAGNER

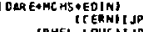

(RHEL LOUC)IJP
TEERHIIJP

-HICKS, KEELY, 5 HIH, JOHNSO

(C MRN+1LL I +ANL)

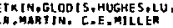

GITCOSKY,HICKS, KELLY

(CARN+ITTHANLIT

(BGNATGLAST,
(LOUT+BARH)

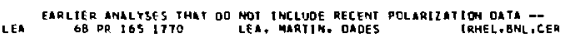

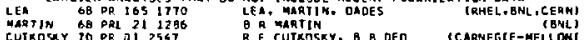

\section{LABEST DEVIEW TALKS NHO PAPERS}

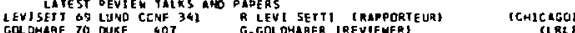

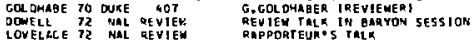

DOHELL 73 POROUE CDAF. 15T DONEL

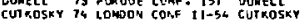

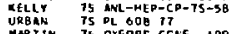

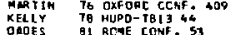

REVIEW VALX IN BARYan SESSION TCERT
(LBL)

(LRe

SIPY)

(BIAM)

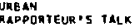

(L9L)

LON . ENTEATEDIAIE ENERGY KN PHYSICS (AMRH)

"ropen

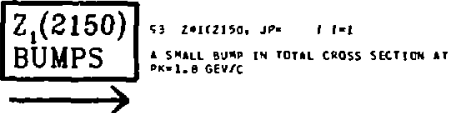

93 I01121501 MASS (MEV)

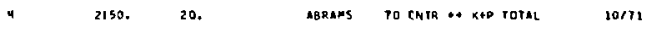

53 2 DII22SOI WIDTH TMEYI

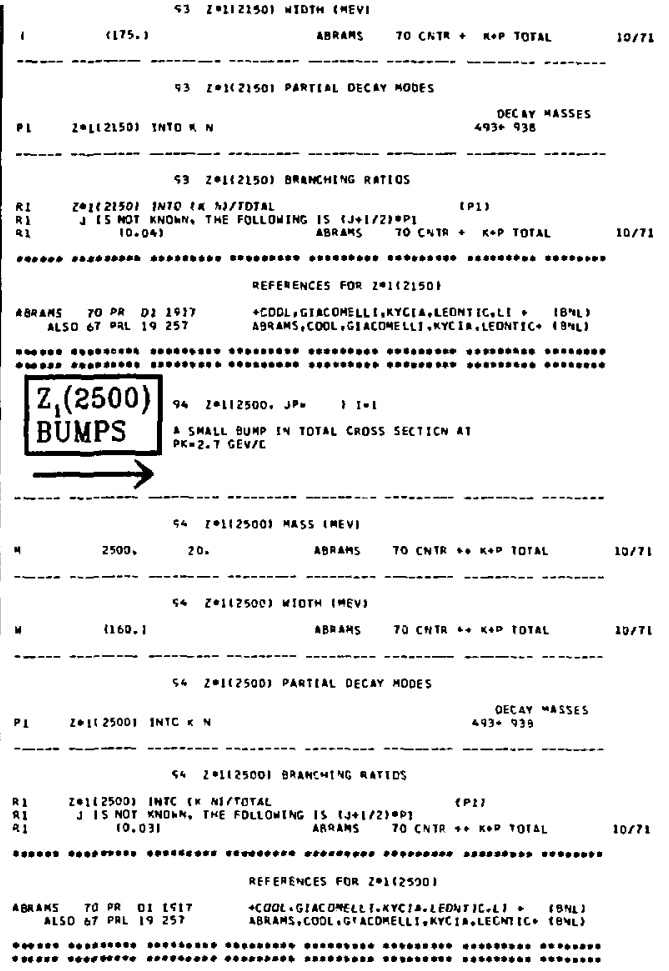

\section{z, CROSS SECTION LIMITS}

SEE mintrevien pReceting i*o

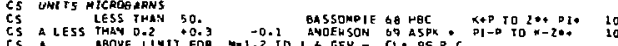

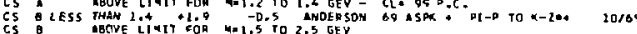

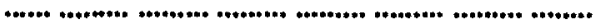
DEFEREMCES FOR Z"L CROSS SECTION LIAITS

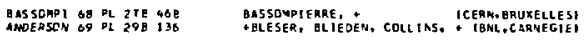

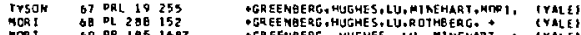

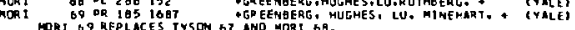

: 
Data Card Listings

For notation, see key at front of Listings.

Note on $\Lambda^{*} s$ and $E^{\prime} s$

The number of established $y^{*}$ resonances is st111 slowly tncreasing. In 1978 two $\Lambda^{\prime} s$ and one $\Sigma$ were promoted to the Baryon Table, In 1980 noiie were promoted, and in this edition two $\Lambda^{\prime} S$ have been promoted. There remain, however, a large number of proposed but unconfirsed regonances in the Data Cerd Listings. Table I is an attempt to evaluate the status, both overall and channel by channel, of each $\mathrm{Y}^{\star}$ in the Listings; the evaluations are of course partly subjective. A blank Indicates there is no evidence at all: elther the relevant couplings are small or the resonance does not really exist. The Baryon Table includes only the well-established resonances (overall status 3 or 4 stars). Any particular one of the questionable resonances may disappear with the next analysis, but there are probably many new resonances underlying those already established. Fone of the $\mathrm{Y}^{*}$ "s proposed in the last few years couple strongly to the main 2-body decay channels $N \bar{K}, A \pi$, and $\Sigma \pi$, and thus they seldom appear in cross sections or Invariant mass distributtons. Rather, when the reactions $\overline{k N}+\bar{k} N$, $\bar{K} N+\Lambda \pi$, and $\bar{K} N+\sum \pi$ are partlal-wave analyzed, some of the amplitudes are found to traverse small, more-or-less resonance-like counterclockwise clrcles. The question in each case 1s: Is this really a resonance, or 18 it an $1 d$ le meander? Is the effect even real, or is th the result of 1mperfect data and analysis procedures?

\section{Formation Experiments}

(by G.P. Gopal, Rutherford and Appleton Laboratories)

Partial-wave analyses have been made mainly for the $N \bar{K}, \Lambda \pi$, and $\Sigma$ is channels, but there are also a few results for the $\equiv \mathrm{K}, \Lambda \omega$, and some quasi-2-body channels. The earliest analyses covered, in the ma1n, rather narrow mass ranges, usually the range of a single bubble chamber experlment. Although the amplitudes obtained of ten did not join smoothly with those frocl other analyses of the same channel performed in neighboring mass ranges, they gave useful and fairly rellable information about the strongly coupled reaonances dominating the middle of the ranges covered. The more recent analyses

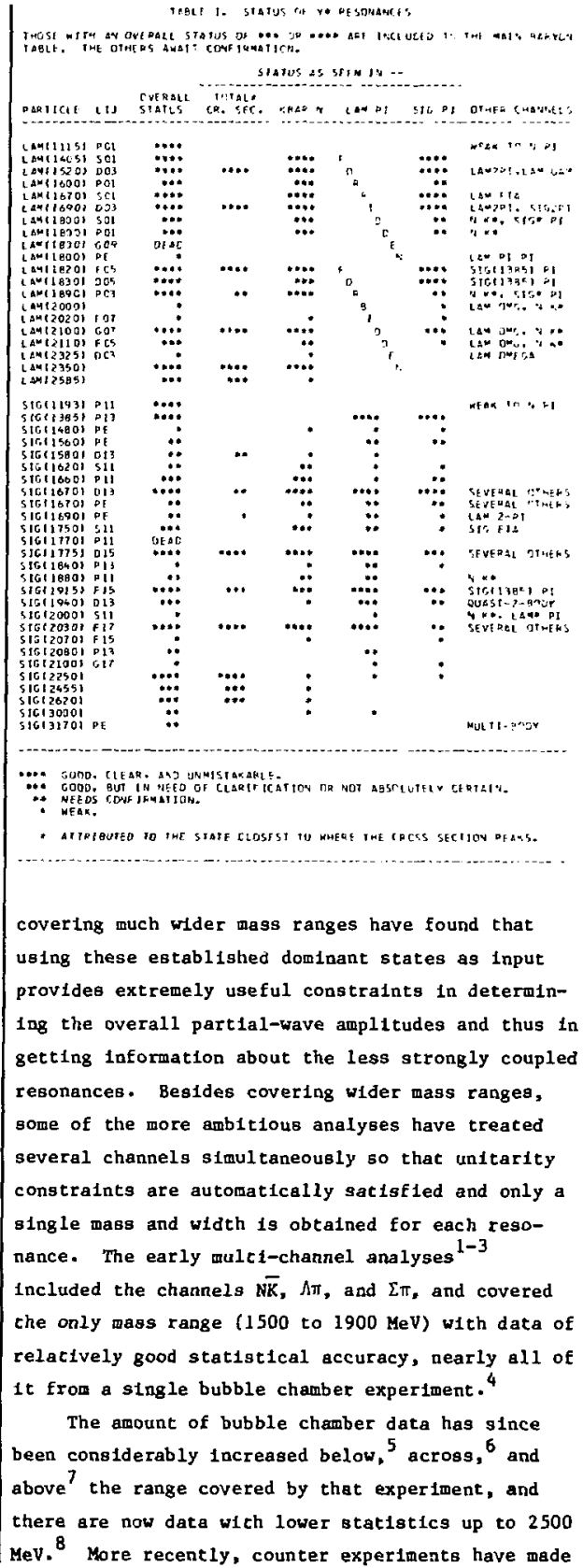




\section{Baryons}

\section{$\Lambda$ 's and $\Sigma$ 's}

mafor contributiona by measuring the $\mathrm{K}^{-} \mathrm{p} \rightarrow \overline{\mathrm{K}}^{0} \mathrm{n}$ total and differential cross sectlons at low energies, ${ }^{9}$ the $K^{-}$p polarizations down to $1630 \mathrm{MeV}$ for the first time, 10 the $K^{-}$p polarizations over the range 1700 to $1900 \mathrm{MeV}$ with an order of aagnitude increase in statistics, ${ }^{11}$ the pure $I=1 \mathrm{~K}^{-} \mathrm{n}$ elastic angular distributions from 1600 to $1800 \mathrm{MeV}^{12}$ and from 1900 to $2300 \mathrm{MeV},{ }^{13}$ and the $180^{\circ} \mathrm{K}^{-} \mathrm{p}$ and $0^{\circ}$ $\Sigma^{-} \pi^{+}$differential croos sections from 1550 to 1900 MeV. ${ }^{14}$ There are also new data with good statiotics on pure $I=1 k_{L}^{0}$ interactions in the $A \pi^{+}$and, more Importantly, the $\Sigma^{0} \pi^{+}$channela. ${ }^{15-17}$ Finally, there are new $K^{-} n \rightarrow(\Sigma \pi)^{-}$data over the range 1750 to $2200 \mathrm{MeV} .^{18}$ All these new data provide sensitive tests of the overall correctness of existing competing partial-wave amplitudes. Some of the new data have yet to be Incorporated Into new analyses.

In the following, we discuss the more recent partial-wave analyses, comparing them with each other and with the new data.

The $\sqrt{\mathrm{N}}$ channel: The most recent analygis of this channel is an update ${ }^{19}$ of the old Rutherford Lab-Imperial College (RLIC 77) analys18. ${ }^{20}$ AB was Its predecessor, it is a conventional energydependent analysis with the added constraint that the masses and widths of the resonances must be consistent with those deterwined in the Inelastic channels analyzed previously -- $\Lambda \pi, \Sigma \pi, \Lambda(1520) \pi$, $\Sigma(1385) \pi$, and $\mathrm{NK}^{k}(892)$. With good angular distributions now avallable at lower energies, 5 the analysis goes closer to threshold: the range covered is 1470 to $2170 \mathrm{MeV}$. With the exception of the very high-statiotics charge-exchange differential cross-section measurements 9 (which are in serfous disagreement with both the earlfer and the latest ${ }^{6}$ high-statistics bubble chamber measurements) and the backward elastic data, ${ }^{14}$ all the new $\mathrm{N} \overline{\mathrm{K}}$ data mentioned above have been included. As before, angular distributions (a total of 5110 data pofnts) have been fitted directly. The new partial-wave amplitudes are not afgnificantly different from the old RLIC 77 amplitudes for this channel. However, the Incluston of the $I=1 \quad K n$ data has removed some of the uncertafaties in the $I=1 \quad Y^{*}$ spectrum.

The LBL-Mt. Holyoke-CERN analyois ${ }^{21}$ covers the

\section{Data Card Listings}

For notation, see key at front of Listings.

narrover range of 1500 to $1940 \mathrm{MeV}$ and also includes most of the new data. It is an energydependent analysis using a unltary background parametrization in terms of Bcattering lengths. The cusp effects at the $A$, and $\sum n$ thresholds are Included by introducing a square-root gingularity in the energy variation of the uidths of the corresponding resonances. The focluston of their own high-statistica charge-exchange data ${ }^{9}$ - admittedly not in good agreement with bubble chamber measurements -- all but kills the less velleatablished resonances.

The University College, London (UCL) K-matrix energy-dependent analys $1 \mathrm{~s}^{22}$ covers the range 1540 to $2000 \mathrm{MeV}$. The NK amplitudes are consistent with those of the other analyses over the greater part of this range. However, at the low-energy end there are considerable differences, Ind icating the lack of strong constralnts fron the domlnant $\mathrm{DO} 3$ $\Lambda(1520)$ state -- just below the range coverec. The new $\mathrm{K}^{-} \mathrm{n}$ angular distributions and $\mathrm{K}^{-} \mathrm{p}$ polarization neasurements are not very well described by the amplitudes from this analysis.

These analyses, all below $2200 \mathrm{MeV}$, are complemented by the College de France-Saclay (CdF-S) energy-dependent analya19 ${ }^{8}$ covering the range 2070 to $2440 \mathrm{MeV}$. Besides the conventional polynomial parametrization of the background amplitudes, they also tried a more economical parametrization with constralnts Imposed from the duality hypothesis (that s-channel backgrounds come exclustvely from the t-channel Pomeron exchange amplitude). With 30 fewer free parameters, the results are consistent with the wore conventional approach.

The $\sum \pi$ channel: There are no new analyses of this channel. There is very 11 thle agreement, particularly in the lover partial uaves, between the asplitudes of the two multi-channel analyses. 20,22 The low energy $k_{L}^{0} p-\Sigma^{0} \pi^{+}$data ${ }^{15,16}$ are better explained by the RLIC 77 amplitudes than by the UCL aplitudes. At the higher mass end there 19 good continuity between the RLIC 77 amplitudes and those of the alngle-channel analyois of the CdF-S collaboration covering the range 2070 to $2440 \mathrm{MeV}$. The two strongly coupled resonances, D03 $\Lambda(1520)$ and $607 \Lambda(2110), 1 y$ ing below and above the mass range covered by the UCL analysis, clearly provide strong 


\section{Data Card Listings}

For notation, see key at front of Listings.

constraints on the amplitudes.

The in channel: Th1s pure I=l channel, where sioultaneous measurements of the polarization and differential cross section are possible, has been the subject of many wide-range energy-dependent and energy-independent analyses (for example, $R L L C$ $77,{ }^{20}$ UCL, ${ }^{22}$ Baillon-Litchfield, ${ }^{23}$ de BellefonBerthon, ${ }^{24}$ and Van Horn ${ }^{25}$ ). However, even the widespread use of the method of Barrelet zeroes has not helped to resolve the $I=1 Y^{*}$ spectrum -- probably because most of the $I=1$ states do not couple strongly to the initial NK channel.

Quas1-2-body channelg: The RLIC group has made energy-dependent analyges of the $\Lambda(1520) \pi$, $\Sigma(1385) \pi$, and $N \bar{K}^{*}(892)$ channels over the widest ranges for which data are avallable. The data are extracted from the appropriate 3-particle final states by making 4-variable fits to an incoherent superposition of quas1-2-body final states and 3particle Lorentz-1nvariant phase space. The qual1ty of the fits guggests a maximum model-dependent systematic uncertalncy of $10 \%$. The errors quoted on the $\mathrm{Y}^{\star}$ couplings do not include this uncertainty. The $N \omega$ channel has been analyzed from threshold to $2440 \mathrm{MeV}$ by the CdF-s collaboration.

F1gures: Argand plots of fifteen $S=-1$ partial waves are shown $1 \mathrm{n} \mathrm{Fig}$. 1(a) through (k). The analyses shown were picked for lllustrative purposes rather than on the basis of our judgment of their quality. For the $\bar{X} N$ channel, we show the amplitudes from RLIC $77^{20}$ and from LBL-Ht. HolyokeCERN, ${ }^{21}$ and for the $\Lambda \pi$ and $\sum \pi$ channels those from RLIC $77^{20}$ and from UCL. 22

Errors on masges and widths: of ten the errors quoted on resonance parameters from partial-wave analyses are only gtatistical, and the parameters can change by more than these errorg when a different parametrization of the waves is used. Furthermore, the different analyses use more or less the same set of data, so it is not really approprlate to treat the different determinations of the resonance parameters as Independent measurements or to average them together. In any case, the spread of the nasses, widtha, and branching fractiong from the different analyses is certainly a better Indication of the uncertainties than are the quoted errors. In the Baryon Table, usually a range reflecting the spread of values obtainad is given rather than a particular value with error. For three stateg, the $\Lambda(1520)$, the $\Lambda(1820)$, and the $\Sigma(1775)$, there is enough information available to make an overall $f$ it of the various branchIng fractions (see Sec. VII $B$ of the main text). It is then necessary to use the quoted errors, but the errors obtalned from the fit are not to be taken seriously.

\section{Production Experiments}

Partial-wave analyses of course sefarate partial waves, whereas a peak in a cross section or an Invariant mass distribution ugualy cannot be d1sentangled from background and apalyzed for its quantum numbers; and more than one resonance may be contributing to the peak. Results from partialwave analyses and $F$ rom production experiwents are generally kept separate in the Listings, and in the Baryon Table results from production experiments are used only for the low mass states. The $\Sigma(1385)$ and $\Lambda(1405)$ of course 11 e below the $\overline{k N}$ threshold and everything about them comes from production experiments; and production and formation experiments agree quite well in the case of $\Lambda(1520)$ and results have been combined. There is soae disagreement between production and formation experiments in the 1600-to-1700 $\mathrm{HeV}$ region: see the $\Sigma(1620)$ and $\Sigma(1670)$ notes for detalls.

The most interesting recent resulc from a production experiment is the observation of a narrow hyperon of mass $3170 \mathrm{MeV}$ decaylng to mult1-atrange final states contalnting five or six particles $(N / K \bar{K}+$ plons, $\Xi K+$ plons $)$. The effect is seen in two high-statisticg bubble chamber experinents studying $K^{-}$p interactions at 6.5 and $8.25 \mathrm{GeV} / \mathrm{C}^{2}{ }^{26}$ The statistical significance of the peak is about 6.5 standard deviations, and the observed wdth is consistent with the experinental resolution. The modes with three strange particles possibly suggest

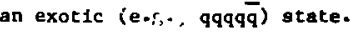

\section{Reierenceg}

(see also the L1stings)

1. J.K. KIm, Phys. Rev. Latt. 27, 356 (1971). 
Baryons

$\Lambda$ 's and $\Sigma$ 's
Data Card Listings

For notation, see key at front of Listings.
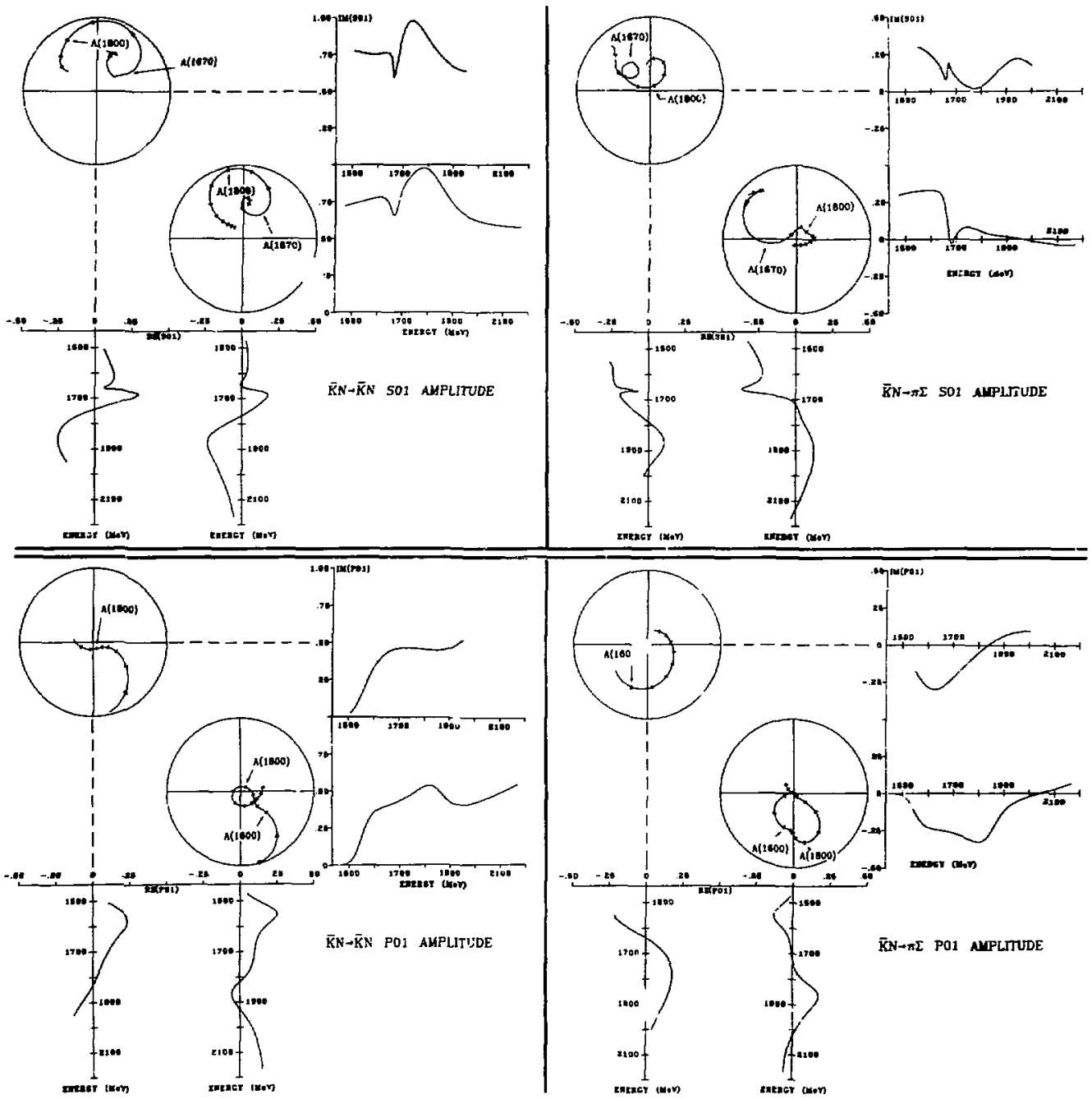

F18. 1(a). The L.I.2J - S01 and P01 partiul-wave amplitudes for $\bar{K} N$ scattering in the elestic and En channela. The lower plot for each anplitude 1s from RLIC 77, the upper plots for the elastic anplitudes are from ALSTON 78, and the upper plots for the $[\pi$ amplitudes are frow MAR'IN 77 . In the Argand plots, the t1cke are at integral wultiples of $50 \mathrm{MeV}$, and the esteblished resonances are hown at their noninal positlone [the SO1 $\mathrm{A}(1405)$ 1s of course below threshold and is not shown]. The real and inaginary parts of the aplitudes as function: of energy are shown profected in alignent with the Argand plots. 


\section{Data Card Listings}

For notation, see key at front of Listings.

Baryons

$\Lambda^{\prime} s$ and $\Sigma^{\prime} s$
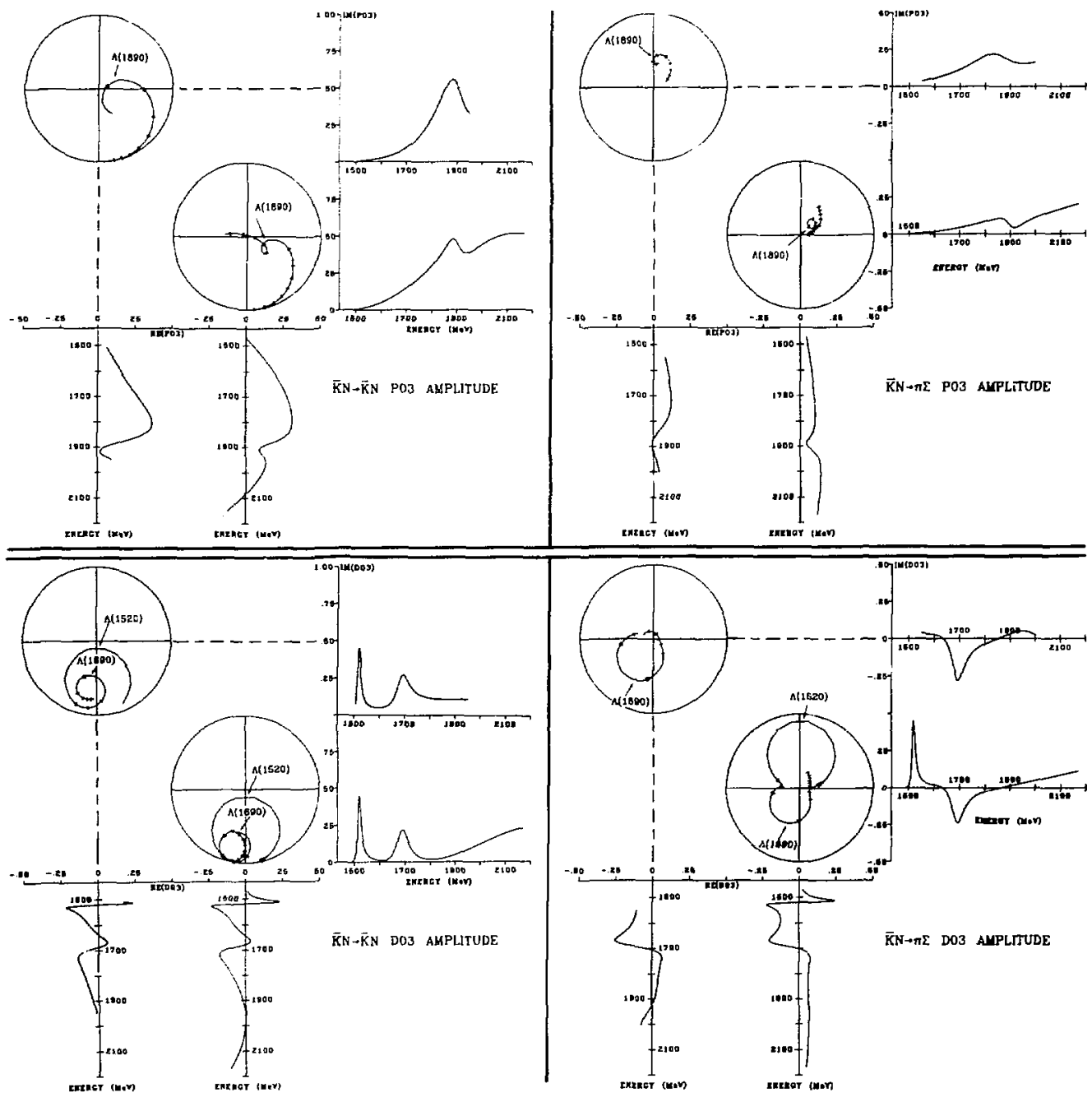

Fig. 1(b). The L.I.2J = P03 and D0 partlal-wave amplitudes for $\bar{K} N$ scart:ring 11, the elastic and $\Sigma \pi$ channels. The lower plot for each amplitude 18 from RLIC 77 , the upper plots for the elastic amplitudes are fron ALSTON 78, and the upper plots for the $\Sigma \pi$ amplitudes are from MARTIN 77 . In the Argand plots, the t1cks are at 1ntegral multiples of $50 \mathrm{MeV}$, and the established resonances are shown at their norinal posit1ons. The real and imaginary parts of the amplitudes as furctions of energy are shown projected in alignment with the Argand plots. 
Baryons

$\Lambda$ 's and $\Sigma$ 's
Data Card Listings

For notation, see key at front of Listings.
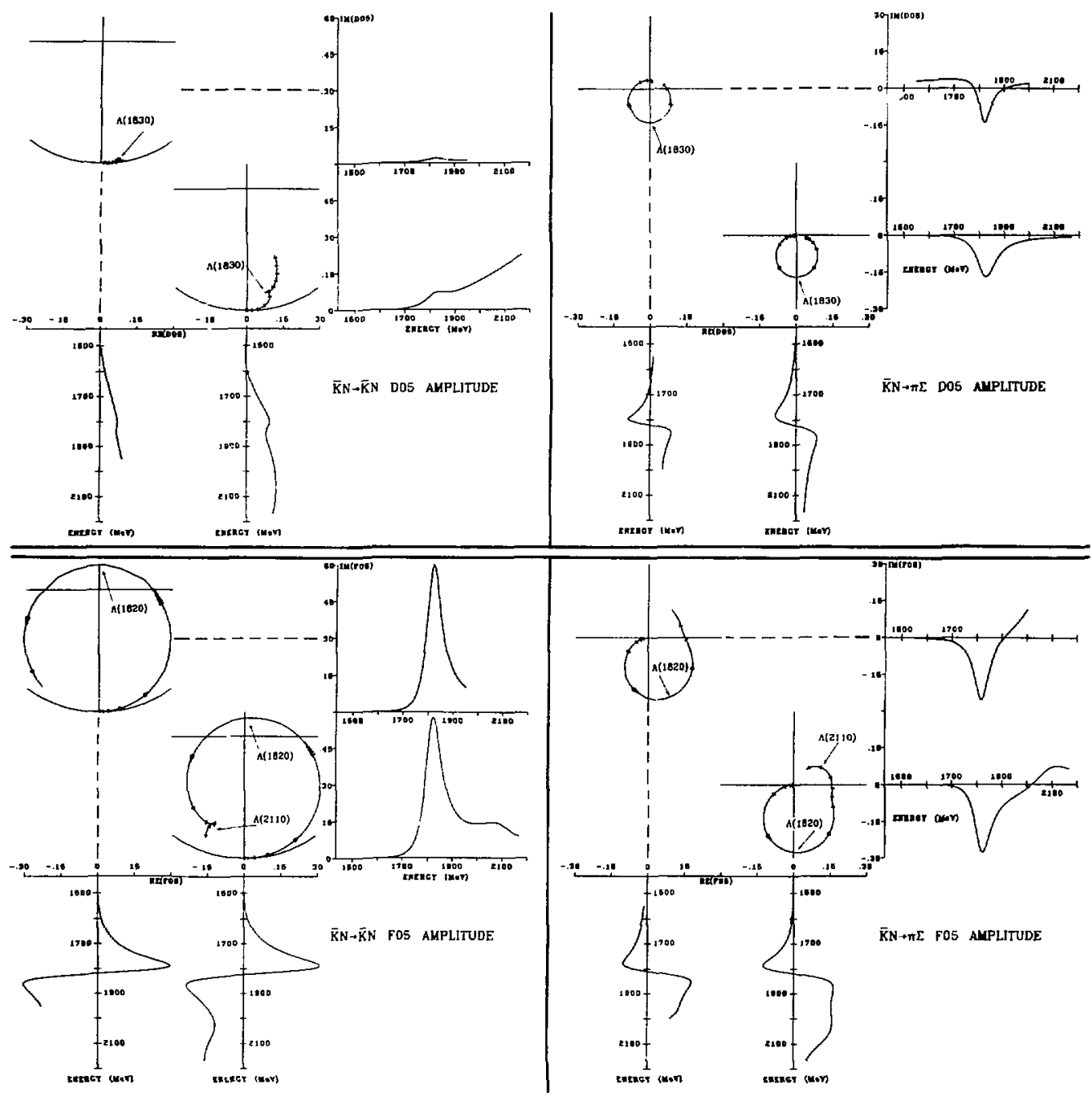

P1g. 1(c). The L.I.2J = D05 and F05 partial-wave amplitudes for $\bar{K} N$ scattering in the elastic and $\sum \pi$ channels. The lower plot for each amplitude is from RLIC 77, the upper plots for the elastic amplitudes are frow ALSTON 78, and the upper plots for the $\varepsilon \pi$ amplitudes are from MaRTIN 77 . In the Argand plots, the ticke are at integral multiples of $50 \mathrm{MeV}$, and the established resonances are shown at the1r nominal positlong. The real and tmaginary parts of the amplitudes as functions of energy are shown profected in alignment with the Argand plots. 
Data Card Listings

For notation, see key at front of Listings.
Baryons

$\Lambda^{\prime} s$ and $\Sigma$ 's
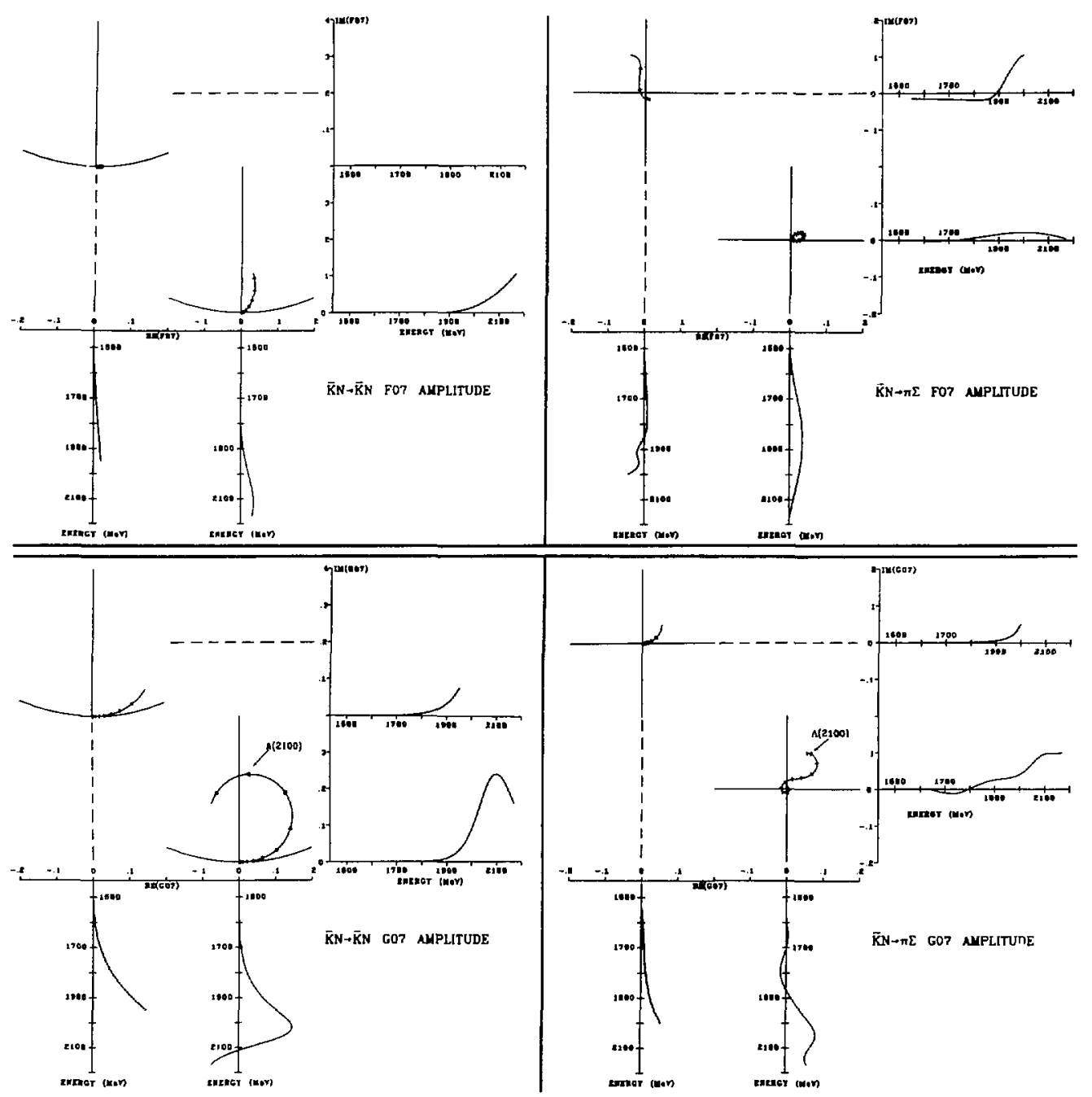

Z1g. 1(d). The L.I.2J = F07 and 607 partial-wave amplitudes for $\bar{k} N$ scattering in the elastic and $\sum \pi$ channeis. The lower plot for each amplitude is frow RLIC 77, the upper plots for the elaatic amplitudeg are from ALSTON 78, and the upper plota for the $\sum \pi$ amplitudes are from MARTIN 77. In the Argand plots, the ticks are at lategral multiples of $50 \mathrm{HeV}$, and the establiahed resonance is shown at itg nominal position. The real and 1maginary parts of the anplitudes as functions of energy are ohown projected in alignment with the Argand plots. 


\section{Baryons}

$\Lambda$ 's and $\Sigma$ 's
Data Card Listings

For notation, see key at front of Listings.
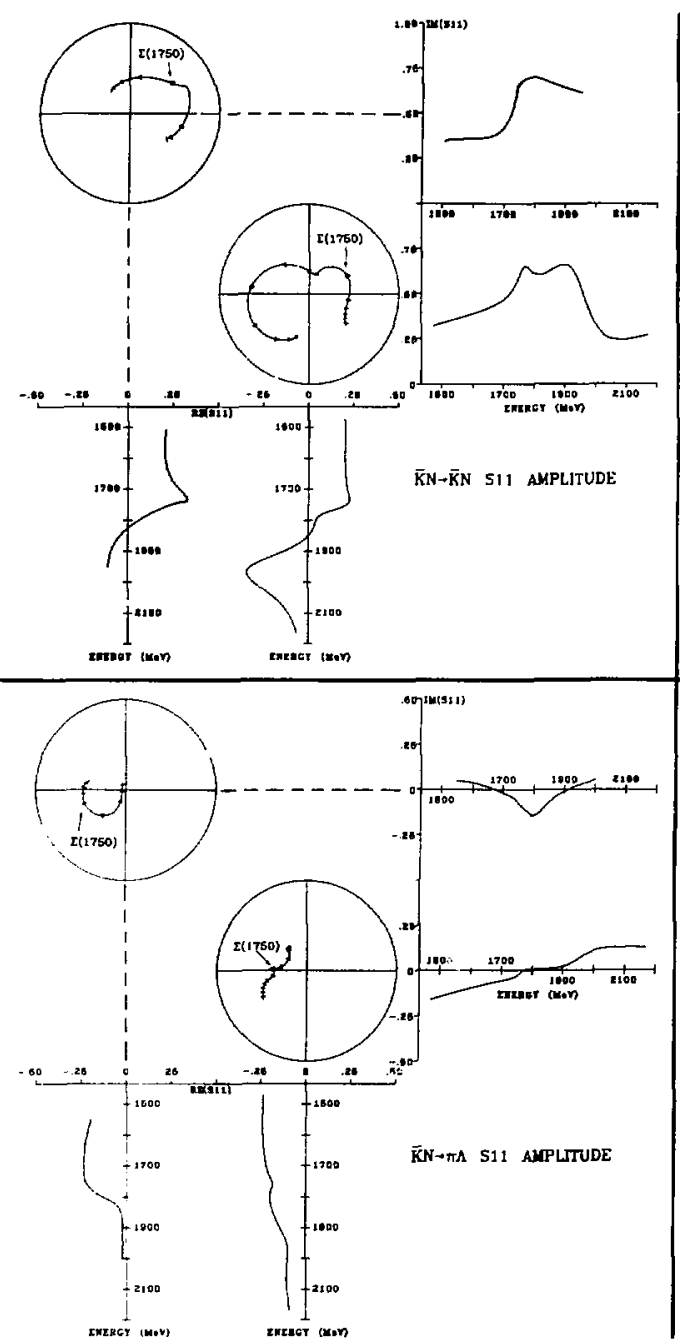

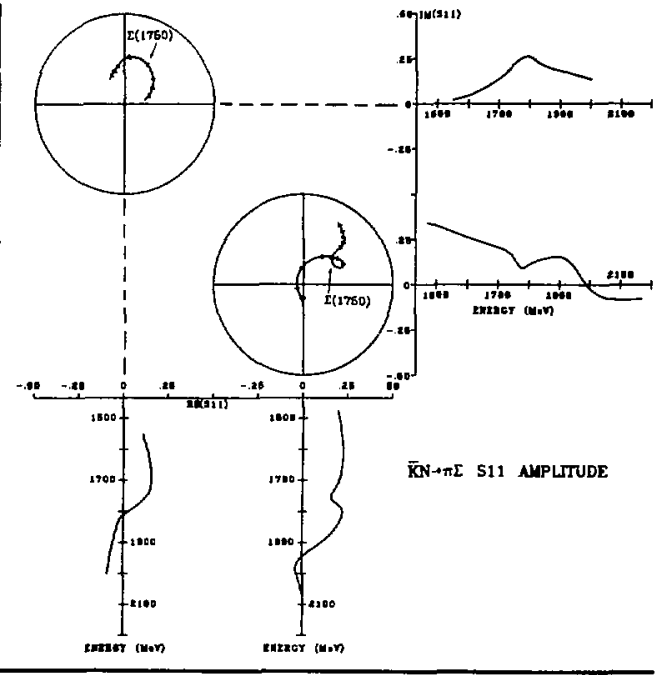

Fig. 1(e). The L.I.2J = S11 partial-wave amplttudes for $\bar{k} N$ scattering in the elastic, $\Lambda \pi$, and $\sum \pi$ channels. The lower plot for each amplitude 1s from RLIC 77, the upper plot for the eiagtic amplitude is from ALSTON 78, and the upper plots for the $\Lambda \pi$ and $\Sigma \pi$ channels are from MARTIN 77 . In the Argand plots, the ticks are at integral multiples of $50 \mathrm{MeV}$, and the established resonance is shown at its nominal position. The resl and imaginary parts of the amplitudea as functions of energy are shown projected in alignment with the Argand plots. 


\section{Data Card Listings}

For notation, see key at front of Listings.

Baryons

$\Lambda$ 's and $\Sigma$ 's

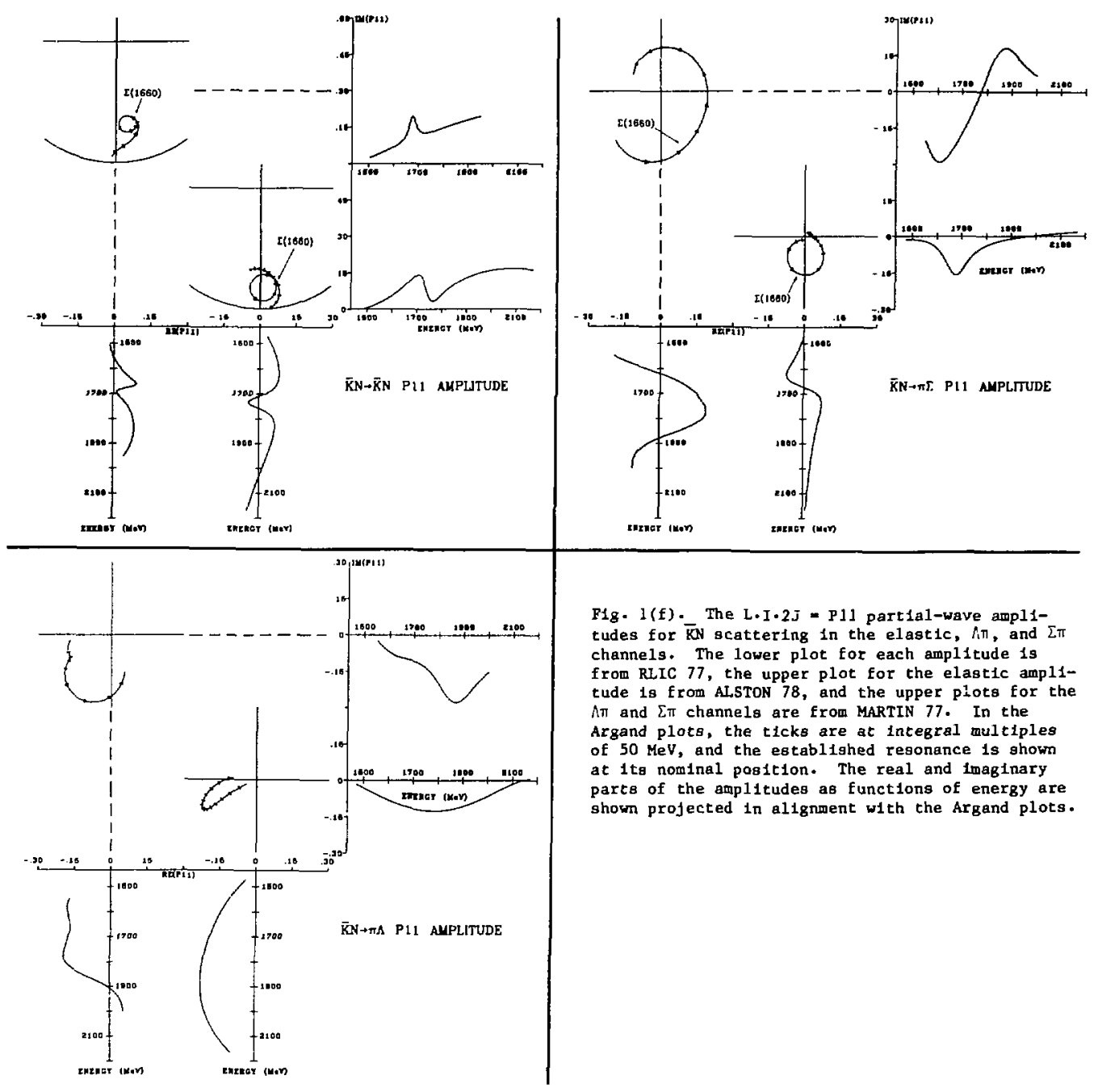


Baryons

$\Lambda$ 's and $\Sigma^{\prime} s$
Data Card Listings

For notation, see key at front of Listings.
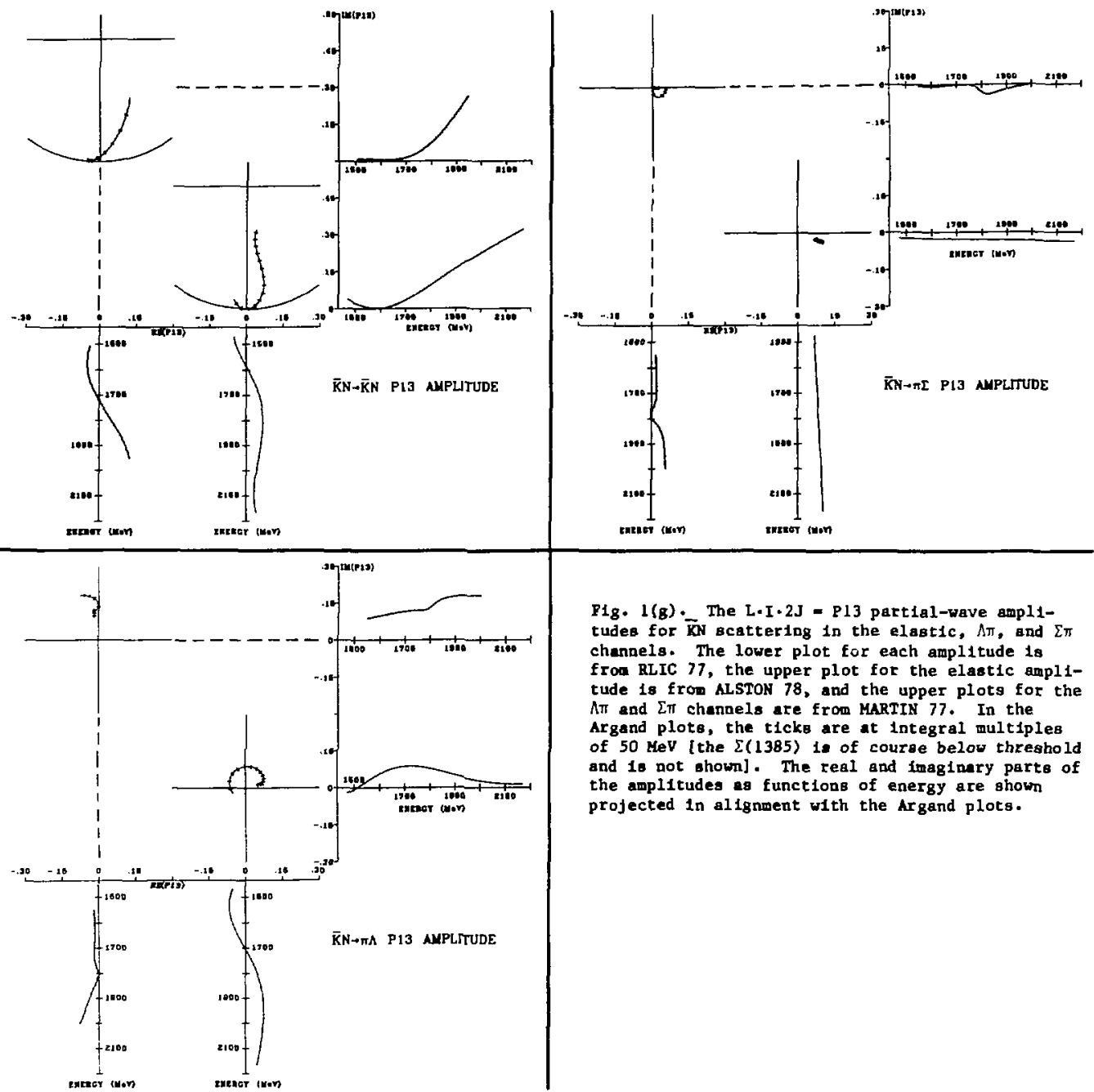

F1g. 1(g). The L.I.2J = P13 partial-wave ampl1tudes for $\overline{\mathrm{K}} \mathrm{N}$ Bcattering in the elastic, $\Lambda_{\pi}$, and $\Sigma \pi$ channels. The lower plot for each amplitude is fron RLIC 77 , the upper plot for the elastic amplitude is from ALSTON 78, and the upper plots for the $\Lambda \pi$ and $\sum \pi$ channels are from MARTIN 77 . In the Argand plots, the ticks are at integral multiples of $50 \mathrm{MeV}$ [the $\Sigma(1385)$ 1. of courae belos threshold and 1s not shown]. The real and inaglnary parts of the amplitudes as functions of energy are shown projected in alignment with the Argand plots. 
Data Card Listings

For notation, see key at front of Listings.

Baryons

$\Lambda ' s$ and $\Sigma$ 's
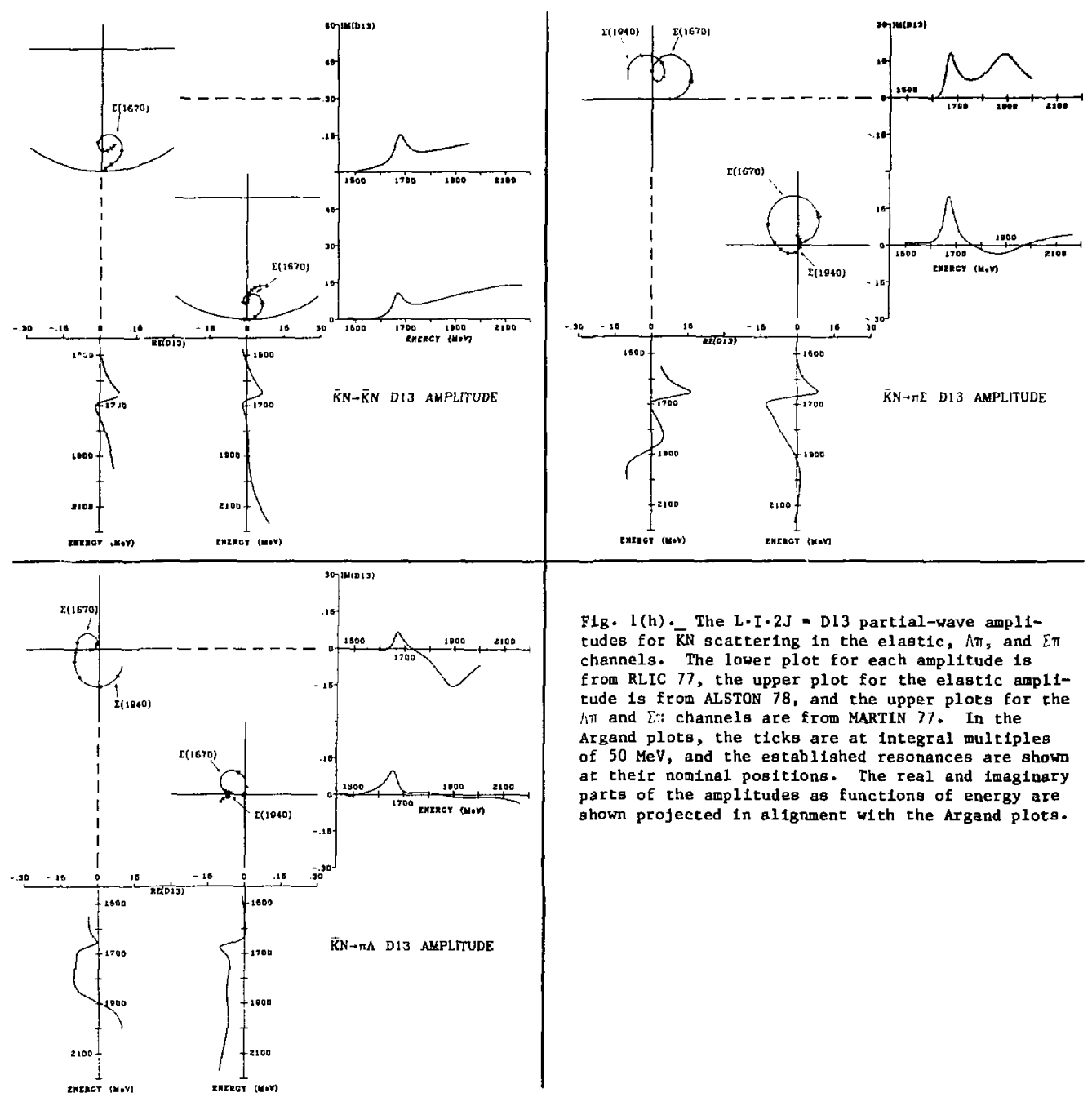

FIg . l(h) . The L.I.2J = Dl3 partial-wave amplitudes for $\overline{\mathrm{kN}}$ scattering in the elastic, $\lambda \pi$, and $\Sigma \pi$ channels. The lower plot for each amplitude is from RLIC 77, the upper plot for the elastic amp11tude is from ALSTON 78, and the upper plots for the $h T$ and Eii channels are from MARTIN 77. In the Argand plots, the ticks are at integral multiples of $50 \mathrm{MeV}$, and the egtablished resonances are shown at their nominal positions. The real and imaginary parts of the amplitudes as functions of energy are shown projected in alignment with the Argand plots. 


\section{Baryons}

$\Lambda$ 's and $\Sigma$ 's
Data Card Listings

For notation, see key at front of Listings.
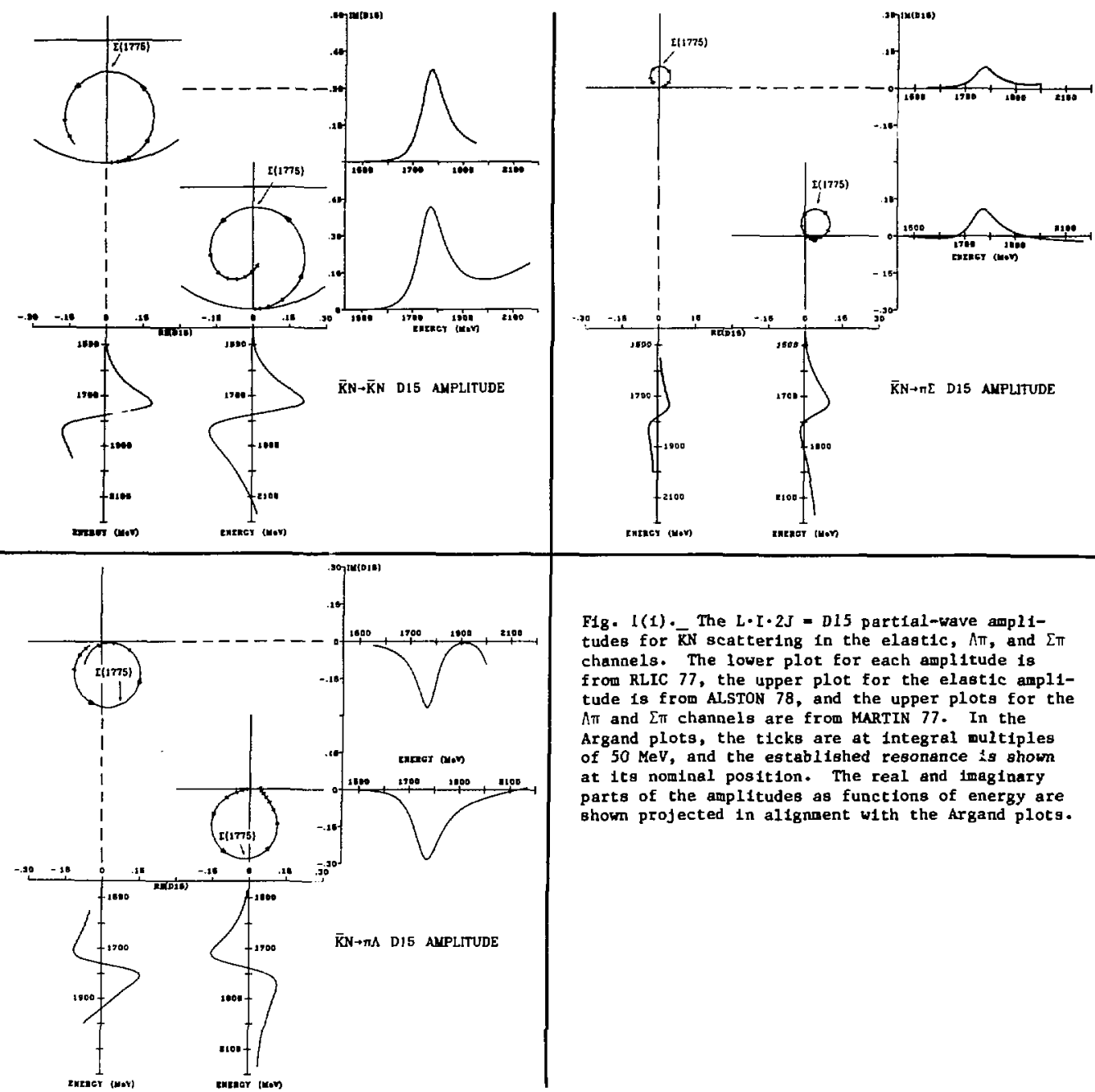

F1g. I(I). The L.I.2J = D15 partial-wave ampl1tudes for $\bar{K} N$ scattering in the elastic, $M \pi$, and $\sum \pi$ channels. The lower plot for each amplitude is from RLIC 77, the upper plot for the elastic anpl1tude is from ALSTON 78, and the upper plots for the $\Lambda \pi$ and $\sum \pi$ channels are from MARTIN 77 . In the Argand plots, the ticks are at integral vultiples of $50 \mathrm{MeV}$, and the established resonance is shown at itg nominal position. The real and imaginary parts of the amplitudes as functions of energy are shown projected in alignent with the Argand plots. 


\section{Data Card Listings}

For notation, see key at front of Listings.

Baryons

$\Lambda$ 's and $\Sigma$ 's

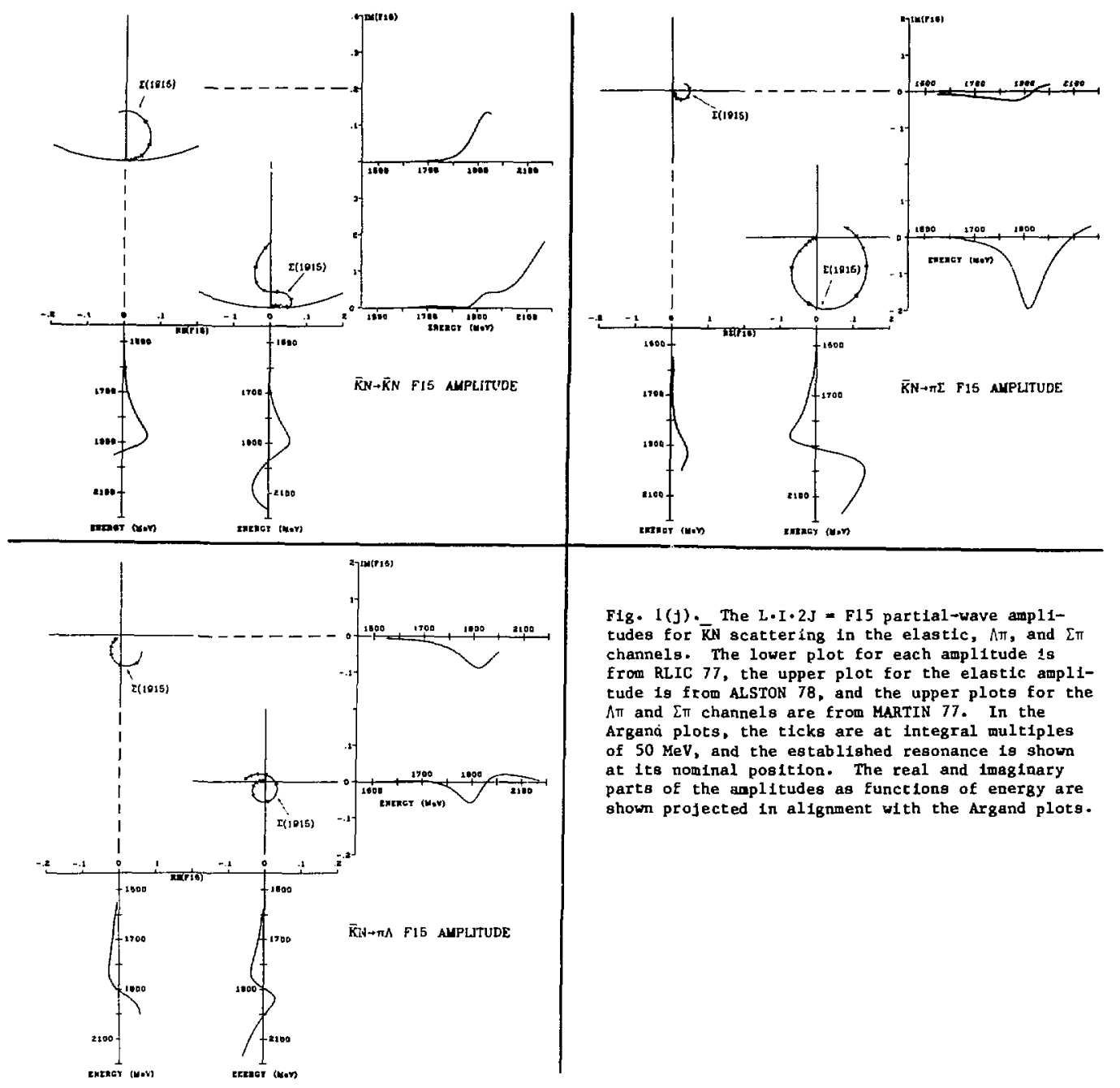


Baryons

$\Lambda$ 's and $\Sigma$ 's
Data Card Listings

For notation, see key at front of Listings.

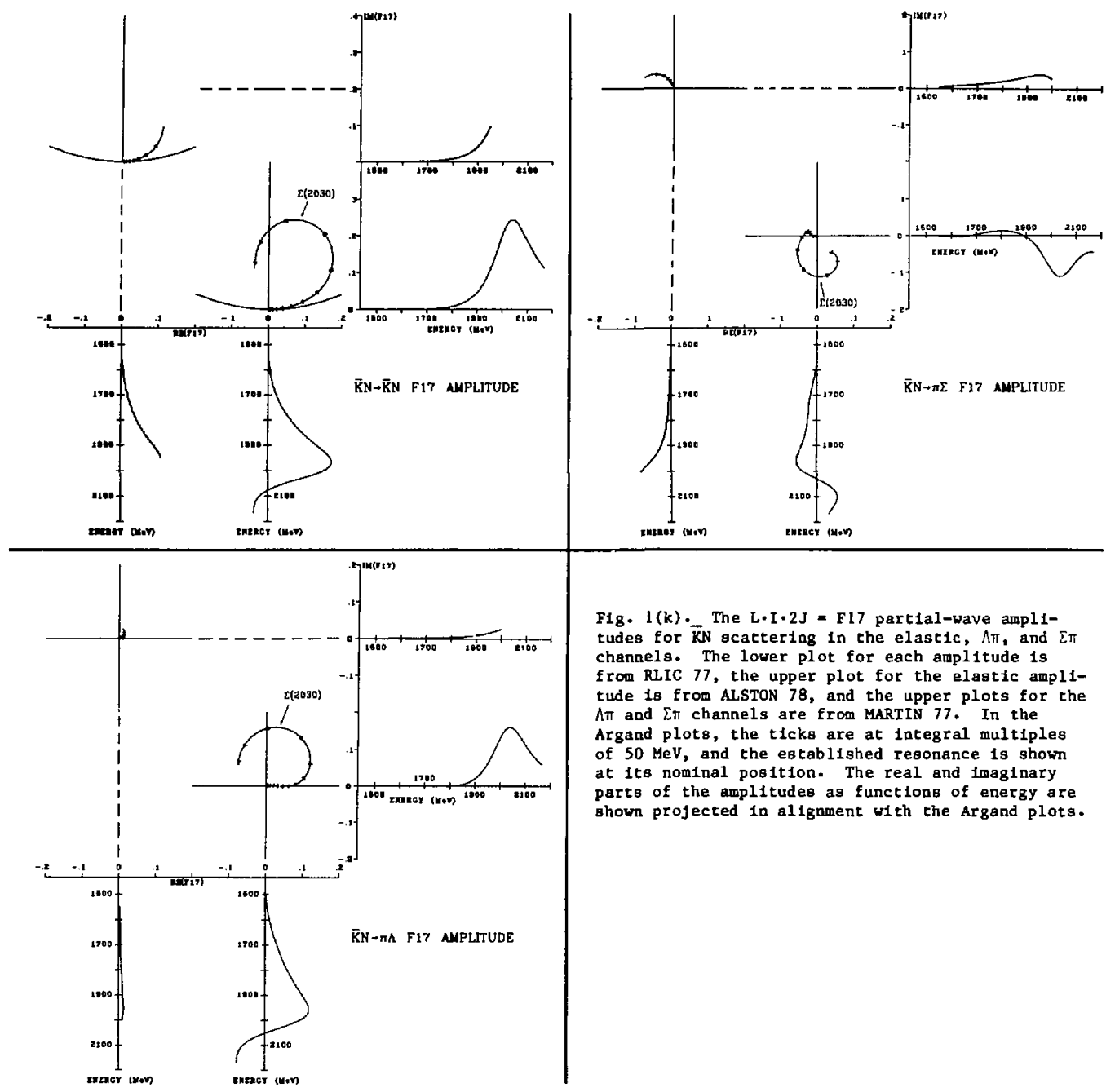




\section{Data Card Listings}

For notation, see key at front of Listings.
Baryons

$\Lambda^{\prime} s$ and $\Sigma^{\prime} s, \Lambda, \Lambda(1405)$
2. W. Langbein and F. Wagner, Nucl. Phys. B47, 477 (1972).

3. A.T. Lea et al., Nucl. Phys. B56, 77 (1973).

4. 3. Armenteros et al., Nucl. Phys, B8, 195 (1968) and B21, $15(1970)$.

5. T.S. Mast et al., Phys. Rev. D14, 13 (1976).

6. B. Conforto et al., Nucl. Phys. B10S, 189 (1976) and $W$. Cameron et al., Nucl. Phys. B193, 21 (1981).

7. R.J. Hemingway et al., Nucl. Phys. B91, 12 (1975).

8. A. de Bellefon et al., Nuovo C1mento 42A, 403 (1977); Nuovo C1mento 37A, 175 (1977); Nuc1. Phys. B90, 1 (1975); and Nuovo Cimento 41A, 96 (1977).

9. M. Alston-Garnjost et al., Phys. Rev. D17. 2216 (1978) and D17, 2226 (1978).

10. R.D. Ehrlich et al., Phys. Lett. 쏘, 455 (1977).

11. H.C. Bryant et al., Nucl. Phys, B168, 207 (1980).

12. C.J.S. Damerell et al., Nucl. Phys. B155, 13 (1979).

13. Y. Decla1s et al., CERN 77-16 (1977),

14. M. Algton-Garnjost et al., Phya. Rev. D21, 1191 (1980).

15. A. Engler et a1., Phys. Rev D18, 3061 (1978).

16. W. Cameron et a1., Nucl. Phys. B132, 189 (1978).

17. M.J. Corden et al., Nucl. Phys. B155, 13 (1979).

18. M.J. Corden et al., Nucl. Phyr. B125, 61 (1977).

19. G.P. Gopal, In Proceedings of the IV ${ }^{\text {th }}$ Internat lonal Conference on Baryon Regonances (Toronto, 1980), ed1ted by N. Isgur, p. 159.

20. G.P. Gopal et al., Nucl. Phys. B119, 362 (1977).

21. M. Alston-Gaznjost e! al., Phys. Rev. D18, 182 (1978).

22. B.R. Martin et al., Nucl. Phys. B126, 266 $(1977) ; \underline{B 126}, 285$ (1977); and B127, 349 (1977).

23. P. Baillon and P.J. Litchfleld, Nucl. Phys. B94, 39 (1975).

24. A. de Bellefon and A. Berthon, Nucl. Phys. B109, $129(1976)$.

25. A.J. Van Horn, Nucl. Phys. B87, 145 (1975).

26. J. Anlrzadeh et al., Phys. Lett. 898,125 (1979).

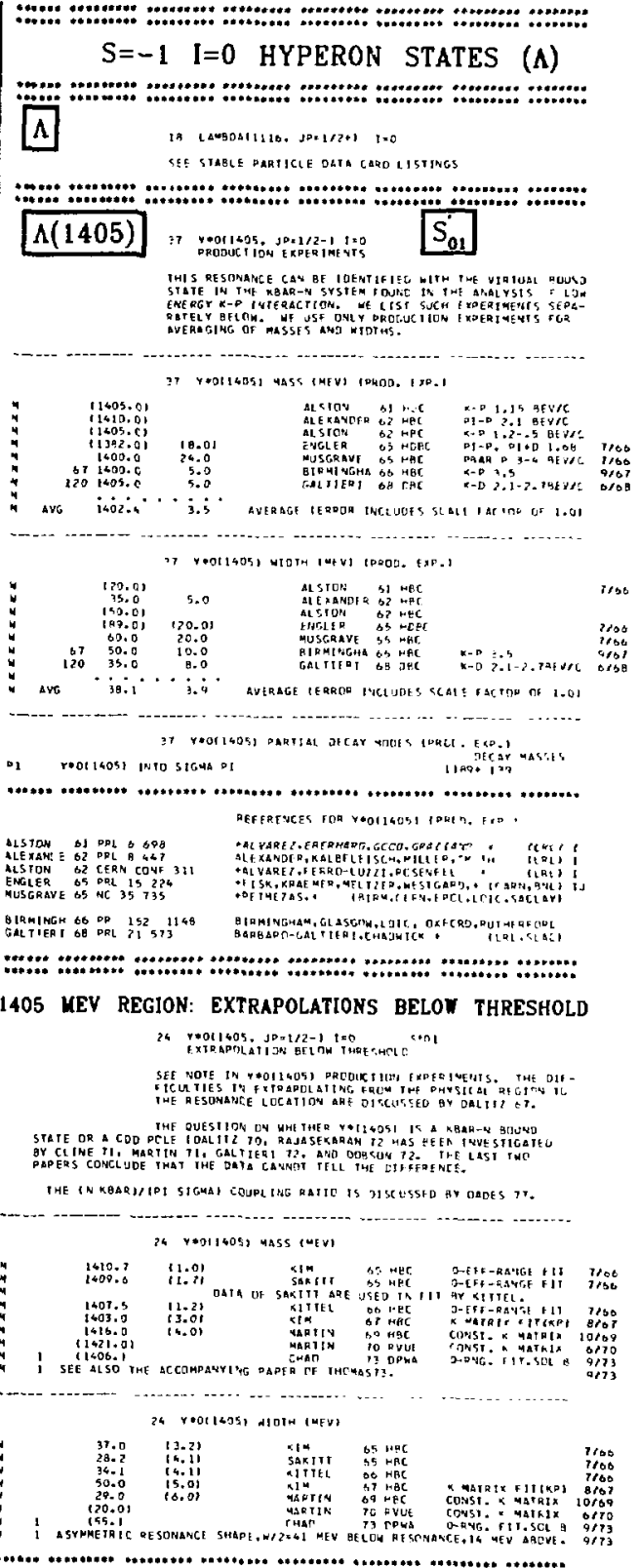


Baryons

$\Lambda(1405), \Lambda(1520)$
Data Card Listings

For notation, see key at front of Listings.

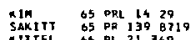

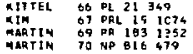

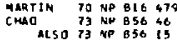

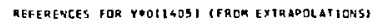
GK KMIM.

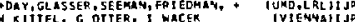

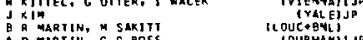

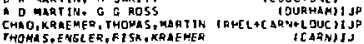
papers nat QefFerReg to IM OATA GaEOS

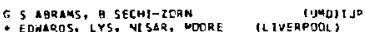

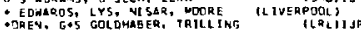

ABRAMS OS PR 1398645

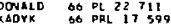

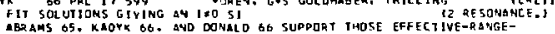

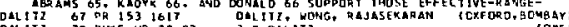

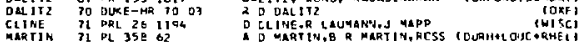

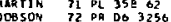

GALTERI 72 LAL 555

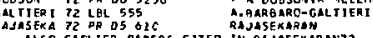

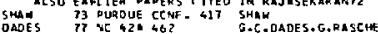

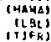

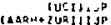

\section{$\Lambda(1520)$;o reoc1520, jax 3/2-1 1=0 $D_{03}^{\prime}$}

DRODUCTION AND F TRHATION EAPER JMENTS ACAEE QUITE MELL

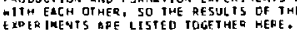

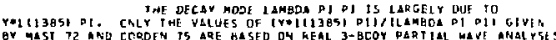

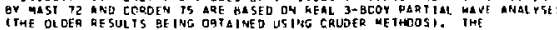

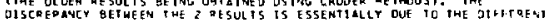

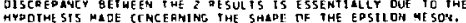

\section{Y.0115201 HASS (4EV)}

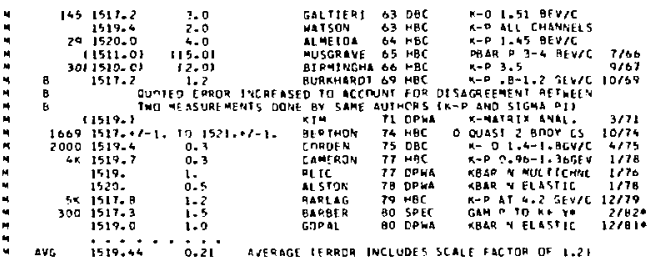

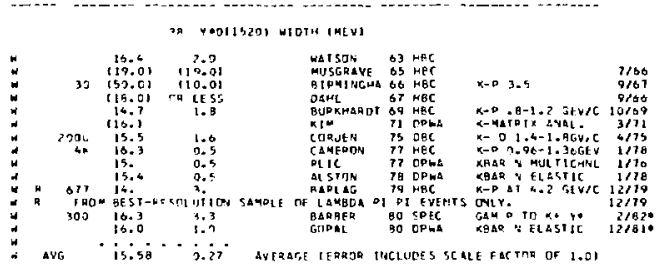

ID roOL15?0I DSEIIAL OFCAY MDOES

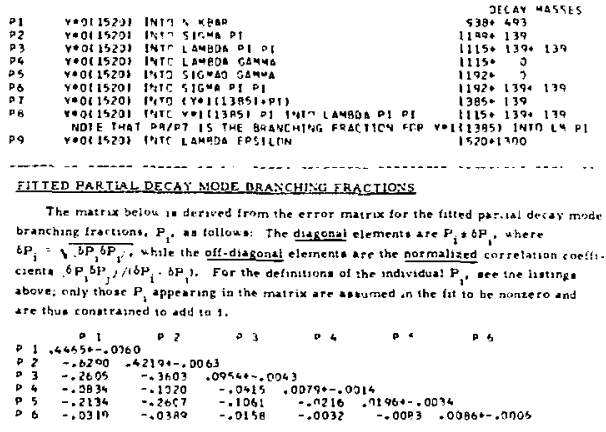

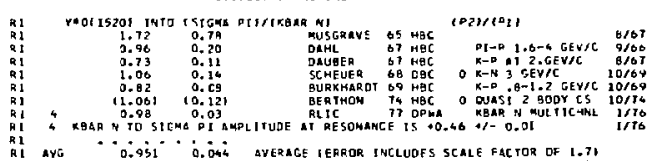

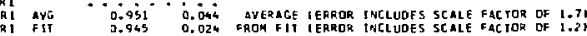

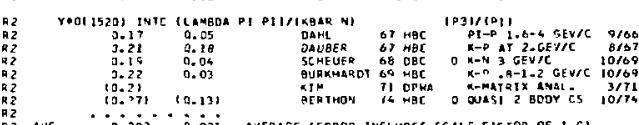

AYG
HIT

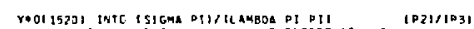

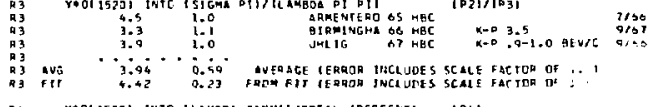

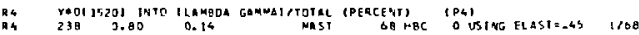

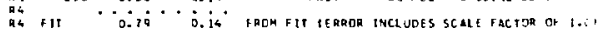

RS
as

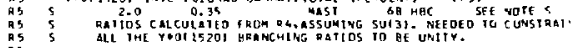

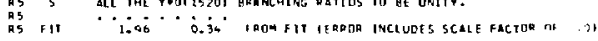

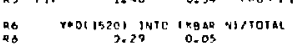

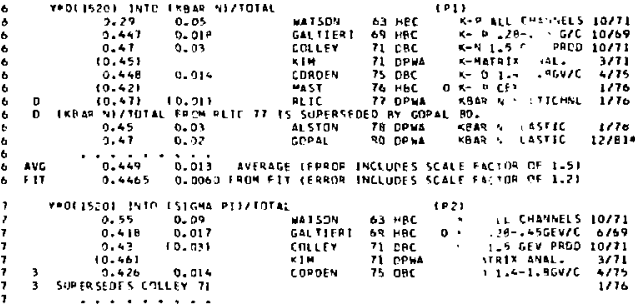

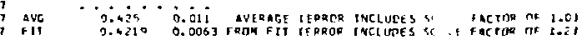

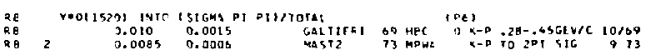

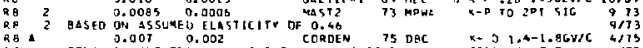

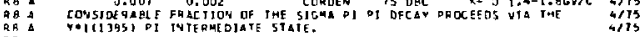

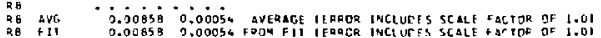

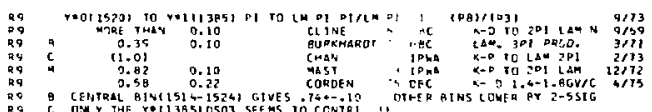

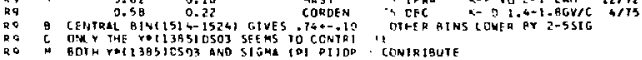

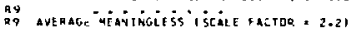

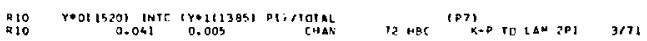

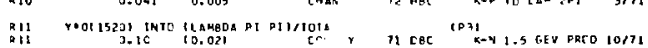

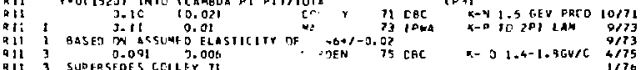

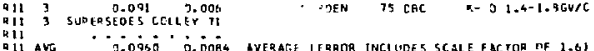

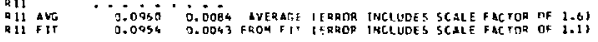

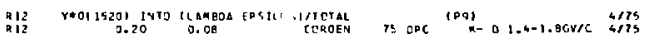

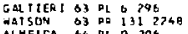

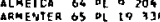

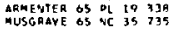

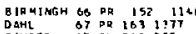

OAUEER G7 DL 269525

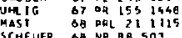

\section{QE RENCES FOR YOOI1520}

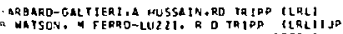

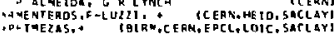

RIAMINGHAM.GL ASGOU,1.C., OXFCHD. QUTHERFORD

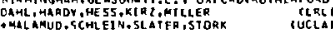

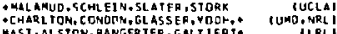
SARRE COLIAB. 


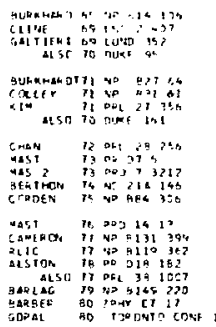

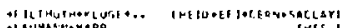

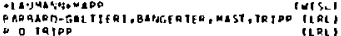

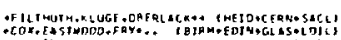

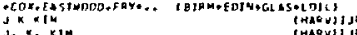
J...$\times 1 \%$ rasovitiog

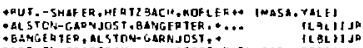

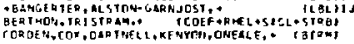
MST, ALSIOH-5LAMJOST. PRKGERTERA ILALI

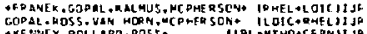

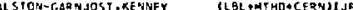

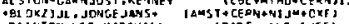

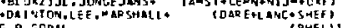

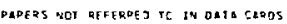

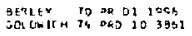

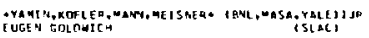

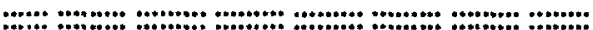

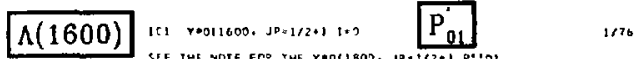

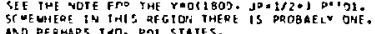
AND DE RMAOS TOO. UOT STATS.

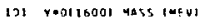

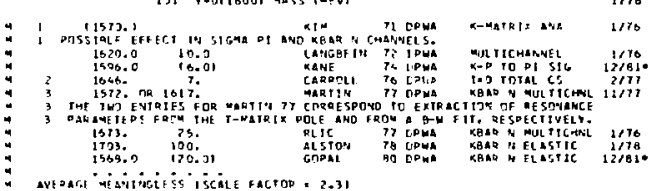

Le1 ror 16901 WIOIH IAEV)

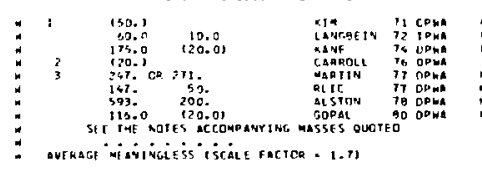

I:L raOt1600S PAKTIAL DECAY mLSES

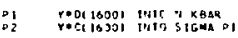
DETAY HSSES $536+603$
$1189+139$

191 Y*O116009 gRARCHING RAITOS

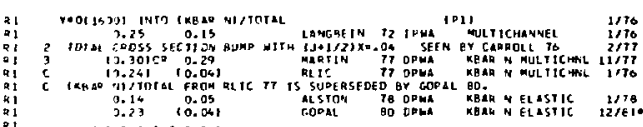
af sverage de aitigifiss iscile factor - 1.01

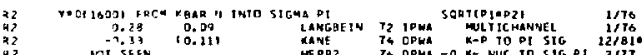

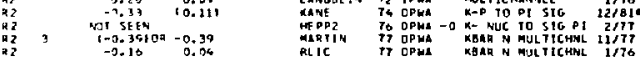

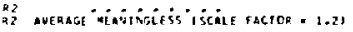

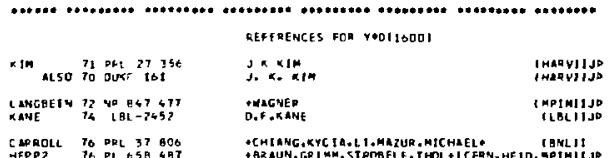

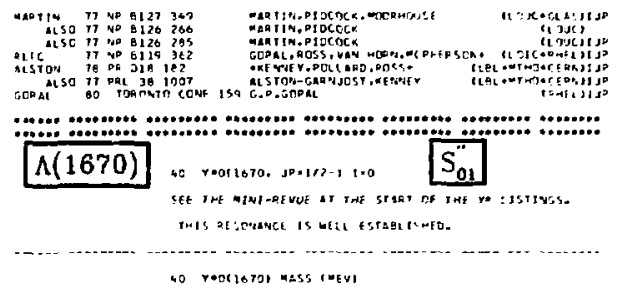

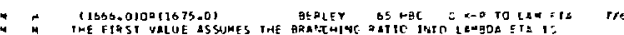

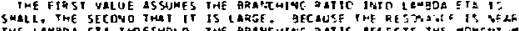
OEPEATES: TF THE TOIA OEPEAJESE MF THE TOTAL WIOTH, ADD THUS

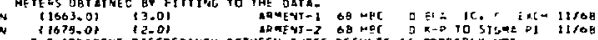

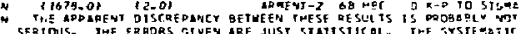

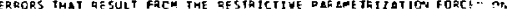

ERE OSRTIOL $=$ WAVE
IOTS.O

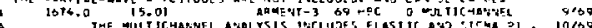

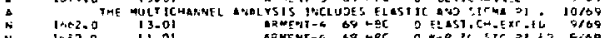

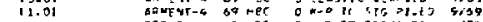

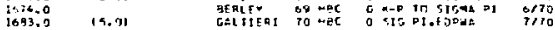

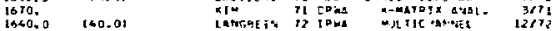

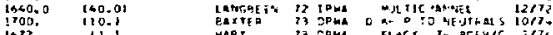

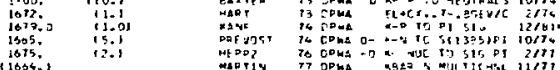

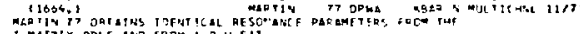

\begin{tabular}{|c|c|c|c|c|c|}
\hline $\begin{array}{l}1670 . \\
\text { 1571: } \\
1607.9\end{array}$ & $\begin{array}{l}15.1 \\
19.8 \\
15.01\end{array}$ & 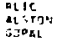 & 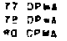 & 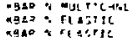 & $\begin{array}{l}1170 \\
1 / 76 \\
\text { limte }\end{array}$ \\
\hline
\end{tabular}

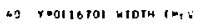

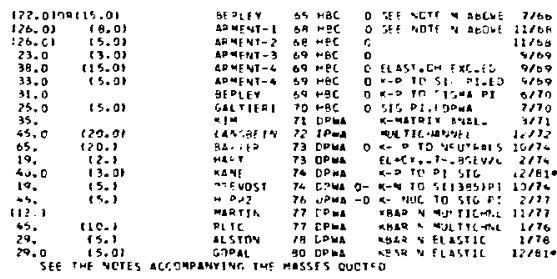

to reat

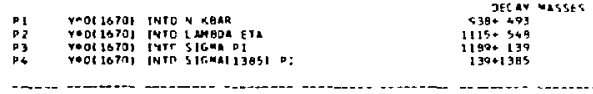

40 rooligtol aranching antlos

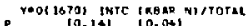

$\begin{array}{lll}0.17 & 0.041 \\ 0.14 & 0.031\end{array}$

$10.391 \quad 10.051$

$0.285 \quad 10.081$

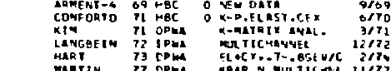

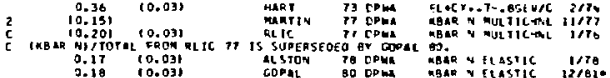

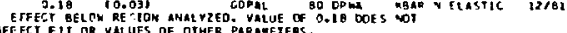

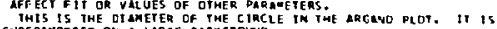
SUPERIMPOSEO OR : LARGE GACKGROUNO

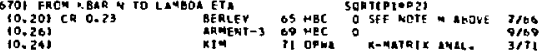

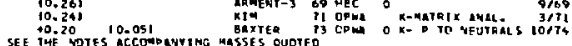




\section{Baryons \\ $\Lambda(1670), \Lambda(1690)$}

Data Card Listings

For notation, see key at front of Listings.

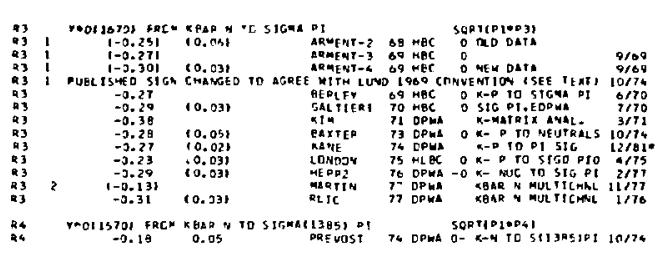

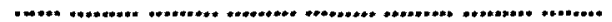

REFERENCES FOR ratio,

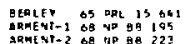

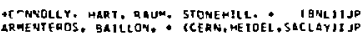

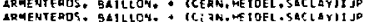

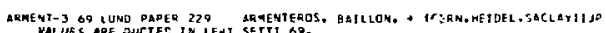

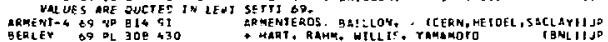
GeriEr Go BL Joe 430

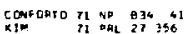

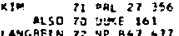

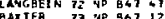

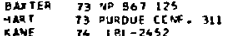
PQTVOST 74 MP BOS 240

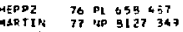

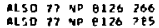
REic 77 NP $B 119362$ also $7 \mathrm{PPL}$ 3s $1 \mathrm{ig}$ CONaL go TOKantis CONF

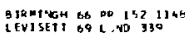

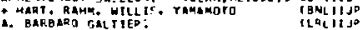

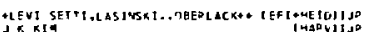
$J K K I A$
$J . K K$ KIA

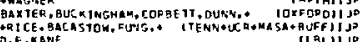
D. F-KANE

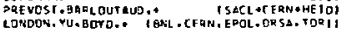

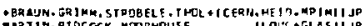

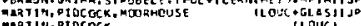

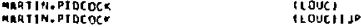

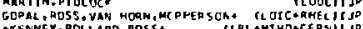

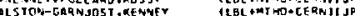

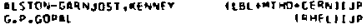
DEPERS NOT REFERECO TO IN ORTL CAOOS

(BIQMIVGHAE. GLASCOW, LCLC, OXFOED, PUTHEREO)

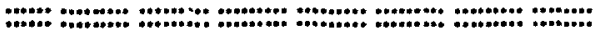

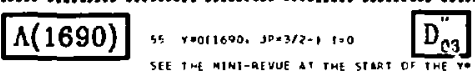
FHIS RESONANCE IS UELL FSTAGISHEO,

55 roDt16901 MASS IAEVI

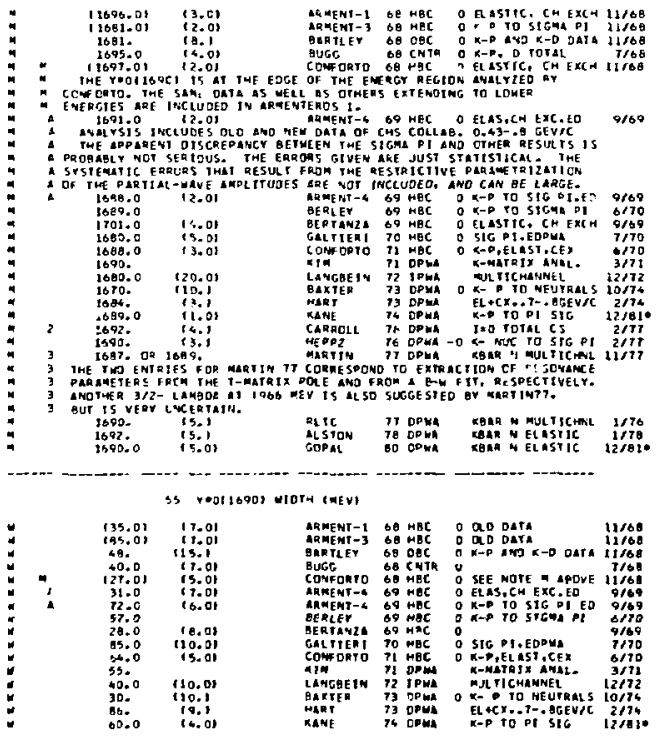

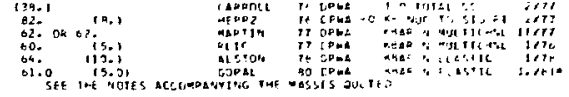

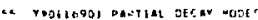

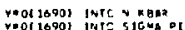

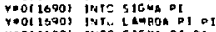

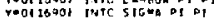

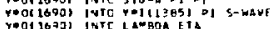

$11,00.130$

1140. 130.120

1199+1103

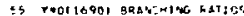

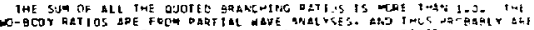

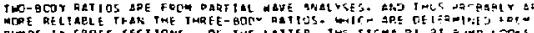

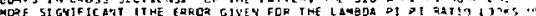

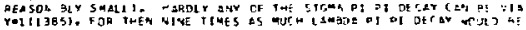
RF QuTAED.

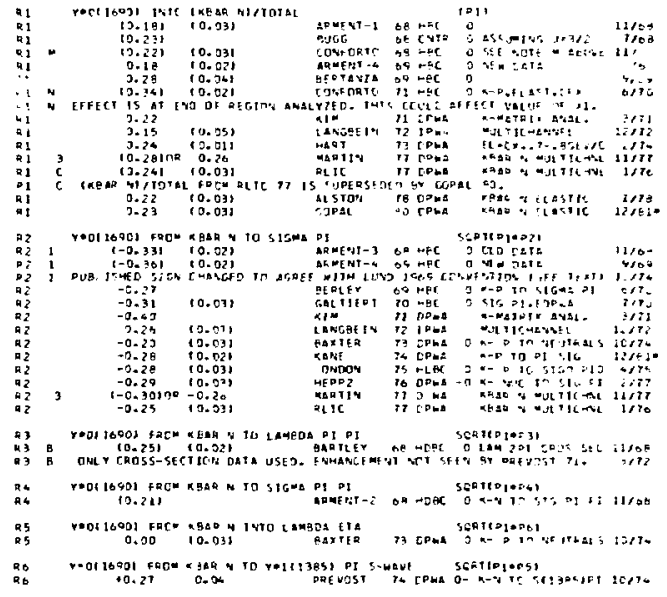

AREENT- 1 SQ NP AB ISS

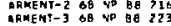

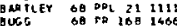

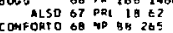
RANENT-C OQ NP 1491

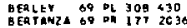
GALTIERI TO DURE 173 CCNE ORTO

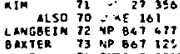

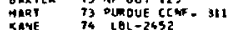
KAYF
PRE VOSS
34

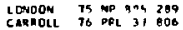
ALATIN 17 \%

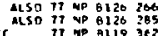
ALIC I1 MD B110 3e ALSTON TO OR OLA 192 HEPP TO PL OSE GET

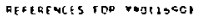

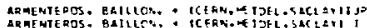

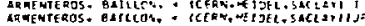

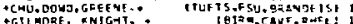

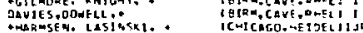

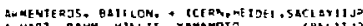

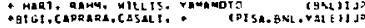

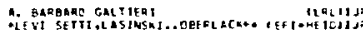

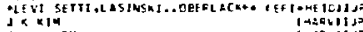
T. $x . x I=$

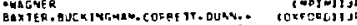

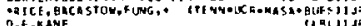

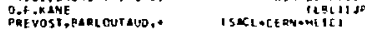

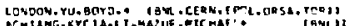

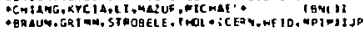

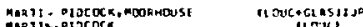

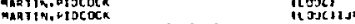

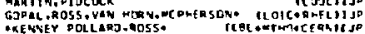

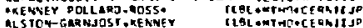

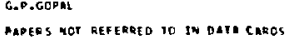

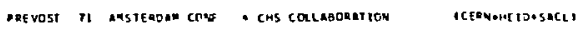




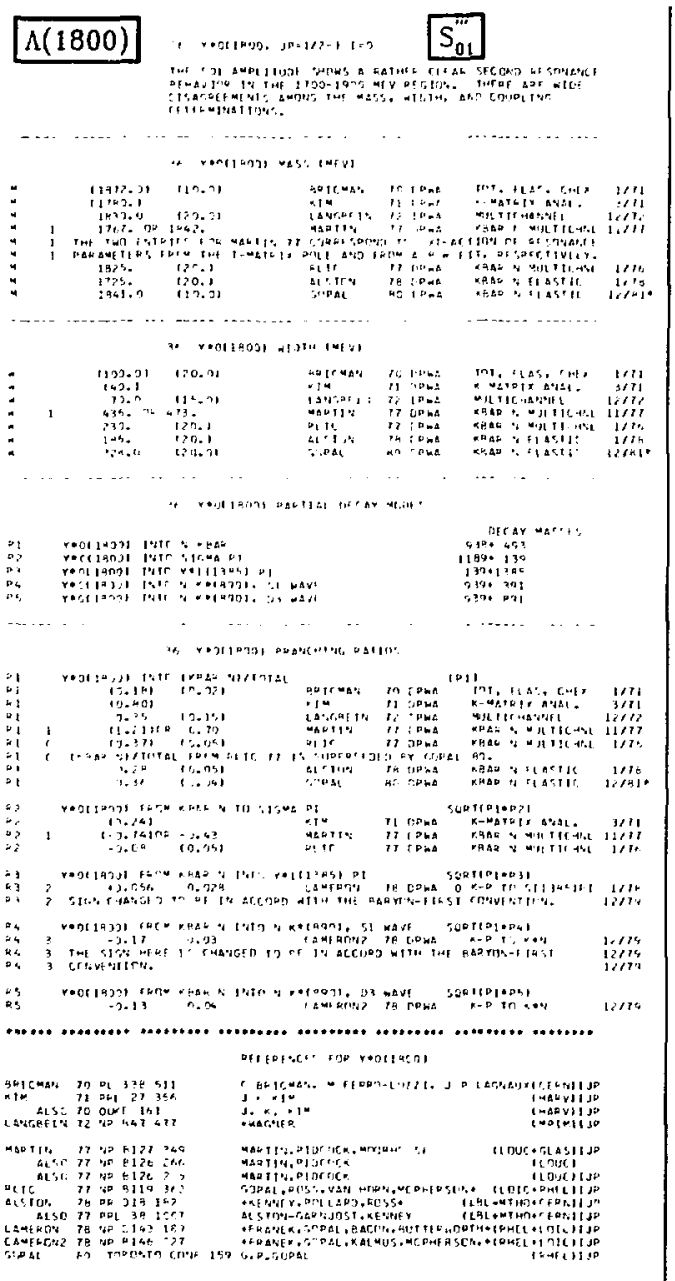

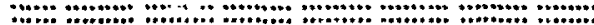

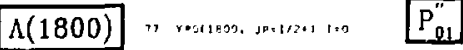

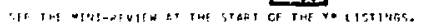

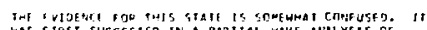

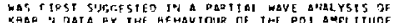

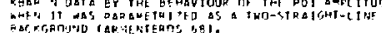

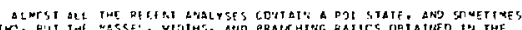

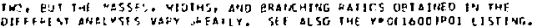

1 reocisgu hass INEVI $\mid$\begin{tabular}{cccc}
$\vdots$ & & 1 \\
$\vdots$ & 1 & \\
$\vdots$ & 3 & \\
$\vdots$ & 3 & T \\
\hline & & & \\
$\vdots$ & &
\end{tabular}

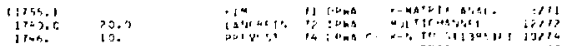

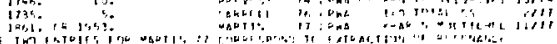

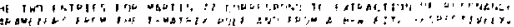

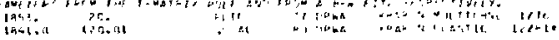

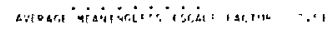

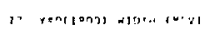
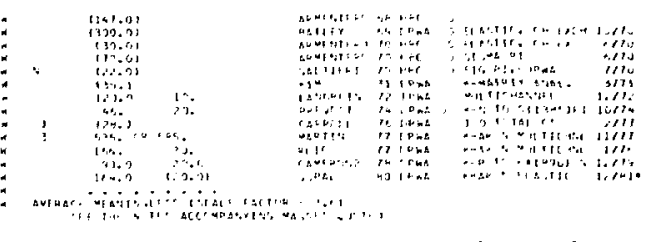

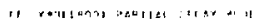

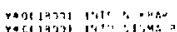

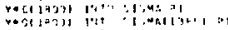

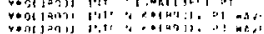

nan $n$

iin: is:

(a)

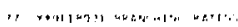

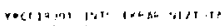

i,....

?ר:?

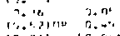

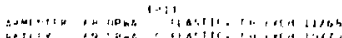

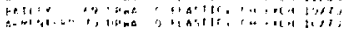

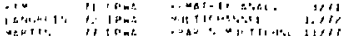
: (10+2

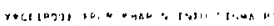

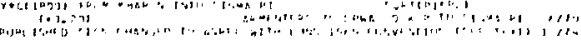

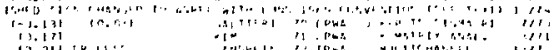
‥11 ard

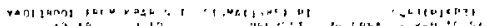

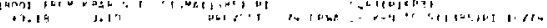

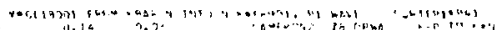

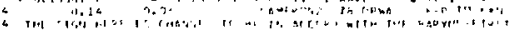

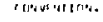

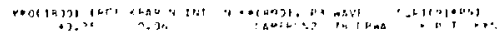

in:

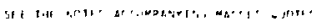
1,1, (.......

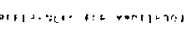

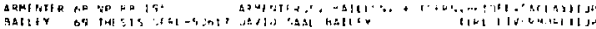

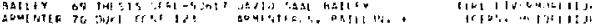

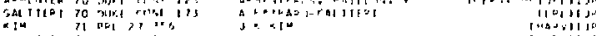

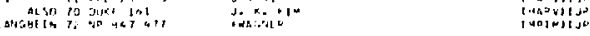

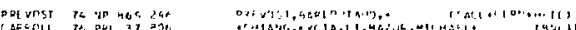

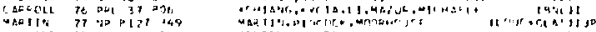

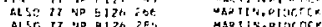

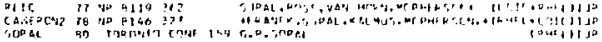

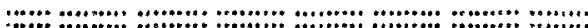

$\Lambda(1800)$ iar $\quad \mathrm{G}_{09}$ $\longrightarrow$ D

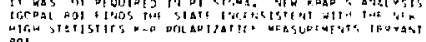

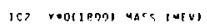

เด0月. 
Baryons

$\Lambda(1800), \Lambda(1820)$

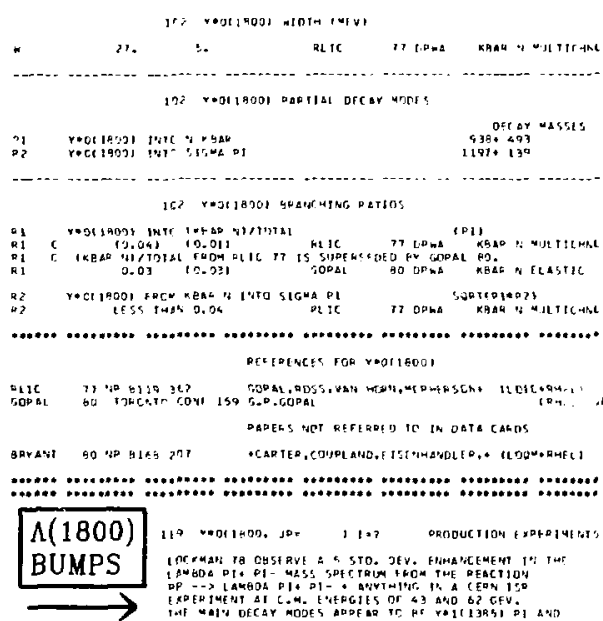

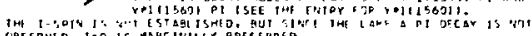

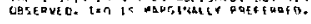

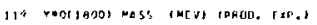

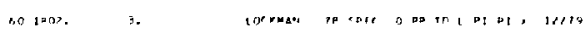

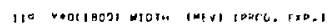

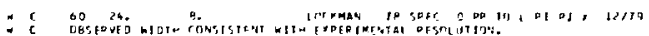

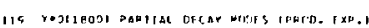
$1560+139$

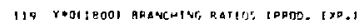

2
01

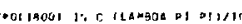
iptiop I P P PI $\times 1 / 278$ $+2$ a. ?

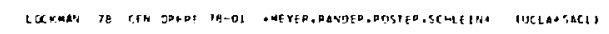

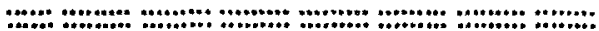

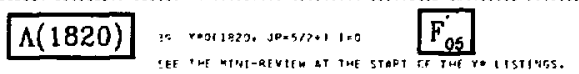

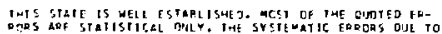

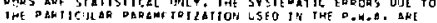

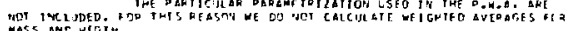

30 YoOTB231 MASS IMEN,
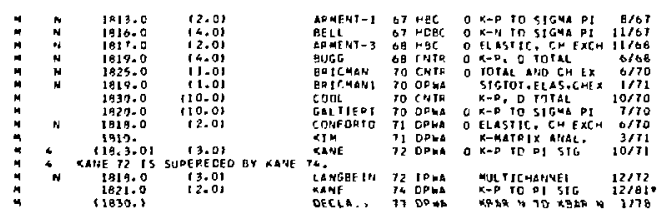

Data Card Listings For.notation, see key at front of Listings.

1170 170

110 $1 / 76$ irgie $1 / 76$

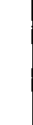

$\mid \begin{array}{ll}3 \\ 01 \\ 03 \\ 03 \\ 03 \\ 03 \\ 03 \\ 3\end{array}$

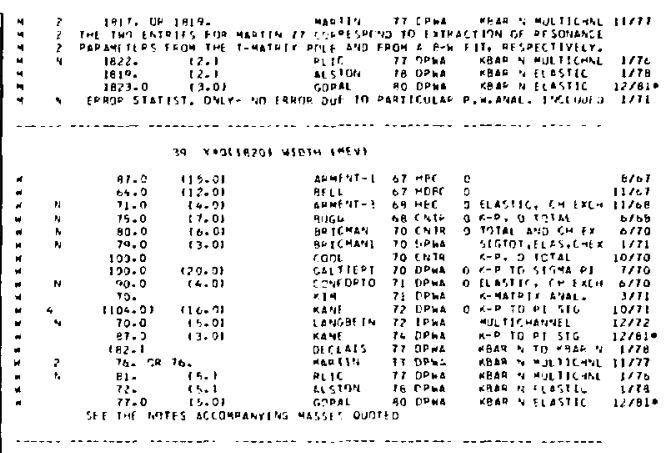

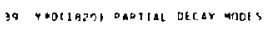

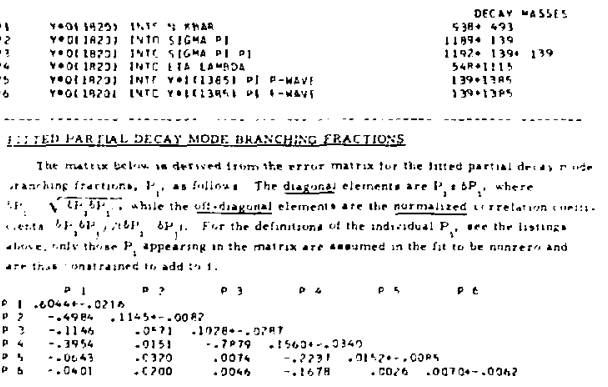

39 Y

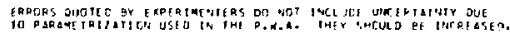

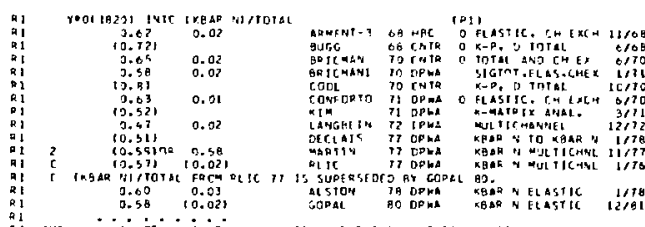

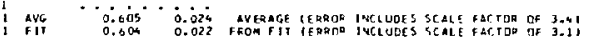

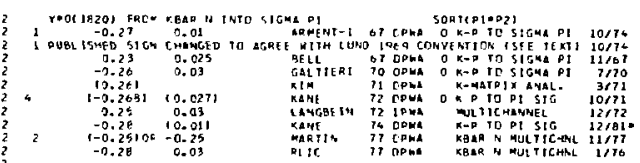

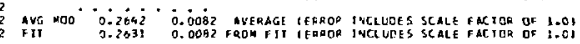

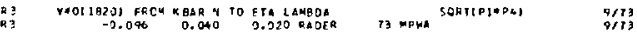

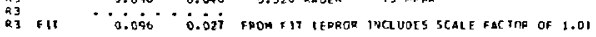

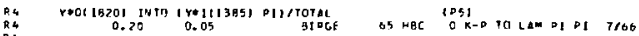

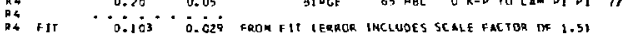

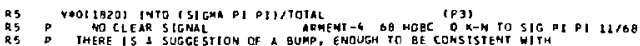

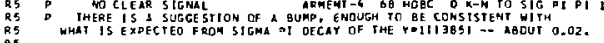


Data Card Listings

For notation, see key at front of Listings.
Baryons $\Lambda(1820), \Lambda(1830), \Lambda(1890)$

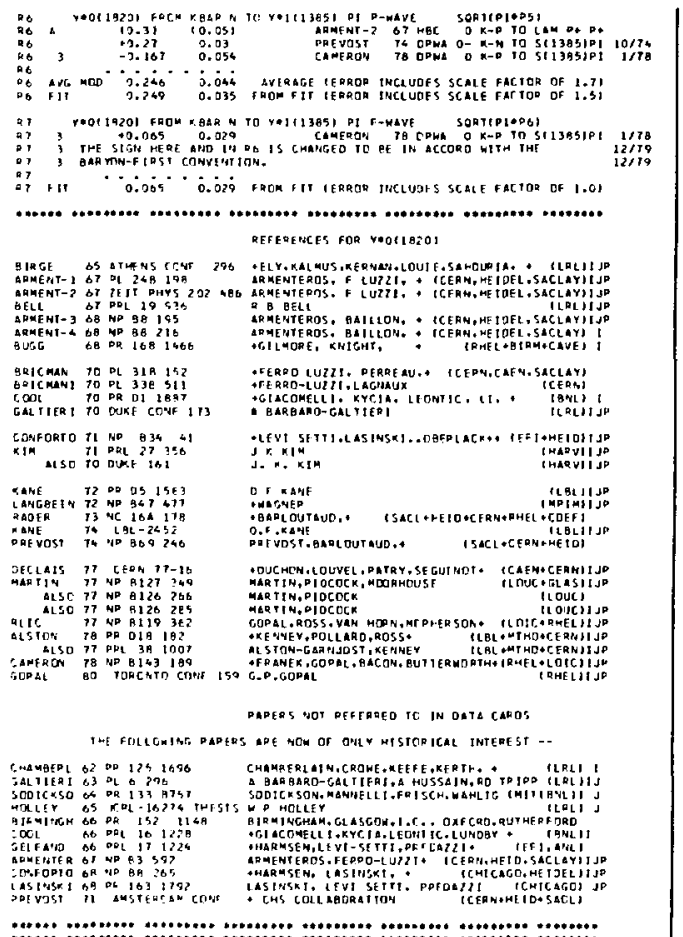

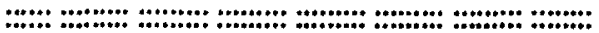

SO YOULIO3OI OAEIJAL DFCAY MODES

01
01
$p 2$
3
04

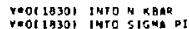

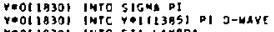

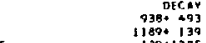

$1169+139$
$139+1395$

SE YOOHSBOS BRANCHINE RATIOS

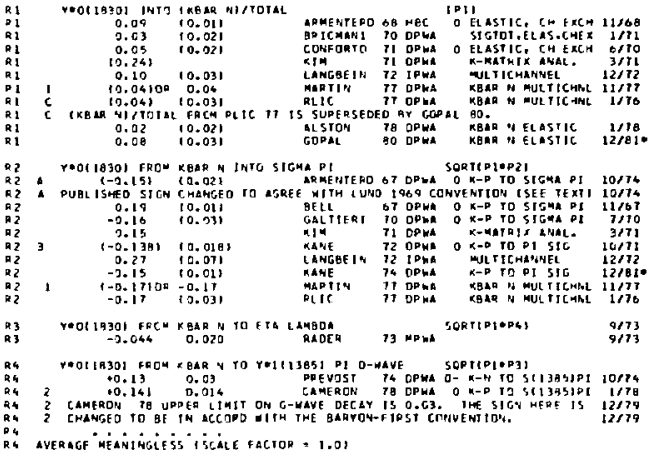

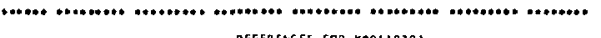

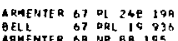

COHFOATO 6 OA APBO 265

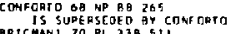

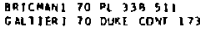

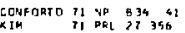

KANE ALSD 70 OUKE 161

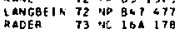

KANE
DAE VOST

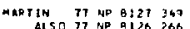

$215077 \mathrm{ND}$ a 128386

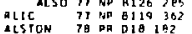

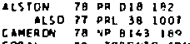

Defeathes for yeortazos

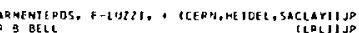

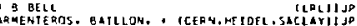

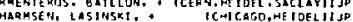

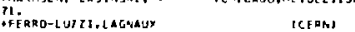

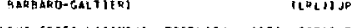

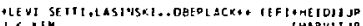

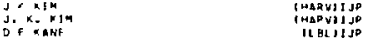

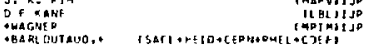

D.F ARANE

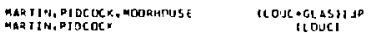

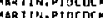

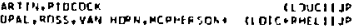

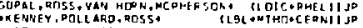

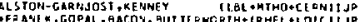

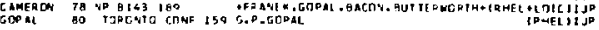
papeos not heferplo it in dat' capos

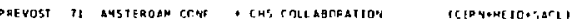

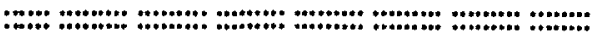

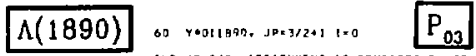

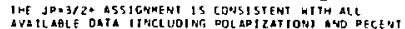

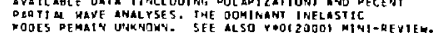

eo reotigao) ahss incri

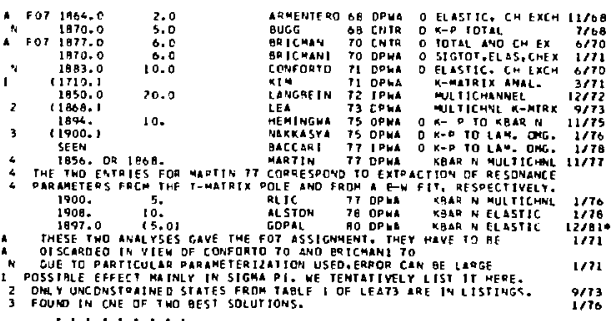

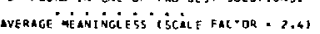




\section{Baryons}

$\Lambda(1890), \Lambda(2000), \Lambda(2020)$
Data Card Listings

For notation, see key at front of Listings.

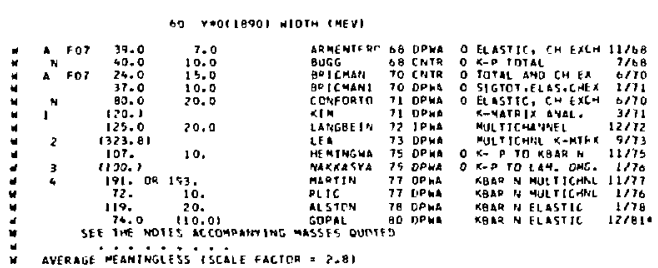

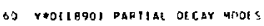

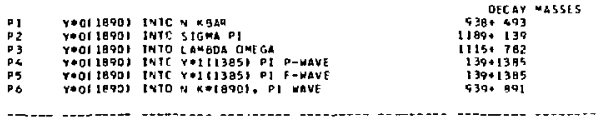

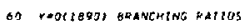

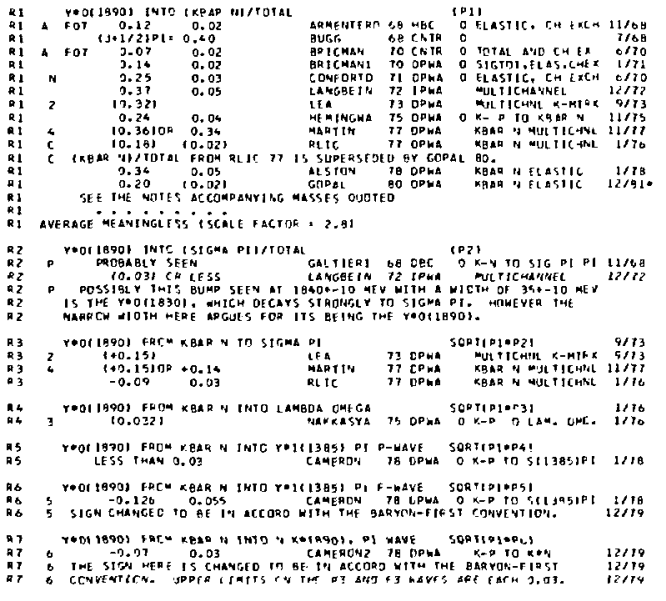

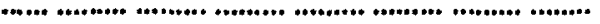

DEFEETCES FID $r=0110901$

APME NTERDEG ND Ba 195

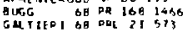

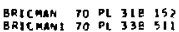
COAFORTO

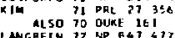

LEA 73 AP a BE 77

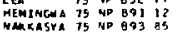

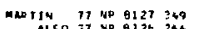

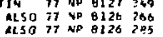
ALIC IT ACSTON 78 PR ODB 197

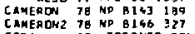

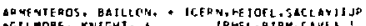

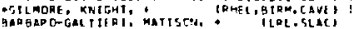

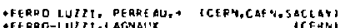

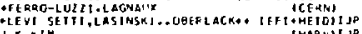

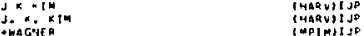

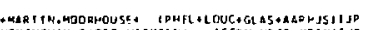

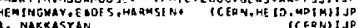
a. NAKKASTAN TCERTIJP

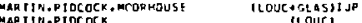

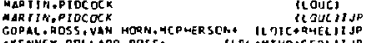

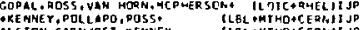

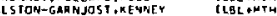

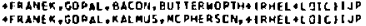
GOPAL

PRPEOS NOT REFERREO TO' II" ORTA GAPQS

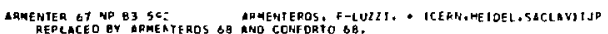

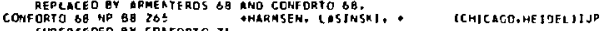

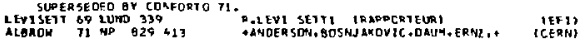

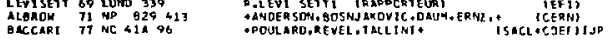

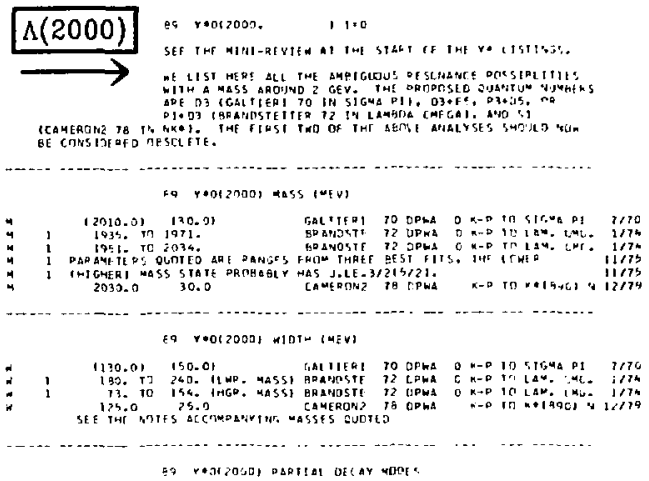

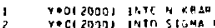

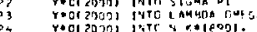

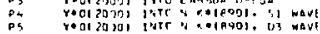

cocencist:

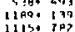

i $39+$ मी

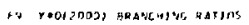

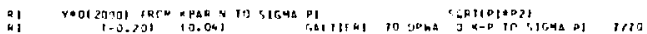

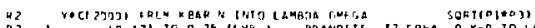

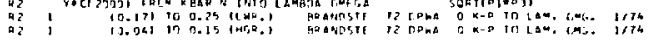

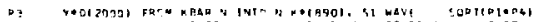

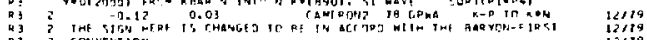

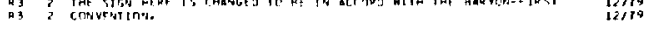

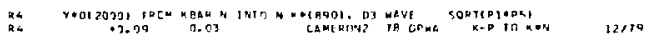

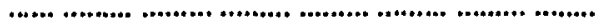

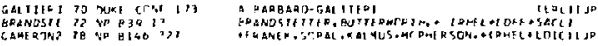

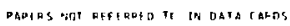

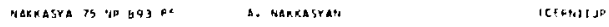

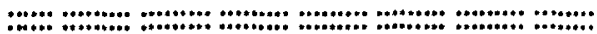

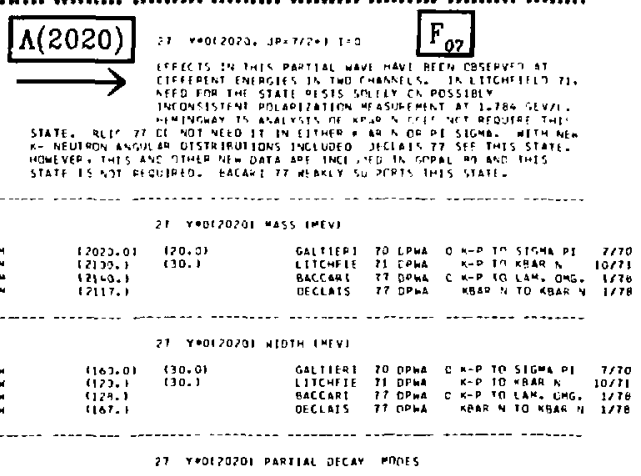

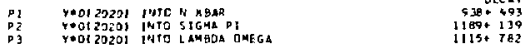


Data Card Listings

For notation, see key at front of Listings.

27 VOOL2020) BH LNCHSME MATIOS

$R 1$
$R I$
$R 2$
$R 2$
$R 2$
$R 3$
$R 3$

Yoot 2020$)$ INTC KKBAR HI/TOTAL
10.051
10.051

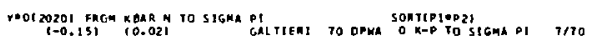

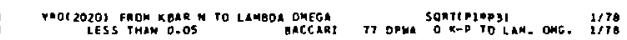

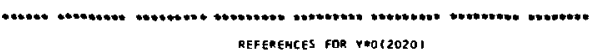

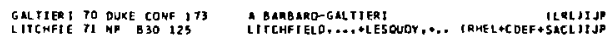

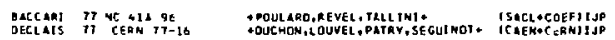
panens mor Referkedo to IM ORTA CaAOS

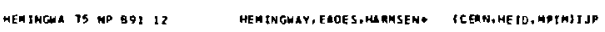

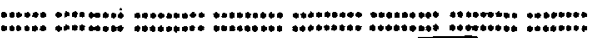

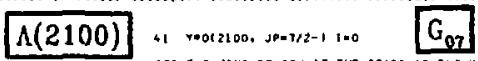
IEe the mint-revien at the stakt DF Ine ve bistings. TH1S ENTRY ONLY THCL UDES MESULTS FROM PARTIAL-WAVE ANALSES. PARARETERS OF DERKS SEEN IN CHOSS-SECTIONS

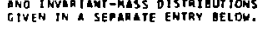

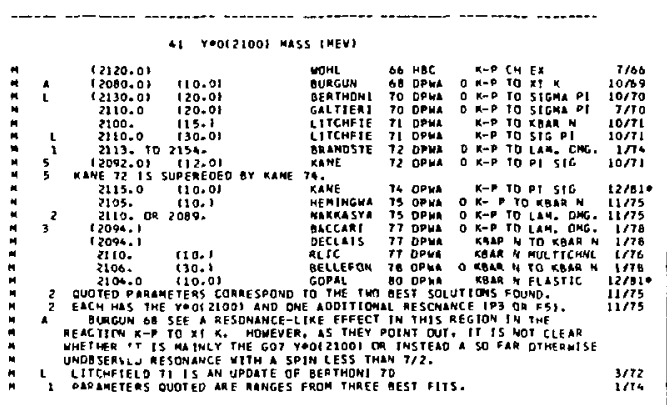

4) VeOtzloO) HIOTK (MEU)

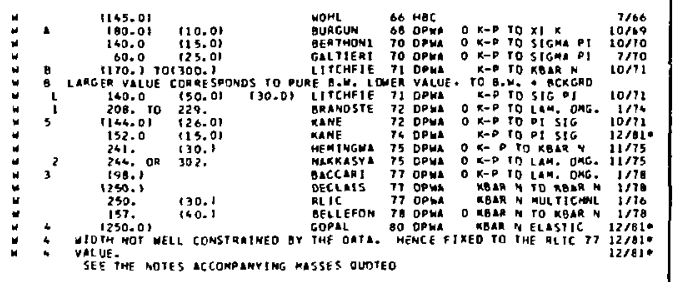

4 roOL2LOO PARTIAL DECAY MOOES

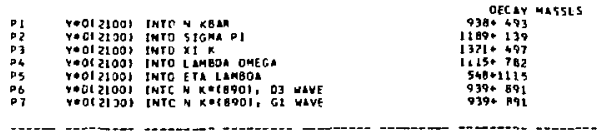

4 TMOIZLODI GRANCHTHG RATIOS

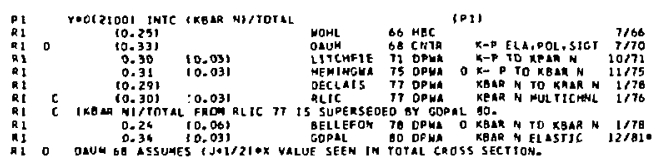

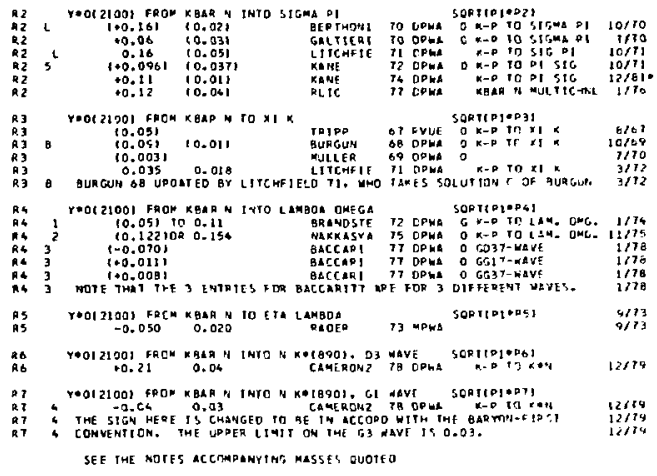

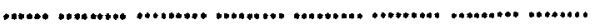

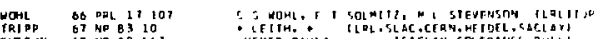

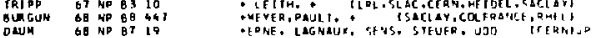

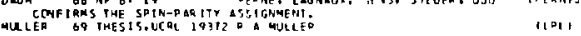

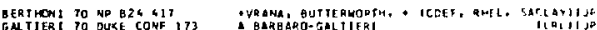

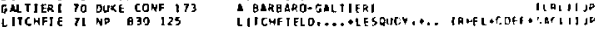

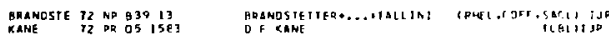

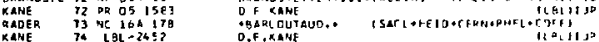

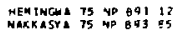
ANCARI $77 \mathrm{MC}$ a1A Se

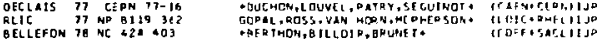
BELLEFDN $78 \mathrm{NC} 22 \mathrm{~A} 403$

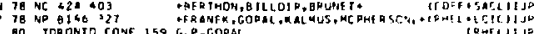

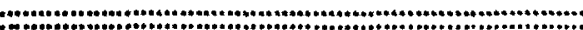

2100 MEV REGION - PRODUCTION AND $\sigma_{\text {TORI }}$ EXP'TS

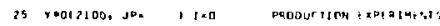

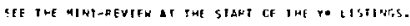

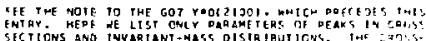

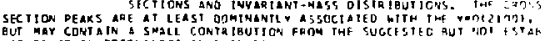

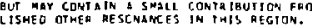

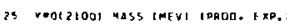

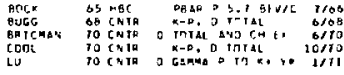

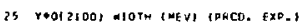

\begin{tabular}{|c|c|c|c|c|c|c|}
\hline $\begin{array}{l}124.01 \\
1400 \\
147.0 \\
185.0 \\
140.01\end{array}$ & $\begin{array}{l}114.01 \\
115.01 \\
115.01\end{array}$ & 124.01 & $\begin{array}{l}\text { BDCK } \\
\text { GUGC } \\
\text { GP ICAA H } \\
\text { COOL } \\
\text { LU }\end{array}$ & $\begin{array}{l}\text { DS HeC } \\
\text { D8 CNTR } \\
70 \text { CNTR } \\
70 \text { CNTR } \\
70 \text { CNTR }\end{array}$ & 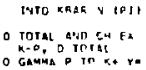 & $\begin{array}{l}7 / 50 \\
0 / 30 \\
6 / 70 \\
10 / 70 \\
1 / 71\end{array}$ \\
\hline
\end{tabular}

25 MOIZLOOI PARTIAL OEGAY MUDES IPROD. FXP,

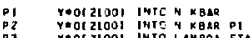

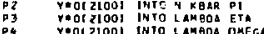
DECAY NASSES $539 * 493$
$978 * 497 * 139$
$1115 * 548$ $1115+548$
$1115+? \mathrm{BO}$

is YOOT2100I BRAHCHING RATIOS IPRDO. EXP.1

roOrzLOOS CNIC TKBAR NITTOTAL

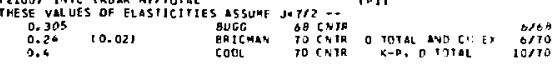

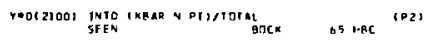


Baryons

$\Lambda(2100), \Lambda(2110), \Lambda(2325), \Lambda(2350)$
Data Card Listings

For notation, see key at front of Listings.

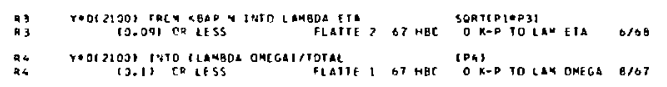

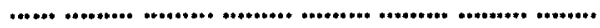

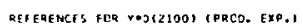

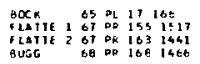

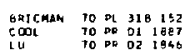

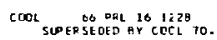

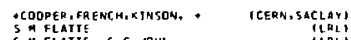

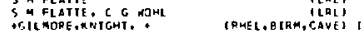

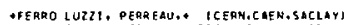

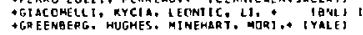
PAPERS NOT REFERPEO TO IN DATA CAFOS

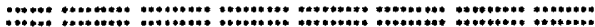
$\Lambda(2110)$ is $\quad$ r=042110, s085/2.1 1.0 $\quad F_{05}$

BEAT DONA TO FIND EITHER FOS OR DOS PESS IBLE IN

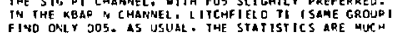

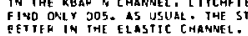

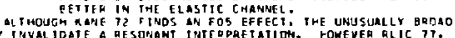

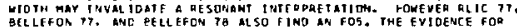

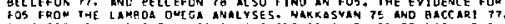

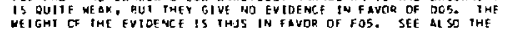
YOOI 20001 MINI-QEVIEN.

\section{Yoot 21801 ness (Mfvi}

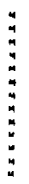

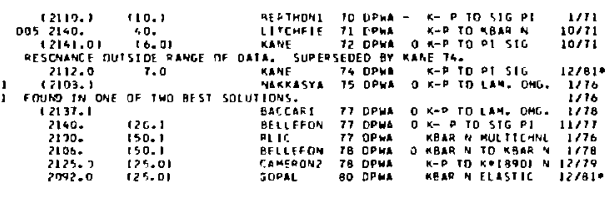

35 reotshios wiorm inevi

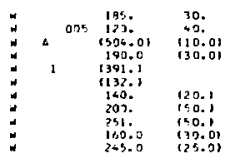

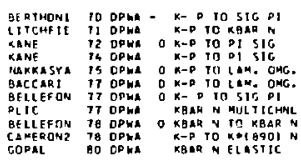

30 VMOI 21101 DAPTIAL OEGAY MOOES

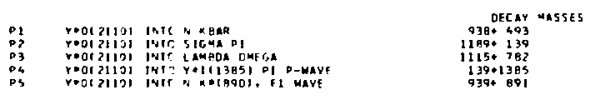

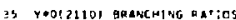

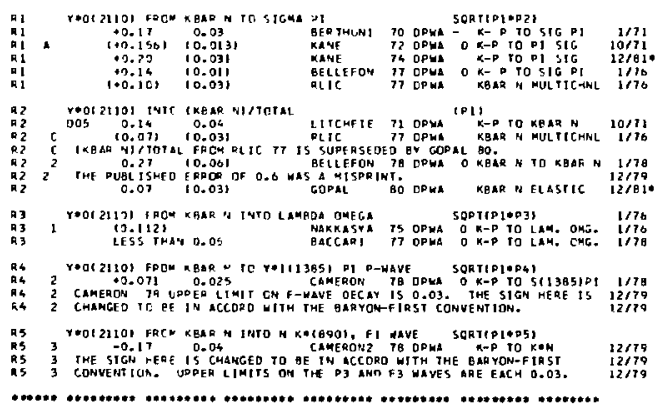

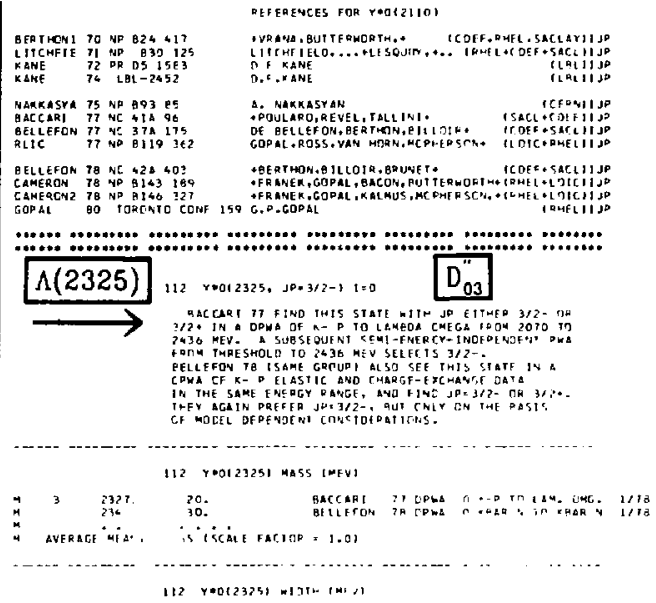

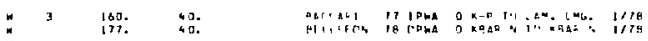

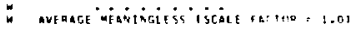

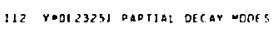

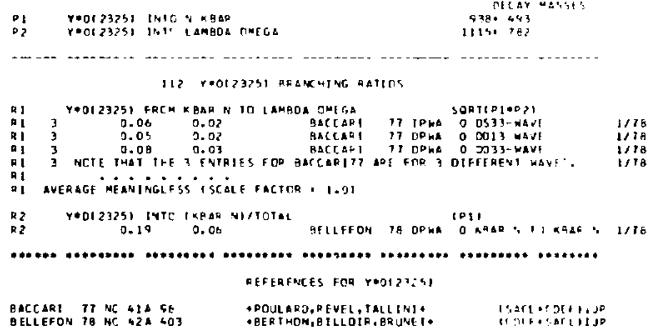

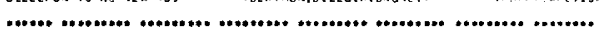

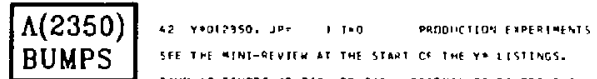

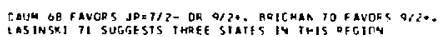

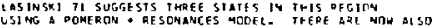

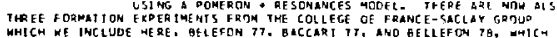

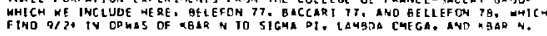

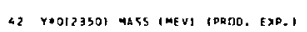

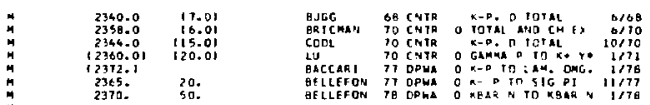

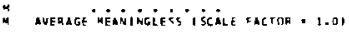

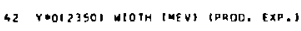

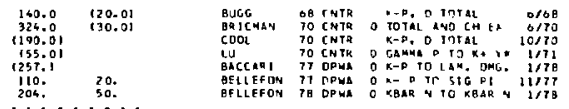

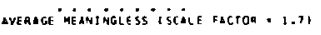




\section{Data Card Listings}

For notation, see key at front of Listings.
Baryons $\Lambda(2350), \Lambda(2585), \Sigma^{+}, \Sigma^{-}, \Sigma^{0}, \Sigma(1385)$

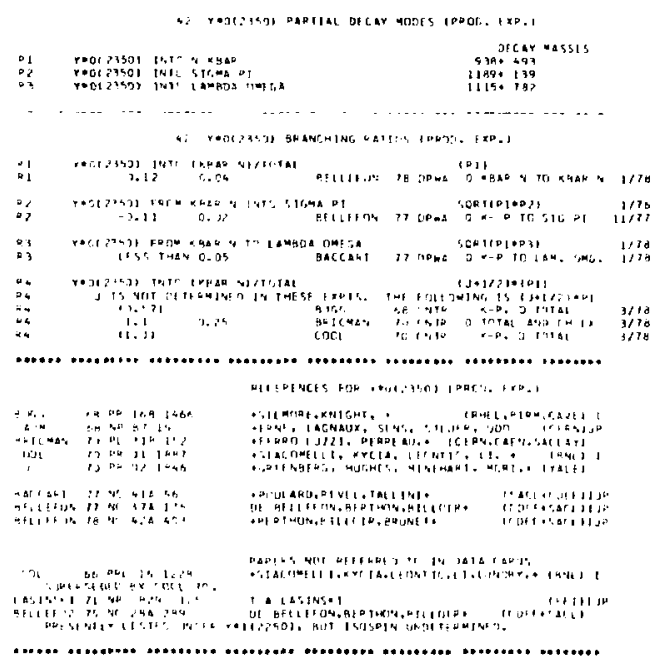

$\Lambda(2585)$
BUMPS

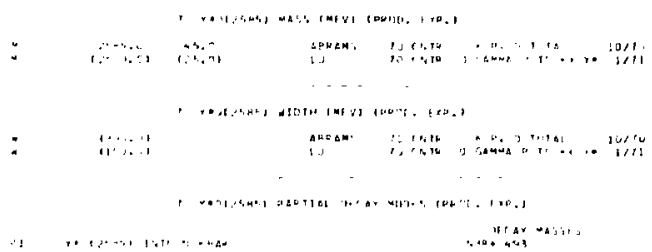

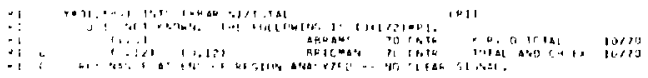

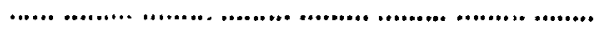

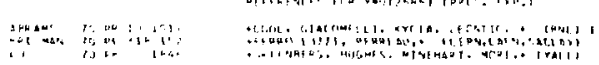

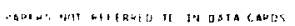

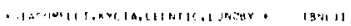

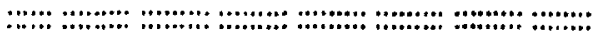

$S=-1 \quad I=1$ HYPERON STATES $(\Sigma)$
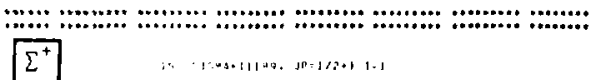

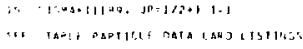

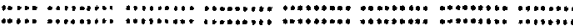

$\Sigma^{-1}$

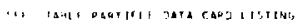

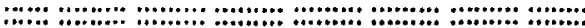

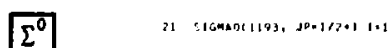

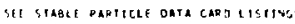

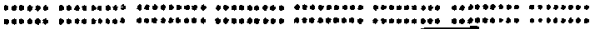

$\sum(1385) \quad \&$ a

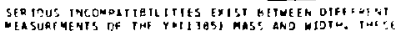

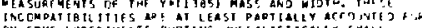

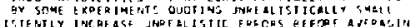

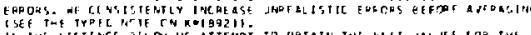

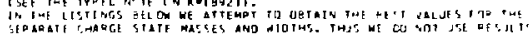

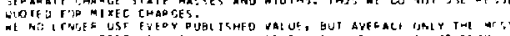

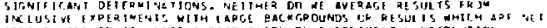

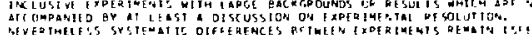

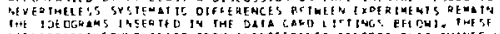

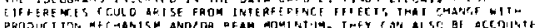

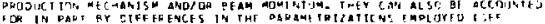

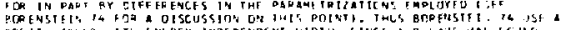

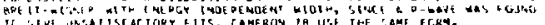

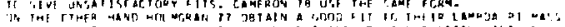
if

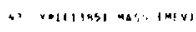

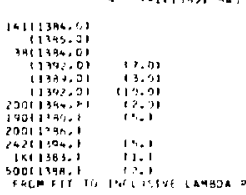

यद

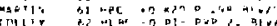

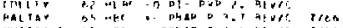

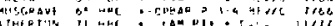
年

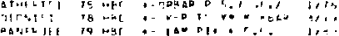

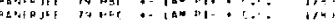

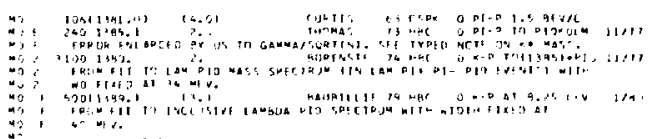

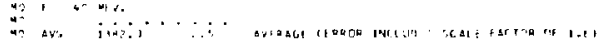

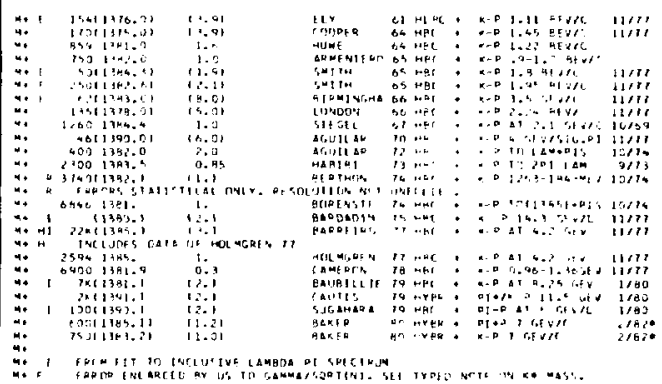

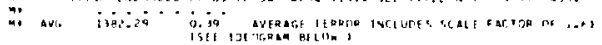

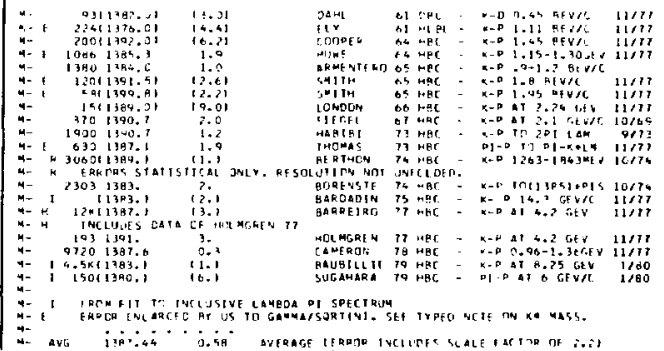

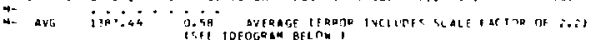


Baryons

$\Sigma(1385)$

WETGHIED AYERAGE $=1302.29 \pm 0.39$ ERROR SCALEO GY 1.6

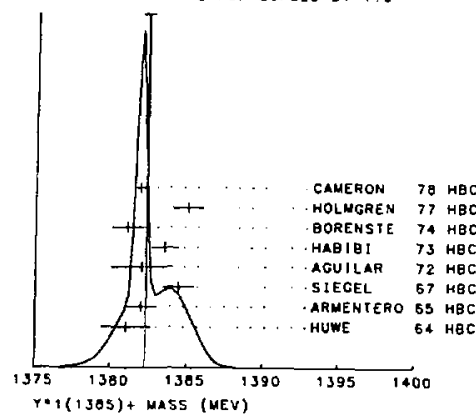

WEIGHTEO AVERAGE $=1387.44 \pm 0.50$ ERROR SCALED EY 2.2

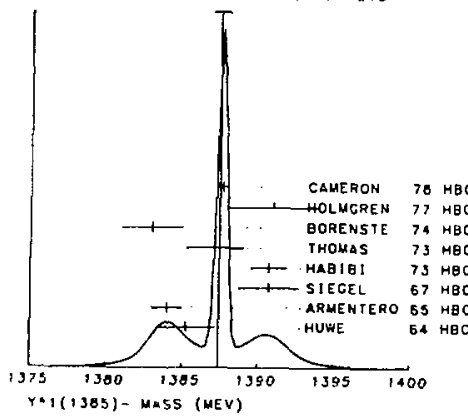

$\frac{\text { CHISO }}{0}$

4. 9

0. 0

7.4

2.7

11.8

$\frac{1.3}{20.4}$

(CONLEV

43 IY"- - Iron! MASS OIFFERENCF (MEV)

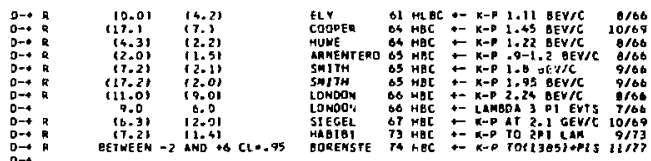

D-: a REOWDENI HITH DATE IN MSS LISTINGS

43 (YMO) - (YNa) MASS OLFFEREHCE IHEYI

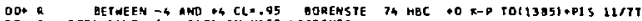

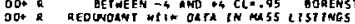

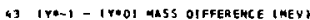

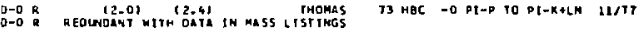

43 VQ1(1385) HIOTH (KEV)

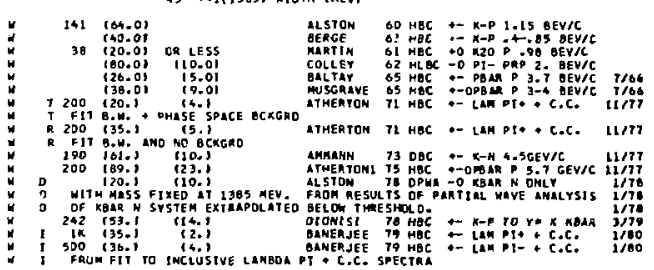

Data Card Listings

For notation, see key at front of Listings.

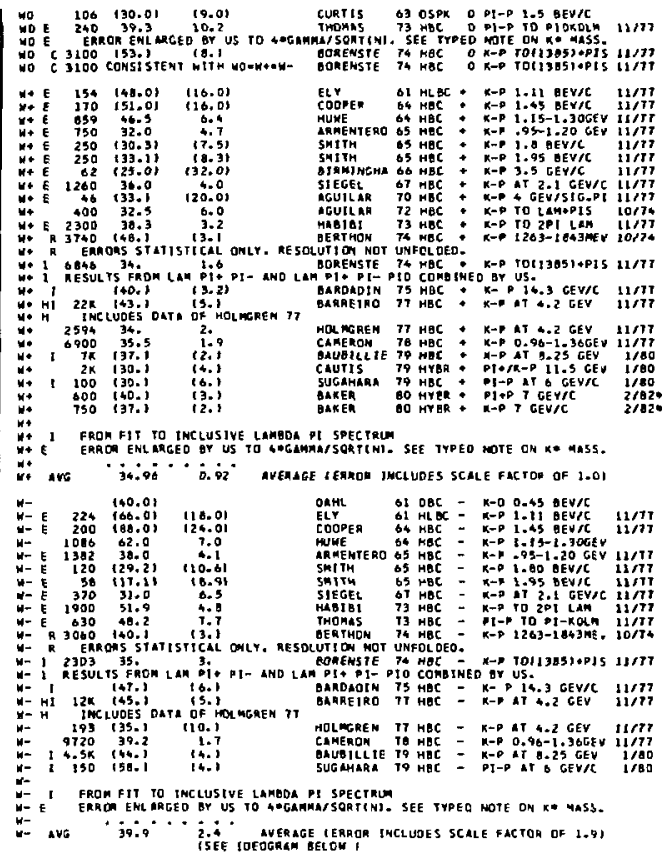

WEIGHTED AVERAGE $-39.9 \pm 2.4$

ERROR SCALED BY 1.9

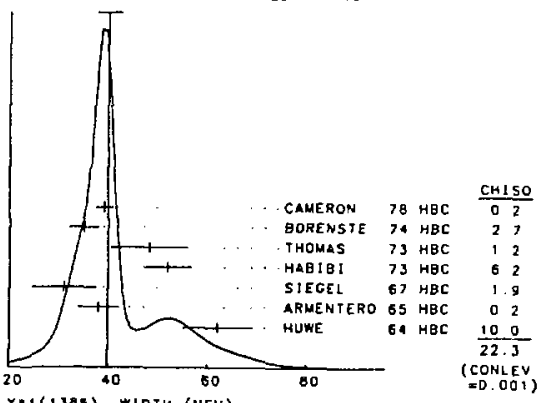

Y०1(1385)- WIOTH (WEV)

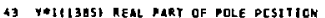

475

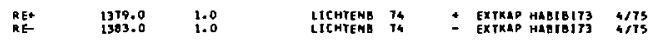

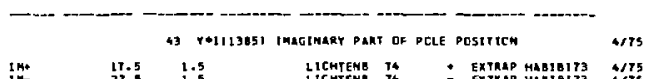

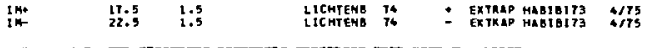

43 reltisesi PART IAL DECAY MODES

\begin{tabular}{|c|c|c|c|}
\hline $\begin{array}{l}1 \\
3 \\
3 \\
34 \\
35\end{array}$ & 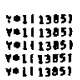 & 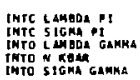 & 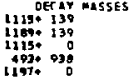 \\
\hline
\end{tabular}


Data Card Listings

For notation, see key at front of Listings.
Baryons

$\Sigma(1385), \Sigma(1480), \Sigma(1560)$
43 PAII3851 SQANCWING RATIOS

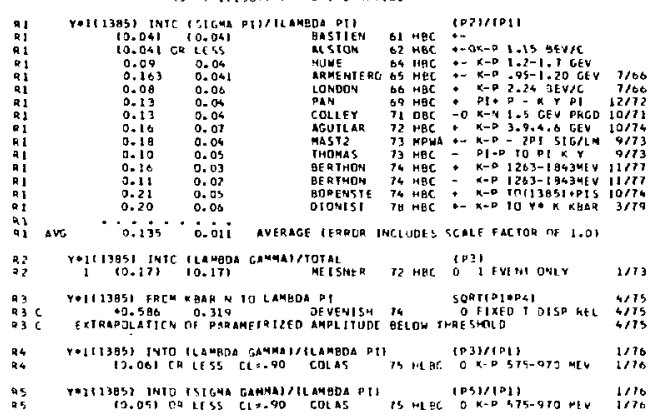

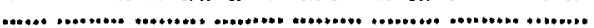

ALSTON 60 POC 5 S 50

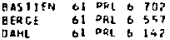

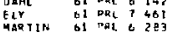

ALSTON OR CEAH CONE 31

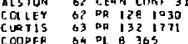

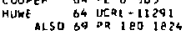

ALMENSER GS PL 19 TS

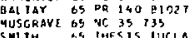

B TRM IFOH GO DD $152 \quad 1148$

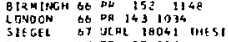

PAN 69 DQL 23 gOD

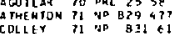

agullap 72 no de 79

MEISNEA T2 NC 124 B 2

AAS 12 T3 08073212

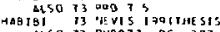

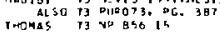

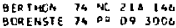

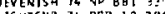

CHTENA 74 PHD $1034 t 4$
AI T4 PAIV. LCH4.

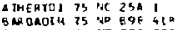

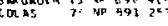

BARREIRO $n 7$ M

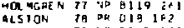

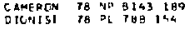

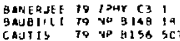

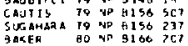

SAL AMUD OA OL 101214

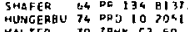

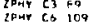

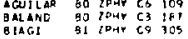

AIFEREACFS FOR YOLII3931

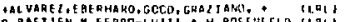

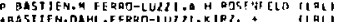
-BASTIEH, OAHL, FFRRO-LUIZI,KIPR, + ILAII

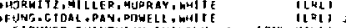

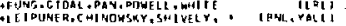

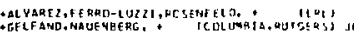

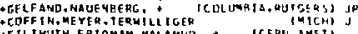

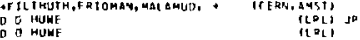

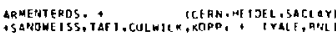

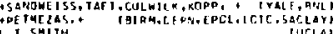

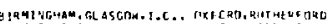

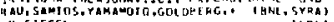
TEPL!
TOORMA:

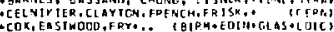

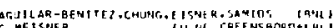

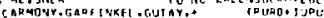

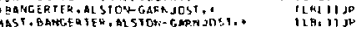

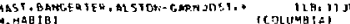

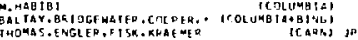

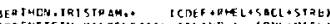

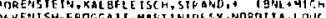

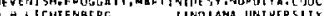

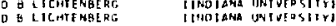

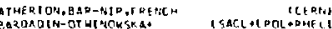

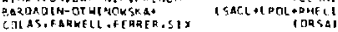

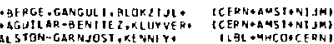

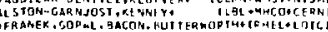

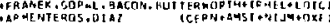

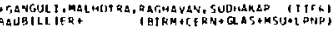
GALLAM.ADUCHE I, CABGOLL, G MAOM ICK. ISLAG

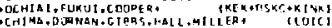
papers vot refenoed it in uata cahos

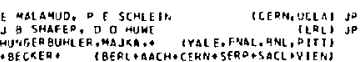

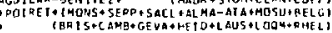

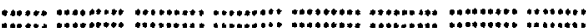

PATDOCTION FXPR IMEN: SFE IHE MINI-AEFUE AT THE START OF IHE YO LISTIMGS.

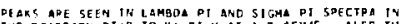
TWE DEACTIOH PI OD TO KO PI AND SIGHA PI SOECTPA TN BUMPS

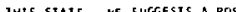
SEE HILLE 7O FOP A OISCUSSION OF IHIS STATE, ME SUFGESTS A POS STBLE ALTERHATE EXPLAHATIDN IN TERHS OF A REFLECT TON OF NA1/2TIGISI

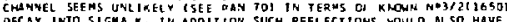
DECAY INTO SIGUA K. I'A ADOITION SUCH REFLECTIOHS WOULO ALSO HAVE TO AC COUAT

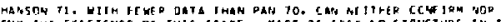

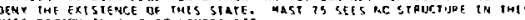

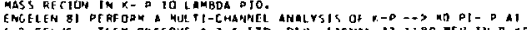

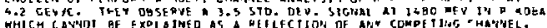

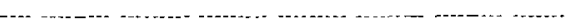

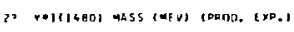

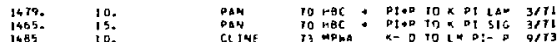

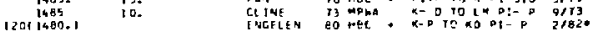
average méntivgiess iscáte tactor =

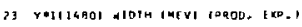

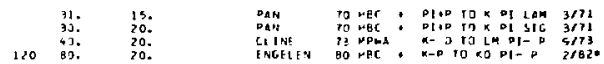
ayerage menifigitśs iscale factor = 1.21

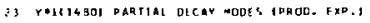

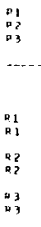

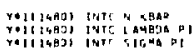

OrCAT USSE: 11154
11 1390.139

3 Y+1114ADI BSANCHING RAIIOS 10 FDO. 120.1

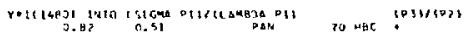

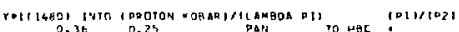

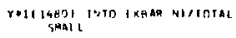

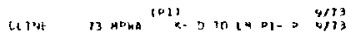

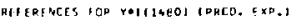

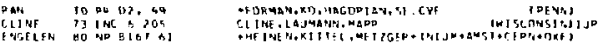

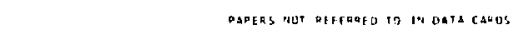

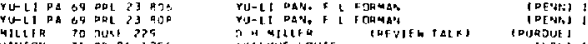

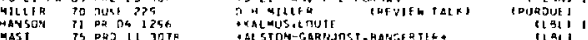

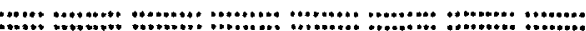

\section{$\Sigma(1560)$ BUMPS}

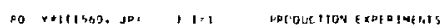

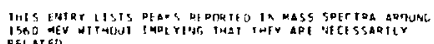

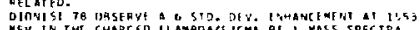

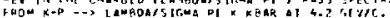

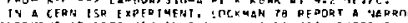

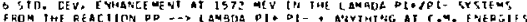
DF 53 a ND 3 ? CEV. IHE SE F FHA'L E WF

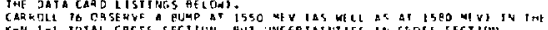

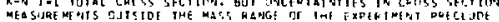

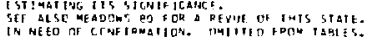

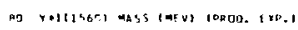

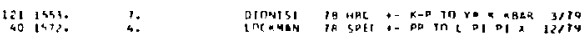
averagr miavi iritiss iscale factor at

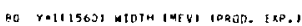

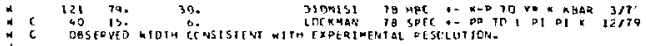

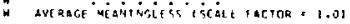

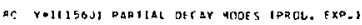

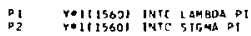
$115+$ MECAY Mas is 
Baryons

$\Sigma(1560), \Sigma(1580), \Sigma(1620)$

BO YELS15801 BHANCHING. OATIDS IPROD. EXP.)

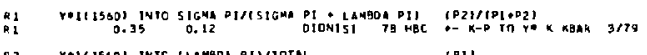

A2 YOL

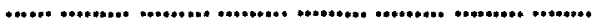

REFEREACES FOR YMISISOOS IPRCO. EXP.)

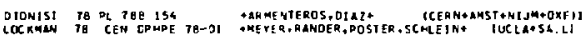

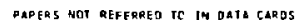

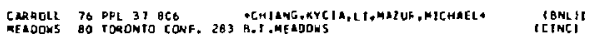

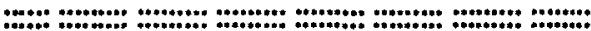

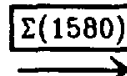

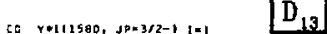

CGSER VEO IN K- N TEL TOTAL CS WITHOUT JP ASSIGHAEAT AT EVL III 73, CARPOKL 73, CLRROLL 7OI AND IN PHL OF $: p$

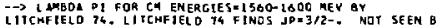

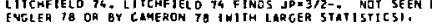
IN KLONA P $T O$ DI L LAMEOA AND PI+ SIGMAO.

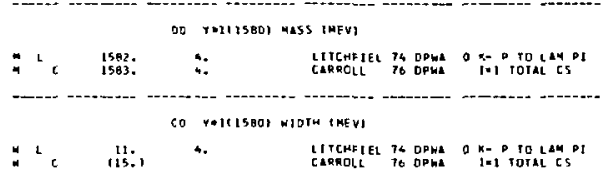

DO reIlISgOI DAPJIAL DEGAY AODES

DI YOLISBOI INIC N KBAR

$P_{2}$
$P_{3}$
Yoll

DECAY MASSES

$938+493$

$1115+130$
$1109+139$

CO reIIISBOI BRATCHING RATHOS

\section{Tas}

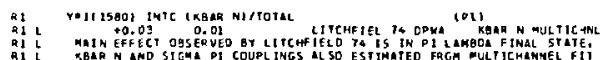

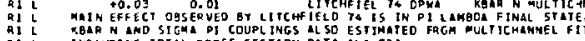

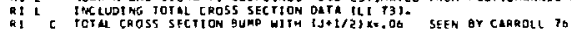

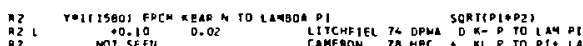

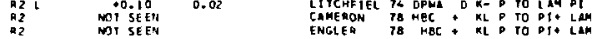

$R 3$
23
$Q 3$
B

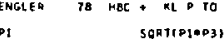

Q 3
R

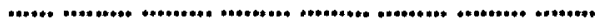

REFEREHCES FOR YMLLLSBOI

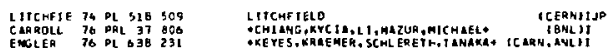

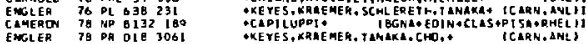

Dadeas mot Rekeared to in oata cardos

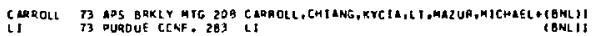

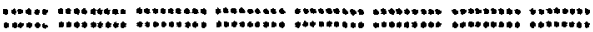

\section{Note on the $\sum(1620)$}

This state was firet suggested by CRENNELL 68 from evidence in the reaction $K^{-} \mathrm{n}+\Sigma(1620) \pm \pi^{\mp} \pi^{-}$at $3.9 \mathrm{GeV} / \mathrm{C}$, with the $\Sigma(1620)^{ \pm}$decaying into $\Lambda \pi \pm$. The situation io atill far from clear.

MILLER 70 is a good review of the production experiwents; there has been no new evidence from them since 1970. The evidence is only in the $\Lambda \pi$ channel. CRENKELL 69 clatmed the effect in this channel with no evidence seen in $\bar{K} M$ or $\bar{K} N \pi$. SABRE
Data Card Listings

For notation, see key at front of Listings.

70 studied the same reaction at $3.0 \mathrm{GeV} / \mathrm{c}$ with comparable statistics and gav no evidence in the $\Lambda \pi$ channel; on the contrary, chey belleve the effect to be a spurious peak resulting from misidentified $\Sigma^{0}$ from the production of $\Sigma(1670)$ decaying into $\Sigma^{0} \pi^{+}$. AMMANN 70 gtudied the same reaction at 4.5 $\mathrm{GeV} / \mathrm{c}$ and reported a otate at $1640 \mathrm{MeV}$, again decaying only into $A \pi$ (no evidence seen in the $\Sigma \pi$ or $\overline{\mathrm{K} N}$ channels). HUNGERBUHLER 74 reported upper 1 inits on production cross sections for a $25 \mathrm{GeV} / \mathrm{c}$ $\Sigma^{-}$beam.

CARROLL 76 measured the $\mathrm{K}^{-} \mathrm{P}$ and $\mathrm{K}^{-} \mathrm{d}$ total cross sections from 0.4 to $1.1 \mathrm{GeV} / \mathrm{s}$ and found three narrow (10-15 MeV wide) bumps in the $I=1 \overline{\mathrm{XN}}$ cross section at 1583, 1608, and $1633 \mathrm{MeV}$.

Several partial-wave analyses have found ev1dence for one or two falrly narrow states within about $50 \mathrm{MeV}$ of the effect seen in production: see the entries for the D13 $\Sigma(1580)$, the $s 11 \sum(1620)$, and the Pll $\Sigma(1660)$. However, the vartous analyses do not agree on widths or branching ratios.

In conclusion, clarification of the $\Sigma(1620)$ question probably muat await more data and a more complete understanding of the entire region from 1600 to $1700 \mathrm{MeV}$. The closeness of the $\Sigma(1620)$ and $\Sigma(1670)$ masses sugsests that the complications of the two reglons may be related (see the "Note on the $\Sigma(1670)$ " below).

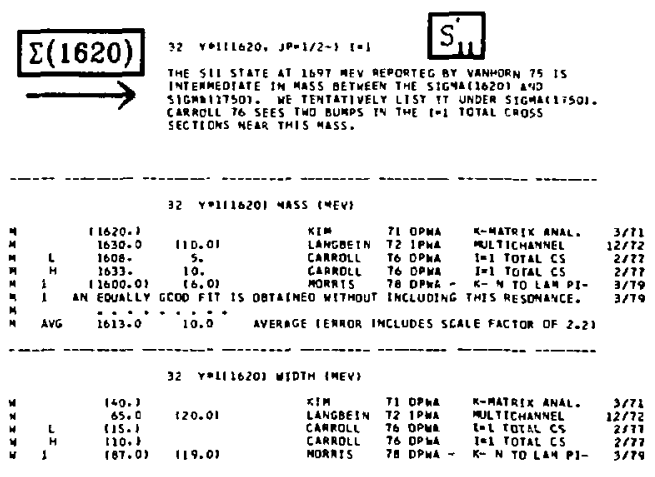

32 rolis

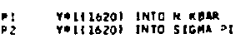

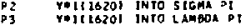

DECAY MASSES

$938 * 493$

$1139+139$ 
Data Card Listings

For notation, see key at front of Listings.
Baryons

$\Sigma(1620), \Sigma(1660)$

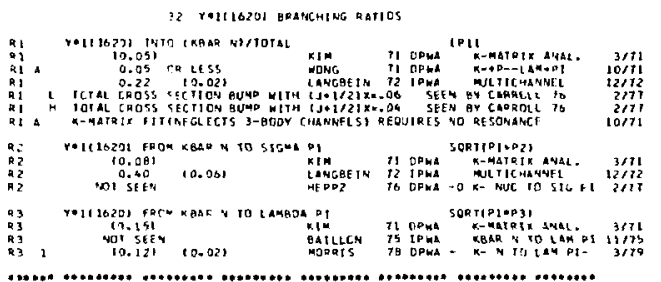

REFEREACES FOR YeII1OZOI

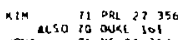

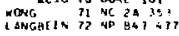

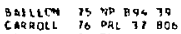

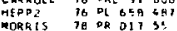

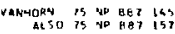

3. $3 x \sin x+4$

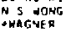

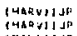

ivalintiju

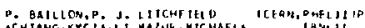

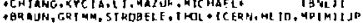

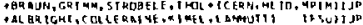

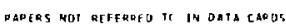

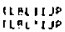

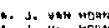

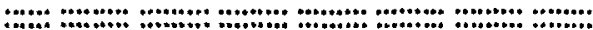

\section{MEV REGION - PRODUCTION EXPERIYENTS}

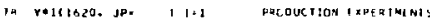

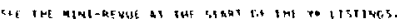

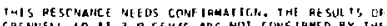

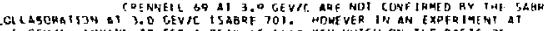

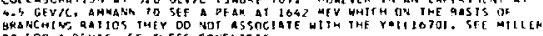

oivien cF thrse contuicts

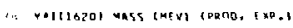

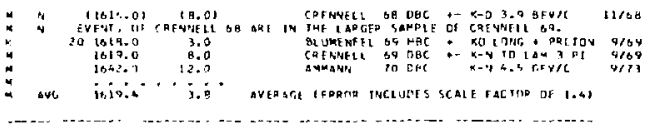

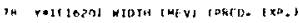

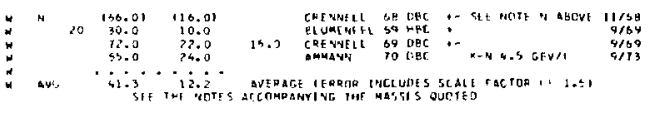

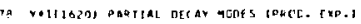

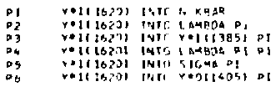

Detcy masse

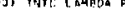

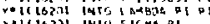

II15: 137

1355,137
$1115,139.139$

11154
11890,1300
14054

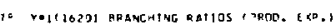

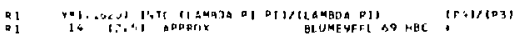

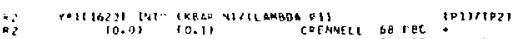

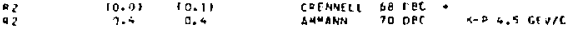

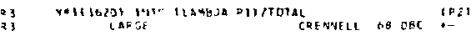

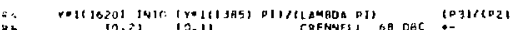

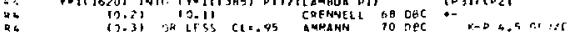

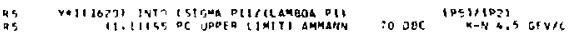

$\Rightarrow 0$
\&O

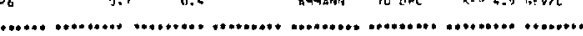

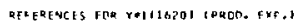

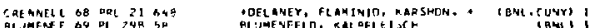

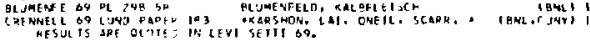

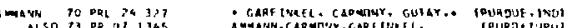

osro

$11 / 00$

$+3 / 68$

$4 / 13$

ar 70
ARECNTEQ BS 49 SB 183

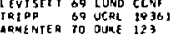

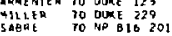

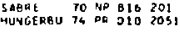

\#.......

$\Sigma(1660)$
PLPES WOR REFERE] IC IN DAFa CaODS

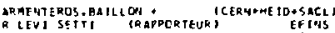
PO TRIDO
TLRLI)

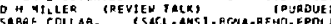

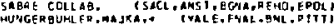

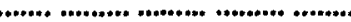

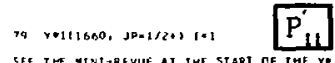

STE THE MUT-REVUE AT IHT START OF THE VA IISTIDIS.

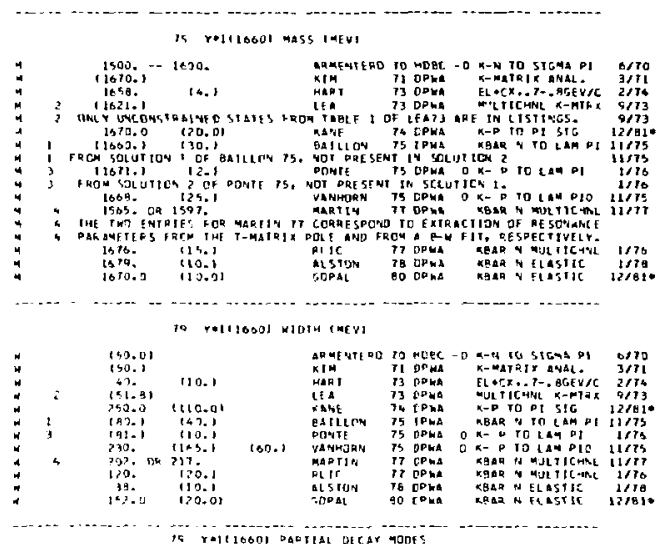

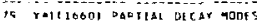

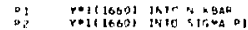

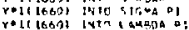

II 1190.199

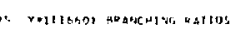

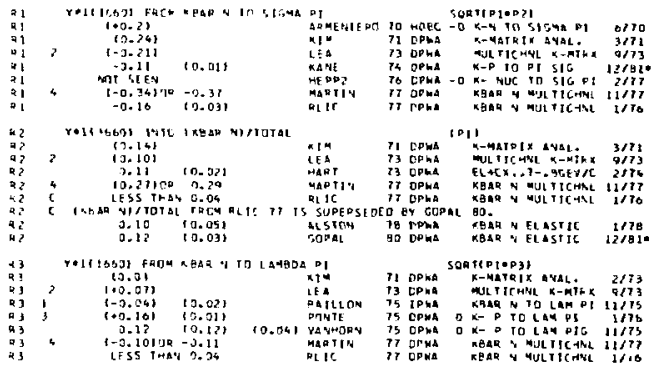

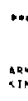

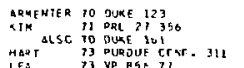

aEFERENCES FDR Volllatol

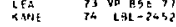

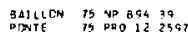

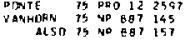

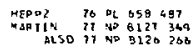

$A 150$
ALSO ND

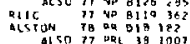

AREETEROS. BatLLON.

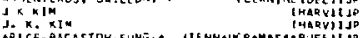

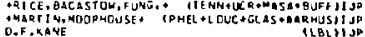

D. BATLLON, P. J. LITCHFIFLO TEERN, RAELIIJP

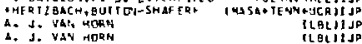

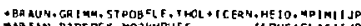

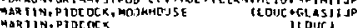
anain, P1

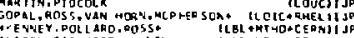

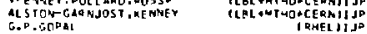

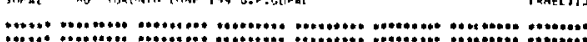


Baryons $\Sigma(1670)$

\section{Note on the $\Sigma(1670)$}

Production experiments: The measured $\sum \pi / \Sigma \pi i$ branching ratio for produced $\sum(1670)$ 's is strongly dependent on momentum trangfer. This was first discovered by EBERHARD 69, who suggested the existence of two $Y_{1}^{*}$ 's with the same wass and quantum numbers: one with a large $\sum \pi \pi$ Ima1nly $\Lambda(1405) \pi]$ decay mode produced peripherally, and the other with a large $\sum \pi$ decay mode produced at larger angles. These rejults were confirmed by AGUILARBEAITEZ 70, ASPELL 74, ESTES 74, and TIMMERMANS 76. When determined, the most likely quantum numbers for both the $\Sigma \pi$ and the $\Lambda(1405) \pi$ obfects are $D 13$. There 1s also possibly a third $Y_{1}^{*}$, the $\Sigma(1690)$ in the Listings, with a large $M \pi / \Sigma \pi$ branch1ng ratio and somewhat larger wass; the large branch1ng rat1o Is the main evidence for 1ts existence, which needs confirmation. These toplcs have been reviewed by EBERHARD 73 and by MILLER 70 .

Formation exper1ments: Two states are also observed near this mass in forwation experiments. One of these, the D13 $\sum(1670)$, has the same quantum numbers as those observed in production and has a large $\Sigma \pi / \Sigma \pi \pi$ branching ratio. It may well be the S(1670) produced at larger angles (see TIMMERMANS

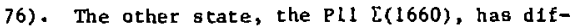
ferent quantum numbers from those seen in production, and its $\sum \pi / \sum \pi f$ branching ratio is unknown. Thus 1 cs relation $c)$ the produced $\left[(1670)^{\prime \prime} \mathrm{s}\right.$ remaing obscure. (See also the "Note on the $\Sigma(1620)$ " above.)

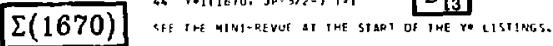

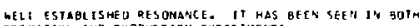
SEE LISTIDE OF DRODUK TION EKPERIMENTS BHC OK

\section{Yel11670) WASS (4IVI}

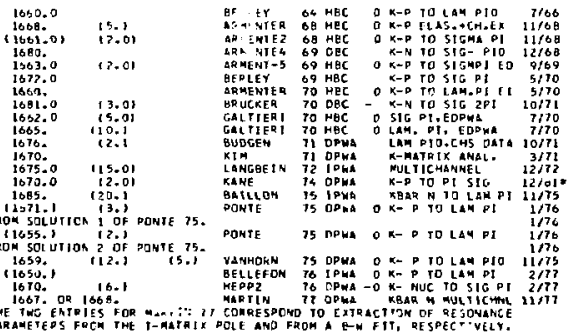

\section{Data Card Listings}

For notation, see key at front of Listings.

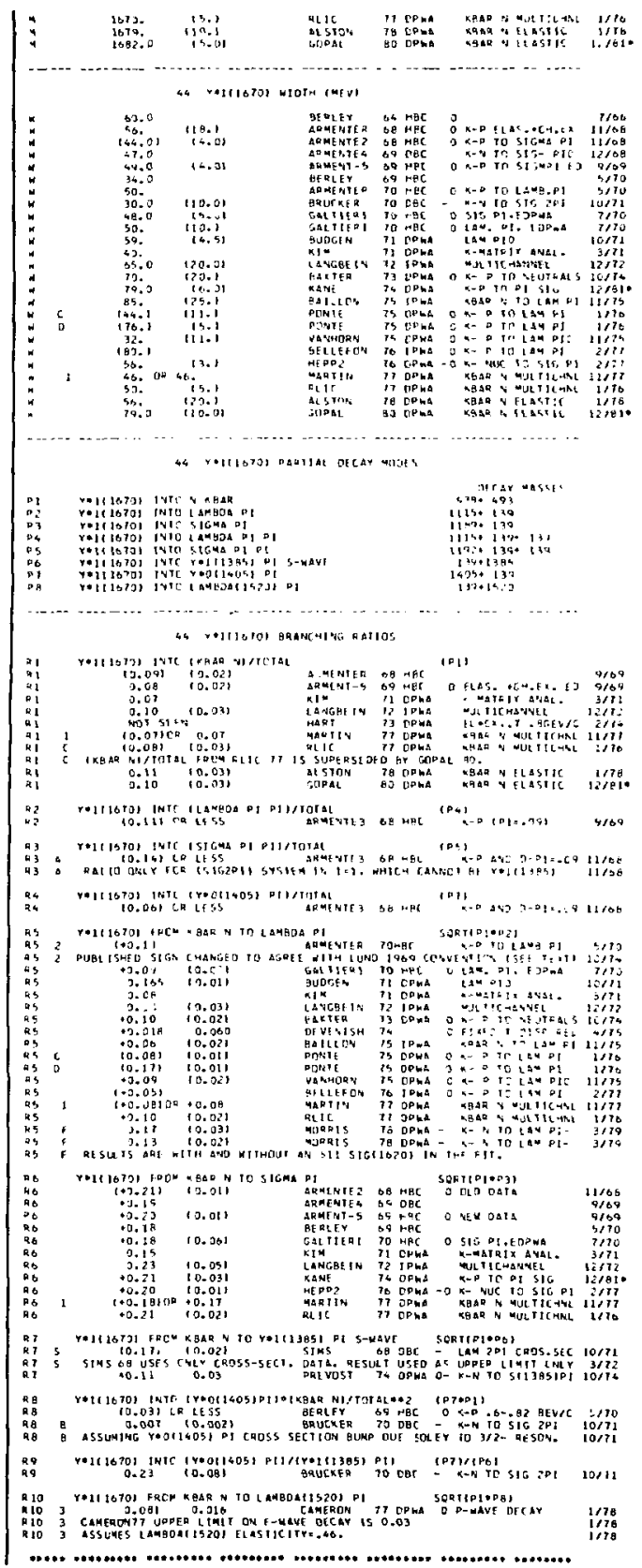




\section{Data Card Listings}

For notation, see key at front of Listings.
Baryons

$\Sigma(1670)$

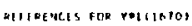

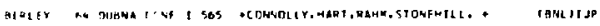

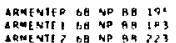

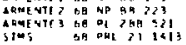

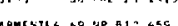

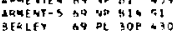
DRMENTE TO JAKA 123

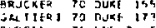

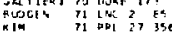
alse to OOF 101

IANGAEIA T2 YP 947417

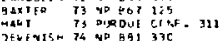
KANE Th $130-262$

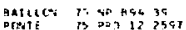

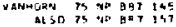

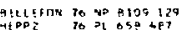

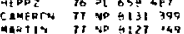
Matilis Ir

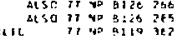

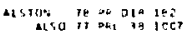

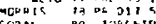

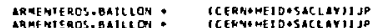

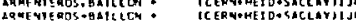

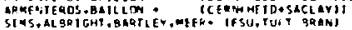

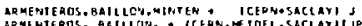

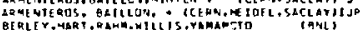

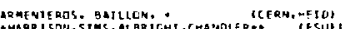

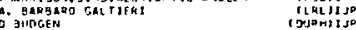
$x \times 1=14$

J. K. Kla

AHAGNF

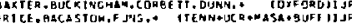

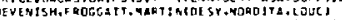

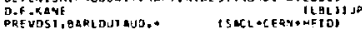

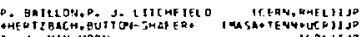

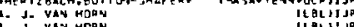
1. J. Vav hodan

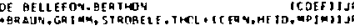

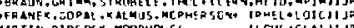

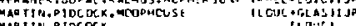

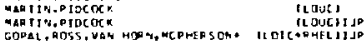

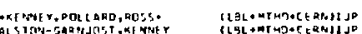

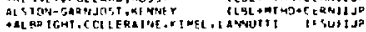

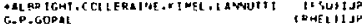

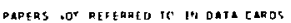

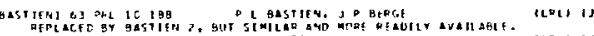

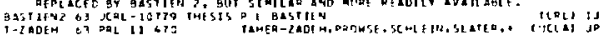

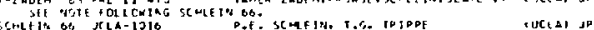

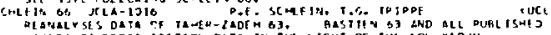

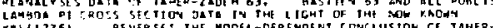

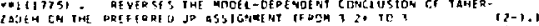

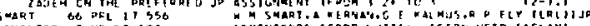

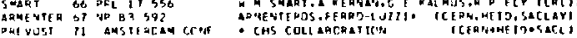

.....

\section{$\Sigma(1670)$ \\ BUMPS}

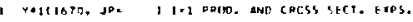

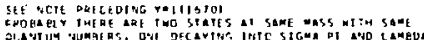

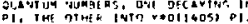

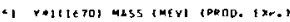

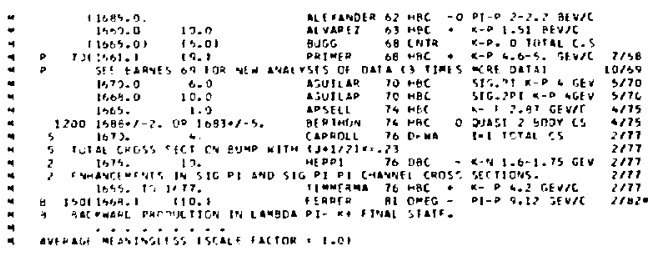

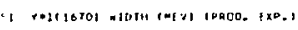

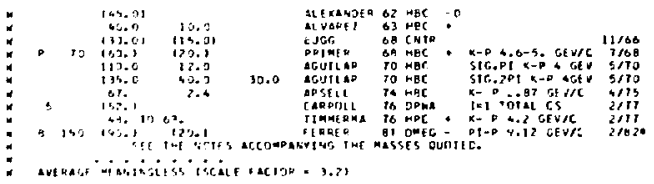

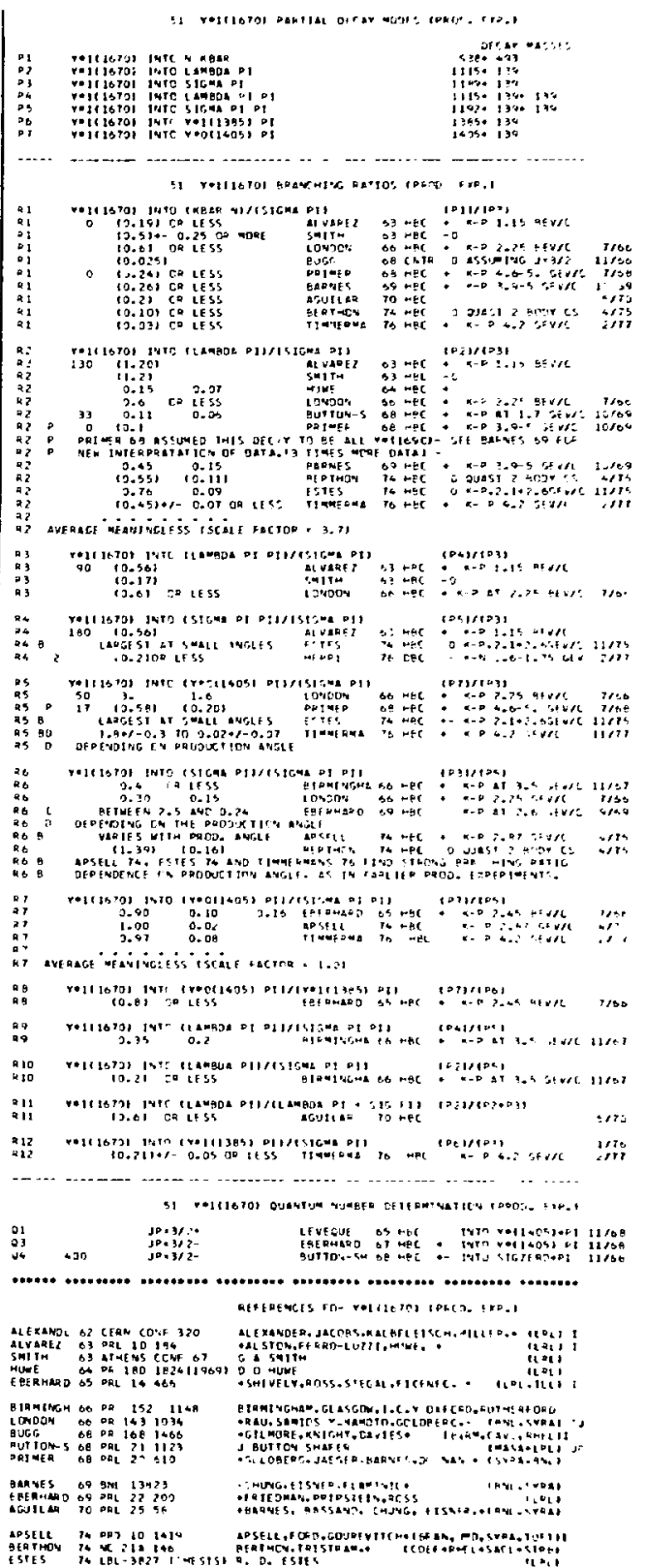


Baryons $\Sigma(1670), \Sigma(1690), \Sigma(1750)$
Data Card Listings

For notation, see key at front of Listings.

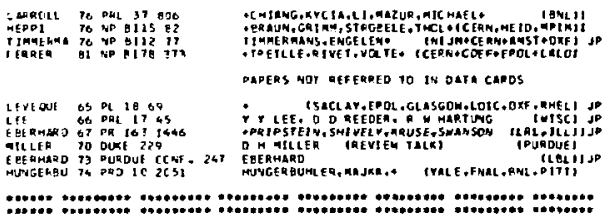

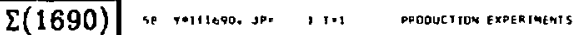
BUMPS

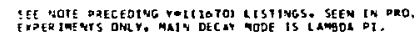

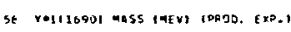

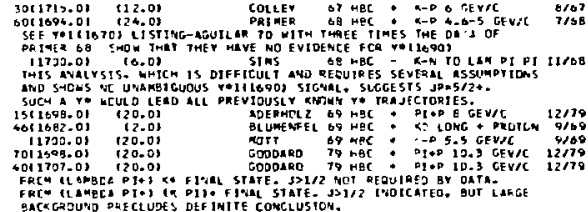

$\Sigma(1750)$

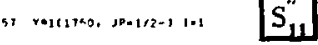

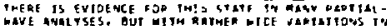

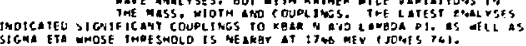

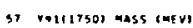

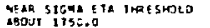

saver 1150.0

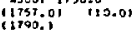

$\begin{array}{rl}11700.01 & 115.01\end{array}$

(1715:) 110.1

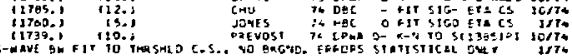

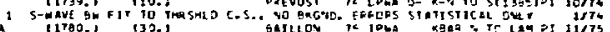

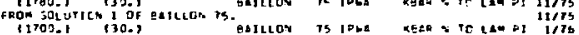

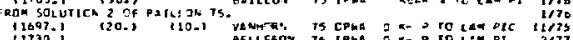
1715: 10. CERCL2 To com

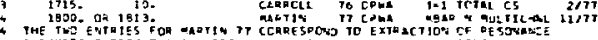

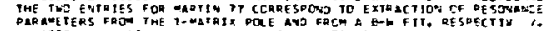

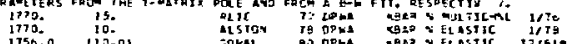
averace meanirgifis iscale tacton = 3.00

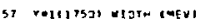

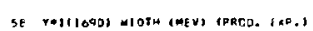

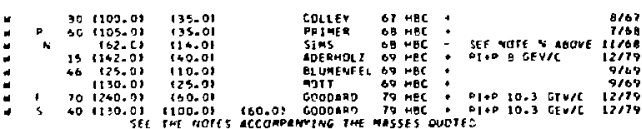

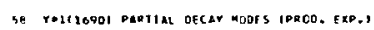

\begin{tabular}{|c|c|c|}
\hline 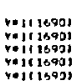 & 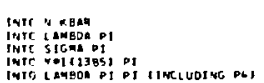 & 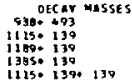 \\
\hline
\end{tabular}

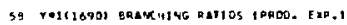

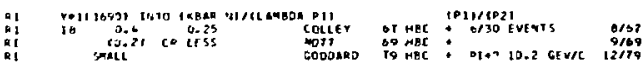

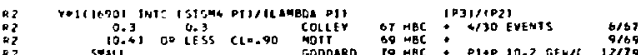

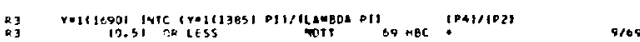

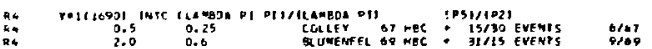

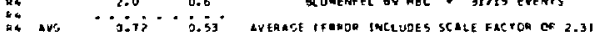

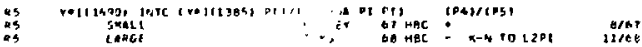

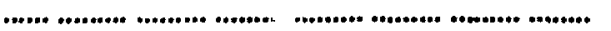

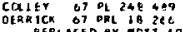
ORIMER
STIS

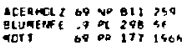
SCDOAKD IT OR OLE isso

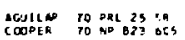

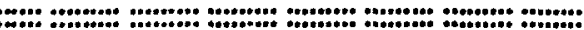

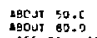

$125,31,113.04$

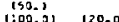

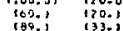

192.19

1109.;

1185, 130.1

rist:

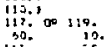

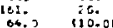

12.1

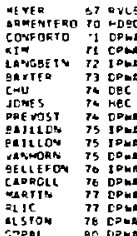

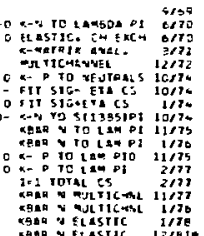

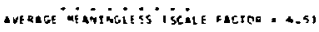

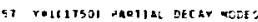

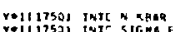

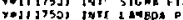

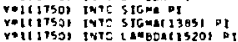

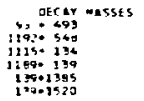

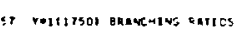

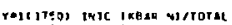
(0.-121 10.0510

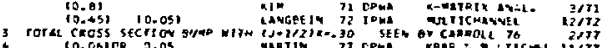

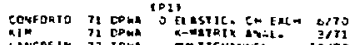

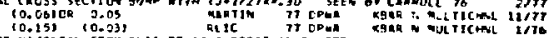

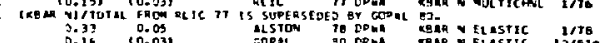

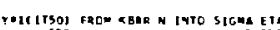

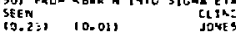

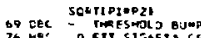

9109

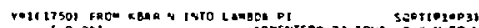

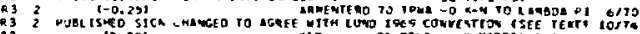

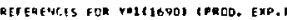

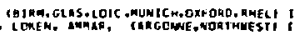

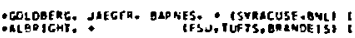

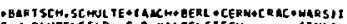

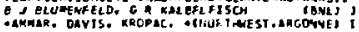

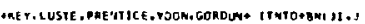

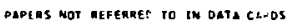

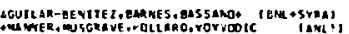
CIn

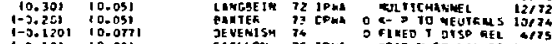

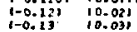

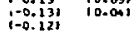

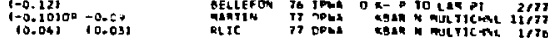

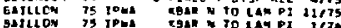

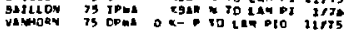

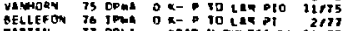

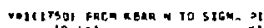

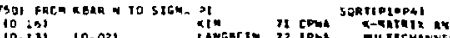
GARGSC19,

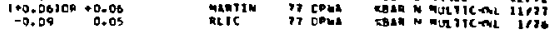

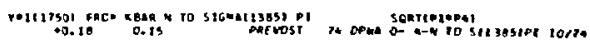

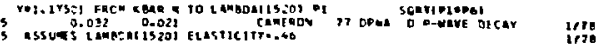




\section{Data Card Listings}

\section{For notation, see key at front of Listings.}

Baryons

$\Sigma(1750), \Sigma(1770), \Sigma(1775)$

\begin{tabular}{|c|c|}
\hline $\begin{array}{l}\text { CLIME } \\
\text { MEYER } \\
\text { ARMEMER } \\
\text { CCMFOERO } \\
\text { KIM ALSO } \\
\text { L ANGEEIN }\end{array}$ & 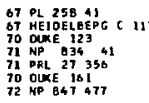 \\
\hline $\begin{array}{l}\text { gNXTER } \\
\text { CHU } \\
\text { JOHES } \\
\text { OEVENISH } \\
\text { PREVOSY }\end{array}$ & 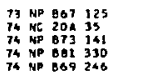 \\
\hline $\begin{array}{l}\text { BA1LLDN } \\
\text { VAMHONW } \\
\text { RLSO }\end{array}$ & 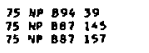 \\
\hline $\begin{array}{l}\text { BELLEFOH } \\
\text { CHAROLL }\end{array}$ & $\begin{array}{l}76 \text { ND } 8108,120 \\
76 \text { PRL } 37 \text { BC6 }\end{array}$ \\
\hline $\begin{array}{l}\text { CAMEAOH } \\
\text { MARTIA } \\
\text { ALSO } \\
\text { MLSC }\end{array}$ & 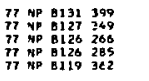 \\
\hline $\begin{array}{l}\text { ALSTON } \\
\cos A L\end{array}$ & 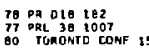 \\
\hline
\end{tabular}

QEFERENCES FQR YMICITSOI

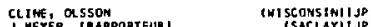

ARMENTEROS. BAILION,! ISACLAY)!JP

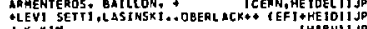

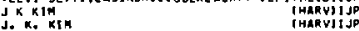
J. K. Kim Kim GAXTER, BUCRINGMAM, COFBE T, DUNM, TOXFOROII JP CHU, BARTLEY, I SUNY PLA TTSE UREH TUF TS + BRANIIJP JOMES S

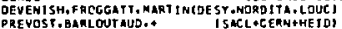

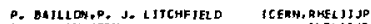
A. J. YAN HORN $Y$ HORN

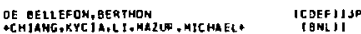

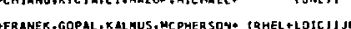
MARTIH, PLOCOCK, MOORHOUSE (LOUT, FLASJIJP MaRTin, Plocace

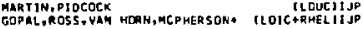
TKEKNEY, POLLLRO-ROSS* TLBL + MTHO+CERNIT JP

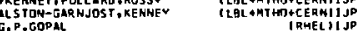

papehs nOt referrev to in DATA Cardos

FERRO-LU OG GEAKELEY CONF IB 3 M ERRO LUZZ1 IRAPPORTEUR, ICERM,

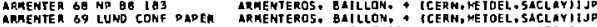

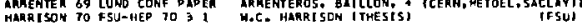

:

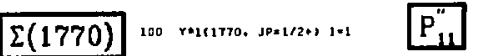

EYTOERCE FIR THIS STATE NOW RESTS SOLELT OH SOLUTION 1

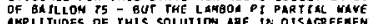
AMPLITUDES OF YHIS SOLUIION TAE IN OISARREEMENT WTTH

ICO TEITLTTOS MLSS (MEYI

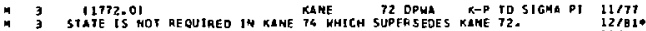

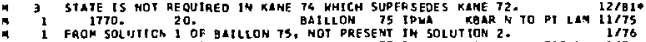

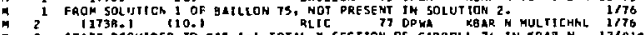

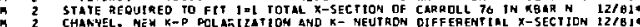

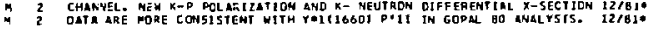
$100 \quad Y=1(1 T 7 D)$ WIOTM (MEV)

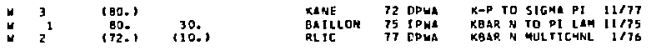
100 PAIt1770) PARTIAL OECAY MODE:

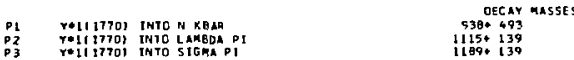

ICO YOI(ITTO) ARANEHING RATIOS

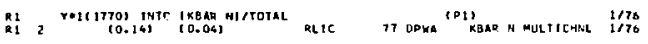

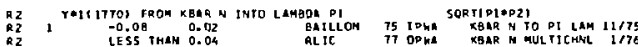

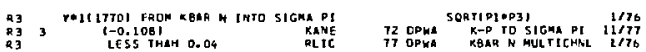

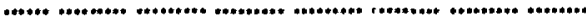
REFEREACES FOR VIIITOI

KANE 72 PR DS 1583 D F KANE (281)

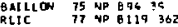

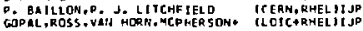
papeas hot Refeoqeo to in orta capas

KANE 74 LRL-2452 D.F-KANE

: $\sum(1775)$ as reatilitis, jo-5/2-1 i-1 D

SEE THE MIHI-REVIEN AT THE STAPT CF THE YP LISTIBGS.

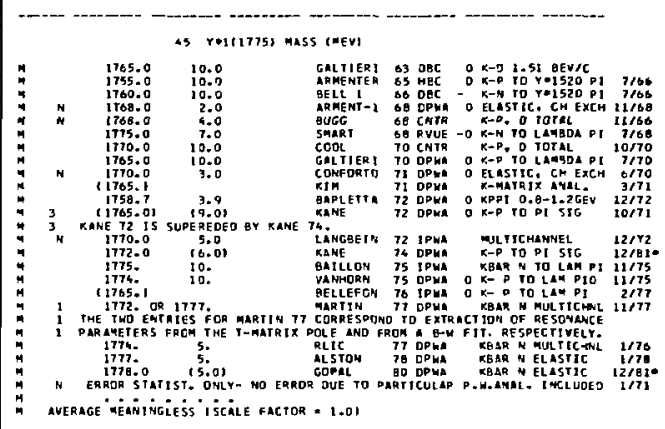

45 rellITI5I WTOTH CMEYI

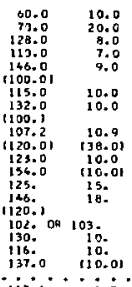

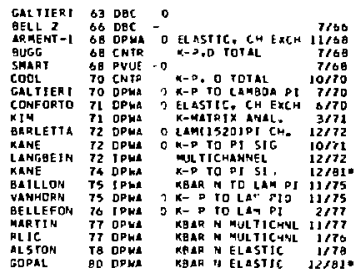

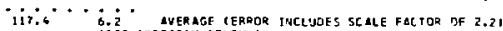

Gi.

WE IGHTEd AVERAGE $=117.4 \pm 6.2$

ERROR SCALEO BY 2.2

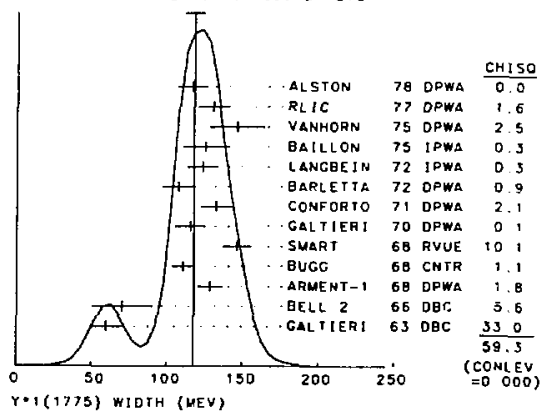

45 Yeititisi patial decar motes

D1 Y*111775) IATE M KBAR

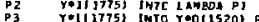

P. Y+111775) INTE Yro(1520) Pf

PS YoII1775) INYO SIGMA PI

Po POH
DECAY nasSES

5394.993

$2115 *$
1520.439
139

11894139

$\begin{array}{ll}1192: & 548 \\ 11920 & 139+139\end{array}$ 
Baryons

$\Sigma(1775), \Sigma(1840)$
Data Card Listings

For notation, see key at front of Listings.

\section{FIT TED PARTML DECAY MODE BRANCHING ERACTIONS}

The matrix below is dexived from the erzor mutrix tot the fitted partiai decay made

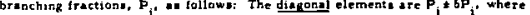

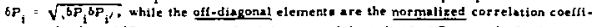

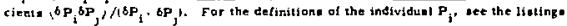
above: only those $P_{i}$ eppearing fn the malrix are ansumed in the fit to be nonsero and ere thue conatraned to add to 1.

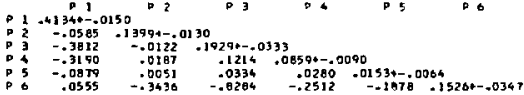

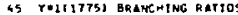

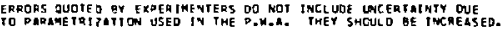

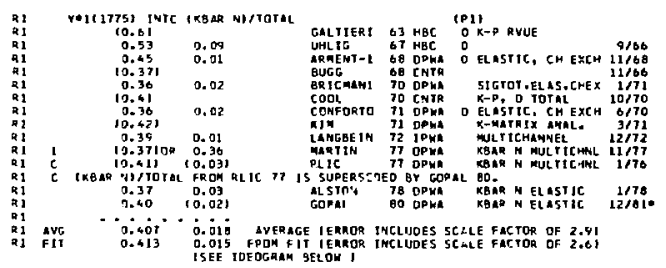

WEIGHTED AVERAGE $=0.407 \pm 0.018$

ERROR SCALED BY 29

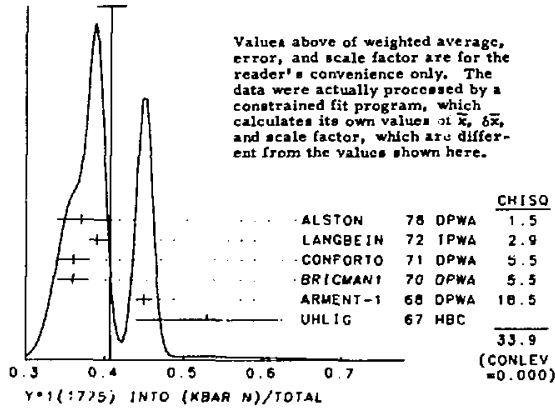

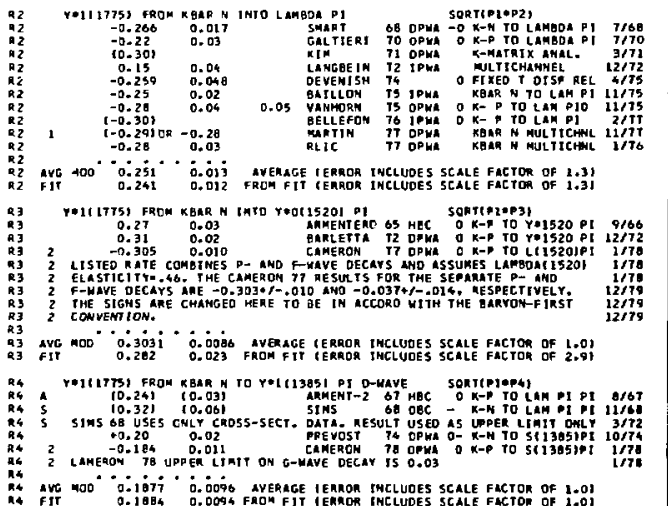

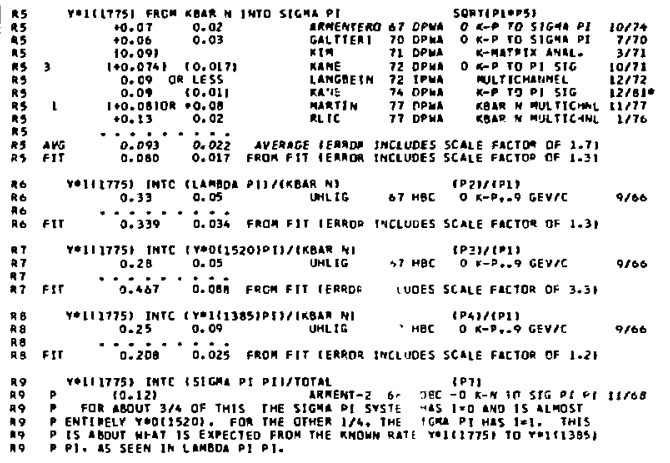

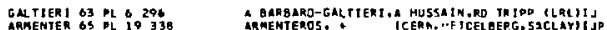

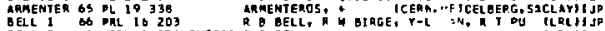

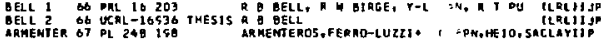

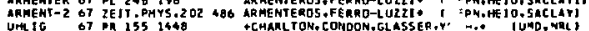

ARAENT-1 OB NP SE $15 \%$

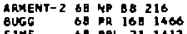
SIMS SMART OQ PRL 211413

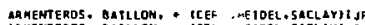
ARAENTEROS. BATLLOH, TCE HE 1DEL, SACL:YT I FILMORE, KNICHT, ORYIES* TM, GAVE, RHELI SIMS.ALGRIGHT, MARTLEY, MEER* SU, TUFT ERANY
ILRL HI JP enjekavi 70 DL $33 E 511$ CODL. TO Ph OL LE87 CONFORTO 72 NP 934 41 CIM ALSO 70 DULE 17 TS1

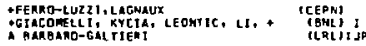

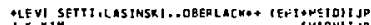
J.K KIT
J. K. KIT GLRLETT 22 NP 84045 KAHE T2 PR O5 $15 \mathrm{E}$

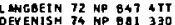
KANE 74 LEL-2452
PREVOST 74 NP 869246 BallLON TE HP 89439 VAKMORH T5 40 OQ1 145 W-a. GanLTTA (EFI) if D F KENE ILQL,J]P

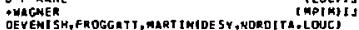
DEF,KAME
MEVOST,BARLOUT AUD.* CSACLCERVAHEIOT P. BRILLOH,D, J. LITCHFIELO ICERH, RMELIIJP

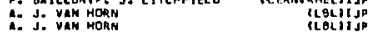

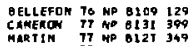

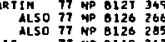

DE OELLLFOH.BERTHON ILELITJ

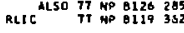
ALSTON TO PR 018 197
ALSO IT PRL 38 IOOT

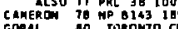

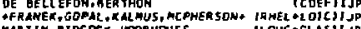
WMUTH.PIDCOCK, HOORHDUSE RLOUC+GLASIT

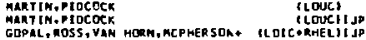

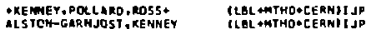
ALS TCH-GARHJOST, RENNEY ILOL + KTHO CERMI IJP

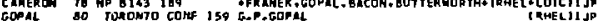

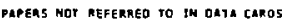

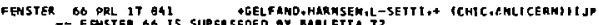

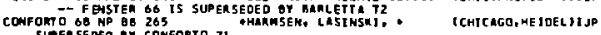

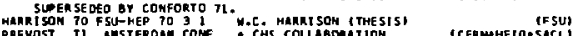
PREYOST TI ATSTEP (x)

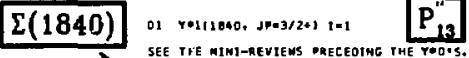

PI3 MAVE IN THE FOK THE TTWE GETHG. WE COST ACL RESONARCE CLAIHS IN THE DI YWLILEOI MASS (NEY)

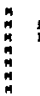

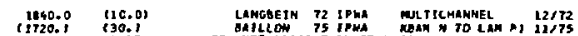

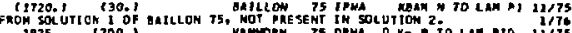 1925. 1200.) 1902.

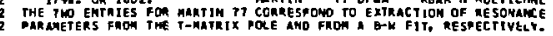




\section{Data Card Listings}

\section{For notation, see key at front of Listings.}

Baryons

$\Sigma(1840), \Sigma(1880), \Sigma(1915)$

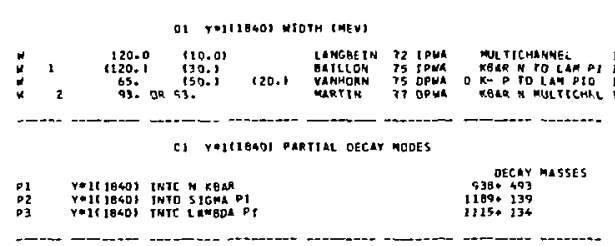

GI YEICLEAOI GPANCHING RATIOS

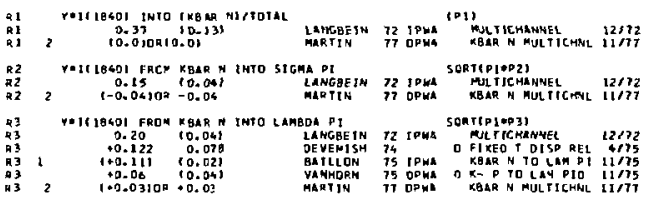

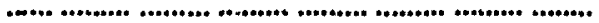

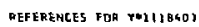

L ANGEEES 72 ND BST 471 DEVENISH 74 ND B81 330

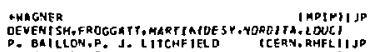

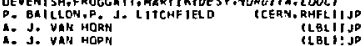

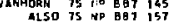

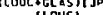

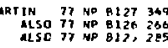

MRRTIN, PIDCOCK, MDORHOUSE MAnTIT, PIDCOCK
MATIIY, PIOCRCK tLONCIIJP

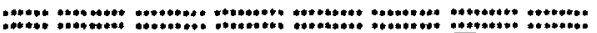

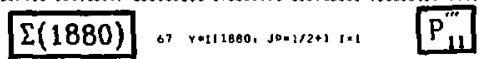

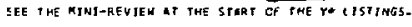
- Pesohanee 15 sugested gy geverme part Ial-have AMALYSES ACROSS YHIS QEGION. GEUT KITH YIOE YaA IATIONS

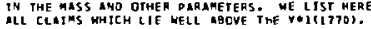

67 YeIt18aOY MasS \{MEVI

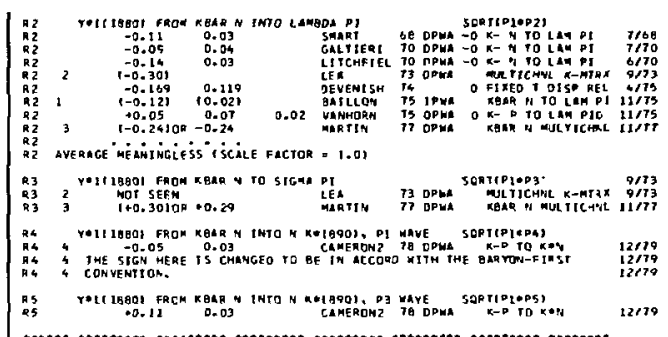

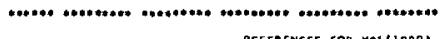

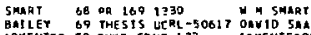
AREFTER 10 DUKE SONF I23 RRAENTEROS. BAILLON, + TCERN. MESDEL HIN

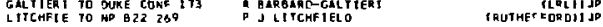

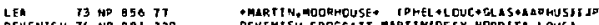

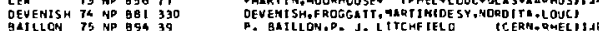

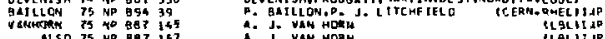
MLSO 75 NO BeT 157 A. J. VAM MOAM TLLITAP

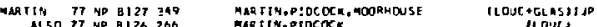

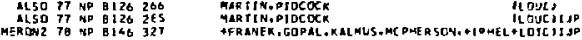

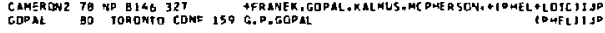
:

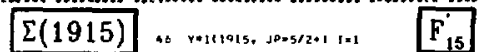
SEE THE HIPT-REYTEN AT TAE STAPT CF THE Ye LISTS NGS. THSS RESONANCE WA FIDST SEEN IN THE TCTAL-COOSSS- SEC-

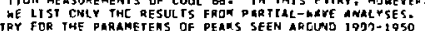

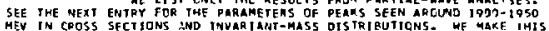

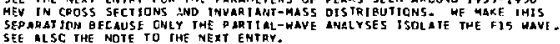

4t Y+111915) MaSS (MFVI

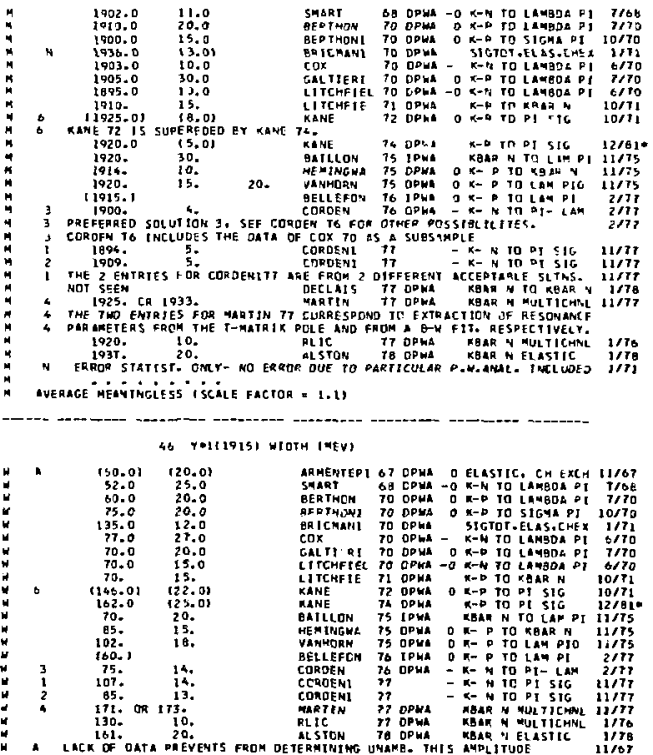

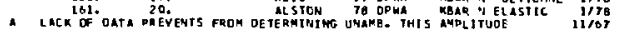
average meanitritjiss i scalle factor : 1.91 
Baryons

$\Sigma(1915), \Sigma(1940)$

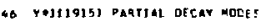

\begin{tabular}{|c|c|c|}
\hline $\begin{array}{l}\text { P1 } \\
02 \\
23 \\
23 \\
05 \\
05\end{array}$ & $\begin{array}{l}r * 1(1215) \\
r+1(1915) \\
r * 1(1915) \\
r * 1(1915) \\
v * 1(1915)\end{array}$ & 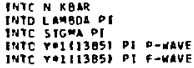 \\
\hline
\end{tabular}

DECEY WASSES

$936 * 493$
$1115 * 139$

$139+1385$

Le $Y$ \#IIIT151 BPAKCHING QATIOS

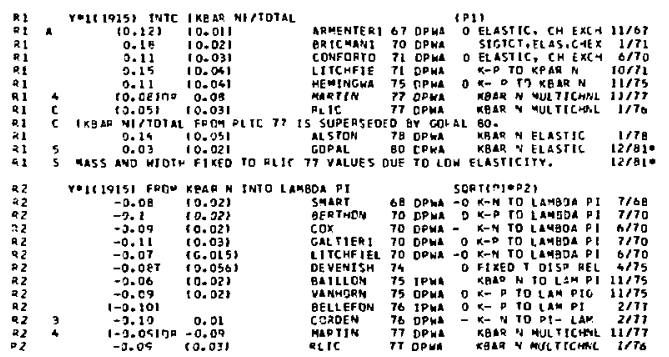

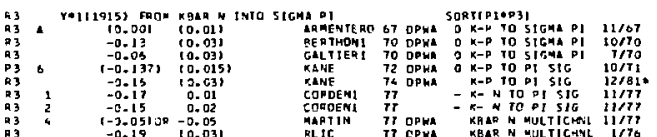

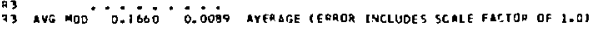

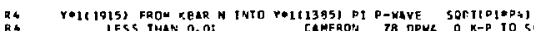

LESS THAN O. OI

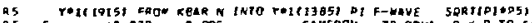

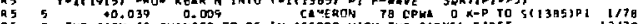

RS 5 THE SICH IS

121790

ARAEAIF Q OT OA 245158

IRMENTE 1 OT NP
SMART

BER INGN TO NP O20 t70

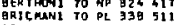

COX TO NP B 19 BI

CAL7IERI TO OUSE CONF 173

CONFOATO II ND B34 41

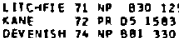

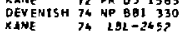

9A1LLON 75 NO 89439

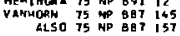

GEL LEFON IS NP DEO9 129

COAOEHI 77 HP BI2S $\$ 1$

OECLAIS IT CERN 77-16

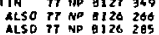

RLIC IT ND 1119362

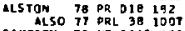

GAMERCN JB NFE143169

\section{REFERENCES FOR Y*1(1015)}

ARMEMTEROS, FERRO-LUZZI * ICERR, HEIO. SACLAY]

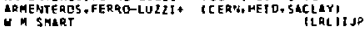

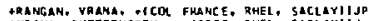
+ VA ANA, GUTTERWORTH, + TCDEF; RHEL, SACLAYIIJ

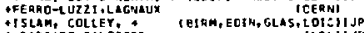

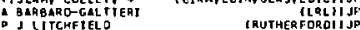

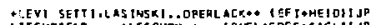

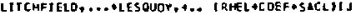

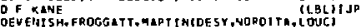

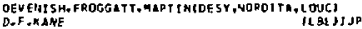
P. BLTLLOH,P. L. LITEHFTELO IGERN, RAELIJ JP

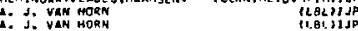

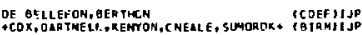

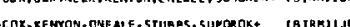
DDUCHOH,LOUVEL, PLTRY,SEGUINDT * ICLENOCERNB IJP MAR TYN,PIOCOCK, HOORMOUSE RUECTGLAIJP MARIIN,PIDGOCK

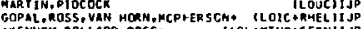

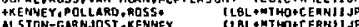

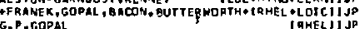
PRDERS NOT REFERRED TO JN DATA CADDS

SMART SE PRL 17556

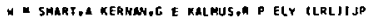
CONFODER GE WD BE 265
SUPERSEDED BY COMFOATO TI.

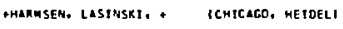

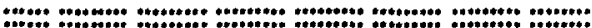

1915 UEV RBGION - PRODUCTION AND $\sigma_{\max }$ EXP'TS

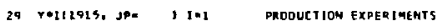
SEE THE MTNI-REVIE AT THE START CF THE V* LISTTMES.

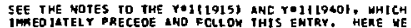
(IST DHEY PAR HMETERS OF DEARS SEEN IH CROSS SECTIONS

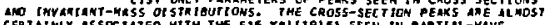

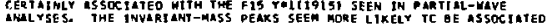
WITH HE D13 Yo 1540 .

59 POLILISI MLSS (MEVI IDROO. EXP.)

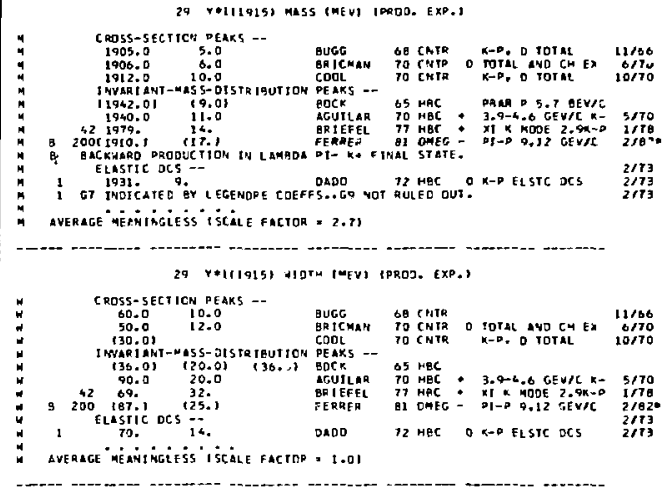

29 riltolsi peptial oecar meOES CPROC. ERr.t

D) roll 19151 InTO N KBaR

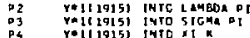

OECAY WASSES

$1215+134$

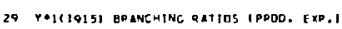

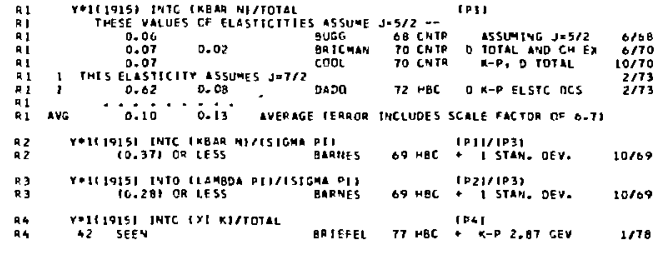

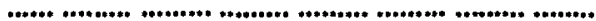

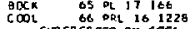

DUGG

BUGG 68 PR 1681460

ACUILAR 70 PRL 255 SE

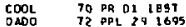

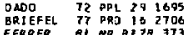

एक

PAT MER 68 PPL 20 GIO REFERENCES FOR Yell(1915) ( PIICO. EXR.)

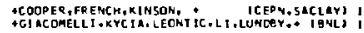

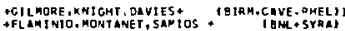

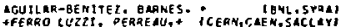

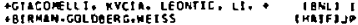

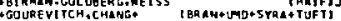

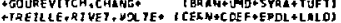
Papeas hot Refenped to In Data capos

-GOI DBE RG, JAEGER,EARHES, DORHAK + ISYRA, BQL I neen $\Sigma(1940)$ so SEE THE NIMT-NEVIEW AT TME STLRT OE THE Y" LISTINAS. SCME. NOT ALL, PARTLAL WAVE AMLLYSES SUGGEST A STLTE IM THIS REGION. IT 15 PERMAPS ASSOC IATED HTH TSE DUMPS SEEN IN DROOUC ITON EXPERTMENTS NEAR 7 HIS MASS ISEE THE PAEEEDING ENTRYI, THIS STATE IS NOT AEDITED TN K- MEUTRON ID TD! 51 GMA!- RN

5Q rHIC1940I MASS IMEVI

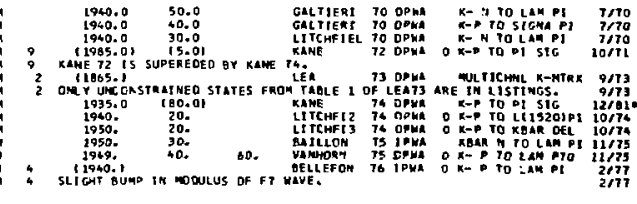


Data Card Listings

For notation, see key at front of Listings.
Baryons $\Sigma(1940), \Sigma(2000)$
5.1800 .001893$.

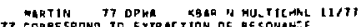

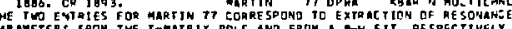

1020.
10.

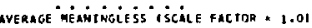

SB Yelt1940) WIOTH TMEVI

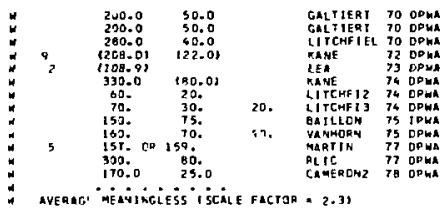

58 YoIL1940) PaRTIAL DECAY MODES

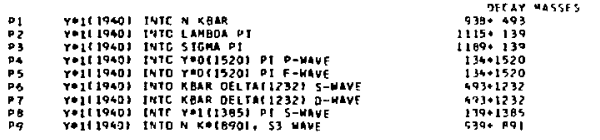

98 PO111940) RRANCHING RETIOS

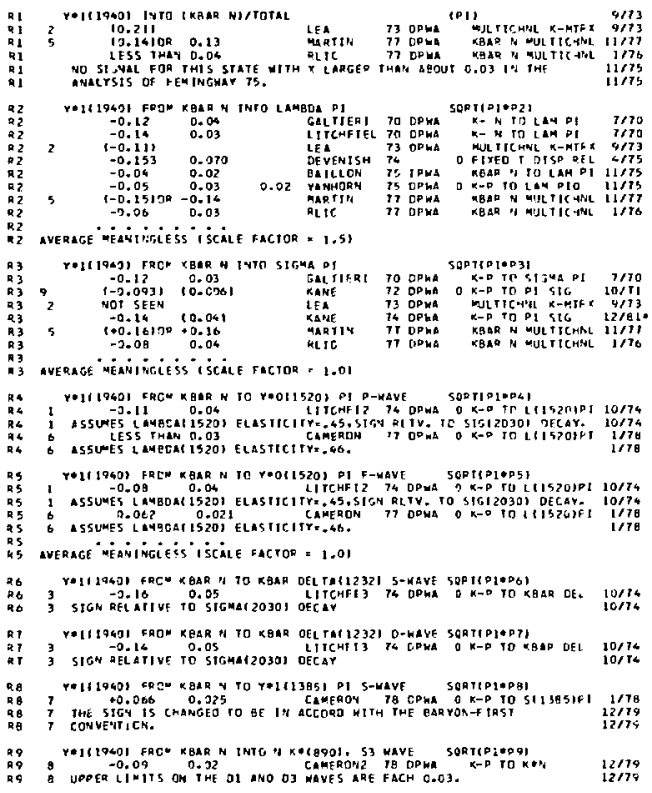

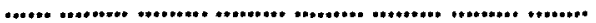
REFERENCES rTE VMIC1940)

GALTIER: TO DURE CONF 173

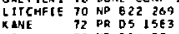

LEA 73 HP BSO 77

DEVENTSH 74 YD PA3 330

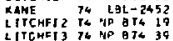

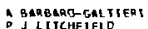

TLOLT) Jo O FANF DEVEHI SH, FDOGGATT, MAPT IMIDESY, YOADI TA.L LOTCI DGF EKME

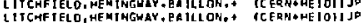

9A1LLCH 75 4P 89439

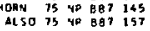
AELLEFON 76 MP 8109129

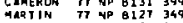
ALSD TT No 8120260

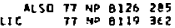

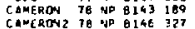

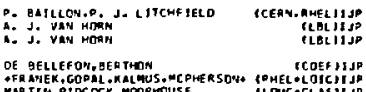

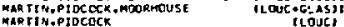

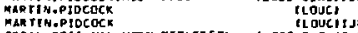

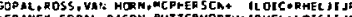

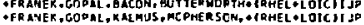
papeas rot Rerekaed to in OATA caros

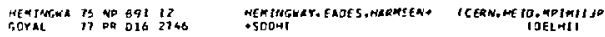

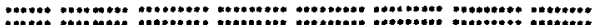
$\Sigma(2000)$ C2 THE LIST HEAE ALL REPDDTEO SII STATES LYING ABOVE

C2 Ya11200O HASS IMEVI

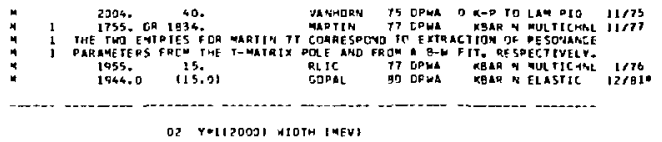

\begin{tabular}{|c|c|c|c|c|}
\hline & $\begin{array}{l}216 . \\
413: 00 . \\
170.040 . \\
215.0 \\
125.01\end{array}$ & 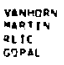 & 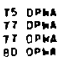 & 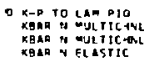 \\
\hline
\end{tabular}

l2 retizoog) patisal dicay modes

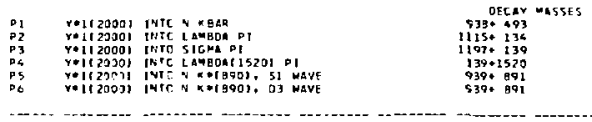

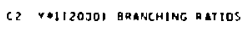

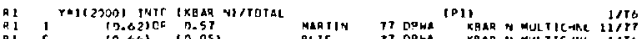

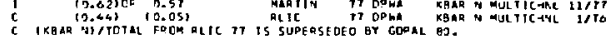

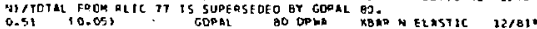

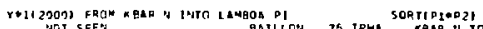

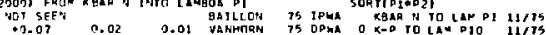

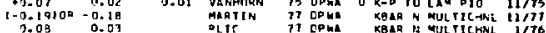

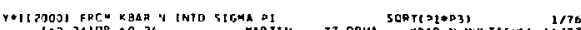

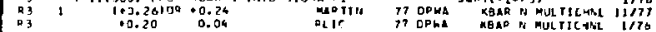

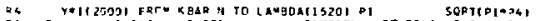

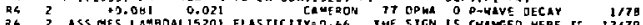

26
26
$R 4$

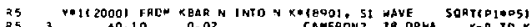

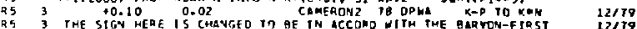

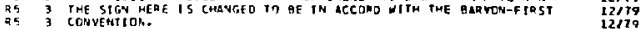

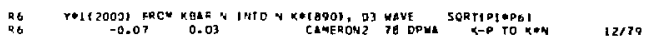

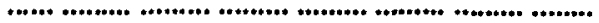

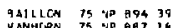

REF EDENCES RDP $r=11 z 0001$

VANHCON TS TP QEY 745

P. BLILLON.P. J. LITCHFIELO

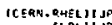

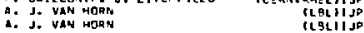

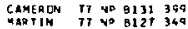

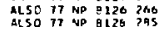

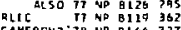

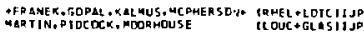

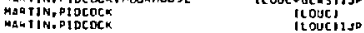

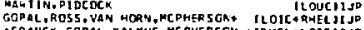

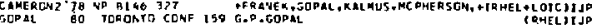

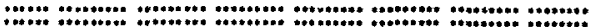




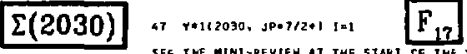

F THE $\mathrm{re}$ LiSTIVIS.

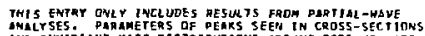

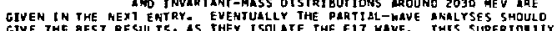

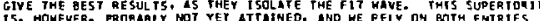

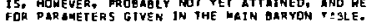

\section{Y 1120301 MASS (MEV)}

\begin{tabular}{|c|c|c|c|c|c|c|}
\hline & \multirow{3}{*}{ 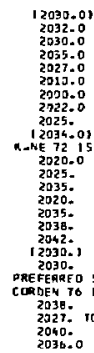 } & \multirow{3}{*}{ 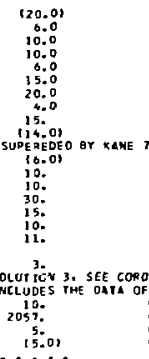 } & \multirow{3}{*}{ 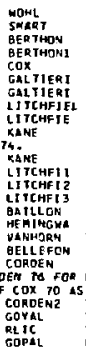 } & \multirow{3}{*}{ 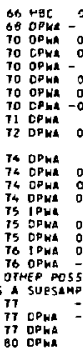 } & \multirow{3}{*}{ 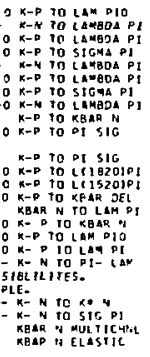 } & 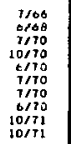 \\
\hline & & & & & & \\
\hline & & & & & & \\
\hline
\end{tabular}

averace déaving íf Śs íscile factor $=1.0$ )

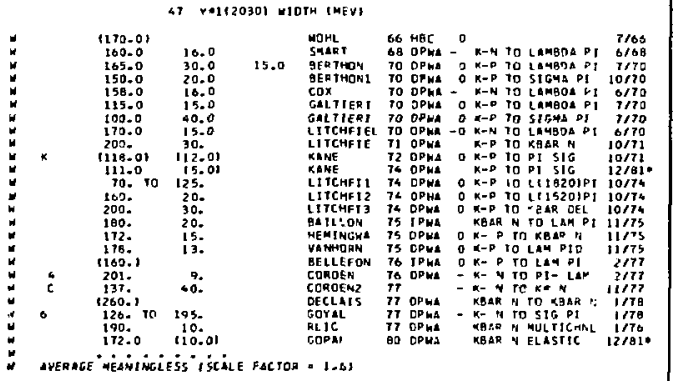

4T YOIIZO3OI PARTILL DECAY YOOES

\begin{tabular}{|c|c|c|}
\hline 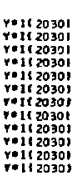 & 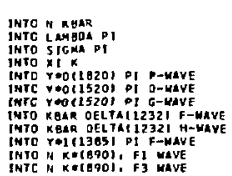 & 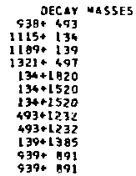 \\
\hline
\end{tabular}

4) TOLI20301 ARAHCHENG RATIOS

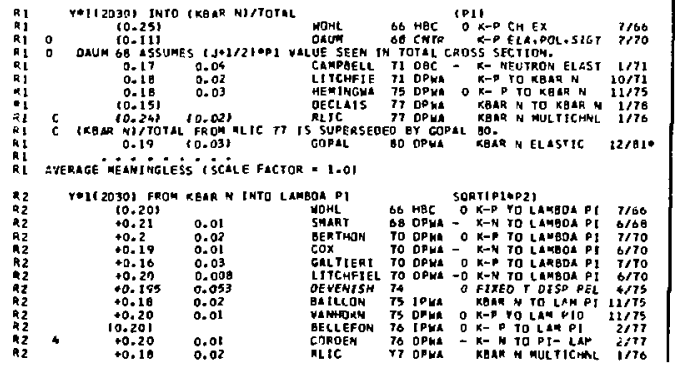

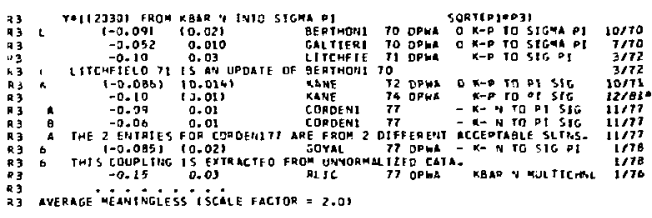

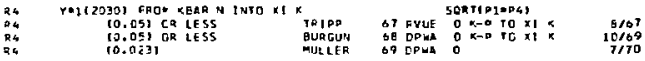

R5
05

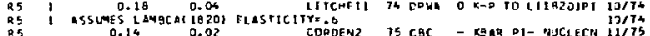

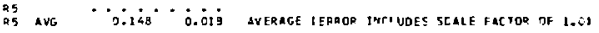

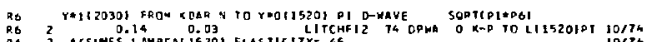

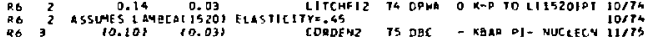

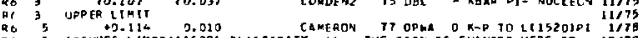

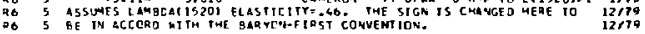

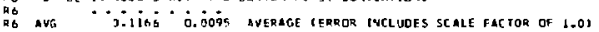

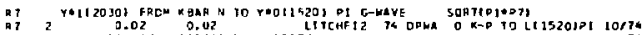

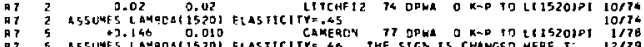

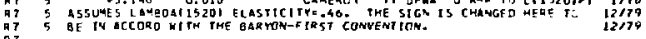

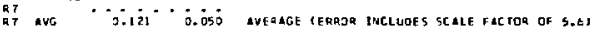

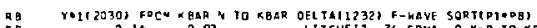

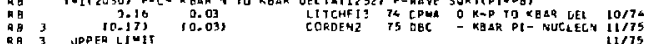

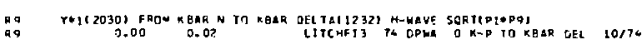

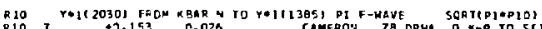

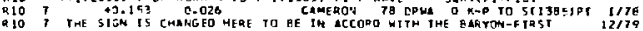

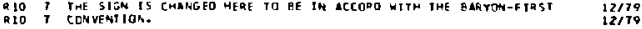

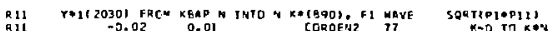

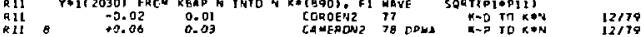
RII 8 THE SIGN HEAE IS CHAVGO TE BE IN ACCORD WTTH THE BAATON-ETRST 12270

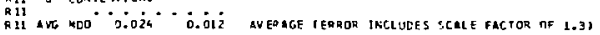

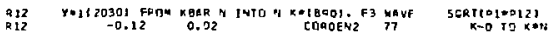

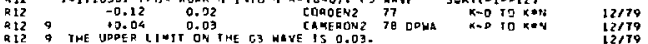

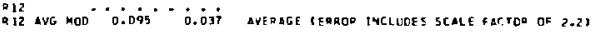

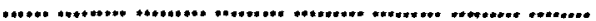

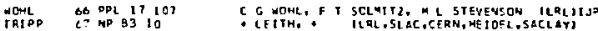

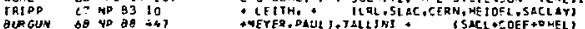

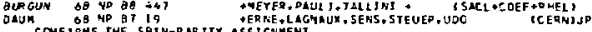

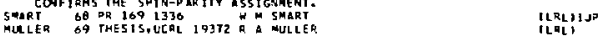

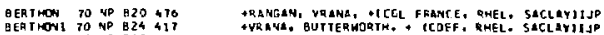

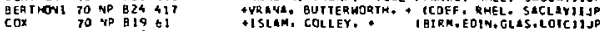

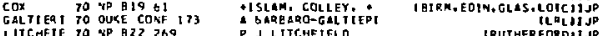

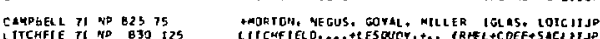
LITCHFIE
KII OEVEATSH TS MP Ba1 330

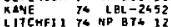

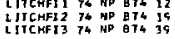
GAILLON 75 NP $\quad$ B 9436

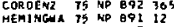
HEAINGW

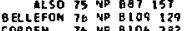
CDMERON 11 KP B B131 399

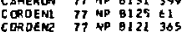
DECLAIS 77 TERN $77-16$

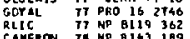

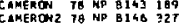
CAMEROH2 78 NP B14S 327
GOPAL
GO TORCNTO CONF

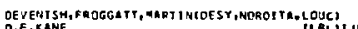

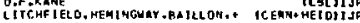

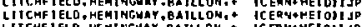

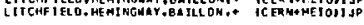
P. ARILLON, P. J. LITCHF IELO TEEGN, AMELIIJP

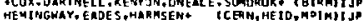
A. J. MAN HERN A. J. VAN MORN cistitijo

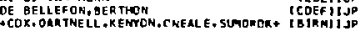

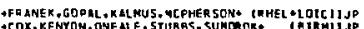

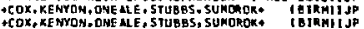

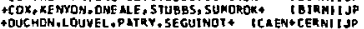

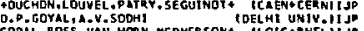
GOPAL, ROSS. VAN HORN .NCPHERSON+ HOTC,*AELLYISP

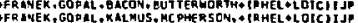

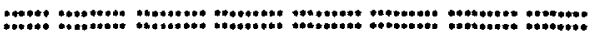




\section{Data Card Listings}

For notation, see key at front of Listings.

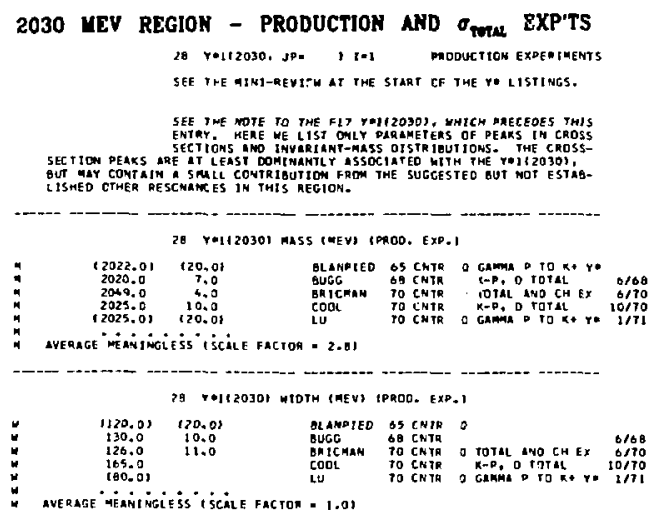

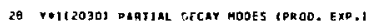

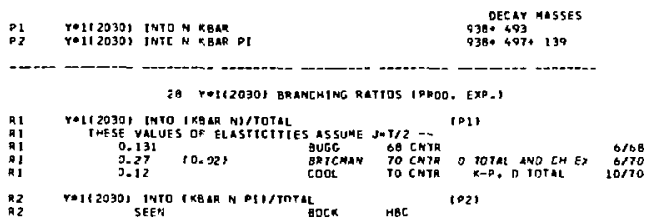

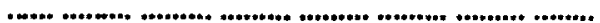

$\begin{array}{lllll}\text { BLANIEO OS PRL } & 14 & 761 \\ \text { COTL } & 66 & \text { PAL } & 10 & 1228\end{array}$

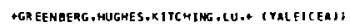

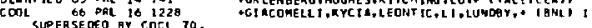

GUSG SUPERSEDEO QV COTL 70

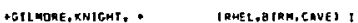

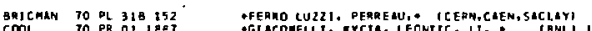

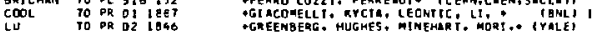

-

$\sum(2070)$ 34 mit2070. Jps5/201 i=2 $F_{15}^{\prime \prime}$

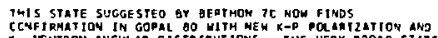

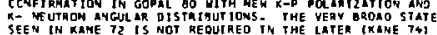

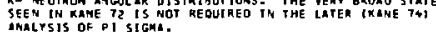

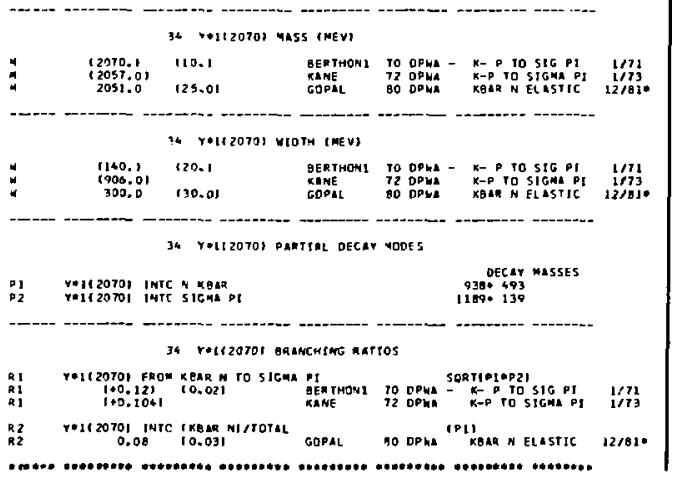

REFERENCES FOR YHisZTOH

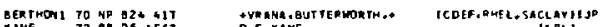

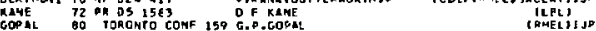
Dos

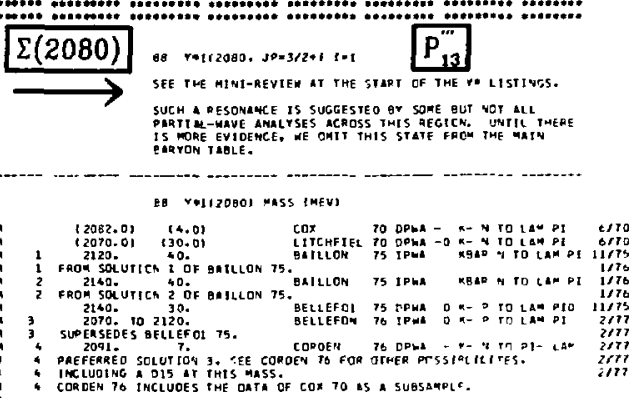

aven

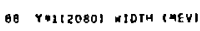

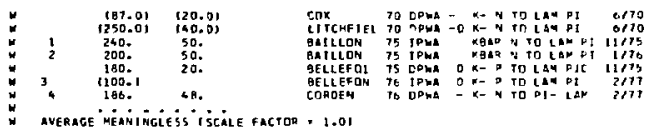

88 r+1LZOQDI DARTIAL DECar MODF 5

plo

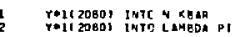
TifCar uasis $\begin{array}{rl}932+ & 493 \\ 1115.130 & 130\end{array}$

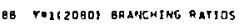

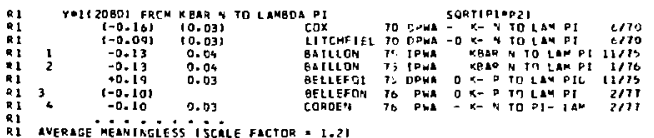

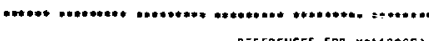

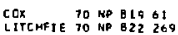

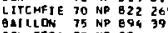

BELLEFOL IS NP $690 \mathrm{I}$

$\begin{array}{llll}\text { BELLEFON } 76 & \text { NP } & 8109 & 129 \\ \text { COROEH } & \text { T6 ND } & \text { BLOS } & 392\end{array}$

RËFERENCES FOR Yat 2000 (

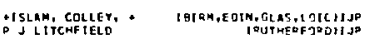
D J LTCHF TELD O I TUT HEOF JPDHJ

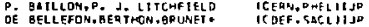

OE BELLEON BEPTHOS ICJFIITP

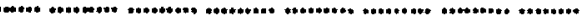

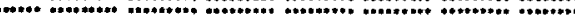

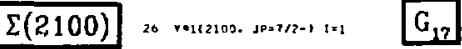

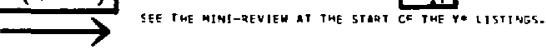

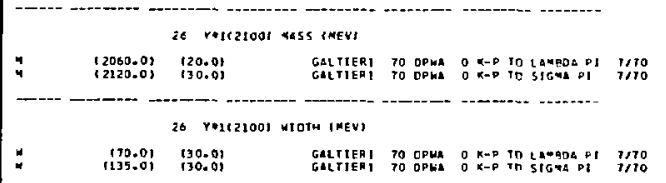

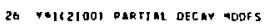

Yaisiogl INTE M KEAR

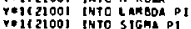
DeCAY Hasses $933+497$
$1115+134$ 
Baryons

$\Sigma(2100), \Sigma(2250), \Sigma(2455), \Sigma(2620)$

20 YHIIZ200I GRANEHING RATIOS

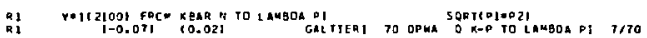

R2 MHIZIODS FROM XGAQ N In SIGMA PI SORTIDINDJI

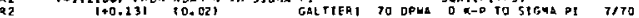

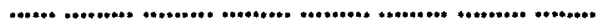

AFFERENCES FOR $r+1(2100)$

GRLTIERI TO DUKE CONF 173 A BARGAPO-GALTIERI RLLIJP

+

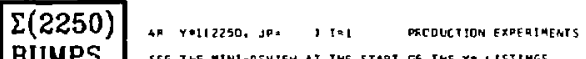

BUMPS

SEF THE MJNI-QEVIEW AT THE START GF THE $Y$ * CISTIMGS.

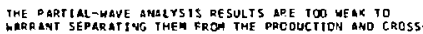
SERTID ERPERIMEQTS.

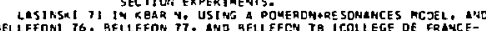

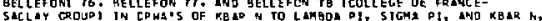

RESOECT IVELY, SUGCEST THE ORESENCE OF TWO RE SÖKNCES APRUNO THES
HLSS VILUE.

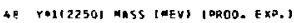

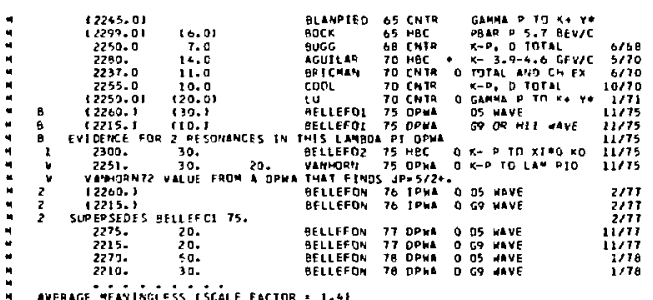

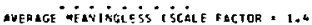

49 REIIZ2591 WIDTH (NEV) (PQOD. EXP.

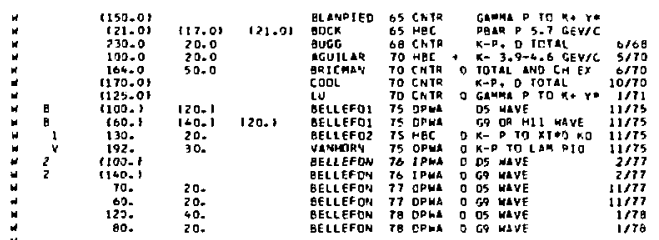

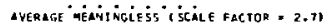

SEE TME NOTES ACTOMPANYING THE -ASSES CIRTED

4 G YWIIZ250I DHATILI DELAY MODFS IPRDO. EXO.)

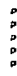

relsz2501 INIC N mene

Pal z250) TNTC L LmEOR PI

Yol 2350 ) INTC SIGMA $P$ !

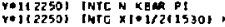

oecar masses

$938+493$
$1125+134$

$935+4974139$
$1533+497$

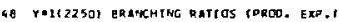

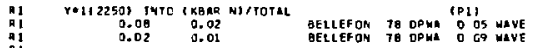

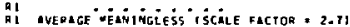

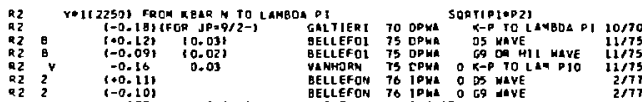

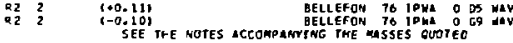

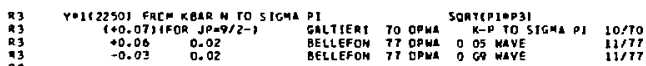
R3 averace héA theiess iscile factor $=1.11$

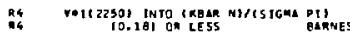

is

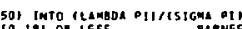

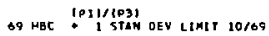

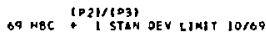

Data Card Listings

For.notation, see key at front of Listings.

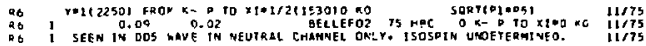

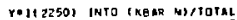

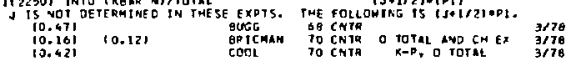

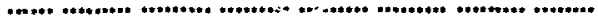

SLANPIED 65 PRL 14741

AOCK 65 PR 17160

QUCG
gARNES
O9 DP

ACUILR 70 ORL 25 50

BRICABN 70 OL 319152

COOL
GALTIERT TO DUSE CONF 173
LU TO PA DE 1940

BELLEFOI 75 WD $890 \mathrm{t}$

BELLEFOZ 75 NE 2 EA 209

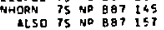

BELLEFON 76 ND EICQ 179

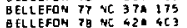

COOL OB DRL 101228
SUTEREOEO BY COLC TO.

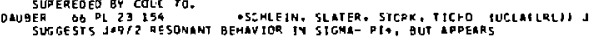

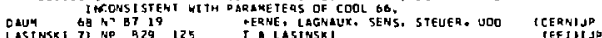
LASTNSXI 71 NP R29 125 TE LASTNSKI

" $\Sigma(2455)$

BUMPS

REFERENCES FOR YEI $12 Z 50)$ (PRCD - EXD.1

+COOPEQ, FRENCH, KINSDH, + "TERA, SACLAYT

TFILMORE, RNIGHT,

AGUILAR-BENITEZ, GAFNES, * CENL, STRAI

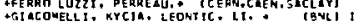

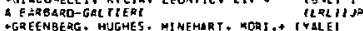

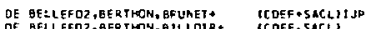

(COEF. SACL)

a. J. YRN HORM

OE BELLEFON. BEDTMON TCDEFIJT

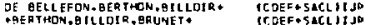

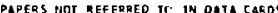

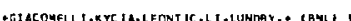

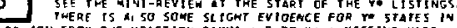

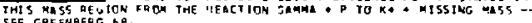

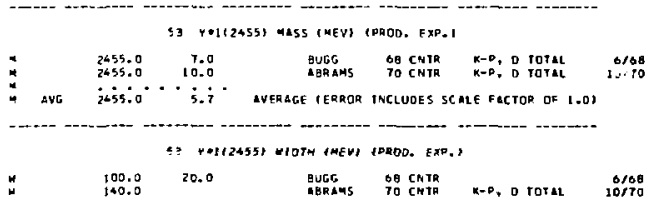

53 YOLI2455I PARTIAL OECAY MDOFS CDRED. EXP.1

Y०162455I INTC $A \times B 48$

OECAY TASSES

53 Y*II2455) ORANEMING RATIOS IPAOD. EXO.)

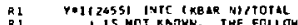

IS MOT KAOW. THE FOLLGMING IS $(1+1 / 2)$ OPI. IPJI

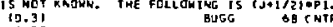

10.31
0.39
10.09

ABRAMS TO CNTR K-D, D TOTAL

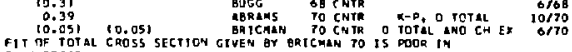

R1 C FIT PF TOTAL CROSS SECTIOH GIVEN OY SRICMAN TO IS POOR IN

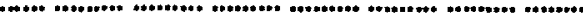

REFERENCES FOA Y\$1124551 IPRCD. EYO-1

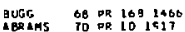

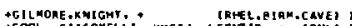

ABRAS TO PR. 10151

tCOOL, G IACOAELLI, KYCIA, LECKI IC. - IBNL)

PAPERS NOT REREREE YO IN OATA CAROS

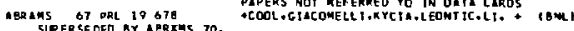

SUP ERSE OED BY A BR KHS 70 .

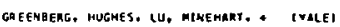

+coten

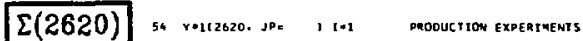

BUNPS See the mint-aeviem at the start dF the ye listings.

\section{Yollzozol MLSS imEVI tPMOD. Exp.}

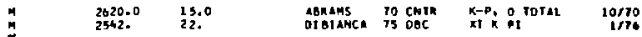

- averagf méningiess iscille facton = 2.91 


\section{Data Card Listings}

For notation, see key at front of Listings.

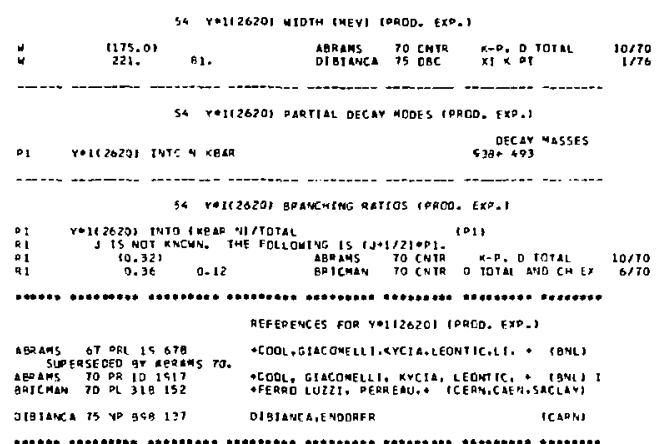

H.e.

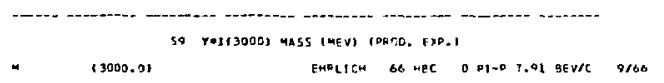

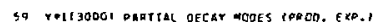

\begin{tabular}{|c|c|c|}
\hline $\begin{array}{l}\because 1 \\
02\end{array}$ & 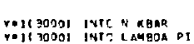 & $\begin{array}{r}\text { Dercay } \\
938 * 493 \\
1115+113\end{array}$ \\
\hline
\end{tabular}

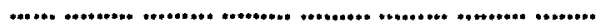

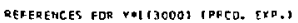

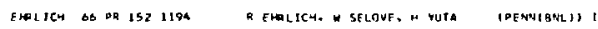
+...
(3170) ra rasion sor

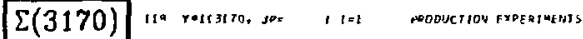

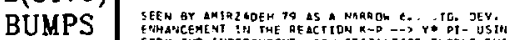

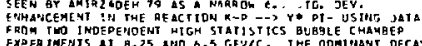 EXPEATMENTS 21 B.25 AHO 6.5 GEV/C. THE ODNIMANT DECAY

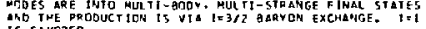 is FA VORED.
IV NEED DF CONETRMATION. OHITFO FFom Tables.

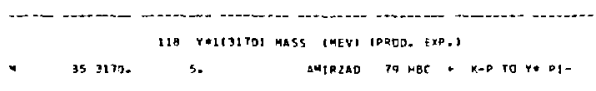

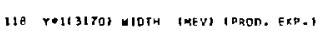

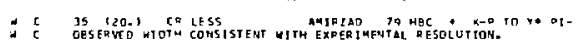
$12 / 79$ - - - - - - - - -

119 Vall31 TOR DARTIAL DECAY MODES IPROO. EXT.I

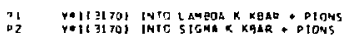

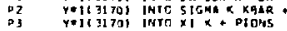

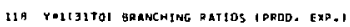

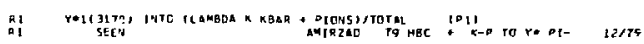

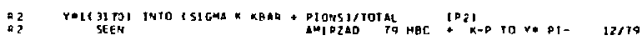

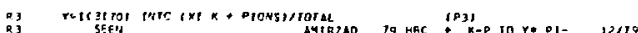

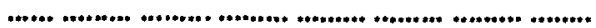

REFERE YCES FOR T*LIJITOS IPRCO. EXP.)

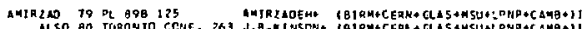

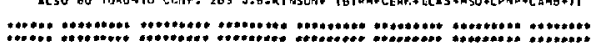

\section{EXOTIC HYPERON CROSS SECTION LILITS}

$$
\begin{aligned}
& \text { THIS IS NOT COCOMPLE TE LIST. WE TABULATE } \\
& \text { CNLT TOTO ON. }
\end{aligned}
$$

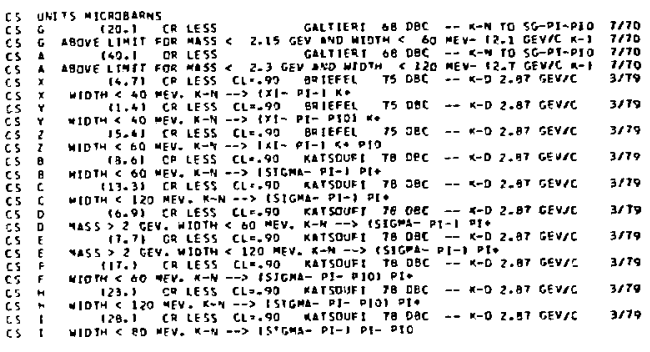

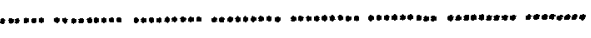

REFEAENCFS FOP. EXETIC MYDERARS

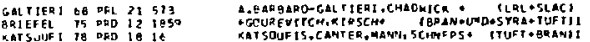

.

Note on $\Xi^{\star}$ Resonances

The $\Xi^{*}$ resonance situation has always been unsettled. Th1s is because: (1) $E^{*}$ 's can only be produced as a part of a final state, and so the analysis is more complicated than if direct formation were possible; (2) they are produced with small cross sections (typlcally a few $\mu \mathrm{b}$ ); and (3) the final states are topologically complicated and difficult to study with electronic techniquea. Thus in we past our knowledge of $\Xi^{\star}$ resonances has come wholly from bubble chamber experiments, where the numbers of events are small.

Until falrly recently only the $=(1530)$ could be considered to be really well established. However, the 1978 edition of this Review ${ }^{1}$ saw a major improvenent in the situation with the regults of GAY 76 and HEMINGWAY 77 . The $\Xi(1820)$ and $\Xi(2030)$ were firmly established as narrow states (widths of about $20 \mathrm{MeV})$, and the spin of the $\equiv(1820)$ was found to be $3 / 2$ (TEODORO 78).

since then, however, little has changed, although there 1s some evidence for a new $\Xi(2370)$ (AMLRZADEH 80, HASSALL 81). There is probably at least one other $\Xi^{*}$ in the $1850-2000 \mathrm{MeV}$ region, and there are Indicationg of several others above 2000 MeV. Indeed, there should be many $\Xi^{*}$ 's below 2500 MeV, and the broad (and not completely eatablished) $\Xi(1940)$ could well be a mixture of several of them. ${ }^{2}$ For now we are forced to group together 
Baryons

$\Xi^{\prime} s, \Xi^{-}, \Xi^{0}, \Xi(1530)$

disparate observations and awalc new reaults. The disagreements among experiments are show In Ideograns in the Listinge.

Results frow experiments using electronic techniques are now beconing avallable. BIAGI 81 used the CERN hyperon bean to study Inclusive $\Lambda \bar{K}$ and $\Xi \pi$ nass spectra from 102 and $135 \mathrm{GeV} / \mathrm{c} \Xi^{-}$ incident on hydrogen and deuterium. They saw a large $\Xi(1820)$ signal in $\bar{\Lambda}$ as well as a peak at about $1700 \mathrm{MeV}$, wich wight be associated with the threshold eahancement seen by DIONISI 78. The $\Xi(1940)$ appears as a broad bump in the $\Xi \pi$ mass spectrum, and there is a very clean $\Xi(1530)$ Bignal. Preliminary results from the Brookhaven multiparticle spectrometer were reported at the Toronto Conference (see Ref. 3). The $\Xi(1820)$ 1s clearly seen as a narrow object decay 1 g to $\Xi(1530) \pi$.

The table below gives our evaluation of the present status of the $\Xi^{*}$ resonances. For a detalled review, see MEADOws $80 .^{3}$

\section{References}

1. Particle Data Group, Phys. Lett. 15B, 1 (1978).

2. R.J. Hemingway, in Proceeding of the Iopical Conference on Baryon Regonances (0xford, 1976), ed. R.T. Ross and D.H. Saxon.

3. B.T. Meadows, In Proceedings of the IV th International Conference on Baryon Resonances (Toronto, 1980), ed. N. Iggur.

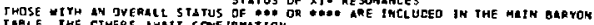
THOLE. THE CTMEPS AHIT CONF IAMATION.

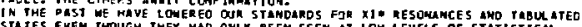
STAYES EVEN THOUGH THEY HAD ONLY BEEN SFEN AT LOH LEVESS OF STATISTICAL ADOTED SOMEHAT STRICTEA CRITERTL

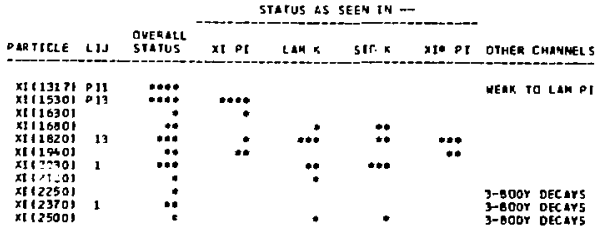

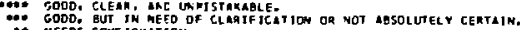

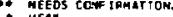

Data Card Listings

For notation, see key at front of Listings.

- comen

$$
S=-2 \quad I=1 / 2 \text { HYPERON STATES }(\Xi)
$$

tos

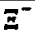

$22 \times 1-(1321, j P=1 / 2,1=1 / 2$

SEE STHBLE PAATICLE DATA CARD LISTINGS

(1)

$\pm 0$

$23 \times 1011315, J P=1 / 2, I=1 / 2$

SEE STABLE PARTICLE DATA CARO LLSTIHGS

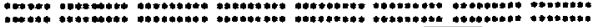
$\mathrm{E}(1530)$ a9 $\times 1+1 / 211530, \mathrm{JP-3/2+1} 1=1 / 2 \quad \mathrm{P}_{13}$

THIS IS THE ONEY WELL-ESTARL ISHED XI* WHOSE PMDPERTIES IRE RLL LT LEAST PEASOHLBLY WELL-KYOWN. SPIA-BRTTY IZZ, IS FAVOUREO BY THE DATA.

WE OC NDT USE DE TERMINETIOAS OF THE MASS AMO TME VIOTH OF THIS

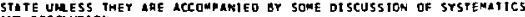
ANO RESGUTION.

49 $x$ J*1/2t1530I MASS IHEV

- MIKEO CHARGES

BERTAHZA OZ MAC -O N-O Z.3 OEVIC

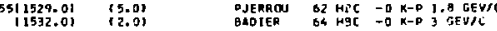

m- Negative CHARCE GRSP

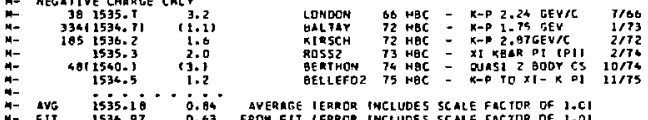

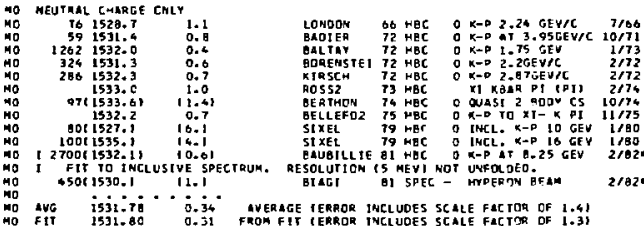

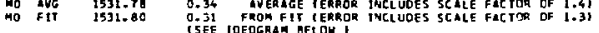

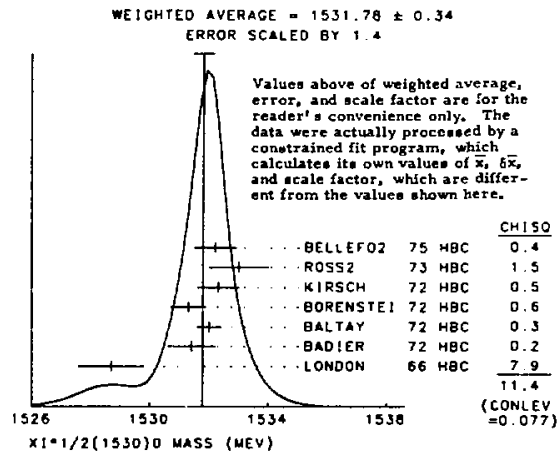


Data Card Listings

For notation, see key at front of Listings.
Baryons

$\Xi(1530), \Xi(1630), \Xi(1680)$

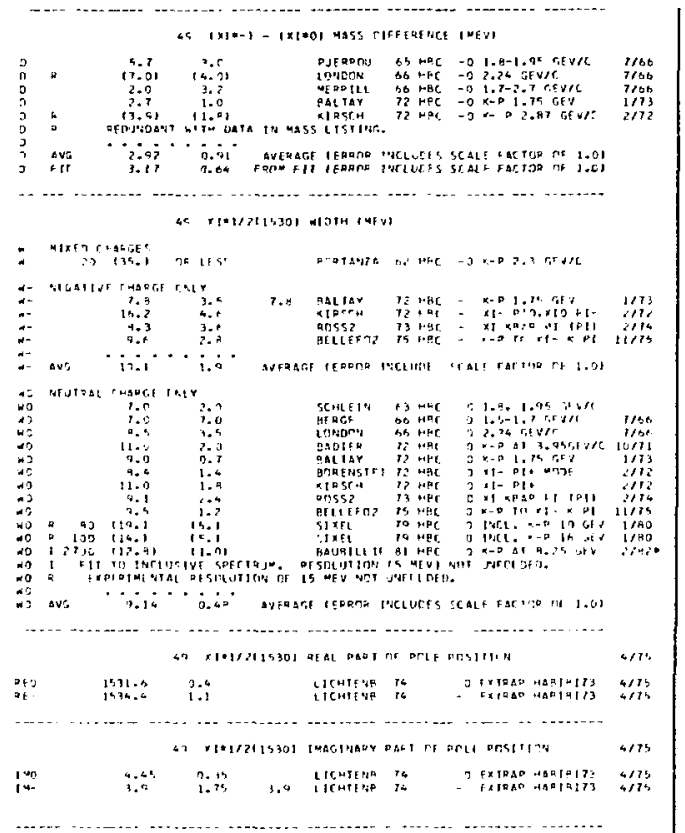

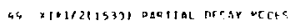

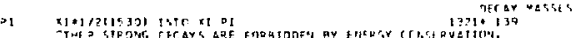

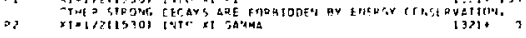

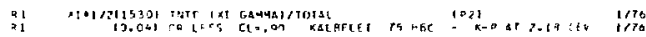

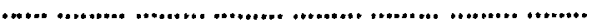
REFEDEACS FOD $\times 1+1 / 201530)$

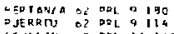

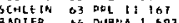

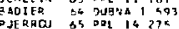

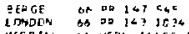

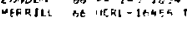

A AOCER VI IP A 374 ?

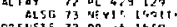

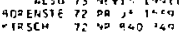

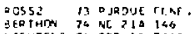

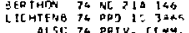

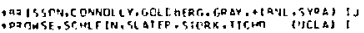

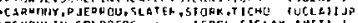

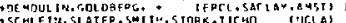

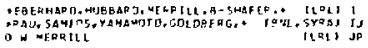

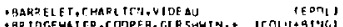

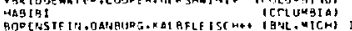

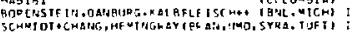

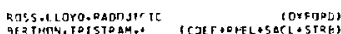

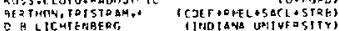

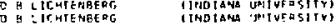

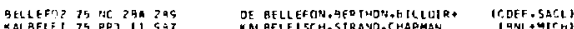

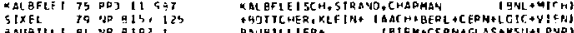

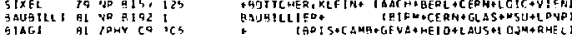
oaperas not feferoio il in data cadjs

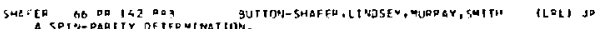

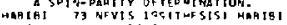

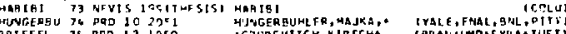

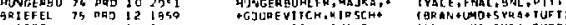

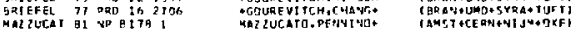

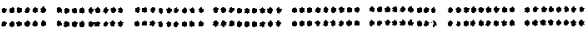

\section{$\Xi(1630)$} $21 \times 1+1 / 211030, \pi p=\quad 101 / 2$

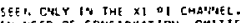

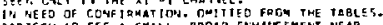

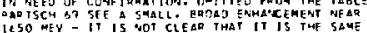

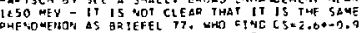

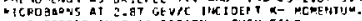

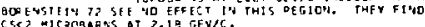

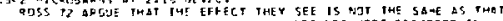

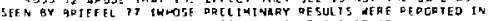

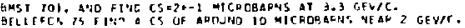

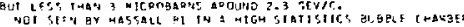

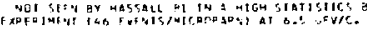

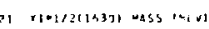

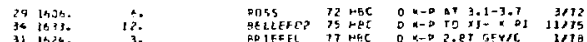

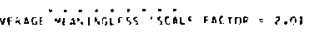

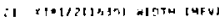

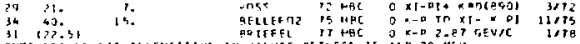

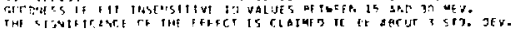

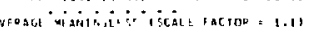

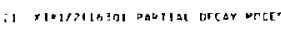

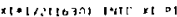

OEC Br UASSE

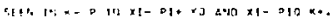

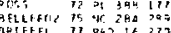

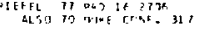

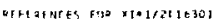

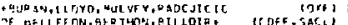

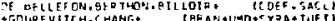

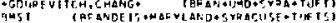

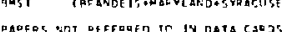

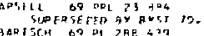

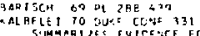

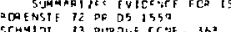

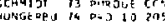

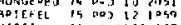

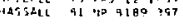

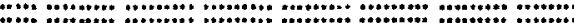

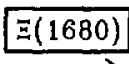

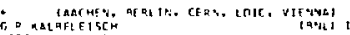

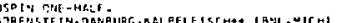
rCHAIJT

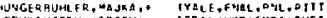
GOUHEVITEH,KIASCHE TBRANAIND+SYZAQ TUFI

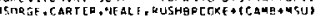

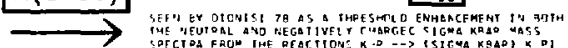

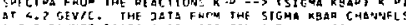
AT C. GEVIC. THE ZATA FWTM THE SIGHA KBAD CME

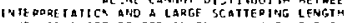

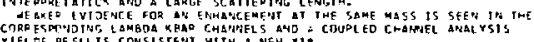

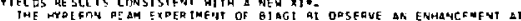

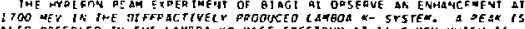

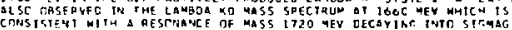

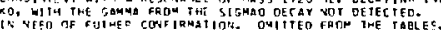

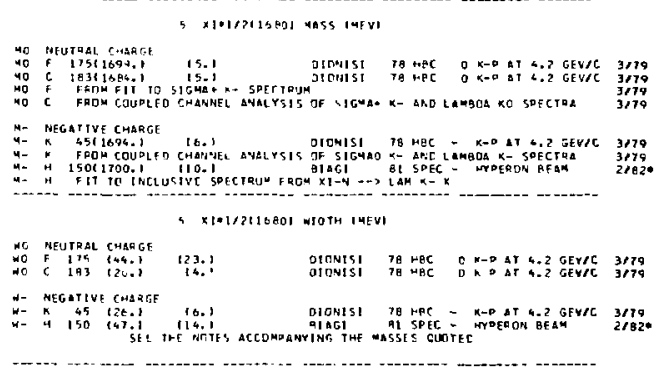


Baryons

$\Xi(1680), \Xi(1820)$

Data Card Listing:

For notation, see key at front of Listings.

5 XI+1/21168Q1 PAPTIAL DEGQY MOEES

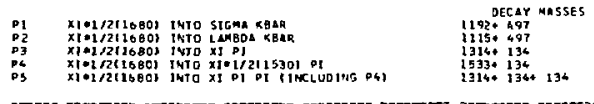

5 XI*I/21]

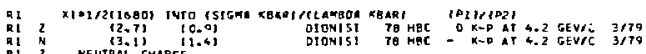

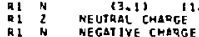

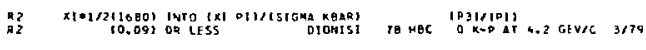

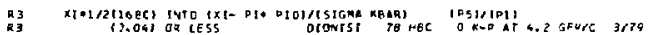

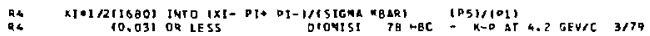

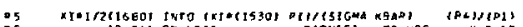

25 W 10.06$)$ OP LFS5

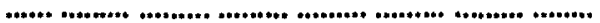

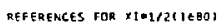

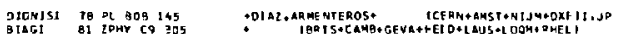

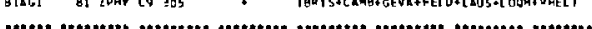

"*ace

$\Xi(1820)$

So $x 1 * 1 / 263820,7 p=3 / 5,1 * 1 / 2$ ME LIST HFRE EVERYTHING REPORTCD T"A THE MASS RANGE

The clearest evidence for this state comes from GAY 76, who saw an B standard-deviation peak In $A K^{-}$as well as signals in $\equiv(1530) \pi$ and $\sum \overrightarrow{\mathrm{K}}$. The peak $1 \mathrm{~s}$ narrow $(\Gamma=2 ! \pm 7 \mathrm{MeV})$, whereas earlier (and wuch smaller) experiments found widths of up to $100 \mathrm{MeV}$ (see the Listings below).

A spin-parity analysis of the GAY 76 data, but with tore events (TBODORO 78), favors sp In $3 / 2$ but cannot make a parity discrimination.

BIAGI BI used the CERN hyperon beam to study $\Xi$ Interactions in hydrogen and deuterium. The diffractively produced $\mathrm{AK}^{-}$syatem has a broad peak $(\Gamma=72 \pm 20 \mathrm{HeV}$ ) at $1830 \mathrm{MeV}$ on top of a substanthal background. There to algo a smaller peak in the Inclusive $A K_{S}^{0}$ ipectrum.

Nelther GAY 76 nor BIAGI 81 saw a peak in the $\Xi \pi$ channel. It is possible that $\Xi \pi$ peaks seen in this region by some lower-momentum experiments are at laast partly due to the $\Xi(1940)$, wth a shape distorted by the 1 inted phase space avallable (SMITH 65). The situation 15 further confuged because some experinents were forced to add several different channels together to overcome poor stat Ist1cs (CRENNELL 70, BADIER 71).

\section{C XTH2/2418201 RASS (MEV!}

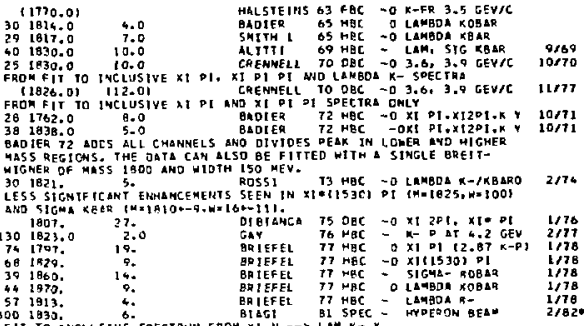
FIT TO INCLLSTVE SPECTRUA FOOM $\times 1-4 \rightarrow$ BL SPEC - HYDEON BEA

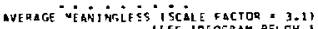

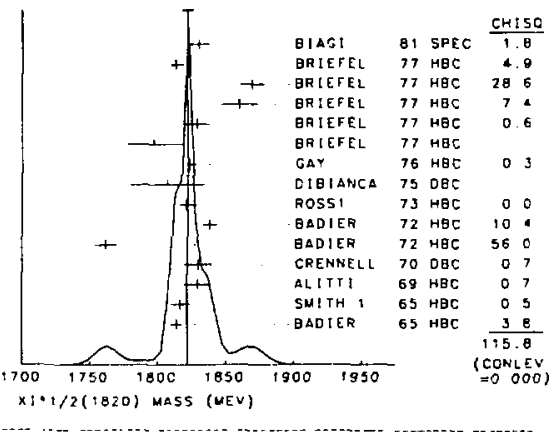

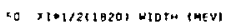

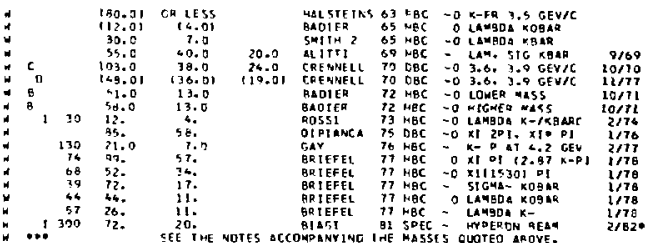

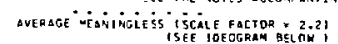

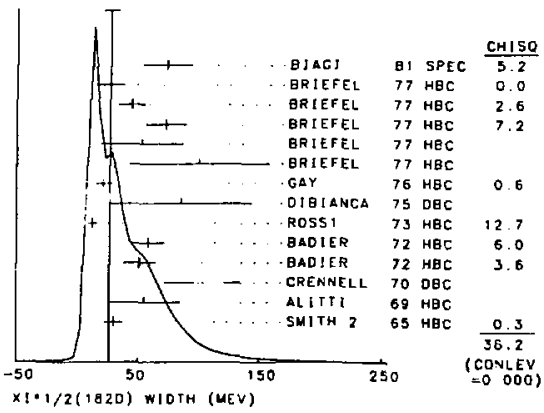


SE XI*1/2CLEZO PAQTIAL DECAY MODE,

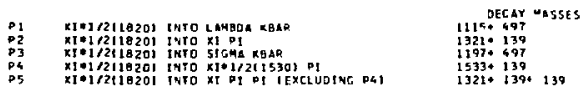

\section{FITTED PARTIAL DECAY MODE BRANCHWE FRUCTIONS}

The matrix below is derived from the er ror matrix for the litted partial decay made branthitg fractions. $P_{i}$, al tollow: The diegonal elements are $P_{i}+5 P_{i}$, where

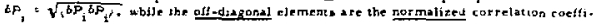

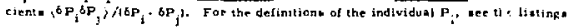
ahove: only those $P_{f}$ appezing in the matrix ate an kumed in the fit to be nonzere and are thus conetrathed 10 add to 9.

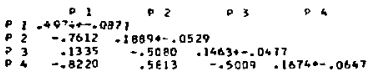

\section{SO K101/212020I BRHCHING RATIOS}

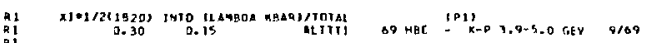

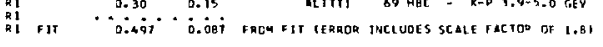

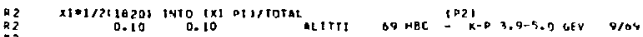

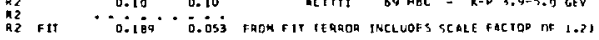

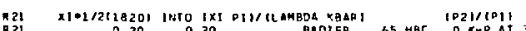

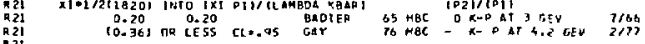

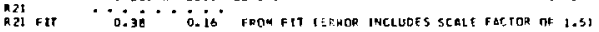

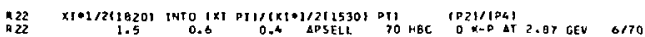

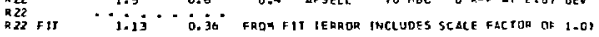

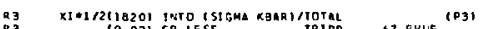

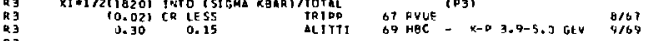

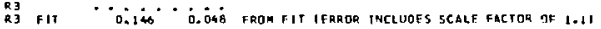

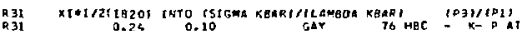

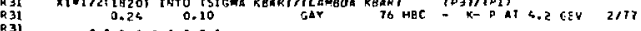

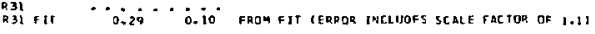

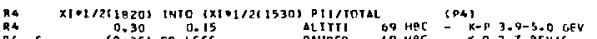

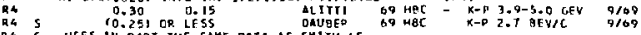

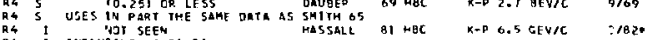
R4 I INCLUDTNE XI PI PI

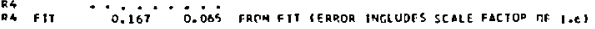

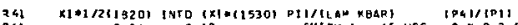

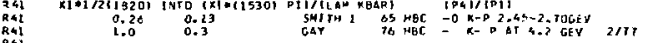

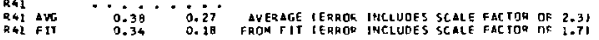

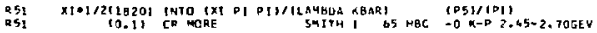

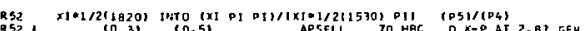

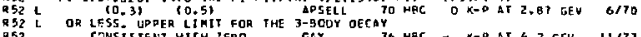

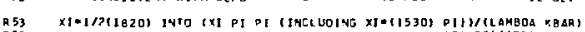

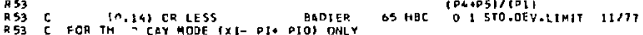

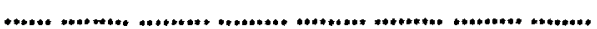

HALSTETM OS SIENE COAF 173

QRDJER BS PL 16172

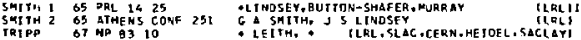
USES OATA OF SHITH L.

ALTTIS 69 PRL 2275

OAUEER O9 OR 1791262

CRENNELL TO OR 10 OQT

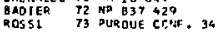

OIBISACX IS HP 8982 ?

GRYEFEL TO DOD 14047708

BRIEFE TO TO DUFE CINF. 31

BJAGt al 7 PHY CQ 305
HASEALL HI

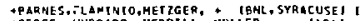
+ PERGE, HUS3ARO, MERPILE; HDLLER ILRLI * TQRARDETS. MARYL ANOD, SYRACUSE. TUFTSI

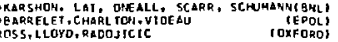

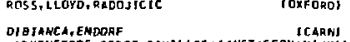

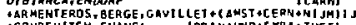

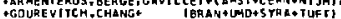
BMST TERAMOET SEMARYLAND +SW ACUSE+TURTS

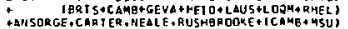

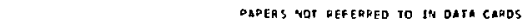

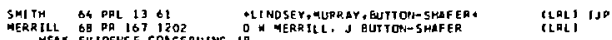
APSELL GQ DFL 23 BQ BQ SUP ERSE CED BY EP IEFEL 37.

Ceratiogls

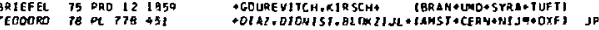

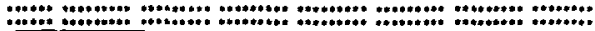

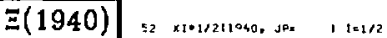

ME LIST UHOER XTM1960I EVEQTINING REPCITTO ITA TME YGSS

$\$ 2$ X1*1/21]7401 MASS IHEVI

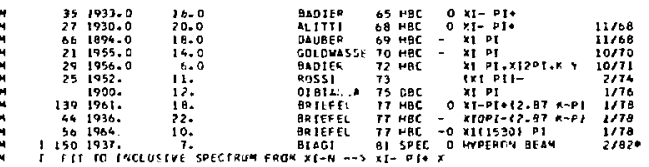

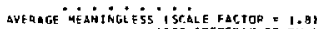

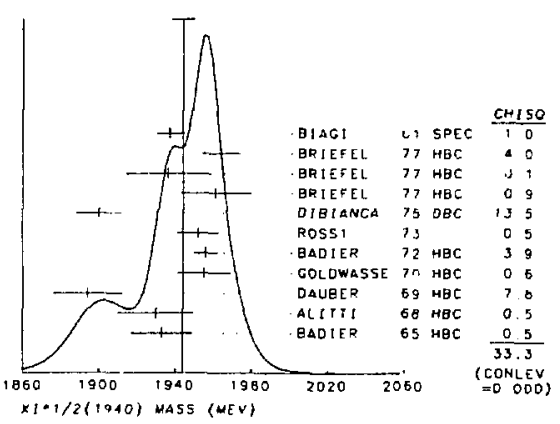

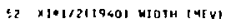
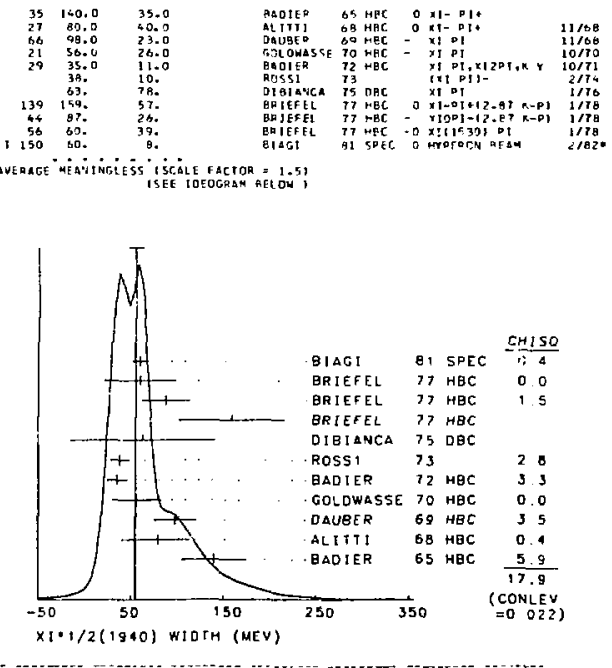
Baryons

$\Xi(1940), \Xi(2030), \Xi(2120)$

$92 \times 1 * 1 / 2678401$ PARTIAL OECAY MOEES

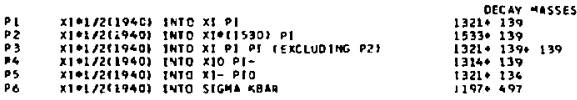

Po $\times 101 / 261940)$ into $x 1-$ PIO

$52 \times[01 / 211940]$ BRANCHING RATIOS

THE XI(1940) IS SEEN MAINEY IN XI PI ANO SOME TN XI11530) 2 . IT HAS BEE LOOKED FCR IN DIHER CHAHHELS BUT ONLY OBSERUEO QY HLSSALL OI

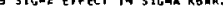

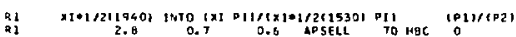

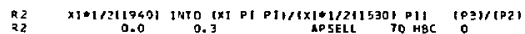

R3 XTA1/21194G) INTO (X10 P1-1/CX1-P10) CPAYCPS)

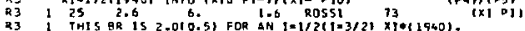

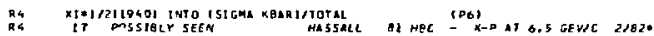

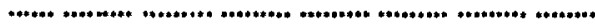

REFERENCFS FOR $X T+1 / 2(1940)$

ADOIEA OS DL 16171

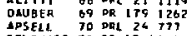

G D OWASS TO PQ 10 1CSD

$\begin{array}{ll}5001 E R & 72 \\ \text { NO } & 337.429 \\ \text { ROS5t } & 13 \text { PISOJUE CCNF. } 365\end{array}$

-DEWGUL IN, GOLDBEAG. A IEPCL, SACLAY, ANSI, I

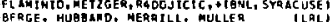

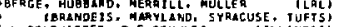
E L GOLDWASSER, P F STHULT 2 TLLIMOIS

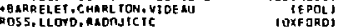

DIBIANC 45 49 940137 ALSC to DUKE CONF. 317

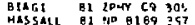

D1B1ATCR,ENOORF

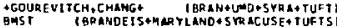

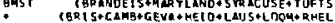
+AMEOREF, CARTER+NEALF, RLSHBFCOKE+1CANB+4SU papeas not neferaed to th oata caros

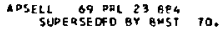

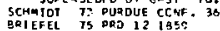

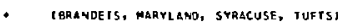

SCMEI DT

IBRaNDEIS

:

$\Xi(2030) \div 9 \times 101 / 212030$. JP.5/2 OP GREMTERI $I=1 / 2$

THF EYIDENCE FDR THIS STATE HAS BFEN MULH TMPRDVED EY HEMINGWAY TT, HMO SEE AM G STC. DEY. ENHANCEMENT

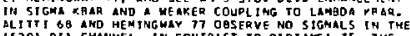

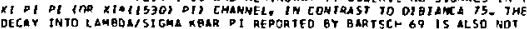
DECAY THTO LAMEDA/SIGMA KOSR

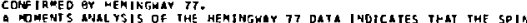

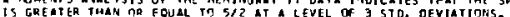

\section{DP $X 1+1 / 2+20302$ Mass (NEY)}

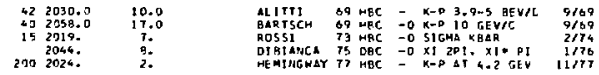

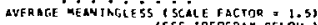

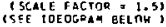

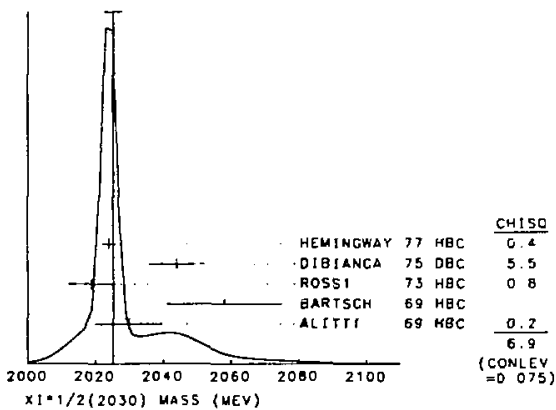

Data Card Listings

For notation, see key at front of Listings.

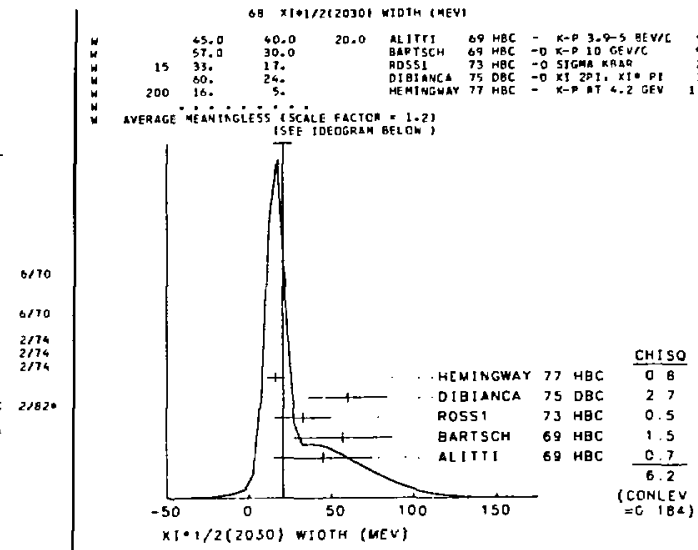

OQ X10172120301 PARTIAL DECAY MOCES

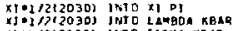

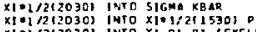

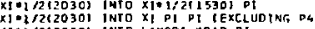

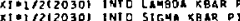

eA XI*1/2IZO3OS BHANCHINC RATICS

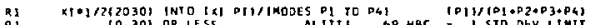

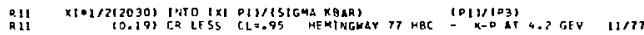

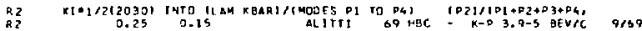

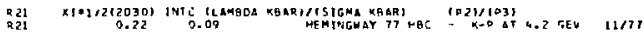

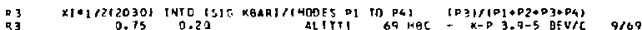

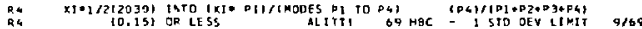

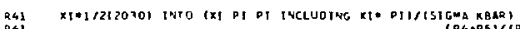

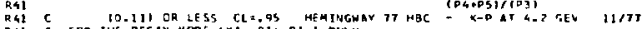

PGI C FDR IHE DECAY MOOE IXI- PIT DI-1 OWLY

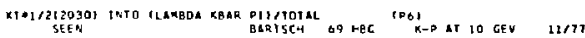

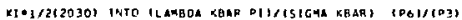

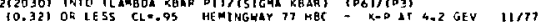

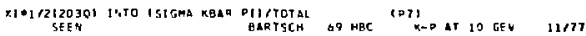

27

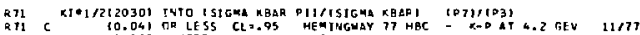

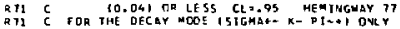

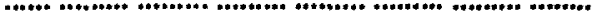

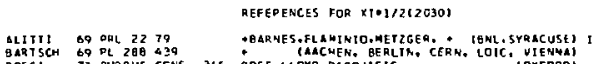

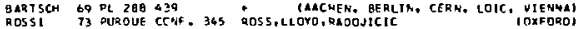

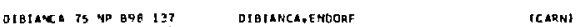

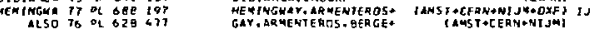

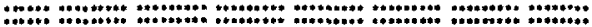

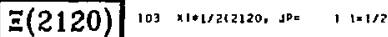

THIS EFFECT IS REPORTEO IN GAY 70 aS A

FIUR STANOARD OEVTATION ENHANCFMENT IN

IAUB OA K-. AN OEMLYSIS OF THE SAPE OATA RY

POINTS OUT THAT THE S1GHIFTCANEF OF THE ENHAMCEMENT IS GAEATIY REOUCEO

IF A RES YRICT IYE FOUA-MOUENTUA CUT IU-CUT) IS AADE. THIS SUGEEST;

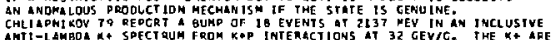

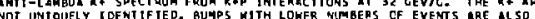

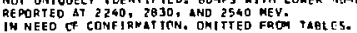


$203 \times 1012021202$ mass the VI

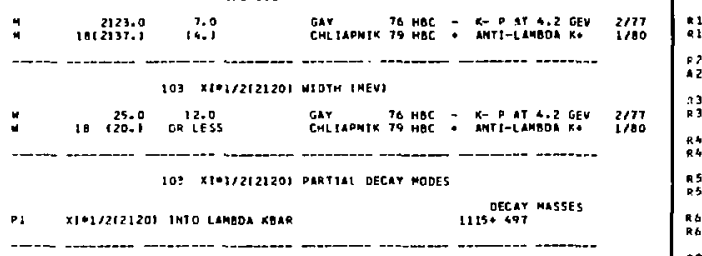

$103 \times 1+1 / 2121201$ BQAHCHING RATIOS

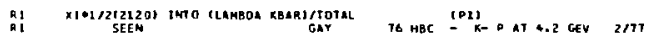

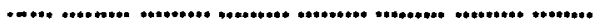
REFERENCES FOR XI*I/2:2120)

GAY TO OL O2B 417 TARMEHTEROS, BERGE. GAVILLET+ I ANST OCERN+NISHII

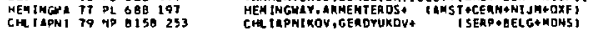

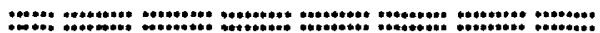
$\Xi(2250)$

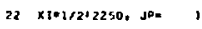

THE EVIDEECE FOR THIS STATE TS WEAK. BRRTSCH BQ FEE 1 BUMP OF NOI MUCH STATISTICAL SI GAIF ICANCE IN LAMUOL

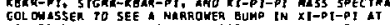
MIGHER MISS. NDT SEEN OY MASALL OI WITH EVERT SIMICGEBRAH AT 6.5 GEVic.

22 XI"1212250) MASS (MEV)

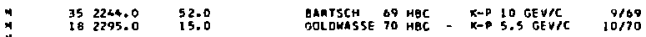

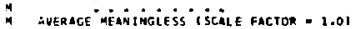

$22 \times 1 * 1 / 2122301$ WIOTH EMEVI

:

\begin{tabular}{|c|c|c|c|}
\hline $\begin{array}{l}130-0 \\
\text { LESS THAN }\end{array}$ & $\begin{array}{l}80.0 \\
30.0\end{array}$ & $\begin{array}{l}\text { BARTSCH } \\
\text { GOL HEC } \\
\text { GOLSE TO HBC }\end{array}$ & $=K-D 5.5 \mathrm{GEV} / \mathrm{G}$ \\
\hline
\end{tabular}

$22 \times[* 1 / 2122501$ PARTIAL DECMY MODES

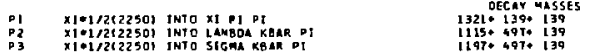

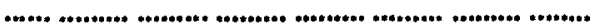
REFERENCES FDR $x: 1 / 2$ (2250)

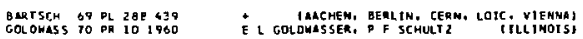
PAPERS NOT REFEREED TD IM DATA CaMOS

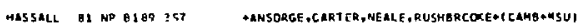

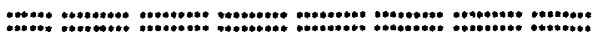

E(2370) 1?1 xi*1/212310, נ.е, $1=1 / 2$

SE EN gY AMIR LADEH 60 AHO HASSALL aI IN THE CHARGEO ANO

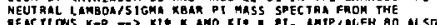

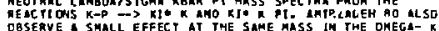

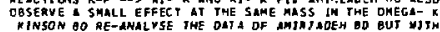

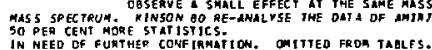

$1: 1 \times 1+1 / 2123701$ MASS (HEVI

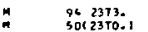

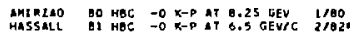

6.

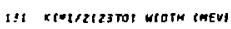

$\quad 94 \underset{180.1}{80.1} 25$.

AMTRZAO BO MEC -0 KOD AT Q.25 GEV 1 HOO

131 XINIRI23TOI PARTIAL OECAN MOOES

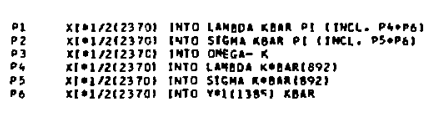

DEC AY masSES

1115. 4970 139

$1197+497$
$1612+407$

$1115+691$

11974891
131 K1०12123701 BRAMCHINF RATICS

XJOLI212370) 1NTO (LAMBDA KEAK PIIITOTAL
SEEN XI-1/262370) INTO ISIGMA KBAR PIJTTOTAL TOZ1

J MTEF

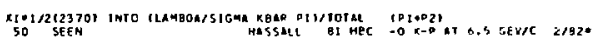

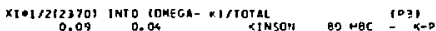

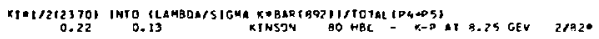

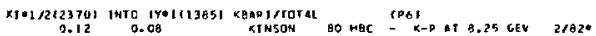

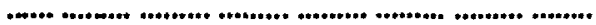

REFERENCES FOR $x I=1 / 2(23 T)$

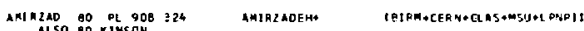

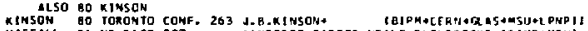

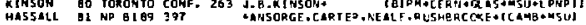

\#\#, $\Xi(2500)$

o3 $\times 1 * 1 / 212500$. JPE $1,1=1,2$

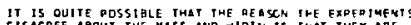

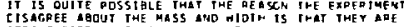

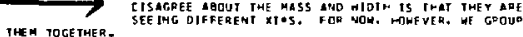

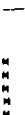
99 $\times 10121250014055$ I4I $v 3$

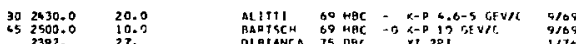
aver age méani nigiçs i ccale factor = 3.21

$29 \times 1012125001$ NJOTH (NE)

150.0 60.0 40.0 ALITIL 60 HAC

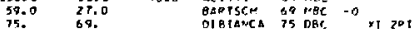

$7 / 60$
7139
1170

avenage meniningless iscal \& factor = 1.11

99 XIN1/262500I DARTIOL, DECAY MTDES

$x !+1 / 2125001$ tN

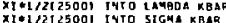

$x$ :

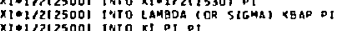

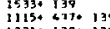

Po

$50 \times 1 * 1 / 212500)$ BDANCHING DATINS

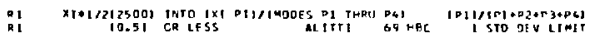

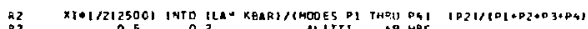

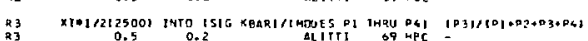

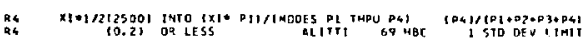

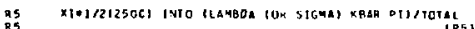

R.5 SEEN PAPTSCH GQ HAC 1 - 05

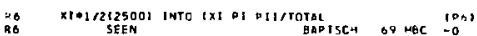

$\begin{array}{lll}\text { ALIIII } & 69 \text { PRL } 2279 \\ \text { BRATSCH } & 99 \text { PL } 286 \text { 16 }\end{array}$

REFERENKES FOA XI*:Z12125001

OTBIANCA IS ND 898 it?

OIBIANGA, ENODPF

[CARN]

+600 $S=-3 \quad I=0$ HYPERON STATE ( $(\Omega)$

:

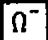

24 OMECA-11075. JPE 3/2+1 $1=0$

SEE STABLE PARTICLE OHTA CARO LTSTIINGS

Qto s/s9 $9 / 69$ $\$ \$ 0$ 9198 $9 / 60$ 


\section{Baryons}

$\Lambda_{\mathrm{c}}^{+}, \Sigma_{\mathrm{c}}(2450), \Lambda_{\mathrm{b}}^{0}$ DIBARYONS

:4*.**

\section{CHARMED BARYONS}

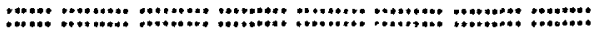
$A_{c}^{+}$

$33 \angle A M B D A / C+125 B Z+7 B=$,

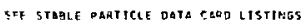

*an

$\sum_{c}(2450)$ ice sicuarcizsso, sot,

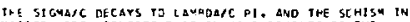

TISSES HEDE DEFLECTS ART IN MEASUREMENTS OF THE

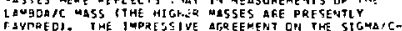

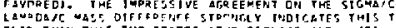

DE THE CASE, RATHEA THA

IHIS POETICLE IS AT AGUT THF Z-ANO-1/2-STAF LEVEL. A DEFINTTIVE EXPEA IAEVI IS NEFDF.

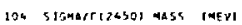

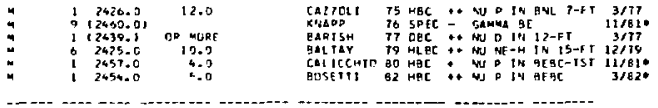

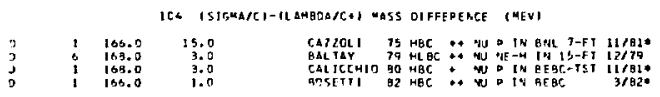

1CG SIGMARCIZLSOS DARTIAL JEGAY MDDES

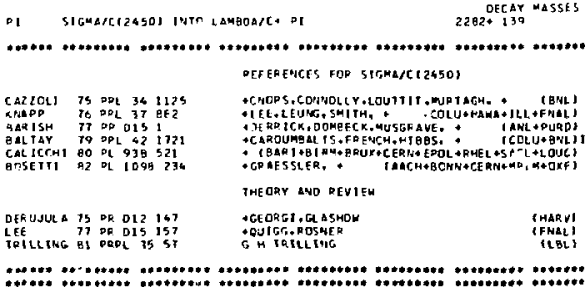

\section{BOTTOM (BEAUTY) BARYON}

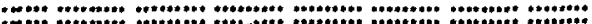

$\Lambda_{b}^{0}$
-30

:

\section{Note on Dibaryon Resonances}

(by L.D. Roper, Virginia Polytechnic Institute and State University)

The first modern theoretical discussion of dibaryon resonances was probably by 0akes. 1 The first experimental hint of them was in a $\Lambda_{p}$ invariant mass distribution by Dahl, ${ }^{2}$ and In NA partialwave analyses by Arnd ${ }^{3}$ for the ${ }^{D_{2}}$ state 10 a $P P$ partial-wave analysis. [The notation is ${ }^{(2 S+1)} \mathrm{L}_{\mathrm{J}}$, where $S$ is the total spin, $L$ is the orbital angular mosentun, and $J$ is the total angular monentum.]
Data Card Listings

For notation, see key at front of Listings.

Dibaryon popularity rose dromatically in 1977 when strong energy dependence was unexpectedly observed In PP polarization experiments at Argonne. ${ }^{4}$ Also, in that year and the next, Hosh1zaki clatmed the existence of dibaryon resonances in a PP partial-wave analysis. 5 In the same year Jaffe gave a detalled theoretical treatment of mult1-quark states. 6

Since the last fssue of this Review some new Pp and np polarization experiments ${ }^{7}$ have been reported which, along with other new and old NN scattering data, have been newly partial-wave analyzed. 8 There are also partial-wave analyses (given in the Listings) of $\pi d$ elastic, $\pi d+\pi p a$, $\mathrm{pp} \rightarrow \pi \mathrm{d}$, and $\mathrm{Yd} \rightarrow$ pn scatcering. Most of these otrongly ind lcate the existence of dibaryon resonances in the ${ }^{1} D_{2}$ and ${ }^{3} F_{3}$ nucleon-nucleon states, and some indicate posible resonances in the ' $\mathrm{S}_{0}$, ${ }^{3} \mathrm{~S}_{1},{ }^{3} \mathrm{P}_{1},{ }^{3} \mathrm{P}_{2},{ }^{3} \mathrm{D}_{3},{ }^{1} \mathrm{~F}_{3}$, or ${ }^{1} \mathrm{G}_{4}$ states.

The Pauli principle restricts two nucleons to be in one of the following states:

$$
\begin{aligned}
& I=0: \quad\left({ }^{3} \mathrm{~S}_{1},{ }^{3} \mathrm{D}_{1}\right),{ }^{\mathrm{I}} \mathrm{P}_{1},{ }^{3} \mathrm{D}_{2},\left({ }^{3} \mathrm{D}_{3},{ }^{3} \mathrm{G}_{3}\right),{ }^{\mathrm{I}} \mathrm{E}_{3},{ }^{3} \mathrm{G}_{4}, \ldots \\
& \mathrm{I}=1: \quad{ }^{1} \mathrm{~S}_{0},{ }^{3} \mathrm{P}_{0},{ }^{3} \mathrm{P}_{1},\left({ }^{3} \mathrm{P}_{2},{ }^{3} \mathrm{~F}_{2}\right),{ }^{1} \mathrm{D}_{2},{ }^{3} \mathrm{~F}_{3},\left({ }^{3} E_{4},{ }^{3} \mathrm{H}_{4}\right), \ldots
\end{aligned}
$$

Here the states that couple together (same $J^{P}$ ) are enclosed in parentheses. Simflarly, only certain quantum number sets are allowed for $M$, etc.

In the Listings below, we separate the determinations of pole posftions and Brelt-Wigner parameters. To be a resonance, the pole must occur on the second sheet for the elastic channel; it may be a bound state or resonance for inelastic channels. The advantages ó pole parameters over Breit-Wigner parameters are discussed briefly in the "Note on $N^{\prime} s$ and $\Delta^{\prime} s . "$

The 1dea that exotic resonanceg are really 'pseudo-resonances ${ }^{-9}$ has recently taken a new turn. 10 The Idea is that box diagrams (e.g., Involving $N \Delta$ in NN Bcattering) create resonancelike loops in the Argand diagram without resonance poles actually existing. The question is whether poles would be created when one unttarlzed the box diagrams in order to calculate physical scattering amplitudes. Kloet and $\mathrm{Tjan}^{10}$ have recently shown that a adel exists in which, lndeed, that is the case. However, resonance hunters should definitely 
Data Card Listings

For notation, see key at front of Listings.

report pole positions rather than Looping Argand diagrame in the future. All who ot 111 auggeat that the NN ${ }^{1} D_{2}$ or ${ }^{3} F_{3}$ resonance-11ke atructure is due to some dynamics other than resonances must take their case to the world collection of wN scattering data in the form of a detalled partial-wave analyeis.

The dinucleon resonances also cominunicate with the $Y d$ and $\pi d$ channels. There 18 not wuch $Y d$ data, and the multipole analysio does not yield much certainty about which dibaryons are 1nvolved. In the rid case, uncertainties abound and the partial-weve analysis yields poor fits compared to the analyses of NN data. Nevertheiess, the existence of dibaryons appears to be corroborated by the $Y d$ and $\pi d$ data.

Only a few papers about strange dibaryon states have appeared since the last lasue of this Review. It appears that the $S=-1$ dominant resonance 18 in the ${ }^{3} S_{1}$ atate, an $S U$ (3) partner of the deuteron. An excellent review of the S-l situation is given by Dalitz, ${ }^{11}$ who cuncludes that the $S=-1{ }^{3} S_{1}$ resonance pole probably exista.

For a more detailed recent leview of dibaryons, see Hoshizak1. 12

\section{References}

1. R.J. Oakes, Phys. Rev. 131,2239 (1963).

2. O.I. Dahl, N. Horwitz, D. H. Mlller, J.J. Kurphy, and P.G. White, Phys. Rev. Lett. $\underline{6}, 142$ (1961).

3. R.A. Arndt, Phys. Rev. $\underline{165}, 1834$ (1968).

4. For a list of references and a review, see $A$. Yokosawa, Phys. Rep. 64, 47 (1980).

5. N. Hoshizak1, Prog. Theor. Phys. 58, 716 (1977), and 60, 1796 (1978).

6. R.L. Jaffe, Phys. Rev. Lett. 38, 195 (1977), and $\underline{38}, 617$ (1977).

7. 1.P. Auer, W.R. Ditzler, D. Hill, H. Spinka, N. Tamura, G. Theodosiou, K. Toshioka, D. Underwood, R. Wagner, and A. Yokosaws, Phys. Rev. Lett. 46, 1177 (1981); I.P. Auer, W.R. Ditzler, D. H111, K. Ima1, H. Sp1nke, R. Stanek, K. Toohioka, D. Underwood, R. Hagner, A. Yokosawa, G. R. Burleson, W.B. Cottingame, S.J. Greene, S. Stuart, E.W. Hoffmann, and J.J. Jarmer, Phys. Rev. D24, 2008 (1981); D. Axen, R. Shypit, M. Conyn, 0. Healey, J. Stanley, N.M. Stewart, D.V. Bugg, J.A. Edgington, and N. Stevenson, J. Phys. G7, L22S (1981).
8. K. Haghtnoto and N. Hogh1zak1, Prog. Theor. Phye. 64, 1678 (1980), and 64, 1693 (1980); R.A. Arnd and L.D. Roper, VPIsSU tN Interactive Dial-In (aanual avallable from Dept. of Phy 1ca, VPIdSU, Blacksburg, VA 24061), 1982 (this interactive progran is also avallable on conputers at Argonne, LAMPF, and TRIUMF); D.V. Bugg, J.A. Edg1ngton, W.R. Gibson, N. Wright, N.K. Stewart, A.S. Clough, D. Axen, G.A. Ludgate, C.J. Oram, L.P. Robertion, J.R. R1chardoon, and c. Amsler, Phys. Rev. C21, 1004 (1980).

9. B.L.G. Bakker, I.M. Narodetsky, and J.A. Simonov, Lett. Nuovo C1m. 19, 265 (1977); Iu. A. Sinonov and $M$. van der Velde, Phys. Lett. 76B, 277 (i978); I.H. Narodetsky and Yu.A. S1monov, Sov. J. Nucl. Phys. 28, 698 (1978); Tu.A. Stwonov and M. van der Velde, J. Phys. 65. 493 (1979).

10. W.M. kloet and J.A. TJon, Phys. Lett. 106B, 24 (1981).

11. R.H. Dal1tz, Nuc1. Phys. A354, 10lc (1981).

12. N. Hosh1zak1, in $\mathrm{VI}^{\text {th }}$ International Seminar on High Energy Phygics Problems (Dubna, 1981).

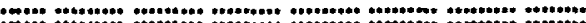

\section{DIBARYONS}

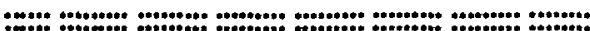

\section{$S=0$ DIBARYONS}

1CB GaRTOH MURBER 2, STRANGEMESS O ETLTES IN THIS SECTION WE USE THE FOLLONING ABBREVIATIONS FOR TYPFS
OF ANALYSES-

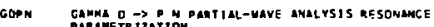

WH PIT TO HN ELASTIC PARTILL-MUE aHALYSTS RE SULTS

MAF FIT TO MK FOHWRO AMPLITUDES

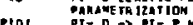

Dlot PI:D $\rightarrow$ PI

DQPO MARET ILATIOK MAMET ILATIOM

NN(2170)

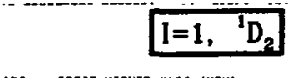

106 O-2, 5=0, 102 -- BREIT-MICNEE MAKS TMFV

BRE IT-WIGHE WASS ADPROXIMATELY EOUALS RELDCLE POSITIONI.

iztro.:

12280.:

12140.)

KAME BO OTD ACT TEY FITS WITH FEUER THAM SIX RESOM ANCES.

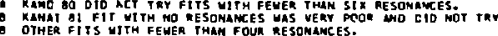

IC6 B=2, 500, 102 -- EAEIT-UIGMER WTOTK (MEVI

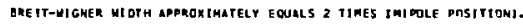

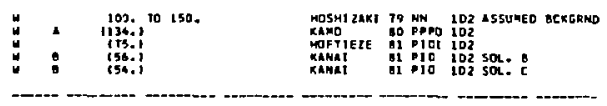

$1 / 020$
$1 / 120$
$1 / 120$
$1 / 1020$
i/aze

ICG OM2, SN0, 102 - BREIT-WIGNER ELASTICITY

GHEIT-WTCMEN ELASTIEITY APMOXXIMATELY EOUALS

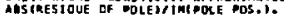

R!

10. 11

HOSHE ZAK1 T9 NM 102

$1102 *$ 
Baryons

DIBARYONS

lob a=2, $5=0$. 102 - REIPOLE POSIJiOHI (MEV) REI NOLE PESITIONY APPROKTMATELY EOUAS ENEIT-WTGHEA MASS.

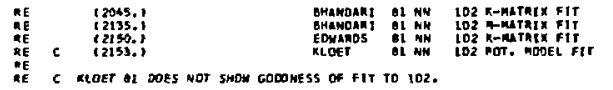

106 G=2. SN0. 102 - IMIPOLE POSIIIOHF ITEVI IMT PDE PCSITION) APPAOXIMATELY EOULL SAETT-WIGNER WIOTH OLVIOEO

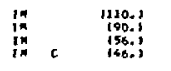

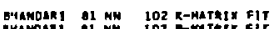

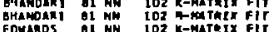

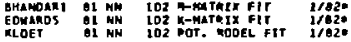

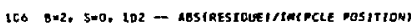

AOS:PESI, INCPOLE MSITION MOOKOXIMLTELY EOUALS EREIT-WIGMER

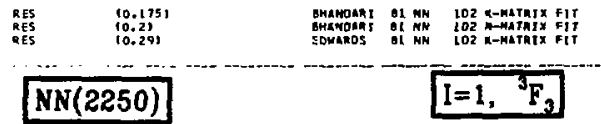

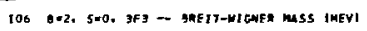
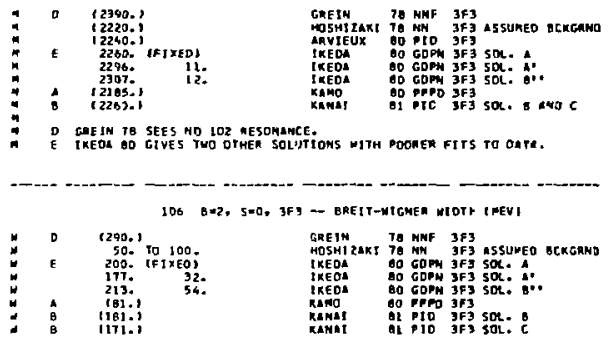

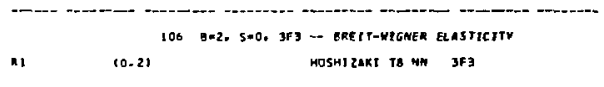

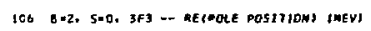

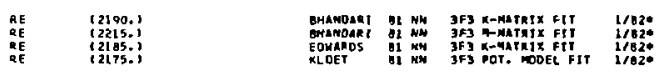

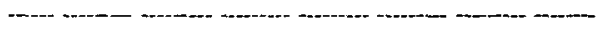

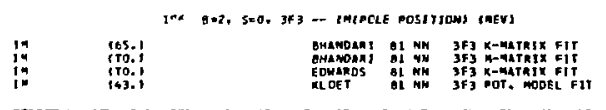

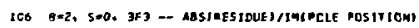

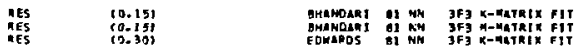

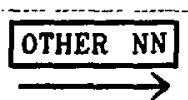

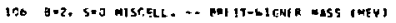
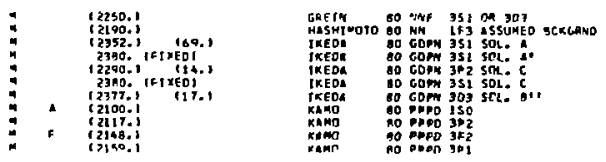

1/82:"

1/102:

1/62:

1,1020

1820

$1 / 620$

inge:
Data Card Listings

For notation, see key at front of Listings.
:

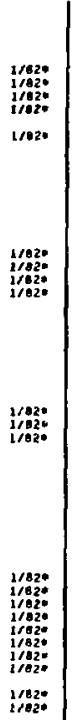

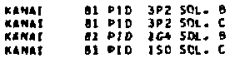

(2510.)

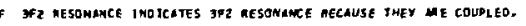

LOS BN2, SMO MISCELL, -- EREIT-WICMEW WIOTH (WEV)

\begin{tabular}{|c|c|}
\hline 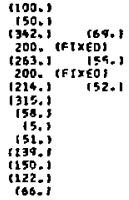 & 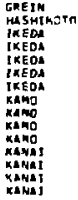 \\
\hline
\end{tabular}

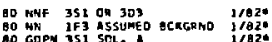

60 GDON 351 SOL. A.

SO GDPM 3P2 SCL. C

OO GODN 351 SOL.
80 GOHN 303 SOL. Bn.

$8 g$ PPPD ISO

80 PPD $3 F 2$

$\triangle D$ PPPD $3 P$

Q1 PID $3 P 2$ SOL.

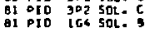

Q1 PID IGG SOL.
GI PID 1 SO SOL.

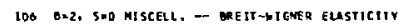

10.221

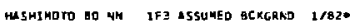

MEFENEHCES FOQ E-2. S=0 STATES

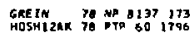

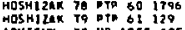

Atrieux

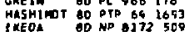

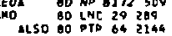

BMNDM 1 \& PAL 46 1111

DOWARS OL OR 1578

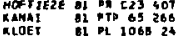

M. GREIN, O.KMOLL L

N. HoSHIZMK!

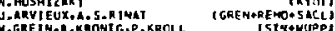

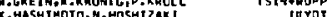

R.HASHTMUT.M. HOSHIZEKI

H.KAMO, W. Wh TAR 1

MAWO.MATAK 1 - YONE ZAWA

roskis?
tosek+hinoi

HARMOT, ROPER. VERHEST TVEI T TENUI

+ GAKER, CLEMENT, OMAGOSET T. IPICE+HOUS + B OHA

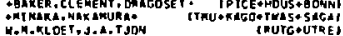

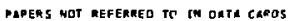

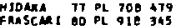

RAOOOER R HPG

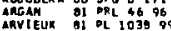

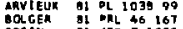

GQGER OH MPL 46167

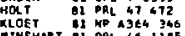

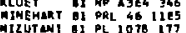

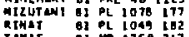

Dapers nat aEFERTED TO TM

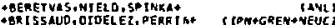

4LOCHER, MYHRER, THOMAS IS[M+NGMD+TRIU]

AHOTI,DEBOTTON,FAURE, MANTIN IN IS MCLI

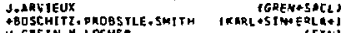

M.GREIN, M.LOCMER , DEICMEN ISTN

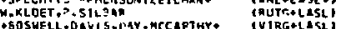

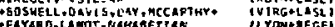

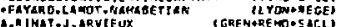

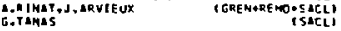

:0en

$S=-1$ DIBARYON

IC T IAYON MUMAER 2. STRLNGENESS - 5 TATES

IH THIS SECTICH WE USE THE FOLLOW:NG AP gREVIATIONS RO TYPES
OF SNALYSES-

LPIN LANGOAT INVARIANT MASS

Solk STEMa

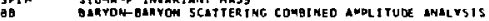

$\Lambda N(2130)$

$\mathrm{I}=1 / 2,3$

QRETT-MIGMER MASS ANDMOXIMATELY EQURLS RE (DOLE DOSITION).

\begin{tabular}{|c|c|}
\hline $\begin{array}{l}2090 . \\
\{2126.1 \\
12230.1 \\
12130.1\end{array}$ & t. \\
\hline $\begin{array}{l}2120-7 \\
12130-0.9 \\
12129.1\end{array}$ & $\left(\begin{array}{l}0.2 \\
0.7\end{array}\right.$ \\
\hline $\begin{array}{r}212 \mathrm{~T} \\
2125,2 \\
12251.41\end{array}$ & $\begin{array}{l}1.5 \\
13.9\end{array}$ \\
\hline $\begin{array}{r}12115.1 \\
2129.0 \\
12130.3 \\
12920.3\end{array}$ & 0.4 \\
\hline
\end{tabular}

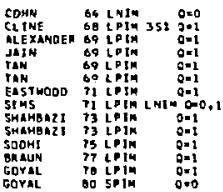

1 SIGMA- D TO LENGOA N T.

Solm

$0=0$

4 I GCY 71 RAISES ODUATS 48001 THE EXPERTMENTAL PROCEDUPE USEO

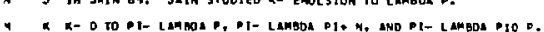

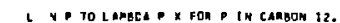

- x- D to D- Dio lamba.

- N GOTML MO SEES ANOTHER UKEERILIH PEAK AT 2195-2210 REY.

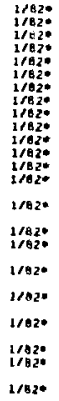

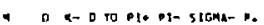




\section{Data Card Listings}

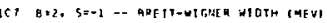

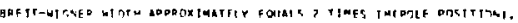

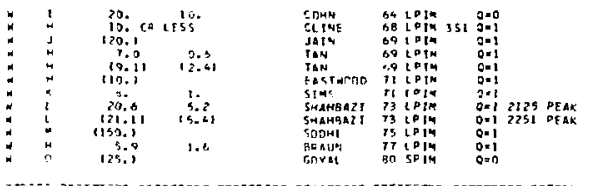

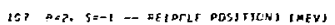

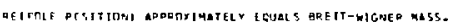

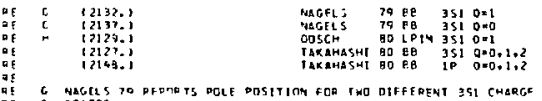

C NAGCLS TO PFONATS DOLE DOSITIOM FOA THO DIFFEPENT 351 CMARCE

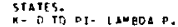

IDT H=2. SH-1 - IMLOLF DOSITIC.N) (MEV)

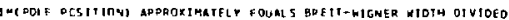
gr 7.

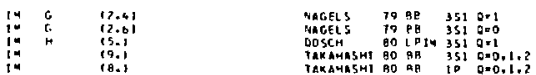

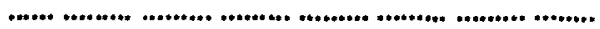

DEFEOEATESS FOA BEz, SE-1 STMTES

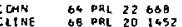

CLEXALE 69 PRL 22893

JAIN $\quad$ TO DR IFT IBIO

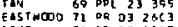

IAS 71 BR 031132

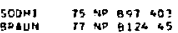

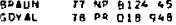

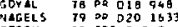

802063244

TAKAHASH BO YO 4338347
H.D.COHN,K,H.BHAIT.M.M.EUGG TORMLATENAI

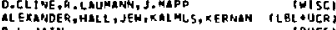
D.L.JaIH

[SLaC)

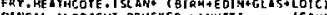

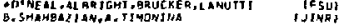

A. SaOHt, D. Goral

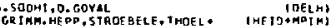

D.GOVAL. A. SOOHI

M. OOSER. T. STAMA TESCU SHART

COYAL Jo 1584

D.FEYHASHT, IHUYURA, K I ITUPA, KUME

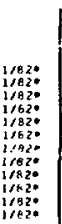

AIEXATOC BG DQ 173 14:2 BLETHEL TO OR 27 OQ CAOYK II ND B T

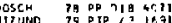

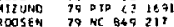

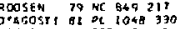

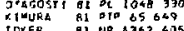

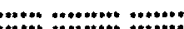

$S=-2$ DIBARYON

\section{$S=-2$ DIBARYON}

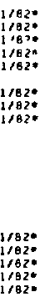

B2:

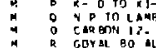

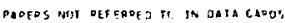

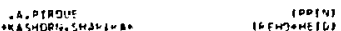

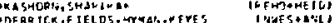

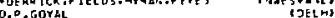

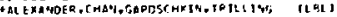

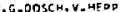

r. at tunc

TTKKY

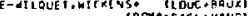

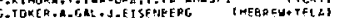

(1)

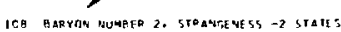

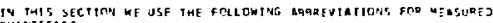

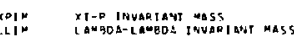

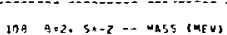

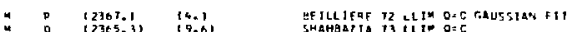

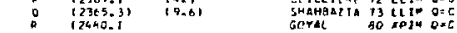

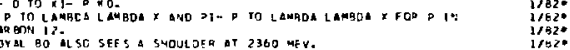

100 P.2. $S=0$ - W HOSH $\{\mathrm{NEV}$

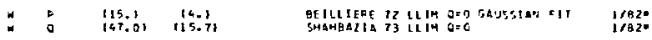

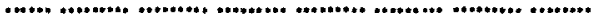

REFEPENCES FOF $Q=2$, 5*-2 STATES

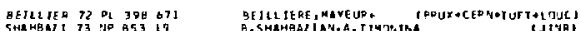

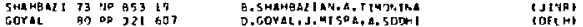

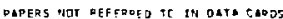

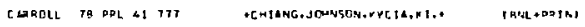

no
1820

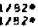
, 


\section{Aprewix I}

TEST OF $\Delta I=1 / 2$ RULE FOR HYPERON DECAYS

\section{o. E. Overseth}

University of Michigan

\section{Nonleptonic decay Amplitudes}

In this edition we again use the new convention for the amplitudes $A$ and $B$ adopred in 1973. Some theorists have suggested that dimensionless amplitudes are more useful to them than the ones appearing in the literature. Bergel used a convention with $A$ and $B$ in units of $\sec ^{-1 / 2}$. Samios ${ }^{2}$ used a convention which gave $A$ and $B$ in units of $(\mathrm{MeV}-\mathrm{sec})^{-1 / 2}$. Following is the convention suggested by Jackson ${ }^{3}$, which gives dimensionless $A$ and $B$.

The effective Lagrangian density for nonleptonic hyperon decays $\left(\mathrm{B}_{1} \rightarrow \mathrm{B}_{2}+\pi\right)$ can be written

$$
\mathbf{L}_{\mathrm{eff}}=\mathrm{G}_{\mu_{\mathrm{c}}}{ }^{2}\left[\bar{\psi}_{2}\left(\mathrm{~A}+\mathrm{B} \gamma_{\mathrm{S}}\right) \psi_{1}\right] \phi_{\mathrm{r}} .
$$

where $G=10^{-5} m_{p}-2$ is a coupling constant characteristic of first-order weak decays, $\mu_{c}$ is the charged pion mass, and $A$ and $B$ are dimensionless compiex numbers giving the relative amplitudes of the parityviolating and parity-conserving decays, respectively. The matrix $\gamma_{S}$ is to be taken in the Pauli form, $r_{5}=\left(\begin{array}{cc}0 & -1 \\ -1 & 0\end{array}\right)$. The invariant amplitude for the decay is

$$
M=G \mu_{c}^{2}\left[\bar{u}(p)\left(A+B \gamma_{S}\right) u(P)\right]
$$

where $\mathbf{P}$ is the 4 monentum of the decaying hyperon of mass $\mathbf{M}$, and $\mathbf{p}$ is the 4 -momentum of the baryon decay product of mass $\mathrm{m}$. With the normalization convention, $\bar{u}_{i} u_{i}=2 m_{j}$, the Pauli form of the matrix element in the rest frame of the decaying hyperon is

$$
M=G \mu_{c}^{2}<x_{2}|\sqrt{2 M(E+m)} A+\sqrt{2 M(E-m)} B \vec{\sigma} . \hat{q}| x_{1}>,
$$

where $E$ is the total energy of the final baryon and $\dot{q}$ is a unit vector in the direction of motion of the final baryon. Comparison with Sec. VI D of :he text shows that the amplitudes $s$ and $p$ defined there are proportinnal to $A$ and $B$ :

$$
\frac{P}{s}=\left(\frac{E-m}{E+m}\right)^{1 / 2} \frac{B}{A}=\left[\frac{(M-m)^{2}-\mu^{2}}{(M+m)^{2}-\mu^{2}}\right]^{1 / 2} \frac{B}{A} .
$$

Here $\mu$ is the mass of the pion entering the decay. The parameters $\alpha$, $\beta$. and $\gamma$ can therefore be expressed in terms of $A$ and $B$, rather than 5 and $p$, if desired.

The decay rate for $B_{1} \rightarrow B_{2}+\pi$ is

$$
\Gamma=\frac{G^{2} \mu_{c}{ }^{4}}{8 \pi} q\left\{\left[\frac{(M+m)^{2}-\mu^{2}}{M^{2}}\right]|A|^{2}+\left[\frac{(M-m)^{2}-\mu^{2}}{M^{2}}\right]|B|^{2}\right\} \text {. }
$$

whese $q$ is the c.m. momentum of the decay products. For reference. the dimensionless constant in this expression has the value $\left(G^{2} \mu_{c}^{4} / 8 \pi\right)$ $=1.9488 \times 10^{-15}$.

Table I summarizes the amplitudes $A$ and $B$ for the nonleptonic decays of the $\Lambda, \Sigma$, and $\Xi$ hyperons. These amplitudes have been $\mathrm{cal}$ culated by using the experimental data for mean lives, branching ratios, and the decay asymmetry a given in the Stable Particle Table of this Review. Time-reversal invariance is issumed and final-state interactions are neglected, so $\mathrm{A}$ and $\mathrm{B}$ are taken to be relatively real. The subscript on the hyperon refers to the sign of the decaying pion. The statistical correlation coefficient

$$
C_{A B}=\frac{\langle\Delta A \Delta B\rangle}{\sqrt{\left\langle\Delta A^{2}\right\rangle\left\langle\Delta B^{2}\right\rangle}}
$$

is also given. The absolute signs of $A$ and $B$ have been assigned, using the following convention. Taking $\mathbf{A}\left(\Lambda^{0}\right)$ as positive, the other $S$-wave decay amplitudes are chosen to give an approximate fit to the triangular relationships

$$
\sqrt{2} \mathrm{~A}\left(\Sigma_{0}^{+}\right)+\mathrm{A}\left(\Sigma_{+}^{+}\right)=\mathrm{A}\left(\Sigma_{-}^{-}\right) \text {and } \sqrt{3} \mathrm{~A}\left(\Sigma_{0}^{+}\right)+\mathrm{A}\left({\Lambda^{0}}^{0}\right)=2 \mathrm{~A}\left(\Xi_{-}^{-}\right) \text {. }
$$

The signs of the B amplitudes relative to those of the corresponding A amplitudes are determined by the sign of the appropriate a decay parameter.

\begin{tabular}{cccc}
\hline \multicolumn{5}{c}{ Table I } \\
\hline$M-m+\mu$ & $A$ & $B$ & $C_{A B}$ \\
$\Lambda_{-}^{0} \rightarrow p+\pi^{-}$ & $1.47 \pm 0.01$ & $9.98 \pm 0.24$ & -0.289 \\
$\Lambda_{0}^{0}-n+\pi^{0}$ & $-1.07 \pm 0.01$ & $-7.14 \pm 0.56$ & -0.740 \\
$\Sigma_{+}^{+} \rightarrow n+\pi^{+}$ & $0.06 \pm 0.01$ & $19.07 \pm 0.07$ & -0.038 \\
$\Sigma_{0}^{+} \rightarrow p+\pi^{0}$ & $1.48 \pm 0.05$ & $-12.04 \pm 0.58$ & 0.982 \\
$\Sigma_{-}^{-} \rightarrow n+\pi^{-}$ & $1.93 \pm 0.01$ & $-0.65 \pm 0.07$ & 0.003 \\
$\Xi_{0}^{0}-\Lambda+\pi^{0}$ & $1.55 \pm 0.03$ & $-5.56 \pm 0.33$ & -0.148 \\
$\Xi_{-}^{-} \rightarrow \Lambda+\pi^{-}$ & $2.04 \pm 0.01$ & $-7.49 \pm 0.28$ & 0.237 \\
\hline \hline
\end{tabular}

2. Tests of the $\Delta \mathrm{I}=1 / 2$ Rule

\section{(a) $\Lambda$ Decay}

For $\Lambda$ decay the $\Delta l=1 / 2$ rule predicts that $\Gamma_{0} / \Gamma_{-}=0.50$ and $a_{0}$ $=\alpha_{-}$. In order to determine the magnitude of possible $\Delta I=3 / 2$ amplitudes present we write the linear expressions 4 for the $\Delta I=3 / 2 \mathrm{~A}$ and $B$ - wave amplitudes in terms of $\Delta \alpha$, where $\Delta \alpha$ is the measured value of $\alpha_{0} / \alpha_{-}$minus the predicted value, and in terms of $\Delta \Gamma$ similarly defined. Evaluating these we find 


$$
\begin{aligned}
& \Delta \alpha=-1.54\left(A_{3} / A_{1}\right)+1.61\left(B_{3} / B_{1}\right) . \\
& \Delta \Gamma=1.84\left(A_{3} / A_{1}\right)+0.25\left(B_{3} / B_{1}\right) .
\end{aligned}
$$

Here the $\Delta I=3 / 2$ amplitudes are expressed relative to the $\Delta I=1 / 2$ amplitudes. The numerical values of the coefficients depend on the ratio $B / A$ The uncertainties in the coefficients are small compared to the uncertainties in $\Delta_{\alpha}$ and $\Delta \Gamma$. Final-state $\pi N$ interactions have been included in these relations but have a very small effect. From the Stable Particle Table.

$$
\Delta a=0.006 \pm 0.066, \Delta \Gamma=0.058 \pm 0.012 \text {, }
$$

and hence

$$
\left(A_{3} / A_{1}\right)=0.027 \pm 0.008
$$

and

$$
\left(B_{3} / B_{1}\right)=0.030 \pm 0.037
$$

The possible $3 \% \Delta \mathrm{I}=3 / 2$ A-wave amplitude is due to the disagreement of decay rates with prediction. At this level the results are sensitive to electromagnetic corrections. However, in A decay the phase space correction and the other radiative corrections appear to be about equal in magnitude and have opposite signs, 5.6 and hence cancel each other in the correction to the decay rates.

\section{(b) E Decay}

The analysis for $\Xi$ decay is very similar to that for $A$ decay. If the $\Delta I=1 / 2$ rule is valid, $r_{0}\left(\Xi^{0}\right) / \Gamma_{-}\left(\Xi^{-}\right)=0.50$ and $\alpha_{0}=\alpha_{-}$. For this case the expressions linear in $\Delta I=3 / 2 A$ - and $B$-wave amplitudes $\mathrm{are}^{4}$

$$
\begin{aligned}
& \Delta a=1.35\left(A_{3} / A_{1}\right)-1.35\left(B_{3} / B_{1}\right) \\
& \Delta \Gamma=-1.43\left(A_{3} / A_{1}\right)-0.07\left(B_{3} / B_{1}\right) .
\end{aligned}
$$

From the Stable Particle Table.

$$
\Delta x=-0.05 \pm 0.06, \Delta \Gamma=0.066 \pm 0.020,
$$

and we find

$$
\left(A_{3} / A_{1}\right)=-0.046 \pm 0.014
$$

and

$$
\left(B_{3} / B_{1}\right)=-0.01 \pm 0.04
$$

(c) $\Sigma$ Decay

The traditional test of the $\Delta \mathrm{J}=1 / 2$ rule in $\Sigma$ decay is that the amplitudes satisfy the relationship

$$
\sqrt{2} \Sigma_{0}^{+}+\Sigma_{+}^{+}-\Sigma_{-}^{-}=0
$$

Graphically this is equivalent to closing the $\Sigma$ triangle when the amplitudes are plotied on $A, B$ axes. Including $\Delta I \geq 3 / 2$ amplitudes in $\Sigma$ decay analysis, the " $\Sigma$ triangle" relationship bermes

$$
\sqrt{2} A_{0}+A_{+}-A_{-}=-3 \sqrt{2 / 5} A_{3}+\frac{2}{\sqrt{15}} A_{5} \text {. }
$$

where $A_{3}$ and $A_{S}$ are $\Delta I=3 / 2$ and $\Delta I=5 / 2$ amplitudes, respectively. There is a similar equation for the $B$ amplitudes. From Table $\mathbf{I}$,

$$
\sqrt{2} A_{0}+A_{+}-A_{-}=0.22 \pm 0.09
$$

and

$$
\sqrt{2} B_{0}+B_{+}-B_{-}=2.7 \pm 1.0
$$

If we neglect the $\Delta I=5 / 2$ amplitudes and assume all amplitudes to be real we can solve for possible $\Delta I=3 / 2$ amplitudes. The result is

$$
\frac{A_{3}}{A_{-}}=-0.061 \pm 0.024
$$

and

$$
\frac{B_{3}}{B_{+}}=-0.074 \pm 0.027
$$

Thus for hyperon decay. present experimental data limit $\Delta \mathbf{j}=3 / 2$ amplitudes to less than about $5 \%$.

\section{The Lee-Sugawara Relation}

From Table I the Lee-Sugawara relation, $7,8 \sqrt{3 \Sigma_{0}^{+}}+\Lambda_{-}^{0}-2 \Sigma_{-}^{-}$ $=0$, is satisfied to $-0.05 \pm 0.11$ for the $A$ amplitudes, and to $4.1 \pm 1.8$ for the $B$ amplitudes.

\section{References}

1. J. P. Berge, in Proceedings of the I3th International Conference on High-Energy Physics, Berkeley, (1966) (University of California Press, Berkeley, 1967), p. 46.

2. N. P. Samios, International Conference on Weak Interactions, Argonne, (1965), p. 189.

3. J. D. Jackson, private communication (1973).

4. See $O$. E. Overseth and S. Pakvasa, Phys. Rev. 184, 1663 (1969). The expression for $\Gamma_{0} / \Gamma_{-}$for $\Lambda$ decay should read

$$
\begin{aligned}
& \frac{\Gamma_{0}}{\Gamma_{-}} \approx \frac{1}{2}\{1+3 \sqrt{2} \\
& \left.\times\left[\frac{S_{11} S_{33} \cos \left(\delta_{1}-\delta_{3}\right)+P_{11} P_{33} \cos \left(\delta_{11}-\delta_{31}\right)}{S_{11}{ }^{2}+P_{11}{ }^{2}}\right]\right\} .
\end{aligned}
$$

5. See A. A. Belavin and I. M. Narodetsky, Yadem. Fiz. 8, 978 (1968) [Sovjet J. Nucl. Phys. 8, 568 (1969)].

6. G. W. Intemann, private communication (1973).

7. See B. W. Lee, Phys. Rev. Lett. 12. 83 (1964).

8. See H. Sugawara. Prog. Theor. Phys. 31. 213 (1964). 


\section{Arpendix II}

\section{A SU(3) CLASSIFICATION OF BARYON RESONANCES}

\section{A. Barbaro-Galtieri \\ Lawrence Berkeley Laboratory}

It is established that a symmetry higher than $\mathrm{SU}(3)$ is necessary to classify the known baryon resonances. However, many highersymmetry schemes have been proposed, and even for SU(6) various versions exist (for a review see Dalitz ${ }^{1}$ ). Since it is not clear which one of these schemes best fits the data, we do not review them here, but we report once again fits of baryon states into $\mathrm{SU}(3)$ multiplets.

For the reader's convenience, we collect here the relevant formulae.

Exact SU(3) symmetry predicts that all the members of a multiplet should have the same mass and the same couplings for decays into other multiplets. It has been found, however, that the members of the actet of stable baryons lie within $20 \%$ of their mean mass; therefore a symmetry-breaking interaction has been introduced by Gell-Mann and Okubo independently. ${ }^{2}$ In addition, for the isospin-0 vector mesons ( $\omega$ and $\phi$ ). an additional symmetry-breaking interaction has been introduced by Sakurai ${ }^{3}$ to take care of octet-singlet mixing. The relevant formulae for masses and decay rates are given below.

\section{Mass Formulae}

Broken SU(3) gives:

\begin{tabular}{|c|c|c|}
\hline Decuplet & $\Delta-\Sigma=\Sigma-\Xi=\Xi-0$ & GMO \\
\hline Octet & $2(N+Z)=3 \Lambda+\Sigma$ & GMO \\
\hline $\begin{array}{l}\text { Octet- } \\
\text { singlet }\end{array}$ & $\sin ^{2} \theta=\frac{\Lambda-M_{8}}{\Lambda-\Lambda^{\prime}}$ & $\begin{array}{l}\text { Mixing } \\
\text { argle }^{4}\end{array}$ \\
\hline mixing & $M_{8}=\frac{2(N+\Xi)-\Sigma}{3}$ & GMO \\
\hline
\end{tabular}

Here GMO stands for the Gell-Minn-Okubo formula; the particle symbol indicates its mass. The formulae would be the same if squared masses were used. For the nonet case, $\Lambda$ is the "mostly-octet" particle, $\boldsymbol{A}^{\prime}$ is the "mostly-singlet" particle.

\section{Decay Rares}

in terms of a relativistically invariant matrix element $T$, the decay iate for 2-body decay of a resonance of mass $M_{R}$ is

$$
\Gamma \propto \frac{|T|^{2} R_{2}}{M_{R}} .
$$

where $R_{2}=k / M_{R}$ is the 2-body phase space factor. Since the numerator is an invariant, and since $\Gamma$ must transform as $1 / \mathrm{E}$, we introduce the denominator $\mathrm{M}_{\mathrm{R}^{-}}{ }^{\mathrm{S}}$

For meson decays (see below) the rates are calculated according to Eq. (5); for baryon resonance decays into $1 / 2^{+}$baryons and $0^{-}$ mesons, one next takes into account the fact that spin sums in $|T|^{2}$ introduce another factor $M_{R}$. canceling the $1 / M_{R}$. We are then left with

$$
\begin{aligned}
\Gamma & =\frac{\mid T\rfloor^{2} k}{M_{R}} M_{N}, \text { for baryons } \\
& =\frac{\left.\perp T\right|^{2} k}{M_{R}^{2}} M_{N}^{2}, \quad \text { for mesons. }
\end{aligned}
$$

The powers of the nucleon mass $\mathbf{M}_{N}$ or $\mathbf{M}_{N}^{2}$ have been introduced so that we can treat $|\mathrm{T}|$ as dimensionless.

$|T|^{2}$ contains centrifugal barrier factors, which we call $B_{\ell}$. We then have

$$
\begin{aligned}
& \left.\begin{array}{l}
\text { Decuplet } \\
\text { Singlet }
\end{array}\right\} \quad \Gamma=(\mathrm{cg})^{2} \mathrm{~B}_{\ell}(\mathrm{k}) \frac{\mathrm{M}_{\mathrm{N}}}{\mathrm{M}_{\mathrm{R}}} \mathrm{k} \\
& \text { Octet } \quad \Gamma=\left(c_{D^{8} \mathrm{D}}+\mathrm{c}_{\mathrm{F}} \mathrm{g}_{\mathrm{F}}{ }^{2} \mathrm{~B}_{\ell}(k) \frac{\mathrm{M}_{\mathrm{N}}}{\mathrm{M}_{\mathrm{R}}} k\right. \\
& \begin{array}{l}
\text { Octet- } \\
\text { singlet } \\
\text { mixing }
\end{array}\left\{\begin{array}{l}
\mathrm{A}=\mathrm{G}_{8} \cos \theta+\mathrm{G}_{1} \sin \theta \\
\mathrm{A}^{\prime}=-\mathrm{G}_{8} \sin \theta+\mathrm{G}_{1} \cos \theta
\end{array}\right. \\
& \text { with } \quad G_{8}=c_{D} g_{D}+c_{F} g_{F} \\
& G_{1}=c_{1} 8_{1} \text {. }
\end{aligned}
$$

Here $c_{i}$ are the $S U(3)$ coefficients with the sign convention adopted in this article [see note in the Table of SU(3) Isoscalar Factors and Fig. 2 in the text]. $M_{N}$ is the nucleon mass, $M_{R}$ is the resonance mass for which $\Gamma$ is calculated, ' , 'ce center-of-mass momentum for the channel being considered, and $8_{\mathrm{i}}$ are the relevant couplings, For the case of singlet-octet mixing, $\mathrm{Eq}$. (8) has to be used in conjunction with (6) and (7). $G_{8}$ and $G_{1}$ represent the couplings for the multiplet, and $A$ and $\boldsymbol{A}^{\prime}$ represent the couplings for the physical states.

The relation between $\mathrm{BD}_{\mathrm{D}} \mathrm{B}_{\mathrm{F}}$ and the parameter $\alpha$ is

$$
\alpha=\left[1+\frac{\sqrt{5}}{3} \frac{\mathrm{g}_{\mathrm{F}}}{\mathrm{g}_{\mathrm{D}}}\right]^{-1} \text {. }
$$

Exact $S U(3)$ predicts that the couplings $g_{i}$ for all the members of a multiplet are the same; however, since the symmetry is broken for the masses, it is probably broken for the widths. In the case of the $3 / 2^{+}$decuplet. broken SU(3) sum rules have been derived by Becechi, ${ }^{6}$ Gupta, ${ }^{7}$ and Konuma ${ }^{8}$ independently. The form derived by Gupta relates the $g_{i}$ for the members of the decuplet by the relation

$$
2(\Delta+\Xi)=3 \Sigma^{*}(\Lambda x)+\Sigma^{*}(\Sigma x)
$$

where $\Sigma^{*}(\Lambda \pi)$ is the coupling for the $\Sigma(1385) \rightarrow \Lambda \pi$ decay and $\Sigma^{\circ}(\Sigma \pi)$ is the coupling for the decay $\Sigma(1385)-\Sigma \pi$.

As mentioned in the text (Sec. IV B) the determination of the relative signt of resonant amplitudes cal, be useful in making an SU(3) 
assignment of resonances. In fact the resonant amplitude $T \propto \sqrt{x_{e} x_{i}}$ $\propto \mathrm{G}_{\mathrm{e}} \mathrm{G}_{\mathrm{i}}$, where the subscript e refers to the elastic channel and the $G_{e}$, $G_{i}$ are the couplings of Eqs. (6) through (9). Assuming that all $b_{i}$ are positive, the sign of the $G_{i}$ are dependent upon the sign of the Clebsch-Gordan coefficients $c_{i}$. Once a sign convention is adopted (we use the Levi-Setti ${ }^{9}$ convention, see Fig. 2 in the text) and the signs for a $\Sigma$ state $(1=1)$ and a $\Lambda$ state $(l=0)$ of known $S U(3)$ assignment have been chosen for reference, the signs of all the other amplitudes can be useful in determining multiplet assignments. For exact $S U(3)$ all the decays of members of a decuplet have the same sign. For octets the relative sign depends upon the value of ${ }_{{ }_{D}} / g_{F}$ and the mixing angle, as seen from Eqs. (7) through (9).

\section{Fits so she Data}

Fits of baryon decay rates within SU(3) can be found in, among others, papers by Tripp. ${ }^{10.11}$ Levi-Setti, ${ }^{9}$ Samios, ${ }^{12}$ and Plane. ${ }^{13}$ More recent fits were made by Barbero-Galtieri ${ }^{14}$ and Samios. 15 A fit of the decay rates within SU(6), may be found in Litchfield et al. ${ }^{16}$ Analysis of the baryon mass spectrum using the quark shell model has been done by Jones et al. ${ }^{17}$ An analysis of beryon cuuplings in a quark model with chromodynamics has been done recently by Konjuk and Isgur. ${ }^{18}$

For our SU(3) analysis in fitting the data a choice for $B_{\ell}$, has to be made. Plane ${ }^{13}$ tried two forms for $B_{\ell}$ :

(a) The form $B_{\ell}=(k r){ }^{L_{2}}(k r)$, $r$ being the radius of interaction and $D_{\ell}$ the nolynomials in kr given by Blatt and Weisskopf. ${ }^{19}$ Usually $r$ is taken to be 1 fermi. ${ }^{10}$ (b) The form $\mathrm{B}_{\ell}=\mathrm{k}^{\ell}$.

However, for final results form (b) was chosen. A discussion of the differences among these two forms has been given by BarbaroGalieri. ${ }^{20}$. As shown in Rer. 20 , not only the values of the couplings, $B_{i}$, depend upon the form used for $B_{\ell}$, but also the value obtained for the mixing angle. For the $3 / 2^{-}$singlet, $\Lambda(1520)$, and the isospin-0 member of the octet, $A(1690)$, the mixing angles obtained in the two cases were

$$
\theta_{\mathrm{a}}=\left(-16.1_{-1.3}^{+1.4}\right)^{\circ}, \theta_{\mathrm{b}}=\left(-27.5_{-3.4}^{+3.6}\right)^{\circ} \text {, }
$$

in disagreement by a few standard deviations. However, if a radius of interaction of $r=0.15$ fermi was used for form (a), the two values of $\theta$ agreed. This valu of $r$ does not fit resonance shapes when used in the Breit-Wigner resonant form Samios 15 used form (b) for $B_{\ell}$.

Table $I$ is a summary of the fits made by us (update of BarbaroGaltieri ${ }^{14}$ ) using the barrier factor form (a) and exact SU(3). The values of the masses, widths, and amplitudes used in the fits are taken from this edition's Tables and Listings.

1/2- Nonet (Boryon-Eta Resonances)

For this nonet Eq. (7) was multiplied by the factor

$$
\left[\frac{M_{R}-M_{B}}{\bar{M}_{R}-\bar{M}_{B}}\right]^{2}
$$

where $M_{B}$ is the decay baryon and $\bar{M}_{R}-\bar{M}_{B}=568 \mathrm{MeV}$ is the difference of the mean $1 / 2^{-}$and $1 / 2^{+}$baryon octet masses. This kinematic factor comes from PCAC arguments (i.e., the assumption that the axial

Table 1. SU(3) baryon multiplets with two or more known members. Values of $\theta$ and $a$ [defined by Eqs. (B) and (10)] are the result of fits made to all the measured 2-body decay

\begin{tabular}{|c|c|c|c|c|c|c|c|}
\hline \multirow{2}{*}{$\frac{J^{P}}{1 / 2^{-}}$} & \multirow[b]{2}{*}{$N(1535)$} & \multicolumn{3}{|c|}{ Octet members ${ }^{a}$} & \multirow{2}{*}{ Singlet } & \multirow{2}{*}{$\begin{array}{l}\theta(\operatorname{deg})^{b} \\
-31 \pm 7 \\
-22 \pm 11\end{array}$} & \multirow{2}{*}{ 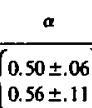 } \\
\hline & & $\Lambda(1670)$ & $\Sigma(1750)$ & $\begin{array}{l}{[\Xi(1737)]} \\
{[\Xi(1850)]}\end{array}$ & & & \\
\hline $3 / 2^{-}$ & $N(1520)$ & $\Lambda(1690)$ & $\boldsymbol{\Sigma}(1670)$ & {$[\Xi(1819)]$} & $\Lambda(1520)$ & $-21 \pm 3$ & $0.31 \pm .04$ \\
\hline $5 / 2^{-}$ & $N(1675)$ & $\Lambda(1830)$ & $\boldsymbol{\Sigma}(1775)$ & & & & $1.18 \pm .04$ \\
\hline \multirow[t]{2}{*}{$5 / 2^{+}$} & $N(1680)$ & $\Lambda(1820)$ & $\Sigma(1915)$ & {$[\Xi(2069)]$} & $\Lambda(2110)$ & $22 \pm 5$ & $0.70 \pm .03$ \\
\hline & & \multicolumn{2}{|c|}{ Decuplet members ${ }^{d}$} & & $8_{10}$ & & \\
\hline $3 / 2^{+}$ & $\Delta(1232)$ & $\mathbf{2}(1385)$ & $\Xi(1530)$ & $\Omega^{-}$ & $1.0-1.5$ & $x^{2} / D F=$ & \\
\hline $7 / 2^{+}$ & $\Delta(1950)$ & $\mathbf{X}(2030)$ & & & & & \\
\hline
\end{tabular}
rates of each mutiplet.

a Masses in parentheses are the nominal masses used in the Baryon Table. The $\Xi$ members have masses as calculated by using Eqs. (1) and (2) with the mixing ang'c $\theta$ derived from the decay widths.

b See text for a discussion of the $1 / 2^{-}$mixing angle.

c The first values of and $\alpha$ are obtained by using a plus sign for the amplitudes of both $N(1535) \rightarrow N_{\eta}$ and $A(1670) \rightarrow A \eta$. The second values use a minus sign for the second amplitude. Both fits, however, have $a$ bed $\chi^{2}$, mostly due to the two baryon-p amplitudes.

d Coupting constants updated from Ref. 14, using new $\Xi(1530)$ data. 
vector current remains an octet in the presence of symmetry breaking) and it was advocated by Graham. ${ }^{21}$ For the $1 / 2^{-}$nonet it was used in this form first by Gell-Mann. ${ }^{22}$ The couplings for the $\Lambda(1405)$ to the $N \bar{K}$ and $\Sigma \pi$ channels are the same as those used in Ref. 14.

$3 / 2^{+}$Decuplet

The agreement among the coupling constants obtained for the four rates in this decuplet is very bad. The fit made using form (a) for $B_{\ell}$ has $x^{2}=58$ for 3 degrees of freedom; the one made with form (b) for $B_{\ell}$ has $\chi^{2} / D F=13 / 3$. The broken $\mathrm{SU}(3)$ relation (11), however, is very well satisfied.

\section{B. SU(3) CLASSIFICATION OF MESON RESONANCES}

All of the discussion above applies, except that for bosons the GMO formula is usually applied to the square of the masses, as opposed to the first power for fermions. Thus for example, Eq. (2) becomes

$$
4 \hat{K}=3 \hat{n}+\hat{x} .
$$

The symbol $\bar{K}$ was introduced by Glashow and Socolow for the square of the $\mathrm{K}$ mass, etc.

Because of the difference between Eqs. $\left(5^{\prime}\right)$ and $\left(5^{\prime \prime}\right)$, there is also an extra factor of $\left(M_{N} / M_{R}\right)$ in Eqs. (6) and (7). The three established nonets $\left(0^{-}, 1^{-}, 2^{+}\right)$and their mixing angles are listed at the bottom of the Meson Table.

\section{FLAVOR SYMMEIRY BREAKING IN A UNITARY MIXING SCHEME}

\section{Roos}

University of Helsinki

Because of unitarity. the GMO formula is an approximation. which breaks Jown especially for broad resonances. The imaginary part of the mass matrix $\mathrm{M}^{2}$ is related to the widths, and for mesons is of the form ${ }^{23-25}$

$$
\operatorname{Im} M_{i j}{ }^{2}(s)=\operatorname{Im} \Pi_{i j}(s)=\sum_{a b} B_{i a b} g_{j a b} k_{a}^{2 \ell+\cdot 1} s^{-1 / 2} F\left(k_{i}\right)
$$

where $i$ and $j$ are resonance indices, $I$ the hadronic self-energy diagram, $g_{\text {iab }}$ the coupling constant for the channel $\mathrm{i} \rightarrow \mathrm{ab}$, and $\mathrm{F}$ a hadronic form factor. Only the real part of $\mathbf{M}^{2}$ approximately satisfies the GMO mass formula. More generally, one can assume Re $\mathrm{M}^{2}$ to be given by quark masses in a bare-mass term plus a hadronic mass shift $\operatorname{Re} \Pi_{i j}(s)$ satisfying analyticity constraints. In general, $\Pi_{i j}(s)$ is nondiagonal and breaks flavor symmetry through the differeni threshold positions. It is diagonalized by a complex orthogonal matrix which determines the generally complex mixing angles. The imaginary part of a mixing angle is related to the overlap between the resonance. ${ }^{23}$
The masses and widths are given respectively by the real and imaginary parts of the eigenvalues of $\mathbf{M}^{2}$.

In this way one can, using both masses and widths as data, within the same framework, determine ${ }^{26}$ both the devialion $\delta$ from ideal mixing (i.e., $\theta=35.3^{\circ}+\delta$ ) and the $Q_{A}-Q_{B}$ mixing angle $\phi$ listed at the end of the Meson Table.

\section{References}

1. R. H. Dalitz, in Fundamentals of Quark Models (Proceedings of the $17^{\text {th }}$ Sectish Universities Summer School in Physies, St. Andrews, August 1976), edited by 1.M. Barbour and A.T. Davies, p. 151.

2. M. Gell-Mann, Phys. Rev. 125, 1067 (1962); S. Okubo. Prog. Theor. Phys. (Kyoto) 27, 949 (1962).

3. J. J. Sakurai, Phys. Rev. Letters 9, 472 (1962).

4. The formula has been calculated from analogy with the formula for mixing of meson states, first put in this form by S.L Glashow and R.H. Socolow, Phys. Rev. Letters 15, 329 (1966).

5. See. R.P. Feynman, Theory of Fundamental Processes, W.A. Benjamin, Inc., New York. 1962.

6. C. Becchi, E. Eberle, and G. Morpurgo, Phys Rev. 136B, 308 (1964).

7. V. Gupta and V. Singh, Phys. Rev. 135B. 1442 (1964).

8. M. Konuma and : Tomozawa. Phys. Lett. 10. 347 (1964).

9. R. Levi-Setti, in Proceedings of the Lund International Conference on Elementary Particles, Lund. 1969.

10. R.D. Tripp, in Proceedings of the 14th Imternational Conference on High Energy Physics, Vienna, 1968, p. 173.

11. R.D. Tripp, in Proceedings of the 3rd Hawaiian Topical Conference on Particle Physics; UCRL-1936l (1969).

12. N.P. Samios, in Proceedings of the ISth International Conference on High Energy Physics. Kiev. 1970. p. 187.

13. D.E. Plane et al., Nuclear Physics B22, 93 (1970). Also J. Meyer and D.E. Piane, Nucl. Phys. B25, 428 (1971).

14. A. Barbaro-Galtieri, LBL-1366 and in Proceedings of the $16 \mathrm{th}$ International Conference on High Energy Physics. National Accelerator Laboratory, Vol. 1. p. 159 (1972).

15. N.P. Samios, M. Goldberg, and B.T. Meadows in Hadrons and SU(3): A Critical Review, BNL Report BNL-1785I (1973).

16. P.J. Litchfield, R.J. Cashmore, and A.J.G. Hey in Proceedings of the Topical Conference on Baryon Resonances. Oxford 1976, edited by R.T. Ross and D.H. Saxon, pg. 477.

17. M. Jones, R.H. Dalitz, and R.R. Horgan, Nucl. Phys. B129, 45 (1977).

18. R. Koniuk and N. Isgur, "Baryon Decays in a Quark Model with Chromodynamics", University of Toronto preprint. November 1979.

19. J.M. Blatt and V.F. Weisskopf, Theoretical Nuclear Physics, Wiley, New York, 1952.

20. A. Barbaro-Galtieri, in Properties of Fundamental Interactions, Erice, July 8-26, 197I, edited by A Zichichi. Editrice Compositori, p. 533 (1973).

21. R. Graham, S. Pakvasa, and K. Raman, Phys. Rev, 163, 1774 (1967).

22. M. Gell-Mann, R. Oakes, and B. Renner, Phys. Rev. 175, 2195 (1968).

23. Y. Dothan and D. Horn, Phys. Rev. D1, 916 (1970).

24. C. Rebby and R. Slansky. Phys. Rev. 185, 1838 (1969).

25. N. Tornquist, Asn. Phys. (N.Y.) 123, 1 (1979).

26. N. Tornquist, RITP-HU-TFT-81-37 and RI-HU-TFT-82-1. 


\section{Appendix III}

\section{GROWTH OF INFORMATION}

From time to time we have presented figures demonstrating the amount of experimental work which has gone into spectroscopy, and the amoun of new information available as a result. The 1982 versions of these figures are shown as Figs. 1 and 2

Figure 1 is a simple count of the number of meson resonances listed in the Tables, categorized as those "understood" -- i.e., all quantum numbers are believed known -- and those simply "listed". A rapid recent increase in both of these categories occurred because of the discovery of the $\mathrm{J} / \psi$ and related particles.

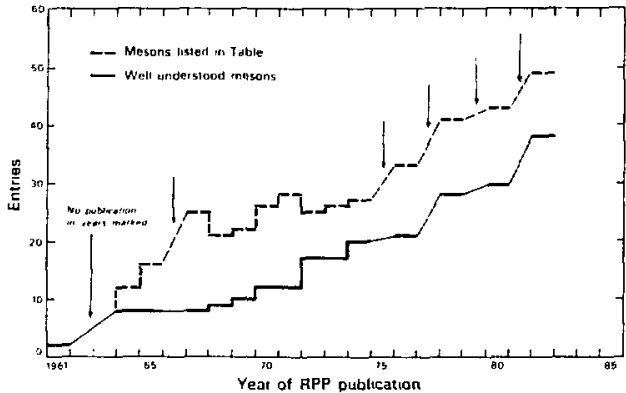

Fig. 1. Number of meson resonances listed in the Tables (dashat line) and those for which all quantum numbers are known (solid line), as a function of year of publication of the Review of Particle Properties.

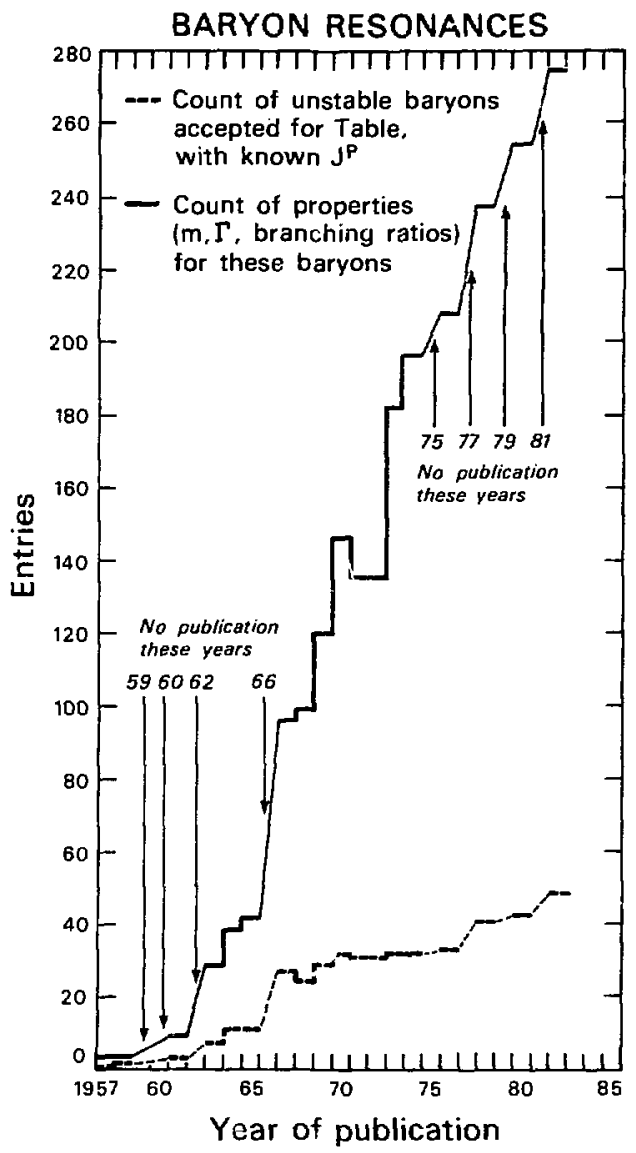

Fig. 2. Total amount of information (mass + width + branch ing ratios) on baryon resonances listed in the Tables, restricted to those with well-established $J^{P}$ (solid line). Dashed line shows numbers of such resonances listed. Abscissa shows year of publication of Review of Particle Properties.

A history of this sort has more than whimsical value. We may use it as a guide to develop a "feel" for the reliability of current values. In Fig. 3 we show how the generally accepted values for the speed of light and a couple of other mustants have changed with time. The "generally accepted value" is usually an ivirage over several experiments, perforned by a compiler (in Fig. 3, the compiler is other deep into his graduate training in 1965. 
than the Particle Data Group in all cases, although we do quote the compiled results). The abscissa on all these figures is the date of publication of the vajue shown. Clearly there is a general progression toward better understanding -- at least as measured by the size of the error bars. However, the sizz of the error bers does not tell the ful] story, as we can see by the frequency with which the "best" value has changed by more than one standard deviation. Changes in these values an come from several sources; a new experimental measurement, roevaluation of an old measusement (which can come about if a prevjously unrecognized source of bias is discovered and cortected, or if a new value for one of the input constants, e.g. the slectric charge is available), or a change in the averaging procedure.

In Fig. 4 we show the history of some masses (including the $A$ for radioactive $\boldsymbol{\Lambda}$ dating of your colleagues), based on averages which we ourseives performed. These are adapted from those originally presented by Rosenfeld ${ }^{t}$ in 1975 . Ti. publication dale refors to the publication of the Review of Particle Properties.

In Fig. 5 we show the best estimates for the lifetimes of some of the particles stable against strong decay. These and subsequent figures have been compiled since publication of the Rosenfeld article. 'In Fig. 6 we show the widths of some of the resonances, and in Fig. 7, the values of some of the branching fractions. All values are taken from the Tables. Before 1964, very few branching fractions were listed in the Tables. In all cases, a representative sample is chosen, usually from those with a iot of activily (a limited number of special requests for a more complete set of such figures may be honored, for those seriously interested in the bistory of the "best" values of physical constants). In each figu:e, the heavy inner error bar represents the statist. ical error computed in the averaging procedure, and the thin outer error bars. when presen1, indicate the increase in the error due to the "scale factor". The scale factor is described in the introductory text, Sec. VII. It represents an attempt to quantify the increase in the uncertainty which is present in the case of experiments which disagree by more than a certain amount. In the case where the error represents an "educated guess," rather than a calculation, the inner error bar is absent.

On the whole, the number of times the values have changed by more than one siandard deviation over the years is remarkably few.
Even those branching fractions which involve rare decays and which are therefore presumably difficult to messuse (Fig- 7) are for the most part, within one or two standard deviations in 1978 of their value in any year since 1960 . This is in spite of the vast amount of new experimental input, and indicates the general reliability of the realts.

Or course, the dath points for a given quantity are hardly independent of each other, but thace differing by several years frequently have quite different experimental input. The relative lack of change is a comment both on the experiments and on the everaging procedures. We, of course, are responsible only for the averages (except Fis. 3). These averages entail considerable exercise of judgment: there are conficting experiments, experiments with impossibly small errors, "pretiminary" results, and so forth. Statistical procedures will tell us that two experiments do not agree; they do not give a clue as to which (if either) is a good representation of the truth. Major decisions, and th-:- mmivations, are usually discussed on a case-by-case basis in the Data Card Listings; general comments may be found in Sec. Il of the text and in Rosenfeldl. Note that, ocasionally, the error bars increase from one publication to the next. This is usually the result of decision making by the compiler, e.g., to cease using a particular result, or because of nuw results in poor agreenent with the old results.

We show these figures not only to demonstrate that there is not much change in these averages in the usual case, but also to show that there exist cases with relatively large changes. There is a psychological danger in preparing tables of "right" answers. The old joke about the experimenter who fights the systematics until he or she gets the "right" answer (read "agres with previous experiments"), and then publishes, contains a germ of truth (presumably, those who compile and average experimental results are also not immune to this disease). A result can disagree with the average of all previous experiments by five standard deviations, and still be right. Hence, perhape it is of value to show that large changes can (and do) sometimes occur.

\section{Reference}

I. A. H. Rosenfeld, Asn. Rer. Nisl. Sci. 25, 555 (1975). 

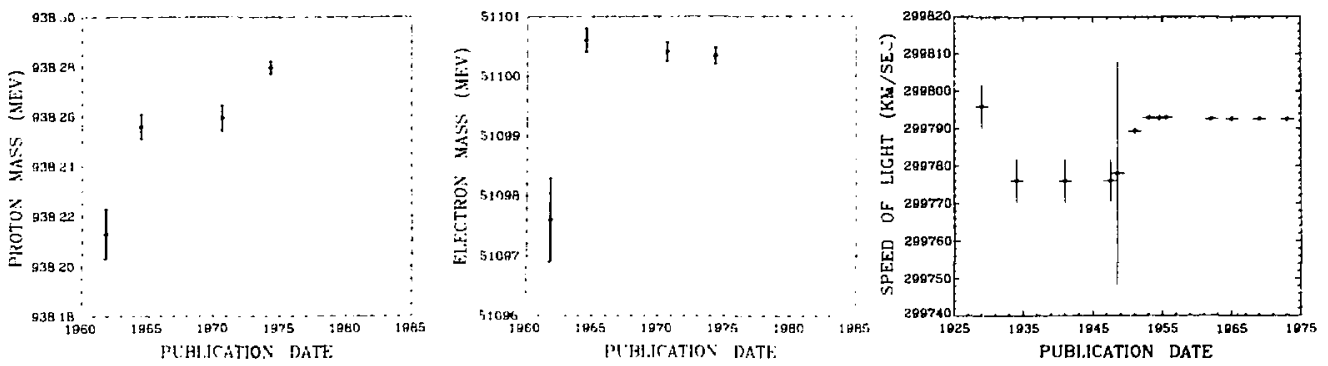

Fig. 3. The "generally accepted values" of the proton mass, the electron mass, and the speed of light, as a function of the publication date of the compilation used (not done by the Particle Data Group). Data for the speed of light plot courtesy of E. R. Cohen, Rociwell International Science Center. See the Stabie Particle Data Card Listings fo: references on proton and electron masses.
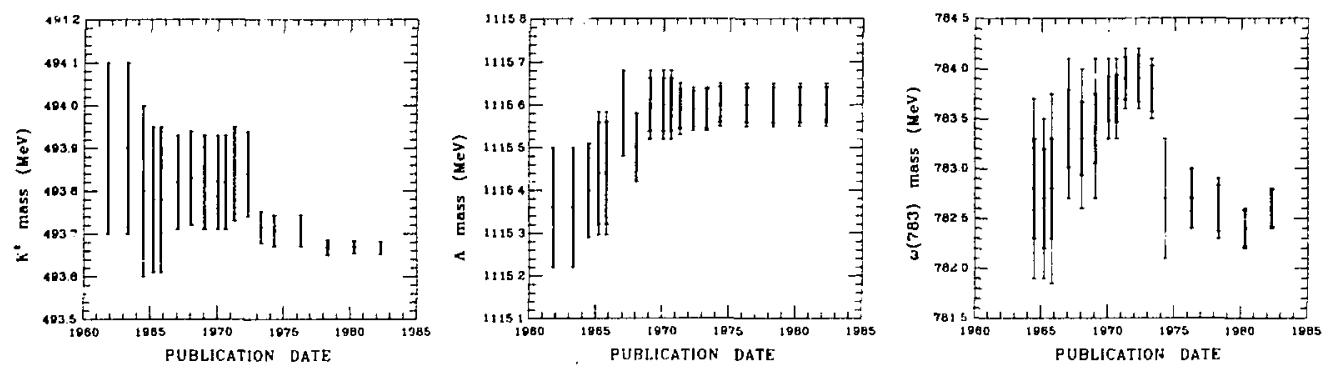

Fig. 4. Particle Data Group averages of the masses of various particles, as a function of date of publication of Review of Particle Properties (Adapted, with permission, from Annual Review of Nuclear Science, Volume 25. Copyright 1975 by Annual Reviews, Inc. All rigbis reserved). Full error bar indicates quoted error; thick-lined portion indicates quoted error with "scale factor" removed (see Sec. VIl of introductory text).
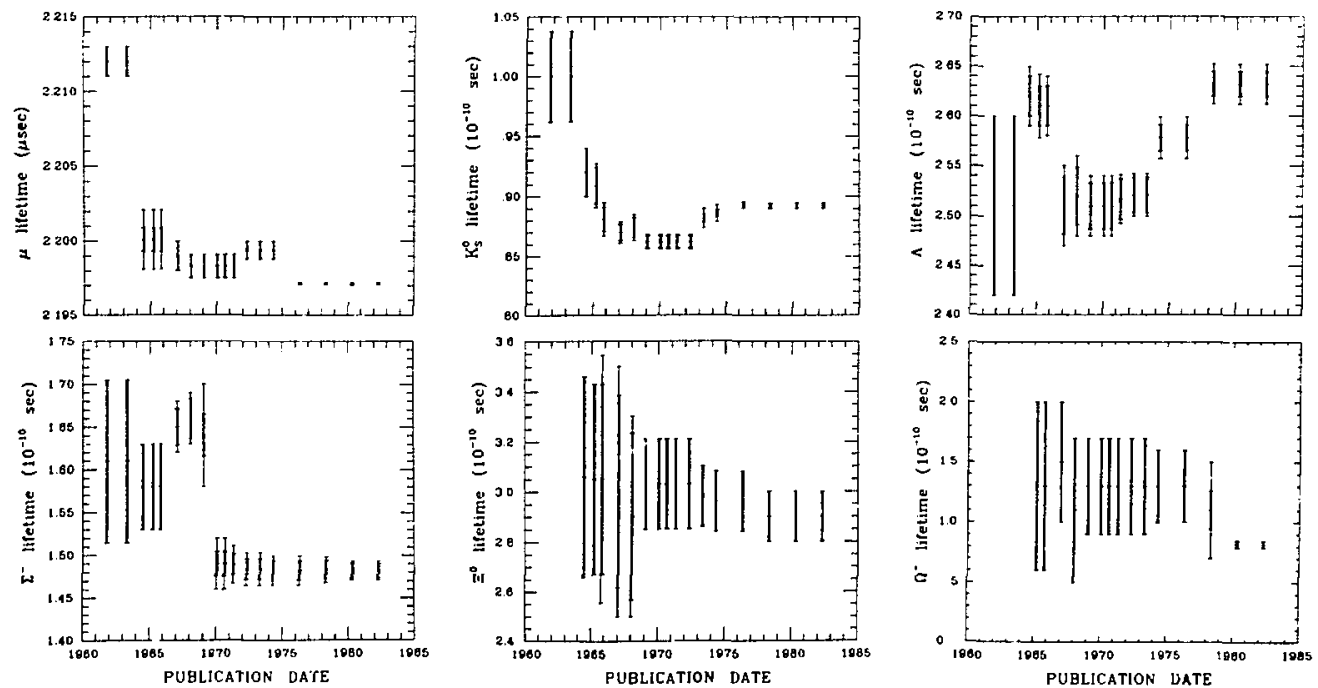

Fig. 5. Particle Data Group averages of the lifetimes of various particles, as a function of publication date of RPP. 

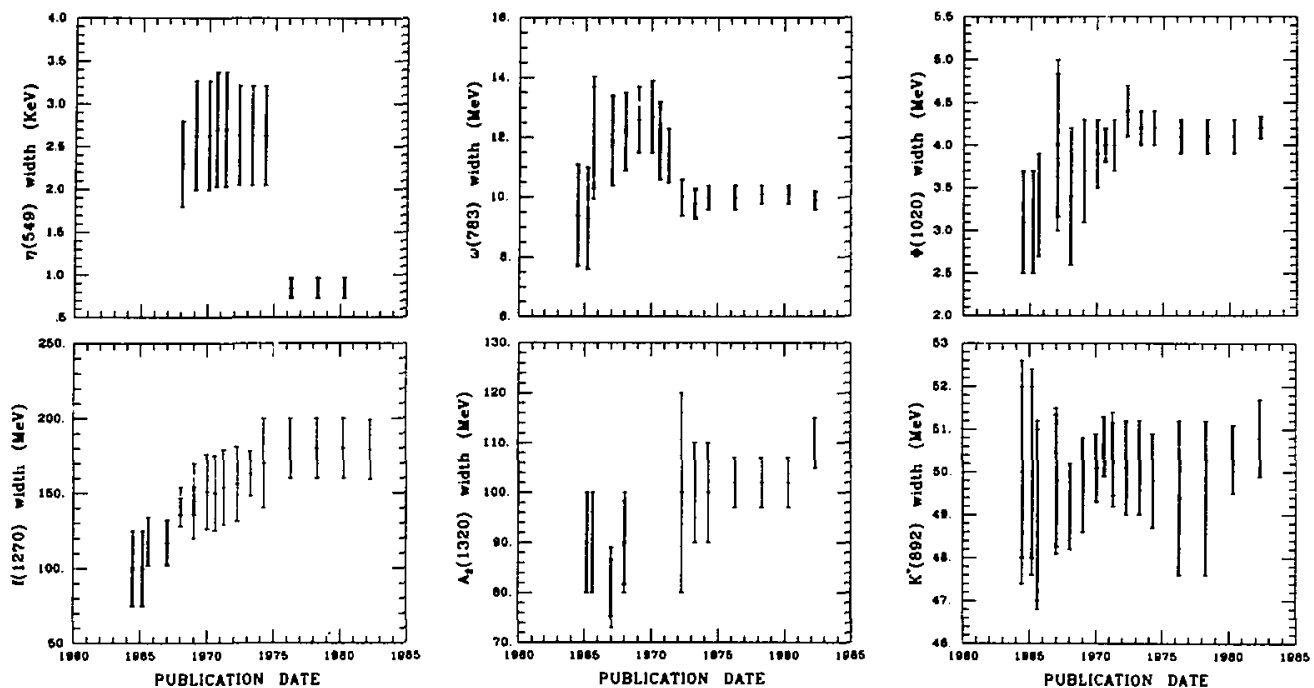

Fig. 6. Particle Data Group averages of the widths of various resonances, as a function of date of publication of RPP. The gap in the $A_{2}$ data indicates the years when the $\lambda_{2}$ was thought to be split.
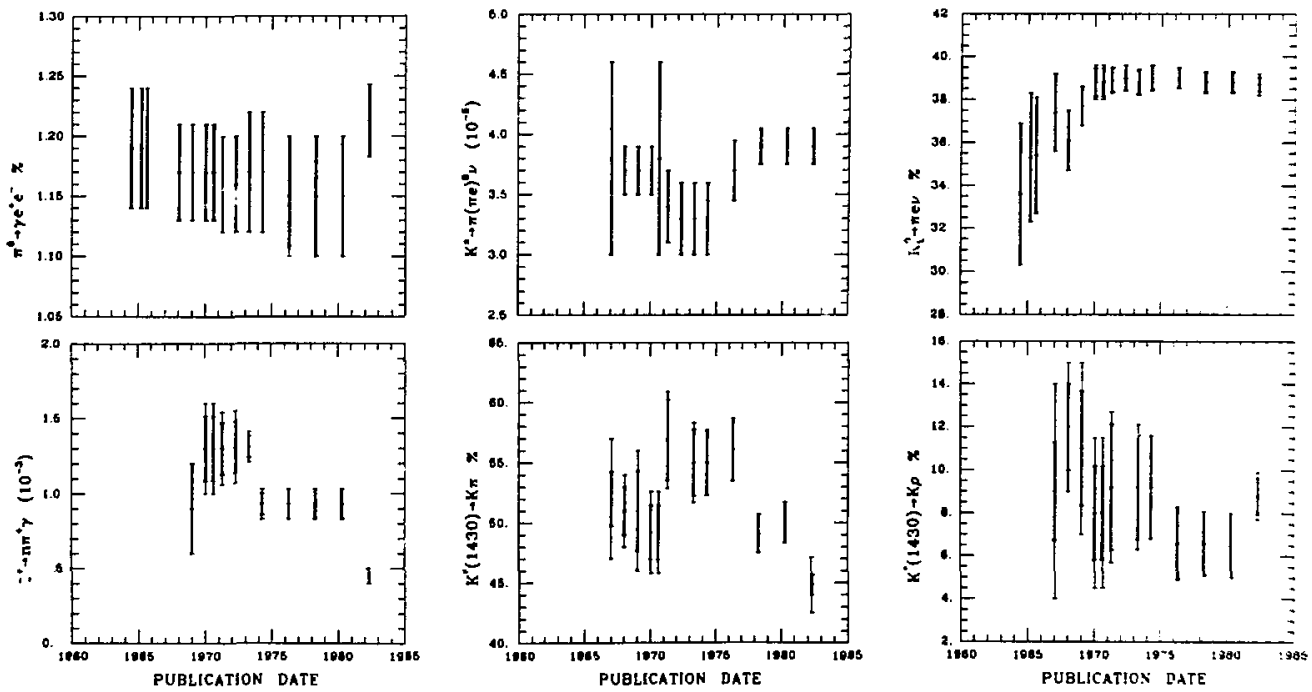

Fig. 7. Particle Data Group averages of various branching fractions, as a function of date of publication of RPP. 